\title{
IntechOpen
}

\section{Recent Advances in Autism Spectrum Disorders Volume I}

Edited by Michael Fitzgerald 



\section{RECENT ADVANCES IN AUTISM SPECTRUM DISORDERS - VOLUME I}

Edited by Michael Fitzgerald 
Recent Advances in Autism Spectrum Disorders - Volume I

http://dx.doi.org/10.5772/46001

Edited by Michael Fitzgerald

\section{Contributors}

Stephen Bustin, Michael Gill, Richard Anney, Elizabeth Sajdel-Sulkowska, Kieran D. O'Malley, Tomislav Benjak, Gorka Vuletic, Esra Guney, Elvan Iseri, Liliana Maria Passerino, Maria Rosangela Bez, Gitta De Vaan, Mathijs Vervloed, Harry Knoors, Ludo Verhoeven, Agnes Cristina Fett-Conte, Olive Healy, Roy McConkey, Sayyed Ali Samadi, Rudimar Riesgo, Michele Becker, Carmem Gottfried, Jennifer Elder, Abdullah Alqallaf, Fuad M. Alkoot, Mashael Aldabbous, Erika G Gisel, Genevieve Nadon, Fernanda Dreux M. Fernandes, Daniela Molini-Avejonas, Juliana Balestro, Cibelle Amato, Danielle Defense-Netvral, Miguel Ángel Romero-Munguía, Maria Urbano, Kathrin Hartmann, Stephen I. Deutsch, Gina Marie Bondi Polychronopoulos, Vanessa Dorbin, Michael Siller, Lindee Morgan, Meghan Swanson, Emily Hotez, Rubina Lal, Rakhee Chhabria, Ponzio, John Connolly, Victorio Bambini-Junior, Diego Baronio, Geancarlo Zanatta, Roberta Silvestrin, Tamara Vaccaro, Ginny Russell, Zsuzsa Pavelka, Efrosini Kalyva, Catherine DeSoto, Robert T. Hitlan, Eric Larsen, Gunilla Thunberg, James R. Lupski, Melanie Lacaria, Jenny Fairthorne, Amanda Langridge, Helen Leonard, Viktoria Lyons, Michael Fitzgerald

\section{(c) The Editor(s) and the Author(s) 2013}

The moral rights of the and the author(s) have been asserted.

All rights to the book as a whole are reserved by INTECH. The book as a whole (compilation) cannot be reproduced, distributed or used for commercial or non-commercial purposes without INTECH's written permission.

Enquiries concerning the use of the book should be directed to INTECH rights and permissions department (permissions@intechopen.com).

Violations are liable to prosecution under the governing Copyright Law.

\section{(cc) BY}

Individual chapters of this publication are distributed under the terms of the Creative Commons Attribution 3.0 Unported License which permits commercial use, distribution and reproduction of the individual chapters, provided the original author(s) and source publication are appropriately acknowledged. If so indicated, certain images may not be included under the Creative Commons license. In such cases users will need to obtain permission from the license holder to reproduce the material. More details and guidelines concerning content reuse and adaptation can be foundat http://www.intechopen.com/copyright-policy.html.

\section{Notice}

Statements and opinions expressed in the chapters are these of the individual contributors and not necessarily those of the editors or publisher. No responsibility is accepted for the accuracy of information contained in the published chapters. The publisher assumes no responsibility for any damage or injury to persons or property arising out of the use of any materials, instructions, methods or ideas contained in the book.

First published in Croatia, 2013 by INTECH d.o.o.

eBook (PDF) Published by IN TECH d.o.o.

Place and year of publication of eBook (PDF): Rijeka, 2019.

IntechOpen is the global imprint of IN TECH d.o.o.

Printed in Croatia

Legal deposit, Croatia: National and University Library in Zagreb

Additional hard and PDF copies can be obtained from orders@intechopen.com

Recent Advances in Autism Spectrum Disorders - Volume I

Edited by Michael Fitzgerald

p. cm.

ISBN 978-953-51-1021-7

eBook (PDF) ISBN 978-953-51-7107-2 


\section{We are IntechOpen, \\ the world's leading publisher of Open Access books}

Built by scientists, for scientists

\section{$4,100+$}

Open access books available

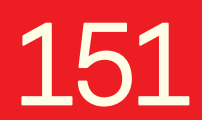

Countries delivered to
$116,000+$

International authors and editors
$120 \mathrm{M}+$

Downloads

Our authors are among the

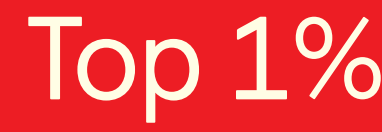

most cited scientists

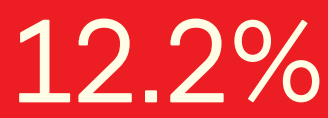

Contributors from top 500 universities

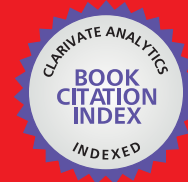

WEB OF SCIENCE ${ }^{\mathrm{TM}}$

Selection of our books indexed in the Book Citation Index in Web of Science ${ }^{\mathrm{TM}}$ Core Collection (BKCI)

Interested in publishing with us?

Contact book.department@intechopen.com

Numbers displayed above are based on latest data collected.

For more information visit www.intechopen.com

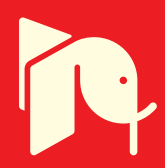





\section{Meet the editor}

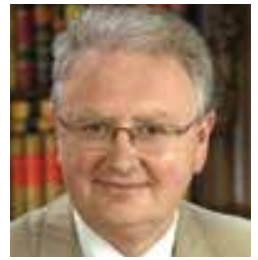

Michael Fitzgerald was the first Professor of Child Psychiatry in Ireland at Trinity College Dublin. He trained at Mount Sinai Hospital, Chicago and Maudsley Hospital, London. He continues to practice in Child and Adult Psychiatry and carries out research on autism and it's co-morbidities. He has diagnosed over 2,500 persons with ASD in the past 40 years. He is on the editorial board of a number of journals and has published extensively in peer-reviewed journals as well as written, edited or co-edited 20 books. 



\section{Contents}

Preface XIII

Section 1 Prevalence and Diagnosis 1

Chapter 1 Promoting Early Identification of Autism in the Primary Care Setting: Bridging the Gap Between What We Know and What We Do 3

Michael Siller, Lindee Morgan, Meghan Swanson and Emily Hotez

Chapter 2 Indicators of Autism in Iranian Children 29

Sayyed Ali Samadi and Roy McConkey

Chapter 3 Prevalence of Pervasive Developmental Disorders - Croatia in Comparison with Other Countries of the World 49

Tomislav Benjak and Gorka Vuletić

Section 2 Aetiological Factors-General Overview 59

Chapter 4 Gut Microbiome and Brain-Gut Axis in Autism - Aberrant Development of Gut-Brain Communication and Reward Circuitry 61

Elizabeth M. Sajdel-Sulkowska and Romuald Zabielski

Chapter 5 Why There Is no Link Between Measles Virus and Autism 81 Stephen A. Bustin

Chapter 6 Vaccine Safety Study as an Interesting Case of "Over-Matching" 99

M. Catherine DeSoto and Robert T. Hitlan 
Chapter 7 Pro-Inflammatory Phenotype Induced by Maternal Immune Stimulation During Pregnancy 113

Nicholas M. Ponzio, Mili Mandal, Stella Elkabes, Pan Zhang, Junichi Sadoshima, Sayantani Basak, Peiyong Zhai and Robert Donnelly

Chapter 8 Valproic Acid in Autism Spectrum Disorder: From an Environmental Risk Factor to a Reliable Animal Model 143 Carmem Gottfried, Victorio Bambini-Junior, Diego Baronio, Geancarlo Zanatta, Roberta Bristot Silvestrin, Tamara Vaccaro and Rudimar Riesgo

Chapter 9 Mnesic Imbalance or Hyperthymestic Syndrome as Cause of Autism Symptoms in Shereshevskii 165

Miguel Ángel Romero-Munguía

\section{Section 3 Aetiological Factors - Genetics 189}

Chapter 10 Genetic Evaluation of Individuals with Autism Spectrum Disorders 191

Eric C. Larsen, Catherine Croft Swanwick and Sharmila BanerjeeBasu

Chapter 11 Genetic Etiology of Autism 215

Agnes Cristina Fett-Conte, Ana Luiza Bossolani-Martins and Patrícia Pereira-Nascimento

Chapter 12 Advances in Autism Research - The Genomic Basis of ASD 249

Melanie Lacaria and James R. Lupski

Chapter 13 Autism Spectrum Disorders: Insights from Genomics 275 John J. Connolly and Hakon Hakonarson

Chapter 14 The Genetic Architecture of Autism and Related Conditions 299

Michael Gill, Graham Kenny and Richard Anney

Chapter 15 Genetic and Environmental Factors in Autism 321 Esra Guney and Elvan Iseri

Chapter 16 Discovering the Genetics of Autism 341

Abdullah K. Alqallaf, Fuad M. Alkoot and Mash'el S. Aldabbous 
Section 4 Aetiological Factors - Co-Morbidity 359

Chapter 17 Co-Occurrence of Developmental Disorders: Children Who Share Symptoms of Autism, Dyslexia and Attention Deficit Hyperactivity Disorder 361

Ginny Russell and Zsuzsa Pavelka

Chapter 18 Pre-Existing Differences in Mothers of Children with Autism Spectrum Disorder and/or Intellectual Disability:

A Review 387

Jenny Fairthorne, Amanda Langridge, Jenny Bourke and Helen Leonard

Section 5 Aetiological Factors - Sensory Issues, Foetal Alcohol Syndrome and Relationships 425

Chapter 19 Relationships, Sexuality, and Intimacy in Autism Spectrum Disorders 427

Maria R. Urbano, Kathrin Hartmann, Stephen I. Deutsch, Gina M.

Bondi Polychronopoulos and Vanessa Dorbin

Chapter 20 Clinical Implications of a Link Between Fetal Alcohol Spectrum Disorders (FASD) and Autism or Asperger's Disorder - A Neurodevelopmental Frame for Helping Understanding and Management $\mathbf{4 5 1}$

Kieran D. O'Malley and Susan D. Rich

Chapter 21 Autism Spectrum Disorders in People with Sensory and Intellectual Disabilities Symptom Overlap and Differentiating Characteristics 479

Gitta De Vaan, Mathijs P.J. Vervloed, Harry Knoors and Ludo Verhoeven

Section 6 Aetiological Factors - Parents and Families 501

Chapter 22 Empowering Families in the Treatment of Autism 503 Jennifer Elder

Chapter 23 Collaboration Between Parents of Children with Autism Spectrum Disorders and Mental Health Professionals 521 Efrosini Kalyva 
Section 7 Aetiological Factors - Intervention in Person with Autism 565

Chapter 24 Early Intensive Behavioural Intervention in Autism Spectrum Disorders 567

Olive Healy and Sinéad Lydon

Chapter 25 Feeding Issues Associated with the Autism Spectrum Disorders 599

Geneviève Nadon, Debbie Feldman and Erika Gisel

Chapter 26 Clinical Approach in Autism: Management and Treatment 633

Rudimar Riesgo, Carmem Gottfried and Michele Becker

Chapter 27 Building an Alternative Communication System for Literacy of Children with Autism (SCALA) with Context-Centered Design of Usage 657

Liliana Maria Passerino and Maria Rosangela Bez

Chapter 28 Addressing Communication Difficulties of Parents of Children of the Autism Spectrum 683

Fernanda Dreux Miranda Fernandes, Cibelle Albuquerque de La Higuera Amato, Danielle Azarias Defense-Netvral, Juliana Izidro Balestro and Daniela Regina Molini-Avejonas

Chapter 29 Early Intervention of Autism: A Case for Floor Time Approach 691

Rubina Lal and Rakhee Chhabria

Chapter 30 Early Communication Intervention for Children with Autism Spectrum Disorders $\mathbf{7 1 9}$

Gunilla Thunberg

Section 8 Aetiological Factors - The Autistic Self and Creativity 747

Chapter 31 Atypical Sense of Self in Autism Spectrum Disorders: A NeuroCognitive Perspective $\mathbf{7 4 9}$

Viktoria Lyons and Michael Fitzgerald

Chapter 32 Critical Evaluation of the Concept of Autistic Creativity 771 Viktoria Lyons and Michael Fitzgerald 


\section{Preface}

The pace of research on Autism Spectrum Disorders (ASD) can only be described as extraordinary as this volume shows. It is extremely difficult for any single professional to keep abreast of all the developments in this area. This volume gathers together leading researchers and expert clinicians from many different parts of the world to produce this "up-to-theminute" volume. It gives an in depth view of many areas of research which may be unfamiliar to the clinician and indeed researcher focused on their own area of interest. The volume gives an in depth overview of the field of Autism Spectrum Disorders.

The best outcome for Autism Spectrum Disorders occurs where there is an early diagnosis at around two years. Unfortunately this is only achieved in a minority of persons with ASD as described in the first section on diagnosis and prevalence. Siller et al describes the strategy for improving the situation. Information from non-English speaking countries is critical to a global understanding of Autism Spectrum Disorders. Samadi and McConkey provide this for Iran. Benjak does this for Croatia.

The brain/gut axis has a long and controversial history in autism. Sajdel-Sulkowska et al produced some new findings in relation to the "leaky gut" during development, abnormalities in the gut microbiome and effects on the brain. This area of research remains an exciting proposition. The non-relationship between vaccines and autism is covered in two important chapters by Bustin and De Soto. The focus on immune dysregulation in ASD by Ponzio et al is an area of increasing importance and a particularly useful method of research is the mouse model. Immune problems have been observed at a clinical level for a long time in ASDs.

Another important chapter using an animal model is by Gottfried et al who focused on Valproac acid in pregnancy and associated autistic features in offspring. Romero-Munguia describes a very interesting mnesic imbalance or hyperthymestic syndrome as a cause of autism symptoms in Shereshevskii, a man whose memory was studied by Luria. The understanding of the aetiology of Autism Spectrum Disorders clearly has to pay large attention to genetic factors. This very rapidly expanding field of study is dealt with in great depth in a number of chapters by some of the foremost researchers on this topic in the world. Larsen et al discusses the genetic evaluation of individuals with ASD including genetic screening tests. Fett-Conte focuses on candidate genes, copy number variations, epigenetics and exome sequencing. Lupski and Lacario focus on mouse models in a number of conditions including autism.

Connolly et al notes the relative scarcity of causal common variants and the growing list of casual rare variants. Gill et al's chapter on the genetic architecture of autism and related disorders gives an excellent overview of genetic linkage and association. They also discuss mutation rates and models of risk in autism as well as next generation sequencing studies. 
Guney et al focus on genetic and environmental factors. They make reference to the intrauterine environment and pre-natal stress.Alqallafet al study the genetic origins of autism.

There is a wide spectrum of interventions for ASD. The earlier the intervention, the better the outcome. Healy et al describes early intensive behavioural intervention (EIBI) which reduces the cost of lifelong care by as much as two thirds. It is an intervention that draws from Applied Behaviour Analysis. They also noted a study where an 'eclectic' treatment group showed significantly greater increase in adaptive functions than the EIBI. I have observed in my clinical practice that where behavioural interventions were applied too intensively that a number of children developed post traumatic stress disorders and of course there are also the problems with generalisation of gains made from behavioural interventions. Lal et al describes the floor time approach that focuses on unique challenges and strengths of children with ASD. The child is the director or leader of activities. It has shown positive results and of course behavioural interventions narrowly applied do not suit all children and families.

Thunberg reviews early communication intervention for children with ASD's and uses evidence/grading which is very helpful. Interventions need to be continuous and include follow ups with the possibility for booster interventions. Nadon et al examines feeding issues with persons with ASDs-a major problem. Graduated exposure food therapy and food fading are described. Passerino et al focuses on building on alternative communication system using context-centred design. It focuses on the re-organisation of the persons with ASD's relations with the world. Developing assistive technology for alternative communication is a very important development. Riesgo et al has an important chapter on the management and treatment of ASD. They provide detailed information on psychopharmacological interventions as well as non- medical approaches and in addition provide a commentary on their effectiveness. Urbano et al focus on the understudied area of sexuality and ASD. They provide an overview and describe treatment in this very sensitive area. I have observed criminal charges being brought against a number of persons with ASD because of errors of judgment in the sexuality area.

A person with Autism Spectrum Disorder places great challenges on the family. The chapter on empowering families in the treatment of autism by Elder is very pertinent. Families are the primary providers of services on the advice of professionals and support groups. This collaboration is explored by Kalyva who provides a parent/professional protocol which is very valuable in improving this relationship. The parental issue is further elaborated in the chapter on addressing communication difficulties in parents of children with Autism Spectrum Disorders by Fernanda Dreux Miranda Fernandes et al.

Another overview chapter by O'Malley on Foetal Alcohol Syndrome Disorders and ASD is particularly important. This is a big global problem in terms of alcohol use during pregnancy. This topic will become of increasing importance in the years to come. De Vaan et al focuses on another somewhat neglected area that is ASD in people with sensory issues. In my experience persons with sensory issues should always be assessed for ASD.

Russell discusses the topic of co-occurrence of developmental disorders. This is extremely common and we now realise that there is much overlap between developmental disorders (Fitzgerald 2012). The categorical approach to diagnosis has now been shown to be limited and the dimensional approach is clearly a way forward. There is also an overlap in some genetic findings between disorders. Fairthorne et al's chapter on ASD and intellectual disability focuses on the pre-existing differences in their mothers. Immigrant mothers were more 
likely to have a child with ASD. Smoking during pregnancy has been associated with ASD and intellectual disability.

There are problems in using the Autism Diagnostic Interview Revised (Lord et al 1994) in clinical practice. It ,not uncommonly, misses high functioning autism and according to Ventola et al (2006) is "under diagnosing toddlers". It is an extremely expensive instrument to train on which makes it almost impossible for under-developed countries because of the major cost of training on the instrument. Professor Dorothy Bishop (Feinstein 2010) points out in relation to the length of this interview that there is no evidence for such a long process "it is more an article of faith to them". I have seen quite a few families with a person with ASD who are negative on the ADI-R come to me in tears because of the missed diagnosis.

An atypical sense of self is central to understanding autism. Lyons et al explores the psychological and neuropathological aspects of self in autism. It is important when discussing autism not to always focus on the negative and on purely the disability (Fitzgerald 2004). Lyons et al examines the positive aspects of ASD in a critical evaluation of the concept of autistic creativity.

Professor Michael Fitzgerald

Consultant Child \& Adult Psychiatrist Trinity College, Dublin

Ireland 

Section 1

Prevalence and Diagnosis 

Chapter 1

\title{
Promoting Early Identification of Autism in the Primary Care Setting: Bridging the Gap Between What We Know and What We Do
}

\author{
Michael Siller, Lindee Morgan, \\ Meghan Swanson and Emily Hotez \\ Additional information is available at the end of the chapter
}

http://dx.doi.org/10.5772/53715

\section{Introduction}

During the last decade, research on Autism Spectrum Disorder (ASD) has made tremendous progress with regard to early identification and diagnosis. These advances were made possible by a growing number of rigorous research studies with large sample sizes that utilized a combination of: (1) retrospective parent report and home video studies [1], (2) prospective studies of infant siblings of children with ASD [2], (3) population-wide studies of ASD screening tools [3], and (4) studies on the early stability of diagnostic classifications [4]. Advances in best practices related to early identification are reflected in a 2006 policy statement published by the American Academy of Pediatrics [5], and a corresponding set of clinical practice guidelines [6]. According to these guidelines, it is recommended that Primary Care Providers (PCPs; e.g., family physicians, pediatricians) administer formal screening tests during every well-child visit scheduled at 18 and 24 months, independent of known risk factors or reported concerns. Moreover, PCPs are urged to promptly refer children for Early Intervention $^{1}$ services as soon as ASD is seriously considered.

Even though the age of first diagnosis has gradually decreased during the last decade [7], population based studies reveal that most children with ASD continue to be diagnosed after three years of age [8]. Given that a reliable diagnosis of ASD is possible by 24 months, and that about $90 \%$ of parents whose children are later diagnosed with ASD express documented concerns before age 2 [9], the gap between best practice guidelines and community imple-

1 Throughout this chapter, we use capital letters when referring to the publicly funded Early Intervention system 
mentation is tangible. This chapter outlines a sequence of four connected activities aimed at improving early identification of ASD and promoting successful referrals for Early Intervention services. For each step in the sequence, this chapter (1) describes the barriers that autism advocates, families, and PCPs face, and (2) showcases novel educational approaches that aim to promote families' access to prompt and appropriate Early Intervention services.

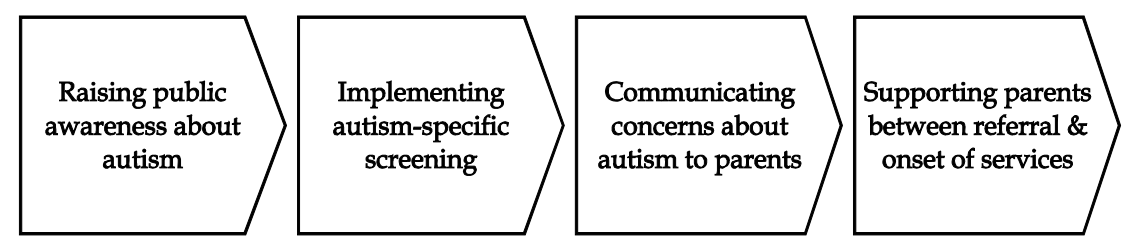

Figure 1. A sequence of four connected activities aimed at improving early identification of ASD and promoting successful referrals for Early Intervention services.

\section{Raising Public Awareness about Autism}

The prevalence of ASD in and of itself has heightened public awareness. What was once considered a rare condition is now reported to affect 1 in 88 children [7]. Recent data from a study that included population-wide screening procedures suggest that the true prevalence of ASD may be even higher [10]. In the case of ASD, awareness is essential because awareness promotes detection, and successful detection efforts result in earlier intervention, maximizing optimal outcomes for this population. Given that there is an average 13-month lag from initial evaluation to diagnosis, and that the average age of initial evaluation is 48 months with diagnosis occurring at 61 months [11], ASD awareness efforts are far from complete. Importantly, limited awareness of the behavioral characteristics of young children with ASD has been noted for both parents and healthcare professionals. For example, data collected in 2004 by Porter Novelli and the Centers for Disease Control and Prevention show that $63 \%$ of parents reported not knowing what behaviors most suggested ASD, and that $57 \%$ did not know the best time to get help for children with ASD [12]. These data also indicated that healthcare professionals need more information on developmental milestones and developmental disabilities in that $30 \%$ recommended that concerned parents should wait to see if their child's development progresses, and only $41 \%$ felt they had the necessary resources to educate parents. Further, awareness campaigns continue to document a considerable gap between best practice and the behaviors of healthcare professionals [13].

\subsection{Barriers to public awareness about autism}

Awareness efforts are critical to improve rates of detection, access to Early Intervention services, and promote optimal outcomes. It is clear that a lack of education and access to accurate information are key barriers to ASD awareness efforts. Before addressing issues related to dissemination of information, it is important to evaluate more emotionally-hinged barriers, 
namely the predominance of negative and stigmatizing stories about ASD in the media and social stigma that may vary across cultures. It is unclear whether stigma and negative stereotypes interfere with people's ability to access accurate information, whether a lack of accurate information promotes stigma and stereotypes, or whether the two are reciprocally linked.

1. Portrayals of ASD in the media

In addition to being a key source of entertainment and news, mass media tends to perpetuate stereotypes and social beliefs in a way that defines and maintains an existing social order [14]. Portrayals of ASD in film (e.g., Rainman, What's Eating Gilbert Grape?), fiction (e.g., The Curious Incident of the Dog in the Night-Time [15]; Daniel Isn't Talking [16]), and non-fiction parent accounts of ASD (e.g., Real Boy: A True Story of Autism, Early Intervention, and Recovery [17]; Let Me Hear Your Voice: A Family's Triumph Over Autism [18]) provide an index of the general increase in ASD awareness. However, there is some disagreement as to whether these representations have a positive or negative impact on awareness efforts $[19,20]$. Claims of 'miracle cures' for ASD that victimize vulnerable families and contribute to unrealistic perceptions of the treatability of ASD are clearly harmful [21]. Jones and Harwood provided a content analysis of 1,228 articles about ASD published from 2002 to 2005 in the Australian media [20]. This analysis revealed some interesting patterns, including a limited amount of factual information in media sources, and descriptions of people with ASD as either dangerous and uncontrollable or unloved and poorly treated. Overall there was a predominance of 'negative' stories about ASD including numerous references to the impact on families, difficulties with diagnosis, and criminal cases. The authors submit that the implication of this type of coverage is likely a reduction in people's willingness to engage with individuals with ASD, creating barriers for social and educational inclusion. Further research is needed to evaluate the impact of media stereotypes of ASD on screening and early identification efforts.

\section{Cultural representations of ASD}

Vast differences in attitudes and approaches to ASD have been reported for culturally and linguistically diverse groups. For example, it has been reported that families in Korea are hesitant to seek help for their children's developmental problems, including ASD, because they are seen as a mark of shame [22]. The effects of stigma have been evaluated for HIV/AIDS, mental illness, ASD, tuberculosis, leprosy, and cancer [23]. Social stigma relating to a condition or disorder can contribute to decreases in willingness to disclose disease status, health-seeking behavior, quality of care received, and social support [24]. Stigma may also be inadvertently perpetuated by healthcare professionals who maintain prejudice and negative stereotypes about ASD and mental health conditions [25,26]. Cultural interpretations of disability can often be found in the context of religion. For example, a qualitative study indicated that Orthodox Jewish Israeli parents view their child with ASD as having a high spiritual status or important religious mission [27]. In contrast, almost half of the Irish families interviewed by Coulthard and Fitzgerald reported that having a child with ASD had prompted them to distance themselves from religion [28]. Cultural perspectives may also influence families' uptake of services. For example, a general lack of trust in service providers has been described as one factor related to African American's underutilization of mental health services [29]. 
3. Financial barriers to successful awareness campaigns

The most practical and often insurmountable barrier to effective awareness campaigns is that of financial restriction. Incredible expense is associated with communicating a message to the public, and campaigns with insufficient funding are often limited in their success. One study, for example, reported that reliance on public service announcements often leads to suboptimal time slots resulting in limitations in the delivery of the message to the intended audience [30]. Autism Speaks collaborated with the CDC and the Ad Council on the 'Learn the Signs. Act Early.' campaign, and initiated 'Light It Up Blue', a campaign to celebrate World Autism Awareness Day. In conducting these and other awareness efforts, Autism Speaks spends more than $\$ 15,000,000$ annually [31].

\subsection{Novel approaches to raising public awareness about autism}

Bertrand and colleagues define health awareness campaigns as programs designed to communicate educational messages to promote awareness and/or behavior change to a target population through large-audience channels such as the Internet (websites and social networking sites), television, radio, and print media (magazines, billboards, and posters) [32]. Support for the effectiveness of awareness campaigns are mixed with some associated with positive behavior change [33], and others having little to no effect [34]. Further, recent events demonstrate that negative awareness campaigns may be ineffective if they are perceived as disturbing or offensive. In December 2007, the New York University Child Study Center initiated an ASD awareness campaign in New York City that utilized advertisement notices resembling ransom notes indicating that 'We have your son. We will make sure he will no longer be able to care for himself or interact socially as long as he lives. Autism.' The ads were immediately met with significant backlash from the disability community as many families and individuals with ASD called and emailed the center to report that the ads were offensive and hurtful. It was suggested that ads such as this would contribute more to the spread of stigma and fear than to improve awareness efforts, and as a result of this outcry the Child Study Center cancelled the awareness campaign [35].

\subsubsection{The 'Learn the signs. Act early.' campaign}

In 2004 the CDC launched an ongoing public health campaign entitled "Learn the Signs. Act Early" (LSAE; [12]). The primary target audience for the campaign is parents of children aged 4 years or less, healthcare professionals (particularly pediatricians), and early educators, including childcare providers and preschool teachers. Campaign objectives are to increase awareness of developmental milestones and early warning signs, to increase knowledge about the benefits of early action, to increase dialogue between parents and providers, and to increase early action when developmental delay is suspected. Very effectively, this campaign incorporated several key features considered crucial for promoting behavior change [36].

1. Using a theoretical framework to conceptualize and guide behavior change. The Transtheoretical Model was used to plot where the target audiences were in terms of a) their awareness and monitoring of developmental milestones, and b) acting early when a delay is first 
suspected [37]. This model assumes that the change of health-related behaviors is a multistage process where individuals move from precontemplation, to contemplation, to preparation, to action, and finally to maintenance. Results from surveys and focus groups revealed that, even though many parents were aware of ASD, they were not aware of relevant developmental milestones, and did not believe that ASD was particularly relevant to them (i.e., precontemplation stage). Healthcare professionals and early childhood educators showed more awareness of relevant developmental milestones, but reported that they did not routinely monitor these milestones or communicate concerns with parents [36].

2. Influencing the information environment. The LSAE campaign developed a comprehensive approach for providing parents of young children with accurate information. Campaign materials include factsheets on developmental milestones (in English and Spanish), a campaign website, print and web banner advertisements, television and radio public service announcements, and a 24/7 live call center (1-800-CDC-INFO). The campaign was launched with a satellite media tour with the CDC Director; the public service announcements aired in collaboration with various TV and radio networks; and the campaign materials were distributed in partnership with local grassroots organizations, private sector companies, and key advocacy organizations (e.g., Autism Society of America, Autism Speaks, and First Signs). Similarly, complex information environments were also created for healthcare professionals and early childhood educators.

3. Creative message framing. One of the biggest challenges for public health campaigns is to stand out in our society's very crowded information environment, so that the message of the campaign gets sufficient exposure [36]. To increase the likelihood that the campaign messages capture the target audiences' attention, LSAE used a creative approach for creating and delivering messages. For example, the resource kit for healthcare professionals featured a picture of a child with the background text, 'A 4-year-old child with autism was once a 3-year-old child with autism, was once...'. Similarly, the TV public service announcement was first aired in New York's Times Square, reaching more than 91 million people.

4. Creating a supportive environment to assist individuals with behavior change. To support the target audiences' transition from contemplation to action, LSAE developed several key tools. For example, the campaign used familiar images such as a growth chart, but modified it to encourage and support the tracking of emotional, cognitive, and social development. Materials for physicians included fact sheets with milestones and red flag warning signs by age as well as informational cards to encourage doctor-parent dialogue.

5. Incorporating process analysis and exposure assessment. The campaign organizers utilized surveys and focus groups to understand the target audience (e.g., their knowledge, beliefs, attitudes, and behaviors) and how best to reach them. In addition, focus groups were also used to develop, test, and refine potential campaign concepts and accompanying images. Results revealed that fear-based messages that focus on the severity of ASD quickly turned parents away. Thus, instead of focusing on 'ASD', messages targeted the parents' natural and strong desire to monitor their child's growth and development. Finally, outcome 
surveys indicate $34 \%$ of parents reported some familiarity with the LSAE campaign. Significant changes in target parent behaviors were detected in that more parents knew of the behaviors likely to be associated with ASD, the best time to get help for ASD, and the developmental milestones their child should be reaching [38]. Similarly, substantially more healthcare professionals believe that they have the resources necessary to educate parents about monitoring child development, and fewer advocated a 'wait and see' approach, indicating that the marketing campaign was effective [39].

\section{Implementing autism-specific screening}

Both the Social Security Act [40] and the Individuals with Disabilities Education Improvement Act [41] reaffirm the mandate for PCPs to play a central role in the identification of developmental disabilities. Between birth and $2^{\text {nd }}$ birthday, children are recommended to participate in a sequence of 11 preventive pediatric healthcare visits (i.e., well-child visits). In the context of these visits, healthcare professionals have a unique opportunity to develop a long-term, trusting relationship with the children and their families. Parents expect that healthcare professionals take an interest in their child's development and behavior, competently identify strengths and weaknesses, and are able to help them access available community resources, if necessary [6].

\subsection{Limitations of developmental surveillance}

Developmental surveillance is defined as a "flexible, longitudinal, continuous, and cumulative process whereby knowledgeable healthcare professionals identify children who may have developmental problems" ([5] p. 407). Surveillance includes eliciting and attending to the parents' concerns, maintaining a developmental history, making accurate and informed observations of the child, identifying the presence of risk and protective factors, and documenting the process and findings. According to the AAP recommendations on developmental surveillance and screening [5], healthcare professionals are recommended to implement developmental screening during all well-child visits. At the same time, this policy statement also asserts that developmental surveillance by itself is insufficient for detecting developmental concerns in a large number of children. Instead, the AAP recommends using a combination of developmental surveillance and formal screening tests. Research has shown that healthcare professionals who rely solely on clinical judgment are much less accurate in estimating developmental status than professionals who implement formal screening tests [42, 43]. That is, the clinical impressions of healthcare professionals tend to have excellent specificity (i.e., if children are identified, concerns tend to be valid). At the same time, their sensitivity in detection developmental delays is quite poor, leading to many un-identified children and revealing significant difficulties in detecting less obvious delays. For example, Hix-Small and colleagues [44] conducted a study where global developmental delays (at 12 and 24 months) were identified based on both, clinical impressions and a formal screening test (Ages and Stages Questionnaire, ASQ; $[45,46])$. Results revealed that $48 \%$ of children with true developmental delays would have been missed if only clinical impressions were used. Similarly, in a 
study that aimed to identify toddlers with ASD, Robins [47] screened a total of 4,797 children, identifying 21 children who were later diagnosed with ASD. Of those 21 children, only 4 were previously red-flagged by the child's pediatrician.

\subsection{Recommended and implemented screening practices}

The use of general developmental screening instruments has been recommended by the AAP since 2001 [48]. Current recommendations for identifying children with developmental disabilities (not specifically ASD) suggest population-wide screening at 9, 18, and 30 months [5]. Empirical information on the extent to which these recommendations have been implemented is limited. Two national surveys of AAP members completed in 2002 and 2009 indicate that implementation has been slow $[49,50]$. When asked about their screening practices, only $23.0 \%$ (2002) and 47.7\% (2009) of physicians reported that they 'always' or 'almost always' administer a formal screening tool. Since many healthcare professionals may administer standard screening tools in a non-standard manner (e.g., by asking some but not other items) or only administer these tools to patients considered 'high risk', these numbers likely overestimate true implementation [51]. Researchers who asked parents to report on the screening practices of their healthcare professionals found that less than $27 \%$ of parents of children between 10 to 35 months recalled completing a developmental screening questionnaire within the last 12 months [52]. Only two survey-based studies have evaluated the implementation of ASD-specific screening instruments. In a 2004 survey of 255 pediatricians licensed in Maryland and Delaware, dosReis and colleagues reported that only $8 \%$ of the respondents screened for ASD [53]. In a 2007 survey of 51 pediatricians licensed in Alabama and Mississippi, Gillis reported that $28 \%$ reported using ASD-specific screening instruments [54]. Importantly, only one pediatrician reported routine screening for ASD at 18- or 24-months, suggesting that many healthcare professionals administer ASD-specific screening tests only to children who are considered 'high risk'.

\subsubsection{Barriers to successful screening in the primary care setting}

Well-child visits are often the only routine, formalized, and longitudinal contact a child has with a healthcare professional and thus is an ideal place to implement population-wide screening. Given this widely acknowledged responsibility, it is striking that many practices do not implement developmental and ASD-specific screening measures as recommended by the AAP. In the following we will consider several key barriers that interfere with healthcare professionals' ability to implement effective screening practices.

1. Parental compliance with the preventive pediatric healthcare schedule

The Medical Expenditure Panel Survey provides nationally representative information on preventive care for children between 0 and 5 years of age [55]. Results indicate that the average compliance ratio is $71.3 \%(S D=1.4 \%)$, indicating that on average, parents attend about 3 out of 4 AAP recommended well-child visits. In addition, findings reveal large variation in wellchild visit attendance based on the families' socioeconomic status, access to resources, and geography. That is, the compliance ratio was significantly below average if the child was 
without health insurance $(M=52.5 \%, S D=3.8)$, lived below the poverty level $(M=62.8 \%, S D$ $=2.4)$, lived with a single parent $(M=65.5 \%, S D=2.7)$, was Hispanic or Black $(M=64.5 \%$, $S D$ $=2.1$, or $M=64.7 \%, S D=2.9$, respectively), or had parents without a high-school degree $(M=$ $60.8 \%, S D=3.1)$. Moreover, in terms of geography, the compliance ratio ranged between $90.9 \%$ $(S D=5.0)$ in New England and 51.3\% $(S D=3.4)$ in the West South Central Census division. To account for this variability in parental compliance with the preventive pediatric care schedule, practices may be able to screen about $30 \%$ more children by flexibly administering screening measures during sick-visits, when necessary [56].

2. Competing priorities, time, and reimbursement constraints

During the last decade, the AAP $[5,6,57]$ has published three separate statements on the early detection of developmental-behavioral problems. Recommended screenings include: (1) screening for maternal postpartum depression (in the first year), (2) broad-band developmental screening (at 9, 18, and 24/30 months), (3) ASD-specific screening (at 18 and 24 months), (4) social-emotional screening (contingent upon abnormal developmental or ASD screeners), (5) kindergarten readiness screening (at 4 years), and (6) mental health/psychological function screening (age 5 years and thereafter). Implementing such a dense screening schedule in the context of short well-child visits filled with competing priorities (e.g., vaccinations, medical surveillance), and limited reimbursement options is often noted as a key challenge by healthcare professionals [53].

3. Availability of high-quality screening instruments

Given that screening tools are being developed, revised, and evaluated on an ongoing basis, the AAP practice guidelines do not recommend any particular ASD-specific screening instrument [6]. Instead, the AAP guidelines include a review of several potential measures, leaving it up to the healthcare professional to select an instrument that fits the particular needs of their practice. To date, the ASD-specific screening measure that has been adopted most widely in primary care settings is the Modified Checklist for Autism in Toddlers (M-CHAT; [47, 58, 59]. The M-CHAT is a 23-item yes/no parent report screener for ASD. Screening positive (i.e., "failing") is defined as failing any three items, or any two of six critical items. Failed items are reviewed with a follow-up interview, typically administered by phone a few weeks after the screener is completed. Most of the research evaluating the efficacy of this measure has focused on the positive predictive value (PPV), defined as the number of true positive cases divided by number of cases that screened positive. Robins reported on the screening results of 4,797 toddlers (screened during their 15-, 18-, or 24-month well-child visits) [47]. The numbers of toddlers who failed the M-CHAT questionnaire, failed the M-CHAT follow-up interview, and were eventually diagnosed with ASD, were 466, 61, and 21, respectively. Thus, if the results of the follow-up interview were considered, the M-CHAT revealed a PPV of.57. However, without the follow-up interview, the PPV of the M-CHAT was as low as.06. These estimates are consistent with data reported by Kleinman and colleagues [59], who reported PPVs of.65 and. 11, depending on whether the follow-up interview was or was not considered. Based on these findings, at least three conclusions seem warranted: (1) without administering the follow-up interview, the M-CHAT is likely to over-identify children by a factor of $17: 1$; (2) over-identifica- 
tion can be dramatically reduced by administering the follow-up interview (3:1); (3) even though the sensitivity of the M-CHAT has not been evaluated, comparisons to prevalence estimates of ASD suggest that the M-CHAT is likely to miss a considerable number of children [7]. Assuming current prevalence estimates (1:88), the sample reported by Roberts [47] likely included about 55 children with ASD. Even after considering participant attrition as a factor (i.e., families who did not complete the follow-up interview or diagnostic evaluations), the number of children with ASD actually identified by the researchers $(n=21)$ is considerably lower than would be expected.

\section{Limited parent literacy}

The extent to which limited parent literacy interferes with the implementation of broad-band developmental and ASD-specific screening measures has not been investigated. However, data presented by Davis and colleagues suggest that limited parental literacy has the potential to pose significant obstacles, particularly in the context of practices that primarily serve indigent or immigrant communities [60,61]. Based on a convenience sample of 396 parents from one large medical center (i.e., Louisiana State University Medical Center, Shreveport, LA), $11 \%$ and $16 \%$ of parents showed a reading level below $4^{\text {th }}$ and $7^{\text {th }}$ grade, respectively. Limited parental literacy may be an important factor in explaining missing data problems, reported across many population-based screening studies. For example, Hix-Small and colleagues reported that only about $54 \%$ of the administered screening questionnaires were completed and returned [44].

5. Need for practice-wide system change

The successful implementation of effective screening practices requires more than educational opportunities for individual staff members. Instead, what is needed is a context that supports organizational restructuring [51,62]. That is, practices need to develop, evaluate, and refine office-wide implementation systems that divide responsibilities among staff members at multiple levels. For example, the screening instrument may be distributed by a member of the front desk staff, scored by a nurse, reviewed with the family by a provider, and possible referrals may be coordinated by a social worker. Developing such an office-wide implementation system requires an 'internal champion' to lead the charge, a process for collecting data to monitor progress, and a seamless integration with the clinics' electronic medical record system. In order to be sustainable, the implementation system also needs to be sufficiently robust to be workable in the context of busy periods (e.g., the onset of the winter viral season) and staff turnover.

\subsubsection{Novel approaches to support autism-specific screening practices}

Research suggests that traditional methods of education, including printed educational materials and didactic, lecture-based continuing medical education (CME) sessions, have little to no effect on the behavior of healthcare professionals [63, 64]. During recent years, several alternative approaches to the traditional CME format have been suggested. These approaches include specialized modules on ASD that are included as part of pediatric residency training programs (e.g., CDC Autism Case Training) $[65,66]$, and academic detailing where a focused 
training session on early identification of ASD is presented to the entire office staff of individual practices [67]. In the following section, we will describe a third approach that has been used widely to improve the delivery of high-quality healthcare, although it has yet to be implemented with a focus on improving early detection of children with ASD.

The Breakthrough Series Collaborative Model

The Breakthrough Series Collaborative Model (BSCM) has been developed by the Institute of Healthcare Improvement (IHI, www.ihi.org) [68], and used successfully to improve the delivery of preventive services by pediatric practices [69], follow-up to newborn hearing screening [70], and child mental health service use [71]. In this model, several healthcare provider teams partner with external experts to overcome specific barriers that impede the delivery of high-quality care within their organization. Figure 2 presents the key elements of a Breakthrough Series model.

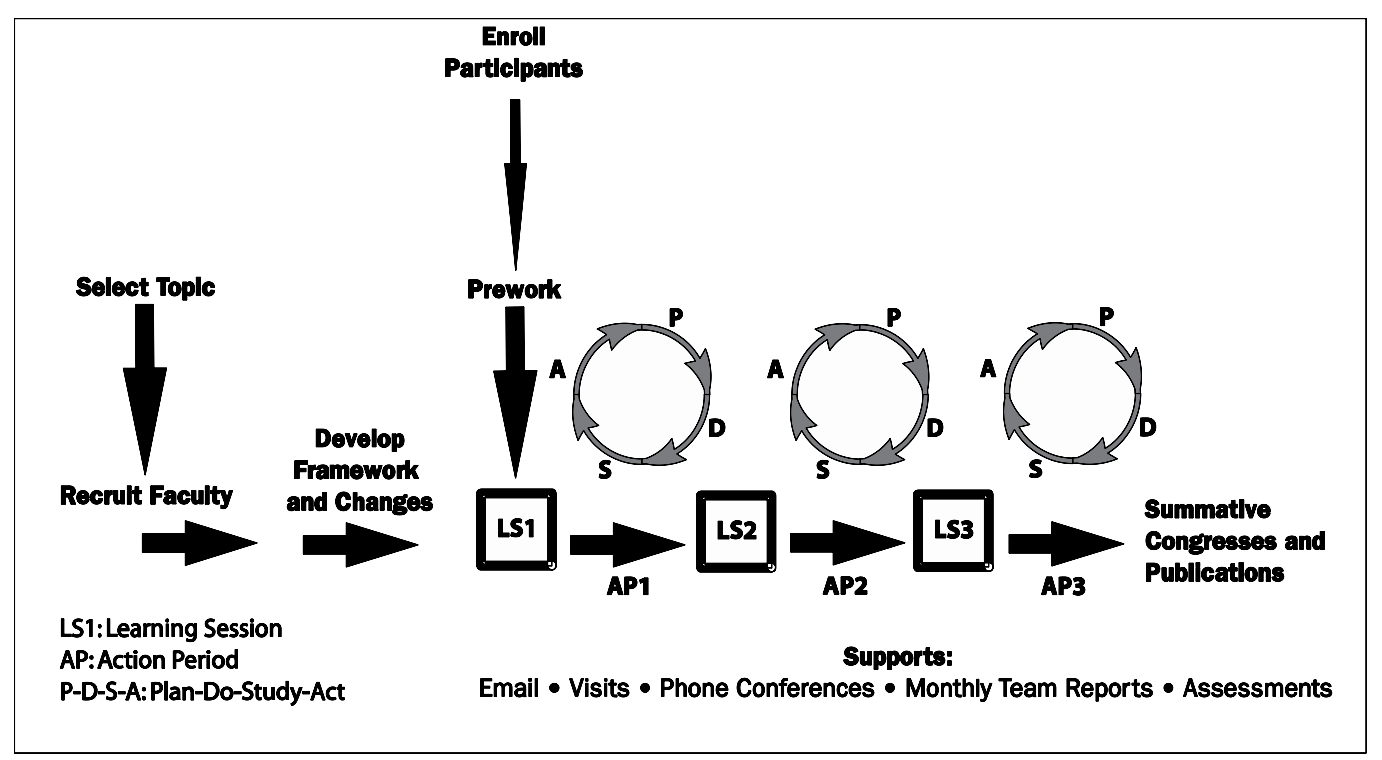

Figure 2. Breakthrough Series Model

The breakthrough series starts with the selection of a specific topic that is considered ripe for improvement. Even though data on the efficacy of learning collaboratives to increase early identification of ASD has not been published to date, we suggest that this would be a very appropriate topic, (1) because of the high prevalence rates of ASD, and (2) because the existing knowledge in this area is sound but not widely used. Once the topic is selected, a faculty team is assembled that combines expertise in the subject area as well as an improvement advisor who coaches teams on improvement methods. Organizations elect to join the collaborative through an application process, appointing multi-disciplinary teams within the organization (a champion's committee). The multi-disciplinary teams from all organizations are then brought together for Learning Sessions that combine the exchange of formal academic 
knowledge with practical voices from peers. In between the Learning Sessions, teams engage in Action Periods during which they implement change in a cyclical fashion: a) teams develop a PLAN to implement change, b) they DO the work to implement the chance, c) they STUDY their progress by measuring clinical behaviors, and d) they ACT upon the results by refining their approach (Plan-Do-Study-Act cycles of learning).

\section{Communicating concerns about autism to parents}

When multiple risk factors are present (e.g., the child has a family history of ASD, the child's parents or other family members are concerned about behaviors related to ASD, the child's pediatrician notes concerns about ASD, or the child fails an ASD-specific screener), the AAP clinical practice guidelines recommend that the child's PCP simultaneously (1) provides parental education on ASD, (2) refers the child for a comprehensive ASD evaluation, (3) refers the child for an audiology evaluation, (4) refers the child to Early Intervention or Early Childhood Education Services, and (5) schedules a targeted follow-up visit within one month [6]. Accomplishing all five actions simultaneously is considered crucial to avoid costly delays.

Little is currently known about how parents experience autism-specific screening, parent education, and referrals provided by the child's PCP in accordance with the AAP clinical practice guidelines. By definition, these interactions between PCP and parent precede a formal ASD diagnosis. Thus, PCPs typically communicate a certain level of uncertainty about the child's ASD diagnosis, emphasizing that the child is considered to be at 'high risk for ASD', and focusing on the need for a comprehensive evaluation to 'rule out ASD'. Even at this tentative level, communicating concerns about ASD requires initiating or engaging parents in a difficult conversation. For some parents, this conversation confirms suspicions that lay dormant for various amounts of time. For other parents, it comes out of nowhere and confronts them with concerns they had not previously contemplated. In either case, parents might not yet be emotionally ready to consider the possibility that their child has autism. Parents often feel responsible for their child's behavior or delays, feel guilty about not noticing or acting upon these concerns more promptly, and fear the stigma all too often associated with developmental disabilities.

When prompted to reflect upon the time when their child was first diagnosed, many parents of older individuals with ASD express significant levels of discontentment, in some cases resentment and anger [72, 73]. In part, these strong emotions stem from the fact that most families experience significant delays between the time when they first note concerns [73], the time when they choose to share these concerns with their child's physician $[9,74]$, the time when their child is first evaluated [11], and the time when the ASD diagnosis is first given [7, 11]. On average, parents report seeing four to five doctors before an ASD diagnosis is made, which occurs on average at age of four or five years [7, 11,72,73]. Parental perceptions of delay in diagnosis due to 'watchful waiting' are associated with (1) lower satisfaction with the diagnostic process, (2) lower satisfaction with the help physicians offer after the diagnosis, and (3) lower parental confidence in the physician's ability to help [72, 73, 75]. 
Parents also express dissatisfaction with the manner in which their child's pediatrician discussed developmental concerns and/or delivered the ASD diagnosis. For example, parents report dissatisfaction if a diagnosis is made but next steps and outcomes are not discussed [26]. Similarly, most parents of children with autism express a preference for receiving a clear ASD label rather than hearing about their child's autism "tendencies" or "trends" [73]. This preference for sincere, prompt, and honest information, even if this means that the physician has to admit a level of uncertainty, can also be found in other medical conditions. For example, cancer patients reported the highest levels of satisfaction and the lowest levels of anxiety when they felt that their healthcare professional prepared them adequately for the diagnosis, when they felt they were being told "everything", when the word "cancer" was used, and when their need to discuss life expectancy had been satisfied [76]. Importantly, high levels of trust in the physician has been linked to high levels of adherence to recommended behavior change [77].

\subsection{Barriers to successful communication between healthcare provider and parents}

The AAP clinical practice guidelines recommend that once ASD is seriously considered (i.e., due to a failed ASD-screener or multiple risk factors) the parent is promptly educated about ASD and referred for Early Intervention services. Research on the implementation of general developmental screening suggests that referral rates for children who fail such screeners vary tremendously across providers $(M=61 \%$; range: $27 \%$ to $100 \%$; [51]). Similarly, two descriptive studies on early identification of ASD in the healthcare setting found that referral to a clinical specialist (e.g., a developmental pediatrician) is the most likely response when autism is first suspected $[53,54]$. In the absence of a simultaneous referral to Early Intervention, referral to a clinical specialist can significantly delay children's access to services due to long waiting lists. In addition, the preference for referring families only to a clinical specialist reveals that, even in the presence of a failed ASD-screener, PCPs may often not feel ready to discuss autism with the child's parents. The reasons why PCPs use their own clinical judgment or uncertainty to override the results from a positive screening test are currently poorly understood [78]. This being said, considering a possible ASD-specific referral requires a delicate balancing act between the PCP's clinical judgment, tolerance for uncertainty, trust in screening tests, expectations about parental reactions, self-efficacy with regard to giving 'bad news' and confidence in the available service system. In the following we will describe select components of this tenuous balancing act, and discuss how each factor challenges the PCPs ability to effectively communicate with parents about autism.

1. Limited, outdated, or incorrect information on ASD and effective interventions

In a recent survey, PCPs reported feeling less competent providing care to children with ASD compared to children with other neurodevelopmental disorders and chronic conditions [79]. As described above, research on early identification and intervention in ASD has evolved rapidly during the last decade. Thus, PCPs may not always have access to the most current information. For example, they may be unaware of recent advances in early diagnosis, they may hold misconceptions about early red flags (e.g., they may erroneously assume that children with ASD never show affectionate behaviors), or they may not be familiar or feel passionate about the effectiveness of early intervention services [80]. 
2. Doubts about the accuracy of ASD-specific screening tests

Available ASD-specific screening tests are far from perfect. As discussed above, if the M-CHAT is administered without the follow-up interview, over-identification is likely to occur at a rate of 17:1 [47]. Several authors have emphasized the utility of second-stage autism-specific screeners in a referral setting to help prevent over-referral and effectively direct high-risk children toward comprehensive ASD evaluations [61, 81, 82]. Even though the M-CHAT follow-up interview has been developed to reduce the number of false positive screens, a feasible process for implementing this interview in the context of children's well-child visits has not been developed. In the absence of an effective second-stage screening process, $\mathrm{PCP}^{\prime} \mathrm{s}$ may use their own clinical judgment to prevent over-referrals.

\section{Expectations about parental reactions}

Even though timely referrals for a comprehensive ASD evaluation and Early Intervention services are important first steps, not all parents choose to comply with their pediatricians' referrals and pursue further evaluations [51, 75, 83]. Importantly, parental compliance rates with ASD-specific referrals tend to be lower in younger, and higher in older children. For example, Pierce and colleagues showed that $40 \%$ of parents refused a comprehensive followup evaluation after failing the CSBS Infant Toddler Checklist at 12 months [3]. Similarly, Pandey and colleagues reported that $37 \%$ of parents of younger children (16 to 23 months) refused a referral for a comprehensive ASD evaluation after a failed M-CHAT follow-up interview [84]. In older children (24 to 30 months), the refusal rate was only $21 \%$. In making decisions about a child's referrals, PCPs may gauge the likelihood of a parents' compliance, and thus be more reluctant to make ASD-specific referrals for younger than for older children.

4. Lack of experience in delivering 'bad news' in uncertain situations

Primary care providers may be reluctant to administer population-wide screening measures for ASD if they have had little or no training in sharing bad news with patients. A review of the relevant literature in cancer has revealed that delivering difficult news can be stressful and physicians may struggle with handling their own emotions of sorrow, guilt, and feelings of failure [85]. Unsurprisingly, physicians report a desire for more training in delivering bad news [85], and physicians who have received such training have expressed increased competence in having conversations where difficult information needs to be delivered [86-88].

\subsection{Novel approaches to improve communication about autism with parents}

How to communicate difficult news to patients has been widely studied, particularly related to cancer. For example, Eid and colleagues [87] tested a standardized intervention to improve communication skills in hematology-oncology fellows and nurses. The fellows and nurses were tested pre-intervention via mock clinical interviews where a "patient" had to be informed of bad news concerning their cancer care. The intervention consisted of one 45-minute interactive lecture that outlined specific methodology for delivering bad news and case scenarios to highlight proper strategies. Fellows and nurses also had the opportunity to watch and score the investigators displaying ideal as well as incorrect practices for deliver- 
ing bad news. A week after the intervention, fellows and nurses participated in another mock clinical interview. Both interviews were then scored using a fidelity checklist to measure skills taught during the intervention. Results indicated that fellows and nurses scored higher on the post-intervention clinical interview when compared to the pre-intervention interview; qualitatively, they also reported that the intervention improved their communication skills while delivering bad news. Similar research has demonstrated that improvements in physician communication skills are associated with improved patient outcomes, including reductions in patient distress [88] and increases in patient satisfaction with the treating physician [89].

Multi-media training materials on communicating with parents about failed ASD-screeners

Given the short history of ASD-specific screening, little research is currently available on how to effectively communicate with parents about failed ASD-specific screening tests. In recent years, at least two groups have developed multi-media training materials that support PCPs in this area. As part of the "Learn the Signs. Act Early." campaign, the CDC developed an in-class curriculum for current and future healthcare professionals, often presented in the context of hospital Grand Rounds (http://www.cdc.gov/ncbddd/actearly/) [65]. As part of this curriculum, learners watch and discuss video examples on how to communicate with parents about concerning screening results as well as various strategies for delivering difficult news. Importantly, learners are also provided with information about the stages of grief experienced by parents of children with disabilities. Similarly, as part of a webcast series on the medical home, the Waisman Center of the University of WisconsinMadison developed a 10-minute webcast on sharing screening results with families (http:// www.waisman.wisc.edu/connections)[90]. The webcast covers topics such as the importance of developmental screening, understanding the difference between screening and diagnosing, and considering specific language to use when sharing concerns. It also discusses why sharing screening results can be difficult, and shares specific steps to follow when sharing screening results.

\section{Supporting parents between referral and onset of services}

Prompt access to Early Intervention can alter the developmental trajectory of individuals with ASD. A recent clinical trial tested the efficacy of a comprehensive developmental behavioral intervention program (Early Start Denver Model) in a sample of toddlers with ASD between 18 and 30 months [91]. Results from this study revealed significant treatment effects on children's IQ, adaptive functioning, and autism symptoms. In many ways, this comprehensive treatment model for toddlers is consistent with the report of the National Research Council, which recommends that services should begin as soon as a child is suspected of having ASD and include a minimum of 25 hours a week, during which the child is actively engaged in systematically planned, and developmentally appropriate educational activity [92-94]. 
Comprehensive early intervention programs, consistent with the NRC recommendations or evidence based intervention practices [92-94] are currently not widely available to families of toddlers with ASD. Significant wait-times may delay children's prompt access to services [95]. In addition, programs are also not providing services at the recommended level of intensity. In a national survey of Early Intervention Coordinators, the vast majority of respondents indicated shortages of ASD-related personnel, including behavioral therapists $(89 \%)$, speech-language pathologists (82\%), and occupational therapists (79\%). Further, Early Intervention Coordinators of almost half of the reporting states (44\%) indicated that children with ASD receive 5 or fewer weekly service hours.

Families' ability to effectively utilize intervention services has also been linked to a range of demographic variables, including race and ethnicity $[9,96]$, immigrant status and cultural beliefs [97, 98], language [68, 99, 100], and SES [101]. Further, many families face geographic barriers in accessing ASD diagnoses and services. Children have been shown to receive diagnoses of ASD at older ages when the family lives in an area with few neurologists and psychiatrists [102]. Similarly, families in remote or rural areas often travel a great distance to reach services that are not available locally [103].

\subsection{PCP behaviors that contribute to delays between referral and onset of services}

The current chapter focuses on the gap between best practice and community implementation with regard to early identification of children with ASD. To fully support the needs of toddlers with ASD and their families, it is important to recognize that a similar gap between 'what we know and do' also exists with regard to early intervention practices. Thus, to ensure that children with ASD have access to appropriate services, PCPs should be mindful of several pitfalls that may contribute to delays between referral and onset of Early Intervention services.

1. Parents need to be reminded about the referrals

As described above, King and colleagues collaborated with 17 primary care practices to implement the AAP policy statement on developmental surveillance and screening [51]. Although a referral-tracking system is not specifically addressed in this policy statement, King and colleagues noted that more than half of the practices (9 out of 17) attempted to implement such a system. Because most practices include multiple healthcare professionals, a clinic-wide referral-tracking system is necessary to monitor which children had been referred and where they had been referred to. On the one hand, King reported that most clinics found referral-tracking to be a "time- and labor-intensive effort that was difficult to maintain over the long-term" (p. 357). On the other hand, clinics that were successful in implementing such a tracking system learned very quickly that many families didn't follow through with the recommended referrals, enabling them to develop strategies for providing additional reminders and supports. In addition, an effective referral-tracking system ena- 
bled the physicians to develop better communication with local referral resources and receive more consistent feedback on the children they referred.

2. Parents need to be educated about ASD

An additional lesson learned by clinics implementing an effective referral-tracking system was that many families did not seem to understand the reason for their child's referral [51]. The parents' limited understanding is concerning, not only because it reduces the chances that the parent will follow through with the pediatrician's referral but also because a comprehensive ASD evaluation will progress more efficiently if parents are familiar with the behavioral characteristics of children with ASD and if they can report accurately about their child's behavioral red flags of ASD [6]. Finally, by educating the parents about ASD at the time when the referral is made, families have the opportunity to prepare themselves emotionally for the evaluation and the possibility that their child may have autism.

3. Early Intervention administrators need to be informed that ASD is suspected

In determining eligibility for Early Intervention services, evaluations may cover multiple areas of development, including (1) physical, including vision and hearing, (2) cognitive, (3) communication, (4) social or emotional, and/or (5) adaptive. The developmental profile of many toddlers with ASD is strikingly uneven with possible strengths in physical and cognitive development and specific delays in several social and communication milestones. Given that ASD symptoms may be observed in the absence of global developmental delays, it is important that the evaluation team is specifically charged with the task to 'rule out ASD'. If ASD symptoms are not specifically addressed during the evaluation, toddlers with ASD who also have strengths in global development may be missed and as a result may not be considered eligible for services.

\subsection{Novel approaches to prevent delays between referral and onset of services}

Several recent projects aiming to implement general developmental or autism-specific screening in the primary care setting found it necessary to create a dedicated staff position, a developmental or autism specialist, to provide second-stage screening services to prevent over-referral, and/or information, support, resources and referrals to families identified with developmental concerns [61, 104]. Other projects provided families with access to a developmental/autism specialist by creating a partnership with a local research group [3,56]. Although this is a rather novel approach in the context of developmental and autism screening, the idea of a patient navigation program has been popular in other medical fields for several decades.

\subsubsection{The patient navigation program}

The American Cancer Society supported the nation's first patient navigation program in 1990 at the Harlem Hospital Center. Founded by Dr. Harold Freeman, patient navigation originally aimed to promote access to timely cancer diagnosis and treatment and to ensure coordinated 
services by assisting patients and their families to navigate through the healthcare delivery system [105]. Ideally, patient navigators are familiar with the specific healthcare system through which the patient must navigate, culturally attuned to the patient, and connected to decision makers in the healthcare system. Patient navigation has the potential to improve the continuity of care [106], promote compliance with recommended referrals by fostering trust between patient and healthcare providers [107], and facilitate access to evaluations and services in underserved populations by connecting them to resources most appropriate for each patient's individual needs [107]. Although a successful patient navigation program bears significant promise for promoting early identification of ASD, such a program has not been implemented to date.

\section{Conclusion}

During the last decade, research on early identification, diagnosis and intervention for toddlers with ASD has made tremendous progress. Moreover, during recent years, the topic of community implementation of best practice strategies has risen to the forefront. In order to develop a sustainable service infrastructure for toddlers with ASD, systems for public awareness, early identification, and early intervention need to be scaled up in tandem with updated priorities in public policy and funding allocation. Eventually, efforts to increase early identification of children with ASD will only be successful if identified children have access to effective Early Intervention services.

\section{Acknowledgements}

Preparation of this manuscript was supported by a grant from The FAR Fund. The themes of this chapter were informed by a public policy roundtable entitled 'Toddlers with Autism in New York City', held at the Roosevelt House Public Policy Institute at Hunter College in March 2011. Roundtable participants included: Peter H. Bell, Autism Speaks; Alice S. Carter, University of Massachusetts at Boston; Susan L. Hyman, University of Rochester; Michael Ganz, Harvard University; Barbara Kalmanson, ICDL Graduate School; Gary Mesibov, University of North Carolina at Chapel Hill; Donna M. Noyes-Grosser, NYS Department of Health; Marilyn Rubinstein, New York Presbyterian Hospital; Laura Slatkin, New York Center for Autism; Wendy Stone, University of Washington; Fred R. Volkmar, Yale University; Amy Wetherby, Florida State University. Information about the event is available online: www.hunter.cuny.edu/autismroundtable.

\section{Author details}

Michael Siller ${ }^{*}$, Lindee Morgan², Meghan Swanson ${ }^{3}$ and Emily Hotez ${ }^{4}$ 
*Address all correspondence to: msiller@hunter.cuny.edu

1 Psychology Department, Hunter College of The City University of New York, New York, USA

2 Autism Institute, College of Medicine, Florida State University, Tallahassee, USA

3 Psychology Department, Hunter College of The City University of New York, New York, USA

4 Graduate Center, The City University of New York, New York, USA

\section{References}

[1] Ozonoff, S., Iosif, A.M., Young, G.S., Hepburn, S., Thompson, M., Colombi, C., et al. Onset Patterns in Autism: Correspondence between Home Video and Parent Report. Journal of the American Academy of Child and Adolescent Psychiatry 2011;50(8): 796-806.

[2] Bhat, A.N., Galloway, J.C., and Landa, R.J. Social and Non-Social Visual Attention Patterns and Associative Learning in Infants at Risk for Autism. Journal of Child Psychology and Psychiatry 2010;51(9): 989-997.

[3] Pierce, K., Carter, C., Weinfeld, M., Desmond, J., Hazin, R., Bjork, R., et al. Detecting, Studying, and Treating Autism Early: The One-Year Well-Baby Check-up Approach. The Journal of Pediatrics 2011;159(3): 458-465.

[4] Kim, S.H. and Lord, C. Restricted and Repetitive Behaviors in Toddlers and Preschoolers with Autism Spectrum Disorders Based on the Autism Diagnostic Observation Schedule (ADOS). Autism Research 2010;3(4): 162-173.

[5] American Academy of Pediatrics. Identifying Infants and Young Children with Developmental Disorders in the Medical Home: An Algorithm for Developmental Surveillance and Screening. Pediatrics 2006;118(1): 405-420.

[6] Plauche' Johnson, C., Myers, S.M., and Council on Children With Disabilities. Identification and Evaluation of Children with Autism Spectrum Disorders. Pediatrics 2007;120(5): 1183-1215.

[7] Center for Disease Control and Prevention. Prevalence of Autism Spectrum Disorders - Autism and Developmental Disabilities Monitoring Network, 14 Sites, United States, 2008. MMWR Surveillance Summaries 2012;61(SS03): 1-19.

[8] Fountain, C., King, M.D., and Bearman, P.S. Age of Diagnosis for Autism: Individual and Community Factors across 10 Birth Cohorts. Journal of Epidemiology and Community Health 2011; 65(6): 503-510. 
[9] Center for Disease Control and Prevention. Prevalence of Autism Spectrum Disorders - Autism and Developmental Disabilities Monitoring Network, United States, 2006. MMWR Surveillance Summaries 2009;58(SS10): 1-20.

[10] Kim, Y.S., Leventhal, B.L., Koh, Y.J., Fombonne, E., Laska, E., Lim, E.C., et al. Prevalence of Autism Spectrum Disorders in a Total Population Sample. American Journal of Psychiatry 2011;168(9): 904-912.

[11] Wiggins, L.D., Baio, J., and Rice, C. Examination of the Time between First Evaluation and First Autism Spectrum Diagnosis in a Population-Based Sample. Journal of Developmental and Behavioral Pediatrics 2006;27(2): S79-S87.

[12] Daniel, K.L., Prue, C., Taylor, M.K., Thomas, J., and Scales, M. 'Learn the Signs. Act Early': A Campaign to Help Every Child Reach His or Her Full Potential. Public Health 2009;123, Supplement 1(0): e11-e16.

[13] Grimshaw, J.M., Shirran, L., Thomas, R., Mowatt, G., Fraser, C., Bero, L., et al. Changing Provider Behavior - An Overview of Systematic Reviews of Interventions. Medical Care 2001;39(8): Ii2-Ii45.

[14] Gataullina, L., Stereotypes in Media. 2003. Available from: http://serendip.brynmawr.edu/local/scisoc/sports03/papers/lgataullina.html.

[15] Haddon, M. The Curious Incident of the Dog in the Night-Time. 1st ed. New York: Doubleday; 2003.

[16] Leimbach, M. Daniel Isn't Talking. 1st ed. New York: Nan A. Talese; 2006.

[17] Adams, C. A Real Boy: A True Story of Autism, Early Intervention, and Recovery. New York: Berkley Books; 2005.

[18] Maurice, C. Let Me Hear Your Voice: A Family's Triumph over Autism. 1st ed. New York: Knopf; 1993.

[19] Farnall, O. and Smith, K.A. Reactions to People with Disabilities: Personal Contact Versus Viewing of Specific Media Portrayals. Journalism \& Mass Communication Quarterly 1999;76(4): 659-672.

[20] Jones, S.C. and Harwood, V. Representations of Autism in Australian Print Media. Disability \& Society 2009;24(1): 5-18.

[21] Baird, G., Cass, H., and Slonims, V. Diagnosis of Autism. British Medical Journal 2003;327(7413): 488-493.

[22] Grinker, R.R. Unstrange Minds: Remapping the World of Autism. New York, NY: Perseus Books; 2007.

[23] Fife, B.L. and Wright, E.R. The Dimensionality of Stigma: A Comparison of Its Impact on the Self of Persons with HIV/AIDS and Cancer. Journal of Health and Social Behavior 2000;41(1): 50-67. 
[24] Brown, L., Macintyre, K., and Trujillo, L. Interventions to Reduce HIV/AIDS Stigma: What Have We Learned? Aids Education and Prevention 2003;15(1): 49-69.

[25] Lyons, M. and Ziviani, J. Stereotypes, Stigma, and Mental-Illness - Learning from Fieldwork Experiences. American Journal of Occupational Therapy 1995;49(10): 1002-1008.

[26] Nissenbaum, M.S., Tollefson, N., and Reese, R.M. The Interpretative Conference: Sharing a Diagnosis of Autism with Families. Focus on Autism and Other Developmental Disabilities 2002;17(1): 30-43.

[27] Shaked, M. The Social Trajectory of Illness: Autism in the Ultraorthodox Community in Israel. Social Science \& Medicine 2005;61(10): 2190-2200.

[28] Coulthard, P. and Fitzgerald, M. In God We Trust? Organised Religion and Personal Beliefs as Resources and Coping Strategies, and Their Implications for Health in Parents with a Child on the Autistic Spectrum. Mental Health, Religion \& Culture 1999;2(1): 19-33.

[29] Nickerson, K.J., Helms, J.E., and Terrell, F. Cultural Mistrust, Opinions About Mental-Illness, and Black-Students' Attitudes toward Seeking Psychological Help from White Counselors. Journal of Counseling Psychology 1994;41(3): 378-385.

[30] Rideout, V., Hoff, T. Shouting to Be Heard: Public Service Advertising in a New Media Age. 2002: Menlo Park, CA.

[31] Autism Speaks. Autism Speaks 2010 Annual Report. 2010; Available from: http:// www.autismspeaks.org/sites/default/files/documents/as_annual_report_2010web_01.pdf.

[32] Bertrand, J.T. and Anhang, R. The Effectiveness of Mass Media in Changing HIV/ AIDS-Related Behaviour among Young People in Developing Countries. Preventing HIV/AIDS in Young People: A Systematic Review of the Evidence from Developing Countries 2006;938: 205-241.

[33] Sood, S. and Nambiar, D. Comparative Cost-Effectiveness of the Components of a Behavior Change Communication Campaign on HIV/AIDS in North India. Journal of Health Communication 2006;11: 143-162.

[34] Hornik, R.C. Public Health Communication: Evidence for Behavior Change. 4th ed. Mahaw: Lawrence Erlbaum Associates, Inc.; 2002.

[35] Shulman, R. Child Study Center Cancels Autism Ads. The Washinton Post 2007, December 20.

[36] Randolph, W. and Viswanath, K. Lessons Learned from Public Health Mass Media Campaigns: Marketing Health in a Crowded Media World. Annual Review of Public Health 2004;25: 419-437.

[37] Prochaska, J.O., Redding, C.A., and Evers, K.E. The Transtheoretical Model and Stages of Change. In: K. Glanz, B.K., Rimer, F.M., Lewis (Eds.) Health Behavior and 
Health Education: Theory, Research, and Practice, San Francisco: Jossey-Bass; 2002. p99-120.

[38] Novelli, P. Healthstyles 2007 Survey. Unpublished dataset. 2007.

[39] Novelli, P. Docstyles 2007 Survey. Unpublished dataset. 2007.

[40] Social Security Act, 42 U.S.C. Chapter 7, Subchapter V, §§ 701-710. (1989).

[41] Individuals with Disabilities Education Improvement Act, Pub. L. No. 108-446. (2004).

[42] Werner, E.E., Honzik, M.P., and Smith, R.S. Prediction of Intelligence and Achievement at Ten Years from Twenty Months Pediatric and Psychologic Examinations. Child Development 1968;39(4): 1063-1075.

[43] Sices, L., Feudtner, C., McLaughlin, J., Drotar, D., and Williams, M. How Do Primary Care Physicians Manage Children with Possible Developmental Delays? A National Survey with an Experimental Design. Pediatrics 2004;113(2): 274-282.

[44] Hix-Small, H., Marks, K., Squires, J., and Nickel, R. Impact of Implementing Developmental Screening at 12 and 24 Months in a Pediatric Practice. Pediatrics 2007;120(2): 381-389.

[45] Bricker, D. and Squires, J. Ages and Stages Questionnaire: A Parent-Completed, Child-Monitoring System. Baltimore, MD: Paul H. Brookes; 1995.

[46] Bricker, D. and Squires, J. Ages \& Stages Questionnaires: A Parent-Completed, Child-Monitoring System (ASQ). 2nd ed. Baltimore, MD: Brookes Publishing; 1999.

[47] Robins, D.L. Screening for Autism Spectrum Disorders in Primary Care Settings. Autism 2008;12(5): 537-556.

[48] American Academy of Pediatrics. The Pediatrician's Role in the Diagnosis and Management of Autistic Spectrum Disorder in Children. Pediatrics 2001;107(5): 1221-1226.

[49] Sand, N., Silverstein, M., Glascoe, F.P., Tonniges, T., Gupta, B., and O'Connor, K. Pediatricians' Reported Practices Regarding Developmental Screening: Are Guidelines Used? Do They Help? Pediatric Research 2004;55(4): 68a-68a.

[50] Radecki, L., Sand-Loud, N., O'Connor, K.G., Sharp, S., and Olson, L.M. Trends in the Use of Standardized Tools for Developmental Screening in Early Childhood: 2002-2009. Pediatrics 2011;128(1): 14-19.

[51] King, T.M., Tandon, S.D., Macias, M.M., Healy, J.A., Duncan, P.M., Swigonski, N.L., et al. Implementing Developmental Screening and Referrals: Lessons Learned from a National Project. Pediatrics 2010;125(2): 350-360. 
[52] Bethell, C., Reuland, C., Schor, E., Abrahms, M., and Halfon, N. Rates of Parent-Centered Developmental Screening: Disparities and Links to Services Access. Pediatrics 2011;128(1): 146-155.

[53] DosReis, S. and Weiner, C.L. Autism Spectrum Disorder Screening and Management Practices among General Pediatric Providers. Journal of Developmental and Behavioral Pediatrics 2006;27(2): S88-S94.

[54] Gillis, J.M. Screening Practices of Family Physicians and Pediatricians in 2 Southern States. Infants \& Young Children 2009;22(4): 321-331 310.1097/IYC. 1090b1013e3181bc1094e1021.

[55] Selden, T.M. Compliance with Well-Child Visit Recommendations: Evidence from the Medical Expenditure Panel Survey, 2000-2002. Pediatrics 2006;118(6): E1766E1778.

[56] Miller, J.S., Gabrielsen, T., Villalobos, M., Alleman, R., Wahmhoff, N., Carbone, P.S., et al. The Each Child Study: Systematic Screening for Autism Spectrum Disorders in a Pediatric Setting. Pediatrics 2011;127(5): 866-871.

[57] American Academy of Pediatrics Task Force on Mental Health. Enhancing Pediatric Mental Health Care: Strategies for Preparing a Primary Care Practice. Pediatrics 2010;125(Supplement 3): S87-S108.

[58] Robins, D., Fein, D., Barton, M., and Green, J. The Modified Checklist for Autism in Toddlers: An Initial Study Investigating the Early Detection of Autism and Pervasive Developmental Disorders. Journal of Autism and Developmental Disorders 2001;31(2): 131-144.

[59] Kleinman, J.M., Robins, D.L., Ventola, P.E., Pandey, J., Boorstein, H.C., Esser, E.L., et al. The Modified Checklist for Autism in Toddlers: A Follow-up Study Investigating the Early Detection of Autism Spectrum Disorders. Journal of Autism and Developmental Disorders 2008;38(5): 827-839.

[60] Davis, T.C., Mayeaux, E.J., Fredrickson, D., Bocchini, J.A., Jackson, R.H., and Murphy, P.W. Reading-Ability of Parents Compared with Reading Level of Pediatric-Patient Education Materials. Pediatrics 1994;93(3): 460-468.

[61] Schonwald, A., Huntington, N., Chan, E., Risko, W., and Bridgemohan, C. Routine Developmental Screening Implemented in Urban Primary Care Settings: More Evidence of Feasibility and Effectiveness. Pediatrics 2009;123(2): 660-668.

[62] Pinto-Martin, J.A., Dunkle, M., Earls, M., Fliedner, D., and Landes, C. Developmental Stages of Developmental Screening: Steps to Implementation of a Successful Program. American Journal of Public Health 2005;95(11): 1928-1932.

[63] Freemantle, N., Harvey, E.L., Wolf, F., Grimshaw, J.M., Grilli, R., and Bero, L.A. Printed Educational Materials: Effects on Professional Practice and Health Care Outcomes. Cochrane Database Systematic Reviews 2000(2): CD000172. 
[64] Farmer, A.P., Légaré, F., Turcot, L., Grimshaw, J., Harvey, E., McGowan, J., and Wolf, F.M. Printed Educational Materials: Effects on Professional Practice and Health Care Outcomes. Cochrane Database of Systematic Reviews 2008(3).

[65] Center for Disease Control and Prevention. Autism Case Training: A DevelopmentalBehavioral Pediatrics Curriculum. 2011; Available from: http://www.cdc.gov/ ncbddd/actearly/act/class.html.

[66] Bauer, S.C., Smith, P.J., Chien, A.T., Berry, A.D., and Msall, M. Educating Pediatric Residents About Developmental and Social-Emotional Health. Infants \& Young Children 2009;22(4): 309-320 310.1097/IYC.1090b1013e3181bc1094da1090.

[67] Honigfeld, L., Chandhok, L., and Spiegelman, K. Engaging Pediatricians in Developmental Screening: The Effectiveness of Academic Detailing. Journal of Autism and Developmental Disorders 2012;42(6): 1175-1182.

[68] Institute of Healthcare Improvement The Breakthrough Series: IHI's Collaborative Model for Achieving Breakthrough Improvement. IHI Innovation Series white paper. Boston: Institute for Healthcare Improvement; 2003. (Available on www.IHI.org)

[69] Young, P.C., Glade, G.B., Stoddard, G.J., and Norlin, C. Evaluation of a Learning Collaborative to Improve the Delivery of Preventive Services by Pediatric Practices. Pediatrics 2006;117(5): 1469-1476.

[70] Russ, S.A., Hanna, D., DesGeorges, J., and Forsman, I. Improving Follow-up to Newborn Hearing Screening: A Learning-Collaborative Experience. Pediatrics 2010;126: S59-S69.

[71] Cavaleri, M., Gopalan, G., McKay, M. M., Apel, A., Bannon, W. M., \& Bigley, M. F. Impact of a Learning Collaborative to Improve Mental Health Service Use among Low-Income, Urban Youth and Families. Best Practices in Mental Health: An International Journal 2006;2(2): 67-79.

[72] Goin-Kochel, R.P., Mackintosh, V.H., and Myers, B.J. How Many Doctors Does It Take to Make an Autism Spectrum Diagnosis? Autism 2006;10(5): 439-451.

[73] Howlin, P. and Moore, A. Diagnosis in Autism: A Survey of over 1200 Patients in the UK. Autism 1997;1(2): 135-162.

[74] Veness, C., Prior, M., Bavin, E., Eadie, P., Cini, E., and Reilly, S. Early Indicators of Autism Spectrum Disorders at 12 and 24 Months of Age: A Prospective, Longitudinal Comparative Study. Autism 2012;16(2): 163-177.

[75] Harrington, J.W., Patrick, P.A., Edwards, K.S., and Brand, D.A. Parental Beliefs About Autism. Autism 2006;10(5): 452-462.

[76] Schofield, P.E., Butow, P.N., Thompson, J.F., Tattersall, M.H.N., Beeney, L.J., and Dunn, S.M. Psychological Responses of Patients Receiving a Diagnosis of Cancer. Annals of Oncology 2003;14(1): 48-56. 
[77] Kosinski, M., Rogers, W.H., Safran, D.G., Taira, D.A., Tarlov, A.R., and Ware, J.E. Linking Primary Care Performance to Outcomes of Care. Journal of Family Practice 1998;47(3): 213-220.

[78] Marks, K.P., Glascoe, F.P., and Macias, M.M. Enhancing the Algorithm for Developmental-Behavioral Surveillance and Screening in Children 0 to 5 Years. Clinical Pediatrics 2011;50(9): 853-868.

[79] Golnik, A., Ireland, M., and Borowsky, I.W. Medical Homes for Children with Autism: A Physician Survey. Pediatrics 2009;123(3): 966-971.

[80] Heidgerken, A.D., Geffken, G., Modi, A., and Frakey, L. A Survey of Autism Knowledge in a Health Care Setting. Journal of Autism and Developmental Disorders 2005;35(3): 323-330.

[81] Warren, Z., Stone, W., and Humberd, Q. A Training Model for the Diagnosis of Autism in Community Pediatric Practice. Journal of Developmental and Behavioral Pediatrics 2009;30(5): 442-446.

[82] Kobak, K.A., Stone, W.L., Ousley, O.Y., and Swanson, A. Web-Based Training in Early Autism Screening: Results from a Pilot Study. Telemedicine and E-Health 2011;17(8): 640-644.

[83] Dietz, C., Swinkels, S.H.N., van Daalen, E., van Engeland, H., and Buitelaar, J.K. Parental Compliance after Screening Social Development in Toddlers. Archives of Pediatrics \& Adolescent Medicine 2007;161(4): 363-368.

[84] Pandey, J., Verbalis, A., Robins, D.L., Boorstein, H., Klin, A., Babitz, T., et al. Screening for Autism in Older and Younger Toddlers with the Modified Checklist for Autism in Toddlers. Autism 2008;12(5): 513-535.

[85] Fallowfield, L. and Jenkins, V. Communicating Sad, Bad, and Difficult News in Medicine. Lancet 2004;363(9405): 312-319.

[86] Baile, W.F., Buckman, R., Lenzi, R., Glober, G., Beale, E.A., and Kudelka, A.P. Spikes - a Six-Step Protocol for Delivering Bad News: Application to the Patient with Cancer. The Oncologist 2000;5(4): 302-311.

[87] Eid, A., Petty, M., Hutchins, L., and Thompson, R. "Breaking Bad News": Standardized Patient Intervention Improves Communication Skills for Hematology-Oncology Fellows and Advanced Practice Nurses. Journal Of Cancer Education: The Official Journal Of The American Association For Cancer Education 2009;24(2): 154-159.

[88] Roter, D.I., Hall, J.A., Kern, D.E., Barker, L., Cole, K.A., and Roca, R.P. Improving Physicians' Interviewing Skills and Reducing Patients' Emotional Distress: A Randomized Clinical Trial. Archives of Internal Medicine 1995;155(17): 1877-1884.

[89] Delvaux, N., Razavi, D., Marchal, S., Bredart, A., Farvacques, C., and Slachmuylder, J.L. Effects of a 105 Hours Psychological Training Program on Attitudes, Communi- 
cation Skills and Occupational Stress in Oncology: A Randomised Study. Br J Cancer 2004;90(1): 106-114.

[90] Waisman Center of the University of Wisconsin-Madison, Wisconsin Medical Home Webcast Series. 2011. Available from: http://www.waisman.wisc.edu/connections.

[91] Dawson, G., Rogers, S., Munson, J., Smith, M., Winter, J., Greenson, J., et al. Randomized, Controlled Trial of an Intervention for Toddlers with Autism: The Early Start Denver Model. Pediatrics 2010;125(1): E17-E23.

[92] National Research Council. Educating Children with Autism. USA: National Academy Press; 2001.

[93] Odom, S.L., Boyd, B.A., Hall, L.J., and Hume, K. Evaluation of Comprehensive Treatment Models for Individuals with Autism Spectrum Disorders. Journal of Autism and Developmental Disorders 2010;40(4): 425-436.

[94] Boyd, B.A., Odom, S.L., Humphreys, B.P., and Sam, A.M. Infants and Toddlers with Autism Spectrum Disorder: Early Identification and Early Intervention. Journal of Early Intervention 2010;32(2): 75-98.

[95] Downs, R.C. and Downs, A. Practices in Early Intervention for Children with Autism: A Comparison with the National Research Council Recommended Practices. Education and Training in Autism and Developmental Disabilities 2010;45(1): 150-159.

[96] Mandell, D.S., Wiggins, L.D., Carpenter, L.A., Daniels, J., DiGuiseppi, C., Durkin, M.S., et al. Racial/Ethnic Disparities in the Identification of Children with Autism Spectrum Disorders. American Journal of Public Health 2009;99(3): 493-498.

[97] Lindsay, S., King, G., Klassen, A.F., Esses, V., and Stachel, M. Working with Immigrant Families Raising a Child with a Disability: Challenges and Recommendations for Healthcare and Community Service Providers. Disability Rehabilitation 2012;34(23):2007-17.

[98] Welterlin, A. and LaRue, R.H. Serving the Needs of Immigrant Families of Children with Autism. Disability \& Society 2007;22(7): 747-760.

[99] Williams, M.E., Atkins, M., and Soles, T. Assessment of Autism in Community Settings: Discrepancies in Classification. Journal of Autism and Developmental Disorders 2009;39(4): 660-669.

[100] Kim, C., Disare, K., Pfeiffer, M., Kerker, B.D., and McVeigh, K.H. Effects of Individual and Neighborhood Characteristics on the Timeliness of Provider Designation for Early Intervention Services in New York City. Journal of Developmental and Behavioral Pediatrics 2009;30(1): 38-49. 
[101] Durkin, M.S., Maenner, M.J., Meaney, F.J., Levy, S.E., DiGuiseppi, C., Nicholas, J.S., et al. Socioeconomic Inequality in the Prevalence of Autism Spectrum Disorder: Evidence from a US Cross-Sectional Study. Plos One 2010;5(7).

[102] Kalkbrenner, A.E., Daniels, J.L., Emch, M., Morrissey, J., Poole, C., and Chen, J.-C. Geographic Access to Health Services and Diagnosis with an Autism Spectrum Disorder. Annals of Epidemiology 2011;21(4): 304-310.

[103] Gething, L. Sources of Double Disadvantage for People with Disabilities Living in Remote and Rural Areas of New South Wales, Australia. Disability \& Society 1997;12(4): 513-531.

[104] Shannon, P. and Anderson, P.R. Developmental Screening in Community Health Care Centers and Pediatric Practices: An Evaluation of the Baby Steps Program. Intellectual and Developmental Disabilities 2008;46(4): 281-289.

[105] Freeman, H.P. Patient Navigation: A Community Centered Approach to Reducing Cancer Mortality. Journal of Cancer Education 2006;21: S11-14.

[106] Bradford, J.B., Coleman, S., and Cunningham, W. HIV System Navigation: An Emerging Model to Improve HIV Care Access. AIDS Patient Care STDS 2007;21 Suppl 1: S49-58.

[107] Natale-Pereira, A., Enard, K.R., Nevarez, L., and Jones, L.A. The Role of Patient Navigators in Eliminating Health Disparities. Cancer 2011;117(15): 3543-3552. 
Chapter 2

\title{
Indicators of Autism in Iranian Children
}

\author{
Sayyed Ali Samadi and Roy McConkey \\ Additional information is available at the end of the chapter \\ http://dx.doi.org/10.5772/52853
}

\section{Introduction}

Autism occurs in every country of the world. However its prevalence varies greatly across nations with higher rates being reported in more affluent, English speaking countries. The lower rates in less developed countries have been attributed to a lack of knowledgeable personnel in child assessment and diagnostic services and their slow response to parental concerns [1]. While this is certainly a major impediment to early identification, other social and cultural factors may play a part. In particular, expectations of children's development and behaviours may mean that parents attach less significance to certain early indicators of $\mathrm{Au}^{-}$ tism across different cultures [2]. If this were so, then screening and other assessment tools developed in Western countries may not be sufficiently sensitive to detect early signs of Autism in other societies [3].

This chapter summarises the findings from two studies in Iran that identified the items that best discriminated children who had a diagnosis of autism. In the first study, the Gilliam Autism Rating Scale - Second edition GARS 11 [4] was used. Comparisons are drawn between three groups of children aged 3 to 16 years: those with a compared diagnosis of Autism; children with intellectual disabilities and those whose development was considered to be normal.

The second study focussed on the Autism Diagnostic Interview- Revised (ADI-R) [5]. This tool was developed for use by clinicians to assist in making a diagnosis of autism usually after referral that follows from the use of a screening tool such as GARS. In all the performance of 333 children (84\%) with a confirmed diagnosis of Autism could be confirmed with those of $64(16 \%)$ who were not given this diagnosis although they had screened positive.

The findings from these two major studies together identify those indicators of autism that appear to be more culturally specific to Iranian or similar cultures. However comparisons are drawn with similar data from other countries to underline the universality of certain au- 
tistic traits [6]. Nevertheless the implementation of tools for the identification of children with autism has to be located within wider considerations; notably the education of parents and professionals around indicators of atypical child development and the possible environmental influences on children's behaviours.

\section{Identifying children with autism in different cultures}

Children with autism have deficits in three developmental domains: communication, socialisation and restricted interests along with repetitive behaviours [7]. These criteria are presumed to hold across all cultures although there is growing evidence of cultural variations in the way children may present with Autism. Indeed there are good theoretical grounds for expecting this to be so. Theories on human development propose that children develop through adapting to the multidimensional, intersecting environments within which they live and grow, the most proximate of which are their parents and families [8]. Parental influences on their child's development may therefore vary in different cultures depending on their beliefs about normative development, the relative value their culture places on different behaviors and the meaning attributed to them[9;10]. For example, in Western societies the absence of direct eye contact is an early indicator of ASD yet in Chinese and Japanese societies, such eye contact is uncommon as looking directly into someone's eyes is considered shameful in these parts of East Asia [11].

Studies done in the Western societies around early signs of autism, [12] found that the most common parental concerns were for delay in speech and language development, followed by abnormal signs of socio-emotional behaviour and medical problems or delay in reaching milestones. By contrast, Daley [13] found that with Indian parents, social difficulties - such as lack of interest in people, poor eye contact and showing no interest in playing with other children - were rated as the first parental concern with delay in speech as a secondary concern. These variations in cultural expectations are likely to exist among indigenous professionals as well as parents.

The diagnosis of autism varies across different ethnic groups within countries. Mandell et al. [14] in a study of over 2,500 eight year-olds in the USA, reported that children from Black, Hispanic and other ethnic or racial groups were less likely than white children to have a documented autism spectrum disorder. Valicenti-McDermott et al. [15] found that children of Hispanic and African American origin, foreign-born children, and children born to foreign mothers were more likely to be diagnosed at an older age than those from white American parentage. Likewise in Holland, fewer children from Moroccan or Turkish immigrants than native born Dutch children were referred for assessments for ASD [16]. Moreover paediatricians $(n=81)$ more often referred to autism when judging clinical vignettes of European majority cases (Dutch) than vignettes of minority cases.

Cultural influences may also explain to some extent the variation in prevalence rate of ASD reported across different countries. A systematic review of 40 studies [17], reported rates varying from 3.8 per 10,000 in Norway, 5.4 in France and 5.6 in Finland through to a high of 
60 per 10,000 in Sweden. However in London, a more thorough study reported a prevalence rate of 389 per 10,000 for autistic disorders and for other ASDs at 772 per 10,000; giving a total prevalence for all ASDs of 1161 per 10,000 [18]. A regression analyses of the prevalence studies found that the most significant influence on ASD rates was the diagnostic criteria used, followed by the age of child when identified, the country of origin and urban/rural location of the sample [17].

These same factors may also account for the variation in prevalence rates that have been reported within countries even when ascertainment method, age group and reporting period are similar. In the USA, the prevalence of all ASDs in eight year old children varied across eleven sites from 42 per 10,000 in Florida to 121 per 10,000 in Arizona and Missouri [19]. Using special education data on students with ASD, Coo at al., [20] reported a prevalence of 43.1 per 10,000 among 4-9 years school children in the British Colombia Province of Canada whereas in Quebec it is reported at less than half this, at 21.6 per 10,000 [21].

One explanation for differences in the prevalence and identification of ASD across cultures and regions is varying awareness of the criteria associated with a diagnosis of ASD [6]. More specifically, it appears that differences may be more likely to arise cross culturally due to various factors such as "when a symptom is perceived, by whom, and what behaviour is noticed first, as well as whether it is perceived as problematic" ([2], p. 538). Moreover cultural attitudes regarding typical behaviours and what is perceived to be normal or abnormal development for that culture would also have an impact on diagnosis of an ASD. Hence children may be more or less susceptible to a diagnosis of an ASD dependent on the cultural expectations of parents and indigenous professionals. Thus screening and other assessment tools for autism developed in Western countries may not be sufficiently sensitive to detect early signs of autism in other societies and could possibly underestimate the prevalence of the condition [3].

\section{Screening and assessing children for autism}

A two-stage process for identifying children with autism is operational in many countries [22]. The first stage involves a universal screening of all children and various tools have been developed for use with children at different ages. They consist of a series of items indicative of autism and use parents as the primary informants. For example the CHAT (Checklist for Autism in Toddlers) is a screening tool developed for use with infants aged 18 months developed in the UK [23] that has also been adapted for use in the USA (M-CHAT: [24]) and which has shown promise for use in Arab countries [25]. More recently, ten item screening tools have been developed for use with children, adolescents and adults based on the Autism Quotient developed in the UK [26]. Those individuals who screen positive their scores exceed a designated cut-off point - are then referred for more detailed diagnostic assessment.

A range of diagnostic tools for autism also have been developed [27]. These are based on DSM-IV/ICD-10 criteria for autism and information about the child is usually obtained 
through a detailed, structured interview with parents. However a multi-disciplinary assessment by experienced clinicians is also recommended including a physical examination along with developmental and/or psychometric evaluations. These will enable a differential diagnosis to be made for autism from other conditions in which there is overlap of symptomology [28].

Such assessments can be time-consuming and costly. Their efficiency is determined by the referrals from the screening tests. If individuals are screened as positive for autism but are not subsequently diagnosed as having autism (false positives) this can mean wasted efforts by the diagnostic team. The converse is also concerning. Children may screen as negative for autism but had they been assessed, they might have been given a diagnosis of autism (false negatives). Thus the effectiveness of the screening test needs to be assessed in terms of its sensitivity (true positives are identified) and its specificity (false negatives are avoided). Thus the choice of items that are included in the screening test is crucial. To date, the screening tools have been developed within Western societies and given the earlier comments about cultural influences, it is conceivable that at least some of these items may not be suited for use in other cultures. The risk then is that the efficiency of the screening tool is diminished within that society [11]. Indeed the same argument may apply when screening individuals from immigrant communities in Western countries.

\section{Autism in Iran}

The Islamic Republic of Iran, formerly known as Persia, is located in the Middle East. The capital city is Tehran. Iranian society is distinct from other Islamic societies of the MiddleEast and Central Eurasia in terms of its long history of civilisation, its geographical location, separate language (Persian) and religious denomination (Shia Muslim).

It is a vast country of 1.65 million sq $\mathrm{km}$, extending in the north from the Caspian Sea to the Persian Gulf, Strait of Hormuz, and Oman Sea in the south, and from Afghanistan and Pakistan in the east to Iraq and Turkey in the west. Persians (51\% population) are the largest ethnic group in the Republic within the total population of 74.8 million. The main minorities are Azeri (24\%), Gilaki and Mazandarani (8\%), and Kurds (7\%). People are mainly Muslims (89\% Shi'a Muslims and 9\% Sunni Muslims). Iran became an Islamic Republic in 1979 and is divided into 31 provinces, each of which is headed by a governor-general appointed by the Minister of the Interior.

Children with ASD will usually be diagnosed by medical doctors either privately or through child and family clinics provided by voluntary organisations. State-funded special schools are provided through the Iranian Special Education Organisation although many parents may opt for private schooling. In addition, parents will arrange private therapy for their children. For children more severely affected or with other conditions such as intellectual disability, day centre placements are available through the Iranian State Welfare Organisation. However, these services are only available in larger cities and probably only for more 
affluent families. Provision for adult services is mostly through private or voluntary organisations that also rely heavily on parental fees.

Iran is one of the few countries that has a national screening programme for autism prior to children's entry to compulsory education at age 6 . An analysis of data obtained on over 1.32 million children aged 5 years of age screened over a three-year period, yielded an overall Iranian prevalence of 6.26 per 10,000 [1]. Although this rate is similar to that previously reported for certain European countries and for Hong Kong, it is much lower than those reported for Sweden, USA and England [29].

\subsection{Indicators of autism in Iran}

The main aim of the two studies reported in this chapter was to identify the most common indicators of autism for Iranian parents whose children had been given a diagnosis of autism. As argued above, this investigation would identify items for use in screening tests that would be culturally sensitive for an Iranian culture. However the initial pool of items would be drawn from those behaviours that define autism internationally according to DSM-IV [7] and ICHD-10 [30]. These were taken from two commonly used scales for autism - the Gilliam Rating Scale for Autism (GARS) and the Autism Diagnostic Interview - revised (ADI$\mathrm{R})$. Although developed in the USA and the UK respectively, they have been translated for use in other countries.

The most common indicators of autism would be chosen as those that best discriminated children with a diagnosis of autism from age peers who were normally developing; from those who were considered to have an intellectual disability and from those who were initially suspected of having autism but were not diagnosed as such following assessment.

The resulting set of indicators would have particular relevance for the development of further screening tools in Iran but these findings might have wider applicability to other Muslim cultures and non-Western societies.

\subsection{Study 1: Screening for autism}

The Gilliam Autism Rating Scale - Second edition GARS 11 [4] was developed in the United States of America and is based on DSM-IV diagnostic criteria for autism. It is widely used in hospitals, school and clinics across the USA with good psychometric properties which subsequent evaluation studies have confirmed [40]. The main reservations concerning its use, centre on the cut-off points that are taken to be indicative of autism. The consensus is that these should be set at a lower level than recommended in the test manual [41].

This tool takes the form of a behaviour checklist developed for use with children and youth aged 3 to 22 years. It consists of 42 items grouped into three subscales: Stereotyped Behaviours, Communication, and Social Interaction which are combined into a standard score called the "Autism Index" with higher scores indicative of ASD. A further 14 items contribute data about the child's development during the first three years of life which are used to supplement information about the child's current level of functioning. Reliability and validi- 
ty data for the English version of the test is available based on a normative sample of 1,107 individuals with ASD and 328 non-ASD persons and those with other developmental disabilities. Coefficients of reliability (internal consistency and test-retest) for the subscales and Autism Index range from 0.80 to 0.90 .

For the purpose of this study the first author translated the GARS II assessment tool from English to Persian. The Iranian version was back-translated and reviewed for language clarity and appropriateness for use in Iranian culture. The tool was then pilot tested with 15 Iranian families with a child who had screened positive for ASD and included parents from different socio-economic backgrounds. Five of the 42 questions were unclear to parents and these items were reworded for greater clarity.

\subsubsection{Recruiting samples}

Three groups of children aged between 3 and 16 years were recruited: those who had been given a diagnosis of autism; those diagnosed as having an intellectual disability and those whose development was considered to be normal. Recruitment took place in four Provinces of Iran in order to achieve a geographical spread.

Children with ASD in the age range 5 to 10 years generally received a confirmed diagnosis from trained diagnosticians from the Iranian Special Education Organisation (see Samadi et al [1] for further details) or were admitted to the ASD special schools based on being at high risk of ASD which meant that they would be re-evaluated one year after their registration. Other children with ASD above or below this age range, had received a confirmed diagnosis from the paediatrician or neurologists based on DSM IV criteria.

All the children with an Intellectual Disability aged 5 to 16 had received an approved diagnosis from ISEO and children under 5 received a confirmation of diagnosis from the paediatrician based on their developmental assessments and clinical presentation (i.e. Down Syndrome or other conditions associated with an intellectual disability).

Parents of children with ASD and ID were recruited from special schools (both public and private) whereas parents of preschool children were recruited from mother and child clinics. The normally developing sample were chosen from mother and child clinics, schools and from membership of the Parents and Teachers Association which has branches in all the cities in Iran.

\subsubsection{Procedure}

All parents were informed about the aims of the study initially through a written notification sent from the clinic or schools but these were repeated verbally when the first author met the parents when their consent to participate was obtained. Parents of children with ASD and children with ID were met individually, the written instructions for completing the scales were explained to them and they were assisted to complete the ratings scales as necessary. Also 30 parents of normally developing children in Alborz province were met personally during eight days in two schools. The remainder of parents whose children were 
developing normally were given the ratings scales at a group meeting and asked to return them within two weeks and $97 \%$ did so.

\subsubsection{Study participants}

In all data was obtained on 532 children: 390 with autism; 55 intellectually disabled and 87 normally developing. Their mean age was 10.5 years (SD 3.1). However those with autism were significantly older (mean age 10.9 yrs) than those in the other two groups (9.4 years). As commonly found with autism; many more boys than girls were identified ( $81 \% \mathrm{v} 19 \%)$; The gender ratio for the children with ID was ( $49 \%$ male v $51 \%$ female) and normally developing (64\% male v $36 \%$ female).

The children were recruited from four provinces in Iran: Tehran (35\%) Alborz (21\%) Razavi Khorasan (25\%) and Western Azerbaijan (19\%). Proportionately more children with ID came from Tehran Province but children with autism and those developing normally came from all four Provinces.

\subsubsection{Item analysis}

In seeking to identify the items that best discriminated the three groups from the 42 items included in the GARS scale the items were arranged into those that the highest percentage of children with autism displayed but with the least percentage of children with intellectual disability and those who were developing normally. The top 16 items were then selected using the following criteria:

- Over one-third of children with ASD showed the behaviour AND

- Normally developing children did not show the behaviour or it was shown by fewer than $12 \%$ of these children AND

- The proportion of children with intellectual disability who showed the behaviour was fewer than half of the proportion of children with a diagnosis of autism.

Our aim was to reflect the range of behaviours that can be indicative of the variation among children with autism and yet maximised their distinctiveness.

Table 1 summarises the percentage of children within each group who were sometimes or frequently observed to show these behaviours. These are ordered by those most commonly seen in children with autism. The subscale from which the item came is also noted.

These 16 items were then tested for their scaling properties. The Chronbach alpha of internal reliability was acceptably high at $0.89(\mathrm{~N}=422)$.

A total score could be calculated for each child on these 16 items with a minimum score of 0 (all items scored as never or rarely seen) and a maximum of 16 (all items scored as sometimes or frequently observed). Table 2 presents the summary statistics for the three groups of children on this computed measure as well as for the total group. With this sample no ceiling effects were present on the scale. 


\begin{tabular}{|c|c|c|c|c|}
\hline Subscale & Item & ASD & $\begin{array}{l}\text { Intellectual } \\
\text { Disability }\end{array}$ & Non-disabled \\
\hline Communication & $\begin{array}{l}\text { Uses gestures instead of speech or uses signs to } \\
\text { obtain objects }\end{array}$ & $67.0 \%$ & $0 \%$ & $2.3 \%$ \\
\hline Social Interaction & $\begin{array}{l}\text { Withdraws, remains aloof or acts stand-offish in } \\
\text { group situations }\end{array}$ & $66.2 \%$ & $21.8 \%$ & $0 \%$ \\
\hline $\begin{array}{l}\text { Stereotyped } \\
\text { behaviour }\end{array}$ & $\begin{array}{l}\text { Makes high-pitched sounds (e.g. eee-eee-eee) or } \\
\text { other vocalizations for self-stimulation }\end{array}$ & $65.4 \%$ & $12.7 \%$ & $6.9 \%$ \\
\hline Social Interaction & $\begin{array}{l}\text { Does not imitate other people when imitation is } \\
\text { required or desirable such as in games or learning } \\
\text { activities }\end{array}$ & $65.0 \%$ & $3.6 \%$ & $0 \%$ \\
\hline Social Interaction & $\begin{array}{l}\text { Stares or looks unhappy or unexcited when } \\
\text { praised humoured or entertained }\end{array}$ & $60.5 \%$ & $0 \%$ & $4.6 \%$ \\
\hline Social Interaction & Laughs, giggles, cries inappropriately & $56.5 \%$ & $21.8 \%$ & $0 \%$ \\
\hline Social Interaction & $\begin{array}{l}\text { Behaves in a unreasonably fearful or frightened } \\
\text { manner }\end{array}$ & $55.6 \%$ & $12.2 \%$ & $0 \%$ \\
\hline Communication & $\begin{array}{l}\text { Does not initiate conversations with peers or } \\
\text { adults }\end{array}$ & $55.4 \%$ & $9.8 \%$ & $11.5 \%$ \\
\hline Social Interaction & $\begin{array}{l}\text { Shows no recognition that a person is present (i.e. } \\
\text { looks through people) }\end{array}$ & $.50 .0 \%$ & $3.6 \%$ & $0 \%$ \\
\hline $\begin{array}{l}\text { Stereotyped } \\
\text { behaviour }\end{array}$ & $\begin{array}{l}\text { Stares at hands, objects or items in the } \\
\text { environment for at least } 5 \text { secs }\end{array}$ & $48.5 \%$ & $16.4 \%$ & $11.5 \%$ \\
\hline Social Interaction & $\begin{array}{l}\text { Avoids eye contact, looks away when someone } \\
\text { looks at him or her }\end{array}$ & $46.7 \%$ & $12.7 \%$ & $4.2 \%$ \\
\hline Communication & $\begin{array}{l}\text { Uses the word I inappropriately e.g. does not say I } \\
\text { to refer to self) }\end{array}$ & $45.7 \%$ & $14.5 \%$ & $0 \%$ \\
\hline $\begin{array}{l}\text { Stereotyped } \\
\text { behaviour }\end{array}$ & $\begin{array}{l}\text { Flicks fingers rapidly in front of eyes for periods of } \\
5 \text { secs or more }\end{array}$ & $43.6 \%$ & $12.7 \%$ & $0 \%$ \\
\hline $\begin{array}{l}\text { Stereotyped } \\
\text { behaviour }\end{array}$ & Flaps hands or fingers in front of face or at sides & $42.4 \%$ & $9.1 \%$ & $0 \%$ \\
\hline $\begin{array}{l}\text { Stereotyped } \\
\text { behaviour }\end{array}$ & $\begin{array}{l}\text { Smells or sniffs objects (e.g. toys, person's hand, } \\
\text { hair) }\end{array}$ & $34.4 \%$ & $3.6 \%$ & $0 \%$ \\
\hline $\begin{array}{l}\text { Stereotyped } \\
\text { behaviour }\end{array}$ & Whirls, turns in circles & $34.1 \%$ & $14.5 \%$ & $4.6 \%$ \\
\hline
\end{tabular}

*Communication items are scored on $\mathrm{N}=422$ for whom these items were rated; otherwise $\mathrm{n}=532$.

Table 1. The percentage of Iranian children in each sample who were sometimes or frequently observed to show the selected behaviours. 


\begin{tabular}{lllllll}
\hline Type of development & $\mathbf{N}$ & Mean & Std. Deviation & Median & Minimum & Maximum \\
\hline $\begin{array}{l}\text { Autism Spectrum } \\
\text { Disorder }\end{array}$ & 294 & 8.31 & 3.71 & 8.00 & 1.00 & 15.00 \\
\hline Intellectual Disability & 41 & 1.41 & 1.67 & 1.00 & .00 & 6.00 \\
\hline Normally developing & 87 & .51 & .76 & .00 & .00 & 2.00 \\
\hline
\end{tabular}

Table 2. Mean, SDs, Median and range scores on 16 items for the three groups ( $N=422)$

The between group differences were statistically significant $(F=252.6 ; \mathrm{p}<0.001)$ as was the variation within each group as indicated by the Standard Deviations with normally developing children showing the least variation and those with autism the most

Using the summary scores it was also possible to check if these indicators varied by age of the child. The Pearson Product Moment correlation was small although significant $r=0.138$ $(p<0.005)$ with older children having higher scores. As regards child's gender, boys had significantly higher scores than girls (Mean 6.46 v 4.72: F=11.06: $\mathrm{p}<0.005$ ). Scores were also higher when fathers were the sole informants (mean 7.69) compared to mothers (mean 5.56) $(\mathrm{F}=4.98: \mathrm{p}<0.01)$. Also those children residing in the Provinces of Tehran and Mashahd (means 6.92 and 6.71) had higher scores than children in two other provinces Alborz (Mean 5.04) and Western Azarbayjan mean 4.61).

A regression analysis was then used to control for the inter-relationships among these variables and with the children's grouping of autism, ID and normal development. Indeed it was children with autism who had the highest Beta scores $(\beta=7.89$ : 95\% Confidence Interval $[\mathrm{CI}]$ 7.25-8.49: $t=24.91 \mathrm{p}<0.001)$ and the effect of child's age and gender were not significant. However children living in Tehran $(\beta=1.53$ : CI 0.75-2.30: $t=3.86 p<0.001)$ and Mashahd Provinces $(\beta=1.45$ : CI 0.60-2.30: $t=3.34 \mathrm{p}<0.001)$ tended to score higher than in the other two provinces.

Finally correlations were computed between the scores on the 16 items with the total scores on the GARS ratings for the three subscales and the total score. All correlations were statistically significant $(\mathrm{p}<0.001)$ but highest with the total score and social interaction subscale and lowest with the communication subscale.

\begin{tabular}{ll}
\hline Correlations $\mathbf{1 6}$ items score with ... & $\begin{array}{l}\text { Pearson Product Moment } \\
\text { Correlations }\end{array}$ \\
\hline Stereotyped behaviours subscale & $r=0.861^{* *}$ \\
\hline Communication & $r=0.445^{* *}$ \\
\hline Social Interactions & $r=0.902^{* *}$ \\
\hline Total score on GARS 42 items & $r=0.903^{* *}$ \\
\hline
\end{tabular}

Table 3. Correlations between 16 item scale and GARS scores 


\subsubsection{Conclusions}

Based on the 42 items included in the GARS Scale, it was possible to identify 16 items based on parental ratings that efficiently discriminated between children with autism and those who were normally developing and those with intellectual disabilities. These items were drawn in the main from the social interaction $(\mathrm{N}=7)$ and stereotyped behaviour subscales $(\mathrm{N}=6)$ with fewer coming from the Communication domain $(\mathrm{N}=3)$. A further paper provides further data on the utility of GARS with an Iranian population and on the sensitivity and specificity of the 16 item as a screening tool [31].

\subsection{Study 2: Diagnosing autism}

In the second study the focus was in identifying the indictors that would distinguish children who were ultimately diagnosed with autism from those who were suspected of having the condition but on further examination were thought not to have autism. To do this, we accessed children's assessments on the Autism Diagnostic Interview-revised (ADI-R). Although widely used by clinicians internationally, this tool has been criticised on the length of time taken to administer and its focus on more severe forms of the condition [42]. However it was the tool chosen by the Iranian Special Education Organisation to assess children who screened positive for autism in the national screening program.

ADI-R takes the form of a structured interview with parents and consists of 93 items arranged in three functional domains: Language/Communication; Reciprocal Social Interactions and Restricted, Repetitive, and Stereotyped Behaviours and Interests. Items are scored for the behaviour that the child has ever showed as well as those showed at present. It is the latter items that were included in this study.

The Persian version ADI-R [32] had been standardised on a sample of 100 children with ASD, 9 children with intellectual disability and 100 normally developing children. The sample age range was from 4 to 14 and they were drawn from different provinces. A Chronbach alpha of 0.85 (for present behaviours) was reported. The test retest reliability on a sample of 33 children (24 with autism and 9 ID) with a 4-6 week interval was 0.99 for items relating to unusual social interaction, 0.99 for Language and Communication and 0.96 for Repetitive and Stereotyped behaviours.

\subsubsection{Procedure}

The ADI-R assessments were obtained for 397 children who had screened positive for autism in the national screening programme for all six-years prior to school entry (see Samadi et al.[1]). The ADI-R Persian version was administered by specialists from the Iranian Special Education Organisation in the form of structured interview with one or both parents supplemented by observations of the child. Also included in this sample were older children who had been admitted to schools for children with ASD, but who needed to be assessed to reconfirm the diagnosis which may have been given by a professional other than those employed by the Iranian Special Education Organisation or by means of other diagnostic tools. 
Following the diagnostic interview, 333 children (84\%) were confirmed in having autism; for $20(5 \%)$ the diagnosis was uncertain and $44(11 \%)$ were thought not to have autism. For the purposes of this study the latter two groups were combined.

\subsubsection{Study participants}

Of the 397 children $80 \%$ were male and $20 \%$ female. Their mean age was 7.3 years (range 5 to 14 years). In all, $32 \%$ were only children and a further $43 \%$ had one sibling with $25 \%$ having two to six siblings. In 23 families (5.8\%), there was another child with a developmental disability although $30 \%$ of families reported having a person with mental or developmental disabilities in the wider family circle.

The mean age of mothers was 35.4 years (range 24 to $53 \mathrm{yrs}$ ) and of fathers 40.8 years (range 25 to 77$)$. Of the mothers, 120 (30.2\%) had completed university education as had 147 fathers (37\%). A further 139 mothers (35\%) and 123 fathers (31\%) had completed high school. The remaining 138 mothers $(27.8 \%)$ and 127 fathers (32\%) had been to middle or elementary school. In 124 families (31\%) the parents were related.

\subsubsection{Item analysis}

As in Study 1, the items relating the children's present behaviours were arranged into those that the highest percentage of children with autism displayed but with the least percentage of children who were thought not to have autism. The top 13 items were then selected so as to reflect the variation among children with autism but also discriminating those with the condition from those unlikely to have it. The following criteria were applied to do this.

- Over $50 \%$ of children with a diagnosis of ASD showed the behaviour AND

- Fewer than $50 \%$ of those children not diagnosed as autism showed the behaviour AND

- The percentage of autism children showing the behaviour was at least double the percentage of those without autism.

The 13 items met these criteria are listed in Table 4 . They are ordered by those most commonly seen in children with autism. The sub-grouping is also noted.

One previous study in Iran had identified the indicators most commonly found in a sample of 61 children (mean age 7 years) assessed clinically assessed as having autism [33]. They were: stereotyped and repetitive behaviours; lack of make-believe play, failure to initiate conversations, use of rituals, motor mannerisms, no spoken language, poor social reciprocity and impaired peer relations. Most of these behaviours are reflected in this study.

The 13 items were tested for their scaling properties and the Chronbach alpha of internal reliability was acceptably high at $0.866(\mathrm{~N}=397)$.

A total score could be calculated for each child on these 14 items with a minimum score of 0 (all items scored as never or rarely seen) and a maximum of 14 (all items scored as observed). Table 5 presents the summary statistics for the two groups of children on this com- 
puted measure as well as for the total group. The differences on scores between the two groups was significant $(\mathrm{F}=238.0 \mathrm{p}<0.001)$.

\begin{tabular}{|c|c|c|c|}
\hline Ref num & Item & $\begin{array}{l}\text { Autism } \\
\mathrm{N}=333 \text { ) }\end{array}$ & $\begin{array}{l}\text { Non-Autism } \\
(\mathrm{N}=64)\end{array}$ \\
\hline Communication 42 & $\begin{array}{l}\text { Does not attempt or limited attempt to express } \\
\text { interest by pointing }\end{array}$ & $80.2 \%$ & $31.7 \%$ \\
\hline Communication 37 & $\begin{array}{l}\text { Mis-uses pronoun ' } I \text { ' and refers to self by name rather } \\
\text { than with pronoun. }\end{array}$ & $80.2 \%$ & $38.3 \%$ \\
\hline Social Interaction 50 & $\begin{array}{l}\text { Uncertain, odd or occasional use of gaze in social } \\
\text { interactions }\end{array}$ & $78.0 \%$ & $28.1 \%$ \\
\hline Repetitive Behaviours 69 & $\begin{array}{l}\text { Play is linked to highly stereotypic use of objects or } \\
\text { attention; interested in infant toys such as music boxes } \\
\text { and rattles,. }\end{array}$ & $73.4 \%$ & $21.9 \%$ \\
\hline Social Interaction 53-2 & No spontaneous sharing or no sharing. & $72.7 \%$ & $21.9 \%$ \\
\hline Social Interaction 59-2 & $\begin{array}{l}\text { Stereotyped, inappropriate, very limited or no } \\
\text { responses to people except parents. }\end{array}$ & $71.6 \%$ & $19.0 \%$ \\
\hline Social Interaction 51-2 & $\begin{array}{l}\text { Little or no smiling at people though may smile at } \\
\text { things; no reciprocal smiling. }\end{array}$ & $68.7 \%$ & $17.2 \%$ \\
\hline Social Interaction 56 & $\begin{array}{l}\text { Little or no coordination of eye gaze and vocalisations } \\
\text { or weakly integrated. }\end{array}$ & $67.0 \%$ & $25.4 \%$ \\
\hline Repetitive Behaviours 68 & $\begin{array}{l}\text { Special or circumscribed interests that can interfere } \\
\text { with social activities }\end{array}$ & $63.6 \%$ & $15.6 \%$ \\
\hline Repetitive Behaviours 77 & $\begin{array}{l}\text { Marked mannerisms of hands and fingers that may or } \\
\text { may not interfere with social activities }\end{array}$ & $62.8 \%$ & $3.1 \%$ \\
\hline Social Interaction 62-2 & $\begin{array}{l}\text { Lack of interest in other children; may watch them but } \\
\text { almost never tries to approach them. }\end{array}$ & $61.9 \%$ & $20.0 \%$ \\
\hline Repetitive Behaviours 71 & $\begin{array}{l}\text { Has one to two unusual sensory interests that may } \\
\text { take major amount of time. }\end{array}$ & $58.3 \%$ & $14.1 \%$ \\
\hline Repetitive Behaviours 78 & Complex and stereotyped bodily movements & $50.8 \%$ & $6.3 \%$ \\
\hline
\end{tabular}

Table 4. The percentage of Iranian children in the two groups who were observed to show the selected behaviours from the ADI-R. 


\begin{tabular}{|c|c|c|c|c|c|c|}
\hline Group & $\mathbf{N}$ & Mean & Std. Deviation & Median & Minimum & Maximum \\
\hline ASD & 333 & 8.65 & 2.63 & 10.00 & .00 & 13.00 \\
\hline Not ASD and uncertain & 64 & 2.97 & 2.89 & 2.00 & .00 & 12.00 \\
\hline Total & 397 & 8.37 & 3.81 & 9.00 & .00 & 13.00 \\
\hline
\end{tabular}

Table 5. Mean, SDs, Median and range scores on 13 ADI-R items for the two groups

Using the summary scores it was also possible to check if these indicators varied by age of the child. The Pearson Product Moment correlation was small although significant $r=-0.162$ indicating that younger children scored more highly on these 13 items $(\mathrm{p}<0.001)$.

However there were no statistically significant differences by child's gender, mother's age, level of education, if the child had siblings, or if there was a another child with developmental problems in the family. This was further confirmed in a regression analysis to control for inter-relationships among the possible predictor variables and with the children's diagnosis. It was children diagnosed with autism who had the highest Beta scores $(\beta=5.59$ : 95\% Confidence Interval [CI] 4.57-6.40: $\mathrm{t}=15.57 \mathrm{p}<0.001$ ) but the child's age was also a significant additional variable $(\beta=-0.20$ : CI $0.03-0.37$ : $\mathrm{t}=2.37$, $\mathrm{p}<0.05)$ with younger children scoring more highly irrespective of their diagnosis.

\subsubsection{Conclusions}

It was possible to identify 14 items on the ADI-R that could reasonably well discriminate between those children who would receive a confirmed diagnosis of autism and those who did not. However these items are also more likely to be found in younger children irrespective of the diagnosis.

\section{Comparison of indicators from study and study 2}

Finally a common set of 'best' indicators could be identified across the two studies although there was some variation in wording and overlap across the two chosen rating scales - see Table 6 . Nonetheless these items reflect the three domains that typify autism although with more emphasis on social interaction and repetitive behaviours than on communication.

However on both scales there were additional items that served to distinguish children with autism (see Table 7) and depending on the intended purpose, these items could be used to supplement those listed in Table 6 for the purposes of screening children for autism (GARS items) or clarifying the diagnosis of autism from other developmental disabilities (ADI-R items). 


\section{Items common to GARS and ADI-R}

\begin{tabular}{|c|c|}
\hline GARS Items & ADI-R items \\
\hline \multirow[t]{2}{*}{$\begin{array}{l}\text { Withdraws, remains aloof or acts stand-offish in group } \\
\text { situations }\end{array}$} & $\begin{array}{l}\text { Lack of interest in other children; may watch them but } \\
\text { almost never tries to approach them. }\end{array}$ \\
\hline & No spontaneous sharing or no sharing. \\
\hline \multirow[t]{2}{*}{$\begin{array}{l}\text { Avoids eye contact, looks away when someone looks at } \\
\text { him or her }\end{array}$} & $\begin{array}{l}\text { Uncertain, odd or occasional use of gaze in social } \\
\text { interactions }\end{array}$ \\
\hline & $\begin{array}{l}\text { Little or no coordination of eye gaze and vocalisations or } \\
\text { weakly integrated. }\end{array}$ \\
\hline $\begin{array}{l}\text { Uses the word I inappropriately e.g. does not say I to refer } \\
\text { to self) }\end{array}$ & $\begin{array}{l}\text { Mis-uses pronoun 'I' and refers to self by name rather than } \\
\text { with pronoun. }\end{array}$ \\
\hline Flaps hands or fingers in front of face or at sides & \multirow{3}{*}{$\begin{array}{l}\text { Marked mannerisms of hands and } \\
\text { not interfere with social activities }\end{array}$} \\
\hline $\begin{array}{l}\text { Flicks fingers rapidly in front of eyes for periods of } 5 \text { secs or } \\
\text { more }\end{array}$ & \\
\hline $\begin{array}{l}\text { Stares at hands, objects or items in the environment for at } \\
\text { least } 5 \text { secs }\end{array}$ & \\
\hline Smells or sniffs objects (e.g. toys, person's hand, hair) & $\begin{array}{l}\text { Has one to two unusual sensory interests that may take } \\
\text { major amount of time. }\end{array}$ \\
\hline Whirls, turns in circles & Complex and stereotyped bodily movements \\
\hline $\begin{array}{l}\text { Stares or looks unhappy or unexcited when praised } \\
\text { humoured or entertained }\end{array}$ & $\begin{array}{l}\text { Little or no smiling at people though may smile at things; } \\
\text { no reciprocal smiling. }\end{array}$ \\
\hline
\end{tabular}

Table 6. Items common to GARS and ADI-R that best discriminated Iranian children with autism

\begin{tabular}{|c|c|}
\hline Items on GARS only & Items ADI-R only \\
\hline Uses gestures instead of speech or uses signs to obtain objects & Does not attempt or limited attempt to express \\
\hline Does not initiate conversations with peers or adults & interest by pointing \\
\hline $\begin{array}{l}\text { Shows no recognition that a person is present (i.e. looks through } \\
\text { people) }\end{array}$ & $\begin{array}{l}\text { Special or circumscribed interests that can interfere } \\
\text { with social activities }\end{array}$ \\
\hline $\begin{array}{l}\text { Does not imitate other people when imitation is required or } \\
\text { desirable such as in games or learning activities }\end{array}$ & $\begin{array}{l}\text { Stereotyped, inappropriate, very limited or no } \\
\text { responses to people except parents. }\end{array}$ \\
\hline $\begin{array}{l}\text { Makes high-pitched sounds (e.g. eee-eee-eee) or other } \\
\text { vocalizations for self-stimulation }\end{array}$ & $\begin{array}{l}\text { Play is linked to highly stereotypic use of objects or } \\
\text { attention; interested in infant toys such as music }\end{array}$ \\
\hline Laughs, giggles, cries inappropriately & boxes and rattles. \\
\hline Behaves in a unreasonably fearful or frightened manner & \\
\hline
\end{tabular}

Table 7. Items particular to GARS and ADI-R that best discriminated Iranian children with autism 


\section{Discussion}

These two studies had a number of strengths. Sizeable samples of children with autism were recruited alongside those who were normally developing and those who had intellectual disability or another form of developmental disorder. In both studies, the selected items discriminated effectively the children with autism. Also the studies were located in Iran; a country on which relatively little published research exists. Equally there are some limitations that need to be acknowledged. No independent verification of the child's diagnosis of autism was possible and reliance was placed on either parental reports or data held by the ISEO. This issue may be of relevance also to children with ID in that some of them may have undiagnosed autism. However even within developed countries, it would have been a costly, not to say difficult undertaking, to obtain independent verification of diagnoses and even more improbable in a country such as Iran.

A further limitation is that the first study was retrospective for parents in that their child had already been diagnosed and hence their ratings on GARS may have been influenced by the increased awareness they had about the indicators of autism which they may not have had prior to the diagnosis. Hence it would be important to replicate the study on a prospective basis especially with parents who had limited contact with professionals or with parents who had lower levels of education. The latter recommendation arises from the finding in Study 1 that parents from two provinces had significantly lower scores on the 16 items. In these two provinces professional services are more limited with fewer parents availing of higher education. These factors were also proposed as reasons for the variations in prevalence rates of autism across Iran that has been previously reported [1].

Nevertheless it would be a major undertaking to repeat the study with an unselected population of children although the existence of the Iranian national screening programme for autism on school entry makes this a possibility for five year olds. An alternative approach is to consider the items identified in these studies as the basis for a referral tool [26]. For example, when concern is expressed about a child by parents or preschool educators, or if a child is already experiencing problems, then these items might serve as a guide for primary health or social care personnel to help them decide as to whether a referral should be made for more specialist assessment for autism.

However the issue of identifying children with autism in other cultures has to be set within a broader context than screening. First increased opportunities need to be provided to parents - and to mothers especially - for them to become more knowledgeable about child development and indicators of potential problems particularly those of relevance to their culture. The desire for increased information about autism is a common request of parents in different cultures [34]. Modern technology provides a cost-effective means for doing this.

Second, the beliefs, knowledge and skills of professions involved in diagnosing developmental problems will need to be expanded in relation to autism so that they can undertake appropriate and thorough assessments of the children and devise relevant intervention programme for them and their families [35]. The development of an indigenous knowledge 
base in relation to autism is especially pertinent as reliance on assessment and intervention tools from other cultures may prove inadequate. Equally there is an onus on professionals in Western countries to become better attuned to the cultural issues they may face with immigrant communities [9] as part of the endeavour to find more effective and efficient tools for the diagnosis of autism spectrum disorders [42].

A third aspect arises from the previous two; namely the relationships between parents and professionals. Recent guidance in relation to autism in the UK, emphases the need for professionals to take seriously parental concerns [36]. Also diagnostic information often has to be obtained through sensitive and careful questioning about the child's typical behaviours in natural settings as well as observations of the child in clinic or school contexts. This is best done through a trusted relationship between parents and professionals. Moreover parents may experience elevated levels of stress and poorer health because of their child's behaviour and will personally benefit from support offered by professional advisers [37]. Thus professionals may need to acquire wider communication and inter-personal skills in order to effectively support families.

These broader issues relating to the identification and diagnosis of autism are not peculiar to Iran and future cross-cultural research would help to identify practical means of addressing them, especially when health, social care and educational resources are limited [38].

The findings also have international application in another sense. They confirm the universality of certain indicators of autism in children; most notably in Iranian culture of repetitive and stereotyped behaviours and atypical social interactions. Items relating to communication and language were apparently less indicative of autism. As yet there is no directly comparable data with that obtained from other cultures although future research might provide this. However studies that have directly compared children in the UK and USA with those from South Korea and Israel [6] found significant differences in the extent to which items relating to socialisation, verbal communication and restricted interests were reported although all were present across the four countries. It is likely that this cultural variation in parental perceptions of autistic traits reflects the behaviours in children that parents value or perceive as being unusual within their particular culture. Future research could usefully explore this proposition further as it may also account for differences between the perceptions of parents from different socio-economic backgrounds.

It is possible though that some of the inter-cultural differences and perhaps the intra-country variation may arise from other environmental factors. Walker et al [39] have identified from international epidemiological studies the key risks that prevent children from attaining their developmental potential, such as lack of cognitive stimulation, intrauterine growth restriction, maternal stress and exposure to societal violence. It is possible that some of these environmental factors could trigger particular behaviour patterns within children that fall within the broad category of autism. To date much energy has been expended on identifying the genetic bases for autism and even if these were to be found, there may well be environmental factors that mediate the genetic disposition for autistic traits. 
Finally cross-cultural research in autism has much to contribute to our wider understanding of this condition and of the factors that may ameliorate its impact on children and families. An essential starting point is to have a common tool for use across countries that not only defines the similarities in children who have the condition but is also sensitive to the cultural variations that may be inherent in its manifestation in varying cultures. These studies in Iran are a contribution to that endeavour and provide a model as to how it could be realised.

\section{Acknowledgement}

Our sincere thanks to Miss Ameneh Mahmoodizadeh from the Iranian Special Education Organisation for her assistance with Study 2.

\section{Author details}

Sayyed Ali Samadi and Roy McConkey

*Address all correspondence to: r.mcconkey@ulster.ac.uk.

Centre for Intellectual and Developmental Disabilities, Institute of Nursing Research, University of Ulster, Newtownabbey, N. Ireland, UK

\section{References}

[1] Samadi SA, Mahmoodizadeh A \& McConkey R. A national study of the prevalence of autism among five-year-old children in Iran. Autism: The International Journal of Research and Practice 2012; 16 (1), 4-15.

[2] Daley, TC. The need for cross-cultural research on the pervasive developmental disorders. Transcultural Psychiatry, 2002; 39 531-550.

[3] Greenfield PM, Ward M \& Jacobs J. You can't take it with you: why ability assessments don't cross cultures. American Psychologist, 1997; 52(10) 1115-1124.

[4] Gilliam JE. Gilliam Autism Rating Scale-Second edition. Austin, Texas: Pro-Ed; 2006.

[5] Lord C, Rutter M \& Le Couteur A. Autism Diagnostic Interview-Revised: A revised version of a diagnostic interview for caregivers of individuals with possible pervasive developmental disorders. Journal of Autism and Developmental Disorders 1994; 24(5) 659-685.

[6] Matson JL, Worley JA, Fodstad JC, Chung K-M, Suh D, Jhin HK, Ben-Itzchak E, Zachor DA, Furniss F. A multinational study examining the cross cultural differences in 
reported symptoms of autism spectrum disorders: Israel, South Korea, the United Kingdom, and the United States of America. Research in Autism Spectrum Disorders 2011; 5 1598-1604.

[7] American Psychiatric Association. Diagnostic and Statistical Manual of Mental Disorders DSM-IV-TR (Text Revision) 4th edition. American Psychiatric Association: Washington, DC; 2000.

[8] Bronfenbrenner, U. The ecology of human development: Experiments by nature and design. Cambridge, MA: Harvard University Press; 1979.

[9] Dyches TT, Wilder LK, Sudweeks RR, Obiakor FR \& Algozzine B. Multicultural issues in autism. Journal of Autism and Developmental Disorders 2004; 34 211-222.

[10] Rogers-Adkinson DL, Ochoa TA \& Delgadom, B. Developing Cross-cultural competence : Serving families of children with significant developmental needs. Focus on Autism and Other Developmental Disabilities 2003; 18 4-8

[11] Wallis, KE \& Pinto-Martin, J. The challenge of screening for autism spectrum disorder in a culturally diverse society. Acta Pædiatrica 2008; 97 539-540.

[12] DeGiacomo A \& Fombonne E. Parental recognition of developmental abnormalities in autism. European Child and Adolescent Psychiatry1998; 7 (3) 131-136.

[13] Daley, TC. From symptom recognition to diagnosis: children with autism in urban Indian. Social Science and Medicine 2004; 58 1323-1335.

[14] Mandell, DS, Wiggins, LD, Carpenter, LA, Daniels, J., DiGuiseppi, C, Durkin, MS et al. Racial/ethnic disparities in the identification of children with Autism Spectrum Disorders American Journal of Public Health 2009; 99 493-498.

[15] Valicenti-McDermott M, Hottinger K, Seijo R, Shulman S. Age at Diagnosis of Autism Spectrum Disorders Journal of Pediatrics 2012; 10.1016/j.jpeds.2012.05.012.

[16] Begeer S, El Bouk S, Boussaid W, Terwogt MM \& Koot HM. Underdiagnosis and Referral Bias of Autism in Ethnic Minorities. Journal of Autism and Developmental Disorders $2009 ; 39: 142-148$.

[17] Williams JG, Higgins JPT \& Brayne CEG. Systematic review of prevalence studies of Autism Spectrum Disorders. Archives of Diseases in Children 2006; 91: 8-15.

[18] Baird G, Simonoff E, Pickles A, Chandler S, Loucas T, Meldrum D and Charman,T. Prevalence of disorders of the autism spectrum in a population cohort of children in South Thames: the Special Needs and Autism Project (SNAP). Lancet 2006; 368 210-215.

[19] Autism and Developmental Disabilities Monitoring Network Principal Investigators (2009) Prevalence of Autism Spectrum Disorders - Autism and Developmental Disabilities Monitoring Network, United States. MMWR. Surveillance summaries, 58 (10), 1-20. Atlanta: Center for Disease Control and Protection; 2006 
[20] Coo H, Ouellette-Kuntz H, Lloyd JEV, Kasmara L, Holden JJA \& Lewis MES. Trends in Autism prevalence: Diagnostic substitution revisited. Journal of Autism and Developmental Disorders 2008; 38 1036-1046.

[21] Fombonne E, Zakarian R, Bennett A, Meng L \& McLean-Heywood D. Pervasive developmental disorders in Montreal, Quebec, Canada: Prevalence and links with immunizations. Pediatrics 2006; 118 (1) 139-50.

[22] Baird G, Charman T, Cox A, Baron-Cohen S, Swettenham J, Wheelwright S. \& Drew, A. Screening and surveillance for autism and pervasive developmental disorders Arch. Dis. Child. 2001;84 468-475.

[23] Baron-Cohen S, Allen J \& Gillberg C. Can autism be detected at 18 months? The needle, the haystack, and the CHAT. British Journal of Psychiatry1992; 161 839-843.

[24] Robins DL, Fein D, Barton ML \& Green, JA. The Modified Checklist for Autism in Toddlers: An initial study investigating the early detection of autism and pervasive developmental disorders. Journal of Autism Developmental Disorders 2001; 31 131144.

[25] Seif Eldin A, Habib D, Noufal A, Farrag S, Bazaid, K, Al-Sharbat M. et al. Use of MCHAT for a multinational screening of young children with autism in the Arab countries, International Review of Psychiatry 2008; 20 (3) 281 - 289.

[26] Allison C, Auyeung B. \& Baron-Cohen S. Toward brief "Red Flags" for autism screening: The Short Autism Spectrum Quotient and the Short Quantitative Checklist in 1,000 Cases and 3,000 Controls. Journal of the American Academy of Child \& Adolescent Psychiatry 2011; 51(2) 202-212.

[27] Lord C \& Corsello C. Diagnostic instruments in autistic spectrum disorders. In: Volkmar FR, Paul R, Klin A, Cohen D. (eds). Handbook of Autism and Pervasive Developmental Disorders. $3^{\text {rd }}$ ed. Vol II. Hoboken, NJ: John Wiley \& Sons; 2005: p730-771

[28] Johnson CP \& Myers SM. Identification and evaluation of children with Autism Spectrum Disorders. Pediatrics 2007; 120 (5) 1183-1215.

[29] Fombonne E. Epidemiology of pervasive developmental disorders. Pediatric Research 2009; 65(6) 591-598.

[30] World Health Organisation. Classification of Mental and Behavioural Disorders ICD-10: Clinical Descriptions and Diagnostic Guidelines. World Health Organisation. Geneva. Switzerland; 1992

[31] Samadi SA \& McConkey R. The utility of the Gilliam Autism Rating Scale for identifying Iranian children with Autism. Paper submitted for publication.

[32] Sasanfar R \& Toloie A. Standardising and Normalizing The Autism Diagnostic Interview- Revised on Iranian population. The Iranian Special Education Organisation. Tehran, The Iranian Special Education Organisation Publication; 2006. 
[33] Ghanizadeh A, Mohammadi MR, Sadeghiyeh T, Shooshtari AA \& Akhondzadeh, S. Symptoms of children with Autism Spectrum Disorder, a clinical sample Iranian Journal of Psychiatry 2009; 4 165-169

[34] Samadi SA, McConkey R \& Kelly G. The information and support needs of Iranian parents of Children with Autism Spectrum Disorders. Early Childhood Development and Care 2011; available online: DOI:10.1080/03004430.2011.616931

[35] Mandell DS, Ittenbach RF, Levy SE, Pinto-Martin JA. (2007) Disparities in diagnoses received prior to a diagnosis of autism spectrum disorder. Journal of Autism and Developmental Disorders 2007; 37(9) 1795-1802.

[36] Baird G, Douglas HR \& Murphy MS. Recognising and diagnosing autism in children and young people: summary of NICE guidance. British Medical Journal 2011; 343:d6360 doi: 10.1136/bmj.d6360.

[37] Samadi SA \& McConkey R. The personal impact on Iranian mothers and fathers who have a child with an Autism Spectrum Disorder. Journal of Intellectual Disability Research 2012 (in press).

[38] Samadi SA, McConkey R \& Kelly G. Enhancing parental wellbeing and coping through a family-centred, short course for Iranian parents of children with an Autism Spectrum Disorder. Autism. The International Journal of Research and Practice 2012; DOI: $10.1177 / 1362361311435156$.

[39] Walker SP, Wachs TD, Grantham-McGregor S, Black MM, Nelson CA, Huffman SL et al. Inequality in early childhood: risk and protective factors for early child development. Lancet 2011; 378: 1325-38.

[40] Lecavalier L. An Evaluation of the Gilliam Autism Rating Scale. Journal of Autism and Developmental Disorders 2005; 35 (6): 795-805.

[41] South M, Williams BJ, McMahon WM, Owley T, Filipek TA, Shernoff E, Corsello C, Lainhart JE, Landa R, Ozonoff, S. Utility of the Gilliam Autism Rating Scale in Research and Clinical Populations. Journal of Autism and Developmental Disorders, 2002; 32 (6): 593-599.

[42] Feinstein A. A History of Autism: Conversations with the Pioneers. New York, Wiley; 2010. 
Chapter 3

\title{
Prevalence of Pervasive Developmental Disorders - Croatia in Comparison with Other Countries of the World
}

\author{
Tomislav Benjak and Gorka Vuletić \\ Additional information is available at the end of the chapter
}

http://dx.doi.org/10.5772/51637

\section{Introduction}

Disabled persons live in every corner of the world and on all social levels. According to UN estimations, disabilities are much more widespread than is believed: at least one in every 10 inhabitants of each country has a certain form of disability, totaling some 450 million people around the world. [1] According to the 2001 census, Croatia had at that time 423891 persons with disabilities (10\% of overall population) [2]. According to the Croatian Disabilities Registry (the Registry), [3], Croatia has 518081 disabled persons (ca. 12\% of overall population). As defined in the Act on the Croatian Disabilities Registry, disability is a limitation or reduction (resulting from damaged health) the capacity to perform a certain physical activity or psychological function normal for a person of a certain age, and refers to the abilities which are manifested in complex activities and behaviors generally accepted as important components of everyday life [4]. Croatia has defined the collection of data on disabled persons in the Act on the Croatian Disabilities Registry (Official Gazette No. 64/01). The Registry includes data on the following types of physical and mental impairments: visual, hearing and speech impairments, impairments of the locomotor, central and peripheral nervous systems, other organs and organ systems, mental retardation, PDDs (autism), mental disorders, as well as data on multiple impairments. PDDs (autism) have been separated as a special disability category. The data on the above disorders is provided by expert evaluation bodies within the education and social welfare system [4]. According to the references, PDD prevalence has registered a continual rise. To illustrate, according to the CDC Report from Atlanta, USA, prevalence numbers 6.6 per 1000 . $[5,6,7,8,9]$. The data on individuals with PDDs in Croatia is registered in the above Registry. However, due to a short history (since 2002), the extent of the potential increase could not be determined. 


\section{Epidemiology of PDDs globally}

\subsection{Classification systems}

For the purpose of learning more about the epidemiology of autism, and, by extension, about the changes in its prevalence, the following part will present two classification systems that have been in international use for many years, together with clearly stated criteria and diagnostic instructions that need to be abided by so as to correctly diagnose autism and PDDs. The two systems are: WHO's ICD-10 (International Statistical Classification of Diseases and Related Health Problems) and DSM (Diagnostic and Statistical Manual of Mental Disorders) by the American Psychiatric Association (APA). ICD-10 defines autism under a three-sign category of F84: Pervasive developmental disorders. This group includes, codes and defines the following: childhood autism (F84.0), atypical autism (F84.1), Rett's syndrome (F84.2), other childhood disintegrative disorder (F84.3), overactive disorder associated with mental retardation and stereotyped movements (F84.4), Asperger's syndrome (F84.5), other pervasive developmental disorders (F84.8), as well as pervasive developmental disorder, unspecified (F84.9) [10]. PDD is also defined by the APA's DSM classification. DSM introduced the term of pervasive developmental disorder for the first time in its third revision from 1980. The term pervasive was selected so as to accentuate the fact that this psychopathological manifestation assumes all of child's spheres of functioning. In DSM Revision III from 1987 PDDs encompass a significantly wider spectrum of symptoms, which has lead to an increased prevalence. The term infantile autism was changed to autistic disorder. All non-autistic pervasive disorders are specially categorized as pervasive developmental disorders not otherwise specified (PDDNOS). Out of a need to maximize the quality of diagnostics and create clearer diagnostic criteria DSM-III has grown into DSM-IV (DSM-IV-TR, APA, 2000). The latter classification calls for an introduction of a new term: autism spectrum disorders (ASDs), which now defines autism [11, 12]. ASDs ultimately include: autistic disorder (AD), Asperger's syndrome (AS) and PDDNOS. An approximation of the two above classification systems is visible in a series of elements. What is significant is that both currently acknowledge a separate category of pervasive developmental disorders, though the two selections of clinical entities encompassed by this category remain diverse. Table 1 outlays nosological categories belonging to PDDs in the ICD-10 and DSM-IV-TR. By analyzing Table 1 a similarity can be noticed between the above classification systems in determining clinical entities belonging to PDDs. Solely hyperkinetic disorder related to mental retardation and stereotyped movements is left out of the DSM-IV-TR [13]. The American Psychiatric Association (APA) has proposed new diagnostic criteria for the fifth edition of the Diagnostic and Statistical Manual of Mental Disorders (DSM-5) for autism. While final decisions are still months away, the recommendations reflect the work of dozens of the nation's top scientific and research minds and are supported by more than a decade of intensive study and analysis. The proposal by the DSM-5 Neurodevelopmental Work Group recommends a new category called autism spectrum disorder which would incorporate several previously separate diagnoses, including autistic disorder, Asperger's disorder, childhood disintegrative 
disorder and pervasive developmental disorder not otherwise specified [14]. Croatia applies ICD-10 codes for PDD diagnoses.

\begin{tabular}{llll}
\hline ICD-10 (WHO, 1992) & & DSM-IV-TR (APA, 2000) \\
\hline F84.0 & Childhood autism & 299.00 & Autistic disorder \\
\hline F84.1 & Atypical autism & & PDDNOS \\
\hline F84.2 & Rett's syndrome & 299.80 & Rett's disorder \\
\hline F84.3 & Other childhood disintegrative disorders & 299.10 & Childhood disintegrative \\
& & & disorder \\
\hline F84.4 & Overactive disorder associated with mental & & \\
\hline F84.5 & retardation and stereotyped movements & \\
\hline F84.8 & Asperger's syndrome & 299.80 & Asperger's disorder \\
\hline F84.9 & Other pervasive developmental disorders & PDDNOS \\
\hline
\end{tabular}

Table 1. Overview of PDD categories in ICD-10 and DSM-IV-TR.

\subsection{Epidemiological studies on autism}

The first epidemiological study of autistic disorder was published in 1966. Viktor Loter, the author, thereby informed the health public that the prevalence of autistic disorder was low, namely four to five autistic children per 10000 inhabitants [15]. Epidemiological studies in 1980s and 1990s registered a significant increase in the prevalence of autistic disorder in different parts of the world. Research done in Wales has shown that autism prevalence in respondents born between 1977 and 1979 was 3.3, while the prevalence in the population born between 1987 and 19899.2 [16]. The prevalence of autism in Iceland grew from 4.2 persons in respondents born between 1974 and 1983 to 13.2 in the population born between 1984 and 1993 [17]. A stable growth in the prevalence of autism during the 1980s and 1990s was also registered in California [18]. Having analyzed a sample of some 300000 respondents born between 1988 and 1996, Honda noted that the incidence of autism grew from an earlier 54 to the present 88 persons, with the highest oscillation being registered in the 1990s [19]. Fombonne conducted a meta-analysis of 32 epidemiological studies on autistic disorder carried out between 1966 and 2001. Data was collected on the prevalence of autistic disorder in 13 different countries on a sample of nearly 5 million people. According to the epidemiological research of the same author, 13 in 10000 liveborn children are born with autism, 3 with Asperger's syndrome, and 60 with PDDs [20]. Similar data are found in studies of other authors $(21,22)$. According to the CDC Report, the prevalence rate for autism numbers 6.6 per 1000 [5, 6, 7, 8]. Autism more commonly appears in boys, accounting for the ratio of 4.3:1 in favor of boys [13]. According the cohort study of children in South Thames prevalance of ASD was about 1\% [23] while in the past year a new epidemological study rates closer to $2 \%$ [24]. Regarding the prevalence of PDDs in EU countries, EUGLOREH 2007 stressed the diversity of the definition of autistic disorder and 
methodology yielding and monitoring prevalence, which is confirmed by Table 2. Specially stressed is the need to approximate the above segments [25].

\begin{tabular}{lcclcc}
\hline \multicolumn{1}{c}{ Area } & Year & $\begin{array}{c}\text { Total children in } \\
\text { age range }\end{array}$ & Criteria used & Age range & $\begin{array}{c}\text { Total rate } \\
\text { per 10 000 }\end{array}$ \\
\hline Aarhus (Denmark) & 1972 & 46500 & Kanner & $2-14$ & 4.3 \\
\hline Camberwell (UK) & 1979 & 34700 & Kanner & $3-17$ & 4.9 \\
\hline Cambridgeshire (UK) & 1999 & 34262 & DSM-IV & $5-11$ & 57 \\
\hline Västerbotten (Sweden) & 1983 & 69600 & Rutter & $0-20$ & 5.6 \\
\hline Göteborg1 (Sweden) & 1984 & 128600 & DSM III & $4-18$ & 4 \\
\hline Göteborg2 (Sweden) & 1986 & 42900 & DSM III & $0-10$ & 7.5 \\
\hline Göteborg3 (Sweden) & 1991 & 40700 & DSM III - R & $4-13$ & 11.5 \\
\hline Rhône (France) & 1989 & 103700 & Rutter & $5-9$ & 10.8 \\
\hline Iceland & 1996 & 38746 & Rutter & $4-12$ & 8.8 \\
\hline Nord-Trondelag (Norwey) & 1998 & n.a. & DSM-IV & $3-14$ & 3.8 \\
\hline Northern Finland & 1997 & 152732 & DSM III - R/DSM-IV & $5-7$ & 20.7 \\
\hline Ireland East & 1997 & 549255 & DSM III /DSM III - R & $0-25$ & 4.94 \\
\hline
\end{tabular}

Table 2. Overview of the prevalence of autistic disorder in EU countries.

The above data leads to the conclusion that the prevalence of autistic disorder in the last 50 years has increased as much as 15 times. Does that mean that the modern world is facing a kind of an autism epidemic? Despite a series of studies conducted to establish a potential cause of the same increase, and, by extension, possible etiology of the disorder, [26, 27, 28, $29,30,31,32,33,34,35,36,37,38]$, many authors continue to believe that the prevalence of autistic disorder in the $21^{\text {st }}$ century remains as it was 50 years ago [13]. The same authors argue that this increment is due to more precise diagnostics, changes in diagnostic criteria and a greater sensitization of the public for the problems of autistic persons. This increase in the prevalence of autism in the past decades was mostly registered in persons of preserved intellectual capacity [39], which would argue that the prevalence in persons with typical autism has remained virtually unchanged.. At this moment it has still not been confirmed or denied that various factors (pollutants, diet, etc.) have a certain effect on the incidence and prevalence of autistic disorder but there is clear scientic evidence that no causal relationship between vaccination and autism [40]. Consequently, no definitive causes of the incurred increase in the prevalence of the above disorder can still be determined [13].

\section{Prevalence of PDDs in Croatia}

Croatia monitors data on persons with PDDs within the scope of the Registry. PDDs are therein separated as a special type of disability and monitored according to the ICD-10 un- 
der the three-sign category of F84. The onset of a systematic epidemiological monitoring of data on PDDs goes back to the introduction of the Registry in 2002. The data on the above disorders is provided by expert evaluation bodies within the education and social welfare system. These expert evaluation bodies hire experts who are to thank for PDD diagnostics. The Registry database, as up until 4 May 2012, contains data on 1109 persons with PDDs, with the largest number of registered under the diagnosis F84.9 (Table 3).

\begin{tabular}{lll}
\hline Pervasive developmental disorders categories & Number of persons \\
\hline F84.0 & Childhood autism & 267 \\
\hline F84.1 & Atypical autism & 57 \\
\hline F84.2 & Rett's syndrome & 24 \\
\hline F84.3 & Other childhood disintegrative disorders & 18 \\
\hline F84.4 & Overactive disorder associated with mental & 30 \\
\hline F84.5 & Asperger's syndrome & \\
\hline F84.8 & Other pervasive developmental disorders & 19 \\
\hline F84.9 & Pervasive developmental disorder, unspecified & 650 \\
\hline Total & & 1109 \\
\hline
\end{tabular}

Table 3. View the number of registered people with pervasive developmental disorders in Croatia, according to ICD-10.

The prevalence of PDDs in the overall population of the Republic of Croatia is about 2-3/10 000, while in children (population aged 0-18) ca. 1/1000, with three times higher incidence in boys. In Register we see increase (2.6x) number of registrated person with ASD in last seven years (304 registrated person until 01.01.2005. and 792 after that date). The largest number of persons with PDDs, namely 759 (70\%), belong to the 5-19 age group (Table 4). Primorsko goranska county and City of Zagreb number the highest prevalence in childhood at just above 1/1000.

\begin{tabular}{|c|c|c|c|c|c|c|c|c|c|c|c|c|c|c|c|}
\hline \multirow{3}{*}{ County of residence } & \multicolumn{15}{|c|}{ Age group } \\
\hline & \multicolumn{2}{|c|}{$0-4$} & \multicolumn{2}{|c|}{$5-9$} & \multicolumn{2}{|c|}{$10-14$} & \multicolumn{2}{|c|}{$15-19$} & \multicolumn{2}{|c|}{$20-29$} & \multicolumn{2}{|c|}{$30-39$} & \multicolumn{2}{|c|}{$40+$} & \multirow[t]{2}{*}{ Total } \\
\hline & $\mathbf{m}$ & f & m & f & m & f & m & f & m & $\mathbf{f}$ & m & $\mathbf{f}$ & m & $\mathbf{f}$ & \\
\hline BJELOVARSKO-BILOGORSKA & 0 & 2 & 4 & 3 & 3 & 2 & 7 & 3 & 2 & 1 & 0 & 0 & 0 & 0 & 27 \\
\hline BRODSKO-POSAVSKA & 0 & 0 & 10 & 1 & 13 & 4 & 9 & 3 & 4 & 5 & 3 & 1 & 1 & 0 & 54 \\
\hline DUBROVAČKO-NERETVANSKA & 2 & 1 & 6 & 0 & 10 & 0 & 0 & 2 & 5 & 3 & 1 & 0 & 0 & 0 & 30 \\
\hline GRAD ZAGREB & 12 & 4 & 48 & 17 & 45 & 11 & 28 & 15 & 33 & 13 & 13 & 5 & 11 & 3 & 258 \\
\hline ISTARSKA & 4 & 0 & 13 & 2 & 13 & 2 & 4 & 5 & 6 & 2 & 5 & 0 & 2 & 1 & 59 \\
\hline KARLOVAČKA & 0 & 0 & 7 & 0 & 6 & 1 & 2 & 1 & 4 & 0 & 1 & 0 & 0 & 0 & 22 \\
\hline
\end{tabular}




\begin{tabular}{|c|c|c|c|c|c|c|c|c|c|c|c|c|c|c|c|}
\hline \multirow{3}{*}{ County of residence } & \multicolumn{15}{|c|}{ Age group } \\
\hline & \multicolumn{2}{|c|}{$0-4$} & \multicolumn{2}{|c|}{$5-9$} & \multicolumn{2}{|c|}{$10-14$} & \multicolumn{2}{|c|}{$15-19$} & \multicolumn{2}{|c|}{$20-29$} & \multicolumn{2}{|c|}{$30-39$} & \multicolumn{2}{|c|}{$40+$} & \multirow[t]{2}{*}{ Tota } \\
\hline & m & f & $\mathbf{m}$ & $f$ & m & $f$ & m & $f$ & m & f & m & $f$ & m & f & \\
\hline KOPRIVNIČKO-KRIŽEVAČKA & 1 & 0 & 7 & 0 & 1 & 0 & 4 & 1 & 3 & 0 & 0 & 0 & 0 & 0 & 17 \\
\hline KRAPINSKO-ZAGORSKA & 1 & 0 & 4 & 0 & 8 & 2 & 9 & 5 & 6 & 1 & 1 & 0 & 1 & 1 & 39 \\
\hline LIČKO-SENJSKA & 1 & 0 & 2 & 0 & 1 & 0 & 1 & 0 & 2 & 0 & 0 & 0 & 0 & 0 & 7 \\
\hline MEĐIMURSKA & 0 & 0 & 10 & 0 & 2 & 3 & 1 & 2 & 2 & 2 & 0 & 0 & 1 & 0 & 23 \\
\hline OSJEČKO-BARANJSKA & 1 & 0 & 20 & 3 & 18 & 3 & 6 & 5 & 12 & 6 & 1 & 3 & 2 & 1 & 81 \\
\hline POŽEŠKO-SLAVONSKA & 0 & 0 & 0 & 0 & 2 & 0 & 1 & 1 & 0 & 1 & 0 & 0 & 0 & 1 & 6 \\
\hline PRIMORSKO-GORANSKA & 0 & 2 & 18 & 7 & 24 & 3 & 15 & 4 & 6 & 2 & 2 & 0 & 1 & 0 & 84 \\
\hline SISAČKO-MOSLAVAČKA & 0 & 0 & 4 & 3 & 8 & 0 & 7 & 1 & 2 & 4 & 1 & 0 & 0 & 0 & 30 \\
\hline SPLITSKO-DALMATINSKA & 7 & 0 & 21 & 4 & 33 & 9 & 18 & 6 & 23 & 8 & 3 & 7 & 8 & 0 & 147 \\
\hline ŠIBENSKO-KNINSKA & 0 & 2 & 5 & 3 & 8 & 1 & 4 & 1 & 7 & 2 & 3 & 1 & 0 & 0 & 37 \\
\hline VARAŽDINSKA & 2 & 0 & 8 & 2 & 6 & 1 & 1 & & 6 & 3 & 0 & 0 & 0 & 0 & 29 \\
\hline VIROVITIČKO-PODRAVSKA & 1 & 0 & 4 & 1 & 4 & 1 & 1 & 0 & 1 & 2 & 0 & 0 & 0 & 0 & 15 \\
\hline VUKOVARSKO-SRIJEMSKA & 1 & 0 & 10 & 3 & 7 & 1 & 4 & 2 & 5 & 2 & 1 & 0 & 1 & 1 & 38 \\
\hline ZADARSKA & 0 & 0 & 17 & 5 & 7 & 0 & 6 & 3 & 5 & 0 & 3 & 0 & 2 & 0 & 48 \\
\hline ZAGREBAČKA & 0 & 0 & 5 & 2 & 11 & 3 & 8 & 4 & 6 & 5 & 4 & 1 & 1 & 1 & 51 \\
\hline Unspecified & 0 & 0 & 1 & 0 & 2 & 0 & 0 & 0 & 3 & 0 & 0 & 0 & 1 & 0 & 7 \\
\hline TOTAL & 33 & 11 & 224 & 56 & 232 & 47 & 136 & 64 & 143 & 62 & 42 & 18 & 32 & 9 & 1109 \\
\hline
\end{tabular}

As amended on 5 May 2012

Table 4. Overview of persons with PDDs (F84) by county of residence, sex and age group.

\section{Conclusion}

All the above leads to the conclusion that there is a difference between the registered prevalences of PDDs in Croatia and other countries of the world. According to the last available CDC data, global prevalence reaches 6.6 per 1000, while in Croatia the rate is ca. 1 in 1000 children (0-18 age group). This great discrepancy, that is under registration of PDDs, could be explained by numerous factors. One of them could be the phenomenon of diagnostic substitution, which was encountered in the USA before 1990 until which time PDDs, as a diagnosis, could not secure children the right to special education. In 1990 the passing of the individuals with Disabilities Education Act was the culmination of a long tradition of state and federal acts promoting the closing of institutions and encouraging state governments to support families in their effort to care for and raise their disabled children in their own homes. Autistic children, especially children with comorbid mental retardation and behavior disorders, who, otherwise, 
would have been institutionalized in the past, consequently, started attending local schools and were included in the data on school prevalence. Before passing of the above Act, children were labeled as having mental retardation, learning disabilities, speech or emotional disorders in order to be granted the right to certain services. The prevalence of autism has, after passing of the same act, experienced a continual growth. This could partly be ascribed to the method of funding the education of autistic persons, as well as to granting rights to supplementary services (e.g. whole-year schooling), as defined by the legal amendment. The effects of these factors on the existing estimations are controversial and illustrative of the reason why the educational administrative data presented in some media-covered studies may affect the display of the number of autistic persons [9]. Whether this phenomenon of diagnostic substitution currently has occurred in Croatia cannot be established with certainty. A proof in favor of its existence could be the fact that there are a total of 141 persons who have official decisions on special education with autism stated under item Orientation list. On the other hand, according to the same decision on special education, 6961 pupils/high school students with unspecified disorders in verbal communication, 3888 pupils/students with mental retardation (whereof some 65\% have light retardation) and a surprising number of 7219 with multiple impairments have a right to special education in regular schools. Some $50 \%$ pupils/students who exercise the right to special education lack the above item of Orientation list so as to escape stigmatization [3]. Each group potentially includes persons with PDDs. A more accurate prevalence of PDDs in Croatia will be available after following the American example and introducing legal regulations to separate autism as a special disability category and granting corresponding rights to persons with PDDs and their families. One should also be reminded of the need of continual professional training of physicians, special education teachers, psychologists and other professionals in new findings in the areas of diagnostics, therapy and rehabilitation of persons with PDDs. By securing this, the advancement of legislation, diagnostics, IT system and the quality of diagnosis recording, as well as sensitizing the public to equation of opportunities for persons with PDDs, will also contribute to advancing the epidemiology of the above disorders.

\section{Author details}

Tomislav Benjak ${ }^{1 *}$ and Gorka Vuletić ${ }^{2}$

*Address all correspondence to: tomislav.benjak@hzjz.hr

1 Croatian National Institute of Public Health

2 School of Public Health, School of Medicine, University of Zagreb, Croatia

\section{References}

[1] http://www.hsuti.hr/EasyWeb.asp?pcpid=32, accessed on 16 Januay 2009. 
[2] "Stanovništvo prema invalidnosti". (2001). Popis stanovništva, kućanstava i stanova 31.ožujak 2001./priredila Mirjana Lipozšćak, Zagreb, Central Bureau of Statistics, Republic of Croatia.

[3] Croatian National Institute of Public Health. (2011). Izvješće o osobama s invaliditetom u RH, http://www.hzjz.hr/epidemiologija/kron_mas/invalidi11.pdf., last accessed on 16 July 2012.

[4] Zakon o Hrvatskom registru o osobama s invaliditetom, NN, br.64/01, Act on the Croatian Disabilities Registry, Official Gazette No. 64/01.

[5] Autism and Developmental Disabilities Monitoring Network Surveillance Year 2000; Centers for Disease Control and Prevention. (2007). Prevalence of autism spectrum disorders. MMWR Surveill Summ., 56(1), 1-11.

[6] Autism and Developmental Disabilities Monitoring Network Surveillance Year 2000; Centers for Disease Control and Prevention. (2007). Prevalence of autism spectrum disorders. MMWR Surveill Summ., 56(1), 1-11.

[7] Van Naarden, Braun. K., Pettygrove, S., Daniels, J., et al. (2007). Evaluation of methodology for collaborative multiple source survaillance network for autism spectrum disorders: Autism and Developmental Disabilites Monitoring Network. 14 sites, United States, 2002., MMWR Surveill Summ., 56(1), 29-40.

[8] Rice, C., Baio, J., Van Naarden, Braun. K., et al. (2007). A public health collaboration for the surveillance of autism spectrum disorders. Paediatr Perinat Epidemiol., 21, 179-190.

[9] Pediatrics. (2007, October). Identification and Evaluation of Children With Autism Spectrum Disorders.

[10] World Health Organization. (1992). International Statistical Classification of Diseases and Related Health Problems,10th Revision Volume , I, 285-287.

[11] American Academy of Pediatrics, Committee on Children With Disabilities. (2001). The pediatrician's role in the diagnosis and management of autistic spectrum disorder in children. Pediatrics, 107, 1221-1226.

[12] American Psychiatric Association. (1994). Diagnostic and Statistical Manual of Mental Disorders (4th Edition), DSM-IV, Washington, DC, American Psychiatric Publishing.

[13] Glumbić, N. (2005). Odrasle osobe s autizmom, Beograd.

[14] American Psychiatric Association. (2012). DSM-5: The Future of Psychiatric Diagnosis. DSM-5 Proposed Criteria for Autism Spectrum Disorder Designed to Provide More Accurate Diagnosis and Treatment, http://www.dsm5.org/Documents/12-03\%20Autism \%20Spectrum\%20Disorders\%20-\%20DSM5.pdf, last accessed on 16 July 2012.

[15] Lotter, V. (1966). Epidemiology of autistic conditions in young children. Social Psychiatry, 1, 124-37. 
[16] Webb, E. J. V., Lobo, S., Hervas, A., Scoudield, J., \& Fraser, W. L. (1997). The changing prevalence of autistic disorder in a Welsh health district. Dev Med Child Neurolo$g y, 39,150-2$.

[17] Maggnusson, P., \& Saemundsen, E. (2001). Prevalence of autism in Iceland. Journal of Autism and Developmental Disorders, 31(2).

[18] Dales, L., Hammer, S. J., \& Smith, N. J. (2001). Time trends in autism and in MMR immunization coverage in California. Journal of American Medical Association, 285(9), 1183-1185.

[19] Honda, H., Shimizu, Y., \& Rutter, M. No effect of MMR withdrawal on the incidence of autism: a total population study. Journal of Child Psychology and Psychiatry, 46(6), 572-579.

[20] Fombonne, E. (2005). Epidemiology of autistic disorder and other pervasive developmental disorders. J Clin Psychiatry, 66(Suppl 10), 3-8.

[21] Baird, G., Charman, T., Baron-Cohen, S., et al. (2000). A screening instrument for autism at 18 months of age: a 6 year follow-up study. Journal of the American Academy of Child and Adolescent Psychiatry, 39, 694-702.

[22] Bertrand, , et al. (2001). Prevalence of autism in a United States population: the Brick Township, New Jersey invastigation. Pediatrics [5].

[23] Baird, G., et al. (2006). Prevalance of Disorders of the Autism Spectrum in a population. Lancet.

[24] Kim, Y. S., et al. (2011). Prevalence of autism spectrum disorders in a total population sample. Am J Psychiatry, 168, 904-912.

[25] European Health Commission. (2007). http://ec.europa.eu/health/ph_information/ dissemination/diseases/autism_1.pdf, last accessed on 16 July 2012.

[26] Volkmar, F. R., \& Pauls, D. (2003, Oct). Autism. Lancet, 362(9390), 1133-41.

[27] Larsson, H. J., Eaton, W. W., Madsen, K. M., Vestergaard, M., Olesen, A. V., Agerbo, E., Schendel, D., Thorsen, P., \& Mortensen, P. B. (2005, May). Risk factors for autism: perinatal factors, parental psychiatric history, and socioecenomic status. AM J. Epidemiol., 161(10), 916-25, discussion 926-8.

[28] Bolton, P. F., Roobol, M., \& Allsopp, Pickles. A. (2001, Sep). Association between idiopathic infantile macrocephaly and autistic spectrum disorders. Lancet., 358(9283), 726-7.

[29] Courchesne, E., Carper, R., \& Akshoomoff, N. (2003, Jul). Evidence of brain overgrowth in the first year of life in autism. JAMA, 290(3), 337-44.

[30] Bolton, P. F., \& Griffiths, P. D. (1997, Feb). Associations of tuberous sclerosis of temporal lobes with autism and atypical autism. Lancet., 349(9049), 392-5. 
[31] Smeeth, L., Cook, C., Fombonne, E., Heavey, L., Rodrigues, L. C., Smith, P. G., \& Hall, A. J. (2004, Sep). MMR vaccination and pervasive developmental disorders: a case-control study. Lancet., 364(9438), 963-9.

[32] Hviid, A., Stellfeld, M., Wohlfahrt, J., \& Melbye, M. (2003, Oct). Association between thimerosal-containing vaccine and autism. JAMA, 290(13), 1763-6.

[33] Fombonne, E., Zakarian, R., Bennett, A., Meng, L., \& Mc Lean-Heywood, D. (2006, Jul). Pervasive developmental disorders in Montreal, Quebec, Canada:prevalence and links with immunizations. Pediatrics, 118, e139-50.

[34] Chih, B., Afrid, S. K., Clark, L., \& Scheiffele, P. (2004, Jul). Disorder-associated mutations lead to functional inactivation of neuroligins. Hum Mol Genet., 13(14), 1471-7, Epub 2004 May 18.

[35] Gillberg, C., \& Wing, L. (1999). Autism : not an extremerly rare disorder. Acta Psychiatrica Scandinavica, 99(6).

[36] Blaxitl, M., et al. (2003). The changing prevalence of autism in California. Commentary on Croen et al. 2002, Journal of Autism and Developmental Disorders, 33(2).

[37] Mc Carthy, P., Fitzgerald, M., \& Smith, M. (1984). Prevalance of Childhood Autism in Ireland. Irish Medical Journal, 77, 129-130.

[38] Fitzgerald, M. (1998, August). A prevalence and psychosocial study of autism in Ireland. Stockholm. 14th International Congress of Child and Adolescent Psychiatry and Allied Professions, 192.

[39] Croen, L. A., Grether, J. K., Hoogstrate, J., \& Selvin, S. (2002). The changing prevalence of autism in California. Journal of Autism and Developmental Disorders, 32(3), 207-215.

[40] Centers for Disease Control and Prevention. (2012). Concerns about Autism-Vaccine Safety, http://www.cdc.gov/vaccinesafety/Concerns/Autism/Index.html, last accessed on 16 July 2012. 


\section{Section 2}

Aetiological Factors - General Overview 

Chapter 4

\title{
Gut Microbiome and Brain-Gut Axis in Autism - Aberrant Development of Gut-Brain Communication and Reward Circuitry
}

\author{
Elizabeth M. Sajdel-Sulkowska and \\ Romuald Zabielski \\ Additional information is available at the end of the chapter \\ http://dx.doi.org/10.5772/55425
}

\section{Introduction}

The function of the gut microbiome and the bidirectional communication between the gastrointestinal tract (GIT) and the brain is increasingly recognized in health and disease and disruption in its composition is not unique to the autistic pathology. However, the bidirectional communication between the gut and the brain, "the gut-brain/brain-gut axis" in autism has been relatively understudied. In general, this communication between gut and brain occurs through a direct neuronal pathway via the vagus nerve, the hormonal pathway of several hormones involved in the regulation of food intake, such as cholecystokinin (CCK), ghrelin, leptin and insulin, and by the immunological signaling pathway involving cytokines. Recent studies indicate that the vagus nerve is involved in immunomodulation as suggested by its ability to attenuate the production of proinflammatory cytokines in experimental models of inflammation (de Jonge and Ullola, 2007). Furthermore, the gut microbiome emerges as a major player not only in the maturation of GIT tissue and the gut brain axis but also in brain maturation, through its effect on both the immune and endocrine systems. Many toxins, toxicants, infectious agents, diet or stress, affect an individual's gut microbiome, which may be especially sensitive during the critical developmental period. Disruption of the developing microbiome may have profound consequences on the developing gut-brain axis including the brain as well as long-term effects on both the physical and psychological development.

This chapter attempts to bridge basic animal studies with clinical findings pertaining to the brain-gut and gut microbiome in autism, and includes a discussion of various strategies in managing autistic symptoms. The discussion also includes possible changes in the reward 
system(s) in autism as a consequence of altered gut microbiome. It is possible that aberrant regulation of the reward system(s) underlines behavioral abnormalities in ASD that could be targeted by future microbiome-targeting therapies.

\section{Effect of perinatal infection and toxicants on the developing brain}

In a continuing quest to understand the nature of gene-environment interactions in ASD, we have recently completed two animal studies examining the effect of perinatal exposure of thimerosal (TM) and lipopolysaccharides (LPS) on the developing rat central nervous system (CNS). Both TM and infections (modeled by LPS exposure) have been implicated in autistic pathology.

Organic mercury compounds are powerful toxicants with a range of harmful neurological effects in humans and animals. TM, which is metabolized to ethyl mercury, has been discontinued as a preservative from infant vaccines but continues to be used in several vaccines including a flu vaccine administered to pregnant and lactating mothers (Sulkowski et al., 2012). Perinatal maternal exposure of two strains of rats, Sprague Dawley (SD) and spontaneously hypertensive (SHR) rats, to thimerosal ( $200 \mathrm{ug} / \mathrm{kg}$ body weight) resulted in both sex- and strain-specific abnormalities in the neonatal rats (Sulkowski et al., 2012). Behavioral abnormalities included delayed startle response and decreased motor learning, with the effects being both sex- and strain-specific. TM exposure also resulted in a significant increase in cerebellar levels of an oxidative stress marker (3-nitrotyrosine) and a decrease in cerebellar type 2 deiodinase, responsible for local intrabrain conversion of thyroxine to the active hormone, 3', 3,5,-triiodothyronine (T3). These effects were associated with an increased expression of several genes negatively regulated by T3 (Sulkowski et al., 2012); Khan et al., 2012) suggesting that perinatal exposure to TM impacts the developing brain at the genetic level. As TM exposure during the postnatal phase coincided with lactation, some of the TM was delivered through the milk to the GIT and may have had an effect on the developing gut microbiome known to be sensitive to heavy metal exposure (Lapanje et al., 2007). This effect may be in part due to competition with zinc resulting in a disturbance in metallothionein function and general chelating capacity for other metals. Thus, at least part of the neonatal impact of TM/mercury could be mediated via its action on the gut microbiome.

In a related study (Xu et al., submitted) we examined the effect of E.coli lipopolysaccharides (LPS) exposure during critical developmental periods on the developing brain employing the animal model of infection. Clinical and epidemiological data suggest that maternal infection during pregnancy and nursing increases the probability of neonatal brain injury and may have a long-lasting impact on brain functions. Maternal infection during pregnancy has been linked to neurological and neurobehavioral disorders in humans such as cerebral palsy (Schendel, 2001; Schendel et al., 2002), neonatal strokes (Ferrieo, 2004), schizophrenia (Watson et al., 1999; Pearce, 2001) and affective disorders (Watson et al., 1999). Animal studies implicate bacterial infection in the pathology of Parkinson's disease (Carvey et al., 2003), and notably, schizophrenia and autism (Patterson, 2002). The triggering signals for cytokine production are endotoxins, major components of the outer membrane of Gram-negative bacteria. 
LPS exposure is one of the most acceptable models of infection; LPS is a sufficient trigger for cytokine production. LPS administered to the pregnant mother are transferred to the fetus through the placenta (Kohmura et al., 2000), and result in increased cytokines levels in the amniotic fluid (Urakabo et al., 2001; Gayle et al., 2004) and the fetal brain (Urakabo et al., 2001). Bacterial infection of lactating mothers also results in an increased level of cytokines in milk (Bannerman et al., 2004). Pretreatment of suckling rats with LPS $(10 \mathrm{mg} / \mathrm{kg}$-day $x 5$ days - the dose which produces weak, transient signs of endotoxemia) results in reduced pancreatic secretion and attenuates acute pancreatitis at adult age due to an increased concentration of the antioxidative enzyme SO in the pancreatic tissue, and to the modulation of cytokines production (Jaworek at al., 2007a, b). This late-effect of LPS is accompanied by dose-dependent reduction of mRNA signal for CCK1 receptor on pancreatic acini as well as modified expression of acinar pro-apoptotic heat shock protein-60 (HSP60) and Bax proteins (Jaworek et al., $2007 b$, 2008). Early postnatal LPS exposure results in inceased expression of toll-like receptor 4 (TLR4) and caspase-3 and 9- proteins in the pancreatic tissue of adult rats (Bonior et al., 2012). These studies clearly indicate that perinatal exposure to LPS may have long lasting consequences on the GIT function, and as expected, though not studied in detail, on the braingut axis.

Perinatal maternal exposure of two strains of rats, SHR or SD rat dams to LPS ( $200 \mu \mathrm{g} / \mathrm{kg}$ body weight) resulted in increased rollover time, delayed startle, and decreased motor learning, with the effects being both strain- and sex-specific. LPS challenge also resulted in a trend towards an increase in cerebellar levels of 3-NT and a decrease in D2 activities in LPS-exposed pups (Xu et al., submitted). Several genes were affected by LPS. Notably Type 2 deiodinase 2 (DIO2) and brain derived neurotrophic factor (BDNF) expression was significantly elevated, while transthyretin (TTR) expression was decreased following LPS exposure. In vitro, acute exposure of cerebellar cultures to LPS resulted in a decreased size of the dendritic area of Purkinje cells. Our data thus demonstrate that perinatal infection impacts the developing cerebellum in a sex- and strain-dependent manner via mechanisms involving oxidative stress, enzymes involved in maintaining local $\mathrm{TH}$ homeostasis, and downstream gene expression. Interestingly, gene changes observed in the brains of LPS-exposed rats were distinct from TMassociated gene effect suggesting that the underlying macromolecular mechanism may be trigger-specific.

Perinatal LPS exposure could have a profound effect on the gut microbiome similar to the effect of repeated treatment with antibiotics. Experiments in healthy mice have shown that disrupting the normal balance of the gut microbiome with antibiotics caused changes in mice behavior and was accompanied by changes in BDNF which has been linked to depression and anxiety (Bercik et al., 2011; Neufeld et al., 2011). Perinatal LPS exposure most likely affects gut motility as suggested by studies of irritable bowel syndrome (IBS), where mild bacterial overgrowth-associated motility disorder can be reversed by antimicrobials (Scarpignato and Pelosini, 1999). Animal studies have also shown that stress can change the composition of the microbiome, where the changes are associated with increased vulnerability to inflammatory stimuli in the GIT. Could gut dysbiosis be induced by recurrent infections? We have observed an increase in neurotrophin levels in the cerebella of rats exposed to LPS (Sajdel-Sulkowska et 
al, unpublished observation) and brain region-specific changes in neurotrophin levels in ASD (Sajdel-Sulkowska et al., 2011). Together these observations suggest that a bacterial infection could trigger the gut microbiome to induce cytokine overproduction leading to an imbalance of brain neurotrophins and contribute to developmental abnormalities.

\section{Effect of environmental perturbations on the developing components of the brain-gut axis: Intestinal permeability, inflammation and gut microbiome}

As indicated above the perinatal development of the CNS structure and function greatly depends on the gastrointestinal GIT function. Little is known about the regulation of embryonic gut epithelium or the effect of prenatal infection. The two key developmental time-points in the regulation of the GIT both occur postnatally, the first few days after birth when all gut digestive functions are launched by first colostrum ingestion and the second at weaning when the digestive system has to modify its function following a switch from mother's milk to solid food. The first time-point is particularly relevant for all mammalian species since it is associated with a complex of dynamic changes in the GIT structure and function leading to a temporary drop in the gut permeability barrier. The secretion of digestive juices (e.g., gastric and pancreatic juice secretion) is obviously close to null before birth. Our studies indicate that in neonatal calves the exocrine pancreas secreted low but measurable amounts of pancreatic juice from the first postnatal day. The secretion responded to colostrum feeding showing a clearcut cephalic phase associated with plasma pancreatic polypeptide (PP) elevation but no gastric or intestinal phase. Further studies involving vagal blockades and pharmacological cholecystokinin receptor antagonists indicated that in neonatal calves pancreatic response to first colostrum feeding is already controlled by a neuro-hormonal mechanism involving CCK and long vago-vagal reflex (Zabielski et al., 2002). Thus, the brain-gut axis control of the exocrine pancreas observed in one- and two-month old milk-fed calves is already present at birth, only the magnitude of the response increases with age reaching its peak at four weeks. The other GIT function in neonatal calves closely associated with the brain-gut axis control include periodic activity of GIT motility (migrating motor complex, MMC) and secretion (pancreatic juice periodic secretion) observed already two-three days after birth along with plasma PP oscillations (Zabielski et al., 2002). Plasma PP, a marker of efferent vagal activity, increased with age indicating that brain-gut axis further develops after birth (and may be potentially sensitive to any environmental modifications).

Intestinal functions in neonates are far more complex than in adults due to intensive developmental processes. The small intestine is one of the fastest body organs to grow in size postnatally as well as the fastest organ in rebuilding its structure. Relatively little is known regarding the development of the large intestine, a major organ inhabited by gut bacteria. At birth, the small intestinal mucosa is lined by enterocytes ready for rapid uptake of colostral macromolecules (open gut). These enterocytes, so called fetal type enterocytes, are equipped with a system of vesicles and cisterns (apical canalicular system, ACS) which 
form large size mobile vacuoles in the upper part of the cell enabling the transfer of intact colostral molecules into the blood (Baintner 2002). Approximately two days after birth, following substantial intake of colostral bioactive substances, the permeability of the gut epithelium is dramatically reduced to macromolecules due to the rapid replacement of fetal type enterocytes by adult type enterocytes, a phenomenon known as gut closure; the cell replacement is made by a receptor-mediated apoptosis involving TGF- $\beta 1$ and TNF- $\alpha$ as mediators (Godlewski et al., 2005, Strzałkowski et al., 2007). Consequently, adult type enterocytes do not contain ACS and large vacuoles. Interestingly, in the gut of neonatal pigs the cells undergoing apoptosis, which is followed by unzipping-zipping events markedly disrupting epithelial cell continuity, are located on the entire length of the villi (Godlewski et al., 2005; see also Fig. 1). In contrast, in adult animals the apoptotic cells are observed only on the villi top, forming a so called extrusion zone. Therefore, in neonates there is a much wider absorptive surface that is potentially subject to environmental stimuli as compared to adults. Though, one population of fetal type enterocytes disappears within the first few days after birth, there is still another population of fetal type enterocytes existing in the lower small intestine, in piglets observed until approximately three weeks after birth. These enterocytes are important for the intracellular digestion of nutrients by lysosomal enzymes, and form digestive vacuoles as a result of non-selective macromolecule uptake. Their massive loss in piglets is observed 2-3 weeks after birth. Nevertheless the protection by intestinal mucus and colostral biologically active peptides and proteins, extensive apoptosis and unzipping-zipping of a great number of epithelial cells at the same time may potentially open epithelial gates for any xenobiotics and harmful bacteria, and thereby facilitate their transfer into blood circulation.

Studies of preterm piglets and intrauterine growth retarded (IUGR) piglets demonstrated that the gut barrier in both groups of animals is open for a longer time than in full-term-appropriate weight piglets. Namely, the lower part of the small intestine of 28 day-old IUGR piglets still contained fetal type enterocytes expressing digestive vacuoles indicating marked delay in gut mucosa development (Mickiewicz et al. 2012 JPP). The gut epithelium continuity in IUGR and preterm neonates is not as finely controlled as in control rats; abnormalities of the gut epithelium may facilitate exposure of the gut and in turn the whole organism to external factors or xenobiotics. It is possible that gut permeability is altered in critically ill children and predispose them to bacterial translocation via a mechanism that creates a hostile environment in the gut and alters the gut microbiome favoring the growth of pathogens that promote bacterial translocation (Papoff et al., 2012).

Recent studies indicate that the vagus nerve is involved in immunomodulation as suggested by its ability to attenuate the production of proinflammatory cytokines in experimental models of inflammation (de Jonge and Ullola, 2007). Furthermore, functional development of the vagus nerve occurs at two stages with the neuronal population in the dorsal motor nucleus of the vagus (DMNV) maturing ahead of the sensory neuron population of the vagal sensory nucleus NTS (Islami et al., 2008). There appears to be an important link between the vagus nerve and memory recall in infancy suggesting that social learning, modulated by autonomic nervous system, may be jeopardized in preterm infants (Haley et al., 2010) 
Fig. 1.

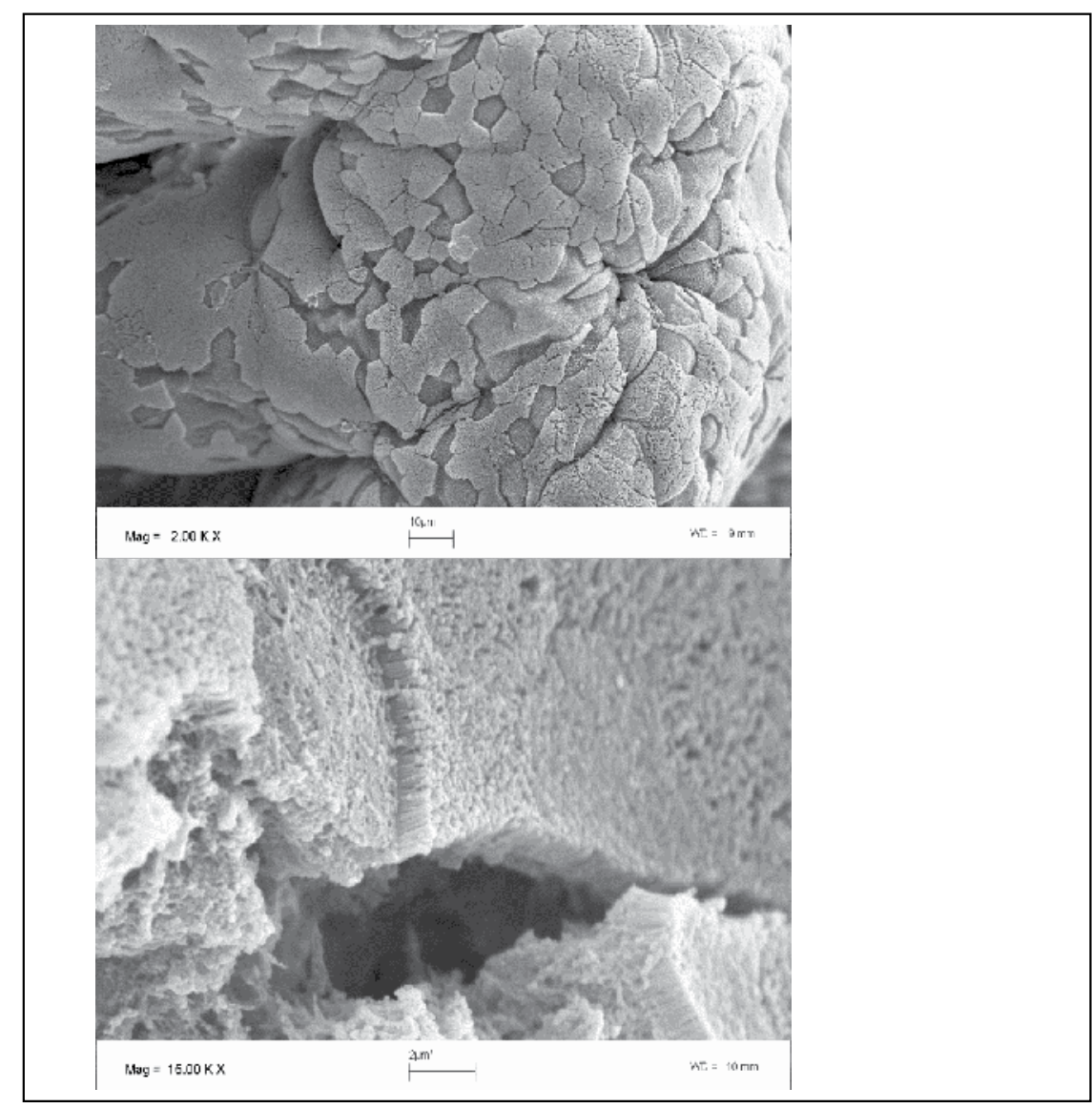

Figure 1. Figkefts Figubeofla Blackettspofeapoptotiaace Hisj|dn. thes epitheliume of neonatal- piglets.

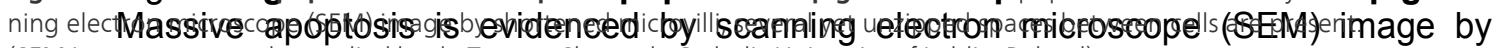

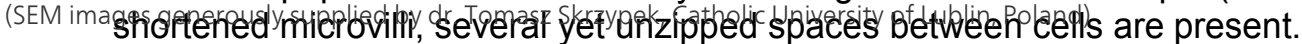

(SEM images generously supplied by dr. Tomasz Skrzypek, Catholic University of In conclusibpin, poblafipdjon of the autonomic nervous system may be delayed in preterm and IUGR animals. Furthermore, delayed development of the GIT in preterm and IUGR animals, including longer gut permeability, facilitates the toxic effect of external factors including bacterial translocation. Furthermore, the immature gut seemingly fails to stimulate the development of the vagus nerve. Importantly, there is some evidence pointing to altered gut permeability (leaky gut) in autism and possibly genetic predisposition to abnormalities in tight junctions in ASD (White, 2003; de Magistris et al, 2010).

\section{Determinants of individual sensitivity of brain-gut axis and gut microbiome to environmental toxins; intrinsic and extrinsic components}

Studies of the human microbiome revealed that even healthy individuals differ remarkably in the microbes of the gut. The gut microbiome is regulated by both extrinsic and intrinsic factors. 
While much of this biodiversity remains unexplained, extrinsic factors such as diet, environment, and early microbial exposure, and the intrinsic factors such as host genetics have been implicated (Human Microbiome Project Consortium, 2012); our own studies (Sulkowski et al., 2012) suggest that sex may play an important role. Diet-derived carbohydrates that are not fully digested in the upper gut are metabolized by bacteria in the human large intestine. These nondigestable carbohydrates influence microbial fermentation and total bacterial number in the colon. Human milk, unlike milk of other mammalian species, contains high amounts of oligosaccharides of yet unknown function, but one can speculate that dietary oligosaccharides may play an important function in the development of the microbiome in human neonates. Evidence exists that the amount and type of nondigestable carbohydrates influence the species composition of the intestinal microbiome. Individual variation in the gut microbiome may, in part reflect differences in dietary intake, but the response of the gut microbiome to dietary change can also differ among individuals (Flint, 2012)

Furthermore, an outcome of the exposure to infectious microbes or their toxins is also influenced by both microbial and host genes. Some host genes encode defense mechanisms, whereas others assist pathogen function. Extensive human diversity in cell lethality dependent on toxin binding and uptake has been observed (Martchenko et al., 2012). Furthermore, there is evidence that individuals may evolve their own specific microbiome (Clayton, 2012).

Results of our recent animal studies (Sulkowski et al, 2012; Khan et al, 2012, Xu et al, submitted; see also Fig. 2) indicate that the sensitivity of the developing CNS to both environmental toxins and infection, are both sex- and rat strain-dependent. It can be extrapolated that the sensitivity of the human microbiome is also sex-dependent. Because of this individual variability in host response it is not surprising that the results of human postmortem studies of ASD brains are difficult to interpret.

\section{Microbiome}

The human GIT harbors a large number (1000 to 1150) of bacterial species and is involved in maintaining homeostasis and well-being. Functions of this microbiome include the regulation of the mucosal immune system, GIT motility, epithelial barrier regulation, gut secretion, digestion and metabolism (Grenham et al., 2011). One of the main functions of gut microbes is to extract nutrients from otherwise indigestible fibers (Tremaroli and Backhed, 2012). The microbiome, absent at birth, is gradually colonized by facultative bacteria and anaerobic bacteria (Grenham et al., 2011).

Several lines of evidence point to both brain-gut axis and gut microbiome abnormalities in autism which are summarized in Fig 3. Children with ASD frequently present a variety of gastrointestinal (GI) symptoms, although some claim that the data supporting increased GI symptomology in autistic children not to be rigorous enough (Erickson et al., 2005). The socalled "bacterial theory" of autism proposes the GIT symptoms are associated with changes in microbial composition and that these changes could be involved in the pathogenesis or progression of several childhood diseases including autism (Somma et al., 2010). 
A. MALES

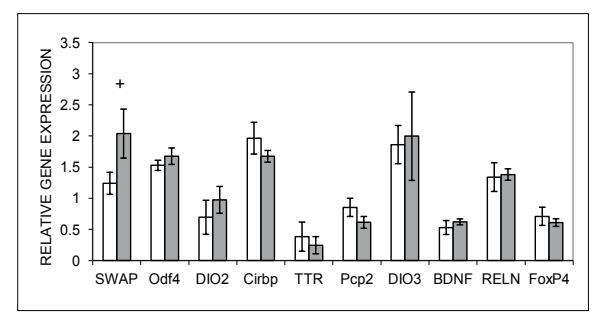

B. FEMALES

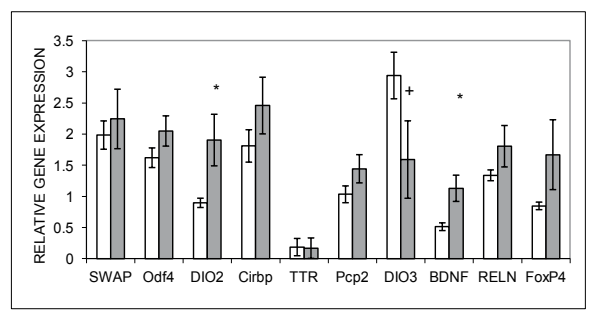

Figure 2. The effect of LPS exposure on cerebellar gene expression. Gene expression was measured by quantitative RT-PCR in cerebellar tissue of rat pups exposed perinatally to LPS $(200 \mu \mathrm{g} / \mathrm{kg} \mathrm{BW})$ and was normalized to cyclophilin A. Panel A: males, Panel B: females. Data are presented as relative gene expression (mean \pm S.E.M.; ${ }^{*}, p<0.05 ;+, p<$ 0.1 ; Xu et al., submitted).

It has been suggested that an abnormal gut microbiome in some ASD children may be due to certain antimicrobial drugs that play a key role in modifying the intestinal bacterial flora and selecting potentially harmful bacteria normally kept at bay by the innate intestinal flora. And so, both Clostridia (Finegold, 2011b) and Desulfovibrio (Finegold, 2011a) have been implicated in autistic pathology. Clostridia form spores and the spores could likely survive antibiotic treatment and subsequently flourish. Desulfovibrio is an anaerobic bacillus that does not produce spores and is resistant to some antibiotics such as cephalosporins used in treatment of common childhood diseases such as ear infections (Finegold, 2011a). An increase in Bacteroides, a decrease in Firmicutes with an overall increase in biodiversity has been observed in IBD, celiac disease and autism (Iebba et al., 2011). An increase in Clostridium histolyticum, a recognized toxin producer with systemic effects, has been observed in fecal samples of ASD children (Parracho et al., 2005). A strong correlation of gastrointestinal symptoms with autism, and a decrease in Bifidobacteria and increase in Lactobacilli, was observed in fecal samples of ASD children (Adams et al., 2011). An association between high levels of intestinal, mucoepithelial-associated Sutterella species and GI disturbances has been detected in intestinal biopsy samples in children with autism (Williams et al., 2012). This latter study may provide the most accurate picture of the gut microbiome as the data were derived directly from the gut.

A response to oral treatment with vancomycin, not absorbed from the GI tract, in autism suggests the importance of gut flora in a disease (Finegold, 2011a). Evidence suggests that ASD may be associated with altered innate immune response; thus children with GI problems may reflect inflammation as a reaction to an endotoxin produced by gut bacteria (Jyonouchi et al, 2002). 


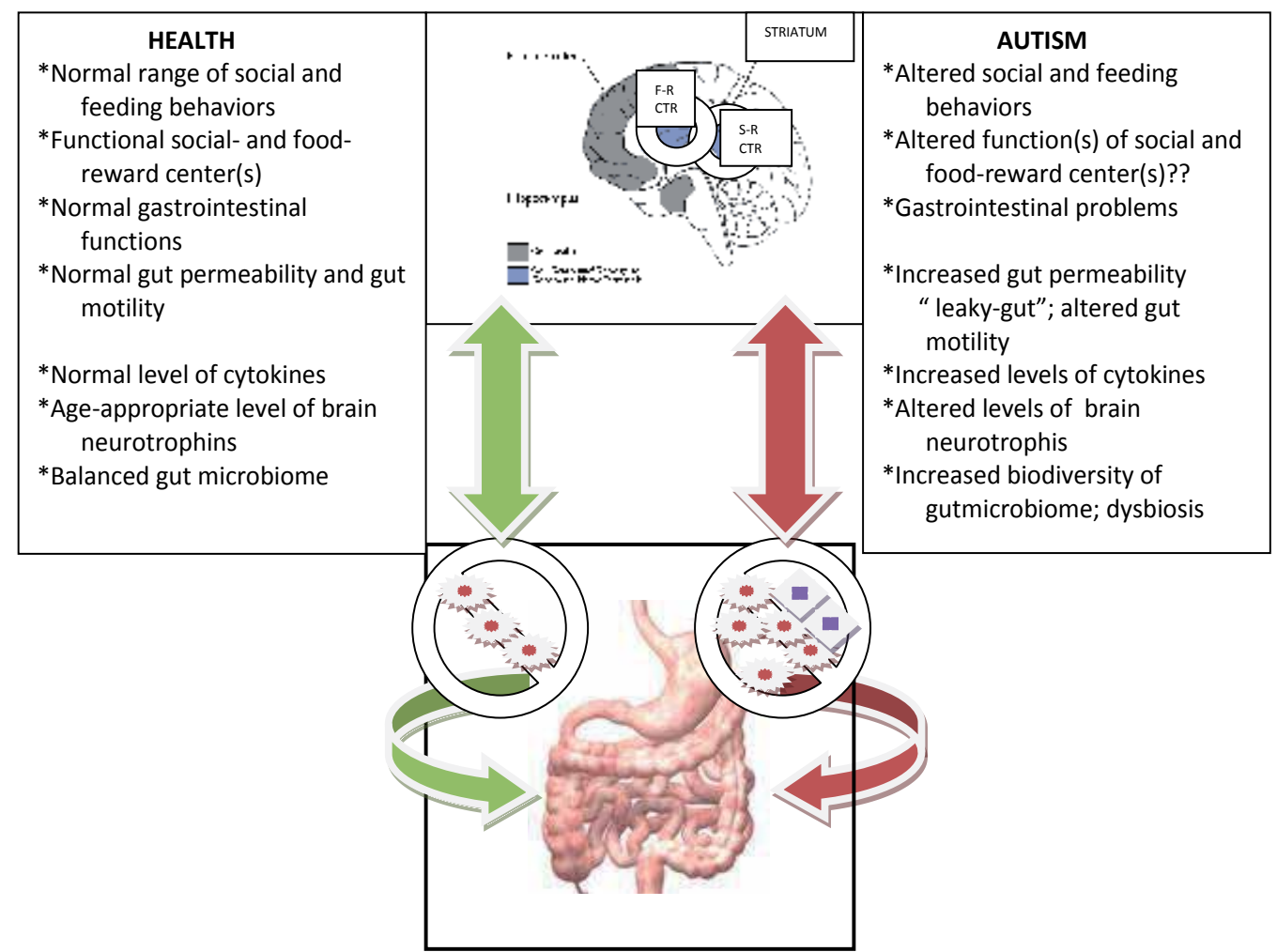

Figure 3. Altered gut microbiome and the brain gut axis in autism. S-R-CTR, social reward center; F-R CTR, food reward center.

Our most recent studies suggest altered expression of ghrelin, the activating enzyme (ghrelin O-acyltransferase, GOAT) and the receptor in several brain areas of autistic children (SajdelSulkowska, unpublished observation). A decrease in ghrelin mRNA has been also observed in the temporal gyrus of Alzheimer patients (Gahete et al., 2010) suggesting ghrelin may contribute to the severity of AD pathology. Since we have measured the levels of ghrelin mRNA, it can be assumed that the changes observed were due to the altered levels of brain ghrelin.

The majority of circulating ghrelin is synthesized by gastric mucosa X/A-like cells in response to negative energy status. These cells are not typical endocrine cells since the oxyntic mucosa cells produce $\mathrm{HCl}$ in the stomach lumen and ghrelin as a hormone. Ghrelin is the most potent orexigenic peptide, and plays an important role in glucose metabolism and also in GIT cytoprotection. In addition to its ability to stimulate appetite, ghrelin stimulates the release of growth hormone release via the growth secretagogue, GHS-R1a receptor. Ghrelin O-acyltransferase, GOAT, is the enzyme that activates ghrelin. The ghrelin/GHS-R/GOAT system may play an important role in metabolic disorders in children (Lim and Korbonits, 2012). In addition to the ghrelin of GIT origin, and the hypothalamus being the main source of brain ghrelin, ghrelin has been detected in the midbrain, hindbrain, hippocampus, spinal cord and 
several organs outside the brain. While the systemic endogenous ghrelin exerts a tonic stimulating effect on hypothalamic CRH (Rucinski et al., 2012), its function in the brain includes the modulation of membrane excitability, control of neurotransmitter release, neuronal gene expression, and neuronal survival and proliferation (Ferrini et al., 2009).

It has been reported that ghrelin of GIT origin interacts with bacterial toxins (Tiaka et al., 2011) and exerts a protective role in experimental colitis; is it possible that the ghrelin of brain origin plays a protective role as well? If so, changes in the level of brain-derived ghrelin could be detrimental to the developing brain.

\section{Existing and emerging therapeutic strategies in autism targeting the gut- brain axis and gut microbiome: Role of individual microbes and dietary amino acids in maintaining gut-brain homeostasis}

Existing therapies targeting the gut microbiome include diet, antibiotics, and probiotics. Dietary restriction, including the removal of dairy casein-containing products, wheat and gluten sources, sugar, chocolate, preservatives, and food coloring have all been found to be therapeutic in autism. Interestingly, dairy casein-containing products stimulate ghrelin (a hunger hormone) and reduce CCK (a satiety agent) production in the periphery and in the brain. Gastrointestinal problems in autism appear to respond to antimicrobial agents. Treatments targeting Candida, and probiotics have been used to reduce disbiosis and control gut permeability (Kidd, 2002). Other strategies include the removal of heavy metals (including mercury) by chelation and sulfur-sulphydryl repletion. Supplementation with dimethylglycine, vitamin B6, magnesium, vitamin B3, C, folic acid, calcium and zinc, cod liver, digestive enzymes, all appear to be beneficial in a number of autistic children (Kidd, 2002). Immune therapies, including pentoxifyllin, immunoglobulin, transfer factors and colostrums appear to work in a limited number of cases,

The initial promising use of secretin, a triggering factor for digestion, in the treatment of autism has been more recently disclaimed. In multiple randomized controlled trials secretin offered no significant benefit (Krishnaswami et al., 2011; Williams et al., 2012).

Abnormalities in the primary pathway for carbohydrate digestion and transporters, involving disaccharidases and hexose transporters, have been reported and found to be accompanied by dysbiosis as evidenced by a decrease in Bactoroidetes and an increase in the ratio of Firmicutes to Bacteroidetes (Williams et al., 2011). These abnormalities respond to probiotic and dietary responses (Williams et al., 2011). Probiotic therapy appears to influence microbiome composition, intestinal barrier function and mucosal immune responses (Critchfield et al., 2011). There is evidence to support alterations of fecal microbiome in autism, and in the majority of cases treatment with vancomycin, an antibiotic that targets gram positive anaerobes and is minimally absorbed by the gut, can improve symptoms (Sandler et al., 2000).

Recently therapies targeting the gut microbiome are emerging as a viable strategy in the treatment of CNS disorders (Forsythe et al., 2010). Preclinical studies of selected probiotics in 
healthy volunteers (Messaoudi et al., 2011) provided encouraging results for further studies exploring the concept of microbial targeting of the GIT under pathological conditions including autism. Individually tailored probiotic formulations, enriched in specific strains of gut bacteria, could one day be used in treatments of ASD even as an adjuvant to other treatments.

\section{Possible connection of gut microbiome and behavior; microbiome and behavioral abnormalities in ASD}

The intestinal microbiome participates in the development of the HPA axis (Sudo et al., 2004) and is critical to the development of appropriate stress response later in life, which occurs during a narrow, critical developmental window. This process involves both the regulation of the levels of brain derived neurotrophic factor (BDNF) and NMDA receptors (Sudo et al., 2004). The microbiome also plays an important role in anxiety-like behavior (Messaoudi et al., 2011), depressive behaviors (Neufield et al., 2011; Messaoudi et al., 2011), but the effects are diminished in vagatomized animals, suggesting either the direct communication between the bacteria and the brain (Bravo et al., 2011) or through the brain-gut axis. The latter possibility is an indirect action of bacteria on an afferent vagal pathway via gut immune, endocrine and enteric nervous system (ENS) controlling mechanisms.

Animal studies have also shown that stress can change the composition of the microbiome, where the changes are associated with increased vulnerability to inflammatory stimuli in the GIT (Gareau et al., 2006); here the microbiome plays an important role in memory dysfunction (Gareau et al., 2011). Stress is known to inhibit gut contraction, one of the crucial defense strategies against bacterial colonization of gut mucosa. Early psychological trauma of maternal separation resulted in persistent mucosal barrier dysfunction in neonatal rats, including host defense to luminal bacteria, by mechanisms involving peripheral CRH receptors (Gareau et al., 2006).

Oral antibiotics disrupt the microbiome and favor environment for opportunistic bacteria. Clostridium tetani, an anaerobic bacillus produces a potent neurotoxin, tetanus neurotoxin (TeNT) that is transported by the vagus nerve from the GI to the CNS. In the brain TeNT disrupts the release of neurotransmitters by the proteolytic cleavage of synaptobrevin, a synaptic vesicle membrane protein. This inhibition may be related to a variety of behavioral deficits characteristic of autism. Some children with autism treated with anti-clostridia antibiotics have shown a reduction in stereotyped behavior (Bolte, 1998).

\section{The role of the reward system in gut-brain communication, the interaction between food-reward and social-reward systems; altered gut- microbiome regulation of the reward loop in autism?}

Autism is characterized by both severe deficits in social interaction and communication and significant eating difficulties with a highly restricted range of food choices (Williams et al., 
2000). It seems logical to hypothesize that altered composition of the gut microbiome under a "leaky gut" condition in autism interferes with the normal activity of the reward circuitry including both social and feeding behavior, as illustrated in Fig. 3. In support of this hypothesis are the neuroimaging, electrophysiological and neurochemical data suggesting a disruption in reward seeking tendencies in ASD, and especially in social contexts (Kohls et al., 2012). It has been proposed that this disruption is caused by abnormalities of the dopaminergicoxytocinergic "wanting circuitry" that includes the ventral striatum, amygdale, and the ventromedial prefrontal cortex (Kohls et al., 2012). Indeed, Individuals with ASD are characterized by low responsiveness to social rewards (Dawson et al., 2005; Schultz, 2005; Neuhaus et al, 2010). Recent studies of the left amygdala and orbito-frontal cortex, which are the main components of the social brain, showed neuronal dysfunctions in these structures in autism (Mori et al, 2012). Furthermore, brain levels of serotonin, the "happy hormone" are regulated by gut bacteria as evidenced by studies involving germ-free animals (Clarke et al., 2012). Abnormalities in blood serotonin levels are consistently altered in a subset of children with ASD.

It is also possible that the abnormalities in vagus nerve functions may further contribute to social deficits in autism (Goetz et al., 2010). ). It is thus of interest (Ito and Craig, 2008) that there is a possibility that the vicerosensory information is sent via the vagus nerve directly to the reward centers. The vagus nerve is involved in our emotional responses and in feelings of compassion as shown in vagal stimulation, suggesting that the social bond is related to the gut-brain axis (Goetz et al., 2010). Studies utilizing single-photon emission tomography (SPET) provide evidence for the limbic system-vagal nerve connection (Barnes et al., 2003). Vagotomy was for decades a method of choice in treating a number of gastric diseases in adults; it would be of interest to address it in context of autistic pathology.

Furthermore, the intestinal microbiome regulates the HPA during both development and adulthood (Sudo et al., 2004) and plays an important role in the stress response. Activation of the HPA axis involves the release of endogenous opioids which are components of the brain reward system (Adam et al., 2007).

In humans, sensory factors, such as taste and smell, have an important role in reward-related feeding (Rolls, 2011); gustatory, olfactory, visual and somatosensory aspects of food are regulated by the orbitofrontal cortex. Environmental cues, as well as cognitive, reward, and emotional factors play an important role in food intake which may override the homeostatic requirements (Berthoud, 2006). Environmental cues regulate endocannabinoid and opioid systems which play an important role in reward-related feeding and have wide receptor distributions within the CNS (Cota et al., 2006). Hypothalamic endocannabinoids increase food intake through a leptin-regulated mechanism. The nucleus accumbens is a key limbic pathway and may be implicated in regulation of hedonistic and homeostatic feeding (Berthoud, 2006). Dopamine appears to be associated with reward-related food intake and with behaviors required to maintain feeding essential for survival (Di Marzo et al., 2001).

The neural circuit mediating reward-related behavior is a complex network that includes the midbrain, substantia nigra, the amygdala, the ventral striatum, the ventromedial prefrontal 
cortex and ventral anterior cingulated cortex with the central relay located in ventral striatum (Kohls et al., 2012).

It is interesting, that the ventral striatum is associated with both social-reward and food-reward circuitry (Adam et al., 2007). Although it is generally assumed that the two centers are separate, the observation of altered sucrose preference and positive correlation with ventral striatum dopamine levels under conditions of social isolation stress in perinatal rats lends support to the speculation of inter-connectivity of the two centers (Brenes and Fornaguera, 2008).

\section{Conclusions}

The "leaky gut" during development may be potentially more vulnerable to environmental insults than the normally developing GIT. Consequently, alterations in the gut microbiome may play an important role in autistic pathology. Evidence is growing that points to an early developmental abnormality in establishing GIT and innate microbial milieu. The gut microbiome, regulated by both intrinsic and extrinsic factors, may be further jeopardized by recurrent infections and/or recurrent use of antibiotics. A developmentally abnormal gut microbiome may in turn affect both the gut-brain axis and brain development and contribute to the etiology of ASD. Abnormalities in the gut-brain axis may further lead to the aberrant development of both the social and the food reward system(s) in autism. Future studies targeting the gut-brain/brain-gut axis in autism and the gut microbiome are warranted, but must take into consideration individual variation in gut microbiomes and intrinsic and extrinsic sensitivities and sex. Results of these studies will likely contribute to our understanding of ASD and advance new and viable therapies.

\section{Author details}

Elizabeth M. Sajdel-Sulkowska ${ }^{1^{*}}$ and Romuald Zabielski

*Address all correspondence to: esulkowska@rics.bwh.harvard.edu

1 Dept. Psychiatry Harvard Medical School and BWH, USA

2 DDept. Physiological Sciences, Warsaw University of Life Sciences, Poland

\section{References}

[1] Adam TC, Epel ES. Stress, eating and the reward system. Physiology and Behavior 2007; 91:449-458. 
[2] Adams JB, Johansen LJ, Powell LD, Quig D, Rubin RA. Gastrointestinal flora and gastrointestinal status in children with autism - comparisons to typical children and correlation with autism severity. BMC Gastroenterology 2011; 11: 22.

[3] Baintner K. Vacuolation in the young. In Biology of the Intestine in Growing Animals, Zabielski R, Gregory PC, Westrom B. (eds). Amsterdam, Elsevier, 2002, pp. 55-110.

[4] Barnes A, Duncan R, Chisholm JA, Lindsay K, Patterson J, Wyper D. Investigation into the mechanisms of vagus nerve stimulation for the treatment of intractable epilepsy, using 99mTc-HMPAO SPET brain images. Eur J Nucl Med Mol Imaging. 2003; 30:301-305.

[5] Bercik P, Denou E, Collins J, Jackson W, Lu J, Jury J, Deng Y, Blennerhassett P, Macri J, McCoy KD, Verdu EF, Collins SM. The intestinal microbiota affects central levels of brain-derived neurotrophic factor and behavior in mice. Gastroenterology. 2011; 141:599-609.

[6] Berthound HR. Homeostatic and non-homeostatic pathways involved in the control of food intake and energy balance. Obesity. 2006; 14:197S-200S.

[7] Bolte ER. Autism and Clostridium tetani. Med Hypotheses. 1998; 51:133-144.

[8] Bonior J, Jaworek J, Kot M, Konturek SJ, Pierzchalski P. Long-lasting effect of infant rats endotoxemia on heat shock protein 60 in the pancreatic acinar cells: involvement of toll-like receptor 4. Int J Inflam. 2012; DOI: 2012:354904. Epub 2012 May 22.

[9] Bravo JA, Forsythe P, Chew MV, Escaravage E, Savignac HM, Dinan TG, Bienenstock J, Cryan JF. Ingestion of Lactobacillus strain regulates emotional behavior and central GABA receptor expression in mouse via the vagous nerve. Proc Natl Acad Sci. doi: 10.1073/pnas.1102999108.

[10] Brenes JC, Fornaguera J. Effects of environmental enrichment and social isolation on sucrose consumption and preference: associations with depressive-like behavior and ventral striatum dopamine. Neurosci Lett. 2008; 436:278-282.

[11] Carvey PM, Chang Q, Lipton JW, Ling Z. Prenatal exposure to the bacteritoxin lipopolysaccharide leads to long-term losses of dopamine neurons in offspring: a potential, new model of Parkinson's disease. Front Biosci 2003; 8:s826-37.

[12] Clarke G, Grenham S, Scully P, Fitzgerald P, Moloney RD, Shanahan F, Dinan TG, Cryan JF. The microbiome-gut-brain axis during life regulates the hippocampal serotonergic system in a sex-dependent manner. Mol Psychiatry. 2012: (Epub 10.1038/mp. 2012.77)

[13] Clayton TA. Metabolic differences underlying two distinct rat urinary phenotypes, a suggested role for gut microbial metabolism of phenylalanine and a possible connection to autism. FEBS Lett. 2012; 586;956-961. 
[14] Cota D, Tschop MH, Horvath TL, Levine AS. Cannabinoids, opioids and eating behavior: the molecular face of hedonism?" Brain Res Rev. 2006; 51;85-107.

[15] Critchfield JW, van Hemert S, Ash M, Mulder L, Ashwood P. The potential role of probiotics in the management of childhood autism. Gastroenterol Res Pract. 2011; 2011:161358.

[16] Dawson G, Webb SJ, Wijsman E, Schellenberg G, Estes A, Munson J, Faja S. Neurocognitive and electrophysiological evidence of altered face processing in parents of children with autism: implications for a model of abnormal development of social brain circuitry in autism. Dev Psychopathol. 2005; 17:679-697.

[17] De Jonge WJ and Ullola L. The alpha7 nicotinic acetylcholine receptor as a pharmacological target for inflammation. British J Pharmacol. 2007; 151:915-929.

[18] de Magistris L, Familiari V, Pascotto A, Sapone A, Frolli A, Iardino P, Carteni M, De Rosa M, Francavilla R, Riegler G, Militerni R, Bravaccio C. Alterations of the intestinal barrier in patients with autism spectrum disorders and in their first-degree relatives. J Pediatr Gastroenterol Nutr. 2010; 51:418-24.

[19] Di Marzo V, Goparaju SK, Wang L et al. Leptin-regulated endocannabinoids are involved in maintaining food intake. Nature 2001; 410:822-825.

[20] Erickson CA, Stigler KA, Corkins MR, Posey DJ, Fitzgerald JF, mcDougle Cj. Gastrointestinal factors in autistic disorder: a critical review. J Autism Dev Disorders 2005; 35:713-727.

[21] Ferriero DM. Neonatal brain injury. N Engl J Med 2004; 351:1985-1995.

[22] Ferrini F, Salio C, Lossi L, Merighi A. Ghrelin in Central Neurons. Neuropharmacol. 2009; 7:37-49.Finegold SM. Desulfovibrio species are potentially

[23] Fingold SM. Desulfovibrio species are potentially important in regressive autism. Med Hypotheses 2011a; 77:270274.

[24] Finegold SM. State of the art : microbiology in health and disease. Anaerobe 2011b; 17:367-368.

[25] Flint HJ. The impact of nutrition on the human microbiome. Nutr Rev 2012; 70:S10S13.

[26] Gahete MD, Rubio A, Cordoba-Chacon J, Gracia-Navarro F, Kineman RD, Avila J, Lugue RM, Castano JP. Expression of the ghrelin and neurotensin systems is altered in the temporal lobe of Alzheimer's patients. J Alzheimers Dis. 2010; 22:819-828.

[27] Gareau MG, Jury J, Yang PC, MacQueen G, Perdue MH. Neonatal maternal separation causes colonic dysfunction in rat pups including impaired host resistance. Pediatr Res. 2006; 59:83-88. 
[28] Gareau MG, Wine E, Rodrigues DM, Cho JH, Whary MT, Philpott DJ, Macqueen G, Sherman PM. Bacterial infection causes stress-induced memory dysfunction in mice. Gut. 2011; 60:307-317.

[29] Gayle DA, Beloosesky R, Desai m, Amidi F, Nunez SE, Ross MG. Maternal LPS induces cytokines in the amniotic fluid and corticotrophin releasing hormone in the fetal rat brain. Am J Regul Integr Comp Physiol 2004; 286:R1024-R1029.

[30] Godlewski MM, Słupecka M, Woliński J, Skrzypek T, Skrzypek H, Motyl T, Zabielski R. Into the unknown--the death pathways in the neonatal gut epithelium. J Physiol Pharmacol. 2005; 56 Suppl 3:7-24.

[31] Goetz JL, Keltner D, Simon-Thomas E. Compassion: an evolutionary analysis and empirical review. Psychol Bull. 2010; 136:351-374.

[32] Grenham S, Clarke, G, Cryan JF, Dinan TG. Brain-gut-microbe communication in health and disease. Front Physiol. 2011; 2:94-

[33] Haley DW, Grunau RE, Weinberg J, Keider A, Oberlander TF. Physiological correlates of memory recall in infancy: vagal tone, cortisol, and imitation In Praterm and full-term infants AT 6 months. Infant Behav Dev. 2010; 33:219-234.

[34] Human Microbiome Project Consortium. A framework for human microbiome research. Nature 2012; 486:215-221.

[35] Iebba V, Aloi m, Civitelli F, CucchiaraS. Gut microbiota and pediatric disease. Dig Dis. $2011 ; 29: 531-539$.

[36] Islami H, Shabani R, Bexheti S, Behluli I, Sukalo A, Raka D, Koliqi R, Haliti N, Dauti $\mathrm{H}$, Krasniqi S, Disha M. In vitro examination of ontogenesis of developing neuronal cells in vagal nuclei in medulla oblongata in newborns. Bosn J Basic Med Sci. 2008; 8:381-385.

[37] Ito S, Craig AD. Striatal projections of the vagal-responsive region of the thalamic parafascicular nucleus in macaque monkeys. J Comp Neurol. 2008; 506; 301-327.

[38] Jaworek J, Konturek SJ, Macko M, Kot M, Szklarczyk J, Leja-Szpak A, Nawrot-Porabka K, Stachura J, Tomaszewska R, Siwicki A, Pawlik WW. Endotoxemia in newborn rats attenuates acute pancreatitis at adult age. J Physiol Pharmacol. 2007a; 58:131-47.

[39] Jaworek J, Nawrot-Porabka K, Leja-Szpak A, Szklarczyk J, Macko M, Bonior J, Stachura J, Konturek SJ, Pawlik WW. Exposition of newborn rats to bacterial endotoxin impairs pancreatic enzyme secretion at adult age. J Physiol Pharmacol. 2007b; 58:287-302.

[40] Jaworek J, Leja-Szpak A, Nawrot-Porabka K, Bonior J, Szklarczyk J, Kot M, Konturek SJ, Tomaszewska R, Pawlik WW. Effect of neonatal endotoxemia on the pancreas of adult rats. J Physiol Pharmacol. 2008; 59 Suppl 4:87-102. 
[41] Jyonouchi H, Sun S, Itokazu N. Innate immunity associated with inflammatory responses and cytokine production against common dietary proteins in patients with autism. Neuropsychobiology 2002; 46:76-84.

[42] Khan A, Sulkowski ZL, Chen T, Zavacki AM, Sajdel-Sulkowska EM. Sex-dependent changes in cerebellar thyroid hormone-dependent gene expression following perinatal exposure to thimerosal in rats. Khan J Physiol Pharmacol. 2012; 63:277-83.

[43] Kidd PM. Autism, an extreme challenge to integrative medicine. Part 2; medical management. Altern Med Rev. 20027:479-499.

[44] Kohls G, Chevallier C, Rrioani V, and Schultz RT. Social 'wanting' dysfunction in autism: neurobiological underpinning and treatment intervention. J Neurodev Disorders. 2012; 4:10.

[45] Kohmura Y, Kirikae T, Kirikae F, Nakano M, Sato I. Lipopolysaccharide (LPS)-induced intra-uterine fetal death (IUFD) in mice is principally due to maternal cause but not fetal sensitivity to LPS. Microbiol Immunol. 2000 ; 44 :897-904.

[46] Krishnaswami S, McPheeters ML, Veenstra-Vanderweele J. A systematic review of secretin for children with autism spectrum disorders. Pediatrics. 2011; 127:1322-1325.

[47] Lim CT, Korbonits M. Paediatric endocrine aspects of ghrelin. Paediatr Endocrinol Rev 2012; 9:628-638.

[48] Matchenko M, Candille SI, Tang H, Cohen SN. Human genetic variation altering anthrax sensitivity. Proc Natl Acad Sci USA 2012; 109:2972-2977.

[49] Messaoudi M, Lalonde R, Violle N, Javelot H, Desor D, Nejdi A, Bisson JF, Rougeot C, Pichelin M, Cazaubiel M, Cazaubiel JM. Assessment of psychotropic-like properties of probiotic formulation (Lactobacillus helveticus R0052 and Bifidobacterium longum R0175) in rat and human subjects. Br J Nutr. 2011; 105:755-764.

[50] Mickiewicz M, Zabielski R, Grenier B, Le Normand L, Savary G, Holst JJ, Oswald IP, Metges CC, Guilloteau P. Structural and functional development of small intestine in intrauterine growth retarded porcine offspring born to gilts fed diets with differing protein ratios throughout pregnancy. J Physiol Pharmacol. 2012; 63:225-39.

[51] Mori K, Toda Y, Ito H, Mori T, Goji A, Fujii E, Miyazaki M, Harada M, Kagami S. A proton magnetic resonance spectroscopic study in autism spectrum disorders: Amygdala and orbito-frontal cortex. Brain Dev. 2012; doi: 10. 1016/j.braindev. 2012.09.016

[52] Neufeld KM, Kang N, Bienenstock J, FosterJ. Reduced anxiety-like behavior and central neurochemical change in germ-free mice. Neurogastroenterol Motil. 2011; 23:255-264.

[53] Neuhaus E, Beauchaine TP, Bernier R. Neurobiological correlates of social functioning in autism. Clin Psychol Rev. 2010; 30:733-748. 
[54] Papoff P, Caccarelli G, d'Ettorre G, Cerasaro C, Caresta E, Midulla F, Moretti C. Gut microbial translocation in critically ill children and effects of supplementation with pre- and pro biotics. Int J Microbiol. 2012; 29012:151393.

[55] Parracho HM, Bingham MO, Gibson GR, McCartney AL. Differences between gut microflora of children with autistic spectrum disorders and that of healthy children. J Med Microbiol 2005; 54:987-991.

[56] Patterson PH. Maternal infection: window on neuroimmune interactions in fetal brain development and mental illness. Curr Opin Neurobiol. 2002; 12:115-118.

[57] Pearce BD. Schizophrenia and viral infection during neurodevelopment: a focus on mechanisms. Mol Psychiatry 2001; 6:634-646.

[58] Rolls ET. Taste, olfactory and food texture reward processing in the brain and obesity. Intern J Obesity. 2011; 35:550-561.

[59] Rucinski M, Ziolkowska A, Szyszka M, Hochol A, Malendowicz LK. Evidence suggesting that ghrelin O-Aceyl transferase inhibitor acts AT the hypothalamus to inhibit hypothalamo-pituitary-adrenocortical axis function in the rat. Peptides. 2012; 35:149-159.

[60] Scarpignato C, Pelosini I. Management of irritable bowel syndrome: novel approaches to the pharmacology of gut motility. Can J Gastroenterol. 1999; 13 Suppl A: 50A-65A.

[61] Schendel DE. Infection in pregnancy and cerebral palsy. J Am Med Womens Assooc. 2002; 56:105-108.

[62] Schendel DE, Schuchat A, Thorsen P. Public health issues related to infection in pregnancy and cerebral palsy. Ment Retard Dev Disabilit Res Rev 2001; 8:39-45.

[63] Schultz RT. Developmental deficits in social perception in autism: the role of amygdale and fusiform face area. In J Dev Neurosci 2005; 23:125-141.

[64] Sajdel-Sulkowska EM, Xu M, McGinnis W, Koibuchi N. Brain region-specific changes in oxidative stress and neurotrophin levels in autism spectrum disorders (ASD). Cerebellum 2011; 10:43-48.

[65] Somma F, Castagnola R, Bollino D, Marigo L. Oral inflammatory process and general health. Part 1: The focal infection and the oral inflammatory lesion. Eur Rev Med Pharmacol Sci. 2010;14:1085-95.

[66] Strzałkowski AK, Godlewski MM, Hallay N, Kulasek G, Gajewski Z, Zabielski R. The effect of supplementing sow with bioactive substances on neonatal small intestinal epithelium. J Physiol Pharmacol. 2007; 58 Suppl 3:115-22.

[67] Sudo N, Chida Y, Aiba Y, Sonoda J, Oyama N, Yu XN, Kubo C, Koga Y. Postnatal microbial colonization programs the hypothalamic-pituitary-adrenal system for stress response in mice. J Physiol. 2004; 558:263-275. 
[68] Sulkowski ZL, Chen T, Midha S, Zavacki AM, Sajdel-Sulkowska EM. Maternal thimerosal exposure results in aberrant cerebellar oxidative stress, thyroid hormone metabolism, and motor behavior in rat pups; sex- and strain-dependent effect. Cerebellum 2012; 11:575-586.

[69] Tiaka EK, Manolakis AC, Kapsoritakis AN, Potamianos SP. Unraveling the link between leptin, ghrelin and different types of colitis. Annals of Gastroentrology 2420-28.

[70] Tremaroli V, Bäckhed F. Functional interactions between the gut microbiota and host metabolism. Nature 2012; 489:242-249.

[71] Urakubo A, Jarskog Lf, Lieberman JA, Gilmore JH. Prenatal exposure to maternal infection alters cytokine expression in the placenta, amniotic fluid, and fetal brain. Schizophr Res 2001; 47:27-36.

[72] Watson JB, Mednick SA, Huttunen M, Wang X. Prenatal teratogens and the development of adult mental illness. Dev Psychopathol. 1999; 11:457-466.

[73] White JF. Intestinal pathophysiology in autism. Exp Biol Med (Maywood).2003; 22:639-649.

[74] Williams BL, Hornig M, Parekh T, Lipkin WI. Application of novel PCR-based methods for detection, quantitation, and phylogenic characterization of Sutterella species in intestinal biopsy samples from children with autism and gastrointestinal disturbances. Mbio. 2012; 3. E00261-11.

[75] Williams K, Wray JA, Wheeler DM. Intravenous secretin for autism spectrum disorders (ASD). Cochrane Database Syst Rev. 2012; 4:

[76] Williams PG, Dalrymple N, Neal J. Eating habits of children with autism. Pediatr Nurs. 2000; 26:259-264.

[77] Xu M, Sulkowski ZL, Parekh P, Khan A, Chen T, Midha S, Iwasaki N, Koibuchi N, Zavacki AM, Sajdel-Slkowska EM. Effects of perinatal lipopolysaccharide (LPS) exposure on the developing rat cerebellum; modeling the effect of maternal infection on the developing human CNS Cerebellum (submitted).

[78] Zabielski R, Morisset J, Podgurniak P, Romé V, Biernat M, Bernard C, Chayvialle JA, Guilloteau P. Bovine pancreatic secretion in the first week of life: potential involvement of intestinal CCK receptors. Regul Pept. 2002; 103:93-104. 

Chapter 5

\title{
Why There Is no Link Between \\ Measles Virus and Autism
}

\author{
Stephen A. Bustin \\ Additional information is available at the end of the chapter \\ http://dx.doi.org/10.5772/52844
}

\section{Introduction}

A report published in the Lancet in 1998 described the case histories of 12 previously normal children who developed symptoms of autism or inflammatory bowel disease after having received the measles, mumps, and rubella (MMR) vaccine [1]. This paper formed the basis for Andrew Wakefield's subsequent claim to have identified a new type of gastrointestinal disease, termed autistic enterocolitis. Despite never explicitly asserting a link between the MMR vaccine and this supposedly new, regressive form of autism, the paper sparked a major health scare in the United Kingdom. It is probable that the uncertainty and controversy surrounding the relationship between measles and autism contributed to the fact that in 2004/05, about 1.9 million school children and 300,000 pre-school children were recorded as incompletely vaccinated against measles in England, including more than 800,000 children completely unvaccinated. Based on this, approximately 1.3 million children aged 2-17 years were susceptible to measles [2]. In 2006, a 13-year old boy, who had not received the MMR vaccine, became the first person in the UK for 14 years to die of measles and as a result of almost a decade of low MMR vaccination coverage across the UK, by 2008 the disease had once again become endemic.

In 2010 the Lancet fully retracted the 1998 publication from the public record, stating that it had "become clear that several elements of the 1998 paper by Wakefield et al are incorrect, contrary to the findings of an earlier investigation". The circumstances surrounding this publication were subject to an extensive investigation and received a huge amount of publicity. Wakefield was found guilty of serious professional misconduct over the way he carried out his research and was struck off the medical register in 2010. A long statement released on 24 May 2010 includes the following key statements: 
- "In all the circumstances and taking into account the standard which might be expected of a doctor practising in the same field of medicine in similar circumstances in or around 1996-1998, the Panel concluded that Dr Wakefield's misconduct not only collectively amounts to serious professional misconduct, over a timeframe from 1996 to 1999, but also, when considered individually, constitutes multiple separate instances of serious professional misconduct. Accordingly the Panel finds Dr Wakefield guilty of serious professional misconduct" and
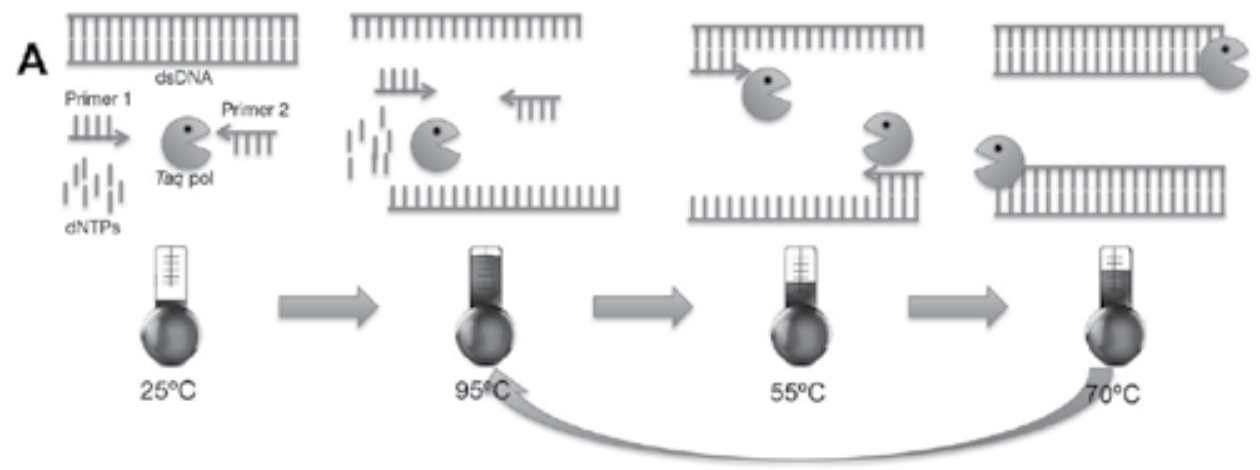

B

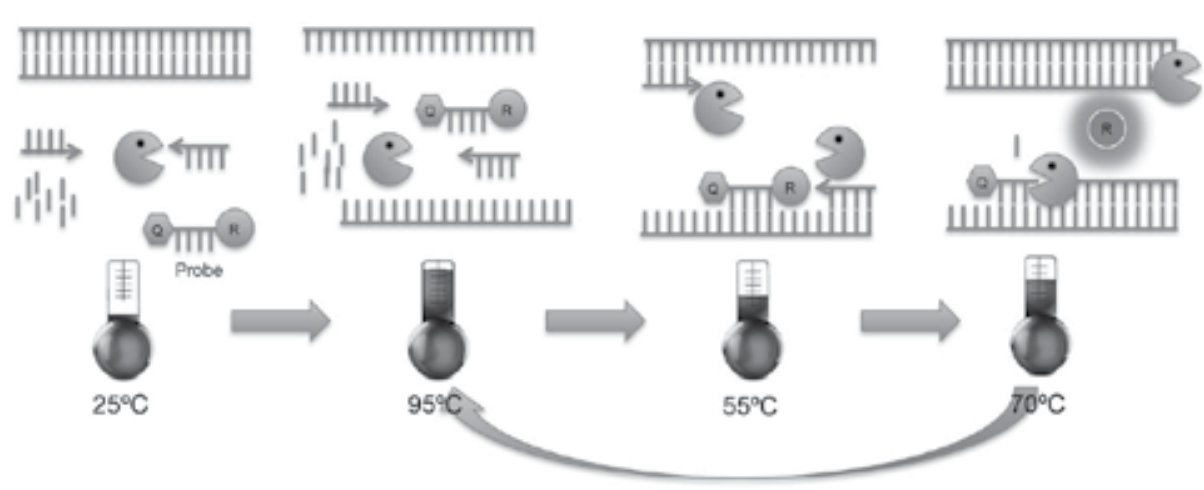

Figure 1. The polymerase chain reaction, a method for copying the same stretch of DNA several million-fold. A. A PCR reaction consists of double stranded DNA, two short DNA molecules ("primers") whose sequence is complementary to opposite strands of the DNA, a DNA synthesis enzyme ("Taq polymerase") and four nucleotide building blocks ("dNTPs"). The reaction mixture is heated to $95^{\circ} \mathrm{C}$ to dissociate the sample DNA strands, then cooled to around $55^{\circ} \mathrm{C}$ to allow the two primers to bind to their targets on the individual strands. Next, Taq polymerase makes two new strands of DNA at its optimal temperature of around $70^{\circ} \mathrm{C}$, using the original strands as templates, hence duplicating the original DNA. This procedure is repeated many times, leading to more than one billion exact copies of the original DNA segment. These can be detected by running the samples on a gel and staining with a DNA-binding dye. B. qPCR obviates the need for gel electrophoresis by using fluorescence to detect copied DNA. The qPCR method used for the detection of MeV uses a target-specific DNA molecule ("probe") that has a fluorescent dye at one end (R) and a quencher $(\mathrm{Q})$ on the other. In the absence of target, the quencher prevents the dye from emitting light. In the presence of target, the probe binds to its target and is degraded by the Taq polymerase. This separates the fluorescent label and the quencher and so results in the emission of light. Both the PCR and light detection are automated and detected in a single step by a dedicated instrument. 
- "Accordingly the Panel has determined that Dr Wakefield's name should be erased from the medical register. The Panel concluded that it is the only sanction that is appropriate to protect patients and is in the wider public interest, including the maintenance of public trust and confidence in the profession and is proportionate to the serious and wide-ranging findings made against him" [3].

There was far less publicity about the attempts to use molecular techniques to corroborate a link between measles virus $(\mathrm{MeV})$ and "autistic enterocolitis". The major technique used was the fluorescence-based real-time polymerase chain reaction (qPCR), a ubiquitous technique used for the sensitive and specific detection of DNA (Figure 1).

\section{Technology and target}

\section{1. $q P C R$}

A key attraction of qPCR technology is its apparent simplicity: an assay consisting of combining oligonucleotides, PCR enzyme and buffer with a nucleic acid template to produce a $\mathrm{qPCR}$ reaction is perceived as undemanding. This practical simplicity is complemented by the absence of any requirement for post-assay handling, as well as the development of user-friendly data analysis software that makes data generation and visualisation in the shape of amplification plots remarkably simple. Indeed, qPCR is often described as a mature technology and as the "gold standard" for nucleic acid quantification. Whilst it is true that the technology is capable of exquisite sensitivity and specificity, coupled with high reproducibility and accuracy, it is essential to understand that qPCR assays are made up of numerous, often divergent protocols that use different instruments, enzymes, buffers and non-identical targets.

Whilst qPCR is the method of choice for the detection of DNA, the enzyme (Taq polymerase) used to copy the DNA template does not work well with RNA. Hence, if the amplification target is RNA, as it is for MeV, an enzymatic RNA-to-DNA conversion (reverse transcription, RT) step must be carried out before the DNA copying step can specifically amplify the target of interest. This variant of the qPCR is termed reverse-transcription (RT)-qPCR and the principle of this reaction is simple: RNA is reverse transcribed into single stranded DNA, either in a separate reaction or as a "one tube" assay that uses a different enzyme (Tth polymerase), as was the case with the experiments discussed below. There are several detection chemistries, but in this case a very specific and widely used probe-based method was used (Figure 1B). Importantly, the use of fluorescent reporter molecules permits concurrent target amplification, detection and quantification as the assay proceeds [4]. Fluorescence is detected using dedicated qPCR instruments, which have (i) a controllable heating block that can hold a variety of temperatures and rapidly change between them, (ii) an excitation light source to excite the fluorochrome, (iii) a detector to register photon emissions and (iv) software that allows analysis of the data. Fluorescence emissions are collected from each sample tube and the levels of background fluorescence detected by the fluorimeter module of the qPCR instrument are established, providing a baseline that defines the sensitivity of the in- 
strument. Instrument-specific algorithms are used to define a fluorescence threshold for each sample. Finally, the algorithm searches the data from each sample for a point that exceeds the baseline and plots a characteristic amplification plot.

Increases in fluorescent signal are proportional to the amount of DNA produced during each PCR cycle and produce a characteristic quantification cycle $(\mathrm{Cq})$ for every test. As a result, the more initial target there is, the sooner the instrument can detect the fluorescence and the lower the Cq. Conversely, a higher Cq denotes less initial target. This correlation between fluorescence and amount of amplified product permits accurate quantification of target molecules over a wide dynamic range in the presence of suitable standards.

The consistency and reliability of RT-qPCR assays depends on the appropriate execution of a number of steps, principally those involving sample selection, template quality, assay design and data analysis.

\subsection{Measles virus}

MeVs, from the family Paramyxoviridae, genus Morbillivirus, have a single negativestrand RNA genome enclosed in a viral envelope associated with three proteins: the matrix (M) lining the inner surface of the envelope and the fusion (F) and haemagglutinin (H) transmembrane proteins. Negative sense means that the RNA is complementary to mRNA and must be copied into the complementary plus-sense mRNA before proteins can be made. Thus, besides needing to code for an RNA-dependent RNA-polymerase, these viruses also need to package it in the virion so that they can make mRNAs upon infecting the cell. The MeV RNA-dependent RNA polymerase generates a full-length positive copy of their genome, the "replicative intermediate" as well as individual RNA copies that serve as mRNA for individual virus-specific proteins (Figure 2). Importantly, at no stage of its replication cycle is $\mathrm{MeV}$ RNA ever reverse transcribed into DNA, i.e. $\mathrm{MeV}$ does not exist as a DNA molecule, a fact that was established in 1964. This is a critical issue, since if it can be demonstrated that a test is amplifying DNA and not RNA, this provides indisputable proof of contamination.

\subsection{What makes a publication credible?}

Before describing the experiments in detail, it is worth reiterating what is expected of a plausible scientific publication. Its credibility depends on a number of conditions that include

- Transparency of reporting

- Reliability of protocols and techniques

- Inclusion of appropriate controls

- Reproducibility of the data

This is especially so for publications that utilises qPCR, since its sensitivity makes experiments susceptible to contamination, which leads to the reporting of false positive results. 
The purpose of this chapter is to demonstrate that the qPCR data claiming to detect $\mathrm{MeV}$ in the intestine of autistic children are unreliable and meaningless because of

- Absence of transparency: the key publication shows no data; hence an expert reader cannot evaluate the reliability of its conclusions

- Unreliable techniques and protocols: analysis of the qPCR data was incorrect

- Disregard for controls: obvious evidenceof extensive contamination was disregarded

- Lack of reproducibility: the data could not be duplicated by several independent investigators

The only conclusion possible is that the assays were detecting contaminating DNA. Since $\mathrm{MeV}$ is an RNA-only virus and never exists in DNA form, these data must be ignored and it it is my opinion that the authors should withdraw this publication from the peer-reviewed literature.

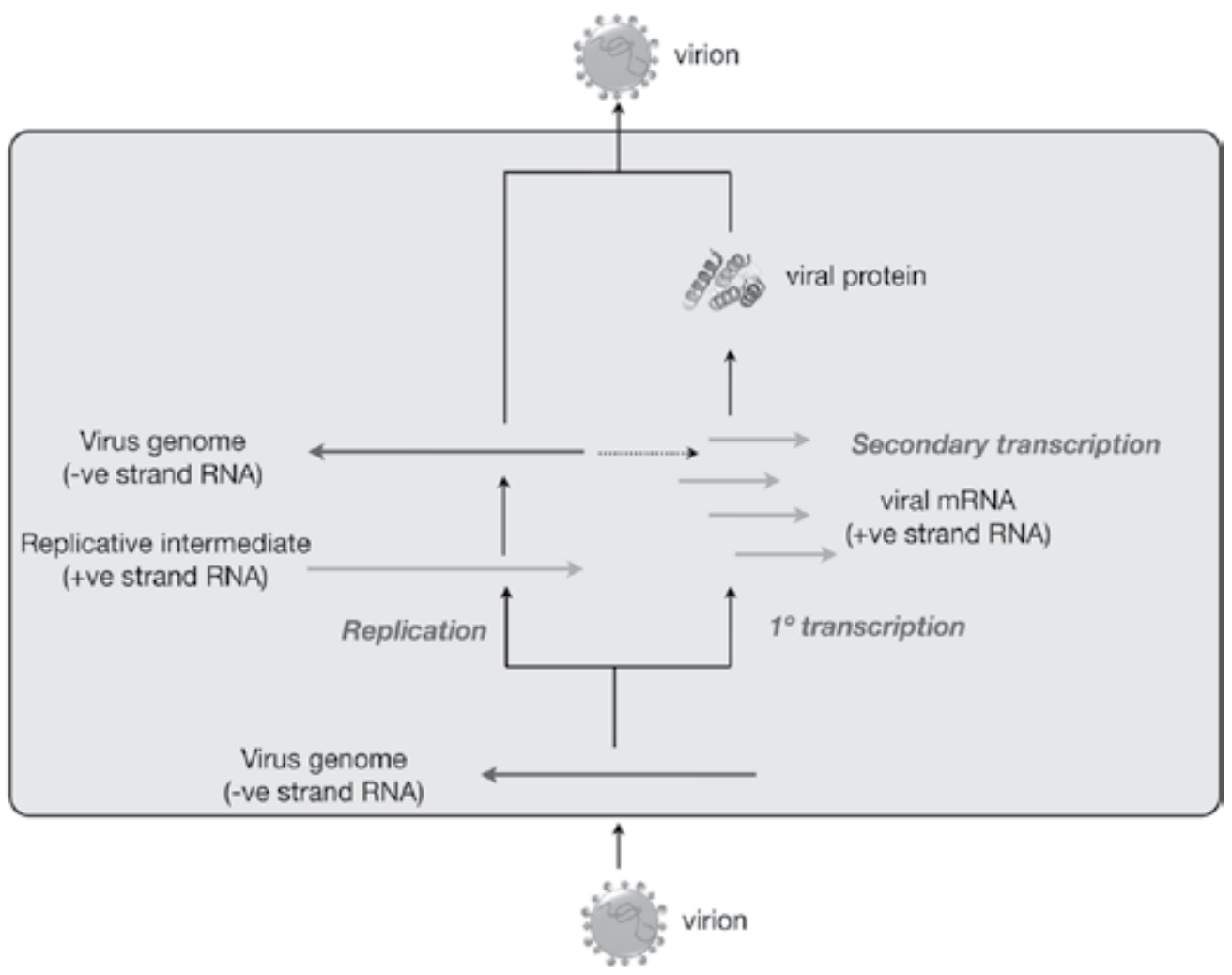

Figure 2. MeV life cycle. The virus attaches to the surface of a host cell, the viral envelope fuses to the plasma membrane and the nucleocapsid is released into the cell. Negative-sense genomic RNA is transcribed into individual messenger RNAs as well as a full-length positive-sense RNA template, which is used to create negative-sense RNA. Viral proteins are translated, assembled around the negative sense RNA and new viruses bud from the cells. 


\section{Timeline}

\subsection{April 2000}

An Irish pathologist, John O'Leary, reports "scientific results in a series of children with autistic enterocolitis... following an approach made by Andrew Wakefield" before the US Congress Committee on Government Reform [5]. He had "compelling" evidence "in relation to the presence of measles virus in children with autistic enterocolitis" and could "confirm that his [Wakefield's] hypothesis is correct". This statement was based on the detection of $\mathrm{MeV}$ using the then rather novel reverse transcription (RT)-qPCR assay as well other methods, in $24 / 25$ "children with autistic enterocolitis", compared to 1/15 control children. O'Leary also emphasised that he went to "desperate lengths" to prove the absence of contamination problems to "outrule the possible generation of false positives". Importantly, he stressed that "nothing in [his] testimony should or must be construed as anti-vaccine; rather it encourages safe vaccine strategies". This final qualification, although very clear, was buried by the headline news of the link between $\mathrm{MeV}$ and "autistic enterocolitis".

\subsection{August 2000}

A brief letter signed by JJ O'Leary, V Uhlmann and AJ Wakefield appeared in the Lancet [6]. It asserted that their "data from molecular virological studies examining the role of measles virus infection in children with autism and enterocolitis have been peer-reviewed, presented, and published at four international scientific meetings". The letter contained references to other publications, with reference 4 listing a publication by Uhlmann et al. entitled "Identification of measles virus genomes in ileo-colonic lymphoid hyperplasia in children" as "in press" in the Journal Laboratory Investigations. However, there is no record of such a publication.

\subsection{April 2002}

Speculation about a possible association between intestinal abnormalities in children with developmental disorders and the MMR vaccine was encouraged by a publication that utilised RT-qPCR assays to screen children's intestinal biopsies for the presence of $\mathrm{MeV}$ [7]. A comparison of terminal ileal samples from 70 normal controls and 91 children with a "new form of developmental disorder, ileocoloniclymphonodular hyperplasia", led to the claim that whereas 75/91 of the affected children patients tested positive for $\mathrm{MeV}$, only 5/70 control patients did. The paper does not reveal whether the autistic children had been given the MMR vaccination, but in the context of the source (Royal Free Hospital), authors (including Wakefield), introduction (reference to Wakefield's paper) and the discussion one is left with the impression that they had. The prominently displayed "take home message" concludes "the data confirmed an association between the presence of measles virus and gut pathology in children with developmental disorder". These results, if true, would constitute hard evidence linking $\mathrm{MeV}$, gut pathology and autism and was indeed used to support the vaccination/autism theory, even though the authors themselves never made that specific link. 


\subsection{May 2004}

An abstract entitled "TaqMan RT-PCR Detection of Measles Virus Genomic RNA in Cerebrospinal Fluid in Children with Regressive Autism" and published on the website of the Association of American Physicians and Surgeons lists two of the authors from the 2002 publication as co-authors, with JJ O'Leary occupying the senior author position[8]. It reports the detection of $\mathrm{MeV}$ in the cerebrospinal fluid of 19/28 children presenting "as autistic regression closely following MMR vaccination" compared with 1/37 controls. In five cases a haemaglutinnin[sic] gene allelic discrimination assay was carried out and showed a result consistent with a vaccine strain being detected. The abstract concludes with the sentence: "The findings confirm a highly significant statistical association between the presence of MV RNA in CSF and autistic regression following MMR vaccination".

\subsection{Summer 2004}

A paper is published that includes six patients (three patients, three controls) whose data were already published in the May 2004 abstract. It reports the detection of $\mathrm{MeV}$ in 3/3 patients but $0 / 3$ controls [9]. Interestingly, $\mathrm{O}^{\prime}$ Leary and Sheils are not listed as authors; instead the publication only thanks them in the acknowledgements.

\subsection{June 2007}

The first of three omnibus autism proceedings took place at the US Court of Federal Claims, where all vaccine claims are managed and are adjudicated by the Office of Special Masters. The trials were designed to establish whether or not autism could be caused by thimerosal containing vaccines, by MMR vaccine, or a combination of the two. I acted as an expert witness for the US Department of Justice (DoJ), presenting evidence based on earlier reports I had prepared for the UK High Court, who gave permission for the release to the US Secretary of the Department of Health and Human Services of two reports authored by myself. These documents had been filed by the three principal defendants in the UK MMR vaccine litigation and constituted an exhaustive analysis of the raw data underlying the results reported by the 2002 O'Leary publication. The evidence presented to the trial is a matter of public record, with the transcript available from the autism omnibus proceedings[10].

\subsection{February 2009-August 2010}

The evidence presented at the 2007 trial was used in further trials that concluded in February 2009 that there was no credible link between the MMR vaccine and autism. These decisions were upheld on appeal in July/August 2009 and then again in August 2010 were read [11].

\section{Analysis of the 2002 O'Leary paper}

The analyses and conclusions shown below were reached after examining all the RT-qPCR data disclosed following a court order. New experimental reports were generated using 
identical analysis software, compared with those disclosed by the O'Leary laboratory and any differences were noted. Any ambiguous or discordant results and all results involving negative controls were further investigated by scrutinising the raw data collected by the qPCR instrument. This permitted a definitive resolution of all ambiguities. All disclosed operator sheets and laboratory notebook entries relevant to the RT-qPCR assay were red and compared with the disclosed and the reanalysed data. Standard operating procedures were examined and inconsistencies with actual procedures were noted.

1. Transparency of reporting

The purpose of publishing a paper in the peer-reviewed literature is to provide adequate information that allows any competent scientist to follow the published protocol and reproduce the published data. Hence it is essential that detailed descriptions of the methods used and of the results obtained are included. It is not acceptable to publish summarised results only without any supporting, relevant data.

a. RNA was extracted from fresh frozen samples as well as formalin fixed, paraffin-embedded tissue (FFPE). However, there is no information on how the fresh samples were frozen, how long they had been stored,what percentage of patient and control samples were fresh frozen or FFPE and whether the same percentage was in each category. This is essential, since it is well established that FFPE treatment modifies and destroys RNA, or in Prof O'Leary's own words "wax and fixation by itself breaks down RNA" [5]. Hence it was well known at that time that RNA-derived data obtained from FFPE samples must be analysed and interpreted with caution [12-14].

b. No information is provided with respect to quantification or quality assessment of the extracted RNA; indeed there is no mention of RNA quality. This is vital information needed to assess the validity of any quantitative or negative result [15].

c. The RT-qPCR results are summarised without providing any actual data; a table simply states that 70/91 children with gut pathology were positive for $\mathrm{MeV}$, as against 4/70 in the control group.

d. $\mathrm{MeV}$ copy numbers in the affected children are reported as ranging from 1 to $3 \times 10^{5}$ copies of RNA/ng total RNA; no corresponding figure is provided for the four positive samples from the control samples. There is also no indication of what the potential error in those copy numbers might be.

e. Despite claims that the authors looked at two viral gene targets, they used only the data from the F-gene, which were discordant with the H-gene results.

2. Reliability of protocols and techniques

a. RNA was extracted from both fresh frozen and FFPE tissue samples and subjected to RT-qPCR analysis. Two RNAs were targeted; a control mRNA specified by the GAPDH gene and the MeV F-gene. As discussed earlier, since FFPE samples are characterised by RNA degradation, the expectation is that the results obtained from the FFPE samples should be different and, in terms of quantification, there should be less RNA present in 
a FFPE sample. This is in fact the results the authors obtain for the control RNA, where there was an approximately 4,000-fold reduction in RNA levels (Figure 3A). In contrast, the results recorded for MeV RNA were the same regardless of its source (Figure 3B).
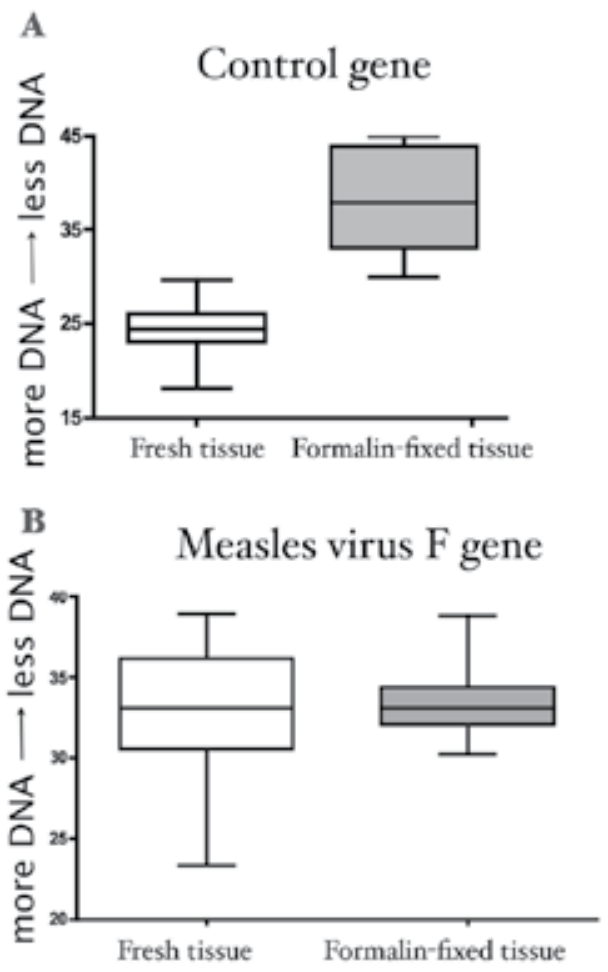

Figure 3. FFPE vs fresh samples. A. Effects of formalin fixation on control gene expression levels showing the difference caused by the formalin fixation process. Due to the exponential nature of the PCR reaction, the difference between the average quantification cycle recorded for fresh tissue and that for formalin-fixed tissue ( 25 vs 37 ) equates to $2^{12}$ or a 4,000-fold reduction of target. B. Absence of an effect of formalin fixation on MeV RNA, with the average quantification cycles very similar.

Since any RNA present during formalin fixation would have been affected in an identical manner, the obvious implication of these results is that whilst the control RNA was indeed present prior to formalin fixation and so was degraded, the MeV target was not degraded and entered the sample after formalin fixation. Consequently, no MeV RNA can have been present in the tissue and the positive result must have been caused by a contaminant.

b. Detection of an internal control following RNA extraction is a useful indicator of whether the extraction has been successful: absence of amplification implies that it has not been. Consequently, the O'Leary laboratory standard operating procedures implemented such an assessment and prescribed the exclusion of such samples from further analysis (Figure 4A). Fortuitously from the investigator's point of view and against the rules 
of their own SOP, the authors did not discard all samples where the control had been negative. Instead, they reported positive $\mathrm{MeV}$ results from samples that contained RNA as well as in autistic patient samples that had been negative for their control (Figure 4B). Since, these samples do not contain RNA by their own definition, their test must be detecting a contaminant.

A

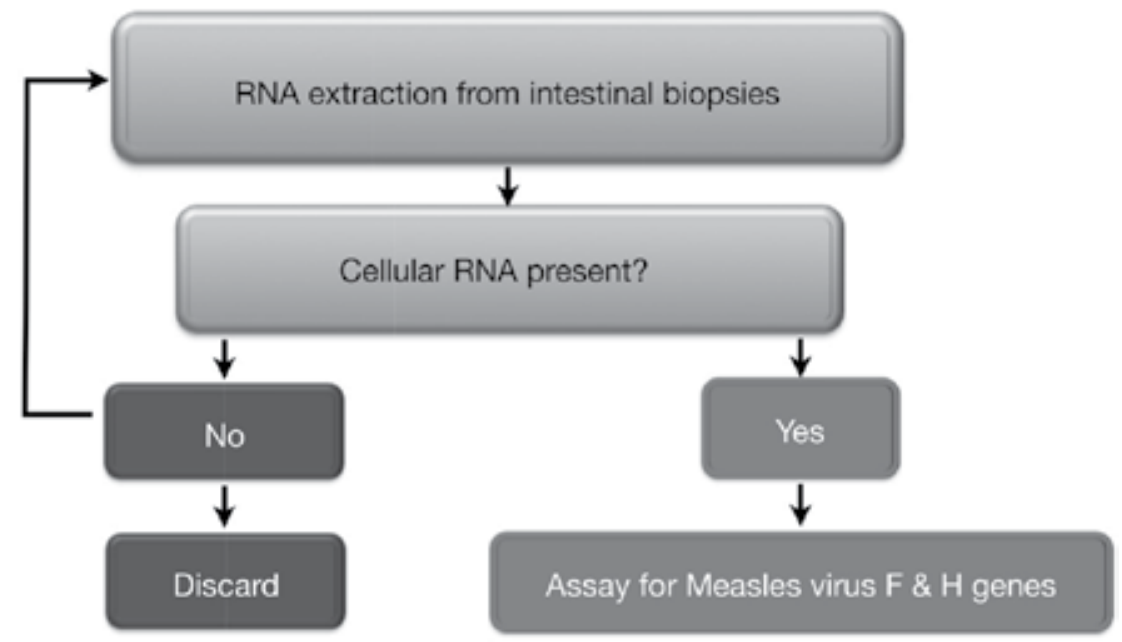

B

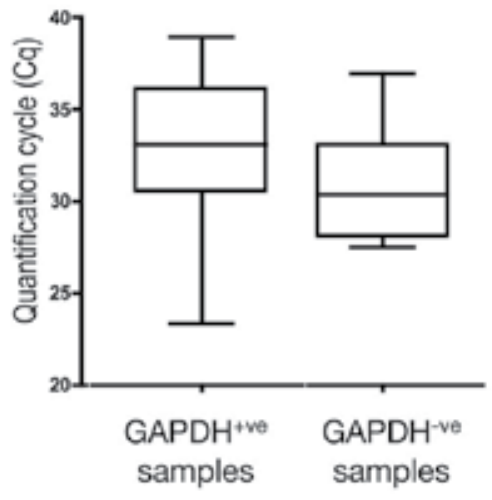

Figure 4. Workflow according to the O'Leary SOP. A. Following RNA extraction, only samples testing positive for GAPDH should have been further analysed for two viral targets. Samples testing negative should have been discarded and fresh RNA extractions attempted. B. MeV target detection from control+ve samples and control-ve samples showing that there is no difference in the quantification cycles. 
c. Two tests accidentally omitted including the RT step before the PCR test. In the case of the control, the results are as expected: the assay works significantly less well (Figure 5A). This is because Taq polymerase is very inefficient at making DNA from RNA. In contrast, the four $\mathrm{MeV}$ samples tested give the same result, regardless, indicating that the test is detecting DNA (Figure 5B). Since MeV does not exist as DNA, the test is not detecting $\mathrm{MeV}$ but a DNA contaminant.

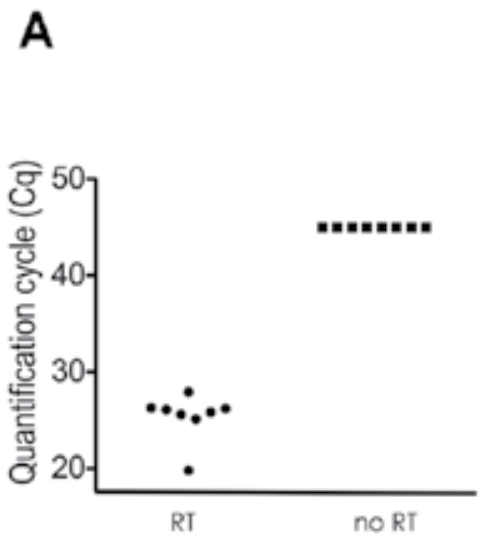

\section{B}

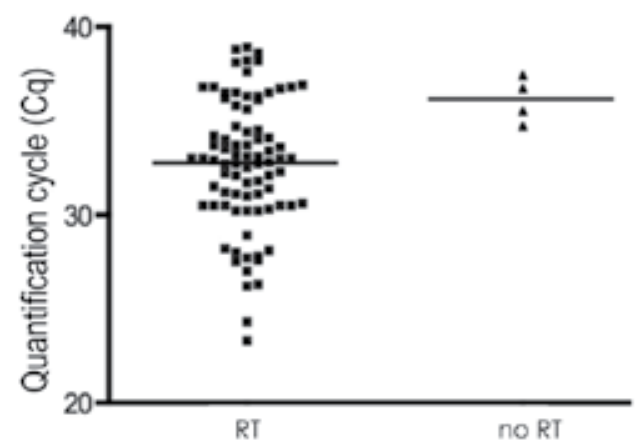

Figure 5. Absence of RT step. A. Control RNA: in the absence of the RT step (no RT), no amplification is observed (a Cq of 40/45 equates by definition to no amplification). B. Measles "RNA": in the absence of the RT step, amplification is observed with Cqs in the same range as in the presence of the RT step (RT).

d. Successful qPCR amplification is characterised by a characteristic "amplification plot", as obtained from sample F4 in Figure 6A and highlighted by the solid arrow. However a second sample (H2) is also recorded as generating a positive result (circled) even though it is clearly not being amplified (dashed arrow). The correct result is obtained by moving the threshold line(dotted arrow) so that well F4 continues to record a positive results, whereas well $\mathrm{H} 2$ now records a $\mathrm{Cq}$ of 40 , which in this setup equates to "no amplification" (Figure 6B). This is a rather elementary mistake. 


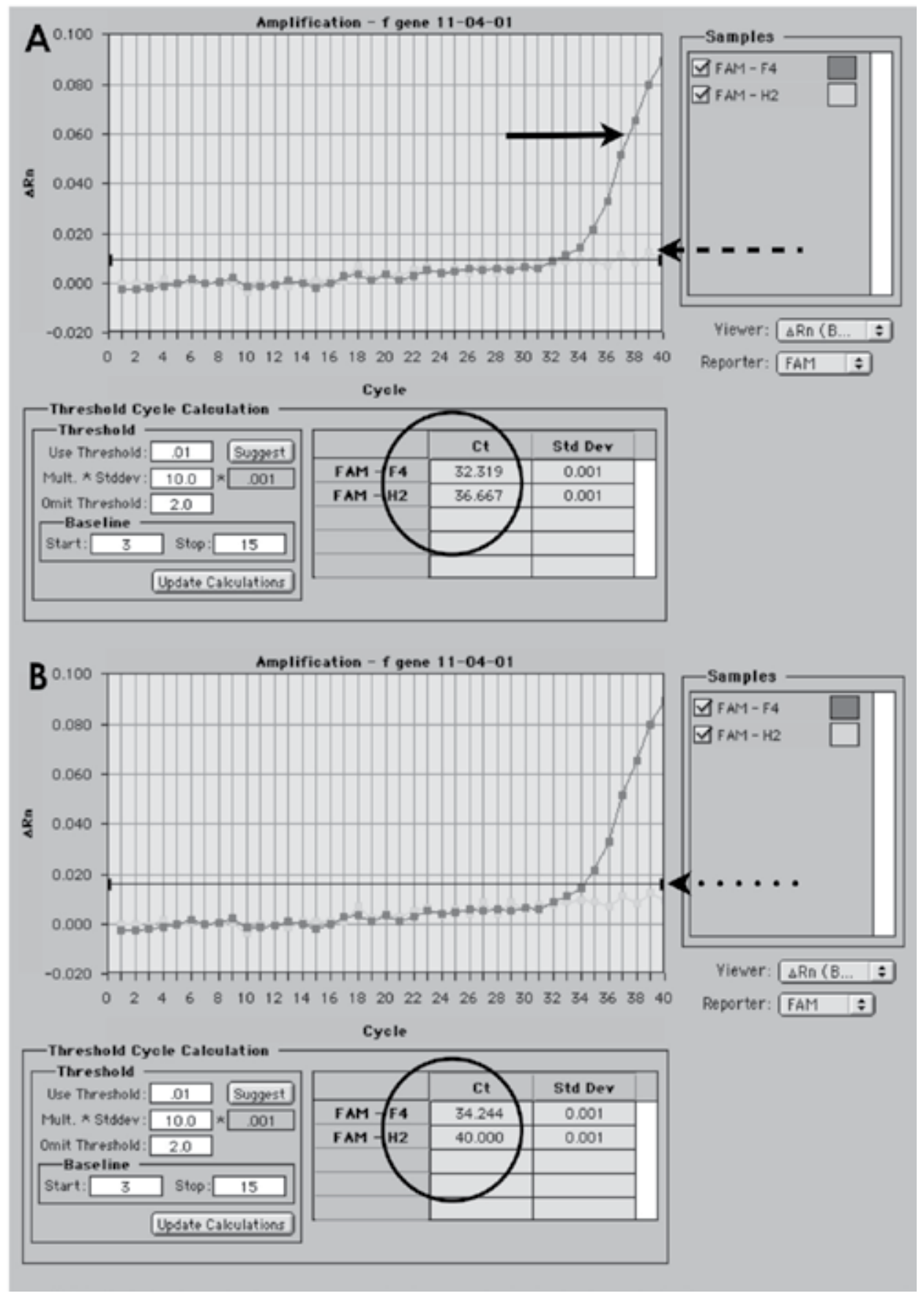

Figure 6. Inappropriate analysis. A. The data as reported by the O'Leary laboratory. B. The re-analysed data showing absence of amplification in well $\mathrm{H} 2$ (circled). 
3. Interpretation of control results

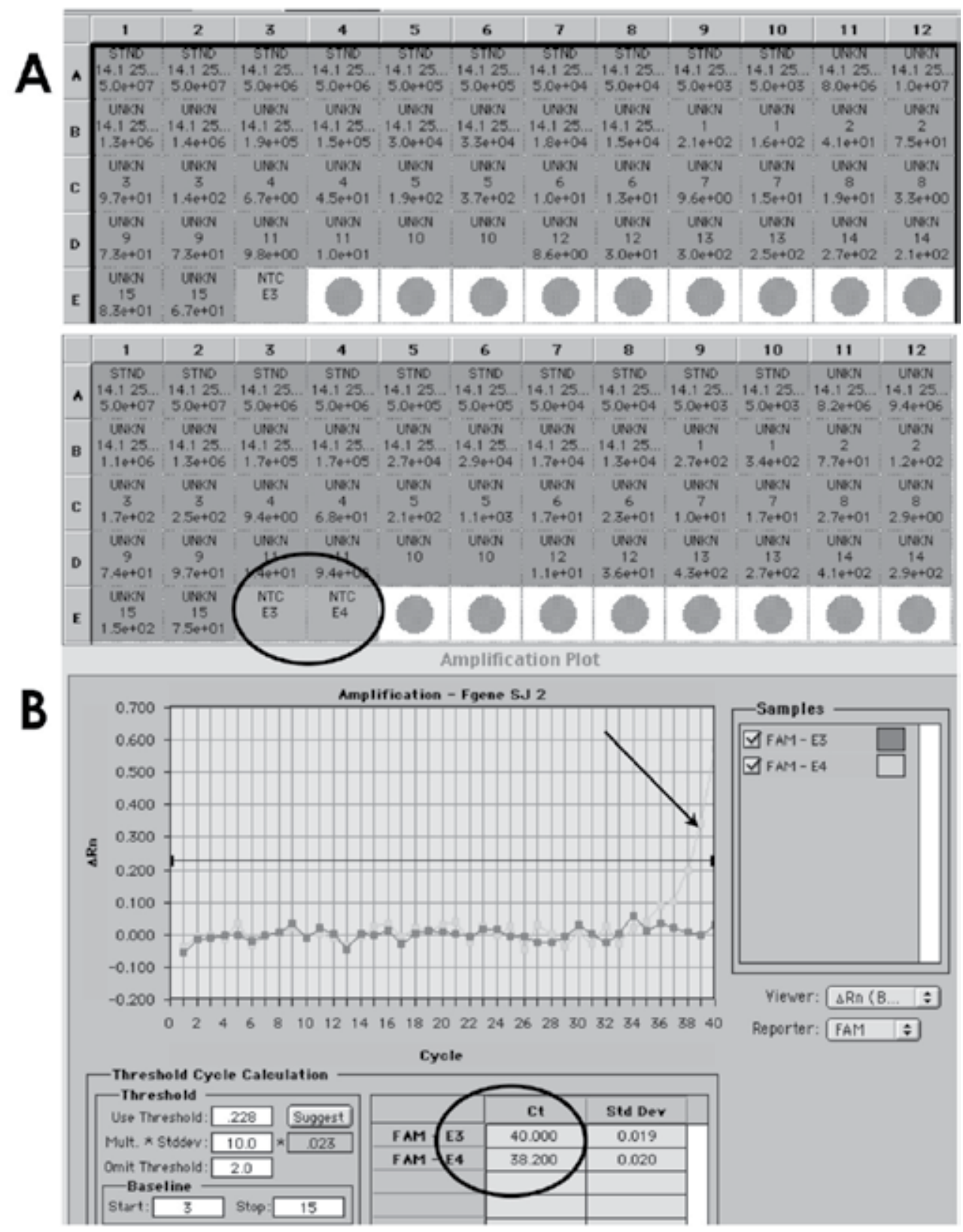

Figure 7. Contamination (1). A. The report submitted by the O'Leary laboratory shows a single negative NTC (circled), with well E4 next to the NTC not analysed, despite all other samples having been analysed in duplicate. B. A re-analysis that includes well E4 shows that this well is contaminated (arrow) and generates a reading (circled), invalidating any results from this run. 
b. Figure 8A shows a similar situation, with all analyses except the NTC in well B11 carried out in duplicate. Again, these were the data disclosed to the investigation, and I had to await the release of the raw data runs to be able to re-analyse those results. This time, a re-analysis of the data including well B12 shows both tests gave positive results, ie both negative controls reported contamination of the test (Figure 8B). The only conclusion from these data can be that the data are unreliable.

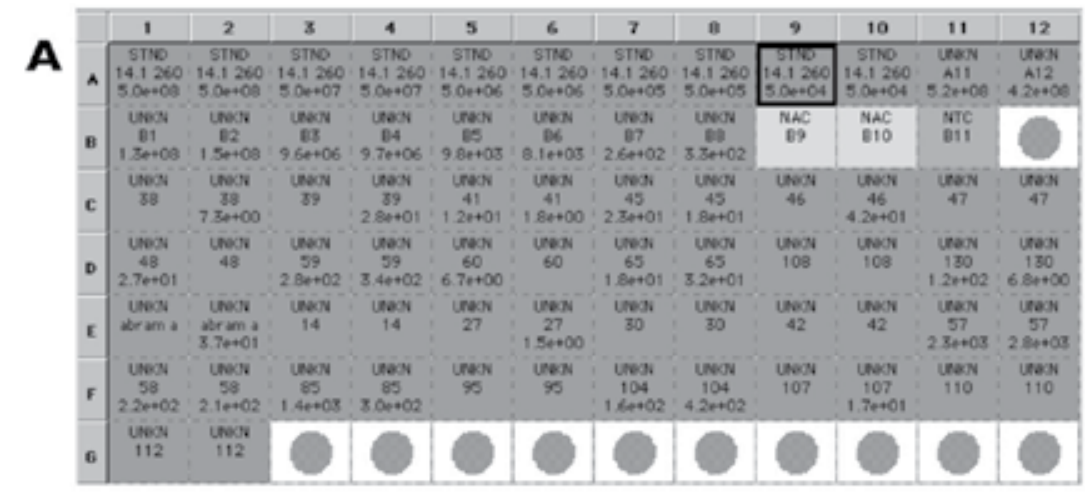

B
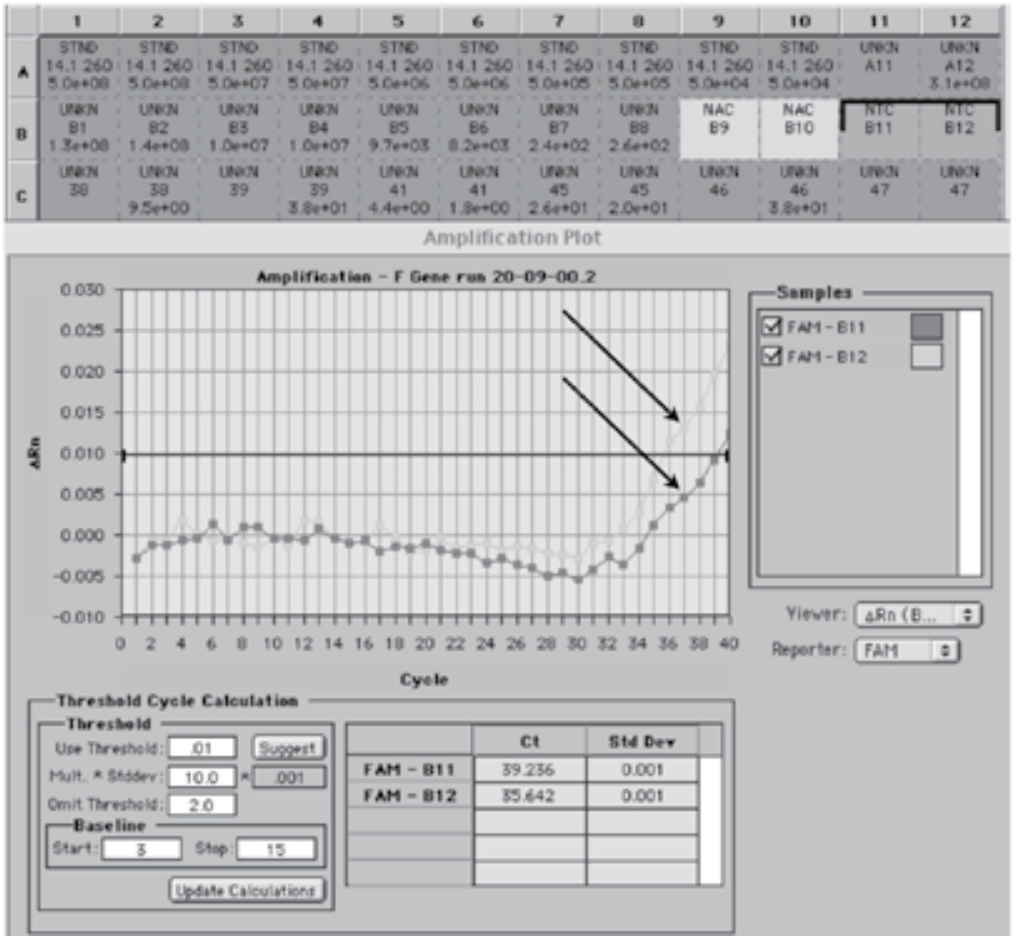

Figure 8. Contamination (2). A. The report submitted by the O'Leary laboratory shows a single negative NTC at B11, with well B12 next to it not analysed, despite all other samples having been analysed in duplicate. B. A re-analysis that includes well B12 shows that both NTCs are contaminated (circled), invalidating any results from this run. 


\section{Reproducibility of the data}

There have been a number of studies attempting to reproduce the findings of the 2002 paper [16-18]. All failed to do so; instead they provided strong evidence for contamination being the cause of the positive findings. However, there were some technical differences between the studies in the choice of tissue (intestine vs blood) or protocols (enzymes, qPCR chemistries). Therefore, whilst there was a strong suggestion that Prof O'Leary's laboratory was detecting contaminants, there was no proof. However, any lingering doubt evaporated with the publication from a multi-centre group of authors that refuted any association between persistent MeV RNA in the gut and autism[19]. Astonishingly, this publication includes the two main authors of the Uhlmann paper, and despite publishing evidence that contradicts their own, they have never retracted their original paper.

\section{The MIQE guidelines}

The problems inherent in the design and reporting of qPCR-based assays had been known for a long time, but there had been no concerted effort to tackle this serious problem. The 2002 O'Leary paper is not unique in omitting sufficient experimental detail, which impedes the readers' ability to evaluate critically the quality of the results presented, repeat the reported experiments, or integrate methodological advances into their own studies. Indeed, a recent survey of qPCR-based publication demonstrates very clearly that this problem persists even in 2010 [20] and that the perceived quality of the publishing journal is not correlated with the actual quality of the qPCR publication. The egregious use of qPCR in the $\mathrm{MeV} / \mathrm{MMR} /$ autism context provided the stimulus to initiate a push towards improving the technical aspects of qPCR assay design and reporting. Consequently, guidelines tackling this issue have recently been published which aim to promote consistency between laboratories, and increase experimental transparency [21]. The Minimum Information for publication of Quantitative real-time PCR Experiments (MIQE) guidelines outline the minimum data set necessary to evaluate effectively RT-qPCR assays, and are designed to be used as a checklist to both accompany manuscript submission and to be available alongside the published manuscript to enhance critical appraisal.

The four key areas of standardisation that define any qPCR experiment are study design, technical detail, analysis methods and statistics. MIQE addresses these under a set of captions that describe a large number of individual elements: "Experimental design, sample, nucleic acids, reverse transcription, target, primers and probes, assay details, PCR cycling and data analysis". At first sight, these look daunting, arduous and over-exacting. In practice, it is clear that most, if not all of these parameters describe information that would be obtained as a matter of course during the experimental design, optimisation and validation stages. Importantly, there is a clear hierarchy with some parameters, labelled " $E$ " (essential) in the published guidelines, indispensable for an adequate description of the qPCR assay, whereas other components, labelled " $\mathrm{D}$ " (desirable) more peripheral, yet constituting an effective foundation for the realisation of best practice protocols. There is increasing recogni- 
tion that the MIQE guidelines provide the basis for much-needed standardisation as well as encourage the publication of essential information that should be accessible to reviewer and reader[22].

\title{
6. Conclusions
}

This exhaustive analysis of the experimental RT-qPCR data generated by the O'Leary laboratory demonstrates:

- Lack of transparency and completeness of reporting

- Persistent and widespread contamination

- The contamination is caused by DNA

- Inept data analysis

As a result, the conclusions put forward by this paper are entirely incorrect and there is no evidence whatever for the presence either of MeV genomic RNA or mRNA in the GI tracts of any of the patients investigated during the course of the studies reported by O'Leary et al. Instead, it is clear that the data support the opposite conclusion: there is no evidence for any $\mathrm{MeV}$ being present in the majority of patients' analysed. Unfortunately, the authors do not report whether any the patients had received the MMR vaccination. However, assuming that a significant proportion had done so, it is also clear that there is no link between the $\mathrm{MMR}$ vaccine and the presence of $\mathrm{MeV}$ in the intestine of autistic children.

\section{Acknowledgement}

This work was carried out for the MMR vaccine litigation trial at the High Court of Justice in London and at the US Vaccine court. The author acted as an expert witness and was paid by the solicitors acting for the principal defendants SmithKline Beecham Plc and Smith Kline \& French Laboratories Ltd, Merck \& Co Inc and Sanofi Pasteur MSD Ltd. The author also acted as an expert witness for the US Department of Justice and was paid.

\section{Author details}

\author{
Stephen A. Bustin
}

Address all correspondence to: stephen.bustin@anglia.ac.uk

Faculty of Health, Social Care \& Education, Anglia Ruskin University, Bishop Hall Lane, Chelmsford, UK 


\section{References}

[1] Wakefield AJ, Murch SH, Anthony A et al. Ileal-lymphoid-nodularhyperplasia, nonspecificcolitis, and pervasive developmental disorder in children. Lancet. 1998;351:637-641.

[2] Choi YH, Gay N, Fraser G, Ramsay M. The potential formeasles transmission in England. BMC Public Health. 2008;8:338.

[3] GMC. Dr Andrew Jeremy WAKEFIELD Determination on Serious Professional Misconduct (SPM) andsanction. http://www.gmc-uk.org/Wakefield_SPM_and_SANCTION.pdf_32595267.pdf (accessed 29th August 2012)

[4] Bustin SA. Absolute quantification of mRNA using real-time reverse transcription polymerase chain reaction assays. Journal of Molecular Endocrinology. 2000; 25:169-193.

[5] O'Leary JJ. House ofRepresentatives, Committee on Government Reform. 106th Cong, 2nd Sess. 2000;Serial No. 106-180:123-137.

[6] O'Leary JJ, Uhlmann V, Wakefield AJ. Measles virus and autism. Lancet. 2000;356:772.

[7] Uhlmann V, Martin CM, Sheils O et al. Potential viral pathogenic mechanism for new variant inflammatory bowel disease. Mol Pathol. 2002;55:84-90.

[8] Bradstreet JJ, ElDahr J, Walker S et al. TaqMan RT-PCR Detection of Measles Virus Genomic RNA in Cerebrospinal Fluid in Children with Regressive Autism 2004; 2004.

[9] Bradstreet JJ, El Dahr J, Anthony A, Kartzinel JJ, Wakefield AJ. Detection of Measles Virus Genomic RNA in Cerebrospinal Fluid of Children with Regressive Autism: a Report of Three Cases. Journal of American Physicians and Surgeons. 2004;9:38-45.

[10] Claims USCoF. Autism Trial Transcript Day 8. 2007 ftp://autism.uscfc.uscourts.gov/ autism/transcripts/day08.pdf (accessed 29th August 2012)

[11] Claims USCoF. Autism Decisions and Background Information. 2009 http:// www.uscfc.uscourts.gov/node/5026 (accessed29th August 2012)

[12] Dakhama A, Macek V, Hogg JC, Hegele RG. Amplification of human beta-actin gene by the reverse transcriptase- polymerase chain reaction: implications for assessment of RNA from formalin-fixed, paraffin-embedded material. J Histochem Cytochem. 1996;44:1205-1207.

[13] Foss RD, Guha-Thakurta N, Conran RM, Gutman P. Effects of fixative and fixation time on the extraction and polymerase chain reaction amplification of RNA from paraffin-embedded tissue. Comparison of two housekeeping gene mRNA controls. Diagn Mol Pathol. 1994;3:148-155. 
[14] Unger ER, Vernon SD, Lee DR, Miller DL, Reeves WC. Detection of human papillomavirus in archivaltissues. Comparison of in situ hybridization and polymerase chain reaction. J Histochem Cytochem. 1998;46:535-540.

[15] Bustin SA, Mueller R. Real-time reverse transcription PCR (qRT-PCR) andits potential use in clinical diagnosis. ClinSci (Lond). 2005;109:365-379.

[16] Afzal MA, Ozoemena LC, O'Hare A, Kidger KA, Bentley ML, Minor PD. Absence of detectable measles virus genome sequence in blood of autistic children who have had their MMR vaccination during the routine childhood immunization schedule of UK. J MedVirol. 2006;78:623-630.

[17] D'Souza Y, Dionne S, Seidman EG, Bitton A, Ward BJ. No evidence of persisting measles virus in the intestinal tissues of patients with inflammatory bowel disease. Gut. 2007;56:886-888.

[18] D'Souza Y, Fombonne E, Ward BJ. No evidence of persisting measles virus in peripheral blood mononuclear cells from children with autism spectrum disorder. Pediatrics. 2006;118:1664-1675.

[19] Hornig M, Briese T, Buie T et al. Lack of association between measles virus vaccine and autism with enteropathy: a case-control study. PLoS ONE. 2008;3:e3140.

[20] Huggett J, Bustin SA. Standardisation and reporting for nucleic acid quantification. Accredit Qual Assur. 2011;16:399-405.

[21] Bustin SA, Benes V, Garson JA et al. The MIQE guidelines: minimum information for publication of quantitative real-time PCR experiments. Clin Chem. 2009;55:611-622.

[22] Bustin SA. Why the need for qPCR publication guidelines?--The case for MIQE. Methods. 2010;50:217-226. 
Chapter 6

\title{
Vaccine Safety Study as an Interesting Case of "Over-Matching"
}

\author{
M. Catherine DeSoto and Robert T. Hitlan \\ Additional information is available at the end of the chapter \\ http://dx.doi.org/10.5772/53876
}

\section{Introduction}

Increasing levels of diagnosed cases of autism have alarmed parents and health officials, but the cause has not been established. It has been hypothesized that vaccination itself, or some component in vaccines, may be somehow related to the onset of autism in some cases (Delong, 2011; Gallagher \& Goodman, 2010). Researchers have sought to alleviate such concerns. Although most studies report null effects, work continues to be published that suggests some reason for concern (Hewiston et al., 2010). Some skepticism of the safety of vaccines still exists, documented by scholars on either side of the issue (Austin, Schandley \& Palombo, 2010, Destafano, 2007). As it is, the topic of vaccine safety and triggering of unintended outcomes is one of the most controversial topics in environmental health and toxicology.

After initial safety studies, case- control designs are often employed to continue to investigate both side effects and efficacy of inoculation. Matching is a technique used to improve signal to noise in research case-control designs. Matching cannot - or should not - be done in a way that artificially increases the chance that within strata exposure is the same. This happens when a matching variable is a strong predictor of exposure and is called overmatching. Here, we report a textbook case of overmatching within a widely - cited article. Focusing on the overmatching as a statistical concept, suggestions are made to standardize when overmatching may have occurred. It is important for statisticians to note when a study that fails to find an effect related to public health outcome has employed a design that would be expected a priori to result in a lack of effect.

It has been noted that some children received exposure to mercury significantly in excess of safety standards during the 1990's, before the level of thimerosal in vaccines was lowered (Geier \& Geier, 2006), this has been suggested to increase odds of various developmental 
disorders (Geier \& Geier, 2006). The research by Price et al. (2010) spans the birth cohort years that saw a decline in thimerosal exposure and reports that thimerosal exposure was not associated with risk outcome of autism. Indeed, many studies have been published that find no negative effect of vaccination on developmental outcomes whatsoever (Parker, Schwartz, Todd, Pickering, 2004; see Destafano, 2007 for a review), indicating a lack of cause and effect between vaccination and autism. Here, we suggest that a recent widely cited study was flawed, and urge statisticians to carefully and critically review outcomes research on high stakes topics. It should be noted and understood that a flaw in such a study does not mean that vaccines cause autism, nor does it follow that one would properly assume that the flaw leads to the conclusion that vaccination is not safe. Rather the weight of scientific research as a whole should be deferred to.

Conditional logistic regression (CLR) is a statistical technique used when the researchers have matched cases with controls on various parameters (e.g., age, gender). CLR is the often-used and appropriate way to analyze matched data sets (Rahman, Sakamoto \& Fukui, 2003). To be clear, matching means that (as an example) for every 'case' that is male and aged 12, there is a control selected from a pool of possible controls that is also male and aged 12. If this were done, the researchers "matched on age and gender." A variant is to have two or three times the number of controls within each condition, or stratum. (Meaning for every male case who is age 12, there are three controls who are male and age 12.) The matched unit is called a stratum. When analyzing the data, CLR analyses are done within strata. When matching is done, only conditions (strata) that have cases and control pairs that vary on the risk factor contribute to the estimate of the effect of the risk factor (Miettinen, 1968). In other words, if exposure level within strata is the same, CLR cannot estimate the effect. As such, matching is a key design feature.

Matching cannot - or should not - be done in a way that artificially increases the chance that within strata exposure is the same; this happens when a matching variable is a significant predictor of exposure and is called overmatching.

Proper design can have important implications and researchers are appropriately cognizant of the possible perils of failing to take enough care in considering the matching design. If matching is used, researchers are wise to give explicit consideration to ensure that the problem of overmatching is avoided when attempting to accurately estimate risk of an exposure of interest (Sasieni and Castanon, 2009; Al-Taiar et al., 2009; Vidal et al., 2008; Agudo \& Gonzalez, 1999; Cullison et al., 2007). And this problem has long been known (see for example, West, Schuman, Lyon, Robison \& Allred, 1984). In their consensus paper on outcomes research, the American Thoracic Society noted that, "Overmatching, matching for a variable that is associated with the exposure but not the outcome, will reduce the statistical power of the study," (p. 364). Improper matching cannot later be undone via analysis and the effect of the matched variables cannot be checked, once matching has been done (Rubenfeld et al., 1999). How could this happen? Usually, this arises when a researcher fails to realize he or she is essentially matching on the exposure variable, and inadvertently the researcher matches the effect out. 
To illustrate overmatching, a fictitious example will be briefly discussed, followed by an actual example from the literature. Assume the question is whether radiation exposure in nuclear plant workers contributes to cancer. A hundred cancer cases are found, and a control group of 700 is identified. Then, each case is matched with one from the control group on gender, smoking, job location, and age. The researchers match on these variables to increase efficiency (because they think these variables might independently account for disease risk). We will keep this as one to one matching for simplicity, but a 1:3 matching would essentially work the same.

In this example, overmatching would happen if the researchers are looking for effects of radiation but fail to consider that while which power plant the worker is employed might have some independent influence on disease risk (which is why it is matched), location could also be a major determinant of radiation exposure. For example, imagine Plant L often had radiation leaks, while Plant $S$ had better safety. If one then matches on where one works, all of the variance unique to a particular plant is matched out. In such a case, an effect for radiation - even if huge could be missed. It will be clear if one considers that this would be like testing if radiation was related to cancer in Japanese nuclear power plant workers after controlling for location with one of the locations being Fukishima (Figure 1). If participants who developed cancer were matched on where they worked - the researchers may not detect any true health effects of the radiation exposure from the nuclear meltdown at Fukushima compared to working at other plants that did not have a meltdown. The researchers would have matched out any effects associated with where they worked.

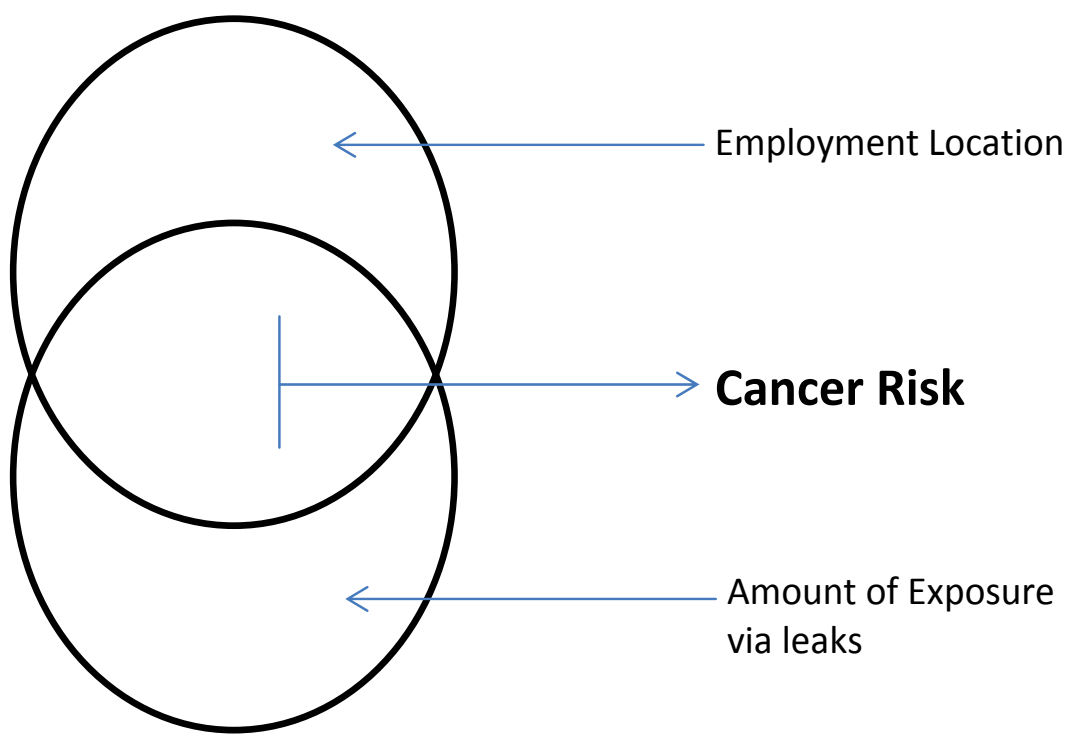

Figure 1. Overlapping variance: Illustration of Overmatching on Radiation Exposure; In this fictitious example, matching on the nuclear power plant of employment in the design of the study would be overmatching because it would remove the largely overlapping variance associated with radiation due to the Fukishima leak, obscuring the effect 
A now classic paper by Marsh, Hutton and Binks (2002) refers to a real research example and is entitled, "Removal of radiation dose response effects: an example of over-matching." It details how a true effect can be missed if the researchers overmatch. According to the authors, "If the exposure itself leads to the confounder or has equal status with it, then stratifying by the confounder will also stratify by the exposure, and the relation of the exposure to the disease will be obscured. This is called over-matching and leads to biased estimates of risk," (p. 1235). After previous work had suggested that radiation did predict leukemia, the more recent case-control study failed to indicate any relation between radiation and leukemia. The matched factors in the new study that showed no increased for leukemia as a result of radiation included: date of birth, gender, and "date of entry". "Date of entry" was a measure of what years the workers worked in the industry. The data was properly analyzed given the matched design by conditional logistic regression, yet failed to find a known effect.

This prompted the study of the statistics used, with a focus on the matching process. It was noted that some things are appropriate to match on, for example, gender. "Because of the underlying difference of the risks of leukemia between the sexes," being male versus female affects the outcome, and it is important not to accidently have more males in the case group as this would be a confound. On the other hand, Marsh et al. clearly showed that radiation exposure varied by year, that is some years were higher than others and this was indeed a major source of radiation variation (see figure 3, Marsh et al., 2001). "The general decline in median dose shows that dose and time are associated. The situation seems to be one where dose is partially 'explained' by date of entry, both being related to time;" in sum, "this seems to have had the effect that workers in the same matched set have broadly similar recorded doses. The apparent over-matching on date of entry has distorted the parameter estimate of the risk of leukemia on cumulative dose by introducing matching (at least partially) on dose," (Marsh et al., 2002).

What is the take home message of this classic report on the problem of overmatching? When researchers match on a variable closely associated with the risk factor exposure, then actual effects will not be-- and cannot be-- detected. This danger is written about by various other authors as well. Richard Monson in his text, "Occupational Epidemiology" notes "over matching is a problem in case control studies." Monson emphasizes that "there should be no possibility that the factor is part of the causal pathway linking exposure and disease under study." (p. 41). If this is even remotely possible, Monsoon advises matching should not be done on that variable. Monson discussed an example where overmatching resulted in underestimating the effect of estrogen use on endometrial cancer. Here the matching was on a correlate of intrauterine bleeding, which in effect controlled for a symptom of the cancer itself.

Price et al. do not mention overmatching as a potential concern. The risk factor of interest is thimerosal exposure via its inclusion in vaccine ingredients. There are two things that have a systematic and predictable effect on how much thimerosal exposure a child would receive: 1) the vaccine schedule a child is born into/national recommendations, and 2) which manufacturer a given provider is using for the vaccines (e.g. for the same years, Smith, Kline and Beecham were using thimerosal in their HepB vaccine, while Merck did not). 


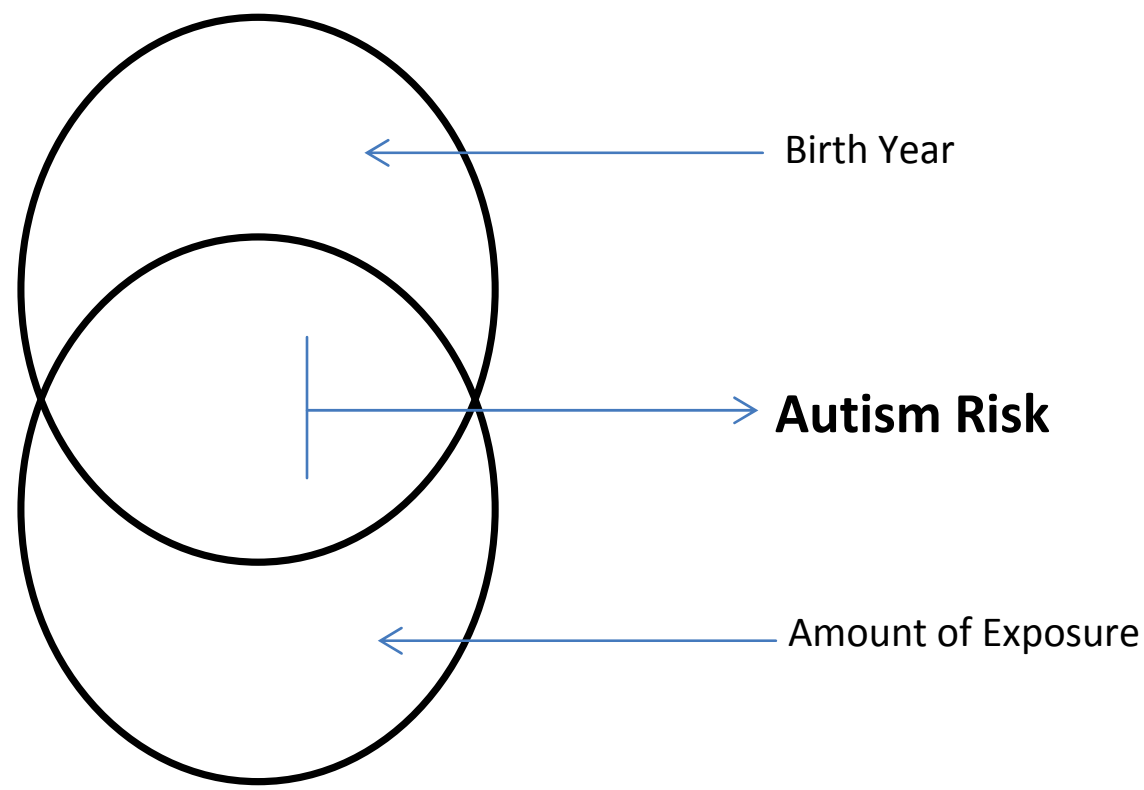

Figure 2. Controlling for Birth Year is overmatching due to the overlap with Amount of Exposure; similar to the radiation risk for leukemia written about by Marsh, controlling for time is (at least partly) controlling for exposure, which varies with birth year. The matching on birth year is matching on the exposure. This seems to have had the effect that children in the same matched set have similar recorded exposures to thimerosal, removing much of the variance

Price et al. matched out both of these variations in exposure. This has the effect of ensuring that the control group is nearly identical with the case group on the risk factor, which prevents its effect from being accurately measured. Considering cumulative exposure for the first 7 months of life, the overall mean for the full data set is 102.88 micrograms $/ \mathrm{Hg}$ and a standard deviation of 42.2. The means for the cases and matched controls is 100.0 and 103.2 micrograms of $\mathrm{Hg}$ : this similarity (less than one tenth of the standard deviation) is forced by the matching on the variables that define exposure. Birth year dictates which vaccine schedule a child is born under as well as which batch brands and formulations are available on the market at a given time. Doctors within a practice will be using the same manufacturer across children (vaccines are ordered in large batches room a given manufacturer; the Vaccine Data Set used by Price et al. documents that the same providers use the same manufacture. Thus, this is a text book case of overmatching: variables were matched on that essentially define exposure. It is well known that matching on a variable that is associated only with exposure, not with disease, reduces statistical efficiency (Zondervan et al, 2002; Rubenfeld et al., 1999; Day, Byar, \& Green, 1980) and that care needs to be taken to avoid this in a case-control research design.

Across the different years, the average cumulative exposure varies from 42.3 micrograms to 125.46 micrograms; while within the birth year stratas, the mean exposures do not vary by more than 15 micrograms. Birth year is a variable that defines exposure due to changes in recommendations regarding the vaccine schedule and changes in vaccine formulas that oc- 
curred at different times. The above panels suggest that variance within the matched variable (year) is small compared to the variance between birth years: birth year is accounting for much variance in thimerosal exposure.
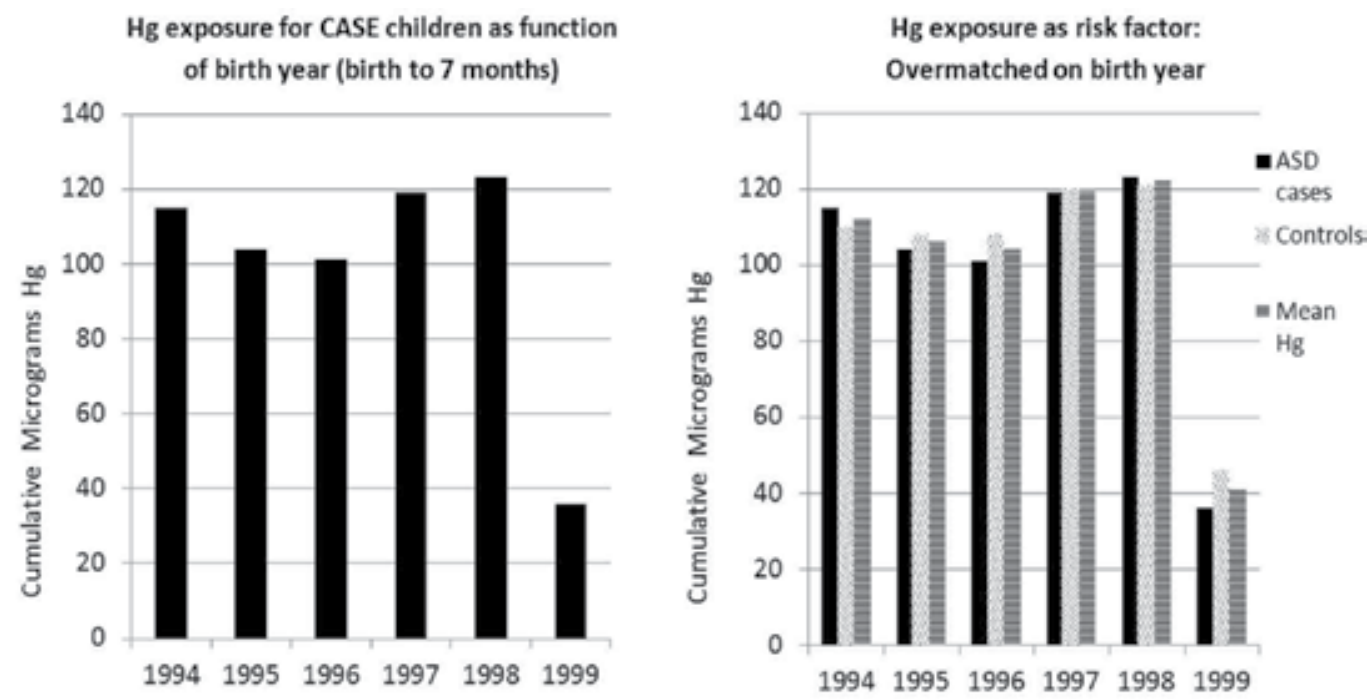

Figure 3. The difference across birth years on the risk factor of interest

During the past decades, there have been three main exposure sources of thimerosal: DPT/ DTaP, then Hepatitis B and Hib vaccines, while flu shots are currently the primary source in the USA today. The Hib/Hep B introductions came in during the late 1980s and early 1990s. The recognition that the cumulative mercury burden may have been too high came in 1999, and mercury levels dropped for most vaccines given to children in the USA. Some people have raised concerns that the increase in autism is associated with the changes in thimerosal exposure; that is, the increase in autism is thought to be a function of the increases in the number and amount of mercury containing vaccines. Whether or not one finds this model persuading, matching on birth year is questionable if the goal is to test the model that differences in thimerosal exposure via vaccine schedule increase ASD risk since -- as most people are aware -- birth year essentially dictates which vaccine guidelines a child is born into. It could be that the authors intended to control for hypothesized changes in diagnostic criteria trends across the six birth years. The problem is that diagnostic effects on risk is not measured while birth year effects on exposure are clear.

Moreover, $\mathrm{HMO}$ is not known to be a significant predictor of the outcome of autism diagnosis, so potential reasons to match on this variable are less clear. As Hansson and Khamis (2008) write in their paper on matched-sample logistic regression, "Generally, matching will increase the efficiency of the study when the matching variable is a strong outcome determinant, but will actually reduce it when the matching variable is strongly related to the exposure variable (over-matching)," (p.595-596). Meittinem (1969) states that, "matching reflects 
the notion that the probability $\mathrm{P}$ of response is related to $\mathrm{M}, "$ (p. 340) meaning that when one matches, one infers that the matching variable effects the probability of risk (here for autism). HMO / health care provider was a major determinant of thimerosal exposure, but we are not aware of papers that identify HMO is an independent risk for autism. Thus, it should not have been matched. What was needed was a design that compared persons with different exposures. "Studies with uniform developmental assessments of children with a range of cumulative thimerosal exposures are needed," (Vertraeten et al., 2003). Here Price

Figurel4, began with such a data set, but then matched on birth year and $\mathrm{HMO}$, matching out exposure differences and negating comparisons of different exposures (see Miettinen, 1969 for a mathematical discussion).

\section{Cumulative Thimerosal first 7 months as a function of $\mathrm{HMO}$}

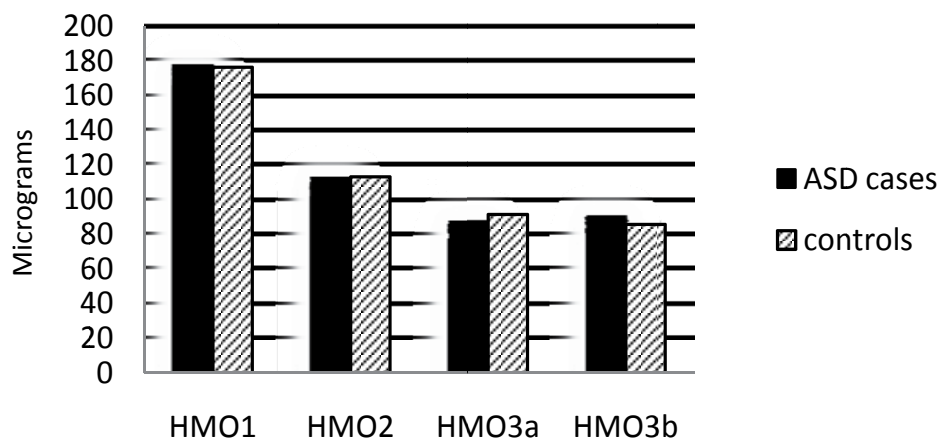

Figure 4. The apparent over-matching on $\mathrm{HMO}$ distorts the estimate of the risk of autism on thimerosol by introducing matching on exposure. If one matches on provider, one is matching on the vaccine manufacturer. There are different manufacturers available, but a given provider will be using one or the other. This seems to have had the effect that children in the same matched set have similar recorded exposures to thimerosal. Again, this removes this variance and obscures the effect

The model Price et al. were trying to test was whether thimerosal exposure via the US vaccination schedule was associated with any increased risk of autism. To do this, they needed to compare persons with and without high levels of exposure. They did not do this because due to the conditional logistic regression matched on both birth year and HMO they have inadvertently made sure that cases were only compared to controls with the same exposure. Because Price et al. did not mention the possibility of overmatching, we assume this did not occur to the research team. We assume this was accidental, but it does underscore the need to have a balanced research team that does not start with assumptions that might flaw the design. For example, assuming that the increase in autism is only due to diagnostic changes would lead to controlling for birth year, which might have been flagged by someone who does not share this assumption. It is harder to understand why HMO would be matched. Overall, this is unfortunate because the question 
of vaccine safety is high stakes. There are concerns that a proper test of the full vaccine schedule has not been properly tested, and that the safety tests that exist have been designed by the vaccine industry itself. Such concerns about conflicts of interest may be preventing otherwise willing parents to adhere to the full vaccine schedule. Vaccines have been and will continue to be a huge benefit to humanity. But this paper is flawed. Unfortunately, there is not an analytic fix for overmatching: it is design flaw.

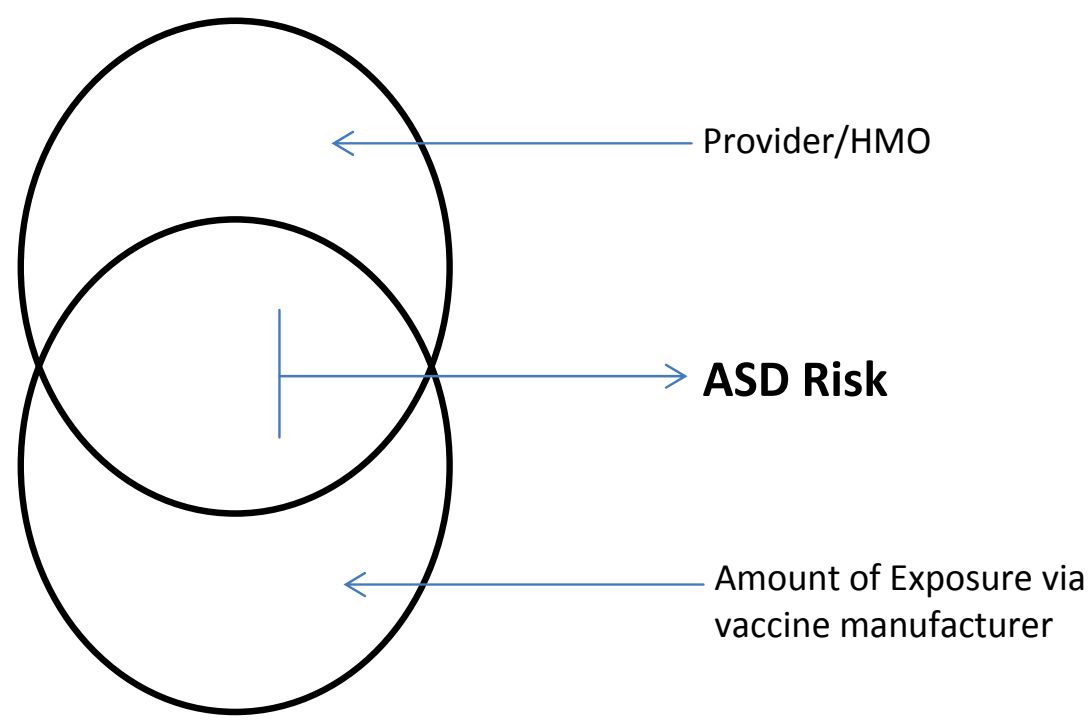

Figure 5. Which manufacturer a given provider used for the vaccines varied by HMO. Manufacturers differed in their thimerosal use. For example, in 2002, Smith, Kline and Beecham were using thimerosal in their HepB vaccine, while Merck did not. While the data set is careful to note manufacturer and $\mathrm{Hg}$ in the associated batch and manufacturer, but CLR matching on $\mathrm{HMO}$ results in comparing cases to controls who had the same levels of exposure

The Price et al. research is an interesting case of overmatching that we think is of general interest in the field of epidemiology. To avoid misunderstanding, we wish to state that this research does not support the argument that vaccines or thimerosal in vaccines cause autism. It is however, uninformative to the question.

\section{Suggestions for avoiding the problem of publishing overmatched results}

One way to conceptualize the problem of overmatching in conditional logistic regression is the preemptive removal of variance that should stay available for the hypothesized predictor variable to attempt to account for. The total variance in a data set can be defined using the average squared distance from the mean score for each participant: $\mathrm{s}^{2}=\mathrm{SS} / \mathrm{df}$. A question related to overmatching concerns how much of the total variance is taken out beforehand (matched out)? How much is too much? 10\%? 90\%? 50\%? We would propose that the per- 
cent removed before testing should normally be small compared to the total. Further, the removal of this variance should only occur when there is authentic need: when the potential matching variable is likely related to the outcome of interest via a path that is distinct from the risk variable of interest in a case-control design.

As elaborated above, matching is appropriate only if the matching variable is a strong predictor of the outcome of interest, but it is not appropriate when the matching variable is strongly related to the exposure risk variable. We offer three suggestions to help objectively identify, and thus avoid, the problem of overmatching.

Empirical Support. Before matching, first and foremost, researchers should locate studies that suggest the potential match is likely correlated to the probability of the outcome occuring. These should be cited to support the need to match on that variable. If there is no reason to think the matching variable relates to the outcome, there is no reason to match it.

Remaining Variance. Next, once the participants have been selected as a matched data set, researchers can check to get an idea how much variance in the exposure variable is actually accounted for by the matching variable $\mathrm{M}$. If only a small amount of the variance is left after the various matching, matching on the variable(s) cannot be justified and an unmatched or lesser matched set of participants is called for. Specifically, a check to see if too much of the total variance in the outcome of interest is matched out could be done by requesting Partial Eta Squared. Partial Eta Squared represents the proportion of the total variance that is explained by the between factor when an ANOVA is performed. Specifically, one can take the extra step of analyzing the variance in the risk factor of interest (e.g., thimerosal exposure) as a function of the matched variable (e.g., HMO or BirthYear). In this example, using thimerosal exposure as the dependent variable, the total SS is 23507522. The SS associated with the Birth Year is 1485471 . This gives Partial Eta Squared $=.456$, meaning that about $46 \%$ of the total variance in thimerosal exposure is fully explainable based on Year of Birth. When one matches on this, only about half (54\%) of the variance is left.

$\mathrm{HMO}$, the other variable matched on, removed about $30 \%$ of the variance.

The percent that should be left would depend on the research question and causal assumptions, but we suggest that if a matched variable is removing more than a fourth (25\%) of the variance (corresponding to a large effect size, Cohen, 1977), matching is unlikely to be warranted for this reason alone and welcome commentary on this benchmark proposal.

Relative relations. Finally, there are times when it could be proper to match on a variable that accounts for variance in the risk factor being tested. A recent case coincidentally also related to vaccines helps to illustrate this more. It had been pointed out that the enormous benefits of the flu vaccine among the elderly appeared to far surpass even the effect that a total eradicating of flu from the vaccinated population could account for (Jefferson, 2006). After additional investigation, much of the original effect appears to be due to the tendency for seriously ill and/or less healthy elderly persons not to have the flu shot. To be clear, most of the flu vaccine effect on mortality was found to be due to health of the participants independent of the flu shot (Jackson et al., 2006). In this case, if this had been a case control design, the risk factor would be flu vaccine and the probability outcome of interest would 
hospitalization or death. In such a case control study, it would be proper to match on preexisting health, even though one would find that health accounts for some of the variability in getting or not getting the risk factor (flu vaccine). BUT: health would also relate to the mortality outcome, and even more strongly. It is this strong relationship that is key. If the variable is more strongly related to the outcome - this serves to justify matching.

To objectively quantify this, one needs to know how strongly $M$ is related to the Risk Factor $\mathrm{R}$; and then how strongly $\mathrm{M}$ is related to the probability of response P. A problem is that different types of data can make precise comparisons of effect size hard to judge.

Assume that $\mathrm{M}$ would be $\mathrm{HMO}, \mathrm{R}$ would be mcg Thimerosal exposure, and $\mathrm{P}$ would be ASD diagnosis. It would be desirable to compare the size of this relationship $M$ to $R$ with the relationship of $\mathrm{M}$ to $\mathrm{P}$. It would be ideal if one could simple compute correlations for $\mathrm{M}$ and $\mathrm{R}$ and for $\mathrm{M}$ and $\mathrm{P}$. However, in most cases this would not work: the scales are not all continual, and even if one were to employ a Spearman correlation, it would not be apparent how to code something like HMO to insure a linear relationship. What if HMO 2 was associated with an increase in thimerosal, and HMO 1 and 3 both had low levels? This would result in a low correlation due to the curvilinear relationship, even IF much of the variance were in fact associated with HMO. On the other hand, the relationship between HMO and thimerosal (M and R) can be checked via ANOVA easily enough since $R$ is continual and $M$ is categorical. ANOVA would not work for testing association between $\mathrm{M}$ and $\mathrm{P}$ because both $\mathrm{M}$ and $\mathrm{P}$ are both categorical in this case. Chi - Square would be appropriate. However, regardless of the correct hypothesis test, all hypothesis tests are in fact unified by the $p$ value.

The $\mathrm{p}$ value is a function of the size of the effect and the sample size. Different types of statistical tests have different probability distributions, but the total area under the curve has a constant meaning across tests. The percent of area covered means the same thing in any test, regardless of the precise shape of the curve associated with a particular statistical test (correlation, ANOVA, Chi-square). A small $\mathrm{p}$ value could be due to a large effect, or it could be due to a very small effect and a very large sample. It should be stated that when sample sizes are similar, it will not be unduly affected by sample size differences. Since the sample will be the same for testing $\mathrm{M}$ to $\mathrm{P}$ or testing $\mathrm{M}$ to $\mathrm{R}$, we propose the $\mathrm{p}$ values are the most readily available means to index the comparison.

Compute a measure for the relationship of $\mathrm{M}$ and $\mathrm{P}$ and the associated $\mathrm{p}$ value. (e.g., $\mathrm{HMO}$ and ASD: $X^{2}(2)=1.59, \mathrm{p}=.45$ )

Compute a measure for the relationship of $\mathrm{M}$ and $\mathrm{R}$ and the associated $\mathrm{p}$ value. (e.g., $\mathrm{HMO}$ and Thimerosal exposure: $\mathrm{F}(2,1090)=237, \mathrm{p}<.0001)$.

The $\mathrm{p}$ value in all cases should be smaller for the $\mathrm{M}$ to $\mathrm{R}$ relationship, compared to the $\mathrm{M}$ to $P$ relationship test. This will serve to demonstrate that even if the Matching variable does bear some relationship to the risk factor for the outcome probability, there is clearly a stronger relationship to the outcome itself, thus objectively justifying the matching. (e.g., the $p$ value of.45 indicates no relationship exists between the matched variable and the outcome of interest, while the $\mathrm{p}$ value $<.0001$ indicates that matched variable is related to the expo- 
sure variable being tested. It is well known that matching on a variable that is associated only with exposure, not with disease, reduces statistical efficiency in a case - control design (Zondervan et al, 2002; Rubenfeld et al., 1999; Day, Byar, \& Green, 1980), and this in essence, defines the problem of overmatching).

To sum, variables such as birth year, $\mathrm{HMO}$, age, gender, address should first and foremost be matched if and only if there is a truly justifiable rationale to expect they have an independent causal pathway to the outcome; "matching will increase he efficiency of the study when the matching variable is a strong outcome determinant, but will actually reduce it when the matching variable is strongly related to the exposure variable (over-matching)," (Hanson \& Khamis, 2008, p.595-596). Second, if the majority of the variance in the risk factor being tested is removed by matching, before the hypothesis is tested, extreme caution in reporting a lack of effect is warranted. Finally, recalling that sample size will be held constant, testing the relationships of $\mathrm{M}$ to $\mathrm{R}$ and $\mathrm{P}$ and comparing the $\mathrm{p}$ values can be used to justify matching in the context of the matching variable removing variance relating to the risk factor. We would propose that overmatching has and will continue to be a problem in matched case control designs, but suggest that employing the three checks above will serve to lessen deleterious effects associated with publishing overmatched results.

We welcome comments on these proposals.

\section{Acknowledgement}

This work was partially funded by a small grant awarded to the second author, Robert T. Hitlan from Safeminds. We thank Safeminds for their support.

\section{Author details}

M. Catherine DeSoto and Robert T. Hitlan

University of Northern Iowa, Cedar Falls, USA

\section{References}

[1] Austin D. W., Shandley K. A., Palombo E. A. 2010. Mercury in vaccines from the Australian childhood immunization program schedule. J. Toxicol. Environ Health A, 73: 637-40.

[2] Agudo A. and González C. A. 1999. Secondary matching: A method for selecting controls in case-control studies on environmental risk factors. Int. J. Epidemiol., 1999: 1130-1133. 
[3] Al-Taiara, A., Assabria, A., Al-Haboria, M., Azazya, A., Algabrib, A., Alganadib, M., Christopher J. M., Whittyc, and Jaffarc, S. 2009. Socioeconomic and environmental factors important for acquiring non-severe malaria in children in Yemen: A case-control study. Transactions of the Royal Society of Tropical Medicine and Hygiene, 1; 72-78.

[4] Bonner, P. C., Schmidt, W. P., Belmain, S. R., Oshin, B., Baglole, D., and Borchert, M. 2007. Poor housing quality increases risk of rodent infestation and lassa fever in refugee camps of Sierra Leone. Am. J. Trop. Med. Hyg., 77; 169-175.

[5] Day, N. E., Byar, D. P., and Green, S. B. 1980. Overadjustment in case control studies. Am. J. Epidemiol., 112: 696-706.

[6] Delong, G. A. 2011. Positive association found between autism prevalence and childhood vaccination uptake across the U.S. population. J. Toxicol. Environ. Health A, 74: 903-16.

[7] DeStefano F. 2007. Vaccines and autism: Evidence does not support a causal association. Clin. Pharmacol. Ther., 82: 756-759.

[8] Gallagher, C. M. and Goodman, M. S. 2010. Hepatitis B vaccination of male neonates and autism diagnosis, NHIS 1997-2002. J. Toxicol. Environ. Health A, 73: 1665-1677.

[9] Geier D. A., and Geier M. R. 2006. An evaluation of the effects of thimerosal on neurodevelopmental disorders reported following DTP and Hib vaccines in comparison to DTPH vaccine in the United States. J. Toxicol. Environ. Health A, 69: 1481-1495.

[10] Hansson, L. and Khamis, H. J. 2008. Matched samples logistic regression in case-control studies with missing values: when to break the matches. Statistical Methods in Medical Research, 17: 595-607

[11] Hewitson, L., Houser, L. A., Stott, C., Sackett, G., Tomko, J. L., Atwood, D., Blue, L., White, E. R. 2010. Delayed acquisition of neonatal reflexes in newborn primates receiving a thimerosal-containing hepatitis $B$ vaccine: Influence of gestational age and birth weight. J. Toxicol. Environ. Health A, 73: 1298-1313

[12] Jackson, L., Kackson M. et al. (2006). Evidence of bias in estimates of influenza vaccine effectiveness in seniors. Int. J. Epidemiol. 35 (2): 337-344.

[13] Jefferson, T. 2006. Influenza vaccination: policy versus evidence. British Medical Journal, 333: 912-915.

[14] Marsh, J. L., Hutton, J. L., and Binks, K. 2002. Removal of radiation dose response effects: an example of over-matching. British Medical Journal, 325: 327-330.

[15] Miettinen, O.S. 1969. The matched pairs design in the case of all-or-none responses. Biometrics, 24: 339-52

[16] Monson, R. R. 1990. Occupational Epidemiology ( $2^{\text {nd }}$ Ed.). BocaRaton, FL: CRC Press. 
[17] Parker, S. K., Schwartz, B., Todd, J., and Pickering, L. K. 2004. Thimerosal-containing vaccines and autistic spectrum disorder: A critical review of published original data. Pediatrics, 114: 793-804.

[18] Price, C. S., Thompson, W. W., Goodson, B., Weintraub, E. S., Croen, L., A., Hinrichsen, V. L., Marcy, M., Robertson, A., Eriksen, E., Lewis, E., Bernal, P., Shay, D., Davis, R. L., and DeStefano, F. 2010. Prenatal and infant exposure to thimerosal from vaccines and immunoglobulins and risk of autism. Pediatrics, 126: 655-664.

[19] Rahman. M, Sakamoto, M. D., and Fukui, T. 2003. Conditional versus unconditional logistic regression in the medical literature J. of Clin. Epidem., 56: 101-102.

[20] Rubernfeld, G. D., Angus, D. C., Pinsky, M. R., Curtis, J. R., Coners, A. F., and Bernard, G. R. 1999. Outcomes research in critical care. Results of the american thoracic society critical care assembly workshop on outcomes research. Am. J. Respir. Crit. Care Med., 160: 358-367

[21] Sasieni, P., Castanon, A., and Cuzick, J. 2009. Effectiveness of cervical screening with age: Population based case-control study of prospectively recorded data. British Medical Journal, 339: b2968.

[22] Vertraeten, T., Davis, R. L., DeStefano, F. 2003. Safety of thimerosal containing vaccines: A two-phased study of computerized health maintenance databases. Pediatrics, 112: 1039-1048.

[23] Vidal, J. S., Vidailhet, M., Elbaz, A., Derkinderen, P., Tzourio, C., and Alpérovitch, A. 2008. Risk factors of multiple system atrophy: A case-control study in French patients. Movement Disorders, 23: 797-803.

[24] West, D. W., Schuman, K. L., Lyon, J. L., Robison, L. M., and Allred, R. 1984. Differences in risk estimation from a hospital and a population-based case-control study. Int. J. Epidemiol., 13: 235-239.

[25] Krina, T., Zondervan1, L. R., Cardon, L. and Kennedy, S. H. 2002. What makes a good case-control study? Human Reproduction, 17: 1415-1423. 

Chapter 7

\title{
Pro-Inflammatory Phenotype Induced by Maternal Immune Stimulation During Pregnancy
}

\author{
Nicholas M. Ponzio, Mili Mandal, Stella Elkabes, \\ Pan Zhang, Junichi Sadoshima, Sayantani Basak, \\ Peiyong Zhai and Robert Donnelly
}

Additional information is available at the end of the chapter

http://dx.doi.org/10.5772/53990

\section{Introduction}

There is consensus among investigators studying Autism Spectrum Disorders (ASD) that the etiological basis involves environmental factors acting on the genetic susceptibility of the individual [1-5]. Over 100 candidate genes that may contribute to ASD susceptibility have been identified, and numerous environmental "triggers" have been suggested. Yet, the cause of ASD eludes clear definition and most likely is, as in most diseases, multi-factorial. However, several common immunological themes emerge from clinical and experimental studies of ASD, including persistent neuroinflammation, immune dysregulation, or autoimmune manifestations in many autistic children. Thus, in addition to genetic and environmental factors, there is compelling evidence that immune factors also play a role in ASD. Abnormalities consistent with immune dysregulation, including abnormal or skewed $\mathrm{T}$ helper (Th) cell subsets and cytokine profiles, decreased lymphocyte numbers, decreased $\mathrm{T}$ cell mitogen responses, and an imbalance of serum immunoglobulin levels have been reported in children with ASD [6-11].

Recent results of transcriptomic analysis of autistic brains [5] provides strong evidence supporting a gene-environment etiology for ASD. These authors demonstrated consistent differences in transcriptome organization in the cerebral cortex of autistic and normal brains, and identified two discrete modules of co-expressed genes associated with autism. The first, a neuronal module of 209 genes, was enriched for known autism susceptibility genes, and the second module of 235 genes was enriched for immune genes and glial markers. Gene enrichment analysis showed that genes in the neuronal module were downregulated and enriched 
for gene ontology categories related to synaptic function, whereas the genes in the immuneglial module were upregulated, and showed enrichment for gene ontology categories implicated in immune and inflammatory responses. The finding of a genetic association for the neuronal module genes, but a non-genetic association for the immune-glial module genes strengthens the gene-environment etiology for ASD.

Compelling clinical data demonstrate that children of mothers exposed to certain infectious organisms during pregnancy have significantly higher frequencies of neurological disorders [12-21], including schizophrenia and ASD, the etiology of which have been linked to activation of the maternal inflammatory/immune responses (reviewed in [9, 22]). Rodent studies in which the maternal immune system is activated during pregnancy replicate these clinical findings, and provide validated mouse models of ASD [14, 15, 19, 23-33]. We have used a well-characterized prenatal mouse model to investigate questions related to the influence of maternal immune stimulation during pregnancy as an environmental risk factor that affects development of the brain and immune system in the offspring.

Injection of pregnant dams with polyclonal immune stimuli, [e.g., polyinosinic-polycytidylic acid (poly(I:C), lipopolysaccharide (LPS)] or direct injection of the pro-inflammatory cytokines these polyclonal stimuli induce (e.g., IL-1, IL-2, IL-6) cause immune dysregulation and behavioral abnormalities in their offspring in comparison to the offspring of pregnant dams given a control [i.e., Phosphate Buffered Saline (PBS)] injection [30, 34-39]. The underlying mechanisms that mediate these abnormalities have not been clearly defined, and are the focus of ongoing studies by us and others. A unique and powerful advantage of this model is the ability to examine subjects for the initiation and persistence of effects and mechanisms over a continuum of time and development from the earliest embryonic stages through the neonatal period and into adulthood.

While it is impossible for any animal model to completely replicate a human condition as complex as ASD, the mouse model of maternal immune stimulation with poly(I:C) has been recognized as an excellent prenatal model for numerous reasons presented in recent reviews by Meyer and Feldon [40] and Patterson [41]. These include (i) face validity (resemblance to the human symptoms) (ii) construct validity (similarity to the underlying causes of the disease) and (iii) predictive validity (expected responses to treatments that are effective in the human disease) [42]. Thus, offspring from poly(I:C)-injected dams exhibit behavioral anomalies reminiscent of those seen in autistic and schizophrenic individuals. In addition to their behavioral abnormalities, our studies show that as a result of in utero exposure to products of maternal immune stimulation these offspring also exhibit a "pro-inflammatory" phenotype that confers a vulnerability to develop immune-mediated pathology after birth and into adulthood [43-45].

In this regard, the results obtained from our investigation of the poly(I:C) mouse model have provided the scientific rationale for an ongoing translational research project to determine if similar molecular pathogenic mechanisms are involved in a cohort of ASD children who also exhibit diagnostic evidence of immune dysregulation [46]. Using DNA obtained from the Autism Genetic Resource Exchange (AGRE) database, we initiated a parallel study to determine if there were polymorphisms in selected maternal cytokine genes that occurred 
more frequently in mothers of these autistic children. Our results show that mothers of autistic children in this cohort have significant increases in pro-inflammatory cytokine gene polymorphisms, thereby conferring the genetic capability to respond more vigorously to immune stimulation by producing the types and amounts of cytokines that promote inflammatory reactions. Thus, results obtained from our investigation of the experimental prenatal mouse model of maternal immune stimulation during pregnancy have already shown biological relevance in humans.

\begin{tabular}{|c|c|c|c|c|c|}
\hline Th cell Type & Surface Markers & Signal Pathways & $\begin{array}{l}\text { Transcription } \\
\text { Factor }\end{array}$ & Inducing Cytokines & $\begin{array}{l}\text { Cytokines } \\
\text { produced }\end{array}$ \\
\hline Th1 & CD4; Tim-3 & STAT1 & T-bet & IL-12 & IFN-Y, IL-2 \\
\hline Th2 & $\mathrm{CD} 4 ; \mathrm{T} 1 / \mathrm{ST} 2$ & STAT3 & GATA3 & IL-4 & IL-4,5,10,13 \\
\hline \multirow{2}{*}{ Th17 } & \multirow{2}{*}{ Not yet defined } & \multirow{2}{*}{ STAT3 } & \multirow{2}{*}{ RORyt } & IL-1, IL-6 & TNF-a \\
\hline & & & & TGF- $\beta$ & IL-1,6,17,21,22 \\
\hline $\mathrm{T}_{\text {reg }}$ & $C D 4 ; C D 25^{\text {hi }}$ & FoxP3 & FoxP3 & TGF- $\beta$ & IL-10, TGF- $\beta$ \\
\hline
\end{tabular}

Table 1. Properties of Thelper (Th) cell subsets

The hypothesis we are investigating in the prenatal mouse model is that maternal immune stimulation during pregnancy acts as a "first hit" that alters the developing immune system in ways that result in more robust pro-inflammatory immune responses by offspring upon subsequent (i.e. second hit) postnatal immune stimulation. Moreover, such fetal programming occurs in elements of both the innate and adaptive immune systems. Therefore, our experiments investigate how maternal immune stimulation during pregnancy influences the development and function of myeloid and lymphoid compartments of the immune system beginning at the level of the progenitor cells, and progressing to functional outcomes in neonates and adult offspring. In the myeloid compartment, we are focusing on the functions of Antigen Presenting Cells (APC), and on those innate immune elements that mediate acute inflammatory responses. With respect to the adaptive immune system, we are focusing on pro-inflammatory $\mathrm{T}$ helper (Th) cell subsets (Th1 and Th17) and anti-inflammatory Th cell subsets [T regulatory $\left(\mathrm{T}_{\text {reg }}\right)$ cells and Th2 cells]. To do this, we are using several well-characterized model systems to document the pro-inflammatory nature of the offspring of poly(I:C)-injected vs. PBS-injected pregnant dams. The results of these studies are forming a solid foundation to investigate how the pro-inflammatory phenotype exhibited by these offspring also contributes to the etiology and neuroinflammation associated with ASD.

Th cell subsets are induced by different cytokines, use different cell signaling pathways, and produce unique cytokine profiles mediated by cytokine-specific transcription factors (Table 1). Th17 and $\mathrm{T}_{\text {reg }}$ cells are dependent on cytokines for their development, maintenance, and function, and have been implicated in modulating the incidence and/or progression of various inflammatory and autoimmune phenomena, including rheumatoid arthritis (RA) [47], Experimental Autoimmune Encephalomyelitis (EAE) [48-50], Inflammatory Bowel Disease 
(IBD) [51-53], diabetes [54, 55], and atherosclerosis [56, 57]. Thus far, however, little is known about the involvement of proinflammatory Th1 and Th17 cells in autism, and how Th cell subsets interact with microglial APC in the brain [58].

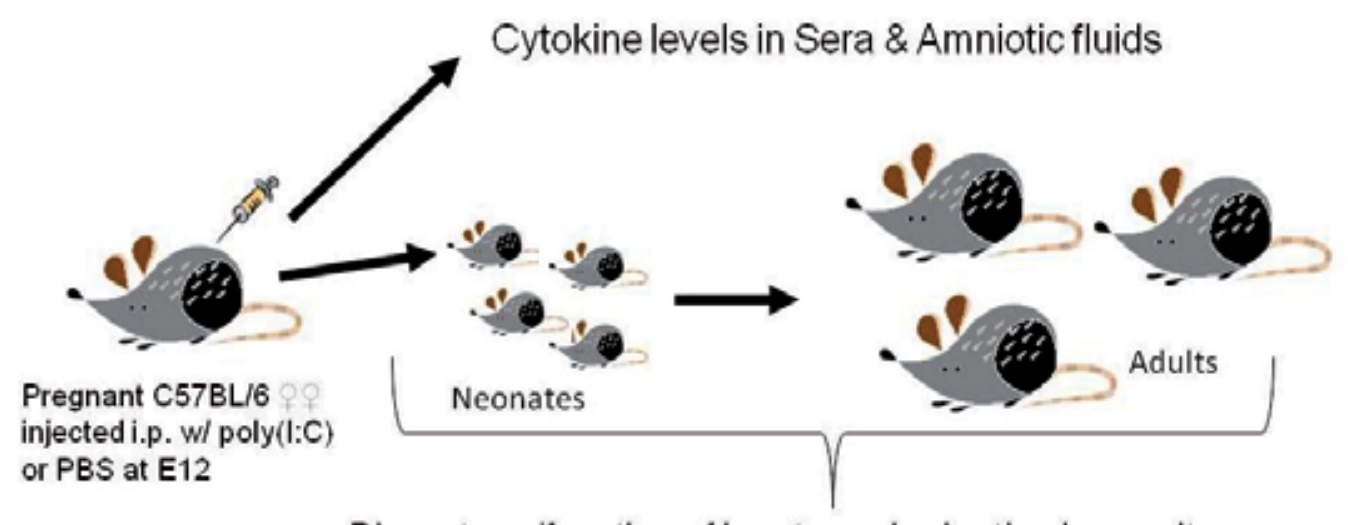

Phenotype/function of innate and adaptive immunity

\begin{tabular}{|c|l|l|}
\hline \multicolumn{2}{|c|}{ PregnantDams } & \multirow{2}{*}{ Phenotype of Offepring } \\
\hline $\begin{array}{c}\text { Immune } \\
\text { status }\end{array}$ & \multicolumn{1}{|c|}{ Injected with } & \\
\hline \multirow{2}{*}{$\begin{array}{c}\text { Non- } \\
\text { immune }\end{array}$} & PBS & Non-immune Control \\
\cline { 2 - 3 } & poly(I:C) & Non-immunePro-inflammatory \\
\hline \multirow{2}{*}{ Immune } & PBS & Immune Control \\
\cline { 2 - 3 } & poly(I:C) & Immune Pro-inflammatory \\
\hline
\end{tabular}

Figure 1. Prenatal models of maternal immune stimulation during pregnancy

\section{Prenatal models of immune stimulation using poly(I:C)}

In the prenatal model of maternal immune stimulation with poly(I:C) (Figure 1), C57BL/6 (B6) females and males are mated, and appearance of a vaginal plug is considered day zero of gestation (E0). At E12, pregnant females are given a single i.p. injection of poly(I:C) or PBS alone as a control. Sera and amniotic fluids are harvested, and stored at $-80^{\circ} \mathrm{C}$ prior to measurement of cytokine levels by Luminex ${ }^{\circledR}$ bead-based multiplex assay [59] that measures up to 32 individual cytokines. In vitro and in vivo analyses are also performed on the neonatal and adult offspring of these poly(I:C)-injected and PBS-injected pregnant dams to assess the phenotype and function of their innate and adaptive immune system components. As also shown in Figure 1, we mate females that are immunologically naïve (i.e., nonimmune), as well as females that possess immunological memory (i.e., immune) with immunologically naïve males. The phenotype of the offspring from these mating schemes reflects the immune status of the of the pregnant dams, and the nature of the prenatal stimulus. Our results demonstrate that offspring of both non-immune and immune poly(I:C)-in- 
jected dams exhibit a pro-inflammatory phenotype in comparison to offspring of PBSinjected dams. In addition, however, T helper (Th) cells from offspring of immune poly(I:C)injected dams show a unique ability to preferentially differentiate to become proinflammatory Th17 cells.

Figures 2A and 2B show the significant increases in IL-6 at 2hr and 16hr after poly(I:C) injection, and similar differences were also seen in levels of IL-1 $\beta$, IL-12, TNF- $\alpha$, and GM-CSF in these samples $[45,60]$.

(A)

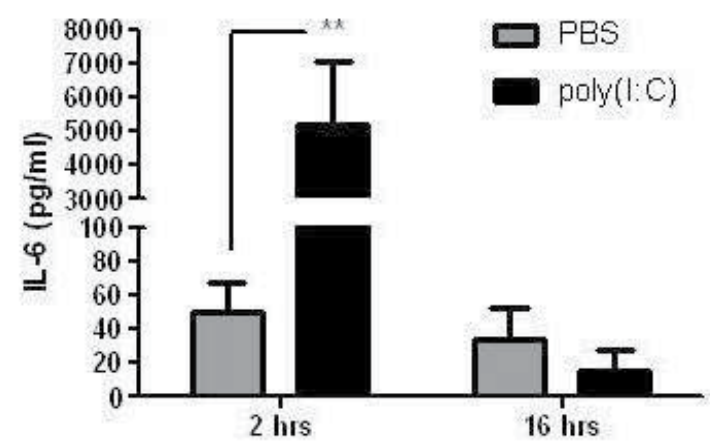

(B)

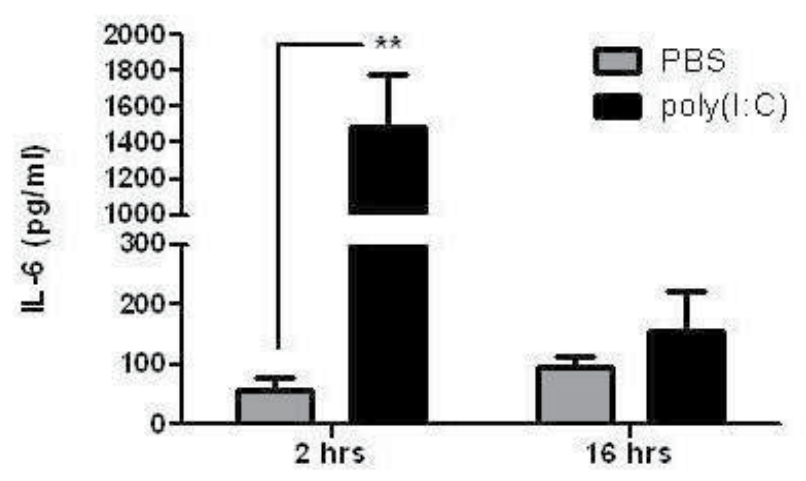

Figure 2. IL-6 levels in sera and amniotic fluids from B6 pregnant dams. Samples collected 2hrs after injection of poly (I:C) or PBS were tested for IL-6 in sera (A) and amniotic fluids (B) using Luminex bead-based multiplex assay. Data show mean \pm SEM. ( $N=3-8 ;{ }^{* *} \mathrm{p}<0.01$ using Tukey's HSD test $)$

In addition to testing sera and amniotic fluids from pregnant dams, we also analyze phenotypic and functional characteristics of lymphoid cells from offspring. To avoid bias due to "litter effects" [61], the number of subjects examined in our experiments not only reflects offspring within a litter, but also offspring from multiple dams, so that the " $\mathrm{N}$ " in our studies considers both the number of dams, as well as the number of offspring. 
Use of immunologically naïve pregnant dams: Immunologically naïve mice are used by most investigators in the standard prenatal model of immune stimulation during pregnancy. These pregnant dams are injected with poly(I:C) on embryonic day 12 (E12), and control pregnant dams are injected with PBS. The embryos and offspring from these dams are then used experimentally to determine the influence of maternal immune stimulation on prenatal development and postnatal function. Since this model was originally developed to investigate neurodevelopmental disorders, such as schizophrenia and autism, a majority of the studies focus on the CNS and behavioral outcomes of offspring. These investigations have shown that maternal immune stimulation during pregnancy with polyclonal stimuli [e.g., poly(I:C) or LPS], infectious pathogens, or specific cytokines (e.g., IL-2 or IL-6) results in expression of ASD-like behavioral manifestations, as well as structural or functional changes in cells in the brain of the offspring [39-41, 61, 62].

However, in the prenatal models that use poly(I:C) as the immune stimulus, the type of poly(I:C) (i.e., sodium or potassium salt), dose of poly(I:C) $(2-20 \mathrm{mg} / \mathrm{Kg})$, and time of injection during pregnancy (E9 through E18) can influence some of the parameters that have been examined in these offspring, including open field exploration, sensorimotor gating (e.g., prepulse inhibition of the startle response), and repetitive/perserverative behavior $([63,64]$. It is thought that poly(I:C)-induced maternal cytokines are primarily responsible for the abnormalities seen in offspring. However, downstream effects induced by these maternal cytokines or trans-placental stimulation of fetal tissues by poly(I:C) itself have not been completely ruled out.

Use of pregnant dams with immunological memory: In addition to the existing model using immunologically naïve dams, we also modified this mouse model of neurodevelopmental disorders by using dams that possess immunological memory prior to mating [43, 44]. This experimental design more closely resembles the human scenario, where women possess immunological memory resulting from immunizations and natural exposure to environmental antigens prior to pregnancy. Using dams with immunological memory yields a more robust mouse prenatal model, which revealed outcomes in offspring that may be significant not only in the etiology and/or pathogenesis of schizophrenia and autism, but also in other disorders that are currently not being considered by use of these prenatal mouse models.

In both of these models, we and others have previously shown that following injection of poly(I:C), pregnant dams produce significantly higher levels of pro-inflammatory cytokines (e.g., IL-1, IL-6, IL-12, TNF- $\alpha$, and GM-CSF) than PBS-injected dams in sera as well as amniotic fluids. Most of the studies involving structural/chemical changes and behavioral abnormalities that are observed after injection of poly(I:C) to pregnant dams have been performed on adult offspring from immunologically naïve pregnant dams. Recently, Hsaio, et al. [65] observed alterations in the peripheral immune system of these offspring. Our results indicate that the adult offspring of immunologically naive poly(I:C)injected pregnant dams also exhibit a more robust acute inflammatory response after injection of the TLR2 ligand, zymosan [45, 60]. 

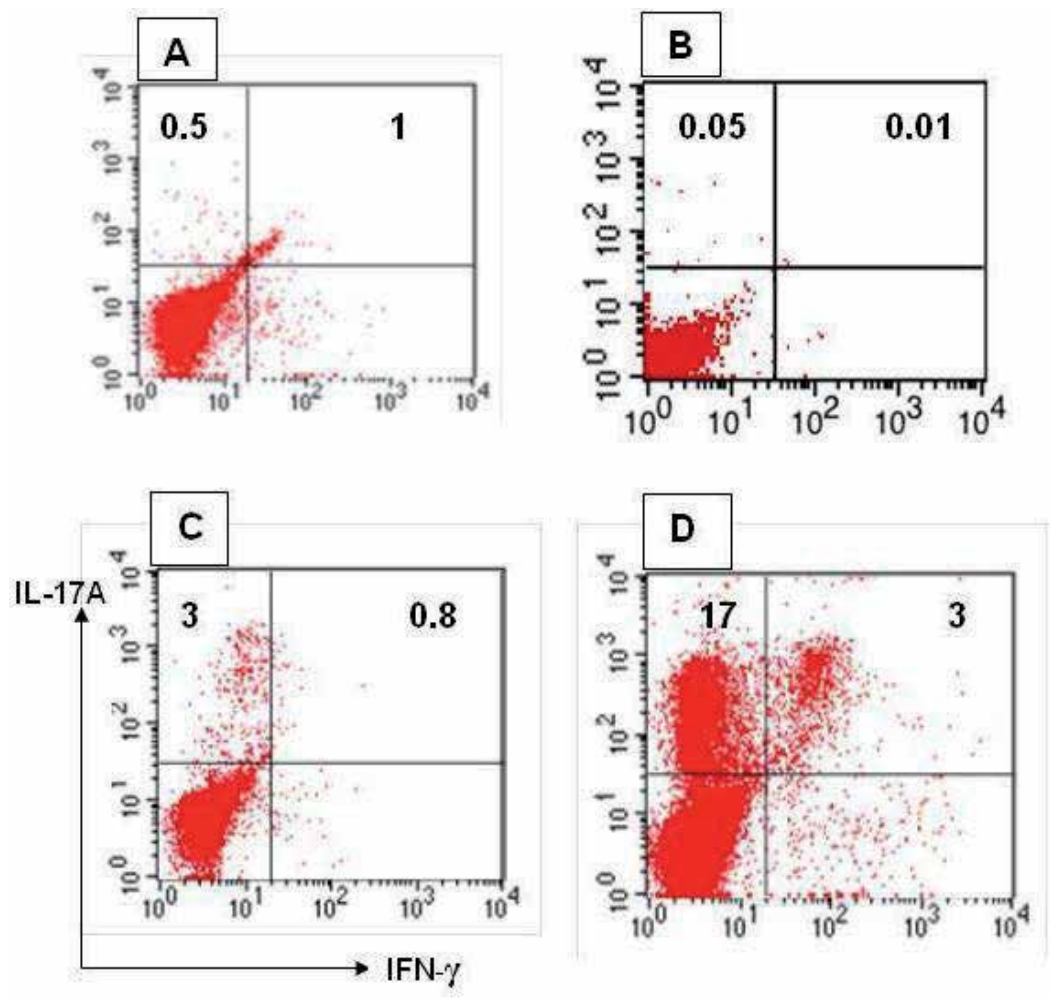

Figure 3. Enhanced production of Th17 cells in offspring poly $(\mathrm{I}: \mathrm{C})$-injected $(20 \mathrm{mg} / \mathrm{Kg})$ immune dams. Spleen cells from 3 wk old offspring of poly(l:C)- and PBS-injected dams were stimulated with $3 \mathrm{ng} / \mathrm{ml}$ PMA and $100 \mathrm{ng} / \mathrm{ml}$ ionomycin for $16 \mathrm{hr}$, the last $4 \mathrm{hr}$ of which were in the presence of $10 \mathrm{ug} / \mathrm{ml}$ Brefeldin A to block cytokine secretion. Cells were harvested, and stained with fluorochrome-conjugated mAbs to detect cell surface molecules and intracellular cytokines by FACS analysis. The spleen cells analyzed in each of the panels were from offspring of PBS-injected immunologically naïve dams (A); offspring of poly(I:C)-injected immunologically naïve dams (B); offspring of PBS-injected immune dams (C), and offspring of poly(I:C)-injected immune dams (D). Numbers in upper left quadrants are percentages of IL-17A ${ }^{+}$(Th17) cells after gating on CD4+ cells. Results shown are representative of seven experiments comparing 18 offspring from 12 different dams. Overall results of percentages of Th17 cells were: $15.1 \pm 7.8$ in offspring from immune poly(I:C)-injected dams vs. $0.8 \pm 0.5$ in offspring from immune PBS-injected dams ( $p=0.05$ using Tukey's HSD test )

The offspring of poly(I:C)-injected (vs. PBS-injected) pregnant dams who possess immunological memory prior to pregnancy exhibit a unique pro-inflammatory phenotype in which there is preferential development of Th17 lymphocytes after T cell activation (Figure 3) [43, 44]. This preferential Th17 cell development is not seen at all in offspring of immunologically naïve poly(I:C)-injected or PBS-injected pregnant dams. Given their role in immune-mediated disorders, it is likely that the potential to produce Th17 cells that we have discovered in offspring of poly(I:C)-injected pregnant dams with immunological memory may also be an important component in the neuroinflammatory pathogenesis of ASD-like changes that have been observed in this prenatal mouse model. Thus, one hypothesis we have tested is that the pro-inflammatory phenotype of offspring induced as a result of embryonic development in a pro-inflammatory cytokine environment in utero make them more susceptible 
(i.e., vulnerable) to develop immune-mediated pathology. Indeed, we have obtained compelling results from in vivo experiments in adult offspring that strongly support this possibility. Using a model of EAE, in which mice are injected with an encephalogenic-peptide, Myelin Oligodendrocyte Glycoprotein peptide $\left(\mathrm{MOG}_{35-55}\right)$, we found that adult offspring of poly(I:C)-injected pregnant dams exhibited a significantly higher frequency and earlier onset of clinical symptoms of EAE compared to offspring of PBS-injected pregnant dams [45, 60]. Our zymosan induced results and the EAE experiments are described in subsequent sections of this chapter.

Maternal vs. fetal sources of cytokines: In this prenatal model, a single i.p. injection of poly(I:C) (or control PBS) is given on gestational day 12 (E12). Convincing evidence from this model by us and others [30, 43, 44, 61, 66-68] has shown that pro-inflammatory cytokines (IL-1, IL-6, IL-12, TNF- $\alpha$, GM-CSF) produced as a result of maternal immune stimulation during pregnancy induce changes in the development of the immune system and the brain of offspring that result in immunological and behavioral manifestations similar to those seen in individuals with ASD. To what degree these changes are induced by cytokines produced by the mother or fetus has not been fully defined. However, our results using IL-6 knock-out (KO) dams mated with wild type males [44], suggests that there is a fetal source for at least some of the cytokines detected in the amniotic fluid of poly(I:C)-injected pregnant dams.

In these experiments, our results from mating IL-6 knock-out (KO) B6 females (IL-6-1-) and wild-type (WT) B6 males $\left(\mathrm{IL}-6^{+++}\right.$) show that despite a maternal genetic deficiency for IL-6 production, fetal components of the heterozygous IL- $6^{+/}$placenta are a source of this cytokine (Figures 4 and 5), and heterozygous neonates can also produce IL-6 [44]. Using similar mating schemes in this prenatal model, however, Hsiao, et al. [66] did not find IL-6 in amniotic fluid of poly(I:C)-injected pregnant IL-6 KO B6 dams. Our results suggest that poly(I:C) (a TLR3 agonist) stimulates fetal placental tissues directly, and contributes to the levels of IL- 6 found in amniotic fluid. This is relevant to the interpretation of data about the source of IL-6 (as well as other cytokines) found in the amniotic fluids and fetal tissues, such as the brain $[9,63,69]$, and also because TLR3 is expressed in the brain during fetal development [70].

\begin{tabular}{ccc}
\hline Pregnant dams injected with & Sickness behavior ratio & N \\
\hline PBS & $1.00 \pm 0.10$ & 9 \\
\hline Poly(l:C) & $0.40 \pm 0.02^{*}$ & 21 \\
\hline
\end{tabular}

Pregnant dams were analyzed for sickness behavior before and 2 and $24 \mathrm{hrs}$ after poly(l:C) injection. All mice in a group were analyzed by calculating a ratio, where each post injection sickness behavior score was divided by its preinjection score. The individual ratios were then used to calculate the means, standard errors, and significance values. ${ }^{*} p<0.0001$ (Tukey's HSD test).

Table 2. Sickness behavior scores of immune poly(I:C)- and PBS- injected pregnant dams 


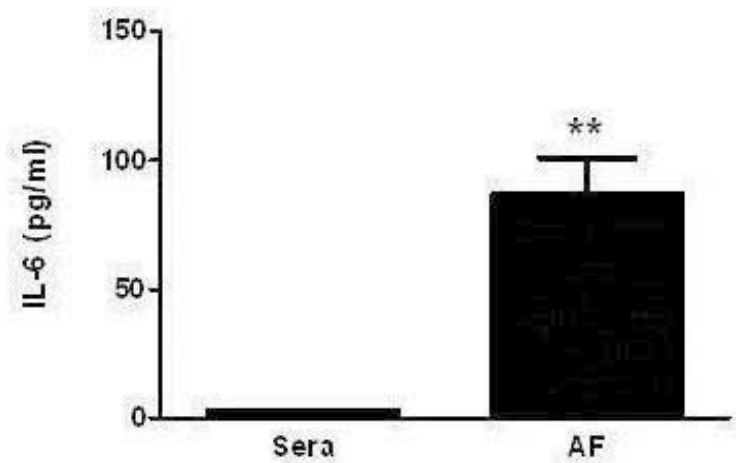

Figure 4. Source of cytokines in pregnant dams. Sera $(N=6)$ and amniotic fluids $(N=17)$ from IL-6 KO pregnant dams were collected $24 \mathrm{hrs}$ after injection of poly(I:C), and IL- 6 levels determined by Luminex assay. ( ${ }^{*} \mathrm{p}<0.0001$ Tukey's HSD test).

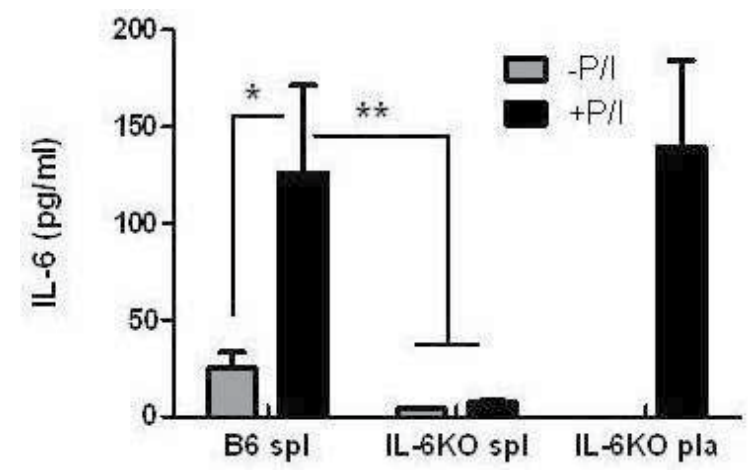

Figure 5. Source of cytokines in offspring. Spleen cells from WT B6 (N=5) and spleen and placental cells from poly(I:C)-injected IL-6 KO females ( $\mathrm{N}=5)$ were cultured with/without PMA and lonomycin (P/I). Supernatants were collected 24hrs later, and tested for the presence of IL-6 by Luminex assay. ( $\mathrm{*} p<0.02$; ${ }^{*} \mathrm{p}<0.01$; Tukey's HSD test)

Regardless of source, however, since in utero exposure to the products of maternal immune stimulation during pregnancy appear to be part of the underlying mechanisms responsible for the changes observed in offspring, it is important to be sure that the pregnant dam responds to the immune stimulus if their offspring are used for experiments. We have addressed this issue by monitoring locomotor activity in a novel environment in every pregnant dam before, and at $2 \mathrm{hrs}$ and $16 \mathrm{hrs}$ after injection as a reliable, non-invasive measure of response to poly(I:C). We opted to use this method in lieu of more invasive procedures that would jeopardize pregnancy in these dams and/or add a level of stress that could influence the cytokine levels and/or fetal development. As shown in Figure 6 and Table 2, there is a consistent and dramatic decrease in activity (indicative of "sickness behavior - [71-74]) in poly(I:C)-injected pregnant dams at 2hrs post injection that is not seen in PBS-injected dams. Moreover, sickness behavior at $2 \mathrm{hrs}$ post poly(I:C) injection correlates very nicely with the increased levels of pro-inflammatory cytokines seen at $2 \mathrm{hrs}$ in the sera and amniotic fluids of pregnant dams (Figure 2). Activity scores 
are measured in every pregnant dam, including those that are brought to term and give birth. In this way, we are confident that the offspring used for subsequent in vivo and in vitro experiments to characterize phenotypic and functional immunological parameters were exposed in utero to a pro-inflammatory cytokine milieu.

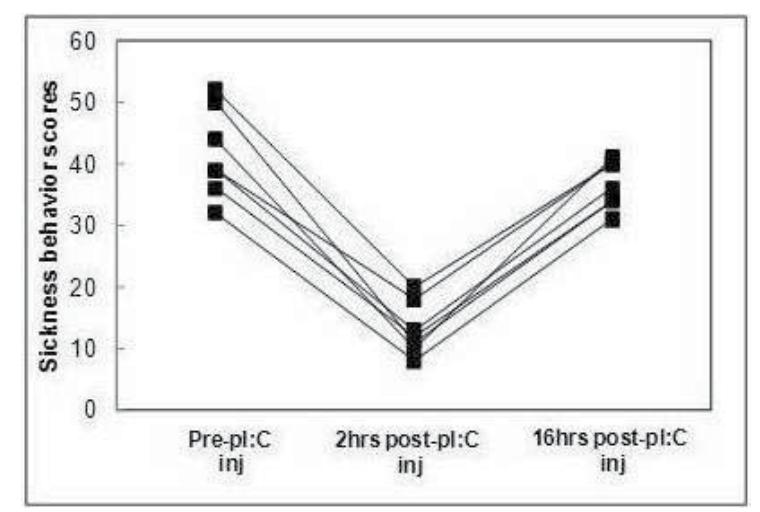

Figure 6. Sickness behavior scores in poly(I:C)-injected pregnant dams. Dams were tested before, and at $2 \mathrm{hrs} \&$ $16 \mathrm{hrs}$ after poly(I:C) or PBS injection. Activity was monitored in a novel environment, and mice were given positive scores for locomotion, rearing, grooming, sniffing, and negative scores for periods of inactivity. The data in this figure show scores for 6 individual poly(l:C)-injected dams at the 3 time periods.

Effects of maternal immune stimulation on Toll-Like Receptors (TLR): We are also examining the effects of poly(I:C) exposure during pregnancy on the expression and function of TLRs during fetal development and in neonates and adult offspring. TLRs are part of a larger family of membrane bound cell surface and intracellular Pattern Recognition Receptors (PRR). Eleven TLRs have been discovered in humans (TLRs 1-11), and 12 TLRs have been found in mice (TLRs 1-12). First discovered in Drosophila [75], this family of molecules is very heterogenous, complex, and highly conserved among species. Individual TLRs bind to particular microbial products, such as LPS, peptidoglycan, lipoproteins, and flagellin on bacteria, as well as viral fusion protein, unmethylated CpG motifs, double- and single-stranded RNAs [76, 77]. TLR expression by cells of the innate and adaptive immune systems allows these cells to recognize and respond to extracellular and intracellular microbial pathogens. Downstream cell signaling pathways are initiated when ligands bind to TLRs, leading to activation of different transcription factors (e.g., NF- $\kappa$ B and others), which stimulate expression of pro-inflammatory cytokine genes (e.g., IL-1, IL-6, TNF, interferons).

Thus, TLRs play an early and important role at the interface between the environment and host tissues by initiating immune responses against pathogens. In addition to expression on cells of the innate and adaptive immune systems, TLRs are also expressed in/on many cell types in various other tissues of the body, including the placenta, embryonic brain, and hematopoietic progenitor cells. In the context of our experimental model of maternal immune stimulation during pregnancy, how the maternal response to poly(I:C) (a TLR3 agonist) during pregnancy affects the normal expression and function of TLR3, as well as other 
TLRs in the developing embryos and offspring is an important question that allows the design of experiments that address underlying mechanisms.

Modulation and desensitization of TLR expression, as well as cross-talk among TLRs has been shown in cells from humans and rodents. There is increasing evidence of TLR expression during embryonic development in the placenta, fetal brain and hematopoietic stem cells [78-81]. In this regard, we have obtained results indicating modulation of TLR expression in $4 \mathrm{wk}$ old neonates from poly(I:C)-injected dams (Figure 7). Using pathway-focused gene expression profiling qRT-PCR arrays, spleen cells from offspring of pregnant dams injected with the TLR3 agonist, poly(I:C), showed a 3.3 to 4.7 -fold increase in constitutive expression levels of TLR2, 4 and 7 over those seen in age-matched control B6 offspring. In contrast, expression levels of TLR3 and 9 were $<2$-fold greater than controls. These results indicate that exposure to poly(I:C) (or poly(I:C)-induced cytokines) during fetal development results in altered TLR expression that persists after birth, consequences of which may relate to differential immune responses to micro-organisms and auto-antigens. These data also suggest that TLR modulation, desensitization, and cross-talk also occur when fetal tissues are exposed to TLR agonists in utero.

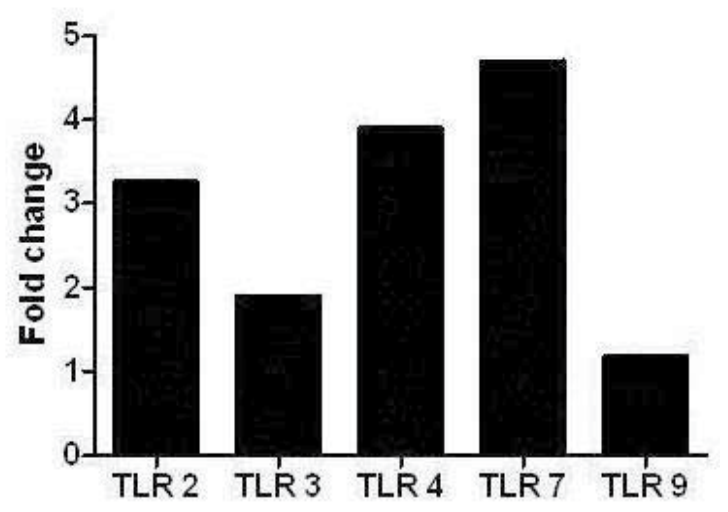

Figure 7. Altered constitutive expression of TLRs in offspring of poly(I:C)-injected dams. RNA was extracted directly from unstimulated spleen cells of 4 wk old offspring of poly(I:C)- and PBS-injected dams, and tested for expression of TLRs using the RT2 Profiler PCR Array system. Data are expressed as fold-changes in gene expression for TLRs in offspring of poly(I:C)-injected dams over PBS-injected dams. The results show $>3$-fold upregulation for TLR2, 4, and 7 and $<2$-fold increase for TLR3 and 9 in offspring of poly(I:C)-compared to PBS-injected dams.

Recent studies have shown expression of TLRs by neural progenitor cells (NPC), neurons and glial cells in the adult brain, which may be important in the responses of these cells to injury or infection [76, 82-88]. Studies of TLR expression in the developing rodent brain have revealed that TLR3 appears as early as embryonic day 12.5 (E12.5) in mouse cortices, but declines over time. By contrast, TLR2 expression appears around E15.5 and increases with time $[70,88]$. Moreover, in standard in vitro neurosphere assays (used to assess developmental potential of NPCs), both TLR2 and TLR3 activation appear to regulate NPC proliferation. These studies raise questions regarding the expression of other TLRs during brain develop- 
ment, and how that expression pattern is altered when dams are exposed to TLR agonists, such as poly(I:C), during pregnancy. The structural and/or functional abnormalities seen in the brains of offspring from poly(I:C)-injected pregnant dams may correlate with alterations of the normal patterns of TLR expression in the developing brain. Therefore, disruption of the normal TLR expression pattern might be involved in the observed structural and/or functional changes in the brain of individuals with neurodevelopmental disorders, such as schizophrenia and autism.

\begin{tabular}{|c|c|c|c|c|}
\hline \multirow[t]{3}{*}{ Cell Marker } & \multicolumn{4}{|c|}{ Percent Positive Cells in Fetuses from } \\
\hline & \multicolumn{2}{|c|}{ PBS-injected dams } & \multicolumn{2}{|c|}{ poly(I:C)-injected dams } \\
\hline & $\# 1$ & \#2 & $\# 1$ & $\# 2$ \\
\hline $\mathrm{Sca}-1^{+}, \mathrm{c}-\mathrm{kit}{ }^{+}(\mathrm{HSC})$ & 0.3 & 0.3 & 1.5 & 1.3 \\
\hline Sca-1; c-kit ${ }^{+}$(CMP) & 24.0 & 25.9 & 21.6 & 24.5 \\
\hline Sca-1+1, c-kit (CLP) & 9.6 & 8.0 & 23.1 & 23.1 \\
\hline
\end{tabular}

Pregnant dams were injected at E12 and fetuses were obtained 24 hrs later. Fetal liver cells from individual fetuses were analyzed by FACS for expression of markers that define HSCs and early common progenitor cells.

Table 3. Hematopoietic Stem Cells in Fetal Liver

TLR expression on hematopoietic stem cells (HSCs): As previously mentioned, maternal exposure to poly(I:C) during pregnancy induces production of pro-inflammatory cytokines, including significant increases in IL-6 in maternal circulation, amniotic fluid, placenta, and fetal brain [51, 89-93]. Direct injection of IL-6 to pregnant dams also results in consequences for the offspring, including structural abnormalities in the brain, as well as behavioral and cognitive abnormalities [30, 34-36, 38]. However, IL-6 also affects the immune system; it is an autocrine growth factor for thymic epithelial cells [94], stimulates fetal hematopoiesis [95], and can alter the balance of $\mathrm{T}_{\text {regs }}$ and Th17 cells towards the pro-inflammatory Th17 phenotype [96-101]. Thus, IL-6 is a key player in the differentiation of cells in the immune system, and may play a role in the immune dysregulation seen in ASD.

Recent studies have also revealed that HSCs not only respond to cytokine signaling to initiate myelopoiesis and lymphopoiesis, but also can sense microbial pathogens directly via TLR signaling [78]. Administration of nanomolar concentrations of the TLR4 agonist, LPS, triggers emigration of monocytes from the BM into the bloodstream, indicating that circulating levels of TLR ligands can also stimulate HSCs within hematopoietic tissues [102]. Additionally, treatment of mice with TLR3 agonist poly(I:C) activates HSCs to proliferate [103]. Therefore, it is likely that in the prenatal model we are studying, HSCs are influenced not only by the poly(I:C) induced cytokines elicited during pregnancy, but also by this TLR3 agonist as well. Therefore, we have examined placentas, fetal livers, and neonatal bone marrow from poly(I:C)-injected (vs.PBS-injected) pregnant dams and offspring to characterize the changes in HSCs, as well as lineage-specific progenitor cells. We examined cells from 
these tissues for surface markers (Sca-1 and c-kit) that define HSCs, and the lineage-specific progenitors for T cells (TCR, CD3), B cells (sIg, CD19), and myeloid cells (CD11b, CD11c).

An example of our results for HSCs in fetal liver is presented in Table 3. Pregnant dams were injected at E12 with either PBS or poly(I:C), and fetuses were examined $24 \mathrm{hrs}$ later. The data show that in comparison to fetuses from PBS-injected dams, fetal livers from poly(I:C)-injected dams had a 4- to 5-fold increase in the percentage of HSCs that were double-positive for Sca-1 and c-kit, and almost a 3-fold increase in the percentage of HSCs that expressed only Sca-1, which are early Common Lymphoid Progenitors (CLP). By contrast, the percent of HSCs that expressed only c-kit, which are early Common Myeloid Progenitors (CMP), was similar in all fetal livers. These results are intriguing because they indicate hyper-proliferation of HSCs and early CLP, which may forecast the preferential changes we have observed in mature Tlymphocytes in the adult offspring of poly(I:C)-injected dams [43-45, 60].

\section{In vivo proof-of-concept experiments}

In addition to our investigation of the consequences of maternal immune stimulation to pregnant dams, embryonic tissues, and 2-4 wk old neonates, we have also extended our studies to adult offspring of poly(I:C)-injected (vs. PBS-injected). Our guiding hypothesis is that as a result of in utero exposure of the fetus to cytokines elicited by maternal immune stimulation (acting as a "first hit"), developmental programming of the immune system occurs in offspring, which persists postnatally and into adulthood. In the case of this prenatal model, such fetal programming results in development of a "pro-inflammatory" phenotype, such that upon subsequent postnatal exposure to an immune stimulus (i.e., second hit) the offspring of poly(I:C)-injected pregnant dams exhibit exacerbated responses in comparison to offspring of PBS-injected dams. Such a scenario is also consistent with the "multiple hit" concept of mental disorders [104, 105]. In the context of ASD, this would mean that abnormalities of behavior and immune dysregulation in some children with ASD could reflect such developmental programming during embryonic development that is manifested postnatally upon encounter with a second hit to their immune system. We tested this hypothesis by using adult offspring of poly(I:C)-injected (vs. PBS-injected) pregnant dams in selected in vivo experimental models that involve activation of their innate and/or adaptive immune systems.

Inflammatory response to TLR2 agonist, zymosan: We induced an antigen non-specific acute inflammatory response in the peritoneal cavity with zymosan (TLR-2 agonist), and assessed the qualitative and quantitative nature of the inflammatory response $4 \mathrm{hrs}$ later [106].

Adult offspring from immunologically naïve poly(I:C)-injected dams were injected i.p. with PBS (control) or zymosan. Adult offspring from immunologically naïve PBS-injected dams were also injected with PBS or zymosan for comparison. Mice were euthanized at $4 \mathrm{hrs,} \mathrm{and}$ $2 \mathrm{ml}$ of cold PBS was used to flush their peritoneal contents. The number and type of peritoneal exudate cells were determined by manual counting and FACS analysis, and the peritoneal fluid was analyzed for the presence of cytokines. 
As shown in Table 4, the $>2$ fold increase in total Peritoneal Exudate Cell (PEC) count in the zymosan-injected poly(I:C) offspring was significantly higher than the count recovered from zymosan-injected PBS offspring. In contrast, there were no significant differences in absolute PEC numbers in control PBS-injected adult poly(I:C) or PBS offspring. The peritoneal cellular infiltrate in offspring injected with PBS was primarily mononuclear cells (monocytes and lymphocytes) (Figure 8A). In contrast, the acute cellular inflammatory response in the peritoneal cavity of zymosan-injected offspring was mostly neutrophils (Figure 8B).

\begin{tabular}{|c|c|c|c|c|c|c|}
\hline \multicolumn{2}{|c|}{ Offspring } & \multirow{2}{*}{$\begin{array}{c}\text { Total PEC } \\
\left(\times 10^{6}\right)\end{array}$} & \multicolumn{2}{|c|}{ Neutrophils } & \multicolumn{2}{|c|}{ IL-6 (pg/ml) } \\
\hline From & Injected with & & Percent & $\begin{array}{c}\text { Absolute } \\
\text { number }\left(\times 10^{6}\right)\end{array}$ & Sera & Peritoneal Fluid \\
\hline \multirow[t]{2}{*}{ PBS-injected dams } & PBS & $0.8 \pm 0.3$ & $<5.0$ & $<0.004$ & $4 \pm 0.6$ & $15 \pm 7.9$ \\
\hline & Zymosan & $6.3 \pm 1.8$ & $70.0 \pm 10$ & $4.40 \pm 1.9$ & $420 \pm 200$ & $1176 \pm 586$ \\
\hline \multirow{2}{*}{$\begin{array}{c}\text { Poly(I:C)-injected } \\
\text { dams }\end{array}$} & PBS & $1.0 \pm 0.4$ & $<5.0$ & $<0.005$ & $8 \pm 2.7$ & $6 \pm 2.5$ \\
\hline & Zymosan & $13.3 \pm 2.1^{\star \star}$ & $81.0 \pm 6$ & $10.8 \pm 2.0^{* *}$ & $2692 \pm 514^{*}$ & $7808 \pm 1306^{*}$ \\
\hline
\end{tabular}

Adult offspring from immunologically naïve poly(I:C)-injected dams were injected i.p. with PBS (control) or zymosan. Adult offspring from immunologically naïve PBS-injected dams were also injected with PBS or zymosan for comparison. Mice were euthanized at $4 \mathrm{hrs}$, and $2 \mathrm{ml}$ of cold PBS was used to flush their peritoneal contents. The number and type of peritoneal exudate cells (PEC) were determined by manual counting and FACS analysis, and sera and peritoneal fluids were analyzed for the presence of cytokines. $N=5-8,{ }^{* *} \mathrm{P}=0.016$ (student's t-test). ${ }^{*} \mathrm{P}<0.05$ (student's $\mathrm{t}$ test)

Table 4. Zymosan-induced acute inflammatory responses in offspring

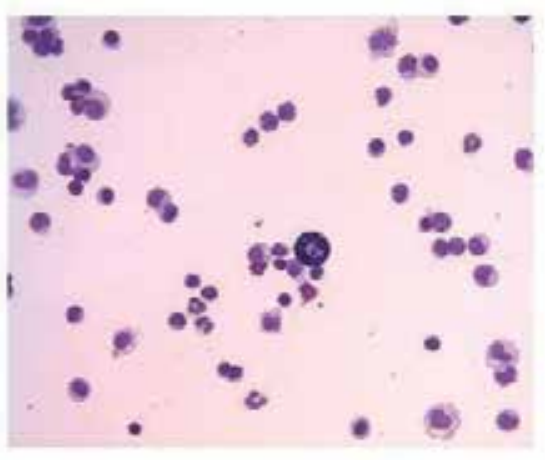

(A)

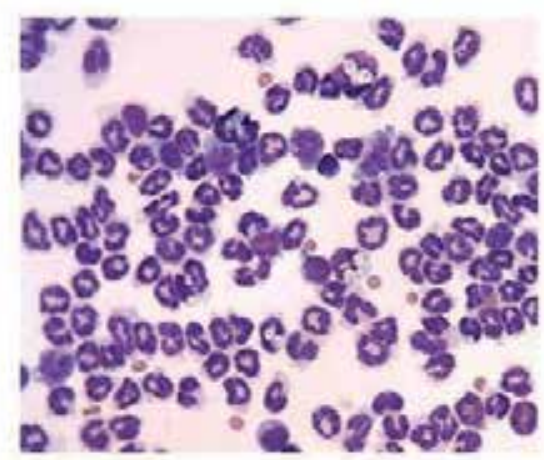

(B)

Figure 8. Acute inflammatory response in zymosan-injected adult offspring. Adult offspring from poly(l:C)-injected non-immune dams were injected i.p. with $1 \mathrm{ml}$ of zymosan suspension or PBS (control). Four hours after injection, peritoneal cavities were flushed with ice cold PBS. Cytospin slides were made from peritoneal exudate cells, and stained with Wright's/Giemsa stain. (A) PBS-injected offspring from poly(I:C)-injected non-immune dams at 630X. (B) zymosan-injected offspring from poly(I:C)-injected non-immune dams at 630X. 
The percentage of neutrophils in zymosan-injected offspring from PBS-injected and poly(I:C)injected offspring (Table 4 ) were similarly high (i.e., $70 \%$ and $81 \%$, respectively). However, because the total number of PEC recovered from zymosan-injected offspring from poly(I:C)injected (vs. PBS-injected) dams was significantly higher, the absolute number of neutrophils from zymosan-injected offspring was also significantly greater in offspring from poly(I:C)-injected dams. Given the huge infiltration of neutrophils into the peritoneal cavity in zymosaninjected offspring, we also examined the bone marrow for evidence of increased myeloid activity, and found evidence of increased myeloid activity in mice showing PEC counts in excess of $10 \times 10^{6}$ cells. As also shown in Table 4, significantly higher levels of IL-6 were observed in fluid obtained from the peritoneal cavity of zymosan-injected poly(I:C) offspring vs. PBS offspring at 4 hrs after zymosan injection. Although not shown in the table, levels of TNF- $\alpha$ and IL-10 were also significantly higher in these zymosan-injected poly(I:C) offspring.

Results from the myocardial Ischemialreperfusion model: Based on the results we obtained using injection of zymosan to mimic the acute inflammatory response induced by an infectious organism, we wished to determine if the offspring of immunologically naïve poly(I:C)-injected dams would also mount a more robust inflammatory responses to endogenous molecules created by non-infectious tissue injury. The persistent neuroinflammation observed in brains of individuals with autism and in rodents from experimental models of neurodevelopmental disorders may be triggered by such endogenous stimuli. For these experiments, we selected a well-characterized cardiac model in which ischemia/reperfusion causes a "sterile" inflammatory response. After an acute myocardial infarction, reperfusion (by thrombolytic therapy or primary percutaneous intervention) is currently the most effective strategy to minimize myocardial damage and improve clinical outcome [107]. Paradoxically, restoring blood flow to the ischemic heart tissue can also induce injury - a phenomenon called myocardial reperfusion injury (R/I). The modes of myocardial cell injury and death following myocardial R/I are apoptosis, autophagy, and necrosis, and several underlying mechanisms have been identified or proposed [108-112]. However, one well-studied cause of myocardial R/I is the host inflammatory response that occurs during reperfusion. Despite the fact that ischemia and reperfusion takes place in a sterile environment, activation of innate and adaptive immune responses occurs and contributes to injury (reviewed in [112]). Contributing factors of reperfusion-induced inflammation include activation of Toll-like Receptors (TLRs), complement activation, free radical generation, cytokine cascade initiated by release of pro-inflammatory cytokines, and chemokine upregulation [113-116]. The presence of these immune mediators leads to recruitment of neutrophils to the ischemic myocardium, which exert cytotoxic effects themselves by release of proteolytic enzymes. Another paradox of myocardial reperfusion is that it may also significantly enhance a healing process. Studies have shown that Monocyte Chemoattractant Protein-1 (MCP-1) is also induced in the infarcted area, which may regulate myeloid cell recruitment, leading to accumulation of macrophages and mast cells that secrete angiogenesis-stimulating factors, which facilitate myocardial repair $[117,118]$.

For these experiments, adult offspring of immunologically naïve poly(I:C)-injected and PBSinjected dams were anesthetized, intubated and ventilated; the heart was exposed by a thor- 
acotomy through the 4th and 5th ribs, and a suture was passed under the left coronary artery. The left coronary artery was occluded for a period of $20 \mathrm{~min}$, and reperfusion applied for $24 \mathrm{hrs}$. Reperfusion was achieved by removal of the occlusion, the thoracotomy incision was closed, and mice were allowed to recover under monitoring in an incubator. After $24 \mathrm{hr}$ of reperfusion, mice were assessed for cardiac injury as previously described $[119,120]$.

As shown in Figure 9, significantly greater cardiac damage was observed in offspring from immunologically naïve poly(I:C)-injected dams than in offspring from control PBS-injected dams. We are currently assessing the underlying mechanisms responsible for the difference in levels of damage in experimental and control offspring. Myocardial I/R induces infiltration of inflammatory cells, such as neutrophils that secrete cytokines/chemokines, including IL-6 and TNF $\alpha$, which in turn contribute to cell death, fibrosis and reduced myocardial contractility [121]. Therefore, it is likely that similar underlying inflammatory mechanisms also occur in the myocardial I/R model as those described above for the acute inflammation induced by zymosan. In the zymosan and myocardial I/R models, the response is measured within hours of the immune stimulus. This indicates that elements of the innate immune system are the primary mediators of the pathology, and suggest that modification of these components has occurred as a result of maternal immune stimulation during pregnancy.

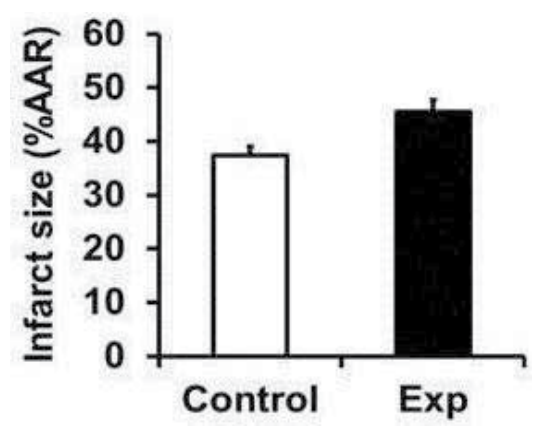

Figure 9. Cardiac damage in offspring of poly(I:C)-injected vs. PBS-injected immunologically naïve pregnant dams. Adult offspring from poly(I:C)-injected (Exp) and PBS-injected (Control) dams were subjected to cardiac ischemia (20 min) and reperfusion (24 hrs), and assessed for cardiac damage (indicated by infarct size). Offspring from poly(I:C)-injected dams exhibited significantly greater cardiac damage ( $p=0.011$; student's t-test) than control offspring. ( $\mathrm{N}=8$ mice/group; 8 weeks of age)

Response to auto-antigens: In order determine if components of the adaptive immune system also mount more robust responses following immune stimulation we chose a well-characterized EAE model of an immune-mediated disease, and used adult offspring of pregnant dams with immunological memory. We have previously shown that T cells from offspring of poly(I:C)-injected pregnant dams with immunological memory (i.e., immune) preferentially differentiate to become Th17 cells after in vitro activation [43-45, 60]. In contrast, T cells from offspring of poly(I:C)-injected immunologically naïve pregnant dams (i.e., non-immune) do not show such Th17 cell preferential differentiation. Th17 cells have been shown to be involved in the neuropathology responsible for the clinical symptoms that develop in Experimental Autoimmune 
Encephalomyelitis (EAE), a mouse model of multiple sclerosis [122-128]. Female offspring of poly(I:C) (vs. PBS) -injected pregnant dams were injected s.c. in each hind flank with an encephalogenic-peptide ( $\mathrm{MOG}_{35-55}$ ) in Complete Freund 's adjuvant (CFA). I.p. injections of pertussis toxin were given after MOG immunization to enhance the immune response and promote $\mathrm{T}$ cell migration into the brain [129]. Typically, 10 - 12 days after injection of MOG and pertussis, $90 \%$ of B6 mice develop progressively: weakness and paralysis in their tail, hindlimb paresis and finally hindlimb paralysis. Controls that receive CFA and pertussis toxin, but no MOG, do not develop clinical signs of EAE.

However, offspring of poly(I:C)-injected immune pregnant dams exhibited clinical signs of EAE significantly earlier and with higher frequency than offspring of poly(I:C)-injected nonimmune dams (Figure 10). More than $70 \%$ of poly(I:C) immune offspring began to show clinical signs of EAE by day 4 after $\mathrm{MOG}_{35-55}$ immunization compared to none seen in poly(I:C) non-immune offspring. On day 7 after immunization, $25 \%$ of poly(I:C) non-immune offspring began to show symptoms, but this was still significantly lower than the $>70 \%$ seen in poly(I:C) immune offspring. By day $9,>60 \%$ of poly(I:C) non-immune offspring showed clinical signs of EAE. In addition to the higher frequency of clinical signs of EAE, poly(I:C) immune offspring also had significantly higher disease severity from days 4-7 post $\mathrm{MOG}_{35-55}$ immunization compared to poly(I:C) non-immune offspring [45, 60]. From days 9-20, the development of EAE among offspring was very similar in both groups. However, the earlier appearance of clinical symptoms affords a window of opportunity to investigate underlying mechanisms in the EAE model that can be applied in future studies of mechanisms of neuroinflammation and pathogenesis in experimental models of autism.

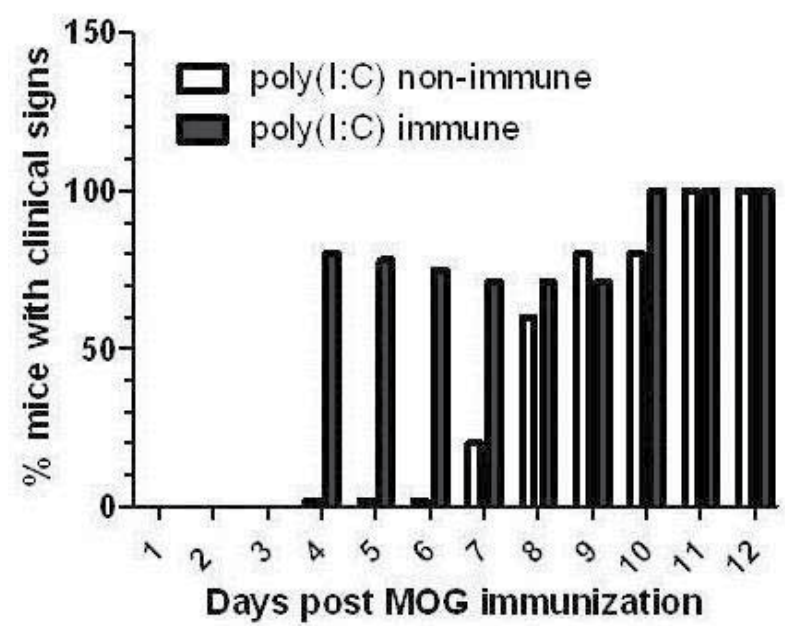

Figure 10. Frequency of mice showing clinical signs of EAE. Adult offspring of of poly(I:C)-injected dams with immunological memory [vs. immunologically naïve poly(I:C)-injected dams] were injected s.c. with MOG in CFA and i.p. with pertussis toxin. Mice were scored for clinical signs of neurological impairment, and the percent of mice showing clinical signs at the indicated times after immunization is shown in this figure. 
These results are consistent with our hypothesis of fetal programming due to effects of maternal immune stimulation during pregnancy, leading to increased susceptibility of offspring to a "second hit" postnatal stimulus. This is likely due to an overall heightened immune responsiveness to develop EAE by MOG-specific Th cells that preferentially differentiate to become Th17 cells in these pro-inflammatory mice and/or a lower antigen threshold for initiation of an immune response. Another contributing factor could be differential responses of these pro-inflammatory offspring to TLR agonists on the mycobacteria in CFA (TLR2) and pertussis toxin (TLR4) used as part of the MOG immunization protocol [130, 131]. Support for this possibility is shown in Figure 7, where offspring of poly(I:C)-injected dams who possess immunological memory showed $>3$-fold higher expression of TLR2 and TLR4 compared to controls.

Overall, our results are consistent with the concept of developmental programming of the immune system [71, 132-136]. These changes persist into adulthood, and increase the vulnerability of offspring from poly(I:C)-injected dams to develop immune-mediated diseases when exposed to subsequent antigen specific, as well as antigen non-specific immune challenges. There is considerable plasticity of the developing immune system, and maternal stressors, such as immune stimulation during pregnancy, can modulate normal development [137]. Immune stimuli during the perinatal period of life can also act as a vulnerability factor for later-life alterations of immune responsiveness [132]. Such fetal programming has been described in relation to abnormalities of metabolism, growth, and behavior in offspring [138-140], as well as in relation to allergic and autoimmune disorders [133-135, 141-143]. The fetal programming of the developing immune system in this prenatal mouse model described herein is most likely mediated by cytokines and/or other inflammatory mediators produced by immune stimulation in response to poly(I:C) given to the pregnant dam. However, as we have previously shown, the sources of these products of immune stimulation are of both maternal and fetal origin [44].

\section{Summary and conclusions}

The results from our investigation of the poly(I:C)-induced prenatal model of neurodevelopmental disorders further identifies and characterizes gene-environment interactions (i.e., maternal immune response genes vs. environmental antigens) that influence fetal development in ways that have consequences for health and disease of offspring. Further characterization of this model presents excellent opportunities to define the underlying mechanisms responsible for the alterations that occur during embryological development, which persist and are manifested in adult offspring. We are using this model to examine the peripheral immune system of offspring to identify mechanisms that explain the immune dysregulation that is characteristic in a significant cohort of children with Autism Spectrum Disorders (ASD). The immunological changes we find in offspring of dams that receive immune stimulation during pregnancy involve significant differences in cyto- 
kines and T helper (Th) lymphocyte subsets. Our investigation of this mouse model has also provided a scientific basis for an ongoing translational research project to determine if similar molecular pathogenic mechanisms are involved in the cohort of ASD children who also exhibit evidence of immune dysregulation. Thus, mothers of autistic children in this cohort have polymorphisms in the same cytokine genes that promote inflammatory reactions in our mouse model, and their children with autism and immune dysregulation inherit the maternal pro-inflammatory phenotype.

Convincing evidence from this model has shown that pro-inflammatory cytokines produced by maternal immune stimulation during pregnancy induce changes in the development of the immune system and brain of offspring that result in similar immunological and behavioral manifestations as those seen in individuals with ASD. Therefore, our results are relevant to the concept of developmental programming of the immune system. In utero exposure to these cytokines produces offspring that exhibit a pro-inflammatory phenotype, which persists throughout the neonatal period and into adulthood. Subsequently, upon postnatal exposure to agents that stimulate the immune system, offspring that exhibit this phenotype mount a more robust immune response in which pro-inflammatory immune elements (i.e., Th17 cells and cytokines) predominate. Th17 cells have been shown to mediate immunopathology in numerous disorders that model human diseases, such as multiple sclerosis, arthritis, inflammatory bowel disease, atherosclerosis, and diabetes. Our use of offspring that have Th cells with the potential to preferentially differentiate into Th17 cells will also determine the contribution of Th17 cells to the etiology and pathogenesis of ASD.

The nature and timing of this second hit to the immune system may also be a critical determining factor in the manifestation of immune outcomes. Thus, if immune stimulation occurs very early in life when organ systems, such as the brain, are still developing, it may lead to neurodevelopmental disorders like ASD. Contrastingly, if the second hit occurs later in life, the outcome may be manifested as an autoimmune disorder. However, possession of a pro-inflammatory phenotype as described in our model is not necessarily a disadvantage. In certain clinical scenarios, such as malignancy or infection with pathogenic micro-organisms, a more robust immune response may provide survival advantage. Indeed, our preliminary results in an infection model indicate that the offspring of poly(I:C)-injected pregnant dams that exhibit a pro-inflammatory phenotype show increased survival time and lower pathogen burden than control offspring from PBS-injected pregnant dams.

As with many other components of the immune system, the effector functions resulting from developmental programming induced by maternal immune stimulation during pregnancy have the potential to be a double-edged sword with outcomes that can be either detrimental or beneficial. The future challenge in studying this prenatal model system will be to sufficiently understand the underlying cellular and molecular mechanisms to enable the design of effective therapeutic interventions to inhibit outcomes that are harmful, and enhance those that are beneficial. 


\section{Author details}

Nicholas M. Ponzio ${ }^{1}$, Mili Mandal ${ }^{2}$, Stella Elkabes ${ }^{3}$, Pan Zhang ${ }^{4}$, Junichi Sadoshima ${ }^{5}$, Sayantani Basak ${ }^{6}$, Peiyong Zhai ${ }^{7}$ and Robert Donnelly ${ }^{8}$

*Address all correspondence to: ponzio@umdnj.edu

1 Department of Pathology \& Laboratory Medicine, University of Medicine and Dentistry of New Jersey - New Jersey Medical School and the Graduate School of Biomedical Sciences, Newark, USA

2 Department of Pathology \& Laboratory Medicine, University of Medicine and Dentistry of New Jersey - Graduate School of Biomedical Sciences, Newark, USA

3 Department of Neurological Surgery, University of Medicine and Dentistry of New Jersey - New Jersey Medical School and the Graduate School of Biomedical Sciences, Newark, USA

4 Department of Pathology \& Laboratory Medicine, University of Medicine and Dentistry of New Jersey - New Jersey Medical School, Newark, USA

5 Department of Cell Biology \& Molecular Medicine, University of Medicine and Dentistry of New Jersey - New Jersey Medical School and the Graduate School of Biomedical Sciences, Newark, USA

6 Department of Pathology \& Laboratory Medicine, University of Medicine and Dentistry of New Jersey - New Jersey Medical School, Newark, USA

7 Department of Cell Biology \& Molecular Medicine, University of Medicine and Dentistry of New Jersey - New Jersey Medical School, Newark, USA

8 Department of Pathology \& Laboratory Medicine, University of Medicine and Dentistry of New Jersey - New Jersey Medical School and the Graduate School of Biomedical Sciences, Newark, USA

\section{References}

[1] Ashwood, P. and J. Van de Water, Is autism an autoimmune disease? Autoimmunity Review, 2004. 3: p. 557-562.

[2] Pardo, C.A., D.L. Vargas, and A.W. Zimmerman, Immunity, neuroglia and neuroinflammation in autism. Int Rev Psychiatry, 2005. 17(6): p. 485-95.

[3] Connolly, A.M., et al., Brain-derived neurotrophic factor and autoantibodies to neural antigens in sera of children with autistic spectrum disorders, Landau-Kleffner syndrome, and epilepsy. Biol Psychiatry, 2006. 59(4): p. 354-63. 
[4] DiCicco-Bloom, E., et al., The Developmental Neurobiology of Autism Spectrum Disorder. J Neurosci, 2006. 26(6): p. 6897-6906.

[5] Voineagu, I., et al., Transcriptomic analysis of autistic brain reveals convergent molecular pathology. Nature, 2011.

[6] Ashwood, P., S. Wills, and J. Van de Water, The immune response in autism: a new frontier for autism research. J Leukoc Biol, 2006. 80(1): p. 1-15.

[7] Ashwood, P., et al., Associations of impaired behaviors with elevated plasma chemokines in autism spectrum disorders. J Neuroimmunol, 2011. 232(1-2): p. 196-9.

[8] Ashwood, P., et al., Altered T cell responses in children with autism. Brain Behav Immun, 2011. 25(5): p. 840-9.

[9] Patterson, P.H., Maternal infection and immune involvement in autism. Trends Mol Med, 2011.

[10] Ashwood, P., et al., In search of cellular immunophenotypes in the blood of children with autism. PLoS One, 2011. 6(5): p. e19299.

[11] Goines, P.E. and P. Ashwood, Cytokine dysregulation in autism spectrum disorders (ASD): Possible role of the environment. Neurotoxicol Teratol, 2012.

[12] Croonenberghs, J., et al., Activation of the inflammatory response system in autism. Neuropsychobiology, 2002. 45: p. 1-6.

[13] Deykin, E. and B. MacMahon, Viral exposure and autism. Am J Epidemiol, 1979. 109: p. 628-638.

[14] Hagberg, H. and C. Mallard, Effect of inflammation on central nervous system development and vulnerability: review. Current opinions in Neurology, 2005. 18: p. 117-123.

[15] Hornig, M., et al., An infection-based model of neurodevelopmental damage. Proc Natl Acad Sci, 1999. 96: p. 12101-12107.

[16] Lipkin, W. and Hornig M, Microbiology and immunology of autism spectrum disorders. Novartis Foundation Symposium, 2003. 251: p. 129-143.

[17] Malek-Ahmadi, P., Cytokines and etiopathogenesis of pervasive developmental disorders. Med Hypotheses, 2001. 56(3): p. 321-324.

[18] Pardo, C. and C. Eberhart, The neurobiology of autism. Brain Pathology, 2007. 17: p. 434-447.

[19] Patterson, P., Maternal infection: window on neuroimmune interactions in fetal brain development and mental illness. Curr Opin Neurobiology, 2002. 12: p. 115-118.

[20] Brown, A.S., Prenatal infection as a risk factor for schizophrenia. Schizophr Bull, 2006. 32(2): p. 200-2.

[21] Brown, A.S. and E.J. Derkits, Prenatal infection and schizophrenia: a review of epidemiologic and translational studies. Am J Psychiatry, 2010. 167(3): p. 261-80. 
[22] Jonakait, G., The effects of maternal inflammation on neuronal development: possible mechanisms. Int J Dev Neurosci, 2007. 25: p. 415-425.

[23] Bell, M. and J. Hallenbeck, Effects of intrauterine inflammation on developing rat brain. J Neurosci Res, 2002. 70: p. 570-579.

[24] Carvey, P., et al., Prenatal exposure to the bacteriotoxin lipopolysaccharide leads to longterm losses of dopamine neurons in offspring: a potential, new model of Parkinson's disease. Front Biosci 2003. 8: p. s826-s837.

[25] Fatemi, S., et al., Prenatal viral infection leads to pyramidal cell atrophy and macrocephaly in adulthood: implications for genesis of autism and schizophrenia. Clin Mol Neurobiol, 2002. 22: p. 25-33.

[26] Hornig, M., et al., Borna disease virus infection of adult and neonatal rats: models for neuropsychiatric disease. Curr Top Microbiol Immunol, 2001. 253: p. 157-177.

[27] Pletnikov, M.V., et al., Rat model of autism spectrum disorders. Genetic background effects on Borna disease virus-induced development brain damage. Ann NY Acad Sci, 2001. 939: p. 318-319.

[28] Pletnikov, M.V., et al., Developmental brain injury associated with abnormal play behavior in neonatally borna disease virus-infected lewis rats: a model of autism. Behav Brain Res, 1999. 100(43-50).

[29] Shi, L., et al., Maternal influenza infection causes marked behavioral and pharmacological changes in the offspring. J Neurosci, 2003. 23(1): p. 297-302.

[30] Smith, S.E., et al., Maternal immune activation alters fetal brain development through interleukin-6. J Neurosci, 2007. 27(40): p. 10695-702.

[31] Weissenbock, H., et al., Microglial activation and neuronal apoptosis in Bornavirus infected neonatal Lewis rats. Brain Pathology, 2000. 10(260-272).

[32] Lancaster, K., et al., Abnormal social behaviors in young and adult rats neonatally infected with Borna disease virus. Behav Brain Res, 2007. 176: p. 141-148.

[33] Rousset, C., et al., Maternal exposure to LPS induces hypomyelination in the internal capsule and programmed cell death in the deep gray matter in newborn rats. Pediatr Res, 2006. 59: p. 428-433.

[34] Dammann, O. and A. Leviton, Maternal intrauterine infection, cytokines, and brain damage in the preterm newborn. Pediatr Res, 1997. 42: p. 1-8.

[35] Conroy, S., et al., Interleukin-6 produces neuronal loss in developing cerebellar granule neuron cultures. J Neuroimmunol, 2004. 155: p. 43-54.

[36] Gilmore, J., et al., Prenatal Infection and Risk for Schizophrenia: IL-1beta, IL-6, and TNFalpha Inhibit Cortical Neuron Dendrite Development. Neuropsychopharmacology, 2004. 29: p. 1221-1229. 
[37] Nawa, H. and N. Takei, Recent progress in animal modeling of immune inflammatory processes in schizophrenia: implication of specific cytokines. Neuroscience Research, 2006. 56: p. 2-13.

[38] Samuelsson, A., et al., Prenatal exposure to Interleukin-6 results in inflammatory neurodegeneration in hippocampus with $N M D A / G A B A(A)$ dysregulation and impaired spatial learning. Amer J Physiol Regul Integr Comp Phyusiol, 2006. 290: p. R1345-1356.

[39] Ponzio, N.M., et al., Cytokine levels during pregnancy influence immunological profiles and neurobehavioral patterns of the offspring. Ann N Y Acad Sci, 2007. 1107: p. 118-28.

[40] Alston, E.N., et al., Cardiac ischemia-reperfusion regulates sympathetic neuropeptide expression through gp130-dependent and independent mechanisms. Neuropeptides, 2011. 45(1): p. 33-42.

[41] Patterson, P.H., Immune involvement in schizophrenia and autism: etiology, pathology and animal models. Behav Brain Res, 2009. 204(2): p. 313-21.

[42] Crawley, J.N., Mouse behavioral assays relevant to the symptoms of autism. Brain Pathol, 2007. 17(4): p. 448-59.

[43] Mandal, M., et al., Preferential development of Th17 cells in offspring of immunostimulated pregnant mice. J Reprod Immunol, 2010. 87(1-2): p. 97-100.

[44] Mandal, M., et al., Maternal immune stimulation during pregnancy affects adaptive immunity in offspring to promote development of TH17 cells. Brain Behav Immun, 2011. 25(5): p. 863-71.

[45] Mandal, M., et al. Maternal immune stimulation during pregnancy facilitates prenatal immuno-developmental changes leading to a pro-inflammatory phenotype in offspring. in International Meeting for Autism Research. 2012. Toronto, Canada.

[46] Ramanathan, M., et al., Maternal cytokine regulation in the pathogenesis of autism. 9th Annual International Meeting for Autism Research, 2010.

[47] Postigo, J., et al., Exacerbation of type II collagen-induced arthritis in apolipoprotein E-deficient mice in association with the expansion of Th1 and Th17 cells. Arthritis Rheum, 2011. 63(4): p. 971-80.

[48] Furuzawa-Carballeda, J., M. Vargas-Rojas, and A. Cabral, Autoimmune inflammation from the Th17 perspective. Autoimmun Review, 2007. 6: p. 169+175.

[49] Bettelli, E., et al., Reciprocal developmental pathways for the generation of pathogenic effector TH17 and regulatory T cells. Nature, 2006. 441: p. 235-238.

[50] Segal, B.M., Th17 cells in autoimmune demyelinating disease. Semin Immunopathol, 2010. 32(1): p. 71-7.

[51] Gayle, D.A., et al., Maternal LPS induces cytokines in the amniotic fluid and corticotropin releasing hormone in the fetal rat brain. Am J Physiol Regul Integr Comp Physiol, 2004. 286(6): p. R1024-9. 
[52] Hundorfean, G., M.F. Neurath, and J. Mudter, Functional relevance of T helper 17 (Th17) cells and the IL-17 cytokine family in inflammatory bowel disease. Inflamm Bowel Dis, 2011.

[53] Liu, Z.J., et al., Potential role of Th17 cells in the pathogenesis of inflammatory bowel disease. World J Gastroenterol, 2009. 15(46): p. 5784-8.

[54] Ankathatti Munegowda, M., et al., A Distinct Role of CD4(+) Th17- and Th17-Stimulated CD8(+) CTL in the Pathogenesis of Type 1 Diabetes and Experimental Autoimmune Encephalomyelitis. J Clin Immunol, 2011.

[55] Emamaullee, J.A., et al., Inhibition of Th17 cells regulates autoimmune diabetes in NOD mice. Diabetes, 2009. 58(6): p. 1302-11.

[56] Cheng, X., et al., The Th17/Treg imbalance in patients with acute coronary syndrome. Clin Immunol, 2008. 127(1): p. 89-97.

[57] Gao, Q., et al., A critical function of Th17 proinflammatory cells in the development of atherosclerotic plaque in mice. J Immunol, 2010. 185(10): p. 5820-7.

[58] Bilbo, S.D., S.H. Smith, and J.M. Schwarz, A lifespan approach to neuroinflammatory and cognitive disorders: a critical role for glia. J Neuroimmune Pharmacol, 2012. 7(1): p. 24-41.

[59] Hulse, R., et al., Optimization of multiplexed bead-based cytokine immunoassays for rat serum and brain tissue. J Neurosci Methods, 2004. 136: p. 87-98.

[60] Mandal, M., et al., Maternal immune stimulation during pregnancy shapes the immunological phenotype of offspring Submitted for publication, 2012.

[61] Meyer, U., J. Feldon, and S.H. Fatemi, In-vivo rodent models for the experimental investigation of prenatal immune activation effects in neurodevelopmental brain disorders. Neurosci Biobehav Rev, 2009. 33(7): p. 1061-79.

[62] De Miranda, J., et al., Induction of Toll-Like Receptor 3-Mediated Immunity during Gestation Inhibits Cortical Neurogenesis and Causes Behavioral Disturbances. MBio, 2010. 1(4).

[63] Meyer, U., et al., The time of prenatal immune challenge determines the specificity of inflammation-mediated brain and behavioral pathology. J Neurosci, 2006. 26(18): p. 4752-62.

[64] Meyer, U., et al., Adult brain and behavioral pathological markers of prenatal immune challenge during early/middle and late fetal development in mice. Brain Behav Immun, 2008. 22(4): p. 469-86.

[65] Hsiao, E.Y., et al., Modeling an autism risk factor in mice leads to permanent immune dysregulation. Proc Natl Acad Sci U S A, 2012.

[66] Hsiao, E.Y. and P.H. Patterson, Activation of the maternal immune system induces endocrine changes in the placenta via IL-6. Brain Behav Immun, 2011. 25(4): p. 604-15.

[67] Shi, L., et al., Activation of the maternal immune system alters cerebellar development in the offspring. Brain Behav Immun, 2009. 23(1): p. 116-23. 
[68] Bitanihirwe, B.K., et al., Late prenatal immune activation in mice leads to behavioral and neurochemical abnormalities relevant to the negative symptoms of schizophrenia. Neuropsychopharmacology, 2010. 35(12): p. 2462-78.

[69] Fortier, M.E., et al., The viral mimic, polyinosinic:polycytidylic acid, induces fever in rats via an interleukin-1-dependent mechanism. Am J Physiol Regul Integr Comp Physiol, 2004. 287(4): p. R759-66.

[70] Lathia, J.D., et al., Toll-like receptor 3 is a negative regulator of embryonic neural progenitor cell proliferation. J Neurosci, 2008. 28(51): p. 13978-84.

[71] Dantzer, R. and K.W. Kelley, Twenty years of research on cytokine-induced sickness behavior. Brain Behav Immun, 2007. 21(2): p. 153-60.

[72] Henry, C., et al., Exaggerated sickness behavior and brain proinflammatory cytokine expression in aged mice in response to intracerebroventricular lipopolysaccharide. J Immunol, 2007. 178: p. B13.

[73] Dantzer, R., et al., From inflammation to sickness and depression: when the immune system subjugates the brain. Nat Rev Neurosci, 2008. 9(1): p. 46-56.

[74] Yirmiya, R. and I. Goshen, Immune modulation of learning, memory, neural plasticity and neurogenesis. Brain Behav Immun, 2011. 25(2): p. 181-213.

[75] Belvin, M.P. and K.V. Anderson, A conserved signaling pathway: the Drosophila toll-dorsal pathway. Annu Rev Cell Dev Biol, 1996. 12: p. 393-416.

[76] Okun, E., K.J. Griffioen, and M.P. Mattson, Toll-like receptor signaling in neural plasticity and disease. Trends Neurosci, 2011. 34(5): p. 269-81.

[77] Kawai, T. and S. Akira, Toll-like Receptors and Their Crosstalk with Other Innate Receptors in Infection and Immunity. Immunity, 2011. 34(5): p. 637-50.

[78] Baldridge, M.T., K.Y. King, and M.A. Goodell, Inflammatory signals regulate hematopoietic stem cells. Trends Immunol, 2011. 32(2): p. 57-65.

[79] Carty, M. and A.G. Bowie, Evaluating the role of Toll-like receptors in diseases of the central nervous system. Biochem Pharmacol, 2011. 81(7): p. 825-37.

[80] Stridh, L., et al., Regulation of Toll-like receptor 1 and -2 in neonatal mice brains after hypoxia-ischemia. J Neuroinflammation, 2011. 8: p. 45.

[81] Koga, K. and G. Mor, Toll-like receptors at the maternal-fetal interface in normal pregnancy and pregnancy disorders. Am J Reprod Immunol, 2010. 63(6): p. 587-600.

[82] Lehnardt, S., et al., The toll-like receptor TLR4 is necessary for lipopolysaccharide-induced oligodendrocyte injury in the CNS. J Neurosci, 2002. 22(7): p. 2478-86.

[83] Babcock, A.A., et al., Toll-like receptor 2 signaling in response to brain injury: an innate bridge to neuroinflammation. J Neurosci, 2006. 26(49): p. 12826-37. 
[84] Ma, Y., et al., Toll-like receptor 8 functions as a negative regulator of neurite outgrowth and inducer of neuronal apoptosis. J Cell Biol, 2006. 175(2): p. 209-15.

[85] Ma, Y., et al., TLR8: an innate immune receptor in brain, neurons and axons. Cell Cycle, 2007. 6(23): p. 2859-68.

[86] Rolls, A., et al., Toll-like receptors modulate adult hippocampal neurogenesis. Nat Cell Biol, 2007. 9(9): p. 1081-8.

[87] Tang, S.C., et al., Pivotal role for neuronal Toll-like receptors in ischemic brain injury and functional deficits. Proc Natl Acad Sci U S A, 2007. 104(34): p. 13798-803.

[88] Okun, E., et al., TLR2 activation inhibits embryonic neural progenitor cell proliferation. J Neurochem, 2010. 114(2): p. 462-74.

[89] Urakubo, A., et al., Prenatal exposure to maternal infection alters cytokine expression in the placenta, amniotic fluid, and fetal brain. Schizophr Res, 2001. 47(1): p. 27-36.

[90] Bell, M., J. Hallenbeck, and V. Gallo, Determining the fetal inflammatory response in an experimental model of intrauterine inflammation in rats. Pediatric Res, 2004. 56: p. 541-546.

[91] Uchide, N., et al., Induction of pro-inflammatory cytokine gene expression and apoptosis in human chorion cells of fetal membranes by influenza virus infection: possible implications for maintenance and interruption of pregnancy during infection. Med Sci Monit, 2005. 11(RA7-16).

[92] Peltier, M. and M. Brown, Experimental genital mycoplasmosis causes increased levels of mRNA for IL-6 and TNF-alpha in the placenta. Am J Reprod Immunology, 2005. 53: p. 189-198.

[93] Ashdown, H., et al., The role of cytokines in mediating effects of prenatal infection on the fetus: implications for schizophrenia. Mol Psychiatry, 2006. 11(1): p. 47-55.

[94] Meilin, A., Y. Sharabi, and J. Shoham, Analysis of thymic stromal cell subpopulations grown in vitro on extracellular matrix in defined medium-- $V$. Proliferation regulating activities in supernatants of human thymic epithelial cell cultures. Int J Immunopharmacology, 1997. 19: p. 39-47.

[95] Suda, T., et al., Effect of interleukin 6 (IL-6) on the differentiation and proliferation of murine and human hemopoietic progenitors. Exp Hematol, 1988. 16: p. 891-895.

[96] Croy, B., et al., Uterine natural killer cells: insights into their cellular and molecular biology from mouse modelling. Reproduction, 2003. 126: p. 149-160.

[97] Stumhofer, J., et al., Interleukin 27 negatively regulates the development of interleukin 17producing $T$ helper cells during chronic inflammation of the central nervous system. Nat Immunol, 2006. 7: p. 937-945.

[98] Batten, M., et al., Interleukin 27 limits autoimmune encephalomyelitis by suppressing the development of interleukin 17-producing T cells. Nat Immunol, 2006. 7: p. 929-936. 
[99] Veldhoen, M., et al., TGFbeta in the context of an inflammatory cytokine milieu supports de novo differentiation of IL-17-producing T cells. Immunity, 2006. 24(2): p. 179-89.

[100] Dominitzki, S., et al., Cutting edge: trans-signaling via the soluble IL-6R abrogates the induction of FoxP3 in naive CD4+CD25 T cells. J Immunol, 2007. 179: p. 2041-2045.

[101] Kimura, A., T. Naka, and T. Kishimoto, IL-6-dependent and-independent pathways in the development of interleukin 17-producing T helper cells. Proc Natl Acad Sci (USA), 2007. 104: p. 12099-12104.

[102] Shi, C., et al., Bone marrow mesenchymal stem and progenitor cells induce monocyte emigration in response to circulating toll-like receptor ligands. Immunity, 2011. 34(4): p. 590-601.

[103] Essers, M.A., et al., IFNalpha activates dormant haematopoietic stem cells in vivo. Nature, 2009. 458(7240): p. 904-8.

[104] Keshavan, M.S., Development, disease and degeneration in schizophrenia: a unitary pathophysiological model. J Psychiatr Res, 1999. 33(6): p. 513-21.

[105] Keshavan, M.S. and G.E. Hogarty, Brain maturational processes and delayed onset in schizophrenia. Dev Psychopathol, 1999. 11(3): p. 525-43.

[106] Ajuebor, M.N., et al., Role of resident peritoneal macrophages and mast cells in chemokine production and neutrophil migration in acute inflammation: evidence for an inhibitory loop involving endogenous IL-10. J Immunol, 1999. 162(3): p. 1685-91.

[107] Yellon, D.M. and D.J. Hausenloy, Myocardial reperfusion injury. N Engl J Med, 2007. 357(11): p. 1121-35.

[108] Buja, L.M., Myocardial ischemia and reperfusion injury. Cardiovasc Pathol, 2005. 14(4): p. 170-5.

[109] Kostin, S., Pathways of myocyte death: implications for development of clinical laboratory biomarkers. Adv Clin Chem, 2005. 40: p. 37-98.

[110] Kajstura, J., et al., Cause of death: suicide. J Mol Cell Cardiol, 2006. 40(4): p. 425-37.

[111] Takemura, G. and H. Fujiwara, Morphological aspects of apoptosis in heart diseases. J Cell Mol Med, 2006. 10(1): p. 56-75.

[112] Eltzschig, H.K. and T. Eckle, Ischemia and reperfusion-from mechanism to translation. Nat Med, 2011. 17(11): p. 1391-401.

[113] Frangogiannis, N.G., C.W. Smith, and M.L. Entman, The inflammatory response in myocardial infarction. Cardiovasc Res, 2002. 53(1): p. 31-47.

[114] Oyama, J., et al., Reduced myocardial ischemia-reperfusion injury in toll-like receptor 4-deficient mice. Circulation, 2004. 109(6): p. 784-9.

[115] Frangogiannis, N.G. and M.L. Entman, Chemokines in myocardial ischemia. Trends Cardiovasc Med, 2005. 15(5): p. 163-9. 
[116] Arslan, F., et al., Bridging innate immunity and myocardial ischemialreperfusion injury: the search for therapeutic targets. Curr Pharm Des, 2008. 14(12): p. 1205-16.

[117] Ren, G., O. Dewald, and N.G. Frangogiannis, Inflammatory mechanisms in myocardial infarction. Curr Drug Targets Inflamm Allergy, 2003. 2(3): p. 242-56.

[118] Frangogiannis, N.G., The role of the chemokines in myocardial ischemia and reperfusion. Curr Vasc Pharmacol, 2004. 2(2): p. 163-74.

[119] Yamamoto, S., et al., Activation of Mst1 causes dilated cardiomyopathy by stimulating apoptosis without compensatory ventricular myocyte hypertrophy. J Clin Invest, 2003. 111(10): p. 1463-74.

[120] Matsui, Y., et al., Distinct roles of autophagy in the heart during ischemia and reperfusion: roles of AMP-activated protein kinase and Beclin 1 in mediating autophagy. Circ Res, 2007. 100(6): p. 914-22.

[121] Del Re, D.P., et al., Proapoptotic Rassf1A/Mst1 signaling in cardiac fibroblasts is protective against pressure overload in mice. J Clin Invest, 2010. 120(10): p. 3555-67.

[122] Kurnellas, M.P., et al., Plasma membrane calcium ATPase deficiency causes neuronal pathology in the spinal cord: a potential mechanism for neurodegeneration in multiple sclerosis and spinal cord injury. FASEB J, 2005. 19(2): p. 298-300.

[123] Murphy, A.C., et al., Infiltration of Th1 and Th17 cells and activation of microglia in the CNS during the course of experimental autoimmune encephalomyelitis. Brain Behav Immun, 2010. 24(4): p. 641-51.

[124] Huppert, J., et al., Cellular mechanisms of IL-17-induced blood-brain barrier disruption. FASEB J, 2010. 24(4): p. 1023-34.

[125] Tesmer, L.A., et al., Th17 cells in human disease. Immunol Rev, 2008. 223: p. 87-113.

[126] Kebir, H., et al., Human TH17 lymphocytes promote blood-brain barrier disruption and central nervous system inflammation. Nat Med, 2007. 13(10): p. 1173-5.

[127] Matusevicius, D., et al., Interleukin-17 mRNA expression in blood and CSF mononuclear cells is augmented in multiple sclerosis. Mult Scler, 1999. 5(2): p. 101-4.

[128] Almolda, B., et al., Increase in Th17 and T-reg lymphocytes and decrease of IL22 correlate with the recovery phase of acute EAE in rat. PLoS ONE, 2011. 6(11): p. e27473.

[129] Hofstetter, H.H., C.L. Shive, and T.G. Forsthuber, Pertussis toxin modulates the immune response to neuroantigens injected in incomplete Freund's adjuvant: induction of Th1 cells and experimental autoimmune encephalomyelitis in the presence of high frequencies of Th2 cells. J Immunol, 2002. 169(1): p. 117-25.

[130] Fujimoto, C., et al., Pertussis toxin is superior to TLR ligands in enhancing pathogenic autoimmunity, targeted at a neo-self antigen, by triggering robust expansion of Th1 cells and their cytokine production. J Immunol, 2006. 177(10): p. 6896-903. 
[131] Stenger, S. and R.L. Modlin, Control of Mycobacterium tuberculosis through mammalian Toll-like receptors. Curr Opin Immunol, 2002. 14(4): p. 452-7.

[132] Bilbo, S.D. and J.M. Schwarz, Early-life programming of later-life brain and behavior: a critical role for the immune system. Front Behav Neurosci, 2009. 3: p. 14.

[133] de Moura, E.G., P.C. Lisboa, and M.C. Passos, Neonatal programming of neuroimmunomodulation--role of adipocytokines and neuropeptides. Neuroimmunomodulation, 2008. 15(3): p. 176-88.

[134] Merlot, E., D. Couret, and W. Otten, Prenatal stress, fetal imprinting and immunity. Brain Behav Immun, 2008. 22(1): p. 42-51.

[135] Phillips, D.I., External influences on the fetus and their long-term consequences. Lupus, 2006. 15(11): p. 794-800.

[136] Bale, T.L., et al., Early life programming and neurodevelopmental disorders. Biol Psychiatry, 2010. 68(4): p. 314-9.

[137] Barouki, R., et al., Developmental origins of non-communicable disease: Implications for research and public health. Environ Health, 2012. 11: p. 42.

[138] Hales, C.N. and D.J. Barker, The thrifty phenotype hypothesis. Br Med Bull, 2001. 60: p. 5-20.

[139] Khashan, A.S., et al., Higher risk of offspring schizophrenia following antenatal maternal exposure to severe adverse life events. Arch Gen Psychiatry, 2008. 65(2): p. 146-52.

[140] Painter, R.C., T.J. Roseboom, and O.P. Bleker, Prenatal exposure to the Dutch famine and disease in later life: an overview. Reprod Toxicol, 2005. 20(3): p. 345-52.

[141] Barrett, E.G., Maternal influence in the transmission of asthma susceptibility. Pulm Pharmacol Ther, 2008. 21(3): p. 474-84.

[142] Bellinger, D.L., C. Lubahn, and D. Lorton, Maternal and early life stress effects on immune function: relevance to immunotoxicology. J Immunotoxicol, 2008. 5(4): p. 419-44.

[143] Conrad, M.L., et al., Maternal TLR signaling is required for prenatal asthma protection by the nonpathogenic microbe Acinetobacter lwoffii F78. J Exp Med, 2009. 206(13): p. 2869-77. 

Chapter 8

\title{
Valproic Acid in Autism Spectrum Disorder: From an Environmental Risk Factor to a Reliable Animal Model
}

\author{
Carmem Gottfried, Victorio Bambini-Junior, \\ Diego Baronio, Geancarlo Zanatta, \\ Roberta Bristot Silvestrin, Tamara Vaccaro and \\ Rudimar Riesgo
}

Additional information is available at the end of the chapter

http://dx.doi.org/10.5772/54824

\section{Introduction}

Autism spectrum disorders (ASD) have attracted public attention by its high prevalence, elevated social cost and large impact on the family [1]. Since the first descriptions of autism made by Hans Asperger in 1938 [2] and by Leo Kanner in 1943 [3, 4], much discussion has focused in the search for the triggering points of autism and identifying risk factors has become a high priority of scientists. Nevertheless, even after almost seventy years since the first reports, the etiology of autism remains unknown and its molecular basis is not well understood. Environmental factors (such as virus, bacteria, drugs, etc.) known to increase the risk of autism have critical periods of action during embryogenesis. Congenital syndromes are found in high rates in patients with autism including somatic changes originated early in the first trimester [5].

The link between rubella and autism came from epidemic rubella in which the incidence of autism diagnosis in prenatally exposed offspring was more than 10 -fold higher than normal. The study describes 243 children exposed to congenital rubella, where $25 \%$ presented mental retardation, $15 \%$ had reactive behavior and $7 \%$ was included in the autism spectrum [6].

Valproic acid (VPA) has traditionally been prescribed for epilepsy, but is increasingly used for psychiatric condition, such as bipolar disease by its modulation on GABA neurotransmission [7]. Furthermore, it has been also shown to be associated with an increased prevalence of autism. In fact, prospective and retrospective studies demonstrate that exposure to VPA during pregnancy is associated with approximately three-fold increase in the rate of major anomalies 
and a possible set of dysmorphic features with decreased intrauterine growth [8, 9], characteristics of Fetal Valprotate Syndrome (FVS) described in item 3. Histone deacetylase (HDAC) inhibition by VPA and changes in gene expression may explain part of the teratogenicity of this drug. In utero exposure of rodents to VPA has been proposed to induce a phenotype with behavioral characteristics reminiscent of those observed in ASD and provides a robust animal model for social cognitive impairment understanding and a potential screen for the development of novel therapeutics for this condition [10]. Other possible explanations include either the effect of VPA through the increase of fetal oxidative stress, affecting mainly the brain in comparison to other fetal organs, or its inhibitory action on the folic acid mechanism [11]. In agreement, it is possible to duplicate a number of anatomic and behavioral features characteristic of human cases by exposing rat embryos to a teratogenic agents at the time of neural tube closure [12].

Thus, in utero exposure to VPA has been used as a reliable model to increase the understanding of behavioral effects evaluated by specific tests as sociability, social preference and stereotypic behavior, also observed in human patients $[9,13,14]$. The present chapter summarizes the current knowledge on the relationship between in utero exposure to VPA in humans and in autism-like animal model phenotypes, highlighting the importance of this model to the neurobiology of autism studies.

\section{Valproic acid}

The compound VPA (Figure 1A) is a fatty acid synthesized in 1882 [15] as an analogue of valeric acid, found naturally in valerian (Valeriana officinalis), used at that time as an organic solvent. The chemical names to VPA and derivatives are shown in Table 1. Antiepileptic properties of VPA, which is structurally unrelated to other antiepileptic drugs, were discovered by chance in 1962, when the French researcher Pierre Eymard in a serendipity discovery observed the anticonvulsant properties of VPA while using it as a vehicle for a number of other compounds that were being screened for anti-seizure activity [16]. He found that it prevented pentylenetetrazol-induced convulsions in rodents. Since then, it has also been used for migraine and bipolar disorder. The U.S. Food and Drug Administration (FDA) approved VPA in 1978 for the treatment of seizure disorder and in 1986 approved its enteric-coated counterpart valproate semisodium (Figure 1B) also named divalproex sodium (USA), for the same indication. Valproate semisodium is a stable co-ordination compound comprised of sodium valproate (Figure 1C) and valproic acid in a 1:1 molar relationship in an enteric coated form. An enteric coating is a barrier applied to oral medication that controls the location in the digestive system where it is absorbed. This compound dissociates to release valproate ions into the gastrointestinal tract. Once in the blood, sodium valproate can be converted also in the acid form or conjugated as valproate semisodium [17]. The acid form is currently used to quantify plasma levels of all three. 


\begin{tabular}{cc}
\hline Valproic Acid & 2-Propylvaleric acid, 2-Propylpentanoic acid or Di-n-dipropylacetic acid \\
\hline Sodium Valproate & Sodium 2-propylvalerate \\
\hline Valproate semisodium & Sodium hydrogen bis(2-propylvalerate) \\
\hline Valproate Pivoxil & Hydroxymethyl 2-propylvalerate pivalate \\
\hline Valpromide & 2-Propylvaleramide \\
\hline
\end{tabular}

Table 1. Chemical names of VPA and derivatives

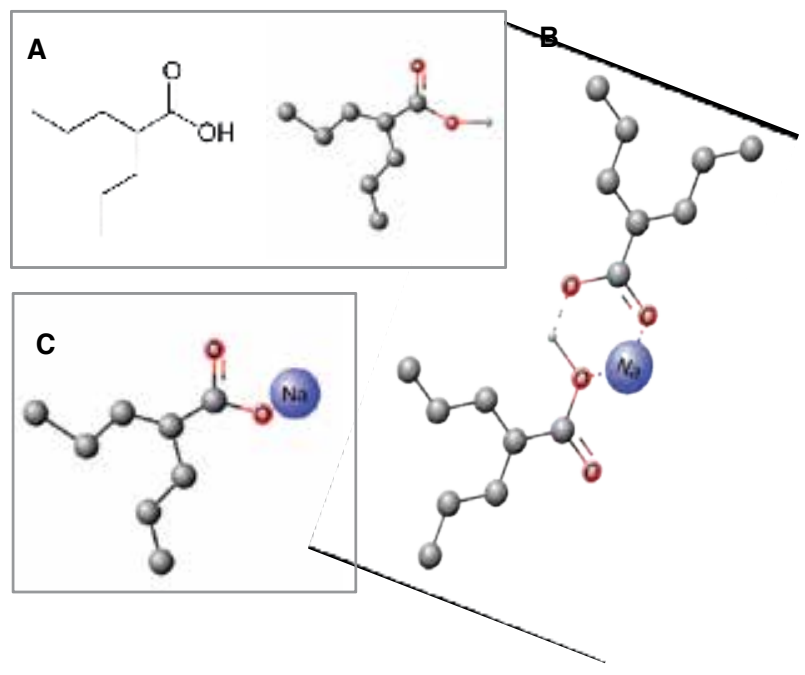

Figure 1. The molecular structure of VPA and derivatives showed in ball and stick view. A. Valproic acid. B. Valproate semisodium, C. Sodium valproate. In A is possible to compare both chemical and ball and stick structures (used also to illustrate derivatives).

The therapeutic concentration of sodium valproate (the sodium salt of VPA) during chronic oral treatment ranges from $40-100 \mathrm{mg} / \mathrm{mL}(280-700 \mathrm{mmol} / \mathrm{L})$ in plasma and from 6-27 mg/g (42-190 $\mathrm{mmol} / \mathrm{g}$ ) in brain [18]. From this point, to simplify the reading throughout the text, the VPA abbreviation will be used when referring to valproic acid and derivatives.

The VPA is marketed under brand names including: Convulex (Pfizer-UK and Byk MadausSouth Africa), Depakene (Abbott Laboratories-USA, Brazil and Canada), Depakine (Sanofi Aventis-France and Sanofi Synthelabo-Romania), Deprakine (Sanofi Aventis-Finland), Encorate (Sun Pharmaceuticals-India), Epilim (Sanofi Synthelabo-Australia), Valcote (Abbot Laboratories-Argentina).

The VPA effects of clinical importance include GABAergic activity increase, excitatory neurotransmission decrease, and modification of monoamines [19]. The biochemical and biological effects of VPA are summarized in Table 2. 


\begin{tabular}{|c|c|c|}
\hline Target of action & Biological effects & Reference \\
\hline HDAC (inhibition) & Open DNA transcription & {$[20]$} \\
\hline Mitochondria & Energy metabolism impairment & [21] \\
\hline Lymphocytes & Modification of the epigenotype & {$[22]$} \\
\hline Neurons from substantia nigra & Reduction in firing rate & [23] \\
\hline c-Jun N-terminal kinase (JNK) & Defective neurite formation & {$[24]$} \\
\hline GSK3 $\beta$ inhibitor & Promotion of hair re-growth & [25] \\
\hline Beta-catenin-Ras-ERK-p21Cip/WAF1 pathway & $\begin{array}{l}\text { Differentiation and inhibition of proliferation in } \\
\text { neural progenitor cells }\end{array}$ & {$[26]$} \\
\hline $\begin{array}{l}\text { Constitutive androstane receptor }(\mathrm{CAR}) \text { and } \\
\text { pregnane } \mathrm{X} \text { receptor (PXR) }\end{array}$ & $\begin{array}{c}\text { Up-regulation of CYP3A4 and MDR1 gene } \\
\text { expression }\end{array}$ & [27] \\
\hline Matrix metalloprotease-9 inhibitor & $\begin{array}{c}\text { Attenuation of blood-spinal cord barrier (BSCB) } \\
\text { after spinal cord injury (SCI) }\end{array}$ & [28] \\
\hline $\mathrm{PI3K} / \mathrm{Akt} / \mathrm{mTOR}$ pathway & Skeletal muscle hypertrophy & [29] \\
\hline
\end{tabular}

Table 2. Biochemical and biological effects of VPA

\section{Valproic acid as an environmental risk factor in the development of autism}

After the VPA license for use in 1978, the first adverse report of a fetus exposed to the drug was published in 1980 [30]. Since then. particular attention has been directed to the occurrence of neural tube defects in infants exposed to VPA in utero [31,32]. The critical period for exposure to teratogens shown to increase the risk of autism is early in the first trimester [33]. Some of the critical teratogens related to autism risk are maternal rubella infection [6], ethanol [34], thalidomide [35] and VPA [8, 9]. Approximately one in 250 pregnancies is known to be exposed to antiepileptic drugs [36] and a significant proportion of these are exposed to VPA, either as monotherapy or as part of a polytherapy drug regimen.

The timing for the teratogenic effect of VPA that increases the risk for autism cannot be estimated directly, as the drug is typically taken throughout the entire pregnancy [33]. Many children exposed in utero to VPA exhibit FVS, first described in 1984 [37] and characterized by a major and minor malformations and developmental and behavioral delays [8, 12, 38-40]. Specific impairments observed in FVS includes neural tube defects, trigonocephaly, radial ray defects, pulmonary abnormalities, coloboma of iris/optic disc, low verbal IQ and features of ASD [41] and has been reported in a number of sibling pairs [12, 21, 37, 42-44] with different degree of severity among affected siblings. Common facial features of FVS include epicanthal folds, broad nasal bridge, short nose with antiverted nares, long upper lip, and low set, posteriorly rotated ears [41]. 
The classical autism was first reported to be one of the behavioral outcomes of VPA exposure [41] through several case reports [12, 39, 45]. The first epidemiological study with drugs as environmental risk factors of autism was described in 2000, with 57 offspring of women taking anticonvulsants (see ref [46], summarized in Tables 3 and 4).

\begin{tabular}{cc}
\hline Features & \% of children \\
\hline Poor social interaction & 53 \\
\hline Poor communication skills & 49 \\
\hline Short attention span & 46 \\
\hline Insistence on routines & 44 \\
\hline Hand flapping & 25 \\
\hline
\end{tabular}

Table 3. Autism features in children exposed in utero to anticonvulsants.

\begin{tabular}{ccc}
\hline & No of children & VPA exposure in mg (weeks of gestation) \\
\hline Neural tube defect & 1 & $1000(0-5), 800(5-40)$ \\
\hline Genitourinary & 1 & $1200(1-17), 1500(17-26), 2000(26-40)$ \\
\hline Extremities & 1 & $1000(0-40)$ \\
& 5 & $1000(0-5), 800(5-40)$ \\
\hline Eyes & 2 & $1500(0-40)$ \\
\hline Teeth & 1 & $1000(0-40)$ \\
& 1 & $1500(0-40)$ \\
\hline Diastasis recti & 1 & $800(0-40)$ \\
\hline
\end{tabular}

Table 4. Congenital malformations in children exposed in utero to VPA

Fifty two children were ascertained through the Fetal Anticonvulsant Syndrome Association (FACS) and five were referred to the Aberdeen Medical Genetics Service (AMGS). The number of patients exposed in utero to each anticonvulsant alone was $34(60 \%)$ to VPA, $4(7 \%)$ to carbamazepine, $4(7 \%)$ to phenytoin, and $15(26 \%)$ to more than one anticonvulsant. The number of patients with behavioral problems was $46(81 \%)$, with hyperactivity or poor concentration was $22(39 \%)$ and with attention deficit and hyperactivity disorder $4(7 \%)$. Autistic features were present in 34 patients (60\%). 


\section{Animal model of autism induced by prenatal exposure to VPA}

Considering human evidences of autism followed by early in utero exposure to teratogens, such as thalidomide and VPA, the next step was to develop a model of autism induced by prenatal exposure of animals to the same drugs, particularly VPA [47]. The in utero exposure to VPA induced patterns of abnormal development across species as demonstrated in Table 5.

\begin{tabular}{|c|c|c|}
\hline Patterns of abnormal development & Specie & References \\
\hline \multirow{3}{*}{ Skeletal abnormalities } & Mice & {$[48,49]$} \\
\hline & Rabbits & {$[50]$} \\
\hline & Rhesus monkey & {$[51]$} \\
\hline Cardiac abnormalities & Mice & {$[52]$} \\
\hline Neural tube defects (including spina bifida) & Mice & {$[53]$} \\
\hline Cranial neural tube defects & Rats & {$[54]$} \\
\hline Behavioral abnormalities & Rats & {$[55]$} \\
\hline
\end{tabular}

Table 5. Patterns of abnormal development across species after in utero exposure to VPA.

The use of animal models allows a wide range of research possibilities including the search for etiologic clues, molecular targets, and biomarkers. The main aspects to take into account in developing animal models, is (i) to reproduce a circumstance that would lead to a certain condition, for example, inducing a genetic disease by manipulating a specific gene; (ii) to induce similar patterns found in the studied condition, for example, observing the same behavioral alterations found in a particular impairment; (iii) to observe if the model has similarities to a human features when exposed to certain treatment [56]. The time of induction, dosage of VPA and the way of administration in rodents are variable in the literature, as demonstrated in Table 6. It is important to observe that in rats, $600 \mathrm{mg} / \mathrm{Kg} \mathrm{VPA}$ at 12.5 days of pregnancy is the most investigated due to similarities in the features of autism. Besides the higher number of studies describing prenatal exposure to VPA, there are some protocols reporting also postnatal exposure and behavioral features of autism [57, 58].

The diagnoses of autism take into account behavioral alterations in three main areas: sociability, communication and behavioral stereotypies and narrow range of interests. Therefore, a consistent animal model must show similar behavioral abnormalities, which might indicate common neural alterations.

Our group has administrated a single intraperitoneal injection of $600 \mathrm{mg} / \mathrm{kg}$ VPA in pregnant rats at the embryonic day 12.5, observing variations in social memory, and flexibility to change strategy [84]. Females were kept separate and with free access to their own litters. Somatic aspects observed during the pups' development, included body weight, ear unfolding and eye opening which were unchanged between groups. In three-chambered-apparatus test, used to 


\begin{tabular}{|c|c|c|}
\hline Embryonic Day(s) & $\begin{array}{l}\text { Procedure } \\
\text { (mg/Kg VPA) }\end{array}$ & References \\
\hline \multicolumn{3}{|c|}{ Mice } \\
\hline 9 & IP $(200,400,800)$ & [59] \\
\hline 9 & SC (400) & [60] \\
\hline $9,12.5,14.5$ & IP (500) & [61] \\
\hline 11 & OA (800) & {$[62,63]$} \\
\hline $12,13,14$ & IP (100) & [64] \\
\hline 12.5 & IP (500) & [65] \\
\hline 13 & SC (600) & {$[66,67]$} \\
\hline \multicolumn{3}{|c|}{ Rats } \\
\hline $7,9.5,12,15$ & IP (400) & [68] \\
\hline $8,9,10,11$ & OA (800) & [69] \\
\hline 9 & IP (600) & [70] \\
\hline 9 & OA (800) & [71-74] \\
\hline 9,11 & $\mathrm{AO}(800)$ & {$[75,76]$} \\
\hline $11,12,13$ & IP (200) & [77] \\
\hline 1.5 & IP (500) & [78] \\
\hline $1.5,12,12.5$ & IP (350) & [9] \\
\hline 12 & IP (400) & [79] \\
\hline 12 & IP (600) & [80-83] \\
\hline 12.5 & IP (600) & {$[10,14,84-93]$} \\
\hline 12.5 & SC (350) & [94] \\
\hline 12.5 & IP (350) & [95] \\
\hline 12.5 & $\mathrm{IP}(400,500,600)$ & [77] \\
\hline 12.5 & IP (500) & [96] \\
\hline
\end{tabular}

Table 6. Prenatal exposure of VPA in rodents: Time of induction and dosage

observe social memory, preferences and interests, the VPA group spent less time in the presence of a stranger rat and more time in the presence of an object, indicating a reduced place preference conditioned by conspecific and an increased preference for the object, revealing sociability impairments. As adults, they showed inappropriate social approach to a stranger rat, decreased preference for social novelty, apparently normal social recognition, no spatial learning deficits and normal resistance to change on Morris water maze. 


\section{Brain alterations induced by prenatal exposure to VPA}

Once prenatal exposure to VPA became a reliable tool to model autism, more brain alterations were investigated in rodents exposed to this teratogen, as summarized in Table 7.

\begin{tabular}{|c|c|c|c|}
\hline Rodent & VPA (mg/kg) & Findings & References \\
\hline \multirow{5}{*}{ Rat } & 500 & Altered distribution of 5-HT neurons in the dorsal raphe nucleus. & [71] \\
\hline & 350 & $\begin{array}{l}\text { Reduction in the number of motor neurons from hypoglossal and } \\
\text { oculomotor nuclei. }\end{array}$ & [9] \\
\hline & 500 & $\begin{array}{l}\text { Reduction in the number of putative synaptic contacts in connection } \\
\text { between layer } 5 \text { pyramidal neurons. }\end{array}$ & [97] \\
\hline & 400 & $\begin{array}{l}\text { Prolonged neuronal progenitor cells proliferation in embrionary } \\
\text { period. }\end{array}$ & [79] \\
\hline & 600 & $\begin{array}{l}\text { Decreased number of purkinje cells, neuronal degeneration and } \\
\text { chromatolysis. }\end{array}$ & [98] \\
\hline \multirow{2}{*}{ Mice } & 500 & $\begin{array}{l}\text { Reduction in the number of Parvalbumin -positive inhibitory neurons } \\
\qquad \text { in the neocortex. }\end{array}$ & [99] \\
\hline & 500 & $\begin{array}{l}\text { Nissl-positive cell loss in the middle and lower layers of the prefrontal } \\
\text { cortex and in the lower layers of the somatosensory cortex. }\end{array}$ & {$[65]$} \\
\hline
\end{tabular}

Table 7. Brain alterations induced by in utero exposure to VPA

Behavioral outcomes started to be studied, demonstrating a number of anatomic and behavioral features characteristic of human cases by exposing rodents' embryos to VPA at the time of neural tube closure. One of the affected structures in the brains is the cerebellum. Magnetic resonance imaging showed that patients with autism have reduced size of the cerebellum when compared to controls, displaying smaller vermal lobules VI and VII. This abnormality is probably an outcome of developmental hypoplasia and not likely shrinkage or deterioration after full development had been achieved [100]. Similar alterations were found in brains from rat model of autism induced by prenatal exposure to VPA [9]. Exposed rats showed a reduction in the number of motor neurons of the earliest-forming motor nuclei (V, XII), and had the VI th and III rd cranial nerve nuclei affected. In the same way, another work found diminished number of cells in the posterior lobe of the cerebellum [86]. In this context, cerebellar anatomy alterations in humans might be due to loss of neurons in the cranial nerve motor nuclei, as demonstrated in rats.

The amygdala is likely to be also linked to autism, due to its involvement in social-emotional behavior. Rats exposed to VPA in utero had longer lasting and harder to extinguish fear memories, which could be explained by the hyperreactivity and hyperplasticity found in the lateral amygdala $[96,101]$. Another work found enhanced long-term potentiation (LTP) in the 
medial prefrontal cortex of rats exposed to VPA, with enhanced synaptic plasticity and shortand long-term fear memories [82].

Synaptic impairments were already described in autism, which may be related to neuroligins alterations. Neuroligins are a family of proteins which play a central role in synaptic maturation and were affected in rats after in utero exposure to VPA. Neuroligin 3 mRNA expression was decreased in the hippocampus, especially in cornu ammonis (CA1) and dentate gyrus [62].

Synaptic plasticity is influenced by brain-derived neurotrophic factor (BDNF), a factor that modulates several neurochemical parameters. High levels of BDNF have been reported in the blood of patients with autism [102]. BDNF acts through TrkB-mediated activation of various signal transduction pathways, including pathways that involve PI3K, mitogen-activated kinase (MAPK), and phospholipase C- $\gamma$ [103]. Infusion of BDNF in the nucleus accumbens of aged rats restored synaptic plasticity and improved cognition [104] and some environmental factors, such social isolation, results in low levels of BDNF in the hippocampus of rats [105]. Animals exposed to VPA in utero display decreased cortical BDNF mRNA expression. It is important to notice that altered levels of the transcript will not necessarily mean an altered protein expression [63]. Diminished BDNF may lead to altered synaptic development; once it is known that this neurotrophic factor is involved in development and function of serotonergic neurons [106].

Several hypotheses have arisen to explain the social deficits in autism. One of these proposals points an alteration in opioidergic mechanisms as a likely causative of behavioral impairments in this disorder [107]. Opioid peptides are involved in stress responses and affective states, and blockage of their receptors causes dysphoria in humans. Enkephalins are part of the opioid family and are distributed in brain areas, like the striatum and the nucleus accumbens, involved in processing emotional information, anxiety and fear. Exposure to VPA reduced proenkephalin mRNA expression in both the core and shell of the nucleus accumbens and dorsal striatum of rats concomitantly to anxious-like behavior [91].

The monoamine system is also altered in patients with autism and their relatives. It was demonstrated that children with autism have increased 5-HT (serotonin) synthesis capacity when compared to children with typical development [108]. Besides, it is widely known that sleep disorders are common in autistic children [109]. Interestingly, increased levels of serotonin was found in pre-frontal cortex of rats prenatally exposed to VPA in association with disrupted sleep/awake rhythm. The elevated levels of 5-HT were found during light phase of animals' circadian rhythm [74]. It was proved that serotonergic neurons have a silent firing rate during REM sleep [110], indicating that the sleep disturbance found in the animals may be related to increased levels of 5-HT found in their prefrontal cortex. In addition, higher levels of 5-HT were also reported in the left side of hippocampus and in blood from rats [111]. However, using the whole hippocampus, it was demonstrated $46 \%$ decrease in 5-HT levels from rats exposed to VPA in utero [70].

Recently, we observed hippocampal reactive astrogliosis in the group of rats exposed in utero to VPA (see ref [84]). After 15 postnatal days, hippocampal astrocytes were intensely immunoreactive to the astroglial marker Glial Fibrillary Acidic Protein (GFAP) (Figure 2). 
Astrocytes are the major cell type in the central nerve system (CNS) and provide a variety of critical supportive functions that maintain neuronal homeostasis, participating of the synapse and the glutamatergic metabolism [112]. These cells become reactive in VPA group, characterized by up-regulation of GFAP and apparently show higher number of processes than the control cells as demonstrated by the squares in A and B.

Seven Fresh-frozen post mortem tissues from individuals with autism and CSF from six living autistic patients were investigated for cytokine protein profiling [113]. This study shows an active neuroinflammatory process in the cerebral cortex, white matter, and notably in the cerebellum. Immunocytochemical studies showed marked activation of microglia and astroglia. The cytokine profiling indicated that the macrophage chemoattractant protein (MCP)-1 and tumor growth factor-beta1, both derived from neuroglia were the most prevalent cytokines in brain tissues. We presumed that microglia/macrophage-derived pro-inflammatory cytokines regulate the transition of astrocytes into reactive astrogliosis. Nevertheless, the mechanisms which regulate the level of astroglial cell activity in the hippocampus from VPA autism model need to be investigated.

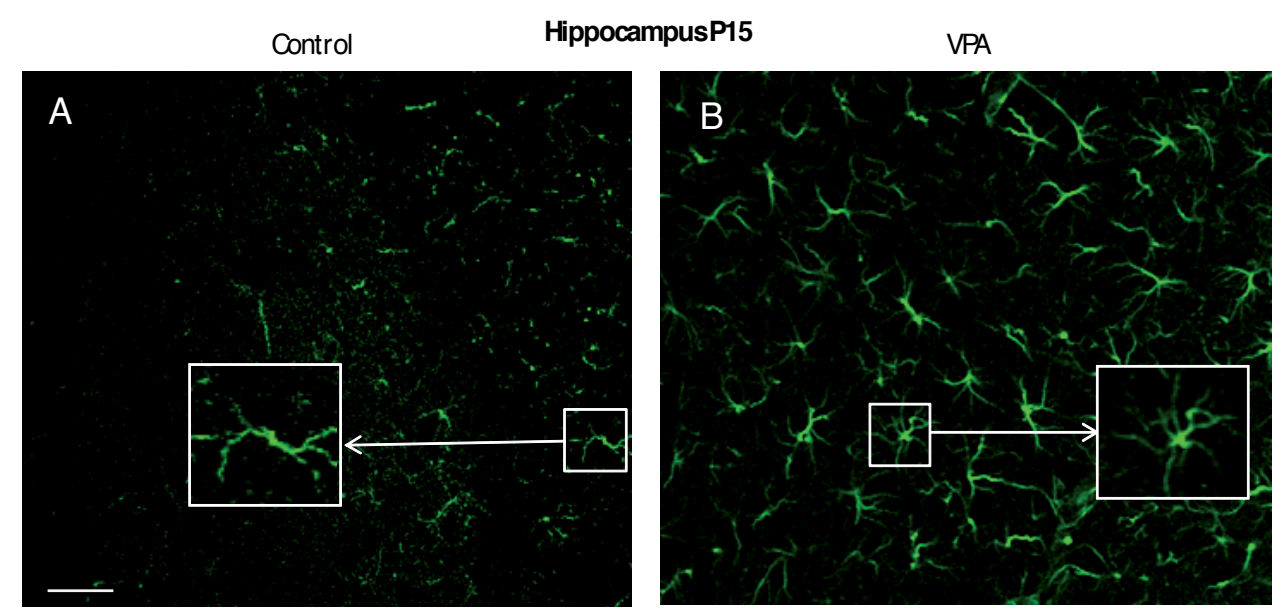

Figure 2. Astrocyte immunoreactive to GFAP in hippocampus from rats. A. Representative image from control group, B. Representative image from VPA group. Scale bar $=50 \mu \mathrm{m}$

Glutamatergic excitatory synapses are the major type of synapses in the brain and it was found that glutamate metabolism is altered in autistic CNS, particularly the glutamate receptors AMPA, NMDA and mGluR5 [114]. In agreement, rats exposed in utero to VPA show impairments in excitatory/inhibitory brain balance [78]. In this context, impairment in excitatory and inhibitory signaling during certain periods of development is proposed to be involved in the autism pathophysiology [115].

Although social impairments are one of the most important features observed in autism, patients present several other symptoms, including motor disturbances. Motor stereotypies are part of the so called autism triad of impairments, but hypotonia, motor apraxia, 
toe-walking, have already been reported [116]. Evaluation of motor cortex neurons of rats exposed to VPA in utero showed no changes in length or volume of either basilar or apical dendrites, but presented greater dendritic arborization in comparison with controls. This data indicates that pruning of neurons is abnormal in animals prenatally exposed to VPA [95]. Evidence suggests that the same may happen in patients with autism, since there are reports of increased brain weight in autopsy cases of autism [117]. However, the involvement of the abnormal pruning in motor cortex neurons with motor disturbances in autism deserves further investigation. Individuals with autism are more likely to present hearing deficits. In a study with a group of 199 children and adolescents, 3.5\% had profound bilateral hearing loss or deafness [118].

The superior olivary complex (SOC) plays different roles in hearing. It is located within the lower brainstem and it is involved in encoding temporal features of sound and descending modulation of the cochlear nucleus and cochlea for listening in background noise. Rats exposed to VPA in utero showed reduced number of neurons and disrupted neuronal morphology in the SOC. Neuronal cell bodies were smaller and more round, indicating that these anatomical feature might have a role in the hearing difficulties that are a common in patients with autism [87]. In a study with brains of patients with autism similar morphological alterations were found, including soma size, shape and number of neurons in the SOC [119].

The cerebellum have been the focus of studies involving active and chronic neuroinflammatory process in autistic patients, demonstrating the presence of proinflammatory chemokines such as MCP-1 as well as antiinflammatory cytokines such as TGF- $\beta 1$ in this brain structure. These findings support the idea that a chronic state of specific cytokine activation occurs in autism [113]. Because neuroimmune responses are influenced by the genetic background of the host, the role of neuroinflammation in the context of the genetic and other factors that determine the autism phenotype remains an important issue to be investigated.

\section{Concluding remarks and scientific challenges}

The spectrum of autism comprises a multifactorial group of disorders, with phenotypic diversity related to the symptoms and increasing prevalence. One of the major challenges of cognitive neuroscience is to understand how changes in the structural properties of the brain affect the plasticity exhibited whenever a person develops, ages, learns a new skill, make social interaction or adapts to a disease. In ASD, it is necessary studies in this field attempting to explain and understand the trigger of autism. In this context, it is not easy to find a single animal model able to captures the entire molecular and cellular alterations observed in patients with ASD.

Studies of in utero interventions in the search for animal models of autism, together with the study of potential clinical markers to ASD are innovative and may generate strategies aiming (a) the prevention of autism; (b) the construction of laboratory kits as new tools to improve and anticipate diagnosis; (c) the study of neuroglial plasticity; (d) the search for new clues to unravel the etiology of ASD. Challenged by these complexities, it is necessary to evaluate the 
most representative animal model to which a research group may address its questions. Considering that neuroimmune responses are influenced by the host, the role of possible neuroinflammation triggered by environmental factors in utero followed by neuroglial alterations in the litters remain an important issue to be investigated.

The present chapter summarizes findings obtained in rodents exposed in utero to VPA which present important similarities to autism features, supporting it as a valuable experimental model to study neurodevelopmental alterations induced by VPA as an environmental risk factor.

\section{Author details}

Carmem Gottfried $^{1 *}$, Victorio Bambini-Junior ${ }^{1}$, Diego Baronio ${ }^{2}$, Geancarlo Zanatta ${ }^{1}$, Roberta Bristot Silvestrin ${ }^{1}$, Tamara Vaccaro ${ }^{1}$ and Rudimar Riesgo ${ }^{2}$

*Address all correspondence to: cgottfried@ufrgs.br

1 Translational Research Group in Autism Spectrum Disorders (GETEA), Brazil

2 Postgraduate Program in Biochemistry at Department of Biochemistry, Institute of Health's Basic Science, Brazil

\section{References}

[1] Wallace, S, Fein, D, Rosanoff, M, Dawson, G, Hossain, S, Brennan, L, et al. A global public health strategy for autism spectrum disorders. Autism Res. (2012). Jun;, 5(3), 211-7.

[2] Asperger, H. Das psychisch abnormale Kind [The psychically abnormal child]. Wien Klin Wochenschr. (1938).

[3] Kanner, L. Autistic Disturbances of Affective Contact. Nervous Child. (1943). , 1943(2), 217-50.

[4] Kanner, L, \& Eisenberg, L. Early infantile autism, Psychiatr Res Rep Am Psychiatr Assoc. 1957 Apr(7):55-65., 1943-1955.

[5] Bello, S. C. Autism and environmental influences: review and commentary. Rev Environ Health. (2007). Apr-Jun;, 22(2), 139-56.

[6] Chess, S. Follow-up report on autism in congenital rubella. J Autism Child Schizophr. (1977). Mar;" 7(1), 69-81.

[7] Haddad, P. M, Das, A, Ashfaq, M, \& Wieck, A. A review of valproate in psychiatric practice. Expert Opin Drug Metab Toxicol. (2009). May;, 5(5), 539-51. 
[8] Ardinger, H. H, Atkin, J. F, Blackston, R. D, Elsas, L. J, Clarren, S. K, Livingstone, S, et al. Verification of the fetal valproate syndrome phenotype. Am J Med Genet. (1988). Jan; 29(1), 171-85.

[9] Rodier, P. M, Ingram, J. L, Tisdale, B, Nelson, S, \& Romano, J. Embryological origin for autism: developmental anomalies of the cranial nerve motor nuclei. J Comp Neurol. (1996). Jun 24;,370(2), 247-61.

[10] Foley, A. G, Gannon, S, Rombach-mullan, N, Prendergast, A, Barry, C, Cassidy, A. W, et al. Class I histone deacetylase inhibition ameliorates social cognition and cell adhesion molecule plasticity deficits in a rodent model of autism spectrum disorder. Neuropharmacology. (2012). Sep; 63(4), 750-60.

[11] Banji, D, Banji, O. J, Abbagoni, S, Hayath, M. S, Kambam, S, \& Chiluka, V. L. Amelioration of behavioral aberrations and oxidative markers by green tea extract in valproate induced autism in animals. Brain Res. (2011). Sep 2; 1410, 141-51.

[12] Christianson, A. L, Chesler, N, \& Kromberg, J. G. Fetal valproate syndrome: clinical and neuro-developmental features in two sibling pairs. Dev Med Child Neurol. (1994). Apr;, 36(4), 361-9.

[13] Alsdorf, R, \& Wyszynski, D. F. Teratogenicity of sodium valproate. Expert Opin Drug Saf. (2005). Mar;, 4(2), 345-53.

[14] Schneider, T, \& Przewlocki, R. Behavioral alterations in rats prenatally exposed to valproic acid: animal model of autism. Neuropsychopharmacology. (2005). Jan;, 30(1), 80-9.

[15] Burton, B. S. On the propyl derivatives and decomposition products of ethylacetoacetate. American Chemistry Journal. (1882). , 18(3), 385-95.

[16] Meunier, H, Carraz, G, Neunier, Y, Eymard, P, \& Aimard, M. Pharmacodynamic properties of N-dipropylacetic acid]. Therapie. (1963). Mar-Apr;, 18, 435-8.

[17] Perry, P. J, Bever-stille, K. A, Arndt, S, \& Gundersen, S. Correlation of valproate plasma concentrations and dose in bipolar affective disorder. J Clin Psychopharmacol. (2000). Apr; 20(2), 277-9.

[18] Silva, M. F, Aires, C. C, Luis, P. B, Ruiter, J. P, Ijlst, L, Duran, M, et al. Valproic acid metabolism and its effects on mitochondrial fatty acid oxidation: A review. J Inherit Metab Dis. (2008). Apr 4.

[19] Chateauvieux, S, Morceau, F, Dicato, M, \& Diederich, M. Molecular and therapeutic potential and toxicity of valproic acid. J Biomed Biotechnol. (2010).

[20] Yildirim, E, Zhang, Z, Uz, T, Chen, C. Q, Manev, R, \& Manev, H. Valproate administration to mice increases histone acetylation and 5-lipoxygenase content in the hippocampus. Neurosci Lett. (2003). Jul 17; 345(2), 141-3.

[21] Clayton-smith, J, \& Donnai, D. Fetal valproate syndrome. J Med Genet. (1995). Sep; 32(9), 724-7. 
[22] Tremolizzo, L, Difrancesco, J. C, Rodriguez-menendez, V, Riva, C, Conti, E, Galimberti, $\mathrm{G}$, et al. Valproate induces epigenetic modifications in lymphomonocytes from epileptic patients. Prog Neuropsychopharmacol Biol Psychiatry. (2012). Oct;, 39(1), 47-51.

[23] Löscher, W, Rohlfs, A, \& Rundfeldt, C. Reduction in firing rate of substantia nigra pars reticulata neurons by valproate: influence of different types of anesthesia in rats. Brain Res. (1995). Dec;702(1-2):133-44.

[24] Yamauchi, J, Torii, T, Kusakawa, S, Sanbe, A, Nakamura, K, Takashima, S, et al. The mood stabilizer valproic acid improves defective neurite formation caused by CharcotMarie-Tooth disease-associated mutant Rab7 through the JNK signaling pathway. J Neurosci Res. (2010). Nov; 88(14), 3189-97.

[25] Lee, S. H, Yoon, J, Shin, S. H, Zahoor, M, Kim, H. J, Park, P. J, et al. Valproic acid induces hair regeneration in murine model and activates alkaline phosphatase activity in human dermal papilla cells. PLoS One. (2012). e34152.

[26] Jung, G. A, Yoon, J. Y, Moon, B. S, Yang, D. H, Kim, H. Y, Lee, S. H, et al. Valproic acid induces differentiation and inhibition of proliferation in neural progenitor cells via the beta-catenin-Ras-ERK-WAF1 pathway. BMC Cell Biol. (2008). , 21Cip.

[27] Cerveny, L, Svecova, L, Anzenbacherova, E, Vrzal, R, Staud, F, Dvorak, Z, et al. Valproic acid induces CYP3A4 and MDR1 gene expression by activation of constitutive androstane receptor and pregnane $X$ receptor pathways. Drug Metab Dispos. (2007). Jul;, 35(7), 1032-41.

[28] Lee, J. Y, Kim, H. S, Choi, H. Y, Oh, T. H, Ju, B. G, \& Yune, T. Y. Valproic acid attenuates blood-spinal cord barrier disruption by inhibiting matrix metalloprotease- 9 activity and improves functional recovery after spinal cord injury. J Neurochem. (2012). Jun; 121(5), 818-29.

[29] Gurpur, P. B, Liu, J, Burkin, D. J, \& Kaufman, S. J. Valproic acid activates the PI3K/Akt/ mTOR pathway in muscle and ameliorates pathology in a mouse model of Duchenne muscular dystrophy. Am J Pathol. (2009). Mar;, 174(3), 999-1008.

[30] Dalens, B, Raynaud, E. J, \& Gaulme, J. Teratogenicity of valproic acid. J Pediatr. (1980). Aug;, 97(2), 332-3.

[31] Mastroiacovo, P, Bertollini, R, Morandini, S, \& Segni, G. Maternal epilepsy, valproate exposure, and birth defects. Lancet. (1983). Dec , 24-31.

[32] Robert, E, \& Rosa, F. Valproate and birth defects. Lancet. (1983). Nov 12;2(8359):1142.

[33] Arndt, T. L, Stodgell, C. J, \& Rodier, P. M. The teratology of autism. Int J Dev Neurosci. (2005). Apr-May;23(2-3):189-99.

[34] Nanson, J. L. Autism in fetal alcohol syndrome: a report of six cases. Alcohol Clin Exp Res. (1992). Jun; 16(3), 558-65. 
[35] Stromland, K, Nordin, V, Miller, M, Akerstrom, B, \& Gillberg, C. Autism in thalidomide embryopathy: a population study. Dev Med Child Neurol. (1994). Apr;, 36(4), 351-6.

[36] Lindhout, D, \& Omtzigt, J. G. Pregnancy and the risk of teratogenicity. Epilepsia. (1992). Suppl 4:S, 41-8.

[37] DiLiberti JHFarndon PA, Dennis NR, Curry CJ. The fetal valproate syndrome. Am J Med Genet. (1984). Nov;; 19(3), 473-81.

[38] Bescoby-chambers, N, Forster, P, \& Bates, G. Foetal valproate syndrome and autism: additional evidence of an association'. Dev Med Child Neurol. (2001). Dec;43(12):847.

[39] Williams, G, King, J, Cunningham, M, Stephan, M, Kerr, B, \& Hersh, J. H. Fetal valproate syndrome and autism: additional evidence of an association. Dev Med Child Neurol. (2001). Mar;, 43(3), 202-6.

[40] Chessa, L, \& Iannetti, P. Fetal valproate syndrome. Am J Med Genet. (1986). Jun; 24(2), 381-2.

[41] Kini, U, Adab, N, Vinten, J, Fryer, A, \& Clayton-smith, J. Dysmorphic features: an important clue to the diagnosis and severity of fetal anticonvulsant syndromes. Arch Dis Child Fetal Neonatal Ed. (2006). Mar;91(2):F, 90-5.

[42] Winter, R. M, Donnai, D, Burn, J, \& Tucker, S. M. Fetal valproate syndrome: is there a recognisable phenotype? J Med Genet. (1987). Nov;, 24(11), 692-5.

[43] Kozma, C. Valproic acid embryopathy: report of two siblings with further expansion of the phenotypic abnormalities and a review of the literature. Am J Med Genet. (2001). Jan $15 ;$; 98(2), 168-75.

[44] Malm, H, Kajantie, E, Kivirikko, S, Kaariainen, H, Peippo, M, \& Somer, M. Valproate embryopathy in three sets of siblings: further proof of hereditary susceptibility. Neurology. (2002). Aug 27; 59(4), 630-3.

[45] Williams, P. G, \& Hersh, J. H. A male with fetal valproate syndrome and autism. Dev Med Child Neurol. (1997). Sep; 39(9), 632-4.

[46] Moore, S. J, Turnpenny, P, Quinn, A, Glover, S, Lloyd, D. J, Montgomery, T, et al. A clinical study of 57 children with fetal anticonvulsant syndromes. J Med Genet. (2000). Jul;, 37(7), 489-97.

[47] Rodier, P. M, Ingram, J. L, Tisdale, B, \& Croog, V. J. Linking etiologies in humans and animal models: studies of autism. Reprod Toxicol. (1997). Mar-Jun;11(2-3):417-22.

[48] Brown, N. A, Kao, J, \& Fabro, S. Teratogenic potential of valproic acid. Lancet. (1980). Mar 22; 1(8169), 660-1.

[49] Bruckner, A, Lee, Y. J, Shea, O, \& Henneberry, K. S. RC. Teratogenic effects of valproic acid and diphenylhydantoin on mouse embryos in culture. Teratology. (1983). Feb; 27(1), 29-42. 
[50] Petrere, J. A, Anderson, J. A, Sakowski, R, Fitzgerald, J. E, \& De La Iglesia, F. A. Teratogenesis of calcium valproate in rabbits. Teratology. (1986). Dec;, 34(3), 263-9.

[51] Mast, T. J, Cukierski, M. A, Nau, H, \& Hendrickx, A. G. Predicting the human teratogenic potential of the anticonvulsant, valproic acid, from a non-human primate model. Toxicology. (1986). May; 39(2), 111-9.

[52] Sonoda, T, Ohdo, S, Ohba, K, Okishima, T, \& Hayakawa, K. Sodium valproate-induced cardiovascular abnormalities in the Jcl:ICR mouse fetus: peak sensitivity of gestational day and dose-dependent effect. Teratology. (1993). Aug;, 48(2), 127-32.

[53] Ehlers, K, Sturje, H, Merker, H. J, \& Nau, H. Valproic acid-induced spina bifida: a mouse model. Teratology. (1992). Feb; 45(2), 145-54.

[54] Turner, S, Sucheston, M. E, De Philip, R. M, \& Paulson, R. B. Teratogenic effects on the neuroepithelium of the CD-1 mouse embryo exposed in utero to sodium valproate. Teratology. (1990). Apr; 41(4), 421-42.

[55] Vorhees, C. V. Behavioral teratogenicity of valproic acid: selective effects on behavior after prenatal exposure to rats. Psychopharmacology (Berl). (1987). , 92(2), 173-9.

[56] Crawley, J. N. Mouse behavioral assays relevant to the symptoms of autism. Brain Pathol. (2007). Oct; , 17(4), 448-59.

[57] Yochum, C. L, Dowling, P, Reuhl, K. R, Wagner, G. C, \& Ming, X. VPA-induced apoptosis and behavioral deficits in neonatal mice. Brain Res. (2008). Apr 8;, 1203, 126-32.

[58] Wagner, G. C, Reuhl, K. R, Cheh, M, Mcrae, P, \& Halladay, A. K. A new neurobehavioral model of autism in mice: pre- and postnatal exposure to sodium valproate. J Autism Dev Disord. (2006). Aug;, 36(6), 779-93.

[59] Downing, C, Biers, J, Larson, C, Kimball, A, Wright, H, Ishii, T, et al. Genetic and maternal effects on valproic acid teratogenesis in C57BL/6J and DBA/2J mice. Toxicol Sci. (2010). Aug;, 116(2), 632-9.

[60] Tung, E. W, \& Winn, L. M. Valproic acid increases formation of reactive oxygen species and induces apoptosis in postimplantation embryos: a role for oxidative stress in valproic acid-induced neural tube defects. Mol Pharmacol. (2011). Dec;, 80(6), 979-87.

[61] Kataoka, S, Takuma, K, Hara, Y, Maeda, Y, Ago, Y, \& Matsuda, T. Autism-like behaviours with transient histone hyperacetylation in mice treated prenatally with valproic acid. Int J Neuropsychopharmacol. (2011). Nov , 18, 1-13.

[62] Kolozsi, E, Mackenzie, R. N, Roullet, F. I, Decatanzaro, D, \& Foster, J. A. Prenatal exposure to valproic acid leads to reduced expression of synaptic adhesion molecule neuroligin 3 in mice. Neuroscience. (2009). Nov 10;,163(4), 1201-10.

[63] Roullet, F. I, Wollaston, L, Decatanzaro, D, \& Foster, J. A. Behavioral and molecular changes in the mouse in response to prenatal exposure to the anti-epileptic drug valproic acid. Neuroscience. (2010). Oct 13; 170(2), 514-22. 
[64] Fucic, A, Stojkovic, R, Miskov, S, Zeljezic, D, Markovic, D, Gjergja, R, et al. Transplacental genotoxicity of antiepileptic drugs: animal model and pilot study on mother/ newborn cohort. Reprod Toxicol. (2010). Dec;, 30(4), 613-8.

[65] Hara, Y, Maeda, Y, Kataoka, S, Ago, Y, Takuma, K, \& Matsuda, T. Effect of prenatal valproic acid exposure on cortical morphology in female mice. J Pharmacol Sci. (2012)., $118(4), 543-6$.

[66] Gandal, M. J, Edgar, J. C, Ehrlichman, R. S, Mehta, M, Roberts, T. P, \& Siegel, S. J. Validating gamma oscillations and delayed auditory responses as translational biomarkers of autism. Biol Psychiatry. (2010). Dec 15; 68(12), 1100-6.

[67] Mehta, MV, Gandal, MJ, Siegel, SJ, \& mGlu, . 5-antagonist mediated reversal of elevated stereotyped, repetitive behaviors in the VPA model of autism. PLoS One. 2011;6(10):e26077.

[68] Kim, K. C, Kim, P, Go, H. S, Choi, C. S, Yang, S. I, Cheong, J. H, et al. The critical period of valproate exposure to induce autistic symptoms in Sprague-Dawley rats. Toxicol Lett. (2011). Mar 5; 201(2), 137-42.

[69] Tashiro, Y, Oyabu, A, Imura, Y, Uchida, A, Narita, N, \& Narita, M. Morphological abnormalities of embryonic cranial nerves after in utero exposure to valproic acid: implications for the pathogenesis of autism with multiple developmental anomalies. Int J Dev Neurosci. (2011). Jun;, 29(4), 359-64.

[70] Dufour-rainfray, D, \& Vourc, h P. Le Guisquet AM, Garreau L, Ternant D, Bodard S, et al. Behavior and serotonergic disorders in rats exposed prenatally to valproate: a model for autism. Neurosci Lett. (2010). Feb 5;, 470(1), 55-9.

[71] Miyazaki, K, Narita, N, \& Narita, M. Maternal administration of thalidomide or valproic acid causes abnormal serotonergic neurons in the offspring: implication for pathogenesis of autism. Int J Dev Neurosci. (2005). Apr-May;23(2-3):287-97.

[72] Nakasato, A, Nakatani, Y, Seki, Y, Tsujino, N, Umino, M, \& Arita, H. Swim stress exaggerates the hyperactive mesocortical dopamine system in a rodent model of autism. Brain Res. (2008). Feb 8; 1193, 128-35.

[73] Narita, M, Oyabu, A, Imura, Y, Kamada, N, Yokoyama, T, Tano, K, et al. Nonexploratory movement and behavioral alterations in a thalidomide or valproic acid-induced autism model rat. Neurosci Res. (2009). Jan;, 66(1), 2-6.

[74] Tsujino, N, Nakatani, Y, Seki, Y, Nakasato, A, Nakamura, M, Sugawara, M, et al. Abnormality of circadian rhythm accompanied by an increase in frontal cortex serotonin in animal model of autism. Neurosci Res. (2007). Feb; 57(2), 289-95.

[75] Kuwagata, M, Ogawa, T, Shioda, S, \& Nagata, T. Observation of fetal brain in a rat valproate-induced autism model: a developmental neurotoxicity study. Int J Dev Neurosci. (2009). Jun;, 27(4), 399-405. 
[76] Ogawa, T, Kuwagata, M, Hori, Y, \& Shioda, S. Valproate-induced developmental neurotoxicity is affected by maternal conditions including shipping stress and environmental change during early pregnancy. Toxicol Lett. (2007). Nov 1;174(1-3):18-24.

[77] Dendrinos, G, Hemelt, M, \& Keller, A. Prenatal VPA Exposure and Changes in Sensory Processing by the Superior Colliculus. Front Integr Neurosci. (2011).

[78] Rinaldi, T, Kulangara, K, Antoniello, K, \& Markram, H. Elevated NMDA receptor levels and enhanced postsynaptic long-term potentiation induced by prenatal exposure to valproic acid. Proc Natl Acad Sci U S A. (2007). Aug 14;, 104(33), 13501-6.

[79] Go, H. S. Chan Kim K, Choi CS, Jeon SJ, Kwon KJ, Han SH, et al. Prenatal exposure to valproic acid increases the neural progenitor cell pool and induces macrocephaly in rat brain via a mechanism involving the GSK-3beta/beta-catenin pathway. Neuropharmacology. (2012). Jul 27.

[80] Stanton, M. E, Peloso, E, Brown, K. L, \& Rodier, P. Discrimination learning and reversal of the conditioned eyeblink reflex in a rodent model of autism. Behav Brain Res. (2007). Jan 10; 176(1), 133-40.

[81] Stodgell, C. J, Ingram, J. L, Bara, O, Tisdale, M, Nau, B. K, \& Rodier, H. PM. Induction of the homeotic gene Hoxa1 through valproic acid's teratogenic mechanism of action. Neurotoxicol Teratol. (2006). Sep-Oct; , 28(5), 617-24.

[82] Sui, L, \& Chen, M. Prenatal exposure to valproic acid enhances synaptic plasticity in the medial prefrontal cortex and fear memories. Brain Res Bull. (2012). Apr 10;, 87(6), 556-63.

[83] Murawski, N. J, Brown, K. L, \& Stanton, M. E. Interstimulus interval (ISI) discrimination of the conditioned eyeblink response in a rodent model of autism. Behav Brain Res. (2009). Jan 23; 196(2), 297-303.

[84] Bambini-junior, V, Rodrigues, L, Behr, G. A, Moreira, J. C, Riesgo, R, \& Gottfried, C. Animal model of autism induced by prenatal exposure to valproate: behavioral changes and liver parameters. Brain Res. (2011). Aug 23;, 1408, 8-16.

[85] Felix-ortiz, A. C, \& Febo, M. Gestational valproate alters BOLD activation in response to complex social and primary sensory stimuli. PLoS One. (2012). e37313.

[86] Ingram, J. L, Peckham, S. M, Tisdale, B, \& Rodier, P. M. Prenatal exposure of rats to valproic acid reproduces the cerebellar anomalies associated with autism. Neurotoxicol Teratol. (2000). May-Jun; 22(3), 319-24.

[87] Lukose, R, Schmidt, E, \& Wolski, T. P. Jr., Murawski NJ, Kulesza RJ, Jr. Malformation of the superior olivary complex in an animal model of autism. Brain Res. (2011). Jun $29 ; 1398,102-12$.

[88] Schneider, T, Labuz, D, \& Przewlocki, R. Nociceptive changes in rats after prenatal exposure to valproic acid. Pol J Pharmacol. (2001). Sep-Oct;, 53(5), 531-4. 
[89] Schneider, T, Roman, A, Basta-kaim, A, Kubera, M, Budziszewska, B, Schneider, K, et al. Gender-specific behavioral and immunological alterations in an animal model of autism induced by prenatal exposure to valproic acid. Psychoneuroendocrinology. (2008). Jul; 33(6), 728-40.

[90] Schneider, T, Turczak, J, \& Przewlocki, R. Environmental enrichment reverses behavioral alterations in rats prenatally exposed to valproic acid: issues for a therapeutic approach in autism. Neuropsychopharmacology. (2006). Jan;, 31(1), 36-46.

[91] Schneider, T, Ziolkowska, B, Gieryk, A, Tyminska, A, \& Przewlocki, R. Prenatal exposure to valproic acid disturbs the enkephalinergic system functioning, basal hedonic tone, and emotional responses in an animal model of autism. Psychopharmacology (Berl). (2007). Sep; 193(4), 547-55.

[92] Wang, Z, Xu, L, Zhu, X, Cui, W, Sun, Y, Nishijo, H, et al. Demethylation of specific Wnt/ beta-catenin pathway genes and its upregulation in rat brain induced by prenatal valproate exposure. Anat Rec (Hoboken). (2010). Nov;, 293(11), 1947-53.

[93] Yochum, C. L, Bhattacharya, P, Patti, L, Mirochnitchenko, O, \& Wagner, G. C. Animal model of autism using GSTM1 knockout mice and early post-natal sodium valproate treatment. Behav Brain Res. (2010). Jul 11; 210(2), 202-10.

[94] Vorbrodt, A. W, Dobrogowska, D. H, Kozlowski, P. B, Rabe, A, Tarnawski, M, \& Lee, M. H. Immunogold study of effects of prenatal exposure to lipopolysaccharide and/or valproic acid on the rat blood-brain barrier vessels. J Neurocytol. (2005). Dec;; 34(6), 435-46.

[95] Snow, W. M, Hartle, K, \& Ivanco, T. L. Altered morphology of motor cortex neurons in the VPA rat model of autism. Dev Psychobiol. (2008). Nov; 50(7), 633-9.

[96] Markram, K, \& Rinaldi, T. La Mendola D, Sandi C, Markram H. Abnormal fear conditioning and amygdala processing in an animal model of autism. Neuropsychopharmacology. (2008). Mar;, 33(4), 901-12.

[97] Rinaldi, T, Silberberg, G, \& Markram, H. Hyperconnectivity of local neocortical microcircuitry induced by prenatal exposure to valproic acid. Cereb Cortex. (2008). Apr; $18(4), 763-70$.

[98] Sandhya, T, Sowjanya, J, \& Veeresh, B. Bacopa monniera (L.) Wettst ameliorates behavioral alterations and oxidative markers in sodium valproate induced autism in rats. Neurochem Res. (2012). May;, 37(5), 1121-31.

[99] Gogolla, N, Leblanc, J. J, Quast, K. B, Sudhof, T. C, Fagiolini, M, \& Hensch, T. K. Common circuit defect of excitatory-inhibitory balance in mouse models of autism. J Neurodev Disord. (2009). Jun;, 1(2), 172-81.

[100] Courchesne, E, Yeung-courchesne, R, Press, G. A, Hesselink, J. R, \& Jernigan, T. L. Hypoplasia of cerebellar vermal lobules VI and VII in autism. N Engl J Med. (1988). May 26;, 318(21), 1349-54. 
[101] Markram, H, Rinaldi, T, \& Markram, K. The intense world syndrome--an alternative hypothesis for autism. Front Neurosci. (2007). Nov; 1(1), 77-96.

[102] Nelson, K. B, Grether, J. K, Croen, L. A, Dambrosia, J. M, Dickens, B. F, Jelliffe, L. L, et al. Neuropeptides and neurotrophins in neonatal blood of children with autism or mental retardation. Ann Neurol. (2001). May;, 49(5), 597-606.

[103] Huang, E. J, \& Reichardt, L. F. Trk receptors: roles in neuronal signal transduction. Annu Rev Biochem. (2003). , 72, 609-42.

[104] Li, M, Dai, F. R, Du, X. P, Yang, Q. D, Zhang, X, \& Chen, Y. Infusion of BDNF into the nucleus accumbens of aged rats improves cognition and structural synaptic plasticity through PI3K-ILK-Akt signaling. Behav Brain Res. (2012). May 16;, 231(1), 146-53.

[105] Scaccianoce, S. Del Bianco P, Paolone G, Caprioli D, Modafferi AM, Nencini P, et al. Social isolation selectively reduces hippocampal brain-derived neurotrophic factor without altering plasma corticosterone. Behav Brain Res. (2006). Apr 3; 168(2), 323-5.

[106] Martinowich, K, \& Lu, B. Interaction between BDNF and serotonin: role in mood disorders. Neuropsychopharmacology. (2008). Jan;, 33(1), 73-83.

[107] Shattock, P, \& Whiteley, P. Biochemical aspects in autism spectrum disorders: updating the opioid-excess theory and presenting new opportunities for biomedical intervention. Expert Opin Ther Targets. (2002). Apr;, 6(2), 175-83.

[108] Chugani, D. C, Muzik, O, Behen, M, Rothermel, R, Janisse, J. J, Lee, J, et al. Developmental changes in brain serotonin synthesis capacity in autistic and nonautistic children. Ann Neurol. (1999). Mar;; 45(3), 287-95.

[109] Hoshino, Y, Watanabe, H, Yashima, Y, Kaneko, M, \& Kumashiro, H. An investigation on sleep disturbance of autistic children. Folia Psychiatr Neurol Jpn. (1984). , 38(1), 45-51.

[110] Jacobs, B. L, \& Fornal, C. A. Activity of brain serotonergic neurons in the behaving animal. Pharmacol Rev. (1991). Dec; 43(4), 563-78.

[111] Narita, N, Kato, M, Tazoe, M, Miyazaki, K, Narita, M, \& Okado, N. Increased monoamine concentration in the brain and blood of fetal thalidomide- and valproic acidexposed rat: putative animal models for autism. Pediatr Res. (2002). Oct; , 52(4), 576-9.

[112] Araque, A. Astrocytes process synaptic information. Neuron Glia Biol. (2008). Feb; 4(1), 3-10.

[113] Vargas, D. L, Nascimbene, C, Krishnan, C, Zimmerman, A. W, \& Pardo, C. A. Neuroglial activation and neuroinflammation in the brain of patients with autism. Ann Neurol. (2005). Jan; , 57(1), 67-81.

[114] Carlson, G. C. Glutamate receptor dysfunction and drug targets across models of autism spectrum disorders. Pharmacol Biochem Behav. (2012). Feb; 100(4), 850-4. 
[115] Rubenstein, J. L, \& Merzenich, M. M. Model of autism: increased ratio of excitation/ inhibition in key neural systems. Genes Brain Behav. (2003). Oct;, 2(5), 255-67.

[116] Ming, X, Brimacombe, M, \& Wagner, G. C. Prevalence of motor impairment in autism spectrum disorders. Brain Dev. (2007). Oct;, 29(9), 565-70.

[117] Bailey, A, Luthert, P, Dean, A, Harding, B, Janota, I, Montgomery, M, et al. A clinicopathological study of autism. Brain. (1998). May;121 ( Pt 5):889-905.

[118] Rosenhall, U, Nordin, V, Sandstrom, M, Ahlsen, G, \& Gillberg, C. Autism and hearing loss. J Autism Dev Disord. (1999). Oct; 29(5), 349-57.

[119] Kulesza, R. J. Jr., Lukose R, Stevens LV. Malformation of the human superior olive in autistic spectrum disorders. Brain Res. (2011). Jan 7;, 1367, 360-71. 

Chapter 9

\title{
Mnesic Imbalance or Hyperthymestic Syndrome as Cause of Autism Symptoms in Shereshevskii
}

\author{
Miguel Ángel Romero-Munguía \\ Additional information is available at the end of the chapter \\ http://dx.doi.org/10.5772/54295
}

\section{Introduction}

Solomon Veniaminovich Shereshevskii (1886-1958) was a man studied by psychologist Aleksandr Romanovich Luria, who thought that Shereshevskii's (S) remarkable memory caused a psychological syndrome with cognitive deficits; consequently, Luria not only described S's memory, but also other aspects of his life such as his synaesthesia, mental imagery, preferences, strengths, weaknesses and personality [1]. Likewise, more recently the phrase "hyperthymestic syndrome" (hyperthymesia) has been coined in order to describe the case of a woman (AJ) whose autobiographical memory is extraordinary, but she has said "it is a burden" [2]. On the other hand, Kanner's clinical description of autistic disorder suggested that excellent rote memory might be involved in its aetiology [3]. In addition, some authors have thought that $S$ could have had an Autism Spectrum Disorder (ASD) $[4,5]$. So the question arises: if $S$ could have had an ASD, then how might his memory have given rise to such an ASD? For this reason, the present chapter begins by reviewing the evidence for the diagnosis of autistic disorder in $S$ using criteria from the Diagnostic and Statistical Manual of Mental Disorders, Fourth Edition, Text Revision (DSM-IV-TR) of the American Psychiatric Association [6] and from proposed criteria for its fifth edition [7], the chapter also compares his symptoms with those from a famous case (DT) with Asperger syndrome [8,9]. It is concluded that S most likely had autistic disorder. Next, I reviewed the literature to determine whether the assumption that a superior memory may be the cause of autism symptoms would be acceptable or not. However, the data seem to show that S's job as a professional mnemonist was associated instead with a gradual decrease in the severity of some of his autism symptoms [1], notwithstanding, there are great mnemonists without ASD [10,11]. Finally, an alternative explanation regarding the possible relationship between superior memories and au- 
tism spectrum disorders is given by the mnesic imbalance theory, which posits that a faulty procedural memory is replaced, in some of its functions, by a relatively preserved declarative memory, resulting in all three diagnostic symptoms of autism [12].

\section{Diagnosis of autism spectrum disorder in Shereshevskii}

\subsection{Diagnostic criteria for autistic disorder by the DSM-IV-TR}

Autistic disorder is the most clinically representative of the ASDs [13]. Currently its diagnosis is based entirely on the observation of behaviors listed in the DSM-IV-TR diagnostic criteria [6]. Such criteria can be assessed by the Autism Diagnostic Interview-Revised (ADI-R), which is conducted with the caregivers of individuals suspected of having autistic disorder or other ASD [14]. Consequently, if one wants to make a retrospective diagnosis of ASD in historical figures, then one might use the ADI-R, although it does not cover all ASDs, on biographical texts. However, there are several difficulties in implementing this strategy. First, since the biographical texts are not usually oriented to describe symptoms of psychiatric disorders, possibly such symptoms were not mentioned even though they had been present. Second, some behaviors from historical figures have been considered autism symptoms [15-17], but this attitude has been questioned because of doubts regarding whether the socio-cultural context was taken into account [18]. Third, it is necessary to find the motivation behind behaviors in order to determine whether they are autism symptoms or not [19].

Fortunately, although the psychologist who wrote the psychobiographical text to be discussed in the present chapter did not specifically search for autism symptoms, he pointed out several of these symptoms in S. This historical figure lived in the twentieth century, hence, his socio-cultural context was relatively similar to ours; the motivation behind behavior from $S$ were examined in detail by the author of that text [1]. In addition, there are three major cognitive theories that attempt to explain autism: mentalizing deficit, that is, deficit to infer mental states [20]; weak central coherence, which is defined as disability to see the overall picture but with ability to see details [21]; and finally, executive dysfunction, which is the disturbed activity of the mental processes which control actions [22], although there is a newer version of the first of them called empathizing-systemizing theory which surmises that autism may be explained by a hyper-developed drive to analyze or construct systems (systemizing) with a hypo-developed drive to infer mental states and to react to them with appropriate emotions (empathizing) [23]. Thus, the presence of the cognitive deficits predicted by such theories may be considered motivations associated with autism. Finally, in the present work, the DSM-IV-TR diagnostic criteria will be mentioned in the order in which they appear in that manual diagnostic [6]; every area of the core triad of impairments (social interaction, communicative capacity and behavioral flexibility) is discussed in order to establish whether $\mathrm{S}$ had autistic disorder. 


\subsubsection{Impairment in social interaction}

A total of six (or more) items from criterion A (core triad) are required for the diagnosis of autistic disorder, with at least two from subcriterion A1 (social interaction), and one each from subcriteria A2 (communicative capacity) and A3 (behavioral flexibility), while each of the three subcriteria contains four items [6]. On the other hand, S had impairment in the use of nonverbal behaviors to regulate social interaction (item A1a). Since the biography of S explicitly describes that some individuals always called him kalter nefesh, which is a phrase in Yiddish that means cold soul, because for instance, he reacted cold-bloodedly to news that other people said to him, so if someone said "Fire!" S did not understand that something was being consumed by flames and consequently he did not show emotions. In this example, the impairment seems secondary to weak central coherence [21].

Besides, $S$ had failure to develop peer relationships (item A1b), which was manifested in various ways. For instance, he had a very good wife and a very intelligent son, but $S$ perceived them as through a haze; also he had difficulties understanding why his fellow men wanted to have any job, which seems to be in accordance with hypo-developed empathizing [23].

Moreover, S showed a lack of socio-emotional reciprocity (item A1d), if his host asked him "How do you like these cigarettes?" he answered "So-so, fair...", that is, S did not respond reciprocally to the kindness of others, which he explained as secondary to his inability to automatically react appropriately because he neeeded to monitor all his own actions consciously [1]. It might be explained as a sort of self-consciousness disturbance through mentalizing deficit [24] but it might also be explained more directly as secondary to hypodeveloped empathizing [23].

In conclusion, the data suggest that $S$ met three of the four items from subcriterion $A 1$, since, unfortunately, the psychobiographical text does not provide enough data to make inferences about item A1c: lack of shared enjoyment $[1,6,14]$.

\subsubsection{Impairment in communicative capacity}

The biography of $\mathrm{S}$ shows evidence that he met item $\mathrm{A} 2 \mathrm{~b}$ (impairment to initiate or sustain conversational interchange), as only about the time $S$ was over fifty years of age did he learn to follow a conversation and stick to the subject; moreover, $S$ had marked difficulty understanding abstract concepts because he understood only what he could visualize and, therefore, he did not understand phrases such as "to weigh one's words" or "the pressure is higher" [1]. This difficulty with abstract thinking (verbal) along with a higher tendency to use visual mental representations seems most easily explained by the mnesic imbalance theory [12] than by the three major cognitive theories, but also such a cognitive profile has been extensively described by the thinking in pictures hypothesis $[25,26]$. Both theorical proposals will later be explained in detail.

On the other hand, S also met item A2c (stereotyped, repetitive or idiosyncratic language), since he at least utilized some words that can be described as neologisms for the ADI-R [14]. For example, S used the word zhuk (Russian: beetle) when he meant chipped part, black bread, 
darkness and negligence, whereas to him the word gis (Yiddish: to spill) assumed the meanings of sleeve and the reflection of a face in the polished surface of the samovar [1]. This is similar to the phrases bread basket instead of home bakery, fifty five instead of grandmother, Annette and Cecile in order to say red and blue, as well as the words Blum instead of a teller of truth and hexagon instead of six, all which were spoken by children described by Leo Kanner, who has always been considered as the first to clinically describe autistic disorder [3,27]. However, a recent communication has suggested instead that Hans Asperger could have done so five years earlier [28]. Furthermore, the pronominal reversal shown by $S$ was not so typical of autistic disorder, since he did refer to himself as "he" but did not show echolalia $[1,14]$. However, his pronominal reversal seems to be related to self-consciousness disturbance shown by individuals with ASD [24].

In summary, there is evidence that $S$ met items $A 2 b$ and $A 2 c$, that is, two of the four items from subcriterion A2, however, there are no data to establish whether $\mathrm{S}$ had a delay in spoken language with failure to compensate through gestures (item A2a), not to say that he had a lack of varied and spontaneous social imitative or make-believe play (item A2d) [6], though $\mathrm{S}$ always had problems with his ability to distinguish between his visual mental representations and the true word [1]; it is difficult, if not impossible, to develop make-believe play without such an ability.

\subsubsection{Impairment in behavioral flexibility}

$S$ had an encompassing preoccupation with an interest that was abnormal in intensity and focus (item A3a), specifically, on very detailed and specific visual mental representations. This unusual and circumscribed interest began in S's early years, for instance, when he was five years old he met a rebe (Yiddish: teacher) but $\mathrm{S}$ thought there was a mistake, since that rebe in his visual mental representation was something white, whereas the teacher was a swarthy man. Furthermore, his preoccupation on visual mental representations took up most of his time, for instance, when $\mathrm{S}$ read this sentence: " $\mathrm{N}$ was leaning up against a tree..." he saw $\mathrm{N}$ (as a slim young man) standing near a big linden tree with grass and woods all around... But then $S$ continued reading: "and was peering into a into a shop window" then he understood that $\mathrm{N}$ was not in a woods but on the street; as a result, $\mathrm{S}$ needed to completely rebuild his visual mental representation. Moreover, $S$ was very anxious if he listened to words but their very detailed images did not appear in his mind, for instance, with the word restaurant $S$ needed to see "its entrance, people sitting inside, a Romanian orchestra tuning up, and a lot else", while with the word airport a $S$ would have seen all details such as the crowd and the police cordon. Also, he did not admit that the words Mariya, Masha, Marusya and Mary (Russian variants of the same name) could all apply to the same woman because each word would elicit visual mental representations, which were very distinct one from the other $[1,6,14]$.

Apparently he showed an inflexible adherence to nonfunctional routines (item A3b). For example, if $\mathrm{S}$ had to remember the phrase American Indian, then he needed to see a very long rope across the ocean from a Russian street to America; these mental journeys were made by him despite the fact that they made him feel exhausted, not by the amount of 
data stored, but because $S$ felt like he really had done those long journeys. Another time he was asked to memorize a table whose numbers were arranged in a simple logical order: $1234-2345-3456-4567$ - etc. Then S produced visual mental representations in which the number six could be represented by a man with a swollen foot and the number seven by a man with a mustache. Later $S$ remarked that if he had been asked to memorize the letters of the alphabet arranged in a similar order, then he would have proceeded with it in the same way because he would not have noticed such an arrangement [1]. Thus, the first example may be explained by executive dysfunction [22], whereas the second may be explained by weak central coherence [21]. On the other hand, his need of adherence to routines was not limited to his job as a professional mnemonist, for instance, when S participated in a lawsuit for which he practiced imagining the judge's table on the right, he entered the courtroom but the judge was sitting on the left, thus, $\mathrm{S}$ lost his head and the case. The latter example may be explained by the mnesic imbalance theory [12,29], which will be explained later.

Besides, S had a persistent preoccupation with parts of objects (item A3d) [6]. For instance, although his synesthesia was present since childhood, even in adulthood he kept showing much preoccupation because of the sensory experiences resulting from it. Thus, even when $S$ was talking with famous people, he would be so interested in their voices that he could not follow what they said [1].

It can be concluded that $\mathrm{S}$ met items $\mathrm{A} 3 \mathrm{a}, \mathrm{A} 3 \mathrm{~b}$ and $\mathrm{A} 3 \mathrm{~d}$ from subcriterion $\mathrm{A} 3$, however, there is insufficient information to determine whether $S$ had stereotyped and repetitive motor mannerisms (item A3c). So, one can claim that $S$ fulfilled all the items required by criterion A for the diagnosis of autistic disorder, such as is summarized in Table 1, although we cannot claim that $S$ has had delays or abnormal functioning in social interaction, language or imaginative play prior to age 3 years (criterion $\mathrm{B}$ ), but it appears that he accomplished criterion $C$, in other words, he suffered neither Rett's disorder nor childhood disintegrative disorder $[1,6]$.

\begin{tabular}{lccccc}
\hline \multicolumn{1}{c}{ Core triad } & item a & item b & item c & item d & total \\
\hline 1. Social interaction & + & + & - & + & 3 \\
\hline 2. Communicative capacity & - & + & + & - & 2 \\
\hline 3. Behavioral flexibility & + & + & - & + & 3 \\
\hline
\end{tabular}

Table 1. Items from the triad of core symptoms for diagnosing autistic disorder: Each addition symbol (+) represents an item met by Shereshevskii, whereas each subtraction symbol (-) represents an item for which there is insufficient information to reach conclusions about it.

\subsection{Proposed diagnostic criteria for autistic disorder in DSM-V}

Currently the DSM-IV-TR guides the diagnosis of ASD [6], but soon its fifth edition (DSM-V) will replace it [7]. For this new edition of the manual, the term Autism Spectrum Disorders (ASD) is proposed instead of Pervasive Developmental Disorders 
(PDDs), which includes Rett's disorder, childhood disintegrative disorder, Asperger's disorder and Pervasive Developmental Disorder Not Otherwise Specified (PDD-NOS) [6], however, according to the American Psychiatric Association (APA) the term ASD excludes Rett's disorder [7], although in a previous use of the term ASD also excludes childhood disintegrative disorder [30]. On the other hand, the APA also proposes to stop using these diagnostic categories and use the name ASD as a single diagnostic category [7]. This proposal is based on the lack of reliability of the clinical distinctions among disorders [31], which is in accordance with the rejection of the assumption of normal early language development in Asperger's disorder [32]. So, it has been suggested to continue the use of the DSM-IV-TR and to regard all the PDDs, with the exception of Rett's disorder, as ASD [33]. However, a study found that only $25 \%$ of those diagnosed by DSM-IVTR with Asperger's disorder, 28\% of those with Pervasive Developmental Disorder Not Otherwise Specified (PDD-NOS) and 76\% of those with autistic disorder met the proposed DSM-V criteria for ASD [34]. This supports the view that the PDD-NOS should not be considered ASD because its diagnosis does not require compliance with the complete diagnostic triad of autism [35]. At first glance, one can assume that it is quite feasible that if the proposed criteria require two symptoms instead of one on impairment in behavioral flexibility (restricted, repetitive patterns of behavior, interests, or activities) any individuals who actually have diagnosis of some ASD will not meet these new criteria; in addition, at present the criteria regarding the impairments in social interaction and communicative capacity are not require to be completely fulfilled. Consequently, the question arises whether $S$ can meet these new criteria just as he met the necessary items from criterion A for the diagnosis of autistic disorder. To answer one might embed the criteria from the DSM-IV-TR among those from the proposed DSM-V criteria and see if $S$ meets the criteria of the latter; thereby items A1c, A1d and A2b of the DSM-IV-TR are integrated into subdomain A1 (social-emotional reciprocity) of the proposed DSM-V criteria, because, indeed, the lack of shared enjoyment (item A1c) evaluates another aspect of the same symptom assessed by item A1d (socio-emotional reciprocity), and item A2b (to initiate or sustain conversational interchange) does too. On the other hand, subdomain A2 (nonverbal communication) includes item A2a (delay in spoken language) since this is evaluated by a failure to compensate through gestures [14]. Besides, subdomain A3 (deficits in relationships) contains the item on social imitative or make-believe play (A2d) because of the importance of these behaviors in early relationships [6]. Furthermore, stereotyped, repetitive or idiosyncratic language (item A2c) is integrated into subdomain B1 (Behavioral stereotypies) and the verbal rituals are integrated into the apparently inflexible adherence to nonfunctional routines according to the ADI-R [14]. Finally, criterion B of the DSM-IV-TR which requires abnormal functioning in social interaction, communication or play is replaced by criterion $C$ in the proposed DSM-V criteria which only requires symptoms in early childhood [6,7].

Using the above method, one can see that $S$ completely met the criteria proposed in the DSM-V for ASD, that is, the new criteria seem to confirm the diagnosis of ASD even more strongly than the DSM-IV-TR (Table 2). 


\begin{tabular}{l|c}
\hline Summarized proposed DSM-V criteria & Summarized DSM-IV-TR criteria \\
\hline A. Social communication and interaction & A: 1. Socialization / 2. Communication \\
\hline 1. Social-emotional reciprocity & $1 \mathrm{c} 1 \mathrm{~d} 2 \mathrm{~b}$ \\
\hline 2. Nonverbal communication & $1 \mathrm{a} 2 \mathrm{a}$ \\
\hline 3. Deficits in relationships & $1 \mathrm{~b} 2 \mathrm{~d}$ \\
\hline B. Restricted and repetitive behavior & A: 3. Restricted and repetitive behavior \\
\hline 1. Behavioral stereotypies & 2c 3c \\
\hline 2. Adherence to routines & $3 b$ \\
\hline 3. Interests and preoccupations & $3 a$ \\
\hline 4. Reaction to sensory aspects & $3 d$ \\
\hline C. Symptoms in early childhood & B. Dysfunction prior to age 3 years \\
\hline D. Impaired everyday functioning & C. No Rett's or childhood disintegrative disorder \\
\hline
\end{tabular}

Table 2. Comparison between the proposed DSM-V criteria for ASD and those from the DSM-IV-TR for autistic disorder: all text in italics represents each criterion and item met by Shereshevskii.

\subsection{Some shared features with a case of synesthesia and Asperger syndrome}

Daniel Tammet (DT) is a British writer born in 1979, who was diagnosed with Asperger syndrome (Asperger's disorder) by researcher Simon Baron-Cohen [8], so this paragraph does not discuss his diagnosis but the characteristics he has in common with S. Indeed DT has been named "the modern-day Shereshevsky" since he not only has an ASD but also phenomenal memory and synaesthesia. For instance, DT memorized and recited 22,514 digits of pi and has an extraordinary ability to learn to speak new languages [9]; similary $S$ had the ability to memorize long chains of digits and reproduce several stanzas of The Divine Comedy fifteen years after having read it and despite not knowing Italian. However, both S and DT showed impaired face memory. Besides, with respect to synaesthesia, DT experiences numbers as having shapes, colour, textures, as well as some words with colour, while $\mathrm{S}$ also had synesthetic reactions whenever he heard tones, voices and speech sounds. Such reactions were puffs of steam, splashes, colourful visual flashes, flavours and even bodily sensations $[1,9]$. On the other hand, DT also has visual mental representations when he hears certain phrases, for instance, with "fragile peace" he imagines a glass dove, whereas with "election triumph" he sees the politician holding a trophy [9].

DT solved mathematical problems such as the following one through mental imagery: "There are twenty-seven people in a room and each shakes hands with everyone else. How many handshakes are there all together?" To solve this problem DT began imagining two men inside a bubble [9]; similarly, S solved the following problem: A husband says to the wife "Give me 7 of your mushrooms and I will have twice as many as you!" To which the wife replies: "No, give me 7 of yours and we will have the same amount". How many mush- 
rooms does each have? In order to solve this problem $S$ imagined himself with them (husband and wife) [1]. On the other hand, DT has also used mental imagery in order to better understand emotions. For instance, in order to empathize with someone who is sad, DT has imagined himself "sitting in the dark hollowness of number 6" [9]; similarly, $\mathrm{S}$ used his mental imagery, for example, during a very difficult performance $S$ could see someone smiling in the audience, which was turned into an image of a sharp spire, so that he felt as if he'd been stabbed in the heart [1].

There are some differences between the symptoms from DT and S. Thus, whereas the neologisms from $S$ were created by simple associations, the neologisms of DT such as "pramble" (to go out for a ramble with a baby in a pram) and "biplets" (twins) were created following more complex rules such as in the last neologism: a bicycle has two wheels and a tricycle three and three sisters might be a "triplets". In other words, the neologisms from S were created through semantic or perceptual association, whereas those from DT were through morphological or syntactic analysis. DT has also shown impairment to initiate or sustain conversational interchange and both DT and S recognized that they were verbose and that this affected the conversations with others. DT also has difficulty understanding language, but whereas S believed that the phrase "the pressure is higher" meant that the gas was moved upward and consequently it could not dissolve in water [Luria], DT believed that the phrase "John is not tall, he is a giant" meant something impossible, although the phrase is not structurally different from the sentence "John does not have ten dollars, he has twenty", that is, it seems $S$ had more problems in understanding the meaning of the words themselves but DT only with whole sentences. DT has shown problems to understand phrases such as "he is not inexperienced in such things" and likewise S could have had the same problems since he said he could not understand the negation of the negation [1,9].

\section{Superior memorization as a cause of autism symptoms}

\subsection{Impaired and preserved memories in ASD}

When Leo Kanner wrote his clinical description of autistic disorder, he pointed out excellent rote memory in children with the disorder and hinted that such an overload may be involved in the development of the disorder [3]. More recently it has been proposed that a mnesic imbalance may be the cause of all symptoms of autism, so the mnesic imbalance theory posits that all three diagnostic symptoms of autism may be explained by faulty procedural memory with relatively preserved declarative memory [12,29]. Procedural memory can be defined as behavioral algorithms that operate at an unconscious level and declarative (explicit) memory is information that is subject to conscious verbal reflection; whereas declarative memory includes episodic, semantic and working memory, procedural memory is only one of the various non-declarative (implicit) memories, such as priming, emotional conditioning and conditioned reflexes [36]. It is important to remember the latter because there are authors who may be referring to different types of memory although with the same term, for instance, procedural memory has been described as all implicit memory [37], 
whereas, other authors have pointed out that it is only a subtype [12,36,38,39]. In addition, a study in high functioning adults with ASD observed that memory for emotionally arousing events was preserved over time [40], and this preservation is particularly important for teaching new abilities to children with low functioning ASD [41].

The mnesic imbalance theory is supported by clinical studies that have shown bad procedural functioning [42-44], or good declarative functioning in individuals with ASD [45,46]. For instance, in a study utilizing the Serial Response Time Task (SRTT) with 10-element sequences to evaluate procedural learning in children and adolescents with autistic disorder, the results showed significant deficit in their procedural learning relative to Intellectual Quotient (IQ)-matched controls [43]. In contrast, some studies have challenged this finding, but it is important to note that all of these later studies had not use the same aforementioned task but rather tasks with 4-element sequences or shorter, as well as other changes in the application of the tasks [47-49], while significant improvement has been found in this sort of tasks in individuals with autistic disorder when the sequence length is short [50]. On the other hand, this deficit in procedural learning of sequences might be related to the poor short-term declarative memory shown on serial recall tasks in adults with ASD during a study, because its experiments demonstrated that their poor performance was due to faulty memory for the order of the items rather than because of memory deficit for the items themselves [51]. In other words, the difficulties in procedural learning of unconscious algorithms might lead to problems in learning explicit sequences, for instance, there are studies that have found impairment in "the delayed self-initiated execution of intentions at designated events" (called prospective memory), such as remembering to turn off the porch light at 11 pm or, to give a message to mom when she arrives [52-53].

Besides, a study utilizing a picture-naming task showed that boys with High Functioning Autism (HFA) responded faster than control boys on lower-frequency words; then, its authors argued that the results support the notion of enhancement in declarative memory of people with ASD [46]. This conclusion is based on the declarative/procedural model that assumes storage of grammar in procedural memory as well as of vocabulary in declarative memory [38]. In addition, such a model is in accordance with a meta-analysis which found that picture vocabulary tests are the peak of ability relative to verbal IQ in high functioning individuals with ASD [54].

Furthermore, a study utilizing a composite measure as an index of procedural learning showed significant negative correlation between procedural learning and autism symptoms [42]. Moreover, in another study, a composite group that included children with autistic disorder, both with non-functional verbal language and no spoken language, a significant positive correlation was observed between autism symptoms and scores of an index of declarative memory, which suggests that the imbalance between declarative and procedural memory in ASD might be more important than the mere faulty procedural memory [44]; such an imbalance might contribute to a global cognitive imbalance. This assumption is in accordance with results from a study in children with autism, which showed that those with a lower verbal than nonverbal IQ profile showed greater social impairment than those without this profile; this result was independent of scores in verbal or full-scale IQ [55]. 
Nevertheless, the mnesic imbalance theory does not suggest that declarative memory has to be above average in individuals with ASD; indeed, an impairment of episodic memory is in accordance with this theory [12]. In addition, the mnesic imbalance theory suggests that although procedural learning is significantly lower relative to declarative learning in individuals with ASD, this procedural memory can be developed [12,50]. Anyway, in this theory the imbalance between procedural and declarative memory is needed to account for the impairments in social interaction, communicative capacity and behavioral flexibility, as well as for the islets of ability and neurobiological findings observed in people with ASD [12,56].

However, it is important note that there are several deficits in declarative memory in individuals with ASD, but such deficits might be explained by overlap in the functioning of the memories. For instance, $S$ and DT showed impaired face memory $[1,8]$, which might be due to the difficulty to verbally describe all elements that make each face unique; consequently, facial memory has to be implicitly acquired through perceptual categorization, which is a type of procedural learning [57]. It is likely that some individuals with ASD see face learning as a task that should be performed by conscious memorization of each element of the face, which might explain the lack of developmental improvement in face learning that has been observed in autism [58]. Besides, utilizing functional magnetic resonance imaging, a study measured the activation over time of the amygdala in adults with ASD and the results showed a delay in the decrease of amygdala activity relative to control adults, which could be an action to improve face learning, since the participants with ASD had a reduction in the number of errors during a second set of the task of facial memory [59], while another study reported an association between increased amygdala activation and better facial memory in healthy people [60]. Furthermore, in individuals with autistic disorder, a study showed deficit of their facial memory awareness, that is, when participants were asked to say how they thought their performance in the task was, the answers did not correspond to reality [61].

The faulty perceptual categorization might be enough to explain the significant preference in individuals with ASD for utilizing an approach oriented to process parts rather than the gestalt during the Rey Osterrieth Complex Figure (ROCF) task [62]; this situation is equivalent to an increased number of elements, which might explain the difficulty for any individual with ASD to memorize the ROCF despite their tendency to use visual mental representations [26]. But the above mentioned deficit in facial memory awareness cannot be explained only by poor perceptual categorization. Another study showed that, whereas in typically developing adults the memory of actions is significantly better if the actions are self-performed (enacted) than if only observed, in adults with Asperger syndrome is not the case (Figure 1). These results were considered secondary to difficulties in encoding specific motor and proprioceptive signals [63], but an alternative explanation might be that during one's actions procedural knowledge is typically created and may aid declarative knowledge during recall, whereas in people with ASD, impairment of procedural learning prevents the appearance of such aid [12]. Consequently, if the latter explanation is used not only for motor actions but also for mental actions, then one might have an account for the deficit in facial memory awareness. 
Impaired procedural memory is not only unable to aid declarative memory, but that declarative memory also has to replace faulty procedural memory in some of its functions, which implies an overload for consciousness. This assumption is in accordance with a study that showed that children with ASD, in comparison to the control group, had significant difficulty in divided attention (ability to simultaneously perform two independent tasks), which was significantly related to everyday working memory [64].

The above findings are in accordance with a review of memory in ASD that pointed out a deficit in episodic memory, but are not in accordance to its assumption of preserved memory for non-social stimuli [65]. On the other hand, one must keep in mind that although there are deficits in encoding and organization of episodic and autobiographical memory in people with ASD, their storage and retrieval are preserved [66,67].

\section{Free Recall Task}

口Enacted $\mathbf{\square}$ Observed

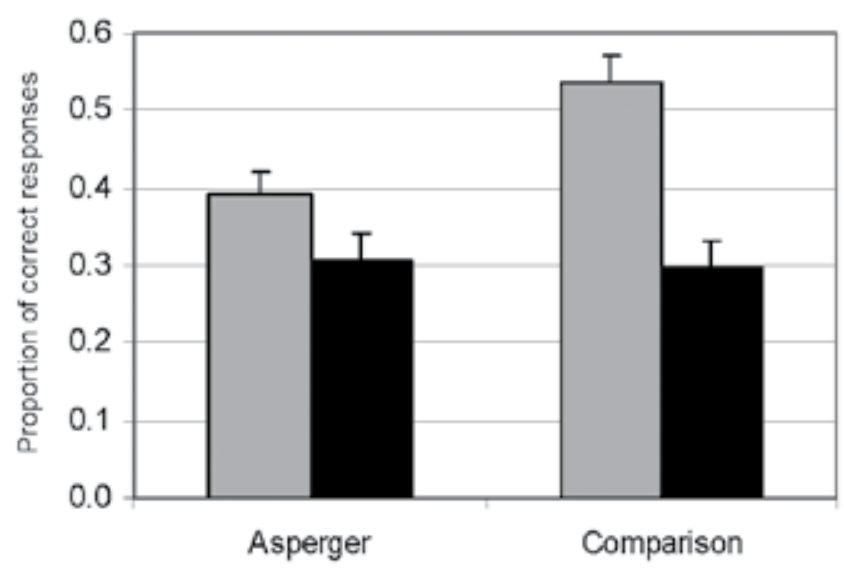

Figure 1. Mean of correctly recalled items of the Free Recall Task of actions (Enacted and Observed) in Asperger and Comparison groups: The bars represent means and the whiskers represent standard error (From Zalla et al., 2010).

\subsection{Reduction of autism symptoms during the mnemonist job}

In the first clinical description of autistic disorder, it was suggested that excellent rote memory might be involved in its etiology, since some children described by him could recite verbatim the "questions and answers of the Presbyterian Catechism", the "Twenty-third Psalm", poems, as well as titles and composers of Victrola record pieces but were unable to initiate or sustain conversations with others [3]. On the other hand, some authors have thought that S could have had an ASD [4,5]; it has been surmised that his astounding memory interfered with his comprehension of what he heard or read [5,68]. However, such an assumption might be inaccurate because his cognitive difficulties could be due to the ASD he suffered, not to his memory. So, the mnesic imbalance memory posits a higher tendency to 
use explicit (visual) mental representations in people with ASD to solve problems [12]. Similarly, a study of individuals with declarative memory deficits due to neurological damage showed that creating mental imagery of events from a personal perspective may improve episodic memory [69], therefore, people with ASD might try to improve their poor episodic memory through mental imagery. In addition, another study found that mental imagery was effective at enhancing the task of relearning after brain injury [70], so mental imagery might be used against motor deficits secondary to faulty procedural memory [12]. Finally, other study reported that understanding expressions (idioms) is associated with more sophisticated and figurative mental images [71]; consequently, the creation of mental images in people with ASD might be a small successful attempt in order to understand abstract thinking as well as mental states of others, for example, mentalizing deficit in children with autistic disorder was initially tested using the unexpected transfer test of false belief [20], but both typically developing 3 year olds and children with autism significantly improve on that test when they use thought bubbles, that is, visual representations of mental states $[72,73]$. In addition, a review supports the "Thinking in Pictures" hypothesis, which posits that a subset of individuals with ASD shows disposition towards using visual mental representations, although the authors of that theoretical proposal make the assumption that in people with ASD, mental imagery creates difficulties for understanding [26]. In view of this scenario, it is possible that neither his astounding memory nor his encompassing preoccupation for very detailed and specific visual mental representations were the cause of the cognitive problems of $S$, but rather some other factor might be the cause of these three features in $\mathrm{S}$ and such a factor might be the mnesic imbalance since procedural memory seems to be required for the development of analogical inference, which is a sort of reasoning performed primarily through unconscious algorithms that might be at the heart of abstract thinking and verbal comprehension [74-76]. So, if it is difficult to perform reasoning through procedural memory then that might be tried through declarative memory using explicit (visual) mental representations [12]. So, it is very likely that this was the reason why S often said "I can only understand what I can visualize" [1].

It has been surmised that the mnemonist job could exacerbate autism symptoms in S [4]. However, the symptoms appear to have decreased precisely because of that job. For instance, the encompassing preoccupation with very detailed and specific visual mental representations that he did for his job was very exhausting, so $S$ decided to make his visual mental representations more and more simple; as a result, the word restaurant was no longer represented by "its entrance, people sitting inside, a Romanian orchestra tuning up, and a lot else", but by "an entrance way with a bit of something white showing from inside", while with the word airport, S could see only "a small segment of the Leningrad Highway". This simplification of the visual mental representations allowed him to better understand and to enjoy what he heard and read. Also, $\mathrm{S}$ showed a decrease of the apparently inflexible adherence to nonfunctional routines, for example, if $S$ heard the word America, he no longer needed to do his mental journeys, but just to imagine "Uncle Sam". In addition, S learned to follow conversations and to understand when his behavior was not tactful [1]; similarly, a man with autistic disorder named Peter Guthrie and another man with an ASD named Kim Peek, who were used as models to create the character with autistic disorder in the movie 
Rain Man also showed significant improvement in socialization and communication after the increase in their public activity $[77,78]$.

\subsection{Relationship between great mnemonists and ASD}

There are several cases of individuals with ASD that have been known for their astounding memory, for example, Kim Peek learned more than 12,000 books and his encyclopedic knowledge covering at least 15 interests such as world and American history, sports, movies, geography, actors and actresses, literature and classical music. Peek as S had significant difficulties with language comprehension, for instance, once when Peek was asked to "lower his voice" he only slid lower into his chair [78,79]. Also, Peter Guthrie has an extraordinary memory: he can say the day of the week for any date between past and future decades, has memorized the Billboard record-sales charts and an extraordinary amount of data from almanacs and newspapers; he reads, writes and pronounces English, Japanese, French, Arabic and Spanish [77]. Moreover, a mental calculator described in his book the case of an elderly man with ASD who could tell what day of the week any given date fell, because he remembered the day of the week and an event of dates that he had lived [80], while another author described twins, John and Michael who also recalled a great amount of events from their own life, but in addition, they could say the day of the week for any date in a period of 80,000 years [81]. It is interesting to note that although bad episodic memory in people with ASD has been mentioned, these cases showed an extraordinary amount of autobiographic remembrances, even $S$ not only remembered the hundreds and thousands of series of numbers, words or syllables, but also the whole scene in which the learning had initially been carried out, so he said "This was a series you gave me once when we were in your apartment... You were sitting at the table and I in the rocking chair... You were wearing a gray suit and you looked at me like this..." [1].

On the other hand, a woman named Jill Price (whose pseudonym in scientific literature is $\mathrm{AJ})$, remembers almost every day of her life since age 11. This feature is considered part of a disorder for which the phrase "hyperthymestic syndrome" (hyperthymesia) has been coined [2]. However, another author has considered the possibility that her hyperthymesia is only a sort of obsessive compulsive disorder since she spends excessive amounts of time reliving past events and although says this is a burden also says "when I think of these things it is kind of soothing" [82]. On the other hand, S showed an apparent obsessed worry by his memories when he said "I both did and didn't want it to appear". However, this was a worry about an apparent real-life problem, because $S$ left his job as a journalist after meeting Luria, then $S$ became a professional mnemonist who performed on stage memory acts to entertain audiences; he often gave several functions each evening and feared he began to confuse the individual functions. Consequently, $\mathrm{S}$ tried to forget what he had memorized in previous functions, but finally, he understood that memories only appear if he wanted it. This latter situation was interpreted by Luria as S had learned to forget [1], but such assumption is inaccurate because it is different forget than to only remember when one wants to. Thus, it is likely that S was finally able to answer the question "Isn't it confusing to remember so much?" as the professional mnemonist without ASD Harry Lorayne, who acted 
before S died and whose response was "No!" [83]; contrary to S, he had a very good empathy and extraordinary memory for faces, so he could memorize the name of 700 individuals in only one function [10].

Hyperthymesia is also called Highly Superior Autobiographical Memory (HSAM); an author with HSAM (and without ASD) has claimed that this is not a disorder, but is "almost like therapy, in that it helps you to better understand yourself and predict how you'll function" [84]. It has been suggested that studying cases of hyperthymesia may provide the basis of potential future treatments for patients with memory disorders [85]. Furthermore, other cases of superior memory studied by science are those individuals without ASD that have set a Guinness World Record by memorizing thousands of digits of pi, for example, Rajan Mahadevan, whose memory allowed him to recited 31,811 decimals of the mathematical constant pi, shows his ability to recite a random sequence of over 40 digits seen by him at the rate of a digit per second during a digit span test [86], whereas Hideaki Tomoyori, could recite the first 40,000 digits of pi, had an average visual digit span of 8 , but 10 in the auditory digit span [87]; likewise, Chao Lu established the Guinness World Record at 67,890 decimals of pi and could remember a mean of 8.83 digits during the visual digit span task [88], whereas Dominic O'Brien (with at least six World Memory Championship titles), memorized a random sequence of 74 digits in the auditory digit span task [11]. These data indicate that the digit span tasks are not useful to show the superior memory of some individuals without ASD, such as Hideaki Tomoyori and Chao Lu. This situation does not seem to be different in people with ASD since DT, who was diagnosed with Asperger syndrome and recited 22,514 digits of pi, had a mean of 11.5 digits on a visual digit span task [8]. In contrast, when these individuals are allowed self-paced learning they can demonstrate their superior memory; for instance, Tomoyori perfectly remembered the 25 digits from a $5 \times 5$ number matrix studied for 233 seconds, that is, at a rate of 9.32 seconds per digit, whereas his control group recalled only a mean of 18.30 digits of that matrix studied at an average of 395.6 seconds (15.82 seconds per digit) [87]. Besides, Chao Lu also perfectly recalled all matrices, such as one of 25 digits which he studied for 35 seconds (1.4 seconds per digit), whereas with a 7 X 7 number matrix he required 260 seconds (5.31 seconds per digit) and on a $9 \times 9$ matrix he used 387 seconds (4.78 seconds per digit) [88]. Likewise, $\mathrm{S}$ also demonstrated his superior memory through self-paced learning, for instance, he correctly recalled all 20 digits from tables of numbers that he memorized in a mean of 37.5 seconds (1.8 seconds per digit), he learned tables of 50 digits in 165 seconds ( 3.3 seconds per digit). He recalled the third vertical column of these later tables in 80 seconds, recalled the second vertical column in 25 seconds, which suggests that $S$ did not memorize the tables as a mental photograph but as a chain of data with a determined order [1]. This same pattern has been observed in Tomoyori who also used mental imagery [87]. Indeed, according to the occipital activation that is observed in mnemonists with and without ASD, even if there is no success in the memorizing, both groups use visual mental representations during recordings [89,90]. In addition, other features of neurobiological similarity between people with ASD have been observed as well as in those whose declarative memory has been significantly enhanced [56]. Even children with Low Functioning Autism (LFA) may show qualitative similarity with the great mnemonists without ASD. For instance, some of them can learn to read whole sentences at pre- 
school age despite their very poor language comprehension $[3,77,78,91]$, besides, the great mnemonists can learn large amounts of text quickly [10,11]. Also the delayed echolalia in children with LFA is a demonstration of good declarative memory [3,27]. On the other hand, skills such as calendar calculation are common among the great mnemonists without ASD and in some individuals with HFA or Asperger syndrome. Indeed DT, Kim Peek and Peter Guthrie made calendar calculations $[9,77,78]$. To summarize, figure 2 illustrates the differences and similarities between people whose declarative memory has been significantly enhanced and that have an ASD regardless of whether or not they have an extraordinary declarative memory.

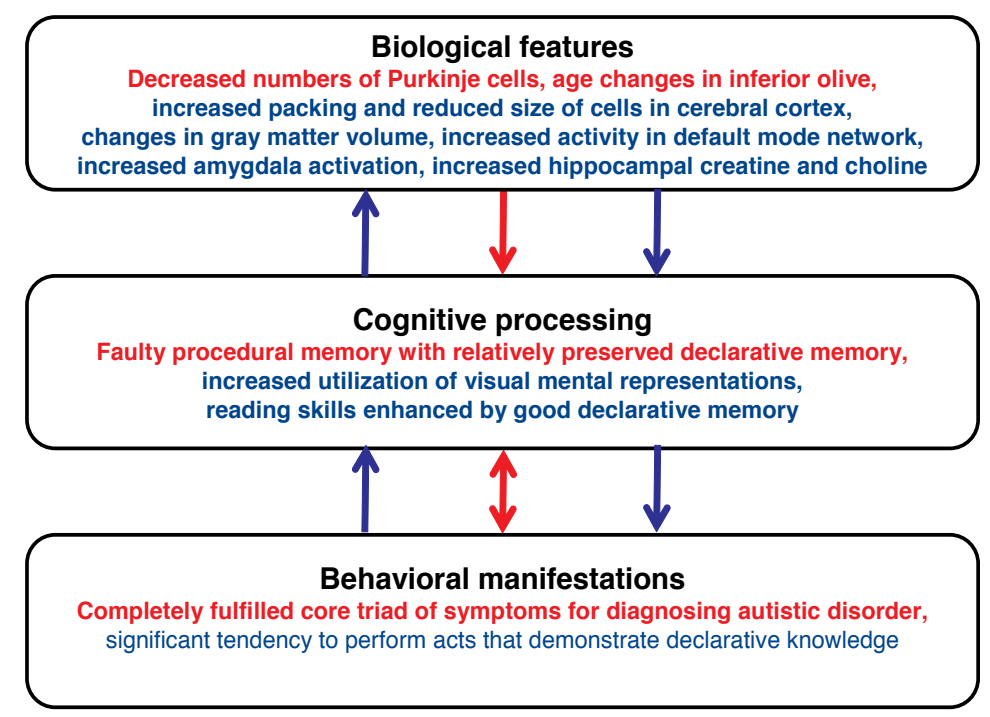

Figure 2. Shared characteristics between people with ASD and those whose declarative memory has been significantly enhanced (in blue text), and those only present in people with ASD (in red text). The blue arrows suggest a reciprocal causal interaction among all shared characteristics, whereas one red arrow indicates unidirectional influence and the other one bidirectional influence.

\section{Mmesic imbalance and superior memory in autism spectrum disorders}

Mnesic imbalance theory predicts a higher tendency in individuals with ASD to use visual mental representations [12,56], these types of representations may be utilized to significantly enhance declarative memory $[11,83,92]$. Luria recognized that this mental imagery was the cornerstone of S's extraordinary memory, whereas the synesthetic experiences were additional information [1]. So for instance, a study of individuals with synaesthesia shows that they have better memory than controls for data that induce synaesthesia, as well as for color 
per se [93]. However, during the World Memory Championships the competitors currently use mnemonic methods, not innate features as synaesthesia; one of these mnemonic methods is "the method of loci" [94], which was used by S, who when he listened to a list of objects to memorize he would distribute them along a street in his home town or the Gorky Street visualized in his mind [1]. "The method of loci" is used by Buenos Aires waiters, who memorize all the orders without written support [95].

Lorayne has pointed out that it is difficult to remember the abstract, he therefore recommends replacing it by tangible objects, for example, 7 might be represented by a flag, whereas 8 by an hourglass [10], while $S$ used a man with a mustache to represent 7 and a very stout woman to represent 8 [1]; O'Brien said that 07 may become James Bond [11]. Finally, it is very difficult to memorize meaningless words, but a solution is to convert those words into intelligible images, for instance, the name "Olczewsky" was imagined by Lorayne as an old man chewing, while he skied [83], while S visualized the word "mavanasanava" as his landlady speaking (Polish: mówić = to speak) from the window, pointing into our guesthouse (Russian: наши = our) and making a sign of negation (Latvian: nava = is not a) with her other hand [1].

\section{Conclusion}

The astounding memory of Shereshevskii has been taken as a paradigmatic example of how the development of a skill can affect the development of others. However, this chapter has offered arguments against such a view and presented evidence that he had an ASD. In addition, the relationship between memory and autism can be better understood if we reanalyze the life of this extraordinary individual under the light of the mnesic imbalance theory.

\section{Acknowledgements}

The author would like to thank Edith Monroy for reviewing the language of the manuscript.

\section{Author details}

Miguel Ángel Romero-Munguía

Address all correspondence to: romero_munguia@yahoo.com

Outpatient Service, “Dr. Samuel Ramírez Moreno" Psychiatric Hospital, Health Secretariat, Mexico 


\section{References}

[1] Luria AR. The mind of a mnemonist: a little book about a vast memory. Solotaroff L (Trans.) from the Russian. New York, NY: Basic Books; 1968.

[2] Parker ES, Cahill L, McGaugh JL. A case of unusual autobiographical remembering. Neurocase 2006;12 (1) 35-49.

[3] Kanner L. Autistic disturbances of affective contact. The nervous child 1943; 2: 217-250.

[4] Elfakir A. Mémoire et autisme: de la neuropsychologie à la psychanalyse. Le cas de Cherechevski. L'Information psychiatrique 2005; 81 (9): 763-770.

[5] Wing L. Asperger's syndrome: a clinical account. Psychological medicine 1981; 11 (1): 115-129.

[6] American Psychiatric Association. Diagnostic and statistical manual of mental disorders (4th edition, text revision).Washington, DC: American Psychiatric Publishing; 2000.

[7] American Psychiatric Association. Neurodevelopmental disorders. In: DSM-5 draft criteria closed for final public comment. Arlington,VA. American Psychiatric Association; 2012. http://www.dsm5.org/ProposedRevision/Pages/NeurodevelopmentalDisorders.aspx (accessed 26 September 2012).

[8] Baron-Cohen S, Bor D, Billington J, Asher J, Wheelwright S, Ashwin C. Savant memory in a man with colour form-number synaesthesia and Asperger syndrome. Journal of Consciousness Studies 2007; 14 (9-10): 237-252.

[9] Tammet D. Born on a blue day: inside the extraordinary mind of an autistic savant. New York, NY: Free Press; 2007.

[10] Lorayne H. Secrets of mind power. Hollywood, FL: Lifetime Books; 1995.

[11] O'Brien D. Learn to remember. San Francisco, CA: Chronicle Books; 2000.

[12] Romero-Munguía MA. Mnesic imbalance: a cognitive theory about autism spectrum disorders. Annals of general psychiatry 2008; 7 (1) e20. http://www.annals-generalpsychiatry.com/content/7/1/20 (accessed 26 September 2012).

[13] Szatmari P. A mind apart: understanding children with autism and Asperger syndrome. New York: The Guilford Press; 2004.

[14] Le Couteur A, Lord C, Rutter M. Autism diagnostic interview-revised (ADI-R). Los Angeles: Western Psychological Services; 2003.

[15] Arshad M, Fitzgerald M. Did Michelangelo (1475-1564) have high-functioning autism? Journal of medical biography 2004; 12 (2): 115-20. 
[16] Fitzgerald M. Einstein: brain and behavior. Journal of autism and developmental disorders 2000; 30 (6): 620-621.

[17] Fitzgerald M. Ludwig Wittgenstein: autism and philosophy. Journal of autism and developmental disorders 2000; 30 (6): 621-622.

[18] Beveridge A. Diagnosis of historical figures. Journal of medical biography 2004; 12 (3): 126-127.

[19] Little C. Which is it? Asperger's Syndrome or giftedness? Defining the differences. Gifted Child Today 2002; 25 (1): 58-63.

[20] Baron-Cohen S, Leslie AM, Frith U. Does the autistic child have a "theory of mind"? Cognition 1985; 21 (1): 37-46.

[21] Frith U. Autism: explaining the enigma. Malden, MA: Blackwell Publishing; 1989.

[22] Ozonoff, S., Pennington, B. F. \& Rogers, S. J. (1991). Executive function deficits in high-functioning autistic individuals: relationship to theory of mind. Journal of child psychology and psychiatry, and allied disciplines, 32 (7), 1081-1105.

[23] Baron-Cohen S. The extreme male brain theory of autism. Trends in cognitive sciences 2002; 6 (6): 248-254.

[24] Frith U, Happé F. Theory of mind and self consciousness: What is it like to be autistic? Mind and Language 1999; 14: 1-22.

[25] Kunda M, Goel AK. Thinking in pictures: a fresh look at cognition in autism. In: Love B, McRae K, Sloutsky V (eds.) Proceedings of the 30th annual conference of the Cognitive Science Society. Austin, TX; 2008. p321-326.

[26] Kunda M, Goel AK. Thinking in Pictures as a cognitive account of autism. Journal of autism and developmental disorders 2011; 41 (9): 1157-1177.

[27] Kanner L. Irrelevant and metaphorical language in early infantile autism. The American journal of psychiatry 1946; 103: 242-246.

[28] Lyons V, Fitzgerald M. Asperger (1906-1980) and Kanner (1894-1981), the two pioneers of autism. Journal of autism and developmental disorders 2007; 37(10): 2022-2023.

[29] Romero-Munguía MA. ¿Es la memoria procesal deficiente la causa del comportamiento estereotipado en el autismo? Psiquiatría, Órgano Oficial de Difusión de la Asociación Psiquiátrica Mexicana, A.C., 1998; 14 (2): 62-65.

[30] Gabis L, Huang W, Azizian A, Devincent C, Tudorica A, Kesner-Baruch Y, Roche P, Pomeroy J. 1H-magnetic resonance spectroscopy markers of cognitive and language ability in clinical subtypes of autism spectrum disorders. Journal of child neurology 2008; 23 (7): 766-774.

[31] Lord C, Petkova E, Hus V, Gan W, Lu F, Martin DM, Ousley O, Guy L, Bernier R, Gerdts J, Algermissen M, Whitaker A, Sutcliffe JS, Warren Z, Klin A, Saulnier C, 
Hanson E, Hundley R, Piggot J, Fombonne E, Steiman M, Miles J, Kanne SM, GoinKochel RP, Peters SU, Cook EH, Guter S, Tjernagel J, Green-Snyder LA, Bishop S, Esler A, Gotham K, Luyster R, Miller F, Olson J, Richler J, Risi S. A multisite study of the clinical diagnosis of different autism spectrum disorders. Archives of general psychiatry 2012; 69 (3): 306-313.

[32] Howlin P. Outcome in high-functioning adults with autism with and without early language delays: implications for the differentiation between autism and Asperger syndrome. Journal of autism and developmental disorders 2003; 33 (1): 3-13.

[33] Kurita H. How to deal with the transition from Pervasive Developmental Disorders in DSM-IV to Autism Spectrum Disorder in DSM-V. Psychiatry and clinical neurosciences 2011; 65 (7): 609-610.

[34] McPartland JC, Reichow B, Volkmar FR. Sensitivity and specificity of proposed DSM-5 diagnostic criteria for autism spectrum disorder. Journal of the American Academy of Child and Adolescent Psychiatry 2012; 51 (4): 368-383.

[35] Mercadante MT, Van der Gaag RJ, Schwartzman JS. Non-Autistic Pervasive Developmental Disorders: Rett's syndrome, childhood disintegrative disorder and pervasive developmental disorder not otherwise specified. Revista brasileira de psiquiatria 2006; 28 (Suppl. 1): 12-20.

[36] Budson AE, Price BH. Memory: clinical disorders. In: Nature Publishing Group. (Ed.). Nature encyclopedia of life sciences. London, UK: Macmillan Publishers Ltd, 2001; 11: 529-536.

[37] Magallón S, Narbona J Detección y estudios específicos en el trastorno de aprendizaje procesal. Revista de Neurología 2009; 48 (Suppl 2): 71-76.

[38] Ullman MT. The declarative/procedural model of lexicon and grammar. Journal of Psycholinguistic Research 2001; 30 (1): 37-69.

[39] Romero-Munguía MA. Trastorno de aprendizaje no verbal frente a trastorno del espectro autista. Revista de Neurología 2009; 49 (8): 448.

[40] Maras KL, Gaigg SB, Bowler DM. Memory for emotionally arousing events over time in autism spectrum disorder. Emotion 2012; doi: 10.1037/a0026679.

[41] Lovaas OI. The autistic child. New York, NY: Irvington Publishers; 1977.

[42] Klinger LG, Klinger MR, Pohlig R. Implicit learning impairments in autism spectrum disorders: Implications for treatment. In: Pérez JM, González PM, Comí ML, Nieto C (eds.) New developments in autism: The future is today. London, UK: Jessica Kingsley; 2007. p76-103.

[43] Mostofsky SH, Goldberg MC, Landa RJ, Denckla MB. Evidence for a deficit in procedural learning in children and adolescents with autism: implications for cerebellar contribution. Journal of the International Neuropsychological Society: JINS 2000; 6 (7): 752-759. 
[44] Romero-Munguía MA. Memoria procesal deficiente y alteraciones de la comunicación en la patogenia del autismo infantil. Neurología, Neurocirugía y Psiquiatría 2002; 35 (4): 203-208.

[45] Beversdorf DQ, Smith BW, Crucian GP, Anderson JM, Keillor JM, Barrett AM, Hughes JD, Felopulos GJ, Bauman ML, Nadeau SE, Heilman KM. Increased discrimination of "false memories" in autism spectrum disorder. Proceedings of the National Academy of Sciences of the United States of America 2000; 97(15): 8734-8737.

[46] Walenski M, Mostofsky SH, Gidley-Larson JC, Ullman MT. Brief report: enhanced picture naming in autism. Journal of autism and developmental disorders 2008; 38 (7): 1395-1399.

[47] Barnes KA, Howard JH Jr, Howard DV, Gilotty L, Kenworthy L, Gaillard WD, Vaidya CJ. Intact implicit learning of spatial context and temporal sequences in childhood autism spectrum disorder. Neuropsychology 2008; 22 (5): 563-570.

[48] Brown J, Aczel B, Jiménez L, Kaufman SB, Grant KP. Intact implicit learning in autism spectrum conditions. The quarterly journal of experimental psychology 2010; 63 (9): 1789-1812.

[49] Nemeth D, Janacsek K, Balogh V, Londe Z, Mingesz R, Fazekas M, Jambori S, Danyi I, Vetro A. Learning in autism: implicitly superb. PloS one 2010; 5 (7): e11731.

[50] Gordon B, Stark S. Procedural learning of a visual sequence in individuals with autism. Focus on autism and other developmental disabilities 2007; 22 (1): 14-22.

[51] Poirier M, Martin JS, Gaigg SB, Bowler DM. Short-term memory in autism spectrum disorder. Journal of abnormal psychology 2011; 120 (1): 247-252.

[52] Altgassen M, Koban N, Kliegel M. Do adults with autism spectrum disorders compensate in naturalistic prospective memory tasks? Journal of autism and developmental disorders 2012; 42 (10): 2141-2151.

[53] Brandimonte MA, Filippello P, Coluccia E, Altgassen M, Kliegel M. To do or not to do? Prospective memory versus response inhibition in autism spectrum disorder and attention-deficit/hyperactivity disorder. Memory 2011;19 (1): 56-66.

[54] Mottron L. Matching strategies in cognitive research with individuals with highfunctioning autism: current practices, instrument biases, and recommendations. Journal of autism and developmental disorders 2004; 34 (1): 19-27.

[55] Joseph R, Tager-Flusberg H, Lord C. Cognitive profiles and social-communicative functioning in children with autism spectrum disorder. Journal of Child Psychology and Psychiatry 2002; 43: 807-821.

[56] Romero-Munguía MA. Mnesic imbalance and the neuroanatomy of autism spectrum disorders. In: Eapen V (ed.). Autism - A Neurodevelopmental Journey from Genes to Behaviour. Rijeka: InTech;2011. p425-444. 
[57] Ashby GF, Crossley MJ. Interactions between declarative and procedural-learning categorization systems. Neurobiology of learning and memory 2010; 94 (1): 1-12.

[58] O'Hearn K, Schroer E, Minshew N, Luna B. Lack of developmental improvement on a face memory task during adolescence in autism. Neuropsychologia 2010; 48 (13): 3955-3960.

[59] Kleinhans NM, Johnson LC, Richards T, Mahurin R, Greenson J, Dawson G, Aylward E. Reduced neural habituation in the amygdala and social impairments in autism spectrum disorders. The American journal of psychiatry 2009; 166 (4): 467-475.

[60] Kleinhans NM, Johnson LC, Mahurin R, Richards T, Stegbauer KC, Greenson J, Dawson G, Aylward E. Increased amygdala activation to neutral faces is associated with better face memory performance. Neuroreport 2007; 18 (10): 987- 991.

[61] Wilkinson DA, Best CA, Minshew NJ, Strauss MS. Memory awareness for faces in individuals with autism. Journal of autism and developmental disorders 2010; 40 (11): 1371-1377.

[62] Tsatsanis KD, Noens IL, Illmann CL, Pauls DL, Volkmar FR, Schultz RT, Klin A. Managing complexity: impact of organization and processing style on nonverbal memory in autism spectrum disorders. Journal of autism and developmental disorders 2011; 41(2): 135-147.

[63] Zalla T, Daprati E, Sav AM, Chaste P, Nico D, Leboyer M. Memory for self-performed actions in individuals with Asperger syndrome. PloS one. 2010; 5(10): e13370.

[64] Yerys BE, Wallace GL, Jankowski KF, Bollich A, Kenworthy L. Impaired Consonant Trigrams Test (CTT) performance relates to everyday working memory difficulties in children with autism spectrum disorders. Child neuropsychology: a journal on normal and abnormal development in childhood and adolescence 2011: 17 (4):391-399.

[65] Boucher J, Mayes A, Bigham S. Memory in Autistic Spectrum Disorder. Psychological bulletin 2012; doi: 10.1037/a0026869.

[66] Southwick JS, Bigler ED, Froehlich A, Dubray MB, Alexander AL, Lange N, Lainhart JE. Memory functioning in children and adolescents with autism. Neuropsychology 2011;25 (6): 702-710.

[67] Crane L, Pring L, Jukes K, Goddard L. Patterns of Autobiographical Memory in Adults with Autism Spectrum Disorder. Journal of autism and developmental disorders 2012; doi: 10.1007/s10803-012-1459-2.

[68] Schacter DL. Searching for memory: the brain, the mind, and the past. New York, NY: Basic Books; 1997.

[69] Grilli MD, Glisky EL. Self-imagining enhances recognition memory in memory-impaired individuals with neurological damage. Neuropsychology 2010;24 (6): 698-710.

[70] Liu KP, Chan CC, Lee TM, Hui-Chan CW. Mental imagery for relearning of people after brain injury. Brain injury 2004;18 (11): 1163-1172. 
[71] Nippold MA, Duthie JK. Mental imagery and idiom comprehension: a comparison of school-age children and adults. Journal of speech, language and hearing research 2003; 46 (4): 788-799.

[72] Kerr S, Durkin K. Understanding of thought bubbles as mental representations in children with autism: implications for theory of mind. Journal of autism and developmental disorders 2004; 34 (6): 637-648.

[73] Wellman HM, Baron-Cohen S, Caswell R, Gomez JC, Swettenham J, Toye E, Lagattuta K. Thought-bubbles help children with autism acquire an alternative to a theory of mind. Autism: the international journal of research and practice 2002; 6 (4): 343-363.

[74] Day SB, Gentner D. Nonintentional analogical inference in text comprehension. Memory \& cognition 2007; 35 (1): 39-49.

[75] de Gortari E. Introducción a la lógica dialéctica. México, DF: Fondo de Cultura Económica; 1956.

[76] Gross WL, Greene AJ. Analogical inference: the role of awareness in abstract learning. Memory 2007; 15 (8): 838-844.

[77] Dalphonse S. Dustin and me. The Washingtonian 1992, 27; (10): 50-55 \& 136-138.

[78] Treffert DA, Christensen DD. Inside the mind of a savant. Scientific American 2005; 293 (6): 108-113.

[79] Foer J. Remember this. National Geographic 2007; (11): 32-57.

[80] Coto A. Entrenamiento mental. Madrid, ES: Editorial Edaf; 2006.

[81] Sacks O. The Twins. In: The man who mistook his wife for a hat and other clinical tales. New York, NY: Harper Perennial; 1990. p195-213.

[82] Marcus G. Total recall: the woman who can't forget. Wired 2009; (17): e04.

[83] Lorayne H. How to develop a super-power memory. New York, NY: Frederick Fell; 1957.

[84] Henner M. Total Memory Makeover: Uncover Your Past, Take Charge of Your Future. New York, NY: Gallery Books; 2012.

[85] Ally BA, Hussey EP, Donahue MJ. A case of hyperthymesia: rethinking the role of the amygdala in autobiographical memory. Neurocase 2012; doi: 10.1080/13554794. 2011.65422 .

[86] Ericsson KA, Delaney PF, Weaver G, Mahadevan R. Uncovering the structure of a memorist's superior "basic" memory capacity. Cognitive psychology 2004; 49 (3): 191-237.

[87] Takahashi M, Shimizu H, Saito S, Tomoyori H. One percent ability and ninety-nine percent perspiration: a study of a Japanese memorist. Journal of experimental psychology. Learning, memory, and cognition 2006; 32 (5): 1195-1200. 
[88] Hu Y, Ericsson KA, Yang D, Lu C. Superior self-paced memorization of digits in spite of a normal digit span: the structure of a memorist's skill. Journal of experimental psychology. Learning, memory, and cognition 2009; 35 (6): 1426-1442.

[89] Neumann N, Dubischar-Krivec AM, Braun C, Löw A, Poustka F, Bölte S, Birbaumer $\mathrm{N}$. The mind of the mnemonists: an MEG and neuropsychological study of autistic memory savants. Behavioural brain research 2010; 215 (1): 114-121.

[90] Maguire EA, Valentine ER, Wilding JM, Kapur N. Routes to remembering: the brains behind superior memory. Nature neuroscience 2003; 6 (1): 90-5.

[91] Goldberg TE. On hermetic reading abilities. Journal of autism and developmental disorders 1987; 17 (1): 29-44.

[92] Buzan T. Use your perfect memory. Ney York, NY: E.P. Dutton, Inc.; 1984.

[93] Yaro C, Ward J. Searching for Shereshevskii: what is superior about the memory of synaesthetes? The quarterly journal of experimental psychology : QJEP. 2007; 60 (5): 681-695.

[94] Ericsson KA. Exceptional memorizers: made, not born. TRENDS in Cognitive Sciences 2003; 7 (6): 233-235.

[95] Bekinschtein TA, Cardozo J, Manes FF. Strategies of Buenos Aires waiters to enhance memory capacity in a real-life setting. Behavioural neurology 2008; 20 (3): 65-70. 



\section{Section 3}

Aetiological Factors - Genetics 



\title{
Genetic Evaluation of Individuals with Autism Spectrum Disorders
}

\author{
Eric C. Larsen, Catherine Croft Swanwick and \\ Sharmila Banerjee-Basu \\ Additional information is available at the end of the chapter \\ http://dx.doi.org/10.5772/53900
}

\section{Introduction}

While the genetic component of Autism Spectrum Disorders (ASD) has been clearly established from various lines of study, the multitude of genes and chromosomal loci associated with ASD has made identification of the underlying molecular mechanisms of pathogenesis difficult to resolve. A range of diverse methodologies and study types have identified both rare and common genetic variants in ASD candidate genes and chromosomal loci. Moreover, the recent development of high-throughput next generation sequencing (NGS) technologies and the increasing usage of chromosomal microarray analysis (CMA) has led to a significant expansion in the number of single nucleotide variants (SNVs) and copy number variants (CNVs) potentially affecting one or more genes that have been identified in ASD individuals. This, in turn, has given critical insight into the molecular and cellular processes that may be preferentially targeted for disruption by genetic lesions in ASD patients.

However, it is important to note that there is no genetic test available for the diagnosis of ASD. Rather, genetic testing is primarily aimed at identifying genetic variants potentially responsible for disease pathogenesis in a given individual diagnosed with ASD. Furthermore, the utility of NGS and CMA in genetic evaluation of ASD individuals is dependent on proper interpretation and reporting of test results. In this chapter we will discuss 1) genetic testing technologies currently available for the identification of genetic variation in ASD cases, 2) the genes and genomic loci targeted by single nucleotide and copy number variants that have been linked to ASD susceptibility, 3) the bioinformatics tools that enable researchers to process the enormous amount of genetic data associated with ASD, and 4) challenges that exist in the interpretation and reporting of genetic evaluation results in ASD cases. 


\section{Genetic screening technologies for the evaluation of ASD cases}

Autism spectrum disorders (ASD) are among the most highly heritable neurodevelopmental disorders, and extensive research has been focused on identifying the underlying genetic basis of these disorders. It has become apparent that ASD is a genetically heterogeneous disorder, with hundreds of genes and chromosomal rearrangements identified that confer varying degrees of risk for disease. Initially, susceptibility genes and genomic loci were identified by costly, low-throughput techniques, such as automated Sanger sequencing and conventional cytogenetic techniques. The need for lower-cost, higher-throughput genetic screening technologies capable of identifying genome-wide variation in individuals with genetically complex diseases, such as ASD, has driven improvements in pre-existing techniques and the development of new technologies. The genetic screening technologies presently available to clinical geneticists and researchers are capable of providing lowercost, high-throughput genetic data that have significantly expanded our knowledge of genetic variation, both in the general population and in ASD individuals in particular. The first major high-throughput studies aimed at identifying CNVs and SNVs in ASD cohorts were published in 2010 and 2011, respectively [1,2]. Here we describe in greater detail two of these genetic screening technologies that have become widely used in the genetic evaluation of ASD cases: next generation sequencing (NGS) and chromosomal microarray (CMA).

\subsection{Next generation sequencing}

Next-generation sequencing (NGS) is a term used to describe a collection of high-throughput sequencing technologies that have enabled clinicans to screen larger amounts of genetic material at lower cost than traditional sequencing technologies, such as automated Sanger sequencing [3]. NGS is typically used to identify single nucleotide variants (SNVs), as well as small insertions or deletions in candidate genes. However, NGS can also be used to identify copy number variants (CNVs), as was recently demonstrated in a report detailing whole exome sequencing in a cohort of ASD cases [4], as well as balanced chromosomal rearrangments, which are typically not detected by genome-wide microarrays [5, 6]. Since 2011, six research articles have been published that have identified rare variants in both existing and novel ASD susceptibility genes using NGS techniques [2, 4, 7-10], a fact that illustrates how extensively these techniques have been adopted by the ASD research community. As a result of these studies, the potential number of potential ASD-linked genes have increased dramatically (Table 1). Furthermore, as demonstrated by the minimal overlap of candidate genes across these studies, the results of these studies further illustrate the genetic heterogeneity of ASD.

NGS techniques are typically divided into three categories, each with its own advantages and disadvantages (summarized in Table 2). These techniques vary in terms of genetic coverage (the size of the sequenced target, which can range in size from one or a few genes to the entire genome) and genetic resolution (sensitivity in detection of variants per sequencing target). In general, the smaller the genetic coverage, the higher the genetic resolution. It should be noted that, as the size of the target for sequencing increases, so does the number 
of both false-positive and false-negative variants [11]; this is one of the many considerations that must be taken into account in deciding which NGS technique to utilize.

\begin{tabular}{cccc}
\hline Exome Report & Total \# of genes & \# of unique genes & \# of overlapping genes \\
\hline O'Roak 2011 & 21 & 21 & 0 \\
\hline Sanders 2012 & 170 & 166 & 13 \\
\hline O'Roak 2012 & 240 & 227 & 5 \\
\hline Neale 2012 & 173 & 168 & 0 \\
\hline Chahrour 2012 & 53 & 53 & 25 \\
\hline Iossifov 2012 & 363 & 338 & 47 \\
\hline Total & 1020 & 973 & \\
\hline
\end{tabular}

Table 1. Six recent scientific articles describing rare genetic variants identified in ASD cases by next generation sequencing (NGS) has led to a dramatic increase in the number of potential ASD candidate genes and further illustrates the genetic heterogeneity of ASD. Overlapping genes are genes in which a rare variant was identified in more than one exome report and are used as a measure of genetic heterogeneity.

\begin{tabular}{|c|c|c|c|c|}
\hline & Availability & Cost & Advantages & Disadvantages \\
\hline $\begin{array}{l}\text { Targeted gene } \\
\text { panels }\end{array}$ & $\begin{array}{l}\text { Commercially } \\
\text { available }\end{array}$ & $\sim \$ 5000$ & $\begin{array}{l}\text {-Highest resolution of NGS } \\
\text { approaches } \\
\text {-Contains a number of well- } \\
\text { characterized ASD } \\
\text { susceptibility genes } \\
\text { (syndromic and non- } \\
\text { syndromic) }\end{array}$ & $\begin{array}{l}\text {-Unable to detect variants } \\
\text { in genes not included in the } \\
\text { panel }\end{array}$ \\
\hline $\begin{array}{l}\text { Whole exome } \\
\text { sequencing }\end{array}$ & $\begin{array}{l}\text { Available only in } \\
\text { research settings }\end{array}$ & $\sim \$ 1000$ & $\begin{array}{l}\text {-Estimated to detect the } \\
\text { majority of disease- } \\
\text { covering variants }\end{array}$ & $\begin{array}{l}\text {-Unable to detect variants } \\
\text { in non-coding regions }\end{array}$ \\
\hline $\begin{array}{l}\text { Whole } \\
\text { genome } \\
\text { sequencing }\end{array}$ & $\begin{array}{l}\text { Available only in } \\
\text { research settings }\end{array}$ & $\sim \$ 4000-\$ 5000$ & $\begin{array}{l}\text {-Greatest coverage of the } \\
\text { genome (both coding and } \\
\text { non-coding regions) }\end{array}$ & $\begin{array}{l}\text {-Lowest resolution of NGS } \\
\text { techniques }\end{array}$ \\
\hline
\end{tabular}




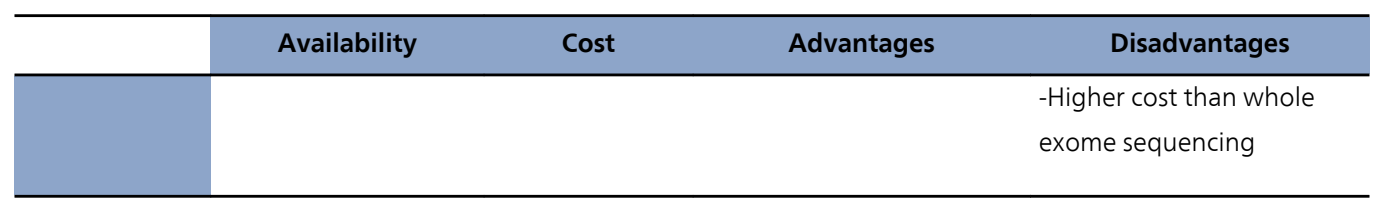

Table 2. A summary of the benefits and drawbacks of the three types of next generation sequencing (NGS) techniques in the genetic evaluation of ASD cases. Cost estimates of whole-exome and whole-genome sequencing in [14].

\subsubsection{Targeted gene panels}

Targeted gene panels generally test for 50-100 genes that have been demonstrated to be strongly associated with a particular disease. Such gene panels are already extensively used to screen individuals for a wide range of cancers and inherited diseases for which causative genes have been identified. A number of commercially-available ASD gene panels have recently been designed to target both genes strongly associated with non-syndromic ASD as well as syndromic genes (genes that cause syndromes in which a subset of affected individuals also develop ASD, such as FMR1, MECP2, and $C A C N A 1 C$, which cause Fragile $\mathrm{X}$, Rett, and Timothy syndromes, respectively). For example, the Greenwood Genetic Center offers a 62-gene syndromic gene panel that covers the coding region and flanking intronic boundaries of ASD-linked 62 genes for $\$ 5500$ (http://www.ggc.org/images/pdfs/syndromicautism62-genengspanel.pdf). While targeted gene panels offer the smallest coverage of the human genome of the three NGS approaches, they offer the highest resolution. One of the major drawbacks to the use of targeted gene panels for a genetically heterogeneous disorder such as ASD is the inability to detect mutations in genes outside of those included in the gene panel.

\subsubsection{Whole exome sequencing}

Whole exome sequencing, which is also known as targeted exome capture, is designed to specifically identify variants in protein-coding regions of the human genome. Although these protein-coding regions, called exons, constitute a very small percentage of the human genome, it is estimated that they contain up to $85 \%$ of disease-causing mutations [12, 13]. However, whole exome sequencing will fail to detect any potentially pathogenic variants in non-coding regions of the human genome. This NGS method also provides lower resolution than targeted gene panels. Nonetheless, whole exome sequencing is increasingly being used to identify potentially pathogenic rare single gene variants in individuals with ASD [2, 4, 7-10].

\subsubsection{Whole genome sequencing}

In contrast to whole exome sequencing, which only covers protein-coding regions of the human genome, whole genome sequencing provide coverage of the entire genome, allowing for the sequencing of both coding and non-coding genomic regions. As such, single nucleotide changes and small insertions/deletions within non-coding regions of the genome can be detected by this method. While whole genome sequencing covers the largest amount of the human genome of all NGS techniques, it offers the lowest resolution of the three NGS technologies. Whole genome sequencing is also more costly than whole exome sequencing, 
although the differences in cost betwen these two techniques have fallen from 10- to 20-fold [13] to 4- to 5-fold [14].

\subsection{Chromosomal microarray}

Microscopically-visible chromosomal rearrangments have long been implicated in the onset and pathogenesis of neurodevelopmental disorders, includng ASD. Indeed, many of the most strongly ASD-linked chromosomal deletions and duplications, collectively referred to as copy number variants $(\mathrm{CNVs})$, were discovered through the use of conventional cytogenetic techniques such as G-banded karyotyping, fluorescent in situ hybridization (FISH), and microsatellite analysis. For example, duplications of chromosome 15q11-q13 were first implicated in ASD in the mid-1990s by these methods [15-17]. Likewise, these methods identified chromosomal rearrangments on the long arm of chromosome 22 in ASD cases [18, 19]. However, conventional cytogenetic techniques are impractical in the identification of copy number variation throughout the human genome in large case cohorts. While G-banded karyotyping is capable of detecting large chromosomal deletions and duplications $(\sim 1 \mathrm{Mb}$ and larger), it lacks the sensitivity to detect smaller CNVs. Alternatively, the use of techniques such as FISH is generally limited to screen a particular chromosomal region, so while they are useful for examining copy number variation in a genomic loci of interest in larger case populations, they are impractical for the purposes of identifying deletions and duplications throughout the genome.

In the last decade, technological and computational advances have allowed clinical geneticists and researchers to detect submicroscopic chromosomal deletions and duplications throughout the human genome in large case cohorts that would not be detected by traditional cytogenetic techniques. Chromosomal microarray (CMA) is a term frequently used to include all types of array-based whole genome copy number analyses, with the two most widely used being arraycomparative genomic hybridization (aCGH) and single nucleotide polymorphism (SNP) arrays. CMA has been demonstrated to provide a higher diagnostic yield than G-banded karyotyping (15-20\% compared to $\sim 3 \%$ ) due to its ability to detect submicroscopic deletions and duplications, and it has been proposed that CMA should replace conventional cytogenetic techniques as a first-tier diagnostic tool for individuals with congential abnormalities and developmental disorders, including ASD [20]. High-throughput genome-wide aCGH and SNP arrays are now regularly used in the detection of CNVs in large ASD cohorts [1,21-24].

aCGH and SNP arrays employ similar methodologies in the detection of CNVs (Figure 1). The first step involves labeling the DNA of the ASD patient with a fluorophore, thereby creating a test sample. The test sample is then mixed with an equal amount of DNA from a normal reference sample that has been labeled with a different fluorophore. This mixed DNA sample is added to a glass slide containing thousands of oligonucleotide probes corresponding to different chromosomal regions that cover the human genome; in the case of SNP arrays, the oligonucleotide probes are specific for common polymorphisms found in the general population. The sensitivity of CMA has been greatly increased in recent years by the development of arrays employing a larger number of smaller oligonulceotide probes; in doing so, clinical geneticists and researchers are able to detect even smaller copy number changes than before 
without compromising genomic coverage. The test and reference DNA samples hybridize with the probes on the slide, and the fluorescence intensities of the test and reference DNA can then be measured. Following analysis with software that is typically specific for the platform being used, one or more algorithms are used to call the $\mathrm{CNV}$. The ratio between the two fluorescence intensities is used to identify copy number changes. For example, if the test-to-reference ratio is 1 (yellow in the example below), then there is no change in copy number at the chromosomal region corresponding to a given probe, If the test-to-reference fluorescence ratio is $>1$ for a particular probe (green in the example below), then the ASD patient carries a duplication in the chromosomal region corresponding to that probe. If the test-to-reference ratio is $<1$ (red in the example below), then the patient carries a deletion at that site of the genome.

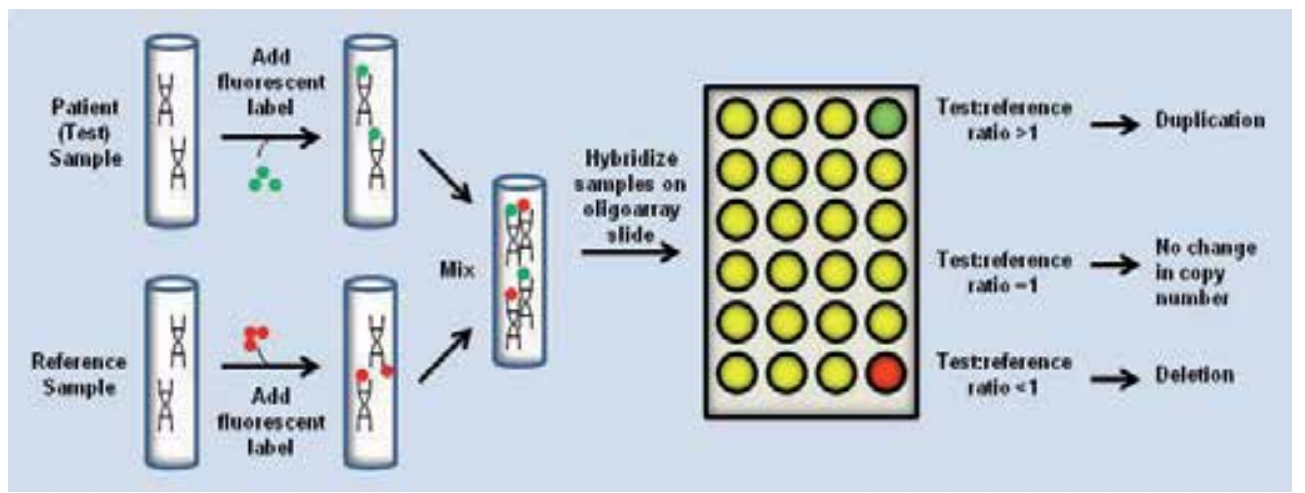

Figure 1. Chromosomal microarray (CMA) analysis involves hybridization of differently labeled test and reference DNA samples with oligonucleotide probes, followed by computerized analysis and identification of copy number variants (CNVs) based on changes in fluorescence intensity ratio.

Despite the recommended use of CMA as a first-tier genetic evaluation tool in place of conventional cytogenetic techniques, it should be noted that aCGH is unable to detect balanced chromosomal rearrangments and other chromosomal abnormalities that have traditionally been detected by karyotype analysis [25]. In addition to their traditional utilization in the detection of risk-conferring common polymorphisms, SNP arrays have the added advantage of being able to detect copy number neutral genetic variation such as uniparental disomy and long contiguous streteches of homozygosity (LCSH) that cannot be detected by aCGH $[25,26]$.

\section{Genetic variation in ASD}

With the advent of NGS techniques and increasing usage of CMA screening, the number of SNVs and CNVs that have been identified in ASD individuals has grown significantly. Based on a survey of recently published exome sequencing studies of ASD cohorts, it was estimated that the number of dosage-sensitive ASD susceptibility genes is approximately 370, with roughly a third of these genes having been identified [10]. However, even this number might be a conservative projection. As shown in Figure 2, the number of ASD susceptibility genes in 
the Human Gene Module of the autism genetic database AutDB [27] has increased from 284 genes in September 2011 to 369 genes in June 2012. A large number of newer susceptibility genes have been annotated from reports employing whole exome sequencing of ASD cases [2, $4,7-10]$, illustrating the increasing usage of NGS techniques in the study of genetic variation in ASD. In addition to the identification of novel ASD susceptibility genes, NGS techniques have identified novel rare variants in previously identified ASD susceptibility genes. The number of ASD-associated CNV loci has also increased significantly, with the CNV module of AutDB expanding from 1034 CNV loci in September 2011 to 1173 loci in June 2012 (Figure 2). In this section we describe the genetic categories into which ASD susceptibility genes have been classified, as well as describe recent studies that have yielded invaluable insight on the functional profiles of ASD-associated genes and CNV loci.

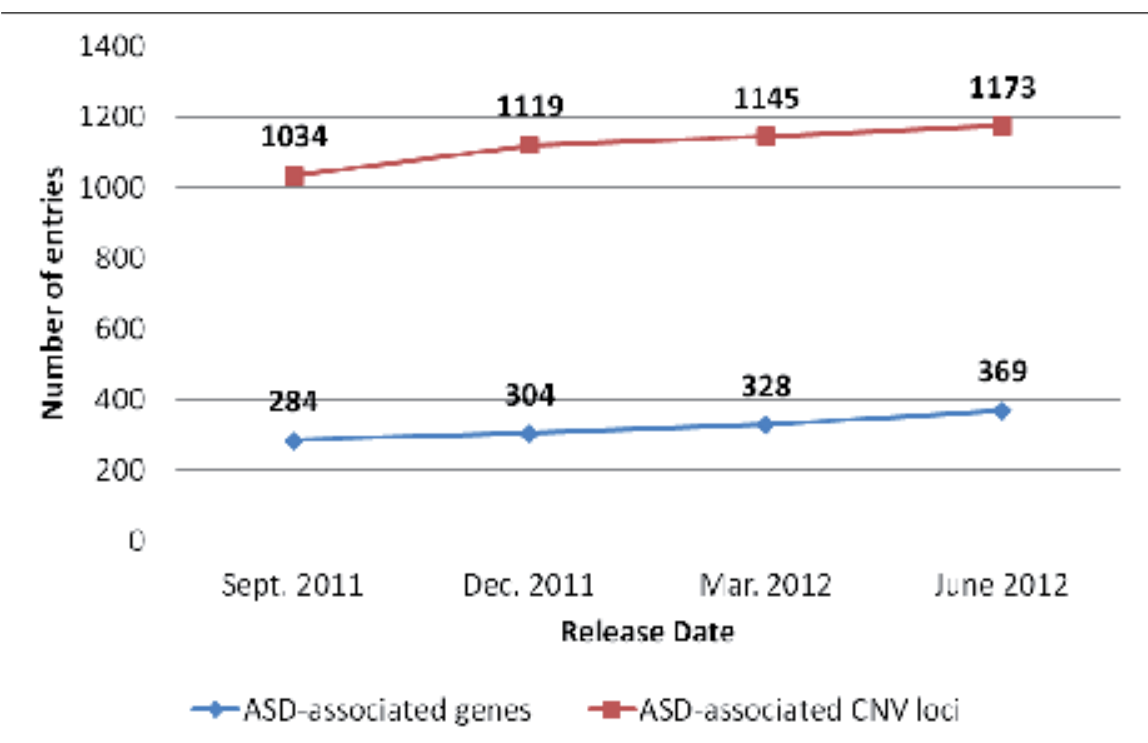

Figure 2. The number of genes and CNV loci associated with ASD in the genetic database AutDB has increased over the last four quarterly release dates.

\subsection{Genetic categories of ASD susceptibility genes}

The earliest ASD susceptibility genes were rare single gene variants in genes associated with syndromes such as Fragile X syndrome and Rett syndrome. The discovery of single gene mutations/disruptions in two neuroligin genes, NLGN3 and NLGN4, in ASD siblings [28] initiated the search for additional ASD susceptibility genes in non-syndromic ASD cases. The continued identification of rare genetic variants associated with both syndromic and nonsyndromic ASD, as well as of risk-conferring polymorphisms enriched in ASD populations 
compared to unaffected controls in genetic association studies, has led to significant increases in the number of ASD-linked genes. While the majority of ASD-associated genes have been linked to disease on the basis of genetic studies in human populations, a number of additional ASD-linked genes have been identified by alternate methodologies, such as gene expression studies in post-mortem brain tissue of ASD individuals.

ASD susceptibilty genes in the Human Gene Module of AutDB are defined into four distinct categories:

1. Rare. This category features genes implicated in rare monogenic forms of non-syndromic ASD. Rare allelic variants within this category include single nucleotide variants, small insertions and deletions, chromosomal rearrangements such as translocations and inversions, and monogenic submicroscopic deletions and duplications. Among the genes within this category are CACNA1H and SHANK1.

2. Syndromic. Syndromic genes were among the first genes for which rare genetic variants linked to autism were identifed. In addition to well-characterized syndromic genes such as FMR1 (Fragile X syndrome), MECP2 (Rett syndrome), and CACNA1C (Timothy syndrome), genes such as CHD7 and SLC9A6 fall into the syndromic category.

3. Association. This category includes genes in which small risk-conferring common polymorphisms have been identified from genetic association studies in idiopathic ASD populations. Among the genes within this category are MET and MTHFR.

4. Functional. This category includes functional candidate genes that have not yet been experimentally linked to ASD by genetic studies. Among the genes in this category are BCL2 and $P D E 4 B$, whose inclusion is based on changes in gene expression in post-mortem brain tissue of ASD subjects.

As shown in Figure 3, while the number of both rare and common ASD-associated variants in the Human Gene module of AutDB has increased over the last four quarterly release dates, the number of rare variants has increased at a much greater rate than the number of common variants. The number of rare variants increased from 1141 in September 2011 to 1675 in June 2012 , an increase of $\sim 146 \%$. In contrast, the number of common variants rose form 508 to 575 over the same span of time, an increase of only $113 \%$. This disparity between the addition of rare and common variants to AutDB is in part due to the increased usage of NGS and CMA and subsequent identification of rare ASD-associated variants in large ASD cohorts.

It should be noted that a given gene can fall under multiple genetic categories, depending on the affected population under investigation and the type of study. For example, both rare variants and risk-conferring common polymorphisms have been identified in the CNTNAP2 gene in ASD individuals across multiple studies [2, 29-31] However, in addition to its role as an ASD susceptibility factor, recent studies suggest that rare variants in CNTNAP2 are responsible for two additional syndromes: cortical dysplasia-focal epilepsy syndrome [32] and Pitt-Hopkins-like syndrome 1 [33]. Therefore, based on the combined evidence from all of these aforementioned studies, CNTNAP2 is classified in AutDB as a syndromic gene, a rare gene, and an association gene. 
The classification of ASD-linked genes into genetic categories is a useful tool in assessing the strength of the evidence for the connection of a given gene with ASD. Genes within the rare and syndromic categories are generally considered to have the strongest link to ASD [34]. Due to the frequent lack of replication in their association with ASD from one study to the next, genes within the association category are considered to have a weaker link to ASD than genes within the rare and syndromic categories. Genes within the functional category have no direct documented connection to ASD and are therefore considered to be among the weakest ASD candidate genes.

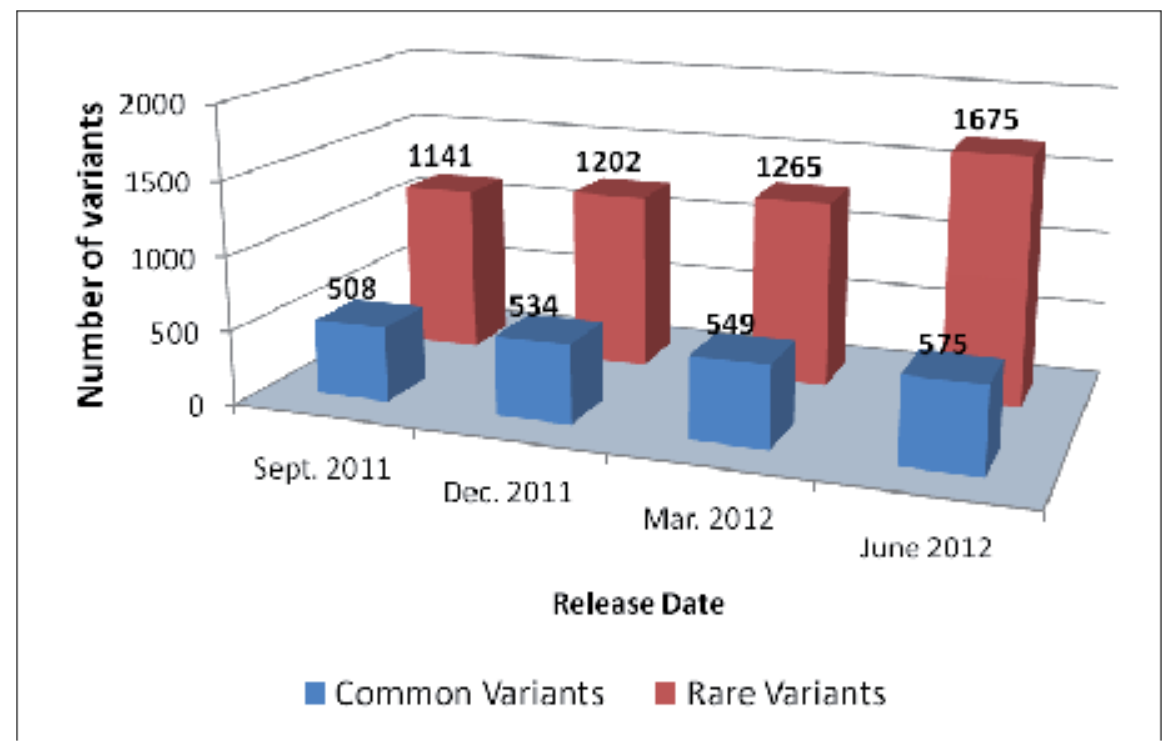

Figure 3. The number of rare and common ASD-associated variants in the Human Gene module of AutDB has increased over the last four quarterly release dates.

\subsection{Functional profiles of ASD-associated genes and CNV loci}

The increasing number of ASD-associated genetic factors, as shown in Figure 2, has only added to the well-established genetic heterogeneity of ASD. In spite of the complexity caused by this genetic heterogeneity, bioinformatic analysis of ASD-linked genes and CNV loci has yielded valuable insight into the molecular interactions and cellular pathways preferentially targeted by genetic lesions in individuals with ASD. Not only can this information be potentially used to design therapeutic approaches targeting disrupted pathways, but it can also aid in assessing the clinical importance of newly-discovered ASD candidate genes and CNV loci in which pathogenic variants are identified by NGS and CMA, respectively. For example, a gene whose encoded gene product resides within a known ASD-associated cellular process or interacts with a known ASD-associated gene is a stronger candidate than a gene that fails to reside within known ASD-associated cellular processes or interact with known ASD-associated genes. 
Recent large-scale ASD genetic studies have used a systems biology approach to translate genetic information into functional profiles that shed light on how genetic variation in ASD may lead to disease onset and pathogenesis. Rare CNVs identified in large ASD cohorts have been shown to be enriched for genes involved in cellular processes of relevance for ASD, including cellular proliferation, projection, and motility, and GTPase/Ras signaling [1], neuronal cell adhesion and ubiquitin-mediated degradation [22], glycobiology [35], axon growth and pathfinding [36], and synapse development, axon targeting, and neuron motility [37]. Gene datasets from genome-wide association studies in ASD populations were demonstrated to be enriched for Gene Ontology (GO) classifications for cellular processes including pyruvate metabolism, transcription factor activation, cell signaling and cell-cycle regulation [38]. A recent report describing gene pathway analysis using single nucleotide polymorphism (SNP) data from the Autism Genetics Research Exchange (AGRE) identified cellular pathways such as calcium signaling, long-term depression and potentiation, and phosphotidylinositol signaling that reached statistical significance in both Central European and Han Chinese populations [39]. More recently, whole exome sequencing studies in large ASD cohorts have demonstrated that proteins encoded by genes in which potentially disruptive de novo mutations were identified showed a higher degree of connectivity among themselves and to previously identified ASD genes based on protein-protein interaction network analysis [4, 8]. Another exome sequencing study in ASD individuals found that many of the genes in which potentially disruptive variants were identified associated with the Fragile X Mental Retardation Protein (FMRP), the encoded product of the syndromic ASD gene FMR1 [10]. Taken together, these functional maps suggest that specific cellular pathways and processes are preferentially targeted by genetic variation in ASD cases, and that association with the encoded products of well-characterized ASD-linked genes offers evidence for pathogenic relevance.

Knowledge of ASD-associated genes can also be used to identify novel ASD candidate genes. Following the construction of functional and expression profiles from a reference set of 84 rare and syndromic ASD-linked genes, we generated a predictive map of novel ASD candidate genes [40]. In total, 460 potential candidate genes were identified that overlapped both the functional profile and the brain expression profile of the initial reference set. The power of this predictive gene map was demonstrated by the capture of 18 pre-existing ASD-associated genes that were not included in the reference gene dataset, with the remaining 442 genes serving as novel ASD candidate genes. Since the publication of our predictive gene map, 12 of the novel ASD candidate genes identified in [40] have been added to AutDB, demonstrating the continued power of this analysis (manuscript in preparation).

\section{Bioinformatics of ASD}

With the rapid growth of genetic data obtained from ASD individuals, there has become a critical need for databases specializing in the storage and assessment of this data. Here we highlight several of the ASD-related genetics databases that are available to researchers. 


\subsection{AutDB}

Our autism database AutDB (http://autism.mindspec.org/autdb/Welcome.do) is a webbased, searchable database of ASD candidate genes identified in genetic association studies, genes linked to syndromic autism, and rare single gene mutations [27]. Evidence regarding ASD candidate genes is systematically extracted from peer-reviewed, primary scientific literature and manually curated by our researchers for inclusion in AutDB. To provide high-resolution view of various components linked to ASD, we developed detailed annotation rules based on the biology of each data type and generated controlled vocabulary for data representation. AutDB is widely used by individual laboratories in the ASD research community, as well as by consortiums such as the Simons Foundation, which licenses it as SFARI Gene.

AutDB is designed with a systems biology approach, integrating genetic information within the original Human Gene module to corresponding data in subsequent Animal Model, Protein Interaction (PIN) and Copy Number Variant (CNV) modules. The Animal Model module contains a comprehensive collection of mouse models linked to ASD [41]. While the Animal Model module initially contained only genetic mouse models of ASD, it has since been expanded to include induced mouse models of ASD in which a chemical or biological agent linked to ASD has been administered. As core behavioral features of ASD such as social interactions and communications can only be approximated in animal models, the annotation strategy for this module includes four broad areas: 1) core behavioral features of ASD, 2) ASD-related traits such as seizures and circadian rhythms that are heritable and more easily quantified in animal models; 3) neuroanatomical features, and 4) molecular profiles. To this end, we developed PhenoBase, a classification table for systematically annotating models with controlled vocabulary containing 16 major categories and $>100$ standardized phenotype terms. The PIN module of AutDB serves as a repository for all known protein interactions of ASD candidate genes, documenting six major types of direct interactions: 1) protein binding, 2) promoter binding, 3), RNA binding, 4) protein modification, 5) direct regulation, and 6) autoregulation. Its content is envisioned to have immediate application for network biology analysis of molecular pathways involved in ASD pathogenesis. For the purposes of genetic evaluation of individuals with ASD, knowledge of the protein interactions of ASD-associated genes can potentially aid in the clinical assessment of novel ASD candidate genes based on their interactions, or lack thereof, with known ASD-linked genes.

\subsection{Gene scoring module of SFARI gene}

As previously mentioned, AutDB is licensed to the Simons Foundation as SFARI Gene. However, unlike AutDB, SFARI Gene includes a unique feature initiated by the Simons Foundation called the Gene Scoring module (https://gene.sfari.org/autdb/GS_Home.do). The Gene Scoring module is a web-based platform detailing the rank of ASD-associated genes in the SFARI Gene Human Gene module [42]. With the increase in the number of genes linked to ASD, a Gene Scoring initiative was launched to assess the ASD candidate genes based on a set of standardized annotation rules. Following evaluation by an expert panel of advisors, the 
gene assessment results are then integrated in the form of Gene Score Cards to display the scores and the evidence in a graphical user interface for the ASD-linked gene. Recently, a community-wide annotation functionality was incorporated into the Gene Scoring module, allowing users to download the Gene Scoring dataset, score genes of their choice, and submit their scores to SFARI for possible inclusion.

\subsection{DECIPHER}

DECIPHER (Database of Chromosomal Imbalance and Phenotype in Humans Using Ensembl Resources) (http://decipher.sanger.ac.uk/) is an interactive web-based database that incorporates a suite of tools designed to aid in the interpretation of submicroscopic chromosomal deletions and duplications [43]. Genetic and phenotypic information is publically available not only for individuals diagnosed with idiopathic ASD, but also for individuals diagnosed with a recognized microdeletion or microduplication syndrome in which a subset of affected individuals also develop ASD.

\subsection{AutismKB}

AutismKB (http://autismkb.cbi.pku.edu.cn/) is a web-based, searchable database hosted by the Center for Bioinformatics, Peking University [44]. AutismKB is an evidence-based knowledge resource for ASD genetics containing information on genes, copy number variants, and linkage regions associated with ASD. Analysis of the gene content in AutismKB is available for users in the form of GO term enrichment analysis using the DAVID functional annotation tool and pathway enrichment analysis. Much like the Gene Scoring Module of SFARI Gene (see section 4.2), the genes within AutismKB are scored.

\subsection{Autism Chromosome Rearrangement Database}

The Autism Chromosome Rearrangement Database (http://projects.tcag.ca/autism/) is a webbased, searchable genetic database of chromosomal structural variation in ASD that is hosted by The Centre for Applied Genomics at the Hospital for Sick Children in Toronto, Canada [21]. The content of this database, which is derived both from published research articles and inhouse experimental results, includes cytogenetic and microarray data from individuals with ASD.

\subsection{Autism Genetic Database}

The Autism Genetic Database (http://wren.bcf.ku.edu/) is a web-based, searchable genetic database developed by researchers at the University of Kansas [45]. In addition to ASDassociated genes and $\mathrm{CNVs}$, this database also includes information on known non-coding RNAs and chemically-induced fragile sites in the human genome.

Recent lines of evidence have placed non-coding RNAs under increased scrutiny with regards to their potential pathogenic role in ASD. A number of small nucleolar RNAs (snoRNAs) reside within the ASD-associated 15q11-q13 region. A mouse model engineered to mimic duplication of the $15 q 11$-q13 region observed in $~ 1 \%$ of ASD cases exhibited overexpression of the snoRNA 
MBII52 (the mouse ortholog of the human snoRNA HBII52), which could potentially alter serotonergic signaling and contribute in part to the ASD-associated traits exhibited by these mice [46]. More recently, it was discovered that a non-coding RNA is transcribed from a genepoor region of chromosome $5 \mathrm{p} 14.1$ identified in genome-wide association studies of ASD cohorts [47]. Expression of the non-coding RNA, designated MSNP1AS, was shown to be higher both in individuals carrying the ASD-associated $\mathrm{T}$ allele and in post-mortem brain tissue of individuals with ASD.

Spontaneous breakage during DNA replication at rare chromosomal fragile sites may also play a role in the pathogenesis of neuropsychiatric disorders such as ASD. The chromosomal fragile site FRAXA has been implicated in fragile $X$ syndrome, and other fragile sites have been identified that associate with ASD, such as FRA2B, FRA6A, and FRA13A [48].

\section{Challenges of genetic evaluation in ASD}

NGS and CMA have expanded the ability of clinical geneticists and researchers to identify potential genetic causes of ASD. However, there are many challenges still present in the field of genetic evaluation. A recent report in the American Journal of Medical Genetics found that many children with ASD fail to get genetic evaluation, and that parents and medical professionals need to be better educated about the potential benefits of genetic evaluation [49]. Educating parents on genetic evaluation is especially critical in light of a recent survey of nine parents regarding their child's participation in genetic research in ASD [50], in which parents valued having had their child enrolled for a variety of reasons, including the potential use of genetic results in tailoring intervention and in family planning, the establishment of connections with experts in the field of ASD, and networking with other families, among others.

Even with the increased sensitivity of genetic evaluation techniques, an underlying genetic cause of ASD is still only identified in a minority $(<25 \%)$ of ASD cases [51]. One of the major challenges in the clinical interpretation of NGS and CMA lies in differentiating between pathogenic and benign genetic variants identified in ASD patients. The pathogenic relevance of the vast majority of ASD-linked genetic variants remains unknown; such variants are frequently classified as variants of unknown significance, or VOUS. While the identification of a genetic lesion in an existing ASD susceptibility gene or CNV locus is suggestive of a possible genetic cause of disease, variants in these genes and CNV loci have also been observed in seemingly unaffected individuals. Furthermore, it is important to note that, while technological advances have expanded the ability of clinical geneticists and researchers to identify these potential genetic causes of ASD, there is no genetic test available for the diagnosis of ASD. A recent report proposed a means of predicting a diagnosis of ASD based on the identification of candidate SNPs [39]. The accuracy of the predictive classifier was found to be $71.7 \%$ in individuals of Central European descent from validation datasets. However, the accuracy of the predictive classifier fell when tested in a Han Chinese cohort, a finding that stresses how genetic heterogeneity across populations complicates the use of such an ap- 
proach. In addition, the overall accuracy of the predictive classifier is likely too low to serve as an effective diagnostic tool.

A number of guidelines have already been proposed to aid clinicians and clinical geneticists in the interpretation and reporting of CNVs. With the increasing use of high resolution NGS technologies, similar guidelines will likely be proposed for the interpretation and reporting of single nucleotide variants (SNVs). Furthermore, tools and prioritization schema have also been developed to aid clinicians in the interpretation of genetic testing results. Here we discuss in greater detail the challenges in interpreting genetic screening results in ASD cases, the strategies that have been proposed for the interpretation and reporting of screening results, and the resources available to aid in that interpretation.

\subsection{Challenges in the interpretation of ASD genetic screening results}

\subsubsection{Technical limitations of NGS and CMA}

As previously mentioned, as the size of the sequenced target increases, so does the potential number of false-positive and false-negative variants identified [11]. Such sequencing artifacts are particularly problematic for the detection of spontaneous, or de novo variants, as false-positive variants would appear to be de novo in origin when they are observed in an offspring's genome but not in parental genomes. Furthermore, the source of DNA used in sequencing studies can introduce sequencing artifacts. DNA from lymphoblastoid cell lines from individuals to be genetically evaluated is a commonly used template for sequencing; however, the creation and culturing of these cell lines can introduce genetic changes that would appear as de novo variants when such cell lines are compared between parents and offspring. In order to remove or reduce the possibility of artifactual results, subsequent variant validation should be performed. In the case of single gene variants identified by NGS, a more targeted sequencing approach limited to the gene or region of interest would confirm the variant previously identified. In the case of CNVs identified by NGS or CMA, a targeted detection method such as quantitative real-time PCR or FISH is frequently used to confirm their discovery.

\subsubsection{Genetic heterogeneity}

While the genetic basis of many human diseases can be traced back to one or a few genes, the genetic basis of complex neuropsychiatric disorders such as ASD has proven to be far more complicated, with hundreds of genes and genomic loci associated with varying risks of disease. The recent utilization of NGS and CMA approaches in genetic evaluation of ASD cases has led to the detection of genetic variation not only in both existing and novel susceptibility genes and genomic loci. However, the strength of evidence for many of these novel candidate genes or genomic loci is minimal, and some degree of replication in follow-up studies will be required to fully assess the relevance of many of these newlyidentified variants. 


\subsubsection{Incompelete penetrance and variable expressivity}

One of the major challenges in identifying potential causative genetic variation in ASD cases lies in the fact that a potentially disruptive variant in a gene or genomic loci may not always associate or segregate with disease. For example, a potentially pathogenic variant in a gene may not only be present in an ASD individual, but it may also be present in seemingly unaffected family members. Similarly, the pathogenic variant may also be observed in seemingly unaffected individuals in the general population. This phenomenon, referred to as incomplete penetrance, complicates the interpretation of genetic evalaution.

Alternatively, a genetic variant may result in a range of disease severity in affected individuals, a phenomenon known as variable expressivity. For example, $\sim 500 \mathrm{~kb}$ deletions and duplications at the 16p11.2 locus are among the most heavily studied ASD-associated CNVs. However, $\mathrm{CNVs}$ at this locus are also responsible for a range of other neurodevelopmental and neuropsychiatric disorders, such as schizophrenia. CNVs at the 16p11.2 locus can also be inherited from seemingly unaffected family members and have been observed in unaffected individuals in the general population. This lack of correlation between genotype and phenotype as it relates to ASD-associated genetic variation may be in part due to differences in gene-environment interactions between individuals carrying such variation.

A recent report highlights some of the challenges inherent in the genetic evaluation of ASD individuals. A putative disruptive variant in the ASD-associated SHANK3 gene was identified in a boy with autism [52]. The variant, which was inherited from a healthy mother, was a small insertion that would be predicted to result in a frameshift and premature stop. Based on this evidence, as well as the relatively high penetrance of SHANK3 mutations in ASD and other neuropsychiatric diseases, one could conclude that this variant in the SHANK3 gene was pathogenically relevant in this autistic male. However, follow-up studies revealed that this variant was unlikely to be present in the majority of SHANK3 transcripts due to alternative splicing events. Furthermore, this variant was observed in 4 out of 382 control individuals without neuropsychiatric conditions, a rate $>1 \%$. This report not only illustrates the necessity of determining the frequency of a given potentially pathogenic variant in the general population but also warns against relying too heavily on computational, or in silico, predictions of the effects of that variant on gene function.

\subsection{Ethical considerations in the reporting of ASD genetic screening results}

Informed consent, and the extent to which participants have been sufficiently informed as to the purpose of a research or clinical study, has long been an issue in the field of genetic evaluation. For example, the extent to which research findings will be released and made available is one that participants in genetic evaluation studies should be informed of beforehand. In some cases, the participants themselves may not be able to gain access to the findings of genetic evaluation. This is an issue that has only been compounded with the rise of NGS and CMA and subsequent explosion in the amount of genetic data generated by these techniques. 
The sheer volume of genetic information generated by NGS and CMA not only leads to the identification of potential genetic causes of a disease of interest, but also frequently leads to the detection of other variants that are no directly related to the disease under investigation but are related to other inherited human diseases. The extent to which these incidental findings should be reported is a subject of some controversy, particularly in those situations in which genetic predisposition to an adult-onset disease is discovered in a child being evaluated for genetic causes of childhood developmental disorders. One such situation was described in a recent news feature in Nature in which the family of a child who had undergone genetic testing for developmental disability had to be informed that the child carried a genetic predisposition to colon cancer after extensive debate between clinical geneticists and ethics reviewers as to the extent to which such genetic information should be reported [53]. The degree to which clinical geneticists should report incidental findings in research participants has been considered by numerous authors [54-57], but as of yet there is not consensus. Many of these same ethical concerns must be considered in the reporting of genetic evaluation results in individuals with ASD.

Another consideration lies in the use of genetic evaluation to determine the recurrence risk in the siblings of children with ASD and in family planning [50]. Given a recent estimate that the recurrence rate of ASD in siblings may be as high as $~ 20 \%$ [58], the identification of inherited variants that potentially impart susceptibility to ASD is of critical importance both in identifying at-risk siblings that have not yet begun to manifest symptoms of ASD and in making informed decisions with regards to family planning.

\subsection{Strategies for ASD genetic screening interpretation and reporting}

The American College of Medicine Genetics released practice guidelines for the use of genetic screening techniques in the evaluation of individuals with ASD in 2008 [59]. In the years that have followed, additional practice guidleines and consensus statements discussing the use of CMA in the genetic evaluation of ASD cases have been published [25, 26]. With its increasing usage in the genetic evaluation of ASD cases, similar practice guidelines and consensus statements regarding NGS will likely be forthcoming, and strategies for the interpretation of NGS data in the evaluation of neurological diseases have recently been proposed [14]. In this section we highlight some of the factors to consider in the interpretation of genetic screening results in ASD cases.

\subsubsection{Variant inheritance and segregation with ASD}

One of the key determinants in the interpretation of ASD genetic screening results is the mechanism of variant inheritance and how closely that variant segregates with ASD. Genetic variation can either arise de novo or be transmitted from one or both parents. There has been considerable interest in the ASD research community in the pathogenic relevance of de novo variants, especially within the context of sporadic ASD cases.

As they have been subjected to less stringent evolutionary selection, de novo variants tend to be more deleterious than inherited variants, making them excellent candidates for sporadi- 
cally-occurring disease [11]. An increased rate of de novo CNVs in sporadic cases compared to familial cases has been reported [21,60], and rare de novo CNVs at specific genomic loci were found to associate with ASD in sporadic cases from the Simons Simplex Collection [24]. Exome sequencing studies using ASD cohorts have reported an increased rate of de novo genedisrupting events (i.e. nonsense, splice-site, and frameshift mutations) in affected children compared to their unaffected siblings [10].

Whereas ASD genetic research is increasingly focused on de novo genetic variation, it should be remembered that the genetic basis of ASD was first established by studies demonstrating the high heritability of the disease, a fact that illustrates the continued importance of identifying inherited genetic variation in ASD cases. A number of inherited single gene variants and CNVs that segregate with disease in ASD families has been recently described [9, 61-64]; these and other findings clearly demonstrate the importance of identifying inherited variants that closely segregate with disease in affected families. It should be noted that determining the extent of variant segregation in ASD families can be complicated by the phenotypic heterogeneity that a given variant can cause from one affected family member to another. Furthermore, a disease-causing variant may exclusively segregate with disease in males, even if the variant does not reside on the $\mathrm{X}$ chromosome, as is the case with a SHANK1 mutation identified in a four-generation ASD family [61]. Detailed family history and genetic evaluation of both affected and unaffected family members is essential in determining the signficance of both de novo and inherited variants in ASD cases.

\subsubsection{Functional impact of variant}

In addition to the mechanism of variant inheritance and variant segregation with disease, another important consideration in interpretation of genetic screening results lies in the functional impact of the variant. In many cases, especially with the use of high-throughput screening technologies, variant function is predicted in silico. In the case of single gene mutations, variation that results in disruption of gene function, such as nonsense mutations, splice-site mutations, or frameshift mutations that introduce premature stop codons, are strong genetic candidates, especially if such gene-disrupting variants are identified in a known ASD suscepibility gene or a gene associated with an ASD-linked pathway. The interpretation of missense mutations is more complicated and requires assessment of evolutionary conservation using phyloP or Genomic Evolutionary Rate Profiling (GERP) conservation scores, as well as scoring of the functional impact using Grantham or PolyPhen-2. However, as previously mentioned [52], dependency on in silico predictions for variant function, even in well characterized ASD-linked genes, can lead to false conclusions. As such, experimental functional assays are essential to accurately determine the impact of a given variant on gene expression or function of the encoded gene product.

\subsubsection{Clinical correlations of the variant with ASD}

Another consideration in the interpretation of genetic screening results in ASD cases is the degree of clinical correlation of a given variant with ASD. Hundreds of susceptibility genes and CNV loci linked with ASD have been identified and catalogued in online genetic databases 
such as AutDB, DECIPHER, and others. The identification of a novel, potentially pathogenic variant in one of these known susceptibility genes or $\mathrm{CNV}$ loci would be strong evidence for a causal role. To a lesser extent, a novel variant in a gene in an ASD-associated pathway or a gene previously shown by gene expression studies to be differentially regulated in ASD tissue would be a strong candidate. Another factor to consider is the frequency of a variant of interest in healthy control populations; the absence or significantly reduced frequency of the variant of interest in unaffected individuals would offer strong evidence for a causal role.

\subsection{Resources for ASD genetic screening interpretation}

A number of online resources are available to aid clinical geneticists in the interpretation of genetic screening results in ASD individuals. Many of these resources are aimed at differentiating between rare, potentially ASD-specific variants and benign variants observed in the general population. In this section we will describe some of these resources in greater detail.

\subsubsection{Genetic variation in control populations}

Differentiating between potentially pathogenic and benign genetic variants in ASD cases requires knowledge of the degree of genetic variation that resides within seemingly unaffected individuals in the general population. A number of online resources, several of which are hosted by the National Center for Biotechnology Information (NCBI) [65], have been developed to allow clinical geneticists to visualize genetic variation identified in the general population. The genetic variation curated in these databases can range from single nucleotide polymorphisms to chromosomal structural variation and has proven invaluable in assessing the potential pathogenic relevance of novel genetic variants.

\subsubsection{1. dbSNP (database of single nucleotide polymorphisms)}

dbSNP (http://www.ncbi.nlm.nih.gov/snp) is a public domain database hosted by NCBI collecting a range of polymorphic genetic variation, including single nucleotide polymorphisms (SNPs), small-scale multi-base deletions or insertions (also called deletion insertion polymorphisms or DIPs), and retroposable element insertions and microsatellite repeat variations (also called short tandem repeats or STRs) [65].

\subsubsection{1,000 Genomes Project}

The 1,000 Genomes Project (http://www.1000genomes.org/) is a consortium employing highthroughput NGS techniques for the purposes of characterizing over $95 \%$ of genetic variants located in genomic regions accessible to sequencing and occurring at an allelic freuqency of $1 \%$ or higher in each of five major population groups [66].

\subsubsection{3. dbVar (database of genomic structural variation)}

dbVar (http://www.ncbi.nlm.nih.gov/dbvar/) is a searchable online database hosted by NCBI containing genomic structual variation, defined by the database as inversions, balanced 
translocations, and CNVs approximately $1 \mathrm{~kb}$ or larger in size, that has been observed in both case and control populations [65].

\subsubsection{Database of Genomic Variants}

The Database of Genomic Variants (http://dgvbeta.tcag.ca/dgv/app/home?ref=NCBI36/hg18) is an curated online database hosted by the Centre for Applied Genomic that contains structural variation, defined by the developers of the database as genomic alterations that involve segments of DNA that are larger than 50bp, in control individuals [67]. Users can search the database for genetic variants such as $\mathrm{CNVs}$, insertions, inversions, and regions of uniparental disomy, as well as download database contents.

\subsubsection{Genotype-phenotype association}

The dbGaP public repository (http://www.ncbi.nlm.nih.gov/gap/) was created by the National Institutes of Health for the purposes of collecting individual-level genotype and phenotype data and associations between them [68]. The studies collected in dbGaP include genome-wide association studies, sequencing and diagnostic assays, and associations between genotype and non-clinical traits. Users can browse association results, utilize the Phenotype-Genotype Integrator (PheGenI) to search for phenotypic traits linked to GWAS data, and download data.

\section{Conclusion}

The development of lower-cost, high-throughput genome-wide genetic screening technologies has revolutionized the field of genetic evaluation and now provides clinical geneticists and researchers the opportunity to detect genetic variation in ASD individuals like never before. In doing so, the evidence for previously identified genetic susceptibility factors will expand, and novel ASD candidate genes and genomic loci will be identified, resulting in a better understanding of the genetic basis of ASD. However, precautions must be taken to ensure that genetic screening results are interpreted and reported properly.

\section{Acknowledgements}

The authors would like to thank the other members of MindSpec, Inc. (Ajay Kumar, M.S., Idan Menashe, Ph.D., Wayne Pereanu, Ph.D., Rainier Rodriguez, and Sue Spence), as well as the Simons Foundation. AutDB is licensed to the Simons Foundation as SFARI Gene.

\section{Author details}

Eric C. Larsen, Catherine Croft Swanwick and Sharmila Banerjee-Basu

MindSpec, Inc., U.S.A. 


\section{References}

[1] Pinto D, et al. Functional impact of global rare copy number variation in autism spectrum disorders. Nature 2010; 466(7304): 368-372.

[2] O'Roak BJ, et al. Exome sequencing in sporadic autism spectrum disorders identifies severe de novo mutations. Nature Genetics 2011; 43(6): 585-589.

[3] Metzker ML. Sequencing technologies-the next generation. Nature Reviews Genetics 2010; 11(1): 31-46.

[4] O'Roak BJ, et al. Sporadic autism exomes reveal a highly interconnected protein network of de novo mutations. Nature 2012; 485(7397): 246-250.

[5] Talkowski ME, et al. Next-generation sequencing strategies enable routine detection of balanced chromosome rearrangements for clinical diagnostics and genetic research. American Journal of Human Genetics 2011; 88(4): 469-481.

[6] Talkowski ME, et al. Sequencing chromosomal abnormalities reveals neurodevelopmental loci that confer risk across diagnostic boundaries. Cell 2012; 149(3): 525-537.

[7] Sanders SJ, et al. De novo mutations revealed by whole-exome sequencing are strongly associated with autism. Nature 2012; 485(7397): 237-241.

[8] Neale BM, et al. Patterns and rates of exonic de novo mutations in autism spectrum disorders. Nature 2012; 485(7397): 242-245.

[9] Chahrour $\mathrm{MH}$, et al. Whole-exome sequencing and homozygosity analysis implicate depolarization-regulated neuronal genes in autism. PLoS Genetics 2012; 8(4): e1002635. http://www.plosgenetics.org/article/info\%3Adoi\%2F10.1371\%2Fjournal.pgen.1002635

[10] Iossifov I, et al. De novo gene disruptions in children on the autistic spectrum. Neuron 2012; 74(2): 285-299.

[11] Veltman JA and Brunner HG. De novo mutation in human genetic disease. Nature Reviews Genetics 2012; 13(8): 565-575.

[12] Cooper DN, et al. The nature of mechanisms of human gene mutation. In: Scriver CR et al. (eds.) The Metabolic and Molecular Bases of Inherited Disease. McGraw-Hill, New York; 1995. 7th edition, p259-291.

[13] Choi $\mathrm{M}$, et al. Genetic diagnosis by whole exome capture and massively parallel DNA sequencing. Proceeding of the National Academy of Sciences U.S.A. 2009; 106(45): 19096-19101.

[14] Foo J-N, et al. Whole-genome and whole-exome sequencing in neurological diseases. Nature Reviews Neurology 2012; doi: 10.1038/nrneurol.2012.148. http:// www.nature.com/nrneurol/journal/vaop/ncurrent/full/nrneurol.2012.148.html 
[15] Baker P, et al. Brief report: duplication of chromosome 15q11-13 in two individuals with autistic disorder. Journal of Autism and Developmental Disorders 1994; 24(4): 529-535.

[16] Bundey S, et al. Duplication of the 15q11-13 region in a patient with autism, epilepsy and ataxia. Developmental Medicine \& Child Neurology 1994; 36(8): 736-742.

[17] Cook EH Jr., et al. Autism or atypical autism in maternally but not paternally derived proximal 15q duplication. American Journal of Human Genetics 1997; 60(4): 928-934.

[18] Carratalá F, et al. A patient with autistic disorder and a 20/22 chromosomal translocation. Developmental Medicine \& Child Neurology 1998; 40(7): 492-495.

[19] Goizet C, et al. Case with autistic syndrome and chromosome 22q13.3 deletion detected by FISH. American Journal of Medical Genetics 2000; 96(6): 839-844.

[20] Miller DT, et al. Consensus statement: chromosomal microarray is a first-tier clinical diagnostic test for individuals with developmental disabilities or congenital anomalies. American Journal of Human Genetics 2010; 86(5):749-764.

[21] Marshall CR, et al. Structural variation of chromosomes in autism spectrum disorder. American Journal of Human Genetics 2008; 82(2): 477-488.

[22] Glessner JT, et al. Autism genome-wide copy number variation reveals ubiquitin and neuronal genes. Nature 2009; 459(7246): 569-573.

[23] Shen $Y$, et al. Clinical genetic testing for patients with autism spectrum disorders. Pediatrics 2010; 125(4): e727-735.

[24] Sanders SJ, et al. Multiple recurrent de novo CNVs, including duplications of the $7 q 11.23$ Williams syndrome region, are strongly associated with autism. Neuron 2011; 70(5): 863-885.

[25] Manning M and Hudgins L. Array-based technology and recommendations for utilization in medical genetics practice for detection of chromosomal abnormalities. Genetics in Medicine 2010; 12(11): 742-745.

[26] Miller DT, et al. Oligonucleotide microarrays for clinical diagnosis of copy number variation and zygosity status. Current Protocols in Human Genetics 2012; Chapter 8: Unit8.12.

[27] Basu SN, et al. AutDB: a gene reference resource for autism research. Nucleic Acids Research 2009; 37(Database issue): D832-836.

[28] Jamain S, et al. Mutations of the X-linked genes encoding neuroligins NLGN3 and NLGN4 are associated with autism. Nature Genetics 2003; 34(1): 27-29.

[29] Arking DE, et al. A common genetic variant in the neurexin superfamily member CNTNAP2 increases familial risk of autism. American Journal of Human Genetics 2008; 82(1): 160-164. 
[30] Bakkaloglu B, et al. Molecular cytogenetic analysis and resequencing of contactin associated protein-like 2 in autism spectrum disorders. American Journal of Human Genetics 2008; 82(1): 165-173.

[31] Li X, et al. Association analysis of CNTNAP2 polymorphisms with autism in the Chinese Han population. Psychiatric Genetics 2010; 20(3): 113-117.

[32] Strauss KA, et al. Recessive symptomatic focal epilepsy and mutant contactin-associated protein-like 2. New England Journal of Medicine 2006; 354(13): 1370-1377.

[33] Zweier C, et al. CNTNAP2 and NRXN1 are mutated in autosomal-recessive PittHopkins-like mental retardation and determine the level of a common synaptic protein in Drosophila. American Journal of Human Genetics 2009; 85(5): 655-666.

[34] El-Fishawy P and State MW. The genetics of autism: key issues, recent findings, and clinical implications. The Psychiatric Clinics of North America 2010; 33(1): 83-105.

[35] van der Zwaag B, et al. Gene-network analysis identifies susceptibility genes related to glycobiology in autism. PLoS One 2009; 4(5): e5324. http://www.plosone.org/article/info\%3Adoi\%2F10.1371\%2Fjournal.pone.0005324

[36] Sbacchi S, et al. Functional annotation of genes overlapping copy number variants in autistic patients: focus on axon pathfinding. Current Genomics 2010; 11(2): 136-145.

[37] Gilman SR, et al. Rare de novo variants associated with autism implicate a large functional network of genes involved in formation and function of synapses. Neuron 2011; 70(5): 898-907.

[38] Anney RJ, et al. Gene-ontology enrichment analysis in two independent family-based samples highlights biologically plausible processes for autism spectrum disorders. European Journal of Human Genetics 2011; 19(10): 1082-1089.

[39] Skafidas E, et al. Predicting the diagnosis of autism spectrum disorder using gene pathway analysis. Molecular Psychiatry 2012; doi: 10.1038/mp.2012.126. http:// www.nature.com/mp/journal/vaop/ncurrent/full/mp2012126a.html

[40] Kumar A, et al. A brain region-specific predictive gene map for autism derived by profiling a reference gene set. PLoS One 2011; 6(12): e28431. http://www.plosone.org/ article/info\%3Adoi\%2F10.1371\%2Fjournal.pone.0028431

[41] Kumar A, et al. Animal model integration to AutDB, a genetic database for autism. BMC Medical Genetics 2011; 4:15. http://www.biomedcentral.com/1755-8794/4/15

[42] Abrahams BS, et al. SFARI Gene 2.0: a community-driven knowledgebase for the Autism Spectrum Disorders (ASDs). Submitted to Nature Genetics, manuscript under review.

[43] Firth HV, et al. DECIPHER: Database of Chromosomal Imbalance and Phenotype in Humans Using Ensembl Resources. American Journal of Human Genetics 2009; 84(4): 524-533. 
[44] $\mathrm{Xu} \mathrm{LM}$, et al. AutismKB: an evidence-based knowledgebase of autism genetics. Nucleic Acids Research 2012; 40(Database issue): D1016-22.

[45] Matuszek G and Talebizadeh Z. Autism Genetic Database (AGD): a comprehensive database including autism susceptibility gene-CNVs integrated with known noncoding RNAs and fragile sites. BMC Medical Genetics 2009; 10: 102. http://www.biomedcentral.com/1471-2350/10/102

[46] Nakatani J, et al. Abnormal behavior in a chromosome-engineered mouse model for human 15q11-13 duplication seen in autism. Cell 2009; 137(7): 1235-1246.

[47] Kerin T, et al. A noncoding RNA antisense to moesin at 5p14.1 in autism. Science Translational Medicine 2012; 4(128): 128ra40. http://stm.sciencemag.org/content/ 4/128/128ra40.short

[48] Smith CL, et al. Genomic and epigenomic instability, fragile sites, schizophrenia and autism. Current Genomics 2010; 11(6): 447-469.

[49] Levenson D. Too many children with autism miss genetics evaluations. American Journal of Medical Genetics, Part A 2012; 158A(8): vii-viii.

[50] Trottier $M_{\text {, }}$ et al. Parents' perspectives on participating in genetic research in autism. Journal of Autism and Developmental Disorders 2012; http://www.springerlink.com/ content/0q6642013v518r31/?MUD=MP

[51] Gurrieri F. Working up autism: the practical role of medical genetics. American Journal of Medical Genetics. Part C, Seminars in Medical Genetics 2012; 160C(2): 104-110.

[52] Kolvezon A, et al. Analysis of a purported SHANK3 mutation in a boy with autism: clinical impact of rare variant research in neurodevelopmental disabilities. Brain Research 2011; 1380: 98-105.

[53] Hayden EC. The broken contract. Nature 2012; 486(7403): 312-314.

[54] McGuire AL, et al. Research ethics and the challenge of whole-genome sequencing. Nature Reviews Genetics 2008; 9(2): 152-156.

[55] Caulfield T, et al. Research ethics recommendations for whole-genome research: consensus statement. PLoS Biology 2008; 6(3): e73. http://www.plosbiology.org/article/ info\%3Adoi\%2F10.1371\%2Fjournal.pbio.0060073

[56] Wolf SM, et al. Managing incidental findings in human subjects research: analysis and recommendations. The Journal of Law, Medicine \& Ethics 2008; 36(2): 219-48.

[57] Bredenoord AL, et al. Disclosure of individual genetic data to research participants: the debate reconsidered. Trends in Genetics 2011; 27(2): 41-47.

[58] Ozonoff $S$, et al. Recurrence risk for autism spectrum disorders: A baby siblings research consortium study. Pediatrics 2011; 128(3):e488-95. http://pediatrics.aappublications.org.ezproxy.nihlibrary.nih.gov/content/128/3/e488.long 
[59] Schaefer GB, et al. Clinical genetics evaluation in identifying the etiology of autism spectrum disorders. Genetics in Medicine 2008; 10(4): 301-305.

[60] Sebat J, et al. Strong association of de novo copy number mutations with autism. Science 2007; 316(5823): 445-449.

[61] Sato D, et al. SHANK1 Deletions in Males with Autism Spectrum Disorder. American Journal of Human Genetics 2012; 90(5): 879-887.

[62] Duong L, et al. Mutations in NRXN1 in a family multiply affected with brain disorders: NRXN1 mutations and brain disorders. American Journal of Medical Genetics. Part B, Neuropsychiatric Genetics 2012; 159B(3): 354-358.

[63] Salyakina D, et al. Copy number variants in extended autism spectrum disorder families reveal candidates potentially involved in autism risk. PLoS One 2011; 6(10): e26049. http://www.plosone.org/article/info\%3Adoi\%2F10.1371\%2Fjournal.pone. 0026049

[64] Shen $Y$, et al. Intra-family phenotypic heterogeneity of $16 \mathrm{p} 11.2$ deletion carriers in a three-generation Chinese family. American Journal of Medical Genetics. Part B, Neuropsychiatric Genetics 2011; 156(2): 225-232.

[65] Sayers EW, et al. Database resources of the National Center for Biotechnology Information. Nucleic Acids Research 2011; 39(Database issue): D38-51.

[66] The 1000 Genomes Project Consortium. A map of human genome variation from population-scale sequencing. Nature 467(7319): 1061-1073.

[67] Iafrate AJ, et al. Detection of large-scale variation in the human genome. Nature Genetics 2004; 36(9): 949-951.

[68] Mailman MD, et al. The NCBI dbGaP database of genotypes and phenotypes. Nature Genetics 2007; 39(10): 1181-1186. 


\title{
Genetic Etiology of Autism
}

\author{
Agnes Cristina Fett-Conte, \\ Ana Luiza Bossolani-Martins and \\ Patrícia Pereira-Nascimento \\ Additional information is available at the end of the chapter \\ http://dx.doi.org/10.5772/53106
}

\section{Introduction}

Autism Spectrum Disorders (ASD) are severe neurodevelopment disorders characterized by impairment in social interaction and communication, and repetitive and stereotyped behaviors. Motor deficits, aggressive behavior, abnormal sleep patterns, gastrointestinal problems, epilepsy and intellectual disability are also observed. Manifestations are observed before three years of age with early stimulation being recommended (Baird et al., 2006; Faras et al., 2010; Bronsard et al., 2011; Miles, 2011; Angelidou et al., 2012). Due to the great phenotypic variability of carriers and the subjectivity of the differential diagnostic criteria of "Pervasive Developmental Disorders" (American Psychiatric Association, 2000), ASD is today considered the most appropriate denomination. The general term, autism, is often used as a synonym for ASD.

ASD were described more than seven decades ago (Sanders, 2009) and many neurobiological changes have been illustrated in carriers, yet the diagnosis is still based on behavioral aspects using diagnostic scales. However, even though there is a detailed "checklist" made up of several scales, most are not translated and validated in different countries, which hinders standardized and efficacious diagnosis (Marteleto et al., 2008; Rapin \& Goldman, 2008; Sato et al., 2009; Biederman et al., 2010).

The prevalence of ASD varies by region, but it is believed to be around 1:150 individuals. However, higher prevalences of up to 1:88 children have been described (Currenti, 2010; Angelidou et al., 2012). The tentatives to explain such high prevalence rates involve changes in diagnostic criteria, greater knowledge of the general population and the exposure of the genetic material of fetus to internal and external toxic agents (King \& Bearman, 2009; Lintas \& Persico, 2009; Avchen et al., 2011). 
Although many environmental factors are related to the pathogenesis of autism, such as the rubella virus whose disruptive effects in the brain may result in autistic behavior, the participation of genetic components is certain. The estimated concordance rate for identical twins ranges from 60 to $90 \%$, while among dizygotic twins and non-twin siblings the rate is from 5 to $31 \%$ (Bailey, et al., 1995; Rosenberg et al., 2009; Hallmayer et al., 2011). Based on studies of twins, heritability was estimated to be between 60 and 80\% (Geschwind, 2011; Hallmayer et al., 2011).

In up to $10 \%$ of ASD cases it is possible to identify etiological, genetic or environmental factors (syndromic autism). Thus, in about $90 \%$ of the cases there is no known cause (non-syndromic autism). A multifactorial etiology can be assigned to these idiopathic cases after the exclusion of environmental and genetic causes, and using specific evaluations (VeenstraVanderweele et al., 2004). Scientific discoveries until now have shown that there are multiple genetic factors (polygenes) involved in the predisposition to ASD which, associated with an external trigger (environmental factor), would result in the behavioral framework for autism. However, these factors alone also result in changes in the brain that lead to autistic behavior. Thus, the same factors may be present in two children with one having autism and the other not. There is no doubt that science has elucidated many biological mysteries about autism, yet for every issue clarified, another, even more complex, appears.

\section{Environmental etiology}

Prenatal or perinatal infections by viral agents such as rubella and cytomegalovirus, as well as exposure to toxic agents, such as thalidomide, valproic acid and alcohol, are some of the best-known environmental causes of ASD (Chess et al., 1978; Christianson et al., 1994).

'Allergenic' environmental factors and autoimmune problems during pregnancy may also be involved in the etiology of autism. The presence of circulating maternal antibodies against fetal brain proteins suggests the possibility of their transposition in the blood-brainbarrier. Studies have demonstrated the presence of pro-inflammatory cytokines in the fetal brain, such as TNF (tumor necrosis factor) which is preformed in maternal mast cells (Vojdani, 2008; Angelidou et al., 2012).

Even premature birth has been implicated as a cause of ASD. Babies from gestations of less than 28 weeks have a high risk of neurological problems. A study in Atlanta, USA, showed that children born in the 33rd gestational week have a greater risk for autistic characteristics (Limperopoulos et al., 2008).

There is a link between oxidative stress and immune response (Viora et al., 2001). There are suggestions that environmental factors trigger oxidative stress in individuals genetically susceptible to autism, which would lead to losses in methylation and secondary neurologic deficits (Dardeno et al., 2010). Increased levels of oxidative stress markers have already been described in the cord blood of mothers who had premature births compared to those of mothers who had full term births (Joshi et al., 2008). 
Premature birth is associated with the formation of reactive oxygen species (Davis \& Auten, 2010). Stress typically results in the release of corticotropin-releasing hormone (CRH) with elevated levels of this hormone in plasma being associated with premature births (Warren et al., 1992; Chrousos, 1995). CRH may stimulate the release of a cytokine, interleukin-6 (IL-6), by mast cells, which are part of the immune system. IL-6, by injuring the blood-brain barrier due to stress related to CRH and mast cells, increases its permeability (Esposito et al., 2001). With the increased permeability, neurotoxic molecules can reach the brain and cause an inflammatory process that contributes to the pathogenesis of ASD (Theoharides, 2008; Valent et al., 2012). This process has suggested a new possibility for the etiology of ASD.

\section{Overlapping genetic etiologies}

Many genomic regions, with genes implicated as candidates, have been associated to the etiology of autistic behavior, although the results of some studies have not been replicated. Even so, it is estimated that all proposed regions together would be involved in the etiology of less than $1 \%$ of cases. In addition, scientific evidence shows that changes in regions reported in ASD have also been described in other neuropsychiatric diseases, which suggests that there is an etiological connection with phenotypes attributed to other neurodevelopment abnormalities (Griswold et al., 2012). For example, some rare mutations associated with increased risk for ASD and schizophrenia have already been reported in 15q13.3, 16p11.2 and 22q11.21 and in the NRXN1 gene (Weiss et al., 2008; Levinson et al., 2011; Sanders et al., 2011).

Moreover, many genes involved in nonsyndromic intellectual disabilities (ID) and in epilepsy have also been implicated in the etiology of nonsyndromic ASD. These genes probably belong to a continuum of neurodevelopment disorders that manifest in different manners depending on associated genetic and environmental factors. The identification of changes is crucial for patients and for counseling of families, as well as for the identification or exclusion of the presence of specific genetic diseases in patients with ASD (Betancour, 2011).

Many patients with chromosomal or monogenic diseases have autistic behavior as one phenotypic manifestation of the disease. A scientific enigma to be elucidated refers to the possible causes of the varying severity of symptoms or the presence/absence of manifestations in carriers in the same family. They are not free from rare or common mutations associated with behavioral phenotype in isolation. Thus, in autism, studies on gene interactions are fundamental and what is called overlapped etiology can be an additive effect between different genes, some of them more significants.

\section{Genetic anticipation}

Genetic anticipation studies are fundamental in the elucidation of inheritance mechanisms for any genetically influenced condition, because, in addition to the clinical importance and 
guidance in genetic counseling, these investigations can assist in the elucidation of the recurrence risks in future generations (Constantino et al., 2010).

Following a promising approach in the investigation of complex disease etiologies, studies on endophenotypes ("intermediate phenotypes") may be used to direct the search for the etiology of ASD (Weinberger et al., 2001). Manifestations related to autistic behavior are often observed in varying degrees of severity in unaffected individuals of previous generations in the same family, thereby characterizing the phenomenon of genetic anticipation in ASD (Losh et al., 2008).

A Swedish study reported that the existence of individuals with schizophrenia and bipolar disorder in the family is a risk factor for the occurrence of autism. The authors found an association between schizophrenic parents or siblings with increased risk of ASD. Bipolar disorder also proved to be a risk factor, but not as strong as schizophrenia (Sullivan et al., 2012).

Studies of autistic families have also shown a significant increase in the recurrence of ASD in first-degree relatives of carriers. For example, siblings of individuals with ASD have a 22- to 25fold higher risk of having the disorder (Lauritsen et al., 2005; Abrahams \& Geschwind, 2008). There are significantly higher risks of ASD in offspring of parents with ASD and those with familial history of psychiatric problems. Depression and personality disorders have been reported to be more common in mothers of children diagnosed with ASD than in mothers of children with normal development (Daniels et al., 2008; Constantino et al., 2010). Even some non-affected individuals of different generations in the same family may show subtle impairment in cognitive development, language changes or in social interaction; this is termed the broad autism phenotype. This phenotypic diversity of autistic behavior and psychiatric manifestations in families of the patients indicate that the genetic factors that influence ASD may be composed of distinct elements that manifest differently between affected and non-affected family members (Pickles et al., 2000; Szatmari et al., 2000; Goldberg et al., 2005).

In the molecular field, studies on genealogies with multiple affected family members and studies on twins suggest that allelic variations are associated with increased susceptibility to ASD and that there are etiological factors common to both ASD and milder autistic phenotypes (Lundstrom et al., 2010; Arking et al., 2008; Wang et al., 2009). Hence, epidemiological studies have been developed with families in an attempt to clarify the relative proportions of cases of autism and broad autism phenotypes in the population that might explain these complex mechanisms of genetic transmission.

\section{Associated syndromes and comorbidity in autism}

Similar to intellectual disabilities or delay in motor developmental, the autistic behavioral phenotype can occur alone or as part of the spectrum of phenotypic manifestations in a particular condition of environmental, genetic or multifactorial etiology. In some situations, the autistic behavioral phenotype is observed in individuals with only one other clinical manifestation, such as epilepsy. This simultaneous occurrence (co-occurrence) has been discussed 
much and is referred to in the literature either as an association or as comorbidity. Probably the truth is, that in most cases, the different manifestations result from the same causal factor, which suggests the term "association" is more appropriate than "comorbidity".

Monozygotic and dizygotic twin studies indicate a variety of neuropsychiatric diagnoses associated with ASD, including attention deficit and hyperactivity disorder (ADHD) and anxiety disorder (Lichtenstein et al., 2010). High frequencies of these diseases have been reported in children with autism, as has bipolar disorder in adolescents and young adults (Munesue et al., 2008; Simonoff et al., 2008). The wide range of clinical behavioral symptoms among carriers may be justified and be a good argument to consider the diagnosis of ASD alone, with all the possible manifestations expected in the spectrum, as no individual is exactly like another. In this way, families would be less anxious and confused on receiving three or four diagnoses for the same child.

There are a number of diseases associated with autism, whose genetic etiology is well established, i.e. autistic behavior is one of the possible manifestations. The most common is Fragile $\mathrm{X}$ syndrome (FRAXA). This is the most frequent form of inherited mental retardation and is considered a monogenic cause of ASD. Symptoms include neurodevelopmental delay, anxiety, hyperactivity, and autistic-like behavior, which are accompanied by macroorchidism and distinct facial morphology. It is caused by the expansion of the CGG trinucleotide repeat in the 5' untranslated region of the fragile $X$ mental retardation 1 (FMR1) gene resulting in loss of the Fragile X Mental Retardation Protein (FMRP), an RNA-binding protein abundant in the brain and gonads of affected men. FMRP has multiple functions in the RNA metabolism, including mRNA decay, dendritic targeting of mRNAs and protein synthesis. In neurons lacking FMRP, a wide array of mRNAs encoding proteins involved in synaptic structure and function are altered. As a result of this complex dysregulation, in the absence of FMRP, spine morphology and functioning is impaired (De Rubeis et al., 2012). Frequencies of between $2 \%$ and $3 \%$ of FRAXA have been observed in studies on boys with ASD, while in boys with FRAXA, the frequencies of ASD range from $20 \%$ to $40 \%$ (Kaufmann et al., 2004; Shibayama et al., 2004). Tuberous Sclerosis, another monogenic disease that results from mutations in the TSC1 and TSC2 genes, is observed in about $1 \%$ of individuals with ASD and is regarded as the second most common genetic cause of the autistic phenotype (Smalley, 1998).

The list of medical conditions associated with the autistic phenotype, including genetic syndromes, is growing. Examples of genetic conditions associated with autism and the gene regions involved are: Angelman Syndrome (UBE3A), Rett syndrome (MECP2), neurofibromatosis (NF1), Timothy syndrome (CACNA1C), Smith-Lemli-Opitz syndrome (DHCR7), CHARGE (CHD7), Cohen syndrome (VPS13B), Noonan syndrome (PTPN11), 2q37 deletion syndrome, Cornelia de Lange syndrome (NIPBL, SMC1A and SMC3), DiGeorge/ Velocardiofacial syndrome (22q11), Smith-Magenis (RAI1), Williams-Beuren syndrome (7q11.23) and Phelan-McDermid syndrome (22q13.3) (Berg et al.,2007; Phelan, 2008; Delahaye et al., 2009; Van der Aa et al., 2009; Laje et al., 2010; Betancour, 2011).

Many inborn errors of metabolism also seem to contribute to at least $5 \%$ of ASD cases as the deficiency of certain enzymes in metabolic diseases can result in the accumulation of substances 
that may have toxic effects on brain development. An example is phenylketonuria, an autosomal recessive disorder that, if untreated, leads to excessively high levels of phenylalanine and toxic metabolites, resulting in intellectual disabilities and ASD (Manzi et al., 2008).

Mitochondria are intracellular organelles that have the function of producing energy. In the mitochondria ATP production, free oxygen radicals and reactive oxygen species (ROS) are produced and then normally removed from the cells by anti-oxidant enzymes. When the production of ROS and free radicals exceeds the limit, oxidative stress occurs, that is, ROS combine with lipids, nucleic acids and proteins in the cells leading to cell death by apoptosis or necrosis. Since brain cells have limited antioxidant activity, a high lipid content and high requirement for energy, it is more prone to the effects of oxidative stress. Some patients with ASD and mutations in mitochondrial DNA have already been reported (Fillano et al., 2002; Dhillon et al., 2011). The first study involving bioenergetic metabolism disorders in ASD was directed by Coleman \& Blass (1995), who reported lactic acidosis in four children with autism. Later Lombard (1998) proposed that mitochondrial oxidative phosphorylation can cause abnormal brain metabolism in children with autism resulting in acidosis. A study by Pons et al. (2004) described five children with ASD who had abnormal respiratory chain enzyme activity, characterized by the A3243G mutation. Graf et al. (2000) described two brothers with autism associated with a mitochondrial DNA G8363A RNA(Lys) mutation.

All these diseases, in addition to peculiar and specific clinical signs and symptoms, have autism as a common manifestation. However, with so different genetic etiologies and probably, the involvement of different interaction mechanisms, what do they have in common that explains the autistic behavior? The autistic behavior is attributed to changes in neurodevelopment and all these diseases cause changes in the brain structure and/or functioning, probably damaging cerebral areas that are linked to autistic symptoms.

In the clinical practice, the recognition of these conditions is fundamental, as it allows the targeting of laboratory tests and assists in the initial breakdown of etiological heterogeneity which categorizes specific cases of autism as syndromic or nonsyndromic. This definition is important because of possible implications in the prognosis and recurrence risk (Miles, 2011; Gurrieri, 2012).

\section{Chromosomal alterations}

Numerical and structural chromosomal alterations, visible by conventional cytogenetic techniques, occur in about 6 to $7 \%$ of ASD cases and have already been described in all autosomal and sex chromosomes. These findings justify karyotyping by GTG banding as part of the etiological work-up protocol of carriers (Castermans et al., 2004; Shen et al., 2010).

Some human chromosomal aneuploidies are known to increase the risk for ASD; the most common, as identified in studies of individuals with autism, are trisomy 21 (Down syndrome), monosomy of chromosome $X$ in women (Turner syndrome), uniparental $X$ disomy in men (Klinfelter syndrome), Y disomy and 45,X/46,XY mosaicism. Structural abnormalities include 15q11-13 duplication and deletions of 2q37, 22q11.2 and 22q13.3 (Betancour, 2011). 
Due to the high number of cases that have been described and the type of genes located in them, the association of eight chromosomal regions is well established in autism including: 1q21, 7q11.23, 15q13, 15q11-13, 16p11.2, 17p11.2, 22q13.3 and 22q11.21. Rearrangements involving these regions are detected by classic cytogenetic techniques but it is recommended that more sophisticated techniques, such as array comparative genomic hybridization (array-CGH), are used for their evaluation (Gillberg, 1998; Griswold et al., 2012).

As expected, unbalanced changes are more frequently found in dysmorphic individuals and with delays in neuropsychomotor development due to the "extent of damage" because they result in significant gains and losses of gene content. Balanced rearrangements, however, are less frequent and can be related to mutations in DNA breakpoints. Some are so rare that it is difficult and risky to consider them a cause of autism. However, some occur at high enough frequencies to be considered risk factors for the disease. Identifying balanced changes is important for genetic counseling, not only due to the etiologic implications, but also because these changes may predispose descendants to unbalanced rearrangements (Carter, 2011; Sherer \& Dawson, 2011; Nowakowska et al., 2012).

However, chromosomic analysis detects only 3-5 megabase abnormalities. New technologies using DNA or chromosomal microarrays can identify submicroscopic abnormalities. Microdeletions and duplications, e.g., may be identified with microarrays in individuals with ASD who previously had normal kariotype. Therefore, if cytogenetic analysis is negative in clinically diagnosed ASD, molecular techniques are necessary.

\section{Candidate genes}

According to recent findings, some common mutations, epigenetic mechanisms, chromosome alterations, rare single gene mutations, copy number variations (CNVs) and single nucleotide polymorphisms (SNPs) result in the autistic phenotype. Because of national and international consortia, many linkage and genome-wide association studies have evolved which elucidated candidate genes and susceptibility of genomic regions relevant to ASD. In contrast to polygenic or complex genetic models for autism, suggested in the majority of cases, a few forms of ASD are known to be caused by single gene defects, such as in FRAXA (Chiocchetti \& Klauck, 2011; Dhillon et al., 2011).

According to a review by Betancour (2011) more than 100 candidate genes for autistic behavior are also related to syndromic or nonsyndromic intellectual disabilities. Many are also associated with epilepsy, with or without intellectual disabilities; this suggests that these neurodevelopment disorders have risk factors in common with ASD.

Mutations in a single gene may be autosomal dominant, recessive or X-linked. Some, not always related to syndromic cases, are highly penetrating and appear at sufficiently high frequencies to be considered monogenic causes of autism. Of the growing list, the most important candidate genes are: NLGN3, NLGN4, SHANK2, SHANK3, NRXN1, NRXN3, PTCHD1/PTCHD1AS, SHANK1, DPYD, ASTN2, DPP6, MBD5, CDH8 and CNTNAP2. It is 
important to note that most of these act on neurotransmission in the central nervous system (Sherer \& Dawson, 2011).

There are reports of more than two hundred candidate genes in the literature. According to Swanwick et al. (2011), they can be classified into four categories: 1) rare - genes involved in rare monogenic forms of ASD. This type of allelic variant includes rare polymorphisms and mutations directly related to ASD (NRXN1 and SHANK3); 2) syndromic - genes related to syndromes with phenotypic manifestations in a significant subpopulation of carriers that include autistic symptoms (FMR1 and MECP2); 3) association - genes with common polymorphisms that confer a small, probably additive risk, for ASD, that were identified from association studies derived from cases of unknown etiology (MET and GABRB1) and 4) functional - genes with functions related to the biology of ASD that are not included in the other categories (CASDP2 and $A L O X 5 A P)$. Among these, the ones that belong to the first two categories are the most strongly related to the pathogenesis of ASD (El-fishawy \& State, 2010). There are indications that de novo point mutations occur in approximately 5 to $20 \%$ of the cases (O'Roak et al., 2011).

Persico and Bourgeron (2008) proposed that there are three main pathways involved in the pathogenesis of ASD. The first entails genes that affect cell migration, the second disruptions of the glutamate-GABA harmony and the third involves synaptic formation and maintenance and dendritic morphology. All these pathways play a fundamental role in the central nervous system, particularly in the serotonergic process (Berkel et al., 2010; Durand et al., 2007).

Recent studies have given more support to evidence that a large subset of genes, involved in the outgrowth and guidance of axons and dendrites, is implicated in the etiology of autism. However, many studies are still needed in order to understand the role of isolated genes and gene regions in ASD and to identify the associations between them and to identify new candidate genes that act within the molecular pathways (Hussman et al., 2011; Griswold et al., 2012).

But despite the large number of previously identified candidate genes, the number of patients with changes in these genes does not reach $1 \%$ of the total cases, which further highlights the extreme heterogeneity seen in the pathogenesis of ASD. The findings have led to a paradigm shift in the concept of the genetic architecture of common neurodevelopmental diseases, stressing the importance of individual patterns, rare mutations and overlapping in genetic etiology. They have also converged on specific neurodevelopmental pathways, providing insights into pathogenic mechanisms (Mitchell, 2011).

\section{Single Nucleotide Polymorphisms (SNPs), Quantitative Trait Locus (QTL) and risk for ASD}

Of the techniques that supported the rapid advance in molecular genetics, comparative genomic hybridization (CGH) and single nucleotide polymorphism (SNP) genotyping array have allowed the qualitatively and quantitatively identification of the genes and genomic rearrangements involved in the autistic phenotype (Alárcon et al., 2008; Whalley, 2011; Mefford 
et al., 2012). The use of these molecular sequencing tools has also enabled the identification of high frequencies of common variants in some genes, such as SNPs, in individuals with SD. SNPs, causing about $50 \%$ of all currently known variations in genetic material, are the most numerous variants in the genome. Moreover, SNPs are considered genetic markers that can be used to identify genes associated with complex diseases (Malhotra \& Sebat, 2012).

Unlike CNVs, SNPs seem to be more penetrating in ASD. De novo SNPs, although less frequent, seem to have more deleterious effects and confer higher risk for autistic behavior (Chahrour et al., 2012). Associations between some SNPs of mitochondrial and nuclear genes and predisposition to ASD have been reported by several studies (Ramoz et al., 2004; Silverman et al., 2008; Smith et al., 2009). Studies have shown SNPs in the GABRA2, GABRA3, GABRA4, SLC25A12, FOXP2, CNTNAP2, CNTNAP2 and BDNF genes (Li et al., 2005; Segurado et al., 2005; Alárcon et al., 2008; Cheng et al., 2009; Scott-Van Zeeland et al., 2010; Jiao et al.,2011). In the Genome-wide Association Studies (GWAS) involving 4305 individuals with ASD and 6941 controls, strong association signals were revealed in six SNPs of two genes encoding neuronal cell-adhesion molecules, cadherin 10 (CDH10) and cadherin 9 (CDH9). These findings were replicated in two independent cohorts and implicated neuronal cell-adhesion molecules in the pathogenesis of ASD (Wang et al., 2009).

It is possible that screening for SNPs may identify new biological mechanisms that are involved in predisposition to ASD. However, GWAS, although promising, has revealed few common alleles and many results have still to be replicated (Ma et al., 2009; Manolio et al., 2009; Klein et al., 2010). It is clear that SNPs may have variable expressions or reduced penetrance. But, while it is apparent that rare variations can play an important role in the genetic architecture of these diseases, the contribution of common variations to risk for ASD is less clear (Jiao et al., 2011; Sherer \& Dawson, 2011). Additionally, a strong Association between SNPs in the 5p14.1 and 5p15.2 regions and ASD has also been reported (Wang et al., 2009).

However, the results of Stage 2 of the Autism Genome Project Genome-Wide Association Study, which incremented 1301 ASD families to the investigation bringing the total to 2705 families analyzed (Stages 1 and 2), showed that no single SNP has a significant association with ASD or selected phenotypes at the genome-wide level and concluded that common variants affect the risk for ASD but their individual effects are modest (Anney et al., 2012).

These controversial results about the role of SNPs in the predisposition for ASD do not rule out participation in the phenotype, but motivate the investigation of biological phenomenon that would explain their participation. Probably a single SNP does not affect the risk, but perhaps the additive effect of several SNPs, in specific combinations, with the participation of environmental determinants cannot be discarded in etiologically complex diseases.

From another standpoint, the Quantitative Trait Locus (QTL) approach is one of the most suitable methods to find susceptibility of loci. This approach follows the assumption that ASD occur as a continuum of severity, a position supported by findings of elevated levels of ASD symptoms in parents and siblings of cases compared to controls, and variations in ASD traits that have been found in the general population. One study to identify the loci that underlie ASD symptoms in children with attention-deficit/hyperactivity disorder (ADHD) in- 
vestigated both the total level of ASD symptoms as well as scores of three ASD symptom domains, thus taking into account potential differential genetic origins of different ASD symptom domains. QTL linkage analyses for the different ASD domains were carried out using 5407 SNPs spanning the entire genome. Findings suggest that some QTLs are ASD specific, although the 15q QTL potentially has pleiotropic effects for ADHD and ASD (Nijmeijer et al., 2011). The genetic analysis of quantitative traits that are phenotypically linked, such as in ASD and ADHD, can reduce the heterogeneity of diagnosis and indicate loci related to susceptibility (Lu et al., 2011).

There is a QTL related to language delay located close to the 7q35-36 region. Interestingly this region is mapped in the CNTNAP2 gene, a strong candidate for predisposition to autistic behavior; it is well known that communication abilities are qualitatively impaired in autistic individuals. The significant delay in language ("age of first word") is observed in about half of affected children (Alárcon et al., 2008). The relationship of SNPs in the CNTNAP2 gene has already been described, as has the association of the gene and its SNPs to language development delay in autistic and non-autistic individuals (Alárcon et al., 2008; Arking et al., 2008; Tan et al., 2010; Stein et al., 2011; Whalley et al., 2011).

Additionally, the transcription factor encoded by the FOXP2 gene has already been linked to the development of language. This factor binds to the promoter of the CNTNAP2 gene regulating its expression during development. There are reports of changes in the FOXP2 binding site in patients with ASD, which suggests that a reduced expression of the CNTNAP2 gene may be the underlying etiology of one of the phenotypic characteristics of ASD (Vernes et al., 2008; Poot, et al., 2009). In addition to these, it has been suggested that WNT2 and EN2 are related to language development in autism (Lin et al., 2012).

Promising results on the influence of genetic bases in neurobehavioral disorders have also been obtained through studies on CNVs. All these emerging genetic technologies have brought more valuable approaches to improve the understanding of the etiology of ASD. Advances in the use of molecular biology tools have provided a promising manner to study gene-gene and gene-environment relationships in disorders (Gurrieri, 2012; Li et al., 2012). This combination of tools in the search for the etiology will reflect in the possibility of targeting the diagnosis, prognosis, early interventions and genetic counseling. However, more data and the reproducibility of findings are necessary to establish the genetic components of these diseases.

\section{Copy Number Variations (CNVs) in ASD}

Copy Number Variations (CNVs), submicroscopic variations that are less than $500 \mathrm{~kb}$ in size in DNA, are widespread in normal human genomes. The most common CNVs tend to be large $(>500 \mathrm{~Kb})$ and contain several genes, while rare or de novo $\mathrm{CNVs}$ are smaller $(<100 \mathrm{~Kb})$, sometimes interrupting a single gene. On average, there are more than $1000 \mathrm{CNV}$ s in the genome, accounting for $\sim 4$ million base pairs of genomic difference. Although SNPs outnumber $\mathrm{CNVs}$ in the genome by three orders of magnitude, their relative contributions to 
genomic variation (as measured in nucleotides) are similar. Thus, in addition to $0.1 \%$ of genetic difference at the nucleotide sequence level, another $0.1 \%$ of genetic difference is apparent at the structural level (Malhotra \& Sebat, 2012).

The rate of genome-wide nucleotide substitutions is estimated at 30-100 per generation and $\sim 1$ per exome. In contrast, the global rate of structural mutation events is lower: CNVs $>10$ $\mathrm{Kb}$ in size occur at a rate of $\sim 0.01-0.02$ per generation (Marshall et al., 2008; Conrad et al., 2011; Levy et al., 2011; Sanders et al., 2011). Nucleotide substitutions probably account for the majority of disease risk alleles, but based on sheer size and potential to impact genes (or multiple genes), structural mutations are, on average, more pathogenic. Thus, CNVs, de novo CNVs in particular, seem to be a class of variants that have large effect on disease risk (Malhotra \& Sebat).

CNVs are gaining importance in the scenario of ASD. They represent a significant source of genetic diversity and seem to significantly contribute to changed behavioral phenotypes (Sebat et al., 2007; Rees et al., 2011). To have an idea, de novo CNVs have already been reported to be three to five times more common in families of individuals with ASD than in controls, and more often presenting the syndromic form of autism, that is, with the most severe phenotypes (Miller et al., 2010; Pinto et al., 2010; Shen et al., 2010; Sanders et al., 2011). In fact, CNVs, in particular de novo CNVs involving many genes, confer risk for ASD. However, although they are important in this respect, they rarely interrupt a single gene or have complete penetrance and many give a wide-ranging risk including risk for other problems such as intellectual deficiency, epilepsy and schizophrenia (Geschwind, 2011; O’Roak et al., 2011).

Up to $40 \%$ of CNVs in autism are inherited from apparently normal parents, consistent with the suggestion of incomplete penetrance. Both de novo (non- inherited) or inherited CNVs occur at the same locus in unrelated individuals, and some of them coincide with those seen in other gene-related diseases associated with ASD, including developmental delay and intellectual deficiency (Cook \& Scherer, 2008; Lee \& Scherer, 2010). Thus, some apparently have a pleiotropic effect.

De novo CNVs have been observed in from $7-10 \%$ of cases in simplex families, in $2-3 \%$ in multiplex families and approximately $1 \%$ of normal controls. Rare de novo CNVs have already been observed in 5.8-7.9\% of carriers and in 1.7-1.9\% of unaffected siblings in simplex families (Levy et al., 2011; Sanders et al., 2011), while de novo mutations in coding regions participate in $<20 \%$ of cases of ASD (Malhotra \& Sebat, 2012). In addition, about $10 \%$ of ASD cases with de novo CNVs have two or more CNVs (Sebat et al., 2007; Christian et al., 2008; Marshall et al., 2008).

Many of the variations occur in gene regions that contain synaptic genes, and it seems that some involve haploinsufficient regions or dominant inheritance. Others seem to express recessive forms as in the cases of the NHE926, PCDH10 and DLA1 genes identified in studies of individuals with consanguineous parents. Other rare variations were found deleted in homozygous (Bourgeron, 2009; Ramocki \& Zoghbi, 2008; Morrow et al., 2010).

There are descriptions of de novo and inherited CNVs, sometimes in combination in a given family, implicating many novel ASD genes such as SHANK2, SYNGAP1, DLGAP2 and the 
X-linked DDX53-PTCHD1 locus, of an enrichment of CNVs disrupting functional gene sets involved in cellular proliferation, projection and motility, and GTPase/Ras signaling, (Pinto et al., 2010). Another pathway with many of these variations is that related to the development of synapses, and maintenance and motility of neurons, the NLGN-NRXN-SHANK pathway (Bourgeron, 2009).

But, CNVs have been regarded as "rare variants" within the panorama of the etiology of ASD and it is important to emphasize the care that should be taken in associating them in the etiology, especially those not previously described. Accuracy is required in the study of these changes during the formation of control groups. On the other hand, when it comes to autism, on finding the same CNVs in carriers and controls is not a reason to dismiss their participation in the phenotype, given the complexity of factors that may be acting jointly and in combination in different cases.

\section{Reduced penetrance and pleiotropic effects}

Incomplete penetrance and variable expressivity are discussed greatly in the literature in an attempt to explain the complexity of the biological phenomena involved in ASD (Zhao et al., 2007; State \& Levtt, 2011; Coleman \& Colbert, 2012).

While some genomic regions seem to play very important roles in predisposition for autism and others not so much, there are some for which there is much divergence in published data. For example, the variations that involve 15q11-13, 16p11.2 and 22q11.2 are among the most commonly found CNVs in patients with autism, however the resulting behavioral phenotype varies from normal to strongly affected (Cook \& Scherer, 2008; Adzhubei et al., 2011; Devlin \& Scherer, 2012). The explanation for the recurrence of rearrangements in these regions is that they are flanked by low repetition segments containing $99 \%$ of the identity, which due to their genomic structure predisposes them to non-allelic homologous recombination and a high mutation rate (Cottrell et al., 2011). However, another four regions in which large deletions are observed (2q22.1, 3p26.3, 4q12 and 14q23) are not flanked by unstable elements and also have a strong potential for involvement in the etiology of autism (Nicholas et al., 2007; Cottrell et al., 2011; Griswold et al, 2011).

With the recent clinical and research use of high resolution array-based strategies for characterizing CNVs in children with developmental disabilities, there has been a significant increase in the diagnosis of interstitial 15q duplications, including small, atypical duplications. The association between ASD and 15q duplications has been described in many case reports since the 1980s and sparked interest in 15q11-13 as a candidate region in autism linkage studies. Prevalence estimates of proximal 15q duplications in cohorts of patients with autism range from $0.5 \%$ to $3 \%$, depending on ascertainment criteria and methods used to identify the duplications (Simon et al., 2010). This region contains receptors of an inhibitory neurotransmitter, gamma-aminobuytric acid (GABA) that acts on a complex channel receptor mainly permeable to chloride anions to reduce neuronal excitability. GABA signaling is also 
established before glutamatergic transmission suggesting that GABA is the principal excitatory transmitter during early development (Takayama \& Inoue, 2004; Ben-Ari et al., 2007).

In multiplex families, i.e. those with more than one affected individual, different individuals have similar microdeletions or microduplications and exhibit autistic, broad or normal phenotypes (Fernandez et al., 2010; Kumar et al., 2008). The microduplication of 15q11-13, which seems to have a major impact on the expression of ASD, is a good example of different effects in carriers. For example, Veltman et al. (2005) reported a female proband referred for evaluation of a possible ASD. Genetic analyses indicated that the proband, her father and one of her sisters, carried a paternally derived interstitial duplication involving 15q11-13. The proband showed evidence of ASD (PDD-NOS), borderline mental retardation, mild hypotonia and joint laxity. Her father and her sister were of normal intelligence and neither was thought to have an ASD, although speech/language difficulties and some autistic type behaviors were reported to have been present early in the development of the sister.

In studies of idiopathic cases, the most common change is the 16p11.2 microduplication/ microdeletion, comprising a region of around $600 \mathrm{~Kb}$. Microduplication of $16 \mathrm{p} 11.2$ is present in autistic and schizophrenic individuals but is also seen in normal individuals. This variation is also observed in cases of ASD with and without dysmorphic signs (Fernandez et al., 2010; Shinawi et al., 2010). Microdeletions of 16p11.2 seem to be more penetrating (around $100 \%$ ) and are associated with the presence of major dysmorphic signs, while microduplications have reduced penetrance $(\sim 50 \%)$ and are associated with minor dysmorphic signs (Ramocki \& Zoghbi, 2008; Bourgeron, 2009; Fernandez et al., 2010).

One possible explanation for these cases, as already mentioned in previous sections, is that probably there are other genetic components that are contributing to the disease in these families that, depending on how they are associated in individuals, result in different phenotypes (Griswold et al., 2012). But it is also very likely that different CNVs display different penetrance depending on the sensitivity of the affected gene to dosage (number of copies), the function of the gene and the affected region.

Endophenotypes (or intermediate phenotypes) are defined as heritable traits that form a causal link between genes and observable symptoms. Brain based endophenotypes offer several important advantages over clinical phenotypes in the search for pleiotropic genes in ASD. They provide insight in the causal chains of action leading from gene to symptom expression, they aid in forming etiologically more homogeneous subgroups of patients (Gould \& Gottesman, 2006).

In addition to the variability of simple and more complex genetic mechanisms proposed in the expression of the ASD phenotype, environmental factors via epigenetic mechanisms seem to be very important. 


\section{Epigenetic in autism}

Particularly in light of recent findings on mutations in the genes that encode synaptic molecules associated with the communication between neurons, genetic factors are considered to be the most important contributors to the pathogenesis of autism. Epigenetic mechanisms, such as DNA methylation and modifications to histone proteins, regulate DNA structure and gene expression, but without changing DNA sequence. Epigenetic abnormalities are associated with several neurodevelopmental diseases. Many features of autism are consistent with an epigenetic dysregulation, such as discordance of monozygotic twins, parental origin and the gender-dependent effects of some alterations. Since epigenetic modifications are known to be affected by environmental factors such as nutrition, drugs and mental stress, ASD are not only caused by congenital genetic defects, but may also be caused by environmental factors via an epigenetic mechanism.

An example of this phenomenon in ASD is the mechanism of action of the SHANK3 gene. SHANK3 is strongly suspected of being involved in the etiology of ASD since several mutations have been identified in a particular phenotypic group of patients with ASD. SHANK3 (also known as ProSAP2) regulates the structural organization of dendritic spines and is a binding partner of neuroligins; genes encoding neuroligins are mutated in autism and Asperger's syndrome (Durand et al., 2007). It codes a synaptic scaffolding protein enriched in the postsynaptic density of excitatory synapses and plays important roles in the formation, maturation and maintenance of synapses. Haploinsufficiency of the SHANK3 gene causes a developmental disorder, 22q13.3 deletion syndrome (known as Phelan-McDermid syndrome), which is characterized by severe language and speech delay, hypotonia, global developmental delay and autistic behavior. Five CpG-islands have been identified in the gene, and tissue-specific expression is epigenetically regulated by DNA methylation. Cumulative evidence in animal models has shown that several SHANK3 variants are expressed in the developing rodent brain with their expression being regulated by the DNA methylation of intragenic promoters (Uchino et al., 2006).

Additionally, oxidative stress in brain cells occurs due to environmental and genetic causes and leads to decreased activity of the methionine synthase enzyme, which participates in DNA methylation processes. So, when the activity of this enzyme is impaired, affected individuals can exhibit attention deficits and other signs, including autistic symptoms, due to defects in the expression of genes that are controlled by this epigenetic mechanism (Naviaux, 2008; Dhillon et al., 2011).Therefore, environmental factors may activate intracellular pathways during embryonic development thereby causing epigenetic changes in neural function that would explain the relationship between environmental signals and genome in the regulation of individual differences in behavior (Zhang \& Meaney, 2010).

Studies carried out in Sweden involving 208 autistic children with Pervasive Developmental Disorder-Not Otherwise Specified (PDD-NOS) and Asperger's syndrome (AS) obtained interesting results. The authors observed that advancing paternal age is associated with an increasing risk for ASD in offspring, that autistic-like traits in the normal population are affected by both young and advancing paternal age and that autistic similarity within pairs 
of twins seems to increase with advancing paternal age. It was suggested that this result may be related to factors such as de novo mutations in germ lines, exposure to toxic agents during life and epigenetic alterations (Lundstrom et al., 2010; Hultman et al., 2011). Advancing maternal age was also reported as a risk factor for autism (Parner et al., 2012). However, the explanations for these phenomena are still only speculative.

\section{Exome sequencing}

The development of the next generation sequencing has radically modified the scientific landscape, making it possible to sequence all exomes of any person. The power of this approach has been demonstrated by a number of studies which have identified pathogenic mutations in diseases that have been difficult to make by traditional genetic mapping.

Exons are coding regions of the genome responsible for the development of functional elements of the body, such as proteins. It has become clear that exome sequencing has great potential with respect to sporadic diseases and the identification of de novo mutations. Whole-exome sequencing (WES) of patient-parent trios has proven to be a successful screening tool for the identification of candidate genes causing complex phenotypes such as in ASD, learning disability, and schizophrenia (Choi et al., 2009; Bilguvar et al., 2010; Vissers et al., 2010; Gilman et al., 2011; Pagnamenta et al., 2012; Sanders et al., 2012).

O'Roak et al. (2012) studied all coding regions of the genome for parent-child trios exhibiting sporadic ASD, under the hypothesis that de novo mutations underlie a substantial fraction of the risk for developing ASD in families with no previous history of ASD or related phenotypes (simplex families). They observed that these mutations are overwhelmingly paternal in origin and positively correlated with paternal age, consistent with the modest increased risk for children of older fathers. It was also possible to estimate 384-821 loci which could be considered pathogenic.

The analysis the homozygosity is used to define loci that may be involved in recessive homozygous mutations that cause diseases characterized by genetic heterogeneity (Lencz et al., 2007; Nalls et al., 2009). Starting from this concept, Chahrour et al. (2012) used homozygosity analysis to identify probands from non-consanguineous families that showed evidence of distant shared ancestry, suggesting potentially recessive mutations. The WES of 16 probands revealed validated homozygous, potentially pathogenic, recessive mutations that segregated perfectly the disease in 4 of 16 families. The candidate genes that were found, UBE3B, CLTCL1, NCKAP5L, and ZNF18, encode proteins involved in neuronal activities.

Neale et al. (2012) studied 175 trios to assess de novo mutations. They found strong evidence that CHD8 and KATNAL2 are genuine autism risk factors. However, the small increase in the rate of de novo events, when taken together with the protein interaction results, were consistent with an important but limited role for these mutations in ASD, similar to that documented for de novo CNVs. According the authors, the data indicated that most of the observed de novo events are unconnected to ASD; those that do confer risk are distributed 
across many genes and are incompletely penetrant. The results support polygenic models in which spontaneous coding mutations in any of a large number of genes increases risk by 5to 20 -fold.

Iossifov et al. (2012) did not find significantly greater numbers of de novo missense mutations in ASD children versus unaffected, but gene-disrupting mutations (nonsense, splice site and frameshifts) were twice as frequent in the first group. Based on this differential and the number of recurrent and total targets of gene disruption they estimated between 350 and 400 autism susceptibility genes. Many of the genes are associated to the FMRP protein, reinforcing links between autism and synaptic plasticity. They suggested that genes associated to FMRP are especially targets of cognitive disorders that are dosage-sensitive.

Another aspect of exomes should also be considered. Mitochondria are cellular organelles that function to control energy production necessary for brain development and activity. Although each individual is typically characterized by a single mitochondrial DNA type, the fact is that each individual is a population of mitocondrial DNA genomes, and the presence of multiple types within an individual is termed heteroplasmy. Although each individual is typically characterized by a single mitocondrial DNA type, in fact to date, more than $400 \mathrm{mi}-$ tochondrial mutations have been associated with human disease and most were observed in heteroplasmic states, with pathogenic mutations coexisting with normal mitochondrial genomes. This suggests that the heteroplasmic level is of particular interest, as the disease phenotype becomes evident only when the percentage of mutant molecules exceeds a critical threshold value. Although this value differs for different mutations and in different tissues, it is usually in the range of $70 \% \sim 90 \%$. However, all the various techniques that have been employed to detect heteroplasmy have disadvantages. WES allows rapid detection of not only nuclear mutations but also mitochondrial mutations that also seem to be involved in the etiology of ASD. In this context, Li et al. (2012) sequenced the mitochondrial genome of 131 healthy individuals of European ancestry. In 32 individuals they identified 37 heteroplasmies at frequencies of $10 \%$ or higher at 34 different sites in the mitochondrial DNA indicating that variations commonly occur in mitochondrial DNA. These variations may impact on energy levels and influence brain development and function. Next generation sequencing should provide novel insights into genome-wide aspects of variation or heteroplasmy useful in the study of human disorders including autism.

All these results show that there are a lot of regions/genes being identified by very advanced methods, but no common etiology can be proposed. It is clear that whatever the proposed model to explain ASD, all aspects such as environmental, oligogenic, de novo mutations, polygenic, multifactorial, pleiotropic effects, combination of locus heterogeneity, heteroplasmy, among others, do not apply to all cases. Perhaps ASD emerge due to highly specific and individual biological patterns. The possibility of distinguishing primary and secondary effects will require a better understanding of the underlying biology and identification of the association between genetic and environmental factors within the phenotypic context of each family. The bottom line is that you must have a systemic view of the problem. 


\section{Conclusion}

Knowledge about the biological mechanisms involved in the etiology of ASD has increased significantly over the past three years. A genetic etiology of these disorders is certain, as certain as is their complexity. An understanding of the genetic factors involved is crucial to establish future intervention strategies. Although the current emphasis on deciphering ASD has demonstrated the necessity of multidisciplinary approaches, clinical geneticists have an important role in diagnosis and research of autism. The interpretation of this new genetic data requires a set of skills. It is important to know how to get and to interpret genetic tests, family pedigrees, to analyze dysmorphic, neurologic, and medical phenotypes, to interpret heterogeneity, develop rational genetic models, and to design researchs.

Despite the numerous known or, at least, allegedly involved causes of predisposition for ASD, the etiology is identified in a few cases ( 10\%) thereby highlighting the importance of genetic testing in affected individuals. The discovery of an etiological agent in a given case will, very probably, not interfere in treatment. However, this will reduce the distress of parents by explaining the cause of the problem and clarify about the possibility of familial recurrence. On identifying the etiologic agent, genetic counseling can be better targeted. Thus, a clinical-genetic evaluation of the patient is important as are the karyotypic analysis, molecular test for FRAXA, the investigation of inborn errors of metabolism, performing imaging tests and multiplex ligation-dependent probe amplification (MLPA) for at least three hot spots in ASD (15q11-13, 16p11.2 and 22q11.2). These are strategies available to better assess the etiology ASD. Certainly, in the not too distant future, other more sophisticated genetic research tools will be commercially available. The question that remains is whether the interpretation of results will accompany the speed of technical advances.

\section{Acknowledgements}

Financial support: Fundação de Amparo à Pesquisa do Estado de São Paulo (FAPESP).

\section{Author details}

Agnes Cristina Fett-Conte ${ }^{1}$, Ana Luiza Bossolani-Martins ${ }^{2}$ and Patrícia Pereira-Nascimento ${ }^{2}$

1 Medical School - FAMERP/FUNFARME, São José do Rio Preto, São Paulo, Brazil

2 IBILCE/UNESP, São José do Rio Preto, São Paulo, Brazil 


\section{References}

[1] Abrahams, B. S., \& Geschwind, D. H. (2008). Advances in autism genetics: on the threshold of a new Neurobiology. Nat Rev Genet, , 9(5), 341-355.

[2] Adzhubei, I. A., Schmidt, S., Peshkin, L., Ramensky, V. E., Gerasimova, A., Bork, P., Kondrashov, A. S., \& Sunyaev, S. R. (2010). A method and server for predicting damaging missense mutations. Nat Methods., , 7(4), 248-249.

[3] Alarcón, M., Abrahams, B. S., Stone, J. L., Duvall, J. A., Perederiy, J. V., Bomar, J. M., Sebat, J., Wigler, M., Martin, C. L., Ledbetter, D. H., Nelson, S. F., Cantor, R. M., \& Geschwind, D. H. (2008). Linkage, association, and gene-expression analyses identify CNTNAP2 as an autism-susceptibility gene. Am J Hum Genet., , 82(1), 150-159.

[4] Anney, R.; Klei, L.; Pinto, D.; Almeida, J.; Bacchelli, E.; Baird, G.; Bolshakova, N.; Bölte, S.; Bolton, P.F.; Bourgeron, T.; Brennan, S.; Brian, J.; Casey, J.; Conroy, J.; Correia, C.; Corsello, C.; Crawford, E.L.; de Jonge, M.; Delorme, R.; Duketis, E.; Duque, F.; Estes, A.; Farrar, P.; Fernandez, B.A.; Folstein, S.E.; Fombonne, E.; Gilbert, J.; Gillberg, C.; Glessner, J.T.; Green, A.; Green, J.; Guter, S.J.; Heron, E.A.; Holt, R.; Howe, J.L.; Hughes, G.; Hus, V.; Igliozzi, R.; Jacob, S.; Kenny, G.P.; Kim, C.; Kolevzon, A.; Kustanovich, V.; Lajonchere, C.M.; Lamb, J.A.; Law-Smith, M.; Leboyer, M.; Le Couteur, A.; Leventhal, B.L.; Liu, X.Q.; Lombard, F.; Lord, C.; Lotspeich, L.; Lund, S.C.; Magalhaes, T.R.; Mantoulan, C.; McDougle, C.J.; Melhem, N.M.; Merikangas, A.; Minshew, N.J.; Mirza, G.K.; Munson, J.; Noakes, C.; Papanikolaou, K.; Pagnamenta, A.T.; Parrini, B.; Paton, T.; Pickles, A.; Posey, D.J.; Poustka, F.; Ragoussis, J.; Regan, R.; Renshaw, K.; Roberts, W.; Roeder, K.; Roge, B.; Rutter, M.L.; Schlitt, S.; Shah, N.; Sheffield, V.C.; Soorya, L.; Sousa, I.; Stoppioni, V.; Sykes, N.; Tancredi, R.; Thompson, A.P.; Thomson, S.; Tryfon, A.; Tsiantis, J.; Van Engeland, H.; Vincent, J.B.; Volkmar, F.; Vorstman, J.; Wallace, S.; Wing, K.; Wittemeyer, K.; Wood, S.; Zurawiecki, D.; Zwaigenbaum, L.; Bailey, A.J.; Battaglia, A.; Cantor, R.M.; Coon, H.; Cuccaro, M.L.; Dawson, G.; Ennis, S.; Freitag, C.M.; Geschwind, D.H.; Haines, J.L.; Klauck, S.M.; McMahon, W.M.; Maestrini, E.; Miller, J.; Monaco, A.P.; Nelson, S.F.; Nurnberger, J.I.Jr,; Oliveira, G.; Parr, J.R.; Pericak-Vance, M.A.; Piven, J.; Schellenberg, G.D.; Scherer, S.W.; Vicente, A.M.; Wassink, T.H.; Wijsman, E.M.; Betancur, C.; Buxbaum, J.D.; Cook, E.H.; Gallagher, L.; Gill, M.; Hallmayer, J.; Paterson, A.D.; Sutcliffe, J.S.; Szatmari, P.; Vieland, V.J.; Hakonarson, H. \& Devlin, B. (2012). Individual common variants exert weak effects on risk for Autism Spectrum Disorders. Hum Mol Genet. 2012 Jul 26. Epub ahead of print.

[5] Angelidou, A., Asadi, S., Alysandratos, K. D., Karagkouni, A., Kourembanas, S., \& Theoharides, T. C. (2012). Perinatal stress, brain inflammation and risk of autism-Review and proposal. BMC Pediatr., , 12(1), 89.

[6] Arking, D. E., Cutler, D. J., Brune, C. W., Teslovich, T. M., West, K., Ikeda, M., Rea, A., Guy, M., Lin, S., Cook, E. H., \& Chakravarti, A. (2008). A common genetic variant 
in the neurexin superfamily member CNTNAP2 increases familial risk of autism. Am J Hum Genet., , 82(1), 160-164.

[7] Avchen, R. N., Wiggins, L. D., Devine, O., Van Naarden, Braun. K., Rice, C., Hobson, N. C., Schendel, D., \& Yeargin-Allsopp, M. Evaluation of a records-review surveillance system used to 880 determine the prevalence of autism spectrum disorders. ((2011). J Autism Dev Disord, , 41(2), 227-236.

[8] Bailey, A., Le Couteur, A., Gottesman, I., Bolton, P., Simonoff, E., Yuzda, E., \& Rutter, M. (1995). Autism as a strongly genetic disorder: evidence from a British twin study. Psychol Med, , 25(1), 63-77.

[9] Baird, G., Simonoff, E., Pickles, A., Chandler, S., Loucas, T., Meldrum, D., \& Charman, T. (2006). Prevalence of disorders of the autism spectrum in a population cohort of children in South Thames: the Special Needs and Autism Project (SNAP). Lancet, , 368(9531), 210-215.

[10] Ben-Ari, Y., Gaiarsa, J. L., Tyzio, R., \& Khazipov, R. (2007). GABA: a pioneer transmitter that excites immature neurons and generates primitive oscillations. Physiol Rev., , 87(4), 1215-1284.

[11] Berg, J. S., Brunetti-Pierri, N., Peters, S. U., Kang, S. H., Fong, C. T., Salamone, J., Freedenberg, D., Hannig, V. L., Prock, L. A., Miller, D. T., Raffalli, P., Harris, D. J., Erickson, R. P., Cunniff, C., Clark, G. D., Blazo, M. A., Peiffer, D. A., Gunderson, K. L., Sahoo, T., Patel, A., Lupski, J. R., Beaudet, A. L., \& Cheung, S. W. (2007). Speech delay and autism spectrum behaviors are frequently associated with duplication of the 7q11.23 Williams-Beuren syndrome region. Genet. Med., , 9(4), 427-441.

[12] Berkel, S., Marshall, C. R., Weiss, B., Howe, J., Roeth, R., Moog, U., Endris, V., Roberts, W., Szatmari, P., Pinto, D., Bonin, M., Riess, A., Engels, H., Sprengel, R., Scherer, S. W., \& Rappold, G. A. (2010). Mutations in the SHANK2 synaptic scaffolding gene in autism spectrum disorder and mental retardation. Nat. Genet., , 42(6), 489-491.

[13] Betancour, C. (2011). Etiological heterogeneityin autism spectrum disorders:more than 100 genetic and genomic disorders and still couting. Brain. Res, , 1380, 42-77.

[14] Biederman, J., Petty, C. R., Fried, R., Wozniak, J., Micco, J. A., Henin, A., Doyle, R., Joshi, G., Galdo, M., Kotarski, M., Caruso, J., Yorks, D., \& Faraone, S. V. (2010). Child Behavior Checklist Clinical Scales Discriminate Referred Youth With Autism Spectrum Disorder: A Preliminary Study. J Dev and Behav Pediatr, , 31(6), 485-490.

[15] Bilgüvar, K., Oztürk, A. K., Louvi, A., Kwan, K. Y., Choi, M., Tatli, B., Yalnizoğlu, D., Tüysüz, B., Cağlayan, A. O., Gökben, S., Kaymakçalan, H., Barak, T., Bakircioğlu, M., Yasuno, K., Ho, W., Sanders, S., Zhu, Y., Yilmaz, S., Dinçer, A., Johnson, M. H., Bronen, R. A., Koçer, N., Per, H., Mane, S., Pamir, M. N., Yalçinkaya, C., Kumandaş, S., Topçu, M., Ozmen, M., Sestan, N., Lifton, R. P., State, M. W., \& Günel, M. (2010). Whole-exome sequencing identifies recessive WDR62 mutations in severe brain malformations. Nature., , 467(7312), 207-210. 
[16] Bourgeron, T. (2009). A synaptic trek to autism. Curr Opin Neurobio., , 19(2), 231-234.

[17] Bronsard, G., Botbol, M., \& Tordjman, S. (2011). Aggression in low functioning children and adolescents with autistic disorder. PLoS One, , 21(5), e14358.

[18] Carter, M., Nikkel, S., Fernandez, B., Marshall, C., Noor, A., Lionel, A., Prasad, A., Pinto, D., Joseph-George, A., Noakes, C., Fairbrother-Davies, C., Roberts, W., Vincent, J., Weksberg, R., \& Scherer, S. W. (2011). Hemizygous deletions on chromosome 1p21.3 involving the DPYD gene in individuals with autism spectrum disorder. Clin Genet, , 80(5), 435-443.

[19] Castermans, D., Wilquet, V., Steyaert, J., Van de Ven, W., Fryns, J. P., \& Devriendt, K. (2004). Chromosomal anomalies in individuals with autism: a strategy towards the Identification of genes involved in. AutismAutism., , 8(2), 141-161.

[20] Chahrour, M. H., Yu, T. W., Lim, E. T., Ataman, B., Coulter, M. E., Hill, R. S., Stevens, C. R., Schubert, C. R., Autism, A. R. R. A., Sequencing, Collaboration., Greenberg, M. E., Gabriel, S. B., \& Walsh, C. A. (2012). Whole exome sequencing and homozygosity analysis implicate depolarization regulated neuronal genes in autism. Plos Genet., , 8(4), e1002635.

[21] Cheng, L., Ge, Q., Xiao, P., Sun, B., Ke, X., Bai, Y., \& Lu, Z. (2009). Association study between BDNF gene polymorphism and autism by threedimensional gel-based microarray. Int J Mol Sci., , 10(6), 2487-2500.

[22] Chess, S., Fernandez, P., \& Korn, S. (1978). Behavioral consequences of congenital rubella. J. Pediatr, , 93(4), 699-703.

[23] Chiocchetti, A., \& Klauck, S. M. (2011). Genetic analyses for identifying molecular mechanisms in autism spectrum disorders. Z Kinder Jugendpsychiatr Psychother, , 39(2), 101-111.

[24] Choi, M., Scholl, U. I., Ji, W., Liu, T., Tikhonova, I. R., Zumbo, P., Nayir, A., Bakkaloğlu, A., Ozen, S., Sanjad, S., Nelson-Williams, C., Farhi, A., Mane, S., \& Lifton, R. P. (2009). Genetic diagnosis by whole exome capture and massively parallel DNA sequencing. Proc the Nat Ac Sci U S A., , 106(45), 19096-19101.

[25] Christian, S. L., Brune, C. W., Sudi, J., Kumar, R. A., Liu, S., Karamohamed, S., Badner, J. A., Matsui, S., Conroy, J., Mc Quaid, D., Gergel, J., Hatchwell, E., Gilliam, T. C., Gershon, E. S., Nowak, N. J., Dobyns, W. B., Cook, E. H., \& Jr , . (2008). Novel submicroscopic chromosomal abnormalities detected in autism spectrum disorder. Biological Psychiatry, , 15(63), 1111-1117.

[26] Christianson, A. L., Chesler, N., \& Kromberg, J. G. (1994). Fetal valproate syndrome: clinical and neuro-developmental features in two sibling pairs. Dev. Med. Child Neurol. , 36(4), 361-369.

[27] Chrousos, G. P. (1995). The hypothalamic-pituitary-adrenal axis and immune-mediated inflammation. N Engl J Med, Vol. Vol. 18, (332), 1351-1362. 
[28] Coleman, M., \& Blass, J. P. Autism and lactic acidosis. ((1985). J. Autism Dev. Disord., , 15(1), 1-8.

[29] Coleman, M., \& Gillberg, C. (2012). The autisms. Oxford: Oxford University Press.

[30] Conrad, D. F., Keebler, J. E., De Pristo, M. A., Lindsay, S. J., Zhang, Y., Casals, F., Idaghdour, Y., Hartl, C. L., Torroja, C., Garimella, K. V., Zilversmit, M., Cartwright, R., Rouleau, G. A., Daly, M., Stone, E. A., Hurles, M. E., Awadalla, P., 10, , \& Genomes, Project. (2011). Variation in genome-wide mutation rates within and between human families. Nat. Genet., , 43(7), 712-714.

[31] Constantino, J. N., Zhang, Y., Frazier, T., Abbacchi, A. M., \& Law, P. (2010). Sibling recurrence and the genetic epidemiology of autism. Am J Psychiatry, , 167(11), 1349-1356.

[32] Cook, E. H., Jr , , \& Scherer, S. W. (2008). Copy-number variations associated with neuropsychiatric conditions. Nature, , 455(7215), 919-923.

[33] Cottrell, C. E., Bir, N., Varga, E., Alvarez, C. E., Bouyain, S., Zernzach, R., Thrush, D. L., Evans, J., Trimarchi, M., Butter, E. M., Cunningham, D., Gastier-Foster, J. M., Mc Bride, K. L., \& Herman, G. E. (2011). Contactin 4 as an autism susceptibility locus. Autism Res., , 4(3), 189-199.

[34] Currenti, S. A. (2010). Understanding and Determining the Etiology of Autism. Cell Molec Neurobiol, , 30(2), 161-171.

[35] Daniels, J. L., Forssen, U., Hultman, C. M., Cnattingius, S., Savitz, D. A., Feychting, M., \& Sparen, P. (2008). Parental psychiatric disorders associated with autism spectrum disorders in the offspring. Pediatrics, , 121(5), e1357-e1362.

[36] Dardeno, T. A., Chou, S. H., Moon, H. S., Chamberland, J. P., Fiorenza, C. G., \& Mantzoros, C. S. Leptin in human physiology and therapeutics ((2010). Front Neuroendocrinol, , 31(3), 377-393.

[37] Davis, J. M., \& Auten, R. L. (2010). Maturation of the antioxidant system and the effects on preterm birth. Semin Fetal Neonatal Med, , 15(4), 191-195.

[38] De Rubeis, S., Fernández, E., Buzzi, A., Di Marino, D., \& Bagni, C. (2012). Molecular and cellular aspects of mental retardation in the Fragile $X$ syndrome: from gene mutation/s to spine dysmorphogenesis. Adv Exp Med Biol, , 970, 517-551.

[39] Delahaye, A., Toutain, A., Aboura, A., Dupont, C., Tabet, A. C., Benzacken, B., Elion, J., Verloes, A., Pipiras, E., \& Drunat, S. (2009). Chromosome 22q13.3 deletion syndrome with a de novo interstitial 22q13.3 cryptic deletion disrupting SHANK3. Eur. J. Med. Genet., , 52(5), 328-332.

[40] Devlin, B., \& Scherer, S. W. (2012). Genetic architecture in autism spectrum disorders. Curr Opin Genet Dev., , 22(3), 229-237.

[41] Dhillon, S., Hellings, J. A., \& Butler, M. G. (2011). Genetics and mitochondrial abnormalities in autism spectrum disorders : a review. Curr Genomics, , 12(5), 322-332. 
[42] Durand, C. M., Betancur, C., Boeckers, T. M., Bockmann, J., Chaste, P., Fauchereau, F., Nygren, G., Rastam, M., Gillberg, I. C., Anckarsater, H., Sponheim, E., GoubranBotros, H., Delorme, R., Chabane, N., Mouren-Simeoni, M. C., de Mas, P., Bieth, E., Roge, B., Heron, D., Burglen, L., Gillberg, C., Leboyer, M., \& Bourgeron, T. (2007). Mutations in the gene encoding the synaptic scaffolding protein SHANK3 are associated with autism spectrum disorders. Nat. Genet., , 39(1), 25-27.

[43] Esposito, P., Gheorghe, D., Kandere, K., Pang, X., Conally, R., Jacobson, S., \& Theoharides, T. C. (2001). Acute stress increases permeability of the blood-brain-barrier through activation of brain mast cells. Brain Res, , 5(888), 117-127.

[44] Faras, H., Ateeqi, N. A., \& Tidmarsh, L. (2010). Autism spectrum disorders. Annals of Saudi Medicine, , 30(4), 295-300.

[45] Fernandez, B. A., Roberts, W., Chung, B., Weksberg, R., Meyn, S., Szatmari, P., Joseph-George, A. M., Mackay, S., Whitten, K., Noble, B., Vardy, C., Crosbie, V., Luscombe, S., Tucker, E., Turner, L., Marshall, C. R., \& Scherer, S. W. (2010). Phenotypic spectrum associated with de novo and inherited deletions and duplications at 16 p11.2 in individuals ascertained for diagnosis of autism spectrum disorder. J. Med. Genet., , 47(3), 195-203.

[46] Fillano, J. J., Goldenthal, M. J., Rhodes, C. H., \& Marin-Garcia, J. (2002). Mitochondrial dysfunction in patients with hypotonia, epilepsy, autism, and developmental delay: HEADD syndrome. J. Child Neurol. , 17(6), 435-439.

[47] Geschwind, D. H. (2011). Genetics of autism spectrum disorders. Trends Cogn Sci, , 15(9), 409-416.

[48] Gillberg, C. (1998). Chromosomal disorders and autism. J. Autism Dev. Disord, , 28(5), 415-425.

[49] Gilman, S. R., Iossifov, I., Levy, D., Ronemus, M., Wigler, M., \& Vitkup, D. (2011). Rare de novo variants associated with autism implicate a large functional network of genes involved in formation and function of synapses. Neuron., , 70(5), 898-907.

[50] Goldberg, W. A., Jarvis, K. L., Osann, K., Laulhere, T. M., Straub, C., Thomas, E., Filipek, P., \& Spence, M. A. (2005). Brief report: Early social communication behaviors in the younger siblings of children with autism. J Autism Dev Disord., , 35(5), 657-664.

[51] Gould, T. D., \& Gottesman, I. I. (2006). Psychiatric endophenotypes and the development of valid animal models. Genes Brain Behav., , 5, 113-119.

[52] Graf, W. D., Marin-Garcia, J., Gao, H. G., Pizzo, S., Naviaux, R. K., Markusic, D., Barshop, B. A., Courchesne, E., \& Haas, R. H. (2000). Autism associated with the mitochondrial DNA G8363A transfer RNA(Lys) mutation. J. Child Neurol., , 15(6), 357-361.

[53] Griswold, A. J., , D., Sacharow, S. J., Robinson, J. L., Jaworski, J. M., Wright, H. H., Abramson, R., K., , Lybaek, H., Oyen, N., Cuccaro, M. L., Gilbert, J. R., \& Pericak- 
Vance, M. A. (2011). A de novo $1.5 \mathrm{mb}$ microdeletion on chromosome 14q23.2- 23.3 in a patient with autism and spherocytosis. Autism Res., , 4(3), 221-227.

[54] Griswold, A. J., , D., Cukier, H. N., Nações, L. D., Schmidt, M. A., Chung, R. H., Jaworski, J. M., Salyakina, D., Konidari, eu., Whitehead, P. L., Wright, H. H., Abramson, R. K., Williams, S. M., Menon, R., Martin, E. R., Haines, J. L., Gilbert, J. R., Cuccaro, M. L., Pericak-M, A., \& Vance, . (2012). Evaluation of copy number variations reveals novel candidate genes in autism spectrum disorders associated pathways. Hum Mol Genet., , 21(15), 3513-3523.

[55] Gurrieri, F. (2012). Working up autism: the practical role of medical genetics. Am J Med Genet C Semin Med Genet. , 160C(2), 104-110.

[56] Hallmayer, J., Cleveland, S., Torres, A., Phillips, J., Cohen, B., Torigoe, T., Miller, J., Fedele, A., Collins, J., Smith, K., Lotspeich, L., Croen, L. A., Ozonoff, S., Lajonchere, C., Grether, J. K., \& Risch, N. (2011). Genetic heritability and shared environmental factors among twin pairs with autism. Arch. Gen. Psychiatry, , 68(1), 1095-1102.

[57] Hultman, C. M., Sandin, S., Levine, S. Z., Lichtenstein, P., \& Reichenberg, A. (2011). Advancing paternal age and risk of autism: new evidence from a populationbased study and a meta-analysis of epidemiological studies. Mol Psychiatr.y, , 16(12), 1203-1212.

[58] Hussman, J. P., Chung, R. H., Griswold, A. J., Jaworkski, J. M., Salyakina, D., , D., Konidari, I.., Whitehead, P. L., Vance, J. M., Martin, E. R., Cuccaro, M. L., Gilbert, J. R., Haines, J. L., \& Pericak-Vance, M. A. (2011). A noise-reduction GWAS analysis implicates altered regulation of neurite outgrowth and guidance in autism. Mol. Autism, , 2(1), 1 .

[59] Iossifov, I., Ronemus, M., Levy, D., Wang, Z., Hakker, I., Rosenbaum, J., Yamrom, B., Lee, Y. H., Narzisi, G., Leotta, A., Kendall, J., Grabowska, E., , B., Marks, S., Rodgers, L., Stepansky, A., Troge, J., Andrews, P., Bekritsky, M., Pradhan, K., Ghiban, E., Kramer, M., Parla, J., Demeter, R., Fulton, L. L., Fulton, R. S., Magrini, V. J., Ye, K., Darnell, J. C., Darnell, R. B., Mardis, E. R., Wilson, R. K., Schatz, M. C., Mc Combie, W. R., \& Wigler, M. (2012). De novo gene disruptions in children on the autistic spectrum. Neuron., , 74(2), 285-299.

[60] Jiao, Y., Chen, R., Ke, X., Cheng, L., Chu, K., Lu, Z., \& Herskovits, E. H. (2011). Predictive models for subtypes of autism spectrum disorder based on single-nucleotide polymorphisms and magnetic resonance imaging. Adv Med Sci., , 56(2), 334-342.

[61] Joshi, S. R., Mehendale, S. S., Dangat, K. D., Kilari, A. S., Yadav, H. R., \& Taralekar, V. S. (2008). High maternal plasma antioxidant concentrations associated with preterm delivery. Ann Nutr Metab, , 53(3-4), 276-282.

[62] Kaufmann, W. E., Cortell, R., Kau, A. S., Bukelis, I., Tierney, E., Gray, R. M., Cox, C., Capone, G. T., \& Stanard, P. (2004). Autism spectrum disorder in fragile X syndrome: communication, social interaction, and specific behaviors. Am. J. Med. Genet A., , 129(3), 225-234. 
[63] King, M., \& Bearman, P. (2009). Diagnostic change and the increased prevalence of 786 autism. Inter J Epidemiol, , 38(5), 1224-1234.

[64] Klein, R. J., Xu, X., Mukherjee, S., Willis, J., \& Hayes, J. (2010). Successes of genomewide association studies. Cell, author reply 353-355., 142(3), 350-351.

[65] Kumar, R. A., Karamohamed, S., Sudi, J., Conrad, D. F., Brune, C., Badner, J. A., Gilliam, T. C., Nowak, N. J., Cook, E. H., Jr , , Dobyns, W. B., \& Christian, S. L. (2008). Recurrent 16p11.2 microdeletions in autism. Hum. Mol. Genet., , 17(4), 628-638.

[66] Laje, G., Morse, R., Richter, W., Ball, J., Pao, M., \& Smith, A. C. (2010). Autism spectrum features in Smith-Magenis syndrome. Am. J. Med. Genet. C Semin. Med. Genet., , $154 \mathrm{C}(4), 456-462$.

[67] Lauritsen, M. B., Pedersen, C. B., \& Mortensen, P. B. (2005). Effects of familial risk factors and place of birth on the risk of autism: a nationwide register-based study. J Child Psychol Psychiatry, , 46(9), 963-971.

[68] Lee, C., \& Scherer, S. W. (2010). The clinical context of copy number variation in the human genome. Expert Rev Mol Med., , 12, e8.

[69] Lencz, T., Lambert, C., De Rosse, P., Burdick, K. E., Morgan, T. V., Kane, J. M., Kucherlapati, R., \& Malhotra, A. K. (2007). Runs of homozygosity reveal highly penetrant recessive loci in schizophrenia. Proc Natl Acad Sci U S A., , 104(50), 19942-19947.

[70] Levinson, D. F., Duan, J., Oh, S., Wang, K., Sanders, A. R., Shi, J., Zhang, N., Mowry, B. J., Olincy, A., Amin, F., Cloninger, C. R., Silverman, J. M., Buccola, N. G., Byerley, W. F., Black, D. W., Kendler, K. S., Freedman, R., Dudbridge, F., Pe'er, I., Hakonarson, H., Bergen, S. E., Fanous, A. H., Holmans, P. A., Gejman, P. V., \& (2011, . (2011). Copy number variants in schizophrenia: confirmation of five previous findings and new evidence for 3q29 microdeletions and VIPR2 duplications. Am J Psychiatry, , 168(3), 302-316.

[71] Levy, D., Ronemus, M., Yamrom, B., Lee, Y. H., Leotta, A., Kendall, J., Marks, S., Lakshmi, B., Pai, D., Ye, K., Krieger, A., Yoon, S., Troge, J., Rodgers, L., Iossifov, I., \& Wigler, M. (2011). Rare de novo and transmitted copy-number variation in autistic spectrum disorders. Neuron., , 70(5), 886-897.

[72] Li, H., Yamagata, T., Mori, M., \& Momoi, M. Y. (2005). Absence of causative mtations and presence of autism-related allele in FOXP2 in Japanese autistic patients. Brain Dev., , 27(3), 207-210.

[73] Li, M., Schönberg, A., Schaefer, M., Schroeder, R., Nasidze, I., \& Stoneking, M. Detecting heteroplasmy from high-throughput sequencing of complete human mitochondrial DNA genomes. ((2012). Am J Hum Genet., , 87(2), 237-249.

[74] Li, X., Zou, H., \& Brown, W. T. (2012). Genes associated with autism spectrum disorders. Brain Res Bull. Epud already print. 
[75] Lichtenstein, P., Carlstrom, E., Rastam, M., Gillberg, C., \& Anckarsater, H. (2010). The genetics of autism spectrum disorders and related neuropsychiatric disorders in childhood. Am J Psychiatry, , 1(11), 1357-1363.

[76] Limperopoulos, C., Bassan, H., Sullivan, N. R., Soul, J. S., Robertson, R. L., Jr , , Moore, M., Ringer, S. A., Volpe, J. J., \& du, Plessis. A. J. (2008). Positive screening for autism in ex-preterm infants: prevalence and risk factors. Pediatrics, , 121(4), 758-765.

[77] Lin, P. I., Chien, Y. L., Wu, Y. Y., Chen, C. H., Gau, S. S., Huang, Y. S., Liu, S. K., Tsai, W. C., \& Chiu, Y. N. (2012). The WNT2 gene polymorphism associated with speech delay inherent to autism. Res Dev Disabil., , 5(33), 1533-1540.

[78] Lintas, C., \& Persico, A. M. (2009). Autistic phenotypes and genetic testing: state-ofthe- 821 art for the clinical geneticist. J Med Genet, , 46(1), 1-8.

[79] Lombard, J., Autism, a., \& mitochondrial, disorder?. (1998). Med. Hypotheses, , 50(6), 497-500.

[80] Losh, M., Childress, D., Lam, K., \& Piven, J. (2008). Defining key features of the broad autism phenotype: a comparison across parents of multiple- and single-incidence autism families. Am J Genet B Neuropsychiatri Genet., , 147B(4), 424-433.

[81] Lu, A. T., Yoon, J., Geschwind, D. H., \& Cantor, R. M. (2011). QTL replication and targeted association highlight the nerve growth factor gene for nonverbal communication deficits in autism spectrum disorders. Mol Psychiatry. Epub ahead of print.

[82] Lundstrom, S., Haworth, C. M., Carlstrom, E., Gillberg, C., Mill, J., Rastam, M., Hultman, C. M., Ronald, A., Anckarsäter, H., Plomin, R., Lichtenstein, P., \& Reichenberg, A. (2010). Trajectories leading to autism spectrum disorders are affected by paternal age: findings from two nationally representative twin studies. J Child Psychol Psychiatry, , 51(7), 850-856.

[83] , D., Salyakina, D., Jaworski, J. M., Konidari, I., Whitehead, P. L., Andersen, A. N., Hoffman, J. D., Slifer, S. H., Hedges, D. J., Cukier, H. N., Griswold, A. J., Mc Cauley, J. L., Beecham, G. W., Wright, H. H., Abramson, R. K., Martin, E. R., Hussman, J. P., Gilbert, J. R., Cuccaro, M. L., Haines, J. L., \& Pericak-Vance, M. A. (2009). A genomewide association study of autism reveals a common novel risk locus at 5p14.1. Ann. Hum. Genet., , 73(Pt3), 263-273.

[84] Malhotra, D., \& Sebat, J. . (2012). CNVs: harbingers of a rare variant revolution in psychiatric genetics. Cell, , 148(6), 1223-1241.

[85] Manolio, T. A., Collins, F. S., Cox, N. J., Goldstein, D. B., Hindorff, L. A., Hunter, D. J., Mc Carthy, M. I., Ramos, E. M., Cardon, L. R., Chakravarti, A., Cho, J. H., Guttmacher, A. E., Kong, A., Kruglyak, L., Mardis, E., Rotimi, C. N., Slatkin, M., Valle, D., Whittemore, A. S., Boehnke, M., Clark, A. G., Eichler, E. E., Gibson, G., Haines, J. L., Mackay, T. F., Mc Carroll, S. A., \& Visscher, P. M. (2009). Finding the missing heritability of complex diseases. Nature, , 461(7265), 747-753. 
[86] Manzi, B., Loizzo, A. L., Giana, G., \& Curatolo, P. (2008). Autism and metabolic diseases. J. Child Neurol., , 23(3), 307-314.

[87] Marshall, C. R., Noor, A., Vincent, J. B., Lionel, A. C., Feuk, L., Skaug, J., Shago, M., Moessner, R., Pinto, D., Ren, Y., Thiruvahindrapduram, B., Fiebig, A., Schreiber, S., Friedman, J., Ketelaars, C. E., Vos, Y. J., Ficicioglu, C., Kirkpatrick, S., Nicolson, R., Sloman, L., Summers, A., Gibbons, C. A., Teebi, A., Chitayat, D., Weksberg, R., Thompson, A., Vardy, C., Crosbie, V., Luscombe, S., Baatjes, R., Zwaigenbaum, L., Roberts, W., Fernandez, B., Szatmari, P., \& Scherer, S. W. (2008). Structural variation of chro- mosomes in autism spectrum disorder. Am. J. Hum. Genet., , 82(2), 477-488.

[88] Marteleto, M. R., Lima, e., Menezes, C. G., Tamanaha, A. C., Chiari, B. M., Perissinoto, J., \& (2008, . (2008). Administration of the Autism Behavior Checklist: agreement between parents and professionals' observations in two intervention contexts. Rev Bras de Psiquiatr, , 30(3), 203-208.

[89] Mefford, H. C., Batshaw, M. L., \& Hoffman, E. P. (2012). Genomics, Intellectual Disability, and Autism. N Engl J Med., , 366(8), 733-743.

[90] Miles, J. H. (2011). Autism spectrum disorders-a genetics review. Genet. Med., , 13(4), 278-294.

[91] Miller, D. T., Adam, M. P., Aradhya, S., Biesecker, L. G., Brothman, A. R., Carter, N. P., Church, D. M., Crolla, , , J. A., Eichler, E. E., Epstein, C. J., Faucett, W. A., Feuk, L., Friedman, J. M., Hamosh, A., Jackson, L., Kaminsky, E. B., Kok, K., Krantz, I. D., Kuhn, R. M., Lee, C., Ostell, J. M., Rosenberg, C., Scherer, S. W., Spinner, N. B., Stavropoulos, D. J., Tepperberg, J. H., Thorland, E. C., Vermeesch, J. R., Waggoner, D. J., Watson, M. S., Martin, C. L., \& Ledbetter, D. H. (2010). Consensus statement: chromosomal microarray is a first-time clinical diagnostic test for individuals with developmental disabilities or congenital anomalies. Am J Hum Genet., , 86, 749-764.

[92] Mitchell, K. J. (2011). The genetics of neurodevelopmental disease. Curr Opin Neurobiol, , 21(1), 197-203.

[93] Morrow, E. M., Yoo, S. Y., Flavell, S. W., Kim, T. K., Lin, Y., Hill, R. S., Mukaddes, N. M., Balkhy, S., Gascon, G., Hashmi, A., Al-Saad, S., Ware, J., Joseph, R. M., Greenblatt, R., Gleason, D., Ertelt, J. A., Apse, K. A., Bodell, A., Partlow, J. N., Barry, B., Yao, H., Markianos, K., Ferland, R. J., Greenberg, M. E., \& Walsh, C. A. Identifyin autism loci and genes by tracing recent shared ancestry. ((2010). Science, , 321(5886), 218-223.

[94] Munesue, T., Ono, Y., Mutoh, K., Shimoda, K., Nakatani, H., \& Kikuchi, M. (2008). High prevalence of bipolar disorder comorbidity in adolescents and young adults with high-functioning autism spectrum disorder: a preliminary study of 44 outpatients. J Affect Disord, , 111(2-3), 170-175.

[95] Neale, B. M., Kou, Y., Liu, L., Ma'ayan, A., Samocha, K. E., Sabo, A., Lin, C. F., Stevens, C., Wang, L. S., Makarov, V., Polak, P., Yoon, S., Maguire, J., Crawford, E. L., 
Campbell, N. G., Geller, E. T., Valladares, O., Schafer, C., Liu, H., Zhao, T., Cai, G., Lihm, J., Dannenfelser, R., Jabado, O., Peralta, Z., Nagaswamy, U., Muzny, D., Reid, J. G., Newsham, I., Wu, Y., Lewis, L., Han, Y., Voight, B. F., Lim, E., Rossin, E., Kirby, A., Flannick, J., Fromer, M., Shakir, K., Fennell, T., Garimella, K., Banks, E., Poplin, R., Gabriel, S., De Pristo, M., Wimbish, J. R., Boone, B. E., Levy, S. E., Betancur, C., Sunyaev, S., Boerwinkle, E., Buxbaum, J. D., Cook, E. H., Jr , , Devlin, B., Gibbs, R. A., Roeder, K., Schellenberg, G. D., Sutcliffe, J. S., \& Daly, M. J. (2012). Patterns and rates of exonic de novo mutations in autism spectrum disorders. Nature. , 485(7397), $242-245$.

[96] Nalls, M. A., Guerreiro, R. J., Simon-Sanchez, J., Bras, J. T., Traynor, B. J., Gibbs, J. R., Launer, L., Hardy, J., \& Singleton, A. B. (2009). Extended tracts of homozygosity identify novel candidate genes associated with late-onset Alzheimer's disease. Neurogenetics., , 10(3), 183-190.

[97] Naviaux, R. K. (2008). Mitochondrial control of epigenetics. Cancer Biol. Ther., , 7(8), 1191-1193.

[98] Nicholas, B., Rudrasingham, V., Nash, S., Kirov, G., Owen, M. J., \& Wimpory, D. C. (2007). Association of Per1 and Npas2 with autistic disorder: support for the clock genes/social timing hypothesis. Mol. Psychiatry, , 12(6), 581-592.

[99] Nijmeijer, J. S., Arias-Vásquez, A., Rommelse, N. N., Altink, M. E., Anney, R. J., Asherson, P., Banaschewski, T., Buschgens, C. J., Fliers, E. A., Gill, M., Minderaa, R. B., Poustka, L., Sergeant, J. A., Buitelaar, J. K., Franke, B., Ebstein, R. P., Miranda, A., Mulas, F., Oades, R. D., Roeyers, H., Rothenberger, A., Sonuga-Barke, E. J., Steinhausen, H. C., Faraone, S. V., Hartman, C. A., \& Hoekstra, P. J. (2011). Identifying loci for the overlap between attention deficit/hyperactivity disorder and autism spectrum disorder using a genome-wide QTL linkage approach. J Am Acad Child Adolesc Psychiatry. , 7(49), 675-685.

[100] Nowakowska, B. A., de Leeuw, N., Ruivenkamp, C. A., Sikkema-Raddatz, B., Crolla, J. A., Thoelen, R., Koopmans, M., den, Hollander. N., van Haeringen, A., van der Kevie-Kersemaekers, , Pfundt, R., Mieloo, H., van Essen, T., de Vries, B. B., Verde, A., Reardon, W., Fryns, J. P., \& Vermeesch, J. R. (2012). Parental insertional balanced translocations are an important cause of apparently de novo CNVs in patients with developmental anomalies. Eur J Hum Genet, , 20(2), 66-170.

[101] O’Roak, B. J., Deriziotis, P., Lee, C., Vives, L., Schwartz, J. J., Girirajan, S., Karakoc, E., Mackenzie, A. P., Ng, S. B., Baker, C., Rieder, M. J., Nickerson, D. A., Bernier, R., Fisher, S. E., Shendure, J., \& Eichler, E. E. (2011). Exome sequencing in sporadic autism spectrum disorders identifies severe de novo mutations. Nat Genet, , 43(8), 585-589.

[102] O’Roak, B. J., Vives, L., Girirajan, S., Karakoc, E., Krumm, N., Coe, B. P., Levy, R., Ko, A., Lee, C., Smith, J. D., Turner, E. H., Stanaway, I. B., Vernot, B., Malig, M., Baker, C., Reilly, B., Akey, J. M., Borenstein, E., Rieder, M. J., Nickerson, D. A., Bernier, R., 
Shendure, J., \& Eichler, E. E. (2012). Sporadic autism exomes reveal a highly interconnect protein network of de novo mutations. Nature., , 485(7397), 246-250.

[103] Pagnamenta, A. T., Lise, S., Harrison, V., Stewart, H., Jayawant, S., Quaghebeur, G., Deng, A. T., Murphy, V. E., Akha, E., Rimmer, A., Mathieson, I., Knight, S. J., Kini, U., Taylor, J. C., \& Keays, D. A. (2012). Exome sequencing can detect pathogenic mosaic mutations present at low allele frequencies. J Hum Genet., , 57(1), 70-72.

[104] Parner, E. T., Baron-Cohen, S., Lauritsen, M. B., Jorgensen, M., Schieve, L. A., Yeargin-Allsopp, M., \& Obel, C. (2012). Parental age and autism spectrum disorders. Ann Epidemiol, , 22(3), 143-150.

[105] Persico, A. M., \& Bourgeron, T. (2008). Searching for ways out of the autism maze:genetic, epigenetic and environmental clues. Trends Neuroscience., , 29(7), 349-358.

[106] Phelan, M. C. (2008). Deletion 22q13.3 syndrome. Orphanet. J. Rare Dis, , 27(3), 14.

[107] Pickles, A., Starr, E., Kazak, S., Bolton, P., Papanikolaou, K., Bailey, A., Goodman, R., Rutter, M., \& (2000, . (2000). Variable expression of the autism broader phenotype: Findings from extended pedigrees. J Child Psychol Psychiatry., , 41(4), 491-502.

[108] Pinto, D.; Pagnamenta, A.T.; Klei, L.; Anney, R.; Merico, D.; Regan, R.; Conroy, J.; Magalhaes, T.R.; Correia, C. \& Abrahams, B.S.; Almeida, J.; Bacchelli, E.; Bader, G.D.; Bailey, A.J.; Baird, G.; Battaglia, A.; Berney, T.; Bolshakova, N.; Bölte, S.; Bolton, P.F.; Bourgeron, T.; Brennan, S.; Brian, J.; Bryson, S.E.; Carson, A.R.; Casallo, G.; Casey, J.; Chung, B.H.;Cochrane, L.; Corsello, C.; Crawford, E.L.; Crossett, A.; Cytrynbaum, C.; Dawson, G.; de Jonge, M.; Delorme, R.; Drmic, I.; Duketis, E.; Duque, F.; Estes, A.; Farrar, P.; Fernandez, B.A.; Folstein, S.E.; Fombonne, E.; Freitag, C.M.; Gilbert, J.; Gillberg, C.; Glessner, J.T.; Goldberg, J.; Green, A.; Green, J.; Guter, S.J.; Hakonarson, H.; Heron, E.A.; Hill, M.; Holt, R.; Howe, J.L.; Hughes, G.; Hus, V.; Igliozzi, R.; Kim, C.; Klauck, S.M.; Kolevzon, A.; Korvatska, O.; Kustanovich, V.; Lajonchere, C.M.; Lamb, J.A.; Laskawiec, M.; Leboyer, M.; Le Couteur, A.; Leventhal, B.L.; Lionel, A.C.; Liu, X.Q.; Lord, C.; Lotspeich, L.; Lund, S.C.; Maestrini, E.; Mahoney, W.; Mantoulan, C.; Marshall, C.R.; McConachie, H.; McDougle, C.J.; McGrath, J.; McMahon, W.M.; Merikangas, A.; Migita, O.; Minshew, N.J.; Mirza, G.K.; Munson, J.; Nelson, S.F.; Noakes, C.; Noor, A.; Nygren, G.; Oliveira, G.; Papanikolaou, K.; Parr, J.R.; Parrini, B.; Paton, T.; Pickles, A.; Pilorge, M.; Piven, J.; Ponting, C.P.; Posey, D.J.; Poustka, A.; Poustka, F.; Prasad, A.; Ragoussis, J.; Renshaw, K.; Rickaby, J.; Roberts, W.; Roeder, K.; Roge, B.; Rutter, M.L.; Bierut, L.J.; Rice, J.P.; Salt, J.; Sansom, K.; Sato, D.; Segurado, R.; Sequeira, A.F.; Senman, L.; Shah, N.; Sheffield, V.C.; Soorya, L.; Sousa, I.; Stein, O.; Sykes, N.; Stoppioni, V.; Strawbridge, C.; Tancredi, R.; Tansey, K.; Thiruvahindrapduram, B.; Thompson, A.P.; Thomson, S.; Tryfon, A.; Tsiantis, J.; Van, Engeland, H.; Vincent, J.B.; Volkmar, F.; Wallace, S.; Wang, K.; Wang, Z.; Wassink, T.H.; Webber, C.; Weksberg, R.; Wing, K.; Wittemeyer, K.; Wood, S.; Wu, J.; Yaspan, B.L.; Zurawiecki, D.; Zwaigenbaum, L.; Buxbaum, J.D.; Cantor, R.M.; Cook, E.H.; Coon, H.; Cuccaro, M.L.; Devlin, B.; Ennis, S.; Gallagher, L.; Geschwind, D.H.; Gill, M.; Haines, J.L.; Hallmayer, J.;Miller, J.; Monaco, A.P.; Nurnberger, J.I.Jr.; Paterson, A.D.; 
Pericak-Vance, M.A.; Schellenberg, G.D.; Szatmari, P.; Vicente, A.M.; Vieland, V.J.; Wijsman, E.M. \& Scherer S.W. ((2010). ). Functional impact of global rare copy number variation in autism spectrum disorders. Nature., Vol. 466, No. 7304, pp.368-372.

[109] Pons, R., Andreu, A. L., Checcarelli, N., Vila, M. R., Engelstad, K., Sue, C. M., Shungu, D., Haggerty, R., de Vivo, D. C., \& Di Mauro, S. (2004). Mitochondrial DNA abnormalities and autistic spectrum disorders. J. Pediatr, , 144(1), 81-85.

[110] Poot, M., Beyer, V., Schwaab, I., Damatova, N., Van't, Slot. R., Prothero, J., Holder, S. E., \& Haaf, T. (2009). Disruption of CNTNAP2 and additional structural genome changes in a boy with speech delay and autism spectrum disorder. Neurogenetics, , 11(1), 81-89.

[111] Ramocki, M. B., \& Zoghbi, H. Y. (2008). Failure of neuronal homeostasi results in common neuropsychiatric phenotypes. Nature., , 455(7215), 912-918.

[112] Ramoz, N., Reichert, J. G., Smith, C. J., Silverman, J. M., Bespalova, I. N., Davis, K. L., \& Buxbaum, J. D. (2004). Linkage and association of the mitochondrial aspartate/ glutamate carrier SLC25A12 gene with autism. Am. J. Psychiatry, , 161(4), 662-669.

[113] Rapin, I., \& Goldman, S. (2008). The Brazilian CARS: a standardized screening tool for autism. Jornal de Pediatr (Rio J), , 84(6), 473-475.

[114] Rees, E., Moskvina, V., Owen, M. J., O’Donovan, M. C., \& Kirov, G. (2011). De novo rates and selection of schizophrenia-associated copy number variants. Biol. Psychiatry, , 70(12), 1109-1114.

[115] Rosenberg, R. E., Law, J. K., Yenokyan, G., Mc Gready, J., Kaufmann, W. E., \& Law, P. A. (2009). Characteristics and concordance of autism spectrum disorders among 277 twin pairs. Arch. Pediatr. Adolesc. Med., , 163(10), 907-914.

[116] Sanders, J. L. (2009). Qualitative or Quantitative Differences Between Asperger's Disorder and Autism? Historical Considerations. J Autism Dev Disord, , 39, 1560-1567.

[117] Sanders, S. (2011). Whole-exome sequencing: a powerful technique for identifying novel genes of complex disorders. Clinical Genetics, , 79(2), 132-133.

[118] Sanders, S.J.; Ercan-Sencicek, A.G.; Hus, V.; Luo, R.; Murtha, M.T.; Moreno-De-Luca, D.; Chu, S.H.; Moreau, M.P.; Gupta, A.R.; Thomson, S.A.; Mason, C.E.; Bilguvar, K.; CelestinoSoper, P.B.; Choi, M.; Crawford, E.L.; Davis, L.; Wright, N.R.; Dhodapkar, R.M.; DiCola, M.; DiLullo, N.M.; Fernandez, T.V.; Fielding-Singh, V.; Fishman, D.O.; Frahm, S.; Garagaloyan, R.; Goh, G.S.; Kammela, S.; Klei, L.; Lowe, J.K.; Lund, S.C.; McGrew, A.D.; Meyer, K.A.; Moffat, W.J.; Murdoch, J.D.; O’Roak, B.J.; Ober, G.T.; Pottenger, R.S.; Raubeson, M.J.; Song, Y.; Wang, Q.; Yaspan, B.L.; Yu, T.W.; Yurkiewicz, I.R.; Beaudet, A.L.; Cantor, R.M.; Curland, M.; Grice, D.E.; Gunel, M.; Lifton, R.P.; Mane, S.M.; Martin, D.M.; Shaw, C.A.; Sheldon, M.; Tischfield, J.A.; Walsh, C.A.; Morrow, E.M.; Ledbetter, D.H.; Fombonne, E.; Lord, C.; Martin, C.L.; Brooks, A.I.; Sutcliffe, J.S.; Cook, E.H. Jr.; Geschwind, D.; Roeder, K.; Devlin, B. \& State, M.W. ((2011). ) Multiple recurrent de novo CNVs, including duplications of the 7q11.23 
Williams syndrome region, are strongly associated with autism. Neuron., Vol. 70, No. 5, pp. 863-885.

[119] Sanders, S. J., Murtha, M. T., Gupta, A. R., Murdoch, J. D., Raubeson, M. J., Willsey, A. J., Ercan-Sencicek, A. G., Di Lullo, N. M., Parikshak, N. N., Stein, J. L., Walker, M. F., Ober, G. T., Teran, N. A., Song, Y., El -Fishawy, P., Murtha, R. C., Choi, M., Overton, J. D., Bjornson, R. D., Carriero, N. J., Meyer, K. A., Bilguvar, K., Mane, S. M., Sestan, N., Lifton, R. P., Günel, M., Roeder, K., Geschwind, D. H., Devlin, B., \& State, M. W. (2012). De novo mutations revealed by whole-exome sequencing are strongly associated with autism. Nature., , 485(7397), 237-241.

[120] Sato, F. P., Paula, C. S., Lowenthal, R., Nakano, E. Y., Brunoni, D., Schwartzman, J. S., \& Mercadante, M. T. (2009). Instrument to screen cases of pervasive developmental disorder: a preliminary indication of validity. Rev Bras de Psiquiatr, , 31(1), 30-33.

[121] Scott Van, Zeeland. A. A., Abrahams, B. S., Alvarez-Retuerto, A. I., Sonnenblick, L. I., Rudie, J. D., Ghahremani, D., Mumford, J. A., Poldrack, R. A., Dapretto, M., Geschwind, D. H., \& Bookheimer, S. Y. (2010). Altered functional connectivity in frontal lobe circuits is associated with variation in the autism risk gene CNTNAP2. Sci Transl Med., , 2(56), 56ra80.

[122] Sebat, J., Lakshmi, B., Malhotra, D., Troge, J., Lese-Martin, C., Walsh, T., Yamrom, B., Yoon, S., Krasnitz, A., Kendall, J., Leotta, A., Pai, D., Zhang, R., Lee, Y. H., Hicks, J., Spence, S. J., Lee, A. T., Puura, K., Lehtimäki, T., Ledbetter, D., Gregersen, P. K., Bregman, J., Sutcliffe, J. S., Jobanputra, V., Chung, W,., Warburton, D., King, M. C., Skuse, D., Geschwind, D. H., Gilliam, T. C., Ye, K., \& Wigler, M. (2007). Strong association of de novo copy number mutations with autism. Science, , 316(5823), 445-449.

[123] Segurado, R., Conroy, J., Meally, E., Fitzgerald, M., Gill, M., \& Gallagher, L. (2005). Confirmation of association between autism and the mitochondrial aspartate/glutamate carrier SLC25A12 gene on chromosome 2q31. Am J Psychiatry., , 162(11), 2182-2184.

[124] Shen, Y., Morre, K. A., Holm, I. A., Bridgemohan, C., Sobeih, M. M., Caronna, E. B., Miller, K. J., Frazier, J. A., Silverstein, I., Picker, J., Weissman, L., Raffalli, P. S., J.este, Demmer. L. A., Peters, H. K., Brewster, S. J., Kowalczyk, S. J., Rosen-Sheidley, B. C., Mc Gowan, Duda. A. W. 3., Lincoln, S. A., Lowe, K. R., Schonwald, A., Robbins, M., Hisama, F., Wolff, R., Becker, R., Nasir, R., Urion, D. K., Milunsky, J. M., Rappaport, L., Gusella, J. F., Walsh, C. A., Wu, B. L., Miller, D. T., autismo, Consórcio., Clínica, Genética. . D. N. A., \& Colaboração, Diagnostics. (2010). Clinical Genetic Testing for Patients With Autism Spectrum Disorders 1, Pediatrics, , 125(4), 727-735.

[125] Sherer, S. W., \& Dawson, G. (2011). Risk factors for autism: translating genomic discoveries into diagnostics. Hum Genet, , 130(1), 123-148.

[126] Shibayama, A., Cook, E. H., Jr , , Feng, J., Glanzmann, C., Yan, J., Craddock, N., Jones, I. R., Goldman, D., Heston, L. L., \& Sommer, S. S. (2004). MECP2 structural and 30- 
UTR variants in schizophrenia, autism and other psychiatric diseases: a possible association with autism. Am. J. Med. Genet. B Neuropsychiatr. Genet., , 128B(1), 50-53.

[127] Shinawi, M., Liu, P., Kang, S. H., Shen, J., Belmont, J. W., Scott, D. A., Probst, F. J., Craigen, W. J., Graham, B. H., Pursley, A., Clark, G., Lee, J., Proud, M., Stocco, A., Rodriguez, D. L., Kozel, B. A., Sparagana, S., Roeder, E. R., Mc Grew, S. G., Kurczynski, T. W., Allison, L. J., Amato, S., Savage, S., Patel, A., Stankiewicz, P., Beaudet, A. L., Cheung, S. W., \& Lupski, J. R. (2010). Recurrent reciprocal 16p11.2 rearrangements associated with global developmental delay, behavioural problems, dysmorphism, epilepsy, and abnormal head size. J Med Genet., , 47(5), 332-341.

[128] Silverman, J. M., Buxbaum, J. D., Ramoz, N., Schmeidler, J., Reichenberg, A., Hollander, E., Angelo, G., Smith, C. J., \& Kryzak, L. A. (2008). Autism-related routines and rituals associated with a mitochondrial aspartate/glutamate carrier SLC25A12 polymorphism. Am. J. Med. Genet. B. Neuropsychiatr. Genet., , 147(3), 408-410.

[129] Simon, E. W., Haas-Givler, B., \& Finucane, B. (2010). A longitudinal follow-up study of autistic symptoms in children and adults with duplications of 15q11-13. Am J Med Genet B Neuropsychiatr Genet., , 153B(2), 463-467.

[130] Simonoff, E., Pickles, A., Charman, T., Chandler, S., Loucas, T., \& Baird, G. (2008). Psychiatric disorders in children with autism spectrum disorders: prevalence, comorbidity, and associated factors in a population-derived sample. J Am Acad Child and Adolesc Psychiatry, , 47(8), 921-929.

[131] Smalley, S. L. (1998). Autism and tuberous sclerosis. J Autism Dev Disord, , 28(5), 407-414.

[132] Smith, M., Spence, M. A., \& Flodman, P. (2009). Nuclear and mitochondrial genome defects in autisms. Ann. N. Y. Acad. Sci., , 1151, 102-132.

[133] State, M. W., \& Levitt, P. (2011). The conundrums of understanding genetic risks for autism spectrum disorders. Nat. Neurosci., , 14(12), 1499-1506.

[134] Stein, M. B., Yang, B. Z., Chavira, D. A., Hitchcock, C. A., Sung, S. C., Shipon-Blum, E., \& Gelernter, J. (2011). A common genetic variant in the neurexin superfamily member CNTNAP2 is associated with increased risk for selective mutism and social anxiety-related traits. Biol Psychiatry, , 69(9), 825-831.

[135] Sullivan, P. F., Magnusson, C., Reichenberg, A., Boman, M., Dalman, C., Davidson, M., Fruchter, E., Hultman, C. M., Lundberg, M., Långström, N., Weiser, M., Svensson, A. C., \& Lichtenstein, P. (2012). Family History of Schizophrenia and Bipolar Disorder as Risk Factors for AutismFamily History of Psychosis as Risk Factor for ASD. Arch Gen Psychiatry., Epub already print., 1(5)

[136] Swanwick, C. C., Larsen, E. C., \& Banerjee-Base, S. (2011). Genetic Heterogeneity of Autism Spectrum Disorders, In: Autism Spectrum Disorders: The Role of Genetics in Diagnosis and Treatment, Deutsh, S. I and Urbano, M. R., Croácia: In Tech., 65-82. 
[137] Szatmari, P., Mac, Lean. J. E., Jones, M. B., Bryson, S. E., Zwaigenbaum, L., Bartolucci, G., Mahoney, W. J., \& Tuff, L. (2000). The familial aggregation of the lesser variant in biological and nonbiological relatives of PDD probands: A family history study. J Child Psychol Psychiatry,. , 41(5), 579-586.

[138] Tan, G. C., Doke, T. F., Ashburner, J., Wood, N. W., \& Frackowiak, R. S. (2010). Normal variation in fronto-occipital circuitryand cerebellar structure with an autism-associated polymorphism of CNTNAP2. Neuroimage. , 53(3), 1030-1042.

[139] Takayama, C., \& Inoue, Y. (2004). GABAergic signaling in the developing cerebellum. Anat Sci Int, , 79(3), 124-136.

[140] Theoharides, T. C., Doyle, R., Francis, K., Conti, P., \& Kalogeromitros, D. (2008). Novel therapeutic targets for autism. Trends Pharmacol, , 29(8), 375-382.

[141] Uchino, S., Wada, H., Honda, S., Nakamura, Y., Ondo, Y., Uchiyama, T., Tsutsumi, M., Suzuki, E., Hirasawa, T., \& Kohsaka, S. (2006). Direct interaction of post-synaptic density-95/Dlg/ZO-1 domain-containing synaptic molecule Shank3 with GluR1 aamino-3-hydroxy-5-methyl-4-isoxazole propionic acid receptor. J Neurochem., , 97(4), 1203-1214.

[142] Valent, P., Akin, C., Arock, M., Brockow, K., Butterfield, J. H., Carter, M. C., Castells, M., Escribano, L., Hartmann, K., Lieberman, P., Nedoszytko, B., Orfao, A., Schwartz, L. B., Sotlar, K., Sperr, W. R., Triggiani, M., Valenta, R., Horny, H. P., \& Metcalfe, D. D. (2012). Definitions, criteria and global classification of mast cell disorders with special reference to mast cell activation syndromes: a consensus proposal. Int Arch Allergy Immunol, , 157(3), 215-225.

[143] Van der , N., Rooms, L., Vandeweyer, G., van den, Ende. J., Reyniers, E., Fichera, M., Romano, C., Delle, Chiaie. B., Mortier, G., Menten, B., Destrée, A., Maystadt, I., Männik, K., Kurg, A., Reimand, T., Mc Mullan, D., Oley, C., Brueton, L., Bongers, E. M., van Bon, B. W., Pfund, R., Jacquemont, S., Ferrarini, A., Martinet, D., SchranderStumpel, C., Stegmann, A. P., Frints, S. G., de Vries, B. B., Ceulemans, B., \& Kooy, R. F. (2009). Fourteen new cases contribute to the characterization of the $7 \mathrm{q} 11.23$ microduplication syndrome. Eur. J. Med. Genet., (2-3), 94-100.

[144] Veenstra-Vanderweele, J., Christian, S. L., \& Cook Jr, E. H. (2004). Autism as a paradigmatic complex genetic disorder. Annu. Rev. Genomics Hum. Genet. , 5, 379-405.

[145] Veltman, M. W., Thompson, R. J., Craig, E. E., Dennis, N. R., Roberts, S. E., Moore, V., Brown, J. A., \& Bolton, P. F. (2005). A paternally inherited duplication in the PraderWilli/Angelman syndrome critical region: a case and family study. J Autism Dev Disord. , 35(1), 117-127.

[146] Vernes, S. C., Newbury, D. F., Abrahams, B. S., Winchester, L., Nicod, J., Groszer, M., Alarcón, M., Oliver, P. L., Davies, K. E., Geschwind, D. H., Monaco, A. P., \& Fisher, S. E. (2008). A functional genetic link between distinct developmental language disorders. N Engl J Med., , 359(22), 2337-2345. 
[147] Viora, M., Quaranta, M. G., Straface, E., Vari, R., Masella, R., \& Malorni, W. (2001). Redox imbalance and immune functions: opposite effects of oxidized low-density lipoproteins and Nacetylcysteine. Immunology, , 104(4), 431-438.

[148] Vissers, L. E., de Ligt, J., Gilissen, C., Janssen, I., Steehouwer, M., de Vries, P., van Lier, B., Arts, P., Wieskamp, N., del Rosario, M., van Bon, B. W., Hoischen, A., de Vries, B. B., Brunner, H. G., \& Veltman, J. A. (2010). A de novo paradigm for mental retardation. Nature Genetics,. , 42(12), 1109-1112.

[149] Vojdani, A. (2008). Antibodies as predictors of complex autoimmune diseases and cancer. Int J Immunopathol Pharmacol, Erratum in: Int J Immunopathol Pharmacol., Vol. 21, No.4., 21(3), 553-566.

[150] Wang, K., Zhang, H., , D., Bucan, M., Glessner, J. T., Abrahams, B. S., Salyakina, D., Imielinski, M., Bradfield, J. P., Sleiman, P. M., Kim, C. E., Hou, C., Frackelton, E., Chiavacci, R., Takahashi, N., Sakurai, T., Rappaport, E., Lajonchere, C. M., Munson, J., Estes, A., Korvatska, O., Piven, J., Sonnenblick, L. I., Alvarez, Retuerto. A. I., Herman, E. I., Dong, H., Hutman, T., Sigman, M., Ozonoff, S., Klin, A., Owley, T., Sweeney, J. A., Brune, C. W., Cantor, R. M., Bernier, R., Gilbert, J. R., Cuccaro, M. L., Mc Mahon, W. M., Miller, J., State, M. W., Wassink, T. H., Coon, H., Levy, S. E., Schultz, R. T., Nurnberger, J. I., Haines, J. L., Sutcliffe, J. S., Cook, E. H., Minshew, N. J., Buxbaum, J. D., Dawson, G., Grant, S. F., Geschwind, D. H., Pericak-Vance, M. A., Schellenberg, G. D., \& Hakonarson, . (2009). H. Common genetic variants on 5p14.1 associate with autism spectrum disorders. Nature, , 459(7246), 528-533.

[151] Warren, W. B., Patrick, S. L., \& Goland, R. S. (1992). Elevated maternal plasma corticotropinreleasing hormone levels in pregnancies complicated by preterm labor. Am J Obstet Gynecol, , 166(4), 1198-1204.

[152] Weiss, L. A., Shen, Y., Korn, J. M., Arking, D. E., Miller, D. T., Fossdal, R., Saemundsen, E., Stefansson, H., Ferreira, M. A., Green, T., Platt, O. S., Ruderfer, D. M., Walsh, C. A., Altshuler, D., Chakravarti, A., Tanzi, R. E., Stefansson, K., Santangelo, S. L., Gusella, J. F., Sklar, P., Wu, B. L., \& Daly, M. J. (2008). Autism Consortium. Association between microdeletion and microduplication at 16p11.2 and autism. N Engl J Med. , 358(7), 667-675.

[153] Weinberger, D. R., Egan, M. F., Bertolino, A., Callicott, J. H., Mattay, V. S., Lipska, B. K., Berman, K. F., \& Goldberg, T. E. (2001). Prefrontal neurons and the genetics of schizophrenia. Biol Psychiatry,. , 1(50), 825-844.

[154] Whalley, H. C., O'Connell, G., Sussmann, J. E., Peel, A., Stanfield, A. C., Hayiou-Thomas, M. E., Johnstone, E. C., Lawrie, S. M., Mc Intosh, A. M., \& Hall, J. (2011). Genetic Variation in CNTNAP2 Alters Brain Function During Linguistic Processing in Healthy Individuals. Am J Med Genet B Neuropsychiatr Genet., , 156B(8), 941-948.

[155] Zhang, T. Y., \& Meaney, M. J. (2010). Epigenetics and the environmental regulation of the genome and its function. Annu Rev Psychol., , 61, 439-449. 
[156] Zhao, X., Leotta, A., Kustanovich, V., Lajonchere, C., Geschwind, D. H., Law, K., Law, P., Qiu, S., Lord, C., Sebat, J., Ye, K., \& Wigler, M. (2007). A unified genetic theory for sporadic and inherited autism. Proc Nati Acad of Sci U S A., , 104(3), 12831-12836. 
Chapter 12

\title{
Advances in Autism Research - The Genomic Basis of ASD
}

\author{
Melanie Lacaria and James R. Lupski \\ Additional information is available at the end of the chapter \\ http://dx.doi.org/10.5772/53689
}

\section{Introduction}

Many of the recent advances in autism research that have provided fundamental insight into this condition have come from the application of genetic/genomic approaches; these advances have been fomented by the advent of new technologies to interrogate the entire genome, such as array comparative genomic hybridization $(\mathrm{aCGH})$, single nucleotide polymorphism (SNP) microarrays, transcriptome sequencing, and whole genome or whole exome sequencing (WGS/WES) [1]. With the recent advancement of these technologies over more traditional, lower-resolution technologies such as cytogenetic analysis, came the ability to interrogate the entire genome at a high-resolution. With the improvement of next-generation sequencing technology, as well as the reduction in the cost of this technique, WGS is becoming more commonplace in the search for novel diseasecausing variants in individual patients. Alternatively, many studies have utilized WES, as it is less costly than sequencing the entire genome and coding simple nucleotide variants (SNVs) can often be more readily interpreted given knowledge provided by the genetic code. While the reduced cost and more readily interpretable variation have proven to be distinct advantages of this method over whole-genome sequencing, it is well known that many other variants in non-coding or regulatory regions can be pathogenic, and they typically cannot be discerned by whole-exome sequencing, which requires a targeted-capture step to enrich for and focus analysis on the coding sequences of all annotated protein-coding genes [2, 3]. Furthermore, repetitive or G-C rich regions or highly homologous sequences are often excluded by WES, and copy number variations (CNVs) usually cannot be accurately called due to the use of PCR-based sample preparation methods. Nonetheless, the utility of WGS/WES in individual patient diagnosis and management has been demonstrated by several recent reports [4-6]. 
Some of the first studies providing a high-resolution view of the entire genome have revealed that a large number of $\mathrm{CNVs}$ are present in the genomes of healthy individuals, and that $\mathrm{CNVs}$ account for a greater proportion of the nucleotide variation between two given individual genomes than can be attributed to SNVs [7-9]. These structural alterations can reach up to several megabases in length, but a much higher frequency is observed for smaller $(<1 \mathrm{~kb}) \mathrm{CNVs}$ [2]. And, as one would expect, the likelihood of CNVs becoming pathogenic rises when they have an increased size and/or occur in gene-dense regions of the genome [8]. Traditionally, structural variation $(\mathrm{CNV})$ was not considered to play a causative role in autism or ASD. However, recent studies have revealed that not only single-gene alterations, but also CNVs can lead to autism or ASD. In fact, it is now becoming increasingly evident that $\mathrm{CNVs}$ account for a larger proportion of new autism diagnoses than single-gene disorders. Recurrent CNVs at specific genomic loci have been associated with autism, including 15q11-q13, 16p11.2, 17p11.2, 22q13.3, 7q11.23, and 2q37, among others [1, 10-16]. While several of these loci are associated with known Centers for Mendelian Genomics, numerous CNVs have also been observed in idiopathic autism, underscoring the importance of these structural variations in the future of all types of autism research [17].

The application of next-generation sequencing technology to evaluate CNVs has also recently been described in a report that utilized whole-transcriptome sequencing analysis of the genomes of a cohort of patients with autism spectrum disorder (ASD) [18]. This approach allows for the evaluation of $\mathrm{CNVs}$ and overcomes some of the problems associated with CNV-calling in WES. With several large-scale projects currently underway, the future of next-generation sequencing and whole-genome analysis in the study of autism will most definitely provide many new insights into the etiology of this disease. Currently, Autism Speaks is working in collaboration with the company BGI to generate the largest database of sequenced genomes of individuals with ASD, a project known as the "Autism Genome 10K." Similarly, the National Institute of Mental Health in the US has funded another large-scale "Autism Genome Project." Mendelian/ syndromic forms of autism are also currently being studied by the Genomic Disorders consortium in the US by WES.

Among the variants identified in the large-scale studies of patients with autism reported to date, many gene networks/pathways have been implicated, including genes for neuronal adhesion [18, 19], ubiquitin degradation [19], chromatin remodelling [5, 20], sodium channels [13], proteolysis [21], cytoskeletal organization [21], signal transduction [18], neuropeptide signalling [18], neurogenesis/synaptogenesis [18], neuronal migration [22], basic metabolism, and RNA splicing [22], among others. While these pathways may seem diverse, repeated "hits" in these networks support the "many genes, common pathway" hypothesis [22]. Importantly, although the biological function of ASD susceptibility genes identified via these whole-genome studies do not appear to lie within the same network, they likely converge to disrupt neuronal function in brain regions that support language, social cognition, and behavioral flexibility, resulting in the phenotypes commonly associated with ASD [22]. 


\section{Chromosome engineering mouse models for ASD}

Since ASD is known to be a highly heterogeneous disorder with both genetic and environmental components, modeling the disease in rodents, where environmental and background effects can be largely controlled and systematically manipulated and studied, is of great advantage in the study of the pathomechanisms underpinning autism. Furthermore, numerous tools for genetic manipulation and for behavioral analysis that are currently available and developed for genetic studies in rodents can be leveraged to facilitate this avenue of research. Importantly, behavioral assays have been developed and validated to objectively quantify the phenotypes relative to autism, including both core and associated autistic-like phenotypes, such as abnormal social behavior, communication deficits, and repetitive behaviors, as well as autism-associated anxiety-like behaviors, motor defects, learning and memory deficits, sleep disorders, sensory hypersensitivity, and seizures, among others [23] (Table 1; adapted from Crawley et al, where a full description of these behavioral tests can be found; [23]).

With the importance of CNVs in the etiology of ASD established, chromosome-engineered mouse models were then generated for the study of autism or ASD. The first such mouse strains were developed over a decade ago using a chromosome-engineering approach [24]. This technique allows for the creation of a targeted duplication or deletion in the desired location by first generating the rearrangement in mouse embryonic stem (ES) cells which can then be established as mouse strains via Cre/loxP site-specific recombination $[25,26]$. To generate the desired rearrangement, two gene-targeting steps are required to prepare each end point for a selectable recombination event (Figure 1). Importantly, the type of rearrangement (deletion, duplication, inversion) depends on the relative orientation of the loxP sites; if the sites are in the same orientation, the region between them can be deleted or duplicated, but if they are in opposite orientation, an inversion results [25]. The cis or trans configuration is also relevant; trans insertion (insertion in each chromosome homologue) of loxP sites enables generation of both deletion and duplication in the same ES cells. Transient transfection of the ES cells with a vector expressing Cre recombinase facilitates the recombination between the targeted loxP sites, and cells containing the event can be selected for using hypoxanthine aminopterin thymidine (HAT)-containing media due to the reconstitution of a functional Hprt cassette as a result of the recombination [25]. The resulting mouse models harbor either a chromosomal duplication or deletion of a defined region that is syntenic to the copy number variable region in humans. Importantly, chromosome-engineered mouse models are distinct from monogenic animal models in that they harbor structural chromosomal rearrangements resulting in specific, targeted $\mathrm{CNVs}$ with genomic intervals that may span several megabases and contain numerous genes, many of which may be of unknown function. In contrast, monogenic animal models primarily utilize a reverse-genetics approach to knock-out or transgenically-overexpress the specific single gene of interest, limiting the study to that one particular gene. A publicly-available resource can facilitate chromosome engineering for the targeted manipulation of the mouse genome; the mutagenic insertion and chromosome engineering resource (MICER) can be utilized to access vectors to create chromosomal rearrangements or to study gene disruptions in a high-throughput manner [27]. (http://www.sanger.ac.uk/resources/mouse/micer/). 


\begin{tabular}{|c|c|}
\hline Core features of autism & Analogous feature/assay in mice \\
\hline \multirow[t]{5}{*}{ Abnormal social interactions } & Preference for social novelty \\
\hline & Social recognition \\
\hline & Home cage monitoring - nesting patterns \\
\hline & Direct social interactions / juvenile play \\
\hline & Social approach to a stranger mouse \\
\hline \multirow[t]{4}{*}{ Impaired social communication } & Vocalizations during direct social interaction \\
\hline & Parental retrieval of separated pups \\
\hline & Ultrasonic vocalizations by separated pups \\
\hline & Response to olfactory cues \\
\hline \multirow[t]{5}{*}{ Repetitive or restrictive behavior } & Perseverative holeboard exploration \\
\hline & Behavioral stereotypies/ home cage observation \\
\hline & Reversal of a position habit in maze tasks \\
\hline & Marble burying \\
\hline & Novel object exploration \\
\hline ASD- Associated features & Analogous feature/assay in mice \\
\hline \multirow[t]{3}{*}{ Anxiety } & Elevated plus maze \\
\hline & Light/dark exploration \\
\hline & Open field analysis \\
\hline \multirow[t]{2}{*}{ Theory of mind deficits } & Social transmission of food preference \\
\hline & Avoidance of aggressive encounters \\
\hline \multirow[t]{3}{*}{ Intellectual disability } & Morris water maze \\
\hline & Contextual and cued fear conditioning \\
\hline & T-maze \\
\hline \multirow[t]{4}{*}{ Motor defects } & Rotarod test for motor learning \\
\hline & Wire hang \\
\hline & Dowel walking \\
\hline & Footprint analysis \\
\hline \multirow[t]{2}{*}{ Aggression } & Resident intruder attack \\
\hline & Tube test for social dominance \\
\hline \multirow[t]{2}{*}{ Sleep disturbances } & Circadian running wheel \\
\hline & Home cage monitoring - activity levels \\
\hline \multirow[t]{3}{*}{ Hypersensitivity to sensory stimuli } & Pre-pulse inhibition \\
\hline & Acoustic startle \\
\hline & Hot plate test for nociception \\
\hline Seizures & Sensitivity to audiogenic or drug-induced seizures \\
\hline
\end{tabular}

Table 1. Behavioral tests for the evaluation of autistic-like features in mice 


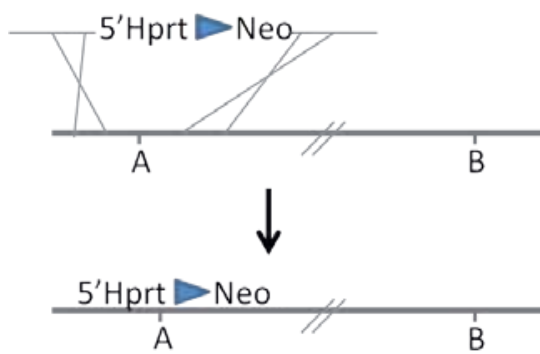

A

B
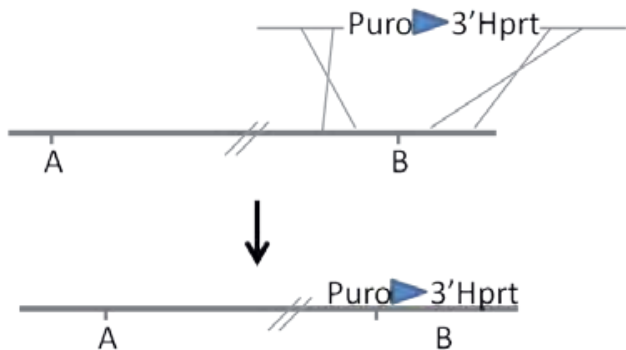

B

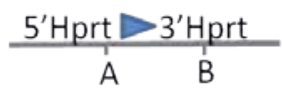

Deletion (Df)
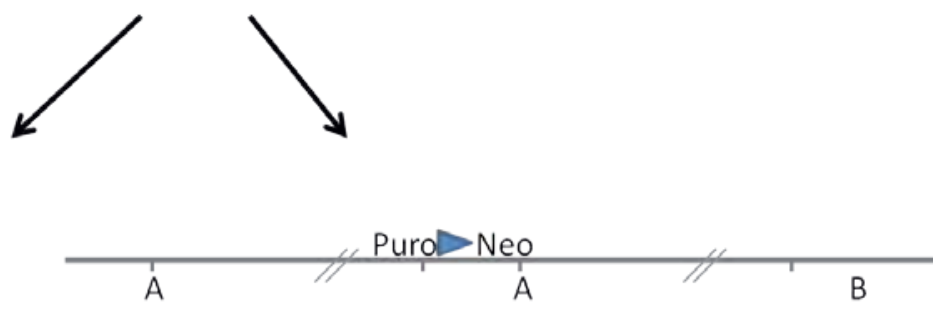

Duplication (Dp)

Figure 1. Generating defined chromosome rearrangements. Defined endpoints of the desired interval for rearrangement $(A$ and $B$ ) are modified by gene targeting in embryonic stem (ES) cells to allow for introduction of a lox $P$ site (blue triangle), a non-functional portion of the HPRT cassette [ $5^{\prime}$ or $3^{\prime}$ ), and a positive selectable marker (Neo or Puro). The targeted ES cells are then transiently transfected with a vector expressing Cre recombinase, which facilitates recombination between the loxP sites, resulting in either deletion (Df) or duplication (Dp) of the intervening region. In this example, reconstitution of the Hprt cassette via recombination between loxP sites lying in a shared intron between the two halves confers resistance to hypoxanthine aminopterin thymidine (HAT), which can be used to select for the deletion event. Two positive selectable markers (Puro and Neo) identify the duplication event. A full explanation of this technology and more examples of chromosome recombineering are outlined by Mills et al [25].

We will discuss specific examples of chromosome-engineered mouse models of ASD for which the neurobehavioral phenotypes have been described using established rodent behavioral assays, including mice harboring duplication or deletion of the genomic interval syntenic to human chromosome 16p11.2, 15q11-q13, and 17p11.2, which all model CNVs that have been identified in patient populations with autism [28-30]. Most of the CNV-based animal models of ASD described to date have focused on syndromic forms of autism, as the underlying genetic cause of these genomic disorders is often well-described.

By definition, genomic disorders result from structural changes in the genome, wherein the genomic instability often reflects a susceptible genome architecture, that leads to disease traits [31]. These structural rearrangements or CNVs commonly cause the disruption or complete loss or gain of dosage-sensitive gene(s). Alternatively, CNVs can cause gene fusion, position effects, transvection effects, or the unmasking of a recessive allele or functional polymorphism [32]. Genomic disorders are therefore distinct from traditional genetic syndromes, which are typically caused by DNA sequence-based changes [33]. Within the past decade, several technologies, including array comparative genomic hybridization $(\mathrm{aCGH})$, next-generation sequencing, and single nucleotide polymorphism (SNP) genotyp- 
ing platforms, have been utilized to detect and analyze CNVs in the genome and to investigate the mechanism by which these CNVs are generated [34]. CNVs can be formed by several mechanisms, such as non-allelic homologous recombination (NAHR), non-homologous end joining (NHEJ), or fork stalling and template switching (FoSTeS) [35]. NAHR, which is often mediated by low copy repeats (LCRs) with high ( 95\%) sequence similarity flanking the rearranged region, is the most common mechanism by which recurrent $\mathrm{CNVs}$ are created. Often this mechanism can result in recurrent genomic rearrangements that are observed in multiple patients with the same disorder, as in Charcot-Marie-Tooth disease type 1A, Prader-Willi syndrome, and Smith-Magenis Syndrome, among many others [32, 33]. The genomic architecture rendering genomic instability at three loci that are enriched for LCRs are shown in Figure 2.
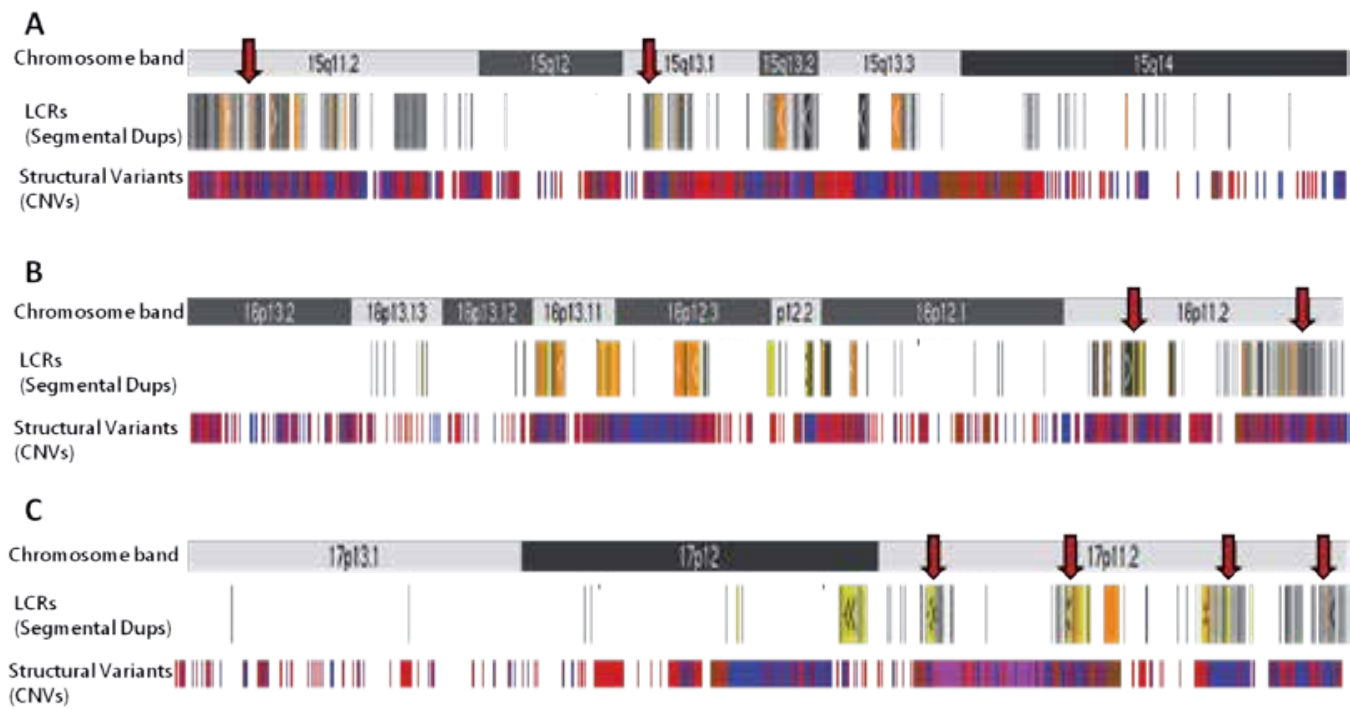

Figure 2. The genomic structure of loci associated with CNV-based ASD. (A) Chromosomes 15q11-13, (B) 16p11.2, and (C) 17p11.2, are enriched for LCRs, or segmental duplications (indicated by red arrows), which facilitate non-allelic homologous recombination (NAHR), resulting in the generation of CNVs (blue and red bars). This figure was generated using the genome browser provided by UCSC (http://genome.ucsc.edu/index.html?org=Human\&db=hg18\&hgsid=289381925].

\section{ASD associated with CNVs on chromosome 16p11.2}

In recent years, whole genome analyses of cohorts of patients with autism have identified recurrent $\mathrm{CNVs}$ on chromosome 16p11.2, effectively linking reciprocal microduplications and microdeletions at this locus with increased susceptibility to ASD [12, 36-39]. Importantly, further studies have revealed that $\mathrm{CNVs}$ at this locus are responsible for $\sim 1 \%$ of all ASD diagnoses, making it the most common $\mathrm{CNV}$ to be associated with ASD identified to date 
[13]. The 16p11.2 locus is flanked by two directly repeated segmental duplications of $\sim 145$ $\mathrm{kb}$, which mediate the NAHR that results in the loss or gain of $\sim 600 \mathrm{~kb}$ intermediate region containing $\sim 27$ protein-coding genes $[9,12,40]$.

Interestingly, the microduplication of this region has also been linked to schizophrenia, suggesting the presence of an underlying biological link between these two disorders [41, 42]. This phenomenon also gives a potential genetic basis for the hypothesis of Crespi et al, which states that autism and schizophrenia represent diametric disorders of the social brain [43]. Thus, schizophrenia and autism might reflect mirror traits of the opposing extremes of behavioral phenotypes reflecting evolution of the social brain [43]. The phenotypes caused by $\mathrm{CNV}$ at the 16p11.2 locus are extremely heterogeneous, and, in addition to ASD, they have been reported to include metabolic disorders [44-47], cardiac anomalies [40, 48], depressive disorder [49], speech delay [50], mental retardation [40, 51, 52], vertebral anomalies [52], syringomyelia [53], abnormal head size [36], and epilepsy [36, 40], as well as other various congenital anomalies and behavioral abnormalities [44]. As the phenotypes of many more patients harboring CNVs in this genomic region are delineated, the full phenotypic spectrum associated with this locus will likely become more well-defined, and the critical genomic interval and dosage-sensitive genes responsible for the phenotypes will be determined. Indeed, a more recent study described a patient pedigree for a family with multiple generations of autism or ASD that also carry a smaller-sized deletion within the common deletion of 16p11.2, thereby reducing the "critical" interval for ASD to a $118 \mathrm{~kb}$ region containing only 5 genes: MVP, CDIPT1, SEZ6L2, ASPHD1, and KCTD13 [54]. To date, none of these genes have been significantly associated with an elevated risk for ASD, which indicates that the situation is likely much more complex [37, 55]. Furthermore, correlation between the phenotypes of patients harboring different- or similar-sized CNVs is confounded by extreme heterogeneity and variability of symptoms. For example, a family with three affected members harboring identical 16p11.2 deletions was recently described to have minimal symptom overlap between family members [56]. Subsequent studies have aimed at using model organisms to identify the key dosage-sensitive genes within this region that give rise to the abnormal phenotypes [29, 57, 58]. Among these, chromosome-engineered mouse models harboring reciprocal deletion or duplication of the mouse chromosome syntenic to human chromosome 16p11.2 have been generated to study the physiological and behavioral phenotypes associated with these chromosome abnormalities [29].

\section{Animal models for human 16p11.2 CNVs}

Mouse models were generated through a chromosome engineering approach for the study of human 16p11.2 deletion and duplication CNVs [29]. It was observed that $\sim 50 \%$ of mice harboring the deletion $\mathrm{CNV}$ die shortly after birth, while duplication mice survive to adulthood, suggesting that the deletion $\mathrm{CNV}$ results in a more severe phenotype than the duplication [29]. A similar phenomenon has been observed in other genomic disorders caused by reciprocal CNVs, including Smith-Magenis and Potocki-Lupski syndromes [15]. Expression of the genes within the 16p11.2 region corresponds to gene dos- 
age in four brain regions that may be relevant for autism, including the olfactory bulbs, cortex, cerebellum, and brainstem [29].

In-cage neurobehavioral phenotypes were assessed in these mice to determine what, if any, affect these CNVs had on autistic-like behaviors. As expected, deletion mice displayed the most abnormal phenotypes, while duplication mice had fewer and milder symptoms. Interestingly, reciprocal phenotypes were sometimes observed for mice harboring reciprocal CNVs. For example, the amount of time spent resting in the cage was lower in deletion mice but higher in duplication mice relative to controls, indicating that $16 \mathrm{p} 11.2 \mathrm{CNVs}$ affect the rate and timing of specific behaviors in a dosage-dependent manner. Deletion mice displayed an abnormal ceiling-climbing behavior where they demonstrated marked stereotypic and nonprogressive motor behaviors, similar to what is often observed in patients with autism or patients with lateral hypothalamic and nigrostriatal lesions in the brain. These abnormal behaviors were accompanied by volumetric and morphological changes in several brain regions, including the lateral hypothalamus. Importantly, the difference between deletion mice and duplication mice was greater than that between deletion mice and controls, indicating that these effects are reciprocal or opposing in nature.

No significant abnormal social behavior was observed in these animal models in the 3chamber test for sociability, indicating either that these animals do not display social abnormalities, or that further investigation into the social behavior of these animals is required. Indeed, with the subtle nature of many social interactions in rodents, it is quite possible that social abnormalities exist in these mice but have not yet been described. It is also distinctly possible that the 'in-cage' environment does not elicit a social deficit that might perhaps be observed in the wild or natural environment of the animal. An extensive battery of tests for social behavior will be required to rule out the possibility of further abnormalities.

Many of the genes mapped to the altered region have unknown function, and therefore, unknown significance or contribution to the disease phenotype. In order to further delineate the function of the dosage-sensitive genes within the common duplication/deletion region, zebrafish models were generated [57,58]. The first study aimed to investigate the diametric head size phenotypes linked to this locus, as in addition to ASD, deletion is known to result in macrocephaly, and duplication gives rise to microcephaly [36, 57]. In this study, zebrafish were utilized for an in vivo overexpression screen, which identified the gene KCTD13 as the likely candidate for the neurodevelopmental phenotypes associated with CNVs at 16p11.2. Interestingly, this gene was also one of the 5 genes found in a minimal critical deletion interval for ASD [54]. Overexpression of this gene in zebrafish resulted in microcephaly, while the reciprocal reduced expression of this locus by morpholino oligonucelotides resulted in macrocephaly, thereby mirroring the phenotypes seen in humans harboring CNVs at this locus [57]. Further study revealed that the function of this gene is likely conserved across species, and it is required to maintain the proliferative status of cortical progenitor cells in mice [57]. Furthermore, this gene is affected in a complex genomic rearrangement identified in a patient with autism [57]. Taken together, these results indicate that KCTD13 is a likely candidate for further study of the neurological phenotypes associated with $\mathrm{CNV}$ at this locus. 
Another study was able to identify homologs of 21 of the known $16 \mathrm{p} 11.2$ human genes in the zebrafish genome by family tree comparisons [58]. These genes were then targeted for loss of function studies by injecting antisense morpholino oligonucleotides into early embryos [58]. Interestingly $\sim 79 \%$ of the genes tested by this method were required for proper brain, eye, or nervous system development, and two of the genes were determined to be dosage-sensitive, with abnormal phenotypes present with a $\sim 50 \%$ reduction in gene expression [58]. The results of this study suggest that at least two genes, aldolase a (aldoaa) and kinesin family member 22 (kif22), are highly dosage-sensitive and are required for proper brain function, making them likely candidates for future studies of the ASD associated with $\mathrm{CNV}$ of this region [58].

These two studies indicate that while kctd13, kif22, and aldoaa are all potentially interesting dosage-sensitive candidate genes, further investigation is needed to determine whether these genes act together in a epistatic manner to contribute to the full neurological phenotype, whether they modify each other via cis interactions, or whether other genes or genetic elements in the 16p11.2 locus are also contributing to the phenotype. The current data, which could not have been obtained without these important studies in model organisms, cannot distinguish between these possibilities, but they provide a starting point for research into the function of these genes and the molecular pathways underpinning the phenotypes associated with 16p11.2 CNVs.

\section{Prader-Willi and Angelman syndromes}

Chromosome 15q11-13 is enriched for LCRs, providing a mechanism for LCR-mediated NAHR, and generating a series of recurrent breakpoints along this chromosome. As a result, interstitial deletion or duplication of this region is common. LCRs can also mediate triplications, or, alternatively, the presence of supernumerary isodicentric chromosome 15 (idic(15)) can lead to crossing over between these LCRs, and ultimately, duplication of the region. A bipartite imprinting center lies at this locus and directs the expression of a number of genes, resulting in a tissue-specific parent-of-origin effect. As a result, many of the phenotypes caused by these structural rearrangements also display parent-of-origin effects.

Paternally- or maternally-inherited deletions of human chromosome 15q11-13 result in Prader-Willi syndrome (PWS) or Angelman syndrome (AS), respectively. Alternatively, these disorders can be caused by uniparental disomy (UPD), or by balanced translocations involving this region. Less frequently, imprinting errors, leading to aberrant methylation of the PWS imprinting center can also cause PWS, and mutations or deletions in the gene UBE3A can cause AS [11,59]. The critical region for AS lies $35 \mathrm{~kb}$ telomeric to the PWS critical region [60]. PWS is characterized by intellectual disability, hypotonia, hyperphagia, obesity, compulsive and repetitive behaviors, skin picking, tantrums, irritability. In addition, congenital abnormalities are often observed, including hypogonadism, facial dysmorphism, and small hands and feet, among others. PWS can also be associated with psychosis, mood disorders, and ASD [61]. 
AS is a neurodevelopmental disorder that is characterized by severe developmental delay, intellectual disability, microcephaly, seizures, lack of speech, ataxia, and dysmorphic facial features [11, 59]. Patients with AS are often described as having happy demeanors, however hyperactivity, attention deficits, aggression, and repetitive or stereotypic behaviors have also been described [59]. AS has been associated with ASD in several studies, however, the severity of the cognitive impairments in most patients with AS may preclude an accurate diagnosis [11,59].

\section{Duplication of 15q11-13}

It has been estimated that up to $\sim 5 \%$ of cases of ASD can be attributed to maternal duplication of the genomic region reciprocal to the PWS-AS critical region on chromosome 15q11-13, making it one of the most common chromosomal abnormalities observed in patients with ASD [10, 62]. Due to the presence of imprinting at this locus (discussed above), parent-of-origin effects are seen, and, for interstitial duplications, maternal origin confers an increased risk for clinical phenotypes. Paternal duplications are much less common, and do not appear to lead to ASD, as familial cases have been described where a seemingly normal carrier mother transmits a paternally-derived duplication to their child [63]. However, a small number of subjects with paternal duplication of 15q11-13 and various clinical phenotypes have been described [64]. Phenotypes are dosage-sensitive at this locus; one extra maternal copy of 15q11-13 results in partial autism penetrance, while two extra copies (caused by idic15 or interstitial triplication) result in a much higher penetrance of autism as well as additional phenotypes that are typically more severe than those seen in patients with duplications [62]. In the case of triplications, parent-of-origin effects are no longer observed, and both paternal and maternal duplications are associated with poor clinical outcomes [65]. This loss of parent-of-origin effects is interesting, and it may be an indication of the underlying mechanism that may give rise to a predisposition for these phenotypes. Many heterogeneous and complex phenotypes can be associated with increased copy number of this region including intellectual disability, apraxia, dyslexia, seizures, hypotonia, developmental delay, gait abnormalities, hyperactivity, schizophrenia, and ASD [66]. Patients with ASD due to duplication of 15q11-13 also display several stereotypic and repetitive behaviors, including rocking, licking, and hand-flapping, among others, that are often directed towards sensory stimulation, suggesting that the underlying cause of these phenotypes may be due to a disregulation of sensory inputs or signaling [63, 64].

Interestingly, recent post-mortem evaluation of the brains of patients harboring maternal duplication of 15q11-13 suggested that accumulation and deposition of abnormal intracellular and extracellular amyloid $\beta$ protein $(\mathrm{A} \beta)$ in the specific regions and neuron types in the brains of patients with maternal duplication of 15q11-13 may underlie or contribute to some of the neurobehavioral phenotypes associated with ASD [67]. However, further studies are needed to confirm this hypothesis. Several animal models for the CNVbased syndromes associated with chromosome 15q11-13 have been developed to facilitate research into these disorders. 


\section{Modeling 15q11-13 CNVs in mice}

The first mouse model for PWS was generated by targeted deletion of part of the imprinting center on chromosome 15q11-13 [68]. While these mice model several aspects of PWS, including hypersensitivity to sensory input in the form of increased acoustic startle response and decreased prepulse inhibition, it is not known whether these mice exhibit other autisticlike behaviors, such as impaired social interactions or altered communication [69]. Further behavioral characterization is needed to determine whether these mice accurately recapitulate the neurobehavioral phenotypes seen in patients with PWS and/or ASD.

Mouse models for Angelman syndrome were initially generated by disrupting maternal expression of Ube3a; these mice exhibit increased anxiety-like behavior that may be due to the disruption of a glucocorticoid receptor transactivation in the brain [70]. They also display various motor defects [71], abnormal cerebellum-driven licking behavior [72], sleep disturbance [73], abnormal EEG patterns, and cognitive defects in the conditioned fear and Morris water maze tests [74]. However, these mice displayed hypoactivity and normal social seeking behavior, in contrast to what is seen in human patients with AS and/or ASD [75]. Importantly, human patients with mutations in UBE3A typically have a milder phenotype than those patients harboring interstitial deletions, so this mouse model may not accurately reflect the majority of AS patients, who have a deletion containing this gene, as well as many other genes at this locus. Indeed, when a larger $1.6 \mathrm{Mb}$ deletion model was generated by chromosome engineering that encompasses the genomic region from Ube3a to Gabrb3, the phenotype of these mice was more severe than that of mice with deletion of Ube3a alone [76]. Similar to Ube3a deficient mice, large deletion mice had significant motor impairment, anxiety-like behavior, and abnormal EEG, but they also had learning and memory defects and abnormal communication [76]. These larger deletion mice may be an appropriate model for $\mathrm{CNV}$-associated ASD, however further investigation into the social neurobehavioral phenotypes is necessary.

Chromosome-engineered mouse models harboring a duplication of $6.3 \mathrm{Mb}$ on mouse chromosome 7 syntenic to the duplication of human chromosome 15q11-13 associated with ASD were developed to study the underlying mechanism behind the phenotypes associated with this CNV in humans [28]. The core features of autism, including abnormal social interactions, stereotypic or ritualistic behavior, and impaired communication were all evaluated in this mouse model, and patDp/+, but not matDp/+ mice were determined to have reduced sociability compared to wild-type mice. Ultrasonic vocalizations were evaluated in pups separated from the dam, and an abnormal USV pattern was observed in patDp/+ but not matDp/+ mice. Specifically, patDp/+ mice appeared to have delayed development of communication, they emitted a greater number of USVs, and some pups emitted vocalizations at abnormally high frequencies $(>70 \mathrm{kHz})$. In order to evaluate communication between older mice, pairs of mice 7-8 weeks of age were observed in a resident intruder paradigm and the pattern of USVs was measured during this interaction. Vocal communication between pairs of pat/Dp/+ mice was significantly reduced compared to the vocalization recorded between WT pairs, giving further support to the notion that patDp/+ mice have a defect in social 
communication. However, no defects in olfactory communication or function were observed in these mice. Restricted behaviors and inflexibility were evaluated with a battery of behavioral tests, including the Morris water maze and the Barnes maze. These tests revealed that patDp/+ mice do not respond as flexibly to a change in situation as wild-type or matDp/+ mice. No overt defects in learning and memory were observed in either the patDp/+ or matDp/+ mice by the Morris water maze or conditioned fear test, although patDp/+ mice did display generalized fear and elevated anxiety-like behavior. The abnormal neurobehavioral phenotypes observed in this CNV-based model of ASD could not be attributed to gross morphological or histological changes in the olfactory bulb, cerebral cortex, hippocampus, amygdala, corpus callosum, or cerebellum. Nor were any abnormalities in the number of Purkinje cells detected in the cerebellum, suggesting that the underlying pathomechanism responsible for these phenotypes is likely due to aberrations in molecular pathways that remain to be determined.

The gene Ube3a (also known as E6-AP) codes for E3 ubiquitin-protein ligase, which belongs to a family of E3 ligase genes that are involved in synaptogenesis and have recently been linked to the pathogenesis of ASD [62]. Given the known association of the gene UBE3A with AS and its maternal-specific expression pattern in neurons, a mouse model that overexpresses this gene was generated to test the hypothesis that $U b e 3 a$ is responsible for many of the phenotypes associated with duplication of 15q11-13 [62]. Transgenic overexpression of Ube3a via bacterial artificial chromosome (BAC) recombineering in mice resulted in autisticlike neurobehavioral phenotypes, including defects in communication, abnormal social behavior, and increased repetitive or stereotypic behavior [62]. Similar to the phenomenon observed in human patients, these effects were also determined to be dosage-sensitive, as the phenotypes were more penetrant in mice with three-fold overexpression of Ube3a than in those observed in mice with two-fold overexpression of this gene. Furthermore, it was determined that glutamatergic synaptic transmission was suppressed, providing a potential mechanism underlying the neurobehavioral phenotypes.

\section{Smith-Magenis and Potocki-Lupski syndromes}

Smith-Magenis syndrome (SMS) and Potocki-Lupski syndrome (PTLS) are two prototypical genomic disorders caused by reciprocal deletion (SMS) or duplication (PTLS) resulting in gene copy number variation on chromosome 17. SMS results from a de novo, recurrent, 3.7 $\mathrm{Mb}$ deletion in $17 \mathrm{p} 11.2-\operatorname{del}(17)(\mathrm{p} 11.2 \mathrm{p} 11.2)$ in $\sim 73 \%$ of cases, that is a consequence of NAHR mediated by LCRs flanking the region [77-79]. However, many of the pleiotropic features of SMS appear to result from haploinsufficiency of a single gene lying in the middle of the SMS critical region, retinoic acid induced 1 (RAI1), as determined by the identification of non-deletion SMS patients with heterozygous point mutations in RAI1 [80-82]. Patients with mutations in RAI1 manifest most of the phenotypes observed in subjects with a chromosomal SMS deletion, demonstrating that the reduced dosage of the RAI1 gene alone may cause much of the SMS phenotype [81, 83]. 
Both SMS and PTLS manifest a broad range of opposing or overlapping phenotypes. SMS is characterized by multiple congenital anomalies, including otolaryngologic, ophthalmologic, brain, cardiac, craniofacial, and renal abnormalities, as well as intellectual disability (ID), brachydactyly, sleep disturbance, hearing impairment, obesity, scoliosis, and other neurobehavioral abnormalities [84, 85]. Specifically, SMS patients display aggressive and self-injurious behaviour, including polyembolokoilamania [84], as well as characteristic repetitive behaviors, including autoamppexation or "self-hugging," which is an identifying feature of the disorder $[86,87]$. More recently, SMS patients have also been described as meeting the criteria for autism spectrum disorder (ASD) [88].

The PTLS duplication was the first predicted reciprocal duplication to be described [89]. PTLS was identified and initially defined much later than SMS, ([89] versus Smith et al. 1986); as a result, fewer PTLS patients have been medically examined and fewer studies of the clinical phenotypes are available in the literature. The clinical features that have been observed in patients with PTLS are distinct from those seen in SMS [15], although cognitive and neurobehavioral abnormalities are present in both disorders. PTLS patients lack the self-injurious behaviors, abnormal facies, and sleep disturbance, as well as some of the congenital anomalies found in most individuals with SMS. The features observed in greater than $90 \%$ of PTLS patients are developmental delay, neurobehavioral abnormalities, language impairment, cognitive impairment, poor feeding, hypotonia, and oropharyngeal dysphasia $[15,90]$. When evaluated by objective clinical assessment, the majority of PTLS patients have autistic features such as decreased eye contact, atypicality, withdrawal, anxiety, and inattention, meeting criteria for a diagnosis of autistic spectrum disorder (ASD) or pervasive developmental disorder not otherwise specified, and making ASD the most common and consistent feature observed in PTLS. [14].

Most PTLS patients have no distinctive facial abnormalities but they can have a triangularshaped face. The other clinical features present in over half of patients include sleep apnea, abnormal EEG, attention deficit, hypermetropia, and cardiovascular abnormalities [15]. These cardiovascular abnormalities can typically include both structural and conduction defects, such as atrial or ventricular septal defects, bicuspid aortic valve, dilated aortic root, dilation of the pulmonary annulus, patent foramen ovale, or hypoplastic left heart [15, 91-93].

Upon molecular analysis, most [22 of 35] PTLS patients included in the first multidisciplinary study were determined to carry a common recurrent $3.7 \mathrm{Mb}$ duplication in 17p11.2 mediated by the same proximal and distal SMS-REPs which also mediate the reciprocal common recurrent SMS deletion [15]. Others have uncommon and sometimes complex genomic rearrangements, all of which involve duplication in 17p11.2 [94]. The smallest PTLS duplication identified to date occurred in a single patient and is $1.3 \mathrm{Mb}$ in size. This duplicated segment contains 14 genes, including both RAI1, the major contributing gene for the reciprocal deletion causing SMS, as well as the steroid-metabolism regulating gene, SREBP1. This patient demonstrates all typical PTLS phenotypes [94]. Whether, or to what extent, PTLS results from RAI1 gene over-dosage still remains to be elucidated, although mouse studies (described below) have shown that it is likely responsible for at least some of the symptoms [95]. 


\section{RAI1: Retinoic acid induced 1}

Spanning over $120 \mathrm{~kb}$, the RAI1 gene consists of six exons, of which the third is the largest, containing $>90 \%$ of the coding region $[80,85,96]$. All of the point mutations identified in SMS patients to date lie within this exon. Most of the mutations are frameshift or nonsense mutations occurring in a specific heptameric poly $\mathrm{C}$ tract hotspot region within $R A I 1$, and thus likely cause loss-of-function alleles [82, 97].

The RAI1 transcript is $7.6 \mathrm{~kb}$, encoding a 1906-amino acid, $200 \mathrm{kDa}$ protein with several known domains, including an extended plant homeodomain (PHD) zinc finger in the carboxyl-terminus (residues 1832-1903; [80]), a polymorphic polyglutamine (CAG) tract in the N-terminus that is associated with the severity of the phenotype and medication response in patients with schizophrenia, as well as the age-at-onset of spino-cerebellar ataxia type 2 (SCA2) [96, 98], two polyserine tracts, two transactivation domains [99], and two bipartite nuclear localization signals (NLS). Importantly, the PHD in Rai1 is highly conserved in the trithorax family of nuclear proteins involved in transcriptional regulation as well as in the formation of a chromatin remodeling complex, suggesting that Rai1 may also function as a transcriptional regulator [100]. Further strengthening this connection, Rai1 is known to be located in the nucleus and have transactivation activity [99], and it shares a similar genomic structure ( $>50 \%$ shared identity and similar zinc finger domains) with another gene, TCF20, or stromelysin1 platelet-derived growth factor (PDGF)-responsive element-binding protein $(S P B P)$, which is known to act as a nuclear transcriptional cofactor [101].

In the human brain, RAI1 is highly-expressed in the hippocampus and the cerebellar cortex, and it is globally-upregulated in the occipital, temporal, and parietal lobes according to expression data from the Allen Brain Atlas (Allen Institute for Brain Science). In contrast, it appears to be down-regulated in the cerebellar nuclei, corpus callosum, dorsal thalamus, and frontal lobe, suggesting that its expression is confined to specific brain regions. Similar to what is seen in humans, Rai1 is also upregulated in the hippocampus and cerebellum of adult mice [102]. Rai1 is critical for development, and the majority of Rai1 mouse embryos are resorbed during development by E15.5 [99]. While Rai1 expression is certainly necessary early in fetal development, according to expression data from mouse embryos, peak Rai1 expression occurs at E18.5 and persists until P4 (Allen Brain Atlas), indicating that it is also required for post-natal development. Although the precise function of RAI1/Rai1 is not currently understood, it is known to be part of a dosage-sensitive pathway that most likely regulates neuronal development and organogensis, that, when perturbed, results in many of the phenotypes observed in both SMS and PTLS. Importantly, RAI1 has been identified in a reconstructed human genenetwork (Prioritizer) as an important candidate gene for involvement in idiopathic autism, suggesting that this gene may function in a common pathway that may influence ASD phenotypes in non-syndromic patients as well [103]. 


\section{Modeling SMS and PTLS in rodents}

Several mouse models interrogating the critical region for SMS and PTLS have been generated in the past decade in order to have an appropriate animal model system to evaluate the phenotypes in SMS and PTLS and to further study the molecular mechanism underlying these disorders. The first of these strains was developed in 2003 using a chromosome-engineering approach described earlier in this chapter [24]. The resulting mouse models harbour either a chromosomal duplication ( $D p(11) 17$, modeling PTLS) or deletion $(D f(11) 17$, modeling SMS) of $\sim 2 \mathrm{Mb}$ that is syntenic to the SMS/PTLS critical region. Soon after, several smaller deletion strains $(\sim 590 \mathrm{~kb}-1 \mathrm{Mb})$ were created using retroviral insertion of loxP sites in ES cells with one fixed end, with the intent to determine which other genes in the critical region may contribute to the complex phenotypes in SMS [104, 105]. Once SMS patients with point mutations in RAI1 were identified, a mouse model harbouring a truncated null allele for Rai1 was generated via gene targeting to further study the function of this dosage-sensitive gene and to compare the phenotype of this model with that of the deletion strains [99, 102]. Likewise, a mouse model harbouring the Rai1 transgene (TgRai1), and globally over-expressing Rai1 at steady-state levels similar to those seen in $D p(11) 17 /+$ mice, was constructed to further study the function of this gene in PTLS [106].

Initial studies of $D p(11) 17 /+$ mice determined that they have reduced weight, reduced abdominal and inguinal fat, and reduced spleen weight [24]. Upon analysis of some of the behavioral traits of these mice, it was determined that they display anxiety-like behaviors, have reduced maximum startle response during the pre-pulse inhibition test, and defects in contextual fear conditioning [107], as well as several other abnormal social behaviors, including decreased nesting, abnormal sociability, and dominant behaviour [108]. Further investigation into the neurobehavioral abnormalities in these mice found that they also have decreased preference for social novelty, motor defects, and increased activity levels in the open field [109]. Many of these behavioral phenotypes are reciprocal or opposing to those seen in $D f(11) 17 /+$ mice, underscoring the dosage-sensitive nature of these disorders [109]. For example, a recent study investigating cerebellum-driven licking behavior in $D p(11) 17 /+$ and $D f(11) 17 /+$ mice found that many of the quantitative licking behavior parameters analyzed were altered in a directly-opposing manner [110]. Specifically, the interval between visits to the waterspout, number of licks per visit, and variability in the number of licks per lick-burst were all altered in duplication and deletion animals in opposite directions compared to wild-type mice (ex: longer versus shorter intervals, etc).

Recently, an extensive battery of behavioral tests were performed and $D p(11) 17 /+$ mice were observed to display complex social abnormalities, including defects in social recognition, dominant and aggressive behavior, as well as abnormal response to social odors [30]. Furthermore, these mice were shown to have altered communication, anxiety-like behavior, disordered circadian rhythm, learning and memory deficits, motor defects, and stereotypic, repetitive behaviors, confirming that these mice model both the core and associated features of autism. In addition, rearing these mice in an enriched environment mitigated or rescued 
certain neurobehavioral abnormalities, suggesting a role for gene-environment interactions in the determination of copy number variation-mediated autism severity [30].

The phenotypic changes observed in $D p(11) 17 /+$ and $D f(11017 /+$ mice are accompanied by changes in gene expression; on average, transcripts in the critical interval are expressed at $138+29 \%$ of wild-type levels in $D p(11) 17 /+$ mice, and at $66+15 \%$ of wild-type levels in $D f(11) 17 /+$ mice [109]. The expression level of these genes can be normalized to roughly that of wild-type mice by crossing $D p(11) 17 /+$ and $D f(11) 17 /+$ mice to create a double heterozygote carrying two copies of the genes within the critical region in cis, (as opposed to the typical trans orientation in wild-type mice). However, the presence of the structural variation itself affects expression of genes outside the affected interval, resulting in "genome regulation" that may ultimately contribute to the phenotype. As a result, these double heterozygous mice display some abnormal behaviors, including elevated activity levels and decreased preference for social novelty [109].

When Rai1 is over-expressed in mice (Rai1-Tg, modeling PTLS), these mice have growth retardation, are underweight, display anxiety-like behavior, social dominance, motor abnormalities, and have increased motor activity in juvenile mice. Furthermore, there is a dosagedependent exacerbation of this phenotype [106]. These mice also display abnormal maternal behavior, altered sociability, reduced reproductive fitness, and impaired serotonin metabolism [111]. Together these results suggest that Rai1- $\mathrm{Tg}$ mice display a complex neurobehavioral and metabolic phenotype similar to that of mice harboring the $D p(11) 17 \mathrm{CNV}$, suggesting that RAI1 is likely responsible for some, if not many of the phenotypes identified in PTLS. Further support for this hypothesis is indicated by studies of $D p(11) 17 / R a i 1$ doubleheterozygous mice with normalized copy number of Rai1, but increased dosage of the surrounding interval; this study revealed that normalization of Rai1 copy number was able to correct weight differences, and at least partially rescue phenotypes on behavioral tests for locomotor activity, anxiety, and learning and memory [95].

\section{Gene-environment interactions}

While genetic defects play a part in the etiology of ASD, environmental effects have long been thought to contribute to these disorders. For example, although the majority of SMS/PTLS patients present with either deletion or duplication of the same $\sim 3.7 \mathrm{Mb}$ genedense region, there is significant variability in the clinical phenotype [112]. Furthermore, while there are some significant differences in the incidence of the abnormalities in patients with the common deletion/duplication compared to those patients with smaller or larger-sized CNVs, a clear distinction between these sub-groups of patients cannot be made; many of these phenotypes are therefore likely strongly influenced by genetic background as well as environmental effects [83, 94]. While gene-environment interactions may potentially explain the source of the variability seen in these syndromes, investigation into the specific environmental factors that may affect outcomes for these genomic disorders has yet to be undertaken. 
Chromosome-engineered mouse models for ASD are ideal for the study of complex disease, as they are mechanistically similar to human patients (targeted duplication/deletion syntenic to human critical interval), they are polygenic (numerous genes are affected), the observed phenotypes equate with common, clinically described features (neurobehavioral phenotypes, sleep disorder, etc), and they can be influenced by environmental factors. In addition, autism is known to be highly variable, and it is suspected to be dependent on both genetic and environmental factors, such as low birth weight and gestational age, prenatal exposure to various agents, parental age at birth, diet, infection, xenobiotic and pesticide exposure, among others [113]. Many of these environmental insults are amenable to study using mouse models, as the interaction of these environmental factors with CNVs can be directly tested in congenic mouse models to control for the effects of genetic background.

Molecular analysis of these mouse models, as well as patient samples, can also be utilized to dissect the role of specific genes or CNVs responsible for the susceptibility to the influence of environmental factors in these autism-related syndromes. Most importantly, the results of these types of studies can provide useful insights as to how genes/CNVs can interact with environmental factors in the context of complex human diseases; this may lead to strategies to alleviate symptoms of not only rare genomic disorders, but also more common idiopathic forms of autism or ASD. Furthermore, these models represent an important resource for future studies of the pathomechanisms underlying ASD, as well as potential treatments for ASD. They may also foster further investigation into the genomic basis of autism and complex behavior, as well the underlying genetic mechanisms leading to these pathogenic CNVs. In studying CNV-based models for complex genomic disorders and ASD, we have come to realize that the ideal animal models of ASD should not only phenocopy relevant human symptoms, but the phenotypes should also be based on similar underlying physiological and genetic mechanisms.

\section{Author details}

Melanie Lacaria ${ }^{1}$ and James R. Lupski ${ }^{1,2}$

*Address all correspondence to: jlupski@bcm.edu

1 Department of Molecular and Human Genetics, Baylor College of Medicine, Houston, TX, USA

2 Department of Pediatrics, Baylor College of Medicine, and Texas Children's Hospital, Houston, TX, USA

\section{References}

[1] Jacquemont ML, Sanlaville D, Redon R, Raoul O, Cormier-Daire V, Lyonnet S, et al. Array-based comparative genomic hybridisation identifies high frequency of cryptic 
chromosomal rearrangements in patients with syndromic autism spectrum disorders. J Med Genet. 2006 Nov;43[11]:843-9.

[2] Gonzaga-Jauregui C, Lupski JR, Gibbs RA. Human genome sequencing in health and disease. Annu Rev Med. 2012;63:35-61.

[3] Bras J, Guerreiro R, Hardy J. Use of next-generation sequencing and other whole-genome strategies to dissect neurological disease. Nat Rev Neurosci. 2012;13([7]): 453-64.

[4] Bainbridge MN, Wiszniewski W, Murdock DR, Friedman J, Gonzaga-Jauregui C, Newsham I, et al. Whole-genome sequencing for optimized patient management. Sci Transl Med. 2011 Jun 15;3[87]:87re3.

[5] O'Roak BJ, Deriziotis P, Lee C, Vives L, Schwartz JJ, Girirajan S, et al. Exome sequencing in sporadic autism spectrum disorders identifies severe de novo mutations. Nat Genet. 2011 Jun;43([6]):585-9.

[6] Neale BM, Kou Y, Liu L, Ma'ayan A, Samocha KE, Sabo A, et al. Patterns and rates of exonic de novo mutations in autism spectrum disorders. Nature. 2012 May 10;485[7397]:242-5.

[7] Ku CS, Polychronakos C, Tan EK, Naidoo N, Pawitan Y, Roukos DH, et al. A new paradigm emerges from the study of de novo mutations in the context of neurodevelopmental disease. Mol Psychiatry. 2012 May 29.

[8] Girirajan S, Campbell CD, Eichler EE. Human copy number variation and complex genetic disease. Annu Rev Genet. 2011;45:203-26.

[9] Sebat J, Lakshmi B, Malhotra D, Troge J, Lese-Martin C, Walsh T, et al. Strong association of de novo copy number mutations with autism. Science. 2007 Apr 20;316[5823]:445-9.

[10] Bolton PF, Veltman MW, Weisblatt E, Holmes JR, Thomas NS, Youings SA, et al. Chromosome 15q11-13 abnormalities and other medical conditions in individuals with autism spectrum disorders. Psychiatr Genet. 2004 Sep;14([3]):131-7.

[11] Hogart A, Wu D, LaSalle JM, Schanen NC. The comorbidity of autism with the genomic disorders of chromosome 15q11.2-q13. Neurobiol Dis. 2010 May;38([2]):181-91.

[12] Weiss LA, Shen Y, Korn JM, Arking DE, Miller DT, Fossdal R, et al. Association between microdeletion and microduplication at 16p11.2 and autism. N Engl J Med. 2008 Feb 14;358([7]):667-75.

[13] Sanders SJ, Ercan-Sencicek AG, Hus V, Luo R, Murtha MT, Moreno-De-Luca D, et al. Multiple recurrent de novo CNVs, including duplications of the 7q11.23 Williams syndrome region, are strongly associated with autism. Neuron. 2011 Jun 9;70([5]): 863-85. 
[14] Treadwell-Deering DE, Powell MP, Potocki L. Cognitive and behavioral characterization of the Potocki-Lupski syndrome (duplication 17p11.2]. J Dev Behav Pediatr. 2010 Feb-Mar;31([2]):137-43.

[15] Potocki L, Bi W, Treadwell-Deering D, Carvalho CM, Eifert A, Friedman EM, et al. Characterization of Potocki-Lupski syndrome (dup[17](p11.2p11.2]) and delineation of a dosage-sensitive critical interval that can convey an autism phenotype. Am J Hum Genet. 2007 Apr;80([4]):633-49.

[16] Berg JS, Brunetti-Pierri N, Peters SU, Kang SH, Fong CT, Salamone J, et al. Speech delay and autism spectrum behaviors are frequently associated with duplication of the 7q11.23 Williams-Beuren syndrome region. Genet Med. 2007 Jul;9([7]):427-41.

[17] Cho SC, Yim SH, Yoo HK, Kim MY, Jung GY, Shin GW, et al. Copy number variations associated with idiopathic autism identified by whole-genome microarraybased comparative genomic hybridization. Psychiatr Genet. 2009 Aug;19([4]):177-85.

[18] Luo R, Sanders SJ, Tian Y, Voineagu I, Huang N, Chu SH, et al. Genome-wide Transcriptome Profiling Reveals the Functional Impact of Rare De Novo and Recurrent CNVs in Autism Spectrum Disorders. Am J Hum Genet. 2012 Jun 21.

[19] Glessner JT, Wang K, Cai G, Korvatska O, Kim CE, Wood S, et al. Autism genomewide copy number variation reveals ubiquitin and neuronal genes. Nature. 2009 May 28;459[7246]:569-73.

[20] Talkowski ME, Mullegama SV, Rosenfeld JA, van Bon BW, Shen Y, Repnikova EA, et al. Assessment of 2q23.1 microdeletion syndrome implicates MBD5 as a single causal locus of intellectual disability, epilepsy, and autism spectrum disorder. Am J Hum Genet. 2011 Oct 7;89([4]):551-63.

[21] Chahrour MH, Yu TW, Lim ET, Ataman B, Coulter ME, Hill RS, et al. Whole-exome sequencing and homozygosity analysis implicate depolarization-regulated neuronal genes in autism. PLoS Genet. 2012 8([4]):e1002635.

[22] Geschwind DH. Autism: many genes, common pathways? Cell. 2008 Oct 31;135([3]): 391-5.

[23] Crawley JN. Mouse behavioral assays relevant to the symptoms of autism. Brain Pathol. 2007 Oct;17([4]):448-59.

[24] Walz K, Caratini-Rivera S, Bi W, Fonseca P, Mansouri DL, Lynch J, et al. Modeling $\operatorname{del}[17](p 11.2 p 11.2]$ and dup[17](p11.2p11.2] contiguous gene syndromes by chromosome engineering in mice: phenotypic consequences of gene dosage imbalance. Mol Cell Biol. 2003 May;23[10]:3646-55.

[25] Mills AA, Bradley A. From mouse to man: generating megabase chromosome rearrangements. Trends Genet. 2001 Jun;17([6]):331-9.

[26] van der Weyden L, Bradley A. Mouse chromosome engineering for modeling human disease. Annu Rev Genomics Hum Genet. 2006;7:247-76. 
[27] Adams DJ, Biggs PJ, Cox T, Davies R, van der Weyden L, Jonkers J, et al. Mutagenic insertion and chromosome engineering resource (MICER). Nat Genet. 2004 Aug; $36([8]): 867-71$.

[28] Nakatani J, Tamada K, Hatanaka F, Ise S, Ohta H, Inoue K, et al. Abnormal behavior in a chromosome-engineered mouse model for human 15q11-13 duplication seen in autism. Cell. 2009 Jun 26;137([7]):1235-46.

[29] Horev G, Ellegood J, Lerch JP, Son YE, Muthuswamy L, Vogel H, et al. Dosage-dependent phenotypes in models of 16p11.2 lesions found in autism. Proc Natl Acad Sci U S A. 2011 Oct 11;108[41]:17076-81.

[30] Lacaria M, Spencer C, Gu W, Paylor R, Lupski JR. Enriched Rearing Improves Behavioral Responses of an Animal Model for CNV-based Autistic-like Traits. Hum Mol Genet. 2012 Jul 15;21(14):3083-96.

[31] Lupski JR. Genomic disorders: structural features of the genome can lead to DNA rearrangements and human disease traits. Trends Genet. 1998 Oct;14[10]:417-22.

[32] Lupski JR, Stankiewicz P. Genomic disorders: molecular mechanisms for rearrangements and conveyed phenotypes. PLoS Genet. 2005 Dec;1([6]):e49.

[33] Lupski JR. Genomic disorders ten years on. Genome Med. 2009;1([4]):42.

[34] Zhang F, Gu W, Hurles ME, Lupski JR. Copy number variation in human health, disease, and evolution. Annu Rev Genomics Hum Genet. 2009;10:451-81.

[35] Gu W, Zhang F, Lupski JR. Mechanisms for human genomic rearrangements. Pathogenetics. 2008;1([1]):4.

[36] Shinawi M, Liu P, Kang SH, Shen J, Belmont JW, Scott DA, et al. Recurrent reciprocal 16p11.2 rearrangements associated with global developmental delay, behavioural problems, dysmorphism, epilepsy, and abnormal head size. J Med Genet. 2009 May; $47([5]): 332-41$.

[37] Kumar RA, Marshall CR, Badner JA, Babatz TD, Mukamel Z, Aldinger KA, et al. Association and mutation analyses of $16 \mathrm{p} 11.2$ autism candidate genes. PLoS One. 2009;4([2]):e4582.

[38] Marshall CR, Noor A, Vincent JB, Lionel AC, Feuk L, Skaug J, et al. Structural variation of chromosomes in autism spectrum disorder. Am J Hum Genet. 2008 Feb; 82([2]):477-88.

[39] Fernandez BA, Roberts W, Chung B, Weksberg R, Meyn S, Szatmari P, et al. Phenotypic spectrum associated with de novo and inherited deletions and duplications at 16p11.2 in individuals ascertained for diagnosis of autism spectrum disorder. J Med Genet. 2010 Mar;47([3]):195-203.

[40] Ghebranious N, Giampietro PF, Wesbrook FP, Rezkalla SH. A novel microdeletion at 16p11.2 harbors candidate genes for aortic valve development, seizure disorder, and mild mental retardation. Am J Med Genet A. 2007 Jul 1;143A[13]:1462-71. 
[41] McCarthy SE, Makarov V, Kirov G, Addington AM, McClellan J, Yoon S, et al. Microduplications of 16p11.2 are associated with schizophrenia. Nat Genet. 2009 Nov; 41[11]:1223-7.

[42] Lupski JR. Insights for autism from Charcot-Marie-Tooth disease. SFARI. September 27, 2011.

[43] Crespi B, Badcock C. Psychosis and autism as diametrical disorders of the social brain. Behav Brain Sci. 2008 Jun;31([3]):241-61; discussion 61-320.

[44] Jacquemont S, Reymond A, Zufferey F, Harewood L, Walters RG, Kutalik Z, et al. Mirror extreme BMI phenotypes associated with gene dosage at the chromosome 16p11.2 locus. Nature. 2011 Oct 6;478[7367]:97-102.

[45] Yu Y, Zhu H, Miller DT, Gusella JF, Platt OS, Wu BL, et al. Age- and gender-dependent obesity in individuals with 16p11.2 deletion. J Genet Genomics. 2011 Sep 20;38([9]):403-9.

[46] Perrone L, Marzuillo P, Grandone A, del Giudice EM. Chromosome 16p11.2 deletions: another piece in the genetic puzzle of childhood obesity. Ital J Pediatr. 2010;36:43.

[47] Walters RG, Jacquemont S, Valsesia A, de Smith AJ, Martinet D, Andersson J, et al. A new highly penetrant form of obesity due to deletions on chromosome 16p11.2. Nature. 2010 Feb 4;463[7281]:671-5.

[48] Puvabanditsin S, Nagar MS, Joshi M, Lambert G, Garrow E, Brandsma E. Microdeletion of 16p11.2 associated with endocardial fibroelastosis. Am J Med Genet A. 2010 Sep;152A([9]):2383-6.

[49] Degenhardt F, Priebe L, Herms S, Mattheisen M, Muhleisen TW, Meier S, et al. Association between copy number variants in 16p11.2 and major depressive disorder in a German case-control sample. Am J Med Genet B Neuropsychiatr Genet. 2012 Apr; 159B([3]):263-73.

[50] Rosenfeld JA, Coppinger J, Bejjani BA, Girirajan S, Eichler EE, Shaffer LG, et al. Speech delays and behavioral problems are the predominant features in individuals with developmental delays and 16p11.2 microdeletions and microduplications. J Neurodev Disord. Mar;2([1]):26-38.

[51] Bijlsma EK, Gijsbers AC, Schuurs-Hoeijmakers JH, van Haeringen A, Fransen van de Putte DE, Anderlid BM, et al. Extending the phenotype of recurrent rearrangements of 16p11.2: deletions in mentally retarded patients without autism and in normal individuals. Eur J Med Genet. 2009 Mar-Jun;52[2-3]:77-87.

[52] Shimojima K, Inoue T, Fujii Y, Ohno K, Yamamoto T. A familial 593-kb microdeletion of 16p11.2 associated with mental retardation and hemivertebrae. Eur J Med Genet. 2009 Nov-Dec;52([6]):433-5. 
[53] Schaaf CP, Goin-Kochel RP, Nowell KP, Hunter JV, Aleck KA, Cox S, et al. Expanding the clinical spectrum of the 16p11.2 chromosomal rearrangements: three patients with syringomyelia. Eur J Hum Genet. 2010 Feb;19([2]):152-6.

[54] Crepel A, Steyaert J, De la Marche W, De Wolf V, Fryns JP, Noens I, et al. Narrowing the critical deletion region for autism spectrum disorders on 16p11.2. Am J Med Genet B Neuropsychiatr Genet. 2011 Mar;156([2]):243-5.

[55] Konyukh M, Delorme R, Chaste P, Leblond C, Lemiere N, Nygren G, et al. Variations of the candidate SEZ6L2 gene on Chromosome 16p11.2 in patients with autism spectrum disorders and in human populations. PLoS One. 2011;6([3]):e17289.

[56] Shen Y, Chen X, Wang L, Guo J, Shen J, An Y, et al. Intra-family phenotypic heterogeneity of 16p11.2 deletion carriers in a three-generation Chinese family. Am J Med Genet B Neuropsychiatr Genet. 2010 Mar;156([2]):225-32.

[57] Golzio C, Willer J, Talkowski ME, Oh EC, Taniguchi Y, Jacquemont S, et al. KCTD13 is a major driver of mirrored neuroanatomical phenotypes of the $16 \mathrm{p} 11.2$ copy number variant. Nature. 2012 May 17;485[7398]:363-7.

[58] Blaker-Lee A, Gupta S, McCammon JM, De Rienzo G, Sive H. Zebrafish homologs of genes within 16p11.2, a genomic region associated with brain disorders, are active during brain development, and include two deletion dosage sensor genes. Dis Model Mech. 2012 Nov;5(6):834-51..

[59] Veltman MW, Craig EE, Bolton PF. Autism spectrum disorders in Prader-Willi and Angelman syndromes: a systematic review. Psychiatr Genet. 2005 Dec;15([4]):243-54.

[60] Lalande M, Calciano MA. Molecular epigenetics of Angelman syndrome. Cell Mol Life Sci. 2007 Apr;64[7-8]:947-60.

[61] Dykens EM, Lee E, Roof E. Prader-Willi syndrome and autism spectrum disorders: an evolving story. J Neurodev Disord. 2011 Sep;3([3]):225-37.

[62] Smith SE, Zhou YD, Zhang G, Jin Z, Stoppel DC, Anderson MP. Increased gene dosage of Ube3a results in autism traits and decreased glutamate synaptic transmission in mice. Sci Transl Med. 2011 Oct 5;3[103]:103ra97.

[63] Roberts SE, Dennis NR, Browne CE, Willatt L, Woods G, Cross I, et al. Characterisation of interstitial duplications and triplications of chromosome 15q11-q13. Hum Genet. 2002 Mar;110([3]):227-34.

[64] Wegiel J, Schanen NC, Cook EH, Sigman M, Brown WT, Kuchna I, et al. Differences between the pattern of developmental abnormalities in autism associated with duplications 15q11.2-q13 and idiopathic autism. J Neuropathol Exp Neurol. 2012 May; 71([5]):382-97.

[65] Ungaro P, Christian SL, Fantes JA, Mutirangura A, Black S, Reynolds J, et al. Molecular characterisation of four cases of intrachromosomal triplication of chromosome 15q11-q14. J Med Genet. 2001 Jan;38([1]):26-34. 
[66] Stewart LR, Hall AL, Kang SH, Shaw CA, Beaudet AL. High frequency of known copy number abnormalities and maternal duplication 15q11-q13 in patients with combined schizophrenia and epilepsy. BMC Med Genet. 2011;12:154.

[67] Wegiel J, Frackowiak J, Mazur-Kolecka B, Schanen NC, Cook EH, Jr., Sigman M, et al. Abnormal intracellular accumulation and extracellular Abeta deposition in idiopathic and Dup15q11.2-q13 autism spectrum disorders. PLoS One. 2012;7([5]):e35414.

[68] Yang T, Adamson TE, Resnick JL, Leff S, Wevrick R, Francke U, et al. A mouse model for Prader-Willi syndrome imprinting-centre mutations. Nat Genet. 1998 May;19([1]): 25-31.

[69] Relkovic D, Doe CM, Humby T, Johnstone KA, Resnick JL, Holland AJ, et al. Behavioural and cognitive abnormalities in an imprinting centre deletion mouse model for Prader-Willi syndrome. Eur J Neurosci. 2010 Jan;31([1]):156-64.

[70] Godavarthi SK, Dey P, Maheshwari M, Jana NR. Defective glucocorticoid hormone receptor signaling leads to increased stress and anxiety in a mouse model of Angelman syndrome. Hum Mol Genet. 2012 Apr 15;21([8]):1824-34.

[71] Mulherkar SA, Jana NR. Loss of dopaminergic neurons and resulting behavioural deficits in mouse model of Angelman syndrome. Neurobiol Dis. 2010 Dec;40([3]): 586-92.

[72] Heck DH, Zhao Y, Roy S, LeDoux MS, Reiter LT. Analysis of cerebellar function in Ube3a-deficient mice reveals novel genotype-specific behaviors. Hum Mol Genet. 2008 Jul 15;17[14]:2181-9.

[73] Colas D, Cespuglio R, Sarda N. Sleep wake profile and EEG spectral power in young or old senescence accelerated mice. Neurobiol Aging. 2005 Feb;26([2]):265-73.

[74] Miura K, Kishino T, Li E, Webber H, Dikkes P, Holmes GL, et al. Neurobehavioral and electroencephalographic abnormalities in Ube3a maternal-deficient mice. Neurobiol Dis. 2002 Mar;9([2]):149-59.

[75] Allensworth M, Saha A, Reiter LT, Heck DH. Normal social seeking behavior, hypoactivity and reduced exploratory range in a mouse model of Angelman syndrome. BMC Genet. 2011;12:7.

[76] Jiang YH, Pan Y, Zhu L, Landa L, Yoo J, Spencer C, et al. Altered ultrasonic vocalization and impaired learning and memory in Angelman syndrome mouse model with a large maternal deletion from Ube3a to Gabrb3. PLoS One. 2010;5([8]):e12278.

[77] Bi W, Lupski JR. RAI1, the Smith-Magenis, and Potocki-Lupski Syndromes. In: Epstein CJ, Erickson RP, Wynshaw-Boris A, editors. Inborn Errors of Development; 2008.

[78] Liu P, Lacaria M, Zhang F, Withers M, Hastings PJ, Lupski JR. Frequency of nonallelic homologous recombination is correlated with length of homology: evidence that 
ectopic synapsis precedes ectopic crossing-over. Am J Hum Genet. 2011 Oct 7;89([4]): 580-8.

[79] Shaw CJ, Withers MA, Lupski JR. Uncommon deletions of the Smith-Magenis syndrome region can be recurrent when alternate low-copy repeats act as homologous recombination substrates. Am J Hum Genet. 2004 Jul;75([1]):75-81.

[80] Bi W, Saifi GM, Shaw CJ, Walz K, Fonseca P, Wilson M, et al. Mutations of RAI1, a PHD-containing protein, in nondeletion patients with Smith-Magenis syndrome. Hum Genet. 2004 Nov;115([6]):515-24.

[81] Slager RE, Newton TL, Vlangos CN, Finucane B, Elsea SH. Mutations in RAI1 associated with Smith-Magenis syndrome. Nat Genet. 2003 Apr;33([4]):466-8.

[82] Bi W, Saifi GM, Girirajan S, Shi X, Szomju B, Firth H, et al. RAI1 point mutations, CAG repeat variation, and SNP analysis in non-deletion Smith-Magenis syndrome. Am J Med Genet A. 2006 Nov 15;140[22]:2454-63.

[83] Edelman EA, Girirajan S, Finucane B, Patel PI, Lupski JR, Smith AC, et al. Gender, genotype, and phenotype differences in Smith-Magenis syndrome: a meta-analysis of 105 cases. Clin Genet. 2007 Jun;71([6]):540-50.

[84] Greenberg F, Lewis RA, Potocki L, Glaze D, Parke J, Killian J, et al. Multi-disciplinary clinical study of Smith-Magenis syndrome (deletion 17p11.2]. Am J Med Genet. 1996 Mar 29;62([3]):247-54.

[85] Greenberg F, Guzzetta V, Montes de Oca-Luna R, Magenis RE, Smith AC, Richter SF, et al. Molecular analysis of the Smith-Magenis syndrome: a possible contiguous-gene syndrome associated with del[17](p11.2]. Am J Hum Genet. 1991 Dec;49([6]):1207-18.

[86] Finucane BM, Konar D, Haas-Givler B, Kurtz MB, Scott CI, Jr. The spasmodic upperbody squeeze: a characteristic behavior in Smith-Magenis syndrome. Dev Med Child Neurol. 1994 Jan;36([1]):78-83.

[87] Arron K, Oliver C, Moss J, Berg K, Burbidge C. The prevalence and phenomenology of self-injurious and aggressive behaviour in genetic syndromes. J Intellect Disabil Res. 2010 Feb;55([2]):109-20.

[88] Laje G, Morse R, Richter W, Ball J, Pao M, Smith AC. Autism spectrum features in Smith-Magenis syndrome. Am J Med Genet C Semin Med Genet. 2010 Nov $15 ; 154 \mathrm{C}([4]): 456-62$.

[89] Potocki L, Chen KS, Park SS, Osterholm DE, Withers MA, Kimonis V, et al. Molecular mechanism for duplication 17p11.2- the homologous recombination reciprocal of the Smith-Magenis microdeletion. Nat Genet. 2000 Jan;24([1]):84-7.

[90] Soler-Alfonso C, Motil KJ, Turk CL, Robbins-Furman P, Friedman EM, Zhang F, et al. Potocki-Lupski syndrome: a microduplication syndrome associated with oropharyngeal dysphagia and failure to thrive. J Pediatr. 2010 Apr;158([4]):655-9 e2. 
[91] Yusupov R, Roberts AE, Lacro RV, Sandstrom M, Ligon AH. Potocki-Lupski syndrome: an inherited dup[17](p11.2p11.2] with hypoplastic left heart. Am J Med Genet A. 2011 Feb;155A([2]):367-71.

[92] Sanchez-Valle A, Pierpont ME, Potocki L. The severe end of the spectrum: Hypoplastic left heart in Potocki-Lupski syndrome. Am J Med Genet A. Feb;155A([2]):363-6.

[93] Jefferies JL, Pignatelli RH, Martinez HR, Robbins-Furman PJ, Liu P, Gu W, et al. Cardiovascular findings in duplication 17p11.2 syndrome. Genet Med. 2012 Jan;14([1]): 90-4.

[94] Zhang F, Potocki L, Sampson JB, Liu P, Sanchez-Valle A, Robbins-Furman P, et al. Identification of uncommon recurrent Potocki-Lupski syndrome-associated duplications and the distribution of rearrangement types and mechanisms in PTLS. Am J Hum Genet. 2010 Mar 12;86([3]):462-70.

[95] Walz K, Paylor R, Yan J, Bi W, Lupski JR. Rai1 duplication causes physical and behavioral phenotypes in a mouse model of dup[17](p11.2p11.2]. J Clin Invest. 2006 Nov;116[11]:3035-41.

[96] Toulouse A, Rochefort D, Roussel J, Joober R, Rouleau GA. Molecular cloning and characterization of human RAI1, a gene associated with schizophrenia. Genomics. 2003 Aug;82([2]):162-71.

[97] Truong HT, Dudding T, Blanchard CL, Elsea SH. Frameshift mutation hotspot identified in Smith-Magenis syndrome: case report and review of literature. BMC Med Genet. 2010;11:142.

[98] Hayes S, Turecki G, Brisebois K, Lopes-Cendes I, Gaspar C, Riess O, et al. CAG repeat length in RAI1 is associated with age at onset variability in spinocerebellar ataxia type 2 (SCA2]. Hum Mol Genet. 2000 Jul 22;9[12]:1753-8.

[99] Bi W, Ohyama T, Nakamura H, Yan J, Visvanathan J, Justice MJ, et al. Inactivation of Rai1 in mice recapitulates phenotypes observed in chromosome engineered mouse models for Smith-Magenis syndrome. Hum Mol Genet. 2005 Apr 15;14([8]):983-95.

[100] Nakamura T, Mori T, Tada S, Krajewski W, Rozovskaia T, Wassell R, et al. ALL-1 is a histone methyltransferase that assembles a supercomplex of proteins involved in transcriptional regulation. Mol Cell. 2002 Nov;10([5]):1119-28.

[101] Rekdal C, Sjottem E, Johansen T. The nuclear factor SPBP contains different functional domains and stimulates the activity of various transcriptional activators. J Biol Chem. 2000 Dec 22;275[51]:40288-300.

[102] Bi W, Yan J, Shi X, Yuva-Paylor LA, Antalffy BA, Goldman A, et al. Rai1 deficiency in mice causes learning impairment and motor dysfunction, whereas Rai1 heterozygous mice display minimal behavioral phenotypes. Hum Mol Genet. 2007 Aug 1;16[15]: 1802-13. 
[103] van der Zwaag B, Franke L, Poot M, Hochstenbach R, Spierenburg HA, Vorstman JA, et al. Gene-network analysis identifies susceptibility genes related to glycobiology in autism. PLoS One. 2009;4([5]):e5324.

[104] Yan J, Bi W, Lupski JR. Penetrance of craniofacial anomalies in mouse models of Smith-Magenis syndrome is modified by genomic sequence surrounding Rai1: not all null alleles are alike. Am J Hum Genet. 2007 Mar;80([3]):518-25.

[105] Yan J, Keener VW, Bi W, Walz K, Bradley A, Justice MJ, et al. Reduced penetrance of craniofacial anomalies as a function of deletion size and genetic background in a chromosome engineered partial mouse model for Smith-Magenis syndrome. Hum Mol Genet. 2004 Nov 1;13[21]:2613-24.

[106] Girirajan S, Patel N, Slager RE, Tokarz ME, Bucan M, Wiley JL, et al. How much is too much? Phenotypic consequences of Rai1 overexpression in mice. Eur J Hum Genet. 2008 Aug;16([8]):941-54.

[107] Walz K, Spencer C, Kaasik K, Lee CC, Lupski JR, Paylor R. Behavioral characterization of mouse models for Smith-Magenis syndrome and dup[17](p11.2p11.2]. Hum Mol Genet. 2004 Feb 15;13([4]):367-78.

[108] Molina J, Carmona-Mora P, Chrast J, Krall PM, Canales CP, Lupski JR, et al. Abnormal social behaviors and altered gene expression rates in a mouse model for PotockiLupski syndrome. Hum Mol Genet. 2008 Aug 15;17[16]:2486-95.

[109] Ricard G, Molina J, Chrast J, Gu W, Gheldof N, Pradervand S, et al. Phenotypic consequences of copy number variation: insights from Smith-Magenis and Potocki-Lupski syndrome mouse models. PLoS Biol. 2010;8[11]:e1000543.

[110] Heck D, Gu W, Cao Y, Qi S, Lacaria M, Lupski JR. Opposing phenotypes in mouse models for Smith-Magenis deletion and Potocki -Lupski duplication syndromes suggest gene dosage effects on fluid consumption behavior. Am J Med Genet Part A. 2012;9999:1-7.

[111] Girirajan S, Elsea SH. Abnormal maternal behavior, altered sociability, and impaired serotonin metabolism in Rai1-transgenic mice. Mamm Genome. 2009 Apr;20([4]): 247-55.

[112] Potocki L, Shaw CJ, Stankiewicz P, Lupski JR. Variability in clinical phenotype despite common chromosomal deletion in Smith-Magenis syndrome [del[17] (p11.2p11.2]]. Genet Med. 2003 Nov-Dec;5([6]):430-4.

[113] Duchan E, Patel DR. Epidemiology of autism spectrum disorders. Pediatr Clin North Am. 2012 Feb;59([1]):27-43, ix-x. 
Chapter 13

\title{
Autism Spectrum Disorders: Insights from Genomics
}

\author{
John J. Connolly and Hakon Hakonarson \\ Additional information is available at the end of the chapter \\ http://dx.doi.org/10.5772/54357
}

\section{Introduction}

The field of genetics has made considerable scientific progress in the past several years and continues to evolve at a rapid pace. This progress parallels developments in genomic technology, where instrumentation and methodology are becoming increasingly sophisticated and cost-effective. Here, we review recent developments in understanding autism spectrum disorder (ASD) from a genomics perspective. A large catalog of common and rare variants has now been associated with ASD, and we are beginning to see some of these discoveries translate into pharmacogenomic intervention. This review provides an overview of genome-wide association studies (GWAS) and common genetic variants, followed by an overview of the status of rare variant research, which have risen to prominence with the proliferation of next-generation sequencing and techniques for identifying copy number variants. While these approaches need not be mutually exclusive, they provide a useful structure for organizing relevant genetic factors. Although there is much work to be done before these discoveries will enter the clinic, the past decade has seen us make major inroads in elucidating the causes of ASD and making tentative steps towards developing treatments.

\subsection{Defining the autism phenotype}

Autism is known to be highly heterogeneous, and this phenomenon has made definitions of the phenotype somewhat problematic. The American Psychiatric Association recently proposed revisions to its Diagnostic and Statistical Manual of Mental Disorders V (DSM-5) criteria for ASD (see Wing et al., 2011) [1], acknowledging the long-observed overlap between social and communication dimensions (previously separate). Thus, ASD will be defined by 1) persistent deficits in social communication and social interaction across contexts, and 2) restricted, repetitive patterns of behavior, interests, or activities. These should impair every- 
day functioning, not be accounted for by general developmental delays, and be present from early childhood.

For large-scale genome analyses, DSM criteria have been considered insufficiently precise, and cases are often selected using scores from the Autism Diagnostic Interview (ADI-R) [2], Autism Diagnostic Observation Schedule (ADOS) [3], and/or Social Responsiveness Scale (SRS) [4]. These instruments offer a more robust psychometric platform, and cases defined as "autism" are required to meet strict threshold criteria (e.g. all sub-dimensions of the ADI$\mathrm{R}$ and ADOS). Individuals not quite meeting these criteria may be subsumed under the "broader" autism phenotype, which also typically includes Asperger syndrome, childhood disintegrative disorder and pervasive developmental disorder not otherwise specified. A diagnosis of Rett syndrome-which has a reportedly distinct pathophysiology, clinical course, and diagnostic strategy (Levy, Mandell \& Schultz, 2009) [5] and will likely be removed in the impending publication of DSM-V-is typically exclusionary. Intellectual impairment, which is often co-morbid with ASD (Dawson et al., 2007; Bölte et al., 2009) [6,7] is not an exclusionary criterion, but is co-varied in statistical analyses. Given the broad range of IQ tests and their associated psychometric properties, this requires considerable finesse.

Standardization of diagnostic criteria has facilitated the accumulation of large ASD samplesets, where institutions can share (de-identified) data. In this vein, initiatives such as the Autism Genome Project include data from several thousand ASD individuals, greatly increasing statistical power of relevant analyses.

\subsection{Heritability of ASD}

Although Skuse (2007) [8] cautions that heritability estimates of ASD may have been skewed by the co-inheritance of (low) intelligence or other variables, there is little doubt that genetic factors play a key role in autism. In the most widely-cited twin study, Bailey et al. (1995) [9] report that monozygotic twins are $92 \%$ concordant on a broad spectrum of cognitive or social abnormalities, compared with only $10 \%$ for dizygotic twins. Parents and siblings of individuals with ASD often exhibit subsyndromal levels of impairment (Piven et al., 1997) [10], and having an affected sibling is the single biggest risk factor for developing an ASD. In an analysis of 943,664 Danish children (Lauritsen et al., 2005) [11], the strongest predictors of autism were siblings with ASD, who conferred a 22-fold increased risk, while Fombonne (2005) [12] suggested that this risk may be even greater.

\subsection{Early genetic studies - insights from Rett syndrome and Fragile $X$}

Early efforts to identify the genetic causes of ASD utilized linkage and association approaches. Linkage studies, more prominent in the 1980s and 1990s, typically focus on families or larger pedigrees and are well powered to identify rarer genetic variants. The most common linkage approach is the affected sib-pair design (see O'Roak \& State, 2008) [13], which examines the transmission of genomic segments through generations. Linkage studies helped define the locus containing FMR1, which is mutated in fragile $\mathrm{X}$ syndrome (e.g. Richards et al., 1991) [14], Approximately 30\% of children with fragile X syndrome meet cri- 
teria for autism (Rogers et al., 2001; Harris et al., 2008) [15,16]. Similarly, linkage studies have been important to identifying MECP2 as the major cause of Rett syndrome (e.g. Curtis et al., 1993) [17].

Association studies take a different approach. Rather than track transmission of specific genomic regions through generations, association studies scan the breadth of the genome. Here, the goal is to determine post-hoc whether identified variants are more or less common in affected individuals. Early association studies (i.e. pre HapMap) were complementary with the linkage approach, and in many designs, linkage primed target loci for this more fine-grained analysis.

These early insights have played an important role in shaping our current understanding of ASD. Functional studies of FMR1 and MECP2 have highlighted the importance of synaptic dysfunction (Ramocki \& Zoghbi, 2008) [18] as a unifying factor that could extend into the more common forms of autism and, as discussed below, remain highly relevant to our understanding of the broader ASD phenotype.

\section{Genome wide association and common variants}

Aside from notable successes with fragile $X$ and Rett syndrome, early linkage and association studies have been inconsistent in resolving more complex genetic correlates of ASD, and candidate genes have often not being replicated between studies. These challenges may in part be accounted-for by their relatively low resolution/coverage, making it difficult to detect candidate loci other than those of major effect. A shift in technology was required to get beyond such challenges, which was engendered by the introduction of high-resolution single nucleotide polymorphism (SNP) arrays. SNP arrays provided coverage of many thousand (now several million) common SNPs, which could be examined at a relatively low cost across large sample sets.

Genome-wide association studies (GWAS) examine the frequency of SNPs in case versus control populations, and can adopt either a case-control or family-based design. The former allows researchers to avoid the often complex process of acquiring diagnostic/phenotype data from a patient's family, and can incorporate very large numbers of control datasets that may be more readily available. The latter controls for the often confounding phenomenon of population stratification, where variants more common to specific racial groups may either be erroneously identified as causal, or obscure actual causal variants. A major caveat with family-based designs is the often unfounded assumption that unaffected family members do not share causal variants.

GWAS test for common variants ( $>1 \%$ population frequency), with the assumption that ASD are at least in part caused by the coinheritance of multiple risk variants, each of small individual effect (odds ratios typically between $\sim 1: 1$ and $\sim 1: 5$ ). This assumption is known as the common disease-common variant (CDCV) model (Risch \& Merikangas, 1996) [20]. 


\subsection{The 5 p14.1 locus}

A 2009 paper from our laboratory (Wang et al., 2009 [21]) was the first to identify common variants for ASD on a genome-wide scale. Our group examined 780 families (3,101 individuals) with affected children, a second, independent group of 1,204 affected individuals, and 6,491 controls. All were of European ancestry. We identified six genetic markers on chromosome 5 in the 5p14.1 region that confirmed susceptibility to ASD. This locus has been replicated in two additional independent cohort studies (Ma et al., 2009; St Pourcain et al., 2010) $[22,23]$, lending further support for 5p14 as associated with ASD risk.

As shown in Figure 1, the region straddles two genes, $C D H 9$ and $C D H 10$. Both genes encode type II classical cadherins, transmembrane proteins that promote cell adhesion. Cadherins represent a large family of transmembrane proteins that mediate calcium-dependent cellcell adhesion, and have been shown to generate synaptic complexity in the developing brain (Redies, Hertel \& Hübner, 2012) [24]. The association of cadherins is consistent with the cortical-disconnectivity model of autism (e.g. Gepner \& Féron, 2009) [25], which postulates that ASD may result from an increase or decrease in functional connectivity and neuronal synchronization in relevant neural pathways. While this hypothesis may yet be confirmed, a recent study by Kerin et al. (2012) [26] suggests a more complex mechanism to explain association between ASD and the 5p14.1 locus.

Basing their analyses on the genomic region surrounding the rs4307059 locus, the authors used a bioinformatics approach (i.e. Genome Browser) to examine relevant expressed sequence tags (ESTs) and RNA (by Tiling Array within the 100-kb linkage disequilibrium at the GWAS peak). Only one functional element-a single noncoding RNA - was located. The 3.9-kb RNA corresponded to moesin pseudogene 1 (MSNP1), and has $94 \%$ sequence identity to the mature mRNA of the protein-coding gene MSN. Located on the $\mathrm{X}$ chromosome (Xq11.2), MSN spans $74 \mathrm{~kb}$ and contains 13 exons. It produces a 4-kb mRNA, and encodes the 577-amino acid moesin protein. The noncoding RNA at 5p14.1 was encoded by the opposite (antisense) strand of MSNP1, and was therefore named moesin pseudogene 1, antisense - MSNP1AS.

Follow-up analyses by the group largely confirm that MSNP1AS is expressed in the brain, providing important functional validation. Using custom TaqMan Gene Expression assays to target the region, they showed that while MSN was widely expressed in all tissues tested, MSNP1AS was expressed variably. Sites of greatest expression were the adult temporal cerebral cortex, adult peripheral blood, and fetal heart, as well as three immortalized cell lines. Moreover, postmortem analyses (qPCR on total RNA) of fresh-frozen, superior temporal gyri of ASD-control pairs ( $\mathrm{n}=10)$ found a 12.7-fold increase of MSNP1AS expression in the temporal cortex of individuals with ASD. Individuals with ASD also showed a 2.4-fold increase in MSN expression in the same region. Interestingly, there was no evidence of increased expression for either $\mathrm{CDH} 9$ or $\mathrm{CDH} 10$.

The group next used genotype-determined from three associated SNPs from the original Wang et al. paper-as the independent variable in expression analyses. All three SNPs, rs7704909, rs12518194, and rs4307059, have a high degree of linkage disequilibrium (LD) 
$\left(r^{2}>0.98\right)$. Using resequencing to compare relevant genotypes, they identified highly significant differences in MSNP1AS expression. Thus, the T/T genotype at rs7704909 corresponded to a 23.3 fold increase in MSNP1AS RNA compared to the C/C genotype. For the rs4307059, the $\mathrm{T} / \mathrm{T}$ versus $\mathrm{C} / \mathrm{C}$ genotype corresponded to a 22.0 -fold increase in MSNP1A expression. For rs12518194, the A/A versus G/G genotype corresponded to a 10.8 fold increase in MSNP1A expression. Again, there was no evidence of increased/decreased expression differences for $\mathrm{CDH} 9$ or $\mathrm{CDH} 10$ in relation to genotype or case/control status.
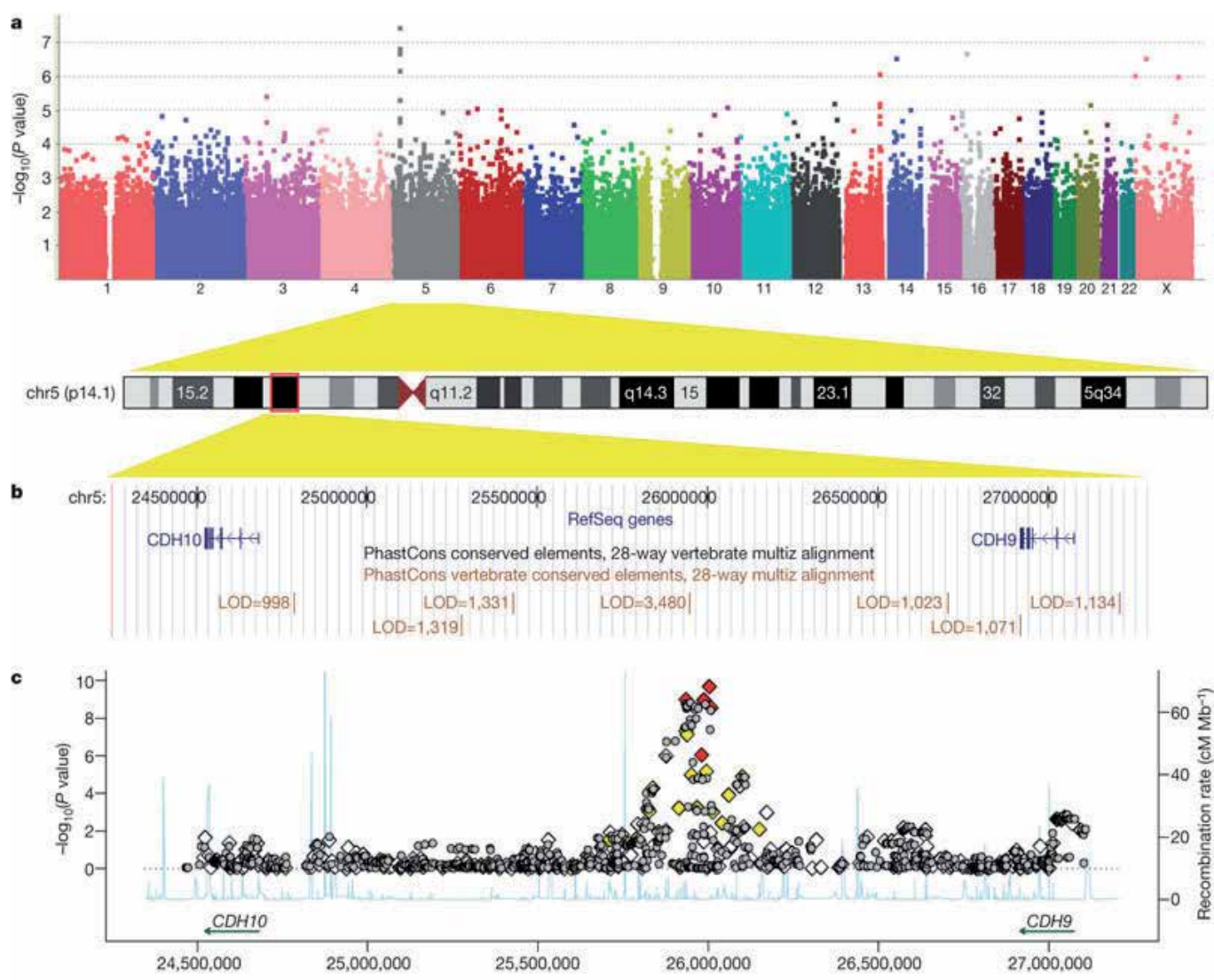

Figure 1. Genome-wide association results at the 5 p14.1 region. a, A Manhattan plot shows the $\log _{10}(p$ values) of SNPs from their combined association analysis. b. The 5 p14.1 region in the UCSC Genome Browser, with conserved genomic elements in the PhastCons track. c, Genotyped (diamonds) and imputed (grey circles) SNPs are plotted with their combined $p$ values. Genotyped SNPs are colored on the basis of their correlation with rs4307059 (red: $r^{2} \geq 0.5$; yellow: $0.2 \leq r^{2}<0.5$; white: $r^{2}<0.2$ ). Estimated recombination rates from HapMap are plotted to reflect local linkage disequilibrium. Adapted from Wang et al., 2009 [21]. Reprinted with permission from Nature Publishing Group.

Although Western blot analyses did not identify significant differences in moesin protein levels between cases and controls, overexpressing MSNP1AS in human cell lines was shown to significantly reduce levels of the moesin protein. The authors speculated that relevant alterations in moesin may occur only during specific development landmarks, which may im- 
pact neurodevelopment. This would explain why moesin levels are not elevated in the ASD samples per se, in spite of the marked differences in MSNP1AS expression. Further work is needed to confirm this hypothesis, and quantification of moesin protein levels at key developmental stages would certainly contribute in this respect.

Taken as a whole, these results provide compelling support for $5 \mathrm{p} 14.1$ as a risk locus for ASD. Although sample sizes for some analyses were small (10 ASD-control pairs for postmortem studies), this quite rigorous series of experiments draws a clear path from GWAS result through functional validation. As such, these results help allay criticism of the GWAS approach as a means of candidate discovery. Thus, a 2010 review by McClellan and King (2010) [27] singled out the 5p14.1 locus as an example of the "perils of cryptic population stratification". These comments seemed somewhat misguided in the light of rigorous methodologies developed by the GWAS community for controlling stratification (e.g. EigenStrat) [28], replication [22, 23], and now functional validation by the Kerin et al. group [26].

Similarly, replication/validation of the 5 p14.1 locus provides an important demonstration of the legitimacy of associations in intergenic regions. Again, McClellan and King had disputed the utility of such results, questioning how "genome-wide association studies come to be populated by risk variants with no known function?" It is important to emphasize that the GWAS approach typically does not tag the disease variant, but rather its approximate location-through linkage disequilibrium, this is typically $100 \mathrm{~kb}$ or less. Moreover, as in the Kerin et al. paper, the significant SNP may be tagging an intergenic regulatory element, which has functional consequences far beyond the associated region, in this case the MSN locus on the X-chromosome.

Finally, these expression analyses provide a reminder about the capabilities of different genomic technologies. In the past twelve months, a number of high-profile next-generation sequencing (NGS) studies have been able to examine genomic correlates of ASD with unprecedented resolution. These types of studies - reviewed in greater depth below-have been interpreted as the future of ASD genetics and, to a large extent, this may be true. However, we note that DNA sequencing in the 5p14.1 region would not have identified the noncoding RNA at this locus. Thus, although NGS platforms used for RNA-sequencing are becoming increasingly sophisticated (Ozsolak \& Milos, 2011) [29], microarray studies retain a place in guiding genomic discovery.

\subsection{Other replicated common variants from candidate gene studies}

A number of other common variants from candidate gene studies have been proposed as ASD risk factors. These include Contactin Associated Protein 2 (CNTNAP2), located on chromosome 7q35, which has been identified as a candidate for the age at first word endophenotype (Alarcón et al., 2002) [30]. A follow-up by the same group (Alarcón et al., 2008) [31] using linkage, association, and gene-expression analyses, found CNTNAP2 to be the only autism-susceptibility gene to reach significance across all approaches. An independent linkage analysis by Arking et al. (2008) [32] also highlighted CNTNAP2 as a significant ASD candidate gene. CNTNAP2 is part of the neurexin family, which have repeatedly been associated with autism (see below). Interestingly, Vernes et al. (2008) [33] showed that 
CNTNAP2 binds to FOXP2, which is a well-established correlate of language and speech disorders (Lai et al., 2001) [34], and are commonly observed in ASD.

Another locus indentified by the candidate gene approach is Engrailed 2 (EN2), a homeobox gene that is critical to the development of the midbrain and cerebellum. Like other homeobox genes, it regulates morphogenesis. EN2 is a human homolog of the engrailed gene, which is found in Drosophila. En2 mouse mutants have anatomic phenotypes in the cerebellum that resemble cerebellar abnormalities reported in autistic individuals (Cheng et al., 2010) [35]. In three separate datasets, Benayed et al. (2005, 2009) [36, 37] have reported and replicated a significant association between EN2 and both broad and narrow ASD phenotypes. Wang et al. (2008) [38] also found an association between EN2 and ASD in a Chinese Han sample, although Zhong et al. (2003) [39] failed to find evidence of an underlying association.

The oncogene MET is also strongly linked to ASD etiology, having been supported by a number of studies in the past decade (e.g. IMGSAC, 2001; Campbell et al., 2006, 2008; Sousa et al., 2009) [40-43]. Recently, Eagleson et al. (2011) [44] reported a role for Met signaling in cortical interneuron development in vitro in a mouse model.

\subsection{Unexplained variance}

For the most significant discovery SNP identified in the Wang et al. study above (rs4307059), the risk allele frequency was 0.65 in cases with an odds ratio of 1.19. This is comparable with common variant discoveries in other psychiatric disorders including schizophrenia (Glessner et al., 2009) [45], bipolar disorder (Ferreira, 2008) [46], and attention-deficit/hyperactivity disorder (Arcos-Burgos et al. 2010) [47]. While it is important not to undermine the significance of these findings, it should be noted that the predictive value of such ratios is relatively low (Dickson et al. 2010) [48], often explaining less than $5 \%$ of the total risk (review at http://www.genome.gov/gwastudies). However, it is also possible that these common SNPs may be tagging a rarer causative variant (i.e. synthetic association), where the effect sizes may be markedly underestimated by the GWAS variant as we recently reported (Dickson et al. 2010) [48]. In one example, Wang et al. (2010) [49] examined the NOD2 locus as a cause of Crohn's disease. Using resequencing data, they found that three causal variants explain $>$ $5 \%$ of the genetic risk, where GWAS had estimated the risk at $\sim 1 \%$. This finding has two potentially important implications. First of all, it highlights the need for careful phenotyping of cohorts, which is important to ensure that the phenotypes produced by rare-variants are not being "filtered-out" and missed as a consequence. A long range haplotype analysis of the GWAS data at respective loci is recommended in an attempt to enrich for individuals with rare-causative variants, who can be selected from the cohort and sequenced for confirmation (Wang et al., 2010) [49].

Second, the results of our Crohn's disease study suggest that in certain circumstances, there may be an explicit relationship between tagged variants and underlying rare variants. Thus, the distinction between loci harboring common versus rare variants is not necessarily concrete. Indeed, the same locus may harbor both common and rare variants (Anderson et al., 
2011). In recent years, we have seen an increased emphasis on the former, which is reflected in an upsurge in the number of copy number variation (CNV) and NGS studies.

\section{Rare variants - CNVs and next-generation sequencing}

\subsection{Copy number variation in ASD}

$\mathrm{CNV}$ s are insertions, deletions, or translocations in the human genome that are universal in the general population (e.g. Pinto et al., 2010) [50]. CNVs can be detected by the same SNP arrays used in GWAS, and vary in length from many megabases to 1 kilobase or smaller. They are often not associated with any observable phenotype.

One of the most widely-known CNVs is Down syndrome, which is characterized by an extra chromosome 21. Rett syndrome is also caused by a CNV, which includes a deletion in MECP2. CNVs can be inherited or occur de novo, the cause of which is thus far unknown. Common disease-causing $\mathrm{CNVs}$ are infrequent but rare $\mathrm{CNVs}$, with a frequency of less than $1 \%$, have been identified for a range of disorders including ADHD (e.g. Williams et al., 2010) [51], schizophrenia (e.g. Glessner et al., 2010; Levinson et al., 2011) [52, 53], bipolar disorder (e.g. Chen et al., 2010) [54] and many others.

Sebat et al. (2007) [55] provided some early insights into the genomic features of CNVs in ASD. Firstly, they noted that de novo CNVs were individually rare - from 118 ASD cases, none of the identified variants were observed more than twice, with the majority seen just once. This confirmed the widely-held assumption that many different loci can contribute to the same ASD phenotype. The sheer volume of loci identified by this approach (multiple loci on 20 chromosomes) affirms the extraordinarily complexity of ASD.

A number of subsequent studies have greatly expanded the number of candidate loci using the CNV approach. Our laboratory (Bucan et al., 2009) [56] reported 150+ CNVs in 912 ASD families that were not found in 1,488 controls. Critically, 27 of these loci were replicated in an independent cohort of 859 ASD cases and 1,051 controls. Some of the rare variants we identified had previously been associated with autism, including NRXN1 and UBE3A, (Guilmatre et al., 2009) [57]. Samaco et al. (2005) [58] previously identified significant deficits in ube3a expression in mecp2-deficient mice, suggesting a shared pathological pathway with Rett syndrome (as well as Angelman syndrome, and autism). Similarly, Kim et al. (2008) [59] associated NRXN1 with a balanced chromosomal abnormality at chromosome 2p16.3 in two unrelated ASD individuals. Rare variants in the coding region included two missense changes.

Glessner et al. (2009) [52] identified and reported CNVs in two major gene networks, including neuronal cell adhesion molecules (such as NRXN1) and the ubiquitin gene family (such as $U B E 3 A$ ). Interestingly, four of the most prominent genes enriched by CNVs in ASD cases (UBE3A, PARK2, RFWD2 and FBXO40) - all of which were uncovered independently - are part of the ubiquitin gene family. Ubiquitination can alter protein function after translation, and degrade target proteins in conjunction with proteasomes. The ubiquitin-proteasome 
system operates at pre- and post-synapses, whose functions includes regulating neurotransmitter release, recycling synaptic vesicles in pre-synaptic terminals, and modulating changes in dendritic spines and post-synaptic density (Yi \& Ehlers, 2005) [60]. As well as implicating an ASD-ubiquitination network, we also identified a second pathway involving NRXN1, CNTN4, NLGN1, and ASTN2. Genes in this group mediate neuronal cell-adhesion, and contribute to neurodevelopment by facilitating axon guidance, synapse formation and plasticity, and neuron-glial interactions. We also note that ubiquitins are involved in recycling celladhesion molecules, which is a possible mechanism by which these two networks are cross linked.

In a similar approach, Pinto et al. (2010) [50] further confirmed the importance of rare CNVs as causal factors for ASD. The group did not observe a significant difference between cases and controls in terms of raw number of CNVs or estimated CNV size. However, the number of CNVs in genic regions was significantly greater in ASD cases. Again, loci enriched for CNVs include a number of genes known to be important for neurodevelopment and synaptic plasticity, such as SHANK2, SYNGAP1, and DLGAP2. Between 5.5\% and 5.7\% of ASD cases have at least one de novo $\mathrm{CNV}$, further confirming the significance of de novo genetic events as risk factors for autism. Similar to the Glessner study, the Pinto group mapped $\mathrm{CNV}$ s to a series of networks involved in the development and regulation of the central nervous system functions. Implicated networks include neuronal cell adhesion, GTPase regulation (important for signal transduction and biosynthesis), and GTPase/Ras signaling, also involved in ubiquitination.

Finally, Gai et al. (2011) [61] took a slightly different approach, focusing exclusively on inherited CNVs. While underlying loci were not necessarily common to those identified by the Glessner and Pinto groups, enrichment in pathways involving central nervous system development, synaptic functions and neuronal signaling processes was again confirmed. The Gai et al. study also emphasized the role of glutamate-mediated neuronal signals in ASD.

Collectively, these CNV studies suggest that certain hotspots on the genome are particularly vulnerable to ASD, which include loci on chromosomes 1q21, 3p26, 15q11-q13, 16p11, and 22q11. These hotspots are part of large gene networks that are important to neural signaling and neurodevelopment and have additionally been associated with other neuropsychiatric disorders. In particular, a number of CNV studies in schizophrenia have highlighted structural mutations incorporating chromosomes 1q21, 15q13, and 22q11 (e.g. Glessner et al., 2010) [62], which are significantly enriched in cases versus controls, with NRXN1 being a standout in this regard. From a phenotype perspective, autism and schizophrenia seem very different, both in behavioral manifestation and age of onset, and it may seem counter-intuitive that associated loci should overlap. Some authors have addressed this peculiarity by proposing that schizophrenia and autism may in fact be different poles of the same spectrum. Thus, Crespi and Braddock (2008) [63] suggest that social cognition is underdeveloped in ASD and over-developed in the psychotic spectrum, with a similar polarization of language and behavioral phenotypes. Although speculative, this hypothesis has gained some traction. In the next several years, genomic, imaging, and model-systems approaches will 
likely shed further light on the relationship between autism, schizophrenia and other neuropsychiatric disorders.

\subsection{Sequencing familial forms of ASD}

To this point, we have focused primarily on the complex interactions of polygenic networks as the major cause of ASD. However, this is not exclusively the case. Paralleling the recent spate of CNV studies is a renewed focus on rare disorders. These include familial forms of complex diseases that are potentially monogenic or with less complex inheritance pattern. At the outset of this chapter, we emphasized the overlap with fragile $X$ syndrome, where one third of cases are co-morbid for ASD. As mentioned, fragile $X$ is caused by a failure to express the protein coded by FMR1. However, mutations in FMR1 do not always result in fragile $X$ and can result in a phenotype more representative of ASD. Thus, Muhle et al. (2004) [64] found that $7-8 \%$ of idiopathic ASD cases may have mutations at the FMR1 locus. Likewise, although mutations in $M E C P 2$ are the common cause of Rett syndrome, certain mutations at the same locus have been associated with idiopathic autism (Carney et al. (2003).

X-linked genes encoding neurologins NLGN3, NLGN4 and SHANK3 (a neuroligin binding partner) are other prominent examples of distinct rare genetic causes. A parallel can be drawn between these studies and studies of mental retardation and epilepsy, which include many rare syndromes that collectively account for a substantial proportion of the two disorders (Morrow et al., 2008). Indeed it is perhaps more than coincidence that autism is heavily co-morbid with these two conditions, with $\sim 40 \%$ of ASD cases meeting diagnostic criteria for mental retardation and epilepsy respectively (Bölte et al., 2009; Danielsson et al., 2005) [7,65]. It is also noteworthy that many of these monogenic-related genes are also major players in neurodevelopment and synapse activity. Other prominent examples include TSC1, TSC2 (Osborne et al., 1991; Franz, 1998) [66, 67], NF1, and UBE3A (see Morrow et al., 2008) [68].

The identification of monogenic or possibly oligogenic autisms is likely to accelerate in the next several years as NGS becomes more widely available. In our group, we recently encountered a family of two parents, six healthy siblings, and two siblings with severe autism suggestive of autosomal recessive inheritance. Unsuccessful attempts using linkage and CNV approaches failed to identify a causal locus, but whole-exome sequencing at 20x coverage identified four genes, including one with a non-synonymous SNP in the protocadherin alpha 4 isoform1 precursor (PCDHA4) gene, which presents a strong candidate gene, currently under validation. Protocadherins are part of the cadherin family that facilitates neuronal cell adhesion and this discovery is consistent with the functional properties of the PCDH family.

Known syndromes with ASD features include fragile $X$, neurofibromatosis type 1, down syndrome, tuberous sclerosis, neurofibromatosis (which confers a 100-fold increased risk for ASD Li et al. (2005) [69], Angelman, Prader-Willi and related 15q syndromes, and at least several dozen others (see Zafeiriou et al., 2007, for a comprehensive review) [70]. Table 1 from Volkmar et al. (2005) [71] lists the most commonly associated syndromes with median rate and range. It is likely that many more unidentified rare syndromes with Mendelian causes have ASD phenotypes. As of September 2012, the Online Mendelian Inheritance in 
Man (OMIM) database listed over 7,000 known or suspected Mendelian diseases (MD), with $\sim 3,500(\sim 50 \%)$ of these having an identified molecular basis (http://omim.org/statistics/ entry). Since OMIM derives its data from published data, these figures likely under-represent rare disorders, which may go unreported. As such, there may be several times more Mendelian disorders that have no defined genetic etiology to date. Given the large-representation of autism phenotypes in known syndromes, we can assume a similar trend in unreported ASD syndromes.

The proportion of ASD accounted for by rare variants remains to be determined. Irrespective, as with many other aspects of scientific inquiry, the study of these events will continue to play an important role in explicating the pathogenesis of ASD. El-Fishawy and State (2010) [72] point to hypercholesterolemia and hypertension (Brown, 1974; Lifton et al., 2001) $[73,74]$ as examples where rare mutations have been successful in driving a molecular understanding of the disease as opposed to identifying risk factors in the general population. Rare mutations, particularly when they are Mendelian, carry large effects and are typically located in genic regions. These characteristics make the resolution of underlying networks distinctly less complex and, moreover, are amenable to modeling in other systems.

Recent groundbreaking studies by Marchetto et al. (2010) [75] and Muotri et al. (2010) [76], who created a cell culture model of Rett syndrome, are potentially exciting developments in this regard. Here, the researchers used skin biopsies from four Rett syndrome patients, each carrying a different $M E C P 2$ mutation, to culture induced pluripotent stem cells (iPS). Once the iPS cells developed into neurons, they showed a decreased number of neurons and dendritic spines, consistent with neurodevelopmental disruptions. Intervention with insulinlike growth factor 1 (IGF1), which is known to regulate neurodevelopment, was subsequently shown to reverse Rett-like symptoms in a mouse model of the disease. This innovative approach is an exciting model of how rare gene approaches can stimulate our understanding of the pathophysiology and potential reversibility of ASD.

\begin{tabular}{cccc}
\hline Syndrome & Number of Studies & Median Rate & Range \% \\
\hline Tuberous sclerosis & 11 & 1.1 & $0-3.8$ \\
FragileX & 9 & 0.0 & $0-8.1$ \\
Down syndrome & 12 & 0.7 & $0-16.7$ \\
Neurofibromatosis 1 & 6 & 0 & $0-1.4$ \\
\hline
\end{tabular}

Table 1. Associated disorders and their rate in autism (from Volkmar et al., 2005 in Zafeiriou et al. 2007) [70,71]

\subsection{Large-scale next-generation sequencing}

In April 2012, Nature simultaneously published three papers that used exome sequencing to probe genomic correlates of ASD. This represented something of a landmark for both ASD and NGS research, as it demonstrated the viability of NGS on a large scale - the three studies combined examined 600 trios (parents and offspring), plus a 935 further ASD cases. Col- 
lectively, these papers suggest that several hundred or more genes may be considered autism candidates, and again highlight the staggering complexity of the phenotype.

O'Roak et al. (2012) [77] sequenced 677 individual exomes from 209 families - primarily from the Simons Simplex Collection [78]. In 189 new probands, they validated 120 severely disruptive de novo mutations, $39 \%$ of which occur in a highly interconnected b-catenin/chromatin remodeling protein network. The group observed a strong paternal bias (41:10) in the rate of de novo mutations, which supports the hypothesis that the germline mutation rate in coding regions is markedly more prominent among males. These de novo events were more common in older fathers, marking paternal age as a significant risk factor for ASD.

Among the identified de novo loci, 62 were identified as top candidate mutations based on severity and/or supporting evidence from the literature. Interestingly, probands with these mutations were broadly distributed in terms of IQ score, with only a modest (non-significant) association with intellectual impairment. Recurrent protein-disruptive mutations were identified in two genes: netrin G1 (NTNG1) and chromodomain helicase DNA binding protein 8 (CHD8). NTNG1 is known to play a role in axon guidance and dendritic organization (Nishimura-Akiyoshi et al., 2007) [79]. CHD8 regulates $\beta$-catenin and p53 signaling, and has not previously been associated with ASD. This gene was emphasized as particularly noteworthy, after follow-up protein-protein interaction (PPI) analyses, showed that $\beta$-catenin and p53 signaling may be features of an ASD-relevant network. In total 49 of proteins in the PPI network were highly interconnected, with a number of underlying genes also previously associated with neurodevelopment.

Neale et al. (2012) [80] exome-sequenced 175 trios and also focused on de novo mutations. As per the O'Roak study, there was a correlation between paternal age and de novo events for offspring $(P<0.0001)$, and also for maternal age $(P=0.000365)$. Across the sample set, the group observed 161 point mutations, of which 101 were missense, 50 silent, and 10 nonsense. Two conserved splice site rare single nucleotide variants and six frameshift insertions/deletions (indels) were also observed. Three genes were found to harbor two de novo mutations: BRCA2 (two missense), FAT1 (two missense) and KCNMA1 (one missense, one silent).

The group next performed PPI analyses to determine whether interactions between genes associated with de novo mutations, as well as existing ASD candidates, was of etiological importance. This pathway approach, which additionally incorporated data from Sanders et al. study (below) [81], found that the distribution of functional de novo mutations is not random. The average distance for non-synonymous variants was significantly larger for controls versus cases (3.78 vs. 3.66; $P=.033$ ). This suggests that a proportion of these de novo events contribute to autism. A model whereby de novo variants in up to $20 \%$ of cases, confer a 10- to 20-fold increased risk was supported.

In the third of these Nature papers, Sanders et al. (2012) [81] performed exome sequencing on 238 families, including 200 quartets (parents, 1 affected and 1 unaffected sibling) from the Simons Simplex Collection [78]. Comparing de novo non-synonymous single nucleotide variants (SNVs) between affected and unaffected siblings, the group observed a significantly 
$(\mathrm{P}=.01)$ higher proportion among the probands (125 total) versus their unaffected sibling (87 total). From simulations, the authors concluded that two or more de novo nonsense/splicesite mutations should be considered significant. The gene sodium channel, voltage-gated, type II, $\alpha$ subunit gene (SCN2A) was the only such gene - with two ASD individuals found to harbor relevant nonsense mutations. Mutations in $S C N 2 A$ have been associated with epilepsy (Kamiya et al., 2004; Ogiwara et al., 2009) [82, 83] and idiopathic ASD in multiplex families (Weiss et al., 2003) [84]. Neither of the probands has a history of seizures.

Combining the exomes from their study with those from O'Roak et al. (n for probands = 414), the groups identified two additional genes that each contained two loss-of-function mutations: the katanin p60 subunit A-like 2 (KATNAL2) and chromodomain helicase DNA binding protein 8 (CHD8). O'Roak et al. also evaluated these three novel candidates using exome sequencing on 935 cases and 870 controls. Three additional loss-of-function mutations each were observed in KATNAL2 and CHD8 in individuals with ASD, while none were identified in controls.

It is important to note, however, that for de novo events in general, there was no evidence to support the hypothesis that multiple events in any individual conferred an increased risk of ASD. As such, the 'two de novo hit' hypothesis is not supported.

In a fourth independent exome sequencing study involving 343 families from the Simons Simplex Collection Iossifov et al. (2012) [85] also reported a relatively equal distribution of de novo mutations in cases and controls. Again however, loss-of-function mutations-nonsense, splice site, and frame shifts - were more common in individuals with ASD (59 versus 28). Of the 59 "likely gene disruptions (LGD)" in ASD cases, none occurred more than once, although two-NRXN1 and PHF2 - had been identified in a previous CNV study by the same group (Gilman et al, 2011) [86]. Intriguingly, the 59-strong LGD shared considerable overlap with a set of 842 proteins that interact with the fragile X protein, FMRP. In total, 14 of the 59 appeared on the FMRP list $(P=.006)$. Furthermore, 13 of $72 \mathrm{CNV}$ candidates from the group's previous $\mathrm{CNV}$ paper were also on the list $(P=.0004)$, meaning 26 of the combined 129 total were FMRP-related $\left(P<1 \times 10^{-13}\right)$.

The authors subsequently screened for de novo mutations in upstream targets of FMR1. One was identified - a deletion in GRM5 that removes a single amino acid and causes an additional substitution at the same site. GRM5 encodes the glutamate receptor mGluR5 (Bear $e t$ al, 2004) [87] and, as noted below, mGluR5 antagonists are currently in clinical trial (Jacquemont et al., 2011) [88] having indicated success in mouse models (Dölen et al., 2007) [89]. Further elucidating the relationship between FMR1/FRMRP and these ASD candidates is clearly an important next step in maximizing the impact of these findings. These are discussed further in the section below.

Collectively, all four of these exome sequencing studies converge upon the conclusion that ASD is highly heterogeneous, with several hundred or more loci potential risk variants. Simulations by the Neale et al. group confirm the statistical implausibility that hundreds of variants with high penetrance are possible, and a model where de novo variants in up to $20 \%$ of cases, confer $\sim 10$ - to 20 -fold increased risk is supported. The studies also converge on the 
conclusion that paternal age (and possibly maternal age) is a significant ASD risk factor, but the frequency and size of de novo mutations per se is not. Evidence for three candidate genes - CHD8, KATNAL2, and SCN2A-would seem quite strong, though further functional studies are needed to help define pathogenesis. Perhaps most exciting is the association between GRM5 and existing/novel candidates. As we have learned from GWAS, larger sample sets are clearly needed to fully harness the power of NGS in relation to such a complex phenotype. While these studies have been important in proposing novel candidates and confirming existing hypotheses of ASD, we await with anticipation results from the sequencing of all 2,648 families from the Simon Simplex Collection.

\section{Toward a treatment?}

Ultimately, the primary goal of genome research should be to propose targets for intervention. As mentioned above, a number of translational studies have begun to probe the metabotropic glutamate receptor, mGluR5, as a potential target for fragile $\mathrm{X}$ syndrome treatment. These studies have a theoretical basis in the hypothesis that protein-synthesis-dependent functions of metabotropic receptors are exaggerated in fragile $X$ syndrome (Bear, Huber \& Warren, 2004) [87]. Thus, the fragile $X$ protein, FMRP, is thought to work in functional opposition to mGluR5 (and mGluR1). Where FMRP is absent, mGluR-dependent protein synthesis becomes over-activated, resulting in neurological and behavioral abnormalities.

Dölen et al. (2007) [89] crossed Fmr1 mutant with Grm5 mutant to produce Fmr1 knockouts who also had a selective reduction in mGluR5 expression. They found that a $50 \%$ reduction in mGluR5 gene dosage rescued a range of deficits in Fmr1 mutants. Relevant measures included protein synthesis in hippocampus, density of dendritic spines (layer 3 pyramidal neurons), visual responsiveness, and cognitive performance (inhibitory avoidance - a hippocampus-dependent memory). This provides confirmation that mGluR5 and FMRP are functionally oppositional. Moreover, it suggests possible pharmacological avenues by which this genetic disease may be treated.

A range of translational studies have begun to target this pathway. These include efforts to inhibit the activity of individual mGluR5 (Jacquemont et al., 2011; Berry-Kravis et al., 2009) [88,90], and FMRP-regulated proteins (Paribello et al., 2010) [91], NMDA (Wei et al., 2012) [92], and GSK3 $\beta$ (lithium, Berry-Kravis et al., 2008) [93], which have shown promise in open label and (in some instances) clinical trials (see Berry-Kravis et al., 2011 for review) [94]. Moreover, these compounds may have clinical application to the broader ASD phenotype. Silverman et al. (2012) [95] recently reported that the mGluR5 antagonist, GRN-529, decreased ASD-related symptoms of autism in two different mouse models of the disease (repetitive grooming/repetitive jumping). In addition to the Iossifov et al. (2012) [85] sequencing study discussed above, Kelleher et al. (2012) [96] recently showed that idiopathic autism cases may have higher burden of mGluR5 variants. The group found that in 209 idiopathic cases, there was significant enrichment for rare functional variants in the mGluR5 pathway-namely the genes TSC1, TSC2 and SHANK3, and HOMER1-relative to controls 
$(n=300)$. It is likely that drugs targeting the mGluR5 pathway, if/when approved for fragile $X$ syndrome, will lead to human clinical trials for ASD. This translational approach - which delineates a direct route from gene discovery, through functional validation to treatment, is clearly the blueprint by which genome research can have tangible clinical impact.

\section{Conclusions}

ASD are clearly highly heritable disorders and advances in gene-finding technology in the past decade have rapidly accelerated gene discovery. As is typically the case, successive developments have made the problem more complex such that there are huge numbers of candidate genes, most of which remain to be replicated. In spite of this complexity, we can observe a number of patterns beginning to unfold 1) the relative scarcity of causal common variants, 2) the growing list of causal rare variants, and 3) the emergence of monogenic disorders with primary and secondary ASD phenotypes.

The monogenic autisms are particularly interesting from a treatment perspective, as they provide a mechanism for studying ASD phenotypes in model systems and are an obvious target for drug intervention. They are also amenable to clinical testing and the decreasing cost of research technologies means that this capacity is more widely available to clinicians. In fact, as the resolution of clinical instruments becomes more sophisticated, it is likely that the clinic will become a primary workplace for syndromic discovery.

A key requirement in driving gene discovery is the necessity of high-quality phenotype data. ASDs are notoriously heterogeneous, and are fractionated in terms of symptoms and trajectory. Mandy \& Skuse (2008) [97] reviewed seven factor analysis studies of ASD symptoms, and found that all but one dissociated social and non-social factors. In a nonclinical sample of 3,000 twin pairs, Happé et al. (2006) [98] examined autistic-like traits and found consistently low correlations $(r=0.1-0.4)$ between each of the core deficits on the autism spectrum. Endophenotypes, sub-components or sub-processes of the broader phenotype, may provide a productive avenue to disentangling some of this complexity. By filtering out all but a few discrete measures, we can theoretically increase the signal-to-noise ratio in genotype-phenotype associations. A number of endophenotypes for ASD have been associated with disease genes, including head circumference (associated with the HOXA1 A218G polymorphism, Conciatori et al., 2004) [99]; age at first word (associated with a quantitative trait locus on 7q35, Alarcón et al. 2005) [100]; delayed magnetoencephalography evoked responses to auditory stimuli (Roberts et al., 2010) [101]; and enhanced perception (Mottron et al., 2006) [102]. The endophenotype approach is arguably more consistent with rare-/mono-genic discovery, where a mutated network may not yield a diagnosis of autism per se, but nevertheless cause associated abnormalities. Note, this approach does not diminish the pleiotropic effects of genes involved in neurodevelopment, and only serves to make the point that the relevant genotype may associate with some but not all ASD features.

The converse, of course, is also true, as a large number of candidate genes contribute to the majority of known ASD. With $\sim 80 \%$ of genes expressed in the brain it is likely that this num- 
ber will continue to grow, and here again careful phenotyping is critical to identifying functional consequences. Ultimately, the primary goal is not to determine the frequency of variation/mutation in cases versus controls, but to determine the pathway(s) and gene networks that lead to pathology. We will also need to identify other major biological players such as epigenetic factors, RNA regulatory elements, and environmental exposures, which are critical components of the ASD equation. While daunting, the elucidation of these elements will doubtlessly take us closer to developing effective treatments for ASD. Given the current rate of progress, we have cause for cautious optimism in this regard.

\section{Author details}

John J. Connolly ${ }^{1}$ and Hakon Hakonarson ${ }^{1,2}$

1 Center for Applied Genomics, The Children's Hospital of Philadelphia, Philadelphia, USA

2 Department of Pediatrics, University of Pennsylvania School of Medicine, Philadelphia, USA

\section{References}

[1] Wing L, Gould J, Gillberg C. Autism spectrum disorders in the DSM-V: better or worse than the DSM-IV? Res Dev Disabil. 2011 Mar-Apr;32(2):768-73.

[2] Lord C, Rutter M, Le Couteur A. Autism Diagnostic Interview-Revised: a revised version of a diagnostic interview for caregivers of individuals with possible pervasive developmental disorders. J Autism Dev Disord. 1994 Oct;24(5):659-85.

[3] Lord C, Rutter M, Goode S, Heemsbergen J, Jordan H, Mawhood L, et al. Autism diagnostic observation schedule: a standardized observation of communicative and social behavior. J Autism Dev Disord. 1989 Jun;19(2):185-212.

[4] Constantino JN, Davis SA, Todd RD, Schindler MK, Gross MM, Brophy SL, et al. Validation of a brief quantitative measure of autistic traits: comparison of the social responsiveness scale with the autism diagnostic interview-revised. J Autism Dev Disord. 2003 Aug;33(4):427-33.

[5] Levy SE, Mandell DS, Schultz RT. Autism. Lancet. 2009 Nov 7;374(9701):1627-38.

[6] Dawson M, Soulieres I, Gernsbacher MA, Mottron L. The level and nature of autistic intelligence. Psychol Sci. 2007 Aug;18(8):657-62.

[7] Bolte S, Dziobek I, Poustka F. Brief report: The level and nature of autistic intelligence revisited. J Autism Dev Disord. 2009 Apr;39(4):678-82.

[8] Skuse DH. Rethinking the nature of genetic vulnerability to autistic spectrum disorders. Trends Genet. 2007 Aug;23(8):387-95. 
[9] Bailey A, Le Couteur A, Gottesman I, Bolton P, Simonoff E, Yuzda E, et al. Autism as a strongly genetic disorder: evidence from a British twin study. Psychol Med. 1995 Jan;25(1):63-77.

[10] Piven J, Palmer P, Jacobi D, Childress D, Arndt S. Broader autism phenotype: evidence from a family history study of multiple-incidence autism families. Am J Psychiatry. 1997 Feb;154(2):185-90.

[11] Lauritsen MB, Pedersen CB, Mortensen PB. Effects of familial risk factors and place of birth on the risk of autism: a nationwide register-based study. J Child Psychol Psychiatry. 2005 Sep;46(9):963-71.

[12] Fombonne E. Epidemiology of pervasive developmental disorders. Pediatr Res. 2009 Jun;65(6):591-8.

[13] O'Roak BJ, State MW. Autism genetics: strategies, challenges, and opportunities. Autism Res. 2008 Feb;1(1):4-17.

[14] Richards RI, Holman K, Kozman H, Kremer E, Lynch M, Pritchard M, et al. Fragile X syndrome: genetic localisation by linkage mapping of two microsatellite repeats FRAXAC1 and FRAXAC2 which immediately flank the fragile site. J Med Genet. 1991 Dec;28(12):818-23.

[15] Rogers SJ, Wehner DE, Hagerman R. The behavioral phenotype in fragile X: symptoms of autism in very young children with fragile $X$ syndrome, idiopathic autism, and other developmental disorders. J Dev Behav Pediatr. 2001 Dec;22(6):409-17.

[16] Harris SW, Hessl D, Goodlin-Jones B, Ferranti J, Bacalman S, Barbato I, et al. Autism profiles of males with fragile X syndrome. Am J Ment Retard. 2008 Nov;113(6): 427-38.

[17] Curtis AR, Headland S, Lindsay S, Thomas NS, Boye E, Kamakari S, et al. X chromosome linkage studies in familial Rett syndrome. Hum Genet. 1993 Jan;90(5):551-5.

[18] Ramocki MB, Zoghbi HY. Failure of neuronal homeostasis results in common neuropsychiatric phenotypes. Nature. 2008 Oct 16;455(7215):912-8.

[19] Klein RJ, Zeiss C, Chew EY, Tsai JY, Sackler RS, Haynes C, et al. Complement factor $\mathrm{H}$ polymorphism in age-related macular degeneration. Science. $2005 \mathrm{Apr}$ 15;308(5720):385-9.

[20] Risch N, Merikangas K. The future of genetic studies of complex human diseases. Science. 1996 Sep 13;273(5281):1516-7.

[21] Wang K, Zhang H, Ma D, Bucan M, Glessner JT, Abrahams BS, et al. Common genetic variants on 5 p14.1 associate with autism spectrum disorders. Nature. 2009 May 28;459(7246):528-33.

[22] Ma D, Salyakina D, Jaworski JM, Konidari I, Whitehead PL, Andersen AN, et al. A genome-wide association study of autism reveals a common novel risk locus at 5p14.1. Ann Hum Genet. 2009 May;73(Pt 3):263-73.

[23] St Pourcain B, Wang K, Glessner JT, Golding J, Steer C, Ring SM, et al. Association between a high-risk autism locus on $5 \mathrm{p} 14$ and social communication spectrum phenotypes in the general population. Am J Psychiatry. 2010 Nov;167(11):1364-72. 
[24] Redies C, Hertel N, Hubner CA. Cadherins and neuropsychiatric disorders. Brain Res. 2012 Aug 27;1470:130-44.

[25] Gepner B, Feron F. Autism: a world changing too fast for a mis-wired brain? Neurosci Biobehav Rev. 2009 Sep;33(8):1227-42.

[26] Kerin T, Ramanathan A, Rivas K, Grepo N, Coetzee GA, Campbell DB. A noncoding RNA antisense to moesin at 5p14.1 in autism. Sci Transl Med. 2012 Apr 4;4(128): $128 \mathrm{ra} 40$.

[27] McClellan J, King MC. Genetic heterogeneity in human disease. Cell. 2010 Apr 16;141(2):210-7.

[28] Li Q, Yu K. Improved correction for population stratification in genome-wide association studies by identifying hidden population structures. Genet Epidemiol. 2008 Apr;32(3):215-26.

[29] Ozsolak F, Milos PM. RNA sequencing: advances, challenges and opportunities. Nat Rev Genet. 2011 Feb;12(2):87-98.

[30] Alarcon M, Cantor RM, Liu J, Gilliam TC, Geschwind DH. Evidence for a language quantitative trait locus on chromosome $7 q$ in multiplex autism families. Am J Hum Genet. 2002 Jan;70(1):60-71.

[31] Alarcon M, Abrahams BS, Stone JL, Duvall JA, Perederiy JV, Bomar JM, et al. Linkage, association, and gene-expression analyses identify CNTNAP2 as an autism-susceptibility gene. Am J Hum Genet. 2008 Jan;82(1):150-9.

[32] Arking DE, Cutler DJ, Brune CW, Teslovich TM, West K, Ikeda M, et al. A common genetic variant in the neurexin superfamily member CNTNAP2 increases familial risk of autism. Am J Hum Genet. 2008 Jan;82(1):160-4.

[33] Vernes SC, Newbury DF, Abrahams BS, Winchester L, Nicod J, Groszer M, et al. A functional genetic link between distinct developmental language disorders. N Engl J Med. 2008 Nov 27;359(22):2337-45.

[34] Lai CS, Fisher SE, Hurst JA, Vargha-Khadem F, Monaco AP. A forkhead-domain gene is mutated in a severe speech and language disorder. Nature. 2001 Oct 4;413(6855):519-23.

[35] Cheng Y, Sudarov A, Szulc KU, Sgaier SK, Stephen D, Turnbull DH, et al. The Engrailed homeobox genes determine the different foliation patterns in the vermis and hemispheres of the mammalian cerebellum. Development. 2010 Feb;137(3):519-29.

[36] Benayed R, Choi J, Matteson PG, Gharani N, Kamdar S, Brzustowicz LM, et al. Autism-associated haplotype affects the regulation of the homeobox gene, ENGRAILED 2. Biol Psychiatry. 2009 Nov 15;66(10):911-7.

[37] Benayed R, Gharani N, Rossman I, Mancuso V, Lazar G, Kamdar S, et al. Support for the homeobox transcription factor gene ENGRAILED 2 as an autism spectrum disorder susceptibility locus. Am J Hum Genet. 2005 Nov;77(5):851-68.

[38] Wang L, Jia M, Yue W, Tang F, Qu M, Ruan Y, et al. Association of the ENGRAILED 2 (EN2) gene with autism in Chinese Han population. Am J Med Genet B Neuropsychiatr Genet. 2008 Jun 5;147B(4):434-8. 
[39] Zhong H, Serajee FJ, Nabi R, Huq AH. No association between the EN2 gene and autistic disorder. J Med Genet. 2003 Jan;40(1):e4.

[40] Campbell DB, Sutcliffe JS, Ebert PJ, Militerni R, Bravaccio C, Trillo S, et al. A genetic variant that disrupts MET transcription is associated with autism. Proc Natl Acad Sci U S A. 2006 Nov 7;103(45):16834-9.

[41] Campbell DB, Li C, Sutcliffe JS, Persico AM, Levitt P. Genetic evidence implicating multiple genes in the MET receptor tyrosine kinase pathway in autism spectrum disorder. Autism Res. 2008 Jun;1(3):159-68.

[42] Sousa I, Clark TG, Toma C, Kobayashi K, Choma M, Holt R, et al. MET and autism susceptibility: family and case-control studies. Eur J Hum Genet. 2009 Jun;17(6): 749-58.

[43] A genomewide screen for autism: strong evidence for linkage to chromosomes $2 q$, 7q, and 16p. Am J Hum Genet. 2001 Sep;69(3):570-81.

[44] Eagleson KL, Campbell DB, Thompson BL, Bergman MY, Levitt P. The autism risk genes MET and PLAUR differentially impact cortical development. Autism Res. 2011 Feb;4(1):68-83.

[45] Glessner JT, Hakonarson H. Common variants in polygenic schizophrenia. Genome Biol. 2009;10(9):236.

[46] Ferreira MA, O'Donovan MC, Meng YA, Jones IR, Ruderfer DM, Jones L, et al. Collaborative genome-wide association analysis supports a role for ANK3 and CACNA1C in bipolar disorder. Nat Genet. 2008 Sep;40(9):1056-8.

[47] Arcos-Burgos M, Jain M, Acosta MT, Shively S, Stanescu H, Wallis D, et al. A common variant of the latrophilin 3 gene, LPHN3, confers susceptibility to ADHD and predicts effectiveness of stimulant medication. Mol Psychiatry. 2010 Nov;15(11): 1053-66.

[48] Dickson SP, Wang K, Krantz I, Hakonarson H, Goldstein DB. Rare variants create synthetic genome-wide associations. PLoS Biol. 2010 Jan;8(1):e1000294.

[49] Wang K, Dickson SP, Stolle CA, Krantz ID, Goldstein DB, Hakonarson H. Interpretation of association signals and identification of causal variants from genome-wide association studies. Am J Hum Genet. 2010 May 14;86(5):730-42.

[50] Pinto D, Pagnamenta AT, Klei L, Anney R, Merico D, Regan R, et al. Functional impact of global rare copy number variation in autism spectrum disorders. Nature. 2010 Jul 15;466(7304):368-72.

[51] Williams NM, Zaharieva I, Martin A, Langley K, Mantripragada K, Fossdal R, et al. Rare chromosomal deletions and duplications in attention-deficit hyperactivity disorder: a genome-wide analysis. Lancet. 2010 Oct 23;376(9750):1401-8.

[52] Glessner JT, Wang K, Cai G, Korvatska O, Kim CE, Wood S, et al. Autism genomewide copy number variation reveals ubiquitin and neuronal genes. Nature. 2009 May 28;459(7246):569-73.

[53] Levinson DF, Duan J, Oh S, Wang K, Sanders AR, Shi J, et al. Copy Number Variants in Schizophrenia: Confirmation of Five Previous Findings and New Evidence for 
3q29 Microdeletions and VIPR2 Duplications. Am J Psychiatry. 2011 Mar;168(3): 302-16.

[54] Chen X, Li X, Wang P, Liu Y, Zhang Z, Zhao G, et al. Novel association strategy with copy number variation for identifying new risk Loci of human diseases. PLoS One. 2010;5(8):e12185.

[55] Sebat J, Lakshmi B, Malhotra D, Troge J, Lese-Martin C, Walsh T, et al. Strong association of de novo copy number mutations with autism. Science. 2007 Apr 20;316(5823):445-9.

[56] Bucan M, Abrahams BS, Wang K, Glessner JT, Herman EI, Sonnenblick LI, et al. Genome-wide analyses of exonic copy number variants in a family-based study point to novel autism susceptibility genes. PLoS Genet. 2009 Jun;5(6):e1000536.

[57] Guilmatre A, Dubourg C, Mosca AL, Legallic S, Goldenberg A, Drouin-Garraud V, et al. Recurrent rearrangements in synaptic and neurodevelopmental genes and shared biologic pathways in schizophrenia, autism, and mental retardation. Arch Gen Psychiatry. 2009 Sep;66(9):947-56.

[58] Samaco RC, Hogart A, LaSalle JM. Epigenetic overlap in autism-spectrum neurodevelopmental disorders: MECP2 deficiency causes reduced expression of UBE3A and GABRB3. Hum Mol Genet. 2005 Feb 15;14(4):483-92.

[59] Kim HG, Kishikawa S, Higgins AW, Seong IS, Donovan DJ, Shen Y, et al. Disruption of neurexin 1 associated with autism spectrum disorder. Am J Hum Genet. 2008 Jan; 82(1):199-207.

[60] Yi JJ, Ehlers MD. Ubiquitin and protein turnover in synapse function. Neuron. 2005 Sep 1;47(5):629-32.

[61] Gai X, Xie HM, Perin JC, Takahashi N, Murphy K, Wenocur AS, et al. Rare structural variation of synapse and neurotransmission genes in autism. Mol Psychiatry. 2011 Mar 1.

[62] Glessner JT, Reilly MP, Kim CE, Takahashi N, Albano A, Hou C, et al. Strong synaptic transmission impact by copy number variations in schizophrenia. Proc Natl Acad Sci U S A. 2010 Jun 8;107(23):10584-9.

[63] Crespi B, Badcock C. Psychosis and autism as diametrical disorders of the social brain. Behav Brain Sci. 2008 Jun;31(3):241-61; discussion 61-320.

[64] Muhle R, Trentacoste SV, Rapin I. The genetics of autism. Pediatrics. 2004 May; 113(5):e472-86.

[65] Danielsson S, Gillberg IC, Billstedt E, Gillberg C, Olsson I. Epilepsy in young adults with autism: a prospective population-based follow-up study of 120 individuals diagnosed in childhood. Epilepsia. 2005 Jun;46(6):918-23.

[66] Osborne JP, Fryer A, Webb D. Epidemiology of tuberous sclerosis. Ann N Y Acad Sci. 1991;615:125-7.

[67] Franz DN. Diagnosis and management of tuberous sclerosis complex. Semin Pediatr Neurol. 1998 Dec;5(4):253-68. 
[68] Morrow EM, Yoo SY, Flavell SW, Kim TK, Lin Y, Hill RS, et al. Identifying autism loci and genes by tracing recent shared ancestry. Science. 2008 Jul 11;321(5886): 218-23.

[69] Li W, Cui Y, Kushner SA, Brown RA, Jentsch JD, Frankland PW, et al. The HMGCoA reductase inhibitor lovastatin reverses the learning and attention deficits in a mouse model of neurofibromatosis type 1. Curr Biol. 2005 Nov 8;15(21):1961-7.

[70] Zafeiriou DI, Ververi A, Vargiami E. Childhood autism and associated comorbidities. Brain Dev. 2007 Jun;29(5):257-72.

[71] Volkmar FR. Handbook of Autism and Pervasive Developmental Disorders. 3 ed. New Jersey: John Wiley \& Sons; 2005.

[72] El-Fishawy P, State MW. The genetics of autism: key issues, recent findings, and clinical implications. Psychiatr Clin North Am. 2010 Mar;33(1):83-105.

[73] Brown MS, Goldstein JL. Expression of the familial hypercholesterolemia gene in heterozygotes: mechanism for a dominant disorder in man. Science. 1974 Jul 5;185(4145): 61-3.

[74] Lifton RP, Gharavi AG, Geller DS. Molecular mechanisms of human hypertension. Cell. 2001 Feb 23;104(4):545-56.

[75] Marchetto MC, Carromeu C, Acab A, Yu D, Yeo GW, Mu Y, et al. A model for neural development and treatment of Rett syndrome using human induced pluripotent stem cells. Cell. 2010 Nov 12;143(4):527-39.

[76] Muotri AR, Marchetto MC, Coufal NG, Oefner R, Yeo G, Nakashima K, et al. L1 retrotransposition in neurons is modulated by MeCP2. Nature. 2010 Nov 18;468(7322): 443-6.

[77] O'Roak BJ, Vives L, Girirajan S, Karakoc E, Krumm N, Coe BP, et al. Sporadic autism exomes reveal a highly interconnected protein network of de novo mutations. $\mathrm{Na}$ ture. 2012 Apr 4.

[78] Fischbach GD, Lord C. The Simons Simplex Collection: a resource for identification of autism genetic risk factors. Neuron. 2010 Oct 21;68(2):192-5.

[79] Nishimura-Akiyoshi S, Niimi K, Nakashiba T, Itohara S. Axonal netrin-Gs transneuronally determine lamina-specific subdendritic segments. Proc Natl Acad Sci U S A. 2007 Sep 11;104(37):14801-6.

[80] Neale BM, Kou Y, Liu L, Ma'ayan A, Samocha KE, Sabo A, et al. Patterns and rates of exonic de novo mutations in autism spectrum disorders. Nature. 2012 Apr 4.

[81] Sanders SJ, Murtha MT, Gupta AR, Murdoch JD, Raubeson MJ, Willsey AJ, et al. De novo mutations revealed by whole-exome sequencing are strongly associated with autism. Nature. 2012 Apr 4.

[82] Kamiya K, Kaneda M, Sugawara T, Mazaki E, Okamura N, Montal M, et al. A nonsense mutation of the sodium channel gene SCN2A in a patient with intractable epilepsy and mental decline. J Neurosci. 2004 Mar 17;24(11):2690-8. 
[83] Ogiwara I, Ito K, Sawaishi Y, Osaka H, Mazaki E, Inoue I, et al. De novo mutations of voltage-gated sodium channel alphaII gene SCN2A in intractable epilepsies. Neurology. 2009 Sep 29;73(13):1046-53.

[84] Weiss LA, Escayg A, Kearney JA, Trudeau M, MacDonald BT, Mori M, et al. Sodium channels SCN1A, SCN2A and SCN3A in familial autism. Mol Psychiatry. 2003 Feb; 8(2):186-94.

[85] Iossifov I, Ronemus M, Levy D, Wang Z, Hakker I, Rosenbaum J, et al. De novo gene disruptions in children on the autistic spectrum. Neuron. 2012 Apr 26;74(2):285-99.

[86] Gilman SR, Iossifov I, Levy D, Ronemus M, Wigler M, Vitkup D. Rare de novo variants associated with autism implicate a large functional network of genes involved in formation and function of synapses. Neuron. 2011 Jun 9;70(5):898-907.

[87] Bear MF, Huber KM, Warren ST. The mGluR theory of fragile X mental retardation. Trends Neurosci. 2004 Jul;27(7):370-7.

[88] Jacquemont S, Curie A, des Portes V, Torrioli MG, Berry-Kravis E, Hagerman RJ, et al. Epigenetic modification of the FMR1 gene in fragile $X$ syndrome is associated with differential response to the mGluR5 antagonist AFQ056. Sci Transl Med. 2011 Jan 5;3(64):64ra1.

[89] Dolen G, Osterweil E, Rao BS, Smith GB, Auerbach BD, Chattarji S, et al. Correction of fragile $X$ syndrome in mice. Neuron. 2007 Dec 20;56(6):955-62.

[90] Berry-Kravis E, Hessl D, Coffey S, Hervey C, Schneider A, Yuhas J, et al. A pilot open label, single dose trial of fenobam in adults with fragile $\mathrm{X}$ syndrome. J Med Genet. 2009 Apr;46(4):266-71.

[91] Paribello C, Tao L, Folino A, Berry-Kravis E, Tranfaglia M, Ethell IM, et al. Open-label add-on treatment trial of minocycline in fragile $\mathrm{X}$ syndrome. BMC Neurol. 2010;10:91.

[92] Wei H, Dobkin C, Sheikh AM, Malik M, Brown WT, Li X. The therapeutic effect of memantine through the stimulation of synapse formation and dendritic spine maturation in autism and fragile X syndrome. PLoS One. 2012;7(5):e36981.

[93] Berry-Kravis E, Sumis A, Hervey C, Nelson M, Porges SW, Weng N, et al. Open-label treatment trial of lithium to target the underlying defect in fragile $\mathrm{X}$ syndrome. J Dev Behav Pediatr. 2008 Aug;29(4):293-302.

[94] Berry-Kravis E, Knox A, Hervey C. Targeted treatments for fragile X syndrome. J Neurodev Disord. 2011 Sep;3(3):193-210.

[95] Silverman JL, Smith DG, Rizzo SJ, Karras MN, Turner SM, Tolu SS, et al. Negative allosteric modulation of the mGluR5 receptor reduces repetitive behaviors and rescues social deficits in mouse models of autism. Sci Transl Med. 2012 Apr 25;4(131): 131 ra51.

[96] Kelleher RJ, 3rd, Geigenmuller U, Hovhannisyan H, Trautman E, Pinard R, Rathmell $\mathrm{B}$, et al. High-throughput sequencing of mGluR signaling pathway genes reveals enrichment of rare variants in autism. PLoS One. 2012;7(4):e35003. 
[97] Mandy WP, Skuse DH. Research review: What is the association between the socialcommunication element of autism and repetitive interests, behaviours and activities? J Child Psychol Psychiatry. 2008 Aug;49(8):795-808.

[98] Happe F, Ronald A, Plomin R. Time to give up on a single explanation for autism. Nat Neurosci. 2006 Oct;9(10):1218-20.

[99] Conciatori M, Stodgell CJ, Hyman SL, O'Bara M, Militerni R, Bravaccio C, et al. Association between the HOXA1 A218G polymorphism and increased head circumference in patients with autism. Biol Psychiatry. 2004 Feb 15;55(4):413-9.

[100] Alarcon M, Yonan AL, Gilliam TC, Cantor RM, Geschwind DH. Quantitative genome scan and Ordered-Subsets Analysis of autism endophenotypes support language QTLs. Mol Psychiatry. 2005 Aug;10(8):747-57.

[101] Roberts TP, Khan SY, Rey M, Monroe JF, Cannon K, Blaskey L, et al. MEG detection of delayed auditory evoked responses in autism spectrum disorders: towards an imaging biomarker for autism. Autism Res. 2010 Feb;3(1):8-18.

[102] Mottron L, Dawson M, Soulieres I, Hubert B, Burack J. Enhanced perceptual functioning in autism: an update, and eight principles of autistic perception. J Autism Dev Disord. 2006 Jan;36(1):27-43. 

Chapter 14

\title{
The Genetic Architecture of Autism and Related Conditions
}

\author{
Michael Gill, Graham Kenny and Richard Anney \\ Additional information is available at the end of the chapter \\ http://dx.doi.org/10.5772/54854
}

\section{Introduction}

Autism is a behaviourally defined developmental disorder characterised by impairments in social communication, restricted interests and repetitive behaviours [1]. Abnormalities in these three developmental areas tend to cluster together in affected individuals. In DSM-IV, Autism is part of a larger continuum of disorders collectively called Pervasive Developmental Disorders. Autism spectrum disorders (ASD) refer to Autism, Pervasive developmental disorder, not otherwise specified, and Asperger syndrome. All individuals with ASDs have qualitative abnormalities of social development in combination with disorders of communication and/or stereotyped repetitive interests and behaviors. The social skills that develop naturally in typically-developing children do not do so in children with ASD. In addition, there are several behaviors and co-morbid symptoms that relate to each of the three classical impairments. Recent studies have reported rates of co-occurring intellectual disability in the range of $25-50 \%$. Neither developmental delay nor cognitive impairment are required for an ASD diagnosis.

Fombonne and colleagues recently estimated the prevalence of strictly-defined autism at approximately 15-20 per 10,000 people [2]. When the definition of autism is relaxed to include Autism Spectrum Disorders, the prevalence estimated expands to approximately 60 in in 10,000 children $[2,3]$.

Little is known of the biological basis of ASD and the future development of rational knowledge based treatments will depend on a comprehensive understanding of innate biological predisposition and its interaction with environmental factors. The identification and characterisation of the genetic variation and genes involved in ASD is a route towards this goal. This chapter outlines the various approaches that have been applied to this task, in the context of rapidly evolving technology and human genome resources, and summarises the state of 
knowledge at this time, anticipating future developments, and outlining the implications for clinical management.

\section{Autism is a heritable disorder}

There is unequivocal evidence to support the role of genetic liability as the basis of familiality in the aetiology of ASD. In their seminal work, Folstein and Rutter [4] observed that 4 of 11 $(36 \%)$ pairs of identical twins were concordant for strictly defined autism - whilst none of 10 pairs of fraternal twins were concordant. In a large follow-up study, which included these original twins, Bailey and colleagues observed a more striking concordance rate in identical twins $(60 \%)$ with no concordance in the fraternal twins [5]. This highly cited study underlined ASD as the most heritable of the neurodevelopmental and psychiatric disorders with heritability estimates of $91-93 \%$. A recent review of over 30 twin studies of ASD [6] noted that the early twin studies, which relied upon a strict diagnosis of autism, show a median identical and fraternal concordance of $76 \%$ and $0 \%$ respectively. The expansion of these and other studies to include a broader definition of ASD revealed a median concordance rate of $88 \%$ and $31 \%$. In both cases, these data reveal that ASD is underpinned by a strong genetic component. More recently, Hallmayer and colleagues [7] using more stringent clinical diagnostic tools, such as the Autism Diagnostic Interview (ADI; [8]) and the Autism Diagnostic Observation Schedule (ADOS; [9]) in a group of 202 twin pairs from the California Twin Registry, identified similar concordance rate of ASD as previously attained; with identical twin boys showing a concordance rate of $77 \%$ and fraternal twin boys $31 \%$.

\section{Genetic linkage and association}

The heritability estimates above calculated from twin studies imply that there is unknown genetic variation in children with ASD that confers risk. Several models may fit the observed family and twin data including, at the extremes, a 'common disorder-common-variant' model, with many DNA variants of small effect combining together to create risk; and a multiple rare variant model where ASD is in reality a large collection of different and individually rare disorders. The true picture is only slowly emerging, and as with many other common disorders, common low penetrant and rare higher penetrant DNA variants combine to jointly contribute to risk. Molecular genetic methods have developed exponentially over the last few decades with ever increasing knowledge of the structure and function of the human genome. For disorders with largely unknown aetiology, such as ASD, using human genetic studies to identify risk genes is a gateway to an understanding of underlying biology. Such studies may focus on individuals or families each using different but related genetic strategies to track down risk or causative genetic variation. These methods include genetic linkage, genetic association, and direct examination of structural and copy number variation.

The basis of genetic linkage is the physical co-location of genes or other DNA variation. Such DNA variation is more likely to be transmitted together (or linked) than those that are further 
apart. Linkage studies take advantage of this non-random assortment of genetic variation. A linkage study calculates whether a known genetic variant and a disease mutation (represented by the disease trait) are linked and if so, roughly localises the causative mutation. Following the successes of using these approaches in the discovery of multiple loci implicate in Mendelian disorders, researchers were encouraged to apply linkage methodology to more complex traits, such as ASD where Mendelian principles may apply, at least in a proportion of families. However, in ASD, only a few scans have highlighted loci with significant linkage, highlighting loci including chromosome $7 q, 2 q$ and $3 q$.

In 1998, the International Molecular Genetic Study of Autism Consortium (IMGSAC) [10] reported modest evidence for linkage. This included significant linkage arising on chromosome 7q32-q34. Supplemented analyses of IMGSAC families provided additional support for linkage at $7 \mathrm{q} 22,16 \mathrm{p} 13$, and $2 \mathrm{q} 31[11,12]$. The long arm of chromosome 7 has received particular attention with additional support reported for 7q21 [13], 7q22 [14], 7q31 [15, 16], 7q32 [17] and 7q36 [16].

Few individual families exist where ASD segregates in an obvious Mendelian fashion that is large enough to provide significant evidence for linkage by themselves. Most linkage studies required the assumption that a significant proportion of the families in the sample might be linked to a given locus and few were sufficiently large to accommodate even modest locus heterogeneity. Under a 'common disorder - common variant' model, multiply affected families will occur but linkage methods would be considerably underpowered. The effects of DNA variation with low penetrance are more easily identified using a genetic association study design in a sample of cases drawn from a population.

Fine-mapping and candidate gene association studies at implicated regions on $7 \mathrm{q}$ have implicated a number of potential susceptibility genes including RELN, MET, CNTNAP2 and EN2. Persico and colleagues [18] studied five DNA variants or polymorphisms across the RELN gene locus, including a GGC repeat variant located close to the RELN gene translation initiator codon. Located at 7q22, RELN encodes an extracellular matrix protein Reelin, which plays a pivotal role in the development of laminar structures including cerebral cortex, cerebellum and hippocampus. Using a genetic association approach, Persico and colleagues identified a nominally significant association with this 5'-UTR GCC-triplet-repeat polymorphism [18]. This finding was further supported by some studies [19-25] but not others [26-31]. MET, located at 7q31 received considerable attention following a high-profile association reported by Campbell and colleagues. The MET gene encodes a protein involved in MET (Mesenchymal epithelial transition factor) receptor tyrosine kinase signalling which has been implicated in brain growth and maturation - offering biological plausibility to its candidature. As with other candidate genes in ASD, the original findings have been supported in some [32], but not other studies [33] [34]. A similar scenario played out for the EN2 homeobox gene located at 7q36 [35, 36]. Arking and colleagues [37] observed an association at CNTNAP2 in the NIHM/AGRE collection. The main association observed was for rs7794745 located in intron 2 of the gene (Discovery $P=0.00002$, Validation $P=0.005)$. The CNTNAP2 (7q35) gene encodes the contactin-associated protein-like 2 protein, which is a member of the neurexin family and thought to play a role in axonal differentiation and guidance. Li and colleagues also found mild support for CNTNAP2 
where they observed a weak association with a haplotype containing the SNP rs7794745 [38]. More recently, a large GWAS of 2705 families identified a strong association for the SNP rs1718101 in the CNTNAP2 gene with a subset of individuals without intellectual disability and of European ancestry $\left(P=7.78 \times 10^{-9}\right)[39]$.

The Paris Autism Research International Sibpair (PARIS) study [15], that supported the findings on 7q, noted additional loci at 2q31-q32, 16p13 and 19p13, that overlapped to some extent with regions identified in the IMGSAC analyses. Fine mapping of the 2q31-q32 led to additional evidence implicating the genes SLC25A12, STK39 and ITGA4 as putative risk genes for ASD. As with most candidate genes examined in small samples, these associations were validated by some [40-42] but not all studies [43-46]. The positional candidature of the 2q31-q32 was further supported by a case study of a young Irish male with high-functioning autism with a complex translocation traversing chromosome $2 \mathrm{q} 32(46, \mathrm{XY}, \mathrm{t}(9 ; 2)(\mathrm{q} 31.1 ; \mathrm{q} 32.2 \mathrm{q} 31.3))$ [47]. Fine mapping and mutation analysis identified an association with a polymorphism within the splice donor sequence of exon 16 of the ITGA4 gene and ASD (rs12690517; P = 0.008) [48].

In a study of Finnish families, Auranen and colleagues [49, 50], who included individuals with autism, infantile autism, Asperger syndrome (AS) and developmental dysphasia reported a significant linkage to 3q25-q27. This was supported by suggestive evidence at 3q25-q27 in a study of AS also in the Finnish population [51] as well as linkage at 3q25-q27 in a single large extended Utah pedigree of Northern European ancestry [52].

Interestingly, the advent of large collaborative studies by the Autism Genome Research Exchange (AGRE), the Autism Genome Project (AGP) and the National Institute of Mental Health (NIMH) has not yielded stronger evidence in favour of specific loci. Liu and colleagues using data from 110 multiplex families from the AGRE collection [16] observed only suggestive linkage on chromosomes 5p13, Xq26-qtel, and 19q12 alongside modest support for previously reported linkage on 7q (7q31 and 7q36) and 16p13. A follow-up analysis including 235 additional AGRE multiplex families was again limited to only suggestive loci at chromosomes 17q11, 5p13, 11p11-p13, 4q21-q22 and 8q24 [53]. In a much larger collection of 1168 multiply-affected families from the AGP (including families previously included in the AGRE, CPEA, IMGSAC, PARIS and Seaver linkage studies), Szatmari and colleagues identified suggestive linkage to chromosome 11p12-p13 and a large region on chromosome 15q23-q25 [14]. Of the regions that were featured prominently in previous linkage analyses, there was only modest support for previously highlighted linkage regions on chromosome 2q31 (female autism-probands) and 7q22 (male ASD-probands) from families of European ancestry [14]. A similarly large linkage study of 1031 families, including 1553 affected offspring, Weiss and colleagues identified suggestive linkage at 6p27 and significant linkage at 20p13 [54].

Early association studies examined whether specific variation in genes was associated with the disease and focused on candidate loci identified from linkage and cytogenetic studies (positional candidate genes) as well as in genes within biological processes that we perceived as having a role in ASD. In the mid-1990's Risch and Merikangas [55] demonstrated that where genetic variants have only small effect on risk the association study is a more powerful ap- 
proach than linkage to identify genetic risk. However, the transition from candidate gene to genome-wide association studies was not realised until technological advances firstly enriched the maps of common variation across the genome and secondly enabled the interrogation of these variants en masse as part of genome-wide SNP arrays.

Wang and colleagues [56] performed one of the first GWAS on individuals in European ancestry individuals from the AGRE collection, the Autism Case Control Collection and unaffected controls from the Children's Hospital of Philadelphia control collection. Neither familybased nor case-control analysis alone yielded genome-wide significant findings. However, in a combined analyses the authors identified genome-wide significant association on chromosome 5p14.1 (rs4307059; $P=3.4 \times 10^{-8}$ ) and a number of additional association signals on chromosome 13q33.3, 14q21.1 and Xp22.32. The 5p14.1 association was validated in the Collaborative Autism Project (CAP) and Centre for Autism Research and Treatment (CART) study. The authors found a modest to strong replication of the association signal on chromosome 5p14.1. In a reciprocal study with the CAP and CART study as the discovery sample followed by validation using the AGRE dataset was published in parallel by Ma and colleagues [57]. The authors examined approximately 500k SNPs, more than in the Wang report and albeit not genome-wide significant, they retained the association signal on chromosome 5p14.1.

A second independent GWAS was reported by Weiss and colleagues [54]. In the initial scan the authors did not find any GW-significant associations. However, as with the previous GWAS, additional supplementation of their family-based studies with a case-control set derived from 90 probands without parental data garnered some additional signal for the top hits. A replication consortium of greater than 2000 trios was genotyped for 45 SNPs across all of the top associated regions. The only marker that showed evidence of replication resides on the short arm of chromosome 5 at $5 \mathrm{p} 15$. Although, like Ma and colleagues, this report has considerable overlap with the AGRE families reported by Wang and colleagues, the authors did not observe strong association at $5 \mathrm{p} 14.1$. The chromosome $5 \mathrm{p}$ association lies in close proximity to TAS2R1. The TAS2R1 gene encodes a G-protein coupled receptor that is involved in bitter taste recognition. The authors highlight a more biologically plausible ASD candidate gene approximately $80 \mathrm{~Kb}$ telomeric, SEMA5A. SEMA5A encodes a gene important in axonal guidance and has been shown to be down regulated in the occipital lobe cortex, lymphoblast cell lines and lymphocytes from individuals with autism.

Two additional GWAS followed by the Autism Genome Project (AGP) [39, 58]. In the first report of 1369 families, Anney and colleagues identified a single GW-significant finding on chromosome 20 at position 20p12 within the MACROD2 (MACRO-domain containing 2) gene locus (rs4141463; $P=2.1 \times 10^{-8} ; \mathrm{OR}=0.56$ ). Weak statistical support was observed for $M A C$ ROD2 in an AGRE validation sample, albeit showing the same direction of effect for the risk allele. In a follow-up study using an additional 1301 families the authors showed little if any signal of association (rs4141463; $P=0.206, \mathrm{OR}=0.91$ ). In a combined analyses the association at MACROD2 was less compelling ( $\mathrm{rs} 4141463 ; P=1.2 \times 10^{-6} ; \mathrm{OR}=0.77$ ). The role of MACROD2 is largely unknown although structural variation in this gene and specifically the region harbouring the ASD association signal have been implicated in schizophrenia $[59,60]$ and epilepsy [61]. However, this same region has been identified as a hotspot for deletions in the genome 
identified as a region of large rare deletions [62]. MACROD-like proteins are highly conserved across evolutionary time, which may indicate an essential role. The MACRO-domain is an ADP-ribose binding module [63] and has been implicated in the ADP-ribosylation of proteins, an important post-translational modification that occurs in a variety of biological processes such as DNA repair [64], heterochromatin formation, histone modification and sirtuin biology [65-67] as well as long-term memory formation [68]. The association signal observed at rs4141463, albeit tagged to the $M A C R O D 2$ gene, resides in an intronic region near an intragenic non-protein-coding-RNA, NCRNA00186 (MACROD2-AS1). Two MACROD2-AS1 transcripts have been reported of $673 \mathrm{bp}$ and $1230 \mathrm{bp}$ in length, located on the reverse strand between exon 5 and 6 of MACROD2. Anti-sense RNAs typically interact with mRNA, resulting in transcriptional or post-transcriptional effects and have been linked to brain development and plasticity [69]. However, unlike MSNP1AS described for the 5p14.1 association observed by Wang and colleagues, a function for this non-coding RNA has not yet been reported.

The strongest association signal observed by the AGP combined analyses was $\operatorname{rs} 1718101(P=$ $7.8 \times 10^{-9}$; OR=2.13 (1.63-2.80)), a SNP within the CNTNAP2 gene which was previously implicated in ASD through linkage analyses. This association was observed in a secondary analysis which was restricted to ASD individuals of European ancestry with a higher IQ. Anney and colleagues suggest that with the current data "few if any common variants have an impact on risk exceeding (an effect size of) 1.2 (or below its inverse)." In an attempt to seek evidence for or against common variants having an impact on risk, the authors constructed an allele-score. The allele-score method, as previously described by Purcell and colleagues [70], calculates a score for each individual based on number of risk associated alleles that the individual possesses. This score is then used to either calculate the predictive value of the score between cases and controls or estimate the amount of variance that this score predicts for the disease. Allelescores derived from the transmission of common alleles from the families described in the AGP stage 1 GWAS [58] could significantly predict case-status in the independent Stage 2 sample. The authors concluded however that despite the limited findings for individual loci from GWAS studies to date, en masse the top results exert a detectable impact suggesting that as the sample sizes increase, additional significant loci will emerge. Putting together samples of the size seen in successful GWAS studies in other disorders $(n>20,000)$ is challenging for a disorder with a complex phenotype such as ASD.

\section{Structural variation: Chromosomal abnormalities in autism}

Autism has been frequently associated with chromosome abnormalities, such as deletions, duplications, inversions, or translocations; with abnormalities on the long arm of Chromosome 15 and with numerical and structural abnormalities of the sex chromosomes. Reddy in a survey of chromosomal abnormality in autism found one in 14 of 421 individuals (3.33\%) [71]. These fourteen cases broke down into 4 supernumerary chromosome markers, 4 deletions, 3 inversions and 3 duplications. Several reviews have since confirmed that such abnormalities can be identified in $\sim 3-5 \%$ of these patients $[72,73]$. The regions commonly reported include $2 q 37$, 
5p14, 5p15, multiple locations on chromosome 7, 11q25, 15q11-q13, 16q22.3, 17p11.2, 18q21.1, 18q23, 22q11.2, 22q13.3 and Xp22.2-p22.3.

Many studies have converged on particular chromosomal abnormalities in autism, the most common of which are maternally inherited duplications at 15q11-13. These duplications are found in as many as $1-3 \%$ of patients diagnosed with autism.

Another relatively common chromosomal variant is the $22 \mathrm{q} 11.2$ microdeletion syndrome, or velocardiofacial syndrome (VCFS) that occurs in $~ 1 / 4000$ live births. This syndrome is also identified in the context of learning disability or in schizophrenia and has a complex phenotypic expression affecting multiple organs. The physical features include a typical facial appearance, (long face, narrow palpebral fissures, flattened malar eminences, prominent nose and small mouth); anatomical and/or functional abnormalities of the palatal shelves such as cleft palate and velopharyngeal insufficiency; lymphoid tissue hypoplasia and heart defects. A wide range of childhood onset developmental symptoms and disorders are described in association with the VCFS mutation [74] including attention deficit hyperactivity disorder (ADHD), oppositional defiant disorder, phobias, anxiety, obsessive compulsive disorder and autism spectrum disorders.

\section{Copy number variation}

The human genome has both sequence and structural variation, the majority of which has no functional consequences and is unrelated to any disorder. Structural variation may be balanced, such as is the case for inversions and balanced translocations or may alter DNA copy number. The later, referred to as copy number variants (CNVs) extend from the duplication or deletion of a single base pair to whole chromosome abnormalities. The term CNV is generally used to indicate the larger changes. Initially, large CNVs were identified with classical chromosomal staining and light microscopy. As many of these abnormalities were initially identified in sub-telomeric regions, there was interest in knowing if such deletions, duplications and other re-arrangements might occur throughout the genome. This hypothesis was confirmed with the development of high resolution array based comparative genomic hybridization (aCGH) [75].

In the arrays, comparative hybridization is performed with DNA immobilized on a platform such as a glass slide. Initially the DNA arrays consisted of human DNA cloned into bacterial artificial chromosomes (BAC arrays) representing the human genome at approximately $1 \mathrm{Mb}$ intervals. The present arrays consist of 25-base pair oligonucleotides (probes). In the last decade the resolution of the arrays have improved to the extent that the number of smaller CNVs identified in the genome that were previously invisible to microscopy has increased enormously. Deletions and duplications at least as small as 10kb are now known to occur throughout the genome.

Interestingly, some CNVs appear to differ from single nucleotide polymorphisms (SNPs) in terms of locus specific mutation rates. Rates for genomic re-arrangements range from $10^{-4}$ to 
$10^{-5}$, a rate that is considerably more frequent than point mutations [76]. This high mutation rate, coupled with reduced fecundity in some carriers, and the fact that comparatively, they affect a larger proportion of the genome, make $\mathrm{CNVs}$ a potentially important source of new and recent mutation in neurodevelopmental disorders.

\section{CNVs in autism}

As discussed above, autism is a phenotypic feature of many genomic disorders. Betancur [77], in her review, lists 103 disease genes and 44 genomic loci where autism or autistic like behaviors have been described. ASD is diagnosed in $\sim 30 \%$ of males with Fragile $X$ syndrome and in reverse, Fragile $X$ mutations are found in as many as $7-8 \%$ of individuals with ASD [78]. Similarly, mutations in MECP2, the Rett Syndrome gene, have been found among cases of autism that do not have the classical Rett phenotype and autism patients have an increased risk for neurofibromatosis and other rare monogenic diseases like tuberous sclerosis and Joubert's Syndrome, again inversely patients with these disorders have an increased risk for having autism $[79,80]$.

The genes and loci listed in the Betancur study are all causally implicated in learning disability (LD), indicating that these two neurodevelopmental disorders share some genetic risk factors. Early use of aCGH in non-syndromic autism suggested the method had promise in detecting hitherto unrecognized CNVs. For example Jacquemont et al [81] identified 6 deletions and 2 duplications in 29 patients presenting with syndromic ASD where previous high resolution karyotyping was reported as normal. Another study showing the potential for CNV analysis was a large linkage study [14] using a 10k SNP array where intensity data was used to determine copy number. The authors highlight some individual findings including a family with two sisters with ASD, both of whom had a $\sim 300 \mathrm{~kb}$ deletion on Ch. 2p16 that included the coding exons of the neurexin 1 gene (NRXN1). A second finding was a recurrent $1.1 \mathrm{Mb}$ duplication at $\mathrm{Ch} .1 \mathrm{q} 2.1$ in four affected individuals from three families, a third was a $\sim 900 \mathrm{~kb}$ de novo duplication at $17 \mathrm{p} 12$ in an affected sib-pair, and with the same region appearing as a maternally inherited deletion in two male siblings, with a paternally inherited deletion in a further female. Duplications in this region cause Charcot-Marie-Tooth 1A (CMT1A) and hereditary neuropathy with pressure palsies when deleted, and overlapping deletions are seen in Smith-Magenis syndrome that includes autism symptoms in many cases [82].

A key development was the report of de novo copy number variants in autism [83] using aCGH. These authors showed that individually rare CNVs, and in particular ones that affect neurodevelopmental genes, were enriched in cases. They further suggested that the rate of de novo CNVs differed between simplex cases, where they occurred in $10 \%$ of families in the sample, and familial cases where they occurred in 3\% of families, suggesting that sporadic and familial cases of ASD might have different underlying genetic mechanisms, although not all studies since then have found this distinction. Several studies of CNVs in large autism case and family series have followed. Marshall et al. [84] examined 427 ASD families using a 500k 
SNP array and karyotyping by standard clinical diagnostic method. A de novo rate of $7.1 \%$ and $2.0 \%$ in simplex and multiplex families respectively was observed, supporting the previous findings [83]. Families occasionally showed more than a single de-novo event where both may combine to produce risk. A further set of loci were identified in two or more unrelated families, increasing the evidence supporting a pathogenic role. As with the LD literature, at some loci, both deletions and duplication were found suggesting a more complex mechanism than simple over or under-expression of gene products. Of the 196 inherited CNVs confirmed experimentally, 90 were of maternal and 106 of paternal origin. The authors list numerous potential ASD candidate genes where a structural change was either de-novo, found in two or more unrelated ASD cases, or, in the case of the X-chromosome, transmitted from an unaffected mother. Given their rarity, very few individual CNVs in this study provided statistical evidence to support their role in autism. For example, $4 \mathrm{CNV}$ s from 427 cases were found at the $D P P 6$ - a subunit that affects the function of Kv4.2 channels at the same site of expression as SHANK3 and NLGN gene products. Only one similar CNV was found in 1652 controls (Fischer's exact test $\mathrm{p}=0.016$ ). In keeping with previous cytogenetic findings and the emerging overlap in disorders involved, CNVs were found in ASD cases that involved known loci or genes in disorders such as Waardenburg Type IIa, Speech and language disorder, learning disability and VCFS. A further study [85] found a total of 51 CNVs in 46 cases and not in the controls. 42 of these were familial and 9 de novo with recurrence in two or more cases at three loci. In total, case specific CNVs were found in $11.6 \%$ of cases, although, in keeping with the Marshall et al [84] study, none were individually associated with case status with the majority being observed in only a single case. Pinto et al [86] compared CNVs in 996 ASD cases of European ancestry to 1,287 matched controls, using the Illumina 1M SNP array. Cases were found to carry a higher global burden of rare, genic CNVs, especially so for loci previously implicated in either ASD and/or intellectual disability. Nearly $6 \%$ of the cases had de-novo mutations with some having two or more events. Novel candidate genes were identified that were de-novo in cases and not controls, including SHANK2, SYNGAP1 and DLGAP2. In keeping with previous studies only one novel CNV (maternally inherited X-linked deletions at PTCHD1) occurred statistically more frequently in cases compared to controls (7 vs 0 ). PTCHD1 involvement in autism and LD was further extended and confirmed in a focused examination of the PTCHD1 locus in cohorts of autism and LD cases and extending the study of CNVs to sequence data, identifying additional maternally inherited missense mutations in 8 probands not seen in controls [87]. In the Pinto et al study [86], certain gene sets were found to be enriched for case deletions but not duplications. These included sets involved in cell and neuronal development, projection, motility and proliferation; GTPase/Ras signaling known to be involved in regulating dendrite and spine plasticity; and kinase activity/regulation. There was additional overlap with gene sets thought to be involved in LD including microtubule cytoskeleton, glycosylation and CNS development/adhesion. More recently, Salyakina and colleagues [88] have shown the value of extended multiply affected families in a CNV study of 42 families. They found 5 deletions and 7 duplications that co-segregated with ASD, two overlapping with known autism CNVs on 7p21.3 and 15q24.1 and two near regions on 3p26.3 and 12q24.32 previously associated with schizophrenia. 
As the resolution of the probe arrays improves, smaller CNVs will be detected, and the boundaries of previously identified CNVs will become more refined [89]. Nord et al. [90], examined genomic DNA of 41 children with autism and 367 healthy controls for rare CNVs using a very high-resolution aCGH platform. They found that cases were more likely than controls to have CNVs as small as $\sim 10 \mathrm{~kb}$, likely to affect genes involved in transcription, nervous system development, and receptor activity. They found that expression of CNTNAP2, ZNF214, PRODH and $A R I D 1 B$ genes affected by CNVs were decreased in probands compared with controls suggesting reduced expression as a potentially aetiological factor during development.

Larger samples, particularly those based on families, will also enable the improved estimation of the overall effects of de-novo mutations and the assessment of rare recurrent events as disease associated mutations [91]. These authors studied 1124 autism families containing probands, unaffected parents and an unaffected sibling using the Illumina 1M SNP array. In a related paper [92] they were able to confirm de novo CNVs identified using the SNP array with those detected using a Nimblegen 2.1M aCGH platform. A combined total of 58 rare de novo CNVs were identified across the two studies with each array type identifying $95 \%$ of the total. However, the sensitivity for smaller CNVs was low for both arrays. Overall, the burden of rare de novo CNVs in the Sanders et al [91] study was greater in probands than in siblings for total number, size and gene content. Using the rate in siblings as a control to evaluate findings in the cases, there was strong individual statistical support for recurrent de novo duplications at 7q11.23, the locus at which deletions cause Williams-Beuren syndrome; deletions at 16p11.2 and duplications at 16p11.2. In addition the authors observed 8 loci at which rare transmitted CNVs, present only in probands, overlapped with one of the 51 regions in probands containing one or more rare de novo CNVs. However, the rare transmitted CNVs were not more likely to be in cases than in unaffected siblings, even when subdivided into genic, exonic, brain-expressed or previously identified as ASD related. This suggested that the excess burden in their sample was due to rare de novo events, although when the gene sets were applied to gene pathway analysis, more pathways showed enrichment in the case set compared to the sibling set. To date, the number of definitive replicated findings for ASD from all studies has been small, with the data suggesting an extreme heterogeneity model with no single risk variant occurring in more than $1 \%$ of cases.

\section{Mutation rates and models of risk in autism}

Given the replicated finding that de-novo mutations are more frequent in simplex cases compared to familial cases, Zhao et al [93] have suggested a model of autism risk in which families fall into two groups; those in which the overall risk for autism is low, representing the majority of families, and those in which the risk is higher due to a disease mutation with a dominant mode of transmission with greater penetrance in males compared to females. Under this model, sporadic cases of autism occur in low-risk families due to a de novo mutation of relatively high penetrance, whereas familial autism occurs due to the inheritance of an existing mutation from a clinically unaffected or asymptomatic parent. In another model, Girirajan et al. [94] proposed the necessity in some families for a second mutation to lead to severe neurodeve- 
lopmental disorder. In this study, individuals with childhood developmental delay are enriched approximately fourfold for a rare 520-kb $16 \mathrm{p} 12$ deletion. In nearly all cases examined (22/23), the deletion was inherited. Thus, $16 \mathrm{p} 12$ deletions appear to be an example of inherited predisposition to neurodevelopmental disorder with dominant transmission. However, these individuals were more likely to carry a second large $(>500 \mathrm{~kb}) \mathrm{CNV}$ compared to matched controls, and clinical features of those with a second large CNV were typically more severe than those with the 16p12 deletion alone. Itsara et al. [95] suggest that multiply affected autism pedigrees segregate an existing inherited mutation of low penetrance which by itself is rarely sufficient to cause disease. Secondary mutations, such as de-novo mutations are required to manifest as disorder. Whether or not these second hits are disease specific remains to be examined. The authors propose that the excess of de novo CNVs among cases may be due to a depletion of second-hits in the unaffected sibling due to the initial low penetrant mutation segregating in the family. The abundance of inherited low penetrance mutations and the high rate of de novo CNVs in the population enable multiple cases to appear within families with apparently unusual patterns of inheritance.

\section{Next-generation sequencing studies}

There is an increasing literature describing the occurrence of single nucleotide mutation in ASD. Unlike the common polymorphisms described earlier, these variants are often rare or private to an individual and considered to have a large impact on the gene's function. Early studies have focused on candidate genes with strong a priori evidence to suggest causation, such as the Neuroligin and Shank encoding genes. Sanger sequencing of the exons of families with ASD have identified many putatively causative mutation in these synaptic proteins [96, 97]. As with the transition from candidate gene association studies to GWAS, the advent of more sophisticated high-throughput next-generation sequencing approaches have enabled many genes to be screened in a single experiment. This has extended to the systematic scanning of the whole exome of individuals with ASD to identify putative risk mutations.

In April 2012, three key manuscripts were published in Nature describing a considerable exome sequencing effort of 622 family trios and 250 unaffected siblings [98-100]. In these studies the focus was on de novo mutation in the exome of individuals with ASD. De novo mutations are those changes that occurred in the child which are not inherited from the parent. Given the natural mutation rate, the number of families studied and the number of genes under investigation it was estimated that three independent mutations would provide strong evidence implicating a gene in ASD, whilst two independent mutations would be suggestive but not definitive. Unfortunately in these studies no gene was burdened with three independent mutations, whilst several revealed two independent de novo mutations. Follow-up analyses across the studies of the two-hit genes in a cohort of over 2600 individuals with ASD and over 1600 typically developing controls identified additional non-synonymous mutations in KATNAL2 (katanin p60 subunit A-like 2), CHD8 (Chromodomain-helicase-DNA-binding protein 8), GRIN2B (Glutamate [NMDA] receptor subunit epsilon-2), LAMC3 (Laminin subunit gamma-3), SCN1A (sodium channel, voltage-gated, type I, alpha subunit) and SCN2A (sodium 
channel, voltage-gated, type II, alpha subunit). Despite the early caution regarding the number of de novo events observed per gene, the burgeoning evidence from focused re-sequencing of these suggests that other families with ASD have damaging loss-of-function mutation in these genes.

\section{Implications of genomic studies for clinical practice}

Genetic findings to date in autism include known genetic syndromes such as Rett and Fragile $\mathrm{X}$; chromosomal structural variation such as duplications on ch15 and deletions on ch22 and increasing numbers of CNVs, many de novo, in a small proportion of cases, especially those with co-morbid developmental abnormalities. Common variants are only slowly emerging from genome-wide studies but sample sizes are still relatively modest compared to other disorders. The diagnosis remains as a clinical one, but genetic testing has diagnostic, prognostic and family planning implications, and in the coming years may also be of importance with respect to potential treatments for specific conditions such as Rett and Fragile X. Therefore, a clinical genetics evaluation should be considered in ASD children in order to identify syndromic forms of autism, identify familial cases, and drive diagnostic testing. Genetic testing using aCGH has been recommended by the American Academy of Pediatrics [101] and the American College of Medical Genetics [102, 103], particularly to evaluate aetiological heterogeneity and identify syndromes. For the vast majority of cases with an identified genetic cause, there is no specific benefit in terms of treatment, but the diagnosis itself may be of value to parents seeking an explanation for the symptoms they see in their child. Acceptance of the disorder may be easier in the presence of a known cause as echoes of social causation and blame can still be heard. Family investigation may also be warranted, particularly where the proband has an identified genetic causation as recurrence risk in siblings may have implications for family planning. If a genetic cause of pathogenic significance is identified, the recurrence risk for sibs may be established according to the particular genetic diagnosis. In the majority of cases, no genetic alteration is found, and an empirical risk for siblings of $10-20 \%$ can be provided. Establishing a clear de novo origin for a given mutation may enable a low risk estimate in siblings although there are caveats relating to the exact origin of the mutation. Finally, and re-stating the main goal of genetic research in ASD, a better understanding of underlying biology may lead to novel and unexpected treatment possibilities. Early signs of this are emerging in human trials for treatments aimed at the specific mutations found in Rett and Fragile $\mathrm{X}$ syndromes.

\section{Author details}

Michael Gill, Graham Kenny and Richard Anney

Department of Psychiatry, School of Medicine, Trinity College Dublin, Ireland 


\section{References}

[1] Lord C. Epidemiology: How common is autism? Nature. 2011 Jun 9;474(7350):166-8. PubMed PMID: 21654793.

[2] Fombonne E. Epidemiology of pervasive developmental disorders. Pediatr Res. 2009 Jun;65(6):591-8. PubMed PMID: 19218885. Epub 2009/02/17. eng.

[3] Fernell E, Gillberg C. Autism spectrum disorder diagnoses in Stockholm preschoolers. Res Dev Disabil. 2010 May-Jun;31(3):680-5. PubMed PMID: 20149593. Epub 2010/02/13. eng.

[4] Folstein S, Rutter M. Genetic influences and infantile autism. Nature. 1977 Feb 24;265(5596):726-8. PubMed PMID: 558516. Epub 1977/02/24. eng.

[5] Bailey A, Le Couteur A, Gottesman I, Bolton P, Simonoff E, Yuzda E, et al. Autism as a strongly genetic disorder: evidence from a British twin study. Psychol Med. 1995 Jan; 25(1):63-77. PubMed PMID: 7792363. Epub 1995/01/01. eng.

[6] Ronald A, Hoekstra RA. Autism spectrum disorders and autistic traits: a decade of new twin studies. Am J Med Genet B Neuropsychiatr Genet. 2011 Apr;156B(3):255-74. PubMed PMID: 21438136. Epub 2011/03/26. eng.

[7] Hallmayer J, Cleveland S, Torres A, Phillips J, Cohen B, Torigoe T, et al. Genetic Heritability and Shared Environmental Factors Among Twin Pairs With Autism. Arch Gen Psychiatry. 2011 Jul 4. PubMed PMID: 21727249. Epub 2011/07/06. Eng.

[8] Le Couteur A, Rutter M, Lord C, Rios P, Robertson S, Holdgrafer M, et al. Autism diagnostic interview: a standardized investigator-based instrument. J Autism Dev Disord. 1989 Sep;19(3):363-87. PubMed PMID: 2793783. Epub 1989/09/01. eng.

[9] Lord C, Rutter M, Goode S, Heemsbergen J, Jordan H, Mawhood L, et al. Autism diagnostic observation schedule: a standardized observation of communicative and social behavior. J Autism Dev Disord. 1989 Jun;19(2):185-212. PubMed PMID: 2745388. Epub 1989/06/01. eng.

[10] International Molecular Genetic Study of Autism Consortium (IMGSAC). A full genome screen for autism with evidence for linkage to a region on chromosome 7q. International Molecular Genetic Study of Autism Consortium. Hum Mol Genet. 1998 Mar;7(3):571-8. PubMed PMID: 9546821. Epub 1998/04/18. eng.

[11] International Molecular Genetic Study of Autism Consortium (IMGSAC), Bailey A. Further characterization of the autism susceptibility locus AUTS1 on chromosome 7q. Hum Mol Genet. 2001 Apr 15;10(9):973-82. PubMed PMID: 11392322. Epub 2001/06/08. eng.

[12] International Molecular Genetic Study of Autism Consortium (IMGSAC), Monaco AP. A genomewide screen for autism: strong evidence for linkage to chromosomes $2 q, 7 q$, 
and 16p. Am J Hum Genet. 2001 Sep;69(3):570-81. PubMed PMID: 11481586. Epub 2001/08/02. eng.

[13] Barrett S, Beck JC, Bernier R, Bisson E, Braun TA, Casavant TL, et al. An autosomal genomic screen for autism. Collaborative linkage study of autism. Am J Med Genet. 1999 Dec 15;88(6):609-15. PubMed PMID: 10581478. Epub 1999/12/03. eng.

[14] Szatmari P, Paterson AD, Zwaigenbaum L, Roberts W, Brian J, Liu XQ, et al. Mapping autism risk loci using genetic linkage and chromosomal rearrangements. Nat Genet. 2007 Mar;39(3):319-28. PubMed PMID: 17322880. Epub 2007/02/27. eng.

[15] Philippe A, Martinez M, Guilloud-Bataille M, Gillberg C, Rastam M, Sponheim E, et al. Genome-wide scan for autism susceptibility genes. Paris Autism Research International Sibpair Study. Hum Mol Genet. 1999 May;8(5):805-12. PubMed PMID: 10196369. Epub 1999/04/10. eng.

[16] Liu J, Nyholt DR, Magnussen P, Parano E, Pavone P, Geschwind D, et al. A genomewide screen for autism susceptibility loci. Am J Hum Genet. 2001 Aug;69(2):327-40. PubMed PMID: 11452361. Epub 2001/07/14. eng.

[17] Schellenberg GD, Dawson G, Sung YJ, Estes A, Munson J, Rosenthal E, et al. Evidence for multiple loci from a genome scan of autism kindreds. Mol Psychiatry. 2006 Nov; 11(11):1049-60, 979. PubMed PMID: 16880825. Epub 2006/08/02. eng.

[18] Persico AM, D'Agruma L, Maiorano N, Totaro A, Militerni R, Bravaccio C, et al. Reelin gene alleles and haplotypes as a factor predisposing to autistic disorder. Mol Psychiatry. 2001 Mar;6(2):150-9. PubMed PMID: 11317216. Epub 2001/04/24. eng.

[19] Skaar DA, Shao Y, Haines JL, Stenger JE, Jaworski J, Martin ER, et al. Analysis of the RELN gene as a genetic risk factor for autism. Mol Psychiatry. 2005 Jun;10(6):563-71. PubMed PMID: 15558079. Epub 2004/11/24. eng.

[20] Zhang H, Liu X, Zhang C, Mundo E, Macciardi F, Grayson DR, et al. Reelin gene alleles and susceptibility to autism spectrum disorders. Mol Psychiatry. 2002;7(9):1012-7. PubMed PMID: 12399956. Epub 2002/10/26. eng.

[21] Serajee FJ, Zhong H, Mahbubul Huq AH. Association of Reelin gene polymorphisms with autism. Genomics. 2006 Jan;87(1):75-83. PubMed PMID: 16311013. Epub 2005/11/29. eng.

[22] Ashley-Koch AE, Jaworski J, Ma de Q, Mei H, Ritchie MD, Skaar DA, et al. Investigation of potential gene-gene interactions between APOE and RELN contributing to autism risk. Psychiatr Genet. 2007 Aug;17(4):221-6. PubMed PMID: 17621165. Epub 2007/07/11. eng.

[23] Li H, Li Y, Shao J, Li R, Qin Y, Xie C, et al. The association analysis of RELN and GRM8 genes with autistic spectrum disorder in Chinese Han population. Am J Med Genet B Neuropsychiatr Genet. 2008 Mar 5;147B(2):194-200. PubMed PMID: 17955477. Epub 2007/10/24. eng. 
[24] Holt R, Barnby G, Maestrini E, Bacchelli E, Brocklebank D, Sousa I, et al. Linkage and candidate gene studies of autism spectrum disorders in European populations. Eur J Hum Genet. 2010 Sep;18(9):1013-9. PubMed PMID: 20442744. Pubmed Central PMCID: 2987412. Epub 2010/05/06. eng.

[25] Kelemenova S, Schmidtova E, Ficek A, Celec P, Kubranska A, Ostatnikova D. Polymorphisms of candidate genes in Slovak autistic patients. Psychiatr Genet. 2010 Aug; 20(4):137-9. PubMed PMID: 20436377. Epub 2010/05/04. eng.

[26] Krebs MO, Betancur C, Leroy S, Bourdel MC, Gillberg C, Leboyer M. Absence of association between a polymorphic GGC repeat in the 5' untranslated region of the reelin gene and autism. Mol Psychiatry. 2002;7(7):801-4. PubMed PMID: 12192627. Pubmed Central PMCID: 1913931. Epub 2002/08/23. eng.

[27] Bonora E, Beyer KS, Lamb JA, Parr JR, Klauck SM, Benner A, et al. Analysis of reelin as a candidate gene for autism. Mol Psychiatry. 2003 Oct;8(10):885-92. PubMed PMID: 14515139. Epub 2003/09/30. eng.

[28] Devlin B, Bennett P, Dawson G, Figlewicz DA, Grigorenko EL, McMahon W, et al. Alleles of a reelin CGG repeat do not convey liability to autism in a sample from the CPEA network. Am J Med Genet B Neuropsychiatr Genet. 2004 Apr 1;126B(1):46-50. PubMed PMID: 15048647. Epub 2004/03/30. eng.

[29] Li J, Nguyen L, Gleason C, Lotspeich L, Spiker D, Risch N, et al. Lack of evidence for an association between WNT2 and RELN polymorphisms and autism. Am J Med Genet B Neuropsychiatr Genet. 2004 Apr 1;126B(1):51-7. PubMed PMID: 15048648. Epub 2004/03/30. eng.

[30] Dutta S, Sinha S, Ghosh S, Chatterjee A, Ahmed S, Usha R. Genetic analysis of reelin gene (RELN) SNPs: no association with autism spectrum disorder in the Indian population. Neurosci Lett. 2008 Aug 15;441(1):56-60. PubMed PMID: 18597938. Epub 2008/07/04. eng.

[31] He Y, Xun G, Xia K, Hu Z, Lv L, Deng Z, et al. No significant association between RELN polymorphism and autism in case-control and family-based association study in Chinese Han population. Psychiatry Res. 2011 May 30;187(3):462-4. PubMed PMID: 20554015. Epub 2010/06/18. eng.

[32] Jackson PB, Boccuto L, Skinner C, Collins JS, Neri G, Gurrieri F, et al. Further evidence that the rs $1858830 \mathrm{C}$ variant in the promoter region of the MET gene is associated with autistic disorder. Autism Res. 2009 Aug;2(4):232-6. PubMed PMID: 19681062. Epub 2009/08/15. eng.

[33] Sousa I, Clark TG, Toma C, Kobayashi K, Choma M, Holt R, et al. MET and autism susceptibility: family and case-control studies. Eur J Hum Genet. 2009 Jun;17(6):749-58. PubMed PMID: 19002214. Epub 2008/11/13. eng. 
[34] Thanseem I, Nakamura K, Miyachi T, Toyota T, Yamada S, Tsujii M, et al. Further evidence for the role of MET in autism susceptibility. Neurosci Res. 2010 Oct;68(2): 137-41. PubMed PMID: 20615438. Epub 2010/07/10. eng.

[35] Benayed R, Gharani N, Rossman I, Mancuso V, Lazar G, Kamdar S, et al. Support for the homeobox transcription factor gene ENGRAILED 2 as an autism spectrum disorder susceptibility locus. Am J Hum Genet. 2005 Nov;77(5):851-68. PubMed PMID: 16252243. Epub 2005/10/28. eng.

[36] Zhong H, Serajee FJ, Nabi R, Huq AH. No association between the EN2 gene and autistic disorder. J Med Genet. 2003 Jan;40(1):e4. PubMed PMID: 12525552. Pubmed Central PMCID: 1735256. Epub 2003/01/15. eng.

[37] Arking DE, Cutler DJ, Brune CW, Teslovich TM, West K, Ikeda M, et al. A common genetic variant in the neurexin superfamily member CNTNAP2 increases familial risk of autism. Am J Hum Genet. 2008 Jan;82(1):160-4. PubMed PMID: 18179894. Epub 2008/01/09. eng.

[38] Li X, Hu Z, He Y, Xiong Z, Long Z, Peng Y, et al. Association analysis of CNTNAP2 polymorphisms with autism in the Chinese Han population. Psychiatr Genet. 2010 Jun; 20(3):113-7. PubMed PMID: 20414140. Epub 2010/04/24. eng.

[39] Anney R, Klei L, Pinto D, Almeida J, Bacchelli E, Baird G, et al. Individual common variants exert weak effects on the risk for autism spectrum disorders. Hum Mol Genet. 2012 Aug 8. PubMed PMID: 22843504. Epub 2012/07/31. Eng.

[40] Segurado R, Conroy J, Meally E, Fitzgerald M, Gill M, Gallagher L. Confirmation of association between autism and the mitochondrial aspartate/glutamate carrier SLC25A12 gene on chromosome 2q31. Am J Psychiatry. 2005 Nov;162(11):2182-4. PubMed PMID: 16263864. Epub 2005/11/03. eng.

[41] Kim SJ, Silva RM, Flores CG, Jacob S, Guter S, Valcante G, et al. A quantitative association study of SLC25A12 and restricted repetitive behavior traits in autism spectrum disorders. Mol Autism. 2011;2(1):8. PubMed PMID: 21609426. Epub 2011/05/26. eng.

[42] Turunen JA, Rehnstrom K, Kilpinen H, Kuokkanen M, Kempas E, Ylisaukko-Oja T. Mitochondrial aspartate/glutamate carrier SLC25A12 gene is associated with autism. Autism Res. 2008 Jun;1(3):189-92. PubMed PMID: 19360665. Epub 2009/04/11. eng.

[43] Correia C, Coutinho AM, Diogo L, Grazina M, Marques C, Miguel T, et al. Brief report: High frequency of biochemical markers for mitochondrial dysfunction in autism: no association with the mitochondrial aspartate/glutamate carrier SLC25A12 gene. J Autism Dev Disord. 2006 Nov;36(8):1137-40. PubMed PMID: 17151801. Epub 2006/12/08. eng.

[44] Rabionet R, McCauley JL, Jaworski JM, Ashley-Koch AE, Martin ER, Sutcliffe JS, et al. Lack of association between autism and SLC25A12. Am J Psychiatry. 2006 May;163(5): 929-31. PubMed PMID: 16648338. Epub 2006/05/02. eng. 
[45] Blasi F, Bacchelli E, Carone S, Toma C, Monaco AP, Bailey AJ, et al. SLC25A12 and CMYA3 gene variants are not associated with autism in the IMGSAC multiplex family sample. Eur J Hum Genet. 2006 Jan;14(1):123-6. PubMed PMID: 16205742. Epub 2005/10/06. eng.

[46] Chien WH, Wu YY, Gau SS, Huang YS, Soong WT, Chiu YN, et al. Association study of the SLC25A12 gene and autism in Han Chinese in Taiwan. Prog Neuropsychopharmacol Biol Psychiatry. 2010 Feb 1;34(1):189-92. PubMed PMID: 19913066. Epub 2009/11/17. eng.

[47] Gallagher L, Becker K, Kearney G, Dunlop A, Stallings R, Green A, et al. Brief report: A case of autism associated with del(2)(q32.1q32.2) or (q32.2q32.3). J Autism Dev Disord. 2003 Feb;33(1):105-8. PubMed PMID: 12708586. Epub 2003/04/24. eng.

[48] Conroy J, Cochrane L, Anney RJ, Sutcliffe JS, Carthy P, Dunlop A, et al. Fine mapping and association studies in a candidate region for autism on chromosome $2 \mathrm{q} 31-\mathrm{q} 32$. Am J Med Genet B Neuropsychiatr Genet. 2009 Jun 5;150B(4):535-44. PubMed PMID: 18846500. Epub 2008/10/11. eng.

[49] Auranen M, Vanhala R, Varilo T, Ayers K, Kempas E, Ylisaukko-Oja T, et al. A genomewide screen for autism-spectrum disorders: evidence for a major susceptibility locus on chromosome 3q25-27. Am J Hum Genet. 2002 Oct;71(4):777-90. PubMed PMID: 12192642. Epub 2002/08/23. eng.

[50] Auranen M, Varilo T, Alen R, Vanhala R, Ayers K, Kempas E, et al. Evidence for allelic association on chromosome $3 \mathrm{q} 25-27$ in families with autism spectrum disorders originating from a subisolate of Finland. Mol Psychiatry. 2003 Oct;8(10):879-84. PubMed PMID: 14515138. Epub 2003/09/30. eng.

[51] Ylisaukko-oja T, Nieminen-von Wendt T, Kempas E, Sarenius S, Varilo T, von Wendt L, et al. Genome-wide scan for loci of Asperger syndrome. Mol Psychiatry. 2004 Feb; 9(2):161-8. PubMed PMID: 14966474. Epub 2004/02/18. eng.

[52] Coon H, Matsunami N, Stevens J, Miller J, Pingree C, Camp NJ, et al. Evidence for linkage on chromosome 3q25-27 in a large autism extended pedigree. Hum Hered. 2005;60(4):220-6. PubMed PMID: 16391490. Epub 2006/01/05. eng.

[53] Yonan AL, Alarcon M, Cheng R, Magnusson PK, Spence SJ, Palmer AA, et al. A genomewide screen of 345 families for autism-susceptibility loci. Am J Hum Genet. 2003 Oct;73(4):886-97. PubMed PMID: 13680528. Epub 2003/09/19. eng.

[54] Weiss LA, Arking DE, Daly MJ, Chakravarti A. A genome-wide linkage and association scan reveals novel loci for autism. Nature. 2009 Oct 8;461(7265):802-8. PubMed PMID: 19812673. Epub 2009/10/09. eng.

[55] Risch N, Merikangas K. The future of genetic studies of complex human diseases. Science. 1996 Sep 13;273(5281):1516-7. PubMed PMID: 8801636. Epub 1996/09/13. eng. 
[56] Wang K, Zhang H, Ma D, Bucan M, Glessner JT, Abrahams BS, et al. Common genetic variants on 5p14.1 associate with autism spectrum disorders. Nature. 2009 May 28;459(7246):528-33. PubMed PMID: 19404256. Epub 2009/05/01. eng.

[57] Ma D, Salyakina D, Jaworski JM, Konidari I, Whitehead PL, Andersen AN, et al. A genome-wide association study of autism reveals a common novel risk locus at 5p14.1. Ann Hum Genet. 2009 May;73(Pt 3):263-73. PubMed PMID: 19456320. Epub 2009/05/22. eng.

[58] Anney R, Klei L, Pinto D, Regan R, Conroy J, Magalhaes TR, et al. A genome-wide scan for common alleles affecting risk for autism. Hum Mol Genet. 2010 Oct 15;19(20): 4072-82. PubMed PMID: 20663923. Epub 2010/07/29. eng.

[59] Xu B, Woodroffe A, Rodriguez-Murillo L, Roos JL, van Rensburg EJ, Abecasis GR, et al. Elucidating the genetic architecture of familial schizophrenia using rare copy number variant and linkage scans. Proc Natl Acad Sci U S A. 2009 Sep 29;106(39):16746-51. PubMed PMID: 19805367. Epub 2009/10/07. eng.

[60] Kirov G, Pocklington AJ, Holmans P, Ivanov D, Ikeda M, Ruderfer D, et al. De novo $\mathrm{CNV}$ analysis implicates specific abnormalities of postsynaptic signalling complexes in the pathogenesis of schizophrenia. Mol Psychiatry. 2012 Feb;17(2):142-53. PubMed PMID: 22083728. Epub 2011/11/16. eng.

[61] Lesca G, Rudolf G, Labalme A, Hirsch E, Arzimanoglou A, Genton P, et al. Epileptic encephalopathies of the Landau-Kleffner and continuous spike and waves during slow-wave sleep types: Genomic dissection makes the link with autism. Epilepsia. 2012 Sep;53(9):1526-38. PubMed PMID: 22738016. Epub 2012/06/29. Eng.

[62] Bradley WE, Raelson JV, Dubois DY, Godin E, Fournier H, Prive C, et al. Hotspots of large rare deletions in the human genome. PLoS ONE. 2010;5(2):e9401. PubMed PMID: 20195527. Pubmed Central PMCID: 2828468. Epub 2010/03/03. eng.

[63] Karras GI, Kustatscher G, Buhecha HR, Allen MD, Pugieux C, Sait F, et al. The macro domain is an ADP-ribose binding module. EMBO J. 2005 Jun 1;24(11):1911-20. PubMed PMID: 15902274. Epub 2005/05/20. eng.

[64] Timinszky G, Till S, Hassa PO, Hothorn M, Kustatscher G, Nijmeijer B, et al. A macrodomain-containing histone rearranges chromatin upon sensing PARP1 activation. Nat Struct Mol Biol. 2009 Sep;16(9):923-9. PubMed PMID: 19680243. Epub 2009/08/15. eng.

[65] Hoff KG, Wolberger C. Getting a grip on O-acetyl-ADP-ribose. Nat Struct Mol Biol. 2005 Jul;12(7):560-1. PubMed PMID: 15999106. Epub 2005/07/07. eng.

[66] Liou GG, Tanny JC, Kruger RG, Walz T, Moazed D. Assembly of the SIR complex and its regulation by O-acetyl-ADP-ribose, a product of NAD-dependent histone deacetylation. Cell. 2005 May 20;121(4):515-27. PubMed PMID: 15907466. Epub 2005/05/24. eng.

[67] Chen D, Vollmar M, Rossi MN, Phillips C, Kraehenbuehl R, Slade D, et al. Identification of macrodomain proteins as novel O-acetyl-ADP-ribose deacetylases. J Biol Chem. 2011 Apr 15;286(15):13261-71. PubMed PMID: 21257746. Epub 2011/01/25. eng. 
[68] Cohen-Armon M, Visochek L, Katzoff A, Levitan D, Susswein AJ, Klein R, et al. Longterm memory requires polyADP-ribosylation. Science. 2004 Jun 18;304(5678):1820-2. PubMed PMID: 15205535. Epub 2004/06/19. eng.

[69] Qureshi IA, Mehler MF. Emerging roles of non-coding RNAs in brain evolution, development, plasticity and disease. Nature reviews Neuroscience. 2012 Aug;13(8): 528-41. PubMed PMID: 22814587. Epub 2012/07/21. eng.

[70] Purcell SM, Wray NR, Stone JL, Visscher PM, O'Donovan MC, Sullivan PF, et al. Common polygenic variation contributes to risk of schizophrenia and bipolar disorder. Nature. 2009 Aug 6;460(7256):748-52. PubMed PMID: 19571811. Epub 2009/07/03. eng.

[71] Reddy KS. Cytogenetic abnormalities and fragile-X syndrome in Autism Spectrum Disorder. BMC medical genetics. 2005 Jan 18;6:3. PubMed PMID: 15655077. Pubmed Central PMCID: 548305.

[72] Gillberg C. Chromosomal disorders and autism. J Autism Dev Disord. 1998 Oct;28(5): 415-25. PubMed PMID: 9813777. Epub 1998/11/14. eng.

[73] Vorstman JA, Staal WG, van Daalen E, van Engeland H, Hochstenbach PF, Franke L. Identification of novel autism candidate regions through analysis of reported cytogenetic abnormalities associated with autism. Mol Psychiatry. 2006 Jan;11(1):1, 18-28. PubMed PMID: 16205736. Epub 2005/10/06. eng.

[74] Gothelf D, Schaer M, Eliez S. Genes, brain development and psychiatric phenotypes in velo-cardio-facial syndrome. Dev Disabil Res Rev. 2008;14(1):59-68. PubMed PMID: 18636637.

[75] Zhang F, Gu W, Hurles ME, Lupski JR. Copy number variation in human health, disease, and evolution. Annual review of genomics and human genetics. 2009;10:451-81. PubMed PMID: 19715442. Epub 2009/09/01. eng.

[76] Lupski JR. Structural variation in the human genome. The New England journal of medicine. 2007 Mar 15;356(11):1169-71. PubMed PMID: 17360997. Epub 2007/03/16. eng.

[77] Betancur C. Etiological heterogeneity in autism spectrum disorders: more than 100 genetic and genomic disorders and still counting. Brain Res. 2011 Mar 22;1380:42-77. PubMed PMID: 21129364. Epub 2010/12/07. eng.

[78] Muhle R, Trentacoste SV, Rapin I. The genetics of autism. Pediatrics. 2004 May; 113(5):e472-86. PubMed PMID: 15121991. Epub 2004/05/04. eng.

[79] Marui T, Hashimoto O, Nanba E, Kato C, Tochigi M, Umekage T, et al. Association between the neurofibromatosis-1 (NF1) locus and autism in the Japanese population. American journal of medical genetics Part B, Neuropsychiatric genetics : the official publication of the International Society of Psychiatric Genetics. 2004 Nov 15;131B(1): 43-7. PubMed PMID: 15389774. Epub 2004/09/25. eng. 
[80] Zafeiriou DI, Ververi A, Vargiami E. Childhood autism and associated comorbidities. Brain \& development. 2007 Jun;29(5):257-72. PubMed PMID: 17084999. Epub 2006/11/07. eng.

[81] Jacquemont ML, Sanlaville D, Redon R, Raoul O, Cormier-Daire V, Lyonnet S, et al. Array-based comparative genomic hybridisation identifies high frequency of cryptic chromosomal rearrangements in patients with syndromic autism spectrum disorders. Journal of medical genetics. 2006 Nov;43(11):843-9. PubMed PMID: 16840569. Pubmed Central PMCID: 2563185. Epub 2006/07/15. eng.

[82] Smith ACM, Boyd K, Elsea SH, Finucane BM, Haas-Givler B, Gropman A, et al. SmithMagenis Syndrome. In: Pagon RA, Bird TD, Dolan CR, Stephens K, editors. GeneReviews. Seattle (WA)1993.

[83] Sebat J, Lakshmi B, Malhotra D, Troge J, Lese-Martin C, Walsh T, et al. Strong association of de novo copy number mutations with autism. Science. 2007 Apr 20;316(5823): 445-9. PubMed PMID: 17363630. eng.

[84] Marshall CR, Noor A, Vincent JB, Lionel AC, Feuk L, Skaug J, et al. Structural variation of chromosomes in autism spectrum disorder. Am J Hum Genet. 2008 Feb;82(2):477-88. PubMed PMID: 18252227. Epub 2008/02/07. eng.

[85] Christian SL, Brune CW, Sudi J, Kumar RA, Liu S, Karamohamed S, et al. Novel submicroscopic chromosomal abnormalities detected in autism spectrum disorder. Biol Psychiatry. 2008 Jun 15;63(12):1111-7. PubMed PMID: 18374305. Epub 2008/04/01. eng.

[86] Pinto D, Pagnamenta AT, Klei L, Anney R, Merico D, Regan R, et al. Functional impact of global rare copy number variation in autism spectrum disorders. Nature. $2010 \mathrm{Jul}$ 15;466(7304):368-72. PubMed PMID: 20531469. Pubmed Central PMCID: 3021798. Epub 2010/06/10. eng.

[87] Noor A, Whibley A, Marshall CR, Gianakopoulos PJ, Piton A, Carson AR, et al. Disruption at the PTCHD1 Locus on Xp22.11 in Autism spectrum disorder and intellectual disability. Sci Transl Med. 2010 Sep 15;2(49):49ra68. PubMed PMID: 20844286. Pubmed Central PMCID: 2987731. Epub 2010/09/17. eng.

[88] Salyakina D, Cukier HN, Lee JM, Sacharow S, Nations LD, Ma D, et al. Copy number variants in extended autism spectrum disorder families reveal candidates potentially involved in autism risk. PloS one. 2011;6(10):e26049. PubMed PMID: 22016809. Pubmed Central PMCID: 3189231. Epub 2011/10/22. eng.

[89] Celestino-Soper PB, Shaw CA, Sanders SJ, Li J, Murtha MT, Ercan-Sencicek AG, et al. Use of array CGH to detect exonic copy number variants throughout the genome in autism families detects a novel deletion in TMLHE. Human molecular genetics. 2011 Nov 15;20(22):4360-70. PubMed PMID: 21865298. Pubmed Central PMCID: 3196886. Epub 2011/08/26. eng.

[90] Nord AS, Roeb W, Dickel DE, Walsh T, Kusenda M, O'Connor KL, et al. Reduced transcript expression of genes affected by inherited and de novo CNVs in autism. European 
journal of human genetics : EJHG. 2011 Jun;19(6):727-31. PubMed PMID: 21448237. Pubmed Central PMCID: 3110052. Epub 2011/03/31. eng.

[91] Sanders SJ, Ercan-Sencicek AG, Hus V, Luo R, Murtha MT, Moreno-De-Luca D, et al. Multiple recurrent de novo CNVs, including duplications of the 7q11.23 Williams syndrome region, are strongly associated with autism. Neuron. 2011 Jun 9;70(5):863-85. PubMed PMID: 21658581. Epub 2011/06/11. eng.

[92] Levy D, Ronemus M, Yamrom B, Lee YH, Leotta A, Kendall J, et al. Rare de novo and transmitted copy-number variation in autistic spectrum disorders. Neuron. 2011 Jun 9;70(5):886-97. PubMed PMID: 21658582. Epub 2011/06/11. eng.

[93] Zhao X, Leotta A, Kustanovich V, Lajonchere C, Geschwind DH, Law K, et al. A unified genetic theory for sporadic and inherited autism. Proceedings of the National Academy of Sciences of the United States of America. 2007 Jul 31;104(31):12831-6. PubMed PMID: 17652511. Pubmed Central PMCID: 1933261. Epub 2007/07/27. eng.

[94] Girirajan S, Eichler EE. Phenotypic variability and genetic susceptibility to genomic disorders. Human molecular genetics. 2010 Oct 15;19(R2):R176-87. PubMed PMID: 20807775. Pubmed Central PMCID: 2953748. Epub 2010/09/03. eng.

[95] Itsara A, Wu H, Smith JD, Nickerson DA, Romieu I, London SJ, et al. De novo rates and selection of large copy number variation. Genome Res. 2010 Nov;20(11):1469-81. PubMed PMID: 20841430. Pubmed Central PMCID: 2963811. Epub 2010/09/16. eng.

[96] Jamain S, Quach H, Betancur C, Rastam M, Colineaux C, Gillberg IC, et al. Mutations of the X-linked genes encoding neuroligins NLGN3 and NLGN4 are associated with autism. Nat Genet. 2003 May;34(1):27-9. PubMed PMID: 12669065. Epub 2003/04/02. eng.

[97] Durand CM, Betancur C, Boeckers TM, Bockmann J, Chaste P, Fauchereau F, et al. Mutations in the gene encoding the synaptic scaffolding protein SHANK3 are associated with autism spectrum disorders. Nat Genet. 2007 Jan;39(1):25-7. PubMed PMID: 17173049. Epub 2006/12/19. eng.

[98] O'Roak BJ, Vives L, Girirajan S, Karakoc E, Krumm N, Coe BP, et al. Sporadic autism exomes reveal a highly interconnected protein network of de novo mutations. Nature. 2012 Apr 4. PubMed PMID: 22495309. Epub 2012/04/13. Eng.

[99] Neale BM, Kou Y, Liu L, Ma'ayan A, Samocha KE, Sabo A, et al. Patterns and rates of exonic de novo mutations in autism spectrum disorders. Nature. 2012 Apr 4. PubMed PMID: 22495311. Epub 2012/04/13. Eng.

[100] Sanders SJ, Murtha MT, Gupta AR, Murdoch JD, Raubeson MJ, Willsey AJ, et al. De novo mutations revealed by whole-exome sequencing are strongly associated with autism. Nature. 2012 Apr 4. PubMed PMID: 22495306. Epub 2012/04/13. Eng.

[101] Johnson CP, Myers SM. Identification and evaluation of children with autism spectrum disorders. Pediatrics. 2007 Nov;120(5):1183-215. PubMed PMID: 17967920. Epub 2007/10/31. eng. 
[102] Schaefer GB, Mendelsohn NJ. Genetics evaluation for the etiologic diagnosis of autism spectrum disorders. Genetics in medicine : official journal of the American College of Medical Genetics. 2008 Jan;10(1):4-12. PubMed PMID: 18197051. Epub 2008/01/17. eng.

[103] Roesser J. Diagnostic yield of genetic testing in children diagnosed with autism spectrum disorders at a regional referral center. Clinical pediatrics. 2011 Sep;50(9):834-43. PubMed PMID: 21525079. Epub 2011/04/29. eng. 
Chapter 15

\title{
Genetic and Environmental Factors in Autism
}

\author{
Esra Guney and Elvan Iseri \\ Additional information is available at the end of the chapter \\ http://dx.doi.org/10.5772/53295
}

\section{Introduction}

Autism is a neurodevelopmental disorder characterized by impaired social interaction, and verbal and nonverbal communication as well as limited and repetitive behaviours. Although symptomatology of autism may be noticed around early months, diagnosis generally occurs around 24-36 months, however in some cases diagnosis may be delayed to adulthood [1]. Since behavioural symptoms and the degree of functional impairment are variable, the autistic disorder is described as a heterogenous symptom cluster of varying etiological and pathological basis [2]. Described as a multifactorial disorder created by interaction of neurologic, immunologic, environmental, and genetic factors, autistic disorder has no definite cause $[3,4]$. In many cases in whom the etiology remains unclear are diagnosed as idiopathic autism or non-syndromic autism $[5,6]$. Seventy percent of cases with idiopathic autism have basic symptoms without physical abnormalities whereas $30 \%$ have complex autism in which dysmorphic features are detected such as microcephaly and/or structural brain malformations [7]. Autism is associated with other syndromes such as Fragile $X$ syndrome, Down Syndrome, and tuberosclerosis in $5-25 \%$ of the cases $([8,9]$. Although phenotypic heterogeneity is the biggest challenge for research efforts directed to identify autism etiology [10], currently it is widely accepted that environmental and genetic factors play essential role in genesis of autistic disorder thanks to a recent advance in research techniques related to biological factors and widespread studies in this field [11, 12].

\section{Genetics}

Autistic disorder is a multifactorial genetic disorder not following classical Mendelian inheritance. Impairment in social interaction and verbal communication as well as genetic differentiation in rigid-repetitive behaviours indicates that different features in autistic disorder 
may be caused by different genes associated with distinct brain regions and be related to cognitive impairment and functional abnormalities [13].

Genetic studies in the field of autistic disorder have mainly focused on molecular genetic studies, assessment of chromosomal abnormalities, twin studies and family studies. In families having an autistic child the recurrence rate has been reported as 3-8\% [14, 15, 16]. The studies on twins and adopted children are important in identifying the actual importance of genetic factors. Concordance among twins enables to measure heritability, and thus to assess what percentage of the phenotype is affected by genetic factors. Monozygotic (identical) twins share $100 \%$ of the genetic material whereas dizygotic (fraternal) twins share $50 \%$ of the genetic material. Monozygotic twins' higher rate of concordance compared to dizygotic twins may be used for calculation of heritability. Twin studies generally showed a higher concordance rate for monozygotic twins compared to dizygotic twins. The concordance rate of monozygotic twins is at least $60 \%$ when diagnostic criteria for autism (DSM-IV) are used whereas the number is as high as $71 \%$ for autism spectrum and $92 \%$ for a broader spectrum of verbal/social interaction disorders $[11,12,16,17]$. On the other hand, the concordance rate of dizygotic twins has been reported as 1-30\% [9, 17-20]. Twin studies demonstrated an average autism inheritance of $90 \%$ [21]. On the basis of these studies autism is considered to be among the most inherited psychiatric diseases [22, 23].

Although autism has a high inheritance rate, its mode of inheritance remains unclear. Multigene interactions and multiple loci are believed to play role in genetic susceptibility to the disease [24]. There are 3 basic approaches in this area: 1) in whole genome scanning method, it is aimed to predict the localization of a disease, about chromosomal localization of which we have no preliminary information, by starting from common genetic determinants in a community composed of multiplex families (families with more than one involved member). 2) cytogenetic studies guide molecular studies by showing inherited or de novo chromosomal anomalies in involved persons or families. 3) candidate gene studies examine the relationship of genes known to affect brain development in associated regions or alternatively, a selected precursor gene considered to hypothetically contribute to autism pathogenesis.

It has been demonstrated that structural chromosomal variations comprising also copy number variations play an important role in etiology of autism. De novo copy number variations have been identified in $7-10 \%$ of sporadic autism cases $[25,26]$.

In studies employing genome scanning method to reveal genetic etiology of autism, cogent evidence for an association with chromosomes $2,7,1$, and 17, especially long arm of chromosomes $2(2 q)$ and $7(7 q)$ has been obtained. Other chromosomes less associated with autism are chromosomes 1, 9, 13, 15, 19, 22, and X chromosome [14, 16, 27]. Although a lot of genomic regions have been explored for etiology, consistent results for a limited number of regions such as 7q11, 7q31, 22q11 have been obtained [16, 28, 29]. Particularly 15q11-q13 region on chromosome 15 has been widely related to autism. It has been suggested that duplications in this region of chromosome 15 may contribute to autism development. There exist in this area a series of potential candidate genes containing gamma aminobutyric acid $\mathrm{A}$ (GABAA) receptor gene complex [30]. These duplications inherited maternally have been reported to be present in 1-3\% of individuals with idiopathic autism [31, 32]. 
Another region related to autism is a deletion region located on chromosome 16p11. This region has also been demonstrated to be in relationship with Asperger Syndrome, mental retardation, and developmental abnormalities [33, 34].

It has been showed that, in individuals with autism, there is a significant increase in the frequency of allelic variations of HOXA1 gene (7p15). HOXA1 and HOXB1, which have a critical role for development of fetal caudal medullary structures, are only expressed at the third week following fertilization, a period when neural tube is formed, and they appear to be partly associated with development of superior olivary, facial and abducens nuclei. It has been suggested that HOXA1 has a role in autism tendency and is associated with early phase of brain stem development in autism etiology [16, 35]. On the other hand, there are studies where no significant association with HOXA1 gene variants and autism could be demonstrated [36, 37].

Engrailed-2 (EN-2) which is the human homologue of drosophila engrailed gene and located on the long arm of Chromosome 7 (7q36) is a homeobox gene having a critical role in midbrain and cerebellar development. Temporal and spatial pattern of engrailed gene expression occurs simultaneously with the development of cerebellar precursor cells. Thus, it has been suggested to be important to determine correct cell number in cerebellum [38]. Petit and his colleagues (1995) reported a significant association between Pvull polymorphism at the 5' region of EN-2 gene and autism [39]. However, this association could not be confirmed in a later family study [40].

MET oncogene coding pleiotropic MET receptor thyrosine kinase is located on the long arm of Chromosome 7. MET signalization has a role in neocortex and cerebellar growth and maturation, and immune functions. MET gene and its ligand, hepatocyte growth factor (HGF), have been related to autism. Studies conducted by Campbell and his colleagues $([41,42]$ showed that $\mathrm{C}$ allele in the promoter region of MET gene decreases MET promoter activity by two fold and decreased MET gene expression is associated with autism tendency.

Another gene on Chromosome 7 is CNTNAP2 (contactin-associated-protein-like 2) gene. CNTNAP2 gene has been associated in various studies with autism, language delay, and epilepsy [43-45].

FOXP2 (forkhead box P2), a forkhead transcription factor gene, is a member of family forkhead known as the key regulators of embryogenesis; it encodes a transcription factor containing polyglutamine and is associated with development of lingual functions. In a study in Chinese society, FOXP2 gene located in the 7q31 region was linked with autism pathogenesis [46]. However, other studies did not replicate these findings [47, 48].

Another gene investigated for autism relationship is Wingless-Int (Wnt2) gene located on the long arm of Chromosome 7 (7q31-33). Wnt genes encode glycoproteins rich in cysteine, which regulate various cellular movements during the embryonic development [49]. It has been shown that Wnt has a role in regulation of activity-dependent dendritic branching in hippocampal pyramidal neurons [50]. Wnt2 gene was linked with autism in a study by Wassink and his colleagues [51]. 
Reelin is an important extracellular matrix glycoprotein that has an important role in development of neuronal migration, lamination, and connection during embryonic brain development and is associated with a signal pathway forming the basis of neuro-conduction, memory formation, and synaptic plasticity [52]. It is responsible for lamination in embryonic period whereas it has a role in cell signalization and synaptic plasticity in adulthood period [53]. Decrease in reelin expression has been associated with autism. RELN gene, which is located in 7q22 region and encodes reelin protein which is important in neurodevelopment, involves a polymorphic GGC repeat in 5' region. Long GGC alleles of RELN gene cause blunt gene expression; therefore, they are considered to be linked with autism [52]. There are studies reporting a significant relationship between RELN alleles with larger numbers of CGG repeats and autism $[52,54]$ while there are also negative studies in terms of such a relationship [55]. Besides the genetic complexity in the etiopathogenesis of this disorder, nonreplication of the results of different studies should also be taken into consideration.

Neuroligins are cellular adhesion molecules located at the postsynaptic side of the synapse. Neuroligins and neuroxins, neuronal cell surface proteins, form an asymmetrical intercellular connection by adhering to each other. Interaction of neuroligins with beta neuroxins forms functional synapses [56]. Neuroligin family is composed of 5 members, i.e. NLGN1, NLGN2, NLGN3, NLGN4, and NLGN4Y. Although all of the neuroligin family is linked with autism spectrum disorder [57], the most robust evidence comes from NLGN3 (Xq13) and NLGN4 (Xp22.3) genes. Jamain and colleagues [58] found that mutations in NLGN3 and NLGN4, two $X$-linked neuroligin genes, are associated with autism spectrum disorders [58]. Following this, it has been demonstrated that a 2-base-pair deletion in NLGN4 gene causes premature stop codon in mental-retarded men with or without autism. This finding indicates that NLGN4 gene is not only associated with autism, but also with mental retardation [59]. Since mutations in neuroligin genes impair the functions of synaptic cell adhesion molecules, they are considered to be related with autism and neurodevelopmental defects in mental retardation [60]. Since neuroligins are abundant particularly in excitatory synapses, a defect in synaptogenesis has been suggested to result in derangement in cognitive development and communication [59]. Nonetheless, some other studies revealed negative results [61].

Genetic studies examining the relationship of neuroxins, the connection partners of neuroligins, with autism revealed that a mutation in neuroxin 1 beta gene results in autism susceptibility [62]. Structural variants of neuroxin 1alpha gene have also been linked with autism [63].

Another protein adhering to neuroligins is SHANK3. Some forms of autism are considered to stem from a single gene, and particularly from a rare allele having a major effect. Doctor Joseph Buxbaum has reported that one of these genes is SHANK3 gene located on Chromosome 22 (22q13) which is responsible for $1 \%$ of autism and some forms of mental retardation, microcephaly, and delay in expressive language [34]. SHANK proteins are believed to be the primary regulator of postsynaptic density thanks to their ability to form multimeric complexes with postsynaptic receptors, signal molecules, and cellular skeleton proteins found in dendritic spikes. Postsynaptic density is the measurement of how synapses are linked to each other. A mutation in SHANK3 gene has been reported to be related with autism spectrum disorders [64]. Role of various mutations in Neuroligin/neuroxin/SHANK3 
complex in development of autism spectrum disorders provide potential evidence for synaptic alterations in etiology of the disorder.

A large-scale study by Wang and his colleagues [65] revealed a significant relationship between a single nucleotide polymorphism located in the 5 p14.1 region and autism spectrum disorders. The associated single nucleotide polymorphism is located in a region placed between cadherin 10 (CDH 10) and cadherin 9 (CDH 9) genes. $\mathrm{CDH} 9$ and $\mathrm{CDH} 10$ encode type II classic cadherins of the cadherin family, which are transmembraneous glycoprotiens responsible for calcium-dependent cell-cell adhesion. This finding shows the role of neuronal cellular adhesion molecules in autism pathogenesis [65].

Neurotrophins have many functions such as neuronal survival, target innervation, and synaptogenesis in development of peripheral and central nervous system. Neurotrophins exert their biologic functions by binding to a Trk tyrosine kinase receptor which is a high-affinity receptor. Neurotrophin family has 4 members. These are nerve growth factor (NGF), brain-derived neurotrophic factor (BDNF), neurotrophin-3 and neurotrophin-4. BDNF is the most important member of neurotrophin family. BDNF has many roles in neuronal differentiation such as neuronal survival, dendritic and axonal growth/branching, synapse formation, and neuronal plasticity [66, 67]. Various studies have investigated the relationship between BDNF gene and autism. Nishimura and colleagues [68] detected an increase in BDNF expression in autistic individuals. Subsequent studies confirmed the potential role of BDNF gene mutations in autism pathogenesis [69]. A recent study in which serum BDNF levels significantly increased in autistic children found no significant impact of genetic variations of BDNF gene on autism risk; however, a significant relationship between neurotrophic tyrosine kinase receptor type 2 (NTRK2) and autism was reported [70]. A large-scale study on patients diagnosed with autism spectrum disorder and mental retardation without autism diagnoses showed that, when compared to control group, autism spectrum disorders and mental retardation had a significant increase in serum neurotrophin 4 and BDNF (both are Trk B ligands) [71, 72]. On the other hand, no changes were observed in NGF (trk A ligand) and neurotrophin 3 (Trk C ligand) levels. In light of these findings, it has been suggested that trkB ligands may be overexpressed or secreted in central nervous system of autistic or mental retarded children during infantile period. It has also been suggested that the effect of BDNF and neurotrophin- 4 on activity-dependent dendritic growth and branching [66] may be related to early and transient brain development seen in autistic infants $[67,73]$. This increase in BDNF expression and/or secretion was suggested to be linked with the role of Metil-CpG-binding protein 2 (MeCP2) gene in BDNF transcription [74]. A mutation in MeCP2 gene encoding a protein functioning as a general transcriptional receptor is responsible for Rett Syndrome. It has been shown that MeCP2 selectively bind to BDNF promoter III and suppresses BDNF gene expression. MeCP2 has an important role in regulation of neuronal activity [75]. It has been suggested that $\mathrm{MeCP} 2$ mutations located on Xq28 locus may be a risk factor for autism by affecting BDNF expression and dendritic differentiation in cortex. In a study investigating $\mathrm{MeCP} 2$ gene mutation in autistic individuals for that purpose, 2 girls exhibited de novo mutations $[67,76]$.

Another gene linked with autism is the Fragile $X$ mental retardation 1 (FMR1) gene encoding Fragile $\mathrm{X}$ mental retardation protein (FMRP). FMR1 is associated with autism secondary to 
Fragile $\mathrm{X}$ syndrome [28]. However, fragile $\mathrm{X}$ mutations may be found in 7-8\% idiopathic autism patients [77]. FMRP is a selective RNA-binding protein; it transports RNAs to dendrites and regulates local translation of synaptic mRNAs as a response to activation of metabotropic glutamate receptors. This protein is considered to have a role in synaptic plasticity and development of synaptic connections between neural cells. Impaired mRNA translation in the absence of FMRP leads to an alteration in protein-synthesis-dependent plasticity [28, 78].

Autism risk is higher than general population in neurofibromatosis, tuberosclerosis, or Cowden Syndrome, a rare syndrome which is characterized by multiple tumor-like growths called hamartomas and affects the intellectual abilities. These diseases develop due to dominant mutations in tumor suppressor genes NF1, TSC1/TSC2, and PTEN. Mutations in these autism-associated genes affect synaptic protein level by impairing cellular translation. Alterations in protein level results in abnormal synaptic functions [28].

Angelman syndrome and Prader-Willi syndrome mainly develop due to genetic deletions in 15q11-q13 locus or disomy (condition where two copies of a chromosome comes from a single parent) belonging to a single parent [79]. Deficiencies in paternal genes cause PraderWilli syndrome; Angelman Syndrome which is more commonly associated with autism may be caused by deletion or mutation in maternal ubiquitin protein ligase gene UBE3A or ATP10C [80, 81]. Other rare single gene defects associated with autism are found in Williams Syndrome, Sotos Syndrome, hipomelanozis Ito, and Moebius Syndrome [82-85].

Since serotonin reuptake inhibitors have favourable effects on rituals and routines in autistic individuals and serotonin transporter gene has important role in serotonergic neurotransmission, serotonin transport gene has been investigated as candidate gene in autism. One of the polymorphisms examined in this gene is the one that is formed by long (L) and short (S) alleles owing to different number of insertion/deletion repeats of a 44-base-pair sequence in the transcriptional control region. Cook and his colleagues [86] reported a significant relationship between autism and short allele while Klauck and his colleagues [87] revealed a significant relationship between autism and long allele. A subsequent study did not duplicate these findings [88]. A different polymorphism investigated at the serotonin transport gene is the variable number of tandem repeats (VNTR) polymorphism due to repeat of a 17base-pair region at 2nd intron of the gene 9,10, and 12 times. This polymorphism could not be related to autism [89]. Evidence has been accumulated on the relationship of many serotonin genes, notably serotonin receptor (HTR) 1B, HTR2A, HTR3A, and HTR5A, with autism [90-93].

Glutamate is the main excitatory neurotransmitter associated with cognitive functions such as memory and learning. Autism has been hypothesized as a hypoglutamatergic disorder by virtue of neuroanatomic studies and the similarities glutamate antagonists generate in healthy persons [94]. It has been demonstrated in genome scanning studies that one of the candidate regions for autism is 6 q21 region [95]. This region contains glutamate receptor 6 (GluR6) gene. A study by Jamain and his colleagues [96] found a significant relationship between GluR6 gene and autism. It has been thought that GluR6 dysfunction may contribute the deterioration of communication and learning process in autism and any dysregulation of GluR expression may be related to an increase in the rate of epileptic disorder in autistic 
children [96]. Other glutamatergic receptor genes associated with autism are metabotropic GluR8 and GRIN2A (glutamate receptor, ionotropic, N-methyl-D-aspartate 2A) [97, 98].

Gama aminobutyric acid (GABA) is the major inhibitor neurotransmitter in the brain. $\mathrm{GABA}_{\mathrm{A}}$ receptors are formed by different homologous subunits. Among GABA receptor subunit genes, GABRA4 with 4p12 location has been shown to play a role in etiology of autism and increases autism risk by interaction with GABRB1 [99]. Other genes associated with autism in some other studies are GABRG3, GABRA5, GABRB3 located on 15q11-q13, and GABRA2 located on 4p [100-102]. Contrary to these findings, there are other studies with negative results in terms of the relationship between GABA receptor genes and autism in various ethnic groups [103].

Proenkephalin, prodinorphine of opioid metabolism; tyrosine hydroxylase, dopamin beta hydroxylase (DBH), D2, D3, and D5 dopamin receptors, monoaminooxidase A (MAOA) and $B$ genes of monoaminergic system have no major role in etiology of autism shown in studies [104, 105]. However, a recent study revealed a significant relationship between MAOA gene and autism [106].

Mutations detected in autism in conjunction with all other genetic factors explored so far have been reported to explain no more than $20 \%$ of cases with autism spectrum disorder. Thus, a gene-dosage model has been proposed according to which the susceptibility for autism is determined by the sum of effects of threshold genetic and non-genetic factors [107, 108]. For autism etiology, it has been suggested that the detected chromosomal abnormalities in combination with other undetected loci cause autism. It has been considered that the inconsistencies between the results of the studies aimed to determine the role of genetic factors may be the product of genetic heterogeneity, clinical heterogeneity, and sample size and ethnic differences among different studies [109].

\section{Environmental risk factors}

In addition to effects of a number of genes of small effect, various environmental factors are believed to be responsible for susceptibility to autism. Development of autism seems to be dependent on interaction of susceptibility genes with each other and with the environment [110]. It has been claimed that among environmental factors related to autism are toxins (environment-polluting matters, insecticides, thimerosal in vaccines, lead), viruses (prenatal exposure to influenza, rubella, and cytomegalovirus infections), and premature birth with premature retinopathy [111-115]. Although there has been a debate regarding the relationship of autism with thimerosal in measles, rubella, and mumps vaccines; further careful evaluation of data could not support the relationship between autism and vaccines [116, 117]. The relationship between exposure to $\mathrm{Rh}$ immune globulin, which contained the preservative thimerosal until 2001 in the United States, and autism has also been investigated; however, no significant association has been revealed between exposure of antepartum RhIg preserved with thimerosal and an increase in risk of autistic disorder. The latter findings are in accordance with the consensus that exposure to ethymercury in thimerosal is not the cause of increased prevalence of autism [118]. 
Other factors related to intrauterine environment are maternal hypothyroxinemia [119], maternal influenza [120], and high levels of sex hormone exposure related to infertility treatment [121].Thalidomide and anticonvulsant exposure in pregnancy is correlated to an increase in autism risk [122, 123]. Rasalam and his colleagues [124] showed that $8.9 \%$ of children exposed to sodium valproate in intrauterine life later develop autistic spectrum disorders such as autism or Asperger syndrome. Recently, Hadjikhani [125] have suggested that serotonin reuptake inhibitor use in pregnancy increases autism risk by causing hyperserotoninemia and indirectly affecting amygdala and oxytocin levels.

In many studies, the pre-perinatal complication rates in autistic disorder have been studied and a higher pregnancy-related complication rate has been demonstrated in autistic children $[126,127]$. In a recent meta-analysis [128], the most strong risk factors for autism were advanced maternal and paternal age, maternal gestational hemorrhage, gestational diabetes, maternal prenatal drug use (particularly psychoactive drugs), and birth in a foreign country following immigration of mother. Both advanced maternal and paternal age are associated with autism. The underlying mechanism is unclear. Maternal age may be related to autism due to increased risk of chromosomal abnormalities in ova of increased age or because of unstable trinucleotide repeats [128]. The relationship between paternal age and autism is considered to result from imprinted genes (genes showing different expression patterns depending on the parent it originates), de novo spontaneous mutations that accumulate with advancing age in spermatagonia, or confounder effects of sociocultural environmental factors [129]. Another potential risk factor for autism is maternal birth abroad [130]. It has been suggested that this factor may result from absence of immunization that mother would develop against widespread infectious agents of the country in which she gives birth. Another possible explanation is about the potential role of maternal stress because of immigration [131]. A more detailed investigation on the relationship between mother immigration and autism is needed. It has been demonstrated in some studies that gestational hemorrhage increases autism risk by causing fetal hypoxia [130]. Among other factors considered to cause hypoxia and associated with increased autism risk in some studies are fetal distress, maternal hypertension, prolonged labor, cord complications, low Apgar score, and cesarean section [132]. Gestational diabetes is another risk factor, with unknown biologic mechanism [128].

Some studies demonstrated that prenatal stress increases autism risk [133, 134]. However, due to limitations that these studies are based on retrospective expressions of mothers and these mothers are generally susceptible for experiencing stressful life events outside pregnancy period, these studies need to be supported by further studies. Spontaneous abortions, pre-perinatal complications, congenital anomalies, and neurologic/immunologic abnormalities are among the negative impacts of prenatal stress. Prenatal stress also has various negative effects on brain development such as a delay in myelinization, an increase in sensitivity of amygdala to glucocorticoids, and abnormal development in dopaminergic system [135-137]. Autistic disorder is associated with a functional derangement in brain areas related to social cognitive functions in which amygdala and orbitofrontal cortex plays an important role. Orbitofrontal cortex is susceptible to effects of prenatal stress especially in the middle of gestation. Normal functioning of orbitofrontal cortex - amygdala axis is very im- 
portant for social cognitive function. Therefore, it has been suggested that damage in orbitofrontal region may cause main deficits in autism that underlies inadequate responses to other people's mental status and that impairs self-organization of social-emotional behaviours $[137,138]$. Prenatal stress may impair brain development by many mechanisms including: a) fetal hypoxia due to reducing of uterine and placental circulation, b) impairment of hypothalamus-hypophysis-adrenal axis by stimulation of secretion of maternal stress hormones that can cross placenta, c) generation of pregnancy and birth complications, d) epigenetic effects on expression of stress response-related genes [137].

It has been reported that exposure of environmental stress factors at 21-32 ${ }^{\text {nd }}$ weeks with a prominent peak at $25-28^{\text {th }}$ weeks is associated with an increase in possibility of development of autism [134]. When data regarding progressively worsening developmental process are considered [139], it has been argued that postnatal environmental exposures in genetically susceptible children may be etiologically important [140]. Expression and the impact of many genes is influenced by environmental factors. Thus, the effect of environmental factors in etiology of autism is believed to be indirect by influencing genetic functions [140, 141].

\section{Conclusion}

In line with studies aimed to understand the neurobiology of autism, the presence of alterations in regional brain anatomy and functional neuronal communicative network has been currently proved. The main role among factors underlying abnormal brain development belongs to genetic factors. Evidence regarding that autism is a primarily genetic disorder is progressively increasing. Although environmental factors alone can explain a few cases, they are believed to increase autism risk by interacting with genetic susceptibility. Although data collected so far contribute to the ever-increasing body of knowledge about neurobiology of autism, they do not influence diagnosis and treatment of autism. Use of these data is aimed in future in differentiation of autism from other neurodevelopmental disorders and in diagnostic and therapeutic processes.

\section{Author details}

Esra Guney $^{1^{*}}$ and Elvan Iseri ${ }^{2}$

*Address all correspondence to: dresraguney@gmail.com

1 Ankara Pediatric \& Pediatric Hematology Oncology Training and Research Hospital, Child and Adolescent Psychiatry Department, Ankara, Turkey

2 Gazi University Medical Faculty, Child and Adolescent Psychiatry Department, Ankara, Turkey 


\section{References}

[1] Filipek PA, Accardo P, Baranek GT, Cook EH Jr, Dawson G, Gordon B et al. The screening and diagnosis of autistic spectrum disorders. J Autism Dev Disord 1999; 29:439- 484 .

[2] Krause I, He XS, Gershwin ME, Shoenfeld Y. Brief Report: Immune factors in autism: A critical review. J of Autism and Developmental Disorders 2002;32:337-345.

[3] Ashwood P, Wills S, Water JV. The immune response in autism: a new frontier for autism research. Journal of Leukocyte Biology 2006;80:1-15.

[4] Chauhan A, Chauhan V. Oxidative stres in autism. Pathophysiology 2006;13:171-181.

[5] Boddaert N, Zilbovicius M, Philipe A, Robel L, Bourgeois M, Barthelemy C et al. MRI findings in 77 children with non-sendromic autistic disorder. PLos One 2009;4: e4415.

[6] Gillberg C, Coleman M. Autism and medical disorders: a review of the literature. Dev Med Child Neurol 1996;38:191-202

[7] Miles JH, Takahashi TN, Bagby S, Sahota PK, Vaslow DF, Wang CH et al. Essential versus complex autism: definition of fundamental prognostic subtypes. Am J Med Genet A 2005;135:171-180.

[8] Rutter M, Bailey A, Bolton P, Le Couteur A. Autism and known medical conditions: myth and subtance. J Child Psychol Psychiatry 1994;35:311-322.

[9] Freitag CM. The genetics of autistic disorders and its clinical relevance: a review of the literature. Mol Psychiatry 2007;12:2-22.

[10] Newschaffer CJ, Fallin D, Lee NL. Heritable and non-heritable risk factors for autism spectrum disorders. Epidemiol Rev 2002;24:137-153.

[11] Folstein S, Piven J. Etiology of autism: genetic influences. Pediatrics 1991;87:767-73.

[12] Bailey A, Le Couteur A, Gottesman I, Bolton P, Simonoff E, Yuzda E et al. Autism as a strongly genetic disorder: evidence from a British twin study. Psychol Med 1995;25:63-77.

[13] Happe F, Ronald A, Plomin R. Time to give up on a single explanation for autism. Nat Neurosci 2006;9:1218-1220.

[14] Shao Y, Wolpert CM, Raiford KL, Menold MM, Donnelly SL, Ravan SA et al. Genomic screen and follow-up analysis for autistic disorders. Am J Med Genet 2002;114:99-105.

[15] Fisher S, Vargha-Kadem F, Watkins K, Monaco A, Pembrey M. Localization of a gene implicated in a severe speech and language disorder. Nat Genetic 1998;18:6-170.

[16] Gadia CA, Tuchman R, Rotta NT. Autism and pervasive developmental disorders. Jornal de Pediatria 2004;80(2 suppl): 83-94. 
[17] Folstein S, Rutter M. Infantile autism: a genetic study of 21 twin pairs. J Child Psychol Psychiatry 1977;18:29-321.

[18] Ritvo ER, Freeman BJ, Mason-Brothers A, Mo A, Ritvo M. Concordance for the spectrum of autism in 40 pairs of affected twins. Am J Psychiatry 1985;142:74-77.

[19] Trottier G, Srivastava L, Walker CD. Etiology of infantile autism: a review of recent advances in genetic and neurobiological research. J Psychiatr Neurosci 1999;24:103-115.

[20] Abrahams BS, Geschwind DH. Advances in autism genetics: on the threshold of a new neurobiology. Nat Rev Genet 2008;9:341-355.

[21] Lichtenstein P, Carlström E, Rastam M, Gillberg C, Anckarsater H. The genetics of autism spectrum disorders and related neuropsychiatric disorders in childhood. Am J Psychiatry 2010;167 (11):1357-63.

[22] Losh M, Sullivan PF, Trembath D, Piven J. Current developments in the genetics of autism: from phenome to genome. J Neuropathol Exp Neurol 2008;67:829-837.

[23] Freitag CM. Genetics of autism. J Intellect Disabil Res 2008;52: 817.

[24] Risch N, Spiker D, Lotspeich L, Nouri N, Hinds D, Hallmayer J et al. A genomic screen of autism: evidence for a multilocus etiology. Am J Hum Genet 1999;65:493-507.

[25] Sebat J, Lakshmi B, Malhotra D, Troge J, Lese-Martin C, Walsh T et al. Science 2007;316: 445-449.

[26] Marshall CR, Noor A, Vincent JB, Lionel AC, Feuk L, Skaug J et al. Structural variation of chromosomes in autism spectrum disorder. Am J Hum Genet 2008;82:477-488.

[27] Gutknecht I. Full genome scans with autistic disorders: a review. Behav Genet 2001;31:113-23.

[28] Toro R, Konyukh M, Delorme R, Leblond C, Chaste P, Fauchereau F et al. Key role for gene dosage and synaptic homeostasis in autism spectrum disorders. Trends in genetics 2010;26(8):363-372.

[29] Cusco I, Medrano A, Gener B, Vilardell M, Gallastegui F, Villa O, et al. Autism spesific copy number variants further implicate the phosphatidylinositol signaling pathway and the glutamatergic synapse in the etiology of the disorder. Hum Mol Genetic 2009;18(10): 1795-1804.

[30] Bass MP, Menold MM, Wolpert CM, Donnelly SL, Ravan SA, Hauser ER et al. Genetic studies in autistic disorder and chromosome 15. Neurogenetics 2000;2(4):219-26.

[31] Cook E Jr, Lindgren V, Leventhal B, Courchesne R, Lincoln A, Shulman C et al. Autism or atypical autism in maternally but not paternally derived proximal $15 \mathrm{q}$ duplication. Am J Hum Genet 1997a;60(4): 928-934. 
[32] Schroer RJ, Phelan MC, Michaelis RC, Crawford EC, Skinner SA, Cuccaro M et al. Autism and maternally derived aberrations of chromosome 15q. Am J Med Genet 1998; 76(4):327-36.

[33] Kumar RA, Karamohamed S, Sudi J, Conrad DF, Brune C, Badner JA. Recurrent 16p11.2 microdeletions in autism. Hum Mol Genet 2008;17:628-638.

[34] Amstadter AB. Selected summaries from the XVII world congress of psychiatric genetics, San Diego, California, USA, 4-8 November, 2009. Psychiatric Genetics 2010;20(5):229-268.

[35] Ingram J, Stodgell C, Hyman S, Figlewics D, Weitkamp L, Rodier P. Discovery of allelic variants of HOXA1 and HOXB1: genetic susceptibility to autism spectrum disorders. Teratology 2000;62:393-405.

[36] Li J, Tabor HK, Nguyen L, Gleason C, Lotspeich LJ, Spiker D et al. Lack of association between HoxA1 and HoxB1 gene variants and autism in 110 multiplex families. Am J Med Genet 2002;114: 24-30.

[37] Talebizadeh Z, Bittel DC, Miles JH, Takahashi N, Wang CH, Kibiryeva N et al. No association between HOXA1 and HOXB1 genes and autistic spectrum disorders (ASD). J Med Genet 2002;39:e70.

[38] Kuemerle B, Zamjani H, Joyner A, Herrup K. Pattern deformities and cell loss in Engraled2 mutant mice suggest two separate patterning events during cerebellar development. J Neurosci 1997;17:7881-7889.

[39] Petit E, Herault J, Martineau J, Perrot A, Barthelemy C, Hameury L et al. Association study with two markers of a human homeogene in infantile autism. J Med Genet 1995;32:269-74.

[40] Zhong H, Serajee FJ, Nabi R, Mahbubul Huq AHM. No association between the EN-2 gene and autistic disorder. J Med Genet 2003;40:e4.

[41] Campbell DB, Sutcliffe JS, Ebert PJ, Militerni R, Bravaccio C, Trillo S et al. A genetic variant that disrupts MET transcription is associated with autism. Proc Natl Acad Sci USA 2006;103 (45):16834.

[42] Campbell DB, Li C, Sutcliffe JS, Persico AM, Levitt P. Genetic evidence implicating multiple genes in the MET receptor tyrosine kinase pathway in autism spectrum disorder. Autism Res 2008;1(3):159.

[43] Arking DE, David JC, Brune CW, Teslovich TM, West K, Ikeda M et al. A common genetic variant in the neuroxin superfamily member CNTNAP2 increases familial risk of autism. Am J Hum Genet 2008; 82(1):160-164.

[44] Alarcon M, Abrahams BS, Stone JL, Duvall JA, Perederiy JV, Bomar JM et al. Linkage, association, and gene-expression analyses identify CNTNAP2 as an autism-susceptibility gene. Am J Hum Genet 2008;82(1):150-9. 
[45] Strauss KA, Puffenberger EG, Huentelman MJ, Gottlieb S, Dobrin SE, Parod JM, et al. Recessive symtomatic focal epilepsy and mutant contactin-associated protein-like 2. N Engl J Med 2006;354(13):1370-7.

[46] Gong X, Jia M, Ruan Y, Shuang M, Liu J, Wu S et al. Association between the FOXP2 gene and autistic disorderin Chinese population. Am J Med Genet B 2004;127(1): 113-116.

[47] Newbury DF, Bonora E, Lamb JA, Fisher SE, Lai CS, Baird G et al. FOXP2 is not a major susceptibility gene for autism or spesific language impairment. AmJ Hum Genet 2002;70(5):1318-27.

[48] Wassink TH, Piven J, Vieland VJ, Pietila J, Goedken RJ, Folstein SE et al. Evaluation of FOXP2 as an autism susceptibility gene. Am J Med Genet B 2002;114(5):566-569.

[49] Wodarz A, Nusse R. Mechanisms of Wnt signaling in development. Annu Rev Cell Dev Biol 1998;14:59-88.

[50] Yu X, Malenka RC. Beta-catenin is critical for dendritic morphogenesis. Nat Neurosci 2003; 6:1169-1177.

[51] Wassink TH, Piven J, Vieland VJ, Huang J, Swiderski RE, Pietila J et al. Evidence supporting WNT2 as an autism susceptibility gene. Am J Med Genet 2001;105:406-413

[52] Persico AM, Levitt P, Pimenta AF. Polymorphic GGC repeat differentially regulates human reelin gene expression levels. J Neural Transm 2006;113(10):1373-82.

[53] Costa E, Chen Y, Davis J, Dong E, Noh JS, Tremolizzo L et al. Reelin and schizophrenia: a disease at the interface of the genome and the epigenome. Mol Interv 2002; 2(1): 47-57.

[54] Zhang H, Liu X, Zhang C, Mundo E, Macciardi F, Grayson DR et al. Reelin gene alleles and susceptibility to autism spectrum disorders. Mol Psychiatry 2002; 7(9): 1012-7.

[55] Krebs MO, Betancur C, Leroy S, Bourdel MC, Gillberg C, Leboyer M et al. Absence of association between a polymorphic GGC repeat in the $5^{\prime}$ untranslated region of the reelin gene and autism. Mol Psychiatry 2002;7: 801-4.

[56] Song JY, Ichtchenko K, Sudhof TC, Brose N. Neuroligin is a postsynaptic cell- adhesion molecule of excitatory synapses. Proc Natl Acad Sci USA 1999;96:1100-1105.

[57] Blundell J, Blaiss CA, Etherton MR, Espinosa F, Tabuchi K, Walz C et al. Neuroligin 1 delletion results in impaired spatial memory and increased repetitive behavior. J Neurosci 2010; 30(6): 2115-29.

[58] Jamain S, Quach H, Betancur C, Rastam M, Colineaux C, Gillberg IC et al. Mutations of the $X$ linked genes encoding neuroglina NLGN3 ve NLGN4 are associated with autism. Nat Genet 2003;34: 27-29. 
[59] Laumonnier F, Bonnet-Brilhault F, Gomot M, Blanc R, David A, Moizard MP et al. Xlinked mental retardation autism are associated with a mutation in the NLGN4 gene, a member of the neuroligin family. Am J Hum Genet 2004;74: 552-557.

[60] Chih B, Afridi SK, Clark L, Scheiffele P. Disorder-associated mutations lead to functinal inactivation of neuroligins. Hum Mol Genet 2004;13:1471-1477.

[61] Kelemenova S, Schmidtova E, Ficek A, Celec P, Kubranska A, Ostatnikova D. Polymorphism of candidate genes in Slovak autistic patients. Psychiatr Genet 2010;20(4): 137-9.

[62] Feng J, Schroer R, Yan J, Song W, Yang C, Bockholt A et al. High frequency of neurexin 1 beta signal peptide structural variants in patients with autism. Neurosci Lett 2006;409(1):10-3.

[63] Yan J, Noltner K, Feng J, Li W, Schroer R, Skinner C et al. Neuroxin 1 alpha structural variants associated with autism. Neurosci Lett 2008;438(3): 368-70.

[64] Durand CM, Betancur C, Boeckers TM, Bockmann J, Chaste P, Fauchereau F et al. Mutations in the gene encoding the synaptic scaffolding protein SHANK3 are associated with autism spectrum disorders. Nat Genet 2007;39(1):25-7.

[65] Wang K, Zhang H, Ma D, Bucan M, Glessner JT, Abrahams BS et al. Common genetic variations on 5p14.1 associate with autism spectrum disorders. Nature 2009;459: 528-533.

[66] Shieh PB, Ghosh A. Neurotrophins: new roles for a seasoned cast. Current Biology 1997;7: 627-630.

[67] Polleux F, Lauder JM. Toward a developmental neurobiology of autism. Mental Retardation and Developmental Disabilities Research Review 2004;10:303-317.

[68] Nishimura K, Nakamura K, Anitha A, Yamada K, Tsujii M, Iwayama Y et al. Genetic analyses of the brain-derived neurotrophic factor (BDNF) gene in autism. Biochem Biophys Res Commun 2007;356(1):200-6.

[69] Cheng L, Ge Q, Xiao P, Sun B, Ke X, Bai Y et al. Association study between BDNF gene polymorphism and autism by three-dimensional gel-based microarray. Int J Mol Sci 2009;10(6):2487-2500

[70] Correira CT, Coutinho AM, Sequeira AF, Sousa IG, Lourenço Venda L, Almeida JP et al. Increased BDNF levels and NTRK2 gene association suggest a disruption of BDNF/TrkB signaling in autism. Genes, Brain and Behavior 2010;9(7):841-8.

[71] Nelson KB, Grether JK, Croen LA, Dambrosia JM, Dickens BF, Jelliffe LL et al. Neuropeptides and neurotrophins in neonatal blood of children with autism or mental retardation. Ann Neurol 2001;49: 597-606.

[72] Miyazaki K, Narita N, Sakuta R, Miyahara T, Naruse H, Okado N et al. Serum neurotrophin concentrations in autism and mental retardation: A pilot study. Brain Dev 2004;26:292-295. 
[73] Lainhart JE, Piven J, Wzorek M, Landa R, Santangelo SL, Coon H et al. Macrocephaly in children and adults with autism. J Am Acad Child Adolesc Psychiatry 1997;36:282-290.

[74] Chen WG, Chang Q, Lin Y, Meissner A, West AE, Griffith EC et al. Derepression of $\mathrm{BDNF}$ transcription involves calcium-dependent phosphorylation of MeCP2. Science 2003;302: 885-889.

[75] Shahbazian M, Young J, Yuva-Paylor L, Spencer C, Antalffy B, Noebels J et al. Mice with truncated $\mathrm{MeCP} 2$ recapitulate many Rett syndrome features and display hyperacetylation of histone H3. Neuron 2002;35:243-254.

[76] Carney RM, Wolpert CM, Ravan SA, Shahbazian M, Ashley-Koch A, Cuccaro ML et al. Identification of MeCP2 mutations in a series of females with autistic disorder. Pediatr Neurol 2003;28: 205-11.

[77] Muhle R, Trentacoste SV, Rapin I. The genetics of autism. Pediatrics 2004;113:472-486.

[78] Kelleher RJ, Bear MF. The autistic neuron: troubled translation? Cell 2008; 135:401-406.

[79] Sutcliffe JS, Nurmi EL, Lombroso PJ. Genetics of childhood disorders: XLVII. Autism, part6: duplication and inherited susceptibility of chromosome 15q11-q13 genes in autism. J Am Acad Child Adolesc Psychiatry 2003;42:253-256.

[80] Akefeldt A, Gillberg C, Larsson C. Prader-Will syndrome in a Swedish rural country: epidemiological aspects. Dev Med Child Neurol 1991;33:715-721.

[81] Fang P, Lev-Lehman E, Tsai TF, Matsuura T, Benton CS, Sutcliffe JS et al. The spectrum of mutations in UBE3A causing Angelman Syndrome. Hum Mol Genet 1999;8:129- 135.

[82] Morrow JD, Whitman BY, Accardo PJ. Autistic disorder in Sotos Syndrome: a case report. Eur J Pediatr 1990;149:567-569.

[83] Reiss AL, Feinstein C, Rosenbaum KN, Borengasser Caruso MA, Gold-smith BM. Autism associated with Williams Syndrome. J Pediatr 1985;106: 247-249.

[84] Zappella M. Autism and hypomelanosis of Ito in twins. Dev Med Child Neurol 1993;35: 826-832.

[85] Stromland K, Sjögreen L, Miller M, Gillberg C, Wentz E, Johansson M et al. Mobius sequence - a Swedish multidiscipline study. Eur J Paediatr Neurol 2002;6:35-45.

[86] Cook EH Jr, Courchesne R, Lord C, Cox NJ, Yan S, Lincoln A et al. Evidence of linkage between the serotonin transporter and autistic disorder. Mol Psychiatry 1997b; 2(3): 247-250.

[87] Klauck SM, Poustka F, Benner A, Lescch KP, Poustka A. Serotonin transporter gene (5-HTT) variants associated with autism? Hum Mol Genet 1997;6(13): 2233-2238. 
[88] Persico AM, Militerni R, Bravaccio C, Schneider C, Melmed R, Conciatori M et al. Lack of association between serotonin transporter gene promoter variants and autistic disorder in two ethnically distinct samples. Am J Med Genet 2000;96(1):123-127.

[89] Betancur C, Corbex M, Spielewoy C, Philippe A, Laplanche JL, Launay JM et al. Serotonin transporter gene polymorphism and hyperserotoninemia in autistic disorder. Mol Psychiatry 2002;7(1): 67-71.

[90] Orabona GM, Griesi-Oliveira K, Vadasz E, Bulcão VL, Takahashi VN, Moreira ES et al. HTR1B and HTR2C in autism spectrum disorders in Brazilian families. Brain Res 2009;1250:14-9.

[91] Cho IH, Yoo HJ, Park M, Lee YS, Kim SA. Family-based association study of 5HTTLPR and the 5-HT2A receptor gene polymorphisms with autism spectrum disorder in Korean trios. Brain Res 2007;1139: 34-41.

[92] Anderson BM, Schnetz-Boutaud NC, Bartlett J, Wotawa AM, Wright HH, Abramson RK et al. Examination of association of genes in the serotonin system to autism. Neurogene 2009;10(3): 209-16.

[93] Coutinho AM, Sousa I, Martins M, Correia C, Morgadinho T, Bento C et al. Evidence for epistasis between SLC6A4 and ITGB3 in autism etiology and in the determination of platelet serotonin levels. Hum Genet 2007;121:243-56.

[94] Carlsson ML. Hypothesis: is infantile autism a hypoglutamatergic disorder? Relevance of glutamate -serotonin interactions for pharmacotherapy. J Neural Transm 1998;105:525-535.

[95] Philippe A, Martinez M, Guilloud- Bataille M, Gillberg C, Rastam M, Sponheim E et al. Genome wide scan for autism susceptibility genes. Paris Autism Research International Sibpair Study. Hum Mol Genet 1999;8: 805-812

[96] Jamain S, Betancur C, Quach H, Philippe A, Fellous M, Giros B et al. Linkage and association of the glutamate receptor 6 gene with autism. Mol Psychiatry 2002;7(3): 302-310.

[97] Serajee FJ, Zhong H, Nabi R, Huq AH. The metabotropic glutamate receptor 8 gene at 7q31: partial duplication and possible association with autism. J Med Genet 2003;40:e42.

[98] Barnby G, Abbott A, Sykes N, Morris A, Weeks DE, Mott R et al. Candidate-gene screening and association analysis at the autism susceptibility locus on chromosome 16p:evidence of association at GRIN2A and ABAT. Am J Hum Genet 2005;76(6): 950-966.

[99] Ma DQ, Whitehead PL, Menold MM, Martin ER, Ashley-Koch AE, Mei H et al. Identification of significant association and gene-gene interaction of GABA receptor subunit genes in autim. Am J Hum Genet 2005;77(3): 377-388. 
[100] Menold MM, Shao Y, Wolpert CM, Donnelly SL, Raiford KL, Martin ER et al. Association analysis of chromosome 15 GABAA receptor subunit genes in autistic disorder. J Neurogenetics 2001;15 (3-4): 245-259.

[101] Cook EH, Courchesne RY, Cox NJ, Lord C, Gonen D. Linkage disequilibrium mapping of autistic disorder, with 15q11-13 markers. Am J Hum Genet 1998;62(5): 1077-1083.

[102] Kakinuma H, Ozaki M, Sato H, Takahashi H. Variation in GABA-A subunit gene copy number in an autistic patient with mosaic 4p duplication (p12p16). Am J Med Genet B 2008;147(6): 973-975.

[103] Tochigi M, Kato C, Koishi S, Kawakubo Y, Yamamoto K, Matsumoto H et al. No evidence for significant association between GABA receptor genes in chromosome 15q11-13 and autism in a Japannese population. J Hum Genet 2007;52(12): 985-989.

[104] Martineau J, Herault J, Petit E, Guerin P, Hameury L, Perrot A et al. Catecholaminergic metabolism and autism. Dev Med Child Neurol 1994;36(8): 688-97.

[105] Philippe A, Guilloud- Bataille M, Martinez M, Gillberg C, Rastam M, Sponheim E et al. Analyses of ten candidate genes in autism by association and linkage. Am J Med Genet 2002;114(2): 125-128.

[106] Yoo HJ, Lee SK, Park M, Cho IH, Hyun SH, Lee JC et al. Family-and populationbased association studies of monoamine oxidase A and autism spectrum disorders in Korean. Neurosci Res 2009;63:172-6.

[107] Cook E, Scherer SW. Copy number variations associated with neuropsychiatric conditions. Nature 2008;455:919-923.

[108] Constantino JN, Todd RD. Intergenerational transmission subthreshold autistic traits in the general population. Biol Psychiatry 2005;57:655-660.

[109] Shastry BS. Molecular genetics of autism spectrum disorders. J Hum Genet 2003;48: 495-501.

[110] Newschaffer CJ, Croen LA, Daniels J, Giarelli E, Grether JK, Levy SE et al. The epidemiology of autism spectrum disorders. Annual Review of Public Health 2007;28: 235-258.

[111] Chess S. Follow up report on autism in congenital rubella. J Autism Child Schzophr 1997;7:69-81

[112] Iversson SA, Bjerre I, Vegfors P, Ahlfors K. Autism as one of several disabilities in two children with congenital cytomegalovirus infection. Neuropediatrics 1990;21:102-103.

[113] Kern JK, Geier DA, Audhya T, King PG, Sykes LK, Geier MR. Evidence of parallels between mercury intoxication and the brain pathology in autism. Acta Neurobiol Exp 2012;72(2):113-53. 
[114] Tsuchiya KJ, Hashimoto K, Iwata Y, Tsujii M, Sekine Y, Sugihara G et al. Decreased serum levels of platelet-endothelial adhesion molecule (pecam-1) in subjects with high-functioning autism: A negative correlation with head circumference at birth. Biol Psychiatry 2007;62(9):1056-58.

[115] Chase JB. Retrolental fibroplasia and autistic symptomatology. American foundation for the blind. Newyork, NY; 1972.

[116] Taylor B, Miller E, Farrington C, Petropoulos M, Favot-Mayaud I, Li J et al. Autism and measles, mumps, and rubella vaccine: no epidemiological evidence for a causal association. The Lancet 1999;353:2026-2029.

[117] Dales L, Hammer SJ, Smith NJ. Time trends in autism and in MMR immunization coverage in California. JAMA 2001;285:1183-1185.

[118] Miles JH, Takahashi TN. Lack of association between Rh status, Rh immune blogulin in pregnancy and autism. American Journal of Medical Genetics 2007;143A:12971407.

[119] Roman GC. Autism: Transient in utero hypothyroxinemia related to maternal flavonoid ingestion during pregnancy and to other environmental antithyroid agents. J Neurol Sci 2007; 262(1-2):15-26.

[120] Smith SE, Li J, Garbett K, Mirnics K, Patternson PH. Maternal immune activation alters fetal brain development through interleukin-6. Journal of Neuroscience 2007;27: 10695-10702.

[121] Croughan M, Schembri M, Bernstein N, Chamberlain N, Purcell N, Camarano L. Maternal and childhool outcomes following infertility and infertility treatments. Paper presented at the American Society for Reproductive Medicine Annual Scientific Meeting, New Orleans, October 21-25, 2006.

[122] Zwaigenbaum L, Szatmari P, Jones MB, Bryson SE, Maclean JE, Mahoney WJ et al. Pregnancy and birth complications in autism and liability to the broader autism phenotype. J Am Acad Child Adolesc Psychiatry 2002;41:572-579.

[123] DeLong GR. Autism: new data suggest a new hypothesis. Neurol 1999;52: 911-916.

[124] Rasalam AD, Hailey H, Williams JH, Moore SJ, Turnpenny PD, Lloyd DJ et al. Characteristics of fetal anticonvulsant syndrome associated autistic disorder. Dev Med Child Neurol 2005;47: 551-555.

[125] Hadjikhani N. Serotonin, pregnancy and increased autism prevalence: Is there a link. Med Hypotheses 2010;74(5): 880-3.

[126] Glasson EJ, Bower C, Petterson B, de Klerk N, Chaney G, Hallmayer JF. Perinatal factors and the development of autism: a population study. Arch Gen Psychiatry 2004;61(6):618-27. 
[127] Larsson HJ, Eaton WW, Madsen KM, Vestergaard M, Olesen AV, Agerbo E et al. Risk factors for autism: perinatal factors, parental psychiatric history, and socioeconomic status. Am J Epidemiol 2005;161(10): 926-8.

[128] Gardener H, Spiegelman D, Buka SL. Prenatal risk factors for autism: comprehensive meta-analysis. The British Journak of Psychiatry 2009;195:7-14.

[129] Reichenberg A, Bresnahan M, Rabinowitz J, Lubin G, Davidson M. Advancing paternal age and autism. Arch Gen Psychiatry 2006;63:1026-32.

[130] Kolevson A, Gross R, Reichenberg A. Prenatal and perinatal risk factors for autism: a review and integration of finding. Arch Pediatr Adolesc Med 2007;161:326-33.

[131] Gillberg C, Schaumann H, Gillberg IC. Autism in immigrants: children born to in Sweden to mothers born in Uganda. J Intellect Disabil Res 1995;39:141-4.

[132] Previc FH. Prenatal influences on brain dopamine and their relevance to the rising incidence of autism. Med Hypotheses 2007;68: 46-60.

[133] Ward AJ. A comparison and analysis of the presence of family problems during pregnancy of mothers of "autistic" children and mothers of normal children. Child Psychiatr Hum Dev 1990;20(4): 279-88.

[134] Beversdorf DQ, Manning SE, Hillier A, Anderson SL, Nordgren RE, Walters SE et al. Timing of prenatal stressors and autism. J Aut Dev Disord 2005;35(4):471-478.

[135] Glover V. Maternal stress or anxiety in pregnancy and emotional development of the child. The British Journal of Psychiatry: the Journal of Mental Science 1997;171:105-106.

[136] Mulder EJ, Robles de Medina PG, Huizink AC, Van den Bergh BR, Buitelaar JK, Visser GH. Prenatal maternal stress: Effects on pregnancy and the (unborn) child. Early Human Development 2002;70 (1-2):3-14

[137] Kinney DK, Munir KM, Crowley DJ, Miller AM. Prenatal stres and risk for autism. Neurosci Biobehav Rev 2008;32(8): 1519-32.

[138] Bachevalier J, Loveland KA. The orbitofrontal-amygdala circuit and self-regulation of social-emotional behavior in autism. Neuroscience and Biobehavioral Reviews 2006;30(1): 97-117.

[139] Dawson G, Munson J, Webb SJ, Nalty T, Abbott R, Toth K. Rate of head growth decelerates and symptoms worsen in the second year of life in autism. Biological Psychiatry 2007;61:458-464.

[140] Dawson G. Early behavioral intervention, brain plasticity, and the prevention of autism spectrum disorder. Development and Psychopathology 2008;20:775-803.

[141] London E, Etzel RA. The environment as an etiologic factor in autism: a new direction for research. Environmental Health Perspectives 2000;108 (supll 3): 401-404. 



\title{
Discovering the Genetics of Autism
}

\author{
Abdullah K. Alqallaf, Fuad M. Alkoot and \\ Mash'el S. Aldabbous \\ Additional information is available at the end of the chapter \\ http://dx.doi.org/10.5772/53797
}

\section{Introduction}

Autism is a complex neurodevelopmental disorder. It is characterized by social isolation, language deficits and repetitive or stereotyped behaviors. Autism spectrum disorder (ASD) has received a great deal of attention in the recent years not only due to the increasing rate of affected children but also because of the social and economical impact of the disorder on their families. Various studies and researches have been proposed to deal with and tackle the ASD. They can be divided into three categories as follows.

1. The basis and causes of the disorder. Different hypotheses have been proposed in an attempt to determine and discover the originality of autism. Genetic risk factors represented by abnormal chromosomal variations and rearrangements, and non-genetic factors represented by environmental agents that have been claimed to contribute to ASD, such as exposure of children to vaccines, infection, certain foods or heavy metals.

2. The methodologies and techniques for characterizing and diagnosing the disorder. Several instrumental diagnostic protocols are commonly used in autism research such as the Autism Diagnostic Interview-Revised (ADI-R) and the Autism Diagnostic Observation Schedule (ADOS). The advances in neuro-imaging techniques such as the functional-Magnetic Resonance Imaging (f-MRI) have allowed scientists to model the structural and functional differences in the human brain tissues of the individuals with ASD. The clinical genetics evaluation provide reliable alternative to the interview-based protocols and screening approaches. It allows geneticists to link an estimate of approximately $40 \%$ of the cases to genetic contributors.

3. The treatments and therapies of autistic patients. The available approaches for treatments include applied behavior analysis (ABA), developmental models, structured 
teaching, speech and language therapy and social skills therapy. When behavioral treatment fails, many medications are used to treat ASD symptoms.

Figure 1 demonstrates the interaction of the autism spectrum disorders researches and studies.

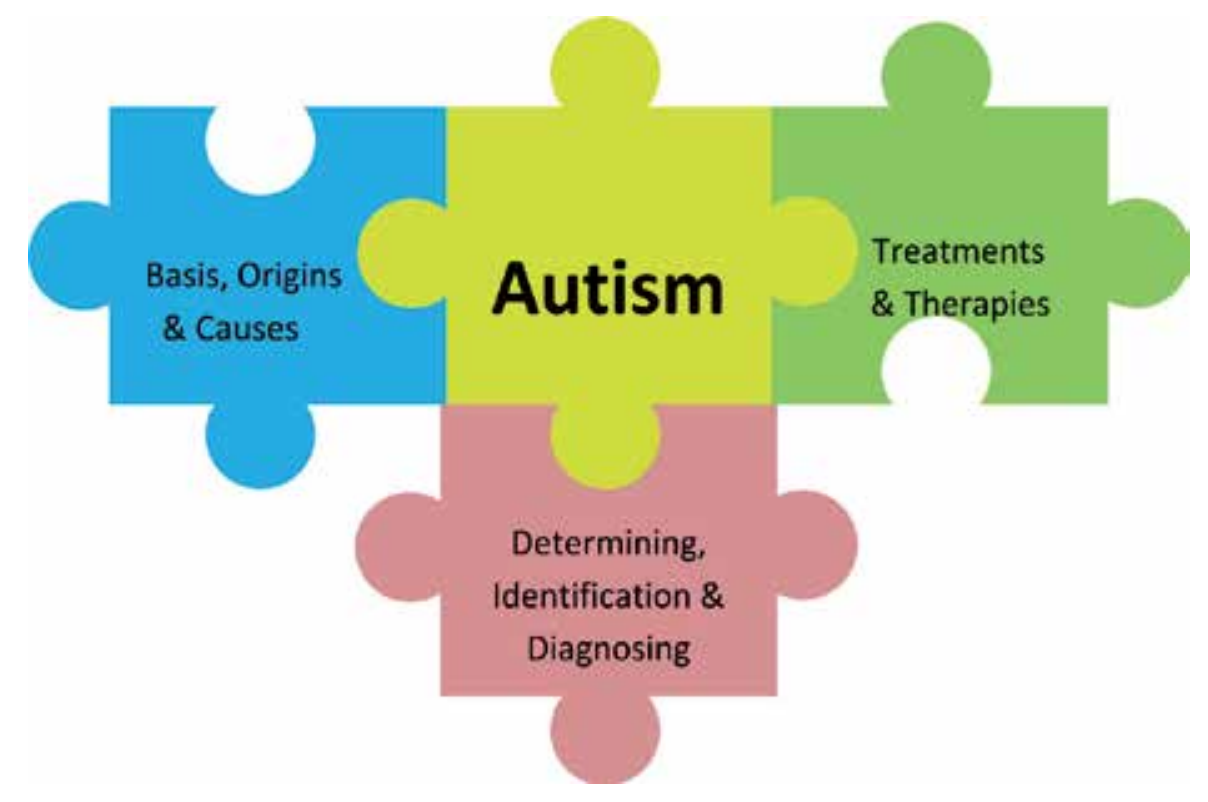

Figure 1. A puzzle-like representation of the interaction process of the researches and studies for autism spectrum disorders.

The advancements of the technologies in the field of genetics provide the opportunities for researchers and scientists to explore in depth the biological information and to convert it into meaningful biological knowledge through computational-based models.

In this chapter, we will investigate the genetics origins of autism and demonstrate the latest techniques and technologies available for diagnosing the complex disorder. We will also propose a robust approach for detecting and identifying the targeted disorder based upon the advantages and strengths of the publically available and commercial approaches while avoiding their weaknesses. The proposed approach is divided into two steps. The preprocessing step is a feature-extraction method used to clearly map and detect the genetic variations and structural rearrangements followed by a statistical-based model as feature-selection to evaluate and measure the statistical and biological significance of the predicted variations. The classification step is to discover the relationship among the tested samples into groups and/or subgroups, and to provide insight into the complex pattern of the genome.

The results suggest that autism is associated with an increased amount of alterations in unstable segments of the genome. The experimental results also show that using high-resolu- 
tion custom-tiled samples improve the accuracy of our proposed approach in determining previously reported and new genetic contributors that warrant investigation.

This chapter aims at utilizing research to bring benefits to individuals and families affected by autism spectrum disorders and to improve the quality of their life. And this can be done by clear mapping and identifying the biomarkers associated with ASD at the early childhood stages which are essential to provide better treatments and therapies. Finally, the proposed approach presented in this chapter is broadly applicable to case-control studies of genetic diseases beyond the ASD.

The chapter is organized as follows. In section 2, we demonstrate the genetic data generating techniques, data modeling and chromosomal variations that are associated with the targeted disorder, ASD. Section 3 is devoted for the methods used to analyze the genetic data trying to discover the variant regions along the genome and to identify the tested samples. In section 4, we apply molecular test to evaluate the predictive power of the proposed approach. Finally, discussion and conclusion based on the results are presented in section 5 .

\section{Genetic data}

\subsection{Genomic structural variations and ASD susceptibility}

Genetic alterations in the form of chromosomal rearrangements are genomic structural variations that lead to changes in the DNA copy number such as duplications and deletions of the DNA copies. However, copy number changes do not include other genomic structural variations such as inversions, insertions and reciprocal translocations. Figure 2 demonstrates different types of chromosomal rearrangements.

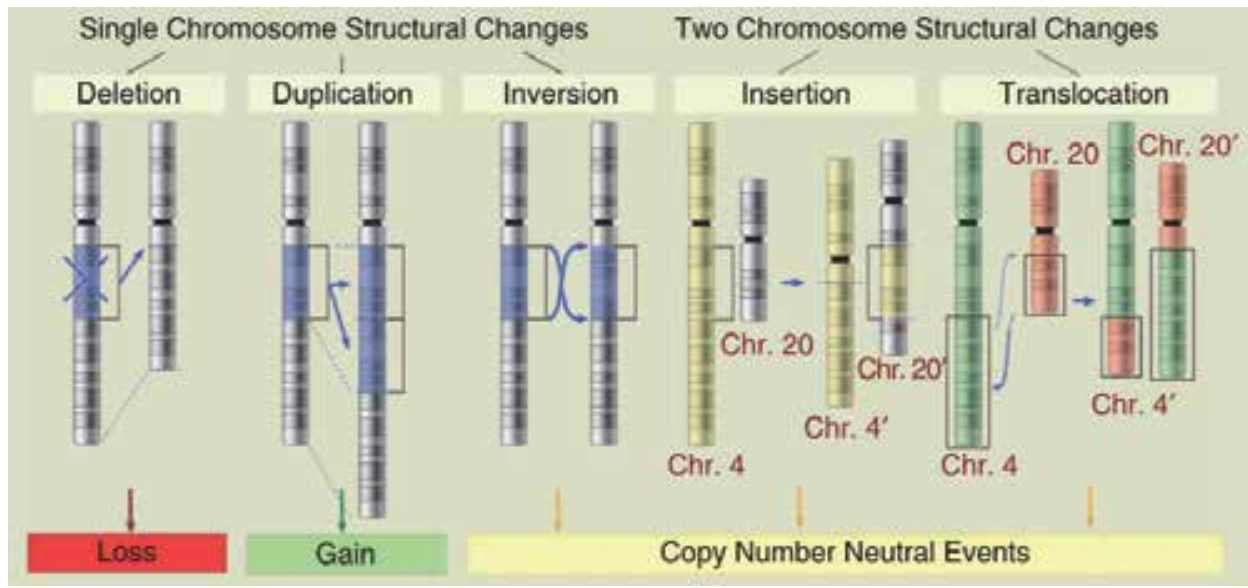

Figure 2. a schematic representation of types of chromosomal rearrangements [67]. 


\begin{tabular}{|c|c|c|c|}
\hline & Chromosome region & Gene & Phenotype \\
\hline \multirow{14}{*}{ 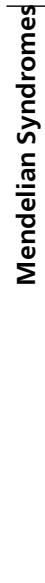 } & $6 q 23.3$ & $\mathrm{AHI1}$ & Joubert syndrome \\
\hline & $7 q 35-q 36.1$ & CNTNAP2 & Recessive EPI syndrome, ASD, ADHD, TS, OCD \\
\hline & $9 q 34.13$ & TSC1 & Tuberous Sclerosis type I \\
\hline & $10 q 23.31$ & PTEN & Cowden disease $^{*}$ \\
\hline & $11 q 13.4$ & DHCR7 & Smith-Lemli-Opitz syndrome \\
\hline & $12 \mathrm{p} 13.33$ & CACNA1C & Timothy syndrome \\
\hline & $15 q 11.2$ & UBE3A & Angelman syndrome \\
\hline & $16 p 13.3$ & TSC2 & Tuberous Sclerosis type II \\
\hline & $17 q 11.2$ & NF1 & Neurofibromatosis \\
\hline & Xp21.2 & DMD & Duchenne muscular dystrophy \\
\hline & Xp21.3 & ARX & LIS, XLID, EPI, ASD \\
\hline & Xp22.13 & CDKL5 & X-linked infantile spasm syndrome \\
\hline & $\mathrm{Xq27.3}$ & FMR1 & Fragile $\mathrm{X}$ syndrome \\
\hline & $\mathrm{Xq28}$ & MECP2 & Rett syndrome \\
\hline \multirow{17}{*}{ 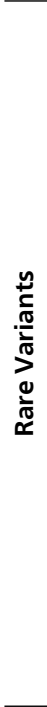 } & $1 q 21.1$ & NBPF9 & ASD, ID, SCZ, ADHD, EPI \\
\hline & $2 p 16.3$ & NRXN1 & ASD, ID, language delay, SCZ. \\
\hline & $3 p 13$ & FOXP1 & ID, ASD, SLI \\
\hline & $6 q 16.3$ & GRIK2 & Recessive ID \\
\hline & $7 q 11.23$ & FKBP6/CLIP2 & ASD, ID, language delay \\
\hline & $\overline{7 q 31.1}$ & FOXP2 & SLI \\
\hline & $11 q 13.3-q 13.4$ & SHANK2 & ASD, ID \\
\hline & $\overline{15 q 11-q 13}$ & MAGEL2/ NDN & ASD, EPI, ID \\
\hline & $16 p 11.2$ & VPS35/ORC6 & ASD, ADHD, ID, EPI, SCZ \\
\hline & $16 p 13.3$ & A2BP1 & ID, ASD, EPI, SCZ, ADHD \\
\hline & $17 q 11.2$ & SLC6A4 & ASD, OCD \\
\hline & $\overline{17 q 12}$ & ACCN1/PNMT & ASD, SCZ, EPI \\
\hline & $22 q 11.21$ & & DiGeorge syndrome, SCZ, ASD, ID.BPAD \\
\hline & $22 q 13.33$ & SHANK3 & ASD, Phelan McDermid syndrome ${ }^{* \star}$ \\
\hline & Xq13.1 & NLGN3 & ASD \\
\hline & Xp22.11 & PTCHD1 & $A S D, I D$ \\
\hline & Xp22.32-p22.31 & NLGN4X & ASD, ID, TS, ADHD \\
\hline \multirow{8}{*}{ 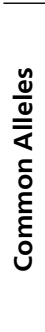 } & $1 \mathrm{q} 42.2$ & DISC1 & SCZ,BPAD \\
\hline & $2 q 31.1$ & SLC25A12 & ASD \\
\hline & $3 p 25.3$ & OXTR & ASD \\
\hline & $7 q 31.2$ & MET & ASD, Diabetes II \\
\hline & $7 q 22.1$ & RELN & ASD \\
\hline & $7 q 36.3$ & EN2 & ASD \\
\hline & $12 q 14.2$ & AVPR1A & ASD \\
\hline & $17 q 21.32$ & ITGB3 & ASD \\
\hline
\end{tabular}

Table 1. Chromosomal regions and genes that are implicated in risk for ASD, and associated genetic disorders and syndroms [68\& 69].Abbreviations: LTD, long-term depression; LTP, long-term potentiation; PPI, prepulse inhibition; E/I, excitatory/inhibitory; PSD, postsynaptic density; ASD, autism spectrum disorders; SCZ, schizophrenia; ADHD, attention deficit hyperactivity disorder; ID, intellectual disability; XLID, X-linked intellectual disability; LIS, lissencephaly; EPI, epilepsy; OCD, obsessive compulsive disorder; TS, Tourette syndrome; SLI, speech and language impairment; USV, ultrasonic vocalization; TF, transcription factor; ECM, extracellular matrix; GPCR, G-protein-coupled receptor;BPAD, Bipolar affective disorder. *A rare autosomal dominant inherited disorder characterized by multiple tumor-like growths, increased risk of certain forms of cancer, and diverse clinical features including neurologic features such as 
autism and Lhermitte Duclos disease [39\& 40].** A genetic syndrome caused by disruption of the SHANK3 gene which codes for the shank3 protein. The protein most important role is in the brain. It is involved in processes crucial for learning and memory. It also has an important role in brain development. It is also known as 22q13.3 deletion syndrome and is highly associated with autism. Human (Homo sapiens) Genome Browser Gateway, http:// genome.ucsc.edu/cgi-bin/hgGateway.

A set of chromosomal regions and genes that are implicated with ASD are listed in Table 1. Some of the regions are associated with known Mendelian syndromes. In some individuals affected with these syndromes, ASD occurs as a secondary diagnosis. In other regions and genes, genetic variations causing ASD include a wide range of possibilities each with very low frequency among the cases (rare variants). In some cases the rare variants are found only once in the population. In contrast to rare variants we see that in other chromosomal regions and genes only few common genetic variations (common alleles) account for ASD susceptibility.

\subsection{Data Generating}

Figure 3 illustrates the process of generating DNA copy number data using Microarraybased comparative genomic hybridization (array CGH) technology.

(a)

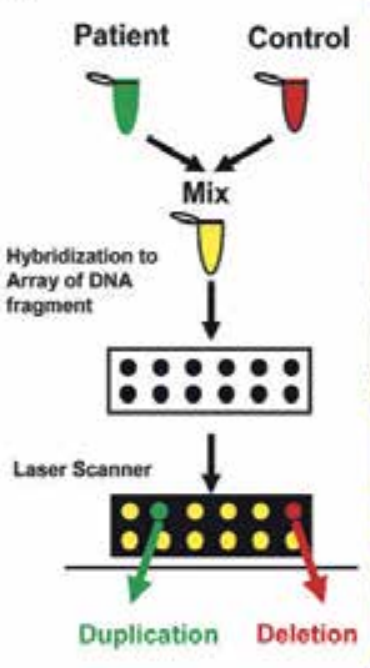

(b) Laser Scanner

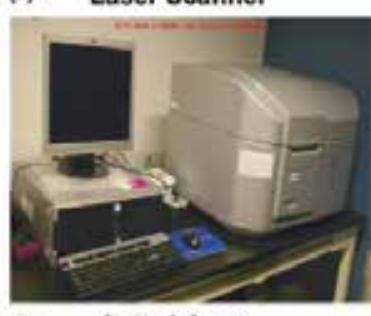

(c)

Actual Array

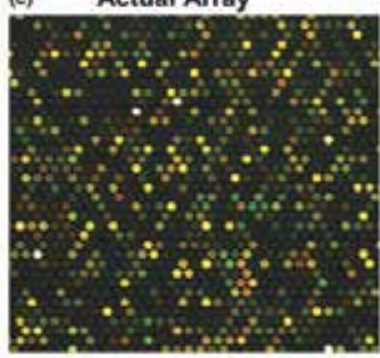

(d) array profile

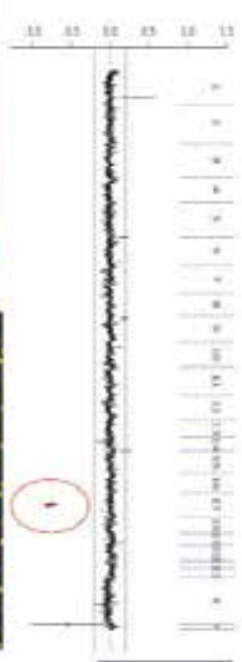

Figure 3. Principles of the aCGH technology. (a) DNA from the sample to be tested and reference DNA are labeled with a green fluorescence dye (Cy3) and red (Cy5), respectively, and competitively co-hybridized to an array containing genomic DNA targets that have been spotted on a glass slide. The resulting ratio of the fluorescence intensities is proportional to the ratio of the copy numbers of DNA sequences in the test and reference genomes measured in a logarithmic scale. (b) The slides are scanned using a specific microarray scanner shown in (c). (d) The output of the scanning process is the ratio of the fluorescence intensities for each spot represented as a point in the relative copy number profile [66]. 


\subsection{Data Modeling}

As illustrated in Figure 3, aCGH technology is an experimental approach for genome-wide scanning of differences in DCN samples. It provides a high-resolution method to map and measure relative changes in DCN simultaneously at thousands of genomic loci. In a biological experiment, unknown (test) and reference (normal) DNA samples are labeled with fluorescent dyes Cy3 and Cy5, respectively. Then, they are combined and competitively cohybridized to an array containing genomic DNA targets that have been spotted on a glass slide. The resulting ratio of the fluorescence intensities is proportional to the ratio of the copy numbers of DNA sequences in the test and reference genomes measured in a logarithmic scale for a certain genomic location. These intensity ratios are informative about DNA copy number changes. We expect to see duplication (gain) for positive ratio, deletion (loss) for negative ratio and normal state for neutral ratio. Due to the logarithmic scale and the probes performance, the data can be approximated as a piecewise function of short and long intervals with different intensity levels that are not equally-spaced along the genome. Moreover, microarray experiments suffer from many sources of error due to human factors, array printer performance, labeling, and hybridization efficiency.

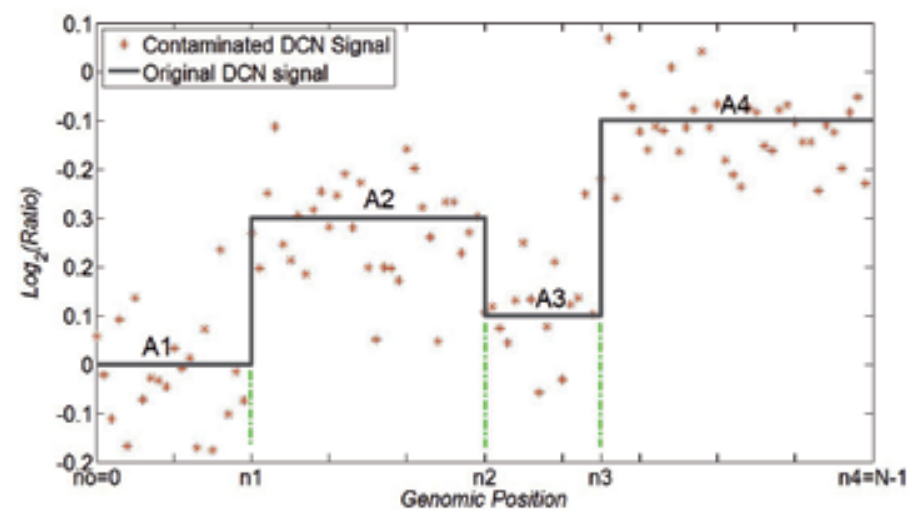

Figure 4. Graphical representation of the generated data using aCGH technology. The red stars represent the raw data as described in (1). The grey solid line represents the true value of 4 variant segments that need to be estimated with intensity levels $A_{i}$ measured in $\log _{2}$ (ratio) and bounded by the breakpoints $n_{i-1}$ and $n_{i}$, respectively.

According to the data description and properties generated by microarray technology, the DCN cell line can be approximated as a one-dimensional piecewise constant (PWC) discretetime signal contaminated with some error. A good model of the genetic data generated by the aCGH technology can be model as follows.

$$
y[n]=x[n]+\varepsilon_{n}, \quad n=1,2, \ldots, N .
$$


where $y[n]$ is the contaminated genetic signal and $x[n]$ is the true value of the genetic variation to be estimated at genomic location $n$ of the length $N . \varepsilon_{n}$ is assumed to be modeled as additive wihte Gaussian noise with zero mean and some variance $\sigma^{2}$.

As described in (1), Figure 4 illustrates the genetic data in the form of DNA copy number generated by aCGH technology where 4 variant segements are presented with different intensity levels.

\section{Methods}

\subsection{Data Filtering}

Although the recent advantecment in microarray technologies and sequencing now make it easy to measure the genetic variations with high-resolution through scanning large number of samples, small changes, particularly at the low copy repeat (LCRs) regions, remain difficult to detect due to different noise conditions. Thus, the challenging problem is to differentiate between the true biological signaling and the noise measurements.

Various methods have been proposed as preprocessing techniques to tackle this problem. These methods have been motivated by either well-known signal processing techniques or statistical-based models.

\begin{tabular}{lc}
\hline \multicolumn{1}{c}{ METHOD } & \multicolumn{2}{c}{ COMPUTATIONAL } \\
SMOOTHING TECHNIQUES & COMPLEXITY \\
\hline SIGMA FILTERING (Alqallaf et al., 2007) & $O(N)$ \\
\hline SMOOTHING AND EDGE DETECTION (Huang et al., 2004) & $O(N)$ \\
\hline WAVELETS (Hsu et al., 2005) & $O(N \log N)$ \\
\hline STATISTICAL-BASED MODELS & $O\left(N^{2}\right)$ \\
\hline CIRCULAR BINARY SEGMENTATION (Olshen et al., 2004) & $O\left(C^{2} N\right)$ \\
\hline HIDDEN MARKOV MODELS (Fridlyand et al., 2004) & $O(N \log N)$ \\
\hline SPARSE BAYESIAN LEARNING (Pique-Regi et al., 2008) & \\
\hline
\end{tabular}

Table 2. Comparison based on the computational complexity of the proposed denoising techniques.

In Table 2, we present a comparison study based on the computational cost of the most recent and successful approaches. As can be noticed that the smoothing techniques are well suited to process very large amount of data such as the genetic signals compared to the statistical-based models. However, these techniques include important features such as the variant regions boundaries in the smoothing process.

Here we present our previously proposed method (Alqallaf et al., 2007), Sigma filter (SF). It is a nonlinear method used as a feature extraction to detect the variant segments edges and 
to smooth the rest of the genetic data. The filter is conceptually simple but effective noise smoothing algorithm. Based on the assumption of the aCGH data modeling, the SF algorithm is well suited to denoise the tested samples before further analysis. SF algorithm is motivated by the sigma probability of the Gaussian distribution, and it smooths the noise by averaging only those neighborhood variant segments which have the intensities within a fixed sigma range of the center data point. Consequently, variant segmets edges are preserved, and subtle details are retained.

\subsection{Statistical significance}

Few studies in the literature have addressed the power of class discovery of the recurrent copy number variations (CNVs) across multiple samples of the genetic data [52\& 53]. However, they did not consider denoising the data prior to applying the statistical analysis.

To reduce the dimensionality of the detected variant regions, we apply a simple statisticalbased approach to measure the significance of the candidate gemonic regions. The approach is based on the frequency difference between the case and control samples at each gemonic location. It is used as a feature selection algorithm to select a small subset of variant segments as features for classification. Figure 5 is an illustration of three RCVNs with different sizes of filtered DCN data for multiple samples of normal control $\left(C_{i}\right)$ and autistic $\left(A_{i}\right)$ individuals, respectively. After selecting the informative segments of the genome, we then applied comparative classification algorithms on the reduced data.

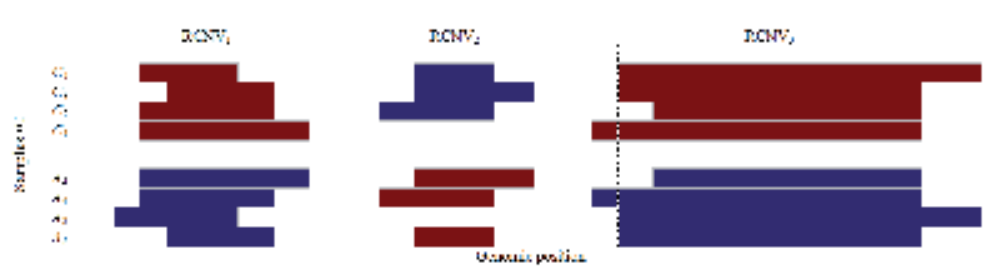

Figure 5. Schematic representation of 3 recurrent copy number variant segments (RCNVs) with different lengths. The $x$-axis represents the genomic position and the $y$-axis represents the indices of the samples, $C_{i}$ is for normal-control samples and $A_{i}$ is for autistic samples, respectively. The vertical dashed lines represent the RCNVs boundaries. The dark red and dark blue bars represent duplication and deletion for the corresponding chromosomal regions.

\subsection{Data classification}

Based on the collected and processed genetic data, we apply a system of classifiers that are used to identify autistic individuals based on their genetic information. This system will help improve detection, identification and diagnosis of autism, which will benefit both the patients and the society in general and will lead to early diagnosis and treatment.

Generally classifiers are used by researchers faced with the task of classification based on a given data. Classifiers are mathematical models that are able to perform the task of classifi- 
cation or decision making, based on a previously provided data. Classifier's ability to spot trends and relationships in large data sets makes it well suited for many applications. In the field of medicine classifiers can be used to classify accurately diseases, genes, tumors, and other medical phenomena $[54 ; 55 ; 56 ; 57 ; 58 ; 59 \& 60]$. Although some attempts were made to use classifiers in genetics [61]. Our attempt is to use three comparative classifiers, namely, $k$ Nearest Neighbor, Neural Network, and Support Vector Machine, to help in diagnosing patients with ASD.

The leave-one-out cross-validation (LOOCV) is applied to evaluate the proposed classifiers by measuring the classification performance to accurately identify the association between the tested samples and the targeted disorder, ASD. The LOOCV involves using a single variant segment from the original sample as the validation data, and the remaining segments as the training data. This is repeated such that each variant segment in the sample is used once as the validation data.

\subsection{1. $k$-Nearest Neighbor Classifier}

The $k$-Nearest Neighbor $(k-\mathrm{NN})$ classifier [64] is a well known nonparametric classifier. To classify a new input $x$, the $k$ nearest neighbors are retrieved from the training data. The input $x$ is then labeled with the majority class label corresponding to the $k$ nearest neighbors. For the $k$-NN classifier, we used the Euclidean distance as the distance metric, and the best $k$ between 1 and 10 was found by performing LOOCV on the training data.

\subsubsection{Neural Network}

Neural networks are another type of classifier or mathematical models used for classification, regression or decision making. Their structure is inspired by the human neural system and brain. It consists of many neurons, interconnected at different stages. The direction of flow of information is usually from the input stage to the output stage. Each neuron has an input and an output, where an activation function converts a neurons input to its output. The output of each neuron is connected to the next stage through a weighted connection. A learning function determines the value of the weights of all the connections. The weights are updated based on a mathematical function that relates the network together. Therefore, a neural network is considered as an adaptive network that changes its structure during the learning or training phase, based on mathematical functions that relate input data to the corresponding class labels. The sum of all neurons at the different layers and the weighted interconnections make up a complex network that is commonly referred to as a black box.

Before its use to classify a test sample, the neural network is trained on a given data set with known classes or labels. During the training phase the weights are updated to minimize the output error. The selected value of the minimum acceptable error determines when the training stops. For a difficult data where it is impossible to reach the set minimum error, the maximum number of epochs is used as criteria for stopping the training process. 


\subsubsection{Support Vector Machine}

The Support Vector Machine (SVM) belongs to a new generation of learning system based on recent advances in statistical learning theory [65]. A linear SVM, which is used in our system, aims to find the separating hyper-plane with the largest margin, defined as the sum of the distances from a hyper-plane (implied by a linear classifier) to the closest positive and negative exemplars. The expectation is that the larger the margin, the better the generalization of the classifier. In a non-separable case, a linear SVM seeks a trade-off between maximizing the margin and minimizing the number of errors.

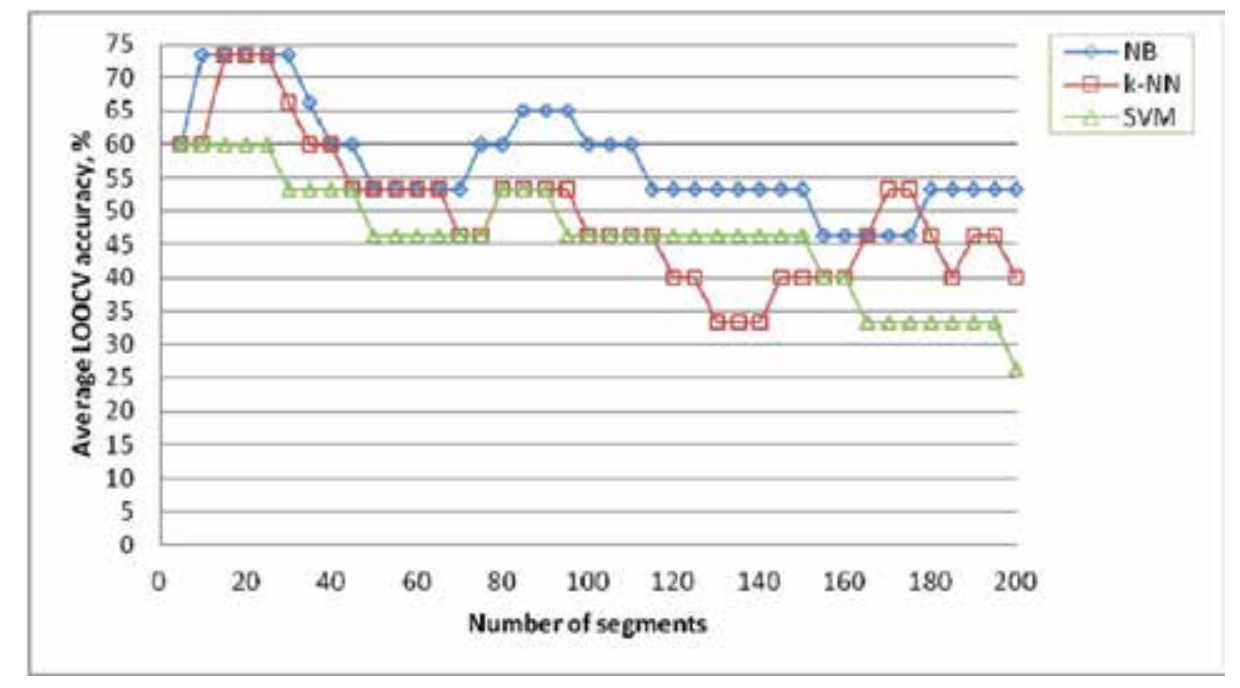

Figure 6. Comparison study of the performance of the three tested classifiers. The $x$-axis represents the number of segments and the $y$-axis represents the percentage average LOOCV accuracy.

Figure 6 illustrates the LOOCV classification accuracies using the tested classifiers, $k$-NN, $\mathrm{NN}$, and SVM. The $x$-axis is associated with the number of selected top-ranked variant segments and the $y$-axis shows the average LOOCV accuracy.

\section{Validation of the predicted variant segments}

To evaluate our predictive power of our method in detecting and identifying patients with ASD, we use molecular test, quantitative Polymerase Chain Reaction (qPCR). It is a very sensitive and precise tool used for the quantification of nucleic acids. It can detect and quantify very small amounts of specific nucleic acid sequence. It is based on the method of PCR, developed by Kary Mullis in the 1980s. It allows the amplification of specific nucleic acid sequence (DNA) more than a billion-fold. Using qPCR allows scientists to quantify the starting amount of a specific DNA sequence in the sample before the amplification by PCR method [62]. 
Quantitative PCR is an indispensable tool for researchers in various fields including fundamental biology, molecular diagnostics, biotechnology, and forensic sciences. Critical points and limitations of qPCR-based assays must be considered to increase the reliability of the obtained data. For the detection of qPCR four technologies are commonly used all of which are based on the measurement of fluorescence during the PCR. One principle is based on intercalation of double-stranded DNA-binding dyes (simplest and cheapest). The other three principles are based on the introduction of an additional fluorescence-labeled oligonucleotide (probe). Detectable fluorescence are only released either after cleavage of the probe (hydrolysis probes) or during hybridization of one (molecular beacon) or two (hybridization probes) oligonucleotides to the amplicon. The introduction of an additional probe increases the specificity of the quantified PCR product and allows the development of multiplex reactions. Other technologies have been described for the detection of qPCR [63].

The qPCR method quickly became the first choice when it comes to quantitative analysis of nucleic acid because of many reasons. It is highly sensitive and it allows the detection of less than five copies (one copy in some cases) of a target sequence. It has good reproducibility. In addition, it has broad dynamic quantification range, at least 5 log units. It is also easy to use and has reasonable good value for money (low consumable and instrumentation costs).

For the purpose of this chapter, we are focusing on one of the many applications of qPCR, which is indispensable for research and diagnostics, the genetic variations.

\begin{tabular}{ccc}
\hline Array & CBS & SF \\
\hline 1 & 14 & 20 \\
\hline 2 & 10 & 20 \\
\hline 3 & 20 & 21 \\
\hline 4 & 13 & 21 \\
\hline
\end{tabular}

Table 3. Representation of the number of events (CNVs) detected by the circular binary segmentation (CBS) and sigma filtering methods, respectively, for 22 qPCR confirmed CNVs.

Table 3 shows that the number of qPCR-confirmed CNVs detected by the sigma filtering (SF) method is considerably higher than those detected using the circular binary segmentation (CBS), ranging from $4.5 \%$ to $36 \%$ more for 4 different array experiments. The results show that applying the averaging window of $2 \mathrm{~Kb}$ allow the algorithms to be well suited for detecting variations in high-density microarray data, especially at the LCR-rich regions.

\section{Conclusion}

The etiology of Autism spectrum disorders involves genetic and environmental risk factors. In this chapter, we have discussed the genetics basis of the complex disorder, autism. With the recent advances in the new screening technologies to investigate the entire genome such 
as array comparative genomic hybridization (aCGH) and whole genome sequencing, provide the opportunities insight into the pattern of the genetic variations and reveal their roles in the genetic diseases. In this study, we have demonstrated an overview for the analysis of genetic variations in the form of DNA copy number changes and their association with autism susceptibility.

Through mathematical-based models and computational-based approaches, we analyze the genetic data trying to discover and identify the relationship between the structural chromosomal rearrangements along the genome and the targeted disorder, ASD. In conclusion, the results show strong evidence that the genetic variations contribute in the complex disorder, autism.

\section{Author details}

Abdullah K. Alqallaf ${ }^{1 *}$, Fuad M. Alkoot ${ }^{2}$ and Mash'el S. Aldabbous ${ }^{3}$

*Address all correspondence to: al.qallaf@ku.edu.kw

1 Electrical Engineering Department, College of Engineering and Petroleum, Kuwait University, Kuwait

2 The Higher Institute of Telecommunication and Navigation, Public Authority for Applied Education and Training, Kuwait

3 Department of Biological Sciences, College of Science, Kuwait University, Kuwait

\section{References}

[1] Freeman, J. L., Perry, G. H., Feuk, L., Redon, R., Mc Carroll, S. A., Altshuler, D. M., Aburatani, H., Jones, K. W., Tyler-Smith, C., \& Hurles, . (2006). Copy number variation: new insights in genome diversity. Genome Research, 16(8), 949-961.

[2] Lee, J. A., \& Lupski, J. R. (2006). Genomic rearrangements and gene copy-number alterations as a cause of nervous system disorders. Neuron, 52(1), 103-121.

[3] Stankiewicz, P., \& Lupski, J. R. (2002). Molecular-evolutionary mechanisms for genomic disorders. Current Opinion in Genetics \& Development on ScienceDirect,, 12(3), 312-319.

[4] Christian, S. L., Brune, C. W., Sudi, J., Kumar, R. A., Liu, S., Karamohamed, S., Badner, J. A., Matsui, S., Conroy, J., \& Mc Quaid, D. (2008). Novel submicroscopic chromosomal abnormalities detected in autism spectrum disorder. Biological Psychiatry, 63(12), 1111-1117. 
[5] Marshall, C. R., Noor, A., Vincent, J. B., Lionel, A. C., Feuk, L., Skaug, J., Shago, M., Moessner, R., Pinto, D., \& Ren, Y. (2008). Structural variation of chromosomes in autism spectrum disorder. American Journal of Human Genetics,, 82(2), 477-488.

[6] Sebat, J., Lakshmi, B., Malhotra, D., Troge, J., Lese-Martin, C., Walsh, T., Yamrom, B., Yoon, S., Krasnitz, A., \& Kendall, J. (2007). Strong association of de novo copy number mutations with autism. Science, $316(5823), 445-449$.

[7] Kirov, G., Gumus, D., Chen, W., Norton, N., Georgieva, L., Sari, M., O’Donovan, M. C., Erdogan, F., Owen, , \& Ropers, H. H. (2008). Comparative genome hybridization suggests a role for NRXN1 and APBA2 in schizophrenia. Human Molecular Genetics, 17(3), 458-465.

[8] Sharp, A. J., Hansen, S., Selzer, R. R., Cheng, Z., Regan, R., Hurst, J. A., Stewart, H., Price, S. M., Blair, E., \& Hennekam, R. C. (2006). Discovery of previously unidentified genomic disorders from the duplication architecture of the human genome. Nature Genetics, 38(9), 1038-1042.

[9] Sharp, A. J., Mefford, H. C., Li, K., Baker, C., Skinner, C., Stevenson, R. E., Schroer, R. J., Novara, F., De Gregori, M., \& Ciccone, R. (2008). A recurrent 15q13.3 microdeletion syndrome associated with mental retardation and seizures. Nature Genetics, 40(3), 322-328.

[10] Cantor, R. M., \& Geschwind, D. H. (2008). Schizophrenia: genome, interrupted. Neuron, $58(2), 165-167$.

[11] Stefansson, H., Rujescu, D., Cichon, S., Pietilainen, O. P., Ingason, A., Steinberg, S., Fossdal, R., Sigurdsson, E., Sigmundsson, T., \& Buizer-Voskamp, J. E. (2008). Large recurrent microdeletions associated with schizophrenia. Nature,, 455(7210), 232-236.

[12] Stone, J. L., O’Donovan, M. C., Gurling, H., Kirov, G. K., Blackwood, D. H., Corvin, A., Craddock, N. J., Gill, M., Hultman, C. M., \& Lichtenstein, P. (2008). Rare chromosomal deletions and duplications increase risk of schizophrenia. Nature,, 455(7210), 237-241.

[13] Walsh, T., Mc Clellan, J. M., Mc Carthy, S. E., Addington, A. M., Pierce, S. B., Cooper, G. M., Nord, , Kusenda, M., Malhotra, D., \& Bhandari, A. (2008). Rare structural variants disrupt multiple genes in neurodevelopmental pathways in schizophrenia. Science, 320(5875), 539-543.

[14] Estivill, X., \& Armengol, L. (2007). Copy number variants and common disorders: filling the gaps and exploring complexity in genome-wide association studies. PLoS Genetics, 3(10), 1787-1799.

[15] Greer, M. K., Brown, F. R., Pai, G. S., Choudry, S. H., \& Klein, A. J. (1997). Cognitive, adaptive, and behavioral characteristics of Williams syndrome. American Journal of Human Genetics, , 74(5), 521-525.

[16] Somerville, M. J., Mervis, C. B., Young, E. J., Seo, E. J., del Campo, M., Bamforth, S., Peregrine, E., Loo, W., Lilley, M., \& Perez-Jurado, L. A. (2005). Severe expressive-lan- 
guage delay related to duplication of the Williams-Beuren locus. The New England Journal of Medicine, 353(16), 1694-1701.

[17] van der , N., Rooms, L., Vandeweyer, G., van den, Ende. J., Reyniers, E., Fichera, M., Romano, C., Delle, Chiaie. B., Mortier, G., \& Menten, B. (2009). Fourteen new cases contribute to the characterization of the $7 \mathrm{q} 11.23$ microduplication syndrome. European Journal of Medical Genetics, 52(2-3), 94-100.

[18] Depienne, C., Heron, D., Betancur, C., Benyahia, B., Trouillard, O., Bouteiller, D., Verloes, A., Le Guern, E., Leboyer, M., \& Brice, A. (2007). Autism, language delay and mental retardation in a patient with 7q11 duplication. Journal of Medical Genetics,, 44(7), 452-458.

[19] Balciuniene, J., Feng, N., Iyadurai, K., Hirsch, B., Charnas, L., Bill, B. R., Easterday, M. C., Staaf, J., Oseth, L., \& Czapansky-Beilman, D. (2007). Recurrent 10q22-q23 deletions: a genomic disorder on $10 \mathrm{q}$ associated with cognitive and behavioral abnormalities. American Journal of Human Genetics,, 80(5), 938-947.

[20] Laumonnier, F., Roger, S., Guerin, P., Molinari, F., M'Rad, R., Cahard, D., Belhadj, A., Halayem, M., Persico, A. M., \& Elia, M. (2006). Association of a functional deficit of the BKCa channel, a synaptic regulator of neuronal excitability, with autism and mental retardation. American Journal of Psychiatry,, 163(9), 1622-1629.

[21] Cook, E. H., \& Jr , . (2001). Genetics of autism. Child and Adolescent Psychiatric Clinics of North America, 10(2), 333-350.

[22] Helbig, I., Mefford, H. C., Sharp, A. J., Guipponi, M., Fichera, M., Franke, A., Muhle, H., de Kovel, C., Baker, C., \& von, Spiczak. S. (2009). 15q13.3 microdeletions increase risk of idiopathic generalized epilepsy. Nature Genetics, 41(2), 160-162.

[23] Papolos, D. F., Faedda, G. L., Veit, S., Goldberg, R., Morrow, B., Kucherlapati, R., \& Shprintzen, R. J. (1996). Bipolar spectrum disorders in patients diagnosed with velocardio-facial syndrome: does a hemizygous deletion of chromosome 22q11 result in bipolar affective disorder? American Journal of Psychiatry ,, 153(12), 1541-1547.

[24] Niklasson, L., Rasmussen, P., Oskarsdottir, S., \& Gillberg, C. (2001). Neuropsychiatric disorders in the 22q11 deletion syndrome. Genetics in Medicine,, 3(1), 79-84.

[25] Antonell, A., de Luis, O., Domingo-Roura, X., \& Perez-Jurado, L. A. (2005). Evolutionary mechanisms shaping the genomic structure of the Williams-Beuren syndrome chromosomal region at human 7q11.23. Genome Research, 15(9), 1179-1188.

[26] Vorstman, J. A., Morcus, M. E., Duijff, S. N., Klaassen, P. W., Heineman-de, Boer. J. A., Beemer, F. A., Swaab, H., Kahn, R. S., \& van Engeland, H. (2006). The 22q11.2 deletion in children: high rate of autistic disorders and early onset of psychotic symptoms. Journal of the American Academy of Child \& Adolescent Psychiatry," 45(9), 1104-1113.

[27] Hertz-Picciotto, I., Croen, L. A., Hansen, R., Jones, C. R., van de Water, J., \& Pessah, I. N. (2006). The CHARGE study: an epidemiologic investigation of genetic and envi- 
ronmental factors contributing to autism. Environmental Health Perspectives,, 114(7), 1119-1125.

[28] Redon, R., Ishikawa, S., Fitch, K. R., Feuk, L., Perry, G. H., Andrews, T. D., Fiegler, H., Shapero, M. H., Carson, A. R., \& Chen, W. (2006). Global variation in copy number in the human genome. Nature, 444(7118), 444-454.

[29] Shen, F., Huang, J., Fitch, K. R., Truong, V. B., Kirby, A., Chen, W., Zhang, J., Liu, G., Mc Carroll, S. A., \& Jones, K. W. (2008). Improved detection of global copy number variation using high density, non-polymorphic oligonucleotide probes. BMC Genet$i c_{,}, 9(27)$.

[30] Conrad, D. F., Pinto, D., Redon, R., Feuk, L., Gokcumen, O., Zhang, Y., Aerts, J., Andrews, T. D., Barnes, C., \& Campbell, P. (2010). Origins and functional impact of copy number variation in the human genome. Nature,, 464(7289), 704-712.

[31] Perry, G. H., Ben-Dor, A., Tsalenko, A., Sampas, N., Rodriguez-Revenga, L., Tran, C. W., Scheffer, A., Steinfeld, I., Tsang, P., \& Yamada, N. A. (2008). The fine-scale and complex architecture of human copy-number variation. American Journal of Human Genetics, 82(3), 685-695.

[32] Berg, J. S., Brunetti-Pierri, N., Peters, S. U., Kang, S. H., Fong, C. T., Salamone, J., Freedenberg, D., Hannig, V. L., Prock, L. A., \& Miller, D. T. (2007). Speech delay and autism spectrum behaviors are frequently associated with duplication of the 7q11.23 Williams-Beuren syndrome region. Genetics in Medicine,, 9(7), 427-441.

[33] Potocki, L., Bi, W., Treadwell-Deering, D., Carvalho, C. M., Eifert, A., Friedman, E. M., Glaze, D., Krull, K., Lee, J. A., \& Lewis, R. A. (2007). Characterization of PotockiLupski syndrome (dup(17)(p11.2p11.2)) and delineation of a dosage-sensitive critical interval that can convey an autism phenotype. American Journal of Human Genetics,, 80(4), 633-649.

[34] Carvalho, C. M., \& Lupski, J. R. (2008). Copy number variation at the breakpoint region of isochromosome 17q. Genome Research,, 18(11), 1724-1732.

[35] Mukaddes, N. M., \& Herguner, S. (2007). Autistic disorder and 22q11.2 duplication. World Journal of Biological Psychiatry, 8(2), 127-130.

[36] Barabash, A., Marcos, A., Ancin, I., Vazquez-Alvarez, B., de Ugarte, C., Gil, P., Fernandez, C., Encinas, M., Lopez-Ibor, J. J., \& Cabranes, J. A. (2009). APOE, ACT and CHRNA7 genes in the conversion from amnestic mild cognitive impairment to Alzheimer's disease. Neurobiol Aging,, 30(8), 1254-1264.

[37] Miller, D. T., Shen, Y., Weiss, L. A., Korn, J., Anselm, I., Bridgemohan, C., Cox, G. F., Dickinson, H., Gentile, J., \& Harris, D. J. (2009). Microdeletion/duplication at 15q13.2q13.3 among individuals with features of autism and other neuropsychiatric disorders. Journal of Medical Genetics, 46(4), 242-248.

[38] Shinawi, M., Schaaf, , Bhatt, S. S., Xia, Z., Patel, A., Cheung, S. W., Lanpher, B., Nagl, S., Herding, H. S., \& Nevinny-Stickel, C. (2009). A small recurrent deletion within 
$15 q 13.3$ is associated with a range of neurodevelopmental phenotypes. Nature Genetics, 41(12), 1269-1271.

[39] Tsuchiya, K. D., Wiesner, G., Cassidy, S. B., Limwongse, C., Boyle, J. T., \& Schwartz, S. (1998). Deletion 10q23.2-q23.33 in a patient with gastrointestinal juvenile polyposis and other features of a Cowden-like syndrome. Genes Chromosomes \& Cancer, 21(2), 113-118.

[40] Zhou, X. P., Woodford-Richens, K., Lehtonen, R., Kurose, K., Aldred, M., Hampel, H., Launonen, V., Virta, S., Pilarski, R., \& Salovaara, R. (2001). Germline mutations in BMPR1A/ALK3 cause a subset of cases of juvenile polyposis syndrome and of Cowden and Bannayan-Riley-Ruvalcaba syndromes. American Journal of Human Genetics, 69(4), 704-711.

[41] Fombonne, E. (2003). Epidemiological Surveys of Autism and Other Pervasive Developmental Disorders: An Update. Journal of Autism and Developmental Disorders, 33(4), 365-382.

[42] Kuehn, B. (2007). CDC: Autism Spectrum Disorders Common. Journal of the American Medical Association,, 297(940).

[43] Abrahams, B., \& Geschwind, D. (2008). Advances in autism genetics: on the threshold of a new neurobiology. Nature Reviews Genetics., 9(5), 341-355.

[44] Alqallaf, A., Tewfik, A., \& Selleck, S. (2009). Genetic variation detection using maximum likelihood estimator. IEEE International Workshop on Genomic Signal Processing and Statistics., 978-1-42444-761-9, Minnesota, USA.

[45] Kallioniemi, A., Kallioniemi, O., Sudar, D., Rutovitz, D., Gray, J., Waldman, F., \& Pinkel, D. (1992). Comparative genomic hybridization for molecular cytogenetic analysis of solid tumors. Science,, 258(5083), 818-821.

[46] Wang, Y., \& Wang, S. (2007). A novel stationary wavelet denoising algorithm for array-based DNA copy number data. International Journal of Bioinformatics Research and Applications, 3(2), 206-222.

[47] Willenbrock, H., \& Fridlyand, J. (2005). A comparison study: applying segmentation to array CGH data for downstream analyses. Bioinformatics, 21(22), 4084-4091.

[48] Rissanen, J. (1978). Modeling by Shortest Data Description. Automatica, 14, 465-471.

[49] Larson, R., \& Castie, J. (1982). Principles of Dynamic Programming. 1-2, Marcel Dekker Inc., NY.

[50] BioDiscovery Inc. (2012). Nexus: Copy Number Professional software package (BioDiscovery), Inc., El Segundo, CA) available from:, http://www.biodiscovery.com.

[51] Venkatraman, E., \& Olshen, A. (2007). A faster circular binary segmentation algorithm for the analysis of array CGH data. Bioinformatics, 23(6), 657-663. 
[52] Grant, G., Manduchi, E., Cheung, V., \& Ewens, W. (1999). Significance testing for direct identity-by-descent mapping. Annals of Human Genetics, 63(5), 441-454.

[53] Diskin, S., Eck, T., Greshock, J., Mosse, Y., Naylor, T., Stoeckert, C., Weber, B., Maris, J., \& Grant, G. (2006). STAC: A method for testing the significance of DNA copynumber aberrations across multiple array-CGH experiments. Genome Research, 16(9), 1149-1158.

[54] Rohlfing, T., Russakoff, D., \& Maurer, C. (2004). Performance based classifier combination in atlas based image segmentation using expectation maximization parameter estimation. IEEE transaction on medical imaging,, 23(8), 983-994.

[55] Guler, I., \& Ubeyli, E. (2005). ECG beat classifier designed by combined neural network model. Pattern Recognition, 38, 199-208.

[56] Sampat, M. ., Bovik, A., Aggarwal, J., \& Castleman, K. (2005). Supervised parametric and non parametric classification of chromosome images. Pattern Recognition, 38, 1209-1223.

[57] Bruijne, M., \& Nielson, M. (2004). Shape particle filtering for image segmentation. Medical Image Computing and Computer-Assisted Intervention (MICCAI),, 1, 168-175.

[58] Shin, H., \& Sohn, S. (2005). Selected tree classifier combination based on both accuracy and error diversity. Pattern Recognition, 38, 191-197.

[59] Tsymbal, A., Cunningham, P., Pechenizkiy, M., \& Puuronen, S. (2003). Search strategies for ensemble feature selection in medical diagnosis. 16th IEEE symposium on computer based medical systems,, 124-129.

[60] Kook, H., Gupta, L., Molfese, D., \& Fadem, K. (2005). Multi-stimuli multi-channel data and decision fusion strategies for dyslexia prediction using neonatal ERPS. Pattern Recognition, 38(11), 2174-2184.

[61] Moon, H., Ahn, H., Kodell, R., Lin, C., Baek, S., \& Chen, J. (2006). Classification methods for the development of genomic signatures from high-dimensional data. Genome Biology, 7(12), 121.

[62] Valasek, M., \& Repa, J. (2005). The power of real-time PCR,. Advances in Physiology Education, 29(3), 151-159.

[63] Klein, D. (2002). Quantification using real-time PCR technology: applications and limitations. Trends in Molecular Medicine, 8(6), 257-260.

[64] Dasarathy, B. (1991). Nearest Neighbor Norms: NN Pattern Classification Techniques. IEEE. Computer Society Press.

[65] Vapink, V. (1998). Statistical learning Theory. Wiley-Interscience.

[66] Shinawi, M., \& Cheung, S. (2008). The array CGH and its clinical applications. Drug Discovery Today, 13(17-18), 760-770. 
[67] Schwab, M., Alitalo, K., Klempnauer, K., Varmus, H., Bishop, J., Gilbert, F., Brodeur, G., Goldstein, M., \& Trent, J. (1983). Amplified DNA with limited homol- ogy to MYC cellular oncogene is shared by human neuroblastoma cell lines and a neuroblastoma tumour. Nature,, 305(5931), 245-248.

[68] Aldinger, K., Plummer, J., Qiu, S., \& Levitt, P. (2011). SnapShot: Genetics of Autism. Neuron, $72,418$.

[69] Sanders, S., et al. (2011). Multiple Recurrent De Novo CNVs, Including Duplications of the 7q11.23 Williams Syndrome Region, Are Strongly Associated with Autism. Neuron, 70, 863-885. 


\section{Section 4}

Aetiological Factors - Co-Morbidity 

Chapter 17

\title{
Co-Occurrence of Developmental Disorders: Children Who Share Symptoms of Autism, Dyslexia and Attention Deficit Hyperactivity Disorder
}

\author{
Ginny Russell and Zsuzsa Pavelka \\ Additional information is available at the end of the chapter \\ http://dx.doi.org/10.5772/54159
}

\section{Introduction}

Children with autism spectrum disorders (ASD) have a higher risk of suffering from several other conditions. In this chapter I review the extent to which autistic individuals can also experience a range of other difficulties, but my focus will be on the common neurodevelopmental disorders. The most common of these include dyslexia, attention deficit hyperactivity disorder (ADHD), dyspraxia, specific language impairment, and dyscalculia. There is considerable symptom overlap in particular between ADHD and dyslexia, and like autism both are described as developmental disorders by psychiatric classification systems (American Psychiatric Association, 2000; World Health Organization., 1992). Overlapping conditions are termed 'comorbidity' by medical practitioners. Co-morbidity may reflect the greater difficulties experienced by children with a combination of deficits. Sometimes it is apparent that many children with a developmental disorder could be classified in several ways. Here I will firstly examine the research evidence that examines how often symptoms of dyslexia and ADHD occur in the population of autistic children, and second, review the various theories that have tried to explain why such co-occurring difficulties are so common.

'Comorbidity', a term used in medical literature to mean a dual diagnosis, or multiple diagnoses, can reflect an inability to supply a single diagnosis that accounts for all symptoms. Children with ASD have been shown to have higher rates of epilepsy, with $30 \%$ of cases having epilepsy comorbid (Danielsson, Gillberg, Billstedt, Gillberg, \& Olsson, 2005). Other conditions that are commonly co-morbid with ASD include hearing impairment (Kielinen, Rantala, Timonen, Linna, \& Moilanen, 2004) mental health and behavioural problems (Bradley, Summers, Wood, \& Bryson, 2004), including anxiety, and depression (Evans, Canavera, 
Kleinpeter, Maccubbin, \& Taga, 2005). It has also been shown that parents of autistic children are twice as likely themselves to have suffered from psychiatric illness than parents of non-autistic children (Daniels et al., 2008).

Most of these problems are distinct from those examined in this chapter: the common developmental disorders of childhood which are also found to co-occur with autism, particularly ADHD and dyslexia.

Before reviewing the evidence that suggests many children share difficulties symptomatic of these conditions, and the theories of why this may be, I will briefly describe how dyslexia and ADHD manifest themselves.

\section{Dyslexia}

Dyslexia is conceptualized by both educational bodies and the psychiatric classification systems as a learning difficulty that primarily affects the skills involved in accurate and fluent word reading and spelling. Characteristic features of dyslexia are difficulties in phonological awareness, verbal memory and verbal processing speed. Dyslexia is developmental delay in literacy and generally slow and inaccurate reading and spelling. The definition of dyslexia has changed over time, and such changes have often been based on the research identifying a range of associated difficulties that occur with dyslexia. Estimates of the prevalence of dyslexia have been complicated because dyslexia cut-offs are contested (Coltheart \& Jackson, 1998) and dyslexia manifests itself differently in various languages according to levels of phonic regularity (Miles, 2004). Research over the last 40 years has focused on phonological skills. These are the reading and de-coding skills used when breaking down language into its component sounds and reassembling the parts in order to read or to spell a word.

Like autism, dyslexic difficulties are considered to exist in a continuum throughout the general population (Fawcett, 2012). There is much interest in the association of cognitive ability with changing symptom profiles and diagnosis. The definition of dyslexia is in flux, and has been recently redefined by many national bodies, for example in the UK, the British Psychological Society, focusing on literacy learning at the 'word level' without attainment discrepancy:

Dyslexia is evident when accurate and fluent word reading and/or spelling develops very incompletely or with great difficulty (British Psychological Society, 1999)

This definition implies that the problem is severe and persistent despite appropriate learning opportunities. This UK definition differs from the ICD-10 diagnosis of developmental dyslexia or 'Specific Reading Disorder', which requires a discrepancy between actual reading ability and the reading ability predicted by a child's IQ. So an intellectual disability, (generally considered IQ below 70) can co-occur with the British Psychological Society definition of dyslexia. This new definition includes the so called 'garden variety' dyslexic chil- 
dren who have difficulties with reading and spelling as well as other generalized intellectual disabilities. The implications of including this group as dyslexic mean that more children with an intellectual disability would also be classified as 'dyslexic'. As ASD includes a large group with intellectual disability the extension is likely to increase the number of children who may be classified as having both conditions. This is important as the clinical and education label may determine the interventions a particular child receives.

In addition to these characteristics, dyslexic children may experience visual and auditory processing difficulties, similar to hyper or hypo sensitivity often associated with ASD. Like the 'islets of ability' seen in many children with ASD, some dyslexic children may also have strengths in particular areas, such as design, logic, and creative skills.

\section{ADHD}

ADHD is known as 'Hyperkinetic Disorder' in ICD-10; there are three subtypes of ADHD according the DSM. In the first, a child will primarily have problems with attention which may manifest as an inability to remain 'on task' for long periods, lack of response to instruction or distractibility. In the second sub-type, symptoms of hyperactivity and impulsivity dominate, which is characterized by wriggling, squirming, being unable to sit still, interrupting and finding it difficult to wait. Children may also be climbing in inappropriate situations and always on the move when free to do so. The third sub-type is simply the coexistence of both attention problems and hyperactivity, with each behavior occurring infrequently alone and symptoms starting before seven years of age.

According to ICD-10, eventually, assessment instruments should develop to the point where it is possible to take a quantitative cut-off score to assess ADHD. Like dyslexia and autism, the symptoms are behavioural in nature, and are part of a continuously distributed pattern that extends into the population at large.

The persistence of ADHD symptoms is not so marked as for autism. Around 70 to 50 percent of those individuals diagnosed in childhood do not continue to have symptoms into adulthood (Elia, Ambrosini, \& Rapoport, 1999). There is evidence suggesting to some extent symptoms of ADHD are expressed in reaction to home (Mulligan et al. 2011) and other environmental contexts. Individuals with ADHD also tend to develop coping mechanisms to compensate for some or all of their impairments. ADHD is diagnosed more often in boys with the reported ratio varying from 2:1 to 4:1 (Dulcan, 1997; Kessler et al., 2005) though some studies suggest this may be partially due to referral bias where teachers are more likely to refer boys than girls (Sciutto, Nolfi, \& Bluhm, 2004). Treatments for ADHD involve a combination of medication, usually methyphenidates which are well established in improving symptoms of inattention, and behavioral intervention in education and at home. The issue of girls being overlooked on identification is a common thread for research in dyslexia, ADHD and autism. Our own results suggest there is some evidence to back up the claim that boys with ASD symptoms are given the diagnosis more frequently than girls with 
equivalent ASD symptoms (Russell, Steer, \& Golding, 2011). This may be because the disorders tend to be conceptualized as 'male' leading to referral bias.

Because ASD, Dyslexia and ADHD are all behaviorally defined, so 'symptoms' are behaviours. All three conditions are conceived as particular behaviours along a spectrum, where traits have a continuous distribution and extend into the general (non-disordered) population. An arbitrary cut off point determines who is considered to be within the various categories and who is not. The clinician giving a diagnosis will be responsible for judging where this cut off may come, guided by diagnostic criteria and standards within disciplines as well as perceived implications: the benfits versus any possible risks of assigning a diagnosis. This is perhaps best established for autism: Constantino and Todd (2003) measured autistic traits in a large community sample, and found no jump in the threshold of autistic behaviours between 'normal' individuals and those with an autism spectrum diagnosis, rather they found a continuous distribution. These findings concurred with those in a Scandinavian study (Posserud, Lundervold, \& Gillberg, 2006). One of our own studies has likewise shown that autistic traits do extend into the 'subclinical' population (Figure 1). As with dyslexia and ADHD, there is not a sharp line separating severity in those with a diagnosis from less severe traits in those without (London, 2007). In both dyslexia, ADHD and the autism spectrum, some children have more severe difficulties than others, and the symptoms extend into the population of children (and adults) as a whole. For dyslexia, there are many people who may have mild dyslexic difficulties but perhaps might not qualify as 'dyslexic'. For autism spectrum disorders, many people without an autism diagnosis do have autistic-type behaviours but the severity and frequency of those behavioural symptoms is less severe than in those deemed to qualify for a diagnosis.

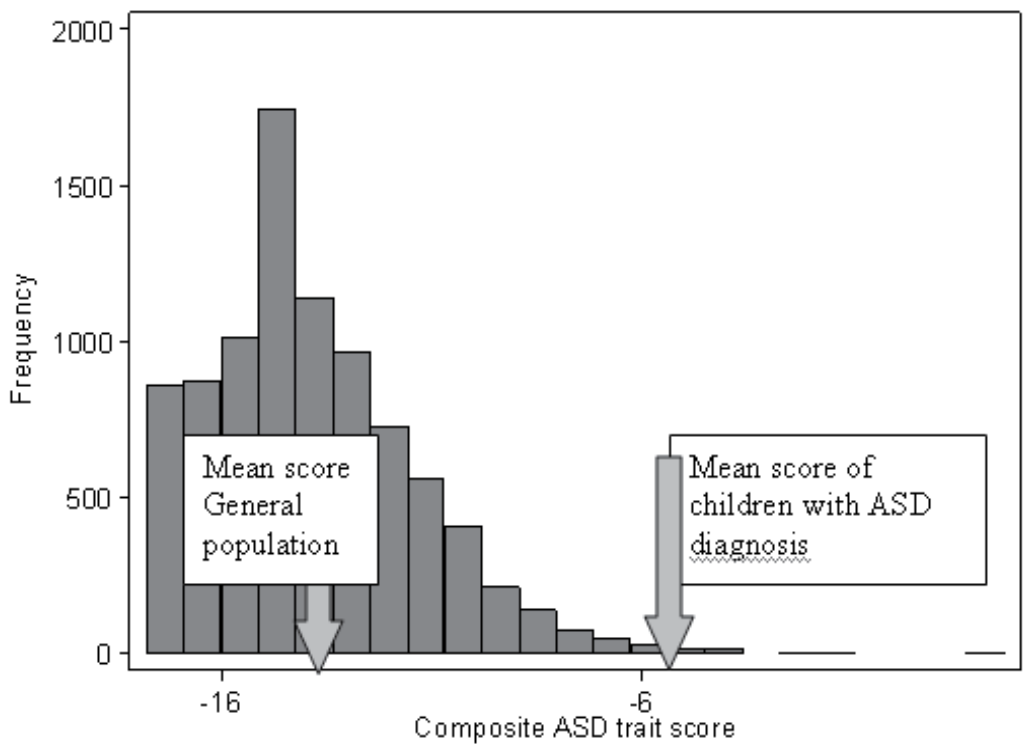

Figure 1. The distribution of an ASD composite trait in the general population from Russell et al.(2012) 
The imposition of a cut off between normality and abnormality is therefore 'an arbitrary but convenient way of converting a dimension into a category' as Goodman and Scott (1997, p. 23) point out.

\section{Evidence of symptom overlap - ASD and ADHD}

Various studies have looked for ADHD or ADHD symptoms in samples of children with autism or ASD. Rates of ADHD have ranged from $28 \%$ to $78 \%$ of these samples (Ronald, Edelson, Asherson, \& Saudino, 2010). Studies that look at ADHD symptoms have reported even higher numbers: for example, Sturm, Fernell, \& Gillberg, (2004) looked at a sample of around 100 high functioning children with ASD and found $95 \%$ had attention problems, $75 \%$ had motor difficulties, $86 \%$ had problems with regulation of activity level, and $50 \%$ had impulsiveness. About three-quarters had symptoms compatible with mild or severe ADHD, or had deficits in attention, motor control, and perception, indicating a considerable overlap between these disorders and high-functioning ASD in children.

In an large analysis of nine hundred forty-six twins, Reierson and colleagues (2008) assigned DSM-IV ADHD diagnoses, and measured autistic traits using the Social Responsiveness Scale. The study showed that there are clinically significant elevations of autistic traits in children meeting diagnostic criteria for ADHD. These findings confirm results in earlier studies (Clark, Feehan, Tinline, \& Vostanis, 1999). Santosh and Mijoovic (2004) which found children with ADHD had elevated levels of impairment in all three autistic symptom domains, namely social deficits, communication and stereotyped behaviors. Clark et al found $65-80 \%$ of parents of children with ADHD reported difficulties in social interaction (particularly in empathy and peer relationships) and in communication (particularly in imagination, and maintaining conversation). So the presence of autistic traits in children with ADHD appears common (Ronald et al., 2010).

In an analysis conducted with Lauren Rodgers at the Peninsula Medical School in the UK using data from the Millennium Cohort Study, a cohort of around 19,000 children who were all born between 2000 and 2002, we noted 44 children had a dual diagnosis of both ASD and ADHD (proportion of total population $0.3 \%$ ) by age seven. The prevalence of children with identified ADHD in the ASD sample was $17 \%$. Conversely, the prevalence of children with ASD in the ADHD sample was higher at $27 \%$. Both figures indicate substantial overlap between these conditions.

Various European research groups have examined co-morbid disorders in adults with diagnosed ASD. An international team lead by Hofvander studied a group of 122 adults with normal IQ from specialist clinics in three European cities: Gothenburg, Paris and Malmö (Hofvander et al., 2009). Here the overwhelming majority had symptoms of ASD. Nonverbal communication problems were also very common, described in $89 \%$ of all their subjects. In this study over half the participants, (52\%) were diagnosed with co-morbid ADHD. Interestingly, participants diagnosed with pervasive developmental disorder. 'Not Otherwise Specified' (PDD-NOS) diagnosis had significantly more symptoms of inattention and 
hyperactivity/impulsivity compared to subjects diagnosed with Asperger's syndrome. However, the prevalence of the categorical diagnosis of ADHD did not differ significantly between the groups, nor were gender differences apparent. Although the study presents clear evidence of many cases where patients display symptoms of both ADHD and ASD, the clinical setting may have led to selection bias as patients with complex needs may be more likely to seek help.

Because behaviours associated with both conditions lie on a spectrum extending into the normal range, some studies have found a range of frequency and severity of symptoms. In Mulligan et al.'s (2009) study, for example, 75 of children with ADHD had severe autism traits, and over half showed sub-clinical autism symptoms. Kadesjö and colleagues (Kadesjö, Gillberg, \& Hagberg, 1999), looked at comorbidity of ADHD in Swedish school-age children and found only 1\% of children meeting the threshold for ADHD had comorbid Aspergers Syndrome (AS). The estimates of co-morbidity of ADHD symptoms with ASD symptoms vary widely because of differing methods of case ascertainment. An additional problem is that the estimate of the prevalence of ASD itself has increased so much in western countries, making ASD itself a 'moving target' (Figure 2).

Patricia Howlin (2000) reviewed the estimated rates of co-existing psychiatric disorders in subjects with high functioning ASD and found these estimates varied from $9 \%$ to $89 \%$ - very substantial differences. However it is possible to generalise; thirty years of research have confirmed that attention deficits and hyperactivity are relatively common in children and adults with ASD even if the exact extent of overlap is dependent on methodology and ascertainment (Hofvander et al., 2009, Sturm, Fernell, \& Gillberg, 2004).

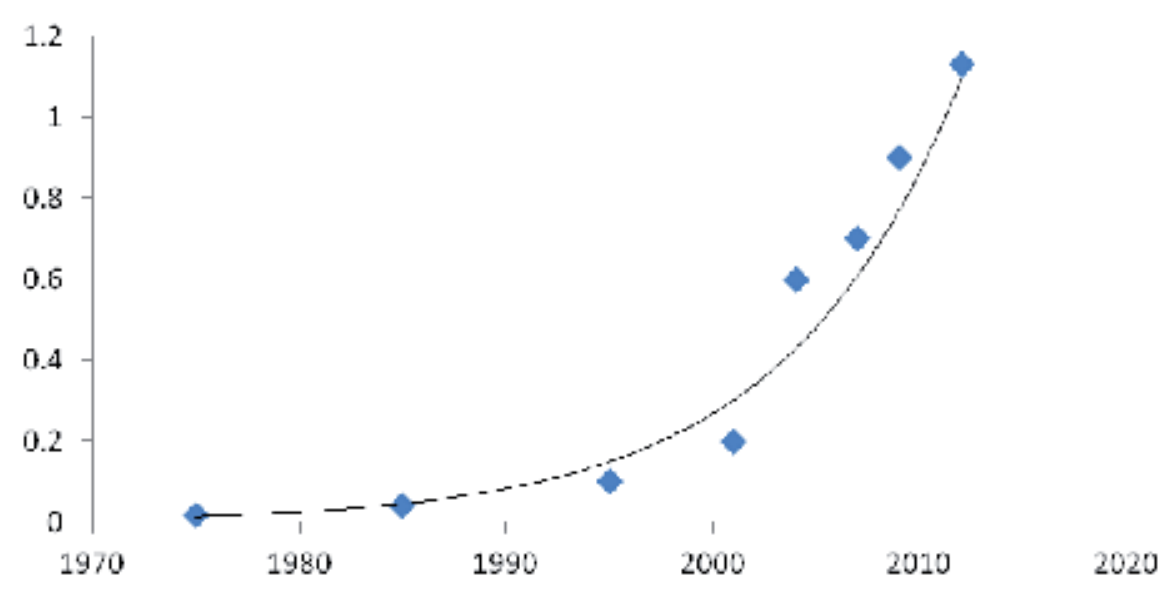

Figure 2. The rising prevalence of autism spectrum disorders over 50 years. (Data from 'Autism Speaks' and CDC, USA)

Recent trends have made categorical diagnosis an integral part of everyday clinical and research practice (Sonuga-Barke \& Halperin, 2010). Christopher Gillberg (2010) points out that clinicians have become focused on dichotomous categories of disorder and that clinics have become increasingly specialized and overlook difficulties not within their immediate juris- 
diction. Gillberg has argued that co-existence of disorders is the rule rather than the exception in child psychiatry and developmental medicine. He has coined the acronym ESSENCE (referring to Early Symptomatic Syndromes Eliciting

Neurodevelopmental Clinical Examinations). This describes cases where a combination of symptoms including inattention, hyperactivity, social and reading difficulties are observed. Major problems in at least one ESSENCE domain before age 5 years often signal major problems in the same or overlapping domains years later.

To summarize, although ADHD and ASD are separate and recognizable, there is good evidence that these conditions co-occur, constituting an amalgam of problems.

\section{Comorbidity between dyslexia and ASD}

There is only a small literature on the overlap in symptomology between autism spectrum disorders with those of dyslexia. Officially, as for ADHD, ASD is an exclusionary criterion for diagnosis of dyslexia and vice versa, but ASD also shows overlap with dyslexia in both cognitive and behavioural features (Reiersen \& Todd, 2008, Simonoff et al., 2008). A proportion of children share symptoms between dyslexia, ADHD and ASD.

The number of children that do share symptoms of ASD and dyslexia is likely to be small (Wright, Conlon, Wright, \& Dyck, 2011). The frequency of reading disorder in combination with disorder of written expression (i.e. dyslexia) was around $14 \%$ in a sample of adults with Asperger's Syndrome (AS) so according to this result around one in seven individuals with AS will have co-occurring dyslexia (Hofvander et al., 2009). However the proportion of individuals with dyslexia who have co-occuring AS is likely to be low as Asperger's Syndrome is much a rarer condition than dyslexia.

A common problem for children with dyslexia is misinterpretation of spoken language, which can also manifest itself in comprehension. This produces further overlap with pragmatic language impairment (PLI) which itself is virtually indistinguishable from communication difficulties associated with high functioning autism. Pragmatic language difficulties may involve literal interpretation so 'run on the spot' would have a child looking for a big black spot to run on, for example. Children with PLI will often fail to interpret the core meaning or saliency of events. This causes a penchant for routine and 'sameness' (also seen in autism and Asperger's Syndrome) as PLI children struggle to generalize and take hold of the meaning of novel situations. Obvious and concrete instructions are clearly understood and carried out, whereas simple but non-literal expressions such as jokes, sarcasm and general social chatting are difficult and may be misinterpretated. PLI may therefore impact on the social abilities of the child who has difficulty interpreting jokes. Current thinking is that PLI is not a problem rooted in language skills but one of social communication and information processing. Griffiths (2007) identified difficulties of this type in dyslexic students, showed they were impaired in making inferences from a story and choosing the right punch-line for a joke. This of course can have implications for written language and examinations under stress, as well as for a range of social interactions. 
It is not just that ASD is co-morbid with dyslexia and ADHD. Other studies have noted high comorbidity with other developmental disorders. Dyspraxia and dyscalculia and conditions with shared symptoms such as specific language impairment are frequently comorbid with autism. Also dyslexia and ADHD themselves co-occur Willcutt and colleagues (Willcutt, Doyle, Nigg, Faraone, \& Pennington, 2005) showed that $40 \%$ of a sample of twins with either dyslexia or ADHD was co-morbid for the other disorder. Reading difficulties were measured with both rating scale and an objective task in a study by Cheung et al. (2012) and correlations were observed among ADHD, reading difficulties and IQ. Over half, (53\%-72\%) of the overlapping familial influences between ADHD and reading difficulties were not shared with IQ. In a school based study Kadesjö and colleagues found $40 \%$ of children with ADHD showed reading problems and 29\% writing problems (2005).

Overall, the literature suggests, there is good evidence to suggest that some children do suffer from symptoms of both dyslexia and ASD, although this is not so well established, and does not occur so frequently as co-morbidity between ADHD and ASD.

\section{Reasons for co-occurrence of ASD with other developmental disorders}

Several theories have been put forward to explain the shared symptoms of the various developmental conditions - in other words why specific learning and language and social disorders are not specific. It is likely that all the explanations below play a part in cooccurrence; the causality of co-morbidity is most probably due to a complex web of interacting factors.

\section{Genetic explanations}

One of the most persuasive explanations is that a genetic predisposition may lead to abnormal neurological development, which in turn may manifest in various different aberrant behaviors and developmental delays. As autism, ADHD and dyslexia and other developmental conditions are all highly heritable, so they all have a large genetic component, the theory seems plausible. The same genetic anomaly may lead to several disorders or psychiatric conditions. In other words one genotype may lead to several (related) phenotypes. This is known as 'pleiotropy'. Researchers have suggested that co-occurrence of autism and ADHD (and other developmental disorders) may reflect such common genetic causes (Reierson et al, 2008). In this model, the origins of both sets of difficulties are due to common genetic anomalies that predispose children to delayed or atypical neurological development. Certainly, specific genetic anomalies have been associated with a range of psychopathologies in adulthood. However, the genetic picture is complex and exact pathways are not established. It is estimated there are more than a thousand gene variations which could disrupt brain development enough to result in social delays (Sanders et al., 2012).

Such a genetic predisposition is almost certainly complex and multi factorial. So far, over 100 candidate genes have been associated with ASD, most of which encode proteins in- 
volved in neural development, but exact mutations within the candidate genes have yet to be identified (Freitag, 2007). Furthermore, different individuals may have mutations in different sets of genes and most of the discovered gene variations are likely to have a low penetrance, thus not all carriers will develop the disorder. There may be interactions among mutations in several genes, e.g. between regulatory genes and coding regions, or between the environment and mutated genes, altering their expression. The effect of a mutation or deletion can depend on processes relating to gene expression and regulation as well as the subsequent effects on the expression of other genes.

The advent of genomics and the emphasis placed on this has led to much research to identify genetic predispositions to ASD. The field of psychiatry as a whole has been 'geneticised' according to some social theorists. This refers to the potential reclassification of psychiatric conditions in the light of findings from molecular biology. For example, a particular sub-category of DSM-IV schizophrenia has been linked to a substitution of a single base in the sequence of DNA of a particular gene localised to a precise place on a particular chromosome, leading to a substitution of one amino-acid for another in an enzyme involved in neurotransmission. Hedgecoe (2001) provides a discussion of the geneticisation of schizophrenia. The debate as to whether the old psychiatric systems of classification should be overhauled in the light of new genomic knowledge which illuminates genetic aetiologies is ongoing (Ericson \& Doyle, 2003).

\section{Gene-environment interactions}

A second theory is that an environmental insult or a stressful event in the life of the fetus or in a young child's life, may trigger a genetic predisposition to be expressed. Thus this constitutes a gene- environmental interaction theory. An example might be the high testosterone levels in the womb that have been observed in some studies. Baron-Cohen's Cambridge group, for example, has carried out work that has suggested high levels of fetal testosterone may be linked to the development of autistic traits (Ingudomnukul, Baron-Cohen, Wheelwright, \& Knickmeyer, 2007). According to the gene-environment explanation, the elevated testosterone might lead to the differential expression of genes controlling the neurological development of the child. Another example that has been quite widely publicized concerns Omega 3 fatty acids. These have been implicated by Richardson (2006), who has argued that attention-deficit/hyperactivity disorder, dyslexia, developmental coordination disorder (dyspraxia) and conditions on the autism spectrum may all share common origins triggered by problems with phospholipid (fatty acid) metabolism. However this is just one genetic / environmental explanation for co-occurrence that vies with several others, and the available evidence is subject to interpretation.

In the majority of cases, the gene-environment hypothesis seems highly plausible. It may be that autism and co-occurring developmental conditions may all be caused by a genetic predisposition which is triggered by an early environmental influence (Trottier, Srivastava, \& Walker, 1999). 
Many environmental factors have been implicated in ASD but the effect of each is poorly established. After the well publicized paper that linked autism to the MMR vaccination, research has repeatedly refuted a link between the MMR jab and ASD (Rutter, 2005). Deykin and MacMahon (1979) found increased risk due to exposure to, and clinical illness from, common viral illnesses in the first 18 months of life. In this study, mumps, chickenpox, fever of unknown origin, and ear infections were all significantly associated with ASD risk. Epidemiological studies have shown there is a higher rate of adverse prenatal and postnatal events in children with ASD than in the general population (Zwaigenbaum et al., 2002). Newschaffer and colleague's (2007) review named associated obstetric conditions that included low birth weight, gestation duration, and caesarean section. It is possible that such an underlying cause partially could explain both autism and the associated conditions (Kolevzon, Gross, \& Reichenberg, 2007). There is evidence to suggest adverse prenatal and perinatal events are also associated with ADHD and cognitive development. Some studies have suggested that the risk of autism may be increased with advancing maternal age (Bolton et al., 1997). Paternal age too has frequently (but not always) associated with autism. There are more mutations in the gametes of older men, and this higher rate of mutation in the genetic material from the paternal side may explain the higher levels of neurodevelopmental disabilities in their offspring. An alternative explanation is that fathers who themselves have autistic traits are less likely to have children young. Using anticonvulsants during pregnancy also appears to increase the risk of ASD (Moore et al., 2000). These drugs are used to combat epilepsy which is commonly often comorbid with ASD. Parental occupational exposure to chemicals during the preconception period has also been higher in ASD families than controls in some studies (Felicetti, 1981).

Environmental risk factors have received widespread media coverage within the last few years, perhaps because of the strong degree of public concern (Russell \& Kelly, 2011). In most health and disease categories, a secondary function of diagnosis is to group together people who have a common aetiology. However, the specific effects of genetic factors and environmental risk factors that might play a part in abnormal neural development are largely unresolved. Goodman and Scott (1997) stress that current understanding of aetiology for childhood developmental conditions will probably look ridiculously simplistic or misguided in years to come. Despite, or perhaps because of, the uncertainty, there is an underlying concern among people involved with children who are diagnosed with developmental conditions that environmental influences may be partially to blame for rising incidence. Novel prenatal and perinatal medical practices, changing diet, shifting family structures and childhood social activities have all been the subject of lay theories to explain rising prevalence not just of ASD, but developmental disorders in childhood more generally, including ADHD and dyslexia (Russell \& Kelly, 2011).

\section{The influence of childcare and the child's environment}

A third possibility is that environmental factors alone may be enough to trigger not just autistic behaviors, but also other maladaptive behaviors such as inattention. Autistic behaviors 
were observed in a study of abandoned Romanian children, conducted by Michael Rutter and colleagues (1999). As well as cases with known genetic causes, in some cases, underlying social factors may predispose autistic symptoms. In this study, Rutter and colleagues noted a very high instance of autism $(6 \%)$ in the Romanian baby cohort, which they put down to poor early care. These children exhibited typical symptoms of autism at four years old, but unlike cases of autism without maltreatment, symptoms by age 6 were much milder. This case is an illustration of how children who share severe autistic symptoms at young ages may have differing developmental trajectories. In this study, the symptoms of autism may have been triggered primarily by the early neglect, rather than by a genetic predisposition, for if a genetic predisposition was involved it would effect $6 \%$ or more of the babies, a very high proportion.

It is not just aetiological environmental factors that seem to lead to increased risks of displaying autistic behaviours. Aetiological causes can be distinguished from proximate determinates which occur at the same time as symptoms, for example, social situations or fluorescent lights may exacerbate the expression of ASD symptoms. There are also those influences in the environment that are sometimes referred to in psychiatry as maintenance factors, including stigmatisation and labelling. Although their influence in perpetuating ASD and other developmental disorders is unclear, an influence in maintaining symptomatic behaviours of autism and co-morbid conditions can not be discounted. Biological causes and behavioural outcomes are mediated by experiential and environmental factors.

\section{Cognitive causes and developmental consequences}

The competing psychological theories that have been put forward concerning the psychological mechanisms of ASD include weak central coherence theory, deficits in executive function and the extreme male brain theory, all were reviewed by Happé in 1994.

The extreme male brain theory as developed by Baron-Cohen (2002) suggests that autistic individuals can systematize-that is, they can develop internal rules of operationbut are less effective at empathizing and handling events that are unexpected or social. The theory was developed from the earlier 'theory of mind' (Baron-Cohen, Leslie, \& Frith, 1985). This suggested that autistic people lack the ability to understand other peoples' mental states, put themselves in another person's place or imagine what they might be thinking or experiencing. This lack of mentalising is discussed by Frith and Happé in their discussion of dyslexia, autism and downstream effects of specific impairments (1998). The 'theory of mind' lines up with the 'mirror neuron theory of autism' (Iacoboni \& Dapretto, 2006) which was based on the discovery that the macaque monkey brain contained 'mirror neurons' that fired not only when the animal is in action, but also when it observes others carrying out the same actions.

An alternative psychological theory for autism is provided by Frith whose 'weak central coherence' theory (Frith, 2003; Happé \& Frith, 2006) describes the ability to place information in a context in order to give it meaning. Most people pull together numerous stimuli to form 
a coherent picture of the world, allowing them to see the 'bigger picture'. In central coherence theory, the failure to appreciate the whole accounts for the piecemeal way in which people with ASD acquire knowledge. People with ASD may also show relative strengths in some areas, known as 'islets of ability'; and this accounts for savant skills. Related to central coherence is the theory that autistic behaviours are due to interference in executive function (Hill, 2004). Executive functions coordinate the flow of information processing in the brain and are the mechanisms of transferring attention from one thing to another flexibly and easily. They allow people to plan strategically, solve problems and set objectives. Their absence means autistic people show an inability to plan and attain overarching goals. This manifests as easily distractible behaviour and reliance on routines. Such psychological theories of ASD are useful models but have also been subject to criticism. Bailey and Parr (2003) describe such theories of psychological mechanisms as 'narrow cognitive conceptualisations' (p. 27), because they cannot accommodate the presence of sub-clinical autistic traits in the general population.

These theories seem very distinct from some psychological theories that explain dyslexic type and attention and hyperactive difficulties. The exception to this is that, deficits in executive function have been suggested as causal for ADHD, as they affect both cognitive and motivational systems (Willcutt et al., 2005). Frith and Happé (1998) focusing on dyslexia and autism, argue that psychological mechanisms could act as 'gateways' to impairment in other domains. These downstream developmental effects have not yet been fully considered, they suggest. Although they focus on autism and dyslexia, ADHD and other developmental disorders could easily be included in their model. As they point out, both dyslexia and autism have genetic origins, an anatomical basis and extremely variable behavioral manifestations. Their idea is that in addition to the genetic and anatomical origins, an additional developmental pathway may contribute to later difficulties. They argue that specific impairments seen in dyslexia or autism (such as dyslexic phonological or autistic mentalising difficulties) may have a 'gatekeeping' function and subsequently lead to difficulties in other areas. Thus impairments in domain-specific functions may have wide ranging developmental effects.

The idea put simply is that during development, one behavior exacerbates problems in other domains. It is perhaps easier to understand given a few concrete examples. Frith and Happé suggest that the core autistic difficulty of social engagement may lead to missed opportunities for learning, including learning vocabulary. This may effect language acquisition and in turn the development of language based skills evident in dyslexia. An easier pathway to understand might be via gatekeeping function of inattention. If a child is inattentive (a core symptom of ADHD) then the likelihood is they may struggle to focus on learning to read. Hence difficulties symptomatic of dyslexia may be expected. Conversely perhaps reading difficulties are primary, in which case inattention might come from frustration and inability to deal with task demands. This direction of causality seems likely in the sub-group of ADHD children whose problems only appear at school, and who are more likely than other groups to show reading problems according to Taylor (2011). Furthermore, an inattentive child may find it difficult to socialize normally, and may have difficulties following instruction. This may lead to the impairment in social skills symptomatic of autism. 
In a similar way, it is possible to theorize that each domain of behavioural impairment in the triad for autism might lead to another. In a review of evidence for single genetic or cognitive causes for autism, Happé, Ronald, and Plomin (2006) note that twin studies suggest combinations of largely non-overlapping genes act on each area of impairment. Their own study found only modest correlations between the three domains of behavioural traits in the triad (namely deficits in social skills and communication and stereotyped behaviour or restricted interests). In the general population, correlations ranged from 0.1- 0.4 for the relationship of each domain to the other. This evidence shows that the three types of autistic traits may be clustered or linked or co-inherited, but with a weak association. These low correlations could be attributed to developmental pathways factors as well as genetic links. Such residual downstream developmental effects are easy to conceptualise. If a young boy is very asocial for example, then his communication skills will not be practised with peers, so he is unlikely to develop as quickly in measures of communication as a more sociable child. The weak correlation between repetitive behaviours is harder to explain. Speculation is possible: repetitive behaviours have been shown to have both self-stimulatory as well as calming functions (Turner, 1999). Repetitive behaviours can therefore be interpreted as responses to unwanted stimuli, e.g. social stimuli with which autistic people have difficulty. Williams (1994) has given a first person account of use of repetitive behaviours to ameliorate the stress of social situations. Conversely, the need for stimulatory repetitive behaviours, concentrating on drawing lines or circles for example, may interfere with social opportunities. Weak associations do not confirm or deny genetic co-inheritance. Developmental pathways where one type of behaviour leads to another may also provide a partial explanation.

In a different but related developmental scenario, Cheslack-Postava and Jordan -Young (2012) suggest that a child's upbringing is highly gendered, and proposed a gendered embodiment model for autism. They cite numerous studies illustrating that the nature of parenting in particular depends on the gender of the child. This they use to describe a gendered theory of development of autism, although the model could also explain the large predominance of boys with other developmental disorders. Cooper (2001) suggests boys are socialized to encourage competition and activity thus a conflict between passivity required at western schools and masculine identity is generated. Some behaviours associated with ADHD when used excessively in school environments, climbing trees for example, are encouraged more often in boys than girls. Cheslack-Postava and Jordan -Young suggest such gendered social processes interact with biology to promote certain 'disordered' behaviours. This they call the 'pervasive developmental environment'.

As well as downstream developmental models, some theorists have suggested one cognitive deficit may underlie several symptomatic behaviours. Although the cognitive/psychological theories of dyslexia and autism seem quite distinct, some research does suggest children with both ADHD and dyslexic difficulties show a distinctive deficit in rapid naming speed, so it may that processing speed underlies the link (Bental \& Tirosh, 2007).

A second example is provided by executive function which is impaired in both autism and ADHD (Willcutt et al, 2005). According to some models, an underlying impairment in executive function prevents children from coordinating information processing in the brain, and 
prevents the transfer attention from one thing to another. It is easy to understand how this absence may translate into symptoms of either autism, due to inability to plan with strategic overarching vision, and hence reliance on routines, or as inattention and distractibility symptomatic of ADHD. Executive functions are neuropsychological processes needed to sustain problem-solving toward a goal. Executive functions allow a resolution of conflict when two responses are simultaneously called for by stimuli. In the laboratory, the Stroop task is an example. The conflicting combination of a word like red written in green ink creates conflict when the task is to say the color of the ink (green), due to the overlearned reading response that automatically elicits the response based on the meaning of the word (red). Executive function allows for the inhibition of the overlearned response and the execution of a response that is more appropriate given the context. Research has confirmed the involvement of deficits in executive functions that are essential for effective self-regulation in people with ADHD. The mental processes most often listed as being part of the notion of executive function are quite diverse so there is no standardized definition. They include: inhibition, resistance to distraction, self-awareness, working memory, emotional self-control, and even self-motivation. Bramham and colleagues (2009) found that both adults with ASD and ADHD had impaired executive function, although they did have distinctive profiles. Nyden and colleagues found that children with Asperger's Syndrome and dyslexia did not differ in tests of executive function: they could not establish any test of executive function that captured the differences in these disorders (1999).

Russell Barkley (2012) conceptualizes executive control as the methods of self-regulation. He writes entertainingly on how a person might use executive functions to resist the temptation to buy a tempting pastry from a shop:

...avert your eyes from the counter, walk to a different section of the shop away from the tempting goodies, engage yourself in mental conversation about why you need to not buy those products, and even visualize an image of the new slenderer version of yourself you expect to achieve in the near future. All of these are self-directed actions you are using to try and alter the likelihood of giving into temptation and therefore increase your chances of meeting your goal of weight loss this month. This situation calls upon a number of distinct yet interacting mental abilities to successfully negotiate the situation. You have to be aware that a dilemma has arisen when you walked into the shop (self-awareness), you have to restrain your urge to order the pastry to go with the coffee you have ordered (inhibition), you redirected your attention away from the tempting objects (executive attention or attentional management), you spoke to yourself using your mind's voice (verbal self-instruction or working memory), and you visualized an image of your goal and what you would look like when you successfully attain it (nonverbal working memory, or visual imagery). You may also have found yourself thinking about various other ways you could have coped effectively with these 
temptations (problem-solving), and may have even used words of encouragement toward yourself to enhance the likelihood that you would follow your plan (self-motivation).

Barkley explains that these and other mental activities are usually included in the understanding of human self-regulation, and it is difficulties in these areas (which are processes in executive function) that may lead to ADHD. Children with ADHD are distractible and selfregulation, the ability to override incoming stimuli, to see the bigger picture and lack the ability to see the consequences of their future actions. Children with ASD have difficulties transferring attention from one thing to another because they also lack overview (and implications of their actions in the future).

Gooch, Snowling and Hulme (2011) note that deficits in time perception (the ability to judge the length of time intervals) have been found in children with both dyslexia and ADHD. These researchers found children with comorbid dyslexia and attention problems performed poorly on measures of executive function as well as on phonological tasks. However, their results were interpreted as the effect of independent underlying cognitive causes. Although deficits in duration discrimination were associated with both dyslexia and attention problems, they concluded the results supported the claim that the two disorders are products of different cognitive defects originating from shared genes with pleiotropic effects.

Developmental models explain comorbidity of developmental disorders by shared cognitive deficits, either as 'gateways' as in Frith and Happés (1998) model, where one difficulty leads to another later in life, or as underlying shared deficits, for example impaired executive function causing both autism and ADHD. The alternative model suggests that cognitive difficulties associated with each disorder are distinct, but multiple cognitive deficits arise from similar genetic/environmental origins. All these theories have some empirical support.

\section{Diagnostic substitution and the influence of society and culture}

When symptoms of two or more conditions are shared, whatever the psychological mechanisms (whether or not there are shared underlying cognitive deficits, and /or genetic and neurological differences) then the area of functioning that is highlighted as a problem may depend on which tests are administered. In our recent research we followed a six year old child who was assessed by three educational psychologists and one multidisciplinary team, each blind to the findings of the others. One concluded that the child had dyspraxia, two that the child had dyslexic difficulties, and a third that borderline AS was likely. We interpreted these differences in the use of diagnostic labels as dependent on settings that varied during assessments, and assessment methods that exposed different types of behaviour (Russell, Norwich, \& Gwernan-Jones, 2012). This work suggests that which diagnosis is assigned depends to some extent on social and cultural factors as well as actual symptoms. If a child has symptoms of several disorders, then one context or test may draw out symptoms associated with one disorder, whereas another setting may expose symptoms of another. 
Thus for co-occurring symptoms it is difficult to differentiate between disorders and the likelihood that a co-morbid disorder will be missed is increased. This emphasizes the need for assessment in multiple settings and reassessment over time.

One of the most compelling cross cultural descriptions of how autism is regarded across various cultures was the book Unstrange Minds. Written by the anthropologist Roy Grinker (2008), Grinker explains how the category of ASD is contingent on the culture through which it is expressed- the condition is associated with differing levels of stigma in different cultures. In the US, several studies have also shown that clinicians may diagnose ASD when resources are targeted at the diagnosis, whereas previously, under other circumstances, they may have diagnosed another category of childhood disorder. Paul Shattuck has written about the extent to which increases in the administrative prevalence of autism have been associated with corresponding decreases in the use of other diagnostic categories, mental retardation and learning disabilities (2006). This process of 'diagnostic substitution' he argues, may partially explain the rise in prevalence in autism in the US.

Our own work suggests that since the 1980s, the recorded prevalence of both ASD and ADHD in the UK has increased dramatically. We examined data from both the Millennium Cohort Study, (the large cohort of around 19,000 children who have been followed from their birth through to seven years old and beyond), and another cohort, called the British Cohort Study, where children were born thirty years previously. Both cohorts were representative of the UK as a whole, and medical reports of both ASD and ADHD were given when children were age seven for in 2007-9 and ten in 1980. The results from 2007 contrasted with the 1980 sample at age 10. Only 11 children in the 1970 British Cohort Study were reported as having ADHD in their medical exam, giving an estimated prevalence of $0.083 \%$. The autism diagnosis was rarely used with just 3 children assigned the label; $0.023 \%$ of children. A number of other child psychiatric diagnoses were available and many of these were diagnosed during the medical exams. Details of these alternative labels are given in Table 1.

\begin{tabular}{lcc}
\hline 1980 Diagnosis (ICD 9 codes) & N of children & Percentage of total examined \% \\
\hline Autism (299.0/1/8/9) & 3 & 0.023 \\
\hline ADHD (314.00/01, 314.9) & 11 & 0.083 \\
\hline Disturbance in emotions (313) & 7 & 0.053 \\
\hline $\begin{array}{l}\text { Delays in development: Reading } \\
\text { (315.0) }\end{array}$ & 13 & 0.098 \\
\hline $\begin{array}{l}\text { Delays in learning \& development } \\
\text { (315.2/8/9/5) }\end{array}$ & 81 & 0.614 \\
\hline $\begin{array}{l}\text { Delays in language } \\
\text { (315.3) }\end{array}$ & 62 & 0.462 \\
\hline $\begin{array}{l}\text { Impulse control (312.3/9) } \\
\text { Mild mental retardation }\end{array}$ & $\begin{array}{l}\text { (1 autism co-morbid) } \\
\text { (317) }\end{array}$ & 0.007 \\
\hline $\begin{array}{l}\text { Other specified delays in } \\
\text { development (318) }\end{array}$ & (1 ADHD co-morbid) & 0.258 \\
\hline
\end{tabular}




\begin{tabular}{lcc}
\hline $\mathbf{1 9 8 0}$ Diagnosis (ICD 9 codes) & N of children & Percentage of total examined \% \\
\hline $\begin{array}{l}\text { Unspecified delays in development } \\
(319)\end{array}$ & 25 & 0.379 \\
\hline Total & (1 ADHD co-morbid) & 1.961 \\
\hline
\end{tabular}

Table 1. Named conditions using ICD-9 categories for 10 year old children in 1980 ( $n=13201)$.

Among the 14,043 children in the 2007 cohort, 209 (1.49\%) were reported to have ASD, and $180(1.28 \%)$ were reported having been given an ADHD diagnosis by a clinician (unweighted figures). There was disproportional stratification in the Millennium Cohort, meaning that all analyses were weighted to account for the clustering and over-inclusion of participants from disadvantaged areas. After weighting, $1.7 \%$ of children were reported as having an ASD (95\% CI, 1.4-1.99). 1.3\% of these were boys, and $0.25 \%$ girls, giving boy girl ratio of approx 5:1 for ASD. Surprisingly, the figure for ADHD was lower. After weighting, $1.4 \%$ of the population were reported as having ADHD (95\% CI, 1.2-1.7). Of these, $2.3 \%$ were boys and $0.25 \%$ girls, giving a gender ratio of approximately of 1 girl to every 4 boys with ADHD.

One interpretation of the historical shift is that diagnostic substitution has occurred: children with similar symptoms in 1980 may have been more likely to receive generalised labels of 'delays in learning \& development' than ASD or ADHD. So changing diagnostic practice, cultural factors and context may do much to explain both co-morbidity and rising prevalence. The steep rise in children assigned these diagnoses cannot be totally explained by the substitution mechanism- twice as many children were given either ASD or ADHD diagnoses in 2009 as the total number diagnosed with any type of developmental disorder in 1980 .

Context also has a big part to play in the identification of difficulties, in terms of what is considered to be 'disordered'. Social constructionists have also pointed out that the conceptualization of difficulties associated with both dyslexia and ASD as 'disorders' is itself a product of social and cultural standards, and of course the definition of each disorder has changed over time. This has prompted calls for the term autism spectrum 'conditions' to replace autism spectrum 'disorders' (2009). Our own analysis of the Millennium Cohort has shown a strong association between ADHD and poverty, reflecting findings from US studies which have also found differing levels of ADHD amongst various ethnic groups- Hispanic children were more likely to be identified with ADHD in a study by Akinbami et al. (2011). It is unclear whether this is entirely due to greater awareness and access to health care in some groups, differential reporting about the same level of difficulties between ethnic groups or whether children in different groups have truly varying symptom levels (Boyle et al., 2011). A study by Cuccaro et al. (1996) showed the nature of diagnosis of developmental disorders varied according to the socio-economic status of the child's family; autism was more likely to be identified in children of higher income families, although no biases of SES were found for identification with ADHD. Cooper (2001) points out that the behaviour symptomatic of ADHD becomes problematic where high value is placed on ability to remain sedentary and sustain attention on tasks, in other words, in schools. Hulme and Snowling (2009) describe how differences of this nature must therefore be thought of as both biological and as a product of the social and environmental world. 


\section{Conclusion}

Two conclusions can be drawn. First, co-morbidities between developmental disorders are common, and second, the causes of these overlapping difficulties are likely to be complex, multifactorial and interacting. Firstly, the high overlap between symptoms of different developmental disorders has been identified in a number of studies and there is an international consensus on this overlap. Studies from Canada, the UK, USA and Scandinavia all show how hard it is provide an unequivocal diagnosis, leading to the quote from Kaplan and her colleagues (2001) in developmental disorders co-morbidity is the rule, not the exception. This was informed by the group's work studying a population-based sample of 179 children receiving special support in Calgary: If the children met the dyslexia criteria, there was a $51.6 \%$ chance of having another disorder. If the children met the ADHD criteria there was an $80.4 \%$ chance of having another disorder. They criticize the term 'comorbidity', as it implies unsubstantiated presumption of independent aetiologies. The authors argue that discrete categories do not exist in real life.

Secondly, in considering the reasons for co-morbidities, a complex bio-psycho-social model is required that leads to symptoms that may result in diagnosis. The nature of the diagnosis itself may depend on social context as well as an individual child's behaviour. A hint of this complexity is achieved in Figure 3, which is a schematic diagram of various potential causal pathways. It is plausible that the same underlying genetic or neurological mechanisms may underlie co-occurrence of dyslexia, ADHD and ASD. The reverse pathways are not at first so obvious. But recent advances in systems biology have shown that the environment of the cell affects gene expression and protein synthesis at molecular levels. Thus environmental influences can alter 'core' biology: for example Mack and Mack (1992) describe how tweaking rats' whiskers changes gene expression in the sensory cortex. In systems theory, genetic influences are conceptualised more like a set of piano keys on which notes may be played or not played, played slowly or quickly, and there is enormous variation in the music produced even with the same basic set of keys. So the cellular environment can affect genetic expression. A simplified model underlying much behaviour genetics research envisages a direct linear relationship between individual genes and behaviours. The reality is likely to be far more complex with gene networks and multiple environmental factors impacting brain development and function, which in turn will influence behaviour (Hamer, 2002). Karmiloff-Smith (2007) emphasizes how learning and experience effects gene expression in humans. Such scholars demonstrate that the social can affect the biological as well as the more intuitive path of genetic origin leading to neurological development leading to aberrant behaviour. Diagnosis itself may influence behaviour too, through differential treatment and interventions. Thus the pervasive developmental environment is composed of many related factors, environmental stresses, and genetic predispositions, and the social contexts all of which may interact to produce developmental outcomes that themselves may contribute to predicting ongoing child development.

Snowling (2012) suggests a new dimensional classification of disorder, where deficits in different components of learning are seen as additive, impacting on the potential for remedia- 
tion, rather than classing children into dichotomous 'disorder' categories. Taylor (2011) notes that for many children, it is better to think of changes in cognitive style, learning and motivation rather than symptoms. Both conclude that it is important to examine children for evidence of co-occurring disorders, and not simply continue to examine the areas which we expect to be impaired according to categorization. The practical application of assessing children for a range of difficulties is that children will be best helped not by any all encompassing diagnosis, but by individual analysis of their strengths and weaknesses. Future research may be wise to focus on the individual profiles of children across a broad range of areas, looking at the unique strengths, as well as the weaknesses of the individual children, so that parents and educators may adapt their support accordingly, regardless of the diagnostic label a child receives.

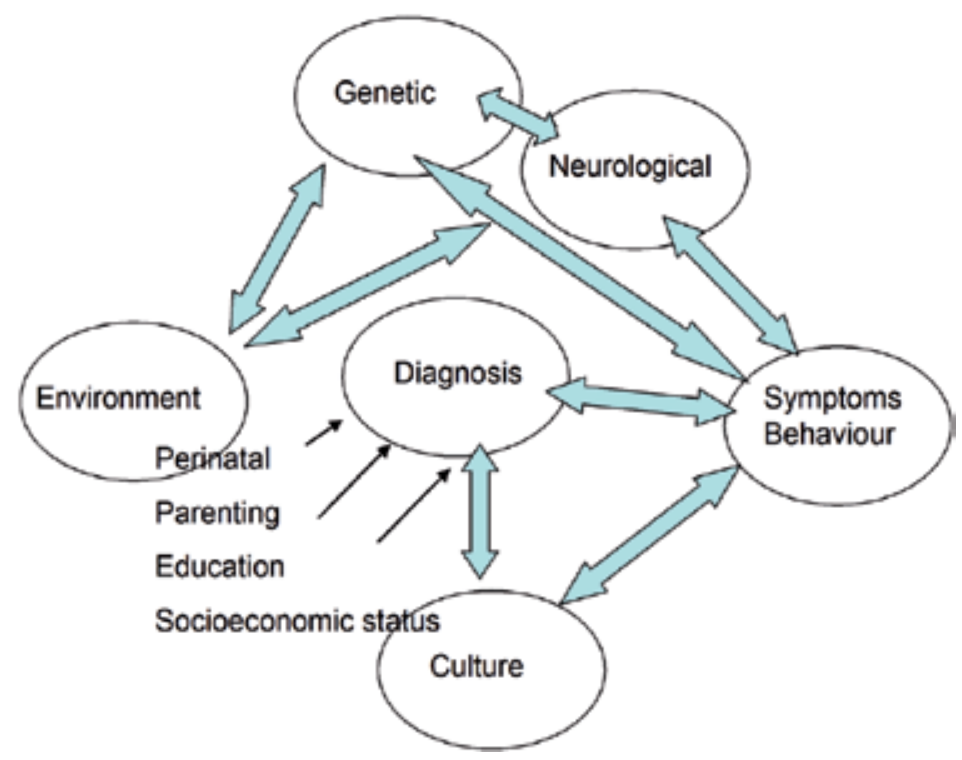

Figure 3. Schematic of interacting causal mechanisms for co-morbidity.

\section{Author details}

Ginny Russell ${ }^{1}$ and Zsuzsa Pavelka ${ }^{2}$

1 University of Exeter Medical School, ESRC Centre for Genomics in Society, UK

2 University of Milan, Italy 


\section{References}

[1] Akinbami, L. J., Liu, X., Pastor, P. N., \& Reuben, C. A. (2011). Attention deficit hyperactivity disorder among children aged 5-17 years in the United States, 1998-2009. NCHS Data Brief, (70), 1-8.

[2] American Psychiatric Association. (2000). Diagnostic and Statistical Manual of Mental Disorders (4th ed., text revision [DSM-IV-TR]

[3] Bailey, A., \& Parr, J. (2003). Implications of the broader phenotype for concepts of autism. Novartis Foundation symposium, 251, 26-35; discussion 36-47, 109-11, 281-97.

[4] Barkley, R. A. (2012). Executive Functions: What They Are, How They Work, and Why They Evolved (1st ed.). Guilford Press.

[5] Baron-Cohen, S, Leslie, A. M., \& Frith, U. (1985). Does the autistic child have a 'theory of mind'? Cognition, 21(1), 37-46.

[6] Baron-Cohen, Simon. (2002). The extreme male brain theory of autism. Trends Cogn Sci, 6(6), 248-254.

[7] Baron-Cohen, Simon, Scott, F. J., Allison, C., Williams, J., Bolton, P., Matthews, F. E., \& Brayne, C. (2009). Prevalence of autism-spectrum conditions: UK school-based population study. The British Journal of Psychiatry, 194(6), 500-509.

[8] Bental, B., \& Tirosh, E. (2007). The relationship between attention, executive functions and reading domain abilities in attention deficit hyperactivity disorder and reading disorder: A comparative study. Journal of Child Psychology and Psychiatry, 48(5), 455-463.

[9] Bolton, P. F., Murphy, M., Macdonald, H., Whitlock, B., Pickles, A., \& Rutter, M. (1997). Obstetric complications in autism: consequences or causes of the condition? Journal of the American Academy of Child and Adolescent Psychiatry, 36(2), 272-81.

[10] Boyle, C. A., Boulet, S., Schieve, L. A., Cohen, R. A., Blumberg, S. J., Yeargin-Allsopp, M., Visser, S., et al. (2011). Trends in the prevalence of developmental disabilities in US children, 1997-2008. Pediatrics, 127(6), 1034-1042.

[11] Bradley, E. A., Summers, J. A., Wood, H. L., \& Bryson, S. E. (2004). Comparing rates of psychiatric and behavior disorders in adolescents and young adults with severe intellectual disability with and without autism. Journal of Autism and Developmental Disorders, 34(2), 151-61.

[12] Bramham, J., Ambery, F., Young, S., Morris, R., Russell, A., Xenitidis, K., Asherson, P., et al. (2009). Executive functioning differences between adults with attention deficit hyperactivity disorder and autistic spectrum disorder in initiation, planning and strategy formation. Autism, 13(3), 245-264. 
[13] British Psychological Society. (1999). Working party of the division of educational and child psychology of the British Psychological Society: Dyslexia, literacy and psychological assessment. British Psychological Society : Leicester.

[14] Cheslack-Postava, K., \& Jordan-Young, R. M. (2012). Autism spectrum disorders: Toward a gendered embodiment model. Social Science \& Medicine (1982), 74(11), 16671674.

[15] Cheung, C. H. M., Wood, A. C., Paloyelis, Y., Arias-Vasquez, A., Buitelaar, J. K., Franke, B., Miranda, A., et al. (2012). Aetiology for the covariation between combined type ADHD and reading difficulties in a family study: The role of IQ. Journal of Child Psychology and Psychiatry,, 53(8), 864-873.

[16] Clark, T., Feehan, C., Tinline, C., \& Vostanis, P. (1999). Autistic symptoms in children with attention deficit-hyperactivity disorder. European Child $\mathcal{E}$ Adolescent Psychiatry, $8(1), 50-55$.

[17] Coltheart, M., \& Jackson, N. E. (1998). Defining Dyslexia. Child and Adolescent Mental Health, 3(1), 12-16.

[18] Constantino, J. N., \& Todd, R. D. (2003). Autistic traits in the general population: A twin study. Archives of general psychiatry, 60(5), 524-30.

[19] Cooper, P. (2001). Understanding ADHD: A brief critical review of literature. Children \& Society, 15(5), 387-95.

[20] Cuccaro, M. L., Wright, H. H., Rownd, C. V., Abramson, R. K., Waller, J., \& Fender, D. (1996). Professional perceptions of children with developmental difficulties: The influence of race and socioeconomic status. Journal of Autism and Developmental Disorders, 26(4), 461-469.

[21] Daniels, J. L., Forssen, U., Hultman, C. M., Cnattingius, S., Savitz, D. A., Feychting, M., \& Sparen, P. (2008). Parental psychiatric disorders associated with autism spectrum disorders in the offspring. Pediatrics, 121(5), e1357-1362.

[22] Danielsson, S., Gillberg, I. C., Billstedt, E., Gillberg, C., \& Olsson, I. (2005). Epilepsy in young adults with autism: a prospective population-based follow-up study of 120 individuals diagnosed in childhood. Epilepsia, 46(6), 918-23.

[23] Deykin, E. Y., \& MacMahon, B. (1980). Pregnancy, delivery, and neonatal complications among autistic children. American Journal of Diseases of Children, 134(9), 860-864.

[24] Dulcan, M. (1997). Practice parameters for the assessment and treatment of children, adolescents, and adults with attention-deficit/hyperactivity disorder. Journal of the American Academy of Child and Adolescent Psychiatry, 36(10 Suppl), 85S-121S.

[25] Elia, J., Ambrosini, P. J., \& Rapoport, J. L. (1999). Treatment of attention-deficit-hyperactivity disorder. New England Journal of Medicine, 340(10), 780-788.

[26] Ericson, R. V., \& Doyle, A. (2003). Risk and Morality. University of Toronto Press. 
[27] Evans, D. W., Canavera, K., Kleinpeter, F. L., Maccubbin, E., \& Taga, K. (2005). The fears, phobias and anxieties of children with autism spectrum disorders and Down syndrome: Comparisons with developmentally and chronologically age matched children. Child Psychiatry and Human Development, 36(1), 3-26.

[28] Fawcett, A. (2012). Introduction to Dyslexia and Co-occuring Difficulties. Dylexia and co-occuring difficulties. Bracknell, UK: British Dyslexia Association.

[29] Felicetti, T. (1981). Parents of autistic children: Some notes on a chemical connection. Milieu Therapy, 1, 13-16.

[30] Freitag, C. M. (2007). The genetics of autistic disorders and its clinical relevance: A review of the literature. Molecular Psychiatry, 12(1), 2-22.

[31] Frith, U. (2003). Autism: Explaining the Enigma (2nd Ed.). Oxford, UK: Wiley-Blackwell.

[32] Frith, U., \& Happé, F. (1998). Why specific developmental disorders are not specific: On-line and developmental effects in autism and dyslexia. Developmental Science, 1(2), 267-272.

[33] Gillberg, C. (2010). The ESSENCE in child psychiatry: Early Symptomatic Syndromes Eliciting Neurodevelopmental Clinical Examinations. Research in developmental disabilities, 31(6), 1543-1551.

[34] Gooch, D., Snowling, M., \& Hulme, C. (2011). Time perception, phonological skills and executive function in children with dyslexia and/or ADHD symptoms. Journal of Child Psychology and Psychiatry, 52(2), 195-203.

[35] Goodman, R., \& Scott, S. (1997). Child Psychiatry. (2nd ed.). Oxford, UK: Blackwell Publishing.

[36] Griffiths, C. C. B. (2007). Pragmatic abilities in adults with and without dyslexia: A pilot study. Dyslexia, 13(4), 276-296.

[37] Grinker, R. R. (2008). Unstrange minds: Remapping the world of autism. Cambridge, MA: Basic Books.

[38] Hamer, D. (2002). Genetics: Rethinking behavior genetics. Science, 298(5591), 71-72.

[39] Happé, F. G. (1994). Current psychological theories of autism: The 'theory of mind' account and rival theories. Journal of Child Psychology and Psychiatry, 35(2), 215-29.

[40] Happé, F., Ronald, A., \& Plomin, R. (2006). Time to give up on a single explanation for autism. Nature Neuroscience, 9, 1218-1220.

[41] Happé, Francesca, \& Frith, U. (2006). The weak coherence account: detail-focused cognitive style in autism spectrum disorders. Journal of Autism and Developmental Disorders, 36(1), 5-25.

[42] Hedgecoe, A. (2001). Schizophrenia and the narrative of enlightened geneticization. Social Studies of Science, 31(6), 875-911. 
[43] Hill, E. L. (2004). Executive dysfunction in autism. Trends in Cognitive Sciences, 8(1), 26-32.

[44] Hofvander, B., Delorme, R., Chaste, P., Nydén, A., Wentz, E., Ståhlberg, O., Herbrecht, E., et al. (2009). Psychiatric and psychosocial problems in adults with normalintelligence autism spectrum disorders. BMC Psychiatry, 9, 35.

[45] Howlin, P. (2000). Outcome in adult life for more able individuals with autism or Asperger syndrome. Autism, 4(1), 63-83.

[46] Hulme, C., \& Snowling, M. J. (2009). Developmental disorders of language learning and cognition. Chichester, UK: Wiley-Blackwell.

[47] Iacoboni, M., \& Dapretto, M. (2006). The mirror neuron system and the consequences of its dysfunction. Nature reviews. Neuroscience, 7(12), 942-51.

[48] Ingudomnukul, E., Baron-Cohen, S., Wheelwright, S., \& Knickmeyer, R. (2007). Elevated rates of testosterone-related disorders in women with autism spectrum conditions. Hormones and Behavior, 51(5), 597-604.

[49] Kadesjö, B., Gillberg, C., \& Hagberg, B. (1999). Brief Report: Autism and Asperger syndrome in seven-year-old children: A total population study. Journal of Autism and Developmental Disorders, 29(4), 327-331.

[50] Kaplan, B. J., Dewey, D. M., Crawford, S. G., \& Wilson, B. N. (2001). The term comorbidity is of questionable value in reference to developmental disorders: Data and theory. Journal of Learning Disabilities, 34(6), 555-565.

[51] Karmiloff-Smith, A. (2007). Atypical epigenesis. Developmental Science, 10(1), 84-88.

[52] Kessler, R. C., Adler, L., Ames, M., Demler, O., Faraone, S., Hiripi, E., Howes, M. J., et al. (2005). The World Health Organization adult ADHD self-report scale (ASRS): a short screening scale for use in the general population. Psychological Medicine, 35(2), 245-256.

[53] Kielinen, M., Rantala, H., Timonen, E., Linna, S.-L., \& Moilanen, I. (2004). Associated medical disorders and disabilities in children with autistic disorder: A populationbased study. Autism, 8(1), 49-60.

[54] Kolevzon, A., Gross, R., \& Reichenberg, A. (2007). Prenatal and perinatal risk factors for autism: A review and integration of findings. Archives of Pediatrics $\mathcal{E}$ Adolescent Medicine, 161(4), 326-33.

[55] London, E. (2007). The role of the neurobiologist in redefining the diagnosis of autism. Brain pathology (Zurich, Switzerland), 17(4), 408-11.

[56] Mack, K. J., \& Mack, P. A. (1992). Induction of transcription factors in somatosensory cortex after tactile stimulation. Molecular Brain Research, 12(1-3), 141-147.

[57] Miles, T. R. (2004). Some problems in determining the prevalence of dyslexia. Electronic Journal of Research in Educational Psychology, 2(2), 5-12. 
[58] Mulligan, A., Anney, R. J. L., O’Regan, M., Chen, W., Butler, L., Fitzgerald, M., Buitelaar, J., et al. (2009). Autism symptoms in Attention-Deficit/Hyperactivity Disorder: A familial trait which correlates with conduct, oppositional defiant, language and motor disorders. Journal of Autism and Developmental Disorders, 39(2), 197-209.

[59] Mulligan, A., Anney, R., Butler, L., O’Regan, M., Richardson, T., Tulewicz, E. M., Fitzgerald, M. Gill, M. (2011). Home environment: association with hyperactivity impulsivity in children with ADHD and their non-ADHD siblings. Child: Care, Health and Development [e-pub ahead of print].

[60] Newschaffer, C. J., Croen, L. A., Daniels, J., Giarelli, E., Grether, J. K., Levy, S. E., Mandell, D. S., Miller, L.A., Pinto-Martin, J, Reaven, J., Reynolds, A.M., Rice, C.E., Schendel, D., Windham, G.C.(2007). The epidemiology of autism spectrum disorders. Annual Review of Public Health, 28, 235-58.

[61] Nydén, A., Gillberg, C., Hjelmquist, E., \& Heiman, M. (1999). Executive function/ attention deficits in boys with Asperger syndrome, attention disorder and reading/ writing disorder. Autism, 3(3), 213-228.

[62] Posserud, M.-B., Lundervold, A. J., \& Gillberg, C. (2006). Autistic features in a total population of 7-9-year-old children assessed by the ASSQ (Autism Spectrum Screening Questionnaire). Journal of Child Psychology and Psychiatry, 47(2), 167-75.

[63] Reiersen, A. M., Constantino, J. N., Grimmer, M., Martin, N. G., \& Todd, R. D. (2008). Evidence for shared genetic influences on self-reported ADHD and autistic symptoms in young adult Australian twins. Twin Research and Human Genetics, 11(6), 579585.

[64] Reiersen, Angela M, \& Todd, R. D. (2008). Co-occurrence of ADHD and autism spectrum disorders: phenomenology and treatment. Expert Review of Neurotherapeutics, $8(4), 657-669$.

[65] Richardson, A. J. (2006). Omega-3 fatty acids in ADHD and related neurodevelopmental disorders. International Review of Psychiatry, 18(2), 155-172.

[66] Ronald, A., Edelson, L. R., Asherson, P., \& Saudino, K. J. (2010). Exploring the relationship between autistic-like traits and ADHD behaviors in early childhood: Findings from a community twin study of 2-year-olds. Journal of Abnormal Child Psychology, 38(2), 185-196.

[67] Russell, G., Golding, J., Norwich, B., Emond, A., Ford, T., \& Steer, C. (2012). Social and behavioural outcomes in children diagnosed with autism spectrum disorders: A longitudinal cohort study. Journal of Child Psychology and Psychiatry, 53(7), 735-744.

[68] Russell, G., \& Kelly, S. (2011). Looking beyond risk: A study of lay epidemiology of childhood disorders. Health, Risk \& Society, 13(2), 129.

[69] Russell, G., Norwich, B., \& Gwernan-Jones, R. (2012). When diagnosis is uncertain: variation in conclusions after psychological assessment of a six-year-old child. Early Child Development and Care, (early on-line version). 
[70] Russell, G., Steer, C., \& Golding, J. (2011). Social and demographic factors that influence the diagnosis of autistic spectrum disorders. Social Psychiatry and Psychiatric Epidemiology, 46(12), 1283-1293.

[71] Rutter, M. (2005). Incidence of autism spectrum disorders: changes over time and their meaning. Acta paediatrica, 94(1), 2-15.

[72] Rutter, M., Andersen-Wood, L., Beckett, C., Bredenkamp, D., Castle, J., Groothues, C., Kreppner, J., et al. (1999). Quasi-autistic patterns following severe early global privation. English and Romanian Adoptees (ERA) Study Team. Journal of Child Psychology and Psychiatry, , 40(4), 537-549.

[73] Sanders, S. J., Murtha, M. T., Gupta, A. R., Murdoch, J. D., Raubeson, M. J., Willsey, A. J., Ercan-Sencicek, A. G., et al. (2012). De novo mutations revealed by wholeexome sequencing are strongly associated with autism. Nature.

[74] Santosh, P. J., \& Mijovic, A. (2004). Social impairment in hyperkinetic disorder - relationship to psychopathology and environmental stressors. European Child $\mathcal{E}$ Adolescent Psychiatry, 13(3), 141-150.

[75] Sciutto, M. J., Nolfi, C. J., \& Bluhm, C. (2004). Effects of child gender and symptom type on referrals for ADHD by elementary school teachers. Journal of Emotional and Behavioral Disorders, 12(4), 247-253.

[76] Shattuck, P. T. (2006). The contribution of diagnostic substitution to the growing administrative prevalence of autism in US special education. Pediatrics, 117(4), 1028-37.

[77] Simonoff, E., Pickles, A., Charman, T., Chandler, S., Loucas, T., \& Baird, G. (2008). Psychiatric disorders in children with autism spectrum disorders: prevalence, comorbidity, and associated factors in a population-derived sample. Journal of the American Academy of Child and Adolescent Psychiatry, 47(8), 921-929.

[78] Snowling, M. J. (2012). Editorial: Seeking a new characterisation of learning disorders. Journal of Child Psychology and Psychiatry 53(1), 1-2.

[79] Sonuga-Barke, E. J. S., \& Halperin, J. M. (2010). Developmental phenotypes and causal pathways in attention deficit/hyperactivity disorder: Potential targets for early intervention? Journal of Child Psychology and Psychiatry, 51(4), 368-389.

[80] Sturm, H., Fernell, E., \& Gillberg, C. (2004). Autism spectrum disorders in children with normal intellectual levels: Associated impairments and subgroups. Developmental Medicine and Child Neurology, 46(7), 444-7.

[81] Taylor, E. (2011). Commentary: Reading and attention problems - how are they connected? Reflections on reading McGrath et al. (2011). Journal of Child Psychology and Psychiatry, 52(5), 558-559.

[82] Trottier, G., Srivastava, L., \& Walker, C. D. (1999). Etiology of infantile autism: A review of recent advances in genetic and neurobiological research. Journal of Psychiatry and Neuroscience, 24(2), 103-15. 
[83] Turner, M. (1999). Annotation: Repetitive behaviour in autism: A review of psychological research. Journal of Child Psychology and Psychiatry, 40(6), 839-849.

[84] Willcutt, E. G., Doyle, A. E., Nigg, J. T., Faraone, S. V., \& Pennington, B. F. (2005). Validity of the executive function theory of attention-deficit/hyperactivity disorder: A meta-analytic review. Biological Psychiatry, 57(11), 1336-1346.

[85] Williams, D. (1992). Nobody nowhere: The extraordinary autobiography of an autistic (1st ed.). New York, NY: HarperCollins.

[86] World Health Organization. (1992). International Classification of Diseases and related health problems (ICD-10). Geneva, WHO. Retrieved from www.who.int/ classifications/icd/en/greenbook.pdf p.179

[87] Wright, C., Conlon, E., Wright, M., \& Dyck, M. (2011). Sub-lexical reading intervention in a student with dyslexia and Asperger's disorder. Australian Journal of Educational \& Developmental Psychology, 11, 11-25.

[88] Zwaigenbaum, L., Szatmari, P., Jones, M. B., Bryson, S. E., MacLean, J. E., Mahoney, W. J., Bartolucci, G., et al. (2002). Pregnancy and birth complications in autism and liability to the broader autism phenotype. Journal of the American Academy of Child and Adolescent Psychiatry, 41(5), 572-9. 
Chapter 18

\title{
Pre-Existing Differences in \\ Mothers of Children with Autism Spectrum \\ Disorder and/or Intellectual Disability: A Review
}

\author{
Jenny Fairthorne, Amanda Langridge, \\ Jenny Bourke and Helen Leonard \\ Additional information is available at the end of the chapter
}

http://dx.doi.org/10.5772/54488

\section{Introduction}

The autism spectrum disorders (ASD) represent a group of severe and chronic neuro-developmental disorders often simply referred to as autism. [1] Using the criteria provided by the Diagnostic and Statistical Manual of Mental Disorders, Fourth Edition, ASD are diagnosed by impairments within the three strands of DSM-4: social interaction, communication and repetitive behaviours or interests. [2] The aetiology of autism is complex. [3] Research has implicated a strong genetic basis [4-7] involving multiple genes [5, 7, 8] and possible gene-environment interactions. [9-13] Advances in chromosomal microarray analysis and gene sequencing technologies have improved diagnoses and suggest that aetiologies of ASD will continue to be uncovered. [9] In addition, a child presenting with autistic symptoms may be found to have a certain genetic mutation which accounts for their true underlying biological diagnosis. For example, a diagnosis of Rett syndrome would be confirmed when a girl with ASD and intellectual disability was found to have a mutation of the $M E C P 2$ gene on the X-chromosome. [14] Children with ASD and intellectual disability have been found to have an expansion of the FMR1 gene confirming a diagnosis of Fragile $X$ syndrome. [15]

Autism and intellectual disability commonly coexist with $30-80 \%$ of persons with ASD reported as also having ID. [16, 17] Currently, the relationship between ASD and comorbid ID is poorly understood. [18] However, it is known that phenotypically, persons with these disorders can be grouped into the three categories of ASD without ID, ASD with ID and ID only. [18] Intellectual disability (ID) is characterized by an intelligence quotient (IQ) of less than 70 which is associated with limitations in at least two areas of adaptive skill and which 
is manifest before 18 years. [19] The level of ID is generally grouped into the five levels of mild, moderate, severe, profound and unspecified by IQ score. In research it is common to stratify ID to the following three levels defined by the American Psychiatric Association [2] (Table 1).

\begin{tabular}{ll}
\hline Descriptor/level of ID & IQ score \\
\hline Mild or moderate ID & $35-40$ up to 69 points \\
\hline Severe or profound ID & $<35-40$ points \\
\hline Unspecified ID & $<70^{\star}$ \\
\hline
\end{tabular}

*Here the person has been assessed as having ID but has not been assessed adequately to determine the level.

Table 1. Levels of intellectual disability

In terms of aetiology, ID can be broadly divided into cases of known biomedical cause and those of unknown cause. The biomedical causes may be divided into genetic and non-genetic causes. Further subdivisions are given in Figure 1.

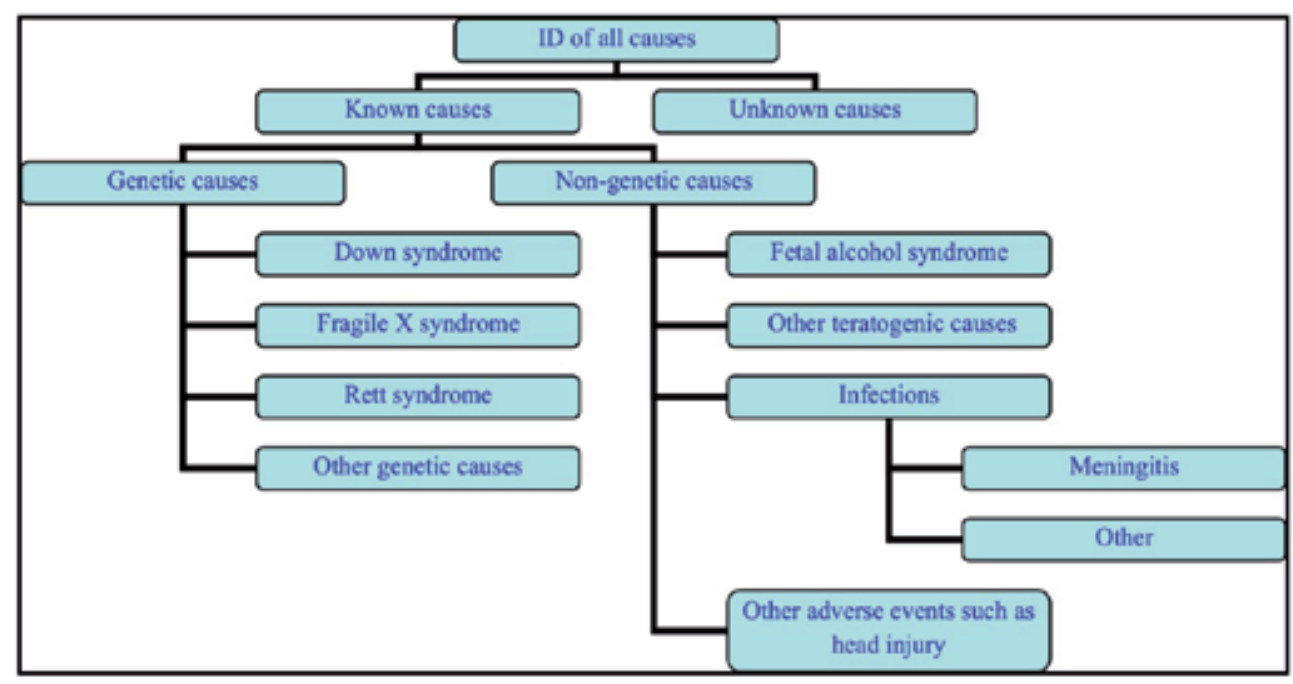

Figure 1. Commonly known aetiologies of intellectual disability

In addition to genetic and non-genetic causes of ASD and ID, relationships with sociodemographic factors such as a mother's education, [20,21] immigration, [17, 22] and ethnicity, [23] have also been identified. Other reported associations involve aspects of a mother's health including physical characteristics [24] physical [25, 26] and mental health [27, 28] and health behaviours. [29, 30]

It has also been reported that milder autistic traits are present in other family members of individuals diagnosed with ASD. This phenomenon has been coined the Broad Autism 
Phenotype [31] and includes qualitatively similar, but milder traits in areas such as language, personality and social behaviour. Some researchers believe that identification of the Broad Autism Phenotype in family members might provide a complementary strategy for detecting genes which contribute to the likelihood of ASD. [32,33] When comparing family members of a child with ASD to persons from the general population, subtle differences within the Broad Autism Phenotype could be associated with specific brain regions, particular neural pathways, and ultimately with particular genes. [33]

The above factors have been used as guides in choosing terms for our literature search to examine pre-existing characteristics of mothers of children with autism and mothers of children with intellectual disability of unknown cause. Inherent characteristics of mothers of children with a specific disability could be associated with the genetic, environmental or genetic-environmental aetiology of their child's condition. It is therefore important to separate pre-existing factors, particularly in relation to mental health, from morbidities such as depression [34] which might develop due to the more intense demands of caring for a child with ASD and/or ID.

The aim of this study is to review research on the pre-existing characteristics which differentiate mothers of children with ASD and/or ID of unknown cause from each other and from mothers of children without these disabilities. Such an investigation may help to further clarify the determinants of ASD and/or ID including the role of genetic and modifiable risk factors. Improving our understanding of the genetic and environmental causes of ASD and ID may reduce the future burden of these disabilities [35] by hastening the development of effective prevention and treatment strategies.

\section{Literature search and selection}

The papers considered for this review resulted from a search of the Medline, Web of Knowledge, Scopus and Google scholar databases. Combinations of the search terms below were chosen.

- Terms associated with ASD and/or ID: autis*, pervasive development disorder*, intellectual disability, mental retardation, disab*;

- Terms associated with ASD and/or ID aetiology: immigra*, migra*, ethnic ${ }^{*}$, age, sociodemographic, prenatal, perinatal, auto ${ }^{*}$, immun $^{*}$, anti $^{*}$, psych $^{*}$ and phenotype ${ }^{*}$; and

- Terms associated with mothers of children with ASD and/or ID: traits, characteristics, parents, mothers, children, persons.

A paper was included in the review if:

- It was accepted for publication between 1st January 1990 and 31st October, 2012 inclusive;

- It was a full text article in English;

- It described new research and was published in a peer-reviewed journal; 
- It described the results of a cohort, case-control, correlation or cross-sectional study of at least 15 subjects;

- It compared a characteristic of parents or mothers of children with ASD and/or ID with parents or mothers of children without disability or with a population norm;

- It assessed characteristics that were pre-existing and not likely to be a result of caring for a child with ASD and/or ID; and

- It used methods of ascertainment and measurement of the characteristic(s) of interest that were assessed as unlikely to lead to bias.

Eighty papers were retained for the review. We stress that these papers do not represent the entire literature pool in the area. Had we chosen different search terms or used different combinations of terms in our searches, the basis for our review would possibly have been different. The three categories for our analyses were; socio-demographic factors, immigrant status and ethnicity and health and physical characteristics and we sorted papers by these categories. An additional 61 articles were used to provide background information and possible explanations of some of the reported associations.

\section{Socio-demographic factors}

There are a number of considerations which impinge on the effect of socio-demographic factors on the prevalence of ASD and/or ID. Firstly, in persons with ASD with ID and those with just ID, the features overlap to some degree. A child with ASD with ID, particularly in the past, may often have been diagnosed with only ID as there have been secular changes in the identification of children with ASD. Secondly, persons who could be diagnosed with ASD without ID are most often able to function independently and may remain undiagnosed in a range of scenarios. Thirdly, the process through which children are assigned a diagnosis of ASD is much more complex than for ID. Whilst elsewhere, the gold standard might be the considered judgement of an expert clinician who had seen many patients with autism [36], in Western Australia, a diagnosis of ASD in a child requires an assessment by a team comprising a paediatrician, psychologist, and speech pathologist. Waiting times for this assessment can be prolonged in the Western Australian public system [37] and even longer in the US. [38] One effect of these considerations is that in some countries a child whose parents are socioeconomically disadvantaged may oft times be diagnosed with ID when a diagnosis of ASD could be more appropriate.

\subsection{Socio-economic status}

Thirteen articles researched the parental socio-economic status (SES) of children with ASD and/or ID [17, 20, 21, 39-48] and all but two [42, 43] supported a different association of SES with ASD than ID. Children with ASD were more likely to be from higher SES families but children with ID were more likely to be from lower SES families.

A range of measures of high SES were consistently associated with ASD. In a large telephone interview study in the US, family wealth was used as a measure of SES. [40] The researchers 
found that children from higher income families were more likely to have a diagnosis of ASD. Similarly, others using family income as a marker for SES, found a significant association between high family SES and ASD in the offspring. [47] Further analyses, using the dual markers of high family income and high maternal education, found a particular association between high SES and ASD without ID. [47] Using population data and deriving SES from mother's place of residence at time of the child's birth, Australian researchers also found that ASD, ASD with ID and particularly ASD without ID were associated with higher SES. [17]

The overall association between high SES and ASD without ID could result from the increased empowerment of parents of high SES to pursue a diagnosis where their children have a milder variant of ASD. [49] In families of low SES, higher functioning children with autistic traits might be informally labelled by family and contemporaries as unusual, difficult or emotionally damaged. In a comparable way, lower functioning children with autistic traits might be formally or informally given a diagnosis of ID. Others have suggested that children of lower SES parents might be more likely to be diagnosed at a later age than those of higher SES and hence not be included in studies of ASD and SES with lower ages of cut-off. [49]

Further evidence of the possible social contributions to the likelihood of an ASD diagnosis was found in a large multi-based national study in the US. [39] Undiagnosed children who met the criteria for ASD had a lower SES than children who had been previously diagnosed. [39] Arealevel SES indicators derived from census data were used in another study where the researchers elucidated that increasing SES and the increasing prevalence of ASD were associated in a dose-response fashion. [39]

King et al. [41] provided evidence that an interaction of social factors was affecting the likelihood of an ASD diagnosis. They examined factors influencing the likelihood of an ASD diagnosis using data on around five million births in Californian cohorts from 1992 to 2000. They found that an interaction between high and low level SES measures influenced the likelihood of an ASD diagnosis. Medi-Cal is a program providing medical assistance to the needy in California and these researchers used family use of Medi-Cal as a binary measure of SES. Property values in the area of a mother's residence were also used as a measure of SES. These researchers reported that children whose families were enrolled with Medi-Cal births and living in wealthier neighbourhoods were two and a half times more likely to receive a diagnosis of ASD than their counterparts living in poorer areas. [41] This could indicate that for parents of limited resources, living in a higher SES neighbourhood had benefits in terms of the likelihood of their child being diagnosed with ASD. Possibly, this results from the parents' increased access to support persons such as paediatricians and child health nurses and to educational programs such as parent classes and interventions for children, compared to that of similar parents in less affluent areas.

In contrast, a Danish study accessing linked population data, used maternal education and parental wealth as a measure of SES and found no association between SES and ASD diagnosis. [42] In neighbouring Sweden, a population-based study published in 2012, used low income, manual occupation and less education as measures of low SES. The researchers concluded that low, not high SES, was a risk factor for ASD. [43] There may be a number of reasons for the differing findings of these studies. The universal health-care and routine screenings offered in 
Denmark and Sweden may eliminate the ascertainment bias associated with high SES which may exist in other Western countries. [43]

By comparison with ASD, low SES was often identified as a risk factor for ID [21, 44-46, 48] and especially mild or moderate ID. [17, 20,48] One of these studies was a cross-sectional study of over five million children. [48] It concluded that children with mild or moderate ID had an increased risk of exposure to social conditions which were detrimental to their development. [48] Another study examined SES and ID prevalence in the 1966 and 1985-6 Finnish birth cohorts. [46] The researchers concluded that the association of low SES with ID was present in both cohorts. Plausible hypotheses for this persisting association are that there had been no improvements in antenatal and obstetric care in those of lower SES over the twenty years in question or, alternatively, there is a prominent genetic involvement in the aetiology of ID. Another, is that the higher risk of exposure to a developmentally unfavourable environment has persisted over the 20 year interval in the children of mothers of lower SES. [48]

In total, ten studies [20, 21, 23, 41, 45, 47, 50-53] used education alone as a measure of SES. All four of the studies investigating ASD reported positive associations between high maternal education and the risk of ASD in the offspring. Three of these studies were from California and each reported that parents of children with ASD were more educated than the general population. [21, 41, 50] The fourth reported that mothers with more than 16 years education were more than twice as likely to have a child with ASD without ID than mothers of a child with only 12 years education. [47] The relationship was reversed with maternal education and ID where all research ascertained a negative association between high maternal education and the risk of ID in the offspring. For instance, with children with unspecified ID [20, 21, 23, 45, 51,53] and developmental delay without ASD [24](which may include those with known genetic syndromes), seven studies concluded that their mothers were of a lower educational status. One of these, a population study, established that mothers of children with ID were less likely to have more than 13 years of education. [23]

The association of maternal education with varying levels of ID has been investigated including for severe ID and on the basis that risk factors for Down syndrome differed from those of other forms of ID, children with Down syndrome were excluded. Mothers of children with severe ID were found to be more likely to have a lower educational status than mothers in the general population. [52] Comparable results were found for mothers of children with mild or moderate ID [20, 21] of unknown cause. These mothers had increased odds of a lower educational status than mothers in the general population. One of these studies used Californian service agency records and a sample of more than 27000 mothers of children with mild or moderate ID or severe or profound ID. [21] Less maternal education was also associated with an increased risk of severe or profound ID in the offspring.

\subsection{Marital status}

Four papers, describing five studies, examined marital status in relation to the odds of ASD and/or ID. [17, 20, 46, 54] At the time of their child's birth, it is uncertain whether a woman's marital status is associated with her odds of a child with ASD. However, mothers of children with ID were more likely to be without partners. 
In Australia, a retrospective cohort study, using linked health registries assessed marital status in terms of living with a partner. They reported that at their child's birth, women living with a partner were $35 \%$ more likely to have a child with ASD and particularly ASD with ID. [17] On the other hand, a similar Canadian study found that mothers not living with a partner at the time of their child's birth were 19\% more likely to have a child with ASD than those mothers who were living with a partner. [54]

With ID, women without a partner had increased odds of having a child with ID [17] and particularly mild or moderate ID. [20] Similarly, a cohort study using UK data, concluded that compared to typically developing children, those with early cognitive delay were less likely to have their biological parents living together during the first five years of their lives compared to families with a typically developing child. [55] However, in Finland, the negative association between living with a partner and the odds of ID in the offspring, present in a 1966 birth cohort, was absent in the 1985-6 cohort. [46] The reduction of the association in the second cohort may have been a reflection of the improved SES of single mothers over the 20 year period.

\subsection{Parental age}

In most studies, increasing maternal age, sometimes along with increasing paternal age, was associated with ASD. A minority of studies found relationships only with paternal age or found no association with either maternal or paternal age. Contrasting results were reported with ID where teenage mothers were more likely to have children with mild or moderate ID were older mothers and particularly likely to have children with severe or profound ID. Socio-demographic and biological explanations are offered.

All ten studies investigating the association of maternal age with the prevalence of ASD found that advanced maternal age was associated with an increasing prevalence of ASD [17, 29, 47, 56-61] and sometimes ASD without ID. [17, 47] Four of these studies, reported an additional association with paternal age. [17, 56, 58, 61] For instance, a population-based study using data from multiple sites throughout the US, found associations with both maternal and paternal age after adjustment for the other parent's age, birth order and maternal education. [58]

Five of the cited studies specifically reported an association between paternal but not maternal age and ASD in the offspring. [58,62-65] One of these studies was a small Japanese case-control study of 84 father-child dyads. The researchers reported that advanced parental age was associated with nearly twice the risk of ASD without ID. [65] Another was a population-based Israeli cohort study which used data from a medical registry. [63] The remaining studies used population data from Sweden and another, population data from Denmark. [62, 64] After an adjustment for maternal age, the Swedish researchers identified a linear association of increasing paternal age and the risk of ASD. These researchers commented that if no adjustment was made for paternal age it would appear as though maternal age, rather than paternal was the risk factor for ASD. They added that paternal age could be a risk factor as generally the male was considered to be the origin of new mutations in the gene pool and their production increased with age. [62] 
By comparison, three studies from Northern Europe, and UK identified that neither of advancing maternal nor advancing paternal age was a risk factor for ASD. [42, 43, 66] One of the studies from Denmark and another from Sweden used linked data from national registries. $[42,43]$ The third was a much smaller UK study of around 5000 participants and parents provided data by completing self-reports. As with broader measures of SES, the results from Denmark and Sweden might reflect the model of health service provision in Scandinavia. Moreover, there is evidence that children with ASD are diagnosed later in younger mothers. [67] Thus there may be a bias of ascertainment in some studies where younger children are included. In the UK study, [66] younger mothers may been included more often since they were recruited when pregnant. Further, a diagnosis of ASD was not required for their child but instead, a parent completed the Social and Communication Disorders Checklist. In other studies from the US, [41, 47, 58, 59] Canada [29] and Australia, [17, 57] ASD may be underascertained in the children of younger parents, possibly as a result of their lesser confidence to be pro-active in the diagnostic process.

Maternal age had a dual association with ID of unknown cause. Firstly, teenage mothers were more likely to have children with mild or moderate ID. [17, 20, 21] Secondly, older women were more likely to have children with severe or profound ID. [21,68] The results of a Finnish cohort study which investigated ID of both known and unknown cause [46] was discounted because of the inclusion of ID of known cause. With Down syndrome, the most common cause of ID, it is known that the risk increases very abruptly with advancing maternal age. [69] This might explain the researchers' finding of an association between increased maternal age and ID in the offspring seen in the 1966 birth cohort. [46] The finding that the association no longer existed in 1985-6 cohort may have been because of the introduction or increased uptake of prenatal screening for Down syndrome.

The association of parental and particularly maternal age with ASD and/or ID suggests that both social and biological forces are operating. Younger parents may find a diagnosis of ASD more difficult to obtain for their children because of inexperience and navigational requirements of local systems. Thus, some of the ID diagnoses of their children may be undiagnosed cases of ASD. Further, the excess of older mothers of children with ASD and to a lesser extent ID may result from increased de novo mutations in older women and their partners [70] or the increase of epigenetic mechanisms which are associated with ageing. [71]

\subsection{Parity}

Parity describes the number of live-born children and stillbirths at more than 20 weeks gestation of a woman. [72] Two strong relationships of low parity with ASD and high parity with ID have been demonstrated in the majority of studies.

In women of lower parity, the risks of ASD, [29, 41, 73] ASD with ID [17] and ASD without ID $[17,74]$ were found to be increased in a number of studies. One of these was a Canadian cohort study using linked data-bases and with nearly 1000 case mothers. [29] The authors identified that nulliparous women (that is women having their first child) were at the greatest risk of having a child with ASD. Moreover, a national, population-based study in the US reported 
that older nulliparous women with older partners were around three times more likely to have a child with ASD. [58]

However, two studies found other associations between parity and the risk of ASD. The first, a Danish case-control study nested in population data, found no association. [42] The second, a prospective cohort study using linked health data of more than 110000 mothers in the US, asserted that mothers of parity greater than two were more likely to have a child with ASD than other mothers. [25] Possibly, socio-demographic factors were also operating in this circumstance. In relation to SES and the odds of ASD, it is possible once again that the disparate findings of this same Danish study may have been due to less ascertainment bias which set them apart from other studies in the area. [42] The second study involved nearly 120000 nurses who were followed via their completion of mailed questionnaires over sixteen years. [25] Hence, all mothers were educated and due to their involvement with nursing, could be expected, on average, to have more knowledge of ASD than other mothers. Further, parity was assessed as a binary variable with the two values of greater than two and less than or equal to two. Commonly, other studies have defined parity as either a continuous variable or one with more than two possible values and this difference might account for variations in study findings.

Mothers of higher parity had increased odds of having a child with mild or moderate ID. [17, $20,21]$ One of the research groups concluded that fourth or subsequent children had an increased risk of mild-moderate ID. [20] A Finnish study of two birth cohorts, twenty years apart, found that high parity persisted as a risk factor for ID over time. [46] A large cohort study compared the parity of the mothers of Californian children with ID to the parity of mothers of typically developing children born between 1987 and 1994. [21] These researchers reported that mothers of parity of three or more were 30-50\% more likely to have a child with mild or moderate ID or unspecified ID. [21] Both this study and another Californian study reported that mothers of children with severe or profound ID had an elevated but not significantly increased parity compared to mothers of typically developing children. [21, 52]

\subsection{Summary}

Socio-demographic factors often operate quite differently for ASD and ID. For example, high parental SES was positively associated with the risk of ASD and negatively associated with the risk of ID in the offspring. Marital status, as defined by living with a partner, has different associations. At the time of their child's birth, there was no consistent association of marital status with mothers of a child with ASD compared to the mothers of typically developing children. On the other hand, mothers of a child with ID were less likely to be living with a partner than mothers of typically developing children. Parity appeared to have reverse associations for ASD and ID. Compared to mothers of typically developing children, mothers of low parity were more likely to have a child with ASD and mothers of high parity were more likely to have a child with ID. Similar patterns exist for maternal age. Mothers of an advanced age were more likely to have a child with ASD than mothers of typically developing children. In contrast, mothers of a younger age were more likely to have a child with ID than mothers 
of typically developing children. However, an additional association exists with older mothers being also more likely to have a child with severe ID.

An under-ascertainment of ASD due to social factors and, to a lesser extent an over-ascertainment of ID could be contributing to the socioeconomic effects seen with ASD and ID. For instance, in terms of the severity of ASD, researchers in California, with birth cohorts from 1992 to 2000, divided the children with ASD into two groups of equal size where the less severe group comprised children in the top $50 \%$ of cases according to level of functioning and the most severe group was the lower 50\%. [41] They found that the children from the less severe group were more often found in neighbourhoods which housed wealthier and more educated individuals. Conversely, the same researchers reported that where low SES was measured by a Medi-Cal payment for the birth, the ratio of more severe to less severe cases was always greater than one. The researchers' interpretation was that the most difficult to diagnose cases of ASD, that is the less severely affected, were under-ascertained in lower SES populations. [41]

The association of high SES with ASD also might be compounded by some of the characteristics known to be related to mothers of children with ASD. Older women with the support of a partner and with fewer children would seem more likely to achieve a more complex diagnosis requiring more assessments for their child than younger single mothers. Socio-demographic associations with ASD in most Western countries do not appear to operate as strongly and might even be absent in some Northern European countries. This might be due to a different social welfare structure in this region and specifically related to the universal screening for developmental disability. In addition to these and other social factors which could bias ascertainment, biological factors may be operating with older parents.

\section{Immigrant status and ethnicity}

Immigrant describes mothers who give birth while residing in a country which is not their own country of birth. Ethnic describes mothers who belong to a minority racial group in their country of residence which may or may not be their country of birth. To some extent, the groups of immigrant and ethnic mothers overlap. When examining social forces in relation to ASD and ID, it is important to take into account the often complex process associated with making a diagnosis of ASD.

\subsection{Immigrant status}

\subsubsection{Immigrant mothers and autism}

In all of the eight studies of immigrant mothers of children and ASD, [17, 22, 30, 57, 62, 75-77] the research concluded that immigrant mothers were more likely to have a child with ASD and particularly ASD with ID. [17, 22] In relation to immigrant mothers from Asia one of these studies was conducted by an Australian research group from New South Wales and used birth records and active surveillance to ascertain children with ASD. The group found that immigrant mothers born in South-East or North-East Asia were more likely to have a child with 
ASD than other immigrant mothers. [57] A Western Australian study, using linked population data, also found that immigrant mothers from South-East or North-East Asia were at increased risk of having a child with ASD with ID. [17] A similar situation was described in Sweden where immigrant mothers from East Asia were more than three times as likely to have a child with ASD. [76]

Black immigrant mothers and immigrant mothers from developing countries were also found to be more likely to have a child with ASD compared to other immigrant mothers. One study from the UK [75] and another from Sweden [76] reported that black immigrant mothers [75] and immigrant mothers from sub-Saharan Africa [76] were much more likely to have a child with ASD compared to non-immigrant mothers. Further, a small Swedish case-control study compared the prevalence of autistic disorder and pervasive development disorder not otherwise specified (PDDNOS) in black African children with at least one parent born in Somali to the prevalence in children without a Somali background. [77] The researchers reported that these 17 black mothers were from three to four times more likely to have a child with ASD compared to the mothers without a Somali background. [77]

There is evidence that 'the intensity of the mother's skin colour' is related to her risk of having a child with ASD. A Swedish study compared the risk of ASD in the children of immigrants from each of North, East and other parts of Africa. [22] The mothers from North Africa were predominantly Moroccan and hence were probably fairer than the other two groups of mothers. For example, the East African group was predominantly from Somalia and Ethiopia while the ethnicity of the group from other parts of Africa was not described. The risk of ASD in the North African group was elevated (1.5) but not significantly higher than that of nonimmigrant parents. On the other hand, the risk in the East African mothers and mothers from other parts of Africa of having a child with ASD was 1.9 and 3.5. [22]

Immigrant mothers from distant countries and those who emigrated during pregnancy were more likely to have a child with ASD than other immigrant mothers. For instance, researchers from the UK and Denmark found that immigrant mothers born outside of Europe were more likely to have a child with ASD. [62,75] Similarly, a Swedish study found that immigrant mothers who were not from either of the US or Europe were nearly three times as likely to have a child with ASD compared to mothers from Nordic countries. [30] Another Swedish study ascertained that immigrant mothers who emigrated during pregnancy were even more likely to have a child with ASD than mothers who emigrated at other times. [22]

There is evidence that immigrant mothers are at different risks of ASD without ID and ASD with ID. Two Swedish studies found that immigrant mothers, excepting those from neighbouring Northern Europe, were less likely to have a child with ASD without ID [22] and Asperger syndrome [76] compared to non-immigrant mothers. One of these studies, along with an Australian study, reported that immigrant mothers were more likely to have a child with ASD with ID. $[17,22]$ In addition, the Swedish study found that the African immigrant mothers were more likely to have a child with ASD with ID compared to non-immigrant mothers. [22] Similar results were found in a small Swedish case-control study, where all seventeen of the Somali children with autism presented with ASD with ID. [77] 
Another group of researchers reported that certain immigrant mothers were less likely to have a child with ASD than non-immigrant mothers. The US study conducted a national telephone survey which chose respondents who resided with their biological child and the child's other parent. [78] These researchers reported that non-immigrant Hispanic children had about twice the prevalence of ASD of immigrant Hispanic children. [78] These results were at variance to those in the previous studies of immigrant mothers. The lower likelihood of ASD in immigrant Hispanics compared to non-immigrant Hispanics could be explained by the relative ease of access of Mexican Hispanics to the US. With many countries, immigrants must meet stringent criteria prior to entry and some of these relate to the health of their offspring, their age, wealth, education and occupation. However, Mexican Hispanics would be less likely to experience the same stress, climatic change and exposure to new infections as most other immigrants groups. Moreover, immigrant parents from some of the other studies have usually relocated from more distant locations. For example, one reported findings which related to immigrants from Somali to Sweden, [77] another to non-European immigrants to Britain [75] and another to immigrants to the isolated continent of Australia. [57]

Overall, immigrant mothers and particularly black or Asian immigrant mothers, mothers from distant, developing countries and those who travelled while pregnant were at a higher risk of having a child with ASD. The mothers at highest risk of a child with ASD were from groups who would be expected to experience the most stress. For example, those relocating from a developing country and those pregnant at the time might be expected to experience higher stress than mothers who are relocating from a developed country or are not pregnant. This stress, along with the environmental changes associated with immigration, may have specific and negative effects on the developing fetal central nervous system. [22]

The risk of immigrant mothers having a child with ASD might be further exacerbated by an increased exposure to novel viruses [75] and intrauterine infections. [57] Other hypotheses to explain this association relate to low vitamin D levels $[75,79]$ and these have been further fuelled by animal studies. One study of rat pups with gross vitamin D deficiencies reported that they had structural brain abnormalities which were similar to those in children with ASD. [80] Furthermore, ASD was particularly common in black or Asian immigrant women and darker women more often have a vitamin D deficiency. [80] Generally, immigrant mothers are more likely to have a child with ASD with ID and less likely to have a child with ASD without ID. This may indicate different aetiologies for these subgroups.

Along with these biologically-based hypotheses, social factors may affect the likelihood of an immigrant mother having a child diagnosed with one of ASD, ASD with ID or ASD without ID. For instance, a diagnosis of ASD without ID would be particularly difficult where the child's parents were in an unfamiliar country, with a different language and where unusual behaviours might be explained by cultural differences. [22] In Australia, excluding the relatively small group of refugees, and in the US, Asian immigrants and their children are more often of a higher SES than other immigrant mothers. [81, 82] This might explain why the association with ASD was greater in this group than in other immigrant groups. 


\subsubsection{Immigrant mothers and intellectual disability}

Compared to ASD, there was a reverse scenario identified with ID. Overall, immigrant mothers were $20-50 \%$ less likely to have a child with mild or moderate ID than non-immigrant mothers. $[17,20,21]$ In Australia, Asian immigrant mothers were less likely to have a child with mild or moderate ID than non-immigrant mothers. $[17,20]$ As with ASD, the reversal with mild or moderate ID might be due to their higher SES compared to other immigrant groups. [81-83]

Differing results were found with the association of ID in the children of Mexican immigrants in a similar manner to the unexpected lower likelihood of Hispanic immigrant mothers having a child with ASD. A study of children with severe or profound ID, and born in California, found that immigrant mothers from Mexico, who would have been likely to be Hispanic, were nearly twice as likely to have a child with severe or profound ID compared to parents born in the US. [52] The idiosyncrasies associated with this immigrant group, compared to those immigrants from more distant locations, might explain this finding. They are likely to be less empowered than their non-immigrant counterparts and, as mentioned previously, their immigration was likely to be less regulated. Hence, from a socio-demographic viewpoint, they are more likely to present with low SES which is a risk factor for ID.

\subsection{Ethnicity}

In many studies, immigrant status and ethnicity were not differentiated. Therefore, within the ethnic group of mothers, there would have been mothers who, due to their country of birth, were both ethnic and immigrant. A recurrent trend of the research is that mothers from minority ethnic groups were less likely to have a child with ASD and more likely to have a child with ID than mothers who were not from minority ethnic groups.

Epidemiologists have found that mothers from ethnic minorities and particularly Aboriginal mothers were less likely to have a child with ASD. For example, in the US, the Hispanic mothers were less likely to have a child with ASD compared to non-Hispanic mothers. [23, 59, 84] In New York, the prevalence in Latinos was around half that in non-Latinos. [40] Some of these mothers from minority ethnic groups would have also been immigrant and, as reported, immigrant mothers usually have higher rates of ASD. This means that mothers from ethnic minority groups and who are native to their country might be expected to have the lowest likelihood of a child with ASD. This is the case in both Australia and Canada, where Aboriginal mothers had about half the odds of having a child with ASD compared to non-Aboriginal mothers $[17,29]$

Compared to ASD, there was a reverse situation with ID since overall mothers from ethnic minority groups were more likely to have a child with ID. Asian mothers giving birth in California were $40 \%$ more likely to have a child with severe or profound ID although this result was not significant. [21] Hispanic mothers were more likely to have a child with either mild or moderate ID or severe or profound ID than Caucasian mothers. Again, in each of the ID groups, the results narrowly failed to achieve significance. [21] By comparison, Australian Aboriginal mothers were more than three times more likely to have a child with mild or moderate ID and were $60 \%$ more likely to have a child with severe or profound ID. [17, 20] 
The higher rates of ID and the lower rates of ASD found in most ethnic minority and particularly indigenous communities may relate to the differing gene frequencies of these groups from the general population. However, differences could be exacerbated by environmental factors such as maternal alcohol consumption [85] without this being specifically identified as an aetiological factor. [86] Another consideration could be that marginalized groups are less empowered than others to pursue a diagnosis of ASD in contrast to a diagnosis of ID and that the infrastructures established for diagnostic assessment do not meet their needs. This second factor may also account for the lower prevalence of ASD and higher prevalence of ID with respect to the Australian Aboriginal community. [87]

In two Californian studies, contrasting findings were found for the previously described associations of ethnicity with ASD. Firstly, a cohort study found that Hispanic mothers were no less likely to have a child with ASD with ID than white mothers. [52] The same study also reported that Californian black mothers were more than five times as likely to have a child with ASD with ID as white mothers. [52] Furthermore, Californian Asian mothers were almost four times as likely to have a child with ASD with ID as white mothers. [52] Again, this may be a reflection of the higher proportion of immigrants in these groups. A second explanation in relation to the Asian mothers could be the fact that Asian mothers in US tend to have a higher SES than most other ethnic mothers. The second of the two Californian studies reported that Asian mothers giving birth in California were 30\% less likely to have a child with mild or moderate ID. [21] This may also be a reflection of their higher SES.

\subsection{Summary}

Generally, immigrant mothers, and especially black and Asian immigrant mothers, were more likely to have a child with ASD compared to non-immigrant mothers. Furthermore, immigrant mothers were more likely to have a child with ASD with ID and less likely to have a child with ASD without ID compared to non-immigrant mothers. Immigrant mothers from distant or developing countries and mothers who emigrated when they were pregnant were even more likely to have a child with ASD. By contrast, in the US, Hispanic immigrant mothers were less likely to have a child with ASD than non-immigrant Hispanic mothers. Furthermore, nonimmigrant mothers and particularly Aboriginal mothers were more likely to have a child with ID and especially mild or moderate ID than mothers who were not ethnic.

\section{Health and associated characteristics}

\subsection{Mental health}

The World Health Organisation describes mental health as a state of mental well-being. [88] This state of well-being can be enhanced by the prevention of mental disorders and the treatment and rehabilitation of those with mental disorders. Compromised mental health has been reported in the mothers of children with ASD and to a lesser extent in the mothers of children with ID compared to mothers of children without these disorders. For example, researchers found that mothers of a child with ASD were more likely to have a pre-existing psychiatric [62, 
$64,89]$ or personality $[62,89,90]$ disorder than mothers of typically developing children. Further, parents of a child with ASD were more likely to have increased rates of disorders which were related to affective disorder, [42] obsessive compulsive disorder, [27] anxiety, [27] paranoia, [27] and somatization [27] than the parents of typically developing children. One of these studies was conducted by a Californian team and recruited 269 parents of children with ASD via an existing university research program and control parents of typically developing children who were students (or their contacts) at the university. Self-reported mental health measures were obtained via questionnaire. [27] Other reported associations with parents of a child with ASD were increased rates of schizophrenia, [42, 89, 91] psychosis [42] and depression, $[27,64,89]$ compared to the parents of typically developing children. Mothers of a child with ASD were more likely to have had pregnancies complicated by depression $[92,93]$ than mothers of typically developing children. Another research group explored mental health by comparing the rates of mental disorders in parents of people with ASD to those in parents of people with Down syndrome. [94] Parents of a child with ASD were more likely to have had an anxiety disorder than the parents of children with Down syndrome.

Studies have most commonly investigated the mental health of mothers of children with disabilities rather than the developmental outcomes in children born to mothers with mental health diagnoses. In one case-control study, the latter approach was employed and linked data from population-based registries was used to compare the likelihood of ASD with ID or ID in the children of more than 3000 mothers with schizophrenia, bipolar disorder or unipolar major depression to the likelihood of these disorders in control mothers. Of these, around 1300 mothers had bipolar disorder and these were assessed as nearly ten times more likely to have a child with ASD with ID than mothers without these disorders. [95] However, there were only four children with a mother with pre-existing bipolar disorder so these large odds are associated with particularly wide confidence intervals and only just reached significance.

The same study found that children of mothers with either schizophrenia, unipolar major depression or bipolar disorder or a combination of these disorders were about three times as likely to have a child with ID as mothers without these disorders. [95] Furthermore, mothers with ID themselves were more likely to have a child with ID compared to mothers with no history of psychiatric disorder or ID. [95]

\subsection{Personality traits}

Personality traits have been more often identified in the parents of children with ASD. For instance, parents of children with ASD were more likely to manifest a range of subtle autisticlike characteristics than parents of typically developing children. These characteristics have been grouped together as the Broad Autism Phenotype and include social cognition deficits, such as reasoning about the emotions of others, [96] autistic-like traits, [97] and impaired aspects of executive function. [98, 99]

A questionnaire entitled the Autism Spectrum Quotient (AQ) [100] was designed to assess the Broad Autism Phenotype in the five domains of social skills, communication, attention to detail, attention switching and imagination. [101] Researchers from the UK conducted a casecontrol study comprising parents of children with and without ASD from more than 1500 
families. Parents of children with ASD were more likely to exhibit autistic-like traits in all domains except that of attention to detail [102] than parents of typically developing children. Furthermore, these researchers and others found that a Broad Autism Phenotype occurred more commonly in parents of children with simplex ASD [97, 102] (where only one family member has ASD) and multiplex ASD [100, 103] (where more than one family member has ASD) than in parents of typically developing children. A dose-response effect was also described with parents in multiplex ASD families expressing a Broad Autism Phenotype significantly more often than parents in simplex ASD families. [32]

Some factors associated with maternal mental health may have a deleterious effect on the fetus and increase the likelihood of a child developing ASD or ID. For example, mothers with schizophrenia may remain on antipsychotic drugs during their pregnancies and these drugs, perhaps along with lower levels of maternal self-care (such as diet and medical care) and genetic factors related to the disease may adversely affect the development in the fetus. The milder autistic features in the parents of children with ASD might also be attributable to genetic factors associated with ASD. [33] In their affected children, these factors, along with additional genetic factors from the other parent, may sometimes produce the clinical phenotype of ASD.

\subsection{Physical characteristics}

Here are a group of diverse findings pertaining to the physical attributes of the mothers of interest. One study identified that mothers of children with ASD were significantly taller, particularly those of children with ASD without ID compared to the mothers of typically developing children. [17] This study population comprised more than 300000 mothers and the mean heights of mothers of children with ASD with ID and mothers of children with ASD without ID were 164.3 and $164.9 \mathrm{~cm}$. These means were significantly higher than the mean of the mothers of typically developing children $(163.4 \mathrm{~cm})$. Another study found that mothers of children with ASD were both taller and heavier than mothers of typically developing children. [93] Similarly, a Canadian population study found that among non-smoking women, taller and heavier women were more likely to have a child with ASD compared to the mothers of typically developing children. [29]

Compared to the mothers of children with ASD, differing associations between maternal height and the mothers of children with ID were identified. Using population data, researchers identified that shorter women and those of medium height were more likely to have a child with mild or moderate ID than other mothers. [20] Further, the shortest group of women were more likely to have a child with severe or profound ID than other women. [20] Others found that mothers of children with mild or moderate ID, with a mean height of $162.1 \mathrm{~cm}$ were significantly shorter then than the mothers of typically developing children whose mean was $164.3 \mathrm{~cm}$. However, the mothers of children with severe or profound ID were not significantly shorter than the mothers of typically developing children. [17] However, associations have also been described between SES and height, [104] and so the observed height differences between the mothers of children with ASD and ID may be a reflection of the different mean SES of these groups. 


\subsection{Health behaviours}

Smoking during pregnancy has been associated with both ASD and ID in the offspring. In one study, mothers who smoked during pregnancy were reported to be more likely to have a child with ASD than mothers who did not smoke. [30] In 2011, a Swedish nested case-control study using medical registry data, found that mothers who smoked during early pregnancy were $70 \%$ less likely to have a child with ASD but almost twice as likely to have a child with Asperger syndrome. [76] This raises the possibility that Asperger syndrome has a distinct aetiology from other forms of ASD.

On the other hand, differing results were found in a US population-based, case-cohort study which explored the association of mothers who smoked during pregnancy and ASD. [105] Data from more than 6000000 mothers and their children were adjusted for potential confounders such as maternal age, education, and marital status. The definition of smoking during pregnancy was not described in the paper, so presumably this encompasses all mothers who had admitted to smoking one or more cigarettes during their pregnancy. The researchers reported that mothers who smoked during pregnancy were no more likely to have a child with ASD than mothers who did not smoke. Two large recently published cohort studies published in 2010 and 2011 also found no association between mothers who smoked during pregnancy and ASD. [29, 106] However, the first study examined only associations between maternal smoking and ASD generally [29] Hence, any associations between the relatively small group of mothers of children with Asperger syndrome may have been lost in the broader analysis. In addition, smoking was defined as any smoking during pregnancy which may have lessened the likelihood of an association with ASD. These were different outcomes to the second study, where mothers of children with ASD with ID and ASD without ID were considered separately and mothers who smoked ten or more cigarettes a day were a distinct group from the less intense smokers. [106] The first research group suggested that associations found with other studies were attributable to a confounding by maternal socio-demographic characteristics. [29]

The findings of associations between smoking during pregnancy and ID are also limited. A population study in the US ascertained that mothers who smoked 20 or more cigarettes a day were more likely to have a male child, but not a female child, with ID than mothers who did not smoke during pregnancy. [107] A large Finnish cohort study found that mothers who smoked after 2 months of pregnancy were no more likely to have a child with ID than mothers who did not smoke after this time. [46] This definition of smoking is broader than that of the first study so the likelihood of identifying an association between maternal smoking and ID may be reduced. Further, smoking during the first two months of pregnancy or gender of the fetus was not considered for inclusion in the model. An alternative study, with a more stringent definition of maternal smoking and which addressed these omissions, would be more likely to discern an association between smoking and ID.

Mothers who consume excessive alcohol during pregnancy were assessed as more likely to have a child with ID (but not ASD). This cause of ID is termed fetal alcohol syndrome (FAS) or fetal alcohol spectrum disorder (FASD). Fetal alcohol syndrome is at the most severe end of the spectrum and is diagnosed by characteristic facial features, brain dysmorphology, intellectual and other disabilities. [108] The milder diagnosis of FASD [109] does not require 
the presence of all of the characteristic physical features required for FAS. [110] Studies from Sweden and the US attributed between $2-10 \%$ of mild or moderate ID to FAS or FASD. [111, 112] While the US study considered that a further $3 \%$ of severe or profound ID was caused by FAS or FASD. [112]

In a Western Australian record linkage study heavy prenatal alcohol exposure was found to be an important cause, accounting for $2.5 \%$ of non-genetic intellectual disability. [85] Underascertainment, particularly of FASD, may result from non-disclosure of alcohol consumption during pregnancy due to the associated stigma. [113] In addition, perhaps due to inadequate training, [114] clinicians may lack awareness and confidence in making this diagnosis. [113] Also, clinicians may be concerned at the psychological effect on the mother of a FASD diagnosis and may not pursue this in situations where it is not conclusive or they feel it would not be beneficial to the mother or child.

Large cohort studies and linked data have provided researchers with the opportunity to study whole populations of mothers and their children with and without ASD and/or ID. Data can also be adjusted for a range of possible confounders such as SES and age. This enables the identification of new risk factors for ASD and/or ID and the elimination of others. For example, the association of smoking during pregnancy with ASD and/or ID in the offspring has weakened in the most recent studies using linked population data. Persisting associations are an increased risk of Asperger syndrome or PDD-NOS in mothers who smoked during pregnancy and an increased risk of ID in the male children of mothers who smoked heavily during pregnancy. Maternal alcohol consumption during pregnancy remains a risk factor for ID.

The remaining associations of maternal smoking with ASD and ID in the offspring could result from the effect of this exposure on overall fetal development and particularly growth restriction, [115, 116] preterm birth [115] and low birth-weight [117] Moreover, sub-optimal fetal growth has been associated with mild or moderate ID in Caucasian children. [118] The association of maternal alcohol consumption with ID might be due the multiple effects of alcohol on the fetus and placenta. [119] For example, alcohol can induce oxidative stress in placental villous tissue. Other demonstrated effects are an increase in neural tube defects and increased heart rate and cortisol levels in the exposed infant.

\subsection{Physical health}

The research literature has provided evidence that maternal physical health, both prior to and during pregnancy is related to the likelihood of a mother having a child with ASD and/or ID. Various pre-existing conditions in the mother and related or unrelated complications of her pregnancy increase the likelihood of a mother having a child with ASD and/or ID compared to mothers who do not have the condition.

Pre-pregnancy obesity is an example of a condition which increases the likelihood of a woman having a child with ASD and/or ID. Obese women were more likely to have a child with ASD, [24, 54, 120] or ID [24,46] than women who were not obese. One of these studies was a Finnish study which used linked data from the birth cohorts of 1966 and 
1985-6 and included around 250 mothers of children with ID in each of the cohorts. [46] In both cohorts, mothers with obesity prior to their pregnancies were more likely to have a child with ID than women without pre-pregnancy obesity. However, the association of ID with pre-pregnancy obesity was an increasing risk reflected in the greater odds in the latter cohort(2.4) compared with the original cohort(1.8). [46] Another of the research groups reported that women with an early age of menarche were more likely to have a child with ASD than other women. [120] Early menarche, along with pre-pregnancy obesity, could indicate the possibility of maternal hormonal involvement in the risk of ASD and ID. [120] Then again, the relationship with ID may be resulting from confounding by the association between socioeconomic disadvantage and obesity in highly developed countries. [121] In the light of the increasing prevalence of obesity in these countries, these associations with ASD and ID are an important future research direction. [122]

Women with an auto-immune disorder or anomalies of the immune system were more likely to have a child with ASD and/or ID than women who did not. [25, 26, 123] Furthermore, the majority of associations in this area were with ASD rather than ID. For example, in a casecontrol study using linked data with more than 1200 cases, mothers with an auto-immune disorder were $60 \%$ more likely to have a child with ASD than mothers without an auto-immune disorder. [26] These findings were supported by a small case-control study of 61 mothers of children with ASD. [123] Other studies have found that women with a particular auto-immune disease were more likely to have a child with ASD than women who did not have the disease. For instance, a case-control study with 407 cases found that women with psoriasis were more likely to have a child with ASD than mothers without this disorder. [124] Another research group used linked population data with more than 3000 mothers of a child with ASD and nearly 700000 control mothers. They reported that women with rheumatoid arthritis or celiac disease were more likely to have a child with ASD than mothers who did not have one of these disorders. [125]

One study found that women with pre-existing diabetes were more likely to have a child with ASD than women without pre-existing diabetes [29] However, a study used linked data from the national birth and inpatient registries and reported that women with preexisting diabetes were no more likely to have a child with ASD than other mothers. [30] In relation to ID, a group of US researchers investigated a possible association with diabetes by comparing more than 160000 mother-child dyads. The researchers identified that mothers with pre-existing diabetes were more than $10 \%$ more likely to have a child with ID. [126] Diabetes during pregnancy was also associated with both ASD and ID. For instance, two studies found that mothers with diabetes during pregnancy were more likely to have a child with ASD [25] and ASD with ID [127] than mothers without the disorder. The first of these was a Canadian population study which included nearly 800 cases of ASD and more than 66000 births. Mothers who developed gestational diabetes were associated with a $76 \%$ increased risk of ASD compared to women who did not develop the condition. [25] The second was an Australian population study which found that mothers who had diabetes during pregnancy were nearly three times as likely to have a child with ASD with ID than mothers without diabetes. [127] More attenuated results were 
ascertained by Californian researchers who conducted a case-control study and compared the mothers of more than 500 children with ASD, 172 mothers of children with developmental disabilities other than ASD to typically developing children. [24] They found that mothers with gestational diabetes were more likely (but not significantly more likely) to have a child with ASD than mothers without the disorder. The lack of significance may be due to the reduced power of this smaller study. Two studies identified an association between gestational diabetes and ID or a condition similar to ID. [24, 127] One was a large retrospective cohort study using linked registry data. Here the researchers found that mothers with diabetes during pregnancy were nearly $70 \%$ more likely to have a child with mild or moderate ID [127] compared to mothers without this disorder. The other research group found that mothers with diabetes during pregnancy were nearly two and a half times more likely to have a child with a developmental disability other than ASD than mothers without diabetes during pregnancy. [24]

Further, differences have been identified relating to the immunological status of mothers of children with ASD prior to their pregnancies. An independent case-control study identified fetal brain antibodies in mothers of children with ASD but not in controls mothers. [128] This study found that mothers of children with ASD were significantly more likely to have an auto-antibody reactivity pattern for human fetal brain proteins than mothers of typically developing children.

Cytokines are regulators of immune response and maternal immune dysfunction has been associated with the neurological development of the fetus. [129] A case-control study identified that mothers of children with ASD and/or ID were more likely to have aberrant cytokine profiles compared to the mothers of typically developing children. [130] In this study, the concentration of serum cytokines at mid-pregnancy in the mothers of children with ASD with ID, developmental disabilities other than ASD and typically developing children were compared. Mothers of a child with ASD with ID were more likely to have higher concentrations of three particular cytokines than mothers of a typically developing child. In addition, mothers of a child with a developmental disability other than ASD were more likely to have higher concentrations of a different set of three different cytokines (to the ASD group) than mothers of typically developing children. [130]

Auto-immune diseases and other immune dysfunction might impinge on the immature nervous system of the developing fetus. This could have a deleterious effect on future cognitive function [131] and increase the likelihood of ASD and ID. [130]

Hypertension or high blood pressure is either a temporary or sustained elevation of the blood pressure in the arteries. [132] Moreover, the elevation is at a level where cardiovascular or other damage may occur. Hypertension during pregnancy was associated with an increased risk of ASD in the child. Three studies provided evidence that women who experienced hypertension during pregnancy were more likely to have a child with ASD [24, 30, 54] than women who had not suffered hypertension. In one of the studies, Swedish researchers conducted a nested, matched case-control study with data from over 400 children with ASD and over 2000 controls. [30] Records of children's hospitalisation over 10 years were linked to birth records. The researchers concluded that mothers who suffered a hypertensive disease 
during pregnancy were $60 \%$ more likely to have a child with ASD than other mothers. In contrast, a large cohort study of more than 650000 nurses found that mothers with hypertension during pregnancy were no more likely to have a child with ASD than mothers without with hypertension during pregnancy. [25] Possibly, these nurses, with their increased medical knowledge, sought treatment before their blood pressure reached a level which would have been damaging to the unborn child.

Hypertension and oedema are two common symptoms of pre-eclampsia or toxaemia [132] which is a condition occurring in about $8 \%$ of first pregnancies. [133] Women who experience this condition, along with those who suffer oedema, were more likely to have a child with ASD and/or ID. Three groups of researchers found that women with pre-eclampsia $[25,29,134]$ and those suffering oedema [92] during their pregnancies were more likely to have a child with ASD than women without these conditions during their pregnancies. In contrast, a much smaller case-control study, found that woman with pre-eclampsia had reduced (though not significantly so) likelihood of a child with ASD. [60] Pre-eclampsia was also associated with ID. [135] This association was found by researchers in a population-based, retrospective cohort study in South Carolina. Here, women who suffered pre-eclampsia were nearly $60 \%$ more likely to have a child with ID.

Associations of maternal epilepsy have been demonstrated with both ASD and ID. Women who experienced epilepsy during pregnancy were more likely to have a child with ASD with ID [127] or mild or moderate ID. [127] These Australian researchers conducted a retrospective cohort study of nearly 3000 mothers of children with ASD and/or ID of unknown cause and around 237000 mothers of typically developing children using linked population data from medical registries. They established that mothers with epilepsy during pregnancy were more than four and a half times as likely to have a child with ASD with ID [127] and more than three and a half times as likely to have a child with mild or moderate ID compared to mothers without epilepsy during their pregnancies. [127] A case-control study in US had only 61 control mothers of a child with ASD. [123] Here, mothers who had experienced seizure prior to their pregnancies were nearly six times as likely to have a child with ASD. However, possibly due to the small size of the study, results did not reach significance.

In addition to epilepsy, mothers who experienced a range of other conditions during pregnancy were found to be more likely to have a child with ASD than other mothers. Overall, health issues during pregnancy were associated with a higher risk of ASD. Researchers reported that women who had allergies, [124] asthma, [124] bleeding, [30] or high body temperature [93] during their pregnancies were more likely to have a child with ASD than women who had not experienced these conditions during their pregnancies. Asthma during pregnancy was also associated with ID, with pregnant women with asthma being more likely to have a child with mild or moderate ID than mothers without this condition during pregnancy. [127]

Other conditions during pregnancy have been associated with ID. For instance, an Australian populationstudy found that women whohad renal or urinary conditions during pregnancy were more than twice as likely to have a child with mild or moderate ID as women without these conditions during pregnancy. [127] Furthermore, women who suffered anaemia during their pregnancies were more than five times as likely to have a child with severe or profound ID than 
women without anaemia during pregnancy. [127] Two research groups ascertained that infections during pregnancy were associated with ASD. They found that women whose pregnancies were complicated by urinary tract infection, [93] or any bacterial or viral infection [93, 136] were more likely to have a child with ASD than mothers who did not experience an infection.

Infections during pregnancy were also associated with ID. For example, one study reported that mothers who suffered trichomoniasis during pregnancy were more likely to have a child with ID than mothers without this condition during pregnancy. [137] A cohort study used Medicaid claims and linked infant records to investigate the association of treated and untreated urinary tract infections during pregnancy with later ID in the child. [138] The researchers reported that pregnant women with untreated urinary tract infections were $30 \%$ more likely to have a child with ID than pregnant women without these infections. Moreover, mothers with untreated urinary tract infections were $22 \%$ more likely to have a child with ID than mothers with antibiotic treated urinary tract infections. [138]

There is always a risk that the use of certain medications during pregnancy may have adverse effects on a developing fetus. This use is likely to be related to a woman's health and the decision to use a particular medication at this time must be difficult. Sometimes, medications initially considered safe have been later implicated to adversely affect the future health of the unborn child. For instance, six studies found that the children of mothers who used antidepressants, [64, 139] anti-convulsants, [140] psycho-active drugs, [64] prescribed medications $[54,93]$ and medications generally [141] had a higher risk of a child with ASD. One of these was a population-based case-control study in Stockholm. [64] Using registry data, the researchers assessed that mothers who took psycho-active drugs or anti-depressants during their pregnancies were more than four times as likely to have a child with ASD.

It is also possible that the increased use of prescribed medications in mothers of children with ASD may have resulted from a bias in data collection. In one of the studies which found an increased use of prescribed medications, case mothers were recruited via their response to an advertisement in a support agency newsletter or via their membership of a support agency. [93] Each of these methods might have resulted in a bias in the direction of a high SES. This, in turn, may have produced an increased use of prescribed medications in the case mothers. On the other hand, the study which found an increased use of medications generally was a population study using medical registries. [141] The reported associations are likely to be mediated by a complex interaction of factors. For instance, in addition to possible SES bias, there could be a genetic association such as the familial link of depressive disorders or epilepsy with ASD. Another possibility is an environmental effect which results from the physiological impact of maternal medication use on the uterine environment.

\subsection{Summary}

Before the birth of their affected children, certain socio-demographic, health and physical attributes differentiate mothers of children with ASD and/or ID from those of mothers in the general population. Further, these attributes often vary by the disability group of their child. In Tables 2 to 4, these differences are grouped into categories according to their associations with groups of mothers. An examination of Table 2 shows that with socio-demographic factors, 
the relationships with ASD and ID are most often reversed. High SES was most often associated with the ASD groups of mothers and low SES, most often associated with ID.

Different associations of marital status were found with each of ASD and ID. With ASD, the only two studies found in the area had opposing results. With all but one study, single mothers were at increased risk of unspecified ID and mild or moderate ID, compared to women who were living with a partner.

In the majority of the studies, increased maternal age, along with increased paternal and parental age, were associated with ASD and ASD without ID. With ID, two associations emerged. Younger mothers had an increased risk of bearing a child with mild or moderate ID. But severe or profound ID was associated with increased maternal age.

Lower parity had a consistent positive association with ASD in most studies. With mild or moderate ID and unspecified ID, the relationship was reversed and the association was with greater parity. However, with severe or profound ID, there was no association.

In Table 3, the associations with immigrant status and ethnicity are summarized. Most often, immigrant mothers are more likely to have a child with ASD or ASD with ID than nonimmigrant mothers. On the other hand, ASD without ID was associated with non-immigrants, excepting those immigrants from nearby countries. The Mexican/Hispanic immigrant mothers in the US were a separate group since these mothers were less likely to have a child with ASD than Mexican/Hispanic non-immigrant mothers.

With ethnicity, the associations differed from those with immigrant status, in spite of the overlap between the groups. Except for Asian and black mothers, mothers from ethnic minority groups were at a lower risk of children with ASD compared to Caucasian mothers. With the exception of Asianmothers, the relationship with ID was reversed since mothers fromethnicminority groups, and particularly Aboriginal mothers, were at an increased risk of a child with ID.

Table 4 shows the many associations of health and behavioural traits with ASD and highlights the quite small proportion common to both ASD and ID. With mental health, ten research groups reported associations with ASD. Contrastingly, only one study found an association with the mothers of children with ID. Autistic-like traits were associated only with the parents of children with ASD.

As with other socio-demographic factors, ASD and ID had an overall reverse association with height. Taller and heavier women were more likely to have offspring with ASD and shorter women to have offspring with ID. The associations with maternal smoking during pregnancy were minimal. Excessive alcohol consumption during pregnancy was only associated with offspring with ID. Obesity though was associated with both ASD and ID.

Both ASD and ID had associations with immune function, though the association with ASD was broader. Both pre-existing diabetes and diabetes during pregnancy were associated with ASD and/or ID. Further, abnormal levels of cytokines during pregnancy were also associated with each of ASD and ID. Other associations were with only ASD and were auto-immune disorder generally, psoriasis, rheumatoid arthritis, celiac disease and maternal fetal brain antibodies. 
Seven studies associated hypertension, oedema and pre-eclampsia with ASD whereas only one study associated pre-eclampsia with ID. Epilepsy and asthma had associations with both of ASD and ID but no other associations during pregnancy were common to both disorders. Medication use during pregnancy was only found to be associated with ASD.

\begin{tabular}{|c|c|c|c|c|c|c|}
\hline \multirow[b]{2}{*}{ Category } & \multicolumn{3}{|c|}{ ASD } & \multicolumn{3}{|c|}{ ID } \\
\hline & $\begin{array}{l}\text { ASD without } \\
\text { ID }\end{array}$ & Undifferentiated ASD & $\begin{array}{l}\text { ASD with } \\
\text { ID }\end{array}$ & Mild ID & Unspecified ID & Severe ID \\
\hline SES & $\begin{array}{l}\text { +veassoc } \\
{[17,47]}\end{array}$ & +veassoc $[17,39-41,47]$ & $\begin{array}{l}\text { +veassoc } \\
{[17]}\end{array}$ & $\begin{array}{l}\text {-veassoc } \\
{[17,20,48]}\end{array}$ & $\begin{array}{l}\text {-veassoc } \\
{[21,44-46,48]}\end{array}$ & \\
\hline $\begin{array}{l}\text { In Denmark \& } \\
\text { Sweden }\end{array}$ & & $\begin{array}{l}\text { No assoc[42] } \\
\text {-veassoc[43] }\end{array}$ & & & & \\
\hline $\begin{array}{l}\text { Education as a } \\
\text { measure of SES }\end{array}$ & +veassoc [47] & +veassoc $[21,41,50]$ & & -veassoc $[20,21]$ & $\begin{array}{l}\text {-veassoc } \\
{[20,21,32,44,45,51,} \\
53] \\
\text {-veassoc (DD) }[24]\end{array}$ & $\begin{array}{l}\text {-veassoc } \\
{[21,52]}\end{array}$ \\
\hline $\begin{array}{l}\text { Marital status } \\
\text { at child's birth } \\
\text { (Women with } \\
\text { partners) }\end{array}$ & & $\begin{array}{l}\text { +veassoc [17] } \\
\text {-veassoc [54] }\end{array}$ & $\begin{array}{l}\text { +veassoc } \\
{[17]}\end{array}$ & -veassoc [20] & $\begin{array}{l}\text {-veassoc[17] } \\
\text {-veassoc (early } \\
\text { cognitive delay) } \\
{[55]}\end{array}$ & \\
\hline Age Maternal & $\begin{array}{l}\text { +veassoc } \\
{[17,47]}\end{array}$ & $\begin{array}{l}\text { +veassoc } \\
{[17,21,29,47,56-61]}\end{array}$ & & $\begin{array}{l}\text {-veassoc } \\
{[17,20,21]}\end{array}$ & No assoc [46] & $\begin{array}{l}\text { +veassoc } \\
{[21,68]}\end{array}$ \\
\hline $\begin{array}{l}\text { Paternal \& } \\
\text { maternal }\end{array}$ & & +veassoc $[17,56,58,61]$ & & & & \\
\hline Only paternal & +veassoc [65] & + veassoc $[58,62-65]$ & & & & \\
\hline $\begin{array}{l}\text { Maternal \& } \\
\text { paternal } \\
\text { (Denmark, } \\
\text { Sweden \& UK) }\end{array}$ & & No assoc $[42,43,66]$ & & & & \\
\hline Lower parity & $\begin{array}{l}\text { +veassoc } \\
{[17,74]}\end{array}$ & $\begin{array}{l}\text { +veassoc }[29,41,73] \\
\text {-veassoc }[25]\end{array}$ & $\begin{array}{l}+ \text { veassoc } \\
{[17]}\end{array}$ & $\begin{array}{l}\text {-veassoc } \\
{[17,20,21]}\end{array}$ & -veassoc $[21,46]$ & $\begin{array}{l}\text { No assoc } \\
{[21,52]}\end{array}$ \\
\hline & & No assoc [42] & & & & \\
\hline $\begin{array}{l}\text { Age \& lower } \\
\text { parity }\end{array}$ & & +veassoc [58] & & & & \\
\hline
\end{tabular}

ASD, autism spectrum disorder; ID, intellectual disability; Mild ID, Mild or moderate ID; Severe ID, Severe or profound ID; SES, socio-economic status; +ve, positive; -ve, negative; assoc, association.

Table 2. Associations of socio-demographic factors in the mothers of children with ASD and/or ID 


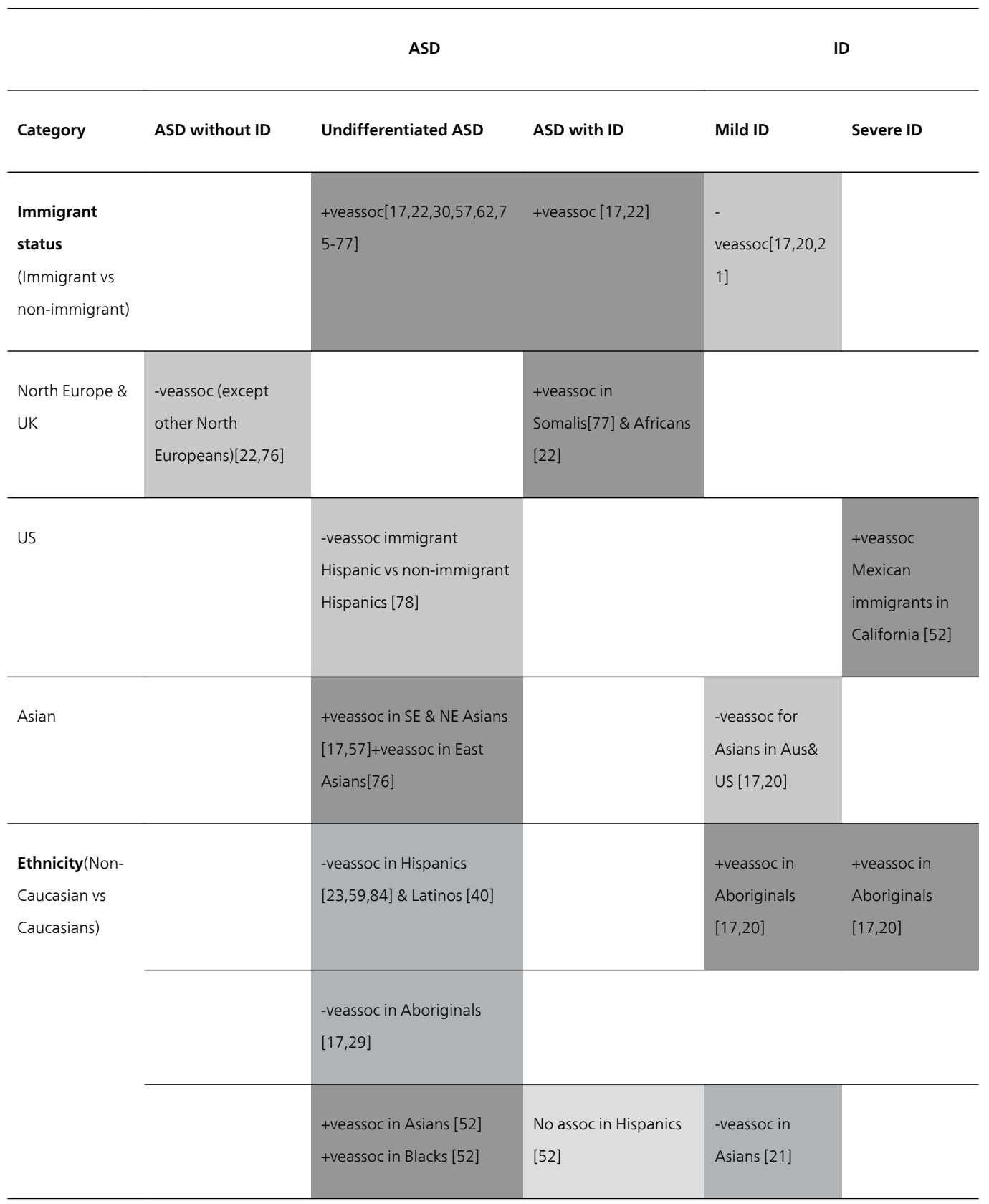

ASD, autism spectrum disorder; ID, intellectual disability; Mild ID, Mild or moderate ID; Severe ID, Severe or profound ID; +ve, positive; -ve, negative;assoc, association; SE, south eastern; NE, north eastern; Aus, Australia; NHW, non-Hispanic white.

Table 3. Associations of immigrant status and ethnicity in the mothers of children with ASD and/or ID 


\section{ASD}

\begin{tabular}{|c|c|c|c|}
\hline Category & $\begin{array}{l}\text { ASD without ID, } \\
\text { [Asperger syndrome] } \\
\text { [ASD with ID] }\end{array}$ & Undifferentiated ASD & Unspecified ID[Mild ID], [Severe ID] \\
\hline \multirow[t]{4}{*}{ Mental health } & & Schizophrenia $[42,89,91]$ & Schizophrenia [95] or \\
\hline & & Depression $[27,64,89]$ & Unipolar major depression [95] or \\
\hline & $\begin{array}{l}\text { Bipolar disorder [ASD } \\
\text { with ID] [95] }\end{array}$ & & Bipolar disorder [95] \\
\hline & & $\begin{array}{l}\text { Psychiatric, }[62,64,89] \text { personality disorders, } \\
{[62,89,90] \text { affective }[42] \text { \& obsessive compulsive }} \\
\text { disorders, }[27] \text { anxiety, }[27,94] \text { \& paranoia } \\
\text { [27]Somatization, [27] psychosis,[42] } \\
\text { depression,[27,64, 89] \& depression during } \\
\text { pregnancy }[92,93]\end{array}$ & ID in mother [95] \\
\hline $\begin{array}{l}\text { Personality } \\
\text { traits }\end{array}$ & & Autistic-like traits $[97,100,102,103]$ & \\
\hline $\begin{array}{l}\text { Physical } \\
\text { characteristics }\end{array}$ & $\begin{array}{l}\text { Taller[ASD without ID] } \\
\text { [17 [17]Taller[ASD } \\
\text { with ID] [17] }\end{array}$ & $\begin{array}{l}\text { Taller and heavier [93] Taller and heavier (non- } \\
\text { smoking mothers only) [29] }\end{array}$ & $\begin{array}{l}\text { Shorter, medium height } \\
\text { [20]Shortest[Severe ID] [20] \& } \\
\text { shorter[Mild ID] [17] }\end{array}$ \\
\hline $\begin{array}{l}\text { Health } \\
\text { behaviours }\end{array}$ & $\begin{array}{l}\text { Smoking[Asperger } \\
\text { syndrome] [76] }\end{array}$ & $\begin{array}{l}\text { Smoking }[30] \text { Smoking(-veassoc) }[76] \\
\text { Smoking (no assoc) }[29,105,106]\end{array}$ & $\begin{array}{l}\text { In male children with } \geq 20 \text { cigs/day in } \\
\text { mother[107]Smoking (no assoc)[46] }\end{array}$ \\
\hline $\begin{array}{l}\text { During } \\
\text { pregnancy }\end{array}$ & & & $\begin{array}{l}\text { Alcohol[85]Alcohol[Mild ID] [111, 112] } \\
\text { Alcohol[Severe ID] [112] }\end{array}$ \\
\hline \multirow[t]{2}{*}{ Physical health } & $\begin{array}{l}\text { Obesity[ASD with ID] } \\
\text { [24] }\end{array}$ & Obesity $[24,54,120]$ & Obesity $[24,46]$ \\
\hline & & Early menarche[120] & \\
\hline \multirow[t]{3}{*}{$\begin{array}{l}\text { Immune } \\
\text { function }\end{array}$} & $\begin{array}{l}\text { Diabetes during } \\
\text { pregnancy [ASD with } \\
\text { ID] [127] }\end{array}$ & $\begin{array}{l}\text { Diabetes }[24,29] \& \text { diabetes during } \\
\text { pregnancy }[25]\end{array}$ & $\begin{array}{l}\text { Diabetes[126] \& diabetes during } \\
\text { pregnancy(Mild ID)[127] \& (DD no } \\
\text { ASD)[24] }\end{array}$ \\
\hline & & Higher conc of 3 cytokines[130] & $\begin{array}{l}\text { Higher conc of } 3 \text { other cytokines(DD) } \\
\text { [130] }\end{array}$ \\
\hline & & $\begin{array}{l}\text { Any auto-immune disorder, }[256,26,123] \\
\text { psoriasis,[124] \& rheumatoid arthritis[125] } \\
\text { celiac disease, [125] \&fetal brain antibodies } \\
\text { [128] }\end{array}$ & \\
\hline
\end{tabular}

ASD, autism spectrum disorder; ID, intellectual disability; Mild ID, mild or moderate ID; Severe ID, severe or profound ID; UTI, urinary tract infection,

Table 4. Associations of mental health, personality traits, physical characteristics, health behaviours and physical health in the mothers of children with ASD and/or ID 


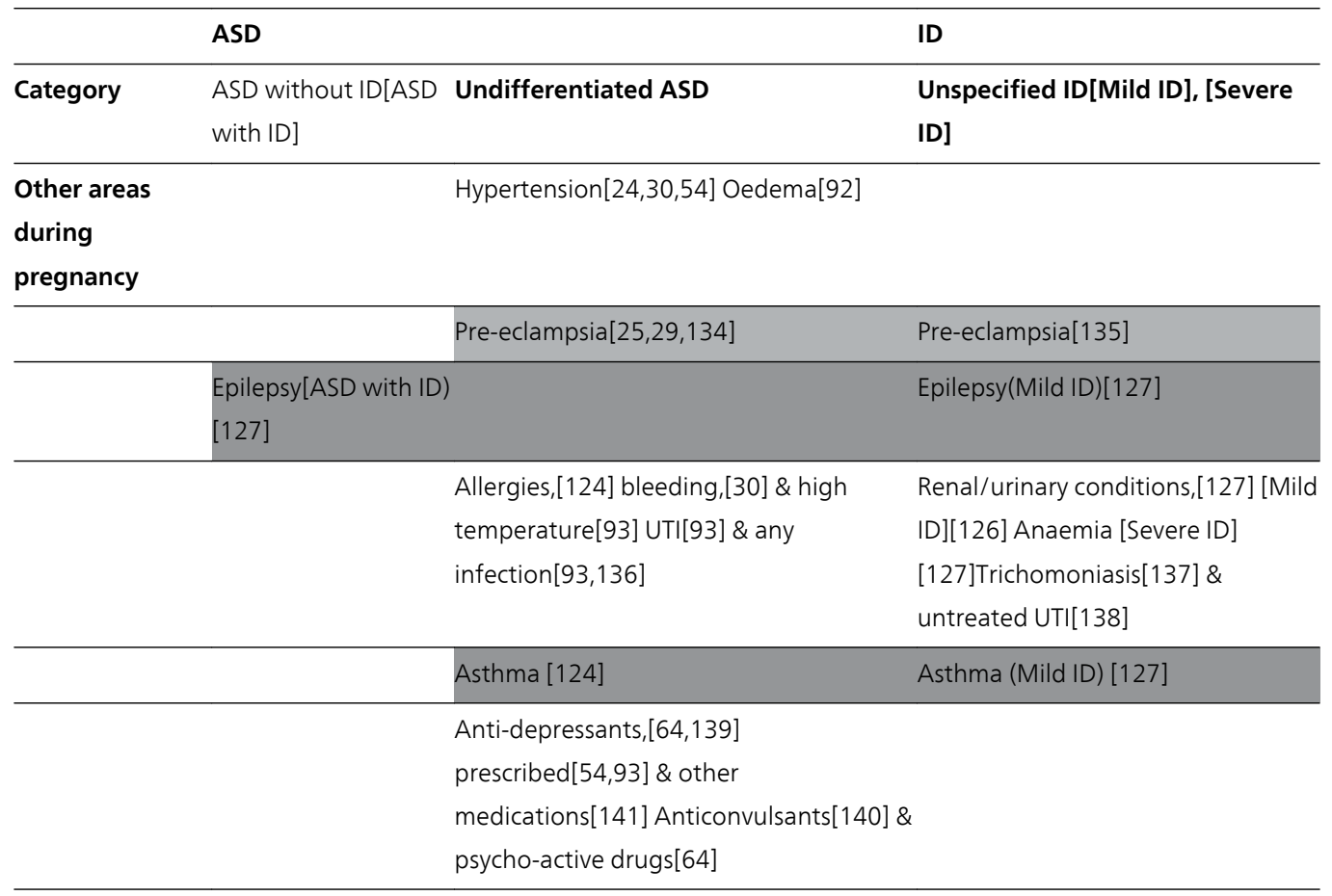

ASD, autism spectrum disorder; ID, intellectual disability; Mild ID, mild or moderate ID; Severe ID, severe or profound ID; UTI, urinary tract infection.

Table 5. Associations of health during pregnancy in the mothers of children with ASD and/or ID

\section{Conclusion}

This chapter provides a review of the research pertaining to the pre-existing characteristics of mothers of a child with ASD and/or ID. Some consistent and enduring associations have emerged across the published reports. With socio-demographic factors, these are the contrasting associations of maternal education, age, immigrant status and ethnicity with ASD and ID. With maternal health; aspects of mental health, personality traits, immune function and the use of medication during pregnancy have stronger associations with the mothers of children with ASD than ID. Some of these differences may be reflections of distinct aetiologies for ASD and/or ID of unknown cause and provide directions for future research. As such, primary and secondary prevention strategies may be refined and/or developed which will contribute to lower prevalence, reduced levels of severity and better outcomes for affected children. 


\section{Author details}

Jenny Fairthorne*, Amanda Langridge, Jenny Bourke and Helen Leonard*

*Address all correspondence to: jfairthorne@ichr.uwa.edu.au

Centre for Child Health, University of Western Australia, Australia

\section{References}

[1] Filipek, P., et al., The screening and diagnosis of autistic spectrum disorders. Journal of Autism and Developmental Disorders, 1999. 29(6): p. 439-84.

[2] American Psychiatric Association, Diagnostic and Statistical Manual of Mental Disorders, Fourth Edition, 2000, American Psychiatric Association: Washington, DC.

[3] Rutter, M., Aetiology of autism: findings and questions. Journal of Intellectual Disability Research, 2005. 49(4): p. 231-38.

[4] Robinson, P., et al., Genetically determined low maternal serum dopamine hydroxylase levels and the etiology of autism spectrum disorders. American Journal of Medical Genetics, 2001. 100(1): p. 30-6.

[5] Trajkovski, V., Etiology of autism. Journal of Special Education, 2004. 5(1-2): p. 61-74.

[6] Bass, M., et al., Genetic studies in autistic disorder and chromosome 15. Neurogenetics, 2000. 2(4): p. 219-26.

[7] Brune, C., et al., 5-HTTLPR genotype-specific phenotype in children and adolescents with autism. American Journal of Psychiatry, 2006. 163(12): p. 2148-56.

[8] Newschaffer, C., et al., The epidemiology of autism spectrum disorders. Annual Review of Public Health, 2007. 28(21): p. 235-58.

[9] Mefford, H., M. Batshaw, and E. Hoffman, Genomics, intellectual disability, and autism. New England Journal of Medicine, 2012. 366(8): p. 733-43.

[10] Stoltenberg, C., et al., The autism birth cohort (ABC): a paradigm for gene-environment-timing research. Molecular Psychiatry, 2011. 15(7): p. 676-80.

[11] Kubota, T., et al., Novel etiological and therapeutic strategies for neurodiseases: epigenetic understanding of gene-environment interactions. Journal of Pharmacological Sciences, 2010. 113: p. 3-8.

[12] Dodge, K. and M. Rutter, Gene-environment interactions: state of the science, in Gene-environment interactions in developmental psychopathology, K. Dodge and M. Rutter, Editors. 2011, Guilford Press: New York. 
[13] Maher, P., Methylglyoxal, advanced glycation end products and autism: Is there a connection? Medical Hypotheses, 2012.

[14] Young, D., et al., The diagnosis of autism in a female: Could it be Rett syndrome? European Journal of Pediatrics, 2008. 167(6): p. 661-9.

[15] Oberlé, I., et al., Instability of a 550-base pair DNA segment and abnormal methylation in Fragile X syndrome. Science, 1991. 252(5009): p. 1097-102.

[16] Fombonne, E., Epidemiology of pervasive developmental disorders. Pediatric Research, 2009. 65(6): p. 591-8.

[17] Leonard, H., et al., Autism and intellectual disability are differentially related to sociodemographic background at birth. PLoS ONE, 2011. 6(3): p. e17875.

[18] Matson, J. and M. Shoemaker, Intellectual disability and its relationship to autism spectrum disorders. Research in Developmental Disabilities, 2009. 30(6): p. 1107-14.

[19] Leonard, H. and X. Wen, The epidemiology of mental retardation: challenges and opportunities in the new millennium. Mental Retardation and Developmental Disabilities Research Reviews, 2002. 8(3): p. 117-34.

[20] Leonard, H., et al., Association of sociodemographic characteristics of children with intellectual disability in Western Australia. Social Science and Medicine, 2005. 60(7): p. 1499-513.

[21] Croen, L., J. Grether, and S. Selvin, The epidemiology of mental retardation of unknown cause. Pediatrics, 2001. 107(6): p. e86.

[22] Magnusson, C., et al., Migration and autism spectrum disorder: population-based study. British Journal of Psychiatry, 2012.

[23] Pinborough-Zimmerman, J., et al., Sociodemographic risk factors associated with autism spectrum disorders and intellectual disability. Autism Research, 2011. 4(5).

[24] Krakowiak, P., et al., Maternal metabolic conditions and risk for autism and other neurodevelopmental disorders. Pediatrics, 2012. 129(5).

[25] Lyall, K., et al., Pregnancy complications and obstetric suboptimality in association with autism spectrum disorders in children of the Nurses' Health Study II. Autism Research, 2012. 5(1): p. 21-30.

[26] Keil, A., et al., Parental autoimmune diseases associated with autism spectrum disorders in offspring. Epidemiology, 2010. 21(6): p. 805-8.

[27] Hodge, D., C. Hoffman, and D. Sweeney, Increased psychopathology in parents of children with autism: genetic liability or burden of caregiving? Journal of Developmental and Physical Disabilities, 2011. 23(3): p. 227-39. 
[28] Morgan, V., et al., What impact do obstetric complications have on the risk of adverse psychiatric outcomes for the high risk children of mothers with schizophrenia and other psychoses? Schizophrenia Research, 2008. 102(Supplement 2): p. 167-8.

[29] Burstyn, I., F. Sithole, and L. Zwaigenbaum, Autism spectrum disorders, maternal characteristics and obstetric complications among singletons born in Alberta, Canada. Chronic Diseases in Canada, 2010. 30(4): p. 125-34.

[30] Hultman, C., P. Sparen, and S. Cnattingius, Perinatal risk factors for infantile autism. Epidemiology, 2002. 13(4): p. 417-23.

[31] Losh, M., et al., Neuropsychological profile of autism and the broad autism phenotype. Archives of General Psychiatry, 2009. 66(5): p. 518-26.

[32] Losh, M., et al., Defining key features of the broad autism phenotype: a comparison across parents of multiple and single incidence autism families. American Journal of Medical Genetics, 2008. 147(4): p. 424-33.

[33] Piven, J., The broad autism phenotype: a complementary strategy for molecular genetic studies of autism. American Journal of Medical Genetics, 2001. 105(1): p. 34-5.

[34] Harvey, J., M. O'Callaghan, and B. Vines, Prevalence of maternal depression and its relationship to ADL skills in children with developmental delay. Journal of Paediatrics and Child Health, 1997. 33(1): p. 42-6.

[35] Hertz-Picciotto, I., et al., The CHARGE Study: an epidemiologic investigation of genetic and environmental factors contributing to autism. Environmental Health Perspectives, 2006. 114(7): p. 1119-25.

[36] Spitzer, R. and B. Siegel, The DSM-III-R field trial of pervasive developmental disorders. Journal of the American Academy of Child and Adolescent Psychiatry, 1990. 29(6): p. 855-62.

[37] Western Australian Autism Diagnosticians' Forum. Diagnosis in Western Australia 2012; Available from: http://waadf.org.au/Waitlist_times_1_December_2011.pdf.

[38] Shattuck, P. and S. Grosse, Issues related to the diagnosis and treatment of autism spectrum disorders. Mental Retardation and Developmental Disabilities Research Reviews, 2007. 13(2): p. 129-35.

[39] Durkin, M., et al., Socioeconomic inequality in the prevalence of autism spectrum disorder: evidence from a US cross-sectional study. PLoS ONE, 2010. 5(7): p. e11551.

[40] Liptak, G., et al., Disparities in diagnosis and access to health services for children with autism: data from the National Survey of Children's Health. Journal of Developmental and Behavioral Pediatrics, 2008. 29(3): p. 152-60.

[41] King, M. and P. Bearman, Socioeconomic status and the increased prevalence of autism in California. American Sociological Review, 2011. 76(2): p. 320-46. 
[42] Larsson, H., et al., Risk factors for autism: perinatal factors, parental psychiatric history, and socioeconomic status. American Journal of Epidemiology, 2005. 161(10): p. 916-25.

[43] Rai, D., et al., Parental socioeconomic status and risk of offspring autism spectrum disorders in a Swedish population-based study. Journal of the American Academy of Child and Adolescent Psychiatry, 2012.

[44] Gissler, M., et al., Social class differences in health until the age of seven years among the Finnish 1987 birth cohort. Social Science and Medicine, 1998. 46(12): p. 1543-52.

[45] Zheng, X., et al., Socioeconomic status and children with intellectual disability in China. Journal of Intellectual Disability Research, 2012. 56(2): p. 212-20.

[46] Heikura, U., et al., Variations in prenatal sociodemographic factors associated with intellectual disability: a study of the 20-year interval between two birth cohorts in Northern Finland. American Journal of Epidemiology, 2008. 167(2): p. 169-77.

[47] Bhasin, T. and D. Schendel, Sociodemographic risk factors for autism in a US metropolitan area. Journal of Autism and Developmental Disorders, 2007. 37(4): p. 667-77.

[48] Emerson, E., Deprivation, ethnicity and the prevalence of intellectual and developmental disabilities. Journal of Epidemiology and Community Health, 2012. 66(3): p. 218-24.

[49] Harris, J., Autism risk factors: moving from epidemiology to translational epidemiology. Journal of the American Academy of Child and Adolescent Psychiatry, 2012. 51(5).

[50] Van Meter, K., et al., Geographic distribution of autism in California: a retrospective birth cohort analysis. Autism Research, 2010. 3(1): p. 19-29.

[51] Yaqoob, M., et al., Mild intellectual disability in children in Lahore, Pakistan: aetiology and risk factors. Journal of Intellectual Disability Research, 2004. 48(7): p. 663-71.

[52] Jelliffe-Pawlowski, L., et al., Risks for severe mental retardation occurring in isolation and with other developmental disabilities. American Journal of Medical Genetics, 2005. 136(2): p. 152-7.

[53] Singhi, P., et al., Psychosocial problems in families of disabled children. British Journal of Medical Psychology, 1990. 63(2): p. 173-82.

[54] Dodds, L., et al., The role of prenatal, obstetric and neonatal factors in the development of autism. Journal of Autism and Developmental Disorders, 2011. 41(7): p. 891-902.

[55] Hatton, E., et al., Changes in family composition and marital status in families with a young child with cognitive delay. Journal of Applied Research in Intellectual Disabilities, 2010. 23(1): p. 14-26. 
[56] King, M., et al., Estimated autism risk and older reproductive age. American Journal of Public Health, 2009. 99(9): p. 1673-9.

[57] Williams, K., et al., Perinatal and maternal risk factors for autism spectrum disorders in New South Wales, Australia. Child-care, Health and Development, 2008. 34(2): p. 249-56.

[58] Durkin, M., et al., Advanced parental age and the risk of autism spectrum disorder. American Journal of Epidemiology, 2008. 168(11): p. 1268-76.

[59] Windham, G., et al., Birth prevalence of autism spectrum disorders in the San Francisco Bay area by demographic and ascertainment source characteristics. Journal of Autism and Developmental Disorders, 2011. 41(10): p. 1362-72.

[60] Stein, D., et al., Obstetric complications in individuals diagnosed with autism and in healthy controls. Comprehensive Psychiatry, 2006. 47(1): p. 69-75.

[61] El-Baz , F., et al., Risk factors for autism: an Egyptian study. Egyptian Journal of Medical Human Genetics, 2011. 12: p. 31-8.

[62] Lauritsen, M., C. Pedersen, and P. Mortensen, Effects of familial risk factors and place of birth on the risk of autism: a nationwide register-based study. Journal of Child Psychology and Psychiatry, 2005. 46(9): p. 963-71.

[63] Reichenberg, A., R. Gross, and M. Weiser, Advancing paternal age and autism. Archives of General Psychiatry, 2006. 63(9): p. 1026-32.

[64] Eriksson, M., et al., First-degree relatives of young children with autism spectrum disorders: some gender aspects. Research in Developmental Disabilities, 2012. 33(5): p. 1642-8.

[65] Tsuchiya, K., et al., Paternal age at birth and high-functioning autistic-spectrum disorder in offspring. British Journal of Psychiatry, 2008. 193(4): p. 316-21.

[66] Robinson, E., et al., Brief report: no association between parental age and extreme social-communicative autistic traits in the general population. Journal of Autism and Developmental Disorders, 2011. 41(12): p. 1733-7.

[67] Frenette, P., et al., Factors affecting the age at diagnosis of autism spectrum disorders in Nova Scotia, Canada. Autism, 2011: p. 1-12.

[68] Drews, C., et al., Variation in the influence of selected sociodemographic risk factors for mental retardation. American Journal of Public Health, 1995. 85(3): p. 329-34.

[69] Hook, E. and A. Lindsjö, Down syndrome in live births by single year maternal age interval in a Swedish study: comparison with results from a New York State study. American Journal of Human Genetics, 1978. 30(1): p. 19-27.

[70] Liu, K., N. Zerubavel, and P. Bearman, Social demographic change and autism. Demography, 2010. 47(2): p. 327-43. 
[71] Hultman, C., et al., Advancing paternal age and risk of autism: new evidence from a population-based study and a meta-analysis of epidemiological studies. Molecular Psychiatry, 2011. 16(12): p. 1203-12.

[72] Mosby's Medical Dictionary 2009: Elsevier. 8th edition.

[73] Glasson, E., et al., Perinatal factors and the development of autism: a population study. Archives of General Psychiatry, 2004. 61(6): p. 618-27.

[74] Schmidt, K., et al., Brief report: Asperger's syndrome and sibling birth order. Journal of Autism and Developmental Disorders, 2012.

[75] Keen, D., F. Reid, and D. Arnone, Autism, ethnicity and maternal immigration. British Journal of Psychiatry, 2010. 196: p. 274-81.

[76] Haglund, N. and K. Källén, Risk factors for autism and Asperger syndrome. Autism, 2011. 15(2): p. 163-83.

[77] Barnevik-Olsson, M., C. Gillberg, and E. Fernell, Prevalence of autism in children born to Somali parents living in Sweden: a brief report. Developmental Medicine and Child Neurology, 2008. 50(8): p. 598-601.

[78] Schieve, L., et al., Association between parental nativity and autism spectrum disorder among US-born non-Hispanic white and Hispanic children, 2007 National Survey of Children's Health. Disability and Health Journal, 2012. 5(1): p. 18-25.

[79] Fernell, E., et al., Serum levels of 25-hydroxyvitamin D in mothers of Swedish and of Somali origin who have children with and without autism. Acta Pædiatrica, 2010. 99(5): p. 743-7.

[80] Cannell, J., Autism and vitamin D. Medical Hypotheses, 2008. 70: p. 750-9.

[81] Zhou, M. and Y. Sao Xiong, The multifaceted American experiences of the children of Asian immigrants: lessons for segmented assimilation. Ethnic and Racial Studies, 2005. 28(6): p. 1119-52.

[82] Grulich, A., M. McCredie, and M. Coates, Cancer incidence in Asian migrants to New South Wales, Australia. British Journal of Cancer, 1995. 71(2): p. 400-8.

[83] Ip, D., C.-T. Wu, and C. Inglis, Settlement experiences of Taiwanese immigrants in Australia. Asian Studies Review, 1998. 22(1): p. 79-97.

[84] Pedersen, A., et al., Prevalence of autism spectrum disorders in Hispanic and nonHispanic white children. Pediatrics, 2012.

[85] O'Leary, C., et al., Intellectual disability: population-based estimates of the proportion attributable to heavy prenatal alcohol exposure. Developmental Medicine and Child Neurology, 2012.

[86] O'Leary, C., Fetal alcohol syndrome: diagnosis, epidemiology and developmental outcomes. Journal of Paediatrics and Child Health, 2004. 40(1-2): p. 2-7. 
[87] Wilson, K. and L. Watson, Autism spectrum disorder in Australian Indigenous families: issues of diagnosis, support and funding. Aboriginal and Islander Health Worker Journal, 2011. 35(5): p. 17-8.

[88] World Health Organisation. Mental health. 2012 [2012 September 3]; Available from: http://www.who.int/topics/mental_health/en/.

[89] Daniels, J., et al., Parental psychiatric disorders associated with autism spectrum disorders in the offspring. Pediatrics, 2008. 121(5): p. 1357-62.

[90] Mouridsen, S., et al., Psychiatric disorders in the parents of individuals with infantile autism: a case-control study. Psychopathology, 2007. 40(3): p. 166-71.

[91] Sullivan, P., et al., Family history of schizophrenia and bipolar disorder as risk factors for autism. Archives of General Psychiatry, 2012: p. 1-5.

[92] Zhang, X., et al., Prenatal and perinatal risk factors for autism in China. Journal of Autism and Developmental Disorders, 2010. 40(11): p. 1311-21.

[93] Wilkerson, D., et al., Perinatal complications as predictors of infantile autism. International Journal of Neuroscience, 2002. 112(9): p. 1085-98.

[94] Piven, J., et al., Psychiatric disorders in the parents of autistic individuals. Journal of the American Academy of Child and Adolescent Psychiatry, 1991. 30(3): p. 471-8.

[95] Morgan, V., et al., Intellectual disability and other neuropsychiatric outcomes in high-risk children of mothers with schizophrenia, bipolar disorder and unipolar major depression. British Journal of Psychiatry, 2012.

[96] Gokcen, S., et al., Theory of mind and verbal working memory deficits in parents of autistic children. Psychiatry Research, 2009. 166(1): p. 46-53.

[97] Hurley, R., et al., The broad autism phenotype questionnaire. Journal of Autism and Developmental Disorders, 2007. 37(9): p. 1679-90.

[98] Wong, D., et al., Profiles of executive function in parents and siblings of individuals with autism spectrum disorders. Genes, Brain and Behavior, 2006. 5(8): p. 561-76.

[99] Hughes, C., M. Leboyer, and M. Bouvard, Executive function in parents of children with autism. Psychological Medicine, 1997. 27(1): p. 209-20.

[100] Bishop, D., et al., Using self-report to identify the broad phenotype in parents of children with autistic spectrum disorders: a study using the Autism Spectrum Quotient. Journal of Child Psychology and Psychiatry, 2004. 45(8): p. 1431-6.

[101] Baron-Cohen, S., et al., The Autism Spectrum Quotient (AQ): evidence from Asperger syndrome/high-functioning autism, males and females, scientists and mathematicians. Journal of Autism and Developmental Disorders, 2001. 31(1): p. 5-17. 
[102] Wheelwright, S., et al., Defining the broader, medium and narrow autism phenotype among parents using the Autism Spectrum Quotient (AQ). Molecular Autism, 2010. 1(1): p. 10.

[103] Bernier, R., et al., Evidence for broader autism phenotype characteristics in parents from multiple incidence autism families. Autism Research, 2011.

[104] Marques-Vidal, P., et al., Secular trends in height and weight among children and adolescents of the Seychelles, 1956-2006. BioMed Central Public Health, 2008. 8(1): p. 166.

[105] Kalkbrenner, A., et al., Maternal smoking during pregnancy and the prevalence of autism spectrum disorders, using data from the autism and developmental disabilities monitoring network. Environmental Health Perspectives, 2012. 120(7): p. 1042-8.

[106] Lee, B., et al., Brief report: maternal smoking during pregnancy and autism spectrum disorders. Journal of Autism and Developmental Disorders, 2011: p. 1-6.

[107] Braun, J., et al., The effect of maternal smoking during pregnancy on intellectual disabilities among 8 year old children. Paediatric and Perinatal Epidemiology, 2009. 23(5): p. 482-91.

[108] Spohr, H., J. Willms, and H. Steinhausen, Fetal alcohol spectrum disorders in young adulthood. Journal of Pediatrics, 2007. 150(2): p. 175-9.

[109] Spohr, H. and H. Steinhausen, Fetal alcohol spectrum disorders and their persisting sequelae in adult life. Deutsches Arzteblatt, 2008. 105(41): p. 693-8.

[110] Malbin, D., Fetal alcohol spectrum disorder (FASD) and the role of family court judges in improving outcomes for children and families. Juvenile and Family Court Journal, 2004. 55(2): p. 53-63.

[111] Hagberg, B. and M. Kyllerman, Epidemiology of mental retardation-a Swedish survey. Brain and Development, 1983. 5(5): p. 441-9.

[112] Yeargin-Allsopp, M., et al., Reported biomedical causes and associated medical conditions for mental retardation among 10 year old children, metropolitan Atlanta, 1985 to 1987. Developmental Medicine and Child Neurology, 1997. 39: p. 142-9.

[113] Quattlebaum, J. and M. O'Connor, Higher functioning children with prenatal alcohol exposure: Is there a specific neurocognitive profile? Child Neuropsychology, 2012: p. $1-18$.

[114] Eyal, R. and M. O'Connor, Psychiatry trainees' training and experience in fetal alcohol spectrum disorders. Academic Psychiatry, 2011. 35: p. 238-40.

[115] Cnattingius, S., The epidemiology of smoking during pregnancy: smoking prevalence, maternal characteristics, and pregnancy outcomes. Nicotine and Tobacco Research, 2004. 6(Suppl 2): p. S125-S140. 
[116] Naeye, R., Effects of maternal cigarette smoking on the fetus and placenta. British Journal of Obstetrics and Gynaecology, 1978. 85(10): p. 732-7.

[117] Wakschlag, L., et al., Maternal smoking during pregnancy and severe antisocial behavior in offspring: a review. American Journal of Public Health, 2002. 92(6): p. 966-72.

[118] Leonard, H., et al., Relation between intrauterine growth and subsequent intellectual disability in a ten-year population cohort of children in Western Australia. American Journal of Epidemiology, 2008. 167(1): p. 103-11.

[119] Ornoy, A. and Z. Ergaz, Alcohol abuse in pregnant women: effects on the fetus and newborn, mode of action and maternal treatment. International Journal of Environmental Research and Public Health, 2010. 7(2): p. 364-79.

[120] Lyall, K., et al., Maternal early life factors associated with hormone levels and the risk of having a child with an autism spectrum disorder in the Nurses Health Study II. Journal of Autism and Developmental Disorders, 2011. 41(5): p. 618-27.

[121] McLaren, L., Socioeconomic status and obesity. Epidemiologic Reviews, 2007. 29(1): p. 29-48.

[122] Mokdad, A., et al., The spread of the obesity epidemic in the United States, 1991-1998. Journal of the American Medical Association, 1999. 282(16): p. 1519-22.

[123] Comi, A., et al., Familial clustering of autoimmune disorders and evaluation of medical risk factors in autism. Journal of Child Neurology, 1999. 14(6): p. 388-94.

[124] Croen, L., et al., Maternal autoimmune diseases, asthma and allergies, and childhood autism spectrum disorders: a case-control study. Archives of Pediatrics and Adolescent Medicine, 2005. 159(2): p. 151-7.

[125] Atladóttir, H., et al., Association of family history of autoimmune diseases and autism spectrum disorders. Pediatrics, 2009. 124(2): p. 687-94.

[126] Mann, J., et al., Children born to diabetic mothers may be more likely to have intellectual disability. Maternal and Child Health Journal, 2012: p. 1-5.

[127] Leonard, H., et al., Maternal health in pregnancy and intellectual disability in the offspring: a population-based study. Annals of Epidemiology, 2006. 16(6): p. 448-54.

[128] Braunschweig, D., et al., Autism: maternally derived antibodies specific for fetal brain proteins. Neurotoxicology, 2008. 29(2): p. 226-31.

[129] Ashwood, P., S. Wills, and J. Van de Water, The immune response in autism: a new frontier for autism research. Journal of Leukocyte Biology, 2006. 80(1): p. 1-15.

[130] Goines, P., et al., Increased midgestational IFN-g, IL-4 and IL-5 in women bearing a child with autism: a case-control study. Molecular Autism, 2011. 2(13): p. e1-e11. 
[131] Derecki, N., E. Privman, and J. Kipnis, Rett syndrome and other autism spectrum disorders-brain diseases of immune malfunction? Molecular Psychiatry, 2010. 15(4): p. 355-63.

[132] McDonough, J., Stedman's Concise Medical Dictionary, 1994: Williams \& Wilkins.

[133] Rudra, C. and M. Williams, Monthly variation in preeclampsia prevalence: Washington State, 1987-2001. Journal of Maternal-Fetal and Neonatal Medicine, 2005. 18(5): p. 319-24.

[134] Mann, J., et al., Pre-eclampsia, birth weight, and autism spectrum disorders. Journal of Autism and Developmental Disorders, 2010. 40(5): p. 548-54.

[135] Griffith, M., J. Mann, and S. McDermott, The risk of intellectual disability in children born to mothers with preeclampsia or eclampsia with partial mediation by low birth weight. Hypertension in Pregnancy, 2011. 30(1): p. 108-15.

[136] Atladóttir, H., et al., Maternal infection requiring hospitalization during pregnancy and autism spectrum disorders. Journal of Autism and Developmental Disorders, 2010. 40(12): p. 1423-30.

[137] Mann, J., et al., Trichomoniasis in pregnancy and mental retardation in children. Annals of Epidemiology, 2009. 19(12): p. 891-99

[138] McDermott, S., et al., Urinary tract infections during pregnancy and mental retardation and developmental delay. Obstetrics and Gynecology, 2000. 96(1): p. 113-9.

[139] Croen, L., et al., Antidepressant use during pregnancy and childhood autism spectrum disorders. Archives of General Psychiatry, 2011. 68(11): p. 11104-12.

[140] Rasalam, A., et al., Characteristics of fetal anticonvulsant syndrome associated autistic disorder. Developmental Medicine and Child Neurology, 2005. 47(8): p. 551-5.

[141] Maimburg, R. and M. Væth, Perinatal risk factors and infantile autism. Acta Psychiatrica Scandinavica, 2006. 114(4): p. 257-64. 



\section{Section 5}

Aetiological Factors - Sensory Issues, Foetal Alcohol Syndrome and Relationships 

Chapter 19

\title{
Relationships, Sexuality, and Intimacy in Autism Spectrum Disorders
}

\author{
Maria R. Urbano, Kathrin Hartmann, \\ Stephen I. Deutsch, \\ Gina M. Bondi Polychronopoulos and \\ Vanessa Dorbin \\ Additional information is available at the end of the chapter \\ http://dx.doi.org/10.5772/53954
}

\section{Introduction}

The purpose of this chapter is to provide a brief overview of Autism Spectrum Disorders (ASD) and sexuality, as there is a paucity of this information in the literature. Specific attention is given to sexuality involving the self, others, and interpersonal relationships. Problematic sexual behaviors, legal concerns, and sexual abuse (including victimization and perpetration) are also discussed. Finally, intervention strategies for ASD children, adults, and families are addressed. The overall aim of this chapter is to highlight major themes regarding Autism Spectrum Disorders and sexuality while contributing to the existing literature.

\section{Autism overview}

Autism Spectrum Disorders, as currently defined by the Diagnostic and Statistical Manual (DSM-IV-TR) criteria, include the diagnoses of Autistic Disorder, Asperger's Disorder and Pervasive Developmental Disorder NOS. The three major diagnostic categories include the following: 1) language impairment, 2) social impairment, and 3) repetitive behaviors/restricted interests, with the impairments present prior to the age of three. Autism has been conceptualized under this diagnostic rubric as a spectrum of disorders with symptoms ranging from severe to minimally impaired [1]. With the advent of the DSM-5, only two major criteria will be included: 1) social communication impairment, and 2) repetitive behaviors/restricted interests. 
The DSM-5 envisions autism as a unitary diagnosis with multiple levels of symptom severity impairing the ability to function [2]. The DSM-5 will use a system of three modifiers to signify level of severity: Level 1 is characterized for patients requiring support as they display difficulty initiating social situations and demonstrate atypical social responses. Rituals and repetitive behaviors cause significant interference for these individuals. They also resist redirection and attempts to be interrupted when involved in restricted interests or repetitive behaviors. Level 2 is characterized for patients "requiring substantial support," as they have marked deficits in verbal and nonverbal social communication skills, which are apparent even with supports in place. They demonstrate limited ability to initiate social interaction and have a reduced or abnormal response to social overtures from others. Repetitive behaviors and restricted interests are obvious enough to be noticed by a casual observer. These patients become distressed or frustrated when they are interrupted or redirected. Level 3 is characterized for patients requiring very substantial support, as they have severe deficits in verbal and nonverbal social communication skills. Repetitive behaviors or rituals markedly interfere with functioning in all spheres. They demonstrate marked distress when routines are interrupted, and they are very difficult to redirect [2].

Proposed changes to the DSM-5 diagnostic criteria include the creation of a single broad autism spectrum disorder (ASD) diagnosis that encompasses current specific DSM-IV-TR diagnoses. Further, the proposed DSM-5 criteria reflect the tension between considering core symptoms from a dimensional perspective (i.e., symptoms are distributed in the population and patients are distinguished from unaffected persons by the severity of their symptoms), as opposed to the presence of discrete symptoms reflecting categorical distinctions between affected and unaffected persons [3]. A dimensional approach suggests that the core symptoms are quantitative traits which vary along a continuum and reflect the expression of, and interactions between, commonly occurring genetic variations and effects of environmental factors, whereas categorical approaches favor models attributing risk of illness to large effects of single genes, especially genes involved in brain development or maintenance of synaptic architecture [3]. In fact, the DSM-5 diagnostic criteria may be best represented by an empirically-derived hybrid model that merges the dimensional and categorical aspects of symptoms of autism (i.e., there are threshold values for numbers and severity of symptoms that define a categorical diagnosis of an ASD). From a biological perspective, although symptoms may be viewed along a continuum, the diagnosis of autism implies the altered, albeit subtle, architecture of the brain. The two core symptom domains of DSM-5, whose severity can vary along a continuum, were validated independently and include 1) impaired social communication and interaction (SCI), and 2) restricted, repetitive behavior (RRB) $[3,4]$. There is still work left to be done with respect to determining the number of criteria that must be satisfied in order to assign an ASD diagnosis. The DSM-5 criteria are clearly being shown as superior to the DSM-IV-TR criteria in terms of specificity. However, a balance must be struck between reducing "false positives," which maximizes specificity, and assuring that criteria are sufficiently sensitive to capture ASD-affected persons that would benefit from intervention and services. This is an especially big concern among caregivers of persons that would have previously received a diagnosis of Asperger's disorder and for children and adolescents with poor historical information about early-life symptoms (e.g., 
children and youth in foster and juvenile justice settings). Inclusion of "subtler" symptoms, such as those reflected in the following items from the Social Responsiveness Scale (C) Western Psychological Services), improved the sensitivity of identifying persons with high-functioning ASD (such as persons diagnosed with Asperger's disorder): impaired social understanding or awareness, literal or pedantic use of language, difficulties in adjusting behavior to various contexts, unusual prosody, and problems with body orientation or social distance [3]. Additional research must be conducted to determine the discriminative diagnostic value should be placed [4].

Along with the proposed diagnostic criteria, estimates of the prevalence of autism have also changed. Recently, the prevalence estimates of the Autism and Developmental Disabilities Monitoring (ADDM) Network for children aged 8 years utilized a consistent "recordsbased" surveillance methodology in 14 sites across the United States, examining both health and education records [5]. The overall estimated 2008 prevalence of autism spectrum disorders was 1 in 88 children, demonstrating a steady increase in prevalence since 2002 [6]. Although the ADDM Network sites are not a nationally representative sample, the methodology used in obtaining prevalence estimates of children aged 8 years has been consistent since the monitoring began, so valid comparisons can be made with earlier years. These comparisons show that the estimated prevalence in 2008 increased by $23 \%$ in comparison to 2006 , and by $78 \%$ when compared to 2002 . The increase in prevalence may simply reflect greater awareness and better ascertainment of autism spectrum disorders by health agencies and schools, as suggested in a community mental health surveillance study in England [7]. The England study showed that the prevalence of autism in adults, when properly diagnosed, was approximately the same as in children.

\section{Normal sexual development}

Sexual development is a complex process that includes sexuality in relation to oneself and others. Sexuality encompasses a broad variety of physical, emotional, and social interactions. It includes sexual beliefs, attitudes, knowledge, values, and behavior and concerns the anatomy, physiology, and biochemistry of the sexual response system. Sexuality involves one's thoughts, feelings, behaviors, relationships, roles, identity, and personality [8].

As with other individuals, those with ASD grow and mature along many developmental lines [9]. The social developmental line includes the development of sexuality, while the physical line includes that of puberty. Sexuality begins in infancy and progresses through adulthood until death. Each life stage brings about physical changes and psychosocial demands that need to be achieved for sexual health to be attained. The capacity for a sexual response, both male and female, has been found as early as in the 24-hour period after birth. The rhythmic manipulation of genitals similar to adult masturbation begins at 2.5 to 3 years of age are a natural form of sexual expression [10]. Also during the first three years of life, a child forms an attachment to his or her parents that is facilitated by physical contact. A stable, secure attachment with parents enhances the possibility of such an attachment when an 
adult is preparing to meet an intimate partner [10]. Gender identity, i.e. one's sense of maleness or femaleness, also forms in the first three years of life. A clear, secure gender identity allows for satisfying, intimate adult relationships. Children may display masturbatory behaviors and engage in a variety of sexual play activities that coincide with the development of socially expected norms in the context of natural curiosity about themselves and their environment. Between the ages of 3 to 7 , children explore their own body parts, recognize them as male or female, and become interested in the genitals of their peers, leading to sexual play [10]. During the latency years, overt sexual play becomes covert, with children beginning to have experience with masturbation, should libidinal urges occur. As latency-age children segregate along sexual lines, any sexual experiences are usually with those of the same gender [10]. More overt behaviors and interests emerge again in adolescence with the onset of puberty. Reports collected by the Centers for Disease Control and Prevention (CDC) in 2000 showed about $52 \%$ of males and $48 \%$ of females in grades 9 to 12 are engaging in sexual intercourse as reported by Delamater and Friedrich in 2002 [10]. Similar statistics were reported as recently as 2011 by the CDC, with $47.4 \%$ of $9-12^{\text {th }}$ graders reporting that they had ever engaged in sexual intercourse [11]. Cultural differences are also apparent among groups regarding premarital intercourse [10].

Pubertal changes can begin as early as 9 years of age or as late as 14 years of age. With the onset of puberty, sexual development moves to the forefront. Puberty, governed by hormonal changes, is defined as the time when a male or female is capable of sexual reproduction. A growth spurt, skeletal changes, increases in muscle and fat tissue, development of breasts, pubic and axillary hair, and the growth of genitalia are all hallmarks of the pubertal process [12]. With the physical maturation of gonads, genitalia and secondary sex characteristics, one's sexual interest increases. Citing a study by Bancroft and colleagues (2003), Delamater and Friedrich noted that many males begin to masturbate between the ages of 13 and 15, whereas the onset for girls is more varied [10]. As older adolescents and young adults develop, more teens engage in sexual intercourse and develop a sexually active heterosexual lifestyle. Between 5 and 10\% of adolescent males, and 6\% of adolescent females, experiment with homosexual behavior. This exploration may be a transient experience, or it may develop into an adult homosexual identity [10]. One of the major psychological developmental tasks of later adolescence is to develop a firm sense of identity, of which one's gender identity is an important aspect [13]. Achieving sexual maturity continues into adulthood with the ability to make informed decisions about one's partner choice, reproduction, and long-term intimate relationships.

\section{Sexuality, disability, and ASD}

Sexual development is an intricate process that examines sexuality in regard to oneself and others. This process is often thought of in terms of normal development; however the developmentally disabled also go through sexual stages as they physically mature. This concept can be difficult to accept for some providers and caretakers, due to their tendency to view the developmentally disabled as perennial children [14]. 
For much of our history, the concept that individuals with any disability as sexual beings was unthinkable [15]. Those with developmental disabilities were frequently subjected to involuntary sterilization in the first half of the $20^{\text {th }}$ century. The sexual nature of those with disabilities has been traditionally denied and/or ignored. It has also been viewed similarly with ASD individuals, whose sexuality is further complicated by social communication and language deficits [15]. Only recently has it been acknowledged that persons with ASD have the universal right to learn about relationships, marriage, parenthood, and appropriate sexuality [8]. A major contribution to the field of autism and sexuality is the TEACCH Report published through the United Kingdom [16]. This article, based on the approach and concepts developed by Mesibov and Schopler [17] in the 1980's, put forth five basic assumptions concerning those with autism and are quoted below.

1. People with autism of all levels of severity experience sexual drives, behaviors, or feelings with which at some point in their lives they need assistance

2. Parent involvement and participation is a crucial ingredient in the area of sexual education

3. Sexual education must be taught in a highly structured, individualized way using concrete strategies with less of an emotional overtone

4. Sexual behaviors must be an important behavioral priority with less tolerance for deviations in this area due to the stringent expectations of society

5. Sexual education must be taught in a specific individualized, developmental manner [16]

This report was one of the first to acknowledge that individuals with autism have the same human sexual urges and behaviors as all humans and that those with ASD have the right to express their sexuality to the greatest level possible. These tenets therefore emphasize the need for sexual education for those with ASD, so they can be integrated into our society's rules concerning what sexual behaviors are considered either appropriate or inappropriate.

Keeping in mind that quite often individuals with ASD may also have an intellectual disability [18], studies of individuals with a disability in general become important for the ASD population as well. The current literature already being conducted for those with disabilities is being applied to the expressed needs for education of those with ASD on how to develop sexual and intimate relationships. One study identified that those under the age of $18 \mathrm{had}$ only limited knowledge about pregnancy and sexual anatomy while most individuals including adults were aspiring to form relationships and marriage [19]. In addition, general reluctance of family members and caregivers to acknowledge and respect the sexual rights of those with an intellectual disability was identified because these concepts created a certain level of anxiety in those family members.

As with others individuals who have a disability, those with an Autism Spectrum Disorder diagnosis possess the right to have a relationship, to marry, and/or to have children. Education about legal rights should be provided to those with ASD and extended especially to those whom they encounter, e.g. teachers, family, policemen, community members, etc. Education 
and awareness are key factors in the ability to identify violations to individuals' basic human rights.

Although those with an ASD diagnosis have the right to date, marry and have children, there is a paucity of empirical research on family units and relationships for this particular group. Though some evidence does exist anecdotally, e.g. through blogs and books, this evidence is not scientifically sound. Therefore, future research should generate empirical studies that focus on interpersonal relationships within the family unit and examine which factors or skills may contribute to their success.

\section{Characteristics common to ASD persons}

The overarching confounding factor for individuals with ASD to develop normative sexual identity, sexual orientation, and sexual behaviors is their core social disability [20] that in turn influences the person's opportunity and availability for romantic and intimate relationships. While levels of romantic and sexual functioning typically increase with age, a developmental lag was reported for individuals with ASD [21]. In a survey of parents of 38 neurotypically developing adolescents and young adults and 25 adolescents and young adults with ASD, Stokes and colleagues found support for their research hypotheses that individuals with ASD had less access to peers and friends, engaged in more unacceptable behaviors in attempting to initiate romantic relationships, and persisted in their pursuit of the relationship even when non-mutual interests were evident [21]. In 2012, Shandra and Chowdhury conducted a study on the first sexual experiences of adolescent girls with and without disabilities and reported that social isolation (not the adolescents' impairment) was the primary contributor to difficulties, based on their review of the literature and analyses of a national longitudinal data bank. Results also suggested that having a mild disability increased the likelihood of having sexual intercourse with a stranger for the first time, rather than with a steady dating partner [22].

Several characteristics of those with ASD interfere with the capacity to develop meaningful adult social relationships, which are necessary for developing sexual, intimate relationships. Foremost is the difficulty with social judgment [8], i.e. missing nonverbal communication, poor eye contact, theory of mind problems, and flexibility in response. Lack of experience in peer relationships prevents the development of the common pathway through which adolescents learn about sexuality [23]. Problematic decision-making skills complicate the capacity to maintain the everyday details of a relationship, such as initiating dates, or remembering plans. Lack of flexibility, along with self-absorption, creates significant areas of conflict in a potential relationship. Emotional dysregulation resulting in feelings that are too intense, or perhaps misplaced, together with a lack of awareness of the other's response can quickly end a relationship. Sensory sensitivities, such as inability to tolerate touch or other physical sensations, sound sensitivities, or food texture issues can cause dating to be fraught with problems [24].

Many persons with ASD have little self-awareness and as noted above, do not understand their impact on others. Another dimension of this issue is that persons with ASD may have 
little knowledge about themselves. Part of what helps us create a sense of self is the ability to create an internal autobiography [25]. Persons with ASD have difficulty in this area, as they frequently cannot describe their own emotions or are unaware of what they are feeling (i.e. alexithymia) or have difficulty controlling their emotional responses (i.e. emotion dysregulation). As a result, many with ASD lack the ability to insightfully understand themselves or respond to the social climate in a meaningful way. Self-advocacy, a crucial skill for maintaining one's function in daily life, is something that can be very difficult for a person with ASD to learn. The ability to maintain personal safety without awareness of the environment or the behaviors of others can pose a significant danger.

Persons with ASD, either as a result of the above difficulties or due to a true lack of social interest, turn away from others into their own world. Self-absorption fosters another type of social disability. Persons with ASD frequently have restricted areas of interest (e.g. computer animation) and may have little to no desire in sharing this interest with others or attending to the interests of others, since there can be a lack of ability to detach from the area of interest without anxiety or distress. The need for sameness and rigidity in daily routines may supersede one's ability to flexibly respond to another person, e.g. being unable to eat at another restaurant when only two specific restaurants are in that person's repertoire [26]. The need for aloneness or "down time" may be greater than the need to be with others, which may seriously jeopardize an attempt to relate to others in a more than superficial manner. Sensory sensitivities can create intolerance of what may be considered part of the human experience. For example, sensitivity to sound may prevent a person with ASD from engaging in activities where airplanes may be heard overhead or babies may be heard crying. Also, sensitivity to touch can be especially difficult in relation to others, as those with ASD may not tolerate someone touching their skin or attempting to hug them. This particular sensitivity may also affect the choice of clothes for someone with ASD, who may be unable to wear clothes with sleeves or tags that they feel are restrictive and might lead one to wear socially inappropriate apparel.

Executive function impairments, i.e. impairments in decision-making skills, cognitive flexibility, impulse control, organizational skills, and planning, create another layer of social dysfunction [27]. Awareness of the passage of time may be compromised for someone with ASD, perhaps secondary to their self-absorption, and is an essential component of everyday function. Everyday memory problems or the ability to remember to plan and organize daily life activities can create social havoc. The ability to problem solve, make informed choices, or plan for the future becomes problematic in what is called "context blindness" [27].

All of the above challenges are magnified when a person with ASD attempts to have an intimate emotional and perhaps sexual relationship. Intimacy is the sharing of emotional, cognitive, and physical aspects of oneself with those of another. A prerequisite for intimacy is the establishment of a firm sense of self-identity. Intimacy requires the flexibility to loosen one's identity in order to feel the pleasure of merging with one's partner in an emotional and physical connection. For all of the reasons above, a person with ASD may be unable to share with another or may be limited in his or her ability to do so. 


\section{Case example: $\mathrm{RJ}$}

$\mathrm{RJ}$ is a 28 year-old female with ASD who was attempting to negotiate an intimate relationship with another woman her age that did not have ASD. First of all, RJ explained that a homosexual relationship was better for her than a heterosexual relationship because her partner was more like her than another man would be, and it was already very difficult to consider an intimate relationship, let alone try to understand someone of a different gender. $\mathrm{RJ}$ was absorbed in her interest in drawing and hoped to get a job at some point in computer animation. She spent most of the hours in a day drawing when she was not at her part time job at the local animal shelter. When she was drawing, it was fine for her partner to sit next to her, but she didn't want to be disturbed or touched. She was unable to do something other than drawing in the evening except on Saturdays, when she was able to include her partner in her schedule. Even on Saturday, she needed to find some time to herself because it took too much energy to be with her partner for a full day. When she attempted to do so, she would experience anxiety and frustration which would frequently culminate in an episode of yelling, stamping her feet, and retreating to her room. On Saturdays, when she was attempting to spend time with her partner, RJ was only able to engage in certain activities. Her partner would frequently ask her to go to the movies, while RJ was unable to tolerate the feel of the seat cushions on her skin, the smell of the popcorn, and the loudness of the sound track. RJ could only eat at two restaurants in the neighboring area but preferred to eat at home. RJ could not understand her partner's frustration with her or her partner's need for physical affectionate contact. RJ was able to tolerate some sexual contact but avoided it whenever possible, as it was adverse to her but she understood from reading that it was an expected part of a relationship. After several months, RJ's partner terminated the relationship, much to RJ's relief. She was very happy to return home to her parents' house where she could have conversation with them at her initiative, and the expectations for social interaction or disruption of her schedule were minimal. It was comforting to return to her family's schedule, which she knew well. She did have the insight to know that her parents wouldn't always be there and knew that she needed to work earnestly to maintain at least some relationship with friends. She understood that even though it may be difficult to do so, she would have to initiate contact and not rely on her friends solely to initiate such contact.

The only significant predictor of romantic functioning among those with ASD is level of social functioning [21]. When meeting someone with ASD, several irregularities are noticeable. Persons with ASD frequently will not look into the eyes of the person with whom they are interacting; instead they may look at their mouths or perhaps even another object in the room [20]. Some of those with ASD would state that looking directly at another's person's eyes is extremely anxiety provoking, whereas others with ASD may be disinterested. Personal physical spatial boundaries, which many people take as second nature, are not part of the social make-up in persons with ASD. They may stand too close to a person with whom they may be interacting, or they may seem distant and uninvolved. Those with ASD may not pay attention to socially acceptable standards of personal appearance and may appear unkempt or inappropriately dressed for an occasion, e.g. wearing a casual, comfortable outfit to a formal event. Persons with ASD have a very difficult time en- 
gaging another person in conversation, i.e. they have difficulty initiating conversation or maintaining conversation through reciprocal social interaction [26]. A person with ASD may answer questions when asked or begin a scripted monologue that is repetitive in nature about an area of interest, with little to no awareness of the reaction of the person with whom they are interacting. Part of the reason for this lack of awareness is that a person with ASD is frequently unaware of the meaning of nonverbal behavior as a means of communication. The concept of theory of mind states that a person cannot understand the thoughts, intentions, and feelings of others or what another person means during an interaction, other than the concrete nature of the words stated [28]. For example, when a mother asked her child to "go sit in the tub", the child sat in the tub with all of her clothes on, when the mother of course meant to prepare for a bath. This may seem obvious to most people but might not be so obvious to a person with ASD. As a corollary, a person with ASD frequently cannot read the emotional meaning behind a verbal or nonverbal communication, i.e. interpret social cues [29]. A study by Izuma supports that people with autism lack the ability to take into consideration what others think of them [30]. Partially due to this lack of awareness, someone with ASD may respond in a very blunt or honest way to a statement of another person with whom they are interacting. For example, when asked a question such as "Do you like my new dress?", the person with ASD might say all the reasons they feel the dress is unattractive, being unaware of the emotional impact such statements might have on the person to whom they are making such comments [31].

\section{Gender identity and sexual orientation}

Gender identity usually develops in neurotypical children by the age of three [10] with ranges of 3-5 years of age [32]. Gender identity may be more rigid in individuals with ASD [33]. For children with developmental disabilities, gender identity in general likely develops in synchrony with many other developmental delays, especially in language, communication and social relatedness, which in turn influences the child's ability to mentally represent their own gender either in images or language. There is no current established literature about gender identity development in children with ASD; however, a recent article on gender dysphoria and identity difficulty found that clinics are reporting an overrepresentation of individuals with ASD in their gender identity referrals [33].

Sexual orientation refers to a person's established patterns of overall attraction to another person, including emotional, romantic, sexual, and behavioral attractions [34] regardless of whether this pattern results in sexual behavior. Research in the last several decades established sexual orientation on a continuum from entirely heterosexual, bisexual, and homosexual to asexual [35-37]. The relatively novel term "sexual fluidity" refers to the situationdependent flexibility in someone's sexual responsiveness and may include both hetero- and same-sex experiences [37]. Same-sex behaviors among adolescents are reported between $5-10 \%$, with similar percentages observed in adults [10].

Sexual identity develops normatively in adolescence related to puberty and overall body changes in the context of societal expectations about partner choices. For most adolescents 
with ASD, this development may occur later than that of their typically developing peers [38] and may include higher percentages of asexuality, but in most aspects of sexual development, the literature identifies similar desires and fantasies [21]. In fact, the literature on sexuality of children and adolescents with developmental disabilities cautions to not erroneously regard people with disabilities as childlike, asexual or as inappropriately sexual [39].

At the same time, several studies were identified by Healy and colleagues [19] that show that people with a disability may hold rather conservative views about their own sexuality related to negative caregiver attitudes toward certain sexual behaviors, including pre-marital sex and homosexual activity. Still, in comparison to caregiving staff, family members may altogether be less inclined to openly discuss issues of sexuality. Family members seemed to prefer low levels of intimacy in the relationships of their child amidst a high acceptance of platonic and non-intimate relationships [40].

\section{ASD and intimacy}

Individuals growing up with ASD have the same human needs for intimacy and relationships as anyone [41]. However, the self-identification of these needs may develop later than same age neurotypically developing peers and become expressed differently depending upon the individual's sexual knowledge, beliefs and values. Understanding of implicit dating rules and the hierarchy of sexual intimacies may become potential barriers for individuals with disabilities in general and particularly for adolescents and adults with ASD. Focus groups have been shown to make a difference in an individual's understanding, especially with involvement of his or her family and caregivers [19].

Intimacy is the sharing of emotional, cognitive and physical aspects of oneself with those of another. Individuals with ASD often have problems with rigidity and the need for repetition, which may limit the spontaneity and playfulness of sexual contact. Sensitivity to physical contact and inability to tolerate internal sensations created by physical intimacy may also create significant anxiety. The inability to read the thoughts, feelings, or expressed sensations of one's partner can lead to miscommunication, emotionally or physically painful experiences, and/or shame and guilt. In the context of navigating intimacy, by adulthood there are several options for types of relationships, typically to include living single, cohabitating with one or several others, and living in a marriage/partnership. Currently, many adult individuals with ASD continue to reside with their family of origin. Due to poor social relationships and lack of employment, living with family provides a comfortable social situation, as observed in the case of RJ. There is no need for continual social contact or concern for others, as family already exists as a group.

When even possible, marital relationships can be very strained, as the ASD spouse (usually a male) frequently has difficulty interpreting the spouse's need for emotional attention. Little to no research has been done on the adult lifestyles of higher functioning persons with ASD other than to say that most of them remain in their parents' home. Most previous research has been with those living in a residential setting. One study whose focus was to sur- 
vey the gender identity of ASD subjects did ask a question pertaining to marital status. Gilmour and colleagues found that the group, which was atypically more female, did not differ from the control group on the basis of marital status. This result was unexpected and may be specific to the group surveyed of 82 persons with ASD [42]. More research is clearly needed in this area, but attaining accurate statistical data will be difficult, as many high functioning individuals with ASD are undiagnosed or misdiagnosed.

\section{Case example: $\mathrm{L}$}

Patient, L is a 35-year-old male engineering student, who was accompanied by his wife for an initial assessment. L's wife believed that he had Asperger's disorder. He did not understand why this potential diagnosis would even matter to his wife. A major concern in their marriage was L's dislike for social situations. His wife worked at a bookstore and was frequently invited to her coworkers' houses to play games, watch movies, or perhaps have dinner. L would begrudgingly attend but would then sit quietly and not interact with anyone. His wife's friends would attempt to include him in conversations, but L would frequently give one-word answers and not reciprocate or would engage in a long monologue about his most recent engineering project. He did not understand his wife's distress at these situations. As a couple, it was their usual routine to have a date on Saturday night consisting of time spent together in an activity, followed by a sexual encounter. L did not understand why his wife would break this routine when she was upset by his lack of social interaction at her co-worker's home. He would become very angry and frustrated, slamming the door, and breaking small nearby items. His wife encouraged him to come to the appointment as a way for her to begin to understand his behavior and to find ways to cope with him.

\section{Potential for abuse}

For all individuals with disabilities, including ASD, there is an increased risk for physical and sexual abuse. In 2006, Murphy and Elias reported a sexual abuse rate that was 2.2 times higher than that of children without disabilities [39]. In a recent study, caregivers of individuals with autism reported that $16.6 \%$ had been sexually abused. Individuals with ASD can be subject to sexual victimization due to their trusting natures, desire to be socially accepted, lack of understanding of the meaning or possible consequences of their behavior, or exposure through internet contacts. Children who experienced sexual abuse were more likely to act out sexually or be sexually abusive toward others [43]. This mindset, although with seemingly honest intentions, places the ASD individual(s) at risk for sexual abuse, due to the lack of available sexual knowledge. Lack of knowledge can contribute to an individual not understanding appropriate boundaries and therefore they may not be able to distinguish when someone is touching them inappropriately. This, coupled with existing social deficits, has resulted in underreported sexual abuse in this population. Therefore, sexual education and public intervention strategies (which will be discussed later in this chapter) are key protective factors and could contribute to healthy sexual development. 


\section{Case example: $M$}

A 17-year-old female patient, $M$, presented for diagnostic evaluation and was diagnosed with ASD. Her cognitive ability was in the low average IQ range. As a student in high school, she was very invested in making friends. She had difficulty managing the intricacies of relationships with other girls in her class, as her hygiene was below average and her clothing choices were not fashionable. M didn't belong to a specific social group of girls, such as cheerleaders, athletes, "Goths," etc. and therefore frequently sat by herself in the lunchroom. As she was failing in her social relationships with girls, she thought she would attempt to make friends with some of the boys in her class. She was coached by her younger sister at home (age 15 without ASD). Her sister was actually aware of M's poor social standing with other girls, as she was frequently asked what was wrong with her older sister by peers. $M$ had previously made positive contact with a boy in her art class, who was drawing a video game character. The art teacher supported this interaction and facilitated their conversations in class. Her contact with another boy, however, was less than positive. He told $\mathrm{M}$ that the best way to make friends was to spend time together after school at the park. The boy then made sexual advances, kissing the patient. She was very confused and did not stop his behavior, which led him to attempt to fondle her genital area. The encounter stopped at that point. $\mathrm{M}$ did not bring this event to the attention of her parents or sister. Fortunately, in her therapy session, she was able to ask if it was OK for a boy to put his hand in her pants. Clearly, M had not received instruction from her parents about "appropriate touch." The parents brought this situation to the attention of the school administrators, who reprimanded the boy but could not address it further, as $\mathrm{M}$ was older than 16 and it was deemed that she consented to the behavior by not stopping him.

\section{Inappropriate sexual behavior}

Along with the concerns of interpersonal intimacy and delayed maturity of sexuality, individuals with ASD may have difficulty determining what and where sexual behaviors are appropriate. Permitted behavior is governed by social appropriateness, which is gathered through social cues. With the limited ability to read and understand social cues, those with an ASD diagnosis can fail to discern between acceptable public behavior and acceptable private behavior [44]. A review article by Stokes and Kaur included masturbatory behaviors in public, removing clothing in public, and touching members of the opposite sex, as reported in previous studies, followed sometimes by the rejection of others due to these problematic behaviors [45]. For example, masturbatory activities are often seen in public when anxiety levels have increased. This in turn could potentially lead to legal implications. Among adolescents with ASD, some concerns include inappropriate courting behaviors, such as stalking or touching the person of interest inappropriately, making inappropriate comments, not always understanding the need for privacy such as knocking on doors [45], making threats against the person of interest, or exhibiting obsessive interest in a person [21], which can lead to both interpersonal and legal consequences. Behavioral and educational interventions must be considered in order to serve as a protective buffer against undesired outcomes. 


\section{Case example: $\mathrm{C}$}

Patient $C$ is a 14-year-old boy diagnosed with autism who had minimal verbal skills. At age 14 , he was $6{ }^{\prime} 2^{\prime \prime}$ tall. Cognitively, he was functioning at the mild intellectual disability range (IQ $\sim 70$ ). C had no friends. His social judgment was poor, so his parents encouraged his interactions and visits with extended family in an effort to improve his social communication. One of the patient's areas of interest was wrestling. He would frequently roughhouse with his other male cousins, who were teenagers as well. On one visit, C was watching a wrestling program with his younger cousin, age 4 , as the older boys had gone to the movies and $\mathrm{C}$ refused to attend. $\mathrm{C}$, not understanding the social implications of his behavior, began to roughhouse with his young cousin. When his mother and aunt entered the room, $\mathrm{C}$ was laying on top of his 4-year-old cousin in what was judged to be an attempt by the patient to molest this young child, whereas $C$ thought he had won the wrestling match like the man on television. When asked, $C$ could not adequately explain his behavior, due to his limited verbal skills. His mother was able to reassure the young boy's mother that $C$ had no sexual intent. However, $C$ and his mother no longer received invitations to visit the home of those relatives.

Masturbation especially in public settings has been the central focus within the developmental disorder literature due to the concerns and personal views of the general public and legal officials. In particular, these groups possess a tendency to label public masturbation as sexual deviancy. This predisposition was greatly reduced when both groups received training on the behaviors of individuals with ASD.

\section{Sexual education}

Sexual education is a core ingredient of successful intervention beginning with body anatomy, physiology and personal hygiene, taught in childhood. As the individual with ASD reaches older adolescence and adulthood, social dictates of what is appropriate sexual behavior in public must be carefully taught with video modeling and social stories [23] to prevent problematic outcomes for the person with ASD and those around him or her [21]. As with all stages of development, sexual development may be delayed, while pubertal development may be chronologically on time. The family needs to be educated about teaching sexuality as well in order to facilitate the knowledge of the individual with ASD throughout his or her development $[8,44]$. Sexual education can also prevent sexual abuse, unwanted pregnancies, and sexually transmitted infections, or STI [8]. A recent article on the sexuality of children and adolescents identified educational needs in the context of parent and health care professionals' expectations [39]. Likewise, a greater educational need was identified for caregivers of individuals with disabilities to help individual better navigate their social environment with implemented help on a societal and political level [44,46,47]. DeLamater and Friedrich cited the Kaiser Family Foundation (1997), noting that young people especially name mass media as a primary source of information about sex and intimacy over information and education provided by parents or professionals [10]. 
This is likely even more true currently, with youth having increased access to information via the Internet and the use of personal electronics. In this sense, the use of electronics may become a useful educational medium and perhaps even an interactive tool to facilitate development of socially expected courting and dating behaviors, with the goal of becoming able to establish longer term romantic relationships.

Education about sexuality is critical for the ASD population. Many persons with ASD have the desire to have friendships and intimate relationships; however it is very difficult for them to make the complex emotional distinctions between friendship, kindness, and romantic interest. In a study by Hellemans, the majority of subjects with ASD expressed sexual interest but lacked the appropriate skills and knowledge to have a successful relationship [48]. Their misinterpretations can lead to emotional pain for themselves and possibly inappropriate behaviors toward others [26]. The most common forms of sexuality education for adolescents and young adults occur through conversations with their peers and/or their families. A study by Realmuto and Ruble suggested that typical children learn about sexuality via casual social experiences, including those in the community, family and school settings [49]. Persons with ASD are at a unique disadvantage as they do not initiate or maintain social contacts to acquire such education. Family members approach sexuality in their children with ASD by denying it and not teaching sexuality at all, or by considering that their ASD children can approach sexuality as any other adolescent would [21]. In a study by Stokes and colleagues, 25 subjects with ASD aged 13-36 were compared to a normal control group of the same age; the study found that persons with ASD relied less upon peers and friends for knowledge but relied more on information they learned through reading and other similar activities [21].

When considering education about sexuality, three content areas need to be included: 1) basic facts and accurate information, 2) formation of individual values with consideration of family values, and 3) application of sexuality to relationships and social situations [15]. More specifically, basic biology of the sexual organs and how they function for males and females, maintenance of hygiene, prevention of pregnancy and sexually transmitted diseases, methods of birth control, how to initiate and maintain intimate sexual relationships, how to prevent unwanted sexual contact, the role of masturbation as a normal sexual bodily function and its social implications, as well as reproductive and parenting rights. What is most essential is to maintain a consistent focus on the social component of sexual behavior [8]. Due to theory of mind deficits, a person with ASD may be unable to understand the actions, feelings and intentions of others, such as not recognizing obvious clues of disinterest and being inappropriately persistent in pursuing a desired person. The person with ASD must learn how to initiate romantic relationships, understand dating behaviors, know appropriate physical boundaries, develop listening skills, and understand the meaning of consensual sexual activity [8]. Frequently, booster sessions are recommended as an individual grows and develops and has the need for additional information and skills or reinforcement of principles already learned that may have been forgotten [8].

Deciding who should teach a person with ASD about sexuality can be confusing. A team approach may be most successful. Parents and caregivers usually provide primary instruction 
but may need the support of a formal sexual education program provided by the school system. Parents provide the foundation for the development of the child's sexuality by modeling relationships in the home. The family's moral values, culture, religion, and other beliefs are clearly a major part of sexuality education. An IEP team can designate a specific component of the health curriculum to sexuality that must be geared to the child's cognitive, emotional, and social level of development. Such a plan should be revisited and revised as a child/adolescent matures with the need for more information, skills, and attitudes [8].

\section{Model programs}

Several models and approaches to sexuality education for those with ASD have been published. One model from a research study in Israel provided treatment through ten bi-weekly sessions, each devoted to topics that included establishment of self-identity, acceptance of one's disability, independence in social life, establishment of friendship and intimate relationships, sexual knowledge and development, and safety skills [50]. The aims of the group were to 1) discuss attitudes and feelings, 2) provide information, 3) advise parents on how to help children manage their sexuality, and 4) encourage independence in their children. The overarching principles of this group treatment were to 1) develop an appropriate self-concept, 2) find a similar social group, 3) develop relations based on equality and reciprocity, and 4) prevent abusive relations, with all of these aims potentially leading to satisfactory intimate romantic relationships. The most improvement in this study was shown in social development and the development of a clearer concept of friendship.

\section{Case example: $\mathrm{H}$}

$\mathrm{H}$ is a 19-year-old female who recently began attending community college. She has an above average IQ and good facility with language. She was able to manage some friendships in high school by being the manager of one of the girls' sports teams. The girls on the team were kind to her and included her in team activities, encouraged by the team's coach. $\mathrm{H}$ also belonged to the Anime club and had some friends there. The structured schedule of high school, along with the academic supports provided by her Individualized education plan, coaching and encouragement from her parents, enabled her success. $\mathrm{H}$ was having a difficult transition to college with no friends, no academic supports, and a less structured schedule. She attended a session provided by the disability services department and sat next to a boy several years older than she with a similar disability, who initiated and maintained a conversation. $\mathrm{H}$ was aware that he was a stranger and was careful in the information she provided. He asked her to meet for lunch at the cafeteria several times. H's mother wanted to meet him because she was unsure of her daughter's social judgment. With her parent's approval and her mother's coaching about dating, they went to a movie. Their relationship slowly progressed over the last six months beyond the handholding stage to the first kiss. H's boyfriend was able to allow her to manage the relationship to assist $\mathrm{H}$ in dealing with the anxiety that this relationship had created for her, though she was beginning to increasingly enjoy their time together. 
Another intervention that shows promise is the development of Social Stories ${ }^{\mathrm{TM}}$ by Carol Gray [51] which can be tailored to each child or adult and written in the person's perspective, so it can be used to prepare persons for dealing with friendships, managing intimacy, and improving safety [23]. Video modeling is another technique where a student watches a video where peers or others demonstrate appropriate behavior. The student then models the behavior he or she just viewed. Video modeling, by providing some distance, helps relieve some anxiety during a practice phase before trying a real time interaction [23].

Concepts from other treatment centers have added to sexuality education. Two precepts from the Devereaux Centers for Autism emphasize that 1) parents are the best sexual educators and 2) it is normal and natural for every person with a body to express their sexuality regardless of their disability [44]. The Benhaven residential program for those with autism emphasizes 1) the need to teach students socially acceptable sexual behavior appropriate for both childhood and adulthood 2) no disapproval of masturbation when done in socially appropriate situations as it may be the only sexual satisfaction some individuals with autism may experience and 3) do not encourage behavior beyond which an individual is capable or that will lead to frustration and disappointment [44].

It is helpful to consider the basic learning needs of those with ASD in general and apply them to sexuality education [26].

1. Use of visual aids, role play

2. Use of concrete, specific examples instead of abstract concepts

3. Dividing large blocks of information into smaller, sequential segments

4. Allowing time for comments and questions

5. Keeping brief any discussions of feelings so as not to confuse or overwhelm

6. Provide overviews and structure to the lesson

7. Include specific problem solving strategies and examples

Especially when considering sexuality education in those with lower functioning ASD, the capacity to make sexually-related decisions must be considered. A study of four adults with moderate intellectual disability (not autism) focused on improving capacity to make sexuality related decisions [52]. Treatment was rendered on a 1:1 basis for 20 sessions. The article by Dukes and colleagues emphasizes that in order to provide valid consent to sexual contact, the person with a disability requires knowledge about sexuality and the understanding of the concept of what is and is not voluntary [52]. Consent must also be individualized and situation specific for decision-making associated with sexual contact. This intervention focused on sexual safety practices, knowledge of the physical self, knowledge of sexual functioning, and knowledge of choices and consequences in sexual matters. The study noted the need for booster sessions, as the memory of topics covered waned with time, perhaps secondary to little opportunity to utilize the information learned [52]. A survey of the sexual behavior of 89 adults with autism living in group homes in North Carolina found that the majority of individuals were engaging in some 
form of sexual behavior [53], with masturbation being the most common sexual behavior. One third of the residents did have other oriented sexual behavior, which mostly consisted of holding hands, touching, and kissing. One third of the residents did not masturbate at all. A major concern with lower functioning individuals is the inappropriate expression of sexual behaviors in a socially unacceptable manner [53].

To improve decision-making related to sexuality in individuals with an intellectual disability, Dukes and McGuire adapted successfully a sexual education program for individuals with special needs called Living Your Life [52]. Possibly such a program could also further be adapted to the specific knowledge and needs of individuals with ASD. In their 2010 article, Travers and Tincani identified Body Awareness, Social Development, Romantic Relationships and Intimacy, Masturbation and Modifying Behavior to Meet Social Norms, and Reproductive and Parenting Rights of Individuals with ASD as crucial components of sexuality education for individuals with ASD [8]. These authors also identified the need for professionals to address sexuality education in an open, confident, and objective manner in a collaborative effort with the individual with ASD and their family.

The TEACCH program [54] has explicit guidelines for teaching sexuality education to the lower functioning person with ASD [16]. An important component is taking an individualized developmental approach, with the goal of matching teaching programs to level of function and development of long range goals (e.g. capacity to have a romantic relationship versus ability to enjoy masturbation in a socially acceptable manner). Another concept is that sexuality cannot be taught in isolation but must be considered in the context of other skills, such as one's ability to verbally communicate or one's cognitive ability, The most basic skill is the ability to have discriminate learning, for example, knowing where and when to touch others or masturbate, and can be taught from a behavioral perspective, with rewards for appropriate behavior. Environmental supports to reinforce appropriate behaviors can be very useful, and environmental changes (e.g. wearing a belt to help prevent a young man from masturbating in public) may allow for intervention prior to a behavior occurring, as slowing down the behavior provides more time to intervene. The next level beyond discriminate learning is managing personal hygiene, followed by understanding body parts and their functions. The highest level is a complete sex education program, including development of sexual relationships with others.

Social skills groups and meet-ups for older adolescents and young adults are essential to continue to build on social skills and allow for facilitated interaction [55]. A recent study with adolescents and young adults with ASD by Stokes and colleagues found that one's level of social functioning predicted romantic functioning [21]. The development of social interaction skills will help promote interest in developing meaningful relationships with others that in turn may lead to intimate relationships and ultimately more independent living arrangements. Equally important are the development of emotion regulation and self-esteem skills that will help to navigate difficulties and changes within significant relationships. In their 2006 article, Murphy and Elias [39] described how children and adolescents with disabilities generally have fewer skills and opportunities to engage in social interactions that could lead potentially into intimate relationships. In particular, this im- 
portant article emphasizes particular skills that are often amiss for individuals with disabilities, Abilities, especially the ability to make eye contact, develop appropriate greetings, recognize personal space, and interpret nonverbal communication, that apply to individuals with ASD [39].

Based on previous studies, and addressing the gap in identified interventions specific to the sexual development of individuals with ASD, a current intervention program called Growing Up Aware is in the process of being developed at Columbia University [14]. The first research component attempted to better understand how parents teach their children with ASD about sexuality. Results of the study showed that the majority of parents indicated a strong interest in learning how to better communicate with their children about sexual and reproductive health [14]. This is met currently by insufficient availability of materials for parents. Many clinical providers appear under-equipped, with normative knowledge and skills themselves about how to address questions of parents regarding their child's changing sexual development based on parental perception. Clinicians need to become better equipped to help families with unusual or inappropriate sexual development.

\section{Medication concerns}

Medication side effects that were not troubling to a child with ASD may cause significant distress in an adult with ASD by decreasing sexual desire or interfering with sexual potency [56]. Self-injury may result if appropriate instruction about masturbation is not provided. Medications such as fluoxetine or sertraline (selective serotonin reuptake inhibitors) are frequently prescribed for persons with ASD to help with anxiety or repetitive behaviors. This group of medications can cause a decrease in sexual desire or make it much more difficult to attain an orgasm. Since masturbation is one of the most frequent sexual behaviors within the ASD population, unintentional self-injury may result from prolonged attempts to reach orgasm. Appropriate instruction in masturbatory behaviors may be necessary in order to prevent self-injury [46]. Alternately, a medication with sexual side effects may be beneficial for a patient who has anxiety and/or excessive inappropriate sexual behaviors by decreasing sexual desire [57] and enhancing the effectiveness of behavioral interventions.

\section{Public intervention}

There are many important reasons for promoting sexuality education for those with ASD including the following: 1) prevention of sexual abuse, 2) preventing inappropriate sexual behavior toward others, 3) promoting health and hygiene and preventing sexually transmitted disease and pregnancy, 4) facilitating the development of intimate relationships, and 5) pre- 
venting self-injury [8]. A basic tenet is that sexuality education for persons with ASD must be geared to their particular level of cognitive, emotional, and social functioning and is most effective when it is highly individualized. Those with ASD have a right to have a sexual life, a right to receive guidance and support, and they need assistance in expressing sexuality in an acceptable way to those in their environment [8].

Public intervention strategies should primarily focus on educating the community about the behaviors and traits common to persons with Autism Spectrum Disorders. Education has been shown to foster tolerance and understanding. In addition to this, education tends to spawn advocacy, thereby facilitating the needed changes in existing policies and law. In particular, advocates of those with ASD have the greatest opportunity to teach others about this population by modeling how best to support persons with ASD in the community.

Particular attention should be given to law enforcement, judicial systems and other populations that traditionally have minimal contact with individuals with ASD [7]. Educational efforts should include a discussion of basic symptomatology, behavioral interventions and treatments. Efforts should also be made to dispel myths, misconceptions and assumptions about those with ASD [58]. In addition, education should include information about potential risks to this population and the available programs and systems that are in place to provide protection for the ASD population [44].

\section{Conclusion}

In summary, our literature review and ample experiences of the families in our clinical practice show that, while every person has the innate basis for developing sexuality in a multitude of expressions and experiences, individuals with disabilities (and especially individuals with an Autism Spectrum Disorder) most often require additional education and help to become able to express their sexuality in a socially appropriate way. While most neurotypically developing peers form intimate relationships beginning in adolescence and into adulthood, along a variety of experiences from dating to partnering in committed relationships, many individuals with an Autism Spectrum Disorder remain living with their family of origin into their adulthood and have significant difficulty navigating the social expectations surrounding relationships. Their difficulty may pertain to recognizing their own needs and wants, as well as to recognizing their partner's wishes coupled with more inexperience than their peers in this arena. Individuals with ASD and their parents and caregivers frequently identify this difficulty when directly asked about it. Sexuality education in a supportive format that includes the individual's family and their particular values and background will be most effective. Interventions need to be individualized with a long-range goal that matches the cognitive, social, and emotional developmental level of the person with ASD. As the prevalence of persons with ASD increases in our society, we are more than ever called to support their ability to mature into adults capable of functioning in all areas of life, including sexuality and intimacy. 


\section{Author details}

Maria R. Urbano, Kathrin Hartmann, Stephen I. Deutsch,

Gina M. Bondi Polychronopoulos and Vanessa Dorbin

*Address all correspondence to: urbanomr@evms.edu

Department of Psychiatry and Behavioral Sciences, Eastern Virginia Medical School, Norfolk, Virginia, USA

\section{References}

[1] Association AP. Diagnostic and Statistical Manual of Mental Disorders (fourth ed., text rev.). Washington, DC: Author; 2000.

[2] American Psychological Association http://www.apa.org2012.

[3] Frazier TW, Youngstrom EA, Speer L, Embacher R, Law P, Constantino J, et al. Validation of proposed DSM-5 criteria for autism spectrum disorder. J Am Acad Child Adolesc Psychiatry. 2012 Jan;51(1):28-40.e3. PubMed PMID: 22176937. Pubmed Central PMCID: PMC3244681. eng.

[4] Mandy WP, Charman T, Skuse DH. Testing the construct validity of proposed criteria for DSM-5 autism spectrum disorder. J Am Acad Child Adolesc Psychiatry. 2012 Jan;51(1):41-50. PubMed PMID: 22176938. eng.

[5] Prevalence of Autism Spectrum Disorders-Autism and Developmental Disabilities Monitoring Network, 14 sites, US 2008. Surveillance Summaries March 30, 2012. Morbidity and Mortality Weekly Report 61(SS03) 1-19.

[6] CDC. Morbidity and Mortality Weekly Report 2012; 61(SS--3): [1-19 pp.].

[7] McCarthy M. Women with Intellectual Disabilities: Finding a Place in the World. Traustadottir RJ, K., editor. London, England: Jessica Kingsley Publishers; 2000.

[8] Travers J, Tincani M. Sexuality education for individuals with Autism Spectrum Disorders: Critical issues and decision making guidelines. Education and Training in Autism and Developmental Disabilities. 2010;45(2):284-93.

[9] Freud A. The Concept of Developmental Lines. Psychoanal Study Child. 1963;18:245-65. PubMed PMID: 14147280. eng.

[10] DeLamater J, Friedrich WN. Human sexual development. J Sex Res. 2002 Feb;39(1): 10-4. PubMed PMID: 12476250. eng.

[11] CDC. Trends in the prevalence of sexual behavior and HIV testing: National YRBS 1991-2011. http://www.cdc.gov/healthyyouth/yrbs/pdf/us_sexual_trend_yrbs.pdf: 2011. 
[12] Nussey SS, Whitehead, SA. Endocrinology: An Integrated Approach. Oxford: Bios Scientific Publishers; 2001.

[13] Erikson E. Childhood and Society. New York: Norton; 1950.

[14] Ballan MS. Parental perspectives of communication about sexuality in families of children with autism spectrum disorders. J Autism Dev Disord. 2012 May;42(5): 676-84. PubMed PMID: 21681591. eng.

[15] Gerhardt, P. Sexuality Instruciton and Autism Spectrum Disorders: Autism Society. Copyright 2006-2012. Education.com. All rights reserved. http://www.education.com/reference/article/sexuality-instruction-autism-ASD/

[16] Sexuality and Autism. TEACCH Report. Autism independent UK. http:www.autismuk.com/index9sub1.htm

[17] Mesibov G, Schopler E. Autism in Adolescents and Adults. New York: Plenum Press; 1983.

[18] Klinger L, Dawson G, Renner P. Autistic Disorder. In: Mash E, Barkley R, editors. Child Psychopathology. 2nd ed. New York: Guilford Press; 2003.

[19] Healy E, McGuire BE, Evans DS, Carley SN. Sexuality and personal relationships for people with an intellectual disability. Part I: service-user perspectives. J Intellect Disabil Res. 2009 Nov;53(11):905-12. PubMed PMID: 19709348. eng.

[20] McPartland JC, Pelphrey KA. The Implications of Social Neuroscience for Social Disability. J Autism Dev Disord. 2012 Mar. PubMed PMID: 22456816. ENG.

[21] Stokes M, Newton N, Kaur A. Stalking, and social and romantic functioning among adolescents and adults with autism spectrum disorder. J Autism Dev Disord. 2007 Nov;37(10):1969-86. PubMed PMID: 17273936. eng.

[22] Shandra CL, Chowdhury AR. The first sexual experience among adolescent girls with and without disabilities. J Youth Adolesc. 2012 Apr;41(4):515-32. PubMed PMID: 21559882. eng.

[23] Chan J, John RM. Sexuality and sexual health in children and adolescents with autism. The Journal for Nurse Practitioners. 2012;8(4):306-15.

[24] Perry N. Adults on the Autism Spectrum leave the nest: Achieving Supported Independence: Jessica Kingsley Publishers; 2009.

[25] Losh M, Capps L. Understanding of emotional experience in autism: insights from the personal accounts of high-functioning children with autism. Dev Psychol. 2006 Sep;42(5):809-18. PubMed PMID: 16953688. eng.

[26] Ray F, Marks C, Bray-Garretson H. Challenges to treating adolescents with Asperger's Syndrome who are sexually abusive. Sexual Addiction \& Compulsivity. 2004;11:264-85. 
[27] Stichter JP, Herzog MJ, Visovsky K, Schmidt C, Randolph J, Schultz T, et al. Social competence intervention for youth with Asperger Syndrome and high-functioning autism: an initial investigation. J Autism Dev Disord. 2010 Sep;40(9):1067-79. PubMed PMID: 20162344. eng.

[28] Baron-Cohen S, Leslie AM, Frith U. Does the autistic child have a "theory of mind"? Cognition. 1985 Oct;21(1):37-46. PubMed PMID: 2934210. eng.

[29] Jellema T, Lorteije J, van Rijn S, van t' Wout M, de Haan E, van Engeland $H$, et al. Involuntary interpretation of social cues is compromised in autism spectrum disorders. Autism Res. 2009 Aug;2(4):192-204. PubMed PMID: 19642087. eng.

[30] Izuma K, Matsumoto K, Camerer CF, Adolphs R. Insensitivity to social reputation in autism. Proc Natl Acad Sci U S A. 2011 Oct;108(42):17302-7. PubMed PMID: 21987799. Pubmed Central PMCID: PMC3198313. eng.

[31] Bauminger N. The facilitation of social-emotional understanding and social interaction in high-functioning children with autism: intervention outcomes. J Autism Dev Disord. 2002 Aug;32(4):283-98. PubMed PMID: 12199133. eng.

[32] Ruble DN, Taylor LJ, Cyphers L, Greulich FK, Lurye LE, Shrout PE. The role of gender constancy in early gender development. Child Dev. 20072007 Jul-Aug; 78(4):1121-36. PubMed PMID: 17650129. eng.

[33] de Vries AL, Noens IL, Cohen-Kettenis PT, van Berckelaer-Onnes IA, Doreleijers TA. Autism spectrum disorders in gender dysphoric children and adolescents. J Autism Dev Disord. 2010 Aug;40(8):930-6. PubMed PMID: 20094764. Pubmed Central PMCID: PMC2904453. eng.

[34] Division 44/Committee on Lesbian G, and Bisexual Concerns Joint Task Force on Guidelines for Psychotherapy with Lesbian, G.y, and Bisexual Clients. Guidelines for psychotherapy with lesbian, gay, and bisexual clients. Am Psychol. 2000 Dec; 55(12):1440-51. PubMed PMID: 11260872. eng.

[35] Kinsey AC, Pomeroy WB, Martin CE. Sexual behavior in the human male. Philadelphia: W.B. Saunders; 1948.

[36] Klein F. The bisexual option. New York: Harrington Park Press; 1993.

[37] Diamond LM. Sexual fluidity: Understanding women's love and desire. Cambridge, Mass.: Harvard University Press; 2008.

[38] Volkmar FR, Carter A, Sparrow SS, Cicchetti DV. Quantifying social development in autism. J Am Acad Child Adolesc Psychiatry. 1993 May;32(3):627-32. PubMed PMID: 7684364. eng.

[39] Murphy NA, Elias ER. Sexuality of children and adolescents with developmental disabilities. Pediatrics. 2006 Jul;118(1):398-403. PubMed PMID: 16818589. eng. 
[40] Evans DE, Rothbart MK. A Two-Factor Model of Temperament. Pers Individ Dif. 2009 Oct;47(6):565-70. PubMed PMID: 20161172. Pubmed Central PMCID: PMC2722842. ENG.

[41] Ousley OY, Mesibov GB. Sexual attitudes and knowledge of high-functioning adolescents and adults with autism. J Autism Dev Disord. 1991 Dec;21(4):471-81. PubMed PMID: 1778961. eng.

[42] Gilmour L, Schalomon PM, Smith V. Sexuality in a community based sample of adults with Autism Spectrum Disorder. Research in Autism Spectrum Disorders. 2012;6:313-8.

[43] Mandell DS, Walrath CM, Manteuffel B, Sgro G, Pinto-Martin JA. The prevalence and correlates of abuse among children with autism served in comprehensive community-based mental health settings. Child Abuse Negl. 2005 Dec;29(12):1359-72. PubMed PMID: 16293306. eng.

[44] Koller R. Sexuality and Adolescents with Autism. Sexuality and Disability. 2000;18(2):125-35.

[45] Stokes M, Kaur A. High Functioning Autism and Sexuality: A Parental Perspective. Autism. 2005;9(3):266-89.

[46] McGuire BE, Bayley AA. Relationships, sexuality and decision-making capacity in people with an intellectual disability. Current Opinion in Psychiatry. 2011;24:398-402.

[47] Kandel I, Morad M, Vardi G, Merrick J. Intellectual disability and parenthood. Scientific World Journal. 2005 Jan;5:50-7. PubMed PMID: 15674450. eng.

[48] Hellemans H, Colson K, Verbraeken C, Vermeiren R, Deboutte D. Sexual behavior in high-functioning male adolescents and young adults with autism spectrum disorder. J Autism Dev Disord. 2007 Feb;37(2):260-9. PubMed PMID: 16868848. eng.

[49] Realmuto GM, Ruble LA. Sexual behaviors in autism: problems of definition and management. J Autism Dev Disord. 1999 Apr;29(2):121-7. PubMed PMID: 10382132. eng.

[50] Plaks M, Argaman R, Stawski M, Qwiat T, Polak D, Gothelf D. Social-sexual education in adolescents with behavioral neurogenetic syndromes. Isr J Psychiatry Relat Sci. 2010;47(2):118-24. PubMed PMID: 20733254. eng.

[51] Gray C. The New Social Story Book. Arlington, Texas: Future Horizons, Inc.; 2010.

[52] Dukes E, McGuire BE. Enhancing capacity to make sexuality-related decisions in people with an intellectual disability. J Intellect Disabil Res. 2009 Aug;53(8):727-34. PubMed PMID: 19527433. eng.

[53] Van Bourgondien ME, Reichle NC, Palmer A. Sexual behavior in adults with autism. J Autism Dev Disord. 1997 Apr;27(2):113-25. PubMed PMID: 9105963. eng. 
[54] Haracopos D, Pederson L. Danish Report. Sexuality and Autism [Internet]. 1992.

[55] White SW, Albano AM, Johnson CR, Kasari C, Ollendick T, Klin A, et al. Development of a cognitive-behavioral intervention program to treat anxiety and social deficits in teens with high-functioning autism. Clin Child Fam Psychol Rev. 2010 Mar; 13(1):77-90. PubMed PMID: 20091348. Pubmed Central PMCID: PMC2863047. eng.

[56] Physicians Desk Reference. 66 ed. Montvale, NJ: Thomson PDR; 2012.

[57] Albertini G, Polito E, Sarà M, Di Gennaro G, Onorati P. Compulsive masturbation in infantile autism treated by mirtazapine. Pediatr Neurol. 2006 May;34(5):417-8. PubMed PMID: 16648008. eng.

[58] Irvine A. Issues in sexuality for individuals with developmental disabilities: Myths, misconceptions, and mistreatment. Exceptionality Education Canada. 2005;15(3): $5-20$. 


\section{Clinical Implications of a Link Between Fetal Alcohol Spectrum Disorders (FASD) and Autism or Asperger's Disorder - A Neurodevelopmental Frame for Helping Understanding and Management}

Kieran D. O'Malley and Susan D. Rich

Additional information is available at the end of the chapter

http://dx.doi.org/10.5772/54924

\section{Introduction}

The teratogenic effect of alcohol was first observed by paediatrician Paul Lemoine in Nantes, France in 1968, when he linked facial dysmorphic and growth features with maternal use of alcohol (wine) in pregnancy. His initial series was 127 infants. Subsequently the syndrome Fetal Alcohol Syndrome was defined in 2 classic papers in 1973 by David smith and Ken Jones in Seattle. Their initial case series were 8 patients. The recognition that prenatal alcohol exposure did not just cause dysmorphic facial features and growth delay was made by Sterling Clarren in Seattle in 1978 with the introduction of the term Fetal Alcohol Effect ( FAE) to describe children with alcohol exposure but no facial features. This descriptive clinical term was changed to Alcohol Related Neurodevelopmental Disorder (ARND) by the Institute of Medicine in 1996.

\section{A conceptual understanding of the spectrum of effects of prenatal alcohol exposure}

In the same vein as Autistic Spectrum Disorders, Fetal Alcohol Spectrum Disorders (FASD, initially described by Streissguth \& O'Malley in 2000 is an umbrella term to describe the continuum of complex neuropsychiatric, cognitive, behavioral, social, language, communication and other multi-sensory deficits. There are, however, two conditions within this spectrum which describe the range of conditions caused by prenatal alcohol exposure. They are Fetal 
Alcohol Syndrome (FAS) a dysmorphic syndrome, and Alcohol Related Neurodevelopment Disorder (ARND) a non dysmorphic condition and by far the more common of the two conditions.

However within FASD there are physical sequellae aside from the facial dysmorphology, which are associated with all levels of prenatal alcohol exposure (Stratton et al 1996). These Alcohol Related Birth Defects (ARBD), as they are called, can occur as early as the first few weeks post conception.So, before most women know they are pregnant (Sulik, et al., 1983).

The central nervous system (CNS) and brain are the most sensitive and vulnerable structures to the effects of alcohol and can be affected by moderate to heavy alcohol use at any point in gestation. There is no safe amount of alcohol (threshold) during pregnancy and the Surgeon General of the United States currently recommends all childbearing age women to avoid alcohol if there is a potential for pregnancy (US Surgeon General, 2005).

Early, frequent, and/or binge exposures with moderate to high blood alcohol concentrations can lead to a range of reproductive outcomes including infertility, miscarriage (spontaneous fetal loss), still birth, sudden infant death syndrome, and a wide range of physical and neurodevelopmental (functional) birth defects. Varying degrees of Fetal Alcohol Syndrome (FAS) may be seen clinically at this range of exposure (Jones \& Smith 1973, Streissguth et al 1987, 1991). Alcohol-Related Neurodevelopmental Disorder (ARND, Stratton et al., 1996) are the neurodevelopmental/functional birth defects that manifest in individuals with FAS, as well as those who do not meet full criteria for FAS but have documented evidence of prenatal alcohol exposure. ARND can be associated with inattention, poor decision making, impulsivity, processing and working memory issues, other areas of executive dysfunction, mood instability, social communication deficits, and difficulties understanding consequences of their actions. These deficits are best evaluated by a thorough neuropsychological exam, including the Vineland Adaptive behavioral scales, IQ testing, and assessment of executive functions, such as BRIEF., FASD includes two conditions FAS which is dysmorphic and ARND, which is non-dysmorphic (See Table 1.).

Both FAS, dysmorphic and ARND. non-dysmorphic conditions can be associated with physical sequellae resulting from alcohol exposure in pregnancy. The Institute of Medicine describes these physical manifestations collectively as Alcohol Related Birth Defects (ARBD), including abnormalities in the developing eye, ear, teeth, heart, kidney, and skeletal system (Stratton et al, 1996; O'Malley \& Streissguth, 2000; Chudley et al, 2005; BMA, 2007). Like the typical FAS facial features, ARBD occur in the first 8 weeks of embryonic development (organogenesis). Many of these conditions may not be diagnosed or evident at the time of a patient's psychiatric evaluation (Iich 2005, Nowick Brown et al 2011).

The major difference between dysmorphic (i.e., FAS) and nondysmorphic (ARND) phenotypes is whether or not the collective cardinal (dysmorphic) facial features are present. The facial features correlate to heavy maternal blood alcohol concentration (e.g., the equivalent of 5 to 6 servings of alcohol) during the earliest points in gestation (late $3^{\text {rd }}$ week to early $4^{\text {th }}$ week of embryonic development) (Sulik, 1983). Both the facial features and ARBD are due to early embryonic changes, disruptions in cell migration, and cell death (apoptosis) due to the 
teratogenic effects of alcohol. Thus, the clinical relevance of both ARBD and the facial features in FAS is that of biomarkers for heavy binge exposure early in pregnancy - and sometimes, but not always, may predict a worse neurodevelopmental prognosis (Riley and McGee, 2005, Coles et al 2011). Since both FAS and ARND have neurodevelopmental (CNS) involvement, essentially ARND is FAS without the characteristic facial features. (Rich\& O'Malley 2012).

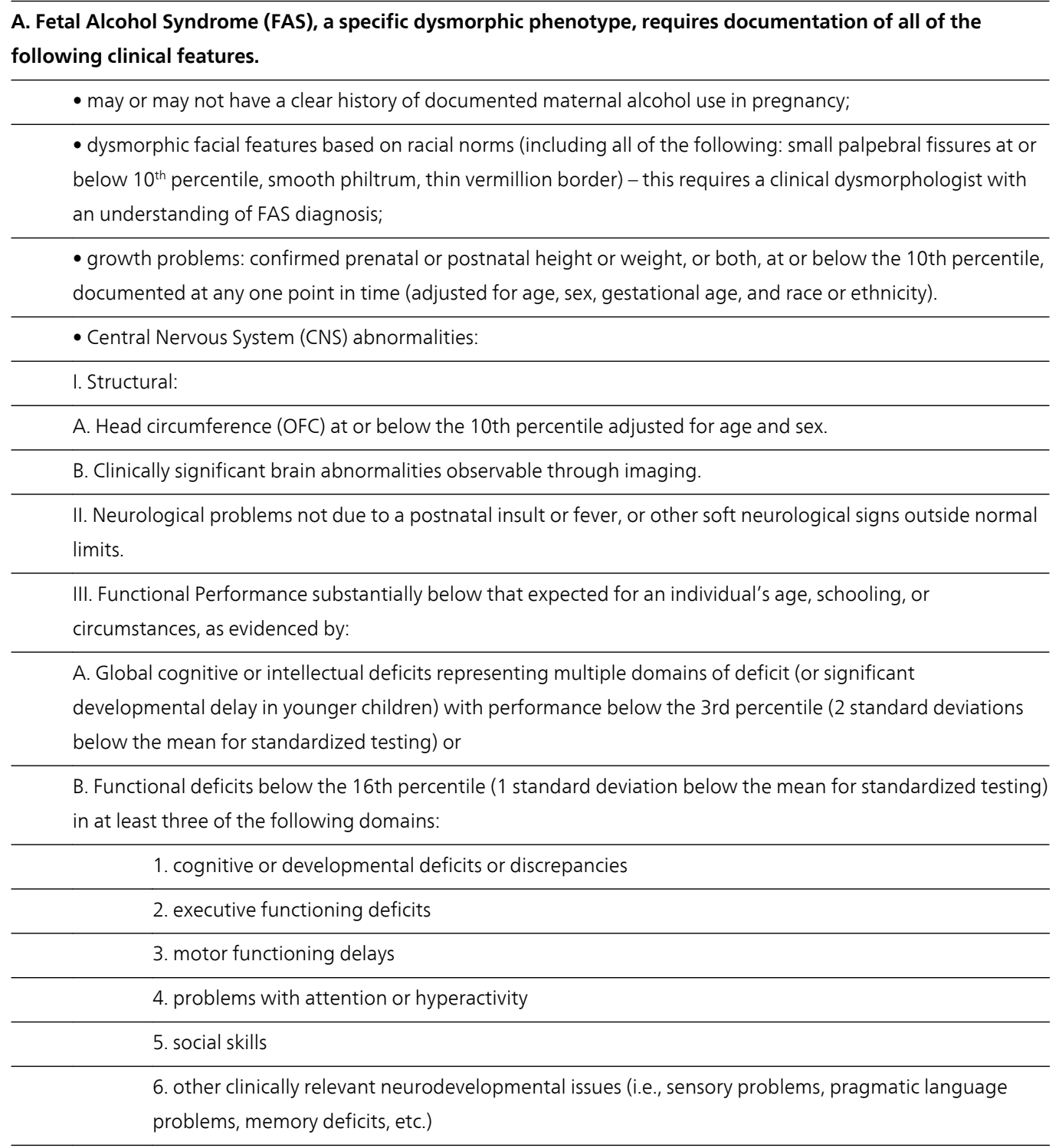

B. Alcohol Related Neurodevelopmental Disorder (ARND) is a non-dysmorphic condition with the following features:

- must have a documented history of maternal alcohol use during pregnancy; 
- none or not all pathognomonic dysmorphic facial features are present;

- no evidence of growth delay, low birth weight, decelerating weight over time, nor other height and weight issues;

- Central Nervous System (CNS) abnormalities:

I. Structural:

A. Head circumference (OFC) at or below the 10th percentile adjusted for age and sex.

B. Clinically significant brain abnormalities observable through imaging.

II. Neurological problems not due to a postnatal insult or fever, or other soft neurological signs outside normal limits.

III. Functional Performance substantially below that expected for an individual's age, schooling, or circumstances, as evidenced by:

A. Global cognitive or intellectual deficits representing multiple domains of deficit (or significant developmental delay in younger children) with performance below the 3rd percentile (2 standard deviations below the mean for standardized testing) or

B. Functional deficits below the 16th percentile (1 standard deviation below the mean for standardized testing) in at least three of the following domains:

\begin{tabular}{l}
\hline 1. cognitive or developmental deficits or discrepancies \\
\hline 2. executive functioning deficits \\
\hline 3. motor functioning delays \\
\hline 4. problems with attention or hyperactivity \\
\hline 5. social skills \\
6. other clinically relevant neurodevelopmental issues (i.e., sensory problems, pragmatic language \\
problems, memory deficits, etc.)
\end{tabular}

(Jones \& Smith 1973, 1975, Stratton et al 1996, Chudney et al 2005, BMA 2007, O’Malley 2008, Novick Brown et al 2011, O'Malley \& Mukarjee 2010, CDC 2011,HHS)

Table 1. Characteristic Diagnostic Features of FAS (dysmorphic) and ARND ( non dysmorphc)

Among both phenotypes, FAS is the less common condition, accounting for only $20-25 \%$ of the affected infants and children exposed to all levels of alcohol exposure. By comparison, nondysmorphic ARND is the more common clinical presentation of affected infants and children, accounting for 75 to $80 \%$ of affected infants exposed to all levels of alcohol in pregnancy. While maternal alcohol use is the leading known preventable cause of mental retardation and birth defects, only $20-25 \%$ of patients with either dysmorphic FAS or nondysmorphic ARND have a total IQ below 70. In other words, 75 to $80 \%$ of patients with FASD are estimated to have a developmental disability or other CNS impairment (acquired brain injury) but are not mentally retarded (Streissguth et al., 1996; Mukarjee et al., 2006). Hence FASD (FAS and ARND) are NOT mental retardation conditions, but are complex neurodevelopmental disorders with 
initial developmental, cognitive, and neurobehavioral outcomes, and higher lifetime risk of psychiatric co-morbidities and substance use disorders.

The dysmorphic facial appearance of an individual is much less an impact than the complex behaviors, psychopathology and developmental disability caused by alcohol's neurotoxicity. Thus, an individual's level of functioning is affected more by behavioral functioning, intellect, cognitive and communication abilities, executive functioning, temperament, social relatedness, emotional regulation, and performance than what his or her face looks like. FAS, the dysmorphic presentation of ARND, is in fact a protective factor for what Ann Streissguth called secondary disabilities of FASD ( Streissguth et al 1996).

Neuroimaging studies suggest that alcohol exposure may be specific rather than global in its teratogenicity, including specific vulnerability in the cerebellum, basal ganglia, and corpus callosum. As well, studies have shown deficits in cognitive functions such as learning and memory, visual-spatial functioning, executive functioning, attention, sequencing, processing and motor control. (Mattson et al 2011) These "functional birth defects" are evidenced by impairment in the brain and central nervous system. Riley and colleagues have shown that functional birth defects are present in children with moderate to heavy prenatal alcohol exposure, even in absence of characteristic (dysmorphic) facial features (Bookstein et al 2001, Riley and McGee, 2005, Coles et al 2011).

It is a critical issue in clinical diagnosis of FASD to understand that the severity of the acquired brain injury is not always correlated with the presence of facial dysmorphology (and FAS facial features commonly change significantly in adolescence and adulthood). Therefore facial features are minimally useful to assess and treat neurocognitive and neurobehavioral deficits associated with prenatal alcohol exposure. (Streissguth, et al., 1991; Steinhausen, et al., 1993, Nowick Brown et al 2011, Kodituwakku et al 2011, O'Malley 2011, Rich \& O'Malley 2012).

The first 30 to 40 years of research in FASD has been driven by animal teratology and the pursuit of minute changes in facial dysmorphology as biological markers for the level of prenatal alcohol exposure. Nevertheless, it is becoming quite clear that it is the central nervous system brain dysfunction that is the kernel of the problem and the guide to diagnostic understanding and management. It is not the face that tells the clinician about the underlying brain dysfunction but the complex mixture of developmental disability and psychiatric disorder. FASD, whether dysmorphic FAS or non dysmorphic ARND are developmental psychiatric disorders which, as Susan Rich and Kieran O'Malley describe in their 2012 paper. These conditions can present a neurodevelopmental mixture of mood dysregulation and autonomic arousal with language and social skills deficits, cognitive and executive decision making dysfunctions and multisensory functional and perceptual deficits

\subsection{The link between FASD and autism or Asperger's disorder}

As far back as 1990, child neuropsychologist Jo Nanson in Saskatoon, Canada, described 6 cases of FAS with autism. As well, the interest in prenatal risk factors contributing to autism has been pursued by a number of authors and this potential aetiological link was published in 1991 by International autism researcher Cathy Lord and colleagues. More recently, since 
2009-2010, adult psychiatrist in London, Raja Mukarjee, has painstakingly clinically analysed the clinical presentation of Autistic Spectrum Disorder in patients with FASD.

In the international paediatric and child psychiatric field the last 5 years have brought a wealth of clinical case descriptions and case studies indicating the presence of ADHD co-morbidly with PDD or Autistic Spectrum Disorder. Clinicians and researchers such as Professor Jeremy Turk in the UK have commented on as much as a 25-30\% co-morbid link between ADHD and PDD/ASD. Furthermore the complexity of diagnostic issues within FASD have been recently illustrated in a 2011 on line book chapter by Natalie Novick Brown, Kieran O'Malley and Ann Streissguth in which the developmental psychiatric presentations of FASD were shown to include sometimes unrecognized Autistic Spectrum Disorder or Asperger's Disorder.

\subsection{Aetiological theories postulated for this link}

It is important to place the possible link between prenatal alcohol exposure and Autism spectrum disorder or Asperger's Disorder in a historical context. Environmental agents, diseases and postnatal interventions have had, it is fair to say, a rather mixed and controversial past, as recently pointed out by Cathy Lord, So Hyun K im and Adriana Dimartino in 2011.

Although as far back as 1971 American child psychiatrist Stella Chess's case review of rubella and thalidomide cases implicated these prenatal infectious and medication exposures as aetiological, the series were small. European researchers Gilberg and Gilberg in 1983 have more rigorously identified a cluster of adverse prenatal complications which may contribute to a clinical presentation of Autism Spectrum disorder in early childhood.

However the most studied, but as well the most problematic, was the potential association between MMR vaccine and Autism Spectrum Disorder. It is not the remit of the chapter to completely review this, ultimately, false trail. Nevertheless it offers a salutary lesson in the emotional reactions that possible environmental agents or interventions can elicit to the public at large, but also the medical profession.

Alcohol has been in society for ever and the acknowledgement of prenatal alcohol and its tertatogenic effect is still relatively a new phenomenon. So it is prudent to not 'scaremonger', but scientifically and clinically carefully piece out the veracity of this possible link.

The science of alcohol teratology continues to advance in leaps and bounds and one of the core findings has been the effect of prenatal alcohol on the dynamic balance of the developing neurotransmitters. In parallel with the more focused autism research on the role of serotoninergic neurotransmitters has been the identified effect of alcohol on the embryological serotoninergic neurotransmitter system. This research branches into the study of the serotonin transporter gene, by groups such as Bonnin et al in 2011, but again parallel work on epigenetics in alcohol has begun to unravel probable trans-generational shifts in genetic transcription through effects on DNA methylation (Haycock 2009).

Another strand of research in alcohol teratogenesis has been identifying brain areas a more sensitive to alcohol damage. Areas such as the corpus callosum, hippocampus, prefrontal cortex, temporal lobe collectively and individually contribute to a clinical presentation of social 
disconnectedness, lack of social cognition and awareness, impulsivity, and inability to understand another person's cognitions or feelings (alexithymia). (Bookstein et al 2001).

The underlying organic brain dysfunction at a cellular, neurotransmitter and structural level related to prenatal alcohol exposure sometimes shares significant congruence with ongoing neuroscience research in Autism Spectrum Disorder and Asperger's Disorder, and awaits collaborative work between the two academic fields.

There is also accumulating research which highlights the biological roots of fundamental functional problems in FASD which relate to sustained impact on working memory, (Congdon et al 2012)

\subsection{Neuropsychological framework of understanding ASD and Aspergers and its relationship to patients with FASD}

1. The psychological deficit in the child must be present before the onset of the disorder and so very early in development.

2. Be pervasive among individuals with the disorder.

3. Be specific to autism

4. Different psychological theories
a. theory of mind theory
b. the executive theory
c. the praxis/imitation theory
d. the emotion theory
e. the emphathizing-systematizing or ' extreme male brain' theory ( Hobson 1989, Russell 1997, Baren Cohen et al 2000, Pennington 2009)

\subsection{Clinical presentations of Aspergers disorder or autism spectrum disorder with FASD}

This is the arena where the divergence between the classic presentations of Autism Spectrum Disorder and Aspergers Disorder are seen, and offer a way to untangle the different aetiological routes to these syndromes.

FASD begin at birth and can be seen in infancy. The Mental Health Classification system, Zero to three (DC 0-3R, 2005) has a diagnostic category of Regulatory Disorders which aptly describes the immediate clinical presentations of Dysmorphic FAS or non dysmorphic ARND. It is the category of Regulatory Disorder, underresponsive type which is the harbinger of autism Spectrum Disorder or Aspergers Disorder diagnoses in early child hood.So the classic time presentation of Autism Spectrum Disorder or Aspergers Disorder is different in the FASD population. 
The stereotypic movements, flapping, posturing are less commonly part of the FASD presentation. However they present more commonly a Developmental Co-ordination Disorder which is diagnosed often Dyspraxia in countries such as Ireland.

The essence of the overlapping clinical presentations comes in the expressive and receptive language area. The qualitative impairments in social awareness, social cognition, social communication are not uncommonly very hard to differentiate whether using clinical assessment by an experienced child psychiatrist or psychologist or using standardized instruments such as ADOS among others. In many countries the ambivalence to accept the true prevalence of FASD( ! in 100 live births) leads school systems and physicians to 'hide' many FASD patients under a Autism Spectrum Disorder or Asperger's Disorder diagnosis because of the expediency of receiving school learning disability services. This is slowly changing, pioneered in countries such as Canada and the USA. Now the UK are acknowledging that FASD are the current biggest challenge for teaching as these pupils display complex learning disabilities with co -morbid psychiatric disorders for which there is no regular curriculum ( Professor Barry Carpenter UK, 2012).

This chapter will include psychiatric clinical analysis of patients with FASD who present autism spectrum Disorder or Aspergers Disorder features. with formal cognitive testing done and not uncommonly differing autism assessments which have proved equivocal. The comorbid ADHD is a more frequent issue in the FASD population and this has critical importance in both understanding and management. For example a successful medication treatment of pervasive distractibility visual and auditory can have a positive effect on the child's social functioning as he/she can now attend sufficiently to read faces and verbal and non verbal cues.

Medication is a change in the FASD patients who present with Autism spectrum disorder or Aspergers Disorder features. The more commonly accepted efficacy of SSRI does not necessarily hold true for FASD children or adolescents with and can lead to unmasking a bipolar diathesis, or in older patients contributing to Extra pyramidal symptoms.. This is especially a problem in Ireland which has a high prevalence of Affective Disorder which is quite common in the mothers who drink alcohol during pregnancy and so this genetic vulnerability can be brought forth by too aggressive use of SSRI for that autism or Aspergers Disorder. As well the psychostimulants can lead to an over focus in the FASD/ ASD group with increased perseveration which can become a source of severe rage if challenged. As well the psychostimulants are more likely to run the risk of bringing a schizoid change in the patient. Atypical agents such as risperidone with its differential effect on $5 \mathrm{HT}$ receptor can also prove problematic in management of Autism or Aspergers with a prenatal alcohol exposure history. In this case the longer and prolonged use of the medicine can make the clinical situation worse by unmasking an affective instability. (Rich \& O'Malley 2012)

Seizure disorders can be related to prenatal alcohol exposure and the effect of alcohol on the GABA ergic system is one hypothesis. (Daniel Bonthius et al 1992, O'Malley and Barr 1998).unexplained explosive episodes, rage attacks in FASD patients with autism Spectrum disorder or Aspergers Disorder may have origins in seizure disorders which are not related to the lower level of cognitive functioning or IQ as is the accepted rule. 
Little comment is made on the family stress in this complex mix population but a family centred therapeutic approach is the kernel of management and Identity issues have a completely different resonance in an adolescent who is bright, has ARND, Aspergers disorder and is trying to cope with the early loss of a birth mother to cirrhosis of the liver at 36 when her/she is aware that the ARND has its roots in the birth mothers drinking during pregnancy. In Ireland the 3/ 4 generations of families with FASD creates a transgenerational challenge to unraveling disorganized parenting from disconnected parenting due to fundamental social communication disorders. (Cummings et al 2000)

Recent international guidelines have included FASD among the environmentally-induced neurodevelopmental disorders. (Sage Handbook of Developmental Disorders, 2011) Such a neurodevelopmental diagnostic framework for children and adolescents with FASD improves outcome and prognosis in many cases, notably for those with persistent aggressive and antisocial behaviors. Neither the dysmorphic, Fetal Alcohol Syndrome (FAS) nor the nondysmorphic,Alcohol Related Neurodevelopmental Disorder (ARND) condition is currently diagnosable as an Axis I disorder.

Therefore Susan Rich and Kieran O'Malley, 2012, have recently proposed an alternative psychiatric formulation based on a neurodevelopmental model. This was suggested in order to improve clinical understanding and treatment of these complex developmental psychiatric patients. Such a paradigm shift would better identify the large numbers of children who fall through the cracks in diagnostic coding, becoming stuck in a revolving door through psychiatric hospitals and institutions. (Brown et al 2011).

These complex cognitive and psychiatric deficits often predispose affected individuals to a high degree of sensitivity to medications, increased risk of overmedication, treatment with medication combinations, susceptibility to changes in dosing regimens, and paradoxical responses to certain drugs.

Increasing clinical experience in using a neurodevelopmental formulation (compared to the traditional multi-axial system) to guide the measured, educated use of psychotropics for treatment of FASD can facilitate dramatic improvements in functioning of this challenging population.

Early and/or multidisciplinary intervention and treatment can prevent or minimize disruptive and risky behaviors, reduce academic failure, improve placement outcomes and reduce chronic involvement in the legal and probation system. ( O'Malley 2011b, Rich \& O'Malley 2012)

\section{The domains of alcohol-related neurodevelopmental disorder}

Although nearly every type of Axis I and II disorder in both DSM IV -TR and ICD 10 Classifications, as well as most disorders from the 0-3 coding manual can be expressed by individuals with effects of prenatal alcohol exposure, there have been efforts to better characterize the common clinical features associated with ARND. While neurodevelopmental deficits may 
exist in a range of severity, all cases of individuals with FAS have some degree of ARND. The following neurodevelopmental domains have been found to be disrupted in clinical psychiatric cases of both FAS and ARND (Figure 1). As indicated in the diagram, prenatal alcohol exposure can lead to mood dysregulation and autonomic arousal, cognitive and executive dysfunctions, language and social skills deficits, and multi-sensory functional and perceptual deficits. Some individuals can have one or more domains of impairment, as indicated by the overlapping areas in the Venn diagram. (Rich et al 2009, Solomon et al 2009, Rich \& O'Malley 2012)

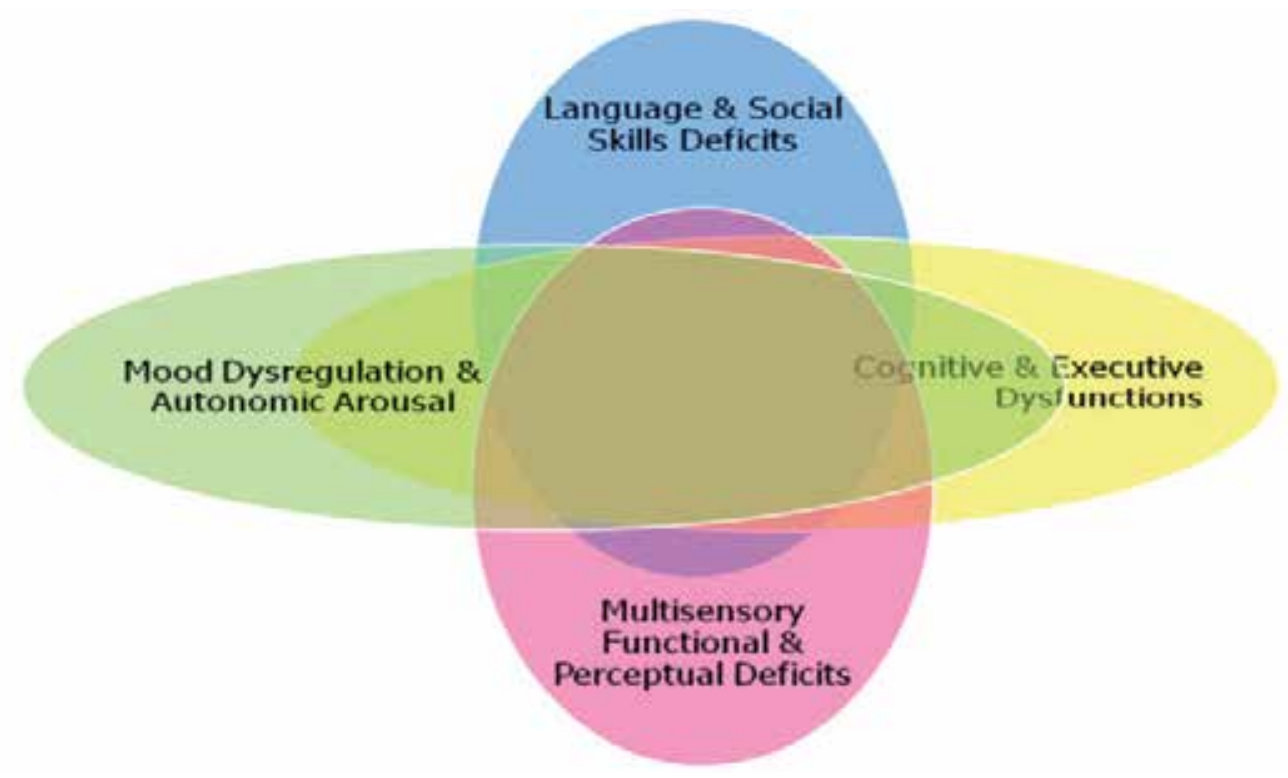

Figure 1. Mood dysregulation and/or Autonomic Arousal:

It is slowly being recognized that the autonomic or involuntary (parasympathetic and sympathetic) nervous system is affected by prenatal alcohol exposure, Animal research indicated this many years ago but human studies are beginning to unravel its effect from the infancy, early child hood and through to adolescence. Regulatory Disorders are prime clinical examples of this effect of prenatal alcohol exposure. The classic dichotomy in temperament is seen in the predisposition for a hyporesponsive infant or child, shy, inhibited, cautious and, anxious or hyperreponsive, a dis-inhibited, impulsive, intense infant or child. the effect of alcohol on the CNS produces a highly mood dysregulated child, having random or easily provoked episodes of frustration, irritability, aggression, and anger. Infants and toddlers with FASD can present with Regulatory Disorder Type I, II, or III (DC Zero to Three, 2005). The type of mood dysregulation may be related to "brain irritability" as epileptiform activity and spike and wave forms can be seen in sleep deprived or 24-hour EEGs for some individuals. This phenomenon is akin to a faulty thermostat which instead of controlling temperature controls emotional and arousal regulation. Thus the patient is unable to adjust their emotional 
or arousal state appropriately in response to sometimes minor challenges i.e. failure of examination, break up from boyfriend. This can lead to emotional incontinence with uncontrollable crying or laughing, or maybe intermittent unpredictable explosive episodes.

These developmental psychiatric disorders presenting from infancy reflect the impact of prenatal alcohol on the developing neurotransmitter system. This teratogenic effect on the serotonin, GABA ergic Glutaminergic and other neurotransmitter systems can lead to anxiety disorders, mood disorders (such as depression), aggression, and possibly later substance abuse. As infants and toddlers, they are often temperamentally difficult to settle and do not seem to enjoy/bond with their parents, birth, foster or adoptive These infants may have pervasive sleep problems (with disruptions in the sleep/wake cycle, initial insomnia and decreased non REM sleep).As well they can display a whole range of regulatory problems in hyper or hyposensivity to auditory, visual,, olfactory, gustatory or tactile stimuli.

As young children, the sensory integration issues involving sensitivity to sounds, environmental noise, lights, fans, easily irritated by voices, loud music, smells, tastes, even touch continue, and are often misunderstood as deliberate defiance.

This is commonly a clinical arena in which so called 'autistic 'features are noticed. In other words, the young child with ARND may either seek out tactile stimulation (touch and/or movement) or may, alternatively, be sensitive to touch and/or easily over-aroused by vigorous proprioceptive stimuli (e.g., movement on swings, roller coasters, etc.).

Generally, transition periods are a challenge, not unlike autistic children. These children require intensive one-on-one adult attention being unable to self-soothe easily, and having difficulty in free /creative play. However, self regulation techniques can be taught and guide play therapy has a role in integration of the child's exploration of self expression.

\subsection{Cognitive and executive dysfunction}

Brain structural, neurophysiological or neurotransmitter abnormalities belie the cognitive deficits in ARND. These include: working memory deficits, difficulty with executive functioning (organization, concentration, auditory processing, processing speed, problem solving, attention and impulse control.); deficits in IQ compared with their biological parents; mathematics disorders, reading disorders (e.g., dyslexia), spelling issues, and other learning disabilities with or without mental retardation due to a hypoaroused, misconnected, or disconnected prefrontal cortex, individuals with ARND may have a variety of deficits in cognitive areas,. A variety of developmental disabilities (speech/language issues, visual integration, gross and/or fine motor skills deficits (i.e., poor handwriting), spasticity, hyperflexibility, etc.) can also be seen in many individuals with ARND.

Disruptions in cognitive functioning often lead to a failure to understand consequences, poor judgment, and limited insight into the origins or the impact of one's behaviors. This subsequently leads to significant and debilitating deficits in basic day to day functional abilities. The child with ARND therefore, rather than thinking through actions, acts impulsively often in a naïve/ primitive manner (as though driven by basic instinct rather than measured intellect). 
Self care is another area of concern. able to care for oneself (e.g., hygiene, meal preparation, scheduling appointments), manage a household (take on responsibilities for chores, balance a checkbook, etc.) and perform other activities of daily living may be limited depending on the extent of a person's ARND.

Time, homework and money management difficulties lead to multitudes of practical daily living problems. Children with ARND are seen as willful, lazy and showing clear oppositional defiant features. The level of IQ does not offer a guide to these cognitive issues and often can suggest a greater capability than is possible. Children with ARND not uncommonly present a mixture of autistic features with ADHD and so are doubly challenged. Medication can have a vital role in this group as they are misunderstood as having faulty 'theory of mind' deficits, whereas their distractibility and lack of focus makes them unable to fully participate in social situation.

It is more common sense in the later grades/years in school to guide the student towards a vocational training certificate rather than a diploma/ A level, Leaving Certificate track and to master the basic life skills to be productive, employed in a semi-skilled trade (e.g., construction worker, brick mason, landscape worker, plumber's assistant, etc.). However, for many individuals with a higher degree of functioning and with appropriate academic/examination support it may not be unreasonable to expect completion of secondary/ high school and even the entering of a two or four year college or university programme. This is especially true for FAS or ARND patients with an autistic profile and average or above average intellectual functioning.

On the other hand, more cognitively impaired patients with FAS or ARND may have frequent rudimentary behaviors (skin picking, pica, compulsive self harm or inappropriate/selfstimulating sexual behaviors). These can be a primitive expression of emotional distress, not unlike non verbal autistic children. The central alexithymia, (inability to understand others feelings or have words for one's own feelings) irrespective of IQ level is a fundamental clinical construct in FASD.(Greene et al 1991)

\subsection{Language and social skills deficits}

The traditional view of language deficits come from the wealth of studies in expressive/ articulation problems and the more complicated so called 'receptive' language problems where the person has fundamental problems in the processing of language. this latter deficits was described by wernicke as long ago as 1874 in his classic treatise on sensory aphasia. It is in this area that patients withFASD truly show their 'autistic type clinical features. misuse of language integral to social cognition and communication are quite common problems in adolescents or young adults with ARND. It is important to understand that prenatal alcohol-induced organic brain damage underpins the language deficits. At times, these patients are misdiagnosed with Autistic Spectrum Disorder or Asperger's Disorder. The term "social language disorder" better fits this population. This does not preclude the fact that medication may engender a positive effect on language functioning, and specifically social communication. Individuals with ARND suffer from indiscriminate or immature behaviors (e.g., telling inappropriate jokes in the classroom or workplace, blurting out what they think of a person even if it is quite 
insulting /silly or negative). These behavior problems range from silly or irritating socially inappropriate behaviors to overtly aggressive and sometimes risky behaviors. Severe social functioning problems may result in lack of long term friendships, being labeled by peers as "weird" or "odd," and/or appearing withdrawn, socially-isolated, and avoidant. At times, ARND may lead to socially indiscriminate behaviors (i.e., individuals engaging in early or promiscuous sexual activity, gang membership, and peer pressure).

The clinical understanding of the effect of pseudo word decoding and alexithymia in management and understanding is critical to the psychiatrist, psychologist and educator. These children and adolescents can be seen in an 'autistic' or 'defiant' light but have specific decoding struggles which effect their receptive).

Case Examples: Two female adolescents with ARND were diagnosed with Autism and Atypical Autism respectively after fulfilling the ADOS criteria. However both had clear documented history of prenatal alcohol exposure. One normal I.Q. I4 year old girl with Atypical Autism had a clinical presentation of ASD and ADD and deteriorated with psychostimulant medication which markedly increased her perseveration. She responded to low dose liquid fluoxetine, and as her attention problems, especially visual, ameliorated, so her 'autistic' features deceased. The other girl 15 years old, with moderate intellectual functioning, had very debilitating social anxiety triggered by oversensitivity to facial cues. She eventually settled for a while with a GABA ergic agent.( Lyrica, pregabulin), but now needs a specialized therapeutic community placement. She had a history of many unexplained physical problems which were Alcohol Related Birth Defects.

\subsection{Multi-sensory functional and perceptual deficits}

Sensory integration issues, including hypo or hypersensitivities to noise, touch, proprioceptive stimuli, smells, tastes, and light may all be seen in children prenatally exposed to alcohol. This may lead to infants and toddlers seeming to be easily agitated, over-stimulated, and overaroused. Adolescents and adults may cope by avoiding or over-reacting in situations or environments which provoke their sensitivities. Adolescents or adults who misread or misunderstand social cues may result in paranoid behaviors, such as over-reactions to the tone of someone's voice or an otherwise harmless look in their direction.

Prenatal alcohol exposure can have very disabling outcomes for alcohol-exposed children and their families due to the interaction between psychosocial risk factors (Mukarjee et al, 2006), cognitive deficits, and neuropsychiatric sequelae ( O'Malley 2011b). In addition to a higher prevalence of chronic exposure to domestic violence, neglect, child abuse, adjudicated youth have higher rates of psychiatric illness, learning disabilities, and academic failure.

The sensory functional and perceptual deficits are commonly' hidden' and included in a generic autistic diagnosis frame. However they are fundamental to understanding the acquired brain damage caused by alcohol, which pervades brain structures, neurotransmitters and electrophysiology (Hagerman 1999, O’Malley 2008, O’Malley \& Mukarjee 2010).

Case example: A 21 year old previously adopted male Caucasian patient presented with a long history of autism and psychotic features. He had been hospitalized a number of times and had 
need restraint because of his reactivity to the environment. He had not responded to high doses of SSRIs (which produced increased suicidality thoughts), and atypical, especially risperidone which made him more affectively unmanageable. When he was assessed in the community his clear history of sensory reactivity to tactile, olfactory, gustatory, visual and auditory stimuli was unraveled as was his history of significant prenatal alcohol exposure. Which had been ignored in previous assessments. A combined multi-modal approach addressing his sensory reactivity combined with low dose buspirione was much more effective and he did not need psychiatric hospitalization. As well he did not present any facial features as adult or as a young child. He had been labeled as having unusual paranoid features but these were really his correct sensitivity to what he perceived as a hostile challenging environment. His adoptive parents recounted many stories of his oversensitivity to noise, light,fabrics food when he was growing op and just saw him as 'over fussy'.

\section{A neurodevelopmenatal approach to management}

\subsection{General diagnostic problems}

Although psychiatrists and mental health professionals treat patients with FASD, there is presently no consistent way within DSM-IV TR to code for either the dysmorphic phenotype (Fetal Alcohol Syndrome, FAS) or the non-dysmorphic condition (Alcohol-related Neurodevelopmental Disorder, ARND). Therefore, treatment of affected individuals is inadequate due to lack of diagnostic clarity, and lack of scientifically tested or accepted psychiatric treatment protocols. FAS, the leading non-hereditary cause of mental retardation and preventable birth defects, is buried in Appendix G of the DSM-IV TR as a congenital malformation (760.71) and the ICD-10 includes Fetus and newborn affected by maternal use of alcohol (P04.3) but excludes FAS (Q86.0) (DSM IV-TR, 2000). (Nowick Brown et al 2011, O'Malley 2011b, Rich \& O'Malley 2012).

At the current time because there is no appropriate DSM-IV TR or ICD 10 diagnostic framework, most psychiatrists and mental health professionals attempt to apply inadequate Axis I diagnoses which are often poorly suited to the clinical understanding of this population. This results in individuals being given a laundry list of psychiatric diagnoses, from ADHD, autism, pervasive developmental disorder and bipolar disorder, to conduct disorder, reactive attachment disorder, personality disorders, and oppositional defiant disorder.

In a large study of secondary disabilities in 415 persons with ARND [FAS or Fetal Alcohol Effect (FAE)], a majority (94\%) had a history of co-occurring mental health problems. Among both adults and children, attention deficits were the most frequently reported problems $(61 \%)$ reported whereas in adults alone, depression was most frequently reported (52\%) (Stratton et al., 1996). A smaller study has shown that the proportion of subjects with a history of psychiatric disorders (74\%) was greater than expected from the general population, including alcohol or drug abuse (60\%), major depressive disorder (44\%), avoidant personality disorder (29\%). Researchers have shown a link between ADHD symptoms and FASD over the past several years, indicating an acquired (non-genetic) etiology for a subtype of children with ADHD. 
Infants and toddlers with FASD can present with Regulatory Disorder Type I, II, or III (DC Zero to 3, 2005). Autistic behaviors have been noted in both younger children as well as school age children prenatally exposed to alcohol (Streissguth et al, 1996; Streissguth \& O'Malley, 2000, Mukarjee et al 2012).

You could build a case that nearly all disorders developing during childhood listed in the DSM IV-TR may be induced by exposure to alcohol in utero. Co-morbidities of FASD include other behavioral, mood, anxiety, and conduct problems. The link between ADHD and FASD is finding more universal acceptance and the link between autism and Aspergers disorder and FASD will not be far behind. (O'Malley 2011a).The lifetime prevalence of mental health or psychiatric disorders in individuals with FASD is as high as 90\% (Streissguth et al 1996, HHS, 2000), highlighting the importance of correct diagnosis and clinical management. Accurate, informed diagnosis is critical in psychiatry to avoid over-medication or inappropriate treatment, leading to worsening of symptoms and poor outcomes.

The current standard of care or "treatment as usual" for individuals with FASD is inadequate due to lack of diagnostic clarity, lack of accepted psychiatric treatment protocols, and further complicated by the presence of Alcohol Related Birth Defects (ARBD) which are multisystem organ involvement (i.e., seizure disorders; renal, eye, cardiac, g.i. problems and skeletal).

Early accurate diagnosis and intervention may be effective in preventing the development of secondary disabilities (i.e., academic or school failure, conduct disorders and antisocial behaviours leading to legal problems, sexually inappropriate behaviours, lack of steady employment and housing).

\subsection{The utilization of a neurodevelopmental formulation}

The utilization of a neurodevelopmental formulation can guide the development of effective multiisystem and multimodal intervention strategies, including appropriate psychopharmacologic management (O’Malley 2008).

1. Thus, shifting diagnostic paradigms in children with prenatal alcohol exposure to the dysmorphic (FAS) and non-dysmorphic (ARND) phenotypic expression of in utero alcohol exposure would allow psychiatrists, pediatricians, and other medical professionals to have a richer, clearer and more holistic interpretation and understanding of the wide range of neurocognitive, neurobehavioral, and neuropsychiatric disorders affecting the individual rather than simply the degree of facial dysmorphology.

2. The social or environmental context includes childhood exposure to domestic or community violence, child abuse/neglect, early institutionalization, community violence, and other early life events that may contribute to development of reactive attachment disorder (RAD), post traumatic stress disorder (PTSD), developmental trauma disorder and other psychiatric (Axis I and II) co-morbidities.

The interaction of the childhood experience on the expressed FASD phenotype cannot be overlooked. Therefore, the neurodevelopmental biological vulnerability profile of FASD during infancy, toddlerhood, childhood, and adolescence predisposes an individual to adverse 
psychological outcomes resulting from early institutionalization, parental loss, physical/ emotional/sexual abuse, neglect, and other forms of trauma(Cummings et al 2000,Elias et al 2011, Rich \& O’Malley 2012).

3. 3.The implications of FASD on development, behavior, academic and adaptive functioning over the life span can be best understood in the context of the interaction of social and familial factors with an individual's neurodevelopmental deficits. Early institutionalization, neglect, abuse, and family violence may engender different nosological (diagnostic) presentations in this patient population, depending on the quality and degree of underlying neurodevelopmental impairment. So co-occurring diagnosis of Reactive Attachment Disorder or Post Traumatic Stress Disorder, Developmental Trauma Disorder are quite appropriate and herald the psychiatric and care complexities of the patient. It is therefore of vital importance that care must be taken to tease out symptoms based on acquired developmental versus social history in order to develop a holistic, appropriate understanding of the interplay between brain-based and environmental (post-natal) origins of psychopathology.

4. Deficits in emotional regulation and mood, implicit ability to comprehend the nuances of social situations, auditory or visual information processing, functional working memory, and/or other executive functions put individuals at risk for further psychopathology in the face of environmental stressors. These neurodevelopmental (CNS) and psychiatric sequellae persist through the life course and may progress to worsening conditions with devastating outcomes and poor prognosis (Streissguth and O'Malley, 2000, Rich \& O’Malley 2012).

\footnotetext{
A neurodevelopmental formulation provides the best option for clinical understanding of these types of FASD complex cases, but especially if autistic features are the presentation, and the patient has borderline or low intellectual function or a marked split ( 12-15 points) between verbal and performance I.Q. (O'Malley 2008, Chapter 1)
}

\subsection{The practical utility of a neurodevelopmental approach}

The challenges in the treatment of FASD currently relate to their clinical presentations having an array of apparent psychiatric co-morbidity, and a general lack of diagnostic clarity. In practice in both North America and Ireland/ UK clinicians who recognize the clinical significance of FAS or ARND, are given a number of psychiatric diagnoses (ADHD, Autism, Aspergers disorder, intermittent explosive disorder, conduct disorder, mood disorder, etc.). It is becoming increasingly evident that early onset dysregulation, social communication and forensic issues are presenting complex mixtures of biological and environmental vulnerabilities which display developmentally changing clinical presentations. The autistic presentation coupled with ADHD symptoms and intermittent unexplained explosive episodes is particularly perplexing and hard to manage. Thus, given that both conditions are common presenta- 
tions of FAS and ARND (Fitzgerald M, 2010). Clinicians using a neurodevelopmental approach will have more success in understanding and treatment of FASD.

Multi-modal treatment can improve the developmental, social, academic, and mental health trajectory of these children (O'Malley 2008, Nowick Brown et al 2011). Brain organization and function is affected in many individuals with FASD/ARND and can be enhanced by appropriate multi-modal treatment strategies.

As with Autistic Spectrum Disorders, FASD diagnosis and treatment involves early intervention with a multimodal team approach (genetics, developmental pediatrics, psychologists, psychiatrists, PT/OT, speech, special education) (O'Malley 2008, 2011b, Kodituwakku et al 2011).

A capacity for consequential thinking is a key requirement for "decisional capacity." This is an expectation for adolescents or young adults in the school, work legal system, who have been involved in antisocial and/or violent acts. Unfortunately, due to the neurocognitive deficits associated with ARND, these individuals are often mentally and emotionally disconnected from the consequences of their actions, misread social cues, are easily frustrated and provoked, and are unable to navigate logical decision making. So called 'high functioning 'autistic patients fit this neurocognitive profile and have the added challenge of unexpected response to medication because of unrecognized brain damage (Coles et al 2009, Kodituwakku et al 2011, Hosenbucus et al 2012).

\subsection{The specific use of medication in FASD with or without autistic features}

Clinical experience has shown that proper medication combined with comprehensive, early intervention services will improve their neurodevelopmental and psychiatric outcomes. To that end, psychotropic medication can be viewed as an integral part of multi-modal management program for dysmorphic and non-dysmorphic ARND (FAS Diagnosis, 2005; Byrne 2008, Coles 2009; Novick Brown, et al., 2011).

\subsubsection{Off - Label or off license use of psychopharmaceuticals in patients with FASD}

No National Institute of Mental Health (NIMH), NICE (UK), or industry-sponsored studies exist on the safety and efficacy of medication in children, adolescents, or adults with FASD, so this continues to be a barrier to measured and safe treatment for all individuals.

There is literature on the pharmacological management of ADHD, Autism, Fragile X, aggression and addictive disorders (Hagerman 1999, Lee et al 2001, Glancy et al 2002 a, b, Vocci et al 2005, Turk 2012), which is often mis-extrapolated to apply to individuals with FASD. Since there is no definitive diagnosis in the current DSM-IV TR for FASD outside of 760.71 (which is embedded in the ICD-9 codes in the Appendices under both "FAS" and "toxic exposure to alcohol in utero"), no impetus exists for large scale clinical trials in psychiatric and mental health research. Such controlled clinical studies are needed to determine "best practices," or even smaller studies to determine safety and efficacy, or to gain FDA or NICE guideline approval for use of the medications in this unique neurodevelopmental psychiatric patient 
population (Turk 2009). Presently, there currently are no FDA, AACAP, APA guidelines for medication usage in adolescents or young adults with FASD who present with neuropsychiatric disorders. Therefore, all medications are used "off label" or 'off license' in this population.

\subsubsection{Caution in use of medication in ARND}

While research is scarce in patients with FASD, this population may be even more vulnerable than those with brain injury sustained in the postnatal period, childhood, adolescence, or adulthood. Individuals with FASD, whether FAS or ARND, have had fetal whole-body exposure during prenatal development, leading to the potential for unrecognized Alcohol Related Birth Defects ( ARBD) in a number of organ systems (kidney, heart, liver/g.i. system, eye, immune system, neurological) (Stratton et al 1996). These underlying problems with physical organs and structures may lead to unanticipated side effects to even low doses of medication.

For example

i. cardiac problems such as conduction anomalies, structural defects, and pathologic murmurs may be linked with adverse events with stimulant medications.

ii. Overt seizure disorders and irritability of the brain (associated with random and triggered electrical discharges) may be present due to neuro-anatomical changes in the ARND brain. Therefore, safety issues related to decreased seizure threshold for certain medications should be considered prior to treatment of this population. (O’Malley \& Barr 1998, Hagerman 1999,Bonthius et al 2001

iii. Other medical complications associated with alcohol-related birth defects (ARBD) need to be considered prior to beginning medication.

iv. Therefore, caution in use of medications should be given due to the unique vulnerability of these patients for severe and catastrophic side effects of certain medications due to:

- differential or paradoxical medication response ;

- prenatal alcohol-induced neurochemical or structural CNS changes (i.e., acquired brain injury);

- complications related to multisystem organ involvement (absorption, metabolic or elimination problems related to kidney, gastro-intestinal or liver problems related to ARBD);

- an increased incidence of seizure disorders in this population (i.e., lower seizure thresh hold);

- overall greater risk of side effects from multiple drug combinations, higher doses of medications, and sensitivity to psychopharmaceuticals. 


\subsubsection{Psychopharmacological targeting of neurodevelopmental deficits to improve current function and general prognosis}

It is well recognized that patients with acquired brain injury respond differently to medications than individuals with no brain injury.

International clinical experience with this population indicates that individuals with alcoholinduced brain damage (FAS and ARND) often respond to medications similarly to those with other types of acquired or traumatic brain injury.

A prudent therapeutic approach in this group of patients is to streamline the numbers of medications the person is taking in order to reduce drug-drug interactions and prevent complications from over-medication (Stratton et al, 1996; O’Malley \& Storoz 2003; Byrne 2008; O’Malley 2008).

Medications need to be started at low doses and increased slowly - with the goal of maximizing efficacy and minimizing side effects to the sensitive/vulnerable central nervous system. This can be achieved more often than not by simplifying the medication regimen, reducing the drug-drug interactions, and providing targeted therapy combined with medication management.

Psychotropic agents do improve brain organization and function (i.e., "neuron glue") and y can facilitate cognitive processes by dampening the spontaneous or random firing of mislaid pathways in the brain. As well, psychotropic medications may improve mood, behavior, and performance in individuals with FASD by altering the physiology of the "injured brain" structure and function. Among these medications are the atypical antipsychotics, such as risperdal, ziprazidone, aripiprizole, and olanzapine. Uses of lamotrigine, carbamazepine, valproic acid, lyrica, frisium have also been anecdotally helpful in patients with generalised anxiety, aggressively, impulsivity, and mood dysregulation. We believe that correctly chosen, appropriately managed medications can have a positive effect on cognitive functioning and decision making. (Hosenbus et al 2012, O’Malley 2010, Turk 2012).

\subsubsection{General principles regarding medication usage in FASD}

The principles guiding use of medications in the organically compromised brain (targeted medications, lower doses, and gradual increases in dosing) combined with psychotherapy and comprehensive community supports, psychiatrists can improve the complex neurodevelopmental issues in these individuals.

Medications may have positive or negative outcomes as patients with FASD are more sensitive to the CNS effects of medications.

i. For example, selective serotonin reuptake inhibitors (SSRI's) such as fluoxetine, paroxetine, or citalopram, used not infrequently in Autism, may be more likely to precipitate agitation, activation, or suicidality in these brain- damaged adolescents due to augmentation of a pre-existing, organically-driven impulsivity. 
At the same time, given that individuals with FASD may have deficiencies and/or differences in neurotransmitter systems such as serotonin and dopamine, low doses of sertraline and fluoxetine have proven anecdotally beneficial for some patients

ii. Adolescents and young adults with ARND and co-occurring seizure disorders have a prenatally kindled organic brain dysfunction as a result of alcohol-induced damage to the corpus callosum, cerebellum or hippocampus. There is anecdotal clinical evidence that antiepileptics (i.e., carbamazepine, valproic acid, lamotrigine, neurontion) can be effective in preventing this kindling effect. The prenatal effects of alcohol can also result in a change in the balance of the developing neurotransmitters. Animal research has shown that prenatal alcohol can induce decrease in inhibitory neurotransmitter GABA in the hippocampus, and this neurochemical imbalance can underpin development of seizures (Hannigan et al 1996, Riley et al 2006). The effects of antiepileptics should be weighed carefully since some medications for seizures may also increase anxiety, affective/mood liability, and reduce learning and cognition.

4.4.5. The future for medication use in FASD including those with common presentations of ADHD, mood disorders and/or autism

i. $\quad$ Obviously multicenter, randomized controlled clinical trials (ideally international) are needed in this vulnerable and clinically complex population. (Turk 2009)

ii. There is a need for scientific testing and evaluation of new clinical instruments which combine cognitive, language, and behavioral response as the 'gold standard' for assessing medication efficacy and safety in patients with FASD. Currently there are no validated clinical instruments to evaluate the developmental, cognitive, language and behavioral response of a patient with FAS or ARND to psychotropic medication. There is a non-specific neuropsychiatric rating scale, but most drug rating scales (with the exception of those used in Alzheimer's disorder) evaluate clinical symptoms related to psychiatric disorder (i.e., Connor's Questionnaire, Beck Depression Inventory, Hamilton Rating Scale, CBCL).

iii. It is long recognized that the use of multiple psychotropic medications is a risk for toxicity and acute confusional state, even in absence of underlying neurocognitive problems. The mechanism of multidrug interaction leading to toxicity relates to individual drugs competing for absorption through the liver cytochrome P450 2D6 enzyme system. In turn, certain medication blood levels increase (i.e., paroxetine is well known to increase blood levels of other psychotropic medications). In a recent lecture at the First European Conference on FASD in Rolduc, Holland (Nov $3^{\text {rd }}$ to $5^{\text {th }}$ 2010), Ken Warren, Acting Director of NIAAA, mentioned concern about medication interactions, but no data was given or studies forthcoming. 


\section{Discussion}

This chapter has attempted to highlight the overlapping clinic al presentations of patients with FASD, whether dysmorphic FAS or non-dysmorphic ARND. Autism and Aspergers Disorder probably rank next to ADHD as the commonest clinical phenotype of FASD.

The lack of DSM or ICD Axis I codes for these individuals means that treating psychiatrists do not usually identify either disorder (dysmorphic or nondysmorphic conditions related to prenatal alcohol exposure as aetiological factors to be considered in diagnosis. The organic brain hypothesis will then inform management at many levels.

Nevertheless, the arrival of DSM V will hopefully herald new diagnostic categories which will capture more correctly some of these patients with FASD who show what are deemed ';autistic' features. Two proposed diagnostic categories, Social Communication Disorder and Alcohol Related Neurobiological Disorder are in serious consideration. It is hoped that Alcohol Related Neurodevelopmental Disorder (ARND), already well recognized, will replace the new category it more correctly captures the essence of the developmental psychiatric disorder (DSM 5 Symposium 2012).

Currently the generic "treatment as usual" prevents individuals with FASD from receiving appropriate multisystem and multimodal services, and further results in predictably poor outcomes for affected individuals and ultimately costly consequences for communities.

Brain imaging such as MRI, fMRI or SPECT scans studies may begin to map more specific areas of brain dysfunction related to prenatal alcohol exposure and psychiatric clinical presentation (Riley, et al 2005, Kodituwakku et al 2011, Coles et al 2011). Historically our scientific knowledge of damaged or diseased brain structure associated with infections such as syphilis, AIDS or lesions associated with cerebrovascular accidents has informed our diagnostic accuracy and informed treatment progress. Therefore it is not unrealistic to expect that correlations between the structural and functional deficits in individuals exposed at certain points during pregnancy could dramatically improve our understanding of brain function.

Finally, the ability to distinguish FASD with an autistic clinical presentation from a geneticallyacquired or non-organic cause of this neurodevelopmental condition. This aetiological knowledge ultimately better describes the pathophysiology and neuropsychiatric phenomenology of the patient's clinical diagnosis. The neurodevelopmental clinical frame expands the clinician's understanding that the autistic presentation is actually a phenotype form of this specific acquired brain injury, The prenatal alcohol exposure creates a chemical, structural, and even electrical CNS environment that is "hard wired" very different from the other aetiological pathways for autism. The child or adolescent psychiatrist (ideally trained in developmental psychiatry) has a long recognized central role as the "case supervisor" for patients with patients with FASD, and is in the better position to manage the unfolding and clarification of the neurodevelopmental formulation, differential diagnosis, psychiatric comorbidities, and psychopharmacology. Increased understanding of these complex patients through a neurodevelopment formulation, unraveling such issues as the hidden aetiology to 
the autism presentation will improve holistic clinical outcomes including the appropriate, targeted use of medication by the treating child/adolescent psychiatrists.

\section{Author details}

Kieran D. O'Malley ${ }^{1}$ and Susan D. Rich ${ }^{2}$

1 Charlemont Clinic/ Our Lady's Children's Hospital Crumlin, Dublin, Ireland 2, USA

\section{References}

[1] Baren Cohen S, Ring, HA, Ballimore ET, Wheelwright, S,Ashwin C, Williams SCR(2000). The amygydala theory of autism. Neuroscience and Biobehavioural Reviews., 24, 3355-3344.

[2] Blumstein, A, Farrington, D. O, \& Mortea, S. D. (1985). Delinquent Careers: Innocence, Desistance and Persistence in an Annual Review of Research, , 6

[3] Bonthius, D, Woodhopuse, J, Bonthius, N. E, Taggrad, D. A, \& Lothman, E. W. (2001). Reduced seizure control and hippocampal cell loss in rats exposed to alcohol during brain growth spurt. Alcohol Exp Clin Res., 25, (1) 70-82

[4] Bookstein, F. L, Sampson, P. D, Streissguth, A. P, \& Connor, P. L. (2001). Geometric morphometrics of corpus callosum and subcortical structures in fetal alcohol effected brain. Teratology: , 4, 4-32.

[5] British Medical Association Board of Science(2007). Fetal Alcohol Spectrum Disorders: A Guide for Professionals. Publisher: BMA, London.

[6] Byrne, C. (2008). April). Psychopharmacology Basics for FASD. Workshop Presentation, $3^{\text {rd }}$ Biennial Conference Adolescents and Adults with FASD. Vancouver, Canada.

[7] Carpenter, B. (2012). ${ }^{\text {st }}$ Intercountry Adoption Conference, Plenary Talk, Education Issues in FASD, Cork, Jan 21st

[8] CDCFAS: Guidelines for Referral and Diagnosis (CDC, HHS, NOFAS, (2005). th Printing. National Center Birth Defects and Developmental Disabilities, Centers for Disease Control and Prevention, Department of Health Human Services, and NOFAS, USA.

[9] Chess, S. (1971). Autism in children with congenital Rubella. Journal of Autism and Developmental Disorders. 1(1), 33-47 
[10] Clarren, S. K, \& Smith, D. W. (1978). The Fetal Alcohol Syndrome: A review of the world literature. New England Journal of Medicine. , 298, 1063-1067.

[11] Clarren, S. K, Astley, S. J, \& Bowden, D. M. (1988). Physical Anomalies and Developmental Delays in Nonhuman Primate Infants Exposed to Weekly Doses of Ethanol During Gestation. Teratology , 37, 561-569.

[12] Chudley, A. E, Conry, J, Cook, J. L, Loock, C, \& Rosales, T. le Blanc N ((2005). March 1) Fetal Alcohol Spectrum Disorder: Canadian Guideline for Diagnosis. Can. Med. Assoc. Journal, 172 (5 supplement).

[13] Coles, C. (2009). IHE Consensus Developmental Conference. FASD: Across the Lifespan. Management Strategies. Westin Hotel, Edmonton, Alberta, Canada.

[14] Coles CD \& Zhihao L ( (2011). Functional neuroimaging in the examination of effects of prenatal alcohol exposureNeuropsychology Review, in press.

[15] Cummings, E. M, Davies, P. T, \& Campbell, S. B. (2000). developmental psychopathology and Family Process. Theory, Research and Clinical Implications. The Guilford Press, London, New York.

[16] DSM 5 Symposium ( (2012). The making of DSMS. Part 1. AACAP, 59th annual meeting Oct $25^{\text {th }}$, San Francisco..

[17] Elias, S. E, Coughlan, B. J, \& O'Malley, KD. (2012). Fetal alcohol spectrum disorders: children, parents and carers of living with the disorder: a mixed methods approach, Poster Presentation SSBP International Conference, Leuven Belgium, Oct $10^{\text {th }}$ to $12^{\text {th }}$

[18] Fitzgerald, M. (2010). Violent and Dangerous to Know. Nova Science Publishers, New York. Available at www.amazon.com.

[19] Gardner H Spiegelman DBaka S ((2011). Perinatal and neonatal risk factors for autism: A comprehensive meta analysis. Pediatrics, , 128(2)

[20] Gilberg, C, \& Gilberg, C. I. (1983). Infantile autism. A total population study of reduced optimality in the pre, peri and neonatal period. Journal of Autism and Developmental Disorders, t, 32(4), 153-166.

[21] Glancy, G. D, \& Knott, T. F. Part I: the Psychopharmacology of Long-Term AggressionToward an Evidence-Based Algorithm. Canadian Psychiatric Association Bulletin, Psychiatry and the Law.

[22] Glancy, G. D, \& Knott, T. F. Part II: The Psychopharmacology of Long-Term Aggression-Toward an Evidence-Based Algorithm, Canadian Psychiatric Association Bulletin. Psychiatry and the Law.

[23] Greene, T, Ernhardt, C. B, Ager, J, Sokol, R, Martier, S, \& Boyd, T. (1991). Prenatal Alcohol Exposure and Cognitive Development in the Preschool Years. Neurotoxicology and Teratology, , 13, 57-68. 
[24] Hagerman, R. J. (1999). Neurodevelopmental Disorders. Diagnosis and Treatment. Oxford University Press, New York, Oxford., 3-47.

[25] Hannigan, J, \& Randall, S. (1996). Behavioural pharmacology in animals exposed prenatally to alcohol. In Abel, EL, editor, Fetal alcohol syndrome. From mechanism to prevention. CRC Press, New York,, 191-213.

[26] Hobson, R. P. (1989). Beyond Cognition. A theory of autism. In Dawson G, ed,.Autism, nature, diagnosis and treatment. Guilford Press, New York,, 222-448.

[27] Hosenbocus, S, \& Chahal, R. (2012). a review of executive function deficits and paharmacological management in children and adolescents. J can acad. Child adolesc. Psychaitry, 21,(3)

[28] National Institute on Alcohol Abuse and Alcoholism (NIAAA(2000). June). Highlights from Current Research: 10th Special Report to the U.S. Congress on Alcohol and Health from the Secretary of Health and Human Services. US Department of Health and Human Services, Public Health Service, National Institutes of Health.

[29] Jones, K. L, \& Smith, D. W. (1973). Recognition of the Fetal Alcohol Syndrome in early infancy, Lancet, , 2, 999-1001.

[30] Jones, K. L, \& Smith, D. W. (1975). The Fetal Alcohol Syndrome, Teratology, 1975,12: $1-10$.

[31] Kodituwaddu FW \& Koditowakku EL ((2011). From research to practiceAn integrative framework for the development of interventions in children with featl alcohol spectrum disorders. Neuropsychology Review,, 21, 204-223.

[32] Lee, R, \& Coccaro, E. (2001). Feb) The Neuropsychopharmacology of Criminality and Aggression. Canadian Journal of Psychiatry, , 46

[33] LemoineP Harousseau H, Borteyru JP ( (1968). Les infants de parents alcoholiques.Anomalies observes a propos de 127 cas. Quest Med. , 21, 476-482.

[34] Lord, C. Mulloy, C Wendelboe M., Schopler E((1991). Pre and perinatal factors in high functioning females and males with autism. J autism Dev Disorder, , 21(2), 197-209.

[35] Lord C Kim SHDimartino A ((2011). Autism Spectrum Disorders. General Overview. Chapter 14, Editors Howlin P, Charman T, Ghaziuddin M, The Sage Handbook of Developmental Disorders, Published London, California, New Delhi, Singapore

[36] Mukarjee, R, Hollins, S, \& Turk, J. (2006). Psychiatric comorbidity in fetal alcohol syndrome. Psychiatric Bulletin. , 30, 194-195.

[37] Mukarjee, R, Hollins, S, \& Curfs, L. (2012). Fetal Alcohol Spectrum Disorders. Is it something we should be more aware of? J R Coll.Physicians, Edin. 21, 2, 42, 143-150

[38] Nanson, J. (1991). Autism in Fetal Alcohol Syndrome. A report of 6 cases. Alcoholism. Clinical and Experimental Research 
[39] Novick BrownN, O'Malley, KD, Streissguth, AP ((2010). FASD: Diagnostic Dilemmas and Challenges for a Modern Transgenerational Management Approach. In Editors, Abubado, S, Cohen D, Prenatal Alcohol Use and Fetal Alcohol Spectrum Disorders: A Model Standard of Diagnosis, Assessment and Multimodal Treatment, Bentham Online Publishing, USA.

[40] O’Malley, K. D, \& Barr, HM. (1998). Fetal Alcohol Syndrome and Seizure Disorder. Letter to editor, Can J Psychiatry.

[41] O'Malley, K. D, \& Storoz, L. (2003). Fetal Alcohol Spectrum Disorder and ADHD. Diagnostic implications and therapeutic consequences. Expert Review of Neurotherapeutics. , 3(4)

[42] O'Malley, K. D. (2008). (Ed.) ADHD and Fetal Alcohol Spectrum Disorders. $2^{\text {nd }}$ Printing, Nova Science Publishers, New York., CHAPTERS 1, 4, 6, 11

[43] O'Malley, K. D. (2010). Fetal Alcohol Spectrum Disorders. in Enyclopedia of Psychopharmacolgy, Springer-Verlag, Berlin, Heidelberg.

[44] O’Malley, K. D, \& Mukarjee, R. (2010). Fetal Alcohol Stndrome, Alcohol Related Neurodevelopmental Disorder, Syndrome Phenotypes, Society Study of Behavioural Phenotypes, UK,www.ssbp.co.uk

[45] O'Malley, K. D. (2011a). ADHD and FASD. From animal research to clinical experience. Invited talk, International CADDRA meeting Toronto, October 16th

[46] O’Malley, K. D. (2011b). Fetal Alcohol Spectrum Disorders. Chapter The Sage Handbook of Devevelopmental Disorders. Howlin P, Charman T, Ghazziuddin M, Editors. Published, London, California, New Delhi, Singapore, 24, 479-496.

[47] Orphan Drug ActUS Congress; Designation of Drugs for Rare Diseases or Conditions; SEC. 526 [360bb]. (a)(1)

[48] Pennington, B. F. (2009). Diagnosing Learning Disorders. A Neuropsychological Framework. $2^{\text {nd }}$ Edition, The Guilford Press, London, New York

[49] Rich, S. D. (2005). Fetal Alcohol Syndrome: Preventable Tragedy. Psychiatric News, Residents' Forum. Page 12., 40(9)

[50] Rich, S. D, Sulik, K. K, Jones, K. L, Riley, E. P, \& Chambers, C. (2009). Nov). Fetal Alcohol Spectrum Disorder: A Paradigm for Neurodevelopmental Formulation and Multidisciplinary Treatment. Presented at the American Academy of Child and Adolescent Psychiatry Annual Conference, Honolulu, Hawaii.

[51] Rich SD \& O'Malley KD(2012). A neurodevelopmental formulation for the psychiatric care of Fetal Alcohol Spectrum Disorders. Journal of psychiatry and the Law, Accepted.

[52] Riley, E. P, \& Mcgee, C. L. (2005). Fetal Alcohol Spectrum Disorders: an overview with emphasis on changes in brain and behavior. Experimental Biology and Medicine, , 230, 357-365. 
[53] Russell, J. (1997). Autism is an executive disorder. Oxford University Press, New York

[54] Sokol, R. J, Delaney-black, V, \& Nordstrom, B. (2003). Dec 10). Fetal Alcohol Spectrum Disorder JAMA, , 290(22)

[55] Solomon, M, Hessl, D, Chiu, S, Olsen, E, \& Hendren, R. (2009). March 1). Towards a Neurodevelopmental Model of Clinical Case Formulation., Psychiatric Clinics of North America. , 32(1), 199-211.

[56] Stratton, K, Howe, C, \& Battaglia, F. (1996). Fetal Alcohol Syndrome. Diagnosis, Epidemiology, Prevention, and Treatment, Institute of Medicine, National Academy Press, Washington DC, USA.

[57] Steinhausen, H-C, Willms, J, \& Spohr, H-L. (1993). Sept). Long-Term Psychophathological and Cognitive Outcome of Children with Fetal Alcohol Syndrome. Journal of the American Academy of Child and Adolescent Psychiatry, 32:5, , 990-994.

[58] Streissguth, A. P, Aase, J. M, Clarren, S. K, \& Randels, S. P. LaDue RA, Smith DF ((1991). April 17). Fetal Alcohol Syndrome in Adolescents and Adults, Journal of the American Medical Association, , 265(15), 1966.

[59] Streissguth, A. P. and LaDue RA ((1987). Fetal Alcohol Teratogenic Causes of Developmental Disabilities. In S. Schroeder (Ed.), Toxic Substances and Mental Retardation, Washington, DC: American Association on Mental Deficiency, , 1-32.

[60] Streissguth, A. P, Barr, H. M, Kogan, J, \& Bookstein, F. L. (1996). Understanding the occurrence of secondary disabilities in clients with fetal alcohol syndrome (FAS) and fetal alcohol effects( FAE) Final Report CDC Grant R04, USA

[61] Streissguth AP \& O'Malley KD ((2000). Neuropsychiatric Implications and Long Term Psychiatric Consequences of Fetal Alcohol Spectrum DisordersSeminars in Clinical Neuropsychiatry, , 5(3)

[62] Sulik, K. K, Johnston, M. C, \& Webb, M. A. (1983). Fetal Alcohol Syndrome: Embryogenesis in a Mouse Model. Science, , 214, 936-38.

[63] Turk, J. (2009). Behavioural Phenotypes in Relation to ADHD. ADHD in Practice. UK., $1(3)$

[64] Turk, J. (2012). Behavioural Phenotypes. Royal College of Learning Disability Psychiatrists residential meeting, Manchester, Sepember 27th

[65] Vocci, F. J, Acri, J, \& Elksahef, A. (2005). Medication Development for Addictive Disorders: The State of The Science. Am J. Psychiatry, , 162(8), 1432-1440.

[66] US Surgeon General (2005, Feb 21). U.S. Surgeon General Releases Advisory on Alcohol Use in Pregnancy: Urges women who are pregnant or who may become pregnant to abstain from alcohol. http://www.surgeongeneral.gov/pressreleases/sg02222005.html. US Department of Health and Human Services, Office of the Surgeon General. 
[67] Bonnin A ,Goedein N ,Chen K, Wilson ML, King J, Shih JC, Blakely RD, Deneris ES, Levitt P ( 2011) A transient placental source of serotonin for fetal forebrain. Nature, April 

Chapter 21

\title{
Autism Spectrum Disorders in People with Sensory and Intellectual Disabilities Symptom Overlap and Differentiating Characteristics
}

\author{
Gitta De Vaan, Mathijs P.J. Vervloed, \\ Harry Knoors and Ludo Verhoeven \\ Additional information is available at the end of the chapter \\ http://dx.doi.org/10.5772/53714
}

\section{Introduction}

Autism Spectrum Disorders (ASD) are developmental disorders that people are burdened with for their whole life. They origin in childhood and are featured by restrictions in social and emotional development, communication, interests and motor skills [1]. People with autism are characterized by three major deficits as defined by the most recent version of diagnostic and statistical manual of mental disorders (DSM-IV-TR). These deficits include qualitative impairments in social interaction, qualitative impairments in communication and restricted, repetitive and stereotyped patterns of behaviour [2]. Behaviours within these main components of ASD may differ per individual because they are expressed in unique ways for each individual. Variations can be found in the way, the intensity and the perseverance with which the symptoms are expressed. Also the core characteristics may vary per individual. Where skills, interests and intellectual levels differ between people, so do the characteristics of autism, only the main problem areas remain the same [3]. In the current chapter, not only autism as defined by DSM-IV-TR, but also all variations within the autistic spectrum will be included.

Several symptoms of ASD are not unique but also found in other groups of people with disabilities. Similar behaviours, overlapping symptoms, or even the exact same behavioural characteristics can be found in people with hearing disabilities [4], visual impairments [5], intellectual disabilities [6] and combinations of these impairments, such as deafblindness [7]. All three of the main components of autism that the DSM-IV-TR describes, are also found in non autistic people with sensory and intellectual disabilities. 
Furthermore, the prevalence of ASD seems to be much higher in people with one or more of these disabilities. In the entire population ASD is estimated to occur in at least between 0,1 and 0,6 percent $[8,9]$ and at most 2,64 percent [10]. In people with intellectual disabilities reported prevalences are much higher, ranging from 4 up to 60 percent [11]. Without giving exact rates the prevalence of ASD and autistic features in people with sensory disabilities is reported to be much higher than in typically developing people [12-14] It is an interesting question what cause this increase in prevalence when other impairments are involved. An obvious explanation could be a relationship between ASD and sensory or intellectual disabilities. An alternative explanation is an overlap of symptoms, but not of the underlying mechanisms, between autistic people without other disabilities and people with sensory and intellectual impairments. If the latter is the case, some people might be unfairly diagnosed as autistic when in fact they are not. False positive diagnoses then causes the increase in prevalence of ASD in sensory, intellectually and multiply impaired people.

The overlap in symptoms between people with ASD and people with sensory, intellectual and multiple impairments interferes with the right classification of the behaviour of people with sensory and intellectual disabilities. Several authors stress that even though the symptoms are similar, the processes that underlie these symptoms are different for autistic versus non autistic people $[4,15,16]$. Nevertheless, when behaviours are the same, there is the risk that ASD is either missed or unjustly diagnosed. A wrong classification may lead to a wrong treatment plan, which is especially problematic if the treatment plan is counterproductive for the true underlying cause. A treatment is most effective if it tackles the cause of the behaviours. An example is the stopping of stereotyped movements. Whereas in the blind these are usually caused by a lack of stimulation from the environment $[17,18]$, in people with ASD stereotyped movements can occur to get away from too much stimulation from the environment $[19,20]$.

The current chapter will give a comprehensive overview of the overlapping symptoms between autistic and non autistic people; it will elaborate on the categories that the DSM-IVTR distinguishes as well as on the overlap within these categories for autistic and nonautistic people, it will describe the differences between the two groups and finally explain why a better differentiation is necessary.

\section{Qualitative impairments in social interaction}

The first characteristic of autism, according to DSM-IV-TR is defined as qualitative impairments in social interaction. These impairments can express through a variety of symptoms: problems in reciprocity and sharing of interests and emotions; impairments in non-verbal behaviours and impairments in joint attention, either in sharing, following or directing [2]. All of these problems in social behaviours contribute to problems in the development of proper peer relations. 


\subsection{Reciprocity and peer relationships}

Some children with ASD prefer doing things alone and might avoid all kinds of social play [2]. Lack of reciprocity is also shown in an aversion to social touch and in problems with responding to your own name [21]. In young children impairments in this area are often expressed as inappropriate responses towards other people and being more interested in objects than people [19].

Autistic people may find it difficult to engage in peer relationships. However, they are not the only ones that have trouble in this area. A recent study about the popularity of deaf children showed that deaf children were less accepted and less popular than their hearing peers. This was explained by them being, amongst others variables, more withdrawn, less prosocial and worse at monitoring a conversation [22], behaviours also typical for ASD in a hearing population.

People with intellectual disabilities show problems in the area of reciprocity and relationships too. Often, intellectual disabilities are caused by abnormalities in the brain. It is not surprising to find that these abnormalities cause problems in people's emotional and social behaviours. However, not everyone with serious intellectual disabilities has social or emotional problems, some of them are even overly interested in social contact. Reciprocity and engagement are definitely present while communicating with them [3]. According to Wing [23] one can spot the difference between impaired social behaviour in intellectually impaired people with ASD versus intellectually impaired people without ASD by looking at the severity of the social impairments.

The problems in reciprocity and developing relationships are not limited to people with ASD, and auditory or mental disabilities. In 1977 Selma Fraiberg described the development of blind children. She noticed that blind children do not reach out to their parents as much as their sighted peers do. This may appear as a lack of reciprocity, when in fact seeing a parent makes sighted children reach out. Blind children obviously lack this ability [24]. This explains their less frequent attempts in reaching out, without any relationship with reciprocity. Moreover, according to Fraiberg, the absence of reaching out could make parents less responsive to their children, restraining them in their development of relationships. She explains that in the sighted, the smallest amount of eye contact with a baby can make an adult talk or play with them [24]. When signals such as reaching out and making eye contact are absent, the development of reciprocity and relationships could be impaired because of this. In fact, because the care for a blind child is so much more challenging and reciprocal signals are easily missed, lack of vision may increase the risk of problems in attachment [18]. However, Warren stressed that despite an increased risk, attachment problems can be avoided if the parents of a blind child respond appropriately. Assessing attachment highlights another problem, that is the reliability and validity of assessment instruments and procedures in children with disabilities. Attachment in sighted children is often tested by the strange situation method [25] where a child's reaction upon reunion with its mother is assessed after it has been left alone or in the presence of a stranger. Children with visual impairments, especially blind children, may not notice the departure and reappearance of their mother and may therefore fail to respond like sighted children would do [18]. In this case 
the perception problems interfere with possible affirmations of attachment problems. The same problems occur when observing people whilst looking for signals of reciprocity or interest in other people. Because of a loss of sight children with visual impairment or blindness may not notice other people or other people's behaviour. In extreme cases they do not show any interest in their surroundings because of poor vision and direct all their attention to objects within arm's reach or to their own body. This is especially the case in deafblind children who have not only problems in vision but also hearing, the two distant senses. Their remaining senses (touch, smell, taste and proprioception) only function in nearby space, giving the impression that deafblind children are ego-centred. This ego-centeredness is however of a different origin than it is in ASD [4].

\subsection{Verbal and non-verbal social behaviours}

In people with ASD, much verbal and non-verbal behaviour is impaired. This can express itself in to unnatural eye-to-eye gaze, a failure to correctly understand and execute facial expressions, atypical body postures and gestures to regulate social interaction. People with ASD often show less eye contact and fewer social smiles to others. They may also show problems in understanding facial expressions and the underlying emotions [19].

Non-verbal behaviours are very important in social communication and are used to make messages more clear. It's hard to imagine communicating without facial expressions, gestures, posture or understanding gaze direction. People with impairments miss a lot of these signals while communicating. In a visually impaired group it may be hard to distinguish autistic people from non-autistic people based on non-verbal behaviours. Non-verbal skills that come natural to people without impairments need to be taught specifically to people with visual impairments [20], for example by explaining gestures in a tactile way and in natural situations. So even though people with sensory impairments show problems in expressing themselves non-verbally, Gense and Gense [20] do believe that many behaviours can be taught. On the other hand, in visually impaired people some behaviours may be impossible to teach. Making eye contact and following gaze direction are simply infeasible for people with visual impairments. One cannot expect them to show these behaviours. Since their impairments make some social behaviours impossible to execute, they may use other signs to show their social skills. A blind person will not look someone in the eye when interested in what they have to say, but they may aim their ears towards this person and will thus aim their face in another direction. This behaviour is inappropriate for someone with adequate visual abilities, but the visually impaired will orient with their ears more than with their eyes and it may even point to social interest in another person.

Another complication is that it is important to take into account the severity of intellectual disability when analysing a person's social behaviours. If mental and chronological age do not match, age inappropriate social behaviours might be seen. An example is that people with intellectual disabilities show few gestures and joint attention signs [26]. On the other hand, people with mental retardation and autism responded to their name much less frequently than did people with mental retardation alone [26], making orientation after hearing ones name a characteristic that may help in differentiating autistic from non-autistic people. 
When trying to differentiate autistic behaviours from behaviours due to multiple impairments, Hoevenaars-van den Boom et al. (2009) showed that even though social behaviours appear similar it is possible to differentiate autistic from non-autistic behaviours. They have found a significant difference between autistic and non-autistic deafblind children with profound intellectual disabilities in the areas of social and communicative behaviours in that these children showed and openness for contact and pleasure while in social contact [7].

\subsection{Joint attention and theory of mind}

Autistic people have trouble sharing interests, emotions and activities [2]. Related to this is problems in joint attention. Joint attention refers to the ability to share your attention, by looking where someone else is looking at and by sharing your own interests through pointing, gazing, or other non-verbal behaviour [19]. People with ASD may fail to share their emotions, feelings and thoughts but they also can have problems in sharing attention, which is expressed in their inability to follow a pointing finger or the direction of a gaze. This is interesting, because in non-autistic children, both pointing and following a finger or gaze not only relates to the object itself, but also to the other person's feelings and interests for this object. Autistic people fail to point or gaze and follow somebody else's pointing or gazing because they fail to understand other people's interests in the objects [19].

Joint attention is often said to be a precursor of theory of mind (ToM) [27]. Someone has a ToM when they are capable of attributing a mental state to themselves and to others [28]. ToM is one of the most important constructs regarding a deeper understanding of ASD [29] and can explain many of the symptoms of ASD. Not only social behaviours as joint attention, but also symbolic play and language problems such as echolalia and reversal of pronouns can be attributed to not having a ToM $[12,30]$. In simple terms, its refers to being able to realize what people think, feel and want [3]. Having a ToM also entails understanding irony and non-literal language, and can therefore also explain some of the deficits in communication. Another aspect of ToM is being able to take someone else's point of view or perspective. Perspective taking is often measured with false belief tasks, such as the Sally-Annetask [31]. Baron-Cohen and colleagues used this task to measure false belief in autistic children by showing them two dolls, one called Sally and the other called Anne. They played out a story where Sally had a marble in her basket. Sally left and Anne put the marble in her own basket. By asking children questions on where the marble really is and where Sally would think the marble is, perspective taking can be measured [32] and give an indication of the development of a ToM. This is a typical false belief task, but many variations have been used since then. Where in sighted children ToM is tested with a false belief task such as the Sally-Anne task [32] or joint attention tasks, these tasks may not be applicable sufficiently enough for children with visual impairment. In addition, joint attention is often measured with gaze direction or pointing, something that blind children are for obvious reasons incapable of showing and is limited in visually impaired children. Peréz-Pereira and Conti-Ramsden do point out that it is not the pointing or gazing what matters, it is the function of this pointing that is of interest [30]. To measure this, things need to be seen from a blind person's perspective. 
Seeing things from a blind person's perspective is difficult when it comes to ToM tasks. Conventional ToM tasks have been carried out on people with impaired vision, showing that visually impaired children invariably performed worse than sighted children. McAlpine and Moore did a false belief task using containers with unexpected contents and asked what another person would think was in it. Many of the blind children failed this task, even though sighted children are able to do this at a younger age [33,34]. A similar study by Minter, Hobson and Bishop (1998) compared visually impaired with sighted children of the same verbal intelligence, and showed similar results. In their first experiment, they did a similar task as the container task McAlpine and Moore used. They used a warm teapot, filled with sand instead of tea. Whereas almost all sighted children were able to pass this task, almost half of the visually impaired children failed to answer false belief questions such as: "What did you think was in here?" and "What would he/she think is in here?" The authors note that blind people may have less experience with hot teapots because of the extra danger their lack of vision provides. Their second experiment was done with three boxes, where the participants helped the experimenter hide a pencil for another experimenter and false belief questions were asked. Again, the visually impaired children performed worse than the sighted, but much better than on the previous task. The authors think this was because they were more involved in this task, because they helped with the hiding [35]. These findings show that children with visual impairments do worse on conventional ToM tasks than do their hearing peers. One could assume that blind children do not have a ToM, or develop it slower. However, other findings indicate that visually impaired children can pass a ToM task, given an adapted task. In line with the notion that things need to be seen more from a blind person's perspective, it could be possible that visually impaired people have just as much a ToM as sighted people do; it's only measured in the wrong way. Peterson and her colleagues confirmed this. They state that blind people may very well rely on completely different features of an object than sighted people do in order to decide what another person thinks about an object [36]. They tested if this was true by adapting frequently used false belief tasks. For example, they have changed the famous Sally-Anne task to a Sally-Bill task. In this task, there were no dolls or pictures of children with baskets and marbles, but it was a purely narrative story. The experimenters performed four ToM tasks, including similar tasks to the container tasks, a location change task and a story. On average, the children performed best on the Sally-Bill task, $73 \%$ of the children passed this task. Despite this result and the careful adaptation of test methods, test methods were not found to be a factor influencing ToM development. Degree of visual impairment was also not found to be of influence in developing a ToM, age was the only significant factor these authors found [36]. These are some interesting findings, firstly because they indicate that visually impaired people can show signs of having a ToM, secondly, because the question is raised where the difference lies between visually impaired and sighted people. According to Minter et al. [35] tasks need to be adapted to the qualities of visually impaired but Peterson et al. [37] did not find a difference between tasks they used. Brambring and Ashbrock [38] elaborated on this question. They used a large variety of different tasks that did not require vision and found that performance was better than with traditional tasks but the blind children were on average 19 months older when they were able to perform the same tasks as sighted children. A 
more recent study [39] found that children with varying levels of congenital visual impairment when compared with sighted children matched on age and verbal intelligence, had a similar performance on advanced ToM stories (second order false belief, that is beliefs about beliefs) and non-literal stories. Despite a limited access to visual information during interactions, children with congenital visual impairment can develop an effective ToM.

Peterson has not only studied ToM in visually impaired children, but also in deaf children $[37,40]$. It looks as if deaf children are strongly delayed or even impaired in their ability to have a ToM. In their 1995 study, Peterson and Siegal tested the Sally-Anne paradigm on several deaf children who were able to communicate in sign language. Even though hearing children with or without intellectual disabilities can pass this task around a mental age of four, only $35 \%$ of these deaf children were able to pass at a mental age of 8 . Furthermore, these results were similar to results of people with ASD, but worse than the performance of children with Down syndrome. Notwithstanding the lack of ToM, these deaf children were not autistic as they did not show any of the other characteristics of ASD [40]. According to Peterson and Siegel deaf children lack a ToM, because of the lack of understanding the communicative signals of others. It also appears that deaf children, especially those with hearing parents, communicate less at home than hearing children. On the one hand this is because a deaf child does not hear nor understand spoken language and on the other hand because their parents are not very fluent in sign language as an alternative for spoken language [41]. A direct consequence of the lower frequency of communication is that deaf children also communicate less about mental states, feelings and thoughts, which hinders the development of a ToM [37, 40]. This idea was supported in a more recent study that assessed the amount of communication in play sessions for pairs of hearing mothers with their deaf children and compared them to hearing mothers with hearing children. They found that these signing mothers of deaf children do not necessarily communicate less than mothers of hearing children, but they do communicate less about mental states. Additionally, a relationship was found between the amount of communication about mental states of mothers of deaf children and the performance on false belief tasks of their children [42]. Despite the similar way in which the lack of ToM expresses itself in people with ASD and in deaf, the cause is different. In children who are deaf it is often attributed to a lack of communication about mental states, thoughts and feelings, whereas in ASD it is caused by inability to take someone else's perspective.

Another possibility for why hearing children outperform deaf children on ToM tasks could be that deaf children do have a ToM but only fail on certain aspects related to ToM and conventional tasks fail to test these aspects. Where normally false belief tests and variations of this are undertaken, a recent study addressed other aspects of ToM as well. Ketelaar, Rieffe, Wiefferink and Frijns [43] assessed deaf children that have received a cochlear implant (CI) at a young age, and compared them to hearing children. They tested other aspects of ToM than false belief, which are the understanding of other's intentions and others desires. The tasks were similar to false belief tasks, only instead of asking what someone would think or believe, it was asked what an other person intended to do with an object (after failing this 
action) or what someone would want to eat (after showing them pictures of food they liked). It appeared that the deaf children and hearing children performed equally well on the intention tasks, but the hearing children outperformed the deaf on false belief tasks and on the desire tasks [43].This study indicates that deaf children may possess some abilities related to a theory of mind. It should be noted, however, that this study only included children with a CI. These children thus had some hearing abilities, though different from hearing children. The study did not include a group that was completely deaf and so conclusions about completely deaf children cannot be drawn.

When children are completely deaf there is, however, still the possibility that, as seen in the visually impaired group, testing methods are not adequate for them. Peterson and Siegal [40] tried to make their intentions more clear in their false belief questions. They reasoned that someone with limited experience in conversation might expect that the experimenter just wants them to tell the location of Sally's marble, when they ask "Where will Sally look for her marble?" For this reason they altered the question to "Where will Sally first look for her marble?" By adding the word "first" they more clearly imply that they are looking for what sally thinks instead of where the marble really is. This slight alteration improved the deaf children's performance slightly, but not enough to overcome differences in ToM development [40] as the different tasks in the study by Ketalaar et al. [43] did. Peterson and Siegal only investigated false belief, though, whereas Ketelaar et al. adressesd other aspects of ToM and tested children with a CI who do have some hearing abilities, instead of children who are completely unable to hear. The question still remains whether a more appropriate methodology for deaf children could increase their scores on conventional ToM tasks and more research has to be done in order to clarify this.

Finally, people with intellectual disabilities often show ToM impairments as well. Typical developing children start to solve ToM tasks around the age of four to five years of age. A general characteristic of people with intellectual disabilities is that they have mental ages not corresponding to their chronological ages. If mental age is below five, which is the case in profoundly and severely intellectually disabled people, and sometimes also in moderately intellectually impaired people they will probably fail ToM tasks irrespective of their chronological age [44]. Interpretations of ToM tasks should be done cautiously, when intellectually disabled people likely fail this task unrelated to the presence of ASD, to prevent unnecessary suspicion of ASD.

\section{Qualitative impairments in communication}

Qualitative impairments in communication form the second criterion that is defined in DSM-IV-TR, and this can refer to the use of language but also to problems in make belief or imitative play. When it comes to language one can find a lack of or delay in language, but also use of repetitive or idiosyncratic language. Autistic people may also find it troubling to initiate and maintain a conversation with others [2]. 


\subsection{Making conversation}

Language is something people use for communication, and so the willingness to communicate is related to their use of language [19].Despite possible technical problems in language the low desire for communication is one of the aspects of ASD that is mentioned in the DSM-IV-TR, that is not only problems in initiating and maintaining a conversation with others but also a lack of an internal willingness or desire to communicate [2]. If people with ASD are simply uninterested in communication, they will not put effort in initiating a social conversation spontaneously. This lack in willingness to communicate also contributes to the language problems found in ASD.

Initiating and maintaining a conversation can be difficult for people with sensory and intellectual disabilities too. The presence of others may go unnoticed for people with visual impairments, and communicative signs may be missed because of blindness or deafness. It has been found that deaf children communicate less with their hearing parents because of their poor skills in spoken language and their parents poor sign language skills [41]. In people with intellectual disabilities conversational skills may be worse than expected based on their chronological age, moreover, their initiations to communicate may be different, inadequate or even awkward.

Even though all of these impaired groups may show impaired conversation making skills, there are differences between autistic and non-autistic people. An example derived from a deaf population shows that despite other problems in the field of communication, such as monitoring a conversation and pragmatic use of language, non-autistic deaf children are not different from their hearing peers in initiating and maintaining a conversation [22]. But even though deaf children without ASD don't seem to have problems in initiating and maintaining a conversation, they still differ from their hearing peers in pragmatics and monitoring, hampering their conversational skills nevertheless. On the contrary, the impaired conversational skills in autistic people lie in the area of the initiation and maintenance of a conversation [2]. It also appeared that one of the areas in which the autistic and non-autistic children with deafblindness and profound intellectual disability differed significantly from each other was the openness and willingness to take initiatives for contact [7]. It is evident that conversation looks different for people with sensory or intellectual impairments versus people without impairments, and conversation skills are hampered by their lack of sensory and intellectual abilities. The difference with autistic people shows itself in the interest for this contact. Non-autistic sensory and/or intellectually impaired people still look for opportunities to make this contact or respond to other people's efforts to make contact, while people with autism lack the interest for this contact.

\subsection{Language}

Besides a lower interest in communication than people without ASD, people with ASD show some technical language impairments as well. Some autistic people do not speak at all and in others the development of language can be seriously delayed or altered [19]. Furthermore, it appears that joint attention and imitation behaviours, which are known to be impaired in ASD, can predict language abilities [27], which raises the question whether 
language is directly or indirectly related to ASD. In addition, ToM can be involved as well, one needs to know that one can influence others with their language and how to do so. Typical ASD language problems include direct or delayed echolalia, reversal of pronouns and lack of understanding of emotional meaning in language. People often describe it as 'robotlike' [45]. People with ASD often interpret the meaning of words literally. The literal meaning of a word does not change over contexts, but the figural meaning does. This is especially vivid in jokes, metaphors and irony. This may also be due to the previously mentioned problems in ToM. Being unable to understand what people mean, people with ASD interpret the words incorrectly [19]. A review about language and communication in ASD confirmed this idea by concluding that the language and communication problems are caused by processing problems when interacting with other people [46].

People with intellectual disabilities show delays in language as well as atypical language skills that can easily be confused with ASD. A study about the language abilities of a group of autistic children showed that there was a relationship between language abilities and IQ [46]. This study was done on autistic people only, but it is a rather expectable finding, even within people without ASD. It makes sense that the language abilities of someone with an intellectual disability are delayed as compared to peers with the same chronological age. This may be confused with the language deficits found in ASD, when in fact they are due to their intellectual disability. For this reason, we should not immediately attribute language issues in people with intellectual disabilities to ASD.

Deaf and people with hearing disabilities often show delays in acquiring language, but can also show peculiar uses of words [4]. Even delays in developing sign language are found for this is often not fully learned until children go to a school for the deaf. Parents are not fluent signers and fail to teach children the full scope of signs they could learn from a signer that is fluent [41]. Atypical language development can also be found in the blind. Without seeing things to potentially talk about, language is centred around other experiences in the blind compared to sighted people [18]. Children with congenital visual impairment have been shown to have difficulties with the use of language for pragmatic and social purposes, while structural language (e.g. articulation, grammar, vocabulary) was good or even superior [47, 48]. This delay or odd language use can be confused with what is found in autistic individuals. However, this language delay may be corrected if it is taught in the right way. It's important to realise that when a child misses its vision, they need to get stimulation through the other senses which affects their understanding of the meaning of words [18].

Several language problems that are found in autistic individuals are also found in people with other impairments. A typical example is echolalia, which is also found in visually and intellectually impaired people [23]. Echolalia is the apparently useless repeating of words or phrases, either immediately after they were spoken or after some time. Even in typically developing children, echolalia is sometimes used to learn language [20], so it's not surprising to find this in people with intellectual disabilities who may have a mental age comparable to when it is normal to use this type of speech. According to Schlesinger, it can be expected for a typically developing 20 month year old to repeat words to indicate more than one (e.g. "apple, apple" for "two apples") [49]. Another author described a child of 15 - 18 months 
old who often repeated her mother's words to learn the names of objects, but also to practice these words [50]. It can therefore be expected that a person with a mental age below two years of age to still show signs of echolalia. These examples consist of people with typically developing vision, but blind children use echolalia even more than typically developing children. In part echolalia serves as a means to stay in contact with people that cannot be seen, but it is also suggested that blind children practice their language by using echolalic speech. In this way they try to get a grip on the meaning of words in the absence of vision [30]. Extra practicing of words and phrases also results in more imitations and use of routines in speech. In the blind, one will also find egocentric speech and reversal of personal pronouns(I, you, he etc.), and improper use of deictic terms (e.g. here, there) which could be mistaken for autistic language, because of its atypical nature. Reversal of personal pronouns, which is found in about a third of the speech of blind children and egocentric speech may be caused by a lack of ToM, resulting in these impairments [12]. However, a logical explanation can also be based on the visual impairment. The direction of speech and who is speaking to whom determines which personal pronoun is used. Absence of vision makes it difficult to understand that the " $\mathrm{I}$ " who is speaking about the self is suddenly referred to as "you" by a person who became the " $\mathrm{I}$ " instead. 'Here' and 'there' are relative terms depending on ones spatial position. Without sight it is hard to adopt an allocentric position, most blind people use an egocentric position in processing spatial information. For instance, in way finding one cannot use landmark information to guide people who are blind, because they cannot see these landmarks. Instead one has to give route information related to the blind person's body position in space [30].

\subsection{Imitation and make-belief or symbolic play}

Finally, imitative and make-belief play are impaired in people with ASD according to the DSM-IV-TR. People with intellectually disabilities normally show delays or absence of imitation too. In one study, the experimenters showed intellectually disabled participants an action that could be done with an object, afterwards they asked the participants what could be done with the object. All participants with intellectual disabilities had trouble recalling what could be done with the object. Participants with intellectual disability and ASD performed the worst [51].

Symbolic play can be troubled in people with intellectual disabilities as well. Wing and colleagues [52] showed that even though only two people of their sample of intellectually disabled people showed the full autistic syndrome, more than half of their participants showed problems in symbolic play. These problems were either characterized as stereotyped play that was a persevering repetitive copy of other's play or no symbolic play at all, but just repetitive manipulations of a part of an object. Despite the fact that only two of their participants had an ASD diagnosis, many showed autistic features. In the group that was able to show symbolic play (43 of 108 participants), only two participants had slight autistic features [52].This finding shows that many intellectual disabled people show impairments in symbolic or make-belief play, and this can therefore not be used as a differentiating characteristic of ASD versus no ASD in this group. 
When these people with intellectual disabilities have an additional sensory impairment, problems in symbolic play and imitation can become more evident. It is reasonable to think that people with impaired vision or hearing have more difficulties in imitating because they are less able to perceive actions of others, than people without these impairments. Similarly, symbolic play can be affected. People have less modalities to perceive a toy with, and therefore also see less ways in which they may use it. Combined with an intellectual impairment they can also have troubles in understanding the function the object is intended to have.

Lack of symbolic play was demonstrated to be related to abnormalities in language development that are typical of ASD, such as repetitive speech [52]. Similar to many of the impairments in ASD that were discussed, this too can be attributed to a lack of ToM. According to Brown et al. [12] ASD is characterized by problems in ToM, symbolic play, and context dependent language. Shared features of these three skills in childhood are: 1) there has to be a communication pattern between parent and child regarding feelings and thoughts; 2) one has to see and understand the direction of someone else's attitudes towards a shared world; and 3) feel inclined to identify oneself with this shared world. People with ASD have problems with all three features. Children who are deaf encounter problems with the first feature. They are offered less ToM related language. Children who are blind have trouble with the second feature and subsequently children who are deafblind have trouble with the first and second feature.

\section{Restricted, repetitive and stereotyped patterns of behaviour}

As the last of three important characteristics, the DSM-IV-TR mentions restricted, repetitive and stereotyped patterns of behaviour, interests and activities. This can refer to motoric stereotypies or mannerisms, preoccupations with objects, parts of objects or interests, or their inflexibility in deviating from routines [2].

\subsection{Stereotyped use of objects}

Uta Frith confirms that autistic people are often very interested in details, which may appear as restricted interests to others [3] and that routines and repetitions are also of importance for them [19].These behaviour can be explained by the central coherence theory. This theory poses that autistic people have a weak central coherence, meaning that they have the tendency perceive objects and situations in parts rather than perceiving the whole picture or combine information to holistic patterns [3]. As a consequence information is often processed out of context [31]. This theory explains the focus on details, but possibly also the need for repetition and routines shown by people with ASD. The ability to generalize parts to the whole keeps situations similar and predictable, and therefore less frightening. If one misses this ability then a coping mechanism is to stick to routines in order to keep situations predictable and safe. If preformed to the extreme these routines become stereotyped behaviours. 
Repetitive and stereotyped use of objects is not only seen in autistic people but also in people with intellectual disabilities. In a study where 108 children with severe and profound mental disabilities were included less than two percent suffered from ASD. However, repetitive routines and stereotyped play were found in 60 percent of this group with a mental age below 20 months [52]. Also in children who are blind strong interest in parts of objects and repetitive use of objects can be seen. Mainly this is the result of the blindness-specific constraints on the use of play material that require visual-manual skills. Blind children, when playing alone, prefer toys and materials that produce distinctive tactile or auditory effects [53]. Toys are often articles of daily living and objects in their surroundings such as spoons, walls and furniture. Activities are often aimed at making noise [53, 54].This behaviour is thought to be a way of getting hold on the function of an object and in contrast to children with ASD this behaviour can be relatively easily stopped or interrupted.

\subsection{Self Stimulation}

Finally, autistic people show stereotyped movements with their own bodies or parts of their body. These are often thought to be self-stimulatory. Stereotyped movements can be performed with every body part but often involve the hands or walking $[55,56]$ and sometimes become self-injurious $[57,58]$. These movements occur in other developmental disorders as well $[55,56]$, but are especially common in ASD. According to Kraijer self-stimulatory behaviours are often caused by lack of stimulation from the environment [44]. In these situations people use their own bodies to provide themselves with the stimulation they need at that moment. He adds to this that the amount of self-stimulatory behaviour and also intensity and severity, that is whether it is self-injurious, is related to the level of functioning. The lower the functional level of the person, the more the self-stimulatory behaviour increases in amount and severity [44].

Stereotyped behaviours occur in people with visual impairments as well. Typical stereotyped behaviours in people who are blind are body rocking, head shaking, eye poking and hand flapping Because these behaviours often occur in the blind, they are sometimes referred to as blindisms, $[18,20]$. Actually this term is not entirely correct, because these stereotyped behaviours are not unique for people who are blind; mannerisms would be a better term. Body rocking and head movements, for instance, are typical examples of behaviours that can be seen in people with visual impairment, intellectual disabilities and ASD [18, 20, 24]. Stereotyped behaviours were seen in nearly all [59] and in all [60] blind children, but in children with visual impairment the prevalence is still 10-45\% [59]. There also seems to be an age dependency in stereotyped behaviours in blind children. In the first two years stereotyped behaviours increase in frequency to decline thereafter [61]. Stereotyped movements are also found in people with multiple disabilities. Heather Murdoch [62] suggests that stereotyped behaviours may be a part of normal motor development but that in people with multiple disabilities, these behaviours do not develop further. In a typically developing child, repetitive behaviours appear as well but develop into conscious movements later on, whereas in people with multiple disabilities they may remain repetitive movements. Trying to stop these behaviours may hamper the development of other motor activities or communicative signs [62]. 
Whereas stereotyped movements in people without ASD are part of a normal development, in people with ASD they are part of their syndrome. Gense and Gense [20] believe that the differences between these behaviours in visually impaired people with or without ASD can be found in the severity and perseverance of this behaviour. People with ASD show higher intensities and stronger persistence in stereotypical behaviours [20, 57]. Similar to the behaviours in the intellectually disabled, this could be due to a lack of external stimulation. Especially in the blind, where stimulation from visual input is missing, self-stimulatory stereotyped movements could provide the necessary sensory stimulation [18]. Another difference between people with ASD and people without, is that stereotyped behaviour can more easily be interrupted or stopped in people with visual impairments alone [20]. Sometimes not much more has to be undertaken than making the blind person conscious of these unconsciously executed stereotyped behaviour patterns.

\section{Differentiation: Why and how?}

\subsection{Overlap and differences}

The overlap in symptoms between autistic and non-autistic people with sensory and intellectual disabilities must be clear after reading this chapter. The diagnoses of ASD is usually based on behavioural characteristics and these can be similar in autistic and non-autistic people with additional impairments. An additional problem is that, although instruments are available for people with intellectual disabilities [63, 64], most of the current test instruments do not have separate norms for people with sensory and/or intellectual disabilities. No valid instruments are available for deaf people according to Jure and colleagues [14], nor for visually impaired people [7]. The overlap in symptoms and trouble in diagnosis cause a distorted representation of ASD in people with sensory, intellectual and multiple impairments. Some people are diagnosed as autistic when they are not, while others do not get the autistic label when they should. So there is both an overdiagnosis [5, 15] of ASD in this group, meaning that more people are diagnosed as autistic than necessary because of these overlapping symptoms, as well as an underdiagnosis $[14,65]$. In a group of deaf children, for example, the diagnosis of ASD was established significantly later than in a group of hearing children. Autistic behaviours were probably missed because of an earlier diagnosis of hearing impairments or other developmental disabilities [65]. The main problem in assessment of ASD can be attributed to a diagnostic overshadowing bias. The diagnostic overshadowing bias was first described for people with intellectual disabilities and is the tendency of clinicians to overlook symptoms of mental health problems in this group and attribute them to being part of "having an intellectual disability" [66]. In the presence of mental retardation it seems that the diagnostic importance of abnormal behaviour decreases. Blindness, deafness or deafblindness all might add an extra overshadowing bias next to intellectual disability, leading to either false positive or false negative diagnoses of ASD in people with these disabilities. 
Despite the obvious similarities between autistic and non-autistic people with sensory and intellectual disabilities, this chapter also outlines that even though the symptoms appear the same, sometimes subtle difference can still be found. This may be due to the possibility that underlying processes of the behaviours are different for autistic and nonautistic individuals $[4,5,15]$. If attempted, a differentiation can thus be made by studying the subtle differences and underlying causes. A couple of years ago, this was done by making a valid instrument to diagnose ASD in people one of the most challenging combination of disabilities, namely deafblindness and profound intellectual disabilities. Hoevenaars-van den Boom and colleagues were able to confirm the huge overlap in behavioural symptoms between autistic and non-autistic people, but were also able to successfully distinguish the autistic from non-autistic people with their approach that was suited to the developmental level of the participants. They found that differences in this group can be found in the social communicative field, mostly in openness for contact, reciprocity and joint attention and communicative functions [7]. It is clear that when using a careful and sophisticated approach, a distinction can be made between autistic and non-autistic people with sensory and intellectual disabilities

\subsection{Interaction, treatment and teaching}

A fair diagnosis of ASD, or no ASD, is very important for the treatment and interaction with people with sensory and intellectual disabilities. An ASD diagnosis or a lack thereof will affect how a person will be treated, as autistic or not. If a child with ASD is placed in a setting where his or her ASD goes unrecognized, the clinicians and care takers might fail to respond to the needs of this person [65]. An important example of why recognition of ASD is so important is the treatment of stereotyped behaviour. Stereotyped movements can be a way to reduce stress $[19,20]$. In someone with no ASD but with blindness or deafblindness, this behaviour is usually caused when the person does not get enough stimulation from their environment $[17,18]$, whereas in persons with ASD stereotyped behaviours can be a way to escape from overstimulation or as a way to ensure the optimal level of arousal. In both cases the way to treat stereotyped behaviour will be different, give extra stimulation or reduce overstimulation, respectively. A valid diagnosis would be very helpful in cases where clinicians or parents have to decide what kind of intervention to give. If it is clear whether someone has ASD or not treatment and interaction can be adjusted. Someone with ASD needs a more structured environment, and needs clear instructions when something needs to be done. In someone with ASD, things need to be re-explained in new situations, because of their difficulties in generalizing [6]. It also seems that the sooner we are aware of ASD the better. People with ASD need to be approached in way that is accommodated to their needs [65], and for the wellbeing of the child, it is best if this is done as soon as possible. A recent meta-analysis on intensive early intervention programs for ASD shows that programs that intervene early are most effective and can produce changes in the area of language and adaptive behaviour [67]. Adaptive behaviour was also found to increase as well when additional behavioural treatments were given to children with ASD and intellectual disabilities [68]. These studies showed that if ASD is treated, successful results can be achieved. 
As can be seen throughout this chapter, people with visual impairments show many behaviours that are similar to ASD, such as the lack of understanding of social situations, ego-centeredness, and lack of understanding gestures and facial expressions. But, according to Gense and Gense [20], these behaviours may still be taught. Teaching appropriate behaviours is especially important, because inappropriate behaviours may interfere with regular social interactions [18], depriving disabled children of these otherwise valuable experiences. And whereas for non-autistic people without visual impairments these behaviours are implicitly learned, in non-autistic visually impaired people, they need to be explicitly taught. With the right type of education, visually impaired people may still learn to interpret social situations, read and understand gestures and facial expressions and learn to play with others [20]. This was also found for two severely mentally disabled deafblind young men, of whom the social interaction became significantly better after tailored training sessions [58]. Although this was only a small study with two participants, it does indicate what a specialized training can mean for children that are not restrained by ASD. The same applies to language. When a delay in language is caused by a lack of seeing things to talk about, parents need to offer more tactile or auditory stimuli [18]. Basically, it is important to take into account everything that singular or multiple disabled people lack. When sensory and intellectual impairments are involved, one needs to try and substitute the missing modality for others as much as possible.

\section{Summary and Conclusion}

Many characteristics of ASD seem to overlap with characteristics that are naturally present in people with sensory disabilities, intellectual impairments or a combination of disabilities. The characteristics appear the same whether ASD is present or not, which makes it difficult to make a valid diagnosis of ASD in this group. All of the criteria that are used in DSM-IVTR to define ASD are, to some extent, also present in people with one or more of these disabilities. However, if one would look closer to these criteria, and the way they are expressed within people with sensory and intellectual impairments, slight and subtle differences can be found. There are differences in the way the symptoms express themselves, the severity of the symptoms and the underlying causes for the behaviours. Problems also occur in methodology. Paradigms that are used to assess problems that are related to ASD, such as ToM tasks, fail to be successful in differentiating people with sensory or multiple impairments. This overlap and these problems in methodology make it a major challenge to diagnose ASD within people with sensory and intellectual disabilities.

The slight differences in the way symptoms are expressed show that a distinction between autistic behaviours and non-autistic behaviours can be made. Making this distinction is very important to do, because the needs of people with ASD differ very much from people without ASD. To make sure the needs of every individual are met, people should be diagnosed in the right way. This is especially important for those groups with problems in communicating their wants and needs. In order to do this, subtle differences need to be taken into account. Up until this day, no instrument is suited to diagnose ASD or assess autistic behaviours within multiply 
impaired people. Ideally, a new way to assess autistic behaviours in sensory and intellectually disabled people that takes into account all the difficulties that assessing this group brings forth will be developed. An instrument that can make accurate diagnosis in people with multiple disabilities should account for all the overlapping symptoms and differences that have been described. First of all, intellectual disabilities should be taken into account. Some behaviours that are typical for ASD in people without intellectual disabilities can be simply explained by a person's mental age or shortcomings in intellectual abilities. An example of this is theory of mind, and related to that joint attention, symbolic play and language abilities, that do not develop until a certain age. If an intellectually disabled person has not reached a sufficient mental age, these behaviours should not be used to assess ASD. Secondly, it's important to realise that sensory disabilities withhold a person from perceiving objects and situations the same way a person without sensory disabilities would and may follow a completely different path. When someone is visually impaired or blind, eye contact, following gaze and sharing attention through pointing cannot be used as differentiating characteristics. Furthermore, it's important to take into account that a person may not always be aware of the presence of objects or people, so failures to respond like a person without ASD can be caused by being unaware of their presence in the first place. Similar precautions should be made for deaf people, who are unable to respond to calling their names, other sounds, and may not even notice the arrival or departure of a person. Finally, a combination of these disabilities can make it more challenging to make diagnostic evaluations of a person. People with multiple disabilities may need more time to process their surroundings and to realise what is expected of them. Furthermore, unexpected and sudden movements or actions, or giving too much information at once may cause a lot of stress that interferes with their performance. Many characteristics that normally differentiate people with ASD from people without ASD should not be assessed or assessed differently in people with multiple impairments. Still, some characteristics of the autistic spectrum are left that can be included in an assessment. Examples that cannot be forgotten include interest in, response to and looking for contact, resistance to change and interest in new items or situations. Sharing of feelings or interests may not occur through pointing or gaze, but may show itself in a more tactile way. It is important to be aware of the different way in which multiply disabled people express themselves. Finally, to account for intellectual disabilities, it is important to assess everything on a level that is suitable for the participants. Do not use complicated questionnaires, but simple toys as much as possible. Only if all of this can be done successfully, autistic people can be differentiated from non-autistic people and personal needs can be met.

\section{Author details}

Gitta De Vaan ${ }^{1 *}$, Mathijs P.J. Vervloed ${ }^{1}$, Harry Knoors ${ }^{1,2}$ and Ludo Verhoeven ${ }^{1}$

*Address all correspondence to: g.devaan@pwo.ru.nl

1 Behavioural Science Institute, Radboud University Nijmegen, NijmegenThe Netherlands,

2 Royal Kentalis, Sint-Michielsgestel, The Netherlands 


\section{References}

[1] Nevid JS, Rathus SA, Greene B. Abnormal psychology in a changing world. 7th ed. Upper Saddle River, NJ: Pearson Education; 2008.

[2] American Psychiatric Association. Diagnostic and statistical manual of mental disorders, fourth edition, text revision (DSM-IV-TR). Washington, DC: American Psychiatric Association; 2000.

[3] Frith U. Autism: A very short introduction. Oxford, United Kingdom: Oxford University Press; 2008.

[4] Knoors H, Vervloed MPJ. Educational programming for deaf children with multiple disabilities: Accomodating special needs. In: Marschark M, Spencer PE, editors. The Oxford Handbook of Deaf Studies, Language and Education. 2 ed. New York: Oxford University Press; 2011. p. 82-96.

[5] Cass H. Visual Impairment and Autism. Autism. 1998 June 1, 1998;2(2):117-38.

[6] De Bildt A, Sytema S, Kraijer D, Minderaa R. Prevalence of pervasive developmental disorders in children and adolescents with mental retardation. Journal of Child Psychology and Psychiatry. 2005;46(3):275-86.

[7] Hoevenaars-van den Boom MAA, Antonissen ACFM, Knoors H, Vervloed MPJ. Differentiating characteristics of deafblindness and autism in people with congenital deafblindness and profound intellectual disability. Journal of Intellectual Disability Research. 2009;53(6):548-58.

[8] Fombonne E. The prevalence of autism. JAMA: The Journal of the American Medical Association. 2003;289(1):87-9.

[9] Fombonne E. Epidemiological Surveys of Autism and Other Pervasive Developmental Disorders: An Update. Journal of Autism and Developmental Disorders. 2003;33(4):365-82.

[10] Kim YS, Leventhal BL, Koh Y-J, Fombonne E, Laska E, Lim E-C, et al. Prevalence of Autism Spectrum Disorders in a Total Population Sample. American Journal of Psychiatry. 2011;168:904-12.

[11] Matson JL, Shoemaker M. Intellectual disability and its relationship to autism spectrum disorders. Research in Developmental Disabilities. 2009;30(6):1107-14.

[12] Brown R, Hobson RP, Lee A, Stevenson J. Are There "Autistic-like" Features in Congenitally Blind Children? Journal of Child Psychology and Psychiatry. 1997;38(6): 693-703.

[13] Hobson RP, Lee A, Brown R. Autism and Congenital Blindness. Journal of Autism and Developmental Disorders. 1999;29(1):45-56. 
[14] Jure R, Rapin I, Tuchman RF. Hearing-impaired autistic children. Developmental Medicine \& Child Neurology. 1991;33(12):1062-72.

[15] Andrews R, Wyver S. Autistic tendencies: Are there different pathways for blindness and Autism Spectrum Disorder? British Journal of Visual Impairment. 2005 May 1, 2005;23(2):52-7.

[16] Hobson RP. Why connect? On the relation between autism and blindness. In: Pring L, editor. Autism and Blindness: Research and reflections. London, United Kingdom: Whurr Publishers; 2005. p. 10-25.

[17] Van Dijk J, Janssen M. Doofblinde kinderen [Deafblind children]. In: Nakken H, editor. Meervoudig gehandicapten: een zorg apart. Rotterdam, the Netherlands: Lemniscaat; 1993.

[18] Warren DH. Blindness and Children: An individual differences approach. Cambridge, UK: Cambridge University Press; 1994.

[19] Frith U. Autism: Explaining the enigma. 2nd ed. Oxford, UK: Blackwell Publishing; 2003.

[20] Gense MH, Gense DJ. Autism Spectrum Disorders and Visual Impairment: Meeting Student's Learning Needs. New York, NY: American Foundation for the Blind Press; 2005.

[21] Baranek GT. Autism During Infancy: A Retrospective Video Analysis of Sensory-Motor and Social Behaviors at 9-12 Months of Age. Journal of Autism and Developmental Disorders. 1999;29(3):213-24.

[22] Wolters N, Knoors HET, Cillessen AHN, Verhoeven L. Predicting acceptance and popularity in early adolescence as a function of hearing status, gender, and educational setting. Research in Developmental Disabilities. 2011;32(6):2553-65.

[23] Wing L, Gould J. Severe impairments of social interaction and associated abnormalities in children: Epidemiology and classification. Journal of Autism and Developmental Disorders. 1979;9(1):11-29.

[24] Fraiberg S. Insights from the blind. New York, NY: Basic Books; 1977.

[25] Ainsworth MDS, Bell SM. Attachment, Exploration, and Separation: Illustrated by the Behavior of One-Year-Olds in a Strange Situation. Child Development. 1970;41(1):49-67.

[26] Osterling JA, Dawson G, Munson JA. Early recognition of 1-year-old infants with autism spectrum disorder versus mental retardation. Development and Psychopathology. 2002;14(02):239-51.

[27] Charman T, Baron-Cohen S, Swettenham J, Baird G, Cox A, Drew A. Testing joint attention, imitation, and play as infancy precursors to language and theory of mind. Cognitive Development. 2000;15(4):481-98. 
[28] Premack D, Woodruff G. Does the chimpanzee have a theory of mind? Behavioral and Brain Sciences. 1978;1(04):515-26.

[29] Noens I, van Berckelaer-Onnes I. Making Sense in a Fragmentary World. Autism. 2004 June 1, 2004;8(2):197-218.

[30] Pérez-Pereira M, Conti-Ramsden G. Language development and social interaction in blind children. East Sussex, UK: Psychology Press; 1999.

[31] Hill EL, Frith U. Understanding autism: insights from mind and brain. Philosophical Transactions of the Royal Society of London Series B: Biological Sciences. 2003 February 28,$2003 ; 358(1430): 281-9$.

[32] Baron-Cohen S, Leslie AM, Frith U. Does the autistic child have a "theory of mind" ? Cognition. 1985;21(1):37-46.

[33] McAlpine LM, Moore CL. The development of social understanding in children with visual impairments. Journal of Visual Impairment \& Blindness. 1995;89(4):349-58.

[34] Pérez-Pereira M, Conti-Ramsden G. Do blind children show autistic features? In: Pring L, editor. Autism and blindness: Research and reflections. London, UK: Whurr Publishers; 2005.

[35] Minter M, Hobson RP, Bishop M. Congenital visual impairment and theory of mind'. British Journal of Developmental Psychology. 1998;16(2):183-96.

[36] Peterson CC, Peterson JL, Webb J. Factors influencing the development of a theory of mind in blind children. British Journal of Developmental Psychology. 2000;18(3): 431-47.

[37] Peterson CC, Siegal M. Insights into Theory of Mind from Deafness and Autism. Mind \& Language. 2000;15(1):123-45.

[38] Brambring M, Asbrock D. Validity of False Belief Tasks in Blind Children. Journal of Autism and Developmental Disorders. 2010;40(12):1471-84.

[39] Pijnacker J, Vervloed M, Steenbergen B. Pragmatic Abilities in Children with Congenital Visual Impairment: An Exploration of Non-literal Language and Advanced Theory of Mind Understanding. Journal of Autism and Developmental Disorders. $1-10$.

[40] Peterson CC, Siegal M. Deafness, Conversation and Theory of Mind. Journal of Child Psychology and Psychiatry. 1995;36(3):459-74.

[41] Vaccari C, Marschark M. Communication between Parents and Deaf Children: Implications for Social-emotional Development. Journal of Child Psychology and Psychiatry. 1997;38(7):793-801.

[42] Moeller MP, Schick B. Relations Between Maternal Input and Theory of Mind Understanding in Deaf Children. Child Development. 2006;77(3):751-66. 
[43] Ketelaar L, Rieffe C, Wiefferink CH, Frijns JHM. Does Hearing Lead to Understanding? Theory of Mind in Toddlers and Preschoolers With Cochlear Implants. Journal of Pediatric Psychology. 2012 July 29, 2012.

[44] Kraijer D. Handboek autismespectrumstoornissen en verstandelijke beperking [Handbook autism spectrum disorders and intellectual disability]. Lisse, the Netherlands: Harcourt; 2004.

[45] Nederlandse Vereniging voor Psychiatrie. Richtlijn Diagnostiek en behandeling autismespectrumstoornissen bij kinderen en jeugdigen [Guideline diagnostics and treatment of autism spectrum disorders in children and youth] Utrecht, Netherlands: de Tijdstroom; 2009.

[46] Tager-Flusberg H, Paul R, Lord C. Language and Communication in Autism. In: Volkmar FR, Paul R, Klin A, Cohen DJ, editors. Handbook of Autism and Pervasive Developmental Disorders. 3rd ed2005.

[47] James DM, Stojanovik V. Communication skills in blind children: a preliminary investigation. Child: Care, Health and Development. 2007;33(1):4-10.

[48] Tadić V, Pring L, Dale N. Are language and social communication intact in children with congenital visual impairment at school age? Journal of Child Psychology and Psychiatry. 2010;51(6):696-705.

[49] Schlesinger IM. Steps to Language. Toward a theory of native language aquisition. Hillsdale, NJ: Lawrence Erlbaum Associates; 1982.

[50] Dore J. A pragmatic description of early language development. Journal of Psycholinguistic Research. 1974;3(4):343-50.

[51] Charman T, Swettenham J, Baron-Cohen S, Cox A, Baird G, Drew A. Infants with autism: An investigation of empathy, pretend play, joint attention, and imitation. Developmental Psychology. 1997;33(5):781-9.

[52] Wing L, Gould J, Yeates SR, Brierly LM. Symbolic play in severely mentally retared and in autistic children. Journal of Child Psychology and Psychiatry. 1977;18(2): 167-78.

[53] Tröster H, Brambring M. The play behavior and play materials of blind and sighted infants and preschoolers. Journal of Visual Impairment \& Blindness. 1994;88(5): 421-32.

[54] Preisler GM. A descriptive study of blind children in nurseries with sighted children. Child: Care, Health and Development. 1993;19(5):295-315.

[55] Goldman S, Wang C, Salgado MW, Greene PE, Kim M, Rapin I. Motor stereotypies in children with autism and other developmental disorders. Developmental Medicine \& Child Neurology. 2009;51(1):30-8.

[56] Militerni R, Bravaccio C, Falco C, Fico C, Palermo MT. Repetitive behaviors in autistic disorder. European Child \& Adolescent Psychiatry. 2002;11(5):210-8. 
[57] Bodfish JW, Symons FJ, Parker DE, Lewis MH. Varieties of Repetitive Behavior in Autism: Comparisons to Mental Retardation. Journal of Autism and Developmental Disorders. 2000;30(3):237-43.

[58] Van Hasselt VB, Hersen M, Egan BS, Mckelvey JL, Sisson LA. Increasing Social Interactions in Deaf-Blind Severely Handicapped Young Adults. Behavior Modification. 1989 April 1, 1989;13(2):257-72.

[59] Jan JE, Freeman RD, Scott EP. Visual impairment in children and adolescents. New York, NY: Grune \& Stratton; 1977.

[60] Tröster H, Brambring M, Beelmann A. Prevalence and situational causes of stereotyped behaviors in blind infants and preschoolers. Journal of Abnormal Child Psychology. 1991;19(5):569-90.

[61] Tröster H, Brambring M, Beelmann A. The age dependence of stereotyped behaviours in blind infants and preschoolers. Child: Care, Health and Development. $1991 ; 17(2): 137-57$.

[62] Murdoch H. Stereotyped Behaviours: How Should We Think About Them? British Journal of Special Education. 1997;24(2):71-5.

[63] Kraijer D, Bildt A. The PDD-MRS: An Instrument for Identification of Autism Spectrum Disorders in Persons with Mental Retardation. Journal of Autism and Developmental Disorders. 2005;35(4):499-513.

[64] Matson JL, Boisjoli JA. Autism spectrum disorders in adults with intellectual disability and comorbid psychopathology: Scale development and reliability of the ASD-CA. Research in Autism Spectrum Disorders. 2008;2(2):276-87.

[65] Roper L, Arnold P, Monteiro B. Co-Occurrence of Autism and Deafness. Autism. 2003 September 1, 2003;7(3):245-53.

[66] Mason J, Scior K. 'Diagnostic Overshadowing' Amongst Clinicians Working with People with Intellectual Disabilities in the UK. Journal of Applied Research in Intellectual Disabilities. 2004;17(2):85-90.

[67] Peters-Scheffer N, Didden R, Korzilius H, Sturmey P. A meta-analytic study on the effectiveness of comprehensive ABA-based early intervention programs for children with Autism Spectrum Disorders. Research in Autism Spectrum Disorders. 2011;5(1): 60-9.

[68] Peters-Scheffer N, Didden R, Green VA, Sigafoos J, Korzilius H, Pituch K, et al. The behavior flexibility rating scale-revised (BFRS-R): Factor analysis, internal consistency, inter-rater and intra-rater reliability, and convergent validity. Research in Developmental Disabilities. 2008;29(5):398-407. 


\section{Section 6}

Aetiological Factors - Parents and Families 

Chapter 22

\title{
Empowering Families in the Treatment of Autism
}

\author{
Jennifer Elder \\ Additional information is available at the end of the chapter \\ http://dx.doi.org/10.5772/54303
}

\section{Introduction}

It is well known that autism is a complex, currently incurable disorder with an unclear etiology, and that individuals with autism typically have normal life expectancies which require parents, and later siblings, to provide varying levels of lifelong care. Because of the complexity of the disease, it is critically important to help families understand the disorder, manage stress, and sift through information that frequently includes erroneous media views and unsubstantiated claims of treatment efficacy. This chapter will help families and advising professionals by providing them with an overview of several topics: first, the common reactions and beliefs about autism and individuals with autism that are held by family members; second, the family-centered as well as complementary and alternative treatment approaches that are currently available; and finally, the best recommendations for helping families adapt to an autism diagnosis and maintain healthy functioning as caregivers - all while planning for, and addressing the lifelong needs of, individuals with autism.

\section{Common reactions and beliefs held by family members}

Families are faced with enormous challenges in caring for children with autism over a lifetime. The first challenge is obtaining the initial diagnosis, which can be difficult despite the fact that autism is better understood today than it was in the past. Indeed, it is common for families to consult a variety of professionals such as pediatricians and primary health care providers before receiving a conclusive diagnosis. Once the diagnosis is made, however, parents face a second, far greater challenge: mourning the loss of their "perfect child" - which can be a long and arduous process that involves coming to terms with the fact that their child, whose physical appearance is normal or even unusually attractive, has a complex, incurable, and frequently debilitating condition. After the family advances through stages of 
grieving that can be characterized using Elizabeth Kübler-Ross's five stages of grief-Denial, Anger, Bargaining, Depression, and Acceptance (DABDA) - they eventually arrive at a "new normal" with family harmony reestablished [1].

The first stage of grieving, denial, is common in parents of children with autism, and can persist even after a child receives a diagnosis. Because fathers are typically less involved in day-to-day care than mothers, they may experience denial more intensely due to fewer opportunities to observe the symptoms. For example, a father may be more likely to say, "he doesn't have autism; he's just quiet," which is supported by stories of other family members who were also "late to develop" yet still "turned out fine". However, as the symptoms of autism become more conspicuous, caregivers notice differences between their child and other, typically developing children whom they encounter in playgrounds, preschools, and family gatherings. Frequently, it is extended family members who identify the autistic symptoms, share their concerns with the primary caregivers and try to convince the caretakers to seek further assessment and follow up as needed. This action is critical to accurate and timely diagnosis, early intervention (< age $3)$, and improved prognosis for overall quality of life.

The next stage, "anger", may result in family members asking "Why us?" or "Why did this have to happen to him?" During this time, family tension is high and anger may also be expressed toward intervening professionals, especially if there has been a prior lack of, or slow responsiveness to, parental concerns. For example, one parent stated, "That pediatrician should have listened to me when I expressed concern about David not speaking at four years old; instead, he told me not to worry about it." This failure to identify the signs sooner can lead to destructive self-blame, resulting in self-talk such as, "If only I had recognized the signs sooner" or "I knew we should have sought other opinions" - comments that may be responded to with active listening (e.g. "You sound as though you are experiencing a lot of regret") and nonjudgmental advice (e.g. "Many parents struggle at this time. What is important is that you are seeking the necessary assistance now.") In addition to self-blame related to behavior, it is also common in this stage for parents to evaluate their genealogy to determine who was genetically responsible for the disorder. Unfortunately, there is no conclusive genetic test for autism and while genetics likely plays a role, environmental factors may also contribute to its development.

The third stage, "bargaining", can place families at great risk because it involves frantically seeking ways to reverse the diagnosis even if those ways are implausible. For example, it is common for parents to directly bargain with a higher power (e.g. "If you cure my child, I will be a better parent") or indirectly, with a lesser power such as the health care profession (e.g. If I find the "right" doctor or medication, my child will be cured). As they desperately seek a "magic bullet", parents may interrogate health care providers about the most useful medications despite the fact that no single medication is effective for all symptoms. In addition, parents may surf the Internet and read testimonials regarding treatments that are not empirically sound; consequently, well-informed professionals need to advise families against these treatments as some are risky and can lead to financial burden. (The most common treatment approaches will be described later in this chapter.) 
The fourth stage, "depression", can take many forms. Parents may at times feel overwhelmed and powerless in their ability to facilitate their child's development or ameliorate difficult, disruptive behaviors such as severe tantrums or self-abuse. Indeed, negative behaviors may intensify to such a degree that families curtail their usual plans or avoid a desired activity all together, leading to feelings of hopelessness that is expressed in statements such as, "I can't do anything right" or "why bother". In addition, because many children with autism have sleep disturbances (e.g., difficulty falling asleep; waking up and becoming active in the middle of the night), parents must be vigilant at night, causing exhaustion and sometimes even deeper depression. At this point, it is important for caregivers to recognize that they may need professional help such as counseling or prescribed medications in order to optimally provide for their child and family.

Many families who advance to acceptance, the final stage, describe having gained spiritual strength, which helped them maintain "hope" - an essential ingredient to successful grieving. In this stage, families recognize that there is no instant cure for their child's autism, but there are credible interventions that can help. Ultimately, families discover that they can be powerful advocates for their children, and after receiving proper education, can implement home interventions that positively affect the family unit and even improve their child's condition. Once they gain confidence in these new approaches, they can serve other families struggling through the grieving process by contributing empathy and wisdom to local family support group meetings. Because grieving is rarely a linear process, these meetings can also help families as they revisit earlier stages by limiting the time they spend in previous ones, thus facilitating a more permanent acceptance.

\section{Families as primary interveners}

\subsection{Parent training}

Historically, development of more family-focused interventions has resulted in a shift from didactic teaching and family therapy models to interactive approaches, in which parents are active participants in all levels of the training process [2,3]. Although parents were once viewed as the cause of their child's problems [4-6], they are now recognized for the key roles they can play in ongoing child training and skill generalization [7-9], which has led to better child prognosis and long-term quality of life.

Because there are now clearer linkages between core constructs such as social reciprocity (e.g. social turn-taking), joint attention (e.g. sharing interest in, and mutually commenting on, an object), and language acquisition, developing these skills can improve a child's communicative capacity. In fact, researchers stress that teaching parents to target pivotal skills such as joint attention may produce positive, sustained effects on social and language development [10]. Similarly, evidence suggests that interventions that require parents to synchronize with the child's attentional focus (i.e. become interested in what the child is interested in) may be more effective than parent-directed approaches (e.g., instructing the child to play with a toy in a certain way) for children who have difficulty responding to, or initiating, joint attention 
[11]. However, there is a need to closely examine the individual parent-training intervention components thought to be linked with these core constructs to determine which components are most effective for a particular child. This would allow researchers to better identify the most convenient and efficient means of teaching these constructs and related intervention components.

\subsection{Research development in parent training}

The author and co-investigators have been following a systematic sequence of research that began in the early 1980's with the development of a play-based, in-home intervention that was initially tested in-depth, over 8-12 weeks, with four mother-child dyads using intrasubject (single subject experimental) methodology [12]. In this initial study, Elder found that mothers figure prominently as recipients of training and other interventions and that even when the focus was on the dyad, mothers "took over" and fathers stayed in the "background," with inadequate diffusion of new learning through the mothers. This lack of father involvement piqued the interest of Elder's research team, who collaborated on new studies directed at fathers. Although a systematic review of the literature revealed only three intervention studies that included fathers, evidence indicated that fathers' interaction styles differed from mothers, possibly resulting in unique contributions to their child's social and language development [13].

Building on Lamb's (1987) seminal work related to fathers and their influence on child development, Elder et al. developed and tested a Father Directed In-Home Training (FDIT) intervention with a total of 36 father-child and mother-child dyads under controlled conditions in two NIH/NINR-funded studies [7,8]. The study was designed so that data from individual training components could be analyzed rather than an entire intervention package. These training components were based on the theoretical constructs in social interaction theory and characterized by the broad concept of social "turn-taking". Because the team had previously observed many fathers sitting passively or aggressively directing interactions and not allowing their child time to respond, the research team created four intervention components: (a) following the child's lead (FCL), which involved allowing the child with autism to direct play, (b) imitating/animating (I/A), which entailed attending to and imitating the ADS child's sounds and/or actions in an animated manner, (c) expectant waiting $(E / W)$, which required signaling the child and waiting for a response, and (d) commenting on the child (CC), which emphasized remarking on the child's actions at appropriate times during play [12]. Fathers were instructed to watch videotaped examples and read written directions about integrating these components into play sessions. After mastering the skills, fathers taught mothers the same techniques using the research team's educational approach, resulting in both parents reporting that training had helped them relax during the in-home play sessions.

After the intervention, fathers significantly increased their use of the skills taught and children with autism responded with greatly increased initiating rates as well as frequencies of child non-speech vocalizations. In follow-up interviews, fathers revealed that the training 
had enhanced their paternal role and the quality of overall family functioning [14]. (Details of these studies can be found in published articles $[7,8])$.

\subsection{Including siblings}

Most children with autism have difficulty with inconsistency as evidenced by their strong adherence to routines and rituals. Therefore, it may be difficult, perhaps even impossible, for these children to effectively modify their interactions if family members are not consistent in their approach. Furthermore, incongruence within the family can distress children with autism, who may express their feelings by engaging in a variety of aberrant behaviors such as tantrums, aggression, and other behavioral expressions of frustration. Present research indicates that training non-affected, typically developing siblings, or other individuals who have ongoing contact with the child with autism, could be beneficial. However, little is known about the effects of training siblings to use theoretically-derived strategies such as those Elder and others have implemented with parents. Also unknown is the effect that training typically developing siblings might have on their own behavior, anxiety, and overall quality of life. Although it seems likely that training would positively affect them, training effects on siblings should be addressed in clinical trials.

In a search of the literature related to non-affected, typically developing (TD) siblings of children with autism, few studies are found describing these children, their relationship with their sibling with autism, or what effect having a sibling with autism has on them [15]. Of the extant reports, the findings are inconsistent, making it difficult to characterize the siblings, identify those who are vulnerable to poor adjustment outcomes, or develop interventions that benefit both the sibling and the entire family [15]. It is clear, however, from both the literature and clinical experience, that TD siblings are often faced with unique challenges related to their affected sibling's autism. Also, because children with ADS rarely have physical disfigurement, it is often difficult for those who are not familiar with autism to understand why these children act the way they do; this, in turn, adds to the stress that TD siblings and the family experience [16-18]. Initial findings are promising because they show that when TD siblings care for their ADS siblings early in life, this can positively affect not only the child with autism but also the intervening sibling [19-22]. This clearly indicates that training and evaluating siblings is an area of research with enormous potential and clinical relevance.

Another important consideration that lends support for training siblings is evidence that children with autism learn best in naturalistic environments such as their homes. In a classic work, Baer, Wolf, and Risley (1968) state that skills taught to children in one setting cannot be expected to generalize to other settings without planned, systematic implementation. In fact, these researchers assert that no deliberate behavior changes, particularly related to language acquisition and socialization, should be made that are not reinforced regularly in the child's primary environment; otherwise, trainers must continue to intervene to maintain the behavior change [23]. If one ascribes to this view, clinic-taught interventions cannot be expected to generalize well to home settings unless: (a) the trainer is always present (an impractical and costly idea), (b) family members are taught to assist with generalization, or (c) 
ideally, intervening family members and children with autism are trained in familiar home environments where naturally reinforcing (caregiving) activities are more likely to occur. Also, children with autism are more likely to exhibit abnormal language in unfamiliar settings than at home [11]. For these reasons, it is important that $\mathrm{AD}$ children acquire communication skills in naturalistic settings where they are most likely to encounter interactions and opportunities to utilize communication skills that are similar to the contexts of their daily routines [11].

\section{Using new technologies to train families}

The use of the Internet has grown substantially over the last few years, with an estimated 260 million people now online in North America [24]. In addition, between 2000 and 2010 the proportion of Internet users who are black or Latino has nearly doubled, causing the Internet population to closely resemble the racial composition of the nation as a whole. Health information is one of the most important subjects researched online, and this is reflected in the autism community, where many families are heavily dependent upon Internet services for education, updates on autism treatment, and peer support via parent chat rooms [15].

However, despite the great interest in using the Internet as a resource for learning about autism, online parent training interventions are rare. Recently, considerable evidence has become available demonstrating that web-based feedback systems may increasingly provide feasible and cost-effective patient education [25] because they are available 24 hours a day and can be used repeatedly to enhance learning. Further, with wide-spread internet technology, it may now be possible to provide much needed training to families living remotely and to those representing previously underserved minorities. Clearly, there is an urgent need for clinicians and researchers who have manualized training interventions to adapt them for online use and systematically evaluate their effectiveness through clinical trials.

\section{Managing family stress}

Until the 1980's, the diagnosis of autism was generally not well-known and most children diagnosed with autism were eventually institutionalized. Today, the majority of these children live with their families, who face enormous challenges in planning for and providing a lifetime of care. Families often experience significant financial burden [26], insecurity regarding long-term family planning, and stress related to the child's social impairments and adverse behaviors that often interfere with family functioning [27, 28]. Because additional care giving has been shown to predict parental distress [29] and parents of children with autism may experience greater stress than parents of children with other disabilities, interventions and techniques that can reduce stress are needed [30-34].

Although caring for a child with ASD can adversely affect quality of life for both parents [35], most research related to parental stress has focused on mothers [36] who have reported 
higher stress levels than fathers [31, 37-40]. However, in two other studies comparing mothers' and fathers' stress levels, no differences were found $[32,41]$. The author and team also found that both mothers and fathers scored very high, over the $90^{\text {th }}$ percentile on the Parenting Stress Index pre-intervention with no statistical significance between the mothers' and fathers' scores [14].

In 2008, Davis and Carter provided more insight regarding how mothers and fathers may react to their child's autism. They noted that although mothers had a higher rate of stress and depression, fathers reported more difficulty interacting with the children. In addition, mothers were more involved with everyday activities and thus, more often affected by their child's inability to perform activities of daily living and self-regulate emotions. In contrast, fathers reacted more to overt behaviors such as tantrums, aggression, and/or loud/peculiar vocalizations, which are particularly difficult to manage and can be embarrassing in public settings. Because the core disability associated with autism is social, it can be stressful for parents to deal with a child who may not like to be held, will not respond to their affection, or even make eye contact.

Although only a few studies have explored effects of child intervention on changes in parental stress levels, [14, 28, 42, 43] results are promising. Parent involvement that results in improved child outcomes can empower parents and lower stress in both mothers and fathers. Also, it is important to consider that although fathers may not appear to be as overtly stressed as mothers, there is evidence that they also experience high levels of stress; therefore, interventions should include both mothers and fathers. Finally, although little is known about stress in siblings, it is likely that their stress is also high and that they could benefit from being included in an intervention.

\section{Alternative and complementary therapies: Helping families}

\subsection{Select credible treatment options}

A report from the American Academy of Pediatrics' Council on Children with Disabilities states that treatment goals for children with autism are to: (a) maximize the child's ultimate functional independence and quality of life by minimizing the core features, (b) facilitate development and learning, (c) promote socialization, (d) reduce maladaptive behaviors, and (e) educate and support families [44]. While standard treatments meet these goals and thus, are generally accepted by the autism research community, the variety of novel approaches are less accepted due to their lack of empirical support. As a result, families, who often become desperate to identify a ready cure for the disorder, must be equipped with the knowledge to avoid scams by fully evaluating the potential of new therapeutic approaches.

While it is not possible to cover the multitude of novel and complementary treatments for autism, the author will provide a critical review of some of the most popular strategies, ferreting out those that are empirically validated from those that are unsubstantiated. This section will include a discussion of findings from the author's previously 
published, randomized clinical trial that evaluated the effects of the popular Gluten-Free, Casein-Free diet on individuals with autism, and subsequently recommend directions for future research.

\subsection{Dietary intervention and nutritional supplements in autism}

Increasingly, parents are using alternative treatments, such as dietary interventions or supplements, which they learn about from internet sites or anecdotal reports from other parents. Perhaps the most well-known dietary intervention is the gluten-free casein-free (GFCF) diet that restricts consumption of wheat and dairy products, and which adherents claim can "cure" autism [45]. This diet is so popular that a person can simply type, "GFCF" and "autism" into Google's search engine, and hundreds of sites appear-from the "GlutenFree Trading Co." to "GFCF Diet Success Stories" with endorsements such as the following: "Three weeks ago, I decided to give it [GFCF diet] a try. After three days without dairy, Wow! Suddenly we had an alert child! He was talking more, making sense of the world, and engaging with us! When I phased out wheat and gluten, he got even better. He is happier; his behavior is better; his muscle tone seems to be improving; his eye contact is great; he is speaking like a normal 4 year old!" [46] Although testimonies like these abound on the Internet, there is limited empirical data to support the claims, resulting in a lack of data that health care providers can use to effectively guide parents in making informed decisions.

This dietary intervention, which has clearly "raced ahead of science," poses health risks as well as financial and social drawbacks. While it is less costly than when it was originally introduced, the GFCF diet can still add financial strain to families and may even compromise nutritional health (e.g., insufficient calcium) in children with autism who already have restricted food repertoires. There are also social costs to the children, who cannot eat foods unless they are prepared at home, ruling out the possibility of eating cake, for example, at a birthday party. Similarly, families experience a social cost because they have to prepare dual meals plans that often consist of time-consuming recipes. Thus, unless families have additional financial or social assistance, the GFCF diet can represent a significant burden to a family already struggling with caring for a child with autism.

Despite the continuing popularity of this diet, only five controlled studies have been published since 1999. Three of these studies-Knivsberg [47], Whiteley [48], and Johnson[49]were not double-blind. That is, parents not only knew when their children were receiving the GFCF diet but were also responsible for implementing it. Of these three single blind studies, Knivsberg [47] and Whiteley [48] reported positive findings but have been criticized for their reliance on reports from parents who were not blinded to the dietary intervention. However, it should be noted that Knivsberg [47] conducted a year-long study and some proponents of the GFCF diet suggest that the short duration of other clinical trials may have been responsible for the insignificant findings.

The other two studies were double blind randomized control trials. In the first study, Elder [50] partnered with researchers and staff at the University of Florida's (UF) General Clinical Research Center [now part of UF's Clinical Translational Science Institute Research (CTSI)] to conduct the first double-blind placebo controlled clinical trial of the GFCF diet that was 
published in The Journal of Autism and Developmental Disorders (2006). The researchers evaluated the effects of the GFCF diet on: (a) autistic symptoms as measured by the Childhood Autism Rating Scale (CARS), Ecological Communication Orientation Scale (ECOS), and behavioral frequencies of child social and language behaviors, and (b) urinary peptide levels of gluten and casein. After videotaping the participating 13 children, aged 2 to 16 years, during in-home play sessions for 15 minutes before the diet's introduction, at the end of the first 6-week period, and at the completion of the 12-week protocol, Elder [50] found that group analysis showed no significant differences in any of the outcomes measured or urinary peptide levels of gluten and casein. Even when they were told that the findings were insignificant, parents of nine children kept the children on the diet, indicating that a strong "parent placebo effect" may exist and be responsible for perpetuating the diet's popularity.

In the second study by Hyman [51], children were given the GFCF diet and provided with food challenges; that is, snacks that contained gluten or casein, and which were disguised so that the participants could not identify if the snacks were GFCF. As in the other clinical trials, these investigators used a variety of well-established outcome measures but like Elder [50], found no significant differences or empirical support for the diet. Despite the insignificant findings, the GFCF diet continues to be popular with parents, leading to the author's published recommendations about how to properly advise families regarding diet: first, parents may use the GFCF diet as long as the child does not have a severely restricted food repertoire that could lead to a nutritional deficiency; and second, the family has the social and financial resources to continue the diet [7].

Similar to dietary interventions, nutritional supplements are frequently used by parents to to treat their child's symptoms although there is little sound empirical evidence to support their efficacy in autism. Vitamins C, D, and the B vitamins are generally known to improve immunity, brain function, and overall nervous system activity [52-55]. As a result, they are often included in special autism supplements, which are specifically blended to treat autism-related symptoms. Other supplements that are frequently used include probiotics and digestive enzymes, which may help treat gastrointestinal problems such as acid reflux and constipation, and melatonin, a natural sleep aid that may help reduce nighttime sleep disturbances [56]. Finally, Omega-3 fatty acids, which have been shown to enhance neurological health in the general population, are currently being evaluated in several clinical trials for the treatment of autism [57]. Despite the lack of empirical support for these supplements, most are generally considered harmless if administered in age-appropriate doses.

\subsection{Other approaches}

Because of speculation that oxygen flow to the brain is reduced in children with autism, "hyperbaric treatments," in which individuals with autism are placed in a chamber and exposed to very high oxygen levels, have become popular. In 2009, the US ABC news network broadcast a story, "The Search for a Cure" describing preliminary results from a trial by Dr. Daniel A. Rossignol, himself a father of two children with autism. He and his colleagues evaluated hyperbaric treatment in 56 children with varying degrees of autism ranging in age from 2 to 7 years $[58,59]$. Reports were positive, indicating that 30 percent of the children 
who received the treatment had greatly increased functioning, while only 8 percent in the control group did. In response to this study, Paul Ott, a M.D., autism expert, and author of Autism's False Prophets commented on the questionable efficacy of the treatment and emphasize its potential to financially drain families [60]. For example, a one-hour treatment can cost $\$ 100$ to $\$ 900$, and generally at least 40 are recommended. Despite his warning, however, the $\mathrm{ABC}$ report concluded on an approving note by stating, "While its positive effects remain unclear, hyperbaric chamber therapy does not present the dangers that other therapies do," thus encouraging parents to consider using an unproven and expensive treatment.

Although hyperbaric treatments are one of the latest alternative therapies to become popular in the autism community, parents have long used other unsubstantiated, pharmaceutical approaches. For example, antibiotics have often been prescribed for children with autism who have frequent respiratory or gastrointestinal infections; similarly, antifungal agents, such as nystatin and fluconazole, have been prescribed for children who suffer from an overgrowth of gastrointestinal yeast (e.g. Candida) [61]. In both situations, the medications are prescribed due to the erroneous belief that an infection or "imbalance" is the root cause of the disorder. Other speculative treatments include the intravenous administration of secretin, a gastrointestinal hormone, and immunoglobulin- $G$, an immune system antibody, which are popular because of a few, uncontrolled studies that demonstrated improvement [62]. Despite their questionable efficacy - several gold standard clinical trials have invalidated the use of secretin-alternative treatments are high in demand, generating countless articles on the Internet, and sparking heated discussion on autism message boards [63]. This prevailing popularity, which shows no sign of slowing in the future, is a testament to the struggle many parents experience in caring for a child with autism.

Another popular, yet more controversial treatment is chelation therapy, which removes mercury - an alleged contributor to autism - from the body. When using this therapy, parents typically have a medical doctor treat their child for lead poisoning or they may also buy unregulated chelation agents from Internet sites. Unlike hyperbaric treatment and other interventions that are intended to complement evidence-based treatments, advocates of chelation therapy espouse it as a cure. Yet, to date, there is no proven link between mercury exposure and autism [64]. Joecker, a researcher from the Mayo Clinic warns that not only is chelation therapy's efficacy unproven, but also that it can be associated with serious side effects, including potentially deadly liver and kidney damage and as a result should be assiduously avoided [65].

\section{Interventions with empirical validation}

After the preceding discussion of popular yet largely unproven interventions, the author would be remiss not to provide at least a brief overview of interventions that are empirically sound. Because autism presentations can vary greatly among individuals, each intervention should be customized to meet the needs of the individual child, and be accompanied with the early speech/language and occupational therapy that are typically indicated. 
In addition to the special education and pharmacological interventions that may be necessary, traditional treatment approaches include providing a child with speech, behavioral, occupational, and physical therapy as indicated in some cases. Although public schools in the United States are required by law to provide such services, the frequency, type, and quality of these services vary considerably. Consequently, parents need to actively participate in meetings where Individualized Educational Plans (IEP), or the equivalent, are developed to specifically address a child's behavioral or learning needs. Furthermore, parents should maintain close contact with educational personnel to help evaluate their children's progress and determine the future direction of treatment.

If the future direction includes medications, parents must carefully analyze the costs and benefits by questioning their health care provider regarding possible improvements and side effects. Although medications do not cure autism, sometimes they can alleviate behavioral symptoms that distress the child and interfere with therapeutic efforts such as intensive education and socialization [66-68]. These behavioral symptoms include hyperactivity, self-injury, aggression, compulsions (repetitive behaviors), mood lability, anxiety, and sleep disturbances [69].

In addition to medication, parents may consider using a behavioral intervention, which researchers have refined over time and developed into a highly successful treatment approach. In particular, two comprehensive behavioral early interventions-Lovaas' Model based on Applied Behavior Analysis (ABA) and the Early Start Denver Model-have been shown to be helpful in improving symptoms related to autism [70, 71]. Mounting evidence also supports the use of other commonly used therapies such as Floortime, Pivotal Response Therapy and Verbal Behavior Therapy [72-74]. For up-to-date information regarding behavioral interventions, visit the website for Autism Speaks, an internationally recognized organization within the autism community, at http://www.autismspeaks.org/what-autism/ treatment. By visiting this site, parents will learn about the many valid treatments available that are safe, effective, and capable of producing a better quality of life for children with autism and their families.

\section{Relationship of family training intervention research to NIH's priorities and NIMH's sponsored work-group recommendations}

Finding ways to improve quality of life for ADS children and their families is one of the top priorities of NIH and congressionally mandated research as noted in the Combating Autism Act of 2006 [75, 76]. A report from a NIMH-supported work group of well-known autism authorities addresses what has traditionally been problematic in the field of autism [77]; namely, that fragmented and isolated individual study approaches have not been effective in systematically advancing the most effective behavioral interventions [78]. In response, Smith et al. proposed a developmental process for designing and conducting studies on psychosocial interventions in autism, which provides a way to systematically validate and disseminate interventions; the process includes the following steps: (a) conduct initial efficacy 
studies that may utilize intrasubject methodology to provide in-depth information about individual responses over time, (b) manualize the intervention and pilot-test it with larger numbers of participants, (c) conduct clinical trials to test the efficacy under controlled conditions, and (d) conduct effectiveness studies to evaluate outcomes in community settings.

The author and team have been following a developmental sequence that is consistent with that of the NIMH work group and especially part of the final step-evaluating outcomes in community settings. Delivering the training to all family members including siblings, and providing training interventions using state of the art internet technology would greatly expand our ability to deliver comprehensive family-centered training in the community, and produce significant gains that would improve the quality of life for individuals with autism and their families.

\section{Author details}

Jennifer Elder

Address all correspondence to: elderjh@ufl.edu

University of Florida College of Nursing, Gainesville, Florida, USA

\section{References}

[1] Ross EK-R, Kessler D. Finding the meaning of grief through the five stages of loss. New York: Scribner; 2007 2007. 256 p.

[2] Gross D, Fogg L, Tucker S. The efficacy of parent training for promoting positive parent-toddler relationships. Research in Nursing and Health. 1995;18(6):486-99.

[3] Webster-Stratton C, Herbert M. What really happens in parent-training? Behavior Modification. 1993;17:407-56.

[4] Bettelheim B. Feral Children and Autistic Children. american Journal of Sociology. 1959;64(5):455-7.

[5] Eisenberg L. THE FATHERS OF AUTISTIC CHILDREN*. American Journal of Orthopsychiatry. 1957;27(4):715-24.

[6] Kanner L. Autistic disturbances of affective contact. Nervous Child. 1943;2:217-50.

[7] Elder J, Donaldson S, Kairella J, Valcante G, Bendixen R, Ferdig RE, et al. In-home training for fathers of children with autism: A follow up study and evaluation of four individual training components. Journal of Child and Family Studies. 2010;20(3): 263-71. 
[8] Elder J, Valcante G, Yarandi H, White D, Elder TH. Evaluating in-home training for fathers of children with autism using single-subject experiementation and group analysis methods. Nursing Research. 2005;54(1):22-32.

[9] Rogers SJ. Evidence-based interventions for language development in young children with autism. Social \& communication development in autism spectrum disorders. T. S. Charman, W. ed. New York: Guildford Press; 2006. p. 143-79.

[10] Mundy P, Sigman M, Kasari C. A longitudinal study of joint attention and language development in autistic children. Journal of Autism and Developmental Disorders. 1990;20(1):115-28.

[11] Wetherby AM. Understanding and measuring social communication in children with autism spectrum disorders. New York: The Guildford Press; 2006. pp. 3-34.

[12] Elder J. In-home communication intervention training for parents of multiply handicapped children. Scholarly Inquiry for Nursing Practice. 1995;9:71-92.

[13] Flippin M, Crais ER. The need for more effective father involvement in early autism intervention. Journal of early intervention. 2011;33(1):24-50.

[14] Bendixen R, Elder J, Donaldson S, Kairella J, Valcante G, Ferdig RE. Perceived stress and family dynamics in fathers and mothers of children with autism following an inhome intervention. American Journal of Occupational Therapy. 2011.

[15] Smith L, Elder J. Siblings and family environments of persons with autism spectrum disorder: a review of the literature. Journal of child and adolescent psychiatric nursing: official publication of the Association of Child and Adolescent Psychiatric Nurses, Inc. 2010;23(3):189-95. Epub 2010/08/28.

[16] Lobato DJ, Kao BT. Integrated Sibling-Parent Group Intervention to Improve Sibling Knowledge and Adjustment to Chronic Illness and Disability. Journal of Pediatric Psychology. 2002;27(8):711-6.

[17] Fisman S, Wolf L, Ellison D, Freeman T. A longitudinal study of siblings of children with chronic disabilities. Canadian journal of psychiatry Revue canadienne de psychiatrie. 2000;45(4):369-75. Epub 2000/05/17.

[18] Hastings RP, Kovshoff P, Ward H, J. N, Espinosa F, Brown T, et al. Systems Analysis of Stress and Positive Perceptions in Mothers and Fathers of Pre-School Children with Autism. Journal of Autism and Developmental Disorders. 2005;35(5):635-44.

[19] Blacher J, Begum G. The Social and the Socializing Sibling: Positive Impact on Children with Autism. Exceptional Parent. 2009;39(5):56-7.

[20] Hodapp RM, Urbano RC. Adult siblings of individuals with Down syndrome versus with autism: findings from a large-scale US survey. Journal of Intellectual Disability Research. 2007;51(12):1018-29.

[21] Maynard AE, Martini MI. Learining in cultural context: Family, peers, and school. New York: Kluwer Academic/Plenum Publishers; 2005. 
[22] Orsmond GI, Kuo HY, Seltzer MM. Father involvement with adolescents and adults with autism. 13th World Congress of the International Association for the Scientific Study of Intellectual Disabilities; Cape Town, South Africa2008.

[23] Baer D, Wolf M, Risley T. Some current dimensions of applied behavior analysis. Journal of Applied Behavior Analysis. 1968;1:92-7.

[24] Internet World Stats, (2010).

[25] Gentles JS, Lokker C, McKibbon AK. Health information technology to facilitate communication involving health care providers, caregivers, and pediatric patients: a scoping review. J Med Internet Res. 2010;12(2):e22.

[26] Fletcher PC, Markoulakis R, Bryden PJ. The costs of caring for a child with an autism spectrum disorder. Issues in comprehensive pediatric nursing. 2012;35(1):45-69. Epub 2012/01/19.

[27] NINDS, (2009).

[28] McConachie H, Diggle T. Parent implemented early intervention for young children with autism spectrum disorder: a systematic review. Journal of evaluation in clinical practice. 2007;13(1):120-9. Epub 2007/02/09.

[29] Lecavalier L, Leone S, Wiltz J. The impact of behaviour problems on caregiver stress in young people with autism spectrum disorders. Journal of Intellectual Disability Research. 2006;50(Pt 3):172-83. Epub 2006/01/25.

[30] Davis NO, Carter AS. Parenting stress in mothers and fathers of toddlers with autism spectrum disorders: associations with child characteristics. Journal of Autism and Developmental Disorders. 2008;38(7):1278-91. Epub 2008/02/02.

[31] Herring S, Gray K, Taffe J, Tonge B, Sweeney D, Einfeld S. Behaviour and emotional problems in toddlers with pervasive developmental disorders and developmental delay: associations with parental mental health and family functioning. Journal of Intellectual Disability Research. 2006;50(12):874-82.

[32] Hastings RP, Kovshoff H, Brown T, Ward NJ, Espinosa FD, Remington B. Coping strategies in mothers and fathers of preschool and school-age children with autism. Autism. 2005;9(4):377-91.

[33] Jones J, Passey J. Family Adaptation, Coping and Resources: Parents of Children with Developmental Disabilities and Behaviour Problems. Journal of Developmental Disabilities. 2005;11(1).

[34] Rao PA, Beidel DC. The Impact of Children with High-Functioning Autism on Parental Stress, Sibling Adjustment, and Family Functioning. Behavior Modification. 2009;33(4):437-51.

[35] Giarelli E, Souders M, Pinto-Martin J, Bloch J, Levy SE. Intervention pilot for parents of children with autistic spectrum disorder. Pediatric nursing. 2005;31(5):389-99. Epub 2005/11/22. 
[36] Phetrasuwan S, Miles MS, Mesibov GB, Robinson C. Defining Autism Spectrum Disorders. Journal for Specialists in Pediatric Nursing. 2009;14(3):206-9.

[37] Sharpley C, Bitsika V, Efremidis B. Influence of gender, parental health, and perceived expertise of assistance upon stress, anxiety, and depression among parents of children with autism. Journal of Intellectual \& Developmental Disability. 1997;22(1): 19-28.

[38] Beckman P. Comparison of mothers' and fathers' perceptions of the effect of young children with and without disabilities. American Journal on Mental Retardation. 1991;95:585-95.

[39] Bristol M, Gallagher J, Schopler E. Mothers and Fathers of Young Developmentally Disabled and Nondisabled Boys: Adaptation and Spousal Support. Developmental Psychology. 1988;24:441-51.

[40] Wolf L, Goldberg B. Autistic children grow up: An eight to twenty-four year followup study. Canadian Journal of Psychiatry. 1986(31):550-6.

[41] Trute B. Gender Differences in the Psychological Adjustment of Parents of Young, Developmentally Disabled Children. Journal of Child Psychology and Psychiatry. 1995;36(7):1225-42.

[42] Hastings RP, Johnson E. Stress in UK families conducting intensive home-based behavioral intervention for their young child with autism. Journal of Autism and Developmental Disorders. 2001;31(3):327-36. Epub 2001/08/24.

[43] Wong VC, Kwan QK. Randomized controlled trial for early intervention for autism: a pilot study of the Autism 1-2-3 Project. Journal of Autism and Developmental Disorders. 2010;40(6):677-88. Epub 2009/12/19.

[44] Myers SM, Johnson CP. Management of children with autism spectrum disorders. Pediatrics. 2007;120(5):1162-82. Epub 2007/10/31.

[45] Christison GW, Ivany K. Elimination diets in autism spectrum disorders: any wheat amidst the chaff? Journal of developmental and behavioral pediatrics : JDBP. 2006;27(2 Suppl):S162-71. Epub 2006/05/11.

[46] Group GDS. Gluten Free Casein Free Diet Success Stories. Gluten Free Casein Free Diet website: The GFCF Diet Support Group; 2012 [cited 2012 August 14]; Available from: http://www.gfcfdiet.com/successstories.htm.

[47] Knivsberg A, Reichelt K, Hoien T, Nodland M. A randomised, controlled study of dietary intervention in autistic syndromes. Nutrition Neuroscience. 2002;5(4):251-61.

[48] Whiteley P, Rodgers J, Savery D, Shattock P. A Gluten-Free Diet as an Intervention for Autism and Associated Spectrum Disorders: Preliminary Findings. Autism. 1999;3(1):45-65. 
[49] Johnson C, Handen B, Zimmer M, Sacco K, Turner K. Effects of Gluten Free / Casein Free Diet in Young Children with Autism: A Pilot Study. Journal of Developmental and Physical Disabilities. 2011;23(3):213-25.

[50] Elder J, Shankar M, Shuster J, Theriaque D, Burns S, Sherrill L. The Gluten-Free, Casein-Free Diet In Autism: Results of A Preliminary Double Blind Clinical Trial. Journal of Autism and Developmental Disorders. 2006;36(3):413-20.

[51] Hyman JM, Zilli EA, Paley AM, Hasselmo ME. Working memory performance correlates with prefrontal-hippocampal theta interactions but not with prefrontal neuron firing rates. Frontiers in Integrative Neuroscience. 2010;4.

[52] Martin A, Cherubini A, Andres-Lacueva C, Paniagua M, Joseph J. Effects of fruits and vegetables on levels of vitamins $E$ and $C$ in the brain and their association with cognitive performance. The journal of nutrition, health \& aging. 2002;6(6):392-404.

[53] Garcion E, Wion-Barbot N, Montero-Menei CN, Berger F, Wion D. New clues about vitamin D functions in the nervous system. Trends in Endocrinology \&amp; Metabolism. 2002;13(3):100-5.

[54] Mora JR, Iwata M, von Andrian UH. Vitamin effects on the immune system: vitamins A and D take centre stage. Nat Rev Immunol. 2008;8(9):685-98.

[55] Reynolds E. Vitamin B12, folic acid, and the nervous system. The Lancet Neurology. 2006;5(11):949-60.

[56] Golnik AEIM. Complementary Alternative Medicine for Children with Autism: A Physician Survey. Journal of Autism and Developmental Disorders. 2009;39(7): 996-1005.

[57] Amminger GP, Berger GE, Schäfer MR, Klier C, Friedrich MH, Feucht M. Omega-3 Fatty Acids Supplementation in Children with Autism: A Double-blind Randomized, Placebo-controlled Pilot Study. Biological Psychiatry. 2007;61(4):551-3.

[58] Brownstein J. Hyperbaric Autism Treatment Shows Possible Promise2009. Available from: http://abcnews.go.com/Health/AutismNews/story?id=7070353\&page=1.

[59] Rossignol DA, Bradstreet JJ, Van Dyke K, Schneider C, Freedenfeld SH, O'Hara N, et al. Hyperbaric oxygen treatment in autism spectrum disorders. Medical gas research. 2012;2(1):16. Epub 2012/06/19.

[60] Offit P. Autism's False Prophets: Bad Science, Risky Medicine, and the Search for a Cure. New York, NY: Columbia University Press; 2008.

[61] Shute N. Alternative biomedical treatments for Autism: How good is the evidence? 2010:[3 p.]. Available from: http://www.scientificamerican.com/article.cfm?id=alternative-biomedical-treatments\&page=2\&WT.mc_id=SA_emailfriend.

[62] Unproven Treatments for Autism2010 August 13, 2012. Available from: http:// www.webmd.com/a-to-z-guides/unproven-treatments-for-autism-topic-overview. 
[63] Krishnaswami S, McPheeters ML, Veenstra-VanderWeele J. A Systematic Review of Secretin for Children With Autism Spectrum Disorders. Pediatrics. 2011;127(5):e1322e5.

[64] Weber W, Newmark S. Complementary and alternative medical therapies for attention-deficit/hyperactivity disorder and autism. Pediatric clinics of North America. 2007;54(6):983-1006; xii. Epub 2007/12/07.

[65] Hoecker JL. Is chelation Therapy an effective autism treatment?2010. Available from: http://www.mayoclinic.com/health/autism-treatment/AN01488.

[66] Malone RP, Gratz SS, Mary Anne D, Hyman SB. Advances in Drug Treatments for Children and Adolescents with Autism and Other Pervasive Developmental Disorders. CNS Drugs. 2005;19(11):923-34.

[67] Rapin I. The Autistic-Spectrum Disorders. New England Journal of Medicine. 2002;347(5):302-3.

[68] West L, Waldrop J. Risperidone use in the treatment of behavioral symptoms in children with autism. Pediatric nursing. 2006;32(6):545-9. Epub 2007/01/30.

[69] King BH, Bostic JQ. An Update on Pharmacologic Treatments for Autism Spectrum Disorders. Child and Adolescent Psychiatric Clinics of North America. 2006;15(1): 161-75.

[70] Dawson G, Rogers S, Munson J, Smith M, Winter J, Greenson J, et al. Randomized, Controlled Trial of an Intervention for Toddlers With Autism: The Early Start Denver Model. Pediatrics. 2010;125(1):e17-e23.

[71] Anderson SR, Romanczyk RG. Early Intervention for Young Children with Autism: Continuum-Based Behavioral Models. Research and Practice for Persons with Severe Disabilities. 1999;24(3):162-73.

[72] Wieder S, Greenspan SI. Climbing the symbolic ladder in the DIR model through floor time/interactive play. Autism. 2003;7(4):425-35. Epub 2003/12/18.

[73] Simpson RL. Evidence-Based Practices and Students With Autism Spectrum Disorders. Focus on Autism and Other Developmental Disabilities. 2005;20(3):140-9.

[74] Sundberg ML, Partington JW. Teaching language to children with autism or other developmental disabilities. Danville, CA: Behavior Analysits, Inc.; 1998.

[75] Combating Autism Reauthorization Act, Stat. S.1094 (2011).

[76] Committee IAC. Summary of Advances in Autism Spectrum Disorder Research: Calendar Year 20102010. Available from: http://iacc.hhs.gov/summary-advances/2010/.

[77] Smith T, Scahill L, Dawson G, Guthrie D, Lord C, Odom S, et al. Designing research studies on psychosocial interventions in autism. Journal of Autism and Developmental Disorders. 2007;37:354-66. 
[78] Committee IAC. 2011 IACC Strategic Plan for Autism Spectrum Disorder Research. Department of Health and Human Services Interagency Autism Coordinating Committee website2011 [cited 2012 May 15]. 
Chapter 23

\title{
Collaboration Between Parents of Children with Autism Spectrum Disorders and Mental Health Professionals
}

\author{
Efrosini Kalyva \\ Additional information is available at the end of the chapter \\ http://dx.doi.org/10.5772/53966
}

\section{Introduction}

When mental health professionals and parents of children with autism spectrum disorders start working together, they bring into this relationship their own personal needs, concerns, priorities and responsibilities, which must be taken into consideration in order to create a mutually satisfactory and functional partnership. A partner is a person that one works with in order to achieve a common goal through shared decision-making and risk-taking. Some partnerships last for a short period of time and include casual encounters, while others last long and evolve through numerous official and unofficial encounters [1]. For a partnership model to work, all involved parties must understand how they feel about each other [2] and to recognize that family operates as a system. When parents and mental health professionals disagree, it is essential to resolve any conflict timely in order to avoid serious confrontations or even legal litigations [3].

Minuchin [4] was the first who introduced the theory of family systems and stated that individuals affect the context where they live and are in turn affected by it through a series of repeated interactions. So, whatever affects one family member affects the whole family in direct or indirect ways. Elman [5] describes families as the mobile that hangs over a baby's crib, with the pressure exerted on one end causing movement throughout. The relationships between family subsystems (spouses, parents and children, and siblings) determine the balance of the entire family [6] and interventions at any subsystem must aim to preserve this balance. For example, an intervention aiming at fostering the mother-child bond could affect the mother's relationship with her husband or her other children if the necessary actions are not taken. Family subsystems describe the interac- 
tions within the family context, whereas cohesion and adaptability describe the way in which family members interact.

Cohesion is inherent to the notions of engagement and disengagement. Some families with high levels of engagement do not have clear boundaries between the subsystems, are overly engaged in the therapeutic process and overprotective [4] and as a result do not allow the individual with autism spectrum disorders to develop a sense of autonomy. On the other hand, families with extremely low levels of engagement adopt rigid boundaries and do not interact with the child sufficiently. So, the child with autism spectrum disorders is left free, but without experiencing the necessary love and support. The degree to which a family adjusts to the diagnosis of autism spectrum disorders depends to a large extent on the pre-existing family cohesion and stability, while the disruption of family cohesion due to the birth of a child with autism spectrum disorders can lead to increased stress [7]. In order to deal with stress, families employ either internal coping strategies that include passive evaluation or active reframing or external coping strategies through social and spiritual support [8].

Adaptability refers to the family's ability to change its functioning when a stressful event occurs [9]. Family adaptability depends on the severity of autism spectrum disorders, as well as on the accumulation of the demands made on parents [10]. Rigid families do not change to face the stress, while chaotic families become unstable and face changes inconsistently. The families that do not manage to adapt successfully are at risk of becoming isolated and dysfunctional [11]. According to family systems theory the disruption of communication among family members is a sign of dysfunction of the whole system and not of a specific individual. Therefore, mental health professionals should aim at changing interaction patterns and not just individuals, without incriminating anyone. Many family members tend to blame the individual with autism spectrum disorders for the difficulties that they experience, but with the appropriate guidance they perceive that miscommunication is often to blame [12].

Most studies conducted with families of individuals with disabilities are based on the assumption that families are homogeneous [13], but there are many features that differentiate families between them. For example, unemployed parents of a child with autism spectrum disorders have access to different resources than high-income parents [1]. Moreover, single mothers of children with autism spectrum disorders experience heightened stress, since they lack the practical, financial and moral support of their partner [14]. Cultural and contextual factors can also affect the ways that families cope with disabilities. First generation Americans with Chinese origin are afraid that their children with autism spectrum disorders will be stigmatized if they use sign language or other alternative forms of communication [15]. Parental reactions to their child's disorders must be viewed and interpreted within the social, historical, and ecosystemic context of every family [16]. Parents initially experience a stage of shock [17], which is followed by a range of reactions that could eventually lead through consecutive reorganizations to adjustment to reality [18]. However, many parents regress to previous stages when they realize that their children with autism spectrum disorders face difficulties that will not disappear and that they need constant care. In order to support parents of children with autism spectrum disorders, mental health professionals 
must know the characteristics of the disorder and set realistic goals both for children with autism spectrum disorders and their families [19].

Many researchers have established that parents of children with disabilities and mental health professionals must cooperate in order to design and implement an effective therapeutic process [20-23]. Therefore, parental involvement in the planning of proper therapeutic intervention for children with autism spectrum disorders was the primary target of many programs since the beginning of the 1980s [24]. Parents have been treated as partners, consultants, advocates, and supporters by the mental health professionals who offer these services. Parents often seek to work together with mental health professionals as they try to help their children overcome the difficulties that they face [25-26]. So, empowering the cooperation between parents and mental health professionals has been a cornerstone for many contemporary care systems for individuals with disabilities [27]. In order to achieve this empowerment, it is important to increase parental autonomy and engagement in decisionmaking regarding the therapeutic goals [28].

It is expected that the cooperation between parents and mental health professionals will result to better services for the children with disabilities, since the knowledge and the experience that each person brings into this relationship are unique [29]. The problem is that many mental health professionals cannot treat parents as equal partners in this process [30]. Through their training, mental health professionals develop an area of expertise that places them almost automatically at the role of the expert. Sharing responsibility with parents, without having a clear hierarchy, creates a new structure that is opposite to the traditional nature of the relationship between parents and mental health professionals. However, the position and the authority of the mental health professionals have been challenged and transformed according to contemporary political and theoretical models, as can be seen below:

\subsection{Professional as experts}

This is the traditional cooperation model that is prominent in doctor-patient relationships, where the professionals use their position and their knowledge to decide what will happen. Parental participation is of secondary importance and compliance with the professionals' suggestions is self-evident. Parents are informed about the decisions that were taken without being allowed to express their opinions, feelings, needs, or wishes. Children are treated as the passive recipients of a therapy, while parents are thought not to have the time, the disposition, the skill or the knowledge to help their children. This relationship is very bureaucratic and rigid, because it disadvantages parents by making them dependent on the professional [31]. Moreover, when mental health professionals do not engage parents actively in their child's treatment there may be a disagreement between the therapeutic goals they set [31-32]. The exclusion of parents from the therapeutic process has been highly criticized since the beginning of the 1970s, since the relationship between parents and mental health professionals becomes impersonal and the sense of trust is lost [33]. Therefore, parents started gradually being involved in the therapeutic process [34] and a lot of emphasis was placed on this involvement [35]. It 
should be pointed out, that even though this kind of relationship is outdated, there are still mental health professionals who impose themselves on parents.

\subsection{The transitional relationship}

Mental health professionals started treating parents as co-therapists and realizing that the house can be used as a learning setting. They shared and transfered their skills to parents to help them become more able, more confident, and more skilled. Parents participate as «co-teachers» or «co-trainers» or «co-therapists» [18]. Mental health professionals have to adapt their methods in order to incorporate and to support their cooperation with parents. So, they have to discover ways to communicate with parents and to engage them in the therapeutic process. Parents who cooperate with mental health professionals become more able, more knowledgeable and more assertive [36]. The main drawback of this model is the underlying assumption that all parents have the motive (and are able) to use this professional knowledge to help their child. It ignores the differences that exist in parenting styles, family relationships, family resources, family values and cultural contexts. For example, some parents may not feel comfortable acting as «teachers» of their children [37]. Many interventions have focused solely on mothers and have left out fathers creating disruption to the family system. This relationship is not truly cooperative, since mental health professionals make the basic decisions and are still in control [31].

\subsection{Parent as consumers}

The consumer model [31] stated that parents should have new rights and be given part of the control. Parents are viewed as consumers, who have the right to choose the appropriate services and interventions for their children. It is the first time that mental health professionals recognize that parents possess specialized knowledge that they lack. Parents use their knowledge to decide what they want and what they need for their child. Mental health professionals guide parents to make more effective and appropriate decisions. Parents may choose not to attend some of the suggested services that they do not consider suitable. Decision-making is reached after mutual exchange of ideas and with mutual respect. The objective is to reach a mutual agreement on the treatment that the child will follow. This model can be quite effective in various intervention settings [38]. The cooperation is very important, since parents have a greater sense of control. The services that adopt this model must be very flexible to provide individualized support [39]. This model presupposes that parents are capable to express and to assert their needs and the needs of their children. However, some parents cannot prioritize their needs or assume the responsibility of making important decisions. The concept of parents as consumers who share resources may not be very realistic in a restrictive financial context that offers minimal services. In this case the consumers do not necessarily buy the best services and many parents cannot afford the increased financial demands of the most effective therapies. This model is similar to counseling that is offered to parents to help them resolve some personal issues. 


\subsection{The empowerment model}

This model has added a social and systemic dimension to the consumer model [39], since parents have the right to choose the services that they will offer to their child and mental health professionals realize that family is a system and a social network. Every family comprises of interconnected social relations within the context of the family itself as well as within the wider social groups (extended family, friends, associates, cultural groups). The system and the network affect the ways in which the family members view the individual with disabilities. Given that each family has different advantages, parents have a unique adjustment method and mental health professionals need to understand and respect that. Mental health professionals should also help parents realize that they can monitor their child's progress and interfere when they identify a problem.

\subsection{The negotiation model}

Partners use negotiation to reach common decisions and to resolve any disagreements that may arise. Negotiation can lead either to a common decision or to disagreement. Disagreements can come up for various reasons, such as the priorities that are set by each interested party [40]. The negotiation model states that the ways that parents and mental health professionals view a situation or a problem, the options that they have to resolve it, and the extent to which they can face it, are affected by their roles - as well as the social, financial, and structural frames where they function. Therefore, according to this model, cooperation may be dysfunctional under the three following conditions:

1. Either parents or mental health professionals do not have the intention or the skill to work with each other and to enter a cooperative relationship. Personal experiences may decrease the likelihood to cooperate [1].

2. Either parents or mental health professionals make all decisions and are not willing to share responsibility [41].

3. If the interests, the views, the priorities and the values of parents and mental health professionals are contradictory, then their relationship may become competitive - even if they apply various conflict resolution strategies.

Some organizations are eager to engage parents in the therapeutic process not because they recognize parental rights but because of staff shortage or scarce financial resources [36]. Parents should be involved in decision-making regarding their children because mental health professionals need their cooperation to do their job properly. Parents will also have a chance to establish and generalize at home the skills that their children have mastered [38]. In order for parents - and especially mothers - to function as therapists, they must devote a lot of time to meeting with mental health professionals to receive the proper training [42]. Parents of children with disabilities need guidance and support to be effective in their role [43-45]; otherwise, they will loose their self-esteem and become ineffective [46].

For the negotiation model to work, it has to operate at five different levels: personal, interpersonal, organizational, institutional, and ideological [40]. The sense of cooperation 
encourages the productive combination of knowledge, skills, and sensitivities from both parents and mental health professionals. The six elements that characterize a cooperative relationship and differentiate it from other types of relationships are [47]: a) cooperation is optional; b) cooperation demands equity among the participants; c) cooperation is based on mutual goals; d) cooperation depends on shared responsibility and decisionmaking; e) people who cooperate share their resources; and f) people who cooperate are equally responsible for the outcome.

Within this model, mental health professionals should have a clearly defined relationship with parents that has four predetermined goals [48]: a) include parents in decision-making about their child; b) train parents to participate in decision-making about their child; c) help parents therapeutically to deal with some issues that stop them from functioning more effectively; and d) render parents capable to work effectively and meaningfully with their child through empowerment.

The negotiation model has many functioning aspects that facilitate the development of a cooperative relationship between parents and mental health professionals, since it is developmental and parents are not viewed as static agents. They are encouraged to develop and improve their skills to become more effective and to work on their personal issues. In order to meet with the demands of this new role, mental health professionals are often called to take on multiple roles and to become more flexible. They may need to act as mediators between the parents and other agents, as well as to fight for the rights of the parents and their children with disabilities - especially in times of financial and moral crisis.

\subsection{How do parents feel about mental health professionals?}

Individuals with autism spectrum disorders depend on their families for daily care and support that are essential for the successful implementation of any therapeutic intervention [49]. Therefore, it has been acknowledged that the needs of all the family members should be taken into consideration when designing an intervention [50]. Many highly recommended treatments for autism spectrum disorders [see 49, for more information] - such as Applied Behavioral Analysis [51], TEACCH [52] and Portage [53] - stress the importance of active parental participation in the therapeutic process, which results from the proper cooperation with mental health professionals. However, many parents claim that their participation in their children's therapy is minimal and restrained to six-monthly briefing meetings, while they are not informed that they could be more actively involved in the treatment process [54]. Parents must be treated as partners during the planning, implementation, and evaluation of the therapeutic approach and not just as observers or clients [55].

Many parents complain because they have to wait a long time to diagnose their children with autism spectrum disorders and they need to visit up to four different mental health professionals [54]. In a small scale study where parents of 25 children with autism spectrum disorders were interviewed, it was found that these parents have to take their children to different therapeutic settings, which is extremely time consuming. They work together with an average of six mental health professionals for a total of approximately 37 hours per week [56]. Since parents are often exposed to many diverse opinions and suggestions expressed 
by mental health professionals, they end up being confused and they need guidance to make the right choices and decisions [1]. Therefore, mental health professionals who work therapeutically with the parents of children with autism spectrum disorders should assume also a counseling role [57].

Parents need to be extremely persistent in order to ensure the services and the provisions that are necessary for their children with autism spectrum disorders [58-59]. Parents started questioning the power of mental health professionals when they formed groups to fight for their rights. An extreme example of disappointment with mental health professionals was the creation of a centre of counseling and support for the parents of children with special needs that was created by parents and to which mental health professionals had no access [2]. The parents who founded this centre stressed that it provided them with the opportunity to talk and to share their experiences - giving them, thus, the strength to deal with their daily problems.

Despite the fact that parents were overall satisfied with the mental health professionals they had worked with in the past, they generally felt that they had to fight in order to access the services that their children needed. They reported that many mental health professionals failed to communicate with each other and with the parents and this created a heightened sense of dissatisfaction. This was due to the fact that most children were monitored simultaneously by several mental health professionals who seemed to work in isolation without sharing information and common therapeutic goals. Furthermore, many parents supported that the services they received did not suffice to address their children's multiple and complex needs [3]. Moreover, some parents claim that they are tired of being accused for the problems that their children face [60] and that constant criticism does not help them become better and more effective parents. Paradoxically, although some mental health professionals view mothers as guilty, they involve them at the same time in their children's therapy [61].

Crawford and Simonoff [62] studied the attitudes of parents of children attending schools for emotional and behavioral disorders. Many parents believed that they felt stigmatized and isolated because of the problems that their children were facing. Although the stigma accompanying mental health problems or other disorders, such as autism spectrum disorders is well recognized, there is limited research on the topic. Parents feel lonely and without any support, but they hesitate to share their concerns with others, because they are afraid that they will be further stigmatized and held responsible for their children's problems. So, it is not surprising that parents were excited to meet with other parents who face similar problems and can offer them valuable support.

Parents of children with special needs are often dissatisfied with the way that mental health professionals behave and with the attitudes that they express. However, most relevant research has not studied the actual interaction between parents and mental health professionals, but they are based on parental anecdotal evidence that is usually negative [63-64]. If the behaviors that parents report are accurate, then they constitute a breach of the professional code of ethics [65] and should be seriously taken into consideration. On the other hand, many parents appreciate that mental health professionals try to understand the family dynamics and to address the individual needs of every family member [66] and there are also 
quite a few parents who mention that mental health professionals have done their best to help them and their children with autism spectrum disorders [54].

The mental health professionals who interact with children with autism spectrum disorders and their families come from different educational and theoretical backgrounds, as well as from different disciplines: specialized professionals (such as psychologists, speech therapists, and social workers), doctors, teachers or students. Despite the fact that the contribution of mental health professionals to the planning and effectiveness of the treatment has been widely acknowledged, more research is needed on identifying how they deal with practical problems that arise during the course of their interactions with parents of children with autism spectrum disorders. The role of mental health professionals and therapists has been approached primarily by the psychoanalytic perspective and most studies have focused only on the role of the teacher of children with autism spectrum disorders.

\subsection{How do mental health professionals feel about parents?}

The beliefs and the assumptions that mental health professionals hold regarding parental contribution to the appearance and maintenance of their children's problematic behaviors and disorders greatly affect their choice of offered therapies and the intervention strategies that they use when interacting with the specific families [67]. Even the term «professional» has been controversial, since some refer to the traditional definition of professional (e.g., doctors, lawyers, architects, university professors), while others use this term to refer to most working people (e.g., nurses, social workers, and teachers) [68]. The term «mental health professionals» is now used to include all the educated people who have received the appropriate training to work with individuals with disabilities. It is used to make the distinction between trained staff and volunteers, carers, or untrained helping staff who work with individuals with disabilities.

There are different sources of «socially acceptable» power for mental health professionals [69]: physical power, power to provide resources, power of profession, power of specialization and personal power. For many years now, the role of mental health professionals is predetermined to provide them with the power and the right to use their knowledge and their experience as they wish. They have resources at their disposal that they can share with children with disabilities and their families, as well as the specialized knowledge that they have acquired through their training. Mental health professionals are usually considered experts, since they are knowledgeable about an area or a topic. In case that some parents disagree or refuse to cooperate with mental health professionals, the latter have the right to stop providing their services. Mental health professionals can have considerable power and so many parents treat them with respect.

The attitudes and perceptions of mental health professionals regarding their relation with the parents of children with disabilities have not been adequately researched [70]. Smets [71] explored staff attitudes regarding parental involvement in a service for individuals with intellectual disabilities and found that staff believed that parents were either unaware or indifferent to their children's problems. Staff believed that parents were limited to the role of the external observer and they were happy to defer the responsibility of caring for their child to 
another person. However, the researcher stressed that the perception of the staff did not correspond to reality and to the actual needs of the families of the service users.

Some mental health professionals recognize the importance of working together with the parents, but they claim that they are not adequately trained or prepared to do so and they receive no support from their services [72-74]. To address this issue, it is important to better understand the skills and the behaviors that mental health professionals need in order to learn how to cooperate with parents [75-76]. Interpersonal skills, such as sensitivity towards the parents, clarity and respect are usually highly appreciated by parents who work together with mental health professionals in early intervention settings [77].

\subsection{Cooperation between parents and mental health professionals}

Cooperation is a term that was recently introduced to literature looking at the relationship between parents and mental health professionals, but is quite difficult to accomplish in practice given that it means different things to different people. Cooperation can be viewed as basic principle or theoretical viewpoint that is based on fundamental power exchange [78]. However, there are many organizational, geographical or financial obstacles in the cooperation between different groups of mental health professionals or between mental health professionals and service users - that is, parents of children with disabilities [79-81]. The potential cooperation between mental health professionals and parents is based mainly on the anticipation that there will be an increase in the number and quality of offered services. However, many mental health professionals feel threatened when they have to choose who will have access to each service, especially when the choices are limited [82].

The cooperation between parents and mental health professionals is not just desirable but also mandatory, since it is enforced by law in many countries [20, 83]. It has been widely accepted that a healthy cooperative relationship between parents and mental health professionals can lead to timely conflict resolution and benefit children with disabilities [84-85]. This cooperation is even more vital in early intervention programs, which are family-centered [86] and through parental empowerment [87] there is a greater sense of parental accomplishment [88].

Most relevant studies show that parents and mental health professionals are familiar with cooperative relationships through their interpersonal experiences [89-90]. Functional cooperative relationships are characterized by trust, respect, communication and shared vision that are essential to make decisions that will lead to increased communication [91], inclusion [92], and appropriate service provision for children with disabilities [93]. Some research also shows that teachers prefer to have a closer and more meaningful relationship with parents of children with disabilities [94]. The existence of supportive relationships among parents and mental health professionals is the most important determinant of a successful cooperative relationship [75].

Despite the existing legislations in some European countries and the wishes of both parents and mental health professionals, it is often extremely difficult to create successful and functional cooperative relationships [95-96]. For example, in the context of family- 
centered early intervention cooperation remains an utopia [86]. Although mental health professionals seem to favor cooperative relationships with parents, research shows that there is a big gap between theory and practice [97]. Relevant studies [98] that were conducted using either focus groups or interviews and questionnaires showed that the basic problem is that mental health professionals do not treat parents as equal partners and continue to maintain control. So, the failure to establish cooperative relationships is due to the fact that that there are no trusting and empowering relationships between parents and mental health professionals [11,99].

This failure to create cooperative relationships could also be caused by the inadequate definition of cooperation [100] that hinders the quest for a common goal through functional interactions [101-103]. There are six factors that are essential for the establishment of a cooperative relationship between parents of children with disabilities and mental health professionals and form the basis of the partnership protocol that will be presented later on in the chapter [104]. These factors are:

a. communication: parents stressed that communication with mental health professionals must be honest, frequent and open, with no hidden agendas. Mental health professionals should inform parents also about unpleasant developments in the therapeutic process but without becoming rude or aggressive and without using jargon. Parents want to have access to information regarding other services that are available for their children. Communication should be a two-way process, with both parents and mental health professionals listening to each other without being critical. Mental health professionals seem to agree about the necessity of open and honest communication with the parents that can form the basis of a trusting relationship.

b. commitment: mental health professionals should not view what they do as a simple job that pays for their expenses and treat children with disabilities just as another client or case that is filed. They must value the individual and pay attention to the relationship with the whole family of the child with disabilities. It is noteworthy that some parents thought that mental health professionals should greet them if they meet somewhere in public as a sign of respect and professional commitment. Many mental health professionals recognized the importance of commitment and argued that they often have to deal with parents who do not want to be involved with the therapeutic process or get involved in decision-making regarding their child. However, this should not stop them from making the effort to work closely with the parents.

c. equity: mental health professionals must make conscious efforts to empower the families that they work with, recognizing the importance of parental knowledge instead of devaluing it. Parents should be encouraged to express their opinions and to be fully engaged in decision-making in the context of a constructive exchange of ideas. Attention is needed to keep the very thin line between empowering the parents and giving them too much independence that could jeopardize the therapeutic process.

d. skills: parents tend to admire the mental health professionals who make the difference by offering practical help both to them and to their children with disabilities and who 
are skilled and well trained. Mental health professionals should have high expectations from the children that they work with if they are going to try hard to make some progress and reach the goals that they have set. Parents appreciate the mental health professionals who have the strength and the will to be constantly updated about the new developments in their areas of expertise. Most mental health professionals referred to the skills that they expect from their colleagues but not from parents (this partly reflects their lack of trust in a cooperative relationship with the parents).

e. trust: this term has three different meanings according to the context where it is used. It means reliability in the sense that mental health professionals should honor their promises any way they can. It is equal to security, in the sense that parents need to feel that their children with disabilities are safe both physically and emotionally when in the company of mental health professionals. The third dimension of trust is the discretion that mental health professionals should possess regarding the information that they share with colleagues about a child.

f. respect: a sign of respect is that mental health professionals treat the child with disabilities as a human being and not as a label or a diagnosis, that they are polite, considerate, punctual, and up-to-date with recent developments in the field. Several parents mentioned that these simple rules of courtesy and proper behavior are often overlooked in daily encounters. Many mental health professionals admit that the lack of respect to parents can cause severe damage to the therapeutic relationship.

It is interesting to note that parents and mental health professionals seem to agree on what they think constitutes a desirable and proper cooperative relationship. They may differ in the importance that they place on each factor and in whether they identify it as essential or not for the success of the cooperation. Both sides recognize that for a cooperative relationship to work, both parents and mental health professionals should do their best keeping in mind the interests of the child with disabilities. This study [104] emphasizes that it is imperative to conduct further research to create guidelines to delineate the relationship between parents and mental health professionals, rendering it thus more satisfactory and more effective. This is the aim of the present study that aspires through the use of a partnership protocol to delineate the relationship between parents of children with autism spectrum disorders and mental health professionals - a need that was identified also by other researchers [16, 105].

Because of the heterogeneity of the symptoms and characteristics of autism spectrum disorders, the diagnosis usually does not provide useful suggestions for the appropriate treatment [106]. Successful therapeutic interventions develop when parents and mental health professionals work together as a coordinated and cooperative team [107]. In order to deal with the needs of children with autism spectrum disorders and their families the program COMPASS was created [54], which aims at the cooperation between staff and parents to design the most appropriate therapeutic intervention for each child. The greatest challenge that mental health professionals who work with the families of children with autism spectrum disorders have to face is to ensure that these children attend the therapeutic interventions that best suit their unique and complicated needs [108]. Parental attitudes and parental 
satisfaction are widely used as indications of the success of early intervention programs [109]. Since parents are the ones caring for their children with autism spectrum disorders, their views should be seriously taken into consideration by mental health professionals. Parental concerns and preferences can be used to improve offered services, while parental satisfaction can be translated into a measure of success of a therapeutic intervention [110].

The interaction between parents of children with autism spectrum disorders and mental health professionals is crucial in special needs education because of the high incidence of autism spectrum disorders in the school population and the lack of resources [111-112]. However, this interaction is often fragmentary and characterized by confusion, disappointment, and tension that result to low levels of cooperation and decreased quality of service provision to the child with autism spectrum disorders [111].

The relationship between parents of children with autism spectrum disorders and teachers is also worth exploring [113-114], especially given that many children with autism spectrum disorders have communication deficits and cannot express themselves and their needs [115-116]. Research so far suggests that trust is built almost exclusively on personal interactions, encounters, and exchanges. Every encounter between parents and teachers turns into an opportunity to expand and to strengthen the bonds of trust between the interested parties. Of course, if parents suspect that teachers are not worthy of their trust, then the bonds that are created are very fragile. Many parents seek to create a strong bond with their child's teachers, because they believe that this will benefit their child [11, 117]. In order to build up their trust, both parents and teachers should state clearly and openly their expectations from this relationship in an effort to minimize misunderstandings [118].

Mental health professionals often have to announce bad news to parents regarding their child's diagnosis and prognosis, which cause drastic and often negative changes in their lives [119-120]. Since parents have the unquestionable right to know the truth about their child's condition, the question is not whether the mental health professionals will share the news but how they will do it [120]. Many mental health professionals have been criticized for the abrupt way in which they communicate upsetting news to the parents [46] and the detrimental effects this can have on the parents is a matter of great concern [121]. However, if the briefing is done properly, then this can be extremely useful for them, since they will be able to understand their child's needs and design the appropriate treatment plan [122].

Despite the significant increase in knowledge about the causes and course of autism spectrum disorders [123] and the appreciation of the importance or early diagnosis [124], there have been no noteworthy changes in the information that parents receive in their first contact with mental health professionals. Some studies [125-126] have looked at the interaction between parents and mental health professionals during the dissemination of the assessment conclusions. It was found that mental health professionals are aware of the dilemma of delivering upsetting news and seek the active participation of the parents in a joint articulation of the problem. Some mental health professionals ask parents first to express their opinions about their child's problems and then they share the diagnosis to corroborate the parents' perspective [127]. Other mental health professionals present a series of related general and specific symptoms that lead to a specific diagnosis and then allow parents to state 
the final diagnosis [126]. Usually, mental health professionals try to bridge the gap between their views and parental views by modifying the diagnostic label, so as to comply with parental wishes and to balance the levels of optimism and pessimism [125].

Parental satisfaction is an important element for the evaluation of the services that are offered to children with disabilities and their families [128] and can be related to other family variables, such as stress or depression [129], increased empowerment [130] or increased school involvement [131]. Some qualitative studies have shown that parents who are not satisfied with their relationship with mental health professionals experience stress and do not feel welcome in the decision-making process regarding their children [59]. There are also some documented cases of parents who were so unsatisfied with the early intervention programs their children attended that they removed them from the program [132]. On the other hand, there are many qualitative studies of families that come from different cultural backgrounds and report that parents who are satisfied with the services provided to their children tend to engage more in their training [133].

Research on parental satisfaction asks parents to evaluate the quantity or the quality of the services that their children receive, as well as the nature of their relationship with mental health professionals $[128,131]$. However, there is still a basic gap in identifying a widely accepted definition of parental satisfaction and which intervention model can be implemented to increase this satisfaction [77]. In a survey of satisfaction among 290 parents of children with autism spectrum disorders [134], it was found that most individualized educational plans were not developed in cooperation with mental health professionals, they did not reflect the views and the concerns of the family and they were not successfully coordinated by the many different people who run the services. In another similar study [108] it was reported that most of the 539 parents had difficulty finding about the available services and accessing them. They also claimed that they were not given any choice, that they had to fight for what they wanted and that ultimately the received services differed greatly from what they had originally asked for. Finally, more than half of the parents who participated in another study [135] complained that they were not fully informed about the available services or the structural changes that were taking place in different agencies and that they were unhappy with their cooperation with mental health professionals. All these problems seem to be even more prominent for the families of children with autism spectrum disorders who have to interact with various mental health professionals, such as pediatricians, psychologists, speech therapists and many others [136].

\subsection{The present study}

The concept of boundaries is inherent in human relations and cooperation and represents the rules and limitations that can create a sense of safety [137]. In strictly professional relationships the involved parties have a clearly defined role that they hesitate to deviate from. However, in many mental health services professionals may fulfill various practical, informative, and emotional needs of the individuals who use these services and their families [105]. Despite the fact that the codes of ethics of different professional bodies offer guidelines for the behaviors that protect mental health professionals against extreme cases of con- 
flict of interest or client exploitation for own purposes, there are no guidelines for the delineation of daily interactions between mental health professionals and service users [138]. The code of ethics in special needs education does not address sufficiently the boundaries in relationships between mental health professionals and parents of children with disabilities [105] and this can hinder the establishment of a cooperative relationship between them [11]. So, it is imperative to create a form for the negotiation of the boundaries in daily interactions between parents and mental health professionals in order to make decisions about how, when and why the involved parties will interact [105]. This is how the partnership protocol that will be presented in this chapter was created on the basis of the codes of ethics of the British Psychological Society [139], the American Psychological Association [140], and the Health and Care Practitioners Council [141]. The aim of this study was to explore whether this partnership protocol could change the perceptions of parents of children with autism spectrum disorders and mental health professionals about their relationship. More specifically, it was hypothesized that parents of children with autism spectrum disorders would hold more positive attitudes about mental health professionals after the implementation of the partnership protocol. Mental health professionals would also express more positive attitudes towards the parents of autism spectrum disorders after the implementation of the partnership protocol.

\section{Methods}

\subsection{Participants}

The participants of this study were 40 mental health professionals working in the private sector with children with autism spectrum disorders and their families (18 men and 22 women): 5 psychiatrists, 10 speech therapists, 12 occupational therapists, 7 psychologists and 6 special educators. Their age ranged from 26 to 55 years old (mean age $=42$ years and 2 months) and they have been working with children with disabilities from 3 to 30 years (mean years of professional experience $=17$ years). The mean time that they have been working therapeutically with a child with autism spectrum disorders was 2 hours per week. Forty mothers and fathers of children with autism spectrum disorders from Northern Greece also took part in the study. There were 33 mothers and 7 fathers, aged 29 to 42 years old (mean age $=34$ years and 7 months). Ten mothers were housewives, 17 were private employees, 9 were public employees, and 4 were self-employed. One quarter of the parents had one child, 24 had two children and 6 had three children. Most parents lived with their spouses, while 4 mothers were divorced and raised their children alone. All the parents had a child diagnosed with autism spectrum disorders from a public child psychiatric or child developmental clinic. The mean age of their child's diagnosis was 4 years and 8 months. Out of the 40 children with autism spectrum disorders there were 7 girls and 33 boys and their age ranged from 3.5 to 14 years old. Ten children attended special schools, 22 attended inclusion classes and the remaining 8 were in mainstream schools. The parents were in contact with more than 5 mental health professionals from the time they started seeking for a diagnosis and visited someone to help their children with autism spectrum disorders for an average of 5 years and 3.5 hours per week. 


\subsection{Measures}

\subsubsection{Partnership protocol}

The partnership protocol (please see Appendix) is a document that aims to delineate the relationship between mental health professionals and the parents of children with autism spectrum disorders. It defines partnership as a «functional relationship characterized by a common goal, mutual respect and desire for negotiation». The protocol is two pages long in order to be handy and to offer condensed information in the 11 following areas: 1) cooperation between parents and professionals, 2) negotiation of boundaries in parent-professional relationship, 3) parental expectations/feelings/needs, 4) parental accuracy and reporting of knowledge, 5) parental understanding of their child's condition, 6) parental participation in decision-making, 7) parents as therapists, 8) parental briefing, 9) disclosure of information to parents or third parties, 10) family discord and 11) negotiation of parent-professional disagreement. The partnership protocol was piloted with five parents and seven mental health professionals.

\subsubsection{Parent measures}

The parents completed a brief questionnaire at baseline, which included the following information: gender, age, educational level, profession, number of children, age of child with autism spectrum disorders, gender of child with autism spectrum disorders, age of diagnosis of child with autism spectrum disorders, agency of diagnosis of the child with autism spectrum disorders, years of cooperation with mental health professionals, weekly contact frequency with mental health professionals and number of mental health professionals with whom they have cooperated so far. Then, parents were asked to define the relationship between parents and mental health professionals; to specify what they expect from cooperative mental health professionals; to mention the problems that they face from uncooperative mental health professionals; to describe what they do in case of disagreement with mental health professionals; to define negotiation and to judge if it is necessary for a successful therapeutic relationship; and to document the three advantages and the three disadvantages of their relationship with mental health professionals.

Parents were asked after the intervention to state whether the protocol was useful or not justifying their answers; whether any points needed further clarification; which were the most important points of the protocol; how often they used it; if it helped them define the nature of the relationship that they had with the mental health professionals; what happened in case of disagreement with mental health professionals; whether the protocol helped them resolve any disagreement with mental health professionals; and whether anything had changed in their relationship with mental health professionals.

In order to measure parental views about mental health professionals, the Helping Behavior Checklist - $(C B C L)$ [142] was used, since it was based on the codes of ethics of six international organizations of mental health professionals. The first part, which was used in this study, consists of 16 statements that parents have to rate on a 4-point scale (where $1=$ almost always true and $4=$ almost never true), such as «the mental health professional clearly ex- 
plained to me what I had to do to help my child», «the mental health professional did not involve me in any decision-making regarding my child's therapy» and «the mental health professional held me responsible for my child's problems». Scores are reversed in some items and the total score for the scale varies from 16 to 64 . This questionnaire is highly correlated to parental satisfaction about their child's progress since they started working with the specific mental health professional. Test-retest reliability varies from 0.48 to 0.89 for a period of 2-3 weeks [142]. The Cronbach $\alpha$ of the scale for this study was high $\alpha=0.89$ and deemed satisfactory.

\subsubsection{Mental health professionals measures}

The mental health professionals filled in a brief questionnaire at baseline with demographic information: age, gender, profession, years of professional experience, as well as weekly frequency of sessions with children with autism spectrum disorders. Then they were asked to define the relationship between parents and mental health professionals and what they expect from cooperative parents; to mention the problems that they face from uncooperative parents; to state whether their cooperation with parents is necessary for successful intervention; to describe what they do in case of disagreement with parents; to define the concept of negotiation and to judge if it is necessary for a successful therapeutic relationship; and to document the three advantages and the three disadvantages of their relationship with parents.

Mental health professionals were asked after the intervention to state whether the protocol was useful or not justifying their answers; whether any points needed further clarification; which were the most important points of the protocol; how often they used it; if it helped them define the nature of the relationship that they had with parents; what happened in case of disagreement with parents; whether the protocol helped them resolve any disagreement with parents; and whether anything had changed in their relationship with parents.

The views of mental health professionals about the parents of children with autism spectrum disorders they worked with were measured using Providers' Beliefs About Parents Questionnaire (PBAP) [143], which is based on some concerns that parents expressed about the attitudes or the behaviors of some mental health professionals who worked with their children with disabilities. It consists of 37 statements that mental health professionals have to rate on a 4-point scale (where $1=$ completely disagree and $4=$ completely agree). There are 5 subscales: a) «parental incrimination», which consists of nine statements, such as «the most common cause of severe emotional disorder in children is their parents' behavior» or «the most common cause of emotional problems in children is their parents' emotional inadequacy»; b) «necessity of informing parents», which consists of ten statements, such as «it is usually advisable to offer parent unlimited access to their child's files» or «all parents must be informed on how exactly a therapy is expected to help their child»; c) «recognition of parental status», which consists of seven statements, such as "parents of children in need of mental health services are usually emotionally involved to such an extent, that they do not accurately report their child's behavior» or "parents possess special knowledge that mental health professionals lack»; d) «attitudes towards drug use», which consists of six statements, such as «drugs usually help to deal with autism» or «the possible merit of drug therapy 
should be taken into consideration in most cases of autism»; and e) «providing guidance to parents», which consists of four statements, such as «it is not usually recommended to inform parents about what they can do exactly to help their child» or «it is therapeutically acceptable to brief parents directly about what they should do to help their child with autism». Scoring is reversed for some statements and the total score varies from 37 to 148 . External validity ranges from 0.60 to 0.87 for each subscale [143] and test-retest reliability for the whole scale is 0.89 for a period of 2-3 weeks. Cronbach $\alpha$ for this study was very high for the whole scale $(\alpha=0.93)$ and is deemed extremely satisfactory.

\subsection{Procedure}

The researcher approached mental health professionals who were working privately with children with autism spectrum disorders in Northern Greece and briefed them about the study. She identified potential participants from the professional phone book and the lists of professional bodies in the area and then randomly pulled 100 papers with names from a container. She contacted them and 68 expressed an initial interest in the study, while 45 ended up agreeing to participate. The next step was to ask these mental health professionals to draw a list with the children with autism spectrum disorders they were working with at that time and the researcher randomly selected one family. The mental health professionals were given the task to brief the families and in case the parents expressed an interest the researcher met with them as well. There were some families who did not want to participate in the study, so another family was selected in their place until every mental health professional was matched to a family of a child with autism spectrum disorders. The parent from each family who participated was the one who was more in charge of the child's therapy and was in more frequent and direct contact with the mental health professional. This was deemed essential in order to follow the partnership protocol and to explore its effectiveness.

Before the beginning of the intervention the researcher informed the participants that they could withdraw at any time without penalty and that all the information that they provided would be confidential. Then, she gave out the baseline questionnaires that were filled out individually in the office of the mental health professionals and in the presence of the researcher. The next step was to present the partnership protocol to the participants in detail, to go through it with them and to answer any questions they might have. The intervention started when all participants reassured the researcher that they had fully understood the content of the partnership protocol and it lasted for six months. During this time the researcher called the participants monthly to check the progress of the data collection and to ask if there were any issues that needed to be addressed. Meanwhile, five parents discontinued the intervention at different points due to time restraints (one parent), health problems (two parents), or because they stopped taking their child to the particular mental health professional. So, the researcher asked the corresponding mental health professionals to stop using the protocol and the final number of participants was decreased to 40 parents of children with autism spectrum disorders and 40 mental health professionals.

Data collection was completed after a series of face-to-face meetings with every participant, who was asked after the intervention to fill in the same questionnaire as in baseline and to 
answer some additional questions about the protocol. The answers of the participants in the open-ended questions were explored with thematic analysis, which led to the categories that are presented. A second rater with experience with this type of analysis looked at approximately half of the data and the interrater reliability was extremely satisfactory (95.7\%).

\section{Results}

Data analysis revealed that after the intervention parents of children with autism spectrum disorders were more satisfied with their cooperation with mental health professionals. Following the implementation of the partnership protocol mental health professionals tended to blame parents less for their children's problems, gave them more information about their children's situation and directions on how they should behave, while they also recognized more their parental status.

\subsection{Parents of children with autism spectrum disorders}

\subsubsection{Baseline data}

The characteristics of a satisfactory cooperative relationship between parents and mental health professionals according to parents were: honesty, mutual briefing, mutual trust and setting common goals. Parents believed that cooperative mental health professionals provide constant briefing on the child's progress (90\%); implement the therapy with consistency $(68 \%)$; are honest with parents $(49 \%)$; recognize parental skills $(40 \%)$; understand parental wishes and problems (35\%); and really want to help (10\%). Uncooperative mental health professionals, on the other hand, do not brief parents about their child's progress ( $88 \%)$; do not implement the therapy consistently (73\%); are dishonest with parents (50\%); do not understand parental wishes and aspirations (43\%); withhold information related to the therapy (23\%); and are not knowledgeable about autism spectrum disorders $(20 \%)$.

The vast majority of the parents $(88 \%)$ thought that it is essential to cooperate with mental health professionals in order for the therapy to succeed and approximately $75 \%$ reported that they should actively participate in their child's therapy. The rest of the parents considered that nothing can be done to alter the predetermined course that their children with autism spectrum disorders will follow. When a disagreement occurred with mental health professionals, most parents insisted and discussed with them when they believe that they were right, while they backed down when they were not certain. Parents defined negotiation as: clear expression of views from both sides; understanding with the child's progress as a common goal; arrangement of a time frame for the accomplishment of some results; and expression of realistic expectation from both parties. Indeed, almost $2 / 3$ of parents $(68 \%)$ reported that negotiation is essential for a successful therapeutic relationship. The advantages and the disadvantages that parents identified in their relationships with mental health professionals are presented in Tables 1 and 2 respectively. 


\begin{tabular}{llc}
\hline & $\mathbf{N}$ & $\%$ \\
\hline 1. Encouragement about the child's progress & 14 & 35 \\
\hline 2. Genuine interest in the child and the family & 10 & 25 \\
\hline 3. Cooperation between parents and mental health professionals & 7 & 17.5 \\
\hline 4. Provision of practical advise to parents & 5 & 12.5 \\
\hline 5. Regular verbal communication with parents & 3 & 7.5 \\
\hline 6. Provision of support in all the areas of the child's development & 1 & 2.5 \\
\hline
\end{tabular}

Table 1. Advantages of Working with Mental Health Professionals According to the Parents of Children with Autism Spectrum Disorders

\begin{tabular}{llc}
\hline & $\mathbf{N}$ & $\%$ \\
\hline 1. Dishonesty about the child's progress & 12 & 30 \\
\hline 2. Lack of genuine interest in the child and the family & 9 & 22.5 \\
\hline 3. No understanding of parental needs & 7 & 17.5 \\
\hline 4. Inability to realize what is promised & 5 & 12.5 \\
\hline 5. Insensitivity to family's needs & 4 & 10 \\
\hline 6. Overcharging for the therapy & 3 & 7.5 \\
\hline
\end{tabular}

Table 2. Disadvantages of Working with Mental Health Professionals According to the Parents of Children with Autism Spectrum Disorders

\subsubsection{Post-intervention data}

Most parents (87\%) stated that the partnership protocol was useful, because it provides a clear context for the relationships between parents and mental health professionals, it defines the roles of both parties, it promotes the positive cooperation that contributes to the child's progress and it familiarizes parents with their rights. The remaining 13\% claimed that the protocol is not useful because it is binding and difficult to adhere to. Some parents said that the protocol should also clarify which qualifications mental health professionals should hold in order to work with children with autism spectrum disorders and what parents can do if mental health professionals do not follow the protocol.

The most important points of the partnership protocol were: ensured cooperation, honest relationships, understanding of parental limitations, parental participation in decision-making, and recognition of parental needs and emotions. Parents referred to the protocol every time that something changed in their child's therapy or a problem came up, as well as in case of disagreement with mental health professionals. The changes that parents observed in their relationship with mental health professionals after the implementation of the protocol were: parents and mental health professionals cooperated more effectively (32\%); mental health professionals offered psychological support to parents $(25 \%)$; mental health profes- 
sionals took parental needs and expectations into account (19\%); parents learned how to act as therapists for their children at home (13\%); a time frame was set for the therapy (9\%); and mental health professionals did not treat parents just as an income source (3\%).

\subsubsection{The attitudes of parents of children with autism spectrum disorders towards mental health professionals}

Paired-samples t-test was use to compare differences in parental attitudes towards mental health professionals before and after the implementation of the partnership protocol. Before the intervention $(M=31.95, S D=8.64)$ parents expressed statistically significant less positive attitudes towards mental health professionals $\left(t_{(1,39)}=107.25, p<0.001, \eta^{2}=0.73\right)$ than after the intervention $(\mathrm{M}=28.65, \mathrm{SD}=7.67)$.

\subsection{Mental health professionals}

\subsubsection{Baseline data}

The characteristics of a satisfactory cooperative relationship between parents and mental health professionals according to mental health professionals were: the exchange of ideas about the child, shared decision-making, mutual trust and respect, will to negotiate, and frequent contact. Mental health professionals believed that cooperative parents provide accurate information about their children (78\%); follow their advise (68\%); are honest about their child's condition (50\%); are interested to learn more about their child (43\%); do not have unreasonable expectations for their child's progress (42\%); and actively participate in their child's treatment. Uncooperative parents, on the other hand, provide inaccurate information about their children (80\%); question the mental health professional $(60 \%)$; do not understand their child's condition (53\%); do not keep certain agreements (45\%); have irrational demands for their child's progress (38\%); do not participate in their child's therapy (25\%); and do not behave consistently $(20 \%)$.

The vast majority of mental health professionals (93\%) claimed that it is necessary to cooperate with parents for the success of the therapeutic intervention, since parents: possess valuable knowledge about their child that can be used in therapy, can complement the therapist's work and spend a lot of time with the child. In order to ensure parental cooperation, mental health professionals make parents feel more comfortable; brief them regularly about their child's progress; show sensitivity to the child's problems; express positive attitudes towards the child and the parents; promote parental beliefs that their child can improve with the proper therapy and support; take parental needs and wishes into account; engage parents in decision-making; gain parental trust; and try to specialize in autism spectrum disorders.

When mental health professionals disagree with parents, they tend usually to have an open and honest discussion with them, to engage them in decision-making, to be discrete, and to present their arguments. They may even resort to another mental health professional and in the end they make the final decisions if they cannot reach an agreement with the parents. Mental health professionals defined negotiation as exchange of ideas with the child's interest in mind, defining the boundaries of the cooperation, determining the expectations from 
the therapy, setting mutual goals, discussing new possibilities, and compromising, if necessary. The advantages and the disadvantages that mental health professionals identified in their relationships with parents are presented in Tables 3 and 4 respectively.

\begin{tabular}{lll}
\hline & $\mathbf{N}$ & $\%$ \\
\hline 1. Interest in their child's therapy & 38 & 95 \\
\hline 2. Willing to devote time to their child & 35 & 88 \\
\hline 3. Realistic expectations about their child's progress & 27 & 68 \\
\hline 4. Active participation in their child's therapy at home & 22 & 55 \\
\hline 5. Source of important information for the child & 19 & 48 \\
\hline 6. Willing to cooperate with mental health professionals & 14 & 35 \\
\hline 7. Trust mental health professionals & 10 & 25 \\
\hline 8. Wish a better future for their child & 9 & 23 \\
\hline 9. Encourage other family members to participate in therapy & 6 & 15 \\
\hline
\end{tabular}

Table 3. Advantages of Working with Parents of Children with Autism Spectrum Disorders According to Mental Health Professionals

\begin{tabular}{lll}
\hline & $\mathbf{N}$ & $\%$ \\
\hline 1. No understanding of the child's condition & 33 & 83 \\
\hline 2. Emotional overload & 30 & 75 \\
\hline 3. Provision of inaccurate information & 29 & 73 \\
\hline 4. Refusal to engage actively in therapy & 25 & 63 \\
\hline 5. Exaggerated and unrealistic expectations & 25 & 63 \\
\hline 6. Unwillingness to cooperate & 50 \\
\hline 7. Lack of trust in mental health professionals & 18 & 45 \\
\hline 8. Feelings of parenting inadequacy & 16 & 40 \\
\hline 9. Insecurity for their child's future & 15 & 38 \\
\hline 10. Parental disagreements about their child & 13 & 33 \\
\hline 11. Rejection of the diagnosis of autism spectrum disorders & 10 & 25 \\
\hline 12. Experience of guilt over the child's condition & 8 & 20 \\
\hline 13. Crossing the boundaries (e.g., calling in the middle of the night) & 6 & 15 \\
\hline 14. Insisting on their views, even when they are wrong & 10 & 4 \\
\hline
\end{tabular}

Table 4. Disadvantages of Working with Parents of Children with Autism Spectrum Disorders According to Mental Health Professionals

\subsubsection{Post-intervention data}

Most mental health professionals (80\%) claimed that the partnership was useful, because it sets the boundaries of the cooperation, it promotes negotiation, it emphasizes mutual respect, it clarifies the need for parental involvement in the therapeutic process and mental health professionals have a reference point in case of disagreement. The remaining $20 \%$ stat- 
ed that the protocol is not useful because it is binding and parents who are not educated cannot understand it. Some mental health professionals said that the protocol should clarify how much time mental health professionals should devote to parents, what happens when parents do not follow the protocol, and for which third parties mental health professionals should get the consent of the parents before they disclose information about their children.

The most important points of the partnership protocol were: the negotiation of the boundaries of the relationship between parents and mental health professionals, the clarification of the roles of both sides, and the emphasis placed on active parental involvement in their child's therapy. Mental health professionals referred to the protocol every time there was a disagreement with parents. The changes that mental health professionals observed in their relationship with parents after the implementation of the protocol were: parental expectations became more relevant to the child's condition (93\%); parents realized that the whole family should be part of the therapy $(80 \%)$; initial tensions were normalized (75\%); parents felt that their expectations and emotions were taken into account $(65 \%)$; parents were more committed to the therapy (58\%); parents were more open to new suggestions and treatments for their child (35\%); parents made less calls of hypothetical crises (23\%).

\subsubsection{The attitudes of mental health professionals towards parents of children with autism spectrum} disorders

Analysis with MANOVA revealed that there was a statistically significant change in four out of the five subscales that measured the attitudes of mental health professionals towards the parents of children with autism spectrum disorders, even after being controlled for age, gender, and years of professional experience. More specifically, there were statistically significant changes in parental incrimination $\left(F_{(1,39)}=5.56, p<0.05, \eta^{2}=0.12\right)$; necessity of informing parents $\left(F_{(1,39)}=5.03, p<0.05, \eta^{2}=0.11\right)$; recognition of parental status $\left(F_{(1,39)}=4.83, p\right.$ $\left.<0.05, \eta^{2}=0.10\right)$; and providing guidance to parents $\left(F_{(1,39)}=5.35, p<0.05, \eta^{2}=0.12\right)$. There was no statistically significant difference in the attitudes that mental health professionals expressed towards drug use before and after the intervention $\left(F_{(1,39)}=0.96, p>0.05, \eta^{2}=002\right)$. Means and standard deviations are presented in Table 5.

\begin{tabular}{llcc}
\hline & Baseline & Post-intervention & \\
\cline { 2 - 4 } & M (SD) & M (SD) & $\boldsymbol{F}$ \\
\hline Parental incrimination & $26.69(3.54)$ & $22.24(3.44)$ & $5.56^{*}$ \\
\hline Necessity of informing parents & $14.15(3.24)$ & $17.93(2.74)$ & $5.03^{*}$ \\
\hline Recognition of parental status & $14.06(2.52)$ & $19(2.04)$ & $4.83^{*}$ \\
\hline Attitudes towards drug use & $6.11(2.18)$ & $8.41(1.54)$ & 0.96 \\
\hline Providing guidance to parents & $3.25(1.07)$ & $8.17(2.34)$ & $5.35^{*}$ \\
\hline$* \rho<0.05$ & & & \\
\hline
\end{tabular}

Table 5. Means and Standard Deviations of the Attitudes of Mental Health Professionals Towards Parents of Children with Autism Spectrum Disorders Before and After the Intervention 


\section{Discussion}

The research hypotheses were confirmed, since both parents of children with autism spectrum disorders and mental health professionals expressed more positive attitudes about each other after the implementation of the partnership protocol. Most participants felt that the partnership protocol was particularly useful and they also identified some points that could be further clarified, while they also pointed out the exact nature of the changes that they have observed in their interactions.

\subsection{The attitudes of parents of children with autism spectrum disorders towards mental health professionals}

Most parents of children with autism spectrum disorders believed from the beginning that it was imperative to work together with mental health professionals in order to enhance their child's progress, while the rest believed that their child's progress was predetermined and there was nothing they could do to change that. Parents define a cooperative relationship as a relationship that is characterized by honesty, mutual briefing, mutual trust, and setting common goals [27]. The information that parents receive from mental health professionals is more important to them than sympathy or psychological support [144-145].

Parents describe cooperative mental health professionals as constant providers of update regarding their child's progress, consistent, honest, understanding, willing to help, and aware that parents possess certain skills. So, the parents in this study have identified essentially the defining characteristics of cooperative relationships [77]. Parents cannot cooperate with mental health professionals who do not brief them, are inconsistent and insincere, ignore them, withhold information and are not well trained - in agreement with other research [24, 28, 117].

The implementation of the partnership protocol helped the parents of children with autism spectrum disorders to redefine their cooperative relationship with mental health professionals. They learned to function as therapists at home, facilitating thus the therapeutic process [25-26]. They received emotional support from mental health professionals and they set a time frame for some therapeutic goals, which could help them feel less stressed [146]. Most parents reported that after the intervention mental health professionals treated them as more equals, briefed them about their child's progress, engaged them more actively in the therapeutic process, and took their feelings and opinions into consideration. This change may be due to the fact that a trusting relationship was created through the protocol, which helped the parents express themselves more freely and become more assertive. Trust is imperative for the creation of a constructive cooperative relationship between mental health professionals and parents of children with disabilities [104]. Many parents actively seek to create this bond of trust, since they feel that it will benefit their child $[11,117]$.

An additional change that was reported by the parents of children with autism spectrum disorders was that the mental health professionals started briefing them more about the ways in which the proposed therapy will help their child and encouraged them to get ac- 
tively involved in decision-making regarding their child's treatment. Active participation in their child's therapy can help parents develop a sense of efficacy and personal control that can help them become even more effective parents [87] and less stressed and concerned [147]. When parents feel heard and respected, then they can cooperate better with mental health professionals to do what is best for their child and this is something that mental health professionals should aim at [66].

\subsection{The attitudes of mental health professionals towards parents of children with autism spectrum disorders}

Mental health professionals tended to incriminate parents of children with autism spectrum disorders less after the implementation of the protocol. This is very important, since it affects greatly the choice of suggested therapies and strategies that mental health professionals employ when interacting with the parents of children with autism spectrum disorders [67]. Since parents of children with autism spectrum disorders have been repeatedly blamed for the problems that their children face [148], the partnership protocol encourages the creation of a relationship that frees parents from guilt and treats them as equal partners. After the implementation of the partnership protocol, mental health professionals realized that it is essential to inform parents about their child's condition and the course of the therapy. The needs of all family members are taken into consideration [50] and, therefore, frictions are decreased $[3,11,99]$. So, it is possible to create a strong cooperative relationship that can benefit both parties [40,47], while children with autism spectrum disorders can also benefit from parental empowerment [149].

The mental health professionals who adopted the protocol said that they recognized more the validity of parental knowledge and information regarding their child with autism spectrum disorders. This change is very positive, since parents of children with autism spectrum disorders know a lot of things about their children that are useful when planning the appropriate intervention [61]. The acknowledgement of the usefulness of parental knowledge by mental health professionals is essential to the cooperative process [60] and is, thus, emphasized in the partnership protocol.

Moreover, mental health professionals understood that they need to provide more clear and more detailed information to parents about the management and upbringing of their children with autism spectrum disorders. The partnership protocol stressed that parents can be trained to satisfy the needs that arise from their parenting role and this is instrumental for various therapeutic approaches [16]. Parents who receive specific guidance on how to deal with challenging and unwanted behaviors believe that they can control them better [150] and so they feel less stressed [146]. However, it is worth pointing out that some parents cannot or do not want to fulfill their instructional role [151] and this is something that mental health professionals should respect.

The statistical analysis revealed that the perceptions that mental health professionals hold about the parents of children with autism spectrum disorders were not affected by their age, gender, or years of professional experience. Therefore, it is likely that they are affected by 
their training and by the «social representations» that they hold about parents of children with autism spectrum disorders [152], as well as by cultural and social factors [18].

Almost all the mental health professionals believed that their cooperation with the parents of children with autism spectrum disorders was essential to the successful course of the therapy [86, 128], since they bring their own knowledge and experience into the therapeutic process [29]. Therefore, they claimed that in order to ensure this cooperation they try to earn parental trust and to make them feel comfortable, while taking into account their needs and wishes. By briefing parents about their child's progress, they engage them more actively in decision-making regarding the treatment course [93]. However, it is worth mentioning that at the beginning of the study parents complained that mental health professionals do not try enough to cooperate with them. This could be due to a wider communication problem that has been documented also in other studies, since good intentions alone are not enough to establish cooperation $[86,94]$. Finally, mental health professionals claimed that they tried to increase their knowledge through training in order to be able to better deal with the problems of children with autism spectrum disorders. This needs to be done if mental health professionals are to design an intervention that is based on the child's needs and skills and is more likely to be successful [49].

Mental health professionals define cooperation as a relationship that is characterized by exchange of views about the child, shared decision-making, mutual trust and respect, desire to resolve disagreement, frequent contact and discussion on equal terms (there is no expert) $[15,59,89,93]$. This definition that they provided includes some of the key characteristics of the negotiation model [40].

Parents should provide accurate information about their child that is essential for the establishment of a cooperative relationship with mental health professionals [77]. Otherwise, mental health professionals have to waste a lot of valuable time and resources to find out what they need to design an effective intervention [1]. Parents who are unaware of their child's actual condition may place irrational demands on both their child and mental health professionals and fail to keep agreements and deadlines, jeopardizing their child's progress [111]. Finally, there are some parents who question the training of mental health professionals and their suitability to work with their child, but may continue to cooperate because they have no other options or because they believe they can motivate the mental health professional [11, 99].

Mental health professionals noted that after the implementation of the partnership protocol parents started to have more realistic expectations that made them realize the importance of engaging the whole family in the therapeutic process [54]. Parents understood that they have to follow the advice of mental health professionals to help their children and became more open to new treatment suggestions [104]. These behaviors are indicative of greater trust for the mental health professionals, who need initially to recognize the shock that parents experience [17] and to help them reach the stage of full acceptance [16]. Parents also seemed to have responded positively to the efforts made by mental health professionals to take their feelings and views into account when designing the intervention [75]. Finally, mental health professionals reported that the parents were better able to judge when they 
needed to communicate in order to resolve an actual crisis, probably as a result of their active therapeutic engagement [98].

\subsection{Evaluation of the partnership protocol}

Most parents of children with autism spectrum disorders and mental health professionals who used the partnership protocol said that it was useful, because: a) it clarifies the relationship between the two parties; b) it defines the roles of both parties; c) it promotes effective cooperation; d) it advances negotiation; and e) it values mutual respect. All these elements were rated by many studies [28, 101, 103] as essential for the creation of a functional and effective cooperative relationship among parents and mental health professionals. It is extremely important to point out that both parents and mental health professionals recognize similar benefits from the implementation of the protocol, corroborating the finding that the needs of parents and mental health professionals are closer that one would think, but they need to be clearly defined in order to be satisfied [100].

The parents of children with autism spectrum disorders reported that the partnership protocol informs them of their rights and helps them fight for provisions and services. Parental participation is instrumental in reassuring the existence of options for adults with autism spectrum disorders [153]. Parents become empowered $[39,86]$ and thus able to negotiate with mental health professionals [40]. Mental health professionals said that they could use this protocol to resolve conflicts or disagreements with parents. This is important, since some mental health professionals do not know how to resolve interpersonal conflicts [3] and they become increasingly stressed [147].

The four parents who thought that the partnership protocol is not useful justified their opinion by saying that it is binding and difficult to adhere to. It is a fact that the implementation of a protocol or any form of agreement requires commitment from all involved parties. Therefore, it is likely that some parents of children with autism spectrum disorders are exhausted from the constant care of a child with multiple and complex needs and do not possess the required strength to enter this process [146]. Moreover, it is plausible that some parents prefer to hold a more distant role from the therapeutic process [54]. The eight mental health professionals who considered the partnership protocol not to be that useful thought as well that it is binding and that parents who are not educated cannot understand it. It seems that they do not want to be committed to a predetermined cooperative relationship with the parents because they have adopted the expert model. Even if some parents are not educated or able to understand some points, it is the role of mental health professionals to explain everything to them in simple and understandable language [56].

Approximately $20 \%$ of the parents of children with autism spectrum disorders identified two things that needed further clarification in the partnership protocol: a) which qualifications mental health professionals should hold in order to work with children with autism spectrum disorders and $b$ ) what parents can do if mental health professionals do not follow the protocol. The first point partly reflects the insecurity that results from insufficient briefing regarding the options that parents have to choose the suitable mental health professional after the diagnosis [55]. They could also result from limited options due to place of residence 
or financial restraints [99]. The second clarification is indicative of feelings of inferiority or intimidation. This partnership protocol has no legal power and it is not a contract with legal ratifications. The whole point of the protocol is to introduce parents to the concept that they can discuss any conflict or disagreement with mental health professionals on equal terms.

Mental health professionals also wanted to know what will happen in case that parents of children with autism spectrum disorders do not follow the protocol. This is something that should be discussed and agreed upon from the beginning between the two parties. Most disagreements are peacefully resolved, while very few have ended up in court [3]. Moreover, it is important that some mental health professionals realize that some parents will not want to cooperate with them [76]. The second question was how much time mental health professionals should spend with parents of children with autism spectrum disorders and this is again to be negotiated between them from the beginning.

The most important positive outcomes of the partnership protocol are: ensured cooperation, honest relationships, understanding of parental limitations, parental participation in decision-making, and recognition of parental needs and emotions. It seems that what parents really need is to feel like equal partners in the therapeutic process and this is something that the partnership protocol offers. Parents who are involved in decision-making about their child and are supported by mental health professionals feel happier [2, 33, 154]. The most important positive outcomes of the partnership protocol are: the negotiation of the boundaries of the relationship between parents and mental health professionals, the clarification of the roles of both sides, and the emphasis placed on active parental involvement in their child's therapy. Mental health professionals need to define their relationships with parents as much as parents do $[28,77-78]$ and they use the protocol to do so.

\subsection{Limitations}

The aim of this study was to create a partnership protocol to delineate the relationship between parents of children with autism spectrum disorders and mental health professionals with the ultimate goal to improve the child's condition [84]. The need for this protocol derived from personal experience and from meticulous literature search [16, 105]. Although the findings were positive and encouraging, there are some limitations that should be taken into consideration:

1. The mental health professionals were working in the private sector and so they might be more willing to follow the protocol in order to keep their clients - things could be different if they worked in a public setting.

2. The mental health professionals who agreed to participate in the study might have been the ones who work better with parents, and the parents who participated might have been the ones who were happy with mental health professionals to begin with. However, analysis showed that all the participants identified some problems at baseline.

3. Some confounding variables, such as the training of mental health professionals or the educational level of the parents were not taken into consideration when analyzing the findings. For example, it was found that single mothers of children with autism spectrum disorders experience more stress than married mothers [14]. 
4. Despite the fact that the participants were reassured that data would be kept confidential, they might be skeptical about expressing very negative attitudes [155].

5. There was no official follow-up, although the researcher had informal contacts with the participants and was informed that many of them continued to follow the partnership protocol after the six-month period.

6. There are many other factors that could have affected the collaboration between parents of children with autism spectrum disorders and mental health professionals that are related to external factors (e.g., financial crisis), internal factors (e.g., depressive mood) or child-specific factors (e.g., severity of the autism spectrum disorders) that were not examined in the present study and could inform further research.

\subsection{Practical implementation and future research}

This research confirmed the findings of previous studies [77, 104, 147] that the relationship between parents of children with autism spectrum disorders and mental health professionals is quite challenging. However, the aim was not just to identify the existing problems, but to propose also some possible solutions. It seems that the implementation of the partnership protocol had a positive effect on the delineation of the relationship of the involved parties and helped them overcome some the existing obstacles by becoming more cooperative and willing to negotiate for the child's benefit.

As mentioned in the introduction, mental health professionals and parents of children with disabilities hold their personal beliefs regarding the kind of relationship that they should have. The partnership protocol helped them understand their rights and obligations, since they might hesitate to discuss them openly. There are many mental health professionals who do not know how to set limits to their relationships with parents and many parents who do not know how to express their opinions or their questions regarding their child to mental health professionals. Therefore, the partnership protocol may be suggested by mental health professionals as a means to negotiate their relationship with parents, while it serves also as an opportunity to discuss further and to resolve any conflicts. However, it should be stressed that the partnership protocol is not a legally binding document and so parents and mental health professionals should follow it because they believe in its value. It can be implemented in any therapeutic framework, where it is essential for parents of children with disabilities and mental health professionals to work together. It could also be used to train mental health professionals who will work with children and adolescents with disabilities and their families [156].

This is the first study in Greece, and worldwide, that introduced the use of a partnership protocol to resolve possible conflicts that arise between mental health professionals and parents of children with autism spectrum disorders. Therefore, future studies should be conducted with parents of children with other disabilities, as well as with parents of children with chronic illnesses and with other mental health professionals, such as doctors, nurses, or teachers. 


\section{Conclusion}

This study confirmed the findings of previous international research regarding the problematic relationships between mental health professionals and the parents of children with disabilities. It was found that Greek mental health professionals are troubled by the parents' demands and their unwillingness to actively engage in their child's treatment. Greek parents of children with autism spectrum disorders claim that mental health professionals are not interested in involving them in decision-making regarding their child's therapy. Similar complaints have been expressed in other studies that have explored the relationship between mental health professionals and parents of children with autism spectrum disorders [77, 104, 147].

Despite the fact that the difficulties that were documented in this study have been identified a long time ago and in several contexts, there has been no published coordinated effort to resolve them. This partnership protocol was based on the codes of ethics of international organizations of mental health professionals, it is written in simple language and it was considered to be useful by most participants. The implementation of the partnership protocol helped mental health professionals and parents of children with autism spectrum disorders to define their interpersonal relationship and to overcome many of the difficulties and the problems that they had identified at the beginning of their cooperation. They started to communicate more honestly, to respect each other more and to resolve their conflicts more effectively. Even though these findings are encouraging, more longitudinal studies with varied participants are needed to explore further the effectiveness of the partnership protocol.

\section{Acknowledgement}

I would like to thank all the participants and Dr Angeliki Gena who was the primary supervisor of my second $\mathrm{PhD}$ that is the basis of this research. I extend my gratitude also to my family and especially my husband and colleague Vlastaris Tsakiris for his constant support throughout this lengthy process.

\section{Appendix}

\section{Parent-Professional Partnership Protocol}

When professionals and parents of children with autism come together for the first time, they bring with them their own worries, concerns, priorities, and responsibilities, which must be woven together into a relationship that could be characterized as a partnership. The roles of the parent and the professional impose certain rights and duties, obligations and anticipated behaviours, as well as expectations. It is extremely difficult to define the exact nature of this partnership, since every parent and every family has its own idiosyncrasies and 
each professional possesses unique characteristics and ways of working and relating. The aim of the present document is to provide some guidelines that could be adopted and implemented by both interested parties in an attempt to define their partnership and it is based on the principles of the negotiating model. The negotiating model defines partnership as "a working relationship that is characterized by a shared sense of purpose, mutual respect and the willingness to negotiate".

\section{Cooperation between parents and professionals}

Professionals need parental cooperation in order to be able to do their job effectively. Parents should recognize that professionals have specialist knowledge and abilities, but they cannot substitute the role of the caregiver. Since both parents and professionals are interested in the child's progress, they need to cooperate to achieve the best results.

\section{Negotiation of boundaries in parent-professional relationship}

Parents and professionals should clarify and negotiate the nature and the limits of their partnership. It would be advisable to make a contract that is not formal or legally binding. It just sets out mutual expectations and intended behaviours. Depending on the situation it may be appropriate to put this in writing and each party should retain their own copy. It is advisable to repeat this process at later stages according to the progress of the child. Parents should refrain from contacting the professional on a regular basis about things that do not concern the child with autism and professionals should be punctual and fulfil their obligations towards the family and the child.

\section{Parental expectations/feelings/needs}

Professionals should identify and evaluate the needs of the family, which cannot be separated from the needs of the child. The child has a relationship with all the other family members and the relationships within the family have an interactive effect with each other. Parents should verbalise and express their urging needs to professionals so that a solution can be sought. These expectations, feelings, and needs should be incorporated into the treatment when the professional believes that is plausible and suitable.

\section{Parental accuracy and reporting of knowledge}

Parents possess a unique and special knowledge and understanding about their child that is valuable for the design of a better intervention. The home is the best available place to consolidate the knowledge that the child acquires and it can also offer multiple opportunities for learning. Parents should be honest with professionals and try to overcome the difficulty they may experience to talk publicly about the condition and the difficulties of their child. If the professional is misled or told half the truth, it is very likely that the suggested treatment will not be the appropriate one.

\section{Parental understanding of their child's condition}

Professionals should use simple language when talking to parents, since they do not have expert knowledge that allows them to familiarise themselves with terms used among professionals. Parents should also express their queries and seek to clarify any misconceptions or 
worries they might have. If parents believe that they are not being listened to, they should make sure that the attitude of the professional changes. Professionals must not focus on the child alone, but they should advise parents on how to care for their child with autism. Even if parents have other children, they may need some practical assistance and tips on how to overcome some of the issues that arise due to the difficulties faced by their child. It is the parents' responsibility to inform professionals on the areas where they believe they need more help and support.

\section{Parental participation in decision-making}

Professionals should allow parents to be more involved in activities and decisions regarding the education and care of their child. For example, professionals should not make drastic changes in the treatment that they follow before consulting with the parents. This is a good way to ensure cooperation and to minimise conflicts in parent-professional relationship. Parents will be able to make an informed choice regarding the future of their own child. Therefore, a common purpose or shared concern or mutual interest should be established in order for the relationship between the interested parties to be productive. Both parents and professionals should be involved in brainstorming regarding potential ideas, plans, or actions that could enhance the development of the child. Parents should make an effort to follow and understand the progress of their child in order to be able to make a decision. This could include reading books, notes, or reports regarding the condition of their child and the treatment that is implemented.

\section{Parents as therapists}

Professionals may train parents to use some behavioral techniques that will allow them to teach their child, complementing thus and supporting the work of professionals. If parents feel confident enough, they may want to assume an active role in furthering their child's learning. However, professionals should be aware that parents may not have enough time to be actively involved in the education of their child if they have a full-time job or other children to look after. Therefore, at the beginning of the partnership professionals and parents should reach an agreement on the amount of time that parents can spend with their child on a weekly basis. Professionals should encourage each member of the family to contribute to the treatment of the child with autism, which may need special assistance to participate in family outings and activities. This can be achieved by encouraging parents to communicate with each other and express openly their concerns and needs.

\section{Parental briefing}

Professionals should inform parents from the beginning about the cost of the treatment, seek their consent when contemplating the acquisition of new material, and brief them about the progress of their child, even if the news are not particularly encouraging or reassuring. It would be a good idea for professionals to keep notes of the meeting with the parents, so that they can refer to them in the future and keep track of the progress of their child.

\section{Disclosure of information to parents or third parties}


Professionals could verbally inform parents about the progress of their child but access to records may be prohibited due to legal issues related to their confidentiality - professionals are called to make individual decisions according to each situation. Parents who advocate their right to have access to the records should be equally responsible in their own record keeping. Professionals should inform parents of any other professionals with whom they discuss the case of their child and elicit their consent before doing so.

\title{
Family discord
}

In case of disagreement between the parents regarding the treatment of their child, professionals should stay neutral and avoid making alliances with one parent or colluding consistently with one parent's preferences. If professionals believe that there are many pressing issues among the family members, they should encourage them to see a counsellor. Parents should realize that professionals working with their child may not have the necessary knowledge and training to deal with these issues.

\section{Negotiation of parent-professional disagreement}

When a disagreement arises, both parents and professionals should try to resolve it. They must express their opinions and feelings openly, keeping in mind that they have the child's best interest in mind. If it is impossible to resolve the disagreement, it might be advisable to discontinue the partnership.

\section{Author details}

\author{
Efrosini Kalyva
}

Address all correspondence to: kalyva@city.academic.gr

Psychology Department, The International Faculty of the University of Sheffield, CITY College, Thessaloniki, Greece

\section{References}

[1] Seligman M, Darling RB. Ordinary Families, Special Children: A Systems Approach to Childhood Disability. 2nd ed. New York: The Guilford Press; 1997.

[2] Swain J, Walker C. Parent-Professional Power Relations: Parents' and Professionals' Perspectives. Disability and Society 2003;18(4) 547-560.

[3] Feinberg E, Beyer J, Moses P. Beyond Mediation: Strategies for Appropriate Early Dispute Resolution in Special Education. Unpublished manuscript. National Center on Alternative Dispute Resolution (CADRE): Eugene, OR; 2002. 
[4] Minuchin S. Families and Family Therapy. Cambridge, MA: Harvard University Press; 1974.

[5] Elman NS. Family Therapy. In Seligman M. (ed.) The Family with a Handicapped Child. 2nd ed. Boston: Allyn \& Bacon; 1991, p369-406.

[6] Turnbull AP, Summers JA, Brotherson MJ. Family Life Cycle: Theoretical and Empirical Implications and Future Directions for Families with Mentally Retarded Members. In Sowers J. (ed.) Making our Way: Promoting Self-Competence among Children and Youth with Disabilities. Baltimore: Brookes; 1986, p45-66.

[7] Weiss S. Stressors Experienced by Family Caregivers of Children with Pervasive Developmental Disorders. Child Psychiatry and Human Development 1991;21(2) 203-216.

[8] Siklos S, Kerns KA. Assessing Needs for Social Support in Parents of Children with Autism and Down Syndrome. Journal of Autism and Developmental Disorders 2006;36(7) 921-933.

[9] Grissom M. "From their own perspective": An Ethnographic Study of Families with Children with Autism. Dissertations Abstracts International: The Sciences and Engineering. 2005;65 3745.

[10] Pakenham KI, Samios C, Sofronoff K. Adjustment in Mothers of Children with Asperger Syndrome: An Application of the ABCX Model of Family Adjustment. Autism 2005;9(2) 191-212.

[11] Turnbull AP, Turnbull HR. Families, Professionals, and Exceptionality: Collaborating for Empowerment. 4th ed. Columbus, OH: Merrill; 2001.

[12] Turnbull AP, Patterson JM, Behr SK, Murphy DL, Maquis JG, Blue-Banning MJ. Cognitive Coping, Families, and Disability. Baltimore: Brookes; 1993.

[13] Turnbull AP, Turnbull HR. Families, Professionals and Exceptionality: A Special Partnership. 3rd ed.Upper Saddle River: Merrill; 1997.

[14] Bromley J, Hare D, Davison K, Emerson E. Mothers Supporting a Child with Autistic Spectrum Disorders: Social Support, Mental Health Status and Satisfaction with Services. Autism 2004;8(3) 409-423.

[15] Parette P, Chuang SJL, Huer MB. First Generation Chinese American Families' Attitudes Regarding Disabilities and Educational Interventions. Focus on Autism and Other Developmental Disabilities 2004;19(1) 114-123.

[16] Gena A. Family and Child with Autism: Parental Reactions and Adjustment. In Gena A, Kalogeropoulou E, Mavropoulou S, Nikolaou A, Notas S, Papageorgiou V. (eds.) The Autism Spectrum: Cooperation between family and professionals. Trikala: The Association of Parents and Friends of Children with Autism; 2006, p45-88.

[17] Gena A. Autism and Pervasive Developmental Disorders. Athens: Author; 2002. 
[18] King Gerlach E. Part 1. The Beginning: How Parents Move Forward and Make Choices after Diagnosis. Exceptional Parent Magazine, New York: www.discovery.org-www.thecenterfordiscovery.org; 2002.

[19] Rolland JS. Mastering Family Challenges in Series Illness \& Disability. In Walsh F. (ed.) Normal Family Processes. 2nd ed. New York: Guildford Press; 1993, p444-473.

[20] Adelman H, Taylor L. Addressing Barriers to Learning: Beyond School-Linked Services and Full Service Schools. American Journal of Orthopsychiatry 1997a; 67(3) 408-421.

[21] Adelman HS, Taylor L. Toward a Scale-up Model for Replicating New Approaches to Schooling. Journal of Educational Psychology Consultation 1997b;5(2)197-230.

[22] Children's Aid Society. Building a Community School. New York: Author; 1997.

[23] McKnight J. The Careless Society: Community and its Counterfeits. New York: Basic Books, 1995.

[24] Osher TW, deFur E, Nava C, Spencer S, Toth-Dennis D. New Roles for Families in Systems of Care. Washington, DC: American Institutes of Research, Center for Effective Collaboration and Practice; 1999.

[25] Bennett J, Grimly LK. Parenting in the Global Community. A Cross-Cultural/International Perspective. In Fine M, Lee S. (eds.) Handbook of Diversity in Parent Education: The Changing Faces of Parenting and Parent Education. San Diego, CA: Academic Press; 2001, p96-132.

[26] Fristad MA, Goldberg-Arnold JS, Gavazzi SM. Multi-Family Psychoeducation Groups in the Treatment of Children with Mood Disorders. Journal of Marital Family Therapy 2003;29(4) 491-504.

[27] Duchanowski AJ, Kurash K, Friedman RM. Community-Based Interventions in a System of Care and Outcomes of Framework. In Burns BJ, Hoagwood K. (eds.) Community Treatment for Youth: Evidence-Based Interventions for Severe Emotional and Behavioral Disorders. New York: Oxford University Press; 2002, p16-38.

[28] Friesen BJ, Huff B. Family Perspectives on Systems of Care. In Stroul B. (ed.) Children's Mental Health: Creating Systems of Care in a Changing Society. Baltimore: Paul H. Brookes; 1996, p41-67.

[29] Ruffolo MC, Kuhn MT, Evans ME. Developing a Parent-Professional Team Leadership Model in Group Work: Work with Families with Children Experiencing Behavioral and Emotional Problems. Social Work 2006;51(1) 39-47.

[30] Bruner C. Thinking Collaboratively: Ten Questions and Answers to Help Policy Makers Improve Children's Services. Washington, DC: Education and Human Services Consortium; 1991. 
[31] Cunningham CC, Davis H. Early Parenting Counseling. In Craft M, Bichnell J, Hollins S. (eds.) Mental Handicap: A Multidisciplinary Approach. London: Bailliere Tindall; 1985, p69-88.

[32] Marteau TM, Johnston M, Baum JD, Bloch S. Goals of Treatment in Diabetes: A Comparison of Doctors and Parents of Children with Diabetes. Journal of Behavioral Medicine1987;10(1) 33-48.

[33] Betz M, O'Connell L. Changing Doctor-Patient Relationships and the Rise in Concern for Accountability. Social Problems 1983;31(1) 84-95.

[34] Pugh G, De'Ath E. Working Towards Partnership in Early Years. London: National Children's Bureau; 1989.

[35] Mittler P, McConachie H. Parents, Professionals and Mentally Handicapped People: Approaches to Partnership. Beckenham: Croom Helm; 1983.

[36] Mittler P, Mittler H. The Transitional Relationship. In Mittler P, McConachie H. (eds.) Parents, Professionals and Mentally Handicapped People: Approaches to Partnership. Beckenham: Croom Helm; 1983, p221-240.

[37] Turnbull AP, Turnbull HR. Parent Involvement in the Education of Handicapped Children: A Critique. Mental Retardation1982;20(2) 115-122.

[38] Davis PB, May JE. Involving Fathers in Early Intervention and Family Support Programs: Issues and Strategies. Child Health Care 1991;20(1) 87-92.

[39] Appleton PL, Minchom PE. Models of Parent Partnership and Child Development Centers. Child: Care, Health and Development 1991;17(1) 27-38.

[40] Dale P. Parent Report Assessment of Language and Communication. In Cole K, Dale P, Thal D. (eds.) Assessment of Communication and Language. Baltimore, MD: Paul H. Brookes; 1996, p161-182.

[41] McConkey R. Working with Parents: A Practical Guide for Teachers and Therapists. London: Croom Helm; 1986.

[42] New C, David M. For the Children's Sake: Making Childcare More than Women's Business. Harmondsworth: Penguin; 1985.

[43] Ayer S, Alaszewski A. Community Care and the Mentally Handicapped: Services for Mothers and their Mentally Handicapped Children. London: Croom Helm; 1984.

[44] Glendenning C. Parents and their Disabled Children. London: Routledge and Kegan Paul; 1983.

[45] Feinberg E, Vacca J. The Drama and the Trauma of Creating Policies on Autism: Critical Issues to Consider in the New Millennium. Focus on Autism and Other Developmental Disabilities 2000;15(2) 130-138.

[46] Hannam C. Parents and Mentally Handicapped Children. Harmondsworth: Penguin; 1975. 
[47] Quine L, Rutter DR. First Diagnosis of Severe Mental and Physical Disability: A Study of Doctor-Patient Communication. Journal of Child Psychology and Psychiatry 1994;35(7) 1273-1287.

[48] Friend M, Cook L. Interactions: Collaborative Skills for School Professionals. White Plains, NY: Longman; 1992.

[49] Fine MJ. The Handbook of Family-School Intervention: A Systems Perspective. Boston: Allyn \& Bacon; 1991.

[50] Kalyva E. Autism: Educational and Therapeutic Approaches. London: Sage; 2011.

[51] Hecimovic A, Gregory S. The Evolving Role, Impact, and Needs of Families. In Zager D. (ed.) Autism Spectrum Disorders: Identification, Education, and Treatment. 3rd ed. Mahwah, NJ: Lawrence Erlbaum Associates; 2005, p111-142.

[52] Lovaas OI. Behavioral Treatment and Normal Educational and Intellectual Functioning in Young Autistic Children. Journal of Consulting and Clinical Psychology 1987;5(1) 3-9.

[53] Schopler E, Mesibov GB, Hearsey KA. Structured Teaching in the TEACCH System. In Schopler E, Mesibov GB. (eds.), Learning and Cognition in Autism. New York: Plenum; 1995, p243-268.

[54] Hardy N, Sturmey P. Portage Guide to Early Education, III: A Rapid Training and Feedback System to Teach and Maintain Mothers' Teaching Skills. Educational Psychology 1994;14(3) 345-358.

[55] Ruble LA, Dalrymple NJ. COMPASS: A Parent-Teacher Collaborative Model for Students with Autism. Focus on Autism and Other Developmental Disabilities 2002;17(1) 76-83.

[56] Friesen BJ. Creating Change for Children with Serious Emotional Disorders: A National Strategy. In Mizrahi T, Morrison J. (eds.). Community Organizations and Social Administration: Advances, Trends and Emerging Principles. New York: Haworth Press; 1993, p127-146.

[57] Kohler F. Examining the Services Received by Young Children with Autism and their Families: A Survey of Parent Responses. Focus on Autism and Other Developmental Disabilities 1999;14(2) 150-158.

[58] Johnson HC. Family Issues and Interventions. In Johnson HC. (ed.) Child Mental Health in the 1990s: Curricula for Graduate and Undergraduate Professional Education. Washington, DC: U.S. Department of Health and Human Services, Public Health Service, National Institute of Mental Health; 1993, p85-101.

[59] Read J, Clements L. Disabled Children and the Law: Research, the Law and Good Practice. London: Jessica Kingsley Publishers; 2001. 
[60] Soodak LC, Erwin EJ, Winton P, et al. Implementing Inclusive Early Childhood Education: A Call for Professional Empowerment. Topics in Early Childhood Special Education 2002;22(1) 91-102.

[61] Collins B, Collins T. Parent-Professional Relationships in the Treatment of Seriously Emotionally Disturbed Children and Adolescents. Social Work 1990;35(5) 522-527.

[62] Smith DE, Griffith AI. Coordinating the Uncoordinated: Mothering, Schooling and the Family Wage. Perspectives of Social Problems 1990;2(1) 25-43.

[63] Crawford T, Simonoff E. Parental Views about Services for Children Attending Schools for the Emotionally and Behaviourally Disturbed (EBD): A Qualitative Analysis. Child: Care, Health and Development 2003;29(6) 481-491.

[64] Francell C, Conn V, Gray D. Families' Perceptions of Burden of Care for Chronic Mentally Ill Relatives. Hospital of Community Psychology 1988;39(12) 1296-1937.

[65] Tarico V, Low B, Trupin E, Forsyth-Stephens A. Children's Mental Health Services: A Parent Perspective. Community and Mental Health Journal 1989;25(3) 313-326.

[66] Johnson HC, Renaud E. Professional Beliefs about Parents of Children with Mental and Emotional Disabilities: A Cross-Discipline Comparison. Journal of Emotional and Behavioral Disorders 1995;5(2) 149-161.

[67] Randall P, Parker J. Supporting the Families of Children with Autism. Chichester: Wiley; 1999.

[68] Hartman A, Laird J. Family-Centered Social Work Practice. New York: Free Press; 1983.

[69] Bennett WS, Hokenstad MC. Full-Time People Workers and Conceptions of the Professional. In Halmos P, (ed.) Professionalism and Social Change. Keele: University of Keele Press; 1973, p36-58.

[70] Handy CB. Understanding Organizations. Harmondsworth: Penguin; 1985.

[71] Cone JD, Delawyer DD, Wolfe VV. Assessing Parent Participation: The Parent/Family Involvement Index. Exceptional Children 1985;51(3) 417-424.

[72] Smets AC. Family and Staff Attitudes toward Family Involvement in the Treatment of Hospitalized Chronic Patients. Hospital Community Psychiatry 1982;33(6) 573-575.

[73] Brand S. Making Parent Involvement a Reality: Helping Teachers Develop Partnerships with Parents. Young Children 1996;51(1) 76-81.

[74] Katz L, Bauch J. The Peabody family involvement initiative: Preparing preservice teachers for family/school collaboration. The School Community Journal 1999;9(1) 49-69.

[75] Tichenor M. Teacher Education and Parent Involvement: Reflections from Preservice Teachers. Journal of Instructional Psychology 1997;24(2) 233-240. 
[76] Dinnebeil LA, Hale LM, Rule S. A Qualitative Analysis of Parents' and Service Coordinators' Descriptions of Variables that Influence Collaborative Relationships. Topics in Early Childhood Special Education 1996:19(4) 322-347.

[77] Dunst CJ, Trivette CM, Johanson C. Parent-Professional Collaboration and Partnership. In Dunst CJ, Trivette CM, Deal AG. (eds.) Supporting and Strengthening Families. Cambridge, MA: Brookline Books; 1994, p197-211.

[78] Summers JA, Hoffman L, Marquis J, Turnbull A, Poston D, Lord Nelson L. Measuring the Quality of Family-Professional Partnerships in Special Education Services. Exceptional Children 2005;72(1) 65-81.

[79] Stevenson O, Parsloe P. Community Care and Empowerment. York. Joseph Rowntree Foundation; 1993.

[80] Loxley A. Collaboration in Health and Welfare: Working with Difference. London. Jessica Kingsley Publishers; 1997.

[81] Malin N. Services for People with Learning Disabilities. London: Routledge; 1995.

[82] Goble R. Multi-Professional Education in Europe. In Leathard A, (ed.) Going InterProfessional: Working Together for Health and Welfare. London: Routledge; 1994, p157-194.

[83] Wistow G. Hospital Discharge and Community Care: Early Days. Leeds: Nuffield Institute for Health; 1993.

[84] Roberts RN, Rule S, Innocenti MS. Strengthening the Family-Professional Partnership in Services for Young Children. Baltimore: Brookes; 1998.

[85] Epstein JL. School, Family, and Community Partnership: Preparing Educators and Improving Schools. Boulder, CO: Westview Press; 2001.

[86] Osher TW, Osher DM. The Paradigm Shift to True Collaboration with Families. Journal of Child and Family Studies 2002;11(1) 47-60.

[87] Bruder MB. Family-Centered Early Intervention: Clarifying our Values for the New Millennium. Topics in Early Childhood Special Education 2000;20(1) 105-115.

[88] Trivette CM, Dunst CJ, Boyd K, Hamby D. Family-Oriented Program Models, Help Giving Practices, and Parental Control Appraisals. Exceptional Children 1995;62(2) 237-248.

[89] Applequist KL, Bailey DB. Navajo Caregivers' Perceptions of Early Intervention Services. Journal of Early Intervention Services 2000;23(1) 47-61.

[90] McWilliam RA, Tocci L, Harbin GL. Family-Centered Services: Service Providers' Discourse and Behavior. Topics in Early Childhood Special Education 1998;18(2) 206-221. 
[91] Park J, Turnbull AP. Service Integration in Early Intervention: Determining Interpersonal and Structural Factors for its Success. Infants and Young Children 2003;16(1) 48-58.

[92] Parette HP, Brotherson MJ, Huer MB. (2000). Giving Families a Voice in Augmentative and Alternative Communication Decision-Making. Education and Training in Mental Retardation and Developmental Disabilities 2000;35(2) 77-90.

[93] Soodak L, Erwin E. Valued Member or Tolerated Participant: Parents' Experiences in Inclusive Early Childhood Settings. Journal of the Association of Parents with Severe Handicaps 2000;25(1) 29-41.

[94] Park J, Turnbull AP. Families Speak Out: What are Quality Indicators of Professionals in Working with Children with Problem Behavior? Journal of Positive Behavioral Intervention 2002;4(2) 118-123.

[95] McWilliam R, Maxwell K, Sloper K. Beyond Involvement: Are Elementary Schools Ready to be Family Centered? School Psychology Review 1999;28(3) 378-394.

[96] Rainforth B, York J, Macdonald C. Collaborative Teams for Students with Severe Disabilities: Integrating Therapy and Educational Services. Baltimore: Brooks; 1992.

[97] Salembier GB, Furney KS. Speaking up for your Child's Future. Exceptional Parent 1998;28(1) 62-64.

[98] Sanders M. Improving School, Family and Community Partnerships in Urban Middle Schools. Middle School Journal 1999;31(1): 35-41.

[99] Blue-Banning MJ, Turnbull AP, Pereira L. Group Action Planning as a Support Strategy for Hispanic Families: Parent and Professional Perspectives. Mental Retardation 2000;38(2) 262-275.

[100] Allen RL, Perry CG. Toward Developing Standards and Measurements for FamilyCentered Practice in Family Support Programs. In Singer GHS, Powers LE, Olson AL. (ed.) Redefining Family Support: Innovations in Public-Private Partnerships. Baltimore: Brookes; 1996. p57-86.

[101] Dunst CJ. Revisiting "Rethinking Early Intervention". Topics in Early Childhood Special Education 2000;20(1) 95-104.

[102] Kalyanpur M, Harry B. Culture in Special Education. Baltimore: Brookes; 1999.

[103] Lynch EW, Hanson MJ. Developing Cross-Cultural Competence: A Guide for Working with Children and Families. 2nd ed. Baltimore: Brookes; 1998.

[104] Sileo TW, Prater MA. Preparing Professionals for Partnerships with Parents of Students with Disabilities: Textbook Considerations Regarding Cultural Diversity. Exceptional Children 1998;64(5) 513-528.

[105] Blue-Banning MJ, Summers JA, Frankland C, Nelson LGL, Beegle G. Dimensions of Parent-Professional Partnerships. Exceptional Children 2004;70(1) 167-184. 
[106] Lord Nelson LG, Summers JA, Turnbull AP. Boundaries in Family-Professional Relationships. Remedial and Special Education 2004;25(3) 153-165.

[107] Ruble L, Sears L. Diagnostic Assessment of Autistic Disorder. In Huebner R. (ed.) Autism and Related Disorders: A Sensorimotor Approach to Management. Maryland: Aspen; 2000, p41-59.

[108] Ruble LA, Dalrymple NJ. An Alternative View of Outcome in Autism. Focus on Autism and Other Developmental Disabilities 1996;11(1) 3-14.

[109] McWilliam R, Young H, Harville K. Satisfaction and Struggles: Family Perceptions of Early Intervention Services. Journal of Early Intervention 1995;19(1) 43-60.

[110] Murphy DL, Lee IM, Turnbull AP, Turbiville V. The Family-Centered Program Rating Scale: An Instrument for Program Evaluation and Changes. Journal of Early Intervention 1995;19(1) 24-42.

[111] McNaughton D. Measuring Parent Satisfaction with Early Childhood Intervention Programs: Current Practice, Problems, and Future Perspectives. Topics in Early Childhood Special Education 1994;10(1) 1-15.

[112] Lake JF, Billingsley BS. An Analysis of Factors that Contribute to Parent-School Conflict in Special Education. Remedial and Special Education 2000;21(4) 240-251.

[113] Minke K, Scott M. Parent Professional Relationships in Early Intervention: A Qualitative Investigation. Topics in Early Childhood Special Education 1995;15(3) 335-346.

[114] Dominique B, Cuttler B, McTarnaghan J. The Experience of Autism in the Lives of Families. In Wetherby AM, Prizant BM. (eds). Autism Spectrum Disorders: A Transactional Developmental Perspective. Baltimore: Brooks; 2000, p369-394.

[115] Lambie R. Working with Families of at Risk and Special Needs Students: A Systems Change Model. Focus on Exceptional Children 2000;32(1) 1-22.

[116] Powers M. Children with Autism: A Parent's Guide. 2nd ed. Bethesda, MD: Woodbine House; 2000.

[117] Katz L. Dispositions: Definitions and Implications for Early Childhood Practices. New York: ERIC; 1993.

[118] Stoner JB, Bock SJ, Thompson JR, Angell ME, Heyl BS, Crowley EP. Welcome to our World: Parent Perceptions of Interactions between Parents of Young Children with ASD and Education Professionals. Focus on Autism and Other Developmental Disabilities 2005;20(1) 39-51.

[119] Bartolo PA. Communicating a Diagnosis of Developmental Disability to Parents: Multiprofessional Negotiation Frameworks. Child: Care, Health and Development 2002;28(1) 65-71.

[120] Buckman R, Kason Y. How to Break Bad News: A Practical Protocol for Health Professionals. London: Macmillan; 1992. 
[121] Siegel B. Coping with the Diagnosis of Autism. In Cohen DJ, Volkmar FR. (eds.) Handbook of Autism and Pervasive Developmental Disorders. New York: Wiley; 1997, p460-483.

[122] Cottrell DJ, Summers K. Communicating an Evolutionary Diagnosis of Disability to Parents. Child: Care, Health and Development 1990;16(2) 211-218.

[123] Cohen DJ, Volkmar FR. Handbook of Autism and Pervasive Developmental Disorders. New York: Wiley; 1997.

[124] Freeman WJ. Neurohumoral Brain Dynamics of Social Group Formation: Implications for Autism. In Carter CH, Lederhendler H, Kirkpatrick B. (eds.) The Integrative Neurobiology of Affiliation. New York: Annals of the New York Academy of Sciences; 1997, p501-503.

[125] Abrams EZ, Goodman JF. Diagnosing Developmental Problems in Children: Parents and Professionals Negotiate Bad News. Journal of Pediatric Psychology 1998; 23(1) 87-98.

[126] Gill VT, Maynard DW. On "Labelling" in Actual Interaction: Delivering and Receiving Diagnoses of Developmental Disabilities. Social Problems 1995;42(1) 11-37.

[127] Maynard DW. Bearing Bad News in Clinical Settings. In Dervin B. (ed.) Progress in Communication Sciences. Norwood, N.J.: Ablex; 1991, p143-172.

[128] Bailey D, Scarborough A, Hebbeler K. Families' First Experiences with Early Intervention. Menlo Park, CA: SRI International; 2003.

[129] King G, King S, Rosenbaum P, Goffin R. Family-Centered Caregiving and Well-Being of Parents of Children with Disabilities. Journal of Pediatric Psychology 1999;24(1) 41-53.

[130] Thompson L, Lobb C, Elling R, Herman S, Jurkiewicz T, Hulleza C. Pathways to Family Empowerment: Effects of Family-Centered Delivery of Early Intervention Services. Exceptional Children 1997;64(1) 99-113.

[131] Laws G, Millward L. Predicting Parents' Satisfaction with the Education of their Child with Down Syndrome. Education Research 2001;43(2) 209-226.

[132] Rao SS. Perspectives of an African American Mother on Parent-Professional Relationships in Special Education. Mental Retardation 2000;38(3) 475-488.

[133] Park J, Turnbull AP. Cross-Cultural Competency and Special Education: Perception and Experiences of Korean Parents of Children with Special Needs. Education and Training in Mental Retardation and Developmental Disabilities 2001;36(2) 133-147.

[134] Able-Boone H, Goodwin I, Sandall S, Gordon N, Martin D. Consumer-Based Early Intervention Services. Journal of Early Intervention 1992;16(2) 201-209.

[135] Covert SB. Whatever it Takes! Excellence in Family Support: When Families Experience a Disability. St. Augustine, FL: Training Resource Network, Inc; 1995. 
[136] Karp D. Speaking of Sadness: Depression, Disconnection, and the Meanings of Illness. Oxford: Oxford University Press; 1996.

[137] Goldstein H. On Boundaries. Family and Society - Journal of Contemporary History 1999;80(5) 435-438.

[138] Strom-Gottfied, K. Professional Boundaries: An Analysis of Violations by Social Workers. Family and Society - Journal of Contemporary History 1999;80(4) 439-449.

[139] British Psychological Society. Code of Ethics and Conduct. Leicester: Author; 2009.

[140] American Psychiatric Association. Diagnostic and Statistical Manual for Mental Disorders. $4^{\text {th }}$ ed. text revision. Washington, DC: American Psychiatric Association; 2000.

[141] Health and Care Professions Council. Fitness to Practice. http://www.hpc-uk.org/ aboutus/committees/ftp/ (accessed 15 June 2008).

[142] Cournoyer DE, Johnson HC. Measuring Parents' Perceptions of Mental Health Professionals. Research in Social Work and Practice 1991;1(3) 399-415.

[143] Johnson HC, Cournoyer DE. Measuring Worker Cognitions about Parents of Children with Mental and Emotional Disabilities. Journal of Emotional and Behavioral Disorders 1994;2(1) 99-108.

[144] Darling RB, Baxter C. Families in Focus: Socio-Logical Methods in Early Intervention. Austin, TX: Pro-Ed; 1996.

[145] Gowen JW, Christy DS, Sparling J. Informational Needs of Parents of Young Children with Special Needs. Journal of Early Intervention 1993;17(2) 194-210.

[146] Hassall R, Rose J, McDonald J. Parenting Stress in Mothers of Children with an Intellectual Disability: The Effects of Parental Cognitions in Relation to Child Characteristics and Family Support. Journal of Intellectual Disability Research 2005;49(6) 405-418.

[147] Summers JA, Gavin K, Hall T, Nelson J. Family and School Partnerships: Building Bridges in General and Special Education. In Obiakor FE, Utley A, Rotatori AF. (eds.). Advances in Special Education: Psychology of Effective Education for Learners with Exceptionalities. Stamford, CT: JAI Press; 2003, p417-445.

[148] Muscott HS. Exceptional Partnerships: Listening to the Voices of Families. Preventing School Failure 2002;42(1) 66-69.

[149] Dillenburger K, Keenan M, Gallagher S, McElhinney M. Parent Education and Home-Based Behaviour Analytic Intervention: An Examination of Parents' Perceptions and Emotions. Journal of Intellectual and Developmental Disabilities 2004;29(1) 119-130.

[150] Longenecker H. Parental Stress - ABA and Traditional Special Education Programs. http://rsaffran.tripod.com/longenecker.html. (accessed 28 June 2006). 
[151] Laborde PR, Seligman M. Counseling Parents with Children with Disabilities. In Seligman M. (ed.) The Family with a Handicapped Child. 2nd ed. Boston: Allyn \& Bacon; 1991, p337-369.

[152] Moscovici S. Social Change and Influence. London: Academic Press; 1978.

[153] Field S, Hoffman A. The Importance of Family Involvement for Promoting Self-Determination in Adolescents with Autism and Other Developmental Disabilities. Focus on Autism and Other Developmental Disabilities 1999;14(1) 36-41.

[154] Renty J, Roeyers H. Quality of Life in High-Functioning Adults with Autism Spectrum Disorder: The Predictive Value of Disability and Support Characteristics. Autism 2006;10(5) 511-524.

[155] Avramidis E, Kalyva E. Research Methods in Special Needs: Theory and Practice. Athens: Papazisis; 2006.

[156] Kalantzi-Azizi A, Besevengkis E. Issues of Training and Sensitization of Mental Health Professionals Working with Children and Adolescents; 2000. 

Aetiological Factors - Intervention in Person with Autism 

Chapter 24

\title{
Early Intensive Behavioural Intervention in Autism Spectrum Disorders
}

\author{
Olive Healy and Sinéad Lydon \\ Additional information is available at the end of the chapter \\ http://dx.doi.org/10.5772/54274
}

\section{Introduction}

Autism spectrum disorder (ASD) is a developmental disorder characterised and diagnosed by behavioural symptoms that mark impairments in social and communication behaviour along with a restricted range of activities and interests. ASD is considered a heterogeneous and complex disorder impacting many areas of development including intellectual, communication, social, emotional, and adaptive (Makrygianni \& Reed, 2010). This disorder can present considerable challenges to both the individual and their family across their lifespan.

A myriad of intervention approaches have been highlighted to treat this condition. Some include therapies that have been developed by parents independent of any particular discipline (e.g., Son-Rise Program and Hanen). Others are based on biological approaches (e.g., special and restricted diets, secretin) or alternative medicine (e.g., homeopathy, chelation therapy). Some more prevalent treatment approaches are available and differ in their etiological, methodological and philosophical interpretation of ASD. These include for example, Applied Behaviour Analysis (ABA; sometimes referred to as behaviour therapy), Treatment and Education of Autistic and related Communication Handicapped Children (TEACCH), Picture Exchange Communication System (PECS), sensory integration therapy, occupational therapy, music therapy, auditory integration therapy and speech therapy. Despite the considerable number of various treatment approaches to ASD available to parents and professionals, the majority of empirical support relating to many of these programs remains at the "level of description" (Makrygianni \& Reed, 2010; Matson \& Smith, 2008), and for many of these proposed interventions there is limited or no evidence provided to demonstrate any effective outcomes with their use (Metz, Mulick, \& Butter, 2005; Mulloy et al. 2010; Lang et al. 2012).

Despite the many debates that exist amongst researchers and practitioners with regard to efficacy of intervention approaches, one consensual fact that is recognised across the board is that 
early intervention is the best response to the treatment of ASD. Providing treatment of symptoms immediately will result in more favourable treatment outcomes (Dawson, 2008; Howlin, Magiati \& Charmin, 2009; Reichow \& Wolery, 2009). Many have argued that this early intervention will allow greater opportunities for a young child to move towards a more typical developmental trajectory because of malleability or plasticity of the developing young brain (see for example Dawson 2008). From a learning theory account, teaching new behaviour or replacement behaviour to a very young child presenting with behavioural deficits or excesses, will result in desirable consequences that impacts behavioural repertoires and learning history from the outset. In this way early intervention for the condition may affect the onset of additional secondary problem behaviours which are often not seen at diagnosis. As such these may be minimised or even prevented (Mundy, Sullivan \& Mastergeorge, 2009).

While a consensus that early intervention for ASD exists amongst researchers in this field, many argue that the actual approach applied during this critical period may be pivotal in producing the greatest outcomes and ensuring the best chance of attaining a typical developmental trajectory. Over the past four decades, interventions based on the science of ABA have been thoroughly evaluated and shown to produce effective outcomes in targeting many of the challenges presented within this condition. Moreover, behavioural interventions drawn from this science can produce substantial gains in cognitive, adaptive and social behaviours in this population (Dillenberger, 2011). Indeed, this approach is internationally recognised as the most effective basis for treatment for children with ASD (Larsson, 2005).

Improving the core symptoms of ASD is a common goal for parents and professionals. Reports of large improvements in this condition have been documented. For example Smith (1999) provided a summary of published peer-reviewed studies involving seven independent groups of researchers documenting dramatic gains when early intervention was applied. Importantly however, in all studies reviewed, interventions were underpinned by $A B A$ methodology and theory and were intensive involving a range of 15 to 40 hours per week across studies. This approach to autism treatment, known as Early Intensive Behavioural Intervention (EIBI) has generated much discussion and excitement, and continues to gather momentum impressing on policy makers the urgency of effective and substantiated provision for individuals and families affected by the condition.

Studies on EIBI have reported the following gains: (1) average increases of approximately 20 points in IQ (e.g., Harris, Handleman, Gordon, Kristoff, \& Fuentes, 1991; Lovaas, 1987; Sheinkopf \& Siegal, 1998) (2) increases in standardised test scores (Anderson, Avery, DiPietro, Edwards, \& Christian, 1987; Birnbrauer \& Leach, 1993; Hoyson, Jamison, \& Strain, 1984; McEachin, Smith, \& Lovaas, 1993; Strauss et al. 2012), (3) increased gains in adaptive behaviour (Eldevik et al., 2012; Strauss et al., 2012); (4) improved language scores (Eldevik et al., 2012; Strauss et al. 2012); (5) the need for less supports in school (Fenske, Zalenski, Krantz, \& McClannahan, 1985; Lovaas, 1987), (6) reduced autism symptomotology (Eikeseth et al,. 2012) and (7) decreased challenging behaviour (Fava et al., 2012). Dillenberger (2011) refers to the increasing evidence of clinical, social and financial efficiency of intensive behavioural intervention in autism treatment which has resulted in "legally enshrining" such intervention in North America. For example, the Autism Treatment Acceleration Act (2010) requires 
that health insurers cover the diagnosis and treatment of autism spectrum disorders, including access to ABA therapy.

\section{What constitutes EIBI?}

EIBI is based on the scientifically applied principles of learning and behaviour, and has the discipline of behaviour analysis (Cooper, Heron, \& Heward, 2007) at its core. The approach generally targets preschool children and is provided intensively, often in a 1:1 student/teacher ratio, for 20-50 hours per week. Dawson (2008) and Green (1996) summarise many of the common and conspicuous features of successful EIBI programs. These include the following:

1. the EIBI program should be initiated as early as 2 years and before the age of four;

2. intensive delivery of the program involving a minimum of 25 hours per week for at least two years;

3. application of a comprehensive curriculum or various curricula, focusing on imitation, language, toy play, social interaction, motor, and adaptive behaviour targets;

4. the curricula and their implementation should show sensitivity to typical developmental sequences;

5. generalisation strategies should be incorporated to ensure new skills are practiced and demonstrated in novel environments outside those in which they were taught;

6. use of supportive and empirically validated teaching strategies and data-driven decision protocols (notably those of Applied Behaviour Analysis);

7. implementation of behavioural strategies to reduce or eliminate major interfering behaviours that are an impediment to learning new skills and repertoires (noncompliance, inattention, impulsivity, tantrum, aggression and self-injurious behaviours are examples of some of the most critical of these behaviours).

8. a functional analytic approach to treating problem behaviours;

9. continual parental involvement and tailored parent education;

10. progressive and gradual transition to increasingly naturalistic environments;

11. qualified and highly trained staff delivering the program and

12. the provision of supervision by qualified over-viewers resulting in ongoing review and systematic progression of the program.

According to Dawson (2008): "When these features are present, results are remarkable for up to $50 \%$ of children" (p.790).

It is important to note that EIBI draws from the bedrock of a science- Applied Behaviour Analysis (ABA). This science constitutes over 300 procedures (Greer, 2002; Steege, Mace, Perry, and Longenecker, 2007) each of which have been tested and demonstrated to produce 
behaviour change. The careful selection and application of these procedures to treat the behavioural symptoms of autism delivered within the scientific framework of ABA (outlined in Baer, Wolf \& Risley, 1968; 1987) is what defines an EIBI approach. It is critical to recognise how $\mathrm{ABA}$ and EIBI are interwoven because the science of $\mathrm{ABA}$ and the various behaviour change strategies therein, have a very long history of substantiated documentation (see for example Matson, Benavidez, Compton, Paclawskyj, \& Baglio, 1996, who reviewed behaviorally based treatments for autism over a 16-year span).

\section{History of EIBI}

The history of this early intervention approach to autism has been well documented over the last three decades. For example, Matson and Smith (2008) trace the origins of this approach in autism treatment to what they refer to as a "seminal paper" (p.61) published as early as 1973 by Lovaas, Koegel, Simmons, and Long (1973). Matson and Smith argue that this paper demonstrated a visionary conceptual framework for early intervention with ASD.

\footnotetext{
"The true significance of the study was the authors' efforts to formulate an overarching treatment of children with autism on a multitude of behaviours including self-stimulation/stereotypies, echolalia, appropriate verbal behaviour, social behaviour, appropriate play, intelligence quotient (IQ), and adaptive behaviour" (Matson \& Smith, 2008, pp. 61-62).
}

Trends in EIBI, to this day, are based on this original template involving the delivery of idiosyncratic treatment packages constituting evidence-based behavioural interventions to target core symptoms as well as expansive groups of behaviours. Numerous studies have been published since this seminal paper in 1973 examining EIBI outcomes in autism. One of the most distinguished and considered published papers which resulted in the acclamation of EIBI involved that of Lovaas (1987). This well-reviewed study which reported an average difference of 31 points on IQ test scores between the ASD treatment group and control group, and classified nine of 19 (47\%) participants as having achieved recovery (defined as post-intervention IQ in the normal range). To this current day, the findings of this study have caused much debate among researchers with criticisms focusing on particular methodological limitations (see for example, Gresham and MacMillan 1998; Short \& Mesibov, 1989). We will return to this study in a later section.

To date, a substantial number of studies have been conducted and published to demonstrate the effectiveness of EIBI in autism treatment. Moreover, six illustrative review papers and one "mega-analysis" (a combination of all of the data into one single analysis) have been published (see below), each providing somewhat varying angles in exploring the outcomes. Steady growing rates of publications on the findings of EIBI in autism have been evidenced and concise descriptions of methodology have appeared to improve in most recent years, particularly with respect to the inclusion of control-no treatment groups and random assignment of participants across experimental conditions.

The current chapter will provide a synopsis of EIBI studies published between 1987-2012. Systematic searches were conducted using the following databases: Scopus, Psychology \& Behavioral Sciences Collection, and PsycINFO 
The searches were carried out using the terms "early intensive behavioural intervention AND autism", and "intensive behavioural intervention AND autism". The inclusion criteria were largely in line with those of Reichow (2012). Studies were reviewed if they included a treatment group who received EIBI and an alternate-treatment control group who received either no treatment, a different treatment or EIBI provided at different intensity levels. Only studies including children with ASD were reviewed. Each study was required to involve original research that was written in English and published in a peer reviewed journal. In the interest of clarity we grouped published investigations under the following headings: Studies published before 2000 (4 studies), studies published from 2000-2010 (12 studies) and studies published between 2011-2012 (5 studies). We provide a summary of factors associated with each published paper including intake characteristics of participants, outcome measures employed, specific treatment characteristics and group differences following intervention. The following sections provide a synopsis of all studies identified.

\section{Studies published before 2000 (4 Studies)}

Lovaas (1987) conducted the first evaluation of EIBI for children with Autism. The outcomes of 19 children receiving EIBI, for a minimum of 40 hours per week, were compared to those of two control groups. The first control group, consisting of 19 children, received low intensity (10 hours or less) behavioural intervention and the second control group, consisting of 21 children, received TAU. After two years of treatment, $47 \%$ of the EIBI group achieved IQ scores in the normal range and were enabled to integrate fully into mainstream educational settings while only $2 \%$ of children in the control group achieved similar outcomes. In this case, almost half of children in the EIBI appeared to recover from their diagnosis of autism.

Birnbauer and Leach (1992) compared the outcomes of nine children receiving EIBI and five children in a control group (no treatment). Children in the EIBI group received an average of 18.7 hours of EIBI per week delivered by trained volunteers in their homes. Children in the EIBI group achieved significantly higher non-verbal IQ scores and language levels. Four of the nine children in the EIBI group achieved IQ scores within the normal range following treatment.

Smith et al. (1997) compared the outcomes of 11 children receiving EIBI to 10 children who received a low intensity behaviour intervention. Children in the high intensity EIBI group received at least 30 hours of clinician-delivered treatment each week while the low intensity group received 10 hours of clinician-delivered behavioural intervention each week. At follow-up, the children in the EIBI group showed greater increases in IQ and expressive language than children in the control group.

Sheinkopf and Siegel (1998) evaluated the outcomes of 11 children receiving EIBI and 11 children receiving Treatment as Usual (TAU). EIBI was delivered by parents, supervised by clinicians, for 27 hours each week. Children in the control group received 11.1 hours of TAU in a school setting each week. Following treatment, the EIBI group achieved significantly higher IQ scores and significantly lower scores on a measure of symptom severity than the control group. 


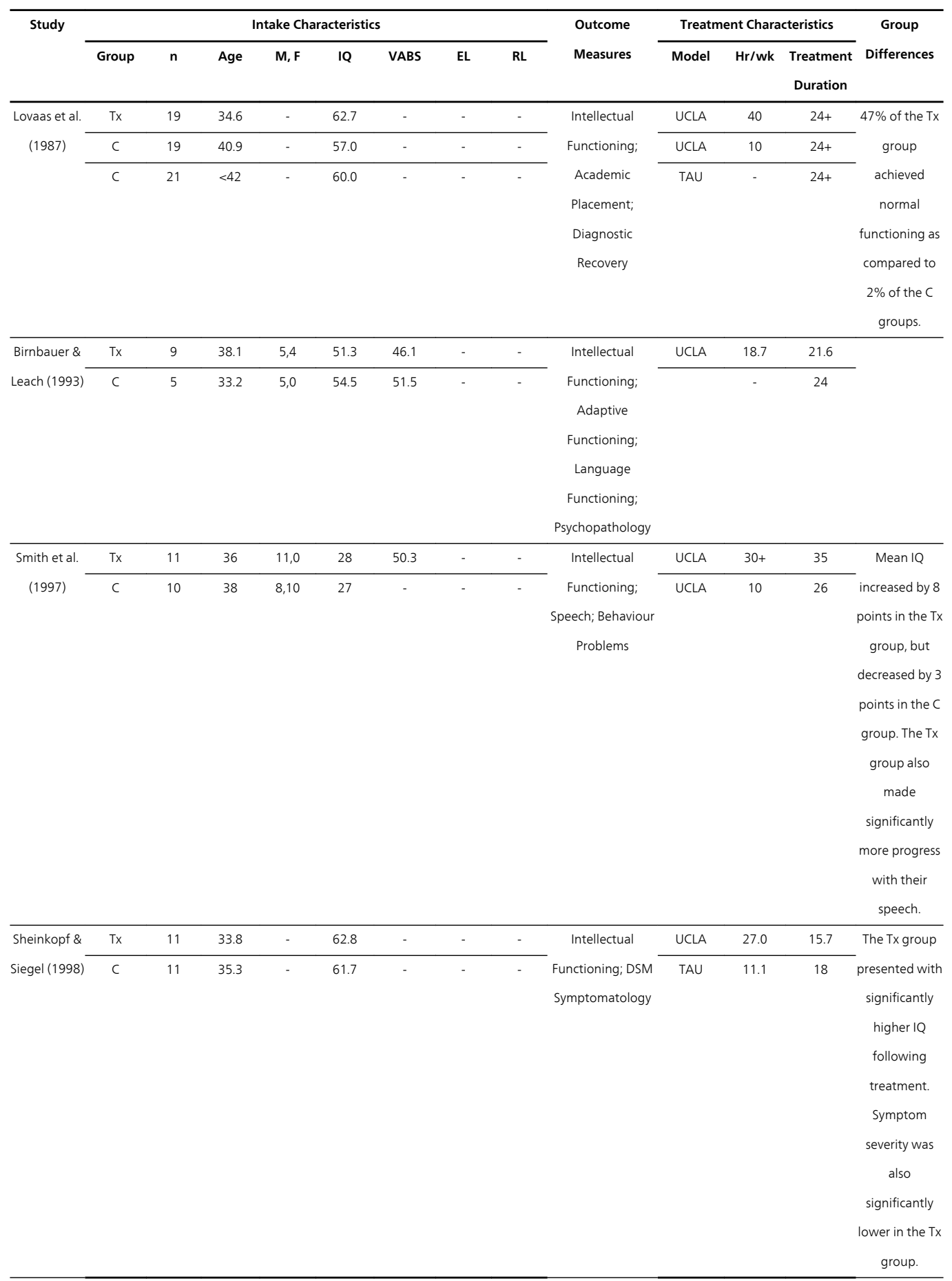

Table 1. Summary of EIBI studies Pre-2000, M, F (male, female), VABS (Vineland Adaptive Behaviour Scale), EL (Expressive Language), RL (Receptive Language) 


\section{Studies published from 2000-2010 (12 Studies)}

Ben-Itzchak et al. (2008) compared the outcomes of 44 children with autism receiving 45 hours of EIBI weekly and 37children with other developmental disabilities receiving TAU. After one year, the children in the EIBI group made significantly greater gains in IQ than the control group. The authors also analysed whether EIBI outcomes were affected by initial cognitive level. Children were categorised as being of normal, borderline, or impaired IQ. They found that baseline cognitive levels did not predict changes in autism symptoms. However, IQ increases due to treatment were correlated with reductions in autism symptoms.

Remington et al. (2007) compared the outcomes of 23 children who received 25.6 hours of EIBI with a control group in which 21 children received an average of 15.3 hours of intervention weekly. After two years of treatment, children in the EIBI group made showed significantly greater increases in mental age, intellectual functioning, language functioning, adaptive functioning and positive social behaviours.

Reed et al. (2007a) compared the impact of high-intensity and low-intensity home-based EIBI. The high-intensity group was composed of 14 children who each received 30.4 hours of intervention per week. There were 13 children in the low-intensity group who each received an average of 12.6 hours of intervention weekly. The high-intensity group made significantly greater gains on measures of intellectual and educational functioning. However, the children in the low-intensity EIBI group did show significant improvements in educational functioning at follow-up.

Reed et al. (2007b) compared the outcomes of children who had received EIBI, "eclectic" intervention, or portage. The 12 children in the EIBI group received an average of 30.4 hours of home-based intervention each week, the 20 children in the "eclectic" group received a mean of 12.7 hours per week, and the 16 children in portage group received 8.5 hours of weekly intervention. At follow-up, the EIBI group outperformed both groups on measures of educational functioning while both the EIBI group and the "eclectic" group scored significantly higher on measures of intellectual functioning than the portage group.

Given the previous considerations, the current study directly compared the impact of existing $\mathrm{ABA}$, special nursery placements, and portage programs on a variety of aspects of the children's abilities. The latter two were selected because special nursery placement is a commonly occurring program offered to children with ASD, which has received little direct assessment in terms of its effectiveness. Portage was chosen as, again, it is increasingly offered to children with ASD (see Reed et al., 2000; Smith, 2000). The portage intervention also allows comparison of a very intensive intervention (ABA) with a less intensive intervention (portage) in a community-based setting. This comparison formed part of the original clinicbased study conducted by Lovaas (1987), and the current comparison allows assessment of the generalization to a community-based sample. However, the intensity of hours of treatment delivery varied greatly between the three interventions and this can make it difficult to "tease out" whether it was the nature of the intervention or simply the duration of treatment that accounted for the differences in outcomes reported. 
Magiati et al. (2007) conducted a prospective comparison of 28 children who received 32.4 hours EIBI each week and 16 children who received 25.6 hours of autism-specific nursery provision each week. The EIBI group received parent-delivered intervention with training and supervision provided by clinicians. At follow-up, both groups achieved similar outcomes although the EIBI group scored significantly higher on the VABS Daily Living Skills subscale.

Eldevik et al. (2006) retrospectively compared the outcomes of 13 children receiving EIBI and 15 children receiving "eclectic" intervention. The EIBI group typically received 12.5 hours of intervention each week. Parent training was also provided to increase maintenance and generalisation of skills. The control group received 12 hours of intervention each week. The EIBI group outperformed the control group on measures of IQ, language functioning, and communication at the follow-up. They also presented with less symptoms of pathology than children in the control group.

Eikeseth et al. (2007) compared the outcomes of 13 children who received 28 hours of EIBI weekly with 12 children who received 29.1 hours of "eclectic" intervention each week. At follow-up, the children who had received EIBI showed significantly greater improvements in IQ, adaptive functioning, and presented with less social and behaviour problems.

Cohen et al. (2006) compared the outcomes of 21 children receiving 35-40 hours of EIBI per week to a control group of 21 children receiving "eclectic" interventions. Parents implementing EIBI received training so that they could use behavioural techniques in the home setting. Following the treatment phase, the EIBI group achieved significantly higher scores on measures of IQ, adaptive functioning, and receptive language. 17 children from the EIBI group transitioned to mainstream education settings as compared to 1 child from the control group.

Sallows and Graupner (2005) compared the effects of clinic-directed EIBI and parent-directed EIBI. This study was the only study we found in our search that directly compared the mode of EIBI delivery. All others either employed an alternate treatment comparison or a control-no treatment comparison. The 13 children in the clinic-directed EIBI group received an average of 37.6 hours of intervention weekly while the10 children in the parent-directed EIBI group typically received 31.6 hours of intervention. Both groups received a UCLAbased intervention (often referred to "Lovaas therapy" based on the original study in 1987). The groups made similar gains on outcome measures suggesting that the less costly parentdirected intervention was equally effective. It was found that $48 \%$ of participants showed rapid learning, achieved normal scores on outcome measures, and, at follow-up, were succeeding in mainstream classrooms. Pre-treatment imitation, language, daily living skills, and socialization were found to be predictive of outcome.

Howard et al. (2005) compared the effects of EIBI, intensive "eclectic" intervention, and low-intensity "eclectic" intervention. The 29 children assigned to the EIBI group received 25-40 hours of EIBI each week and their parents received training so that teaching could extend to the home setting. The 16 children in the intensive "eclectic" intervention group received 25-30 hours of intervention each week, while the 16 children in the low-intensity "eclectic" group received 15 hours of intervention each week. The EIBI group achieved significantly higher scores on measures of intellectual functioning, visual spatial skills, language functioning and adaptive functioning. The outcomes of the two "eclectic" control groups did not differ. 


\begin{tabular}{|c|c|c|c|c|c|c|c|c|c|c|c|c|c|}
\hline \multirow[t]{2}{*}{ Study } & \multicolumn{8}{|c|}{ Intake Characteristics } & \multirow[t]{2}{*}{ Outcome Measures } & \multicolumn{3}{|c|}{ Treatment Characteristics } & \multirow{2}{*}{$\begin{array}{c}\text { Group } \\
\text { Differences }\end{array}$} \\
\hline & Group & $\mathrm{n}$ & Age & $M, F$ & IQ & VABS & EL & $\mathbf{R L}$ & & Model & $\mathrm{Hr} / \mathrm{wk}$ & $\begin{array}{l}\text { Treatment } \\
\text { Duration }\end{array}$ & \\
\hline \multirow{15}{*}{$\begin{array}{l}\text { Smith et al. } \\
\text { (2000) }\end{array}$} & Tx & 15 & 36.1 & 12,3 & 50.5 & 63.4 & 41.9 & 37.3 & Intellectual & UCLA & 24.5 & 33.4 & The Tx group \\
\hline & $C$ & 13 & 35.8 & 11,2 & 50.7 & 65.2 & 45.6 & 38.3 & Functioning; Visual- & UCLA & $15-20$ & 24 & made significantly \\
\hline & & & & & & & & & Spatial Skills; & & & & greater gains in \\
\hline & & & & & & & & & Language & & & & IQ, visual-spatial \\
\hline & & & & & & & & & Functioning; & & & & skills, and \\
\hline & & & & & & & & & Adaptive & & & & language \\
\hline & & & & & & & & & Functioning; & & & & development. The \\
\hline & & & & & & & & & Socioemotional & & & & Tx group tended \\
\hline & & & & & & & & & Functioning; & & & & to make greater \\
\hline & & & & & & & & & Academic & & & & academic \\
\hline & & & & & & & & & Achievement; Class & & & & achievements and \\
\hline & & & & & & & & & Placement; Progress & & & & to be in less \\
\hline & & & & & & & & & in Treatment; Parent & & & & restrictive \\
\hline & & & & & & & & & Evaluation & & & & academic \\
\hline & & & & & & & & & & & & & placements. \\
\hline \multirow{17}{*}{$\begin{array}{c}\text { Eikeseth et al } \\
\text { (2002). }\end{array}$} & Tx & 13 & 66.3 & 8,5 & 61.9 & 55.8 & 45.1 & 49.0 & Intellectual & UCLA & 28.0 & 12.2 & The Tx group \\
\hline & C & 12 & 65.0 & 11,1 & 65.2 & 60.0 & 51.2 & 50.4 & Functioning; Visual- & Eclectic & 29.1 & 13.6 & achieved \\
\hline & & & & & & & & & Spatial Skills; & & & & significantly \\
\hline & & & & & & & & & Language & & & & higher scores that \\
\hline & & & & & & & & & Functioning; & & & & the $\mathrm{C}$ group on all \\
\hline & & & & & & & & & Adaptive & & & & measures, except \\
\hline & & & & & & & & & Functioning & & & & the VABS \\
\hline & & & & & & & & & & & & & socialization \\
\hline & & & & & & & & & & & & & subscale and the \\
\hline & & & & & & & & & & & & & daily living \\
\hline & & & & & & & & & & & & & subscale. Children \\
\hline & & & & & & & & & & & & & in the Tx group \\
\hline & & & & & & & & & & & & & had significantly \\
\hline & & & & & & & & & & & & & fewer disruptive \\
\hline & & & & & & & & & & & & & behaviours than \\
\hline & & & & & & & & & & & & & the $C$ group at \\
\hline & & & & & & & & & & & & & follow-up. \\
\hline \multirow{10}{*}{$\begin{array}{l}\text { Howard et al. } \\
\text { (2005) }\end{array}$} & Tx & 29 & 30.9 & 25,4 & 58.5 & 70.5 & 51.9 & 52.2 & Intellectual & EIBI & $25-40$ & 14.2 & The outcomes of \\
\hline & C & 16 & 37.4 & 13,3 & 53.7 & 69.8 & 43.9 & 45.4 & Functioning; Visual- & Eclectic & $25-30$ & 13.3 & the two eclectic C \\
\hline & C & 16 & 34.6 & 16,0 & 59.9 & 71.6 & 48.8 & 49.0 & Spatial Skills; & Eclectic & 15 & 14.8 & groups did not \\
\hline & & & & & & & & & Language & & & & differ. The Tx \\
\hline & & & & & & & & & Functioning; & & & & group performed \\
\hline & & & & & & & & & Adaptive & & & & significantly \\
\hline & & & & & & & & & Functioning & & & & better on all \\
\hline & & & & & & & & & & & & & measures, except \\
\hline & & & & & & & & & & & & & motor skills than \\
\hline & & & & & & & & & & & & & the $C$ groups. \\
\hline
\end{tabular}


576 Recent Advances in Autism Spectrum Disorders - Volume I

\begin{tabular}{|c|c|c|c|c|c|c|c|c|c|c|c|c|c|}
\hline \multirow[t]{2}{*}{ Study } & \multicolumn{8}{|c|}{ Intake Characteristics } & \multirow[t]{2}{*}{ Outcome Measures } & \multicolumn{3}{|c|}{ Treatment Characteristics } & \multirow{2}{*}{$\begin{array}{c}\text { Group } \\
\text { Differences }\end{array}$} \\
\hline & Group & $n$ & Age & $M, F$ & IQ & VABS & EL & RL & & Model & $\mathrm{Hr} / \mathrm{wk}$ & $\begin{array}{l}\text { Treatment } \\
\text { Duration }\end{array}$ & \\
\hline $\begin{array}{c}\text { Sallows \& } \\
\text { Graupner } \\
(2005)\end{array}$ & $T x$ & 13 & 35.0 & 11,2 & 50.9 & 59.5 & 47.9 & 38.9 & $\begin{array}{l}\text { Intellectual } \\
\text { Functioning; } \\
\text { Language } \\
\text { Functioning; } \\
\text { Adaptive } \\
\text { Functioning; Social } \\
\text { Functioning; } \\
\text { Academic } \\
\text { Functioning }\end{array}$ & UCLA & 37.6 & 48 & $\begin{array}{l}\text { Both Tx groups } \\
\text { performed } \\
\text { similarly on all } \\
\text { outcome } \\
\text { measures. }\end{array}$ \\
\hline $\begin{array}{c}\text { Cohen et al. } \\
\text { (2006) }\end{array}$ & $\frac{T x}{C}$ & $\begin{array}{l}21 \\
21\end{array}$ & \begin{tabular}{|l|}
30.2 \\
33.2
\end{tabular} & \begin{tabular}{|l|}
18,3 \\
17,4
\end{tabular} & $\begin{array}{l}61.6 \\
59.4\end{array}$ & $\begin{array}{l}69.8 \\
70.6\end{array}$ & $\begin{array}{l}52.9 \\
52.8\end{array}$ & $\begin{array}{l}51.7 \\
52.7\end{array}$ & $\begin{array}{c}\text { Intellectual } \\
\text { Functioning; Visual- } \\
\text { Spatial Skills; } \\
\text { Language } \\
\text { Functioning; } \\
\text { Adaptive } \\
\text { Functioning; } \\
\text { Academic } \\
\text { Placement }\end{array}$ & $\begin{array}{c}\text { UCLA } \\
\text { Eclectic }\end{array}$ & $\frac{35-40}{-}$ & 36 & $\begin{array}{l}\text { The Tx group } \\
\text { made significantly } \\
\text { greater gains in } \\
\text { IQ, receptive } \\
\text { language, and } \\
\text { adaptive } \\
\text { functioning. } 17 \\
\text { children from the } \\
\text { Tx group were } \\
\text { included in } \\
\text { mainsteam } \\
\text { education settings } \\
\text { as compared to } 1 \\
\text { child in the C } \\
\text { group. }\end{array}$ \\
\hline $\begin{array}{c}\text { Eldevik et al. } \\
\text { (2006) }\end{array}$ & $\begin{array}{l}\mathrm{Tx} \\
\mathrm{C}\end{array}$ & $\begin{array}{l}13 \\
15\end{array}$ & $\begin{array}{l}53.0 \\
49.0\end{array}$ & $\begin{array}{l}10,3 \\
14,1\end{array}$ & $\begin{array}{l}41.0 \\
47.2\end{array}$ & $\begin{array}{l}52.5 \\
52.5\end{array}$ & $\begin{array}{l}33.8 \\
41.6\end{array}$ & $\begin{array}{l}37.3 \\
33.2\end{array}$ & $\begin{array}{c}\text { Intellectual } \\
\text { Functioning; } \\
\text { Language } \\
\text { Functioning; } \\
\text { Adaptive } \\
\text { Functioning; Visual } \\
\text { Spatial Skills; } \\
\text { Pathology; Degree } \\
\text { of Intellectual } \\
\text { Disability }\end{array}$ & $\frac{\text { UCLA }}{\text { Eclectic }}$ & $\begin{array}{l}12.5 \\
12.0\end{array}$ & $\begin{array}{l}20.3 \\
21.4\end{array}$ & $\begin{array}{l}\text { The Tx group } \\
\text { significantly } \\
\text { outperformed the } \\
\text { C group on } \\
\text { intellectual } \\
\text { functioning, } \\
\text { language } \\
\text { functioning, and } \\
\text { the } \\
\text { communication } \\
\text { subscale of the } \\
\text { VABS. The Tx } \\
\text { group also } \\
\text { showed } \\
\text { significantly less } \\
\text { pathology at the } \\
\text { follow-up }\end{array}$ \\
\hline
\end{tabular}




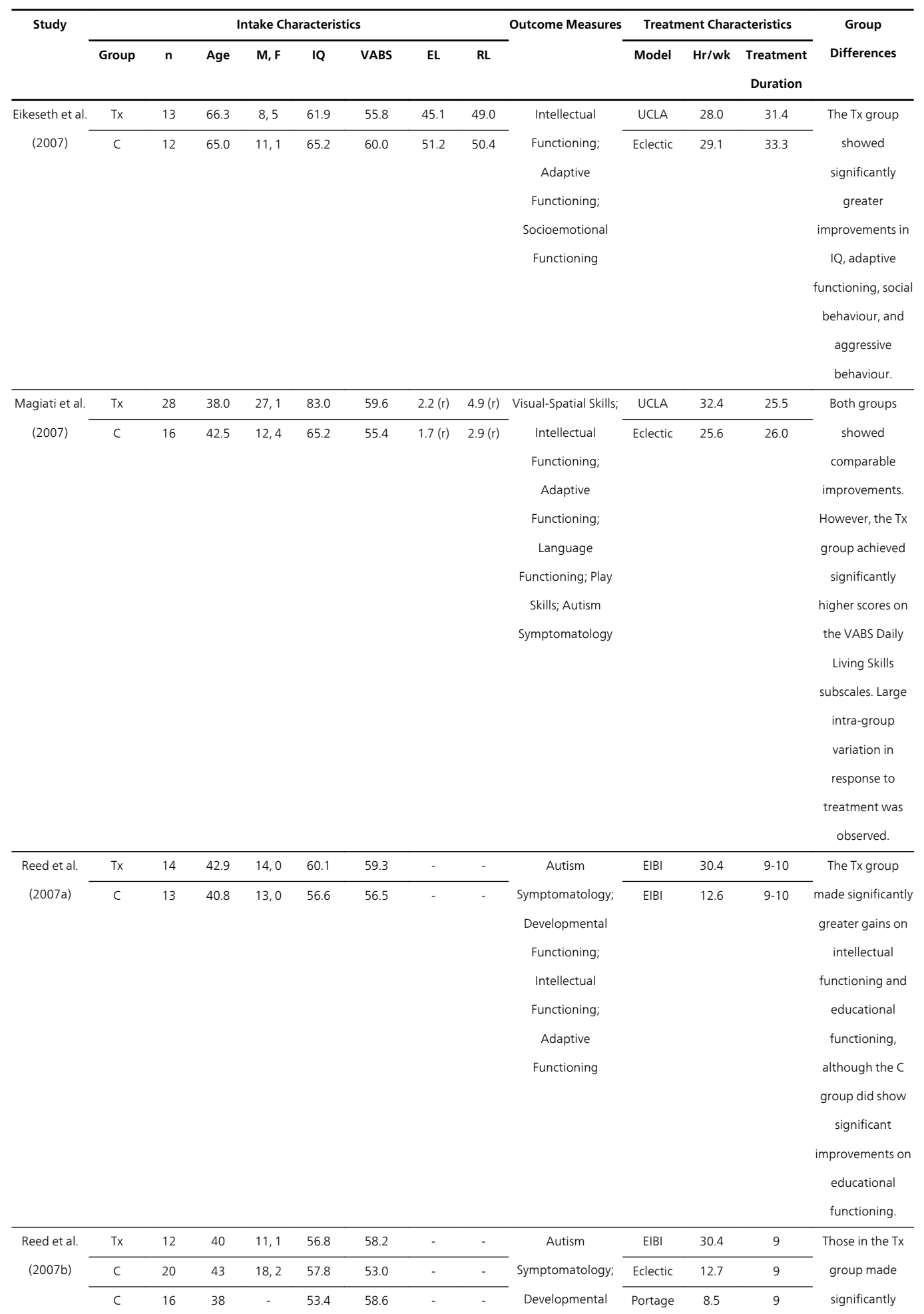




\begin{tabular}{|c|c|c|c|c|c|c|c|c|c|c|c|c|c|}
\hline \multirow[t]{3}{*}{ Study } & \multicolumn{8}{|c|}{ Intake Characteristics } & \multirow[t]{2}{*}{ Outcome Measures } & \multicolumn{3}{|c|}{ Treatment Characteristics } & \multirow{2}{*}{$\begin{array}{c}\text { Group } \\
\text { Differences }\end{array}$} \\
\hline & Group & $\mathrm{n}$ & Age & $M, F$ & IQ & VABS & EL & RL & & Model & Hr/wk & $\begin{array}{l}\text { Treatment } \\
\text { Duration }\end{array}$ & \\
\hline & & & & & & & & & $\begin{array}{l}\text { Functioning; } \\
\text { Intellectual } \\
\text { Functioning; } \\
\text { Adaptive } \\
\text { Functioning; } \\
\text { Comorbid Problems }\end{array}$ & & & & $\begin{array}{l}\text { greater gains than } \\
\text { the portage } \\
\text { group on } \\
\text { intellectual } \\
\text { functioning and } \\
\text { made greater } \\
\text { gains than both C } \\
\text { groups on } \\
\text { educational } \\
\text { functioning. }\end{array}$ \\
\hline $\begin{array}{c}\text { Remington et } \\
\text { al. (2007) }\end{array}$ & $\begin{array}{l}\mathrm{Tx} \\
\mathrm{C}\end{array}$ & $\begin{array}{l}23 \\
21\end{array}$ & $\begin{array}{l}35.7 \\
38.4\end{array}$ & - & $\begin{array}{l}61.4 \\
62.3\end{array}$ & $\begin{array}{l}114.8(r) \\
113.6(r)\end{array}$ & - & - & $\begin{array}{c}\text { Intellectual } \\
\text { Functioning; } \\
\text { Language } \\
\text { Functioning; } \\
\text { Adaptive } \\
\text { Functioning; } \\
\text { Behaviour; } \\
\text { Nonverbal Social } \\
\text { Communication; } \\
\text { Parental Wellbeing }\end{array}$ & $\begin{array}{l}\text { EIBI } \\
\text { TAU }\end{array}$ & $\begin{array}{l}25.6 \\
15.3\end{array}$ & $\begin{array}{l}24 \\
24\end{array}$ & $\begin{array}{c}\text { The Tx group } \\
\text { showed } \\
\text { significantly } \\
\text { greater increases } \\
\text { in mental age, } \\
\text { intellectual } \\
\text { functioning, } \\
\text { language } \\
\text { functioning, } \\
\text { adaptive } \\
\text { functioning, and } \\
\text { positive social } \\
\text { behaviours. }\end{array}$ \\
\hline Ben-Itzchak & $T x$ & 44 & 27.3 & 43,1 & 74.8 & - & - & - & Intellectual & EIBI & 45 & 12 & The Tx group \\
\hline et al. (2008) & c & 37 & 24.2 & 23,14 & 71.0 & - & - & - & $\begin{array}{c}\text { Functioning; Autism } \\
\text { Symptomatology (Tx } \\
\text { group only) }\end{array}$ & TAU & - & 12 & $\begin{array}{l}\text { made significantly } \\
\text { greater gains in IQ } \\
\text { than the C group. }\end{array}$ \\
\hline
\end{tabular}

Table 2. Summary of EIBI studies 2000-2010, M, F (male, female), VABS (Vineland Adaptive Behaviour Scale), EL (Expressive Language), RL (Receptive Language), (r) (raw scores)

Eikeseth et al. (2002) compared the outcomes of EIBI and "eclectic" treatment for children with autism after one year of intervention. The 13 children in the EIBI group received an average of 28 hours of intervention each week in a school setting. Parents were trained for a minimum of four hours each week for three months so that they were able to extend their child's treatment to the home setting. Children in the "eclectic" group received an average of 29.1 hours of intervention each week. Following treatment, the EIBI group outperformed the control group on measures of intellectual functioning, visual-spatial skills, and language functioning. They also engaged in fewer disruptive behaviours than the "eclectic" group. However, the "eclectic" group showed significantly greater increases in adaptive functioning than the EIBI group. 
Smith et al. (2000) evaluated the outcomes of children with autism or pervasive developmental disorder not otherwise specified who were assigned to an EIBI group or parentdelivered behavioural intervention group. The 15 children in the EIBI group received, on average, 24.5 hours of intervention each week delivered by trained student therapists while parents were included in five hours of teaching each week. The 13 children in the parent-delivered behaviour received 15-20 hours of intervention each week. Their parents received bi-weekly training for 3-9 months and a minimum of one hour of supervision each week. At the end of the treatment phase, the EIBI group performed significantly better than the parent-trained group on measures of intellectual functioning, visual-spatial skills, language, and academic functioning. The groups did not differ on measures of adaptive functioning or challenging behaviours. Children with pervasive developmental disorder not otherwise specified tended to respond better to treatment than children with autism.

\section{Studies published between 2011-2012 (5 Studies)}

Strauss et al. (2012) compared the outcomes of 24 children receiving 35 hours of EIBI each week and 20 children receiving 12 hours of a mixed "eclectic" intervention each week after six months of treatment. EIBI was delivered by staff and by parents, following initial comprehensive parent training. At follow-up, the EIBI group outperformed the control group on IQ measures, early language measures, and also showed greater reductions in autism severity. Both groups made significant gains in adaptive behaviour and receptive language. However, it was found that the "eclectic" intervention led to significant reductions in parental stress while parental stress in the EIBI group did not change over the course of treatment.

Flanagan et al. (2012) conducted a retrospective comparison of the outcomes of 61 children receiving EIBI for over two years and 61 children, matched on chronological age, who were on a treatment waitlist. Children in the EIBI group received, on average, 25.8 hours of treatment each week, typically at community treatment centres, and parent training was available and encouraged. The EIBI group made significantly greater gains in intellectual functioning and adaptive function, and scored lower on a measure of autism symptomatology. Furthermore, younger age at treatment onset, and higher adaptive skills, were found to predict better EIBI treatment outcomes.

Eldevik et al. (2012) analysed the outcomes of 31 children receiving EIBI in a mainstream pre-school and 12 children receiving TAU in the form of an "eclectic" mix of interventions. The EIBI group typically received 13.6 hours of intervention each week and parents were encouraged to use behavioural procedures at home to promote generalisation and maintenance. The TAU group received a minimum of five hours of treatment each week. After two years, the EIBI group achieved significantly greater scores on measures of intellectual and adaptive functioning. 


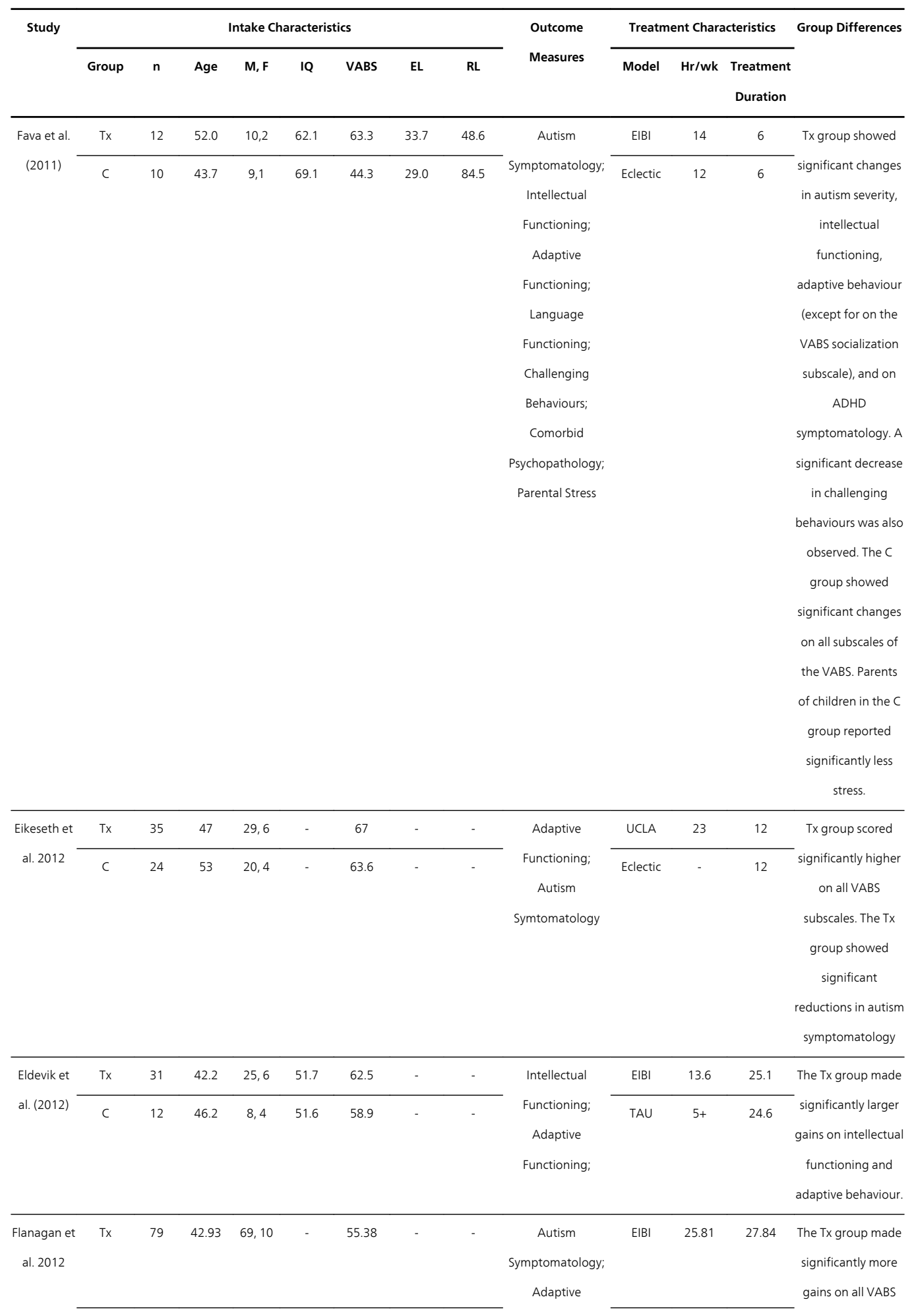




\begin{tabular}{|c|c|c|c|c|c|c|c|c|c|c|c|c|c|}
\hline \multirow[t]{3}{*}{ Study } & \multicolumn{8}{|c|}{ Intake Characteristics } & \multirow{2}{*}{$\begin{array}{l}\text { Outcome } \\
\text { Measures }\end{array}$} & \multicolumn{3}{|c|}{ Treatment Characteristics } & \multirow[t]{2}{*}{ Group Differences } \\
\hline & Group & $\mathrm{n}$ & Age & $\mathbf{M}, \mathbf{F}$ & IQ & VABS & EL & RL & & Model & $\mathrm{Hr} / \mathrm{wk}$ & $\begin{array}{l}\text { Treatment } \\
\text { Duration }\end{array}$ & \\
\hline & Control & 63 & 42.79 & 53,10 & - & 55.49 & - & - & $\begin{array}{l}\text { Functioning; } \\
\text { Intellectual } \\
\text { Functioning }\end{array}$ & $\begin{array}{l}\text { Waitlist } \\
\text { Control }\end{array}$ & - & 17.01 & $\begin{array}{l}\text { subscales. They } \\
\text { achieved } \\
\text { significantly higher } \\
\text { IQ scores and } \\
\text { scored significantly } \\
\text { lower on a measure } \\
\text { of autism } \\
\text { symptomatology. }\end{array}$ \\
\hline $\begin{array}{l}\text { Strauss et } \\
\text { al. } 2012\end{array}$ & $\frac{T x}{C}$ & 23 & $\begin{array}{l}55.67 \\
41.94\end{array}$ & 22,2 & 66.91 & 78.33 & 32.95 & $\begin{array}{l}52.60 \\
47.87\end{array}$ & $\begin{array}{c}\text { Autism } \\
\text { Symptomatology; } \\
\text { Intellectual } \\
\text { Functioning; } \\
\text { Adaptive } \\
\text { Functioning; } \\
\text { Language } \\
\text { Functioning; } \\
\text { Challenging } \\
\text { Behaviours; } \\
\text { Parental Stress }\end{array}$ & $\begin{array}{c}\text { EIBI } \\
\text { Eclectic } \\
\end{array}$ & $\frac{35}{12}$ & $\frac{6}{6}$ & $\begin{array}{l}\text { Tx group showed } \\
\text { significantly greater } \\
\text { gains in intellectual } \\
\text { functioning, } \\
\text { expressive } \\
\text { language, and } \\
\text { social interactions. } \\
\text { They showed } \\
\text { significantly greater } \\
\text { reductions in autism } \\
\text { symptomatology } \\
\text { and challenging } \\
\text { behaviour. Both } \\
\text { groups made } \\
\text { significant gains in } \\
\text { receptive language } \\
\text { and adaptive } \\
\text { behaviour. Parents } \\
\text { in the Tx group } \\
\text { were significantly } \\
\text { more stressed. }\end{array}$ \\
\hline
\end{tabular}

Table 3. Summary of EIBI studies between2011-2012, M, F (male, female), VABS (Vineland Adaptive Behaviour Scale), EL (Expressive Language), RL (Receptive Language)

Eikeseth et al. (2012) examined the outcomes of 35 children receiving EIBI and 24 children receiving TAU after one year of treatment. Children in the EIBI group received 23 hours of intervention per week, on average, and parent training was provided. Children in the "eclectic" group were attending special education settings where teachers incorporated a variety of interventions. The children in the EIBI group made significantly greater gains in adaptive functioning. They also demonstrated reduced autism symptomatology. 
Fava et al. (2011) compared the outcomes of 12 children receiving EIBI and 10 children receiving "eclectic" intervention after six months of treatment. EIBI was delivered by trained therapists, in a clinic-based setting, and by intensively trained and supervised parents, in a home-based setting, with children receiving 14 hours per week on average. Children in the "eclectic" group typically received approximately 12 hours per week. After six months of intervention, the EIBI group showed significantly greater increases in intellectual functioning, and significantly greater decreases in autism symptomatology and challenging behaviour. Both groups, however, showed significant gains in adaptive functioning. Parents in the "eclectic" group showed significant reductions in stress over the course of treatment while no changes in parental stress were observed for the EIBI group.

\section{Challenges to EIBI}

Ongoing analysis of the outcomes of EIBI in comparison to other treatment programs is clearly continuing to capture the interest of many researchers with five studies alone demonstrating outcomes between 2011 and 2012. Indeed, given the growing international recognition of EIBI as the recommended approach to autism intervention, this ongoing investigation and demonstration of effects is vital. Such demonstrations and continuous rigor in testing this approach with children with autism diagnoses, substantiates the view that intensive early intervention using the scientific precision of behaviour analysis, can be a very powerful intervention (Howlin, 2010; Granpeesheh, Tarbox \& Dixon, 2009).

However, despite publication of the numerous studies outlined above, criticism of methodological stringency and dependent variables analysed within and across them, has been documented.

\footnotetext{
"Remarkably, despite thousands of ABA-EIBI studies on specific core deficits, and related challenging behaviours and skills, and EIBI studies as well, some researchers still question the efficacy of these methods" (Matson, Tureck, Turygin, Beighley \& Rieske, 2012, p.1413).
}

One of the most pronounced criticisms of EIBI research for some time is that large multi-element randomized clinical trials are required to provide a definitive scientific demonstration of its effectiveness in autism treatment (Spreckley \& Boyd, 2009). We, and others, (e.g., Keenan \& Dillenberger, 2011; Matson et al. 2012) do not support this view and we encourage the reader to examine an excellent rebuttal of the reasons that the gold standard, randomized controlled trial in research evaluation, is in actual fact inappropriate for the design and evaluation of individualised treatment protocols (see Keenan \&Dillenberger, 2011 for a thorough analysis).

One criticism presented in relation to the overall interpretation of the studies outlined in this chapter involves the issue that large idiosyncratic differences occur across children diagnosed with autism. Because of the extensive discrepant features and their expression across the condition, Howlin (2010) stresses the need to determine which components of the inter- 
vention work best for specific individuals and under what set of circumstances. Smith et al. (2010) also suggest that ongoing research is necessary in identifying key moderating variables in EIBI outcomes. Specifically, they pose the question of what are the most effective components, and the amount of such components, in producing marked changes in core autism symptoms and additional problems. Other researchers have also emphasised this point (Alessandri, Thorp, Mundy, \& Tuchman, 2005; Granpeesheh et al. (2009). For some, determining predictor variables such as personal characteristics affecting outcomes has been a focus. For example, Itzchak and Zachor (2009) demonstrated that the presence of an intellectual disability and significantly delayed adaptive skills in young children with autism was a major risk factor and a predictor of weaker outcomes for EIBI. They also showed that children who were 30 months of age or younger responded significantly better to early intervention. A more recent study by Perry et al. (2011) showed that variables including younger age and higher intellectual functioning at onset of intervention were predictors of greater positive effects. Not surprisingly, Perry et al. (2011) also found that duration of intervention was a predictor of positive outcomes for young children undergoing EIBI- the longer the child participated in the intervention, the better the outcome.

While EIBI programs provide strong adherence to the framework and foundational principles of learning within ABA, some investigators have followed a particular "brand name" approach (Healy, Leader \& Reed, 2010). There are a number of different ABA approaches that have been outlined in a variety of sources (some examples include: Greer, Keohane \& Healy, 2002; Koegel \& Koegel, 2006; Lovaas, 1981; Lovaas \& Smith, 1989; Sundberg \& Michael, 2001). Often this "branding" can lead to obfuscation for the reader in interpreting what "type" of EIBI/ABA program is best. However, these approaches are all built on the same bedrock sharing important common features- intensity in program delivery (up to 40 hours weekly for at least three years), one-to-one teaching where the individual requires such intensive instruction, and discrete-trial reinforcement-based methods (in both massed trial formats and natural environmental teaching opportunities) incorporated within the scientific stringency of a behaviour analytic framework (Matson et al. 2012).

Magiati and Howlin (2001) have argued that many of the EIBI studies employ different measurements across participants and at baseline and follow up thereby compromising interpretation and reliability. For example, Eikeseth et al. (2002) and Howard et al. (2005) did not use the same tests at baseline and at follow up phases. Inconsistencies in participant characteristics across groups (lack of matching: (e.g., Eldevik, Eikeseth, Jahr, \& Smith, 2006; Fenske, Zalenski, Krantz, \& McClannahan, 1985) have also been critiqued within the studies. In addition, different investigators examined various settings for EIBIsome were clinic-based (Ben-Itzchak et al., 2007; Eldevik et al., 2006) others were community-based (Cohen et al., 2006; Eikeseth et al. 2002; Eikeseth et al., 2007; Eikeseth et al., 2012; Eldevik et al., 2012; Flanagan et al., 2012; Howard et al. 2005; Magiati et al., 2007), while others were home-based (Reed et al., 2007a; Reed et al., 2007b; Remington et al., 2007; Sheinkopf \& Siegel, 1998;Smith et al., 2000). This variation in measures/settings across studies may provide challenges in the generalisation of intervention outcomes to different environments (Mudford et al., 2009). 
However, we believe that it is critical to be able to assess the effectiveness of EIBI across participants who may reflect different tracts on the spectrum i.e., those with more severe core autism symptoms, presence of challenging behaviours, less linguistically able; impaired IQ; co-morbid or co-occurring problems etc. In this sense it appears important to utilise a wide range of instruments in the assessment procedure, not only to examine autism severity but also measures of intellectual functioning, adaptive behaviour, challenging behaviour, comorbid psychopathology and educational functioning.

Treatment integrity including initial training of therapists and parents along with continual supervision is often not reported in studies yet many authors have written on the importance of adherence to the scientific rigor of ABA (Symes, Remington, Brown \& Hastings, 2006). While many of the studies reviewed referred to training either for therapists or parents, detail on the fidelity of treatment delivery was not measured. Where some have investigated adherence to strict training protocols, highly effective outcomes can be demonstrated using EIBI (see McGarrell, Healy, Leader, O'Connor \& Kenny, 2009).

Critiques of the initial results reported by Lovaas (1987) concerning the effectiveness of EIBI were dominant amongst the most vociferous arbiters, especially given that exact replication of such results is not evident to date. Indeed, this is one of the greatest challenges faced by many EIBI researchers. The children undergoing EIBI treatment in the Lovaas study made remarkable gains of up to 30 IQ points and were not noticeably different from neuro typical developing children after 3 years of the intervention. Replications of this original study have certainly attempted to address the methodological criticisms by incorporating more rigorous experimental design including random assignment to groups (Sallows and Graupner 2005; Smith et al. 2000). However, studies to date have yet to achieve the extent of the outcomes reported by Lovaas (1987).

It is clear that over time the methodological criticisms of the earlier studies have been addressed by more recent investigators. Some of the recent published studies have employed larger small sample sizes, comparison groups, random assignment of the children to groups, matched characteristics across groups and standardising measures used for assessment between and within children (e.g., Flanagan et al., 2012)

Certainly, consistency in measures at baseline and follow-up has improved with most of the studies published between 2011-2012 implementing the same measures at entry and output for the majority of variables measured (Eikeseth, et al., 2012, Eldevik, et al, 2012; Fava et al., 2011; Flanagan et al., 2012; Strauss et al., 2012). Furthermore, it is worth noting that most recent studies on EIBI are employing a more extensive battery of measures to assess the effects of EIBI- in addition to IQ and adaptive behaviour which was the focus of earlier research. For example, Fava et al. (2011) and Strauss et al. (2012) measured autism symptomatology, language functioning, challenging behaviour, comorbid psychopathology, and parental stress as outcomes of EIBI. Eikeseth et al. (2012) and Flanagan et al. (2012) also examined autism symptomatology as a dependent variable. This focus on increasing evaluation of treatment outcomes is a welcome development in EIBI research. Examining the impact of EIBI on the core symptoms of autism, challenging behaviours and comorbid psychopathology provides an exciting avenue for future research. 
While some authors have provided criticism in response to their interpretation of the EIBI outcome studies summarised within this chapter (e.g., Shea, 2004), others have acknowledged the long-term effects of such an intervention resulting from the best empirically validated interventions (e.g., Granpeesheh, Tarbox \& Dixon, 2009).

Prior to 2009 six EIBI descriptive review papers were published each analysing methodologies, variables and outcomes from different perspectives (e.g., Eikeseth 2009; Granpeesheh et al. 2009; Howlin, Magiati \& Charman, 2009; Matson and Smith 2008; Reichow \& Wolery, 2009; Rogers and Vismara, 2008). As well as these research reviews, Eldevik et al. (2010) gathered individual participant data from 16 group design studies on behavioural intervention for children with autism, resulting in individual participant data for 309 participants in an EIBI group, 39 participants in an alternate treatment comparison group, and 105 in a control group-no treatment group. Their analysis revealed that more children who underwent behavioral intervention achieved significantly greater change in IQ and adaptive behaviour compared with the comparison and control groups (see Eldevik et al. 2010). We encourage the reader to examine these papers in order to discern the conventional acclaim of EIBI as an acknowledged intervention for ASD.

More importantly, since 2009 EIBI research for young children with ASD has been subject to six meta-analytic reviews (Eldevik et al. 2009; Makrygianni and Reed 2010; Reichow and Wolery 2009; Peters-Scheffer, Didden, Korzilius \& Sturmey, 2011; Spreckley and Boyd 2009; Virue's-Ortega, 2010). A meta-analysis is a particular type of statistical method for integrating results from many individual studies. This type of statistic can be useful for obtaining an overall estimate of whether or not an intervention is effective and, if so, what the size of the benefits are (i.e., the effect size). The overwhelming findings from five of the six meta-analyses conducted between 2009 to 2012 (Eldevik et al. 2009; Makrygianni and Reed 2010; PetersScheffer et al., 2011; Reichow \& Wolery 2009; Virue's-Ortega 2010) concluded that EIBI was an effective intervention strategy for many children with ASD, accelerating development, improving IQ and adaptive skills compared to those receiving no intervention or alternate diverse standard care treatments.

Most recently, Reichow (2012) presented an overview of the five meta-analyses on EIBI for young children with ASD. He concluded that the collective and accumulating evidence supporting EIBI from meta-analytic studies cannot be dismissed. Reichow's impressive dissection of the investigations of EIBI to date achieves the following assertion:

"Furthermore, the current evidence on the effectiveness of EIBI meets the threshold and criteria for the highest levels of evidencebased treatments across definitions ... Collectively, EIBI is the comprehensive treatment model for individuals with ASDs with the greatest amount of empirical support and should be given strong consideration when deciding deciding treatment options for young children with ASDs" (Reichow, 2012, p. 518.) 


\section{Screening for ASD and EIBI provision}

It is accepted in the field of autism that there now exists enough evidence to recognise the disorder at a very early age (Feldman et al. 2012; Matson, Boisjoli, Rojahn, \& Hess, 2009). While many screening instruments exist for the disorder, the most thoroughly examined of these is the BISCUIT (Matson et al., 2009; Matson, Fodstad, \& Mahan, 2009; Matson, Fodstad, Mahan, \& Sevin, 2009; Matson, Wilkins, Sevin, et al., 2009; Matson, Wilkins, Sharp, et al., 2009). In addition to providing clinicians with a measure of the very early signs of autism symptomology, the BISCUIT also provides a measure of emotional/behavioural disorders and comorbid psychopathology. We believe that providing EIBI to young infants showing early signs of autism, before the condition is fully manifest, will target core skills by accelerating developmental sequences, halting deteriorating behavioural repertoires, and preventing additional secondary problems. Provision of EIBI at the time when symptoms are initially detected, may in tandem, alter the course of early behavioural and brain development increasing the likelihood that children attain a rate of typical development (Dawson, 2008).

We advocate for the need to screen children for this disorder during routine health and developmental checks. Screening in Ireland is currently haphazard and often depends on a parent showing concern for some area of their child's development. In particular, prevention entails detecting infants at risk before the full diagnostic criteria are present and it has been recognised that early signs may emerge as soon as 9 months in infants with siblings who have ASD (Ozonoff et al. 2010; Zwaigenbaum et al. 2005). Screening these biologically "at risk" children in early infancy should allow greater access to the effective methods demonstrated by EIBI. We strongly believe that the availability of both standardised screening techniques and EIBI provision to such children will impact on a more promising prognosis in the long-term.

\section{The benefits of EIBI}

There is no doubt that the cost of an intensive and accomplished EIBI program is expensive. For example, cost analysis studies revealed that the average annual cost of an EIBI program in North America to be $\$ 33,000$ per year with the average duration being three years (Jacobson, Mulick \& Green, 1998). However, further analysis of this cost-effectiveness and saving over time has also been provided. For instance, the Autism Society of America reported in 2008 that the cost of lifelong care could be reduced by up to as much as two thirds with early diagnosis and EIBI.

Dillenberger (2011) provides a synopsis of recent cost-benefit analyses showing the savings that can be achieved by implementation of EIBI in autism treatment. She puts forward the following:

1. in Ontario, Canada, an estimated annual CA $\$ 45$ million can be saved if EIBI is made available to all children diagnosed with ASD (see Motiwala et al., 2006); 
2. in Texas, USA, a total of US $\$ 208,500$ per child is saved by the education system through the use of EIBI (see Chasson, Harris \& Neely (2007);

3. and in Pennsylvania, USA, average savings per child are estimated even higher to range from US\$274,700 to US\$282,690 (see also Chasson, Harris \& Neely (2007).

Based on these cost-saving analyses increasing change has been shown in policy regarding the role of EIBI in early intervention. For example, the state of Ontario in Canada, has legislated to make EIBI services available for all children diagnosed with ASD (Perry \& Condillac, 2003). In the USA, 32 States have passed legislation to ensure that ABAbased interventions are either state-funded or provided through medical insurance companies (Dillenberger, 2011; Market Watch, 2012). It remains to be seen whether government policy in the United Kingdom or Ireland will catch up with that of Canada and the USA and provide government funded EIBI once children are deemed at risk for or indeed presenting with this condition. Interestingly, the use of trained volunteers to deliver EIBI has been shown to produce effective outcomes (Birnbrauer \& Leach, 1993) and may be an option for some parents/services to consider when cost is an issue. Many university students who train on third level post-graduate programmes in Applied Behaviour Analysis could make strong contributions in a voluntary capacity, to EIBI in autism treatment, as part of their ongoing accreditation process as Board Certified Behaviour Analysts with the international certification body (Behaviour Analyst Certification Board $($ ) $)$. Alternatively providing parents of children with autism with training in behavioural interventions (demonstrated by Sallows and Graupner, 2005) can result in cost-saving and important positive outcomes for children with autism.

\section{Controversies related to EIBI efficacy}

The published studies outlined in this chapter highlight the possible positive outcomes for young children diagnosed with autism. EIBI continues to be investigated internationally as a treatment intervention for this condition and as a result of these investigations attracts many critics and controversies. In the past, some authors have criticised a behavioural approach to autism intervention with regard to "robotic" teaching and behaviour patterns that lack generalisation to naturalistic settings (Jordan, Jones \& Murray, 1998; Shea, 2004) along with the use of negative consequences in acquisition teaching and behaviour reduction (Carr, Robinson \& Palumbo, 1990). Others have highlighted the concerns with regard to claims of "recovery" or a "cure" for autism (Offit, 2008). However, the improvements shown over the last decade in EIBI refinement and provision, particularly with regard to training and regulatory protocols with its delivery (Behavior Analyst Certification Board $\AA$, 2012) has addressed many of these issues. Indeed, professional training in behaviour analysis and behavioural intervention has never been as well regulated as it is today.

No doubt there are still many issues that continue to require analysis in the EIBI and autism field of research. We would like to draw the reader's attention to a recent publication by Matson and Smith (2008) providing an analysis of the current status of intensive behavioural 
intervention for young children with autism. We believe that this paper provides an excellent summary of the criticisms provided on EIBI and we will highlight these here. Firstly, many of the studies providing analysis of EIBI outcomes fail to report the severity of ASD across participants and groups. This makes it difficult to decipher which children will show greatest susceptibility to the intervention. Those with greater severity of symptoms may show slower progress or less gains. It has been reported that a milder degree of autism is related to better prognosis (e.g., Bartak \& Rutter, 1976) and therefore it is essential that variables at intervention onset include such a measure. Secondly, Matson and Smith (2008) highlight the fact that researchers often do not take into account the additional, co-morbid, problems that present with autism (e.g., ADHD symptoms or anxiety disorders). Psychopathological problems can co-occur with the condition and may exacerbate the challenges and deficits for many children. The impact this can have on treatment susceptibility is underreported and often not addressed in treatment research. For example, only two studies in our review provided outcome measures of co-morbid psychopathology (Birnbrauer \& Leach, 1993; Fava, 2011). Matson and Smith (2008) provide a strong argument for the assessment of psychopathology before, during, and after EIBI, to determine ongoing changes in child profiles or to address any required adjustments to the delivery of EIBI (e.g., increasing or decreasing the duration of intervention, removing skills acquisition teaching from artificial environments, less emphasis on massed trial instruction etc.). Perhaps not enough attention has been given to these issues in EIBI research. The young age of onset of EIBI and the intensity of the intervention may have undesired side effects such as anxiety, stress, "burn out" or indeed refusal to participate. Other controversial issues involving EIBI include parent and sibling involvement which can often induce stress and family strain when highly intensive intervention is provided within the family home. The negative side effects of this kind of intensive intervention certainly warrant separate analysis.

Unfortunately, like any professional practice or therapeutic intervention, there will be those who claim to provide EIBI without adhering to the scientific demonstrations of what is, and is not, effective within an intervention protocol. We have heard of anecdotal accounts of the applications of behavioural interventions in autism treatment that are outdated and often lack individualisation. Treatment fidelity is often a major problem in the field and often authors fail to demonstrate or report adherence to effective and current practice in many of the published studies on EIBI. Such problems can lend support to a negative view of the use of EIBI with young children with autism diagnoses.

An analysis of changes in adaptive functioning of young children has become an added focus of EIBI studies in more recent years. Traditionally, studies tended to focus on changes in intellectual and social functioning and language and communication abilities. Some authors have criticised EIBI for overly focusing on cognitive skills with 1:1 teacher/student ratios and a focus on desk-top instruction and intensive "drills" (e.g., Shea, 2004). Increasingly, EIBI curricula and instructional protocols have grown to ensure inclusion of adaptive skills teaching and acquisition of novel skills in natural environments. Studies evaluating outcomes of EIBI have also focused more on adaptive functioning changes as a result of the intervention. In 2002, Eikseth et al. reported greater increases in adaptive functioning in a 
group of young children who received "eclectic" intervention than those receiving EIBI. Furthermore, Fava et al. (2011) and Strauss et al. (2012) showed that both groups receiving EIBI and "eclectic" intervention showed significant gains in adaptive functioning. Two more recent studies by Eldevik et al.(2012) and Eikseth et al., (2012) reported the opposite findings to Eikseth et al. (2002) in relation to adaptive functioning when comparing both interventions.

Another variable that has been increasingly analysed in early intervention autism research includes parental stress. Interestingly, two comparison studies (Fava et al., 2011; Strauss et al., 2012) showed significant reductions in parental stress for those parents whose children were receiving "eclectic" intervention. The same effect was not shown for parents of children receiving EIBI. This is another important area of analysis particularly in light of the demands that EIBI places on parents and family.

\section{Conclusion}

EIBI as an approach to autism treatment is one of the most intensively analysed interventions in paediatric clinical psychology (Matson \& Smith, 2008).

Substantial objective evidence for EIBI has been demonstrated at an experimental, descriptive and meta-analytic level of analysis (Reichow, 2012). We support the contention of many authors in the field of autism treatment, that EIBI prevails by adhering to a principle of evidence-based practice, incorporating standardised objective measurement of outcomes along with implementation of robust experimental design. This robust demonstration of effectiveness is driving policy change on the international stage and some authors (e.g., Dawson, 2008) suggest that one of the most important goals of investigations in the domains of autism and behaviour analysis research, is to become more effective communicators of scientific findings to the general public/government bodies/advocacy groups/related professionals, not only to harvest their support, but to ensure the dissemination of accurate and effective intervention to so many who require it.

\section{Author details}

Olive Healy and Sinéad Lydon

National University of Ireland, Galway

\section{References}

[1] Alessandri, M., Thorp, D., Mundy, P., \& Tuchman, R. F. (2005). Can we cure autism? From outcome to intervention. Revista de Neurologia, 40, S131-S136. 
[2] Anderson, S. R., Avery, D. L., DiPietro, E. K., Edwards, G. L., \& Christian, W. P. (1987). Intensive home-based early intervention with autistic children. Education and Treatment of Children, 10, 353-366.

[3] Autism Treatment Acceleration Act (ATAA). (2010). Summary of the Autism Treatment Acceleration Act of 2009 (ATAA) (S. 819 and H.R. 2413) Retrieved from http:// autismtreatmentaccelerationact.org/4.html.

[4] Behavior Analyst Certification Board® (2012). http://www.bacb.com.

[5] Baer, D. M., Wolf, M. M. \& Risley, T. R. (1968). Some current dimensions of applied behavior analysis. Journal of Applied Behavior Analysis, 1, 91-97.

[6] Baer, D. M., Wolf, M. M. \& Risley, T. R. (1987). Some still-current dimensions of applied behavior analysis. Journal of Applied Behavior Analysis, 20, 313-317.

[7] Ben-Itzchak, E., Lahat, E., Burgin, R., \& Zachor, A. D. (2008). Cognitive, behavior and intervention outcome in young children with autism. Research in Developmental Disabilities, 29, 447-458.

[8] Birnbrauer, J. S., \& Leach, D. J. (1993). The Murdoch early intervention program after 2 years. Behaviour Change, 10, 63-74.

[9] Carr, E.G., Robinson, S., \& Palumbo, L.W. (1990). The wrong issue: Aversive versus nonaversive treatment. The right issue: Functional versus non-functional treatment. In A. Repp \& N. Singh (Eds.), Perspectives on the use of nonaversive and aversive interventions for persons with developmental disabilities (pp. 361-379). Sycamore, IL: Sycamore Publishing Co.

[10] Chasson, G. S., Harris, G. E., \& Neely, W. J. (2007). Cost comparison of early intensive behavioural intervention and special education for children with autism. Journal of Child and Family Studies, 16, 401-413.

[11] Cohen, H., Amerine-Dickens, M., \& Smith, T. (2006). Early intensive behavioral treatment: Replication of the UCLA model in a community setting. Developmental and Behavioral Pediatrics, 27, S145-S155.

[12] Cooper, J. O., Heron, T. E., \& Heward, W. L. (2007). Applied behavior analysis (2nd ed.). Upper Saddle River, NJ: Prentice Hall.

[13] Dawson, G. (2008). Early behavioral intervention, brain plasticity, and the prevention of autism spectrum disorder. Development and Psychopathology 20, 775-803.

[14] Dillenberger, K. (2011). The Emperor's new clothes: Eclecticism in autism treatment. Research in Autism Spectrum Disorders, 5, 1119-1128.

[15] Eikeseth, S. (2009). Outcome of comprehensive psycho-educational interventions for young children with autism. Research in Developmental Disabilities, 30, 158-178.

[16] Eikeseth, S., Hayward, D., Gale, C., Gitlesen, J. P., \& Eldevik, S. (2009). Intensity of supervision and outcome for preschool aged children receiving early and intensive 
behavioral interventions: A preliminary study. Research in Autism Spectrum Disorders, 3, 67-73. doi: 10.1016/j.rasd.2008.04.003

[17] Eikeseth, S., Klintwall, L., Jahr, E., \& Karlsson, P. (2012). Outcome for children with autism receiving early and intensive behavioral intervention in mainstream preschool and kindergarten settings. Research in Autism Spectrum Disorders, 6, 829-835.

[18] Eikeseth, S., Smith, T., Jahr, E., \& Eldevik, S. (2002). Intensive behavioral treatment at school for 4- to 7- year old children with autism: A 1-year comparison controlled study. Behavior Modification, 26, 49-68.

[19] Eikeseth, S., Smith, T., Jahr, E., \& Eldevik, S. (2007). Outcome for children with autism who began intensive behavioral treatment between ages 4 and 7: A comparison controlled study. Behavior Modification, 31, 264-278.

[20] Eldevik, S., Hastings, R. P., Hughes, C., Jahr, E., Eikeseth, S., \& Cross, S. (2009). Metaanalysis of early intensive behavioral intervention for children with autism. Journal of Clinical Child and Adolescent Psychology, 38, 439-450.

[21] Eldevik, S., Eikeseth, S., Jahr, E., \& Smith, T. (2006). Effects of low-intensity behavioral treatment for children with autism and mental retardation. Journal of Autism and Developmental Disorders, 36, 211-224.

[22] Eldevik, S., Hastings, R. P., Jahr, E., \& Hughes, J. C. (2012). Outcomes of behavioral interventions for children with autism in mainstream pre-school settings. Journal of Autism and Developmental Disorders, 42, 210-220.

[23] Eldevik, S., Hastings, R. P., Hughes, J. C., Jahr, E., Eikeseth, S., \& Cross, S. (2010) Using participant data to extend the evidence base for intensive behavioral intervention for children with autism. American Journal on Intellectual and Developmental Disabilities, 115, 381-405.

[24] Fava, L., \& Strauss, K. (2011). Cross-setting complementary staff and parent-mediated Early Intensive Behavioral Intervention for young children with autism: Aresearch-based comprehensive approach. Research in Autism Spectrum Disorders, 5, 512 522.

[25] Fava, L., Strauss, K., Valeri, G., D’Elia, L., Arima, S., \& Vicari, S. (2011). The effectiveness of a cross-setting complementary staff- and parent-mediated early intensive behavioral intervention for young children with ASD. Research in Autism Spectrum Disorders, 5, 1479-1492.

[26] Feldman M.A., Ward R.A., Savona D., Regehr, K., Parker, K., Hudson, M., Penning, H. \& Holden, J.J.A. (2012). Development and initial validation of a parent report measure of the behavioral development of infants at risk for autism spectrum disorders. Journal of Autism and Developmental Disorders, 42, 13-22.

[27] Fenske, E. C., Zalenski, S., Krantz, P. J., \& McClannahan, L. E. (1985). Age at intervention and treatment outcome for autistic children in a comprehensive intervention program. Analysis and Intervention in Developmental Disabilities, 5, 49-58. 
[28] Flanagan, H. E., Perry, A., \& Freeman, N. L. (2012). Effectiveness of large-scale community-based intensive behavioral intervention: A waitlist comparison study exploring outcomes and predictors. Research in Autism Spectrum Disorders, 6, 673-682.

[29] Granpeesheh, D., Tarbox, J., \& Dixon, D. R. (2009). Applied behavior analytic interventions for children with autism: A description and review of treatment research. Annals of Clinical Psychiatry: Official Journal of the American Academy of Clinical Psychiatrist, 21, 162-173.

[30] Green, G. (1996). Early behavioral intervention for autism: What does research tell us? In C. Maurice, G. Green, \& S. Luce (Eds.). Behavioral intervention for young children with autism: A manual for parents and professionals (pp. 29- 44). Austin, TX: PRO-ED.

[31] Greer, R.D. (2002). Designing teaching strategies: an applied behavior analysis systems approach. Academic Press: Dan Diego, CA.

[32] Greer, R. D., Keohane, D. D. \& Healy, O. (2002). Quality and applied behavior analysis. The Behavior Analyst Today, 3 (2), 120-132. www.behavior-analyst-today.org.

[33] Gresham, F.M. \& MacMillan, D.L. (1998). Early intervention project: Can its claims be substantiated and its effects replicated? Journal of Autism and Developmental Disorders, 28. 5-13.

[34] Harris, S. L., Handleman, J. S., Gordon, R., Kristoff, B., \& Fuentes, F. (1991). Changes in cognitive and language functioning of preschool children with autism. Journal of Autism and Developmental Disorders, 21, 281-290.

[35] Healy, O., Leader, G., \& Reed, P. (2009). Applied Behavior Analysis and the Treatment of Autism Spectrum Disorders in the Republic of Ireland. In Leon V. Berhardt (Ed.) Advances in Medicine and Biology, Volume 8. Nova Science Publishers.

[36] Howlin, P. (2010). Evaluating psychological treatments for children with autismspectrum disorders. Advances in Psychiatric Treatment, 16, 133-140.

[37] Hoyson, M., Jamieson, B., \& Strain, P. S. (1984). Individualized group instruction of normally developing and autistic-like children: The LEAP Curriculum Model. Journal of the Division for Early Childhood, 8, 157-172.

[38] Howard, J. S, Sparkman, C. R., Cohen, H. G., Green, G., \& Stanislaw, H. (2005). A comparison of intensive behavior analytic and eclectic treatment for young children with autism. Research in Developmental Disabilities, 26, 359-383.

[39] Howlin, P., Magiati, I., \& Charman, T. (2009). A systematic review of early intensive behavioural interventions (EIBI) for children with autism. American Journal on Intellectual and Developmental Disabilities, 114, 23-41.

[40] Jacobson, J. W., Mulick, J. A., \& Green, G. (1998). Cost-benefit estimates for early intensive behavioral intervention for young children with autism: General model and single state case. Behavioral Interventions, 13, 201-226. 
[41] Jordan R, Jones G, Murray D. (1998). Educational Interventions for Children with Autism: A literature review of recent and current research. School of Education, University of Birmingham, UK.

[42] Keenan, M. \& Dillenberger, K. (2011). When all you have is a hammer ... : RCTs and hegemony in science. Research on Autism Spectrum Disorders, 1-13.

[43] Kelley, E., Naigles, L., \& Fein, D. (2010). An in-depth examination of optimal outcome children with a history of autism spectrum disorders. Research in Autism Spectrum Disorders, 4, 526-538.

[44] Klintwall, L., \& Eikeseth, S. (2012). Number and controllability of reinforcers as predictors of individual outcome for children with autism receiving early and intensive behavioral intervention: A preliminary study. Research in Autism Spectrum Disorders, 6, 493-499.

[45] Koegel, Robert L. and Lynn Kern Koegel (2006). Pivotal Response Treatments for Autism: Communication, Social, and Academic Development. Baltimore, Md.: Paul H. Brookes.

[46] Kovshoff, H., Hastings, R. P., \& Remington, B. (2011). Two-year outcomes for children with autism after the cessation of early intensive behavioral intervention. Behavior Modification, 35, 427-450.

[47] Lang, R., O’Reilly, M., Healy, O., Rispoli, M., Lydon, H., Streusand, W., Davis, T., Kang, S., Sigafoos, J., Lancioni, G., Didden, R., \& Giesbers, S. (2012). Sensory integration therapy for autism spectrum disorders: A systematic review. Research in Autism Spectrum Disorders, 6, 1004-1018

[48] Larsson, E. (2005). Resources for parents. In M. Keenan, M. Henderson, K.P. Kerr, \& K. Dillenburger. Applied behaviour analysis and autism. Building a future together (pp. 255-287). London: Jessica Kingsley Publishers.

[49] Lovaas, O. I. (1981). Teaching developmentally disabled children: The me book. Baltimore: University Park.

[50] Lovaas, O. I. (1987). Behavioral treatment and normal educational and intellectual functioning in young autistic children. Journal of Consulting and Clinical Psychology, 55, 3-9.

[51] Lovaas, O. I., Koegel, R., Simmons, J. Q., \& Long, J. S. (1973). Some generalization and follow-up measures on autistic children in behavior therapy. Journal of Applied Behavior Analysis, 6, 131-166.

[52] Lovaas, O. I., \& Smith, T. (1989). A comprehensive behavioraltheory of autistic children: Paradigm for research andtreatment. Journal of Behavior Therapy and Experimental Psychiatry, 20, 17-29. 
[53] Love, J. R., Carr, J. E., Almason, S. M., \& Petursdottir, A. I. (2009). Early and intensive behavioral intervention for autism: A survey of clinical practices. Research in Autism Spectrum Disorders, 3, 421-428.

[54] Magiati, I., Charman, T., \& Howlin, P. (2007). A two-year prospective follow-up study of community-based early intensive behavioural intervention and specialist nursery provision for children with autism spectrum disorders. Journal of Child Psychology and Psychiatry, 48, 803-812.

[55] Magiati, I., \& Howlin, P. (2001). Monitoring the progress of preschool children with autism enrolled in early intervention programmers. Autism, 5, 399-406.

[56] Market Watch (2012). http://www.marketwatch.com/story/department-of-defenseand-tri-care-ordered-to-provide-applied-behavioral-therapy-to-autistic-children-ofmilitary-dependents-2012-07-26.

[57] Makrygianni, M. K., \& Reed, P. (2010). A meta-analytic review of the effectiveness of behavioural early intervention programs for children with autism spectrum disorders. Research in Autism Spectrum Disorders, 4, 577-593.

[58] Matson, J. L. Benavidez, D. A., Compton, L. S., Paclawskyj, T. R., \& Baglio, C. S. (1996). Behavioral treatment for autistic persons: A review of research from 1980 to the present. Research in Developmental Disabilities, 17, 433-466.

[59] Matson, J. L., Boisjoli, J., Rojahn, J., \& Hess, J. (2009). A factor analysis of challenging behaviors assessed with the Baby and Infant Screen for Children with aUtIsm Traits (BISCUIT-Part 3). Research in Autism Spectrum Disorders, 3, 714-722.

[60] Matson, J. L., Fodstad, J. C., \& Mahan, S. (2009). Cutoffs, norms, and patterns of comorbid difficulties in children with developmental disabilities on the Baby Infant Screen for Children with aUtIsm Traits (BISCUIT-Part 2). Research in Developmental Disabilities, 30, 1221-1228.

[61] Matson, J. L., Fodstad, J. C., Mahan, S., \& Sevin, J. A. (2009). Cutoffs, norms, and patterns of comorbid difficulties in children with ASD on the Baby Infant Screen for Children with aUtIsm Traits (BISCUIT-Part 2). Research in Autism Spectrum Disorders, 3, 977-988.

[62] Matson, J. L., Wilkins, J., Sevin, J. A., Knight, C., Boisjoli, J. A., \& Sharp, B. (2009). Reliability and item content of the Baby and Infant Screen for Children with aUtIsm Traits (BISCUIT): Part 1-3. Research in Autism Spectrum Disorders, 3, 336-344.

[63] Matson, J. L., \& Smith, K. R. M. (2008). Current status of intensive behavioral interventions for young children with autism and PDD-NOS. Research in Autism Spectrum Disorders, 2, 60-74.

[64] Matson, J.L., Tureck, K., Turygin, N., Beighley, J., Rieske, R. (2012). Trends and topics in Early Intensive Behavioral Interventions for toddlers with autism. Research in Autism Spectrum Disorders, 6, 1412-1417. 
[65] McEachin, J. J., Smith, T., \& Lovaas, O. I. (1993). Long-term outcome for children with autism who received early intensive behavioral treatment. American Journal on Mental Retardation, 97, 359-391.

[66] McGarrell, M., Healy, O., Leader, G., O'Connor, J., \& Kenny, N. (2009). Six reports of children with autism spectrum disorder following intensive behavioural interventions using the Preschool Inventory of Repertoires for Kindergarten (PIRRK®). Research in Autism Spectrum Disorders, 3, 767-782.

[67] Metz, Mulick, J., and Butter, E. (2005). Autism: A late-20th-century fad magnet. In J. W. Jacobson, R. M. Foxx, and J. A. Mulick (Eds.), Controversial therapies for developmental disabilities: Fad, fashion, and science in professional practice. Mahwah, New Jersey: Lawrence Erlbaum Associates.

[68] Motiwala, S. S., Gupta, S., Lilly, M. B., Ungar, W. J., \& Coyte, P. C. (2006). The costeffectiveness of expanding intensive behavioural intervention to all autistic children in Ontario. Health Policy, 1, 135-151.

[69] Mudford, O., et al., (2009). Technical review of published research on applied behaviour analysis interventions for people with autism spectrum disorders: Auckland Uniservices Ltd. Wellington, New Zealand: Ministry of Education.

[70] Mulloy, A., Lang, R., O’Reilly, M., Sigafoos, J., Lancioni, G., \& Rispoli, M. (2010). Gluten-free and casein-free diets in the treatment of autism spectrum disorders: A systematic review. Research in Autism Spectrum Disorders, 4, 328-339.

[71] Mundy, P., Sullivan, L., \& Mastergeorge, A. (2009). A parallel and distributed-processing model of joint attention, social cognition and autism. Autism research, 2, 2-21.

[72] Offit, Paul (2008). Autism's False Prophets: Bad Science, Risky Medicine, and the Search for a Cure. Columbia University Press: New York.

[73] Ozonoff, S., Iosif, A.-M., Baguio, F., Cook, I. C., Hill, M. M., Hutman, T., et al. (2010). A prospective study of the emergence of early behavioral signs of autism. Journal of the American Academy of Child \& Adolescent Psychiatry, 49(3), 258-268.

[74] Perry, A., \& Condillac, R. (2003). Evidence-based practices for children and adolescents with autism spectrum disorders: Review of the literature and practice guide. Ontario, Canada: Children's Mental Health Ontario.

[75] Perry, A., Cummings, A., Geier, J. D., Freeman, N. L., Hughes, S., Managhan, T., et al. (2011). Predictors of outcome for children receiving intensive behavioral intervention in a large community-based program. Research in Autism Spectrum Disorders, 5, 592603.

[76] Peters-Scheffer, N., Didden, R., Korzilius, H., \& Matson, J. (2012). Cost comparison of early intensive behavioral intervention and treatment as usual for children with autism spectrum disorder in the Netherlands. Research in Developmental Disabilities, 33, 1763-1772. 
[77] Reed, P., Osborne, L. A., \& Corness, M. (2007a). Brief report: Relative effectiveness of different home-based behavioral approaches to early teach intervention. Journal of Autism and Developmental Disorders, 37, 1815-1821.

[78] Reed, P., Osborne, L. A., \& Corness, M. (2007b). The real-world effectiveness of early teaching interventions for children with autism spectrum disorder. Exceptional Children, $73,417-433$.

[79] Reichow, B. (2012). Overview of Meta-Analyses on Early Intensive Behavioral Intervention for Young Children with Autism Spectrum Disorders. Journal of Autism and Developmental Disorders, 42, 512-520.

[80] Reichow, B., \& Wolery, M. (2009). Comprehensive synthesis of early intensive behavioral interventions for young children with autism based on the UCLA Young Autism Project model. Journal of Autism and Developmental Disorders, 39, $23-41$.

[81] Remington, B., Hastings, R. P., Kovshoff, H., degli Espinosa, F., Jahr, E., Brown, T. \& Ward, N. (2007). Early intensive behavioral intervention: Outcomes for children with autism and their parents after two years. American Journal on Mental Retardation, 112, 418-438.

[82] Rogers, S. J., \& Vismara, L. A. (2008). Evidence-based comprehensive treatments for early autism. Journal of Clinical Child and Adolescent Psychology, 37, 8-38.

[83] Sallows, G. O., \& Graupner, T. D. (2005). Intensive behavioral treatment for children with autism: Four-year outcome and predictors. American Journal on Mental Retardation, 110, 417-438.

[84] Schopler, E., Short, A., \& Mesibov, G. (1989). Relation of behavioral treatment to normal functioning: Comment on Lovaas. Journal of Consulting and Clinical Psychology, 57, 162-164.

[85] Sheinkopf, S. J., \& Siegel, B. (1998). Home-baseed behavioral treatment of young children with autism. Journal of Autism and Developmental Disorders, 28, 15-23.

[86] Schreibman, L. (2000). Intensive behavioral/psychoeducational treatments for autism: Research needs and future directions. Journal of Autism and Developmental Disorders, 30, 373-378.

[87] Shea, V. (2004). A perspective on the research literature related to early intensive behavioral intervention (Lovaas) for young children with autism. Autism, 8, 349-367.

[88] Smith, T. (1999). Outcome of early intervention for children with autism. Clinical Psychology: Science and Practice, 6, 33-49.

[89] Smith, T., Eikeseth, S., Klevstrand, M., \& Lovaas, O. I. (1997). Intensive behavioral treatment for preschoolers with severe mental retardation and pervasive developmental disorder. American Journal on Mental Retardation, 102, 238-249.

[90] Smith, T., Eikeseth, S., Sallows, G. O., \& Graupner, T. D. (2010). Efficacy of applied behavior analysis in autism. The Journal of Pediatrics, 155, 151-152. 
[91] *Smith, T., Groen, A. D., \& Wynn, J. W. (2000). Randomized trial of intensive early intervention for children with pervasive developmental disorder. American Journal on Mental Retardation, 105, 269-285.

[92] Spreckley, M., \& Boyd, R. (2009). Efficacy of applied behavioral intervention in preschool children with autism for improving cognitive, language, and adaptive behavior: A systematic review and meta-analysis. The Journal of Pediatrics, 154, 338-344.

[93] Steege, M. W., Mace, C., Perry, L., \& Longenecker, H. (2007). Applied behavior analysis: Beyond discrete trial teaching. Psychology in the Schools, 44, 91-99.

[94] Strauss, K., Vicari, S., Valeri, G., D’Elia, L., Arima, S., \& Fava, L. (2012). Parent inclusion in early intensive behavioral intervention: The influence of parental stress, parent treatment fidelity and parent-mediated generalization of behavior targets on child outcomes. Research in Developmental Disabilities, 33, 688-703.

[95] Sundberg, M. L., \& Michael, J. (2001). The value of Skinner's analysis of verbal behavior for teaching children with autism. Behavior Modification, 25, 698-724.

[96] Symes, M. D., Remington, B., \& Brown, T. (2006). Early intensive intervention for children with autism: Therapists' perspective on achieving procedural fidelity. Research in Developmental Disabilities, 27, 30-42.

[97] Virue's-Ortega, J. (2010). Applied behavior analytic intervention for autism in early childhood: Meta-analysis, meta-regression and dose-response meta-analysis of multiple outcomes. Clinical Psychology Review, 30, 387-399.

[98] Zwaigenbaum, L., Bryson, S., Rogers, T., Roberts, W., Brian, J., \& Szatmari, P. (2005). Behavioral manifestations of autism in the first year of life. International Journal of Developmental Neuroscience, 23, 143-152. 

Chapter 25

\title{
Feeding Issues Associated with the Autism Spectrum Disorders
}

\author{
Geneviève Nadon, Debbie Feldman and Erika Gisel \\ Additional information is available at the end of the chapter \\ http://dx.doi.org/10.5772/53644
}

\section{Introduction}

Feeding issues are prevalent in young children. Feeding will be defined here as the process of ingesting food and drink in social environments where such activities take place. Estimates of problems may range from 13 to $50 \%$ in typically developing children, but may be as high as $80 \%$ in children with developmental disabilities [1-7]. In 1 to $10 \%$ of these children problems may become chronic and may affect their health and development [1,8]. Anatomical, metabolic, gastrointestinal, motor or sensory problems may be the cause of or may contribute to some of these feeding problems [8]. A global medical assessment is necessary when feeding problems persist, because some medical symptoms may not be recognized as associated with feeding at first sight, such as asthma. Even if the association remains unclear, a high prevalence of asthmatic children, particularly with nocturnal asthma, have gastro-esophageal reflux (GER) [9]. Both feeding and eating, the processing of food and drink in the mouth and swallowing, are also known as activities of daily living (ADL) and studies examining the specific problems of children with Autism Spectrum Disorders (ASD), found that 46 to $89 \%$ have feeding problems [10-18].

While these studies are important to determine the nature and extent of such problems, results have to be interpreted with caution. First, small and heterogeneous sample sizes do not permit generalization to the entire population of children with ASD. There is also no consensus regarding the terminology and definitions used to describe these problems, i.e. feeding problem, eating problem, food refusal, selective/picky eating, mealtime problems, etc... Furthermore, authors use different instruments to measure these problems. Caregiver questionnaires are the most commonly used tools for this purpose; however, their psychometric properties are not well established. Further, observational studies of these subjects' eating skills or self-reports from them are lacking. This makes it difficult to compare studies or to replicate their results. 
Despite these methodological limitations, it is clear that feeding problems constitute a frequent and significant preoccupation for many parents of children with ASD [18]. In support of such concerns, some studies found that children with ASD are more susceptible to feeding problems than children with other developmental disabilities [19-23]. There is as yet no defined etiology for feeding problems in children with ASD neither is there for the pediatric population in general. Significant associations have been found between oral-motor, gastrointestinal and sensory problems in children with ASD [19, 24-26]. According to Skinner [27], individuals' responses to environmental stimuli shape their behaviors and this interaction constitutes the foundation for learning. When feeding is described as a struggle in the family environment, behavioral approaches such as escape extinction and positive reinforcement are used by professionals and gradually assumed by the caregiver. However, feeding problems may also arise from a limited ability to communicate or from poor social and cognitive skills. Eating skills and mealtime manners are learned by observation and imitation, yet these associations have not been correlated with ASD. More recent studies have found similarities between anorexia nervosa (AN) and ASD, on the basis of global processing deficits, inflexible style of thinking, communication difficulties and impairment of interpersonal functioning and social interactions [28-30]. Hence, treatment approaches used for AN might also be suitable for ASD.

Considering the impact feeding problems can have on children's health, the stress experienced by parents, as well as the impact on social participation of child and family, it will be crucial to continue documenting feeding problems in this group, to better understand them and thereby, offer better treatment. Similarly, it will be just as important to provide professionals with better guidelines to evaluate feeding problems, as well as to appreciate the consequences they have on family function.

\section{Essentials of diagnosis}

The severity of pediatric feeding problems can range from mild to severe. Despite this wide range, there are no clear indicators to determine which problems will be transient and those that will persist over the long term and may have an impact on children's health [1]. The DSM-IV-TR, a classification for psychiatric disorders, describes criteria for feeding disorder of infancy and early childhood; however, this particular diagnosis is rarely used in research or clinical practice. There are several reasons for this. A majority of the children who are referred for feeding problems, in general, do not meet all of the criteria outlined in the DSM-IVTR (Table 1) [7, 31]. For example, children do not qualify even if they have severe feeding problems but normal weight (e.g. eating foods of poor nutritional value; eating only purees or being tube fed) [7]. It is also not clear which medical or mental conditions, including ASD, would exclude a child from a diagnosis of feeding disorder of infancy and early childhood. Other diagnostic classifications and screening criteria appear promising. These are: Feeding Behavior Disorder [32, 33], Avoidant/Restrictive Food Intake Disorder [34] and Feeding Disorder [35-37], the Wolfson Diagnostic Criteria [38] and the framework proposed by Davies et al.[39]. 
Criterion A. Feeding disturbance as manifested by persistent failure to eat adequately with significant failure to gain weight or significant loss of weight over at least 1 month.

Criterion B. The disturbance is not due to an associated gastrointestinal or other general medical condition (e.g. esophageal reflux).

Criterion C. The disturbance is not better accounted for by another mental disorder (e.g., Rumination Disorder) or by lack of available food.

Criterion $D$. The onset is before age 6 years.

Table 1. Diagnostic Criteria for Feeding Disorder of Infancy or Early Childhood from the DSM-IV-TR [31]

\subsection{DC: 0-3R and Proposed DSM-V}

Feeding Behavior Disorder [32] applies «when the child does not regulate his feeding in accordance with physiological feelings of hunger or fullness» and comprises six categories to be described below. The future DSM-V [34] Avoidant/Restrictive Food Intake Disorder will include a description of three main subtypes that will map onto the first three categories of the DC:0-3R [32]. The reader is referred to the APA DSM-V website for further details on the inclusion criteria [34]. A clarification has been made to consider severe feeding problems, when they exceed what is normally expected with a concurrent medical condition or another mental disorder, which may include ASD [34]. The criterion has been further modified to also include children that do not loose or fail to gain weight.

\subsubsection{Infantile anorexia}

The central problem of infantile anorexia is a lack of appetite, as manifested by a lack of interest in eating and food refusal, and issues of control and autonomy that may exist between the parent and the child [33, 35]. Parent recall indicates that the child will be easily distracted by environmental stimuli, which interfere with nursing from the bottle or breast from the very first weeks of life. Later, children in this category never complain of hunger and are satisfied with only a few bites. Parents worry when their child does not eat enough and often try different strategies to encourage their child to eat. Early on distraction manoeuvres may work, but they do not last, and parents are forced to invent new strategies to entice their child to eat. They may coax the child and sometimes use forcefeeding. Despite these efforts the child does not eat enough to maintain normal growth, which may later lead to malnutrition, but will come to attention when the child does not follow his expected growth curve.

\subsubsection{Sensory food aversions}

In contrast to infantile anorexia, children with sensory based feeding problems are not lacking in appetite and eat an adequate diet as long as it meets their preferences which are consistent and stable over time [33]. These food preferences may be based on food texture, taste, smell, temperature or appearance. Sensory aversions may range from mild to severe, with some children refusing only a few items and others a whole food category. The varying in- 
tensities of these aversive reactions may lead to food refusals that may get generalized to foods with similar characteristics or to all new foods. Some children are so sensitive to the sensory characteristics of the rejected food that they will not eat any other food that comes in contact with the refused food, or refuse that certain foods be placed in their line of vision, or refuse to eat when others, seated next to them, eat a food that has been rejected or it may trigger an aversive reaction (Figure 1). What distinguishes Sensory Food Aversions from normal food preferences is the degree of severity of the food refusal and the presence of nutritional deficiencies or oral-motor delays arising from a lack of exposure to more demanding food textures [33]. Some studies have shown a significant relationship between food selectivity or mealtime problems and problems with sensory modulation $[26,40]$.

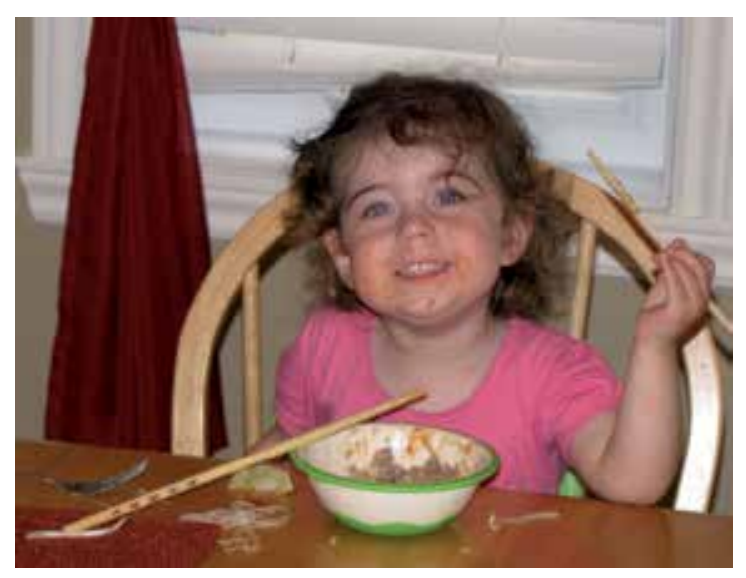

Figure 1. Children learn about food through exploration with their senses

\subsubsection{Feeding disorders associated with insults to the gastrointestinal tract}

This diagnosis, later renamed posttraumatic feeding disorders by Chatoor [33], has a sudden onset and results in severe food refusal. Young children with this diagnosis refuse to be fed, and often cry, hyper-extend their trunk and refuse to open their mouth when food is offered. Posttraumatic feeding problems are the result of a traumatic event or chronic, repeated traumatic events that affect the oropharynx or the esophagus. The event may have been aspiration of solid food into the trachea, related to force-feeding, due to medical procedures, such as placement of a nasogastric tube or enteral feeding. The refusal of food may manifest itself in different ways, depending on the type of feeding that is associated with the trauma. Depending further on the situation where the trauma occurred, such as the location or the positioning associated with feeding, the child may show signs of anxiety and marked distress at the approach of the bottle or the spoon, or when the food is placed in the mouth. Fear will override any sense of hunger and the effects on the child's health may vary, depending on the duration and extent of the food refusal, and the adequacy and adaptations made for nutritional compensation. If the food refusal extends over a prolonged period of time, delay in oral-motor skills, or overall development may be the result [33]. 


\subsubsection{Feeding disorder associated with concurrent medical conditions}

The DC: 0-3R [32] also lists feeding problems that are associated with medical conditions whereas the DSM-V [34] will only deal with mental health issues, not medical problems. Children with medical conditions and associated feeding problems are able to initiate eating; however, they may soon show signs of distress and/or fatigue and may not be able to finish their meal [33]. This inability may vary according to the severity of the medical condition. Heart and respiratory problems, as well as allergies and gastro-esophageal reflux are frequently associated with this type of feeding disability. Resolving the medical issues often improves the feeding related problems, although the latter may not always be eliminated completely. Several symptoms such as gagging, lack of appetite, food refusals, weight loss or growth faltering, may also be found in conjunction with other medical diagnoses. It is essential therefore, that children with feeding problems be carefully examined for associated medical problems.

\subsubsection{Feeding disorder of state regulation}

This feeding disorder is characterized by its onset in infancy, difficulty in establishing a quiet alert state necessary for feeding, weight loss, and absence of any medical condition that could explain these problems. This disturbance in state regulation, similar to disturbances in sleep or crying, will not be included in the next issue of the DSM-V [34]. Feeding is the first competent motor skill of infants [41] and is also an early indicator of self-regulation [33]. Therefore, specific aspects of feeding problems that also coincide with the behavior characteristics of ASD, might become 'red flags' for its diagnosis. Infants must be alert and able to maintain a calm state during feeding right from birth, i.e. the infant should not fall asleep at the onset of feeding, be too agitated or too distressed to feed [33]. Infants triple their body weight in the first year of life [41]. Therefore, a child who does not gain weight will not be able to maintain his established growth curve, or tends to cross over into a lower growth curve, which is interpreted as 'losing weight'. Mother and infant are 'mutual caregivers' [42]; the child who engages the mother visually, smiles or babbles and gains weight provides feedback to the mother that she is 'doing a good job', whereas a child who is colicky, cries, arches away from the mother and does not seem to eat enough causes the mother to worry and to try to compensate. She may feed the child more frequently than a child who eats well and she will also feed the child longer to compensate for the emerging weight loss [43]. The recent development of the P.O.P.S.I.C.L.E Center Infant and Child Feeding Questionnaire $\bigcirc$ (Parent Organized Partnerships Supporting Infants and Children Learning to Eat), an age-specific questionnaire available on the web, gives parents information regarding typical feeding development and helps them identify whether referral to a health professional (feeding specialist) is indicated [44]. Future studies will need to determine whether the constellation of early weight loss, lack of reciprocity, and distractibility during feeding may be an early indicator of ASD. 


\subsubsection{Feeding disorder of caregiver-infant reciprocity}

Feeding disorders of caregiver-infant reciprocity have their onset in the infant's first year of life and may come to attention through a problem that needs medical attention. The infant's developmental progression shows growth retardation and a lack of age appropriate engagement with the primary caregiver. Careful examination of the child-family relationships often point toward child neglect that may have its origin in the caregiver's history. These difficult problems will need to be addressed in conjunction with the original feeding problems of the infant [33]. In the DSM-V, this problem will be classifiable under a V code (i.e. a relational problem) [34].

\subsection{Feeding Disorders as classified by Dovey and collaborators}

The classification by Dovey et al. [35, 36] is built on an older classification by Chatoor and Ganiban [45]. Of the five types of feeding disorder, which will be further described below, four are similar to the classifications mentioned above. Learning-dependant food refusal is added and will include many children seen briefly in clinical practice. In their decision-making model [36, 37], Autism-Related Food Refusal is mentioned as a distinct category, but not further elaborated. In an earlier paper Dovey et al. [35] briefly describe feeding problems associated with ASD and touch upon the importance of the cognitive and social aspects of these problems. However, at that time, the authors seemed to include Autism-Related Food Refusal in their selective food refusal category. For clarity we will present a brief definition of the $A u$ tism-Related Food Refusal as a sixth category in this chapter.

\subsubsection{Medical complications-related food refusal}

Similar to the Feeding Disorder Associated with Concurrent Medical Conditions [32], food refusal is associated with one or more medical conditions. Medical professionals (e.g. gastroenterologist, general practitioner, health visitor, etc.) are needed to address these issues. The child may lack developmentally appropriate experiences with food because of major medical interventions that may have required nasogastric tube feeding which is often followed by gastrostomy feeding until the medical issues are resolved. Periods of longer than 1 week of tube feeding put the child at risk for 'oral deprivation,' i.e. they deprive the child of the daily practice of oral behaviors which in turn seem to have a detrimental effect on the associated brain development $[46,47]$. If it occurs in infancy children will experience great difficulty in making the transition to oral feeding. If children have had oral feeding experience before intubation there will be a transition time where they will have 'to learn to eat' again, but the transition will be shorter than in infants who have not had any feeding experience [46]. Dovey et al. [35] describe these children as not interested in eating but generally as happy to explore and play with food. Food refusal can also be present due to an association of food and/or eating with pain or discomfort. Many children who were tub-fed for extended periods will require additional support when making the transition to oral feeding [48]. 


\subsubsection{Learning-dependent food refusal}

According to Dovey et al. [35], the feeding disorder defined in this category is «completely dependent on the child's experience with it (eating)». Children in this category may have temper tantrums when new foods are offered to them and the usual response of the parent is to take their plate away and replace it with something they know their child will eat. Eventually, parents adapt the family menu to better fit their child's preferences and avoid unfamiliar foods or ones they know will trigger aversive reactions. Children between 2 and 6 years of age often refuse to taste new foods, which generally improves as the children get older. Food refusal based on novelty is called food neophobia and is considered an evolved behavior from human ancestry that protects the organisms from poisoning, at a time when children begin to leave their parents' supervision and gain more autonomy [35]. Repeated exposure, a positive experience and social influence will help children to overcome food neophobia. Therefore, caregiver education will be the first strategy to use when a learning dependent feeding disorder is suspected and rapid change can be expected.

\subsubsection{Selective food refusal}

Initially the picture of the «selective child» will be similar to learning dependent food refusal but for various reasons will evolve into a significant decrease in dietary variety. Here, exposure and social facilitation will have little to no effect on food acceptance and the child will not play with food. His diet will rely mostly on hedonic foods, e.g. foods that have a high salt, sugar and fat content. The child may eventually develop gastrointestinal problems as a result of a lack of fiber in his diet. Similar to Chatoor's Sensory Food Aversion category [33], these children have some sensory sensitivities, both tactile and/or oral defensiveness. Enlargement of dietary variety is a long process for children in this category and needs collaboration of parents, other caregivers and professionals. Dovey et al. [35] suggest that children in this category be referred for diagnostic work-up because of the high prevalence of sensory problems in the ASD population.

\subsubsection{Appetite-awareness-autonomy-based food refusal}

This category is the same as Infantile Anorexia described earlier by Chatoor and her colleagues [33] and is included in the above classification. The reader is referred to it for further details.

\subsubsection{Fear-based food refusal}

Fear-Based Food Refusal is also called Food Phobia. This category is identical to Posttraumatic Feeding Disorders of Infancy [33]. Some authors believe that, for many of these children, food phobia might be associated with a more general anxiety or affective disorder [49]. Food refusal in this category can be distinguished from other categories by the intensity of the emotional reaction when the child is asked to eat the target food. 


\subsubsection{Autism-related food refusal}

Dovey et al. [35] describe children with ASD and feeding problems as having «seemingly illogical rules around what constitutes an acceptable meal». We are not aware of any studies that have examined these children's rationale for their eating behaviors and the cognitive decisions that have led to them. Although, we do not know whether Dovey and collaborators have studied these, they acknowledge that these children must make decisions whether to eat something or not. This constitutes an important gap in our understanding of these children's feeding behavior and has important consequences on how we treat them. We observe children's behaviors and decide to manipulate them without understanding the underlying rationale that has led to these behaviors. An interpretation based on the hyper-systematization theory of Baron-Cohen et al. [50] will be discussed in the intervention section of this chapter. Meanwhile, perhaps one approach would be to study adults with autism and/or higher functioning children with ASD where some communication and insight is present, in order to access this very challenging domain. Even here, we must be sensitive to the fact that many other domains of ADL might be affected, besides eating, and that children and their family must be treated holistically.

\subsection{The 'Wolfson group' diagnostic criteria of infantile feeding disorders}

The 'Wolfson Group', a collaboration of medical professionals from Israel, has studied infantile feeding problems for a number of years [38, 51,52] and has shown considerable success in discriminating between infantile feeding disorders (non-organic) and medically based feeding problems (organic). Levine et al. [38] compared the diagnostic criteria of the Wolfson group to DC: 0-3R [32], and the DSM-IV [31] classifications in a group of children referred for food refusal. Results discriminated 100\%, 77\% and $47 \%$ respectively. The Wolfson criteria (Table 2) successfully identified a substantial proportion of treatable patients that the two other existing classifications could not identify [38].

1. Persistent food refusal "/>1 month

2. Absence of obvious organic disease leading to food refusal or lack of response to medical treatment of an organic disease

3. Age of onset $<2$ years, age at presentation $<6$ years

4. Presence of at least one of the following:

a. Pathological feeding or

b. Anticipatory gagging

Table 2. The Wolfson Diagnostic Criteria 


\subsection{Davies and collaborators' framework for classification}

Finally, another convincing argument for a reconceptualization of diagnostic criteria of feeding problems has been advanced by Davies and collaborators [39], suggesting that feeding problems are the result of a relational disorder of the child in his social context. The authors propose that feeding problems be diagnosed along 6 axes that define 1) a feeding disorder between parent and child, characterized by the child's persistent failure to eat foods in accordance with his developmental stage and the cultural or sub-cultural expectations. 2) a character/developmental disorder of parent and/or child, where the feeding relationship may be disrupted due to the caregivers' own psychopathology or life demands, and/or where the child may have a difficult temperament or medical and developmental issues that interfere with feeding, 3) This axis describes medical disorders of parent and/or child, that need to be addressed before feeding problems can be resolved 4) psychosocial stressors need to be identified "through use of the multi-axial diagnosis, to reflect the multi-determined nature of feeding disorders" 5) global functioning of parent and child will be examined separately for each, and 6) the global parent-infant relationship is classified through assessment of the quality of the parent-child relationship.

\subsection{Parent reported feeding problems}

A systematic literature review of feeding problems reported by parents, shows that many symptoms are similar to those associated with sensory feeding problems $[4,5,10,11,14,17$, $20,26,53-55]$. The peculiar ways in which sensory input is treated by persons with ASD are well documented in the literature [56-59]. The most frequently mentioned problems are texture, color, and smell selectivity, refusal of new foods, and food refusals in general. These problems were not associated with weight loss in a study comparing body mass index between typically developing children and those with ASD [60]. One study even reported a large proportion of children with ASD to be obese [61].

Parents' perception of their child's feeding problem often leads them to seek professional advice. In terms of nutritional deficiency, results vary widely [62-64]. Application of standards of reference with different criteria for severity levels may explain these discrepancies [65]. Furthermore, none of the studies reviewed so far have exclusively studied children with clinically significant feeding problems. Within a normally distributed population one would expect some children with, but the majority to be without nutritional deficiencies. Thus, it is not yet possible to determine whether or not children with ASD and severe feeding problems would also have nutritional deficiencies. Some case studies and larger studies report negative health effects due to selective eating or restrictive diets [66,67]. Therefore, it is always advisable to refer a child to a qualified nutritional expert when the child presents with selective eating.

Some parents report problems with chewing and abnormal drooling even after the child's developmental age has been taken into account $[13,68]$. Nadon et al. [13] compared children with ASD and their typically developing siblings of the same mean age and found that only the children with ASD had problems with eating related drooling, chewing, moving their tongue or swallowing. While parents interpreted these problems as a source of their child's 
feeding problem, this study showed that these motor behaviors were associated with tactile sensitivity but independent of mental retardation, attention deficit disorder or hyperactivity that is often present in these children [13]. These oral-motor problems are often overlooked, because it is generally a small group when compared to the whole population of children with ASD. However, careful evaluation may be particularly helpful for this group of children, because specific treatments exist, and have been shown to be effective for other neurologically based feeding problems [41].

Anticipatory behavior is an early indicator of social engagement. Kanner [69] noted that infants who later were described as 'autistic' did not reach out to an adult who was engaged in picking them up. Brisson and colleagues [70] made use of this characteristic by studying anticipatory behavior associated with feeding. The authors performed a retrospective review of home movies of infants, 3 to 6 months of age, who were later diagnosed with autism, expecting that they would perform poorly on opening their mouth (the anticipatory behavior) in response to an approaching spoon. Results were compared to an age matched typically developing group. While typically developing children, as a group, achieved $79 \%$ correct responses, only $46 \%$ of the children with autism did so. There was a clear learning curve in both groups, with younger infants showing fewer mouth opening responses than the older ones, and a larger proportion of typically developing infants opening their mouth to an approaching spoon than did infants with autism. These results are consistent with parent descriptions that infants were easily distracted when feeding, right from birth, and this behavior may indeed become an early diagnostic indicator, in conjunction with other behaviors that characterize the ASDs.

\section{Evaluation}

Feeding at mealtimes occurs as the result of the interaction between a child's body functions and structures, his health condition and some contextual factors (i.e. environmental factors as well as personal factors). An illustration of these interactions, using the model of the International Classification of Functioning, Disability and Health (ICF) is illustrated in Figure 2. The complexity of these interactions may be the reason why many investigators developed their own assessment tool, because existing ones did not adequately cover the domains to meet the authors' needs [71]. To have a complete picture of a child's problems, it is necessary to combine various methods of evaluation and to collaborate with professionals who have different domains of expertise.

There are a number of methods and feeding assessments, with varying content as well as different psychometric properties (e.g. caregiver questionnaires, interviews, child observations). The following review will be selective and is not intended to be exhaustive. For a more complete review the reader is referred to Nadon et al. [71] and Seiverling, Williams and Sturmey [72]. Other evaluations may be performed using standardized assessments if the child's condition suggests additional problems. 


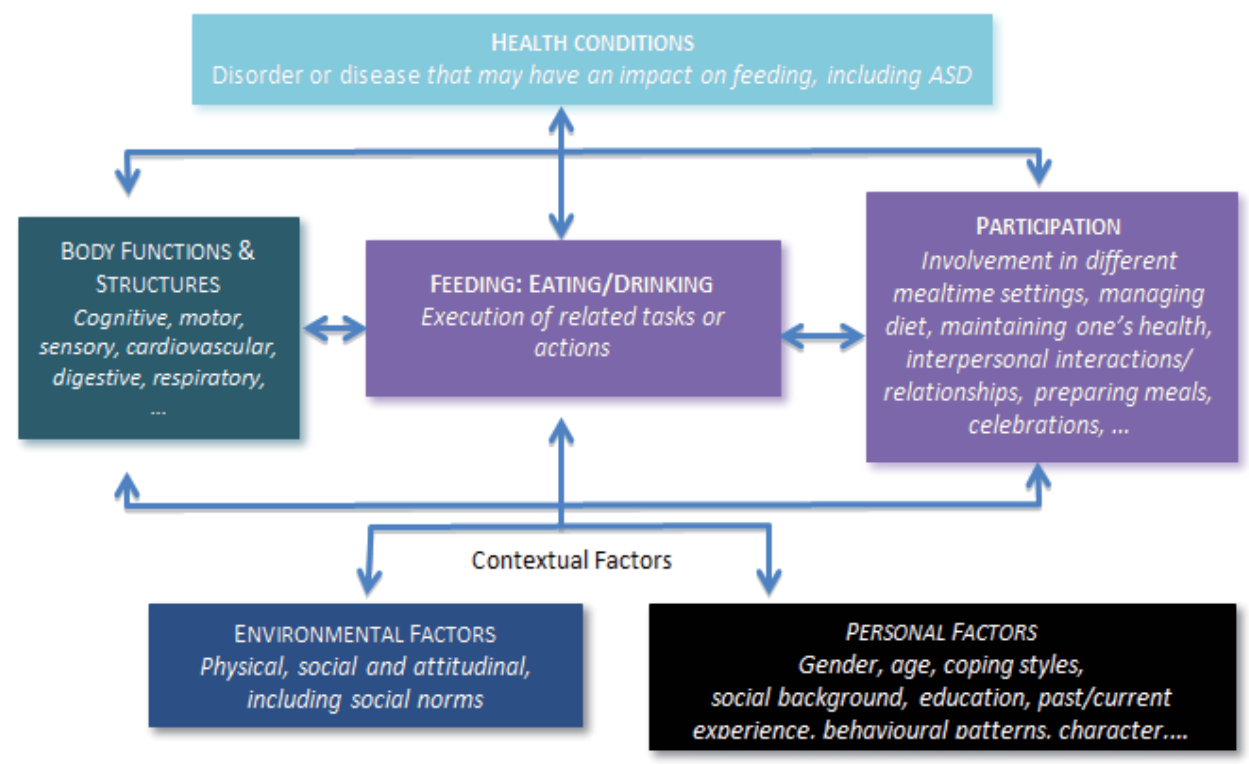

Figure 2. Adapted from the International Classification of Functioning, Disability and Health (ICF)

\subsection{Parent questionnaires}

Parent questionnaires with interview formats are commonly used [71-74]. These have the advantage that they can cover an extended period of the child's life. As well, documenting the feeding history during the first year of life, and again around 18 months when most of the problems become apparent, sheds light on the evolution of the presenting problems. $\mathrm{Pa}$ rents can provide a more complete picture of the child's feeding behaviors because they usually provide their child's daily meals. The advantage for the evaluator is that he is not dependent on a single meal observation that may not be representative of the daily routine in the home. It is also very important to get to know parents' motivation for consultation, their perception of the problems and their priorities for resolving them. It is important likewise, to learn what strategies parents have tried to solve the feeding problems and how the child reacted to them [75]. A detailed description of the child's food preferences is in order to determine the adequacy of the nutritional content. As well, common sensory characteristics of his preferred and non-preferred foods need to be assessed (color, texture, consistency, taste, smell, appearance,...) to better understand the nature of the problem and assist in the formulation of a treatment plan [76]. A sensory profile of the child is essential and, to understand his way of communicating, his motor abilities, learning strategies, and his cognitive and sensory information processing. It is also important to assess whether the child has a minimal understanding of negotiation, and what his play skills and interests are. These abilities will determine how best to approach his treatment. 


\subsection{Mealtime behavior questionnaires}

The Children's Eating Behavior Inventory (CEBI-R) [77] covers eating and behavioral problems at mealtimes in 2 groups: typically developing children (non-clinic) and a " clinic » group, aged 2 to 12 years. It uses a 5 point Likert scale to identify whether a problem occurs between "never" and "always", and a dichotomized scale for parents to note whether the behavior is perceived as a problem or not. Construct validity was demonstrated by significant differences between the two groups in total eating problems and the number of items perceived as problematic. Internal reliability or item consistency ranged from.58 to.76 using Cronbach's alpha, and test-retest reliability was 84 (parent score) and 87 (total eating problems).

The Brief Autism Mealtime Behavior Inventory (BAMBI) [73] measures the frequency of mealtime behavior problems in children with ASD between the ages of 3 and 11 years. The BAMBI contains 18 questions based on a 3 factor structure that identifies limited variety of foods (8 items), food refusals ( 5 items) and autistic behaviors (5 items). The BAMBI has good internal consistency. 88 , high inter-rater 0.78 and test-retest reliability 0.87 , as well as strong construct and criterion-related validity [73].

The Behavioral Pediatric Feeding Assessment Scale (BPFAS) is a 35 item, standardized caregiver report inventory that was developed for children with feeding problems, 9 months to 8 years of age, but who are otherwise typically developing. The tool has a 5-point Likert scale and caregivers indicate how frequently children show a behavior, i.e. "never happens » to " always happens », parents" frequency of feelings or strategies is noted and a total frequency is computed. Higher scores indicate more problems. Internal consistency of the BPFAS is. 88. Test-re-test reliability for the total score is.78 in both normal and clinical samples. The BPFAS was shown to have adequate reliability and validity [78].

The 31-item Parent Mealtime Action Scale (PMAS) [75] measures parents' actions regarding their child's mealtime behaviors in nine dimensions: snack limits, positive persuasion, daily fruit and vegetable availability, use of rewards, insistence on eating, snack modeling, special meals, fat reduction and many food choices. Parents also provide three-point ratings ( $1=$ never, 2 = sometimes, 3 = always) how often they use each of these actions in a typical week. The clinician then provides specific recommendations to caregivers regarding actions they can implement to improve their child's feeding. The PMAS was developed with a sample of over 2000 typically developing children [75] but a recent study [79] examined it's applicability with a clinical sample including 49 children with ASD. Mean internal reliability was.62 and convergent validity was demonstrated with expected associations between parent mealtime actions measured by the PMAS and some children's feeding problems. The five PMAS dimensions most associated with children's feeding problems were snack limits, insistence on eating, fat reduction, many food choices, and special meals [79].

The Feeding Demands Questionnaire is a parent report questionnaire that measures parents' belief how their child should eat [80]. Mothers of 3 to 7-year old children completed the 8item Feeding Demands Questionnaire, the Child Feeding Questionnaire, measures of depression and fear of fat. The Feeding Demands Questionnaire revealed 3 factors: anger/ 
frustration, food amount demandingness, and food type demandingness, for which subscales were computed. The Feeding Demands Questionnaire showed acceptable internal consistency (.70 to.86). The authors concluded that different demand beliefs influence different feeding practices.

About Your Child's Eating (AYCE) [81] was developed to document positive mealtime environment, parent aversion to mealtime and child resistance to eating in a sample of typically developing and a group of chronically ill children, aged 8 to 16 years. The AYCE is scored on a Likert scale from "never » to " nearly every time " describing the frequency of the child's mealtime behaviors, the caregiver's interaction with the child and the caregiver's reaction to the meal. While the constructs evaluated would seem to be similar in a group of younger children, validity for the ASD diagnostic group remains to be determined. The AYCE internal consistency is -.24 for child resistance, positive mealtime environment.55, and parent aversion -.37. There is also evidence for convergent validity with the Family Environment measure. Other psychometric properties still need to be developed.

The Eating Profile [13] covers eleven domains (145 items): 1) dietary history of the child, 2) child health, 3) family dietary history, 4) mealtime behaviors of the child, 5) food preferences, 6) autonomy with respect to eating, 7) behaviors outside of mealtimes, 8) impact on daily life, 9) strategies used to resolve difficulties encountered at mealtimes, 10) communication abilities of the child and 11) socio-economic factors of the family. The psychometric properties of this questionnaire have been studied to a limited degree [13, 82]. It was used to compare sibling mealtime behavior (ASD vs typically developing) in the same family. It showed that although typically developing children also had some mealtime problems (mean of 5.0), children in the same social and physical environment but with ASD, had significantly more such problems (mean of 13.0) than their siblings. Lack of variety of foods, i.e. less than 20 items, an inadequate number of meals, not eating at the table, or not staying seated during the meal, as well as showing some oral-motor deficits were the most significant differences between the two groups [13]. Even after developmentally related behaviors were excluded the difference between the number of mealtime problems in the two groups persisted. These results suggest that the impact of the diagnosis on mealtime behavior is greater than that of the environment.

\subsection{Nutritional assessments}

The Youth/Adolescent Questionnaire (YAQ) [83] is a self-report inventory for food frequency with 148 items to determine the nutritional intake of 9 to 18 year-olds and the average food serving frequency of six food groups. It provides an estimate of the average serving frequency per day for 6 food groups as well as the average intake over one year. In a validation study a correlation of.54 was achieved between the YAQ and 24-hour food recall interviews [83]. Test-retest reliability ranged from.26 to.58 for nutrients and from.39 to.57 for food groups. A modified version of the YAQ was used with children with ASD to quantify food refusal and food selectivity (i.e. 'High-Frequency Single Food Intake') on a daily basis [64]. Although food frequency questionnaires are known to commonly over-report dietary intake [84], they are useful to analyze children's preferences as it is required when using graduated exposure therapies. 
Food records are routinely used by nutritionists to measure energy intake. A systematic review [84] suggests that the 24-hour multiple pass recall conducted over at least a 3-day period that includes weekdays and weekend days, using parents as reporters is the most accurate method for children aged 4 to 11 years and that weighted food records provided the best estimates of Estimated Intake for younger children aged 0.5 to 4 years. Cornish [54] used a three day food record to study a small group of children with ASD, aged 3 to 16 years where 8 had followed a gluten and/or casein free diet for various lengths (1 to 6 months) and 29 consumed a regular diet. Caregivers filled out a 3-day diary of all foods and drinks consumed. Nutrient intakes in 12 children were lower than recommended in 'Lower Reference Nutrient Intake' for zinc, calcium, iron, vitamin A, $\mathrm{B}_{12}$ and riboflavin in the regular diet group and in 4 for zinc and calcium in the diet group although these differences were not statistically significant between the 2 groups. The median daily energy intake was $93 \%$ of Estimated Average Requirements (EAR) in both groups, and did not differ in the contribution of proteins, fats, or carbohydrates. Fruit and vegetable intake was higher and consumption of starches was lower in the diet group. The author notes that parents who followed the exclusion diet found that it isolated the family socially, food substitutes were difficult to find and costly, meals required longer preparation time, and it was very difficult for the child to make the change to the new diet.

\subsection{Direct observations}

Observation of the child's mealtime routine in his familiar environment provides insight into the family's daily life and the accommodations made for coping with problems [74]. The family may be so enmeshed in this routine that it does not always realize how it has adapted to the child's problem and to what extent the child's behavior or the environmental setting may contribute to the maintenance of problems. By making several visits to the home and by changing different variables (person, environment, social demands, sensory stimuli, liked vs non-liked foods...) the observer gets a clearer picture of the situation. If possible the evaluator should have a discussion with the child regarding his global understanding of eating and his recognition of any problems. If a home visit is not possible, the parent/caregiver should come to the clinic and bring some of the child's liked and non-liked foods. Familiar plates or utensils can also be brought. Evaluation will then focus on oral-motor skills, reaction to foods, intensity of food aversions, and acceptability of food modifications. A systematic presentation of foods was used by Ahearn et al. [10] for children with ASD. However, a major criticism with this type of evaluation is that it does not measure the severity or the problem experienced in the home, because the context is far from what the child is used to. It is more likely measuring the child's reaction to novelty or the influence of different contexts.

The Multidisciplinary Feeding Profile (MFP) was developed by Kenny and collaborators [85] with a group of 18 children, 6 to 18 years of age, who had neurological disabilities and were 'dependent feeders.' The evaluation is divided into six sections covering: I) Physical/Neurological factors such as posture, tone and reflex activity, 2) Oral-Facial 
Structure, consisting of 'an evaluation of the face and mouth at rest to identify variations from normal, using surface anatomy exclusively', 3) Oral-Facial Sensory Inputs: a subjective evaluation of sensory and reflex motor activity produced by stimulation of selected cranial nerves, 4) Oral-Facial Motor Function: a series of voluntary oral facial postures such as puckering the lips or deviating the jaw to the right or left, 5) Ventilation/Phonation: 'a subjective evaluation of breathing and sound production' and 6) Functional Feeding Assessment: an 'evaluation of oral-motor skills during specific feeding tasks' examining spoon feeding, biting, chewing, cup- and straw drinking and swallowing. Overall rater agreement, among 3 raters, was 0.83, and overall rater consistency was 0.90. Other psychometric properties such as validity, item consistency and test-retest reliability still need to be determined. As well, examination of the suitability for the population with ASD will be needed.

The Schedule of Oral-Motor Assessment (SOMA) by Reilly et al. [86] measures the oral-motor and feeding skills of children 8 to 24 months of age. A sample of 127 children constituted the original sample, $90 \%$ were typically developing and $10 \%$ were children with cerebral palsy. Differently textured foods and liquids are offered to the child in a pre-determined order: liquid, puree, semi-solids, solids, biscuits and dried fruit and scored in 6 sections. Scores are based on the quality of oral-motor, mandible, lip and tongue movements. Inter-rater reliability was 0.75 and internal consistency 0.85 [87]. The predictive validity was $>95 \%$ and sensitivity $>.85$ [88]. The age range of the SOMA makes it particularly attractive for use with young children because of the benefits of early intervention [41]. While a diagnosis of ASD may only be confirmed by two years or later, feeding problems are often recognized by parents from the first year. Treatment of feeding problems is not dependent on the diagnosis of ASD. Therefore, early intervention may prevent aggravation of feeding problems with time when not treated promptly.

\section{Treatment}

Treatment must take the complete evaluation into account, including the interaction of the person with his familiar environment. Feeding cannot be treated as an isolated problem and the strategies employed should not be limited to mealtimes only. To illustrate: the stress a child experiences during mealtime may decrease his appetite or decrease his tolerance for tactile or olfactory stimuli. Also, if functional analysis reveals environmental contributions to the feeding problems, like inappropriate parental strategies to cope with behavioral issues during mealtime, treatment needs to include these routines as well.

Whether the treatment approach will be interdisciplinary or trans-disciplinary [19, 89] collaboration between different professionals is desirable, given the complex nature of feeding problems and the many factors to be considered [21, 39, 90, 91]. The degree of involvement may vary, depending on the expertise of the individuals involved, the etiology of the problem to be addressed or the relationship the professionals have with the parents $[89,90]$. 


\subsection{Parent education}

When professionals help parents recognize the source of the feeding problem, the course of its evolution, and what contributes to its maintenance, the parent can become the professional's strongest ally, because parents know their child best. Engagement is better when parent and professional come to an agreement regarding treatment goals and parent involvement is essential in a family-centered approach to treatment [92]. If the child is still young, one would anticipate that the child will become more food selective around the age of two or three years, as seen in typical development, and that this stage will be more challenging for a child with ASD. When parents first learn of the child's diagnosis, it may be wise to show them a number of coping strategies (Table 3), in order to prepare them for the developmental food refusals, or to prevent some problems from getting worse. Particular attention must be paid to prevent intrusive feeding and to assure the child is regularly exposed to variation in the menu and presentation of foods [38, 93]. Parents' actions and their relationship with their child influence the course of mealtimes. Eating is not only about food. At the table members of the family enjoy each other's company, the meal, exchange feelings and family/cultural values. When mealtimes are not pleasurable and some members do not feel respected with regard to their needs and choices, the relationship may become affected. When the strategies chosen by the parent affect the relationship and contribute to the maintenance of a feeding problem, alternatives may be suggested and modeling used, to demonstrate different actions and attitudes in response to the child's behaviors. Regardless which treatment approach is chosen, parents must learn it and the transfer to different social environments must be carefully planned. It is hoped that regular follow-up with the family can be established, so that parent support is ensured and that changes can be made when needed.

\footnotetext{
- Never force feed children

- Never require that children empty their plate

- Schedule regular meal times, to establish a cycle of hunger and satiety

- Include activities before and after meals, to establish an anticipated routine.

- Use adapted communication and/or visual cues to establish clear expectations

- Limit the amount of liquids (especially juice and milk), because very small amounts can decrease appetite.

- Each child needs a designated seat at the table.

- Avoid commercial containers to facilitate the use of other brands

- Praise 'good' behavior.

- Change constituents of menus regularly.

- Introduce changes that are big enough to be recognized, but small enough that they will be tolerated.

- Adapt portion size to the age of the child and to his average appetite.

- Plan for generalizations
}

Table 3. Principles of feeding 


\subsection{Nutritional supplementation}

Feeding problems may be long-term problems. This is one reason why nutritional supplements may be useful to ensure that the child's health is not compromised [94]. This will require a nutritionist's evaluation, to determine whether supplementation or modification of the existing diet is indicated. These changes have to be made carefully and follow-up is needed to ensure that they don't suppress the child's appetite or interfere with digestion. Another option is to introduce supplements in small quantities after each meal, or before bedtime. Modification of preferred recipes or the introduction of nutritional supplements to a child who refuses any change to his established mealtime routine may present a major challenge and considerable risk. It is best to try such changes under professional supervision because, if done wrong, children may eliminate another food from their already limited repertoire. To increase the chance of success, it will be best not to change the sensory properties of preferred foods and to present modifications as similar as possible to the taste and texture of preferred foods [76]. Fading and desensitizing techniques are usually best for introducing food supplements [94]. These will be described below.

\subsection{Behavioral treatment approaches}

Behavioral treatments must be based on a functional analysis in order to determine which behaviors contribute to the maintenance of feeding problems and what function these behaviors serve (avoidance, attention seeking, pleasure seeking, obtaining a reward). Qualified professionals must supervise interventions so that no undesirable behaviors are reinforced [95].

A number of studies have shown the effectiveness of behavioral interventions for increasing acceptance of new foods $[15,16,96]$. However, there are only a few studies demonstrating that acceptance of new foods generalizes to other foods or other environments and that preferences of the child have been taken into account [97, 98]. Different types of behavioral interventions are often used in varying combinations [8,97]. Positive reinforcement, for example, consists of rewarding the child when he shows the desired response. Sequential presentation is a form of positive reinforcement. In this type of intervention acceptance of a non-preferred food is immediately followed by a preferred food. In simultaneous presentation the new, non-preferred food is presented together with the preferred food. Although not mentioned in the literature, clinical practice requires great care with this approach. If parents report that their child can detect the slightest change to a familiar recipe, or reacts negatively to different commercial brands when they look exactly alike, the child may be hypersensitive to flavors. When new foods are hidden in what is familiar to the child, they are often detected by the child. The danger of this approach is, that if the child has limited communication abilities, he may not understand what happened to his food and thus, may refuse to ever eat it again for fear that this problem will repeat itself. It is best not to use this method without the knowledge of the child. First, the caregiver will want to decrease the risk that the child would eliminate one of his preferred foods from an already limited repertoire. Second, the child should be aware that a new food is being introduced, if we want to gradually diminish anxiety toward this food and new foods in general. 
In food fading a similar approach is used. The intensity of the taste or texture is decreased by mixing the new food with something that the child likes. For instance, one can mix a teaspoon of home-made applesauce in a cup of commercial applesauce. If the child tolerates it, one can add a second teaspoonful at the next meal, and so on. If the child reacts mostly to visual changes he may be a good candidate for this particular approach.

Antecedent manipulation aims likewise at modifying the characteristics of a novel food or its presentation (e.g. texture, bite size, utensil, etc.), to make it more acceptable to the child and to fit the oral-motor skills of the child [8].

Escape extinction is used when the functional analysis shows that problem behaviors during a meal result in avoidance of encountering or having to eat a certain food. Physical guidance and non-withdrawal of the spoon are the general methods used for this situation [15]. The spoon is presented to the child and kept near his mouth until the food is accepted. Physical guidance consists of exerting slight pressure on the chin, to elicit opening of the mouth. Bad behavior is ignored. This approach is very difficult to accept for parents because it can be very taxing emotionally for both parent and child. Professional supervision is strongly recommended, to prevent post-traumatic feeding problems or adverse effects on the parentchild relationship. We do not recommend using this approach on a long-term basis but rather for specific identified behavioral problems.

\subsection{Treatment based on the theory of sensory integration}

Sensory modulation describes a component of the theory of sensory integration [99]. It is defined as the ability of adjusting responses to the degree, nature, or intensity of the sensory environment [100]. Sensory modulation disorders (SMD) describe responses that are inconsistent, inflexible and fail to meet the demands and expectations of the environment or a task [100, 101]. One or more sensory systems may be involved, such as touch, vision, hearing, proprioception, vestibular, smell and taste. According to Miller et al. [101], there are three subtypes of SMD; over-responsivity, under-responsivity, and seeking/craving. Children who are over-responsive react to sensory input more rapidly, and with greater intensity and duration than the majority of their peers [101]. Over-responsivity can lead to avoidance or aggressive behavior, to escape discomfort caused by sensory input. Tactile defensiveness is part of this subtype and is probably the most documented SMD [40, 99, 100, 102-104]. Under-responsivity describes slower, less intense responses to sensory stimuli [101]. Children in this subtype are difficult to engage, they seem lethargic and lack the inner drive to explore their environment or initiate social contact. Sensory seeking/craving is defined as an 'intense, insatiable desire for sensory input' [100]. Available inputs are not enough for children in this subcategory. They need input of greater intensity. They may take risks and engage in socially unacceptable behaviors, and may have unusual olfactory or gustatory preferences.

People, in general, react differently to intrinsic (e.g. hunger, pain) or extrinsic (e.g. texture, taste of food) sensations. Reaction thresholds and sensory preferences are part of each individual's unique characteristics. However, these are not easy to measure objectively. Some investigators do measure them, but their tools are not readily available to the clinician [105]. 
Through analysis of a person's emotional as well as physiologic and autonomic reactions, professionals make a decision whether these constitute a SMD or not. The impact of this sensory excitatory state on activities of daily living, or on general development, must be significant in order to constitute a problem. The association of SMD with feeding problems has been documented by some investigators [26, 40, 106-108].

Analysis of the sensory components associated with meals is essential. For a child to function optimally at meal times, he has to be in a calm and alert state. The clinician assesses the child's overall level of arousal prior to mealtime and may intervene to ensure an optimal state for eating. Exploration and tactile desensitization activities may be recommended, such as exploration of new foods through touch, smell, and taste [109, 110]. Some investigators have found a correlation between anxiety and over-responsivity; heightened sensitivity to sensory information mediates the impact of anxiety on selective eating [106]. If the child's anxiety level is too high, it is appropriate to start tactile exploration of familiar foods or items outside the eating domain. Manipulating foods with subtle differences in texture, smell, temperature and taste can help the child feel more comfortable in their presence and is often a preliminary step before accepting them to eat. When these exercises are done playfully they are often less stressful for the child and facilitate participation. Finally, all components of a meal, e.g. utensils used or food textures, must be included in the analysis and can be modified to better suit the child's sensory profile. Despite the extensive use of these techniques in the clinical environment, research using appropriate controls is still lacking to corroborate results from anecdotal reports [8].

\subsection{Graduated exposure food therapy}

Graduated exposure food therapy is similar to systematic desensitization, a type of behavioral therapy used for specific phobias and other anxiety disorders. Treatment consists of systematic and gradual exposure to the fear producing stimulus (food), the learning and application of coping strategies, observation of the development of tolerance and maintenance of the engagement of the child; finally, acceptance without adverse reaction, so that the targeted food eventually becomes an integral part of the child's diet. Another goal of desensitization is to gradually eliminate the fear/anxiety that is associated with eating and to replace it with more positive sentiments such as pleasure. Graduated exposure may commence with two types of hierarchy: 1) introducing foods that share some sensory properties with the child's preferred food (e.g. visual, taste, texture) as suggested by Fraker et al. [76] in Food Chaining $\odot$ or 2) increasing the food's proximity, e.g. tolerating the presence of the food on the table - on the plate - observing it - touching it - smelling it - tasting it - chewing it and, finally, swallowing it [110, 111]. It may be necessary to use both types of hierarchy when introducing a target food. Change can take a long time and it is important that the child does not refuse foods that he previously accepted which can happen when familiar food is modified without his knowledge. 


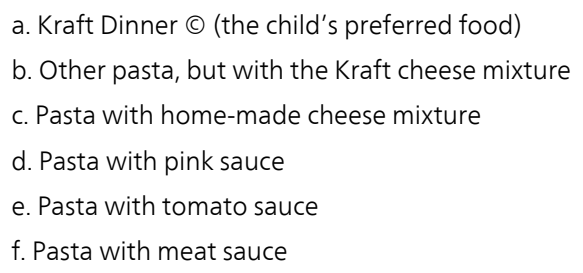

Table 4. Example of Desensitization Based on Sensory Properties as in Food Chaining @ [76]

In Food Chaining@ [76], the child's food preferences are analyzed in detail to establish a point of departure from which professionals can enlarge the child's food repertoire (Table 4). No studies were found to support the effectiveness of this approach for children with ASD [112]. Nevertheless, it is currently in use in North American clinical environments and several documents addressed to parents and professionals mention it [76, 110, 113]. Hierarchical exposure, based on proximity, associated with individualized positive reinforcement showed promising results for some children with ASD [98]. Validation is needed with a more representative sample of the ASD population [98]. Graduated exposure may be used in combination with other approaches, mentioned earlier, to determine which foods may be easier to introduce first, to structure the progression of treatment, and to ensure that treatment does not progress too rapidly. Some use these strategies in combination with other trans-disciplinary interventions in group therapies as a means of exploring foods through games [111, 114]. This may be an interesting approach for children who have good symbolic play and imitation skills. Other authors have used graduated exposure with more cognitivebehavioral methods, such as in a competition table or a diary to describe the child's progress, or with positive reinforcement or strategies to decrease stress [115]. All children in this last study were 7 years old or older. Some had autistic features, but none had a definitive diagnosis of autism. A self/auto-evaluation scale for the child who has fair insight, as well as an observation scale, are suggested by the authors of Food Chaining $₫$ to rate reactions when exposed to a new food [76].

\subsection{Cognitive approach}

Sensory hypersensitivities are very prevalent in ASD [56, 116, 117]. Baron-Cohen et al. [50] suggest that excellent attention to detail observed in ASD results from this sensory hypersensitivity and that it leads to hyper-systemizing, an exceptional capacity to recognize repeating patterns in stimuli; i.e. recognition of the rules that define a system. This theory explains «savantism» as well as non-social features of autism, like narrow interests, or resistance to change. When applied to feeding, some food selectivity or «illogical rules», like wanting food prepared exactly the same way every day, may be the expression of a strong systemizing capacity; i.e. sameness helps the child build concepts. According to Baron-Cohen et al. [50] a concept is a system and helps to define what items to include as members of the system. Therefore, a child's concept of 'French fries' may rely on visual systemizing (i.e. visual properties of the food are used to categorize: homogeneous light brown, thin and long, in a specific container), or alternatively on social/environmental sys- 
temizing (i.e. categorizing according to who is present or when it is eaten: 'French fries' are eaten after swimming class, at McDonalds, with dad). Because the child with ASD is also hypersensitive, a minor change in cooking duration, a different tablemat, is immediately detected and the presence of this new feature (often more than one, considering all the variations possible during mealtime) may no longer allow the child to include the 'new' food in his concept of 'French fries'. This interpretation of autism-related feeding problems could explain amelioration of feeding problems as children with ASD get older, as well as 'miraculously resolved' feeding problems observed sometimes in clinic or reported by parents. When the child understands and has a better global conceptualization of food and mealtime situations, his feeding issues may resolve very rapidly. Other approaches such as sensory integration and graduated exposure may be complementary, because the first addresses sensory hypersensitivity, which leads to hyper-systemization, and the second supports how changes can be introduced. According to Baron-Cohen et al. [50], changing one variant at a time is better to support the child in building general concepts. Another avenue may be inspired by cognitive remediation therapy used for children and adolescents with anorexia nervosa but it would have to be adapted to ASD, and maybe also focus more on food concepts and feeding situations [118].

Developing a teaching method to learn global concepts of food and eating specific to ASD may be needed. Baron-Cohen et al. [119] found an «autism-friendly» way to teach emotions to children with ASD which may potentially be adapted to the feeding domain. Eating and the socialization associated with it, touches a spectrum of emotions. Children with ASD seem to only recognize 'like vs dislike' and not the broader spectrum of 'tolerate, appreciate, enjoy, love, or crave.' Understanding these may also help them to explore and eat a larger number of foods.

\subsection{Adaptation of commonly used tools/approaches to ASD}

To achieve acceptable table manners, Social Stories ${ }^{\text {tm }}$ [120] may be used to describe a mealtime situation, a skill or a food concept, that includes expected table manners, and aims at helping the individual with ASD better understand social expectations at mealtimes. A Social Story ${ }^{\mathrm{tm}}$ may be illustrated such that it explains to the child how meals are set up, why one has to eat, or even to explain what table manners are and what is expected at home or outside the home (i.e. formal and informal rules). This type of intervention was shown to be effective in a young boy with Asperger syndrome, for decreasing unacceptable table manners, such as spilling food and increasing desirable behaviors such as mouth wiping [121]. The TEACCH approach seeks to promote understanding and independence by adapting the environment to better fit the learning style of children with ASD [122]. Visual supports used in TEACCH to enhance predictability and understanding of a task would also be appropriate for eating. For example, one would place only a tiny amount of a new food on the child's plate, if the goal is only to taste the food. To help the child understand the sequence of the meal, one could place a visual sequence next to the plate, to illustrate what he is expected to do, how/when the meal will end and what will happen after the meal (e.g. sit at the table eat foods on your plate - drink beverage from your glass - wipe your hands - return to play). 
Use of alternative and augmentative communication such as the Picture Exchange Communication System (PECS) [123] may enhance communication and understanding of social settings between the child and members of the family at mealtimes.

\section{Discussion}

Much progress has been made in our ability to discriminate between constellations of apparently similar feeding behaviors, and thereby establish differential diagnoses for children with ASD and feeding problems [33-35, 38, 39]. However, each new insight gained seems to beg new questions that call for an answer. The evaluation and treatment for these feeding problems has experienced a similar evolution. We will discuss these in the same order as the chapter has been presented so far, starting with diagnoses, followed by evaluation and treatment.

\subsection{Differential diagnoses}

One of the basic needs for the classification of a problem is the use of a nomenclature that is understood and used consistently by the professionals who work in the same domain. There is still no universal consensus what defines a 'feeding problem, eating problem, food refusal, selective/picky eating, mealtime problems' etc., in terms of their characteristics, duration, and severity. It may be the source of confusion and disagreement in the interpretation of results from research. Therefore, such a classification would do much to advance the field, by minimizing the need for defining terms by individual investigators in the course of their work. Consensus building of this type is usually called upon by nationally recognized professional organizations which in the case of feeding problems will need to ensure that as broad a spectrum of professionals is represented in the discussions and formulation of such a classification of this complex topic.

Several classification systems are currently in use. These may contribute to some of the inconsistencies of results, but each makes a unique contribution, and so, a comparison may be helpful to conclude the discussion on differential diagnoses. The DC: 0-3R [32], the proposed DSM-V [34] and Dovey et al.' [35] classifications have several advantages over the current DSM-IV-TR classification. These are the addition of the constructs of appetite, self-regulation, and the sensory and post-traumatic feeding problems. Despite these advances, there will always be children who will not exactly fit these new definitions. It must also be noted that the authors of these classifications do not exclude the possibility that a child may present with more than one diagnosis at a time. Nonetheless, there are still gaps. For example, much attention has been paid to nutritional deficiencies and weight loss, whereas normal weight gain or over-weight due to hyper-caloric diets associated with high hedonic value from sugars, fats and salt are not yet covered. These diets are quickly becoming an important societal problem. Certain symptoms and diagnostic criteria sometimes overlap and standardized tools are not yet available, especially for sensory food aversions [124]. The recognition of sensory based feeding problems is new and studies will be needed to validate 
criteria for sensory based food aversions. It is also not yet clear whether some of these feeding problems are specific to the population with ASD, if they are an associated condition or a learned behavior complicated by their diagnosis.

\subsection{Evaluation}

In the section on evaluation we noted that the age ranges of evaluations vary from infancy to late adolescence. New assessments may be needed if the age range for a particular domain is not yet available. While a diagnosis of ASD is often not confirmed until a child is 3 to 4 years old, feeding problems are prevalent and often come to attention in infancy [70]. Treatment of a feeding problem does not depend on a diagnosis of ASD. Therefore, it can be dealt with as early as it comes to attention. Such an approach may prevent the serious longterm consequences in terms of weight gain and brain development [46]. Whether early feeding behaviors may become predictors for a diagnosis of ASD will need further study. However, inclusion of feeding evaluation at the time of the diagnostic work-up is highly recommended for the many reasons that have been stated throughout this chapter.

We proposed the International Classification of Functioning (ICF; WHO) as the model for evaluation, in order to ensure that the interactions between the child's body functions and structures, his health condition, and some contextual factors (i.e. environmental as well as personal factors) will be included in the global evaluation. As of this writing no standardized evaluation exists that covers all domains of this model. Some evaluations may cover some domains, e.g. activity/participation and environment, or personal factors and activity/ participation and so, feeding assessments based on all domains have to be accomplished by using several evaluations that in combination cover these domains. Another problem is that some of these evaluations have been developed for typically developing children or children with other diagnoses, and will need to be validated for children with ASD. As stated in the discussion of differential diagnoses, collaboration by an interdisciplinary team to develop a tool comprising all three domains would move the field forward substantially.

Many of the evaluation tools reviewed above are questionnaires and may have satisfactory psychometric properties $[73,77,78]$, while others have only limited psychometric properties [13, 85] and need further development. Questionnaires offer the advantage of describing the child's usual abilities. These behaviors are described by a person who is familiar with the child, usually a parent or teacher, and reflects the observer's perception of the child's performance. Direct observations of the child's performance in his familiar environment are still lacking. This constitutes a significant gap in the treating professional's knowledge, because the treatment plan will be based on results obtained from a questionnaire or from contexts unfamiliar to the child.

Evaluation of children's nutritional state is based on caloric and nutrient sufficiency of the diet. These are commonly evaluated by food frequency questionnaires, and by 1, or 3-day food records [84]. Food intake is very individual, depending on the child's age and activity level, as well as the cultural environment of the family unit. To judge intake adequacy, results are compared to established national standards such as Estimated Average Requirements, or National Recommended Intake Standards (NRIS). Evaluation of nutrient adequacy 
(vitamins and micronutrients) should be part of a nutritional evaluation. A particular challenge is the peculiar eating habits of children with ASD; they may be of normal weight, hence pose no major 'medical problem', but their diet may consist predominantly of sugars, fats and salt. Such diets may also eventually lead to obesity. To assist parents in their daily mealtime struggles a number of evaluations also focus on parent perceptions of their successes and difficulties [80, 81]. We are not aware of any standardized dietary evaluation for children with ASD.

Although some standardized sensory evaluations exist [125-127], they cover sensory reactions in many domains of ADL and so, only a few items deal directly with feeding and eating. Hence, the greatest need in this area is an assessment that will fully cover the sensory domains associated with feeding and eating. Preliminary data are available from a study by Tessier [124], but now need to be subjected to a full psychometric evaluation. Common sensory characteristics should include color, texture, consistency, taste, smell, shape, size of a bite, and appearance which may have an influence on the variety of foods eaten. A study of how language and social skills affect feeding ability and the cognitive aspects that are involved for or against eating a certain food may be appropriate to include, since meals are social events. We are also still lacking self-report studies from individuals with ASD who may help us understand the challenges they associate with eating. An update of evaluation guidelines or even new guidelines for clinicians and researchers may well arise from the research suggested in this section and would immediately benefit the population we are trying to serve.

\subsection{Treatment}

A combination of treatment strategies, based on a holistic evaluation is, in our opinion, the most promising approach to intervention. Regardless which treatment approaches are chosen, intervention based on the contextual factors suggested by the ICF will facilitate the transfer to different environments, maintenance of the gains over time and hopefully further improvement. Early parent education may be critical to prevent the establishment of feeding problems but will need further study.

We have seen that careful manipulation of foods, the mealtime environment or the nutrient content of the diet may lead to some success in the acceptance of a new food, but one of the challenges remaining is that success with one food or domain does not necessarily generalize to other foods or domains. Such progress will probably only happen once children's cognitive decisions/intuitive reactions for acceptance/rejection of foods will be more clearly understood. We have suggested that one approach may be to study the more highly functioning children with ASD or Asperger's syndrome where some communication skills and insight are present. It may also be helpful to begin work by letting the child determine his food preference to facilitate co-operation.

Typically developing children also go through food jags, i.e. phases where they will only eat a limited variety of foods over an extended period of time. One of the authors (E.G.) recalls a parent telling her that his three-year-old son ate only pasta for three months and once he had his fill he 'returned' to eating the well balanced family diet. What this 'fill' was, the pa- 
rent could not tell, but it upset the family considerably. Therefore, the definition of what is 'normal' or 'abnormal' at different stages of development has not yet been adequately defined. Despite the extensive use of behavioral and sensory integration techniques in the clinical environment, research using appropriate controls is still lacking to corroborate results from anecdotal reports [8]. Most of the behavioral approaches are 'patient' centered and so, may not take the whole family unit into account. This point has been particularly emphasized by Davies et al. [39]. With an activity that is so 'family/culture' centered as mealtimes are, a further challenge will be to integrate the family into our treatment approaches.

\section{Conclusion}

This literature review has illustrated how common feeding problems are in children with ASD. However, it is not yet definitively established whether these problems are different from the general pediatric population. There is no consensus yet on the terminology to be used to describe these problems, on evaluation methods, and use of different diagnostic classification systems. This makes comparisons of different studies very difficult at present. Some feeding problems are similar to the sensory problems described in the DC: $0-3 R$. This would justify the use of the sensory integration approach, as well as hierarchic desensitization in the treatment of children with ASD and feeding problems. Updating guidelines for diagnoses and clinical practice will contribute to knowledge translation from research to general practice. Preventive approaches, and teaching parents how to handle feeding problems also seems promising. Further research is needed to support these beginnings.

\section{Author details}

Geneviève Nadon ${ }^{1}$, Debbie Feldman ${ }^{1}$ and Erika Gisel ${ }^{1,2}$

*Address all correspondence to: erika.gisel@mcgill.ca

1 Université de Montréal and Center for Interdisciplinary Rehabilitation Research, Montreal, Quebec, Canada

2 Faculty of Medicine, School of Physical \& Occupational Therapy, McGill University, Canada

\section{References}

[1] Aldridge VK, Dovey TM, Martin CI, Meyer C. Identifying clinically relevant feeding problems and disorders. Journal of Child Health Care 2010;14 261-270.

[2] Bryant-Waugh R, Markham L, Kreipe RE, Walsh BT. Feeding and eating disorders in childhood. International Journal of Eating Disorders 2010;43 98-111. 
[3] Carruth BR, Ziegler PJ, Gordon A, Barr SI. Prevalence of picky eaters among infants and toddlers and their caregivers' decisions about offering a new food. Journal of the American Dietetic Association 2004;104 s57-64.

[4] Field D, Garland M, Williams K. Correlates of specific childhood feeding problems. Journal of Paediatrics and Child Health 2003;39 299-304.

[5] Manikam R, Perman JA. Pediatric Feeding Disorders. Journal of Clinical Gastroenterology 2000;30 34-46.

[6] Mascola AJ, Bryson SW, Agras WS. Picky Eating during Childhood: A longitudinal study to age 11 years. Eating Behavior 2010;11 253-257.

[7] Williams KE, Riegel K, Kerwin ML. Feeding Disorder of Infancy or Early Childhood: How often is it seen in feeding programs? Children's Health Care 2009;38 123-136.

[8] Sharp WG, Jaquess DL, Morton JF, Herzinger CV. Pediatric feeding disorders: a quantitative synthesis of treatment outcomes. Clinical Child and Family Psychology Review 2010;13 348-365.

[9] Molle LD, Goldani HAS, Fagondes SC, Vieira VG, Barros SGS, Silva PS, et al. Nocturnal reflux in children and adolescents with persistent asthma and gastroesophageal reflux. Journal of Asthma 2009;46 347-350.

[10] Ahearn WH, Castine T, Nault K, Green G. An assessment of food acceptance in children with autism or pervasive developmental disorder-not otherwise specified. Journal of Autism and Developmental Disorders 2001;31 505-511.

[11] Martins Y, Young RL, Robson DC. Feeding and eating behaviors in children with autism and typically developing children. Journal of Autism and Developmental Disorders 2008;38 1878-1887.

[12] Ledford JR, Gast DL. Feeding problems in children with Autism Spectrum Disorders: A review. Focus on Autism and Other Developmental Disabilities 2006;21 153-166.

[13] Nadon G, Ehrmann Feldman D, Dunn W, Gisel E. Mealtime problems in children with autism spectrum disorder and their typically developing siblings: A comparison study. Autism 2011;15 98-113.

[14] Schreck KA, Williams K, Smith AF. A comparison of eating behaviors between children with and without autism. Journal of Autism and Developmental Disorders 2004;34 433-438.

[15] Volkert VM, Vaz PCM, Piazza CC. Recent studies on feeding problems in children with Autism. Journal of Applied Behavior Analysis 2010;43 155-159.

[16] Williams KE, Seiverling L. Eating problems in children with Autism Spectrum Disorders. Topics in Clinical Nutrition 2010;25 27-37.

[17] Williams PG, Dalrymple N, Neal J. Eating habits of children with autism. Pediatric Nursing 2000;26 259-264. 
[18] Rogers LG, Magill-Evans J, Rempel GR. Mothers' challenges in feeding their children with Autism Spectrum Disorder-Managing more than just picky eating. Journal of Developmental and Physical Disabilities 2011;24 19-33.

[19] Cermak SA, Curtin C, Bandini LG. Food selectivity and sensory sensitivity in children with autism spectrum disorders. Journal of the American Dietetic Association 2010;110 238-246.

[20] Collins MSR, Kyle R, Smith S, Laverty A, Roberts S, Eaton-Evans J. Coping with the usual family diet: Eating behaviour and food choices of children with Down's Syndrome, Autistic Spectrum Disorders or Cri du Chat Syndrome and comparison groups of siblings. Journal of Learning Disabilities 2003;7 137-155.

[21] Provost B, Crowe TK, Osbourn PL, McClain C, Skipper BJ. Mealtime behaviors of preschool children: Comparison of children with Autism Spectrum Disorder and children with typical development. Physical \& Occupational Therapy in Pediatrics 2010;30 220-233.

[22] Schmitt L, Heiss CJ, Campbell EE. A Comparison of nutrient intake and eating behaviors of boys with and without Autism. Topics in Clinical Nutrition 2008;23 23-31.

[23] Williams KE, Gibbons BG, Schreck KA. Comparing selective eaters with and without developmental disabilities. Journal of Developmental and Physical Disabilities 2005;17 299-309.

[24] Badalyan V, Schwartz RH. Feeding problems and GI dysfunction in children with Asperger Syndrome or Pervasive Developmental Disorder not Otherwise Specified: Comparison with their siblings. Open Journal of Pediatrics 2011;1 51-63.

[25] Page J, Boucher J. Motor impairments in children with Autistic Disorder. Child Language Teaching and Therapy 1998;14 233-259.

[26] Nadon G, Ehrmann Feldman D, Dunn W, Gisel E. Association of sensory processing and eating problems in children with Autism Spectrum Disorders. Autism Research and Treatment DOI:10.1155/2011/541926, 2011. Article ID541926, 8 pages.

[27] Skinner B. The operational analysis of psychological terms. Behavioral and Brain Sciences 1984;7 547-553.

[28] Coombs E, Brosnan M, Bryant-Waugh R, Skevington SM. An investigation into the relationship between eating disorder psychopathology and autistic symptomatology in a non-clinical sample. British Journal of Clinical Psychology 2011;50 326-338.

[29] Oldershaw A, Treasure J, Hambrook D, Tchanturia K, Schmidt U. Is anorexia nervosa a version of autism spectrum disorders? European Eating Disorders Review 2011;19 462-474.

[30] Pepin G, Stagnitti K. Come play with me: An argument to link Autism Spectrum Disorders and Anorexia Nervosa through early childhood pretend play. Eating Disorders: The Journal of Treatment and Prevention 2012;20 254-259. 
[31] American Psychiatric Association. Diagnostic and Statistical Manual of Mental Disorders: DSM-IV-TR. Washington, DC: American Psychiatric Publishing, Inc.; 2000.

[32] ZERO TO THREE. Diagnostic Classification of Mental Health and Developmental Disorders of Infancy and Early Childhood: Revised edition (DC: 0-3R). Washington, DC: Zero To Three; 2005.

[33] Chatoor I. Diagnosis and Treatment of Feeding Disorders in Infants, Toddlers, and Young Children. Washington, DC: Zero To Three; 2009.

[34] American Psychiatric Association. DSM-V Development. Avoidant/Restrictive Food Intake Disorder. http://www.dsm5.org/ProposedRevisions/Pages/proposedrevision.aspx?rid=110: American Psychiatric Association (accessed 24 August 2012).

[35] Dovey TM, Farrow CV, Martin CI, Isherwood E, Halford JCG. When does food refusal require professional intervention? Current Nutrition and Food Science 2009;5 160-171.

[36] Dovey TM, Isherwood E, Aldridge VK, Martin CI. Typology of feeding disorders based on a single assessment system. Infant, Child, and Adolescent Nutrition 2010;2 52-61.

[37] Southall A, Martin C. Decision-making model. Feeding problems in children: A practical guide, Second edition. Oxford, New York: Radcliffe Publishing; 2010. p. 314-315.

[38] Levine A, Bachar L, Tsangen Z, Mizrachi A, Levy A, Dalal I, et al. Screening criteria for diagnosis of Infantile Feeding Disorders as a cause of poor feeding or food refusal. Journal of Pediatric Gastroenterology and Nutrition 2011;52 563-568.

[39] Davies W, Satter E, Berlin KS, Sato AF, Silverman AH, Fischer EA, et al. Reconceptualizing feeding and feeding disorders in interpersonal context: The case for a relational disorder. Journal of Family Psychology 2006;20 409-417.

[40] Smith AM, Roux S, Naidoo NTR, Venter DJL. Food choices of tactile defensive children. Nutrition 2005;2114-2119.

[41] Gisel E. Interventions and outcomes for children with dysphagia. Developmental Disabilities Research Reviews 2008;14 165-173.

[42] Anderson GC. The mother and her newborn: Mutual caregivers. Journal of Obstetric, Gynecologic, \& Neonatal Nursing 1977;6 50-57.

[43] Ramsay M, Gisel E, McCusker J, Bellavance F, Platt R. Infant sucking ability, Nonorganic failure to thrive, maternal characteristics, and feeding practices: A prospective cohort study. Developmental Medicine and Child Neurology 2002;44 405-414.

[44] P.O.P.S.I.C.L.E.Center®. P.O.P.S.I.C.L.E. Center Infant and Child Feeding Questionnaire@ ${ }^{\circ}$ http://www.popsicle.org: P.O.P.S.I.C.L.E. Center ${ }^{\circledR}$ (accessed 18 August 2012).

[45] Chatoor I, Ganiban J. Food refusal by infants and young children: Diagnosis and treatment. Cognitive and Behavioral Practice 2003;10 138-146. 
[46] Senez C, Guys J, Mancini J, Paredes AP, Lena G, Choux M. Weaning children from tube to oral feeding. Child's Nervous System 1996;12 590-594.

[47] Dunitz-Scheer M, Levine A, Roth Y, Kratky E, Beckenbach H, Braegger C, et al. Prevention and treatment of tube dependency in infancy and early childhood. Infant, Child, and Adolescent Nutrition 2009;1 73-82.

[48] Daveluy W, Guimber D, Mention K, Lescut D, Michaud L, Turck D, et al. Home enteral nutrition in children: An 11-year experience with 416 patients. Clinical Nutrition 2005;24 48-54.

[49] McNally RJ. Choking phobia: A review of the literature. Comprehensive Psychiatry 1994;35 83-89.

[50] Baron-Cohen S, Ashwin E, Ashwin C, Tavassoli T, Chakrabarti B. Talent in autism: Hyper-systemizing, hyper-attention to detail and sensory hypersensitivity. Philosophical Transactions of the Royal Society B: Biological Sciences 2009;364 1377-1383.

[51] Levy Y, Levy A, Zangen T, Kornfeld L, Dalal I, Samuel E, et al. Diagnostic clues for identification of nonorganic vs organic causes of food refusal and poor feeding. Journal of Pediatric Gastroenterology and Nutrition 2009;48 355-362.

[52] Zangen T, Ciarla C, Zangen S, Di Lorenzo C, Flores AF, Cocjin J, et al. Gastrointestinal motility and sensory abnormalities may contribute to food refusal in medically fragile toddlers. Journal of Pediatric Gastroenterology and Nutrition 2003;37 287-293.

[53] Cornish E. A balanced approach towards healthy eating in autism. Journal of Human Nutrition and Dietetics 1998;11 501-509.

[54] Cornish E. Gluten and casein free diets in autism: A study of the effects on food choice and nutrition. Journal of Human Nutrition and Dietetics 2002;15 261-269.

[55] Kerwin MLE, Eicher PS, Gelsinger J. Parental report of eating problems and gastrointestinal symptoms in children with Pervasive Developmental Disorders. Children's Health Care 2005;34 217-234.

[56] Baranek GT, David FJ, Poe MD, Stone WL, Watson LR. Sensory Experiences Questionnaire: Discriminating sensory features in young children with autism, developmental delays, and typical development. Journal of Child Psychology and Psychiatry 2006;47 591-601.

[57] Leekam SR, Nieto C, Libby SJ, Wing L, Gould J. Describing the sensory abnormalities of children and adults with Autism. Journal of Autism and Developmental Disorders 2007;37 894-910.

[58] Harrison J, Hare DJ. Brief Report: Assessment of sensory abnormalities in people with Autistic Spectrum Disorders. Journal of Autism and Developmental Disorders 2004;34 727-730. 
[59] Jones R, Quigney C, Huws J. First-Hand Accounts of Sensory Perceptual Experiences in Autism: A qualitative analysis. Journal of Intellectual and Developmental Disability 2003;28 112-121.

[60] Emond A, Emmett P, Steer C, Golding J. Feeding symptoms, dietary patterns, and growth in young children with Autism Spectrum Disorders. Pediatrics 2010;126 e337e342.

[61] Ho HH, Eaves LC, Peabody D. Nutrient intake and obesity in children with Autism. Focus on Autism and Other Developmental Disabilities 1997;12 187-192.

[62] Levy SE, Souders MC, Ittenbach RF, Giarelli E, Mulberg AE, Pinto-Martin JA. Relationship of dietary intake to gastrointestinal symptoms in children with Autistic Spectrum Disorders. Biological Psychiatry 2007;61 492-497.

[63] Geraghty ME, Depasquale GM, Lane AE. Nutritional intake and therapies in Autism - a spectrum of what we know : Part 1. Infant, Child, \& Adolescent Nutrition 2010;2 $62-69$.

[64] Bandini LG, Anderson SE, Curtin C, Cermak S, Evans EW, Scampini R, et al. Food selectivity in children with Autism Spectrum Disorders and typically developing children. Journal of Pediatrics 2010;157 259-264.

[65] Altenburger JL. The quality of nutritional intakes in children with Autism. MS thesis, Ohio State University, OH; 2010.

[66] Hediger ML, England LJ, Molloy CA, Yu KF, Manning-Courtney P, Mills JL. Reduced bone cortical thickness in boys with Autism or Autism Spectrum Disorder. Journal of Autism and Developmental Disorders 2008;38 848-856.

[67] McAbee GN, Prieto DM, Kirby J, Santilli AM, Setty R. Permanent visual loss due to dietary vitamin A deficiency in an autistic adolescent. Journal of Child Neurology 2009;24 1288-1289.

[68] Dailey SA. Oral motor skills in children with food refusal behaviors. PhD thesis, The University of Iowa, IA; 2009.

[69] Kanner L. Autistic disturbances of affective contact. Nervous Child 1943;2 217-250.

[70] Brisson J, Warreyn P, Serres J, Foussier S, Adrien-Louis J. Motor anticipation failure in infants with Autism: A retrospective analysis of feeding situations. Autism 2012;16 420-429.

[71] Nadon G, Ehrmann Feldman D, Gisel E. [Review of assessment methods used to evaluate feeding in children with pervasive developmental disorder] in French. Archives de Pediatrie 2008;15 1332-1348.

[72] Seiverling L, Williams K, Sturmey P. Assessment of feeding problems in children with Autism Spectrum Disorders. Journal of Developmental and Physical Disabilities 2010;22 401-413. 
[73] Lukens CT, Linscheid TR. Development and validation of an inventory to assess mealtime behavior problems in children with autism. Journal of Autism and Developmental Disorders 2008;38 342-352.

[74] Evans-Morris S, Dunn Klein M. Mealtime Assessment. In: Evans-Morris S, Dunn Klein M. (eds.) Pre-Feeding Skills. Austin, TX: Pro-Ed. 2000. p. 157-186.

[75] Hendy HM, Williams KE, Riegel K, Paul C. Parent mealtime actions that mediate associations between children's fussy-eating and their weight and diet. Appetite 2010;54 191-195.

[76] Fraker C, Fishbein M, Walbert L, Cox S. Food Chaining: The proven 6-step plan to stop picky eating, solve feeding problems and expand your child's diet. Cambridge, MA: Da Capo Press; 2007.

[77] Archer LA, Rosenbaum PL, Streiner DL. The children's eating behavior inventory: Reliability and validity results. Journal of Pediatric Psychology 1991;16 629-642.

[78] Crist W, Napier-Phillips A. Mealtime behaviors of young children: A comparison of normative and clinical data. Journal of Developmental \& Behavioral Pediatrics $2001 ; 22279$.

[79] Williams KE, Hendy HM, Seiverling LJ, Can SH. Validation of the parent mealtime action scale (PMAS) when applied to children referred to a hospital-based feeding clinic. Appetite 2011;56 553-557.

[80] Faith MS, Storey M, Kral TVE, Pietrobelli A. The Feeding Demands Questionnaire: Assessment of parental demand cognitions concerning parent-child feeding relations. Journal of the American Dietetic Association 2008;108 624-630.

[81] Davies W, Ackerman LK, Davies CM, Vannatta K, Noll RB. About Your Child's Eating: Factor structure and psychometric properties of a feeding relationship measure. Eating Behaviors 2007;8 457-463.

[82] Nadon G. Le profil alimentaire des enfants présentant un trouble envahissant du développement: un lien avec l'âge et le diagnostic. MSc Thesis, Université de Montréal, Montreal: 2007.

[83] Rockett HRH, Breitenbach M, Frazier AL, Witschi J, Wolf AM, Field AE, et al. Validation of a youth/adolescent food frequency questionnaire. Preventive Medicine $1997 ; 26$ 808-816.

[84] Burrows TL, Martin RJ, Collins CE. A systematic review of the validity of dietary assessment methods in children when compared with the method of doubly labeled water. Journal of the American Dietetic Association 2010;110 1501-1510.

[85] Kenny DJ, Koheil RM, Greenberg J, Reid D, Milner M, Moran R, et al. Development of a multidisciplinary feeding profile for children who are dependent feeders. Dysphagia 1989;4 16-28. 
[86] Reilly S, Skuke D, Wolke D. SOMA: The schedule of Oral Motor Assessment. London: Whurr Publisher; 2000.

[87] Reilly S, Skuse D, Mathisen B, Wolke D. The objective rating of oral-motor functions during feeding. Dysphagia 1995;10 177-191.

[88] Skuse D, Stevenson J, Reilly S, Mathisen B. Schedule for oral-motor assessment (SOMA): Methods of validation. Dysphagia 1995;10 192-202.

[89] Bruns DA, Thompson SD. Feeding challenges in young children: Toward a best practices model. Infants and Young Children 2010;23 93-102.

[90] Twachtman-Reilly J, Amaral SC, Zebrowski PP. Addressing feeding disorders in children on the autism spectrum in school-based settings: Physiological and behavioral issues. Language, Speech, and Hearing Services in Schools 2008;39 261-272.

[91] Geraghty ME, Bates-Wall J, Ratliff-Schaub K, Lane AE. Nutritional interventions and therapies in Autism - a spectrum of what we know: Part 2. Infant, Child, and Adolescent Nutrition 2010;2 120-133.

[92] Angell A. Selective eaters and tactile sensitivity: A review of classification and treatment methods that address anxiety and support a child's need for a sense of control. Infant, Child, and Adolescent Nutrition 2010;2 299-303.

[93] Schreck KA, Williams K. Food preferences and factors influencing food selectivity for children with Autism Spectrum Disorders. Research in Developmental Disabilities 2006;27 353-363.

[94] Williams KE, Foxx RM. Treating Eating Problems of Children with Autism Spectrum Disorders and Developmental Disabilities. Austin, Texas: Pro-ED Inc.; 2007.

[95] Kodak T, Piazza CC. Assessment and behavioral treatment of feeding and sleeping disorders in children with Autism Spectrum Disorders. Child and Adolescent Psychiatric Clinics of North America 2008;17: 887-905, x-xi.

[96] Matson JL, Fodstad JC. Issues in identifying the etiology of food refusal in young children. Journal of Pediatric Gastroenterology and Nutrition 2009;48 274-275.

[97] Kozlowski AM, Matson JL, Fodstad JC, Moree BN. Feeding therapy in a child with Autistic Disorder: Sequential food presentation. Clinical Case Studies 2011;10 236-246.

[98] Koegel RL, Bharoocha AA, Ribnick CB, Ribnick RC, Bucio MO, Fredeen RM, et al. Using individualized reinforcers and hierarchical exposure to increase food flexibility in children with Autism Spectrum Disorders. Journal of Autism and Developmental Disorders 2011;42 1574-1581.

[99] Ayres AJ. Sensory Integration and the Child. Los Angeles, CA: Western Psychological Services; 1979.

[100] Schaaf R, Schoen S, Smith Roley S, Lane S, Koomar J, May-Benson T. A Frame of reference for sensory integration. In: Kramer P \& Hinojosa J. (eds.) Frames of Reference 
for Pediatric Occupational Therapy. Baltimore, MD: Wolters Kluwer Lippincott Williams \& Wilkins; 2010. p. 99-186.

[101] Miller LJ, Anzalone ME, Lane SJ, Cermak SA, Osten ET. Concept evolution in sensory integration: A proposed nosology for diagnosis. 2007.

[102] Baranek GT, Foster LG, Berkson G. Tactile defensiveness and stereotyped behaviors. The American Journal of Occupational Therapy 1997;51 91-95.

[103] Ghanizadeh A. Sensory processing problems in children with ADHD, a systematic review. Psychiatry Investigation 2011;8 89.

[104] Dovey TM, Staples PA, Gibson EL, Halford JCG. Food neophobia and 'picky/fussy' eating in children: A review. Appetite 2008;50 181-193.

[105] Reynolds S, Lane SJ. Diagnostic validity of sensory over-responsivity: A review of the literature and case reports. Journal of Autism and Developmental Disorders 2008;38 516-529.

[106] Farrow CV, Coulthard H. Relationships between sensory sensitivity, anxiety and selective eating in children. Appetite 2012;58 842-846.

[107] Naish KR, Harris G. Food Intake Is Influenced by Sensory Sensitivity. PloS one 2012;7:e43622.

[108] Coulthard H, Blissett J. Fruit and vegetable consumption in children and their mothers. Moderating effects of child sensory sensitivity. Appetite 2009;52 410-415.

[109] Flanagan MA. Improving Speech and Eating Skills in Children with Autism Spectrum Disorders: An Oral-Motor Program for Home and School. Shawnee Mission, Kansas: Autism Asperger Publishing Company; 2008.

[110] Ernsperger L, Stegen-Hanson T. Just Take a Bite: Easy, Effective Answers to Food Aversions and Eating Challenges! Arlington, VA: Future Horizons Inc; 2004.

[111] Toomey K, Nyhoff A, Lester A. Picky Eaters vs. Problem Feeders: The SOS Approach to Feeding: Conference Proceedings, February 2007, Manchester, NH: Education Resources Inc. 2007.

[112] Cox S, Fraker C, Walbert L, Fishbein M. Food chaining: A systematic approach for the treatment of children with eating aversion. Journal of Pediatric Gastroenterology and Nutrition 2004;39 S51.

[113] Fraker C, Walbert L. Treatment of selective eating and dysphagia using pre-chaining and food chaining® therapy programs. Perspectives on swallowing and swallowing disorders. Dysphagia 2011;20 75-81.

[114] Toomey KA, Ross ES. SOS approach to feeding. Perspectives on swallowing and swallowing disorders. Dysphagia 2011;20 82-87.

[115] Nicholls D, Christie D, Randall L, Lask B. Selective eating: Symptom, disorder or normal variant. Clinical Child Psychology and Psychiatry 2001;6 257-270. 
[116] Lane AE, Dennis SJ, Geraghty ME. Brief report: Further evidence of sensory subtypes in Autism. Journal of Autism and Developmental Disorders 2011;41 826-831.

[117] Lane AE, Young RL, Baker AEZ, Angley MT. Sensory processing subtypes in Autism: Association with adaptive behavior. Journal of Autism and Developmental Disorders 2009;40 112-122.

[118] Lindvall C, Owen I, Lask B. Cognitive Remediation Therapy (CRT) for Children and Adolescents with Eating Disorders: Resource Pack. 2011.

[119] Baron-Cohen S, Golan O, Ashwin E. Can emotion recognition be taught to children with Autism Spectrum conditions? Philosophical Transactions of the Royal Society B: Biological Sciences 2009;364 3567-3574.

[120] Gray C. The New Social Story Book: 10th Anniversary Edition. Arlington, VA: Future Horizons Inc.; 2010.

[121] Bledsoe R, Myles BS, Simpson RL. Use of a social story intervention to improve mealtime skills of an adolescent with Asperger syndrome. Autism 2003;7 289-295.

[122] Mesibov GB, Shea V, Schopler E. The TEACCH Approach to Autism Spectrum Disorders. New York: Kluwer Academic/Plenum; 2005.

[123] Bondy A, Frost L. The Picture Exchange Communication System. Behavior Modification 2001;25 725-44.

[124] Tessier MJ. Marqueurs précoces des problèmes sensoriels chez le jeune enfant présentant des problèmes d'alimentation. MSc thesis, Université de Montréal, Montreal; 2011.

[125] Dunn W. The Sensory Profile Manual. San Antonio TX: Psychological Corporation; 1999.

[126] Miller-Kuhaneck H, Henry DA, Glennon TJ, Mu K. Development of the sensory processing measure-School: Initial studies of reliability and validity. The American Journal of Occupational Therapy 2007;61 170-175.

[127] Parham L, Ecker C, Kuhaneck HM, Henry D, Glennon T. Sensory Processing Measure. Los Angeles, CA: Western Psychological Services; 2006. 
Chapter 26

\title{
Clinical Approach in Autism: \\ Management and Treatment
}

\author{
Rudimar Riesgo, Carmem Gottfried and \\ Michele Becker \\ Additional information is available at the end of the chapter \\ http://dx.doi.org/10.5772/54784
}

\section{Introduction}

The terms Autism and ASD (Autism Spectrum Disorders) can be interchangeable in the clinical setting, and have been used to describe one of the most intriguing neurobehavioral syndromes, that include the so-called "triad of Wing": problems in communication, social skills, and restrict repertoire of interests. However, it is somewhat difficult to precisely define autism, because of the imprecise boundaries between different kinds of ASD as well as the fact that there is no biological marker to date (Gottfried and Riesgo 2011).

By definition, in autism the social deficits are characterized by lack of interest in spontaneously sharing feelings, different levels of communication deficits, difficulties in imaginative plays, restrictive repertoire of interests, non-functional routine fixations, as well as stereotypies and other motor alterations, such as flapping with hands, circular movements and others (Nikolov, Jonker, and Scahill 2006; Gadia, Tuchman, and Rotta 2004).

While the criteria of the DSM-V (Diagnostic and Statistical Manual of Mental Disorders - Fifth Edition) are not yet published, we still have to use the "older version". According with the DSM-IV criteria, there are five clinical situations that could be encompassed by the term "PDD" (Pervasive Developmental Disorders) or "ASD" (Autism Spectrum Disorders) with the same meaning of PDD or autism (Association 2002).

Although it will change in the near future, the five current clinical ASD diagnosis admitted by DSM-IV-TR (Gadia et al., 2004) are: a) Autistic Disorder; b) Asperger Disorder (AD); c) Rett Disorder; d) Childhood Disintegrative Disorder; e) PDD-NOS (Pervasive Developmental Disorder - Not Otherwise Specified). 
According with DSM-IV-TR, and in agreement with previous epidemiological data, our group found that the most prevalent ASD is the PDD-NOS, followed by Autistic Disorder, and then by Asperger Disorder. Accordingly, the Rett Disorder and the Childhood Disintegrative Disorder for sure are less frequently seen in the clinical practice (Longo et al. 2009).

The increasing levels of prevalence in ASD probably is due to several reasons, such as the changes in diagnostic criteria, the high level of awareness, the underestimation of former data, the massive information exchange regarding ASD, the public strategies, etc. The first description of autism was made by Hans Asperger, in 1938. In 1943, when Leo Kanner described a sample with 11 children, autism was a rare condition affecting not more than 4 in 10.000 children (Kanner 1943).

However, childhood autism is much more frequent and is identified in at least one in each 100 children nowadays. For instance, a recent paper describes prevalence of $2.6 \%$ of ASD in children aging from seven to twelve years of age (Kim et al. 2011).

Autism and ASD certainly have different kinds of approaches. These neurobehavioral syndromes can be addressed, for example, both from the clinical and from the experimental field. To our knowledge, at least in the academic environment, the best approach could be the translational type because it made us able to rapidly build a bridge between the experimental and the clinical field (Gottfried and Riesgo 2011).

Obviously, the earlier results usually came from the experimental research for several reasons. In general, the time spent in each one experiment can be shorter compared to clinical research; the environmental variables can be in part controlled, etc. By the other side, clinical research can be more time consuming and potentially more complicated to be performed. There is no doubt that both approaches are not mutually exclusive. Actually they are complementary.

Strictly speaking from the clinical perspective in autism, we can divide the clinical approach into two basic and complementary issues. The first one is the general management, including the confirmation of the correct diagnosis, the determination of the intensity of the compromise, and the evaluation of intensity level of eventual core behavioral symptoms. The last one encompasses several treatment options, which includes psychopharmacotherapy and different types of non-medical treatments.

As the first cases of autism were described in the early 40's, now we have adults with ASD. That is the reason to keep in mind how ASD symptoms usually change during lifetime. As time pass, different symptoms change differently and it is crucial to clinicians to know these differences.

In this context, the present chapter aimed to review (i) the general management of ASD from the clinical perspective; (ii) the lifetime changes in ASD symptoms; and (iii) the evidence-based treatment options.

\section{General management of ASD from the clinical perspective}

The general management of ASD from the clinical perspective encompasses both interventions in the family/environment as well as interventions addressed to the patient. Ideally, after 
diagnosis confirmation, the best initial approach could be done by an interdisciplinary team including professionals coming from medicine, psychology and social sciences.

Obviously, before initiating any kind of intervention, several steps must be done as follows. First of all, the final diagnosis must be confirmed by a careful anamnesis as well doublechecked using the DSM-IV criteria as well as a reliable clinical instrument such as Autism Diagnosis Interview-Revised (ADI-R) (Becker et al. 2012). The ADI-R is frequently used as a gold standard instrument for publication purposes, but it is problematic in the clinical practice for several reasons, such as it can miss same ASD cases as well as it need at least two hours to be completed. Then, the intensity of the ASD could be defined both from the clinical perspective and by one instrument such as CARS (Pereira, Riesgo, and Wagner 2008). Another critical issue is to delimitate if there is any associated mental disability and its degree of intensity. As clinicians, we know the prognostic importance of an unaffected intelligence in ASD patients.

The second step includes the definition of the parent's doubts, fears, and degree of awareness. Usually, after diagnosis confirmation, parents became stressed. Not infrequently they go to internet in order to search every kind of available information regarding autism. Because some information coming from internet can be inaccurate, at this point, it is very important to clarify which are the evidence-based types of therapies to date.

The third step could be the delimitation of environmental variables that needs to be addressed, starting from the home and family. Neighborhood and school needs to be evaluated both in terms of potential stressors and also because they can facilitates choosing a given type of therapy on an individual basis.

The next step is done by the identification of the target behaviors needing treatment. After core symptoms definition in each case, the different professional specialties that need to be involved are selected. In general, the team includes a physician specialized in ASD patients as well as one speech therapist and others professionals arising from health care and/or education with experience in children with ASD.

\section{How ASD symptoms change during lifetime}

All professionals who treat children and adolescents, both coming from health care as well as from education must know how behaviors can normally change during the normal neuropsychological development. In other words: there is an ontogenetic evolution on each one of the behavioral manifestations in the normally developed children.

For example: in terms of gender versus behavior, usually hyperactivity is more prevalent in normal boys when compared with normal girls. The humor control, the language skills and the social competence usually improves in normally developed children as long as time passes. Usually, normal girls tend to improve faster their language skills and their social competence when compared with normal boys. This knowledge is crucial to identify how different behavioral symptoms change during lifetime in ASD patients. 
When the issue is childhood autism symptoms, there are no major problems in terms of information, because of most of the available publications are directed to pediatric patients. As a consequence, adult ASD symptoms are less frequently accessed in the available literature.

Researchers had noted that the prevalence of adult ASD may be underestimating and most of these patients reach adulthood without any diagnosis or treatment. This is especially true to patients with Asperger. (Szatmari et al. 1995; Arora et al. 2011).

More recently, an increasing interest is observed in prevalence and clinical presentation of ASD in adults. The few available prospective studies indicate a diagnostic stability through life (Billstedt, Gillberg, and Gillberg 2005), and near of $80 \%$ of individuals with ASD diagnosed in childhood continues to present scores within this spectrum during adolescence and adulthood (Rutter, Greenfeld, and Lockyer 1967).

It is important to mention the difficulties in making diagnosis of ASD in adult patients, because many of them have no information regarding their first years of life. If the diagnosis of ASD is hard to be made in adults, then the prognosis is equally affected. The prognostic studies in adults with ASD had includes patients with very different levels of cognitive, linguistic, social, and behavioral functioning (Howlin et al. 2004). Additionally, most of available the prognostic studies in adult ASD use small samples, which make impossible to obtain definitive conclusions.

Where searching literature regarding how ASD symptoms change during lifetime, a paucity of published information is promptly identified. Although the lack of publications, at least two different timelines could be identified in ASD patients: a) how ASD core symptoms change as time pass; b) how ASD-associated symptoms change with time.

\subsection{How ASD core symptoms change during lifetime}

The three core symptoms of ASD, the so-called "triad of Wing" are the following: social deficits, communication deficits, and restrict and repetitive behavior.

The social deficits persist as an important problem in adolescence and adult age and usually are accessed by the Autism Diagnostic Interview (ADI) and also by the Vineland Adaptive Behavior Scale (VABS). Our group translated into Brazilian Portuguese the ADI-R, considered the "gold-standard" in autism diagnosis and is extremely useful identifying social deficits (Becker et al. 2012). One study found that only $16.7 \%$ of adults with autism presented high scores in social domain of VABS. Additionally, more than half of patients had no social contact at all and one third showed strange social contact (Howlin, Mawhood, and Rutter 2000). In general, social deficits do not improve significantly as time pass.

The communication skills tend to improve. As a group, ASD patients tend to keep almost unchanged the idiosyncratic use of language as well as the inappropriate patterns of communication in adulthood. More recent research had shown that more than half of ASD patients present language below the level of ten years of age, when adults. When comparing ASD versus AD patients with similar age and cognition, it is identified a 
slight superiority in language skills in the AD patient group (Mawhood, Howlin, and Rutter 2000; Howlin et al. 2004).

The restrictive repertoire of activities and interests do not change in intensity as long as time passes, but certainly the type of interest do change during lifetime. Only few studies address the restrictive repertoire of interests. According with Rutter and colleagues (1967), in a cohort study, although some improvement was identified, all of patients with repetitive behaviors during infancy continued presenting it 10 years later, with a trend to increasing frequency and intensity of such symptoms (Rutter, Greenfeld, and Lockyer 1967). Subsequent research showed that near of $90 \%$ of adolescents and adults with autism persisted with restrictive repertoire of activities and interests (Seltzer et al. 2003; Howlin et al. 2004).

Another recurrent preoccupation in ASD follow up is regarding the Intellectual Quotient (IQ). Although some studies revealed lifelong IQ stability, it seem to have a performance IQ decline and a verbal IQ increase as time pass. In reality, there is a paucity of studies regarding IQ changes lifelong in ASD patients. In patients with verbal and performance IQ above 70, these changes seem to be less intense (Howlin et al. 2004).

\begin{tabular}{lll}
\hline Core symptom & \multicolumn{1}{c}{ In adolescence } & \multicolumn{1}{c}{ In adulthood } \\
\hline Social deficits & $\begin{array}{l}\text { Persistence of social deficits. A } \\
\text { discrete improvement can occur }\end{array}$ & $\begin{array}{l}\text { Persistence of social deficits. A discrete improvement can } \\
\text { occur }\end{array}$ \\
\hline $\begin{array}{ll}\text { Communication deficits Can improve, but some deficits } \\
\text { persists }\end{array}$ & Can improve, but some deficits persists \\
\hline $\begin{array}{ll}\text { Restrict repetitive } \\
\text { behavior }\end{array}$ & $\begin{array}{l}\text { Increase in frequency and } \\
\text { complexity }\end{array}$ & $\begin{array}{l}\text { Persists in 90\%. Uncommon concerns and complex } \\
\text { stereotypies can decrease. The focus of interests can vary }\end{array}$ \\
\hline
\end{tabular}

Table 1. How ASD core symptoms change during lifetime.

\subsection{How ASD-associated symptoms change with time}

There are few epidemiologic studies of the ASD-associated comorbidities changes as time pass. Consequently, to date any estimate need to be taken with caution.

In general, the comorbidities found in classic autism are different from the identified in Asperger patients, which is probably associated with cognition. As a result, classic autism is more associated to violent behavior, and psychosis. By the other side, Asperger disorder can be more linked to anxiety and/or depression.

The more prevalent psychiatric diagnosis in ASD patients is depression that seems to become more intense with age and frequently associated with anxiety (Howlin, Mawhood, and Rutter 2000). In our experience, the dyad depression/anxiety is more frequent in intelligencepreserved ASD patients, such as those with Asperger disorder. Additionally, anxiety seems to increase in stress situations and also during lifetime (Gottfried and Riesgo 2011). Because of their ability to identify their own difficulties (Cederlund, Hagberg, and Gillberg 2010), patients with Asperger are more prone to became depressed. 
The second more frequent psychiatric disorder in ASD patients is probably bipolar disorder (Howlin, Mawhood, and Rutter 2000). Young ASD children experience more difficulties in mood stabilization. In addition, mood's changes occur more rapidly in children when compared with adults. As a result, in very young ASD children the humor can change almost instantaneously.

The prevalence of bipolar disorders as a whole can reach up to $33 \%$ in ASD patients (Abramson et al. 1992). Obsessive and compulsive symptoms are frequently identified in ASD, although is difficult to distinguish the pure obsessive-compulsive disorder from bizarre concerns common in patients with autism (Howlin, Mawhood, and Rutter 2000).

Adults with Asperger disorder can experience occasional episodes of psychosis, such as persecutory ideas, auditory hallucinations, paranoid idea or delusional thoughts. But schizophrenia is not common and must remain as a differential diagnosis (Howlin, Mawhood, and Rutter 2000). The abovementioned episodes of psychosis can be identified in up to $15 \%$ of Asperger patients after adolescence (Hofvander et al. 2009).

Hyperactivity is a frequent symptom in children with ASD, is more prevalent in boys than in girls, and can decrease as time passes. Although the concomitant aggressiveness itself usually decrease with aging, the consequences of aggressiveness can be worse with age increasing in patients with autism because of their increase of muscle strength. An overlap between ADHD and ASD is relatively common in childhood, but this association is rarely described in manuscripts with ASD adults (Stahlberg et al. 2004).

\subsection{Prognosis for ASD patients in adulthood}

Although there are no doubts regarding a substantial improvement in the management of autism in the last three decades, unfortunately even nowadays a minority of adults with autism is able to work, to live independently, as well to develop appropriate social skills. Most of these patients still live with their parents or other caregivers (Howlin et al. 2004).

It is known by far that the most important prognostic value is defined by the cognitive functioning in childhood. In this sense, the clinical problem eventually is to access intelligence in non-verbal ASD children. According with literature, children with autism and IQ above 70 had better global prognosis in adulthood (Howlin et al. 2004).

The ability to acquire functional language until the age of six years is also another prognostic landmark (Howlin et al. 2004). Better language and more preserved cognition are the two probably reasons to explain the best prognosis in Asperger disorder when compared with classical forms of autism.

\section{Psychopharmacological treatment of ASD patients}

Since to date there is no specific medication developed to autism itself, the psychopharmacologic approach is addressed to some core symptoms, such as hyperactivity, anxiety, depres- 
sion, etc. Actually, medication is frequently required to decrease the "noise" surrounding autism, including a wide range of maladaptive behaviors and/or associated problems (Benvenuto et al. 2012). To our knowledge, psychopharmacotherapy can eventually improve adhesion to non-medical treatment of ASD patients (Gottfried and Riesgo 2011).

In our experience, we usually identify 2-5 ASD associated symptoms and/or diagnosis, including epilepsy. We have found disruptive behavior more frequently in ASD patients with cognitive impairment, as well as symptoms related with depression and/or anxiety in preserved intelligence ASD children (Gottfried and Riesgo 2011). Other related symptoms are: aggression, self-injury, impulsivity, decreased attention, anxiety, depression, and sleep disruption, among others.

Because ASD are chronic and markedly impairing situations in many cases, there is justifiably a high desire for effective treatments. By the other side, it is important to mention that there is a paucity of well conducted evidence-based studies of medications used in ASD patients. Not infrequently, this desire leads to premature enthusiasm for agents and interventions that appear promising in early reports but later do not withstand the rigor of randomized controlled trial (RTC).

Another critical issue is the co-occurrence of epilepsy in ASD patients which is almost twenty times more frequent when ASD patients are compared with children with typical development. The management of combined epilepsy can represent a challenge for clinicians. Several anti-epileptic drugs can determine an exacerbation of behavioral symptoms, and some psychotropic medications used in ASD patients may lower the seizure threshold (Benvenuto et al. 2012). In our experience, risperidone can be safely used up to $3 \mathrm{mg} / \mathrm{Kg} / \mathrm{day}$, and higher doses can lead to seizures in susceptible patients. That is the reason why we prefer to perform an electroencephalogram before using psychoactive drugs in ASD children (Gottfried and Riesgo 2011). Therefore, it's mandatory to search a treatment strategy with the minor negative impact on this subgroup of patients

It should be noted that most psychotropic use in ASD is actually off-label, as currently there are only two medications approved for use in ASD children by the FDA (Food and Drug Administration). These drugs are risperidone and aripiprazole, which are effective to associated behaviors, but not to autism itself. The general principles for the pharmacotherapy in ASD are similar to the used in other neuropsychiatric conditions (Weinssman and Bridgemohan 2012).

In summary, the use of psychotropic medications, alone or in combination, should follow some guidelines, such as: be focused on specific targets, be used at the minimum effective dosage, as well as be used for short period of time (Benvenuto et al. 2012). Ideally, medications should be initiated only after behavioral and educational interventions are in place.

\subsection{Disruptive behaviors}

Disruptive behaviors in ASD children may include irritability, aggression, explosive outbursts (tantrums), and/or self-injury. These symptoms can be identified in almost two thirds of ASD patients and certainly have the biggest impact on the care of affected individuals, as well as 
marked distress for their families (Benvenuto et al. 2012; Kanne and Mazurek 2011). Although behavioral and environmental approaches are recommended as the initial treatment, more severe or even dangerous behaviors usually result in requests for urgent pharmacologic intervention (Kaplan and McCracken 2012; Weinssman and Bridgemohan 2012). In our experience, this type of symptoms is more frequently found in intelligence disabled ASD patients (Gottfried and Riesgo 2011).

In the past, conventional neuroleptic agents such as haloperidol have been used in disruptive behaviors of autistic patients (Benvenuto et al. 2012; Miral et al. 2008; Kaplan and McCracken 2012). Our group showed that risperidone is superior when compared with haloperidol in one experimental research using hippocampal cells (Quincozes-Santos et al. 2010). Additionally, in the clinical research, at least one study proved that risperidone is more effective than haloperidol in ASD patients (Miral et al. 2008). There are two RTC suggesting that haloperidol is effective in disruptive behaviors of ASD children (Campbell et al. 1982; Miral et al. 2008), but sedation and other side effects including dyskinesia and extrapyramidal symptoms limits its use (Weinssman and Bridgemohan 2012).

As a result, to date atypical antipsychotic seem to be more helpful in treatment of disruptive behaviors. Currently, risperidone and aripiprazole are the only second-generation antipsychotic drugs that have shown to decrease disruptive behaviors in large-scale, controlled, double-blind studies (Benvenuto et al. 2012; Kaplan and McCracken 2012; Weinssman and Bridgemohan 2012).

Before the approval by FDA in 2006, risperidone was carefully studied by the NIMH Research Units on Pediatric Psychopharmacology (RUPP) Autism Network. A multiphasic trial comparing risperidone with placebo was performed by RUPP for the treatment of aggressive behaviors in patients aged 5 to 17 years with ASD. There was an initial double-blind, 8-week RCT study (McCracken et al. 2002).

The studies found that risperidone, in mean doses of 2,08mg/d, was effective for reducing moderate to severe tantrums, aggression, and self-injurious behavior in children with autism. There wasn't evidence of side effects such as dyskinesia or dystonia. However, the observed weight gain of $5,6 \mathrm{~kg}$ for the risperidone group was more than twice the expected weight gain over a 6-month period (McCracken et al. 2002; Kaplan and McCracken 2012).

Risperidone was approved by the FDA in 2006 for the treatment of disruptive symptoms in children and adolescents aged from 5 to 16 years with autism, with a maximum recommended dose of $3 \mathrm{mg} / \mathrm{d}$. In our experience, risperidone was initially used in dose up to $6 \mathrm{mg} / \mathrm{d}$. As time pass, we noted that if no response was obtained with $3 \mathrm{mg} / \mathrm{d}$, no more increments were useful. Coincidentally, this daily regimen seems to be the seizure threshold in susceptible patients (Gottfried and Riesgo 2011).

Aripiprazole was approved by the FDA for the treatment of disruptive behavior in ASD patients aged 6 to 17 years in 2009. Two large controlled studies documented the short-term efficacy of aripiprazole at 5, 10 or $15 \mathrm{mg} / \mathrm{d}$ for severe aggression and irritability in young subjects with autistic disorder. The most commonly reported adverse events were drowsiness and weight gain, with extrapyramidal symptoms mostly in the fixed-dose study, but these 
events rarely led to treatment discontinuation (Marcus et al. 2009; Owen et al. 2009). Aripiprazole dosing and response can vary considerably; the usual recommended clinical dose for maintenance is between 5 and $15 \mathrm{mg} / \mathrm{d}$ (Kaplan and McCracken 2012).

Other atypical antipsychotics lack large-scale controlled studies. Small open-label reports suggest variable benefits of olanzapine (Potenza et al. 1999), clozapine (Beherec et al. 2011), and ziprasidone (Malone et al. 2007), which have possible support, versus quetiapine, which has not appeared to be beneficial. Other medications of different classes have been used, such as alpha-2 agonists, mood stabilizers, beta blockers, SSRI (selective serotonin reuptake inhibitors), all of them without evidence-based studies of efficacy in disruptive behavior to date (Weinssman and Bridgemohan 2012).

Probably due the co-occurrence of epilepsy in ASD, the use of some antiepileptic drugs has been used in the management of maladaptive behaviors (Gottfried and Riesgo 2011). Divalproex sodium has been demonstrated to be efficient not only in decreasing irritability/ aggression, but also in improving of repetitive behaviors, social relatedness and mood instability (Hollander et al. 2006; Hollander et al. 2010).

Adjunctive topiramate therapies can decrease irritability, hyperactivity and inattention (Hardan, Jou, and Handen 2004; Mazzone and Ruta 2006). Moreover, the combination of topiramate with risperidone has been proved superior to risperidone monotherapy in reducing irritability and severe disruptive symptoms (Rezaei et al. 2010). In our experience, this specific combination would be helpful in preventing or at least decreasing the weight gain due to risperidone usage in ASD patients.

Although preliminary data of open-label studies showed that levetiracetam may reduce hyperactivity, impulsivity, mood instability and aggression in autistic children, a RCT suggest that levetiracetam does not improve behavioral disturbances of ASD (Weinssman and Bridgemohan 2012), as well lamotrigine (Belsito et al. 2001).

\subsection{Hyperactivity and inattention symptoms}

These symptoms are frequently identified in ASD patients. Inattention, hyperactivity and impulsivity may be related to comorbid ADHD (attention deficit hyperactivity disorder) and/ or to baseline anxiety of these children (Murray 2010; Rommelse et al. 2010; Benvenuto et al. 2012) Weinssman \& Bridgemohan, 2012). It is known that children with ASD and ADHD have more clinical impairments than children with ASD alone (Gadow, DeVincent, and Pomeroy 2006; Kaplan and McCracken 2012).

The potentially useful drugs for inattention and hyperactivity in ASD could be stimulants, alpha-2 adrenergic agonists, atypical antipsychotics as well as anticonvulsant mood stabilizers. To date, there is strong evidence that both stimulants and risperidone are effective for hyperactivity. If the inattention and/or hyperactivity behaviors are due to anxiety, SSRI may be a useful choice (Weinssman and Bridgemohan 2012).

Psychoestimulants and other medications used in typically developing children with ADHD have been evaluated as a therapeutic option for treatment of ADHD symptoms 
in patients with ASD. The largest trial undertaken by RUPP Autism Network has demonstrated that methylphenidate $(\mathrm{MPH})$ was reasonably efficacious in patient with both ASD and ADHD (RUPP 2005). Convergent evidence from different studies confirms a positive effect on social behaviors (joint attention, response to bids for joint attention, self-regulation, and regulated affective sate), hyperactivity, inattention and impulsiveness (Di Martino et al. 2004; Jahromi et al. 2009). However, response rate to MPH is lower in ASD children compared with children with ADHD without ASD (Weinssman and Bridgemohan 2012). In ASD children, MPH should be started at the lowest dosage ant titrated slowly because of these patients are more prone to experience side effects.

As the same observed with MPH, atomoxetine has initially demonstrated a lower efficacy in ASD patients with ADHD than in ADHD children without autism (Posey et al. 2007; Charnsil 2011). Nevertheless, more recent studies showed significant reductions in ADHD symptoms in high-functioning ASD boys (Zeiner, Gjevik, and Weidle 2011).

Regarding the use of antipsychotic drugs in inattentive/hyperactive ASD patients, secondary analyses from large RTCs demonstrated that risperidone and aripiprazole are associated with large reduction of hyperactivity in children with ASD (McCracken et al. 2002; Owen et al. 2009; Weinssman and Bridgemohan 2012).

Despite the small number of RCT, another option is the use of alpha-2 agonists drugs in ASD children with inattention, hyperactivity, and impulsivity. The use of guanfacine in autistic children has showed modest improvement in the domains of hyperactivity, inattention, insomnia, and tics (Scahill et al. 2006; Handen, Sahl, and Hardan 2008; Weinssman and Bridgemohan 2012). Clonidine is effective in reducing sleep disorders of children with ASD, with a consequent daily improvement of attention deficits, hyperactivity, mood instability and aggressiveness (Jaselskis et al. 1992; Ming et al. 2008). However, only two RCT have been conducted for this class of agent (Weinssman and Bridgemohan 2012).

\subsection{Stereotypy and repetitive behaviors}

One of the core symptoms in ASD children is perseverative or repetitive behaviors usually associated with difficulties in change interests, which can interfere in the quality of life of patients and parents. Stereotypies and repetitive behaviors are not unique to ASD and can be found in other developmental disorders, although clinicians and researchers agree that these tend to be more frequent in ASD (Kaplan \& McCracken, 2012; Leekam et al., 2011). By the other hand, difficulties in changing interests, in the context of a developmental disorder, is one of the hallmarks of autism.

Before use of medication, behavioral therapies should be performed. In our experience, poor cognitive performance can be one of the limitations to behavioral therapy. If the child is mentally disabled, the non-medical approach can be unsuccessful. In this situation, when these symptoms are intense enough to cause impairments to academic performance and/or interpersonal relationships, pharmacologic treatment is often considered. 
Because of the similarity of this cluster of autistic symptoms to anxiety as well as other serotonin-related disorders such as obsessive compulsive disorder has led clinicians to use and researchers to investigate the efficacy of SSRI in the treatment of repetitive behaviors and rigidity. Other possibilities in terms of medication include clomipramine, atypical antipsychotics and valproate (Weinssman \& Bridgemohan, 2012).

To date, although the lack of high quality evidence that SSRI are effective to stereotypy and repetitive behaviors, we still use this class of medication in clinical practice. In a meta-analysis of published trials with different classes of antidepressants, including SSRI and tricyclic antidepressants, the small benefit of these drugs on repetitive behavior disappeared after statistical adjustment (Carrasco et al., 2012).

Other types of SSRI were tested in ASD children with stereotypy and repetitive behaviors, for example: fluvoxamine, sertraline, paroxetine, citalopram and escitalopram. There is one unpublished trial of fluvoxamine, which was poorly tolerated by children (McDougle et al., 1996). There are no RCT of sertraline and paroxetine in ASD children (Weinssman \& Bridgemohan, 2012). The largest published trial of citalopram (mean dose 16mg/d) found no effect at all on repetitive or compulsive behavior but found a possible effect on challenging behaviors (King et al., 2009). Others RCT didn't show strengths of evidence for effect of citalopram or escitalopram to reduce repetitive or challenging behavior (McPheeters et al., 2011).

Concerning antipsychotic drugs, in the RUPP studies, stereotypies and repetitive behaviors were examined as secondary outcomes and then risperidone achieved levels of statistical significance in reduction of repetitive behavior (McDougle et al., 2005). Similarly, aripiprazole studies showed that the agent significantly improved repetitive behaviors over placebo (Marcus et al., 2009; Owen et al., 2009).

There is only one small RCT which shows the efficacy of valproate in repetitive behaviors of ASD children (Hollander et al., 2006). Our group avoids the usage of valproate in such symptoms. In summary, from the clinical point of view, it is hard to improve stereotypy and repetitive behaviors with pharmacotherapy. As a matter of fact, sometimes these symptoms can be more uncomfortable to parents than patients.

\subsection{Mood instability}

In clinical practice, mood instability is more difficult to control in ASD patients compared with typically developed children (Gottfried \& Riesgo, 2011). Different drugs have been used, including antipsychotics, SSRI, and lithium. The problem is that none of these medications have been studied with RCT specifically for mood regulation in ASD pediatric patients (Weinssman \& Bridgemohan, 2012).

If mood lability is associated with disruptive behavior, the best choice could be atypical antipsychotics. If this symptom is associated with depression and or anxiety, the use of SSRI could be considered. It is important to remember the higher possibilities of behavioral activation in ASD patients after SSRI use, leading to hypomaniac states in susceptible children. 


\subsection{Sleep disorders}

Sleep disorders can be identified years before an unequivocal diagnosis of autism. Not infrequently, we face with sleep complaints in very young babies who lately will develop the whole clinical picture compatible with ASD. By the other hand, sleep disorders occur more frequently in ASD patients compared with developing children (Benvenuto et al., 2012; Miano \& Ferri, 2010).

Sleep disorders tend to be under-recognized valued in the ASD patient group, probably because they can be considered less disabling than aggression and repetitive behaviors; however, ongoing abnormal sleep patterns are very disruptive to the overall quality of family life and interfere with patient daytime functioning. Parents frequently ask for medication and then physicians are confronted with the lack of FAD-approved treatments for this problem (Kaplan \& McCracken, 2012; Weinssman \& Bridgemohan, 2012).

Before use of medication, is important to ensure appropriate sleep hygiene as well as to use behavioral intervention. Pharmacology is recommended only when psychosocial treatments fail. Melatonin administration in ASDs is reported to be safe, well tolerated and efficient in improving sleep parameters and daytime behavior, and in decreasing of parental stress (Malow et al., 2011; Rossignol \& Frye, 2011).

\begin{tabular}{|c|c|c|}
\hline Core symptoms & Medications & Level of evidence \\
\hline Aggressiveness & Risperidone* & Large scale double blind RCT \\
\hline Irritability & Aripiprazole* & Large scale double blind RCT \\
\hline elf-injury & Olanzapine & Double blind RCT \\
\hline \multirow[t]{4}{*}{ Other disruptive behaviors } & Clozapine & Small open label reports \\
\hline & Ziprazidone & Small open label reports \\
\hline & Valproic acid & $\mathrm{RCT}$ \\
\hline & Topiramate & $\mathrm{RCT}$ \\
\hline Hyperactivity & Metilfenidate & Crossover RCT \\
\hline \multirow[t]{5}{*}{ Inattention } & Atomoxetine & Crossover RCT \\
\hline & Risperidone* & Large scale double blind $\mathrm{RCT}^{\star * *}$ \\
\hline & Aripiprazole* & Large scale double blind $\mathrm{RCT}^{\star * *}$ \\
\hline & Guanfacine & $\mathrm{RCT}$ \\
\hline & Clonidine & Small open label reports \\
\hline Repetitive behavior & Risperidone* & Large scale double blind $\mathrm{RCT}^{\star \star}$ \\
\hline \multirow[t]{3}{*}{ Stereotypies } & Aripiprazole* & Large scale double blind $\mathrm{RCT}^{\star *}$ \\
\hline & Fluoxetine & $\mathrm{RCT}$ \\
\hline & Valproic acid & $\mathrm{RCT}$ \\
\hline Sleep disorders & Melatonin & $\mathrm{RCT}$ \\
\hline
\end{tabular}

Table 2. Psychopharmacological treatment in ASD patients 


\section{Non-medical treatment of ASD patients}

The treatment of ASD evolves professionals coming from different area and usually is characterized by comprehensive and intense programs encompassing both patients and families. Early identification is critically important to ensure that families have the opportunity to reap the many unique benefits that may arise from early intervention efforts. For example, intervention efforts that occur early during a child's development may have the advantage of increasing brain plasticity, which may enhance outcomes (LeBlanc \& Gillis, 2012).

In our experience, children with low intensity ASD treats, when early-treated can eventually get out from de ASD diagnosis when accessed by CARS, a rating scale of autism symptoms (Gottfried \& Riesgo, 2011).

The non-medical intervention programs are directed to the core social, communication and cognitive issues in autism. The objectives of each one program are selected according with the specific abilities and difficulties as well as the actual neurodevelopmental phase of the ASD patient. As a result, this kind of intervention needs to be customized (Dawson \& Burner, 2011; LeBlanc \& Gillis, 2012).

In general, the following types of therapy can be used both isolate or in different combinations: behavioral, occupational, speech therapy as well as psychopedagogic therapy. Although the non-medical treatments for ASD patients can be different from each other, they usually had the same goals, such as to give the child the best degree of independent functioning as well as to improve quality of life from the patient and family (Myers \& Johnson, 2007).

There is a consensus that facing a suspicious case of ASD in children the treatment must be promptly initiated, independently of the type of non-medical treatment, because of the brain plasticity in the developing child (LeBlanc \& Gillis, 2012; Lord \& McGee, 2001).

Besides the large number of non-medical type of treatment, there are some of them with good level of evidence. According with the National Autism Center's Standard Report, after a systematic review of literature available from 1957 to 2007, at least 11 treatment methods for ASD were considered with good level of evidence.

Additionally, there are some problems in evaluating the efficacy of non-medical treatments in ASD patients. For example, the small sample sizes, the different methodologies, the difficulty in the outcome measures, etc.

\subsection{Behavioral treatment}

The therapies involving behavioral and educational strategies are the main components of the non-medical treatments of ASD children. The only psychoeducational treatment that meets the criteria as well-established and efficacious intervention for ASD to date is the behavior treatment (Dawson \& Burner, 2011; LeBlanc \& Gillis, 2012). 
There is consensual that behavioral therapy must be intensive with at least 25 hours per week, all year long. There are two main types of behavioral treatments: interventionists and non-interventionists. Among the first group of available therapies, there are three principal methods: a) Applied Behavior Analysis (ABA); b) Treatment and Educational of Autistic and related Communication-handicapped Children (TEACCH); c) developmental/relationship-based therapy (Floortime). Some of these strategies use combinations of different models and are denominated integrative models. To date, there is no evidence that integrative models are better than the original models (Weinssman \& Bridgemohan, 2012). By the other side, one example of non-interventionist behavioral therapy is the Picture Exchange Charts System (PECS).

\subsubsection{ABA (Applied Behavior Analysis)}

Aims to teach the absent child skills through the introduction of these skills in stages. Usually, each one of the skills is individually showed, presenting it coupled with an indication or instruction. When necessary, any support that is offered should be removed as soon as possible. (Ospina et al., 2008; Warren et al., 2011). In the clinical setting, we have identified problems in terms of improvement from the classroom as well as a trend to overestimate the efficacy of ABA.

\subsubsection{Treatment and Educational of Autistic and related Communication-handicapped Children (TEACCH)}

Use structured activities and environment to help ASD patients to improve compromised area. The model is adapted to each one child and addresses environment organization as well as predicable routines in order to adapt the environment to make it easier for the child to understand it, and understand what is expected of her. TEACCH programs are usually given in a classroom, but can also be made at home. Parents work with professionals as co-therapists for techniques that can be continued at home. It is used by psychologists, special education teachers, speech therapists and trained professionals (Myers \& Johnson, 2007).

\subsubsection{Floortime}

The main objective is to teach fundamental skills expected to the level of development which were not acquired in a given ASD patient age, but to date the efficacy evidences are still inconclusive (Ospina et al., 2008). Our group is conducting an evidence-based research to find out if this treatment is reliable.

\subsubsection{Picture Exchange Communication System (PECS)}

This non-interventionist behavioral therapy enables non-verbal children to communicate by using figures. PECS can be used at home, in the classroom or in several others environments (Bondy \& Frost, 2001). A meta-analysis showed that PECS is a promising intervention (Ganz et al., 2012). 


\begin{tabular}{|c|c|c|}
\hline $\begin{array}{l}\text { Psychoeducational } \\
\text { treatments }\end{array}$ & Example & Effectiveness \\
\hline Interventional Models & $\begin{array}{c}\text { ABA }^{*} \\
\text { TEACCH } \\
\text { Denver model } \\
\text { Floortime }\end{array}$ & $\begin{array}{l}\text { Well established } \\
\text { Insufficient evidence to recommend one over } \\
\text { another }\end{array}$ \\
\hline Specific behaviors & Focal behavior intervention & Well established \\
\hline Communication & PECS & Promising results \\
\hline Social skills instruction & & Promising results \\
\hline Integrative Models & Focal behavior intervention & $\begin{array}{l}\text { Insufficient evidence to recommend one over } \\
\text { another }\end{array}$ \\
\hline Parental role & $\begin{array}{l}\text { Parent-mediated intervention } \\
\text { programs }\end{array}$ & $\begin{array}{l}\text { Inconsistent results } \\
\text { Small size studies }\end{array}$ \\
\hline Sensory integration therapy & & Inconsistent results \\
\hline Occupational therapy & & Little research \\
\hline
\end{tabular}

Table 3. psychoeducational treatment of ASD patients

\subsection{Complementary and alternative therapies}

Complementary and alternative medicine (CAM) encompasses different kinds of medical and healthcare systems, practices, and products usually not considered to be a part of the conventional medicine. There are several proposed CAM systems to treat ASD children, but to date still without recognized efficacy by FDA. As a result, they are considered "off label". Interestingly, more than $70 \%$ of ASD patients are treated by CAM (Rossignol, 2009).

It is important to note that the definition of CAM is slightly different when used in ASD when compared with other medical disorders. That difference is due the fact of many of the psychoeducational therapies used in ASD children, although not considered conventional medical therapies; they are well accepted methods treating this group of patients.

In terms of scientific support, there are three main groups of CAM: a) promising treatments; b) treatments with some degree of scientific evidence; c) treatments with no scientific proved efficacy to date (Rossignol, 2009).

\subsubsection{Promising CAM}

These types of treatment showed the highest level of evidence and include music therapy, naltrexone, and acetyl-cholinesterase inhibitors (Rossignol, 2009). Concerning music therapy, there is evidence that it is able to improve social interaction as well as communication skills (Gold et al., 2006; Kim et al., 2008). Our group conducted a RCT using music therapy in ASD patients and we identified the promising effect of this treatment (Gattino et al., 2011). There is 
a comprehensive RCT been done testing the efficacy of music therapy in ASD patients (Geretsegger et al., 2012).

\subsubsection{CAM with little evidence}

This group of therapies may include the use of carnitine, ocytocin, vitamin C, tetrahydrobiopterin, adrenergic alfa- 2 agonists, hyperbaric oxygen therapy, immune-modulatory treatment, and anti-inflammatory treatment (Rossignol 2009). Caution is needed with the hyperbaric oxygen therapy because of the potential adverse effects, such as barotrauma, reversible myopia, oxygen toxicity, and seizures (Weinssman \& Bridgemohan, 2012).

\subsubsection{CAM with no proved efficacy to date}

Several of the proposed CAM for ASD had no proved efficacy to date, for example: use of carnosine, multi-vitamin and mineral complexes, piracetam, omega-3 fatty acids, selective diets, vitamin B6, magnesium, chelation, cyproheptadine, glutamate antagonists, acupuncture, auditory integration training, massage, neuro-feedback, and others (Rossignol, 2009).

\section{Clinical recommendations in ASD}

The following clinical recommendations can be done as a result of more than twenty years of personal clinical practice in Child Neurology dealing with ASD children, among other neuropediatric situations. For instance, our Child Neurology Unit (http://www.ufrgs.br/ neuropediatria) usually makes more than 16,000 neuropediatric evaluations per year.

From the clinical point of view, it is important to remember the ongoing changes in DSM criteria for ASD diagnosis. To date, we still deal with five different diagnosis of autism, according with DSM-IV criteria. Even after modifications due the new DSM-V classification, ASD children will remain as a heterogeneous group, making difficult the exact clinical diagnosis.

It is important to remember that ASD diagnosis can be catastrophic to parents. As a result, an incorrect diagnosis would be even worse. That is the reason to be careful in terms of making ASD diagnosis as well as to make a double check if diagnosis is really correct.

After finishing a list of the prominent symptoms, the next step is to decide if they are intense enough to deserve treatment, which is not easy. Some symptoms seem to be more unpleasant to parents than the ASD child. At this point, there is no guideline to follow, and the previous clinical experience is extremely helpful.

Usually the non-medical treatment is started earlier than the use of medications. It is important to remember the relevance of evidence-based CAM, since there are a great number of proposed non-medical treatments.

In general, medications are used in addition to non-medical treatments. The best medication approach would be monotherapy, but it is not always possible in the real clinical 
world. Another critical problem in terms of psychopharmacotherapy is the paucity of well-conducted RCT, as pointed before in this chapter, especially in the table 2. To date, there are only two FDA-approved antipsychotic medications for ASD in children: risperidone and aripiprazole.

Risperidone was approved by FDA in 2006. The usual dose varies from 1 to $3 \mathrm{mg} /$ day. In our practice, $3 \mathrm{mg} /$ day of risperidone seems to be the cutoff dose in terms of seizure susceptibility. We have identified patients who experienced seizures with doses higher than 3mg/day. Aripiprazole was FDA-approved in 2009 and the daily dose is up to $15 \mathrm{mg}$.

Because of ASD patients are almost twenty times more prone to have epilepsy when compared with normally developing children, and because of many of the drugs used in autism can decrease the seizure threshold in susceptible children, it is important to assure that there is a previous normal EEG before prescribing psychopharmacotherapy.

\section{Conclusions and future remarks}

The clinical approach includes a general management as well as two types of not excluding treatment strategies: one with medication and another without medication. From the clinical point of view, these two types of treatment are, in fact, complementary.

In the clinical practice, numerous types of treatment have been proposed and there is urgent need to choose any one of them in short period of time. Searching literature, a lack of well conducted RCT was identified. As a result, caution is the best form to approach ASD cases.

Future perspectives in the treatment of ASD probably will include immunomodulation, quantic biochemistry, stem cell therapy and other forms of approach after careful RCT attesting its efficiency.

\section{Author details}

Rudimar Riesgo ${ }^{1,2}$, Carmem Gottfried ${ }^{1,3}$ and Michele Becker ${ }^{1,2}$

1 Translational Research Group in Autism, (UFRGS) Federal University of Rio Grande do Sul, Porto Alegre, RS, Brazil

2 Child Neurology Unit, HCPA (Clinical Hospital of Porto Alegre), UFRGS, Porto Alegre, RS, Brazil

3 Neuroglial Plasticity Laboratory, Department of Biochemistry, Postgraduate Program of Biochemistry, Institute of Basic Health Sciences, UFRGS, Porto Alegre, RS, Brazil 


\section{References}

[1] Abramson, R. K.,Wright, H.H., Cuccaro, M.L., Lawrence, L.G., Babb, S., Pencarinha, D., Marstelle, F., \& Harris, E.C. 1992. Biological liability in families with autism. Journal of the American Academy of Child and Adolescent Psychiatry, 31, 2, pp. 370-1.

[2] Arora, M., Praharaj, S.K., Sarkhel, S., \& Sinha, V.K. 2011. Asperger disorder in adults. Southern Medical Journal, 104, 4, pp. 264-8.

[3] Association, American Psychiatric, ed. 2002. Manual Diagnóstico e Estatístico de Transtornos Mentais: DSM-IV-TR. Artmed, Porto Alegre.

[4] Becker, M. M., Wagner, M.B., Bosa, C.A., Schmidt, C., Longo, D., Papaleo, C., \& Riesgo, R.S. 2012. Translation and validation of Autism Diagnostic Interview-Revised (ADI-R) for autism diagnosis in Brazil. Arquivos de Neuro-Psiquiatria, 70, 3, pp. 185-90.

[5] Beherec, L., Lambrey, S., Quilici, G., Rosier, A., Falissard, B., \& Guillin, O. 2011. Retrospective review of clozapine in the treatment of patients with autism spectrum disorder and severe disruptive behaviors. Journal of Clinical Psychopharmacology, 31, 3, pp. 341-4.

[6] Belsito, K. M., Law, P.A., Kirk, K.S., Landa, R.J., \& Zimmerman, A.W. 2001. Lamotrigine therapy for autistic disorder: a randomized, double-blind, placebo-controlled trial. Journal of Autism and Developmental Disorders, 31, 2, pp. 175-81.

[7] Benvenuto, A., Battan, B., Porfirio, M.C., \& Curatolo, P. 2012. Pharmacotherapy of autism spectrum disorders. Brain \& Development, http://dx.doi.org/10.1016/j.braindev.2012.03.015.

[8] Billstedt, E., Gillberg, I.C., \& Gillberg, C. 2005. Autism after adolescence: populationbased 13- to 22-year follow-up study of 120 individuals with autism diagnosed in childhood. Journal of Autism and Developmental Disorders, 35, 3, pp. 351-60.

[9] Bondy, A., \& Frost, L. 2001. The Picture Exchange Communication System. Behavior Modification, 25, 5, pp. 725-44.

[10] Campbell, M., Anderson, L.T., Small, A.M., Perry, R., Green,W.H., \& Caplan, R. 1982. The effects of haloperidol on learning and behavior in autistic children. Journal of Autism and Developmental Disorders, 12, 2, pp. 167-75.

[11] Carrasco, M.,Volkmar, F.R., \& Bloch, M.H. 2012. Pharmacologic treatment of repetitive behaviors in autism spectrum disorders: evidence of publication bias. Pediatrics, 129, 5, pp. e1301-10.

[12] Cederlund, M., Hagberg, B., \& Gillberg, C. 2010. Asperger syndrome in adolescent and young adult males. Interview, self- and parent assessment of social, emotional, and cognitive problems. Research in Developmental Disabilities, 31, 2, pp. 287-98. 
[13] Center, National Autism. 2009. The National Autism Center's National Standards Report [cited 08/23/2012. Available from www.nationalautismcenter.org/pdf/NAC \%20Standards\%20Report.pdf

[14] Charnsil, C. 2011. Efficacy of atomoxetine in children with severe autistic disorders and symptoms of ADHD: an open-label study. Journal of Attention Disorders, 15, 8, pp. 684-9

[15] Dawson, G., \& Burner, K. 2011. Behavioral interventions in children and adolescents with autism spectrum disorder: a review of recent findings. Current Opinion in Pediatrics, 23, 6, pp. 616-20.

[16] Di Martino, A., Melis, G., Cianchetti, C., \& Zuddas, A. 2004. Methylphenidate for pervasive developmental disorders: safety and efficacy of acute single dose test and ongoing therapy: an open-pilot study. Journal of Child and Adolescent Psychopharmacology, 14, 2, pp. 207-18.

[17] Gadia, C. A., Tuchman, R., \& Rotta, N. T. 2004. Autism and pervasive developmental disorders. Jornal de Pediatria (Rio J), 80, 2, pp. S83-94.

[18] Gadow, K. D., DeVincent, C. J., \& Pomeroy, J. 2006. ADHD symptom subtypes in children with pervasive developmental disorder. Journal of Autism and Developmental Disorders, 36, 2, pp. 271-83.

[19] Ganz, J. B., Davis, J.L., Lund, E.M., Goodwyn, F.D., \& Simpson, R.L. 2012. Meta-analysis of PECS with individuals with ASD: investigation of targeted versus non-targeted outcomes, participant characteristics, and implementation phase. Research in Developmental Disabilities, 33, 2, pp. 406-18.

[20] Gattino, G., Riesgo, R., Longo, D., Leite, J.L., \& Faccini, L.S. 2011. Effect of relational music therapy of communication of children with autism: a randomized controlled study. Nordic Journal of Music Therapy, 20, 2, pp.142-154.

[21] Geretsegger, M., Holck, U., \& Gold, C. 2012. Randomised controlled trial of improvisational music therapy's effectiveness for children with autism spectrum disorders (TIME-A): study protocol. BMC Pediatrics, 5, 12, pp. 2.

[22] Gold, C., Wigram, T., \& Elefant, C. 2006. Music therapy for autistic spectrum disorder. Cochrane database of systematic reviews, 2, CD004381.

[23] Gottfried, C., \& Riesgo, R. 2011. Antipsychotics in the treatment of autism. In Autism spectrum disorders: from genes to environment, T. Williams, Intech: Rijeka. pp.23-46

[24] Handen, B.L., Sahl, R., \& Hardan, A.Y. 2008. Guanfacine in children with autism and/or intellectual disabilities. Journal of Developmental and Behavioral Pediatrics, 29, 4, pp. 303-8.

[25] Hardan, A.Y., Jou, R.J., \& Handen, B.L. 2004. A retrospective assessment of topiramate in children and adolescents with pervasive developmental disorders. Journal of Child and Adolescent Psychopharmacology, 14, 3, pp. 426-32. 
[26] Hofvander, B., Delorme, R., Chaste, P., Nyden, A., Wentz, E., Stahlberg, O., Herbrecht, E., Stopin, A., Anckarsater, H., Gillberg, C., Rastam, M., \& Leboyer, M. 2009. Psychiatric and psychosocial problems in adults with normal-intelligence autism spectrum disorders. BMC Psychiatry, 9, pp. 35.

[27] Hollander, E., Chaplin, W., Soorya, L., Wasserman, S., Novotny, S., Rusoff, J., Feirsen, N., Pepa, L., \& Anagnostou, E. 2010. Divalproex sodium vs placebo for the treatment of irritability in children and adolescents with autism spectrum disorders. Neuropsychopharmacology : official publication of the American College of Neuropsychopharmacology, 35, 4, pp. 990-8.

[28] Hollander, E., Soorya, L.,Wasserman, S., Esposito, K., Chaplin, W., \& Anagnostou, E. 2006. Divalproex sodium vs. placebo in the treatment of repetitive behaviours in autism spectrum disorder. The international journal of neuropsychopharmacology / official scientific journal of the Collegium Internationale Neuropsychopharmacologicum, 9, 2, pp. 209-13.

[29] Howlin, P., Goode, S., Hutton, J., \& Rutter, M. 2004. Adult outcome for children with autism. Journal of child psychology and psychiatry, and allied disciplines, 45, 2, pp. 212-29.

[30] Howlin, P., Mawhood, L., \& Rutter, M. 2000. Autism and developmental receptive language disorder--a follow-up comparison in early adult life. II: Social, behavioural, and psychiatric outcomes. Journal of child psychology and psychiatry, and allied disciplines, 41,5, pp. 561-78.

[31] Jahromi, L.B., Kasari, C.L., McCracken, J.T., Lee, L.S., Aman, M.G., McDougle, C.J., Scahill, L., Tierney, E., Arnold, L.E., Vitiello, B., Ritz, L., Witwer, A., Kustan, E., Ghuman, J., \& Posey, D.J. 2009. Positive effects of methylphenidate on social communication and self-regulation in children with pervasive developmental disorders and hyperactivity. Journal of Autism and Developmental Disorders, 39, 3, pp. 395-404.

[32] Jaselskis, C.A., Cook, E.H., Fletcher, Jr., K.E., \& Leventhal, B.L.1992. Clonidine treatment of hyperactive and impulsive children with autistic disorder. Journal of Clinical Psychopharmacology, 12, 5, pp. 322-7.

[33] Kanne, S.M., \& Mazurek, M.O. 2011. Aggression in children and adolescents with ASD: prevalence and risk factors. Journal of Autism and Developmental Disorders, 41, 7, pp. 926-37.

[34] Kanner, L. 1943. Autistic disturbances of affective contact. Nervous Child, 2, pp. 217-250.

[35] Kaplan, G., \& McCracken, J.T. 2012. Psychopharmacology of autism spectrum disorders. Pediatric Clinics of North America, 59, 1, pp. 175-87.

[36] Kim, J., Wigram, T., \& Gold, C. 2008. The effects of improvisational music therapy on joint attention behaviors in autistic children: a randomized controlled study. Journal of Autism and Developmental Disorders, 38, 9, pp. 1758-66. 
[37] Kim, Y.S., Leventhal, B.L., Koh,Y.J., Fombonne, E., Laska, E., Lim, E.C., Cheon, K.A., Kim, S.J., Kim, Y.K., Lee, H., Song, D.H., \& Grinker, R.R. 2011. Prevalence of autism spectrum disorders in a total population sample. The American Journal of Psychiatry, 168,9 , pp. 904-12.

[38] King, B.H., Hollander, E., Sikich, L., McCracken, J.T., Scahill, L., Bregman, J.D., Donnelly, C.L., Anagnostou, E., Dukes, K., Sullivan, L., Hirtz, D.,Wagner, A., \& Ritz, L. 2009. Lack of efficacy of citalopram in children with autism spectrum disorders and high levels of repetitive behavior: citalopram ineffective in children with autism. Archives of General Psychiatry, 66, 6, pp. 583-90.

[39] LeBlanc, L.A., \& Gillis, J.M. 2012. Behavioral interventions for children with autism spectrum disorders. Pediatric Clinics of North America, 59, 1, pp. 147-64.

[40] Leekam, S.R., Prior, M.R., \& Uljarevic, M. 2011. Restricted and repetitive behaviors in autism spectrum disorders: a review of research in the last decade. Psychological Bulletin, 137, 4, pp. 562-93.

[41] Longo, D., Schuler-Faccini, L., Brandalize, A.P., Riesgo, R.S., \& Bau, C.H. 2009. Influence of the 5-HTTLPR polymorphism and environmental risk factors in a Brazilian sample of patients with autism spectrum disorders. Brain Res, 1267, pp. 9-17.

[42] Lord, C., \& McGee, J.P. eds. 2001. Educating children with autism. Washington, DC: National Academy Press.

[43] Malone, R.P., Delaney, M.A., Hyman, S.B., \& Cater, J.R. 2007. Ziprasidone in adolescents with autism: an open-label pilot study. Journal of Child and Adolescent Psychopharmacolog, 17, 6, pp. 779-90.

[44] Malow, B., Adkins, K.W., McGrew, S.G., Wang, L., Goldman, S.E., Fawkes, D., \& Burnette, C. 2011. Melatonin for sleep in children with autism: a controlled trial examining dose, tolerability, and outcomes. Journal of Autism and Developmental Disorders, 42, 8, pp. 1729-37.

[45] Marcus, R.N., Owen, R., Kamen, L., Manos, G., McQuade, R.D., Carson, W.H., \& Aman, M.G. 2009. A placebo-controlled, fixed-dose study of aripiprazole in children and adolescents with irritability associated with autistic disorder. Journal of the American Academy of Child and Adolescent Psychiatry, 48, 11, pp. 1110-9.

[46] Mawhood, L., Howlin, P., \& Rutter, M. 2000. Autism and developmental receptive language disorder--a comparative follow-up in early adult life. I: Cognitive and language outcomes. Journal of child psychology and psychiatry, and allied disciplines, 41,5 , pp. 547-59.

[47] Mazzone, L., \& Ruta, L. 2006. Topiramate in children with autistic spectrum disorders. Brain \& Development, 28, 10, pp. 668.

[48] McCracken, J.T., McGough, J., Shah, B., Cronin, P., Hong, D., Aman, M.G., Arnold, L.E., Lindsay, R., Nash, P., Hollway, J., McDougle, C.J., Posey, D., Swiezy, N., Kohn, A., 
Scahill, L., Martin, A., Koenig, K., Volkmar, F., Carroll, D., Lancor, A., Tierney, E., Ghuman, J., Gonzalez, N.M., Grados, M.,Vitiello, B., Ritz, L., Davies, M., Robinson, J., \& McMahon, D. 2002. Risperidone in children with autism and serious behavioral problems. The New England Journal of Medicine, 347, 5, pp. 314-21.

[49] McDougle, C.J., Naylor, S.T., Cohen, D.J., Volkmar, F.R., Heninger, G.R., \& Price, L.H. 1996. A double-blind, placebo-controlled study of fluvoxamine in adults with autistic disorder. Archives of General Psychiatry, 53, 11, pp. 1001-8.

[50] McDougle, C.J., Scahill, L., Aman, M.G., McCracken, J.T., Tierney, E., Davies, M., Arnold, L.E., Posey, D.J., Martin, A., Ghuman, J.K., Shah, B., Chuang, S.Z., Swiezy, N.B., Gonzalez, N.M., Hollway, J., Koenig, K., McGough, J.J., Ritz, L., \&Vitiello, B. 2005. Risperidone for the core symptom domains of autism: results from the study by the autism network of the research units on pediatric psychopharmacology. The American Journal of Psychiatry,62, 6, pp. 1142-8.

[51] McPheeters, M.L., Warren, Z., Sathe, N., Bruzek, J.L., Krishnaswami, S., Jerome, R.N., \& Veenstra-Vanderweele,V. 2011. A systematic review of medical treatments for children with autism spectrum disorders. Pediatrics, 127, 5, pp. e1312-21.

[52] Miano, S., \& Ferri, R. 2010. Epidemiology and management of insomnia in children with autistic spectrum disorders. Paediatric Drugs, 12, 2, pp. 75-84.

[53] Ming, X., Gordon, E., Kang, N., \& Wagner, G.C. 2008. Use of clonidine in children with autism spectrum disorders. Brain \& Development, 30, 7, pp. 454-60.

[54] Miral, S., Gencer, O., Inal-Emiroglu, F.N., Baykara, B., Baykara, A., \& Dirik, E. 2008. Risperidone versus haloperidol in children and adolescents with $\mathrm{AD}$ : a randomized, controlled, double-blind trial. European Child \& Adolescent Psychiatry, 17, 1, pp. 1-8.

[55] Murray, M.J. 2010. Attention-deficit/Hyperactivity Disorder in the context of Autism spectrum disorders. Current Psychiatry Reports, 12, 5, pp. 382-8.

[56] Myers, S. M., \& Johnson, C.P. 2007. Management of children with autism spectrum disorders. Pediatrics, 120, 5, pp. 1162-82.

[57] Nikolov, R., Jonker, J., \& Scahill, L. 2006. Autistic disorder: current psychopharmacological treatments and areas of interest for future developments. Revista Brasileira de Psiquiatria, 28, Suppl 1, pp. S39-46.

[58] Ospina, M.B., Krebs Seida, J., Clark, B., Karkhaneh, M., Hartling, L., Tjosvold, L., Vandermeer, B., \& Smith, V. 2008. Behavioural and developmental interventions for autism spectrum disorder: a clinical systematic review. PloS one, 3,11, pp. e3755.

[59] Owen, R., Sikich, L., Marcus, R.N., Corey-Lisle, P., Manos, G., McQuade, R.D., Carson,W.H., \& Findling, R.L. 2009. Aripiprazole in the treatment of irritability in children and adolescents with autistic disorder. Pediatrics, 124, 6, pp. 1533-40. 
[60] Pereira, A., Riesgo, R.S., \& Wagner, M.B. 2008. Childhood autism: translation and validation of the Childhood Autism Rating Scale for use in Brazil. Jornal de Pediatria (Rio J), 84, 6, pp. 487-94.

[61] Piven, J., Harper, J., Palmer, P., \& Arndt, S. 1996. Course of behavioral change in autism: a retrospective study of high-IQ adolescents and adults. Journal of the American Academy of Child and Adolescent Psychiatry, 35, 4, pp. 523-9.

[62] Posey, D.J., Aman, M.G., McCracken, J.T., Scahill, L., Tierney, E., Arnold, L.E., Vitiello, B., Chuang, S.Z., Davies, M., Ramadan,Y., Witwer, A.N., Swiezy, N.B., Cronin, P., Shah, B., Carroll, D.H., Young, C., Wheeler, C., \& McDougle, C.J. 2007. Positive effects of methylphenidate on inattention and hyperactivity in pervasive developmental disorders: an analysis of secondary measures. Biological Psychiatry, 61, 4, pp. 538-44.

[63] Potenza, M.N., Holmes, J.P., Kanes, S.J., \& McDougle, C.J. 1999. Olanzapine treatment of children, adolescents, and adults with pervasive developmental disorders: an openlabel pilot study. Journal of Clinical Psychopharmacology, 19,1, pp. 37-44.

[64] Quincozes-Santos, A., Bobermin, L.D., Tonial, R.P., Bambini-Junior, V., Riesgo, R., \& Gottfried, C. 2010. Effects of atypical (risperidone) and typical (haloperidol) antipsychotic agents on astroglial functions. European Archives of Psychiatry and Clinical Neuroscience, 260, 6, pp. 475-81.

[65] Rezaei, V., Mohammadi, M.R., Ghanizadeh, A., Sahraian, A., Tabrizi, M., Rezazadeh, S.A., \& Akhondzadeh, S. 2010. Double-blind, placebo-controlled trial of risperidone plus topiramate in children with autistic disorder. Progress in Neuro-Psychopharmacology \& Biological Psychiatry, 34, 7, pp. 1269-72.

[66] Rommelse, N.N., Franke, B.,Geurts, H.M., Hartman, C.A., \& Buitelaar, J.K. 2010. Shared heritability of attention-deficit/hyperactivity disorder and autism spectrum disorder. European Child \& Adolescent Psychiatry, 19, 3, pp. 281-95.

[67] Rossignol, D.A. 2009. Novel and emerging treatments for autism spectrum disorders: a systematic review. Annals of Clinical Psychiatry : official journal of the American Academy of Clinical Psychiatrists, 21,4, pp. 213-36.

[68] Rossignol, D.A., \& Frye, R.E. 2011. Melatonin in autism spectrum disorders: a systematic review and meta-analysis. Developmental Medicine and Child Neurology, 53, 9, pp. 783-92.

[69] RUPP. 2005. Randomized, controlled, crossover trial of methylphenidate in pervasive developmental disorders with hyperactivity. Archives of General Psychiatry, 62, 11, pp. 1266-74.

[70] Rutter, M., Greenfeld, D., \& Lockyer, L. 1967. A five to fifteen year follow-up study of infantile psychosis. II. Social and behavioural outcome. The British Journal of Psychiatry : the journal of mental science, 113, 504, pp. 1183-99.

[71] Scahill, L., Aman, M.G., McDougle, C.J., McCracken, J.T., Tierney, E., Dziura, J., Arnold, L.E., Posey, D., Young, C., Shah, B., Ghuman, J., Ritz, L., \& Vitiello, B. 2006. A prospec- 
tive open trial of guanfacine in children with pervasive developmental disorders. Journal of Child and Adolescent Psychopharmacology, 16, 5, pp. 589-98.

[72] Seltzer, M.M., Krauss, M.W., Shattuck, P.T., Orsmond, G., Swe, A., \& Lord, C. 2003. The symptoms of autism spectrum disorders in adolescence and adulthood. Journal of Autism and Developmental Disorders, 33, 6, pp. 565-81.

[73] Stahlberg, O., Soderstrom, H., Rastam, M., \& Gillberg, C. 2004. Bipolar disorder, schizophrenia, and other psychotic disorders in adults with childhood onset AD/HD and/or autism spectrum disorders. Journal of Neural Transmission, 111, 7, pp. 891-902.

[74] Szatmari, P., Archer, L., Fisman, S., Streiner, D.L., and Wilson, F. 1995. Asperger's syndrome and autism: differences in behavior, cognition, and adaptive functioning. Journal of the American Academy of Child and Adolescent Psychiatry, 34, 12, pp. 1662-71.

[75] Warren, Z., McPheeters, M. L., Sathe, N., Foss-Feig, J.H., Glasser, A., \& VeenstraVanderweele, J. 2011. A systematic review of early intensive intervention for autism spectrum disorders. Pediatrics, 127, 5, pp. e1303-11.

[76] Weinssman, L., \& Bridgemohan, C. 2012. (last updated: 06/05/2012) Autism spectrum disorder in children and adolescents: pharmacologic interventions. In, Up To Date, acessed on 08/30/2012, Available from http://www.uptodate.com/contents-autismspectrum-disorders-in -children-and-adolescents-pharmacological-interventions

[77] Zeiner, P., Gjevik, E., and Weidle, B. 2011. Response to atomoxetine in boys with highfunctioning autism spectrum disorders and attention deficit/hyperactivity disorder. Acta Paediatrica, 100, 9, pp. 1258-61. 
Chapter 27

\title{
Building an Alternative Communication System for Literacy of Children with Autism (SCALA) with Context-Centered Design of Usage
}

\author{
Liliana Maria Passerino and Maria Rosangela Bez \\ Additional information is available at the end of the chapter
}

http://dx.doi.org/10.5772/54547

\section{Introduction}

Human language is a system of linguistic symbols acquired through a long ontological process of cultural learning [1]. It serves two functional aspects, communication and cognition [2]. The communicative function of language emerges in the indicative function and allows the establishment of the communication process through choice and combination of symbols [2], whereas, the cognitive function of language allows the representation of beliefs and intentions through linguistic symbols; thus, acts on one's own mental states and that of others [1]. Our view of autism and the way it affect communication is discussed along those lines.

As our conception of language development, it is assumed that communicating is more than speaking. Communicating means skillfully using a powerful tool of mediation ${ }^{1}$ human language. In addition, human language is taken here with all its possible modes of expression, including verbal and non-verbal symbols. Communication is neither regarded as a linear process of direct use of a symbolic system (language) nor as a process of language acquisition of grammatical and phonetic items. The complex process behind language acquisition includes social, cultural, historical, and intersubjective dimensions and is interactional in essence. Interaction, the fuel for development, occurs within scenes of joint attention, in which interacting agents intentionally use linguistic symbols to express intentions, beliefs and representations from their own perspective in several ways [3]. These are the premises underlying our research.

1 From a sociohistorical perspective, mediation is regarded as a scene of joint attention [1] between two or more subjects intentionally using tools and signs (such as language) to promote a process of appropriation with differentiated responsibility and competence among participants. 
Human primates' natural trend to understand others as intentional agents with goals and perceptions is the basis for the engagement in collaborative activities and joint attention [1]. Different from other primates, humans have developed a specific capacity to share attention and establish a unique type of social interaction. Hence, scenes of joint attention constitute social interactional processes in which: 1 ) agents are reciprocally responsible; 2 ) there is a shared goal, that is, each partner is aware of the goal to be achieved together; and, 3) participants coordinate their plans of action and intentions mutually so that each participant can anticipate the roles in the interaction and potentially help others with their role if necessary ${ }^{2}[4]$.

Scenes of joint attention contribute with the locus for the negotiation needed for the construction of intersubjective and perspectivated meanings [1]. This is what characterizes the process of communication as a relational and systemic phenomenon. Subjects are actively involved in interaction with a particular dynamics of implicit or explicit rules over which none of the subjects have complete control.

Such intersubjective and perspectivated construction of meanings reveals the uniqueness of human language as, upon the specific use of a particular linguistic symbol, it carries a local, historical and social meaning jointly constructed. This is also to say that in each interaction, participants quickly update possible meanings.

By extension, learning a language is a process situated relationally, historically and culturally. In each interactional process where two individuals engage, there is an intersubjective reconstruction of the perspectives of the others in the representation of their own intentions and beliefs, which requires interacting individuals to select, filter and reconfigure symbols, according to the context, intentions, beliefs and mental representations of co-participants in the communication process.

Communication implies reorganization and coordination of social, cultural and mental representations of subjects in interaction. It is precisely by means of linguistic symbols, namely signs, that it is possible to build and share meanings. That dialectical dimension of the use/ understanding/acquisition of a sign is a feature of the linguistic symbol which always involves two dimensions, language and thought. As a consequence, the attainment of a linguistic symbol constitutes a real and complex act of thought, represented by the word. It is not simply acquired by memorization or association [2].

Language acquisition is realized through the use of the symbol in actions of mediation (triadic) by which participants negotiate and construct meaning in an intersubjective way, because "[...] the meaning of a word is given through the process of verbal and social interaction with adults. Children do not build their own concepts freely. They derive them through the process of understanding the speech of others " [5] (p. 121). It is precisely within those triadic scenes, called joint attention scenes [1], that the interlocutors share some Aspect of their context ${ }^{3}$ and where intersubjectivity occurs [4]. It is also important to note that interlocutors may reach different levels intersubjectivity depending on the extent of their exchanges $[5,6]$.

2 Especially in interactions between subjects with different levels of experience or knowledge about the situation. 3 The context refers to the way objects and events are represented and meant in a situation [6]. 
Besides intersubjectivity, linguistic symbols require an ability to understand perspectivation. Understanding a symbol is a prerequisite to understanding the intentions, beliefs and background knowledge of others, as well as a particular perspective about an object or event that is incorporated into the symbol [1]. Human ability to adopt different perspectives for the same symbol or to treat different objects as if they were the same for some communicative purpose is only possible because all of those perspectives are incorporated into the symbol. So, this perspectivated nature of linguistic symbols sets forth an endless array of possibilities to manipulate the attention of others with implications for the nature of its cognitive representation [1].

\section{Communication in autism: Some considerations}

Autism belongs in the group of Pervasive Developmental Disorders (PDDs). Literature highlights a triad of elements [7] for the identification of the disorder: behavior, social interaction, and language and communication [8]. In the presence of autism, such elements portray qualitative features which prove to be peculiar or bear deficits. This session aims at discussing the characteristics related to communication and language in autism in more detail without deepening other inter-related aspects of the syndrome (such as interaction and behavior). Presenting a state of the art of on autism is not intended here, but rather, a brief review of some researches concerning language use and communication.

The field of language and communication in autism presents a great potential for researches. Although there have been many recent studies on autism, there is a gap in what concerns language and communication. So far, emphasis has been on aspects of social interaction, diagnostic and prevalence.

It is widely known that there are certain deficits in communication, such as, the absence of expressive body cues (in non-verbal communication), deficits in understanding colloquial exchanges, and speech that is not adjusted the context (in verbal communication). Several elements in the speech of a subject with autism account for it being regarded as strange, unproductive, monotonous and unusual, such as a) the difficulty in using pronouns properly, particularly, with pronoun inversions; $b$ ) the repetition of questions which have already been answered or of fixed sentences in a mediated echolalic process; $\mathrm{c}$ ) the literal understanding of metaphors or idiomatic slangs; and, d) the difficulty in using predicative abbreviations ${ }^{4}$ [9].

In a study involving with neurotypical, mentally impaired and children with autism, the kind of gestures children use to communicate have been analyzed [10] and three main categories of gestures emerged: deictic (pointing); instrumental, to organize others' behavior; and, expressive, to share emotions. The study reveals that while typical and mentally impaired children use the three types of gestures, the group of children with autism only uses deictic

4 Predicative abbreviations consist of replacind the subject of a sentence so that the predicative remains as a hidden subject. For example: "Laura always buys bread at the grocery at the corner. She takes a bag and some change her mother leaves on the fridge." The second statement has a predicative abbreviation. It contains the action and the subject is implicit (WERTSCH, 1988). 
and instrumental gestures. Besides that, other studies have established that children with autism face difficulties when it comes to using time and space pragmatic markers [11, 12], expressing mental states [13, 14], using adequate expressions and gestures [15], organizing more complex and "if-so" statements [16].

As for stories and narratives, the greatest difficulty for children with autism seems to lie in the ability to follow a narrative with multiple characters and organize each character's specific traits and personality. It is also hard for them to follow a character's way of thinking and to put themselves into the character's position $[9,10,17,18]$.

Such deficits in symbolization affect communication because it requires an active use of symbols for representation, especially, when situations involve more abstract elements such as feelings and emotions. Narratives demand the narrator to organize information for a potential listener and to select relevant aspects from the listener's perspective. Researchers have tried to explain such deficits for understanding narratives through the Theory of Mind $[11,13,14,16,19,20]$. In those researches, it is hypothesized that people with autism fail to read other people's state of minds and understand their intentions, beliefs and emotions.

From another perspective, problems in communication could be associated with joint attention [1] or mutual imitation [21]. A recent research, consistent with previous studies, has focused adults diagnosed with high functioning autism or Asperger's syndrome [22]. It has not found deviation in phonology and syntax or deficits in the subjects' ability to understand and extract the plot of narratives. There was significant difference in the use of referents, though. As a consequence, narratives have been less coherent and less organized. Just as in [9], the research has identified pronominal inconsistencies, preference for simple and unbound sentences, disregard for the relationship of a specific event with what happened previously, and limited use of time expressions.

In another research [22], however, subjects are able to apprehend the structure of the story and follow the main plot as they mention all the relevant events of the narrative. Such outcome confirms that adults diagnosed with high functioning autism or Asperger's syndrome do not present difficulties with morphosyntactic aspects but rather a limited perception of a character's intentions and inner states in the story, that is, in pragmatics of communication.

Other study, aiming at the identification of the symbolic understanding of images with three children aged 7 to 9 years old [23], has shown it is possible to use functional communication successfully. In that study, non-verbal children not employing any type of visual/symbolic communication previously have undergone a process of systematic visual literacy consisting of understanding family, people, actions and sequences. Each category has been composed by a set of 10 symbols (or photographs); and, after nine weeks the proposal of intervention has shown positive results as children begin using the images to communicate requests, define tasks and other communicative activities. Such research adds to other of the kind focusing functional communication in autism [24].

Functional communication has started in the 90s with the Picture Exchange Communication System (PECS). PECS is an Alternative Communication (AC) system with a behavioral methodology for children with social communication deficits. Its main goal is to teach 
functional communication by means of hierarchical organization, basic principles of behavior such as modeling, differential reinforcement, stimulus control, control transference of stimulus through delayed strategies of questions [23].

PECS is one of several psychoeducational programs for people with autism available nowadays [25]. It seeks to stimulate spontaneous communication through potential reinforcers, images and physical exchanges. The system is organized into six hierarchical phases interwined with a method of behavior analysis and teaching. Although PECS seems functionally efficient, our critique is targeted at the strong behavioral control imposed by the system and its disregard for prerequisite abilities for language use, such as joint attention, imitation or visual contact as previously discussed.

Summing up, it is important to note that even children with autism who do not evidence language impairment might benefit from communication support systems at times, as a way to compensate for their lack of understanding of language semantics or pragmatics [26]. Is such cases, where speech is not present, the use of alternative communication systems can promote and develop processes to facilitate communication (facilitated communication). This way, the use of technology may contribute to the sociocognitive development of those subjects [27]; and, alternative communication systems may assist them in developing meaningful communication $[9,17,23]$.

\section{Alternative communication: Methodology and resource in an approach to autism}

Alternative communication (AC) in one of the most important areas within the field known as Assistive Technology. It encompasses technical aids for communication, be it to complement, supplement or provide alternatives to make the communicative process happen.

There are several systems of AC. These provide a vast repertoire of representative elements, such as photographs, drawings and pictograms. Support for those systems may require either low (concrete material) or high (computational systems) technological devices. The importance of $\mathrm{AC}$ lies in the communicative strategies and techniques to promote subjects' autonomy for communicative instances so; it is secondary whether support is mediatic or not.

Given the impact language development on human development, subjects presenting deficits in communication can benefit from the use of AC systems as application of techniques and technology go beyond its instrumental character to enhance the development of abilities to use linguistic symbols intentionally. Considering that in the case of autism, communication deficits may exhibit alterations in language use, form or content in pragmatic level, and to a lesser extent, in syntactic, morphosyntactic, phonological or phonetic level, the importance of employing an AC system is more concerned with adequate reception and production processes, and those play an inter-related role [28].

Although the use of AC dates from the 90s, researches are recent. One of the first works was the adaptation and standardization of the PECS [29, 30]. It is also worthy of note the research 
on language development and meaning construction supported by AC with three children with autism [31], which revealed relevant outcomes.

More recent studies [32, 33, 34, 35] have involved the use of AC with Global Development Disorders (autism and Cornelia-Lange's syndrome). Those studies have also come to important results, particularly, when AC processes are supported by digital technologies.

According to the studies cited above, there is significant improvement in communication processes both in enunciation and pragmatics for subjects with autism when an AC system acts as tool of mediation between subjects in interaction. We believe that when AC is adapted to the needs of subjects with autism, it serves as a factor of facilitation and proximity because it contributes with an alternative for communication and establishes a "bridge" between people.

AC systems may function as bridge for human communication. However, it cannot be naively assumed that a technological support can stand on its own. On the contrary, an AC resource must be clearly founded on a methodology epistemologically grounded. The AC system presented in the following session (SCALA) is constituted of technological, human and social elements which are interwined to build an integral relationship, as highlighted by a researcher when stating that "[...] more important than [...] any resource to intermediate dialogue, it is time, attention to listen and dedication granted to one another" [36] (p. 139).

Two versions of SCALA have been developed by our team: web and tablet. Development has started in 2009 and the version for tablets is currently undergoing experimentation in a study with three children aged 4 diagnosed with autism.

\section{Technology and autism: SCALA project}

Researches on the use of technological applications for subjects with autism is frequent in the literature. One of the first studies involving technology and autism used computer-aided instruction (CAI) to stimulate the language development of children with autism [37]. Other studies have been published reporting the positive effects of technological applications in the process of teaching how to read and write [38, 39, 44], problem-solving [40, 41], social interaction [27, 42], and, cognition and learning [27, 39, 43].

Improvement of language use and children's learning how to read and write have been reported by [38] and [44]. In Brazil, the first study dates from 1975 [45]. It has been developed in the Laboratory of Artificial Intelligence of Edinburgh with a seven-year-old child with autism, with the use of Logo ${ }^{5}$. It has pointed out the use of Logo facilitated the process of interaction of the child with other people because "[...] the turtle assumed the role of mediator in the interaction of D. with other people and served as an object to help the development of mental schemes [...]" [45] (p. 73).

5 LOGO is a computational tool in which a student controls an icon represented by a turtle through simple commands. Designed by Seymour Papert in the 70s, it was the first computational tool especially projected to be constructivist and grounded on Genetic Epistemology. 
Other positive outcomes of the studies above include gains in communication and motivation. Such correlations are often observed in a structured and communicative environment supported by technology and organized for interventions with subjects with autism [27]. Even when more guided [38] or more flexible [45] softwares are employed, results are productive. Such outcome could be traced back to the pedagogical strategy adopted by the teacher, who acts as a more experienced partner because the use of computers, particularly in virtual learning environments designed to adapt to the subject's interests and needs, becomes relevant and important tools of mediation as advocated under a sociohistorical conception [3].

Once strategies are added to the flexibility, adaptation and complexification processes inherent to digital technologies, they help to promote sociocognitive development of participants. Nevertheless, it is necessary to establish strategies for different learning environments, learning situations and subjects in interaction so that the introduction of technology can contribute with its qualitative differential for the enhancement of social interaction of subjects with autism [46]. In doing so, researches corroborate that the use of technologies can help people with autism communicate and interact [47].

Mobile devices also represent a possibility for the use of applications to assist users as well. Their utility extends into day by day activities as they are easy to handle, can be used in different places and allow connectivity to other devices. Connectivity in particular can be quite useful to enable communication and learning in groups and, as a consequence, can help foster the integration of subjects in their social environment [48].

On sociability, a recent study with high functioning autism and Asperger's syndrome adults users of online social networks has found that the structuring features of computer-mediated communication (CMC) help and promote their participation in social interactional processes [49]. Similar results have been identified in a study focusing chat room interaction [27]. In addition, a research by [50] highlights that engagement in mediated communication may not only foster participation, but also enhance learning of social rules of turn taking and dialog maintenance when supported by intelligent computational systems. Despite the likely advantages of $\mathrm{CMC}$, it is important to consider its potential limitations and complications when it intensifies problems associated with trust, secrecy, inflexibility and perspectivation [51].

Turn-taking, which underlies unstructured social talk, poses a challenge which can be even greater if some sensorial hypersensibility (to lights, sounds, smell and touch) is associated with the syndrome. In those cases, communication controlled by a computational device may play a role in the maintenance of social relationship and management of feelings of loneliness and depression [52].

Adults with Asperger's may reveal intense isolation and difficulties to initiate social interaction [53]. They often lack a model of behavior socially acceptable and, as a result, may behave in a way that impacts their communication with other people negatively. Hence, alternative means of communication, like $\mathrm{CMC}$, and other platforms easily availa- 
ble such as Orkut, Facebook, and other social networks, can be useful for the promotion of interaction of people with autism $[27,49,50]$.

CMC offers users with autism control over their environment as well as over problems with prosody and intonation [49]. A study developed by e-mail with adults with Asperger's Syndrome has found that visual anonymity, time flexibility and the permanence allowed by the internet help diminish the social and emotional pressures of interpersonal communication as well as the cognitive complexity of the processes involved [49]. In particular, the authors state that online communities provide a space for interchanges and talks for people with similar interests or problems, so people with autism do benefit from those possibilities and structuring characteristics of CMC. In their study, 16 adults with high functioning autism or Asperger's syndrome have been interviewed on their daily activities and participation in social networks and attested that CMC tends to be beneficial for the initiation of social interactions - more than half of the interviewees participated in some type of social network. However, limitations and drawbacks in the interactions in social networks have been reported as well, which often refer to initiation of contact, maintenance of interaction for long periods and issues of security and trust. As a result, those users seek to interact with people already known from other spaces ${ }^{6}$.

In spite of the benefits reported in studies, few address AC in technological systems with nonverbal subjects with autism. We know that communication with subjects with autism can resemble the "Tower of Babel" and challenges are greater when subjects are non-verbal". In this case, we are in a rather complex situation which requires the adoption of strategies and resources to "climb up the tower". In the researches presented here, we notice that the use of technology is promising for the processes of communication and interaction. That brings us to some important questions: is it possible to identify the same benefits when allying the potential of CMC with AC? And, if so, how to use AC with mobile devices with non-verbal children with autism?

In this specific research node (AC, technology and autism), there are few studies on mobile devices for $\mathrm{AC}$ that focus people with the syndrome. In the literature review, in addition to the work of our research group, we found a research with the system Sc@ut [47]. Sc@ut is an AC system adapted to be a communicator for Pocket PC and Nintendo DS. According to the authors, the use of the system in groups of children with autism has shown an improvement in the behavior of subjects in oral language. With some subjects, the models of communication provided by the system were used to train social skills and daily life activities ${ }^{8}$ [48]. The studies developed by our research group are reported in the following session.

6 This is typical within other social groups investigated. In general, confidence is stronger and more consistent among stigmatized group minorities [54].

7 Researches point out that a third of children with autism are non-verbal. Such proportion fells to 14 to $20 \%$ when they receive early intervention [55].

8 Another product under development by [47] is a platform for the creation of pedagogic activities for Ipad and Iphone. Activities are diverse including navigation, association, memory games, puzzles, sequencing, visual and aural perception, vocabulary, visuo-spatial coordination, among others. However, this product (Picaa) has not been tested with children with autism yet. 


\section{Development centered in contexts of use}

Since 2009, when we started the development of SCALA, we have aimed at supporting language development of children with autism presenting deficits in communication. The epistemological basis of the sociohistorical theory, which we adopt, implies a conceptual reorganization of the software development process known as User-Centered Design (UCD). In $\mathrm{UCD}$, the objective goes beyond subject-object interaction and focuses on designing strategies to allow interaction and communication between children with autism and other interlocutors $[32,34,56]$. SCALA is composed of three modules: board, narratives and free communication and follows a UCD approach.

From a sociohistorical perspective and the theoretical premises already presented, our approach not only contemplates the subject with disabilities, but that subject in interaction, which broadens our focus of investigation to (a) the social contexts in which (b) cultural practices of communication and literacy are developed by (c) different participants through (d) mediating actions.

The general guidelines of the UCD consider the macro context of human development in social interaction as the basis for the analysis of cases. Characteristics and needs of individuals cannot be understood apart from the contexts in which they belong. As a consequence, the development of assistive technology goes beyond a functional view of the human being. In spite of so, we do not ignore functional aspects in our proposal, we consider them within the cultural spectrum in which the AC is to operate. Each individual "inhabits" many contexts in which is more or less active in face of different cultural practices. In some cases, those practices happen within a triadic mediated action (individual-mediator-object) which entails learning and development. So, our focus of analysis is always the individual in relation with his/her different contexts. On its turn, each context impacts our relationships, consequently, the mediations that are possible. In time, we do not regard the cultural context as something that limits the individual, but as an element that shapes the relationship. This is why it is of importance to consider social contexts in the development of technology that will be employed as a qualitative resource and psychological tool in the mediating action.

Context, under such conception, exceeds the notion of physical space. It constitutes a condition that represents the action and is crossed by a space-time dimension. This dimension includes not only physical space but social space, and four types of time: a) present, which is the microgenetic time, i.e., the time now, b) lived, which refers to human history, or the ontogenetic time c) historical, which refers to one's personal story and is related to culture; and, at last, d) future, which is a projection, what one imagines will happen, one's own expectations and that of others, the wishes, the intentions that projects oneself to the future. Those four times frames pervade contexts and are constantly updated, so, they must be taken into consideration when we project assistive technology systems.

The analysis of the different frames of time starts with the present one - microgenetic - and through it, the others are recovered. An ethnographic approach is central in that analysis because "informants" provides information about lived and historical time that allows us to 
project into the future [57]. As ethnographic research is strongly based on discourse, therefore, discourse itself is a powerful informant. Because discourse is imbued with subjectivity, a report of memories and expectations, it may be argued that it does not provide a wholly truthful account. However, as subjectivity is an important aspect within the sociohistorical research, triangulation of the data is adopted as a regulating mechanism [58].

The configuration of contexts underlies sociohistorical research. The nature of contexts is a discursive one, in which language emerges and allows us to analyze various elements: persons, situations, cultural practices and mediating actions within those practices (Figure 1) in relation to time. Thus, context cannot be regarded as a static element as it plays a role in the interaction too. Besides the agents (people) - subject A1, subject A2 -, overlapping contexts need to be included in the analysis of contexts of use.

The overview of contexts constitutes the macro level of investigation necessary to deepen the understanding of the phenomenon of communication within educational spaces. In the micro level, triads (subject-mediator, non-verbal subject and mediating actions) represent the starting point for the understanding of the processes of mediation with technologies. Such methodological perspective supports the development of technological resources (for instance, SCALA) in a differentiated way, that is, different from traditional processes of development and different from processes based on UCD, which involve users in the process of development and take their needs, expectations and experiences into consideration.

In SCALA, there is not a single model of user but a diverse range of agents involved with many peculiarities that differ in expectations and experiences, this is why we propose a broader view. We are not only interested in the user, as in the UCD, but in the peculiarities and specificities of the various agents in interaction as well. Our focus encompasses the action implied in the interaction, the cultural practices in which agents and technological resources are embedded.

Besides UCD, another proposal is the Activity-Centered Design (ACD). ACD focuses the activity that is performed and, as in the UCD, tries to create a model of activity. Considering that literacy practices cannot be thought of as an activity but as a set of practices that vary across different situations, we propose a Context-Centered Design (CCD). In this sort of development, differentiated sociohistorical contexts provide the guidelines to orient system development. In other words, what people do in different contexts, with different objectives and scenarios is what guides this project development. Figure 1 shows a scheme of CCD.

Three multi-case researches underlie SCALA developed. The first research allowed us to identify mediation strategies and validate the methodological proposal for intervention with communication with children with ASD [33]. The second case study was concerned with the interaction and intervention with a child aged 5-6 diagnosed with ASD and presenting deficits in communication. This case study was a follow-up to the previous one and derived strategies for the development of communication with the use of a first prototype in this phase [35]. Interventions allowed a broader understanding of the process of use implementation of AC with children with autism, and provided input on how a tool for such purpose should be developed. 


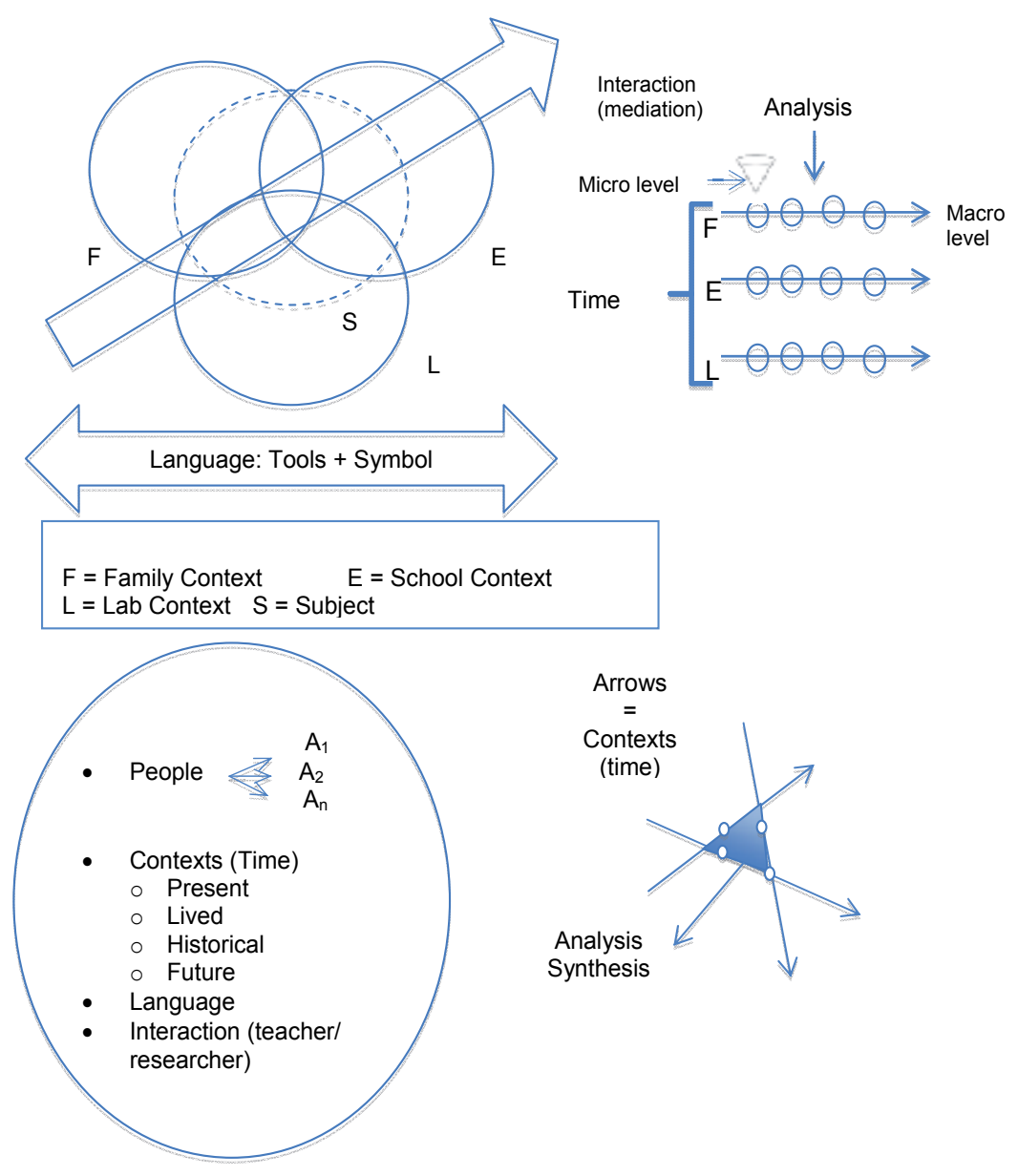

Figure 1. Scheme of Context-Centered Design (CCD)

Requirements, like touch screens and the adaptation of the size of the figures among other aspects, have significantly contributed to the second version of SCALA. A new version, now considering mobile devices and fast connection with the internet is under development (2011-2012) and has been informed by the third study, currently involving three subjects from three different cultural contexts. From the family context, the study took into consideration daily interactions, hygiene, leisure time, among other information. Besides family, other contexts include school and laboratory. The proposal of the controlled context is to investigate interaction with peers by inserting 3 children with autism in the same social space and at present time.

As it is possible to observe, development and investigation imbricate in a spiral process where each process repeats itself reaching greater complexity, and thus, improving the system which proceeds development according to the principles of CCD. To develop SCALA, on the one hand, we address the needs of communication of non-verbal children, the expectations of their 
teachers as mediators of educative practices and count on the intense participation of family to use and adapt those strategies and resources $[32,34,59,60]$. On the other hand, research trajectory involves several investigation projects developed by the research group in different spaces and moments, with points of intersection and team consolidation through regular meetings to keep investigation on track. Spiral development starts from a deep analysis of existing systems ${ }^{9}$ adding to the results of the multi-case researches, which gradually informs the construction of requirements for the system and is constantly adjusted.

From a technological standpoint SCALA has as its main features, a module for building communication boards, a module for the construction of stories, and a module for free communication. It also encompasses common application features such as the ability to import files, edit sounds, save, export, and manage the various files generated by the system (Figure 2). The menu on the left to the user presents the categories of images that can be used with all the three modules and the horizontal menu bar displays the features.

From a predefined layout one can fill each card by clicking on the categories of images. Each image has a caption pattern which can be edited. For each card it is possible to record sounds and hear them. If the user does not want to record a sound, a speech synthesizer will read the caption (otherwise, the sound recorded by the user will be supplied).

In addition to the existing images in the system, it is possible to add personal images allowing customization and adaptation to the sociohistorical context of the user. Finally, the last feature designed was the animation of actions. This feature was introduced as empirical studies have shown evidence that animated actions may be more suitable to forge understanding of metaphorical and symbolic elements with autism [46, 61, 62].

SCALA is currently available for two platforms (web e android), which allows its use with mobile devices. In the next session, some preliminary results are presented.

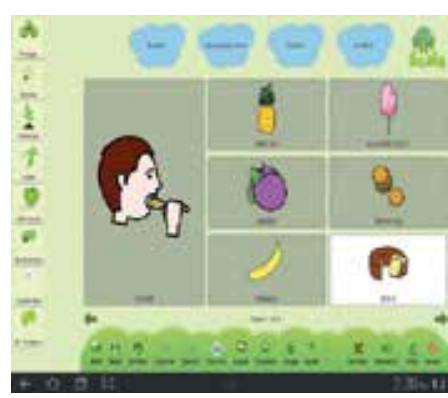

(a) board module

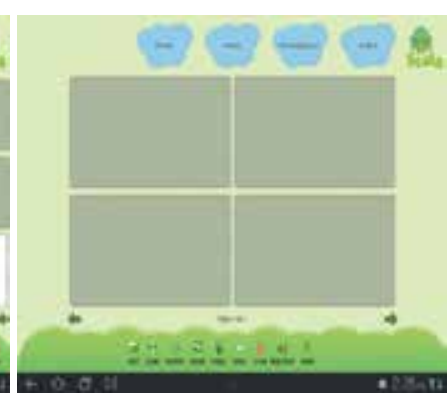

(b) story module

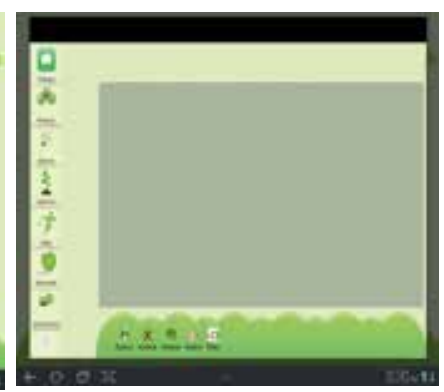

(c) story module (editing)

Figure 2. Board and Story modules in the version for Android for tablets

\footnotetext{
9 The main softwares available in the market have been explored, for example, Amplisoft, Boardmaker,and other free systems whose traits concerning interaction and narrative building were relevant to think about the system's requirements. A complete synthesis of such assessment was developed by [35] as part of her masters research.
} 


\section{Development and preliminary results}

In the session, an extract of some preliminary results of our research is presented. It comprises the period of time raging from August 2011 to May 2012. The subjects are three non-verbal children diagnosed with autism aged 4 years old. Three contexts have been taken into account: family, school and laboratory, but, in this report, we mainly focus on the interactions in the context of the laboratory, so, just a few considerations about other contexts are referred.

Interactions have been planned upon the methodology of mediating actions, with particular focus on the promotion of scenes of joint attention in order to accomplish communication and social interaction of the subjects involved. Those interactions happened simultaneously with the three subjects once a week in the laboratory. Interactions in the laboratory took place weekly and duration was flexible in the beginning ${ }^{10}$ to adapt to the needs of individuals. In addition, there were visits to each subject's home and school followed by observation and initial guidance to mothers and school personnel. This way, the subjects' contexts encompassing greater social experiences and participation were accessed. The first contact was with the mothers, school and teachers with the distribution of some instruments for data collection ${ }^{11}$ to help set up an initial profile of the subjects.

The interventions of the researcher in the three contexts do not follow a linear fashion. Visits are scheduled according to opportunity and the needs identified in the course of the research. Interventions are filmed for later analysis and to subsidize reconstruction and development of new possibilities of interactional arrangements so as to contribute to subjects' development across contexts.

Besides the scenes of joint attention, which have been promoted as part of the methodology of intervention, subjects had the opportunity to interact with physical AC materials as instruments of mediation of those scenes. Then, during the first weeks, AC material employing both low and high technology was used along with other resources, as Presented in the figure 3.

Mediating actions had a focus on the triadic interactions of the mediator with subjects and objects acting as instruments of mediation to further expand the interactions with the other subjects. With the first interventions in the lab, it was possible to establish bonds with the children and get to know their needs and potential. Furthermore, with the help of their mothers and school teachers, it was possible to outline a descriptive profile of their forms of communication, social interaction and initial potentialities.

In spite of the same diagnosis, the three children have very distinctive characteristics within the symptoms of the syndrome and are accompanied by diverse professionals in therapeutic interventions. They are referred to as Case 1, 2 and 3 in the table 1.

10 In the beginning, meetings were shorter and gradually increased in time.

11 Open interviews, anamnesis and consent forms 

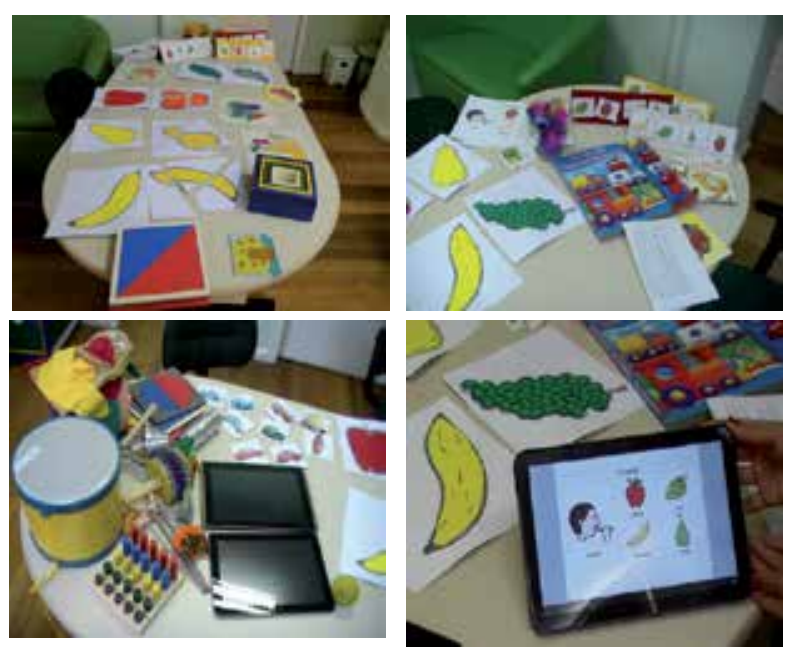

Figure 3. Example of $A C$ material of low and high technology

Case 1

Boy, 3.10 y.o., living with his parents and two older sisters; attends nursery (level 3) school in the afternoon.

Some abnormality in his development was noticed at the age of $1.3 \mathrm{y}$. 0 . as he did not show any vocabulary. ASD was diagnosed at the age of 1.9 by a team of professionals (pediatrician, neuropsychologist and a psychiatrist).

Communication Makes some sounds, makes meaningful facial expressions (looks) to pay attention, when he is called, to get to know the environment and closes his eyes in protest. He smiles to demonstrate satisfaction and joy and cries, grumbles and mumbles to show contrariety. Body expressions involve pointing and touching what he wants with his finger and waving. Is starting AC with speech therapist.

Communicates spontaneously through gestures in order to have his wishes realized. Does not present stereotyped behavior.

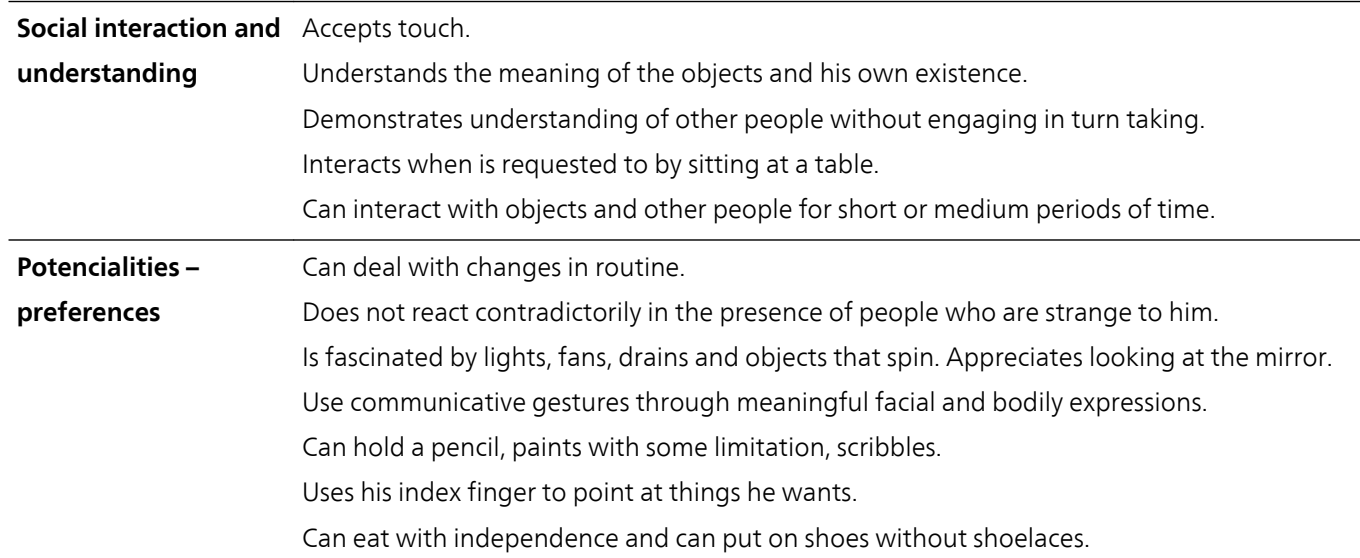


Boy, 3.10 y.o., living with his parents and two older sisters; attends nursery (level 3) school in the afternoon.

Some abnormality in his development was noticed at the age of $1.3 \mathrm{y}$. O. as he did not show any vocabulary. ASD was diagnosed at the age of 1.9 by a team of professionals (pediatrician, neuropsychologist and a psychiatrist).

\begin{tabular}{ll}
\hline Is in the process of toilet training and learning to dress and undress with independence. \\
\hline Boy, 4.2 y.o. lives with parents and a brother. Some abnormality in his development was \\
noticed at the age of 2.3 y.o.. ASD was diagnosed at the age of 2.3 by a neuropediatrician \\
and psychologist. Attends nursery school in the afternoon. \\
\hline Communication & Presents some language delay. It is difficult to understand what he says. Uses a proper \\
& language. Understands speech but does not engage in turn taking. \\
& Facial expressions are observed when he is upset, cries and grumbles to show contrariety. \\
& Does not sustain visual contact. Knocks his head to call attention or squeezes his arms and \\
& legs. Moves his hands and fingers in a strange way. \\
& To get what he wants, uses other people's arm or hand. Pointing is not part of his routine. \\
& Has difficulty in sitting still or remaining in an activity. \\
Likes to scribbles, but with no apparent meaning. \\
Does not use any form of alternative communication.
\end{tabular}

Social interaction and Resists to be touched. Contact is accepted only by family members.

understanding Limited understanding of the meanings of objects or people.

Does not get attached to his environment or shows a sense of belonging.

Interaction is restricted to objects when they are interesting to him and gets attached to them.
Potencialities - $\quad$ Appreciates music, fascination for lights, mirrors and bright eyes.
preferences Hyposensitive in relation to senses, laughs for no apparent reason, shows good coordination.
Likes to jump, lie on the floor and run.
Food compulsiveness needs to be managed.
Depends on other people to dress, undress and for hygiene.

Case 3 Boy, 3.5 y.o. lives with parents. Some abnormality in his development was noticed at the age of 1.3 y.o.., neurologist attested ASD. Uses anti-psychotic (Resperidal) and anticonvulsive medication. Attends nursery school in the afternoon..

\section{Communication Oral communication is expressed through few grumbles.}

Communicates through gestures with people who are familiar to him. To get what he wants, uses other people's arm or hand. Does not point at objects.

Facial expressions are observed when he is displeased, cries and grumbles to show contrariety. Frustration is expressed through aggression (beating himself and others, pulling one's hair, bites).

Shows great difficulty in demonstrating what he wants to communicate.

Does not use any form of alternative communication. 


\begin{tabular}{|c|c|}
\hline Case 1 & $\begin{array}{l}\text { Boy, } 3.10 \text { y.o., living with his parents and two older sisters; attends nursery (level 3) school in } \\
\text { the afternoon. } \\
\text { Some abnormality in his development was noticed at the age of } 1.3 \mathrm{y} \text {. o. as he did not show } \\
\text { any vocabulary. ASD was diagnosed at the age of } 1.9 \text { by a team of professionals (pediatrician, } \\
\text { neuropsychologist and a psychiatrist). }\end{array}$ \\
\hline $\begin{array}{l}\text { Social interaction and } \\
\text { understanding }\end{array}$ & $\begin{array}{l}\text { Does not accept physical contact and does not make eye contact. } \\
\text { Elects small spaces to stay. } \\
\text { In some moments, he seems to "unplug" and becomes apathetic to everything and } \\
\text { everyone } \\
\text { Does not accept the mediation of the researcher and in rare moments, it happens with } \\
\text { some object he is interested in. }\end{array}$ \\
\hline $\begin{array}{l}\text { Potencialities - } \\
\text { preferences }\end{array}$ & $\begin{array}{l}\text { Loud noises call his attention, shows fascination for lights, interest in small details of } \\
\text { objects. } \\
\text { Keeps a fixed and strange look at his fingers and hands. } \\
\text { Often puts objects in his mouth. } \\
\text { Faces difficulty to run, jump, climb and go down the stairs. } \\
\text { Exaggerated attachment and attraction to certain objects, likes to spin them and does not } \\
\text { use games properly. } \\
\text { Changes in routine are not well accepted. Sometimes he is too active and other times too } \\
\text { passive. Is afraid of wide spaces and symmetric floor. }\end{array}$ \\
\hline
\end{tabular}

Table 1. Initial profile of communication, social interaction and potentialities of subjects.

As can be noticed, only one of the subjects used the pointing function. Due to that, initial sessions focused on actions to make that gesture meaningful. SCALA software was used in two versions with symbols and boards with tablets. In the beginning, there was a great need to associate concrete material with the symbols in the boards and, afterwards, the gesture of pointing emerged with the fascination for the tablet technology.

The subject from case 2 accepts to be touched and soon learns to point. He also increases lateral visual contact. Although we accomplished only a few instances of mediation, he started interacting with the technological tool and increased attention span through the observation of details. On its turn, subject 1 improves pointing and eye contact and starts participating in scenes of joint attention in response to the employed mediating actions (mediator-subject-object). He soon shows great autonomy in dealing with the tablet. At last, subject 3 required more time to accept some physical contact and to fix his eyes on the activities proposed. Pointing was initially motivated by the sound produced by this touch on the screen.

Together with SCALA, several free applications have been tried with the children (Figure 4). Applications were picked according to the profile of each of the subjects and that was important to promote the appropriation and understanding of the technology, as seen in Figure 5, along with the use of AC boards (Figure 6). 

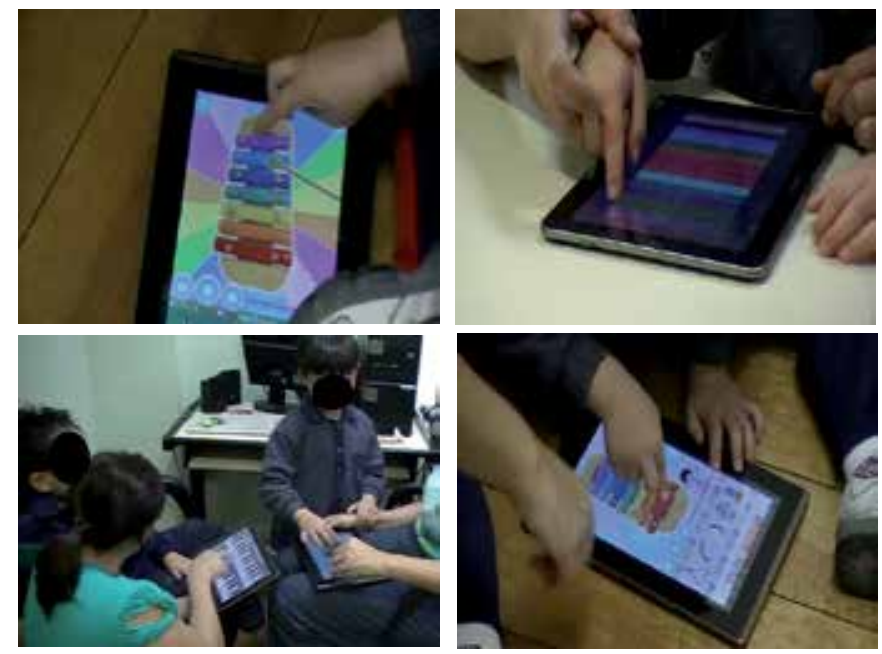

Figure 4. Using different tablet applications
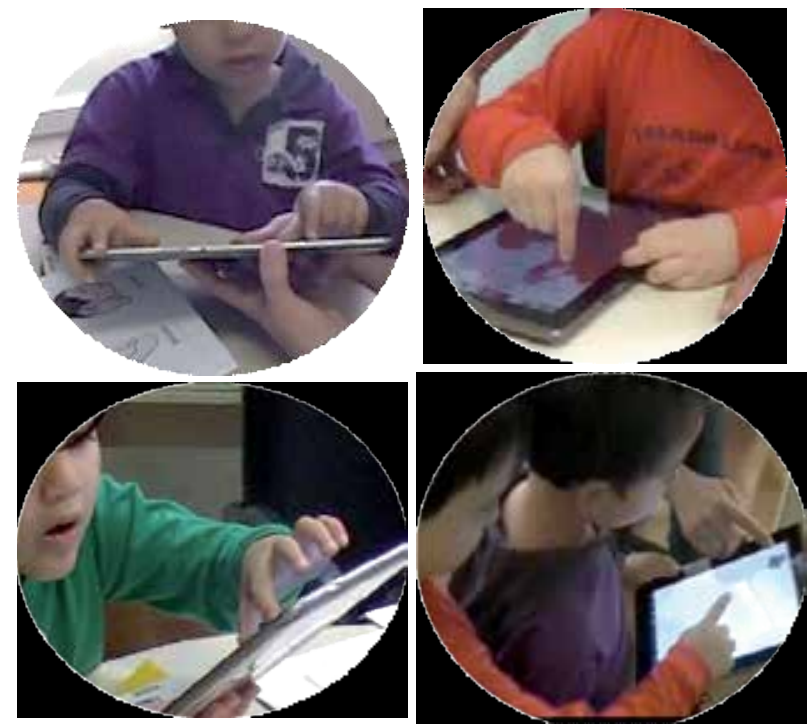

Figure 5. Using tablets with subjects with autism

With the use of tablets, we could notice attention spans increased for all the subjects. Speech was also prompted in all mediations. So, subjects' range of vocabulary has increased. Subject 1 showed easiness with the technology and the participation as an intentional agent in mediated actions. He is currently producing more words with two syllables and participating in scenes of joint attention in the mediations with other subjects. 
Although subject 2 demonstrates he prefers to interact with the equipment on his own, he also starts participating in scenes of joint attention in the mediated actions. In some few instances, he initiates interactions with the other subjects spontaneously. It is possible to notice the verbalization of some isolated words and that he accepts being touched and demonstrates affection through hugs and kisses.

Subject 3, through mediating actions accepts touch and demonstrates affection through kisses and hugs. Aggression is only expressed when he feels some pain. His interactions with the object increase and he starts participating in some mediating actions with the researcher. Only one word was said after great insistence, but the symbols of alternative communication start being understood, which is likely to contribute to his way of communication soon.

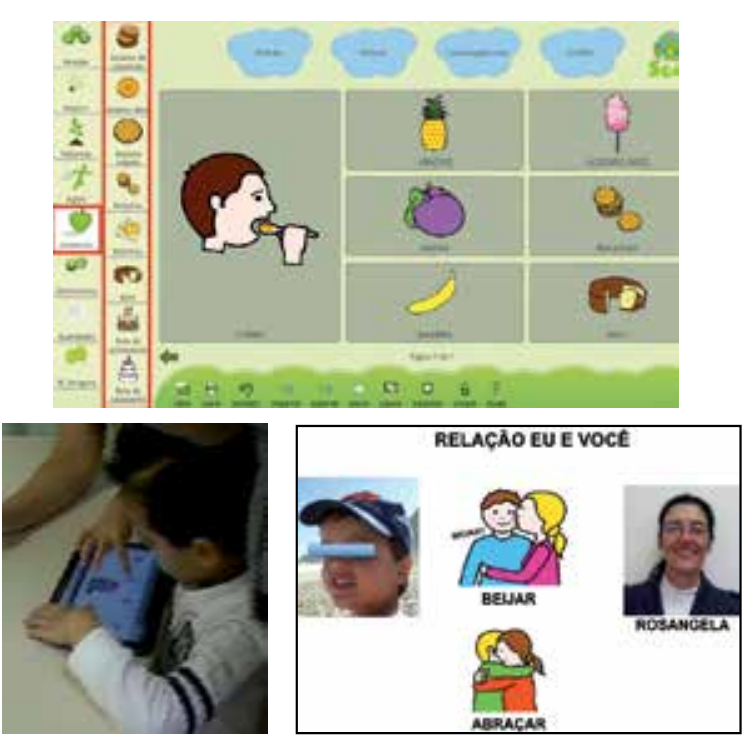

Figure 6. the use of SCALA with subjects with autism in the tablet and a board with symbols

The first image of Figure 3 shows one of the subjects interacting with AC software - SCALA. The second shows a board constructed with the software and meant to be used in the mediated actions. The third image is a board adopting low-technology with printed material.

Apart from the work in the laboratory, mothers were asked to use alternative communication at home. As needs came up, mothers turned to us and together we constructed boards. A tablet was purchased by two families (subjects 1 and 2), so the children started using it in family contexts too. As for schools, the teachers of subjects 1 and 2 have requested some boards to use in that environment too, but we perceived a lack of understanding about how to integrate $\mathrm{AC}$ in the school context. Therefore, we are providing two training courses, for teachers and assistants and for the school team.

The results referred here are preliminary as the project stretches until 2013. However, they are consistent with previous research $[27,33,50,63]$ showing relevant outcomes for the social and 
cognitive development of subjects with autism through the use of digital learning environment as instruments of mediation. Just as the present study, they have also adopted a sociohistorical view where mediating actions widen the level of development through the use of symbols and tools in a way that the zone of proximal development is adjusted until internalization of concepts is complete.

In fact, we can consider the significant improvement in both social interaction and cognitive development of subjects with autism with the introduction of technology from a sociohistorical perspective as it allows more flexible adaptive and abstraction processes with increasing levels of complexity.

\section{Conclusion}

To sum up, it is important to highlight that developing assistive technology for alternative communication as proposed in this chapter, that is with Context-Centered Design, implicates a multidimensional process involving technological innovations, pedagogic mediation, cultural practices and contexts, as well as, specific formations pervaded by critical analysis to favor the creation of new technologies with differentiated theoretical and methodological proposals.

The introduction of alternative communication can go far beyond the specialized spaces in the scope of Health and Education, such as the rooms of multifunctional resources ${ }^{12}$, for instance. For those who need it, alternative communication is a tool to be used in varied social spaces and systematically in daily life.

\section{Acknowledgements}

We would like to thank

- CAPES (Coordination for higher Education Staff Development), which through PROESP (Special Education Support Program), has funded graduate students involved in this project;

- CNPq (National Counsel of Technological and Scientific Development) for research scholarships to undergraduate students and grants to research professors;

- FAPERGS (Foundation of Research Support of Rio Grande do Sul) for the financial support through through the Edict Pesquisador Gaúcho 2009 that funded the development of phases II and III of SCALA Project; and

12 Rooms of multifunctional resources are equipped with diverse assistive technologies applications that are distributed by the Ministry of Education to regular public schools that serve students with disabilities or special needs through a specialized educational support outside school hours. 
- PROPESQ/UFRGS (Research Dean Office of Federal University of Rio Grande do Sul) for the infrastructure and financial support to SCALA project.

Project funded by CAPES and FAPERGS. Article developed from researches funded by CAPES (PROESP program), CNPq (Grant for Productivity in Technological Development and Innovative Extension) and FAPERGS ("Gaucho" Researcher Edict 2010).

\section{Author details}

Liliana Maria Passerino ${ }^{1}$ and Maria Rosangela Bez $^{2}$

*Address all correspondence to: liliana@cinted.ufrgs.br

1 Graduate Program in Education (PPGEDU) and Computer Science and Education (PGIE) and the Interdisciplinary Center of Technologies in Education - CINTED/UFRGS, Computer Science and Education, Brazil

2 Interdisciplinary Center of Technologies in Education - CINTED/UFRGS, Brazil

\section{References}

[1] Tomasello, M. Origens culturais da aquisição do conhecimento humano. São Paulo: Martins Fontes, 2003.

[2] Vygotsky, L. S. A Construção do Pensamento e da Linguagem (texto integral traduzido do russo). São Paulo: Martins Fontes, 2001.

[3] Vygotsky, L. S. Formação Social da Mente. 6.ed.- São Paulo: Martins Fontes, 1988.

[4] Tomasello, M; Carpenter, M. The Emergence of Social Cognition in Three Young Chimpanzees. Monogr Soc Res Child Dev. 2005;70(1):vii-132.

[5] Wertsch, J. Vygotsky y la formación social de la mente. Serie Cognición y desarrollo humano. Barcelona: Ed. Paidós, 1988.

[6] Wertsch,J. La Mente en Acción. Buenos Aires: Aique, 1999.

[7] Wing, L. El Autismo en niños y adultos: Una guía para la família. Buenos Aires. Argentina: Paidós, 1998.

[8] Rivière, A. Colección Estructura y Procesos. Serie Pensamiento, Psicopatologia y Psiquiatría. Madrid, 2001.

[9] Jordan, R. \& Powell, S. Understanding and Teaching Children with Autism. West Sussex, England: John Wiley\&Sons, 1995. 
[10] Peeters, T. Autism: From Theoretical Understanding to Educational Intervention. Whurr Publishers, 1998.

[11] Bruner, J., \& Feldman, C. Theory of mind and the problem of autism. In S. Baron-Cohen, H. Tager-Flsberg \& D. Cohen (Eds.), Understanding other minds: Perspectives from autism. Oxford: Oxford University Press, 1993.

[12] Loveland, K. \& Tunali, B. Narrative language in autism and the theory of mind hypothesis: A wider perspective. In S. Baron-Cohen, H. Tager-Flusberg, and D. Cohen (Eds.), Understanding other minds: Perspectives from autism, pp. 247-266, Oxford University Press, 1993.

[13] Baron-Cohen, S. Without a theory of mind one cannot participate in a conversation. Cognition, 1988b, 29, 83-84.

[14] Baron-Cohen, S. Autismo: uma alteração cognitiva específica de "cegueira mental". Revista Portuguesa de Pedagogia, Ano XXIV, 1990, p.407-430.

[15] Loveland, K. A., Mcevoy, R. E., Tunali, B., \& Kelley, M. L. Narrative story telling in autism and Down's syndrome. British Journal of Developmental Psychology, 1990, 8, 9-23.

[16] Tager-Flusberg, H., \& Sullivan, K. Attributing mental states to story characters: A comparison of narratives produced by autistic and mentally retarded individuals. Applied Psycholinguistics, 1995, 16, 241-256.

[17] Sigman, M. \& Capps, L. Niños y Niñas autistas. Série Bruner. Madrid: Morata, 2000.

[18] Hobson, P.R. El autismo y el desarrollo de la Mente. Madrid: Alianza Editorial, 1993.

[19] Baron-Cohen, S. Perceptual role taking and protodeclarative pointing in autism. British Journal of Developmental Psychology, 1989, 7, 113-127.

[20] Happé, F. An advance test of theory of mind: understanding of story characters' thoughts and feeling by able autistic, mentally handicapped, and normal children and adults. Journal f Autism and Development Disorders, 1994, 24, 129-154.

[21] Meltzoff, A. N., \& Gopnik, A. (1993). The role of imitation in understanding persons and developing a theory of mind. In: S. Baron-Cohen, H. Tager-Flusberg, \& D. J. Cohen (Eds), Understanding other minds. Oxford Medical Publications, 1993, p. 335-366.

[22] Colle, L.; Baron-Cohen, S.; Whellwrigth, S; van der Lely, H. Narrative Discourse in Adults with High-Functioning Autism or Asperger Syndrome. Journal Autism Dev Disord. 2008, 38:28-40 DOI 10.1007/s10803-007-0357-5.

[23] Cihak, D. Teaching students with autism to read pictures. Research in Autism Spectrum Disorders 1(2007) 318-329.

[24] Bondy, A., \& Frost, L. The picture exchange communication system. Focus on Autistic Behavior, 1994, 9, 1-19. 
[25] Bergeson, T.; Heuschel, M.; Harmon, B.; Gill, D.; Colwell, M.L. Los aspectos pedagógicos de los trastornos del espectro autista. Office of Superintendent of Public Instruction, (OSPI), 2003. Disponível on-line em: http://www.k12.wa.us.

[26] Bosa, C. Intervenções psicoeducacionais. Revista Brasileira de Psiquiatria. 2006;28(Supl I):S47-53.

[27] Passerino, L, M. \& Santarosa, L. Autism and Digital Learning Environments: processes of interaction and mediation. Computers and Education, v. 51, p. 385-402, 2008.

[28] Passerino, L. M. A Comunicação Aumentativa e Alternativa no espaço do Atendimento Educacional Especializado: trajetórias imbricadas de investigação e desenvolvimento tecnológico. In: Anais VI Seminário Nacional de Pesquisa em Educação especial: Práticas Pedagógicas na educação Especial: multiplicidade do atendimento educacional especializado, 2011. v. 1. p. 1-17.

[29] Walter, C. A adaptação do sistema PECS de comunicação para o Brasil: uma comunicação alternativa para pessoas com autismo infantil. In: Marquezine MC, Almeida MA, Tanaka EDO, Mori N, Shimazaki, E., organizadores. Perspectivas multidiciplinares em educação especial. Londrina:Ed UEL. 1998. p.277-80.

[30] Walter, C. Os efeitos da adaptação do PECS associada ao Curriculum funcional em pessoas com autismo infantil [dissertação]. São Carlos (SP): Universidade Federal de São Carlos; 2000.

[31] Orrú, S. E. A constituição da linguagem de alunos autistas apoiada em Comunicação Suplementar Alternativa. Tese de Doutorado. Piracicaba: UNIMEP, 2006.

[32] Bez, M. R.; Passerino, L. M. Applying Alternative and Augmentative Communication to an inclusive group. In: WCCE 2009 - Education and Technology for a Better World Monday, 2009, Bento Gonçalves. WCCE 2009 Proceedings - Education and Technology for a Better World Monday. Germany : IFIP WCCE, 2009. v. 1. p. 164-174.

[33] Bez, M. R. Comunicação Aumentativa e Alternativa para sujeitos com Transtornos Globais do Desenvolvimento na promoção da expressão e intencionalidade por meio de Ações Mediadoras. Dissertação. Programa de Pós-Graduação em Educação Faculdade de Educação. Universidade Federal Do Rio Grande Do Sul. Porto Alegre, 2010.

[34] Avila, B. G; Passerino, L. M. Comunicação Aumentativa e Alternativa e Autismo: desenvolvendo estratégias por meio do SCALA. In:Anais VI Seminário Nacional de Pesquisa em Educação especial: Práticas Pedagógicas na educação Especial: multiplicidade do atendimento educacional especializado, 2011. v. 1. p. 1-10

[35] Avila, B. G. Comunicação Aumentativa e Alternativa para o Desenvolvimento da Oralidade de Pessoas com Autismo. Dissertação de Mestrado. PPGEDU/UFRGS. 2011.

[36] Walter, C. O PECS adaptado no ensino regular: ma opção de comunicação alternativa para alunos com autismo. In: Nunes, L. Quiterio, P; Walter, C.; Schimer, C.;Braun, P. 
(Org.) Comunicar é preciso: em busca das melhores práticas na educação do aluno com deficiência. Marilia: ABPEE, 2011 [192 p.] (p. 127-140).

[37] Colby, K. M. The Rationale for Computer-Based Treatment of Language Difficulties in Nonspeaking Autistic Children, Journal of Autism and Childhood Schizophrenia 1973, 3: 254-60.

[38] Heimann, M., Nelson, K.E., Tjus, T. \& Gillberg, C. Increasing reading and communication skills in children with autism through an interactive multimedia computer program. Journal of Autism and Developmental Disorders, 1995, 25(5):459-80.

[39] Tjus, T.; Heimann, M. \& Nelson, K. E. Gains in Literacy through the Use of a Specially Developed Multimedia Computer Strategy: Positive Findings from Thirteen Children with Autism, Autism 1998, 2: 139-56

[40] Jordan, R. \& Powell, S. Improving Thinking in Autistic Children Using Computer Presented Activities. Communication, 1990, 24: 23-5.

[41] Jordan, R. \& Powell, S. Teaching Autistic Children to Think More Effectively', Communication, 1990b, 24: Wiley\&Sons, 1995.

[42] Parsons, S; Chell, P.; Leonard, A. Do adolescents with autistic spectrum disorders adhere to social conventions in virtual environments?. SAGE Publications and The National Autistic Society, 2005, Vol 9(1) 95-117.

[43] Bernard-Opiz, V.; Ross, K.; Tuttas, M.L. Computer assisted instruction for autistic children. Annals of the Academy of Medicine, 1990, v. 19, p. 611-616.

[44] Nelson, K. E., Heimann, M. \& Tjus, T. Theoretical and Applied Insights from Multimedia Facilitation of Communication Skills in Children with Autism, Deaf Children, and Children with Other Disabilities. In: Adamson, L. \& Romski, M. (eds) Communication and Language Acquisition: Discoveries from Atypical Development. Baltimore, MD: Brookes, 1997, p. 295-325.

[45] Valente, J. A. Informática na Educação Especial. In: VALENTE, J. A. (Org.) Liberando a Mente: Computadores na Educação Especial. Campinas, SP: Unicamp. 1991. 62-79.

[46] Passerino, L. M. Pessoas com autismo em ambientes digitais de aprendizagem : estudo dos processos de interação social e mediação. 2005. Universidade Federal do Rio Grande do Sul. Faculdade de Educação. Programa de Pós-Graduação em Informática na Educação. Disponível em http://hdl.handle.net/10183/13081.

[47] Rodríguez-Fórtiz, M. J., Fernández-Lopez, A., Rodriguez, M. L. Mobile Communication and Learning Applications for Autistic People. IN: Williams, Tim (Ed.) Autism Spectrum Disorders - From Genes to Environment. Rieka, Croatia: Intechweb.org, 2011 (p. 349-362).

[48] Rodríguez-Fórtiz, M. J., González, J. L., Fernández, A., Entrena, M., Hornos, M. J., Pérez, A., Carrillo, A. \& Barragán, L. “Sc@ut: Developing Adapted Communicators for Special 
Education". Procedia - Social and Behavioral Sciences, 2009, 1 (1), pp. 1348-1352. Elsevier.

[49] Burke, M. and Kraut, R. Mopping up: modeling wikipedia promotion decisions. Proc. CSCW ACM (2008), 27- 36.Colby, K. M. (1973). The Rationale for Computer-Based Treatment of Language Difficulties in Nonspeaking Autistic Children, Journal of Autism and Childhood Schizophrenia 3: 254-60

[50] Rabello , R. S.; Passerino, L. M.; Vicari, R. M.; Silveira, R. A. Interação e Autismo: Uso de Agentes Inteligentes para Detectar Déficits de Comunicação em Ambientes Síncronos. Revista Brasileira de Informática na Educação (2011) v. 19, n. 01.

[51] Seltzer, M.M., Krauss, M.W., Shattuck, P.T., Orsmond, G., Swe, A., \& Lord, C. The Symptoms of Autism Spectrum Disorders in Adolescence and Adulthood. Journal of Autism and Developmental Disorders 33, 6 (2003), 565-581.

[52] Lainhart, J.E. and Folstein, S.E. Affective disorders in people with autism: a review of published cases. Journal of Autism and Developmental Disorders 24, 5 (1994), 587-601.

[53] Muller, E., Schuler, A., and Yates, G.B. Social challenges and supports from the perspective of individuals with Asperger syndrome and other autism spectrum disabilities. Autism, 2008, 12, 2, 173.

[54] Passerino, L \& Montardo, S. P. Inclusão social via acessibilidade digital: proposta de inclusão digital para Pessoas com Necessidades Especiais (PNE). E-Compós (Brasília), 2007 , v. 8, p. $1-18$.

[55] Thunberg, G.; Ahlsén, E.; Sandberg, A. D. Autism, communication and use of a speechgenerating device in different environments - a case study, Journal of Assistive Technologies, 2011, Vol. 5 Iss: 4, pp.181 - 198Tjus, T.;

[56] Passerino, L. M.; Avila, B. G.; Bez, M. R. SCALA: um Sistema de Comunicação Alternativa para o Letramento de Pessoas com Autismo. RENOTE. Revista Novas Tecnologias na Educação, v. 1, p. 1-10, 2010.

[57] Goetz, J. P. Etnography and qualitative design in educational research. Orlando, EUA: Academic Press, 1984.

[58] Yin, R. K. Case study research, design and methods, 3rd ed. Newbury Park: Sage Publications, 2003.

[59] Rodrigues, G. F. E se os outros puderem me entender? Os sentidos da Comunicação Alternativa e Suplementar produzidos por educadores especiais. 2011. Dissertação (mestrado) - Universidade Federal do Rio Grande do Sul. Faculdade de Educação. Programa de Pós-Graduação em Educação, Porto Alegre, BR-RS, 2011.

[60] Rodrigues, G. F.; Passerino, L. Formação permanente de professores e Comunicação Alternativa: uma aproximação necessária. In: I Seminário de Políticas Públicas de inclusão escolar no Rio Grande do Sul, 2010, Porto Alegre. Anais do I Seminário de Políticas Públicas de inclusão escolar no Rio Grande do Sul, 2010. p. 01-18. 
[61] Barth, C; Passerino, L.M.; Santarosa, L. M. C. Descobrindo emoções: software para estudo da teoria da mente em sujeitos com autismo. CINTED. Porto Alegre, v.3, n.1, 2005. Disponível em: <http://www.cinted.ufrgs.br/>. Acesso em: 20 de mai. 2007.

[62] Barakova, E.; Gillessen, J.; Feijs, L. Social training of autistic children with interactive intelligent agents. Journal of Integrative Neuroscience, Vol. 8, No. 1 (2009) 23-34

[63] Bez, M. R. ; Passerino, L. M. Tecnologias Assistivas, salas de recursos e Inclusão escolar a partir da perspectiva sócio-histórica. In: VI Seminário Regional de Formação de gestores e Educadores do Programa Educação Inclusiva: Direito à Diversidade, 2010, Uruguaiana. VI Seminário Regional de Formação de gestores e Educadores do Programa Educação Inclusiva: Direito à Diversidade. Uruguaiana: Prefeitura Municipal de Uruguaiana, 2010. v. 1. p. 31-40. 

Chapter 28

\title{
Addressing Communication Difficulties of Parents of Children of the Autism Spectrum
}

\author{
Fernanda Dreux Miranda Fernandes, \\ Cibelle Albuquerque de La Higuera Amato, \\ Danielle Azarias Defense-Netvral, \\ Juliana Izidro Balestro and \\ Daniela Regina Molini-Avejonas
}

Additional information is available at the end of the chapter

http://dx.doi.org/10.5772/53964

\section{Introduction}

The autism spectrum includes major developmental disorders that, by definition, involve early and severe disorders in the areas of social, communicative and cognitive development. The resulting disorders are frequently severe and persistent with large individual variations. Therefore its impact on the families should not be overlooked.

On the other hand, the intervention aimed towards children with Autism Spectrum Disorders (ASD) should be comprehensive, intensive and long term. It leads to the notion that the families' participation in these processes should be a systematic focus of therapeutic proposals and studies involving children of the autism spectrum.

However, a recent literature review [1] about the papers published in three of the most important journals with specific focus on autism revealed a different reality. Only $0.7 \%$ of the papers published between 2005 and 2009 referred to studies about families with ASD children, comprising a total of 4883 participants. It is interesting to note that more than half of the papers about families with children of the autism spectrum were published in the last 18 months of the considered period. The themes of those studies involved issues about stress and emotional problems (13 papers); support groups and quality of life (7 papers); characterization of the families and their members (7 papers); intervention processes and their re- 
sults (5 papers) and how the parents consider their children with autism spectrum disorders (8 papers).

The growing interest in the area may be a result of the recognition that families should be included in any plan for intervention designed towards ASD children. A recently published research [2] studied the experiences that were shared by families during the diagnostic process that identified an ASD. Reports about 16 children identified that there was an average 2year lag between the first doubts about the child's development and the ASD diagnosis. These processes were more difficult and more painful to families of older children.

Another recent study [3] assessed schooling problems of ASD children and their families. The results confirmed the difficulties frequently observed in adaptation of ASD children in regular schools. The authors point out that opportunities for establishing friendship groups and peer acceptance seem to be the key elements to successful adaptations. [4] studied the opinion of parents of ASD children in the search for treatments - a process that often demands time, money and energy - in six different countries. The most significant issues that emerged were the effectiveness of treatments, relationships with professionals, access to treatments, costs, medication and stress. Early inclusion in a regular school, whenever possible, should be part of the resources provided for the development of children with autism spectrum disorders.

A Brazilian study [5] proposed a questionnaire to the identification of perceptions of caregivers of ASD children about the quality of their communication with their children, regardless of the concrete disorders presented by the child and the specific diagnosis within the spectrum. The questionnaire had a specific focus on the caregivers and was divided in four domains regarding their impressions about themselves, about other people, about their children and about their attitudes with their children. Caregivers report difficulties with other people's reactions to their children's behavior, communicative stile of the adult-child dyad, concern about the child's future and the need for more information about their child communication and instructions on how to face their difficulties.

The atypical communication development of individuals with ASD is related to difficulties with the various communicative roles (as speaker and as listener); disorders in the use of the different communicative means; a restricted repertoire of communicative functions; lack or few demonstrations of communicative intent, imitation, joint attention and other disorders in the social cognitive and symbolic development and social communicative adaptation $[6,7]$.

Verbal communication may be absent. Language delays, discursive or narrative disorders may also be observed. Social impairments may also vary from lack of visual contact or social reciprocity to severe behavior disorders (including aggressive and disruptive behaviors and eating and sleeping idiosyncrasies).

The aim of this chapter is to present some specific points and strategies to cope with autistic children's communication inabilities and suggestions on how to improve opportunities for communicative development and improvement. Not all the suggestions will be useful to all parents at any moment of their child's development; but probably some of them will answer 
to doubts of many parents at some point. Hopefully they will help parents and caregivers of ASD children to think about how their child communicates, which can be the key elements of successful communication experiences and the triggers of critical situations. This way they will be able to increase the occurrence of good and pleasant communication while decreasing the number of stressful situations. However, it should be remembered that some amount of misunderstanding and frustrating communicative situations is part of the everyday life of every person and therefore it is not reasonable to plan to completely eliminate them form the ASD child's communicative experiences.

\section{Specific focus and action options}

The broad themes considered address possible strategies to improve opportunities for communication, favor language development, improve social contact and improve the quality of communication with family and peers.

\subsection{Improve opportunities for communication}

Observing the communicative style of the ASD child parents may identify new ways of fostering the development of new abilities and their use in different situations or with different functions.

Identifying if there are specific situations when the child uses preferentially a certain communicative mean (speech, gestures, vocalizations, writing).

Showing the possibilities of expressing a certain meaning - or improving the communication's efficiency - by the use of alternative communicative means or by the combination of more than one mean.

Stimulating the use of new gestures, sounds or words in familiar situations.

Depending on the child's abilities, it may be important to exercise various situations and opportunities for expressing a certain content or intent.

In other situations it may be important to improve the creativity in communication. Sometimes it can be useful to show, in familiar situations, the various forms to express a certain message or intent.

Visual contact requires a delicate balance where the person must look at other people but shouldn't stare at them. Parents should be supportive in the development of strategies to improve social visual contact. Simple strategies, as being at the child's eye level, consistently maintaining and requesting eye contact and responding to the child's eye contact initiatives may produce significant results to the child's social adaptation.

Being aware of all the communication the child expresses, regardless of the communicative mean. Shouts, murmurs, vocalizations and gestures may convey meaningful contents and therefore lead to productive interactions. On the other hand, if a communicative attempt 
made by the child is ignored it may send a confusing feedback about communication strategies and their results.

Exploring natural and routine situations (such as baths, meals, outings) to increase the repertoire of words and expressions that constitute the common ground for communication.

\subsection{Stimulate language development}

Simple but consistent activities and attitudes may have an important role in building an environment that will stimulate language development and provide comfortable contexts where the child may use his/her communication abilities.

Defining a time or a place to be with the child in a pleasant situation (playing, talking, exchanging impressions about something that happened during the day or planning a future event or activity). The situations should be simple enough so they can happen every day. Its duration may depend on the participants' interest, but should not vary too much, so the child may be comfortable, knowing what will happen next. If the mother has more time during daily routines, she can spend, for example, two fifteen-minute periods playing, talking or working on a project with the child. But if she is overwhelmed by the routine, the father may include these activities in his routine. If both parents are available, the three may be involved in the activities. Siblings and other relatives (even pets) may be included. What is essential is to consider that these short periods of time should be part of a routine that the whole family respects and enjoys.

Adapting adult's language to the child's level of understanding. Sometimes the ASD child present a speech level far superior to his/her understanding level. Parents should consider how well the child understands language and adapt their own language to it.

Associating language to actions (movements of body or objects) or sensorial experiences, such as sounds, smells or tactile sensations (especially those associated with extremes and graduations between them; for example, hot, warm, cold and freezing) will improve the whole experience and therefore increase its meaning.

Waiting for the child's own time to answer a question or perform a task. Also being aware to the fact that sometimes the child will perform a requested action or answer to a question after a quite large time-gap. It can be useful to retrieve the question or the request and show the child if it is still meaningful of if it lost its function. For example: the mother is cooking, asks the child to get a spoon but when the child doesn't do it immediately she goes and get it - and it is reasonable that it should occur on a natural situation -; if the child gets the spoon some minutes later the mother should appraise it, thank for the help, but also tell the child that she needed it a few minutes before, or the food would get burned.

Using linguistic expressions in natural situations while enjoying an activity, such as one, two, three, go!; or bye, bye; or pick-a-boo. Using onomatopoeic sounds when playing with animals, vehicles or other objects with characteristic sounds, or when telling stories.

Use routine situations such as baths, meals or organizing a drawer to use known and new words and expressions, building a repertoire of words or expressions that will be always 
used during these situations. For example, always saying wash both feet during the bath; ate it all when finishing a meal; socks go together when organizing a drawer. But also sometimes introducing new elements to familiar situations, for example saying use your hands to wash both feet, or I ate it all, my bely is full or socks go together and boxers (or panties) on the other side.

Use varied and even exaggerated facial expressions, associating them with communicative functions, demonstrating awareness to the child's facial expressions, commenting about them, identifying different facial expressions in pictures and films.

Stimulate the child to use language to express his/her own emotions.

\subsection{Improve social contact}

When the family engages in social activities and includes the ASD child in them, there are more opportunities for social interaction. Sharing social situations with their ASD child parents will be able to eventually identify focus of more difficulties and also productive strategies to help the child to cope with them.

Taking the child to parks and playgrounds and stimulating the child to interact with other children. Situations such as sharing a swing, respecting the line for the slide or playing on the sand along with other children, may provide interesting and pleasant experiences that can represent opportunities to experience communicative strategies and exercise recently acquired abilities. Parents should be aware however, that cooperative and competitive activities are frequently stressful and cause of major disagreement between children with normal development also. Said disagreements are also important experiences to the development of social and communicative repertoires as long as they are accompanied by a soothing and supportive attitude by the parents.

Including the child in family activities such as travels, outings, visits to different places and social activities. Planning these activities with the child will help him/her to prepare for new situations, talking about what to expect, how to react in specific contexts. Including relaxing time and places to unwind or calm down will probably be useful to the whole family and can be adjusted to each child's needs and rhythm.

Helping the child to cope with problematic places and situations. Do not avoiding stressful situations but trying to identify and reduce the stressing factors. They may be related to loud noises, flashing light, crowds, specific characters (such as clowns or Santa Claus) or previous unpleasant experiences. Parents should try gradual approximation to the situations, explaining the source of lights and sounds, increasing time or proximity according to the child's response.

Including the child in household routine activities. The child should share house chores as all other family members. Depending to his/her abilities and on the family's routine, the child may unpack shopping bags, organize a drawer, write the shopping list, sort out mailing, measure the dog's food or taking it for a walk. This routine should be part of the child's responsibilities as well as an opportunity to a feeling of accomplishment. 


\subsection{Improve the quality of interaction within the family and with peers}

Attention to the child's interests, interactive strategies, communication breaks or triggers to disruptive behavior may enable parents to improve the quality of the family's interaction. All must be willing to proceed to changes in routines, responses and automatic reactions.

Observing and identifying child's interests, behaviour and communicative attempts are essential to attribute effective value to the interaction and to respond to it in a productive way, building contexts of joint attention and shared experiences.

Including the child in the family's dynamic means to comply with the same rules and limits that are applied to the other members. But close observation may also indicate that for some children more clear routines and limits may be useful. An agreement about a sequence of activities (such as choosing a toy or game, playing with it and storing it away when finished) may help the child to organize his/her expectations. Specific strategies may be needed to maintain the agreed procedure, but it may be essential to be consistent with it until the child can build his/her own rhythm and behavioural organization.

In the presence of peers (siblings, cousins, friends' children) take advantage of the opportunity to encourage the child to share objects and toys and to respect collective rules (waiting for his/her turn, complying with rules of specific games or sports).

Use unexpected situations to help the child to learn how to cope with them. Depending on the child's level, it can involve: searching for a lost item, fixing a broken toy, asking for help when needed or arguing a point of view.

Encourage the child to engage in organized activities or combinatory play, such as building blocks, puzzles, logical sequences of pictures, narrating stories. Using concepts as time, space, rhythm and position.

Use and encourage the combined use of speech and gestures and facial expressions to improve communicative efficiency.

Try to adapt the length and complexity of phrases and language to the child's language level and to guarantee the child's attention (using eye contact, physical contact of other sensory clue to the communicative situation).

Be aware of the result of your communicative initiatives, as shown by the child's reactions or answers. Try to identify what are the most difficult points to the child's comprehension and be prepared to communicative breaks and alternatives to solve them.

\section{Conclusions}

The suggestions presented are mostly examples on how everyday life activities and routines can be used to increase the opportunities to improve the ASD child's communicative abilities and their creative use as an important part of the whole development. 
The notion that the family's routine is an important part of the child's developmental environment, however, should not lead parents or other family members to transform it on a permanent training field. The best intentions and the undeniable stress involved in wishing to provide the better developmental opportunities to their ASD child may result on a stressful and over-stimulating situation. It will probably increase the stress level of the whole family decreasing the opportunities for relaxed, joyful interaction with affectively meaningful persons, which is also very important to the child's development.

The inclusion of parents and other family members in the education and intervention processes with ASD children should not be a responsibility attributed just to therapists or educators. Families can have an active part in it, by asking questions and demanding for instructions.

However, families can also need a "time-out" when they are not requested to collaborate on any structured activity. Each family should be allowed and encouraged to develop its own coping strategies and supported when a more direct approach is needed.

Groups of parents of children attending the same service or going to the same school can be very helpful in building a supportive network that includes persons that share similar problems and may also share some solutions.

\section{Author details}

Fernanda Dreux Miranda Fernandes*, Cibelle Albuquerque de La Higuera Amato, Danielle Azarias Defense-Netvral, Juliana Izidro Balestro and Daniela Regina Molini-Avejonas

*Address all correspondence to: fernandadreux@usp.br

Department of Phisical Therapy, Speech-Language Pathology and Audiology and Ocupational Therapy, School of Medicine, Universidade de São Paulo, São Paulo, Brazil

\section{References}

[1] Fernandes FD. Families with autistic children: international literature. Rev Soc Bras Fonoaudiol. 2009; 14(3): 427-32.

[2] Sansosti FK, Lavik KB, Sansosti JM. Family Experiences through the Autism Diagnostic Process. Focus on Autism and Other Developmental Disabilities, 2012. 27 (2): 81-92 doi 10.1177/1088357612446860

[3] Dillon GV, Underwood JDM. Parental Perspectives of Students with Autism Spectrum Disorders Transitioning from Primary to Secondary School in the United King- 
dom. Focus on Autism and Other Developmental Disabilities, 2012. 27(2): 111-121. doi 10.1177/1088357612441827

[4] Mackintosh VH, Goin-Kochel RP, Myers BJ. What do you Like/Dislike about the Treatments you're Currently Using? A Qualitative Study of Parents of Children with Autism Spectrum Disorders. Focus on Autism and Other Developmental Disabilities, 2012. 27: 51-60. doi 1

[5] Balestro JI, Fernandes FDM. Questionnaire about communicative difficulties perceived by parents of ASD children. Rev Soc Bras Fonoaudiol. 2012; 14:

[6] Fernanda FDM, Amato CAH, Molini-Avejonas DR. Language Assessment in Autism in Mohammad-Reza M (ed) A comprehensive Book on autism Spectrum Disorders. Ryjeka: Intech; 2011. p1-20.

[7] Hurlbutt KS. Experiences of Parents who Homeschool their Children with Autism Spectrum Disorders. Focus on Autism and Other Developmental Disabilities. 2011; 26(4): 239- 249, doi 101177/1088357611421170 
Chapter 29

\title{
Early Intervention of Autism: A Case for Floor Time Approach
}

\author{
Rubina Lal and Rakhee Chhabria \\ Additional information is available at the end of the chapter \\ http://dx.doi.org/10.5772/54378
}

\section{Introduction}

Autism is a developmental disorder that affects a child's perception of the world and how the child learns from his or her experiences. Even among the most complex disabilities, autism remains an enigma. Autism is the frequently occurring form of a group of disorders known as Autism Spectrum Disorders (ASD). The term Autism Spectrum Disorders (ASD) covers diagnostic labels which include Autistic Disorder, High Functioning Autism, Asperger's Syndrome, and Pervasive Developmental Disorder - Not Otherwise Specified (PDD-NOS).

Autism Society of America [1] defines autism as a complex developmental disability that typically appears during the first three years of life and is the result of a neurological disorder that affects the normal functioning of the brain, impacting development in the areas of social interaction and communication skills. Autism has also been defined as a neurological disorder characterized by qualitative impairment in social interaction and communication as well as the presence of restricted, repetitive, and stereotyped patterns of behaviors, interests and activities [2]. Children with ASD share the social and communicative symptoms which are the core of autism, but they vary in severity of symptoms and in level of functioning.

The first three years of life are critical to a child's development. Parents take their child to the pediatrician, during this period for general health check up, screening and vaccinations. Although child with autism can be screened by 18 months by a pediatrician, parents often are the first ones to suspect behavioral deviations in their child. The mean age for such screening is approximately 15 months and in some cases it can be as early as 11 months [3]. According to the parents, children manifest patterns of extreme reactivity, either by getting upset when new stimulus is shown or by completely ignoring it. The infants often fail to copy verbal behavior of others and do not babble by 12 months. 
Research reports a significant difference between age-matched infants with autism and typically developing infants with respect to visual attention to social stimuli, smile frequency, vocalization, object exploration engagement, facial expression, use of conventional gesture, and pointing to indicate interest [4].

Identifying autism in toddlers is a recent practice. A large number of children have been diagnosed reliably at 2 years. Professionals can now predict autism from the behaviors observed in a child younger than 2 years. Providing therapeutic intervention at this age would improve developmental and adaptive outcomes. The global trend in early intervention of autism is to provide training to parents so they can help the children develop in key areas of social responsiveness, attention skills, early communication skills, and interactive behavior.

\section{Autism and social behavior}

Difficulties in social relationships and interactions have been the defining features of autism. Hence, the need to understand the nature of these difficulties and to find effective treatments for them has been central to autism research and educational practices [5]. Unlike neuro-typical children who learn how to be social and interactive by watching how others talk, play and relate to each other, enjoy the give-and-take of social engagement and initiate, maintain and respond to interactions with others, children with autism often do not show the expected development of early social interaction skills. They are often socially avoidant, socially indifferent and awkward. Autistic children avoid social contact by having a tantrum or running away from people who attempt to interact with them. They seek social contact with people only when they want something. Factors that may affect development of social behavior are described below.

- Theory of Mind: Many children with autism also show profound empathy deficits. They develop a limited appreciation or no appreciation at all, of other people's feelings and ideas. They don't recognize and respond to faces as do normal children, and they thus do not learn that each face belongs to an individual separate person. To the severely autistic child, his/her own feelings and ideas are the only feelings and ideas that appear to exist. Autistic children may have no reaction to another person's crying, for example. They may have no idea that their words and actions affect other people. Many autistic children are completely unaware of their surroundings and other people in their surroundings. It is impossible for some autistic children to take another person's perspective without deliberate training. For individuals with autism, it does not come naturally to consider other people's perspective. This makes it difficult for them to understand how others think and feel [6]. Clinicians and researchers call this inability to consider other's perspective as deficit in theory of mind. Theory of mind, the ability to attribute mental states to self and others in order to understand and predict behavior, is an area of weakness among individuals in the autism spectrum. The development of theory of mind begins in infancy, as does the shift from the typical course that is seen in children with autism spectrum disorders. While the peak in theory of mind development occurs in typical children from the 
age of 3 to 4 , mental state understanding in individuals within the autism spectrum often continues to be conspicuously absent throughout the lifespan and leads to significant social and communicative challenges.

- Play Behavior: Play is considered a key social behavior. Children play, regardless of age, so this is a behavior that is typically found in the behavioral repertoires of all children. To teach play to children with autism is to teach them skills that other typically developing children have and give them a common ground, a common language to engage with others. Play phases occur in developmental stages that typically developing children go through, so play is not only for fun, but for a purpose. Children learn about social interaction and language through play. As children with autism have trouble in symbol use and joint attention, understanding another's perspective, participating in pretend play and using imitative skills are difficult for many of them. They are more self-centered than selfish. When involved in joint play, there can be a tendency to impose or dictate the activity. Social contact is tolerated as long as other children play their game according to their rules. Children with ASD play in a 'bubble' and can resent other children intruding into their activity. They prefer to be left alone and continue their activity uninterrupted. There is a strong preference to interact with adults who are far more interesting, knowledgeable and more tolerant and accommodating to their lack of social awareness. It is often hard for them to enter into play with other children, maintain that play, and be appropriate. The children do not see themselves as members of a particular group and follow own interest rather than that of other children in the group. In fact, while other children have mastered the rules of simple childhood games, these children may not understand what is expected of them in team sports. They are often not interested in competitive sports or team games. Even understanding basic turn-taking may elude them. Most of them are unable to comprehend how or why one would have a sense of satisfaction in knowing that one's opponents felt inferior.

- Comprehending Emotions: Inability to empathize with people may be misinterpreted as a complete lack of the ability to care for others. It is more often a lack of understanding of emotions. The child is either confused by the emotions of others or has difficulty expressing own feelings. The child does not display the anticipated range and depth of facial expression. As interaction continues, one is aware that the child is not recognizing or responding to changes in the other person's facial expression or body language. Hands may be moved to describe graphically what to do with objects or express anger or frustration, but gestures or body language based on an appreciation of another person's thoughts and feelings- e.g. embarrassment, consolation or pride- are conspicuously diminished or absent [7]. Subtle clues may not be recognized by a child with Asperger's Syndrome. The child can then be confused and offended when criticized for not complying with the signals of hidden intention. Not only are there problems with the understanding of the emotional expressions of others, but the child's own expression of emotions are unusual, and tend to lack subtlety and precision. A complete stranger may be given a kiss on the lips, or distress is expressed quite out of proportion to the situation. Sometimes they cannot express their anger appropriately. When they are anxious or 
stressed, they may not be able to let others know how they are feeling and may react violently or aggressively. Additionally, appropriate social interaction in autism is hampered by a tendency to become fascinated by special interest that dominates the child's time and conversation, and the imposition of routines that must be completed. The interest is a solitary pursuit and not that evinced by age peers. A lack of completion of the activity in a routine can lead to distress and anxiety. Researches indicate that insistence on completing an activity in a particular way may be the child's attempt to find patterns and look for rules and organization within environment [8]. Once a pattern has emerged it must be maintained. Thus, establishment of a routine ensures that there is no opportunity for change. As social situations are inherently dynamic, this adherence to routine and limited interest deeply impacts the child's ability to be socially active in appropriate manner.

\section{Early intervention}

Early intervention (EI) is a system of services provided to children who are disabled, have delayed development or are at risk of delayed development, from birth until about five years of age. To help children with autism it is essential to focus on the earliest years of development, since this is a critically important time for early learning which powerfully affects the child's future life course.

Early intervention, also known as early childhood education, provides a support system for children with developmental disabilities and their families. Early intervention may start as soon as it is evident that the child has a developmental disability or is at risk of acquiring it. The early intervention services ensure that infants and pre-school children develop the core skills in physical, cognitive, communication, socio-emotional and self help domains. Early intervention (EI) services are coordinated so that they enable child's growth and development and support families during the critical early years. For the family, such services help in overcoming the feelings of isolation, stress and frustration, and reduce the cost of providing for special education, rehabilitation and health care needs of the child. EI services follow a multidisciplinary approach, with a variety of therapists and teachers working in collaboration to improve the child's prognosis in every area of development.

To help children with autism it is essential to focus on the earliest years of development, since this is a critically important time for early learning which powerfully affects the child's future life course. The children are actively engaged in an instructional program three to five times a week, through the year. It involves planned intervention organized around relatively brief periods of time for the very young children so that they may receive sufficient adult attention. Since children with autism find it difficult to work in large groups, the EI services for them should follow a structured program of one-on-one training or training in small groups to help attain individual goals.

EI is the most dynamic and critical period in the treatment of autism for one very simple reason: the younger they are, the more 'elastic' their brains are [9]. Recognizing and diagnosing autism before pre-school age has been uncommon until the last few years. But increas- 
ingly autism is being identified very early in development. It has been shown that diagnosis can be valid and reliable at 2 years of age, and signs can be recognizable and predictive of autism even from early in the second year of life. In future it is likely that autism will be diagnosed for most children in the toddler age period [18 - 30 months). Very early therapeutic intervention is likely to improve developmental and adaptive outcomes. Trials of early intervention need to focus on training parents to work with their very young children in the key areas of social responsiveness, attention skills, early communication skills, and interactive play. The findings of a study by Ivar Lovaas [10] on early behavioral intervention of children with autism in 1987 showed a significant gain in IQ and that $49 \%$ of children who received EI were mainstreamed in regular classrooms.

The guidelines for best practice in early intervention for children with autism [11] recommend the following:

- Preparation: All children on entering intervention programs should have had a comprehensive, multidisciplinary diagnostic assessment from an interdisciplinary team of experienced clinicians and based on national and internationally agreed criteria. Diagnostic evaluations should include interviews with parents/care givers to review the child's developmental history, family history, previous assessments and interventions; collection of information from all professionals involved in the care of the child; paediatric, psychological, and speech pathology examinations to assess communication, relevant health conditions including motor skills, vision, and hearing, and any associated problems such as intellectual disability and anxiety. Additionally, direct observation of the child is important in the assessment of cognitive, social, and communicative (verbal \& nonverbal) domains, fine and gross motor, and adaptive functioning using both standardised tests and informal procedures.

- Timing: Intervention should begin as early as possible in the child's life. Since a child at risk of autism can be screened by 16 months the intervention may start immediately.

- Process: All children should have an Individual Family Service Plan (IFSP), for their education, designed to best fit their and their family's needs and strengths, developed in consultation with parents, and reviewed and revised regularly in light of the child's progress and ongoing needs.

- Intensity: Ideally the intervention should be provided for 20 hours a week for two years, with continuing support into, and through the school age years.

- Content and Focus: The content should be autism specific and include teaching joint attention skills, play, and imitation skills; building communication through Alternative and Augmentative Communication (AAC) techniques such as pictures, symbols and signs; developing social interaction and daily living skills; and management of sensory issues and challenging behaviors.

- Settings: The intervention should be delivered in various settings, individually and with peers. Implementation should happen both at the centre and at home. Including age peers 
with no disability enhances the quality but it should be done so that peer interaction is adequately supported.

- Program Design and Methods: A high degree of structure in the program is essential, i.e. well organized, regular and predictable, focused on specific objectives, and consistently managed. A supportive teaching environment with modeling, prompting, praise, shaping, and generalization strategies will maximize learning.

- Challenging Behaviors: A functional approach to modifying challenging behaviors includes positive behavior support that consists of teaching alternative appropriate behavior and communication skills to replace challenging behaviors.

- Personnel: Teachers and therapists should be adequately trained in working with children with autism and have knowledge and skills required for their special needs.

- Family Collaboration: Parents need information about autism and services, especially at key times like first diagnosis and school entry. Programs should include parent involvement, such as provision of support, counseling, and parent education to help the child with play, social, and communication skills development, and with management of challenging and repetitive behaviors

- Research and Evaluation of Program: Evaluation of treatment outcomes should be built into EI programs using systematic assessment of the child's social, cognitive, and adaptive functioning before, during, and at the end of the program. Regular and systematic documentation of program process and outcome helps in evaluation.

Collaboration with family or parents is a component of best EI practices. Parents of children who have autism play an important role; they are critical components of the intervention process, without whom gains are unlikely to be maintained. The involvement of parents in implementing intervention strategies designed to help their autistic children has a history stretching back at least three decades [12]. Parental involvement is an integral part of the success of early intervention programs for children with autism. The collaboration between the parent and the professional working with the child in the program is critical to the effectiveness of programs.

Traditionally, the EI for autism has been premised on the use of applied behavioral methods such as discrete trials. However, at times parents find the structure, organization and protocol of behavioral intervention difficult to implement and maintain. Consequently, the program receives inadequate follow up in the child's home. There is a need for interventions that do not require a rigid structure and ensure parental involvement. Hence, in the recent years, EI practices for autism have seen a shift from behavioral methods to developmental approaches.

\section{Developmental approach}

In a developmental approach, development of a child with autism is compared with the developmental sequence seen in non-disabled children. Early childhood assessment tools are 
used to determine the patterns of typical development. The skills that the child demonstrates are indicative of his or developmental level. The intervention goals are set for the skills the child failed or partially accomplished during assessment. A developmental approach to intervention is also referred to as child centered approach in which the adult follows the child's lead. It uses materials and activities that suit the child's level in a given area of development. The materials are provided to the child, and the adult facilitates the child' interaction with them so that the child moves towards achieving the pre-set developmental goal. But it is the child's initiative with the material or activities that serves as guideline for the adult's interaction. For example, if a child picks up a toy, the adult may show what can be done with it by demonstration and prompts. Child's preferences decide what should be selected as material, and the adult plays a supportive role to encourage the child's interaction with the material. Unlike the behavioral methods, developmental approach does not require the child to interact with material or carry out an activity in a pre-specified structured manner. The consequences of such interactive behaviors are reinforcements that occur naturally in child's environment. The reinforcements may be internal, such as, happiness at being able to complete a task successfully.

\section{The DIR model and floor time}

The Developmental, Individual, Relationship-based (DIR) Model, designed by Stanley Greenspan in 1989, provides a framework to understand the functional emotional development and unique profile of every child, and a guide to create emotionally meaningful learning interactions that promote critical functional emotional developmental capacities. The objectives of the DIR Model are to build healthy foundations for social, emotional, and intellectual capacities rather than focusing on skills and isolated behaviors [13]. The DIR describes six milestones as crucial to a child's development. Parents and professionals involved with the child must comprehend how the milestones affect a child's emotional and intellectual growth. The six milestones, namely, Self regulation and interest, Intimacy, Two-way communication, Complex communication, Emotional ideas, and Emotional thinking, are explained below. Individual difference is the unique biologically-based ways each child takes in, regulates, responds to, and comprehends sensations such as sound, touch, and the planning and sequencing of actions and ideas. While children may be very hyper sensitive to touch and sound, others may be hypo sensitive, and still others seek out these sensations. Relationship is described as the learning relationships with caregivers, educators, therapists, peers, and others who tailor their affect based interactions to the child's individual differences and developmental capacities to enable progress in mastering the essential foundations.

Floor time, is central to the DIR model of early intervention. It enables professionals and parents to assess and implement intervention programs that address the unique developmental needs of children with autism. The major element of this approach entails that (a) professionals do floor time with the child (b) parents observe floor time being done with their child, and (c) parents change their style of relating to the child with regard to a given milestone. Floor time is a systematic way of working with a child with autism to help him or 
her climb the developmental ladder. Floor time intervention aims at taking the child back to the first milestones that the child may have missed in the process of development. With the help of the therapists and parents the child works towards achieving the milestones. This is done through intensive one to one sessions for which parents share equal responsibility with the therapists. According to the DIR/Floor time framework, due to individual processing differences, children with autism do not master the early developmental milestones that are the foundations of learning. Floor time [14] describes six core developmental stages that children with autism have often missed or not mastered:

- Regulation and interest in the world: Infants try and process what they see, hear, and feel. They respond to pleasant face and soothing voice. They learn to enjoy, understand and, use the pleasant feelings and sensations to calm themselves. This helps them learn to take in and respond appropriately to the world around them. This ultimately develops the ability to self regulate.

- Engagement and relationship: Babies learn to bond with their parents very soon. They recognize the parents' face and voice, and want to touch them or be close to them. They enjoy being cuddled and loved by their parents. This process of bonding also builds a relationship of trust between babies and their parents. This trusting relationship enables the child to become a well-adjusted adult later in his or her life. It also forms a stable base for all future relationships. The baby learns that relationships with people can be joyful.

- Two-way communication: Once relationship with parents is developed, the baby realizes that he or she can have an impact on parents. The baby's smile can produce a smile from the parents. If the baby reaches out to mother, she picks him or her up. The baby learns that adults can understand and respond to its communication intents and feelings. A dyad of communication starts slowly. When the baby looks at the mother and reaches out to her - the mother responds by giving eye contact and a hug. In turn the baby may smile, vocalize or touch the mother. Thus a non-verbal dialogue or a two-way communication process may be completed. The baby soon transfers this new ability to other things in the environment. He bangs a toy, it makes a noise, and if he drops his bottle, it breaks. His actions can have an impact not only on his parents but others too. Hence, two-way communication helps babies to learn about them and about the world.

- Complex communication: The non-verbal two way communication slowly becomes complex in nature. While earlier the baby was initiating or responding to a communication by a simple gesture of reaching out or smiling, now he may run towards the mother, and squeal with pleasure. Anger and displeasure may be expressed by pulling, kicking and grabbing or throwing things. Similarly, hugs and kisses are used to express affection. Since, the baby is ambulatory by now, he may take the parent by hand and show them what he wants. Complex communication ability also aid development of creativity. The toddler adds his own ideas to the games that parents play with him. This leads to the emergence of the child's own personality.

- Emotional ideas: Play is a fertile ground for ideas. Using toys and playthings, a child creates a world where toys play roles. So, a teddy is a friend, a doll is a baby and a shoe box 
is a car garage. This idea-filled play provides a strong basis for language development. Besides learning to label things, the child now uses dialogue during play with help of the parents. Eventually, he is able to manipulate the ideas to meet his needs. When hungry, he can ask for food; if he needs help he can call his mother instead of crying. He learns about object permanence - that although not visible to him, object do not disappear. Hence, he can feel secure thinking about his parents even when they are not with him. With this ability to use symbols, the child moves on to a higher level of communication and awareness.

- Emotional thinking: When he reaches this stage, a child is ready to connect various ideas into a logical sequence. While in the previous stage he was able to carry out symbolic activities, such as dressing a doll, and banging a toy car into another to simulate a crash, the child is now able to think emotionally. He may dress up the doll for a car ride. At this stage, the child is able to express a wide range of emotions, and through this learns to recognize self. The child now comprehends concept of space and time at a personal level. For example, the child understands that grandmother's house is different from his own, or that if he grabs another child's toys, his own favorite car may later be taken away by that child. The child, by this time, is fully verbal and can use words to express ideas and feelings.

\subsection{Floor time method}

A typical floor time session is conducted in a child's naturalistic environment and requires the therapist or parent to sit on the floor and work with the child. The purpose is to help the child achieve the stages of development, by taking him back to the milestones that he may have missed. During a session, the parent or therapist follows the child's lead. This helps in establishing relationship between the child and the adult. It is this relationship that slowly enables the child to develop the basic social, emotional and communication abilities. During a floor time session the child learns to engage with others, initiate actions, make own wishes and desires known and the realization that his actions can elicit responses from others. Floor time creates opportunities for children to have dialogues, which are called circles of communication, first without words and later with them, and eventually to imagine and think. Since floor time sessions are child-centered, the activities are motivating to the child as it is he who has chosen them. Additionally, the selecting the child's natural environment for the session also contributes to calming him and improving his comfort level. A floor time session follows the steps given below.

1. Observation: Before starting a session, the adult observes the child. This requires watching the child while he is in the room, observing what interests him, assessing his level of interaction - is he running around or is he sitting quietly. This observation helps the adult determine the child's current emotional state.

2. Approach: Once the adult understands the child's level of emotional functioning, he or she joins the child in whatever the child is doing. If the child sits and merely twirls a toy, the adult follows this play behavior. However, the adult adds value to it by label- 
ing the activity in gestures and words. The adult also uses appropriate facial expression and tone of voice to convey own enjoyment in what the child is doing. Such measures enable the adult to open the circle of communication with the child

3. Child's Lead: During a floor time session, the child is the director or leader of activities. The adult's role is to follow the child. The aim here is to support the child's activities and initiatives, and through this to take him to a higher level of emotional functioning.

4. Expand Ideas: As the sessions progress, the adult builds on the child's play initiatives. Now the adult associates daily experiences with the experiences during the play activities. For example, the adult may say "give teddy a bath, like mommy gives you". This planned expansion and addition to child's activities help in development of emotional ideas.

5. Close Circle of Communication: Once the adult engages the child at a level the child currently enjoys, enters the child's activities, and follows the child's lead, he or she now attempts to move the child from a mutually shared engagement toward more increasingly complex interactions, a process known as "opening and closing circles of communication." In a circle of communication, the adult opens the circle by approaching the child, and the child closes the circle by giving a reaction to the adult's comments and gestures. During session many circles may open and close in quick successions as the adult interacts with the child. The process leads to two-way communication.

\section{Research support for floor time}

The Floor time approach examines the functional developmental capacities of children in the context of their unique biological profile and their family relationships and interactive patterns. A longitudinal study [15] was conducted to determine if children with ASD could overcome the core deficits in social behavior and become empathetic and reflective with floor time intervention. A follow-up study of 16 children diagnosed with (ASD) revealed that with the DIR/Floor time approach, a subgroup of children with ASD can become empathetic, creative, and reflective, with healthy peer relationships and solid academic skills. This suggests that some children with ASD can master the core deficits and reach levels of development formerly thought unattainable with a family-oriented approach that focuses on the building blocks of relating, communicating, and thinking [16].

In another study undertaken by Greenspan and Weider [15] where the progress of 200 children who had earlier received Floor time sessions, was reviewed showed that majority of the children learned to relate and engage with warmth, trust and intimacy; they were able to interact, read and respond to social signals; a subgroup of children developed the capacity for imaginative play, creative use of language and reflective thinking. This sub group was included in mainstream schools where the children developed meaningful relationships with peers. 
Josefi and Ryan [17] conducted a case study on a 6 year old boy with severe autism. Video recordings of 16 sessions of play therapy with the child were analyzed qualitatively and quantitatively. The study concluded that this child was able to enter into a therapeutic relationship and demonstrated attachment behavior towards the therapist. Key areas of improvement were in the child's development of autonomy and pretend play, while ritualistic behaviors showed only mild improvement. Changes were also noted in the boy's behavior at home of increased independence and empathy. One implication of this preliminary research is that non-directive play therapy may enhance and accelerate emotional/social development of children with severe autism.

Children with ASD differ from one another-in the ways they engage, relate, and communicate and in the ways they respond to sensations, and plan and sequence their actions. These differences mean that each child requires an intervention approach tailored to his uniqueness, an intervention that must also consider the home setting. According to Costa and Witten [18] the goals of such a program, regardless of the approach used, must be to strengthen the child's core deficits, namely: building the foundations for relating, communicating and thinking. The DIR/Floor time Model is especially beneficial to children with ASD and other developmental and/or emotional challenges.

Solomon et al [19] published an evaluation of The PLAY Project Home Consultation, a widely disseminated program that trains parents of children with autism spectrum disorders in the DIR/Floor time model. Sixty- eight children, 2 to 6 years old (average 3.7 years) completed an 8-12 month program where parents were encouraged to deliver 15 hours per week of 1:1 interaction. Pre/post ratings of videotapes by blind raters using the Functional Emotional Assessment Scale (FEAS) showed significant increases in child subscale scores. That is, 45.5 percent of children made good to very good functional developmental progress. Overall parents' satisfaction with program was 90 percent.

\section{Method}

The study was experimental in nature and employed a pre-test post-test control group experimental design. It was conducted on children with ASD residing in Mumbai, India. The objectives were to determine the efficacy of floor time approach for developing social behavior in pre-school children with ASD, and to compare the levels of social skill achievement by children who received floor time intervention with those who did not.

\subsection{Subjects}

Children with ASD within the age group of 3 to 6 years were randomly selected from five pre-schools and intervention clinics located across the city and suburban areas of Mumbai. A total of 26 children participated in the experiment. After selection the children were randomly assigned to treatment and control groups so that both groups had 13 children each. 


\subsection{Instruments}

The Behavioral Scale for Social Skills (BSFS) and Floor time intervention were the primary instruments used in the study. They were developed for the purpose of the research. A brief description of both is given below.

- Behavioural Scale for Social Skills: The BSFS was used as a measure at both pre and post tests. The instrument measured social behaviour under 4 domains -

a. Turn taking: This is one of the bases for development of social skills and inferring others' intentions correctly [20]. Turn taking includes use of play material with an adult and with peers.

b. Two-way communication: As a child enjoys intimacy in a safe and calm manner, he realizes he can have an impact on others. The child expresses a feeling or intention, and his partner responds. This is the beginning of communication. Two-way communication enables the child to enjoy intimacy and initiate interaction through gestures at first and then with words.

c. Understanding of cause and effect: This is a basis for development of thinking skills. The ability to see the relationship between an event and the factors leading to it helps a child decode the world around him. Understanding of cause and effect relationship improves by providing the child the opportunity to explore the environment.

d. Emotional thinking: According to Greenspan, emotional thinking is the ability to build bridges between ideas to make them reality-based and logical. Ideas are linked together into logical sequences and play, and imagination is also more rational.

The BSFS had a total of 20 items. Each item was measured on a 4-point scale based on the category of response, namely, correct response; response with verbal prompt; response with gestural prompt; and response with physical prompt. Whereas correct responses were scored as 4 , responses with physical prompts were scored as 1 . The selection of items under each sub head of BSFS was done after detailed discussions with developmental psychologists, pre-school teachers, and many parents. In addition, several observations of pre-school children with and without ASD were also made for selection of items. The instrument was pilot tested on children with ASD belonging to the same age group as the subjects.

- Floor time Intervention: Floor time is a comprehensive program for infants, young children, and families with a variety of developmental challenges including ASD. The program aims at enhancing the functional emotional developmental levels and creating those learning relationships that will help the child move ahead in social skills acquisition. Floor time can be tailored to suit the individual needs of children with ASD. Floor time approach was used for treatment in the study. As stated earlier, Floor time approach helps an infant/young child reach the 6 milestones crucial for development of social behavior, namely, self regulation; intimacy; two way communication; complex communication; emotional ideas,; and emotional thinking. However, in this study, the treatment was directed toward achievement of 4 milestones - Turn taking (a component skill in intimacy), Two way communication, Understanding of cause and effect relationship (an impor- 
tant skill for problem solving that enhances complex communication), and Emotional thinking. Various activities were developed for the purpose of enhancing the target skills. Some of the activities are mentioned below.

a. Turn taking: Here the activities selected were done with the authors and then done with peers. Such activities as building block tower, bead stringing, rolling a ball, and throwing ball in a bucket were used for teaching turn taking skills to children.

b. Two-way communication: Training a child to respond to his name, reach out to a plaything, and respond to non-verbal communication such as gestures, facial expressions etc was undertaken to develop the ability for two way communication.

c. Cause and effect: A series of simple activities were done to explain the relationship between an outcome and its cause. Tapping a spoon on a surface, shaking a bell, pressing a toy to produce sound or movement, squeezing a wet sponge, opening a transparent box to obtain a desirable object within, etc. were undertaken to help the children establish the connection between a cause and its effect.

d. Emotional thinking: Pretend play was primarily used for this purpose. Hence, pretend play such as talking on telephone, dressing or feeding the doll (where the authors would at times play out the doll's emotions in the right tone of voice), and playing a shopkeeper etc. were included. The focus was on recognition of emotions. Thus, flash cards of happy and sad faces were used too during the pretend play so that the child was able to understand what did it mean when the 'doll' was 'crying' or the the shopkeeper was 'happy'.

\subsection{Procedure}

The intervention started after assessing the children's baseline behavior on BSFS. The 26 children were then randomly assigned to experimental and control groups so that both groups had 13 children each. As per the recommended floor time protocol, the researchers observed each child in the experimental group to determine his or her current emotional level, before the commencement of intervention. Each child in the experimental group received 20 sessions of floor time intervention. Each session was of 30 minutes duration. Each session included at least one activity relevant to the pre selected social skills. The sessions started by getting the child's attention by showing a desired object. The researchers used word and simple phrases to describe each activity. The activities were done as given below, and parents were encouraged to observe the sessions.

- Building a block tower began by demonstrating how to make a tower from the four blocks provided on the floor. The child was then asked to lay a block over the one put by the adult. Subsequently, the adult would put another block over it. The adult would then prompt the child to take his or her turn to put a block on top. The activity was repeated with a peer. Now the peer would take the adult's role. The adult would call out each child's name and say ' your turn now ', as they put one block over the other to make a block tower. 
- A number of colorful beads were placed on the floor along with a string for the bead stringing activity. The task was first demonstrated, and then used to encourage the child to take turn with an adult and later with a peer in slipping a bead through the string.

- The ' ball rolling ' activity was done by rolling the ball to a child and asking him or her o roll it back to the adult. In case of 5 or 6 year old children, a slight variation was made. The activity was introduced with a peer. Both the child and peer were asked to take turns in throwing the ball to the adult and to each other.

- Throwing the ball in the bucket required that the child identify the bucket first. Subsequently, the task was demonstrated before the child taking turn with the adult and with peer to throw a ball in the bucket.

- Cause and effect activities such as ringing a bell (to produce sound), squeezing a sponge (for water to drip), and opening a box (to get what is inside) were demonstrated and subsequently, taught with prompts and cues. Some fun activities such as blowing soap bubbles were also included as soap bubbles excited the children.

- Calling out the child's name, seeking his attention by showing a preferred object or toy helped in initiating two-way communication. Preferred activities served a dual purpose. They could get the child's attention, but they were also helpful in teaching the child a way to communicate. The adult would have a picture of the preferred activity or toy. The child would be asked to point or pick up the picture in order to get the activity. The adult also used facial expression cards to help the child understand what each expression meant.

- Pretend play was a strong medium for teaching emotional thinking. Pretend play was encouraged using a variety of toys such as dolls, telephone, car, kitchen set, and doctor set etc. The adult would pretend to call the child, and ask the child to pick up the phone and say something. While the child was holding the doll, the adult would prompt him or her to hug and kiss the doll. If the child put the doll away, the adult would convey in appropriate tone and affect how sad the doll was feeling. The child would then be prompted to hold the doll again.

- Taking the lead from the child, the adult would stand at the window if the child was standing there. The adult would then softly describe what they could both see.

- Though all activities were pre-planned, the adult would at times digress to include activities that suited the need of the child on a given day.

While the experimental group children received floor time intervention, the children in the control group received the usual early intervention sessions provided in their educational settings. Post intervention, BSFS was administered again.

Figures 1 to 7 illustrate some of the floor time activities done with the children. 


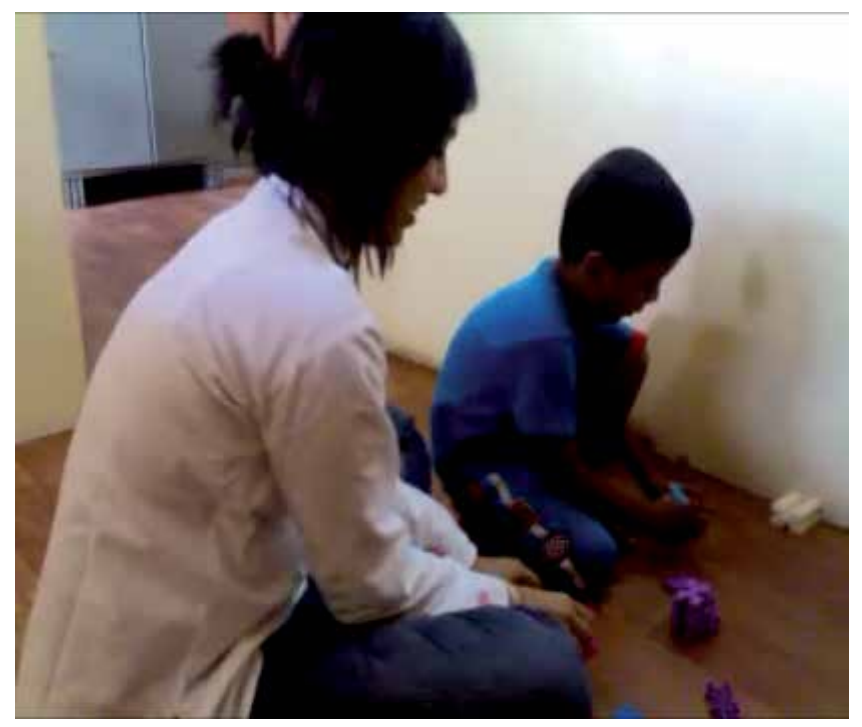

Figure 1. Block tower activity

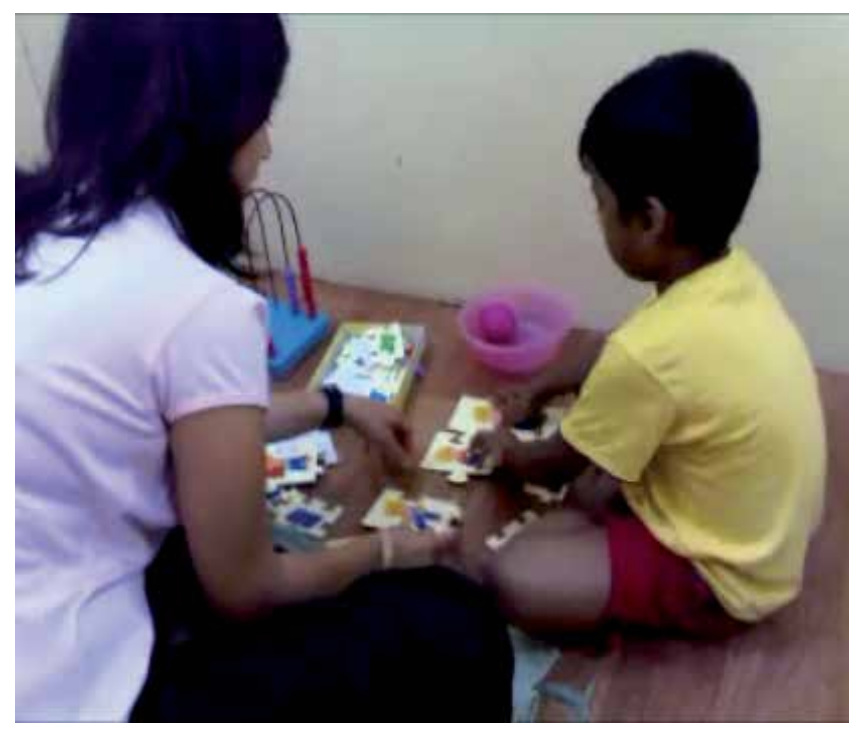

Figure 2. Picture matching 


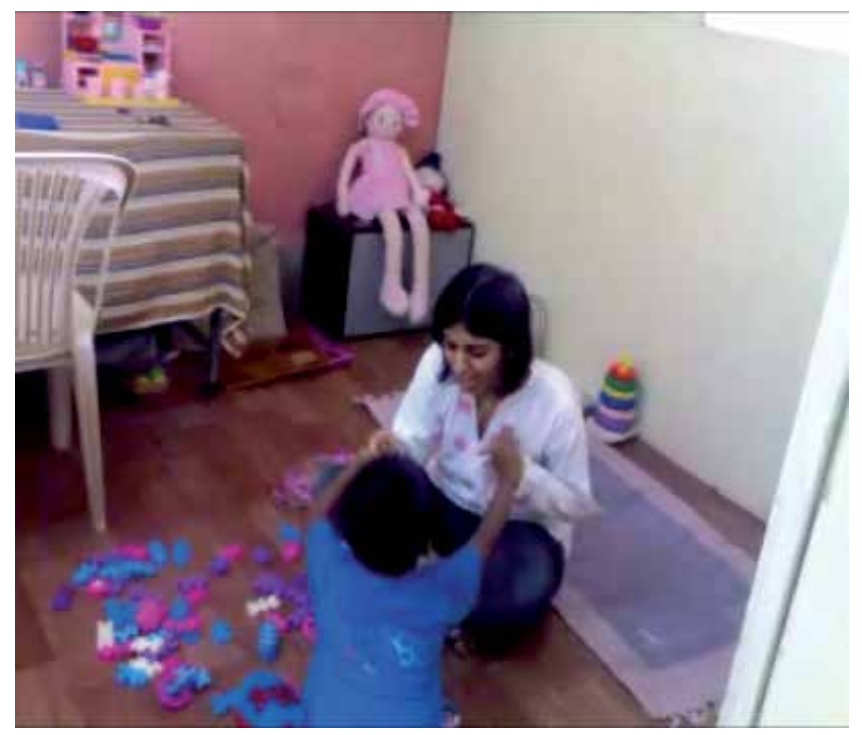

Figure 3. Getting a child's attention

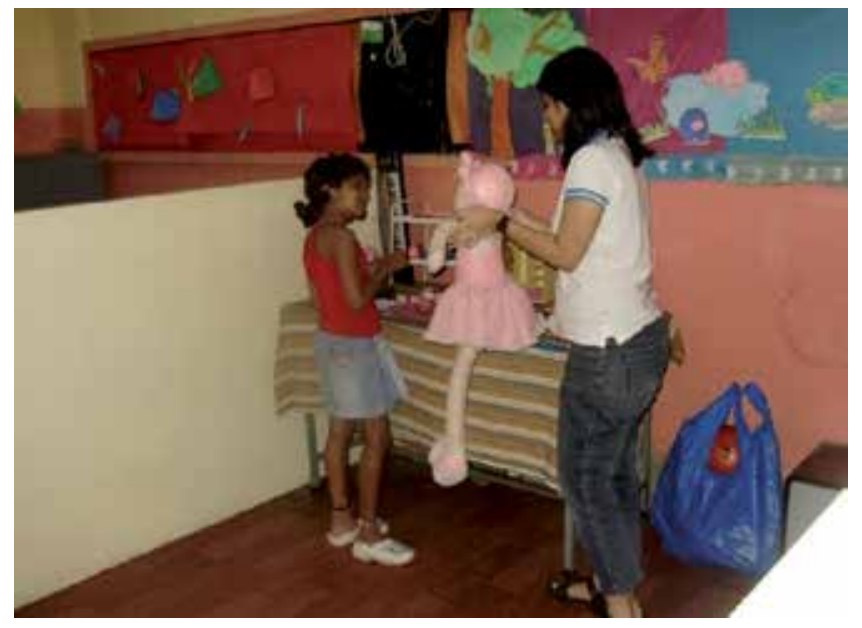

Figure 4. Pretend play with doll 


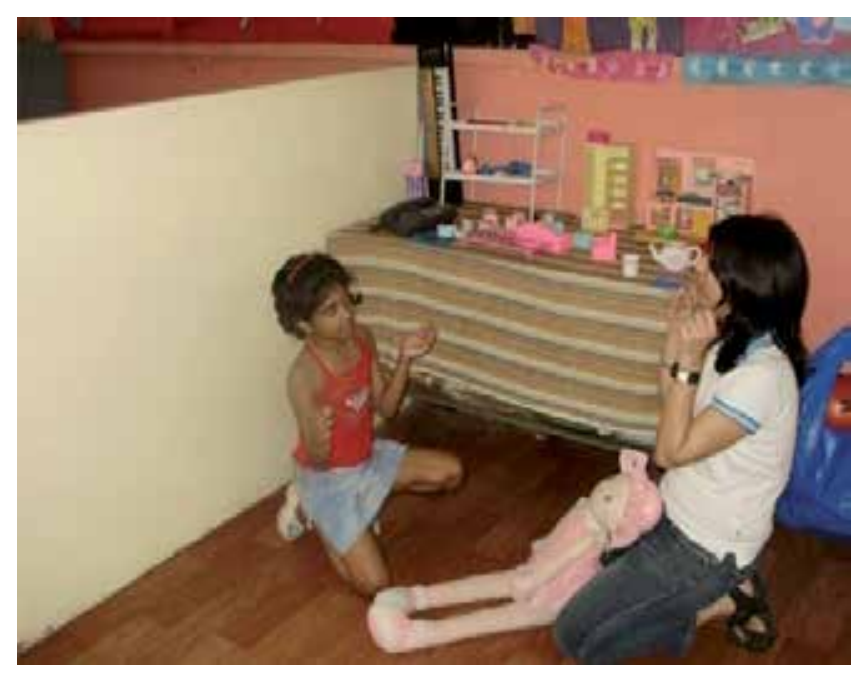

Figure 5. Teaching facial expression

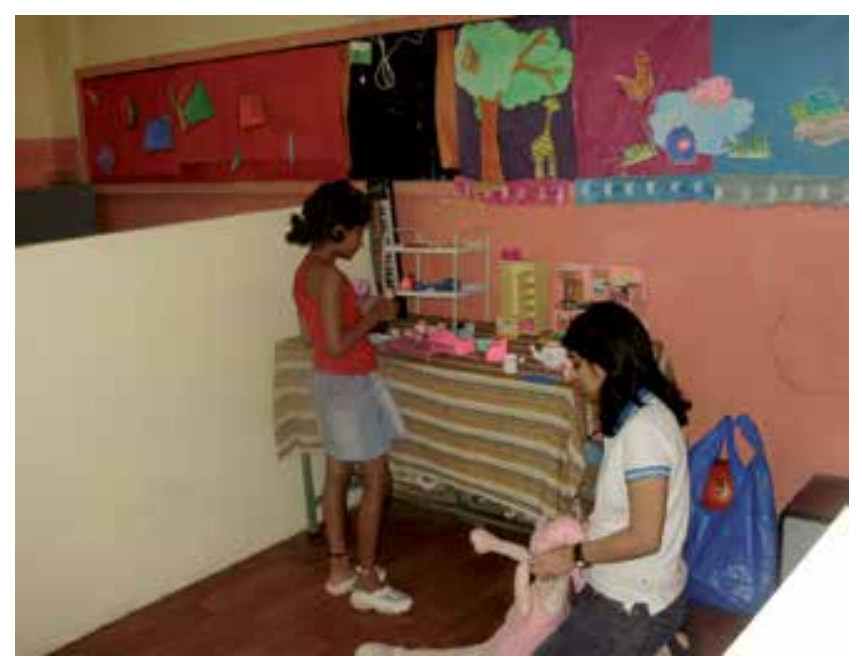

Figure 6. Choice of play things 


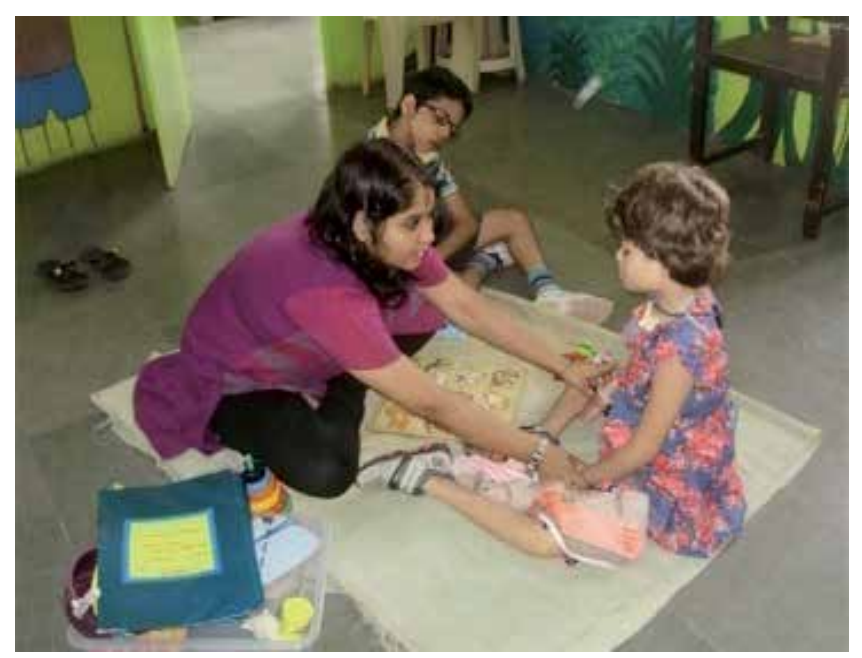

Figure 7. Activity with peer

\section{Results}

The study was conducted to establish the efficacy of floor time for development of social behavior in pre-school children with ASD. The children who received intervention showed a qualitative change in their interactive behavior. A comparison of their composite mean score on BSFS at baseline with that at post intervention showed a significant difference. The data was analyzed using t-test, as the selection of children was random. Table 1 presents the details.

\begin{tabular}{cccccc}
\hline & Mean & $\mathbf{N}$ & df & t-value & Significance \\
\hline Pre test & 34.92 & 13 & 12 & 9.56 & $p<.0001$ \\
\hline Post test & 48.38 & 13 & & & \\
\hline
\end{tabular}

Table 1. Comparison of Composite Mean Scores on BSFS at Pre and Post Tests

The statistical analysis of data indicated the overall effectiveness of floor time. The average score on BSFS at baseline [34.92] increased post intervention [48.38]. This increase was significant as evident from the obtained t-value [9.56, $\mathrm{p}<.0001]$. That the intervention was effective for all children in the group may be seen from Figure 8 which shows the performance of each child at pre and post intervention conditions 


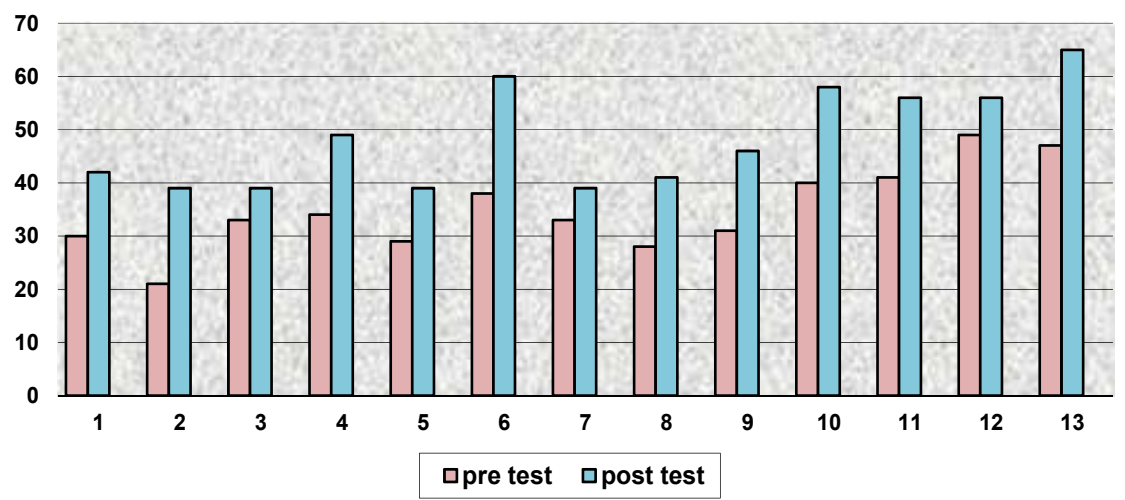

Figure 8. Comparison of individual performance on BSFS

From Figure 8 it is evident that floor time intervention enhanced the social behavior of children, though some gained more from the treatment than others. This variance may be due to initial intra group differences in the children's functioning levels.

Children's scores on selected components of BSFS of turn taking, two way communication, cause and effect and emotional thinking were analyzed individually. On Turn taking skill, the baseline mean [12,38] was significantly lower than the mean score [17.69] post intervention. The derived $t$-value [5.02] was statistically significant $(p<.0002]$. An illustration of each child's performance on turn taking skill is provided by Figure 9.

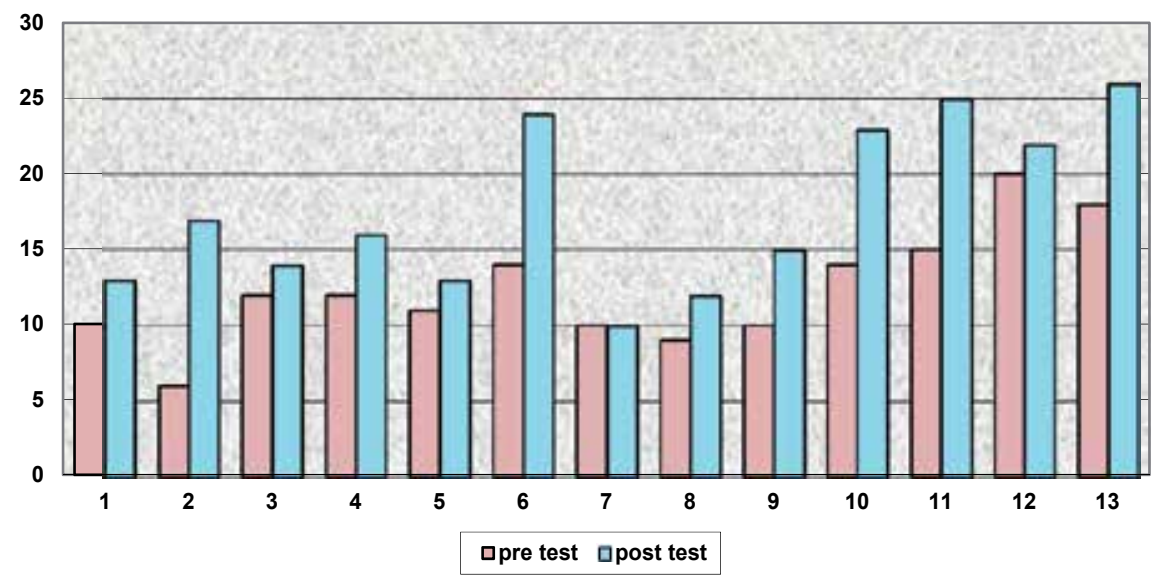

Figure 9. Comparison of individual performance on turn taking

It is evident from Figure 9 that the treatment was effective for all children in the experimental group. All of them gained significantly, except child no. 7 who showed a marginal improvement only. The children's ability to understand the relationship between cause and its 
effect also improved. Their mean performance on this sub skill post intervention [13.30] was higher than the baseline [8.61]. The derived t-value was significant [7.17, p<.0001]. Each child's performance on cause and effect is depicted in figure 10. The data indicates the effectiveness of floor time as a method to develop the understanding of cause and effect relationship in children with ASD.

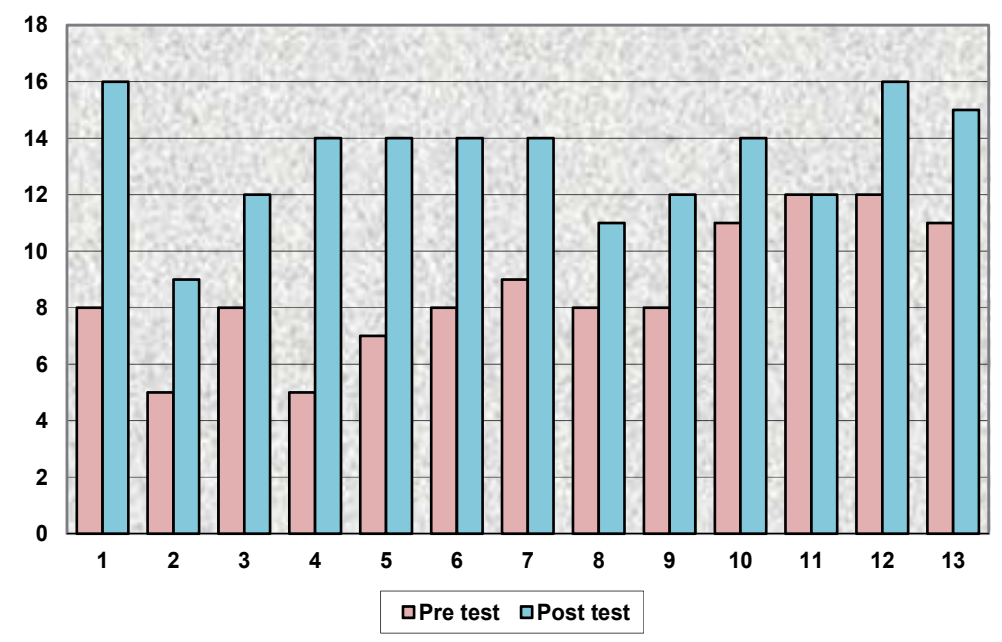

Figure 10. comparioson of individual performance on cause and effect relationship

When performances on two-way communication skills were compared, a similar trend was evident. The mean score at baseline [6.31] was lower than that post intervention [8.69] and the difference was statistically significant $(\mathrm{t}=5.72, \mathrm{p}<.0001]$. Individually too, children improved as may be seen from figure 11. All children gained on the ability for two-way communication.

Lastly, when the data from BSFS were analyzed for performance on emotional thinking, a significant gain was seen in this area too. The difference between baseline mean score [7.38] and post intervention mean score [8.84] was significant ( $\mathrm{t}$-value $=3.5, \mathrm{p}<.004$ ]. Though this difference was significant when means were compared, individually all children did not gain from the intervention. Whereas most children showed an enhancement in emotional thinking from pre to post intervention, performance of some remained the same as what it was at baseline. Figure 12 presents the data on emotional thinking. Since, emotional thinking is the last and the most complex of the six milestones; it is possible that these children required more time to achieve this skill than what was given during the 20 sessions of intervention. However, these children improved their performance on the earlier sub-skills of turn taking, two-way communication and understanding of cause and effect relationship. 


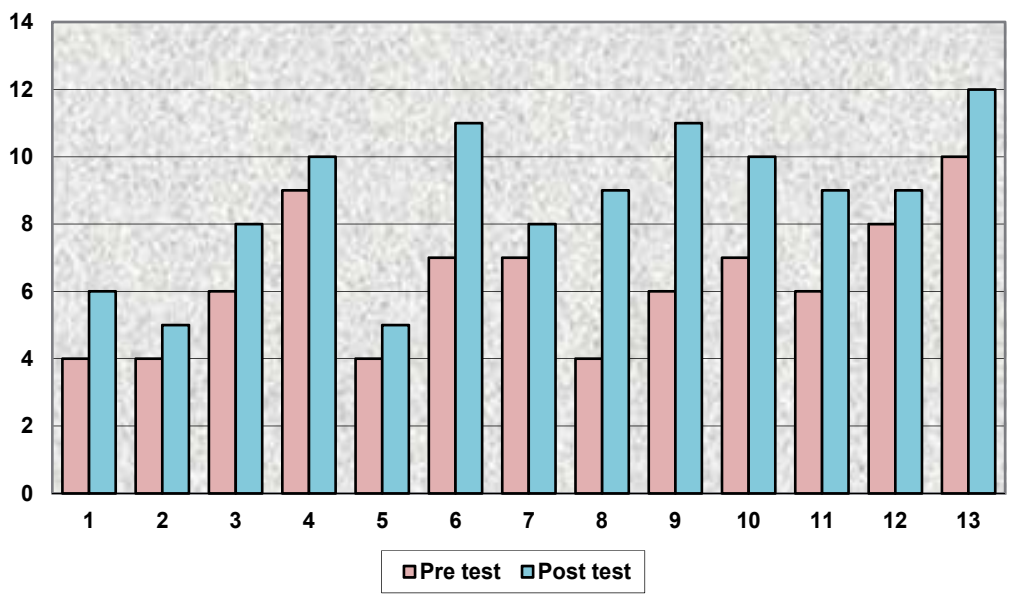

Figure 11. Comparison of individual performance on two-way communication

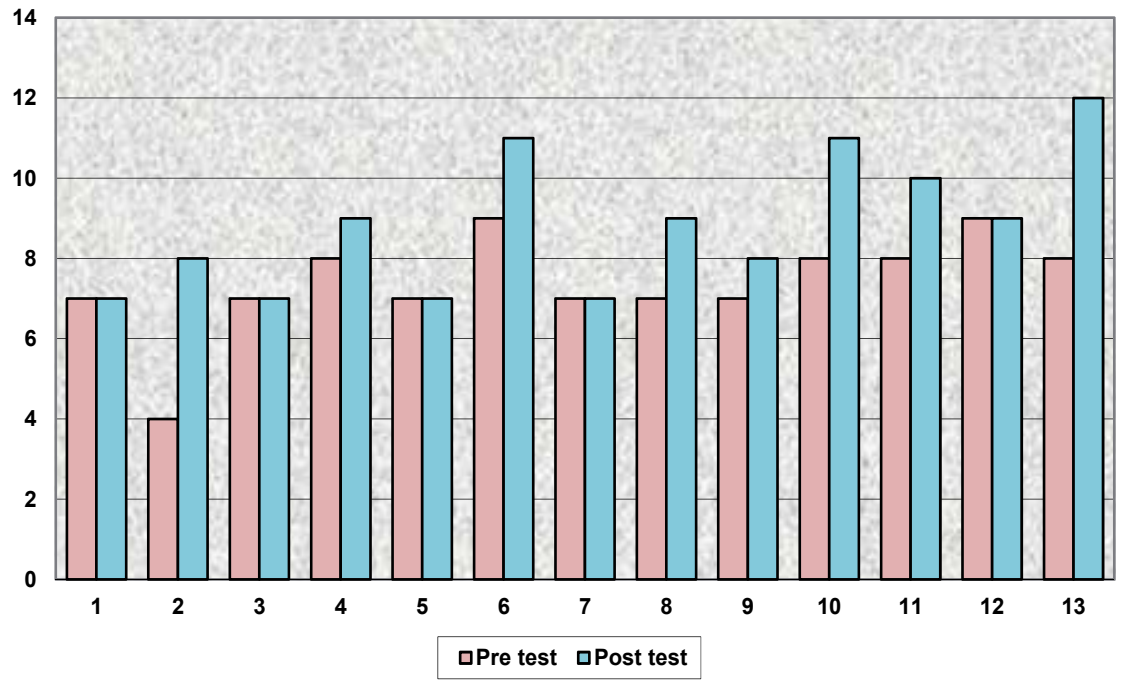

Figure 12. Comparison of individual performance on emotional thinking

The second objective of the study was to compare the performance of children in the experimental group with that of those in control group. As mentioned earlier, the participant children were randomly selected from 5 pre-schools and intervention clinics. Hence, when the study commenced all children were on some kind of early intervention program. The study, in effect, determined the efficacy of floor time in comparison with other early intervention strategies. In order to do this, the post intervention performance on BSFS by both groups was analyzed. The mean score of experimental group was compared with that of control group. The data analysis is presented in Table 2. 


\begin{tabular}{cccccc}
\hline & Mean & $\mathbf{N}$ & $\mathbf{d f}$ & t-value & Significance \\
\hline Experimental & 48.38 & 13 & 24 & 3.08 & $p<.0 .005$ \\
\hline Control & 37.46 & 13 & & & \\
\hline
\end{tabular}

Table 2. Comparison of Post test performance of experimental and control groups

Comparison of post intervention mean scores of experimental and control groups showed a significant difference between the two, in favour of the experimental group. The resultant $t$ value [3.08] was statistically significant $(p<.005]$. This indicated that in comparison to other measures for early intervention, floor time was more effective in development of social behavior of children with ASD. Figure 13 provides a graphic representation of this difference

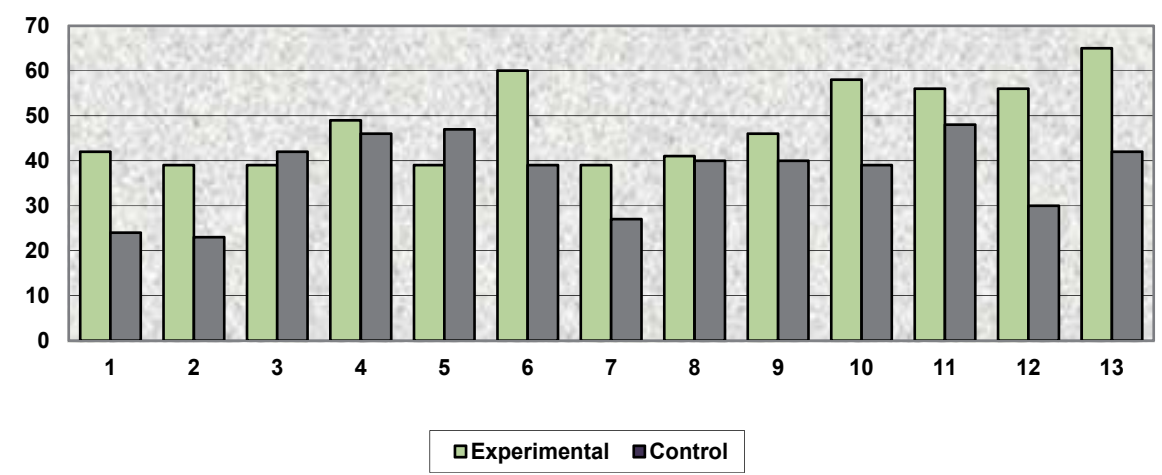

Figure 13. Comparison of post intervention performance of experimental and control groups

It is evident from Figure 13 that except for child no. 3 and child no. 5, all children in experiment group achieved higher scores on BSFS than the control group children. Most children's scored significantly higher than their control group peers. A comparative analysis of both group's mean performance on each sub skill i.e. turn taking (TT), two-way communication (TWC), cause and effect (C\&E), and emotional thinking (ET), within BSFS is presented in Figure 14.

The children who received floor time intervention performed better on an average than those who were in the control group. However, the performance gap between the two groups was not uniform across all sub skills. On emotional thinking skill, the average performance of both groups was nearly same with control group's mean less than 2 points below that of experimental group. 


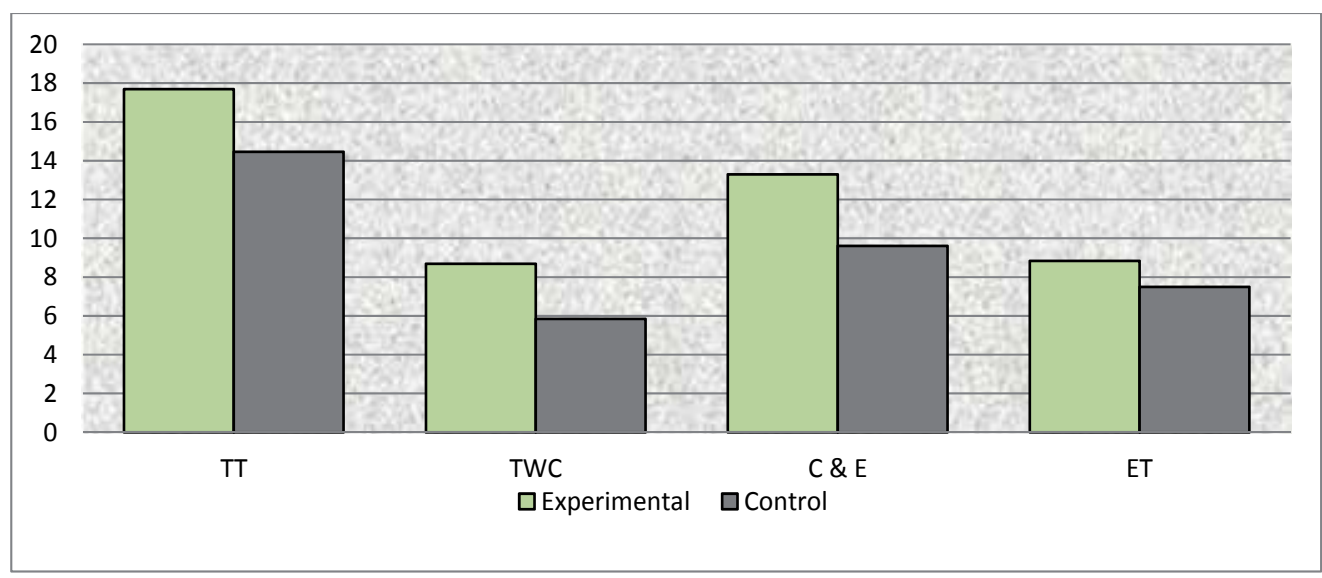

Figure 14. Comparison of experimental and control group on sub skills of BSFS

\section{Discussion}

Unlike neuro-typical children who learn how to be social and interactive by watching how others talk, play and relate to each other, enjoy the give-and-take of social engagement and initiate, maintain and respond to interactions with others, children with autism often do not show the expected development of early social interaction skills. Promoting the social development of infants and toddlers with ASD is one of the primary goals of early intervention services, as is facilitating the ability of young children with social delays to develop appropriate friendships. With early and intensive intervention, the seemingly pervasive social skill deficits of many children with ASD can be remediated[21]. To successfully target these important skills, intervention efforts, even within early intervention, should include: (a) regular access to typical peers, (b) thoughtful planning of meaningful social situations embedded throughout the day, (c) the use of "social" toys, (d) multiple-setting opportunities (home inclusive, community-based) to practice emerging social skills, and (e) intensive data collection in order to make midcourse corrections to existing intervention plans [22]. Poor social skills are an impediment to child's success in classroom, and can also be the cause of behavioral problem. Accordingly, teaching social skills is a common educational objective for children who have autism [23]. However, while teaching variables such as age, developmental and functional levels and sensory profile of each should be considered. Floor time which is based on the developmental approach takes care of the child's developmental level and emphasizes building the milestones that the child may have missed during his or her period of growth. Rather than focusing on teaching a child to speak a few words to interact, Greenspan suggests that the child's gestural system should be worked upon first for language to flow in naturally rather than by rote, thus focusing on the developmental ladder. As the child climbs the developmental ladder he or she becomes more and more regulated and forms a 
sense of self. In the study the authors chose age and functionally appropriate activities for helping a child achieve the given milestones for social behavior. The individual sessions during which the adult followed the child's lead, prompted and encouraged the child effort to participate, and provided the opportunity to practice the skill with a peer contributed to the significant increase in each child's performance from pre to post intervention on BSFS.

According to the Colorado guidelines [24] early intervention strategies must involve building of positive relationships between adults (parents and caregivers) and the infant or toddler. The intent should be to teach the child that parents and caregivers can be relied on as stable, secure, and safe figures that provide nurturance, comfort, pleasure and guidance. Developing attachments is a challenge for a young child with ASD, so special efforts are required, even when signs of a child's interest are not apparent. This might require that a parent or caregiver identify the activities, objects, settings, and interactions that the child finds pleasurable and provide those events and items to the child contingent on a social interaction behavior (rather than non-contingently in a manner meant to keep a child satisfied without social interaction). A tickle game might be initiated with a child and then interrupted by the caregiver with the expectation that the child look at the adult or repeat a gesture to continue. A key objective of efforts to form positive relationships is to ensure that the interactions are pleasurable and that they are associated with the child receiving input that is consistent with needs and interests. Importantly, successful efforts to form strong, positive bonds when a child is very young result in a subsequent relationship in which an adult has considerable influence over a child's behavior and this influence can be essential for the guidance and instruction that the adult (parent or other caregiver) must provide on an ongoing basis. The floor time intervention addressed the issues mentioned above. Activities selected were simple and manageable for the children. Most activities were demonstrated before the child was required to participate. For children with autism, visually organized tasks are easier to learn [25]. During intervention the adult often provided model/picture of a task to be done e.g. block tower, completed puzzle, picture and symbol cards etc. Intervention sessions were built around child's motivation and interests. Most early intervention programs for children with ASD are based on behavioral approach and use discrete trial training. Though evaluations have shown acquisition of learning and behavioral development in several children [26], behavioral approach does not suit all children and families. Strict protocol of timing, intensity, structure, and quality of therapist training influences the success of behavioral interventions. In contrast, floor time encourages naturalistic interactions to develop the core skills. It takes into account the inherent bonding and affection parents have for the child, and guides the parent to modify and channelize their interactions to suit the developmental level of the child. As stated earlier, the children selected for the study attended pre-school and intervention clinics. Thus control group children also received early intervention while floor time intervention was given to the experimental group. However, the experimental group children performed better on selected social skills at the end of the intervention period. The significantly higher achievement of social skills by experimental group children may be attributed to the child-centric naturalistic interactions that occurred during the floor time intervention. 


\section{Conclusion}

Early intervention is very important for enhancing the development of infants and toddlers with disabilities, and they are especially crucial in determining the future language, social and behavioral outcomes of very young children with ASD [27]. A primary consideration of programs for young children with ASD is to provide an environment that is designed to prevent problem behaviors, promote engagement and participation, and facilitate successful interactions with typically developing peers. Getting the child to engage with materials and activities may prevent challenging behavior occurrence and promote appropriate social behavior [28]. Results of this research support the above findings. Floor time principles state that development begins with a shared world between the caregiver and the young child. The goal is to help the child with ASD emerge from its own world and enter this shared world in order to develop his or her functional and emotional capacities. Floor time achieves this by encouraging child to engage in age and level appropriate play activities with adults and later with peers. The outcomes indicate the effectiveness of Floor time as a method for early intervention of children with autism. The findings of the study may be useful for families who are in need of evidence based and suitable early intervention for children with ASD.

\section{Acknowledgements}

The authors wish to thank the children who participated in this study, and are grateful to the children's parents, teachers, therapists, and administrators of the schools and intervention clinics for their support.

\section{Author details}

Rubina Lal and Rakhee Chhabria

Department of Special Education, SNDT Women's University, Mumbai, India

\section{References}

[1] Autism Society of America (2006). Defining Autism. Available from http:// www.autismsociety.org (accessed May. 8, 2012)

[2] American Psychiatric Association (2000). Diagnostic and Statistical Manual of Mental Disorders ( $4^{\text {th }}$ ed. TR). Washington DC

[3] Young, R. Brewer, N. Pattison, C. (2003). Parental identification of early behavioralabnormalities in children with autistic disorder. Autism, 7, 125-143. 
[4] Volkmar, F. Chawarska, K. Klin, A. (2005). Autism in infancy and early childhood. Annual Review of Psychology, 56, 315-36.

[5] Lal, R. and Ganesan, K. (2011) Children with Autism Spectrum Disorders:Social Stories and Self Management of Behaviour British Journal of Educational Research1(1): 36-48, 2011SCIENCEDOMAIN international

[6] Richard, G. J. (2000). The source for treatment methodologies in autism. East Moline, IL: Lingui Systems

[7] Attwood, T. (1988). Asperger's syndrome: A guide for parents and professionals. London. Jessica Kinsley.

[8] Baron-Cohen, S. (2003a). The essential difference: The truth about the male and female brain. New York: Basic Books

[9] Martin, N. (2009) Art as an early intervention tool for children with autism. Jessica Kinsley Publishers, PA.USA

[10] Lovaas OI. Behavioral treatment and normal educational and intellectual functioning in youngautistic children. J Consult Clin Psychol. 1987;55(1):3-9

[11] Prior,M \& Robert, J. (2006) Early Intervention for Children with Autism Spectrum Disorders: for Best Practices http://www.health.gov.au/internet/main/publishing.nsf/ content/D9F44B55D7698467CA257280007A98BD/\$File/autbro.pdf (accessed June 20, 2012)

[12] Diggle, T.T.J, and McConachie, H.H.R. (2009) Parent-mediated early intervention for young children with autism spectrum disorder (Review) http://onlinelibrary.wiley.com/doi/10.1002/14651858.CD003496/pdf/standard (accessed on August, 2012)

[13] http://www.icdl.com/DIRFloortime.shtml (accessed on Sept. 1, 2012)

[14] Greenspan, S.I., and Wieder, S. (1998). The child with special needs. Encouraging intellectual and emotional growth. Perseus Publishing, Massachusetts

[15] Wieder, S. and Greenspan, S.I., (2005). Can children with autism master the core deficits and become empathetic,creative, and reflective?:A Ten to Fifteen Year Follow-Up of a Subgroup of Children with Autism Spectrum Disorders (ASD) Who Received a Comprehensive Developmental, Individual-Difference, Relationship-Based (DIR)Approach. Journal of Developmental and Learning Disorders Vol 9: 39-60

[16] Greenspan, S.I., and Wieder, S. (1997b). developmental patters and outcomes in infants and children with disorders in relating and communicating: A chart review of 200 children with autistic spectrum diagnoses. Journal of Developmental and Learning Disorders. 1: 87-141

[17] Josefi, O.,Ryan, V (2004). Non-directive play therapy for young children with autism: A case study Children. Clinical Child Psychology \& Psychiatry. Vol 9 (4), 533-51. 
[18] Costa G \& Witten MR(2009). Pervasive Developmental Disorders. In Mowder, Rubinson \& Yasik (Eds), Evidence-Based Practice in Infant and Early Childhood Psychology. Wiley.

[19] Solomon, R., J. Necheles, C. Ferch, and D. Bruckman (2007) Pilot study of a parent training program for young children with autism: The P.L.A.Y. Project Home Consultation program. Autism 11, no. 3 205-224.

[20] Nadel, J.: Early imitation and a sense of agency. In: Proceedings of the 4th international workshop on epigenetic robots (2004) http://tivipe.com/TVPresearch/ 62430115.pdf (accessed on Sept.19, 2012)

[21] McGee, G., Daly, T. \& Jacobs, H.A. (1993). Walden preschool. In S. L. Harris \& J.S. Handleman (Eds.), Preschooleducation programs for children with autism. Austin, TX:Pro-Ed.

[22] Strain, P. S., \& Danko, C. D. (1995). Caregivers' encouragement of positive interaction between preschoolers with autism and their siblings. Journal of Emotional and Behavioral Disorders, 3, 2-12.

[23] Weiss, M.J., \& Harris, S.L. (2001). Teaching social skills to people with autism. Journal of Behavior Modification. 25, 785-802

[24] Early Intervention Colorado Autism Guidelines for Infants and Toddlers (2010). Developed by the Universityof Colorado Denver, PELE Center, under contract with the Colorado Department of Human Services, Division for Developmental Disabilities. www.eicolorado.org (accessed on July 2,2012)

[25] Lal, R. and Shahane, A. (2011). TEACCH Intervention for autism. In T.Williams (Ed.) Autism Spectrum Disorders-From Genes to Environment. In Tech, Croatia

[26] Lal, R and Lobo, S. (2007). Discrete trial training and development of pre learning skills in intellectually impaired children with autism. Journal of Rehabilitation Council of India. 3, 1\&2: 15-23

[27] National Resource Council, (2002). Educating Children with Autism. National Academic Press, Washington

[28] Strain, P., \& Schwartz, I. (2009). Positive behavior support and early intervention for young children with autism: Case studies on the efficacy of proactive treatment of problem behavior. In W. Sailor, G. Dunlap, G. Sugai, \& R.H. Horner (Eds). Handbook of positive behavior support (pp. 107-123). New York 



\title{
Early Communication Intervention for Children with Autism Spectrum Disorders
}

\author{
Gunilla Thunberg \\ Additional information is available at the end of the chapter
}

http://dx.doi.org/10.5772/54881

\section{Introduction}

The main purpose of this chapter is to present the results of a review of communication interventions for children aged 0-6 years with autism spectrum disorders and to formulate recommendations for an evidence-based practice. The study, including 20 reviews and 27 primary studies, specifically focus interventions targeting children with diagnosis within the autism spectrum being on an early communicative level.

\section{Communication in children with Autism Spectrum Disorders (ASD)}

\subsection{Difficulties with communication and language as part of the spectrum}

Major advances have been made over the two past decades in understanding the socialcommunication difficulties of children with ASD, resulting in greater emphasis on early socialcommunication features in the diagnostic criteria. Most parents of children with autism first begin to be concerned that something is not quite right in their child's development because of early delays or regressions in the development of speech [1]. Problems with communication, in terms of both understanding and expression, are often said to be one of the main causes of the severe behaviour problems that are common among persons with severe autism and mental retardation [2]. The lack of meaningful, spontaneous speech by age five has been associated with poor adult outcomes $[3,4,5,6]$. Certainly, communication and communication problems are at the heart of what ASD is all about.

Although all persons diagnosed with autism have problems with communication, their type and degree vary a lot and the work of identifying different subgroups has just begun. It has 
been estimated that between one-third [7] and one-half [8] of children and adults with autism have no speech. However, more recent research results indicate that the proportion of nonspeaking children with ASD is much smaller, approximately $14 \%$ to $20 \%$, among those who received very early intervention [9].

Two phenotypes of speaking children with ASD were identified by Tager-Flusberg and Joseph [10]: children with normal linguistic abilities (phonological skills, vocabulary, syntax, and morphology) and children with impaired language that is similar to the phenotype found in specific language impairment. Another potential subgroup may experience verbal dyspraxia or dyspraxia of speech [11;12;13]. Voluntary motor control is disturbed in children with dyspraxia, which also affects their ability to imitate. The new research on the role of the 'mirror neurons' in the parietal and frontal lobes may provide some answers on the relationships between motor control and imitation but also on the possible link with the development of intersubjectivity [11].

In spite of the heterogeneity of language abilities in children with ASD, social-communication or pragmatic impairments are universal across all ages and ability levels [14]. According to Wetherby [15], the social-communication deficits in children with ASD can be organized into two major areas: (1) the capacity for joint attention and (2) the capacity for symbol use. Since joint attention emerges before words, this deficit may be more fundamental and a number of longitudinal studies provide evidence of a relationship between joint attention and language outcomes [16, 17]. According to Wetherby [15] p. 11, 'deficits in initiating and responding to joint attention have a cascading effect on language development since language learning occurs within the context of the modelling by the caregiver of words that refer to objects and words that are jointly regarded'. Wetherby [15] states that deficits in imitation and observational learning are other main causes of the problems with symbol use experienced by children with ASD. Learning shared meanings, imitating and using conventional behaviours, and being able to decontextualize meaning from the context constitute the symbolic deficits in children with ASD [13].

\subsection{Development of communication and language in children with ASD}

Because autism is usually not diagnosed until age three or four, there is relatively little information about language in very young children with autism [10]. Retrospective studies using parent reports and/or videotapes collected during infancy, together with studies of children considered likely to develop autism, show severely delayed language acquisition with respect to both receptive and expressive skills $[18,19,20]$. Another typical phenomenon described by $25 \%$ of parents of children with ASD is language loss after initially developing some words [21]. Lord, Schulman, and DiLavore [22] found that this language regression is unique to autism and does not occur in other children with developmental delays. Chawarska et al. [21] hypothesize that these early-acquired speech-like productions are lost by children with ASD because the link between these expressions and a network of symbolic communication fails. There is significant variability in the rate at which language progresses among children with ASD who do acquire speech. 
The few longitudinal studies of language acquisition in children with ASD suggest that progress within each domain of language follows similar pathways as it does in typically developing children $[9,12]$. However, the speech of children with ASD is also characterized by some typical deviations. One of the most salient aspects is the occurrence of echolalia, which can be either immediate or delayed. Although some echolalia seems to be self-stimulating, both types of echolalia can serve communicative purposes for the speaker [12]. At an early stage of language development, this may be the only way in which the child can actually produce speech. Tager-Flusberg et al. (1990) found that, over the course of development, echolalia rapidly declined for all the children with ASD and Down's syndrome in their study. Another prominent feature of language in children with ASD is general problems with deixis, which are most often manifested as pronoun confusion [10]. Features such as vocal quality, intonation and stress patterns often result in problems for persons with ASD, although there is a lack of research in this field. Taken together, the findings suggest that the difficulties are due not only to problems in social intent but also to problems affecting a more basic aspect of vocalization [12].

Less research attention has focused on the comprehension skills of individuals with ASD although deviations in response to language and comprehension have been found to be strong indicators of ASD [18]. According to Tager-Flusberg et al. [14], it seems that ASD children 'not only may have limited ability to integrate linguistic input with real-world knowledge but also may lack knowledge about social events used by normally developing children to buttress emerging language skills and to acquire increasingly advanced linguistic structures' $[12$, p. 350].

The pragmatic aspects of language have been studied in numerous ways. Children with autism share important similarities across different language levels [12]. The speech acts that are missing or rarely used in the conversations of children with autism often concern social, rather than regulatory, uses of language [22]. Ramberg, Ehlers, Nydén, Johansson, and Gillberg [24] found that children with ASD were impaired in taking turns during dyadic conversations. A higher proportion of initiations rather than responses was found in a study [25]. Although the basic intention to communicate often exists, the person with autism has impaired skill in participating in communicative activities involving joint reference or shared topics [12, p. 354].

\section{Interventions to support communication and language development in children with ASD}

\subsection{History and different theoretical approaches}

The first reports on language interventions were published in the mid-1960s. The intervention at that time built on the operant tradition developed by Skinner during the 1950s. The teaching sessions in this method, referred to as discrete trial teaching or didactic teaching, are marked by a high level of adult control and direction, massed-practice periods for preselected tasks, 
and precise antecedent, teaching, and reinforcement practices. The learner is in a responder role, and the teacher has a directive role [11]. The strength of the didactic behavioural approach is primarily that it has demonstrated efficacy in many studies, using a variety of treatment settings and treatment deliverers, with both single-subject and group designs [11]. Limitations on this approach as a language-training method were recognized early on, with the children's lack of generalization being a core problem [26].

The pragmatic understanding of communication was fully developed after the operant teaching methods were first developed [11]. The current scientific understanding of communication and language development stems from the 1970s and 1980s, when it was demonstrated that language develops from the preverbal social exchanges of infants with important others (Bates, 1976). According to Rogers [11 p. 149], 'current research, building primarily on the work of Wetherby [13, 15, 23], Prizant [13], and Mundy, Sigman and Kasari [17], has demonstrated that young children with autism lacked these early building blocks of communication, involving social initiative, joint attention, social and emotional reciprocity, and the use of gestures to co-ordinate social exchanges.

In 1968, an important study was published by Hart and Risley [27]. Very positive results were obtained with an intervention in which the principles of operant teaching were applied in the child's natural environment. The term 'incidental teaching' was used for this approach, in which the natural environment is deliberately structured to highlight the function of the targeted language form. This intervention produced much better results with respect to maintenance and generalization and stimulated development and research in the field [11]. According to Rogers [11, p. 153], the effectiveness of this approach results from four factors: (1) child language functions to achieve child-chosen goals and child-chosen reinforcers, which strengthen their power; (2) the focus is on child communication skills that are functional in all settings; (3) the social functions of language are highlighted; (4) emphasis on child motivation and natural reinforcers adds a positive element to the interactions, which may enhance memory for learning.

The third major approach in the field of communication intervention for children with ASD is the developmental pragmatic approach. The most elaborated programme for treatment, the SCERTS (Social-Communication, Emotional Regulation, Transactional Support) model [28] focuses on functional communication. This approach bears many resemblances to the behavioural naturalistic teaching methods. More emphasis is, however, placed on developing nonverbal behaviours prior to verbal communication and on the use of Augmentative and Alternative Communication (AAC) systems to assist in the development of verbal communication [11]. Today many models combine behavioural techniques and social-interactionist approaches, such as Enhanced Milieu Teaching, developed by Kaiser and colleagues [29], The Denver Early Start [30], Caregiver Mediated Joint Engagement Intervention for Toddlers with Autism [31], Focus parent training for toddlers with autism [32]. The strength of the developmental model is its strong basis in the science of communication development. Its weaknesses include the lack of treatment manuals and the fact that it requires considerable knowledge on the part of the therapist [11]. 


\subsection{Early communication intervention}

\subsubsection{Why early communication intervention?}

Several new research findings point to the importance of an early start of communication intervention. The most essential of these are:

- Difficulties in understanding and expressing communication is very closely linked to the development of challenging behaviors in individuals with autism [2].

- Communication and language are pivotal for the development of several other cognitive constructs or competencies, such as:

- Reading and writing.

- Theory of Mind [33].

- Communication ability predicts outcome with respect to functioning and quality of life in adults with autism spectrum disorders [3].

- The severity of communication difficulties in preschool-aged children is correlated to the perceived level of stress in their parents [34].

- Communication is one of the most important factors for the participation in daily activities of young disabled children [35].

- According to several guidelines, among these NRC (National Research Council) in the United States, functional communication and social interaction should be prioritized in early intervention programs given to children with ASD.

Furthermore, interactional research done on children with communicative impairments and their parents has shown that the responsive communication style that characterizes parents of typically developing children is often replaced by a more directive style in parents of children with communicative impairments. Besides this impact on quality, quantity is also affected, in that the rate of communication occasions in these families tend to decrease. This adds a cumulative negative effect on the communication development due to less stimulation and experience [36]. Research has also shown that children with ASD whose parents used a responsive style during preschool years in general had better communication and languages skills when they were followed up as teen-agers [37].

\subsubsection{Early communication intervention methods or programs}

There are many intervention programs for children with ASD that focus on communication. Some of these are more specifically aimed at communication whilst others include communication and language as a part of a comprehensive early intervention program. Some programs (indirect interventions) focus on the parent or partner usually by guiding and teaching parents, individually or in groups (courses). Other intervention programs focus more on the training of the child (direct interventions). Today it is common 
that early intervention programs include both indirect and direct aspects: education and tutoring of parents and training of the child.

Another dimension of great importance in early communication intervention concerns the degree of child focus. To have a child focus means that the motivation of the child and the developmental level is decisive in what is done during intervention. The adult follows the lead of the child and the place for training is where the child is, often the floor. In this way it's not necessary to use reinforcements or rewards since the child is already interested and motivated. To get the child to train and focus the intended skills or functions different behavioural techniques are often used. At the other end of this dimension we find the more traditional didactic training situation where the adult trainer or therapist follows and uses a predefined set of activities and materials during a training session. The specific behavioural techniques; prompts and reinforcements used during the session are often also specified or planned. The child is expected to follow the lead of the adult and it is typical that the training is held the child and the adult sitting face-to-face at a table. It is more typical that child-focused interventions are provided during daily activities in the natural environment of the child; at home and/or in preeschool, whilst didactic training is provided at a clinic, at least during the introduction of new materials and training activities.

Still another difference between programs that might be seen as a dimension is the degree to which augmentative and alternative communication (AAC) is included. In some programs these strategies, in the form of manual signs, symbols and pictures and speech-generating devices (SGDs, today often Apps used on an iPad, smartphone or other platform), are included already from the start to promote communication and build language, whilst in other programs AAC strategies are not included, but instead seen as a last resort when training of speech has failed.

\subsubsection{Education and tutoring of parents and staff}

The most common intervention of this type is parental education. The internationally most wide-spread parental education programs most probably are the courses developed by the Canadian Hanen Centre [38]. The course being developed for children within the autism spectrum is called More Than Words and includes eight group sessions for the parents and three "home-visits" by the Speech-language pathologist. During these visits the interaction between the child and the parent is videotaped and the parents are given feed-back and further guidance how to improve communication and use of the strategies being taught during the course. The Hanen courses is a developmental approach and teaches responsive strategies to the parents adding some behavioural techniques to stimulate communication learning within the frames of child-focused natural interaction in the home [38]. A new parental course called ComAlong has been developed in Sweden and now is spreading in northern Europe [39]. ComAlong include eight group sessions focusing on responsive strategies and environmental teaching but also puts a large focus on the use of augmentative communication strategies in 
the home setting [39]. The parents are provided with picture boards so they can use aided language modeling in their homes $[39,40]$.

\subsubsection{Comprehensive intervention programs}

Training of communication, language and speech is most often an important part in the different comprehensive programs, addressing different skills and problems, that has been developed for young children within the autism spectrum. Some of these are built on behavioural theories, others on developmental pragmatic approaches. There seems to be a trend that the programs being developed and researched during the last decade, specifically for young children with autism, are more eclectic. The background theories are often described as developmental pragmatic whilst ABA (Applied Behavior Analysis) techniques are used to strengthen the teaching practices. Most often these comprehensive programs include both direct training to the child and indirect intervention parts in that parents and/or staff in the close network of the child are given education, training and/or guidance.

\subsubsection{Augmentative and Alternative Communication - AAC}

AAC comprises different methods and modes of communication such as body communication, concrete objects, manual signs, graphic symbols or speech-generating devices. Historically, the first studies describing AAC techniques being used for individuals with autism appeared in the 1970s; they reported on the use of sign language to improve communication [41]. These studies appeared at the same time as the unsatisfactory results of spoken-language-training programmes were being published. Studies by, for example, Lovaas et al. [26] reported little change after many hours of intensive treatment, and the results were particularly poor for the children whose comprehension and vocal skills were most impaired [41]. Initially, most signing programmes were built on formal sign language systems, but it became evident that these were often too complex and abstract, and so specially adapted systems were developed and implemented. Sign-based programmes spread rapidly in schools for children with autism in many countries.

During the $80^{\prime}$ s and the $90^{\prime}$ s a gradual change in AAC intervention for persons with autism, was seen, as visual-graphic communication was more in focus. Mirenda and Erickson [42] explain that the shift away from the use of signing to visual-graphic communication occurred as a result of research findings in three main areas: imitation, iconicity, and intelligibility. In addition to the evidence of a generalized imitation deficit in autism, there were also studies showing that some children with ASD had extremely poor sign imitation skills [43] due to difficulties with motor planning, control and execution [44]. According to Howlin [41], the shift from the use of manual signs to visual methods was also due to the fact that visual methods had proven to be effective in enhancing general skill acquisition, mainly within the TEACCH programme (Treatment of Education of Autistic and related Communicationhandicapped CHildren; [45]) developed during the 1970s. A variety of symbol systems were also developed, beginning with Blissymbolics and Rebus followed by Pictogram and Picture Communication Symbols. The improvements in computer technology made these symbol sets easily available in the form of practical software packages. The development of digital cameras 
during the 1990s also increased the possibility of including personal photos in AAC systems, which, according to clinical reports, seemed to increase motivation and facilitate understanding of pictures, particularly for individuals with ASD [46].

There are, however, also reports of problems in teaching symbols to children with ASD, mainly in teaching them to use the pictures spontaneously and for communicative functions other than requesting [41]. It was precisely these problems that led Bondy and Frost [47] to develop the method called Picture Exchange Communication System (PECS). PECS is a systematic approach to communication training specifically developed for children with autism. The elements that make PECS different from other visual-graphic techniques are the use of the concrete hand-to-hand exchange of the picture and also the highly prescriptive user manual with its six levels to follow in sequence.

Historically, the use of speech output technologies with individuals with ASD has not been a matter of course [48]. Computer technology was introduced into educational settings for children with autism late, not only in North America, but also in other countries. Professionals feared that people with ASD would become even more aloof if they were encouraged to sit in front of a computer screen. Concerning speech-generating devices (SGDs), a common view was that they would only stimulate echolalia in children with ASD, and that there would be too much noise in the classroom. By the end of the 1990s, scepticism had decreased. This was probably due to reports of some studies of successful computer-assisted instruction (CAI) carried out. The introduction of "app technology "has meant a revolution to the field of speechgenerating devices and the first studies of the effects of apps are now being published.

\section{Evidence-Based Practice - EBP}

The term evidence-based used as a prefix and a denominator of interventions and methods comes from medicine. The term evidence based means that the choices of interventions and assessments are based on a research literature not simply professional experience or previous practice. Evidence-based practice has been important within the area of early communication intervention. The behavioural intervention tradition with its roots in the research clinic has produced a lot of high-quality research during the years. Other types of interventions has been less researched and sometimes have used methods and produced data that are different so that comparisons of effects are hard or impossible to do. This has also led to an interesting discussion of how to do EPB within the field of communication intervention. Ralph W. Schlosser, professor at NorthEastern University, USA, has been of great importance in this respect. Partly because he is spreading knowledge about evidence-based practise (EBP) and due to the many thorough compilations of research that he has done, but also in demonstrating the problems and shortcomings using EBP in relation to the field of augmentative communication intervention [49]. One of these problems concerns the use of the Randomized Controlled Trial or Study (RCT) as the golden standard, as RCTs are almost non-existent within the AAC field. There are many reasons to this but the main ones are that (1) children with communicative disabilities are so heterogeneous and (2) that randomization is extremely difficult to put through due to ethical reasons. 
Schlosser has therefore suggested an alternative evidence hierarchy placing the meta-analysis on top $[49,50]$. Schlosser and several other prominent authors within the field of communication intervention research designs recommend the use of well-controlled single-subject research designs that can form the base for systematic meta-analyses.

\section{Method}

\subsection{EBP-group}

The review of research within the field of early communication intervention that is presented in this study was initiated by the Swedish association of Habilitation directors as part of a project concerning EBP that was started 2002. Within the frames of this project several reports have been produced with respect to interventions for children and adults with disability. The author of this chapter was appointed scientific leader for a group of five speech-language pathologists and one special educator in Sweden, that applied for taking part in the project. The group has worked together during recurrent two-day-sessions and in between, work has also been done separately and in pairs.

\subsection{EBP-method and search question}

The group decided to use the EBP-model of Ralph Schlosser [49]. As mentioned above the hierarchy of evidence of Schlosser is a bit different compared to the traditional ones, in that it places the meta-analysis on top of the hierarchy beside the RCT-study. Schlosser also includes perspectives of the stakeholder and the influence of environment into his model of EBP and defines EBP as follows: "The integration of best and current research evidence with clinical/educational expertise and relevant stakeholder perspectives to facilitate decisions for assessment and intervention that are deemed effective and efficient for a given stakeholder". The classical model of formulation of a evidence question shortened PICO - Problem, Intervention, Comparison, Outcome - has accordingly been revised into PESICO - Problem, Environment, Stakeholders, Intervention, Comparison, Outcome [49]. The question that was formulated in this review was: A young child with severe communicative disability, living with his/her parents and being placed in a pree-school group: which intervention is most effective; indirect or direct interventions.

\subsection{Procedure}

When the clinical question had been formulated the group identified search terms to use. These were: Early Intervention, Communication, Communication Disability/ies, Direct intervention, Indirect intervention, Early childhood, Kindergarten, Pree-school, AAC, Augmentative Communication, Alternative Communication, Early Communication, Language, Meta-analysis, Review. The terms were searched separately and in combinations using four scientific data bases: PubMed, PsycInfo, CINAHL and ERIC. It was seen that CINAHL generated significantly more results than the other three. All abstract were browsed and the studies considered as relevant were downloaded. The reference lists of these studies led to some new findings. A few 
studies and book chapters were found through the group's different contacts and readings of literature. The studies were read and reviewed using a protocol and a manual that was developed. The factors that were examined in each study were: Research methods, participants, environment, intervention, results, evidence grading and a final category called notes. This column included judgements of (a) ICF domain/s that the study involved, (b) validity: internal, external, social and ecological, (c) importance of discussion and suggestions of future studies.

Each study was first reviewed by two group members separately and then discussed and graded by the group altogether. The group graded the studies according to three systems: Schlosser [49], Nordenström [51] and Golper [52]. Schlosser's system was seen as the most important for this study due to the fact that it was developed for the field of communication intervention for people with disability. Nordenström represent the classical medical evidence hierarchy whilst the Golper was included for its ambition to catch or grade the level or depth of evaluation that the study represents.

\begin{tabular}{|c|c|c|}
\hline System & Level & Definition \\
\hline \multirow[t]{9}{*}{ Schlosser } & 1 & Meta-analyses of SSRD /RCT \\
\hline & 2 & Well designed non-RCT group study \\
\hline & & SSRD - one intervention \\
\hline & & SSRD - several interventions \\
\hline & & Subgroups to/variants of the types above \\
\hline & 3 & Narrative quantitative reviews (except of meta-analyses) \\
\hline & 4 & Narrative reviews \\
\hline & 5 & Pre- experimental group studies (i.e. before-after) and case studies \\
\hline & 6 & Expertise: educational books, journals, expert opinion \\
\hline \multirow[t]{4}{*}{ Nordenström } & A & Strong scientific evidence (meta-analysis, well-done and large RCT) \\
\hline & $\mathrm{B}$ & $\begin{array}{l}\text { Moderate evidence (smaller or non-randomized studies, cross-sectional } \\
\text { studies, case studies, cohort studies) }\end{array}$ \\
\hline & C & $\begin{array}{l}\text { Week evidence (expert opinion, concensus reports, case studies and other } \\
\text { descriptive reports) }\end{array}$ \\
\hline & D & Non-existent scientific evidence (No studies of sufficient quality exists). \\
\hline \multirow[t]{3}{*}{ Golper } & Phase I & $\begin{array}{l}\text { Hypotheses about treatment efficacy are being developed for later testing. } \\
\text { Often this involves experimental manipulations to test potential benefits } \\
\text { or activity of a particular treatment. }\end{array}$ \\
\hline & Phase II & $\begin{array}{l}\text { The goals are to formulate and standardize protocols, validate } \\
\text { measurement instruments, optimise dosage of treatment, and so on. } \\
\text { Includes case reports and small group studies with no control groups or } \\
\text { treatment comparisons. }\end{array}$ \\
\hline & Phase III & $\begin{array}{l}\text { Treatment efficacy of a specified protocol is formally tested either with } \\
\text { SSRD or group studies with controls such as control groups or treatment } \\
\text { comparisons. }\end{array}$ \\
\hline
\end{tabular}

Table 1. Systems for evidence-grading being used in this study. SSRD=Singel Subject Research Design, $\mathrm{RCT}=$ Randomized Controlled Study 


\begin{tabular}{|c|c|c|c|}
\hline Author\&year & Study design & Intervention & Evidence grading \\
\hline \multirow[t]{3}{*}{ Aldred, Green \& Adams, 2004 [54] } & $\mathrm{RCT}$ & Education and guidance of parents in & Schlosser: 1 \\
\hline & & the use of responsive strategies & Nordenström \\
\hline & & & Golper. III \\
\hline \multirow[t]{3}{*}{ Callenberg och Ganebratt, 2009 [56] } & Pre-experimental & ComAlong parental education; & Schlosser: 5 \\
\hline & group-study & responsive strategies and $A A C$ & Nordenström: B \\
\hline & & & Golper. II \\
\hline Drew, Baird, Baron-Cohen, Cox, & Pilot RCT & Focus parent training; joint attention & Schlosser: 1 \\
\hline Slonims, Wheelwright, Swettenham, & & & Nordenström: A \\
\hline Berry \& Charman, 2002 [32] & & & Golper. III \\
\hline Elder, Valcante, Yarandi, White \& Elder, & Large-scale SSRD & Education and guidance of fathers: & Schlosser: 2 \\
\hline \multirow[t]{2}{*}{2005 [57] } & & imitation and responsive strategies & Nordenström: B \\
\hline & & & Golper. III \\
\hline Ferm, Andersson, Broberg, Liljegren \& & Group study; mixed & Parents and course leaders' experiences & Schlosser: 5 \\
\hline \multirow[t]{3}{*}{ Thunberg, 2011 [55] } & methods & of the ComAlong augmentative and & Nordenström: B \\
\hline & & alternative communication early & Golper. II \\
\hline & & intervention course & \\
\hline Girolametto, Sussman \& Weitzmann, & Case study, & Hanen More than Words parental & Schlosser: 5 \\
\hline \multirow[t]{2}{*}{$2007[58]$} & Interaction analyses & education and guidance: responsive & Nordenström: C \\
\hline & & strategies & Golper. III \\
\hline Howlin, Gordon, Pascoe, Wade \& & $\mathrm{RCT}$ & PECS - training of pree-school teachers & Schlosser: 1 \\
\hline \multirow[t]{2}{*}{ Charman, 2007 [59] } & & (and also some older children) & Nordenström: A \\
\hline & & & Golper. III \\
\hline Jonsson, Kristoffersson, Ferm \& & Pre-experimental & ComAlong parental education; & Schlosser: 5 \\
\hline \multirow[t]{2}{*}{ Thunberg, 2011 [40] } & group study; mixed & responsive strategies and $A A C$ & Nordenström: B \\
\hline & methods & & Golper. II \\
\hline \multirow[t]{3}{*}{ Karlsson \& Melltorp, 2006 [62] } & Pilot group study, & ComAlong parental education; & Schlosser: 5 \\
\hline & mixed methods & responsive strategies and AAC & Nordenström: B \\
\hline & & & Golper. I \\
\hline \multirow[t]{3}{*}{ Lennartsson och Sörensson, 2010 [60] } & Group study, small & ComAlong parental education; & Schlosser: 5 \\
\hline & control group & responsive strategies and $A A C$ & Nordenström: B \\
\hline & & & Golper. II \\
\hline McConachie, Randle, Hammal \& & Controlled group & Hanen More than Words parental & Schlosser: 2 \\
\hline \multirow[t]{2}{*}{ LeCouteur, 2005 [61] } & study & education and guidance: responsive & Nordenström: B \\
\hline & & strategies & Golper: III \\
\hline \multirow[t]{3}{*}{ Oosterling et al., 2010 [63] } & RCT & Focus parent training; joint attention & Schlosser: 1 \\
\hline & & & Nordenström: A \\
\hline & & & Golper. III \\
\hline Seung, Ashwell, Elder \& Valcante, 2006 & Group study & Verbal outcomes after training of & Schlosser: 2 \\
\hline \multirow[t]{2}{*}{ [64] } & & fathers as analyzed by video & Nordenström: B \\
\hline & & interactions & Golper. III \\
\hline Sharry, Guerin, Griffin \& Drumm, 2005 & Group study & Evaluation of the parental plus progam & Schlosser: 5 \\
\hline \multirow[t]{2}{*}{ [65] } & & including responsive strategies & Nordenström: B \\
\hline & & & Golper. II \\
\hline
\end{tabular}

Table 2. Studies of education and guidance to parents or staff 
The results were analysed and grouped primarily according to the formulated search question but also according to the identified areas of intervention and methods being evaluated in the studies. Building on these results, recommendations and a model for early communicative intervention was suggested. These results were documented in a report being published on the website of the Association of Swedish Habilitation directors [53]. A new literature search using the same procedure as described above led to some revision of results and recommendations in a new version of the report that was recently published [53].

The results that will be shared in this book chapter concerns the studies that specifically involved children on the autism spectrum, which in total involved about half of the studies, or exactly 47 studies. The data from both literature searches was used: 30 studies from the review published in 2011 and 16 studies from the updated version of 2012.

\section{Results}

The number of studies that were included in the review totalled 106. Of these, 39 were reviews, while the other 67 were primary studies. 46 of the studies involved children diagnosed within the autism spectrum. This means that about half the research on interventions for children with communicative disabilities have focused children with ASD. 31 of the studies were included in the report published 2011 while 14 were added in the review done 2012. 20 of the publications were reviews while 27 were primary studies. There were comparatively more primary studies, often of high research quality, to be found in the more recent search (2012). Only publications where the children were clearly described as having ASD were included in this review. There were most probably even more studies of the 106 that included children with ASD since sometimes participants were described according to type and/or severity of disability (such as severe communicative disability), and not diagnose..

\subsection{Indirect interventions - Education and guidance to parents}

14 primary studies were found. The evidence is moderately to strong since there are also some studies with a high level of scientific control. Many of the studies were noted as showing high validity with respect to external validity as well as social and ecological validity. In several studies the parents were involved in the evaluation procedure and measures of natural interactions were included.

In general the results of education and guidance to parents and staff are very positive although this review shows that there seems to be a lack of research when it comes to education and guidance of staff. Only one study was found where pree-school teachers were educated and guided how to use the PECS-method [32]. The results of the parental interventions indicate that they are effective in that positive results can be seen very quickly with respect to different areas and with comparatively little amount of intervention. This is also probably one of the reasons behind the trend that parental education seems to be included as a part of the more recently developed intervention programs. In the second literature search in this study more interventions were found that included 
guidance of parents (for example 31, 63, 74, 75, 81). Several of these interventions included education that was combined with home-visits when the therapist interacted with and trained the child during natural play situations. The parents observed these play activities and the therapist's use of behavioural strategies, which were then discussed an practiced during the sessions. The results of these comprehensive programs are included in the section of direct interventions below (table 3), but it is important to also recognize the fairly large amount of indirect instruction in these programs.

In several studies of the interventions more specifically aimed at parental education, it was seen that the parent's use of responsive strategies increased [54, 58, 60, 61, 62] and some studies showed that interaction between the parent and the child was positively affected [57, 58, 62, 65]. Some studies report that the development of communication and language in the child seems to be increased when the parents are provided with education and guidance $[32,54,56,61,62,64]$. Several studies have tried to measure parental stress and other family related parameters that are expected to be affected, also out from parental interviews $[54,55,56,61,65]$. Most studies failed in proving effects in this respect, at least on a level of statistical significance. In some studies the researchers speculate that the questionnaires given before and after an intervention seems to fail in catching an effect. In qualitative studies parents report that they can see the problems of the child more clearly after the course and can be more open about the family problems [55]. This means that items related to family issues even might "get worse" comparing questionnaires filled in blindly before-after intervention.

So far very little is known of the long-term effects of indirect intervention. The few studies with this focus show that the effects seem to fade over time. Both clinicians and researchers hypothesize that there probably is a need to do follow-ups and/or provide booster interventions to maintain the intervention effects over time. There are also indications that the effects of a parental education on the development of the child seems to be further enhanced when the education is complemented with direct intervention to the child.

\subsection{Direct interventions - Provision of training of the child}

19 studies were found of which 10 were reviews (1 meta-analysis) and the rest primary studies. The scientific level of evidence varies, but the recently published primary studies being of high quality certainly strengthen evidence in the area of direct communication intervention.

Direct interventions or training of the child has proved to have a positive impact on the development of the child with ASD as is stated in most, but not so sure in all, of the studies in the table. Exactly what is described to be affected differs in different studies, depending on the focus of the study, but to a large extent also on what have been measured in a particular study. It is more common that classical didactic programs report outcomes within the function- or activity-domain, often by the use of measures of intelligence (IQ) or language (different language tests). The child-directed naturalistic interventions more often describe outcomes in terms of activity or participation and use data of communication or interaction from video analyses, parental questionnaires and interviews. 


\begin{tabular}{|c|c|c|c|}
\hline Author\&year & Study design & Intervention & Evidence grading \\
\hline \multirow[t]{3}{*}{ Charman, 2010 [76] } & Review & Review of developmental approaches to & Schlosser: 4 \\
\hline & & understanding and treating autism & Nordenström: C \\
\hline & & & Golper. I \\
\hline \multirow[t]{3}{*}{ Corsello, 2005 [66] } & Review & Review and discussion of interventions 0-3 & Schlosser: 4 \\
\hline & & years & Nordenström: C \\
\hline & & & Golper. I \\
\hline \multirow[t]{3}{*}{ Dawson et al, 2010 [30] } & RCT & Study of the effects of : The Early Start & Schlosser: 1 \\
\hline & & Denver Model for toddlers with ASD & Nordenström: A \\
\hline & & & Golper. III \\
\hline \multirow[t]{3}{*}{ Delprato, 2001 [67] } & Review & Comparison of discrete trial interventions & Schlosser: 4 \\
\hline & & and naturalistic language interventions & Nordenström: B \\
\hline & & & Golper. II \\
\hline Diggle \& McConachie, 2009 & Review & Review of parent-mediated intervention/ & Schlosser: 3 \\
\hline \multirow[t]{2}{*}{ [68] } & Cochrane-report & training of children with ASD & Nordenström: B \\
\hline & & & Golper. III \\
\hline \multirow[t]{3}{*}{ Fernell et al, 2011 [77] } & Comparative group & Comparison of effects of 1 ) intensity and & Schlosser: 2 \\
\hline & study & form of intervention 2) intelligence on & Nordenström: B \\
\hline & & adaptive behaviour on children with ASD & Golper. II-III \\
\hline \multirow[t]{3}{*}{ Goldstein, 2002 [69] } & Review & Review and comparison of communication & Schlosser: 4 \\
\hline & & intervention to children with ASD & Nordenström: B \\
\hline & & & Golper. II \\
\hline \multirow[t]{3}{*}{ Kasari et al (2010) [31] } & RCT & Study of the outcomes of an intervention for & Schlosser: 1 \\
\hline & & joint attention & Nordenström: A \\
\hline & & & Golper: III \\
\hline Kasari, Paparella \& Freeman, & Randomized group & Comparison of interventions for play and & Schlosser: 2 \\
\hline \multirow[t]{2}{*}{$2008[70]$} & study & joint attention in children with ASD & Nordenström: A \\
\hline & & & Golper. III \\
\hline \multirow[t]{3}{*}{ McConkey et al, (2010) [74] } & Controlled group study & Evaluation of the impact of home-based & Schlosser: 2 \\
\hline & & intervention to promote communication & Nordenström: B \\
\hline & & & Golper. III \\
\hline \multirow[t]{3}{*}{ McConnell, 2002 [71] } & Review & Review of interventions to promote social & Schlosser: 4 \\
\hline & & interaction in young children in educational & Nordenström: B \\
\hline & & settings & Golper. I \\
\hline \multirow[t]{3}{*}{ Paul \& Roth , 2011 [78] } & Narrative review/ & Characterizing and Predicting Outcomes of & Schlosser: 6 \\
\hline & expertise & Communication Delays in Infants and & Nordenström: C \\
\hline & & Toddlers: Implications for Clinical Practice. & Golper. I \\
\hline \multirow[t]{3}{*}{ Rogers, 2006 [11] } & Review & Review of and historic description of & Schlosser: 6 \\
\hline & & communication intervention to young & Nordenström: C \\
\hline & & children with ASD & Golper. I \\
\hline \multirow[t]{3}{*}{ Schuit et al, 2011 [79] } & Controlled group study & Evaluation of a program aimed at stimulate & Schlosser: 5 \\
\hline & (small groups) & language learning in disabled children & Nordenström: B \\
\hline & & & Golper. III \\
\hline \multirow[t]{3}{*}{ Spreckley \& Boyd, 2009 [80] } & Meta-analysis & Meta-analysis of discrete-trial-interventions & Schlosser: 1 \\
\hline & & for children with ASD & Nordenström: A \\
\hline & & & Golper. III \\
\hline
\end{tabular}




\begin{tabular}{llll}
\hline Author\&year & Study design & Intervention & Evidence grading \\
\hline Vismara et al, 2009 [81] & $\begin{array}{l}\text { Group study Non- } \\
\text { concurrent multiple } \\
\text { baseline design }\end{array}$ & $\begin{array}{l}\text { Evaluation of the effects of "start-kit" of 12 } \\
\text { individual sessions teaching parents } \\
\text { communicative strategies }\end{array}$ & $\begin{array}{l}\text { Schlosser: } 2 \\
\text { Nordenström: B } \\
\end{array}$ \\
& RCT (small groups) & Evaluation of Autism 1-2-3-progam & Schlosser: 1 \\
\hline Wong \& Kwang, 2010 [75] & & Nordenström: B \\
& & & Golper. III \\
\hline Woods \& Wetherby, 2003 [72] & Review, clinical report & Review of methods of identification and & Schlosser: 4 \\
& & intervention for young children at risk of & Nordenström: C \\
& & ACD & Golper. II \\
\hline Yoder \& Stone, 2006 [73] & Randomized & Comparison of RPMT and PECS on spoken & Schlosser: 2 \\
& comparison & communication & Nordenström: B \\
& & & Golper. III \\
\hline
\end{tabular}

Table 3. Studies of direct interventions and comprehensive programs

As mentioned in earlier paragraphs generalization and maintenance has been a big issue within the field of communication and language intervention for years. Generally the childfocused interventions show better generalization and maintenance in younger children with ASD $[67,72,76]$. These studies discuss that the use of the inborn motivation of the child and the use of natural context and natural play context make the difference-all according to current theories of development of cognition and communication. Proponents of didactic training hold that the use of learned words and phrases might be a start of a positive social spiral where the child gets more response and is treated differently. Some reviews come to the conclusion that we still do not have enough evidence to tell which type of program is best, didactic or childfocused, but that the important factors seem to be early start and intensity [66, 69]. According to the meta-analysis of six RCT studies of didactic interventions [80] these however fail in reporting better outcomes than the control groups when it comes to cognition, language and adaptive functioning. Generally the children in didactic training programs also were older [68]. Didactic training in its intensive and comprehensive form seems less effective on younger children and children at early communicative levels [77]. The involvement of the parents in recurrent didactic training activities in the home is also questioned in some studies [68]. There are indications of a high degree of stress in these parents and a comparative study showed that parental stress was lowered when the training was done by others and furthermore that the results with respect to communication development was enhanced [68].

Several recent studies report outcomes from eclectic comprehensive interventions [30, 31, 74]. These programs are built upon current theories of cognitive, communicative and neurophysiological development but also adds knowledge from the behaviourist tradition or rather Applied Behavior Analysis (ABA) in optimizing the learning situation. More concretely this means that these programs are child-focused in that it makes use of the child's motivation and interests and focus the communication between the parent and the child and are often implemented in the home setting, sometimes after some introductory sessions on a clinic. An analysis of the child's communication development forms the decision of what is going to be focused during interaction. Prelinguistic competencies such as imitation, joint attention and 
use of symbol play and symbols are seen as basic and pivotal. The behavioural techniques are used to arrange the environment and chose strategies to refine and enhance learning in the natural interaction. The trainer serve as model to the parent and then guide and coach the parent, often in the home.

The majority of the primary studies in the table above report excellent outcomes [30, 3170,73 , $74,75,81]$. In general the research quality of evaluations of these interventions were high since many were of RCT type or Randomized Group studies. External, social and ecological validity was also considered as generally high partly due to the use of more interactional data and information from the stakeholders. The studies show that these interventions seem to be very effective in proving positive outcomes with respect to interaction, parental communication style and child development. Some of these intervention programs are of comparatively low intensity and short, which is interesting and important, as high intensity traditionally have been said to be essential to success in children with autism

Some articles compare interventions and discuss recommendations with respect to different needs of the child or family. A comparison of the AAC-method PECS and RPMT (a comprehenesive program containing parental education in the use of responsive strategies and training of the child and guidance to parents in their home) showed interesting results with respect to communication outcomes in the children [73]. The children at the earliest communicative stage, not yet being interested in objects, seem to develop more with RPMT. At the next communicative stage when the children has an interest of objects, an understanding of cause and effect and some emergent understanding of joint attention PECS is more effective. When joint attention is more established the Prelinguistic Mileu Teching strategies (behavioral techniques implemented in natural interaction) in the RPMT seems to be more operant. It was also seen that the PMT-training had better effect for those children whose mothers used a responsive communication style. The focus on development of joint attention is emphasized as the primary goal in this study with a successive introduction of symbol play as joint attention is being established [73].

Finally, one review studies the effect of different types of interventions to promote social interaction in pree-school settings and conclude that there is good evidence that it is important to work both with the child with disability as well as with his/her friends in the school environment [71].

\subsection{AAC intervention}

The field of AAC is a fairly new field of knowledge that has gradually grown as there is a increasing interest in functional communication and in ensuring the communicative rights of individuals with disability. There has also been an explosion of available communication technologies and methods that can support and improve communication for individuals with autism. We have probably and hopefully only seen the dawn of these new options. It is also possible to see that we are moving from using one technique or approach at the time to working with multimodal techniques or approaches where different tools and methods combined with an understanding of communication and use of interactional strategies build a total system of communication. 


\begin{tabular}{|c|c|c|c|}
\hline Author\&year & Study design & Intervention & Evidence grading \\
\hline Binger, Berens, Kent-Walsh \&Taylor, & Review & The impact of AAC-intervention on use & Schlosser: 4 \\
\hline \multirow[t]{2}{*}{2008 [82] } & & of AAC, symbolic gestures and speech & Nordenström: B \\
\hline & & & Golper. II \\
\hline \multirow[t]{4}{*}{ Bopp, Brown \& Mirenda, 2004 [83] } & Review & Review of FCT and use of visual & Schlosser: 4 \\
\hline & & strategies and discussion of the role of & Nordenström: B \\
\hline & & speech-language pathologist in & Golper. I \\
\hline & & working with challenging behaviors & \\
\hline \multirow[t]{3}{*}{ Brady, 2000 [84] } & Case study & Study of the impact of use of SGDs on & Schlosser: 5 \\
\hline & & the understanding of speech & Nordenström: C \\
\hline & & & Golper. I \\
\hline \multirow[t]{3}{*}{ Branson \& Demcak, 2009 [85] } & Meta-analysis & Evaluation of AAC interventions for & Schlosser: 1 \\
\hline & & toddlers and infants with disability & Nordenström: A \\
\hline & & & Golper. III \\
\hline Ganz, Simpson \& Corbin-Newsome, & SSRD - Multiple & Impact of PECS on requesting and & Schlosser: 2 \\
\hline \multirow[t]{2}{*}{2008 [86] } & baseline & speech & Nordenström: B \\
\hline & & & Golper. III \\
\hline \multirow[t]{3}{*}{ Mancil et al(2009) [95] } & SSRD - multiple & Study of the effects of a picture- & Schlosser: 2 \\
\hline & baseline & exhange-intervention using milieu & Nordenström: B \\
\hline & & teaching in the home & Golper. III \\
\hline \multirow[t]{3}{*}{ Millar, Light \& Schlosser 2006 [87] } & Meta-analysis & The impact of AAC-interventions on & Schlosser: 1 \\
\hline & & speech development & Nordenström: B \\
\hline & & & Golper. I \\
\hline \multirow[t]{3}{*}{ Papparella \& Kasari, 2004 [88] } & Review & Study of the relationship between joint & Schlosser:4 \\
\hline & & attention and language - manual & Nordenström: C \\
\hline & & signing & Golper. I \\
\hline \multirow[t]{3}{*}{ Preston \& Carter, 2009 [89] } & Meta-analysis & Study of the effects of PECS & Schlosser: 1 \\
\hline & & & Nordenström: B \\
\hline & & & Golper. III \\
\hline \multirow[t]{3}{*}{ Schlosser \&, 2006 [90] } & Quantitative review & The impact of AAC on children with & Schlosser: 4 \\
\hline & & developmental disabilites & Nordenström: B \\
\hline & & & Golper. II \\
\hline \multirow[t]{3}{*}{ Schlosser \& Wendt, 2008 [91] } & Meta-analysis & Effects of different types of AAC on & Schlosser: 1 \\
\hline & & speech in children with ASD & Nordenström: B \\
\hline & & & Golper: II \\
\hline \multirow{3}{*}{$\begin{array}{l}\text { Sigafoos, Drasgow, Reichle, O'Reilly \& } \\
\text { Tait, } 2004 \text { [92] }\end{array}$} & Review & Study of the effectiveness of training of & Schlosser: 4 \\
\hline & & rejecting using $A A C$ & Nordenström: B \\
\hline & & & Golper. I \\
\hline Snell, Chen, Lih-Huan \& Hoover, 2006 & Review & Review of AAC-interventions to children & Schlosser: 4 \\
\hline \multirow[t]{2}{*}{ [94] } & & with severe communicative disabilities & Nordenström: B \\
\hline & & & Golper. I \\
\hline \multirow[t]{3}{*}{ van der Meer et al, 2011 [93] } & Controlled group & Evaluation of a program using AAC (not & Schlosser: 5 \\
\hline & study & further described) & Nordenström: B \\
\hline & & & Golper. II \\
\hline
\end{tabular}

Table 4. Studies of education and guidance to parents 
The research base with respect to AAC used by young children with autism has grown in recent years. This research mostly consists of singe-subject-design studies and case studies, with very few controlled group studies being done. On the other hand there are some welldone meta-analyses published that compile results from singe-subject research studies. Due to the difficulties of conducting RCT studies within the field of AAC-intervention the metaanlyses are important and can be seen as the golden standard. In total 14 studies were identified as focusing the use of $\mathrm{AAC}$ and of these 10 were reviews or meta-analyses.

In conclusion, meta-analyses and other studies show that AAC-interventions are cost-effective and give fast results and furthermore tend to stimulate speech development $[82,84,85,86,87$, 91]. The best results seem to be reached when the social network surrounding a child is given support and resources, to be able to use responsive strategies and provide communication opportunities and direct training using AAC in natural daily interactions. AAC intervention should be started as soon as communication difficulties are displayed or suspected since AAC promotes communication, language and speech. AAC-intervention has also been proved to effectively decrease challenging behaviour [83]. There is today no mode of AAC that is known to be better than any other for young children with autism. Instead multimodal approaches seem to be the most effective [93]. However, graphic AAC seem to be acquired at a faster rate and also easier to generalize to other situations [90]. PECS has been proved to be an effective AAC method, specifically at early stages of communication and with respect to the first three phases of the method [89].

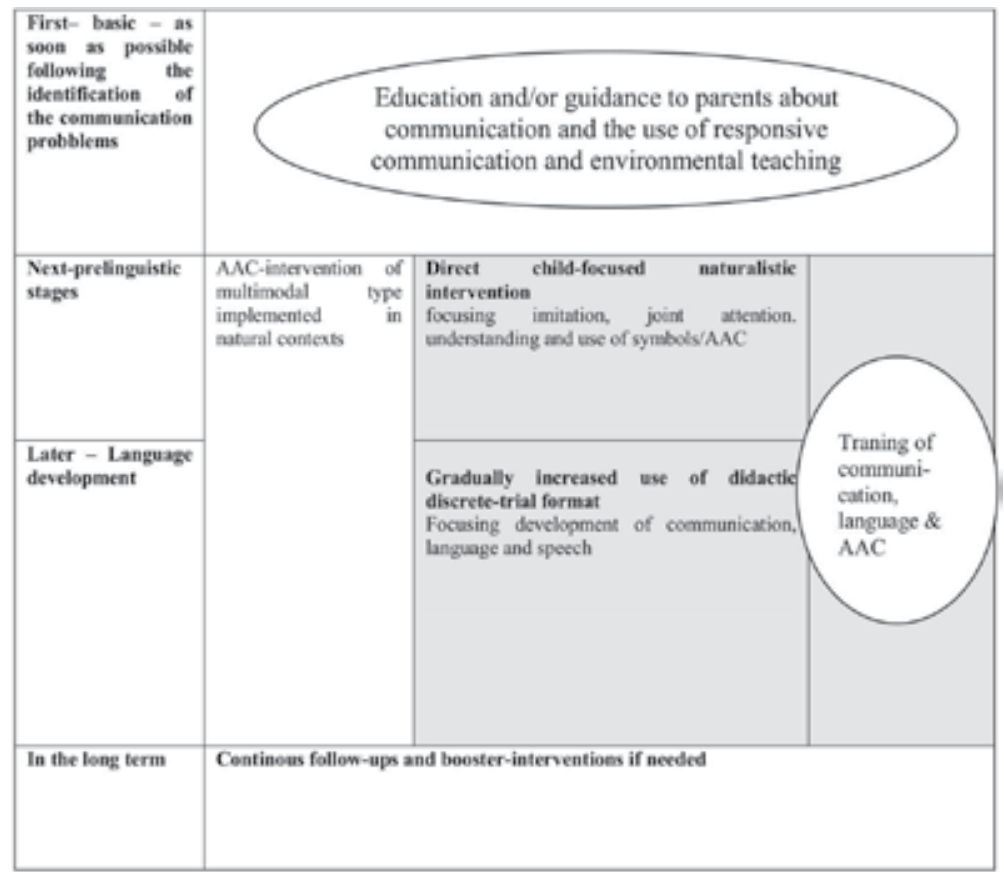

Figure 1. Model for early communication intervention 


\section{Conclusion}

The conclusion of this chapter is presented in the form of eight recommendations and of a model for early communication intervention answering the question that was initially formulated in this study: "A young child with autism and severe communicative disability, living with his/her parents and being placed in a pree-school group: which intervention is most effective; indirect or direct interventions?"

1. A combination of indirect and direct interventions. There is strong evidence that the combination of education and guidance to the parents and direct child-focused intervention to the child in a naturalistic context leads to good outcomes with respect to several parameters such as: development of communication and language, interaction between the parent and the child where the parent uses a responsive communication pattern,

2. Parental education should include knowledge of and training in the use of responsive strategies and behavioural/environmental teaching techniques within the frames of natural interaction in the home. Several studies show that parents change their communicative style after a few education sessions and that this positively affect the interaction pattern with the child and enhance language development in the child. Guidance or coaching of the parents in natural interactions in the home environment is included in most of the recently presented studies and show very good results in short time.

3. Direct interventions provided to children on early communicative stage should be child-focused and implemented in daily natural interactions. The intervention should focus imitation, joint attention and symbol use (speech, symbols, manual signs). Didactic intervention is not effective for young children since maintenance and generalization of training is low.

4. Interventions need to be continuous and include follow-ups and possibilities of booster-intervention. The few long-term follow up-studies all show that interventions (of different type) tend to wear off by time. The recent published studies show that lowintensity interventions also could yield good results. The engagement of the parent also might be an important success factor.

5. Children at early communicative stages should be provided with AAC as early as possible. There is no age-limit or prerequisites that need to met before AAC is introduced. There is strong evidence that AAC decreases challenging behaviour. There is moderate to strong evidence that AAC facilitates development of speech.

6. AAC-intervention should ideally be multimodal. All modes of AAC are effective. There is some evidence that symbols (specifically combined with speech output) are learned faster than manual signs and that iconic symbols are learned faster.

7. PECS (Picture Exchange Communication System) is an effective AAC-method for children at early communicative stages. There is strong evidence that PECS has a positive effect on interaction and behaviour and that functional communication is increased. 
8. The AAC-modes should be used and modelled by the child's communication partners (aided language stimulation or modelling) to promote learning and spontaneous use of the symbols.

These recommendations means that the child in our formulated question should be provided with intervention according to the model below.

\section{Acknowledgements}

Parts of chapter two, three and four was first published in the author's thesis [96]. Thanks to my collegues in the ebp-group: Lena Nilsson, Maria Nolemo, Barbara Eberhart, Jessica Forsberg, Ruth Breivik and Anna Fäldt.

\section{Author details}

Gunilla Thunberg*

DART - Centre for AAC and Assistive Technology, Sahlgrenska University Hospital, Sweden

\section{References}

[1] Short C, Schopler E. Factors relating to age of onset in autism. Journal of Autism and Developmental Disorders $1988 ; 18$ 207-216.

[2] Carr EG, Levin L, McConnachie G, Carlson JI, Kemp DC, Smith CE. Communicationbased intervention for problem behavior. Baltimore, MD: Paul H. Brookes Publishing; 1997.

[3] Billstedt E. Children with autism grow up: Use of the DISCO (Diagnostic Interview for Social and COmmunication disorders) in population cohorts. Göteborg: Göteborg University; 2007.

[4] Billstedt E, Gillberg IC, Gillberg C. Autism after adolescence: Population-based 13-22-year follow-up study of 120 individuals with autism diagnosed in childhood. Journal of Autism and Developmental Disorders 2005 ; 35, 351-360.

[5] Howlin P, Goode S, Hutton J, Rutter M. Adult outcomes for children with autism. Journal of Child Psychology and Psychiatry, 2004; 45, 212-229.

[6] Shea V, Mesibov G. Adolescents and adults with autism. In F. Volkmar, R. Paul, A. Klin \& D. Cohen (Eds.), Handbook of autism and pervasive developmental disorders (pp. 288-311). Hoboken, NJ: John Wiley \& Sons; 2005. 
[7] Bryson S. Brief report: Epidemiology of autism. Journal of Autism and Developmental Disorders 1996; 26, 165-167.

[8] Bryson S, Clark BS, Smith TM. First report of a Canadian epidemiological study of autistic syndromes. Journal of Child Psychology and Psychiatry 1988; 29, 433-445.

[9] Lord C, Risi S, Pickles. Trajectory of language development in autism spectrum disorders. In R. M \& S. Warren (Eds.), Developmental language disorders: From phenotypes to etiologies (pp. 7-29). Mahwah, NJ: Lawrence Erlbaum; 2004.

[10] Tager-Flusberg H., Joseph RM. Identifying neurocognitive phenotypes in autism. Philosophical Transactions of the Royal Society of London, Series B: Biological Sciences 2003; 358, 303-314.

[11] Rogers S. Evidence-based interventions for language development in young children with autism. In T. Charman \& W. Stone (Eds.) Social and communication development in autism spectrum disorders (pp. 143-179). New York: The Guildford Press ; 2006.

[12] Tager-Flusberg H, Paul R, Lord C. Language and communication in autism. In F. Volkmar, R. Paul, A. Klin \& D. Cohen (Eds.) Handbook of autism and pervasive developmental disorders (Vol. 1, pp. 335-364). Hoboken, NJ: John Wiley \& Sons ; 2005.

[13] Wetherby A M, Prizant BM, Schuler AL. Understanding the nature of communication and language impairments. In AM Wetherby \& BM Prizant (Eds.), Autism spectrum disorders: A transactional developmental perspective pp. 109-141. Baltimore, MD: Paul H. Brookes Publishing; 2000.

[14] Tager-Flusberg H, Joseph R, Folstein S. Current directions in research on autism. Mental Retardation and Developmental Disabilities Research Reviews 2001; 7, 21-29.

[15] Wetherby AM. Understanding and measuring social communication in children with autism spectrum disorders. In T. Charman \& W. Stone (Eds.), Social and communication development in autism spectrum disorders (pp. 3-34). New York: The Guildford Press: 2006.

[16] Charman T, Baron-Cohen S, Swettenham J, Baird G, Drew A, Cox A. Predicting language outcome in infants with autism and pervasive developmental disorder. International Journal of Language and Communication Disorders 2003; 38, 265-285.

[17] Mundy P, Sigman M, Kasari C. A longitudinal study of joint attention and language development in autistic children. Journal of Speech and Hearing Research 1990; 38, 157-167.

[18] Dahlgren SO, Gillberg C. Symptoms in the first two years of life: A preliminary population study of infantile autism. European Archives of Psychiatric and Neurological Science 1989; 283, 169-174. 
[19] Osterling J, Dawson G. Early recognition of children with autism: A study of first birthday home videotapes. Journal of Autism and Developmental Disorders 1994; 24, 247-258.

[20] Watson LR, Baranek GT, Crais ER, Reznick SJ, Dykstra J, Perryman T. The first year inventory: Retrospective parent responses to a questionnaire designed to identify one-year olds at risk for autism. Journal of Autism and Developmental Disorders 2007: 37, 49-61.

[21] Chawarska K, Paul R, Klin A, Hannigen S, Dichtel L E, Volkmar F. Parental recognition of developmental problems in toddlers with autism spectrum disorders. Journal of Autism and Developmental Disorders 2007; 37, 62-72.

[22] Lord C, Schulman C, DiLavore P. Regression and word loss in autistic spectrum disorders. Journal of Child Psychology and Psychiatry 2004; 45, 936-955.

[23] Wetherby AM. Ontogeny of communicative functions in autism. Journal of Autism and Developmental Disorders 1986; 16, 295-316.

[24] Ramberg C, Ehlers S, Nydén A, Johansson M, Gillberg C. Language and pragmatic functions in school-age children on the autism spectrum. European Journal of Disorders of Communication 1996; 31, 387-413.

[25] Bishop D, Hartley J, Weir F. Why and when do some language-impaired children seem talkative? A study of initiation in conversation of children with semantic-pragmatic disorders. Journal of Autism and Developmental Disorders 1994 ; 24, 177-197.

[26] Lovaas OI, Koegel RL, Simmons JQ, Long JS. Some generalization and follow-up measures on autistic children in behaviour therapy. Journal of Applied Behavior Analysis 1973; 6, 131-166.

[27] Hart BM, Risley TR. Establishing use of descriptive adjectives in the spontaneous speech of disadvantaged preschool children. Journal of Applied Behavior Analysis 1968; 1, 109-120.

[28] Prizant BM, Wetherby AM, Rydell P. Communication intervention issues for children with autism spectrum disorders. In A. M. Wetherby \& B. M. Prizant (Eds.), Autism spectrum disorders: A transactional developmental perspective. Baltimore, MD: Paul H. Brookes Publishing; 2000.

[29] Hancock TB, Kaiser AP. The effects of trainer-implemented enhanced milieu-teaching on the social communication of children with autism. Topics in Early Childhood Special Education 2002; 22, 29-54.

[30] Dawson G, Rogers S, Munson J, Smith M, Winter J, Greenson J, Donaldson A, Varley J. Randomized, Controlled Trial of an Intervention for Toddlers With Autism: The Early Start Denver Model. Pediatrics 2010; 125; e17. 
[31] Kasari C, Gulsrud AC, Wong C, Kwon S, Locke J. Randomized controlled caregiver mediated joint engagement intervention for toddlers with autism. Journal of Autism and Developmental disorders 2010; 40, 1045-1056.

[32] Drew A, Baird G, Baron-Cohen S, Cox A, Slonims V, Wheelwright S, Swettenham J, Berry B, Charman T. A pilot randomised control trial of a parent training intervention for pre-school children with autism: preliminary findings and methodological challenges. European Child \& Adolescent Psychiatry 2002; 11(6) s. 266-272.

[33] Dahlgren S, Dahlgren-Sandberg A. The non-specificity of theory of mind deficits: Evidence from children with communicative disabilities. European Journal of Cognitive psychology 2004; 15, 129-155.

[34] Ello LM, Donovan SJ. Assessment of the relationship between parenting stress and a child's ability to functionally communicate. Research on Social Work Practice 2005;15(6), 531-544.

[35] Wilder J, Granlund M. Behaviour style and interaction between seven children with multiple disabilities and their caregivers. Child: care, health and development 2003; 29, 559-567.

[36] Warren S, Brady N, Sterling A, Fleming K, Marquis J. Maternal responsivity predicts language development in young children with fragile $\mathrm{X}$ syndrome. American Journal on Intellectual Disabilities 2010;115 (1), 54-75.

[37] Siller M, Sigman M. The behaviors of parents of children with autism predict the subsequent development of their children's communication. Journal of Autism and Developmental Disorders 2002; 32, 77-89.

[38] Hanen centre. http://www.hanen.org (accessed 23 October 2012).

[39] AKKtiv. http://www.akktiv.se (accessed 23 October 2012).

[40] Jonsson A, Kristoffersson L, Ferm U, Thunberg G. The ComAlong communication boards: Parents' use and experiences of aided language stimulation. Augmentative and Alternative Communication 2011; 27 (2), 103-116.

[41] Howlin P. Augmentative and alternative communication systems for children with autism. InT. Charman \& W. Stone (Eds.), Social and communication development in autism spectrum disorders (pp. 236-266). New York: The Guildford Press: 2006.

[42] Mirenda P, Erickson KA. Augmentative communication and literacy. In B. M. Prizant \& A. M. Wetherby (Eds.), Autism spectrum disorders: A transactional developmental perspective (pp. 369-394). Baltimore, MD: Paul H. Brookes Publishing. 2000.

[43] Yoder PJ, Layton TL. Speech following sign language training in autistic children with minimal verbal language. Journal of Autism and Developmental Disorders 1988: 18, 217-230. 
[44] Seal BC, Bonvillian JD. Sign language and motor functioning in students with autistic disorders. Journal of Autism and Developmental Disorders 1997; 27, 437-466.

[45] Schopler E, Reichler R, Lansing M. Teaching strategies for parents and professionals. Austin, TX: PRO-ED Inc: 1980.

[46] Danielsson H, Jönsson, B. Pictures as language. Paper presented at the International Conference on Language and Visualisation, Stockholm: 2001.

[47] Bondy A, Frost L. The Picture Exchange Communication System. Focus on Autistic Behavior, 1994: 9, 1-19.

[48] Schlosser RW, Blischak DM. Is there a role for speech output in interventions for persons with autism. Focus on Autism and Other Developmental Disabilities 2001;16, 170-176.

[49] Schlosser RW. The Efficacy of Augmentative and Alternative Communication: Towards Evidence-Based Practice. Baltimore: Paul Brookes: 2003.

[50] Schlosser RW, Raghavendra P. Evidence-Based Practice in Augmentative andAlternative Communication. Augmentative and Alternative Communication 2004; 20, $1-21$.

[51] Nordenström J. Evidensbaserad medicin: I Sherlock Holmes fotspår. Stockholm: Karolinska University Press; 2006.

[52] Golper LAC, Wertz RT, Frattali CM, Yorkston K, Myers P, Katz.R, Beeson P, Kennedy MRT, Bayles K, Wambaugh J. Evidence-based practice guidelines for the management of communication disorders inneurologically impaired individuals: Project introduction. ANCDS: 2001.

[53] Sveriges habiliteringschefer [Swedish habilitation directors]. http://www.sverigeshabiliteringschefer.se (accessed 23 october 2012).

[54] Aldred C, Green J, Adams C. A new social intervention for children with autism: pilot randomised controlled treatment study suggesting effectiveness. Journal of Child Psychology and Psychiatry 2004;45(1) 1420-1430.

[55] Ferm U, Andersson M, Broberg M, Liljegren T, Thunberg G. Parents and course leaders' experiences of the ComAlong augmentative and alternative communication early intervention course. Disability Studies Quarterly: Mediated Communication 2011; 31(4) http://dsq-sds.org

[56] Callenberg A, Ganebratt P. Utvärdering av AKKtiv föräldrautbildning: föräldrars bedömningar av barnens kommunikativa utveckling. [Evaluation of ComAlong parental course: parents' views and development in children]. Unpublished masters' thesis in Speech-language Pathology, University of Gothenburg: Department of Neuroscience and Physiology, Gothenburg: 2009. 
[57] Elder JH, Valcante G, Yarandi H, White D, Elder TH. Evaluating In-Home Training for Fathers of Children With Autism Using Single-Subject Experimentation and Group Analysis Methods. Nursing Research 2009; vol 54 no 1.

[58] Girolametto L, Sussman F, Weitzmann E. Using case study methods to investigate the effects of interactive intervention for children with autism spectrum disorders. Journal of Communication Disorders 2007; 40, 470-492.

[59] Howlin P, Gordon K, Pasco G, Wade A, Charman T. The effectiveness of Picture Exchange Communication System (PECS) training for teachers of children with autism: a pragmatic, group randomised controlled trial. J Child Psychol Psychiatry. 2007; 48(5):473-81.

[60] Lennartsson E, Sörensson K. Föräldrars sätt att kommunicera med sina barn före och efter KomIgång kommunikationskurs. [Parental communication style before and after ComAlong parental course]. Unpublished masters' thesis in Speech-language Pathology, University of Gothenburg: Department of Neuroscience and Physiology, Gothenburg: 2010.

[61] McConachie H, Randle V, Hammal D, Le Couteur A. A controlled trial of training course for parents of children with suspected autism spectrum disorders. The Journal of Pediatrics 2005; 14, 335-40.

[62] Karlsson E, Melltorp M. Utvärdering av AKKtiv: Tidig intervention till föräldrar som har barn med omfattande kommunikationssvårigheter. [Evaluation of ComAlong: Early intervention to parents of children with communicative disability]. Unpublished masters' thesis in Speech-language Pathology, University of Gothenburg: Department of Neuroscience and Physiology, Gothenburg: 2006.

[63] Oosterling I, Visser J, Swinkels S, Rommelse N, Donders, R, Woudenberg T, Roos S, van der Gaag RJ, Biotelaar J. Randomized controlled trial of the Focus parent training for toddlers with autism: 1-year outcome. Journal of Autism and Developmental Disorders 2010; 40, 1447-1458.

[64] Seung HK, Ashwell S, Elder JH, Valcante G. (2006). Verbal communication outcomes in children with autism after in-home father training. Journal of Intellectual Disability Research, 2, (50), 139-150.

[65] Sharry J, Guerin S, Griffin C, Drumm, M. (2005). An evaluation of the Parents Plus Early Years Programme: A video-based early intervention for parents of pre-school children with behavioural and developmental difficulties. Clinical Child Psychology and Psychiatry, 10, 319.

[66] Corsello CM. Early Intervention in Autism. Infants \& Young Children 2005; 18(2) 74-85. 
[67] Delprato, D. J., (2001) Comparisons of Discrete-Trial and Normalized Behavioral Language Intervention for Young Children with Autism. Journal of Autism and Developmental Disorders, 31, 315-325.

[68] Diggle TTJ, McConachie HHR. Parent-mediated early intervention for young children with autism spectrum disorder (Review). The Cochrane Library 2009, Issue 4.

[69] Goldstein H. Communication Intervention for Children with Autism: A Review of Treatment Efficacy. Journal of Autism and Development Disorders 2002; 32(5), 373-396.

[70] Kasari C, Papparella T, Freeman S. Language Outcome in Autism: Randomized Comparison of Joint Attention and Play Interventions. Journal of Consulting and Clinical Psychology 2008; 76(1) 125-137.

[71] McConnell S. Intervention to Facilitate Social Interaction for Young Children with Autism: Review of Available Research and Recommendations for Educational Intervention and Future Research. Journal of Autism and Developmental Disorders 2002; 32(5) 351-372.

[72] Woods J, Wetherby A. Early Indentification of and Intervention for Infant and Toddlers Who Are at Risk for Autism Spectrum Disorder, Language, Speech and Hearing in Schools, 2003; 34 180-193.

[73] Yoder P. Stone WL. A Randomized Comparison of the Effect of Two Prelinguistic Communication Interventions on the Acquisition of Spoken Communication in Preschoolers With ASD. Journal of Speech, Language and Hearing Research 2006; 49 698-771.

[74] McConkey R, Truesdale-Kennedy M, Crawford H, McGreevy E, Reavey M, Cassidy A. Preeschoolers with autism spectrum disorders: evaluating the impact of homebased intervention to promote their communication. Early Child Development and Care 2010; 180(3) 299-315.

[75] Wong VCN, Kwan QK. Randomized Controlled Trial for Early Intervention for Autism: A Pilot Study of the Autism 1-2-3 Project. Journal of Developmental Disorder 2010; 40 677-688.

[76] Charman, T. (2010). Developmental Approaches to Understanding and Treating Autism. Folia Phoniatrica et Logopaedica, 62, 166-177.

[77] Fernell E, Hedvall Å, Westerlund J, Höglund Carlsson L, Eriksson M, Barnevik Olsson M, Holm A, Norrelgen F, Kjellmer L, Gillberg C. Early Intervention in 208 Swedish preschoolers with autism spectrum disorder. A prospective naturalistic study Research in developmental Disabilities 2011; 32 2092-2101.

[78] Paul R, Roth FP. Characterizing and Predicting Outcomes of Communication Delays in Infants and Toddlers: Implications for Clinical Practice. Language, Speech, and Hearing Services in Schools, 42, 331-340. 
[79] Van der Schuit M, Segers E, Van Balkom H. Verhoeven L. Early language intervention for children with intellectual disabilities: A neurocognitive perspective. Research in Developmental Disabilities 2011; 32 705-712.

[80] Spreckley M, Boyd R. Efficacy of Applied Behavioural intervention in preschool children with autism for improving cognitive, language and adaptive behaviour. A systematic review and meta-analysis. J Pediatr 2009; 154 338-344.

[81] Vismara LA, Colombi C, Rogers SJ. Can one hour per week of therapy lead to lasting changes in young children with autism? Autism 2009; 13(1) 93-115.

[82] Binger C, Berens J, Kent-Walsh J, Taylor S. The Effects of Aided AAC Interventions on AAC Use, Speech, and Symbolic Gestures. Seminars och Speech and language 2008; 29 101-111.

[83] Bopp K, Brown K. Mirenda P. Speech-Language Pathologists' Roles in the Delivery of Positive Behaviour Support for Individuals With Developmental Disabilities. American Journal of Speech-Language Pathology 2004; 13 5-19.

[84] Brady NC. (2000). Improved Comprehension of Object Names Following Voice Output Communication Aid Use: Two case Studies. Augmentative and Alternative Communication, 16, 197-204.

[85] Branson D Demchak M. The use of Augmentative and Alternative Communication Methods with Infants and Toddlers with Disabilities: A Research Review. Augmentative and Alternative Communication 2009; 25 274-286.

[86] Ganz JB, Simpson RL, Corbin-Newsome J. The impact of the Picture Excange Communication System om requesting and speech development in preschoolers with autism spectrum disorders and similar characteristics. Research in Autism Spectrum Disorders 2008; 2 157-169.

[87] Millar DC, Light JC Schlosser RW. The Impact of Augmentative and Alternative Communication Intervention on the Speech Production of Individuals with Developmental Disabilities: A Research Review. Journal of Speech, Language and Hearing Research 2006; 49 248-264.

[88] Papparella T. Kasari C. Joint Attention Skills and Language Development in Special Needs Populations Translating Research to Practice. Infants and Young Children 2004; 17(3) 269-280.

[89] Preston D. Carter M. A Review of the Efficacy of the Picture Exchange Communication System Intervention. Journal of Autism and Developmental Disorders 2009; 39 1471-1486.

[90] Schlosser R. Sigafoos J. Augmentative and Alternative communication interventions for persons with developmental disabilities: narrative review of comparative singlesubject experimental studies Research in Developmental Disabilities 2006; 27 1-29. 
[91] Schlosser R. Wendt O. Effects of Augmentative and Alternative Communication Intervention on Speech Production in Children With Autism: A Systematic Review. American Journal of Speech-Language Pathology 2008; 17 212-223.

[92] Sigafoos J, Drasgow E, Reichle J, O’Reilly M, Green V, Tait K. Forum on Intervention Strategies for Severe Disabilities Tutorial: Teaching Communicative Rejecting to Children With Severe Disabilities. American Journal of Speech-Language Pathology 2004; 13 31-42

[93] van der Meer L, Sigafoos J, O'Reilly M. Lancioni, G. Assessing preferences for AAC options in communication interventions for individuals with developmental disabilities: A review of the literature. Research in Developmental Disabilities 2011; 32 14221431.

[94] Snell M, Chen L-Y \& Hoover K. Teaching Augmentative and Alternative Communication to Students With Severe Disabilities: A Review of Intervention Research 1997-2003. Research and Practice for Persons with Severe Disabilities 2006; 3, 203-214.

[95] Mancil RG, Conroy AC, Haydon TF. Effects of a Modified Milieu Therapy Intervention on the Social Communicative Behaviours of Young Children with Autism Spectrum Disorders. Journal of Autism and Developmental Disorders 2009; 39 149-163.

[96] Thunberg, G. Using speech-generating devices at home. A study of children with autism spectrum disorders at different stages of communication development. Gothenburg Monographs in Linguistics 34. Göteborg, Sweden: Göteborg University: 2007. 


\section{Section 8}

Aetiological Factors - The Autistic Self and Creativity 

Chapter 31

\title{
Atypical Sense of Self in Autism Spectrum Disorders: A Neuro-Cognitive Perspective
}

\author{
Viktoria Lyons and Michael Fitzgerald \\ Additional information is available at the end of the chapter \\ http://dx.doi.org/10.5772/53680
}

\section{Introduction}

\subsection{Self - Definitions and concepts}

The concept of self is notoriously difficult to define and different notions and theories of the self have been proposed by a variety of disciplines all interpreting concepts of self and identity in various ways. We adopt the definition advanced by neuroscientists Kircher and David [1] who interpret the self as 'the commonly shared experience, that we know we are the same person across time, that we are the author of our thoughts/actions, and that we are distinct from the environment' (p.2). In cognitive neuroscience literature, operational definitions of the self are used which are measurable by experimental methods including self recognition, self and other differentiation, body awareness, awareness of other minds, awareness of self as expressed in language and important concepts such as autobiographical memory and self narrative. There is significant interest in the role of the self and possible abnormalities associated with the self, as causally implicated in autism. In this paper we review developmental perspectives of self and self-related functions with reference to their neuroanatomical basis and investigate the possible causes for atypical self-development in Autism Spectrum Disorders (ASD).

\section{Review of studies investigating the self in ASD}

The question whether individuals with ASD have a different sense of self than people without ASD, i.e. 'neurotypicals' has fascinated researchers and clinicians for decades. Kanner [2] in 1943 already noted self-deficits in the children he described, including their difficulties in maintaining a constant self-concept, and associated problems of adapting their fragile 
self-concept to changing environments. Asperger [3] (1974, p. 2026) refers to a disturbance and a weakness of the self in children with 'autistic psychopathy'. Psychoanalytic theories considered autism as a disorder of the self [4] based on the lack of ability in establishing stable internal representations of themselves and of others. The German psychiatrist Lutz [5] (1968) interpreted autism as a disturbance of self-consciousness, self-related activities and self-perception. Powell and Jordan [6] (1993) suggested that individuals with ASD lack an 'experiencing self' that provides a personal dimension for ongoing events. This is consistent with Frith's [7] suggestion of an 'absent self' in autism. Hobson [8] put forward a developmental account of the self in autism emphasising impaired interpersonal relatedness, 'intersubjectivity' and its far-reaching consequences for the development of the self. In a recent study investigating whether children with autism show abnormal self-other connectedness, Hobson and Meyer [9] found a failure in autism to identify with another person. These authors suggest that this process of identifying with others is crucial for a normal development of self-other relationship and the basis for understanding other minds.

\section{Atypical development of self-related processes in ASD}

There is general consensus among developmental theorists [e.g. 10,11] that early interpersonal communication is central to the establishment of the self in normal development. A large body of research literature indicates that early processes underlying self-other awareness are impaired or delayed in autism including gaze following, abnormal response to sound and deficits in attention [12], showing of objects, responding and orienting to own name [13], looking at other's faces [14], pretend play, protodeclarative pointing and gaze monitoring [15], empathy and imitation [16], joint-attention behaviour [17], affect and personal relatedness [18,19].

\subsection{Self recognition and awareness}

The ability to recognize one's own face in the mirror is considered a test for 'self-awareness'. Self-recognition as measured by mirror tests [20] in 18 months old children has been depicted as a developmental milestone in self-conception and described as the 'achievement of a cognitive self' [21]. Not only is self recognition essential for developing a concept of self, and self-other differentiation, it is also a prerequisite for later developing theory of mind abilities, as a stable self concept is the basis for being able to read the mental states of others. Research data on self-recognition reveals that autistic children's responses to their mirror images are qualitatively different from those of normal children [22]. Children with ASD have deficits of self-awareness as measured by a self-recognition test [23] they show little interest in their own mirror images and have been described as relatively 'face inexperienced' [24].

The prefrontal cortex, especially the Right Hemisphere $(\mathrm{RH})$ plays a critical role in the recognition of one's own face [25] as evidenced by functional imaging studies. Face perception studies in individuals with ASD suggest abnormal functioning in the fusiform 
face area as well as amygdala, brain regions associated with the 'social brain' (e.g. 26,27] involving the $\mathrm{RH}$.

\subsection{Self/other differentiation}

The ability to differentiate between self and other is also essential for the development of self-awareness, which appears to be impaired in autism. In particular, the recognition of a separate existence of other people seems to be delayed in children with autism [28,29]. Attentional abnormalities, such as 'tunnel vision', the tendency to think in a monotropic manner have been suggested by some as the cause of 'self-other problems' in ASD [30]. Donna Williams [31] interprets monoprocessing as the inability to process simultaneously information of oneself and others.

In neurotypicals the middle cingulate cortex and ventromedial prefrontal cortex are involved in self/other processing. In contrast, atypical neural responses have been reported in individuals with ASD. A recent fMRI study [32] investigating the attribution of behavioural outcomes to either oneself or others while playing an interactive trust game revealed a lack of brain activity in the cingulate cortex indicating diminished 'self responses' in individuals with ASD. However, 'other responses', attributing actions to other people were intact. Previous research data using trust games [33] had demonstrated that cingulate cortex activation is consistent with self-response patterns generated during interpersonal exchanges. Chiu et al. [34] interpreted their data in terms of a deficit in ASD in monitoring their own intentions in social interactions and thus contributing to impaired theory of mind abilities, lack of introspection and self-referential processing. Of particular interest is the fact that the 'impaired self-responses' in the ASD group correlated with their behavioural symptom severity, i.e. the lesser activity along cingulated cortex the more serious were their behavioural symptoms. Similar results have been reported by Lombardo et al. [35] also demonstrating atypical neural responses from the middle cingulate cortex during a self-referential processing task. This study also provided a link between these deficits and early social impairments in autism. In addition, these authors also demonstrated reduced functional connectivity between ventromedial prefrontal cortex and lower level regions (e.g. somatosensory cortex) in individuals with ASD during these self- representation tasks.

Previous studies identified the right inferior parietal lobe, along with frontopolar and somatosensory regions $[36,37]$ as critical for distinguishing between self and other. Additional data [e.g. 38] demonstrate that SI and SII cortices, which contribute to the mirror-neuron system, are also crucial for preserving a sense of self.

\subsection{Body awareness, sense of agency}

Knowing oneself and knowing one's body are closely related concepts. In his review on body image and the self, Goldenberg [39] argues that the acquisition of body image is not innate but acquired through experiences of one's own and other bodies. Likewise, Jordan and Powell [40] believe that a body concept develops from interacting with others. Anecdotal reports indicate that some children and adults with autism have an insecure body image 
or totally lack body awareness. Russell [41] suggested that the 'body schemas of persons with autism are poorly specified' resulting in an atypical experience of agency. A sense of agency is a central aspect of human self-consciousness and refers to the experience of oneself as the agent of ones actions. Based on his executive function account of impaired action monitoring Russell [42] put forward the hypothesis that individuals with autism are impaired in distinguishing between self and others. In contrast, a recent study by Williams and Happè [43] suggests that individuals with ASD are aware of their agency as indicated by their ability to monitor their own actions.

Support for a dominant role of the right hemisphere in the above processes is substantial. The right posterior parietal lobe is generally associated with spatial and bodily awareness $[44,45]$. Activation of right inferior parietal lobe correlates with a sense of ownership in action execution [46]. Additional research evidence [47] based on transcranial magnetic stimulation (TMS) supports the significance of the right temporo-parietal junction in the maintenance of a coherent sense of one's body.

\subsection{Theory of mind, emotions and self-awareness}

An essential component of self-awareness is the ability to be aware of other minds. A multitude of studies have provided evidence that theory of mind is lacking or delayed in ASD, or develops differently than in neurotypicals [for a substantive review see 48]. Deficits in mindreading may also affect the ability to reflect on one's own mental states [49] resulting in diminished self-awareness. There is some evidence suggesting that the ability to think about one's own thoughts depends on the same cognitive and neural processes as mindreading [50]. Equally, emotions play an important role in self-awareness. The development of the capacity to experience, communicate and regulate emotions is considered to be the most important event in infancy [51]. One of the main characteristics of autism is lack of empathy and emotional engagement with others $[52,53]$. Children with autism have difficulties with interpreting emotions, are deficient in processing their own emotional experiences and pay little attention to emotional stimuli in general [e.g. 54-57]. Due to this inability to empathize and emotionally engage with others individuals with ASD are totally focussed on their own interests and concerns.

A network of structures important for theory of mind processing includes the superior temporal sulcus and the adjacent temporo-parietal junction, the temporal poles and the medial prefrontal cortex [58; see also 59 for a review). Research evidence implicated the RH in theory of mind reasoning across various tasks $[60,61]$. The neural substrates for emotions and empathy are complex [62] involving amygdala, ventral medial prefrontal cortex. Recent imaging studies point to an involvement of a 'mirror neuron circuit' for empathy $[63,64]$.

\subsection{Egocentrism/Allocentrism}

In apparent contrast to the mentalizing impairment even among very high functioning individuals with ASD is their often-documented increased sense of self or total focus on the self $[65,66,67]$ that is also reflected in numerous biographical accounts. The term 'autism' is de- 
rived from the Greek word 'autos' ('self') and since Kanner's time this focus on the self as atypical applies to all individuals with ASD. Extreme egocentricity was one of the diagnostic criteria for Asperger Syndrome proposed by Gillberg \& Gillberg [68]. Frith and de Vignemont [69] suggest that there are differences to reading other minds depending on whether the other person can be understood using an 'egocentric' or an 'allocentric' standpoint. From an egocentric point of view other people are understood only relative to the self whereas from an allocentric stance the mental state of a person is independent from the self. These theorists suggest that individuals with ASD suffer from an imbalance between both point of views, 'they are unable to connect an egocentric to an allocentric stance and can only adopt extreme forms of either' [70]. This very detailed analysis of mindreading further illustrates the different and unique aspects of awareness of self and others in individuals with ASD.

\subsection{Self awareness across time}

Awareness that we are the same person across time, also defined as temporally extended self-awareness [71] is an essential part of one's self-concept. Although the results of two recent studies [72,73] indicate that individuals with ASD have undiminished temporally extended self-awareness as assessed by the delayed self-recognition task, this task may not adequately measure self-awareness as suggested by Lind \& Bowler [74]. Indirect evidence suggests that temporally extended self-awareness is impaired in ASD based on their problems with theory of mind as well as some aspects of temporal cognition [75]. Alternative explanations are the autobiographic memory difficulties [76] and also the well-documented language impairments in ASD. Language is a medium with which we monitor ourselves and it allows us to experience past, present and future [77].

\subsection{Language and awareness of self}

Conceptions about oneself and others develop from an early age and depend largely on the emergence of language. At around 18 months of age children start referring to themselves as ' $\mathrm{I}$ ' and begin using the word 'you' for others, indicating a further development in their selfother awareness. According to Kircher and David [78] 'the symbolic presentation of the self in language is the personal pronoun I'. Language difficulties such as pronoun reversal, use of third person perspective, impoverished inner speech, and impaired narrative have a negative effect on mental processes and also restrict certain aspects of self-awareness.

There is substantial clinical and research evidence of impaired pragmatic language use in children with ASD as indicated by pronoun reversal errors ('I'/'me/'you') [79-82] reflecting general difficulties with their sense of self, as well as problems in self-other differentiation.

Peeters et al. [83] suggested that the reason children with autism sometimes communicate from a third-person perspective instead of a first- vis-à-vis second person perspective is that in contrast to typically developing children they possess a non-social basis of self-other categorization. Use of a third person perspective also has consequences for attribution of mental states, and mentalizing ability in general. As argued by Northoff and Heinzel [84] a third person perspective is an indication of a fragmented image of self and other. Adults with 
ASD also appear to have difficulties with first person pronoun usage [85]. Of particular interest might be the fact, that Hans Asperger often used to refer to himself from a third person perspective [86].

A fundamental role in self-awareness is attributed to inner speech [87] that is impaired in ASD [88]. When asked about the nature of their thoughts, a group of adults with Asperger syndrome reported mainly images and actions as their only inner experience and made no reference to inner speech or emotions [89]. Many individuals with ASD are visual thinkers and rely heavily on visualization to process information [90].

A recent fMRI study [91] provided evidence of underintegration of language and imaging in autism by showing that individuals with ASD are more reliant on visualization to support language comprehension. These authors suggest that cortical underconnectivity is the reason for the lack of synchronization between linguistic and imaginal processing in autism. Supporting these findings are the results of an imaging study on daydreaming [92] indicating that autistic individuals do not 'daydream' about themselves or other people. This study also points to a link between daydreaming and the construction of self and self-awareness.

In summary, language is of fundamental significance to self-awareness and necessary for forming a clear identity of self and others. Another important dimension in the formation of the self that is also dependent on language is autobiographical memory as well as the construct of a narrative self.

\subsection{Autobiographical memory}

Many influential theorists $[93,94]$ associate the development of self with the emergence of autobiographical or episodic memory. The components necessary for a fully functioning autobiographical memory are a basic memory system, spoken or signed language, understanding and production of narrative, temporal understanding, self-awareness and theory of mind [95]. Autobiographical memory not only depends on these cognitive constructs but is also specifically concerned with events that have specific meaning to the individual. This personal significance evolves through emotions and motivations that are constructed in interaction with others. Autobiographical disturbances can arise from combined deficits in the realms of memory, emotion and self-related processing which are intricately connected, both behaviourally and neurologically [96].

The majority of components that make up an autobiographic memory system are impaired in autism. There is significant evidence that individuals with ASD have circumscribed episodic memory impairments, e.g. they have an impaired recall for personally experienced events [97-101]. As suggested by Millward et al [102] individuals with ASD remember real-life episodes less well than other people because they have no 'experiencing self'. Wheeler et al [103] in their investigation of episodic memory in autism concluded that remembering of personal events requires the 'highest form of consciousness, autonoetic consciousness (self-knowing), which is dependent of self-awareness'. 
The prevailing view is that episodic memory is created in the neocortex and subsequently stored in the medial-temporal lobes and after a time becomes independent and is distributed in neocortical networks [104]. Whereas the left temporal lobe is dominant for the acquisition of new verbal information, episodic information involves mainly the right fontal lobes $[105,106]$. Neuroimaging studies provide evidence for right frontal involvement in the processing of autobiographic memories [e.g. 107]. The RH is especially important for memories with emotional contents.

\subsection{The narrative self}

Many theorists [e.g. 108, 109] have highlighted the importance of the narrative self and argued that the autobiographical self is a similar construct as the narrative self. Individuals create their own identity by constructing autobiographical narratives or life stories [110]. The benefits of a personal or narrative self are significant; a narrative mediates self-understanding and creates coherence out of life's experiences. Narrative emerges early in development and narrative and self are inseparable [111]. The creation of a narrative self depends on various cognitive capacities, including working memory, self-awareness, episodic memory and reflective metacognition, a sense of agency, the ability to attribute action to oneself together with a capacity for temporal integration of events, a fully functioning pronoun system, an ability to differentiate between self and non-self as well as a sense of one's own body that is based on proprioceptive-motor processes [112].

The mechanisms responsible for each of the above dimensions are impaired in autism and as a consequence individuals with ASD have great difficulties in constructing a self-narrative. If autobiographical material cannot be provided, the narrative is disoriented and confused and in many cases is no narrative at all but only confabulation [113], which is often the case in autism. As a result, the narrative of individuals with ASD, and the self that is represented in this narrative, is quite vague and not representing the true self. Research evidence confirms deficits in narrative abilities in individuals with ASD [114-116].

Language and symbolic functions are localized in the left hemisphere, whereas narrative abilities are considered to be a function of the right hemisphere. There is a significant evidence for RH contribution to social language and many of the functions associated with autobiographic memory specifically those with emotional contents. In addition, narrative organisation depends on coordination of activity among various brain regions [117] and as suggested by Belmonte [118] malfunction in neural connectivity may be the underlying problem with autistic narrative.

To summarize, there is substantial evidence that the main components of self-awareness including self recognition, self-other differentiation, body awareness, theory of mind, intersubjectivity, emotion processing, language (pronoun reversal, inner speech, third person perspective), autobiographical memory and narrative self are impaired in ASD. Our review of neural substrates underlying these processes has highlighted the significance of the Right Hemisphere. 


\section{Self neuropathology in ASD}

From a neural point of view the self can be viewed as a complex and dynamic representation consisting of multiple brain networks $[119,120]$. The origins of self begin in infancy and over the first several years of life normally developing children acquire an understanding of different dimensions of self and other. Deviant development in autism is likely to result in a cascade of developmental impairments including dysfunctional self-related processes as outlined above. Various brain regions have been indicated in the pathogenesis of autism including frontal lobes [e.g., 121] cerebellum [122], parietal lobes [123], hippocampus [124] and amygdala [125]. The extent of anatomical and functional abnormalities in autism points to a possible core dysfunction in neural processing. In addition, the vast amount of potential genetic risk factors suggests that multiple or all-emerging functional brain areas are affected during early development [126]. This theory is supported by widespread growth abnormalities in the brain of children with autism [127, 128].

In the following sections we will explore three neural theories implicated in the development of an atypical or different sense of self in individuals with ASD. Apart from the involvement of the $\mathrm{RH}$ in self-related processes a dysfunctional mirror neuron system as well as abnormal connectivity may have a role to play in the atypical developmental trajectories in ASD.

\subsection{Right hemisphere hypothesis}

The prefrontal cortex plays a vital role in the development of the self as it generates a sense of self and facilitates many links with other parts of the brain. Cognitive neuroscience studies have shown that the RH plays a special role in personal relatedness, which is intimately linked to the development of the self. Based largely on recent neuroimaging research evidence an increasing number of cognitive neuroscientists have emphasized the specific role of the RH in self-related functions [129-132]. Specifically the right dorsomedial prefrontal cortex seems to play a critical role in the development of models of the self [133]. This has been confirmed by several imaging studies, including a recent study of self-evaluation [134]. As described in the previous paragraphs there is substantial research evidence that the $\mathrm{RH}$ may be dominant for self-awareness and self-related functions. The psychiatrist and philosopher Iain McGilchrist [135] provided an extensive exploration of the dominance of the RH in self-related processes.

Elsewhere, Lyons and Fitzgerald [136] put forward the theory that RH impairment leads to a dysfunctional self-development in ASD. There is substantial research evidence linking Asperger syndrome to right hemisphere dysfunction [e.g. 137, 138]. The RH is dominant in the first years of human life when the major brain development during critical periods takes place. Results of a cerebral metabolism study in children (aged between 18 days to 12 years) showed that the RH is prominently activated, suggesting that the RH develops earlier than the LH [139]. The RH in implicated during early social interactions [140] including early attachment processes [141], maternal face and voice recognition [142] as well as the ability to view others in a similar way as the self [143]. The developing self depends on relations with 
others and these early experiences are vital for the maturation of the right brain system. Substantial behavioural evidence of infants who later developed autism is supporting the theory of disrupted intersubjective behaviour. We argue that impairments in neurobiology affecting particularly the $\mathrm{RH}$ both cause and interact with defects in personal relatedness and later developing self processes.

\subsection{Abnormal connectivity}

The 'Abnormal Neural Connectivity Theory' proposes that autism is a distributed systemwide brain disorder that restricts the coordination and integration among various brain areas. The original positron emission tomography (PET) study by Horwitz et al. [144] found reduced correlations among frontal cortex, parietal and other brain regions and suggested that autism involves impairment in functional connectivity between frontal cortex and other brain systems. More recent studies proposed that autism is a disorder of neural underconnectivity [145], overconnectivity [146, 147] or both under and overconnectivity in which local connectivity may be relatively dense whereas long-range connectivity between brain regions may be reduced or abnormally patterned [146-151].

Studies of the cerebral cortex in autism show abnormalities of synaptic and columnar structure. Cortical minicolumns are fundamental units of cerebral cortical information processing. Examination of neurons revealed abnormalities in the size of cortical minicolumns particularly in the frontal and temporal lobes in $\operatorname{ASD}[152,153]$ that could alter overall levels of connectivity within the brain. These findings are in accordance with the observed white matter abnormalities reported particularly in people with ASD [154]. A recent study using functional connectivity MRI (fcMRI) [155] provided further evidence of atypical enhanced functional connectivity suggesting that abnormal connectivity may be linked to developmental brain growth disturbances in autism.

These studies suggest that connectivity among diverse brain areas may be the core problem in autism. In autism the network connectivity through which various brain areas communicate with each other are limited, particularly the connections to the frontal cortex [156] which is dominant for self-related processing particularly in the RH. The network model of the self proposed by Stuss et al. [157] suggests that the self is hierarchically organized, with the highest level of the self involved in self-awareness being subserved by frontal lobes. Early developmental impairments in minicolumnar microcircuitry in the frontal cortex in autism could be the reason for the deficits found in higher order frontal processes [158] which are likely to result in fragmented self awareness and identity formation in autism.

\subsection{Mirror neuron system}

Another recent neural theory of autism suggests that a dysfunctional mirror neuron system may be fundamental to the aetiology of autism $[159,160]$. The existence of mirror neurons in humans has been demonstrated by a number of EEG and imaging studies [e.g. 161]. Mirror neurons are activated in relation both to specific actions performed by self and matching actions performed by others, providing a potential bridge between minds [162] and might 
have a role to play in self related processes. Mirror neurons may enable us to understand the actions of others by mapping the actions of other people to our own motor system and so allow a shared representation of actions. In addition to understanding the action of others this so-called 'mirroring' might also allow the automatic experience of the intention and emotion of the other person as suggested by Kaplan and Iacoboni [163].

Research has demonstrated that mirror neuron activity correlates with empathy [164] and social competence in general [165]. It has been suggested that mirror neurons are a prerequisite for the normal development of self-recognition, imitation, theory of mind, empathy, intersubjectivity and language $[166,167]$. Furthermore, mirror neurons are likely to play a central role in self-awareness. To quote Ramachandran and Oberman [168] 'they may enable humans to see themselves as others see them, which may be an essential ability for selfawareness and introspection' (p.41). Developmental data suggest that there is higher imitative behaviour in children that can self-recognise, possibly facilitated by mirror neurons, in contrast to those who cannot [169]. Providing support for a RH hypothesis in self-related functions are recent imaging studies $[170,171]$ indicating that a frontoparietal 'mirror' network is associated with self-recognition processes.

Several recent functional brain-imaging studies have found evidence of mirror neuron dysfunction in individuals with ASD in social mirroring tasks [172], motor facilitation [173], and imitation [174]. A fMRI study [175] revealed that individuals with autism showed a different pattern of brain activity during cognitive tasks relating to self-referential processing. The authors concluded that a core deficit in autism might be related to the construction of a sense of self in its relation with others. Echoing Hobson [176] Iacoboni [177] suggests that primary intersubjectivity is the basis for the development of the neural systems associated with internal and external self-related processes. Failure or abnormal development of a fully functioning mirror neuron system in the autistic infant is likely to result in a cascade of developmental impairments including dysfunctional self-related processes.

\section{Conclusion}

The centrality of an impaired sense of self in autism has been the focus of research for many decades. The development of self-awareness is a complex process that involves integration of information from many sources and coordination across the brain systems involved in self-related concepts. A sense of self emerges from the activity of the brain in interaction with other selves. There is substantial evidence that early deficits in self-development including impaired relations with others result in a fragmented and atypical sense of self in ASD. In this review we have presented evidence that a great majority of self-related processes that are mediated to a significant extent by the right hemisphere are impaired in individuals with ASD. Additional lines of investigation indicate that an unintegrated sense of self in autism is also potentially associated with abnormal functional connectivity and an impaired mirror neuron system. Consequences of this atypical sense of self are the well-documented impairments individuals with ASD experience in the social and communication 
domain. In contrast, there have been suggestions that this different sense of self might be a contributory factor to the significant talents and special skills present in a majority of individuals with ASD. Happè \& Vital [178] put forward the notion that diminished self-awareness in ASD might be advantageous in the development of these special gifts.

\section{Author details}

Viktoria Lyons $^{1}$ and Michael Fitzgerald ${ }^{2}$

*Address all correspondence to: viktorialyons@yahoo.co.uk

1 Blackrock, Co. Dublin, Ireland

2 Trinity College Dublin, Ireland

\section{References}

[1] Kircher T, David AS. Introduction: The Self And Neuroscience. In: Kircher T, David A.S. (eds) The Self in Neuroscience and Psychiatry, Cambridge: Cambridge University Press; 2003. p2.

[2] Kanner L. Autistic Disturbances of Affective Contact. Nervous Child 1943;2: 217-50.

[3] Asperger H. Frühkindlicher Autismus, Medizinische Klinik 1974;69: 2024-27.

[4] Cohen DJ. The pathology of the Self in primary childhood autism and Gilles de la Tourette Syndrome. Psychiatric Clinics of North America 1980; 3 (3): 383-402.

[5] Lutz J. Zum Verstaendnis Des Autismus Infantum Als Einer Ich-Bewusstseins-, IchAktivitaets- Und Ich-Einpraegungsstoerung. Acta Paedopsychiatrica 1968; 35, 161.

[6] Powell S, Jordan R. Being Subjective About Autistic Thinking And Learning To Learn. Educational Psychology 1993;13: 359-370.

[7] Frith U. Autism: Explaining The Enigma. Oxford: Blackwell; 1989, $2^{\text {nd }}$ Edition; 2003.

[8] Hobson RP. On The Origins Of Self And The Case Of Autism. Development And Psychopathology 1990;2: 163-81.

[9] Hobson RP, Meyer JA. Foundations For Self And Other: A Study In Autism. Developmental Science 2005;8: 481-91.

[10] Stern DN. The Interpersonal World Of The Infant. New York: Basic Books; 1985.

[11] Neisser U. Five kinds of self-knowledge. Philosophical Psychology 1988;1(1): 35-58. 
[12] Dahlgren SO, Gillberg C. Symptoms in the first two years of life. A preliminary population study of infantile autism. European Archives of Psychiatric and Neurological Science 1989; 283: 169-74.

[13] Nadig AS, Ozonoff S, Young GS, Rozga A, Sigman M, Rogers SJ. A Prospective Study Of Response To Name In Infants At Risk For Autism. Archives Pediatric Adolescent Medicine 2007;161: 378-83.

[14] Osterling J, Dawson G. Early Recognition Of Children With Autism: A Study Of First Birthday Home Videotapes. Journal Of Autism And Developmental Disorders 1994;24: 247-53.

[15] Baron-Cohen S, Cos A, Bird G, Swettenham J, Nightingale N, Morgan K, Drew A. Charman T. Psychological markers in the detection of autism in infancy in a large population. British Journal of Psychiatry 1996;16: 158-63.

[16] Dawson G, Adams A. Imitation and social responsiveness in autistic children. Journal of Abnormal Child Psychology 1984;12 (2): 209-26.

[17] Mundy P, Crowson M. Joint Attention And Early Communication: Implications For Intervention With Autism. Journal Of Autism And Developmental Disorders 1997;6: 653-76.

[18] Hobson RP. Autism And The Development Of Mind. Hillsdale, NJ: Lawrence Erlbaum; 1993.

[19] Hobson, 1990.

[20] Gallup GG. Jr. Chimpanzees: Self-Recognition. Science 1970;167: 86-87.

[21] Lewis M., Ramsay D. Intentions, Consciousness And Pretend Play. In: Zelazo PD, Astington JW, Olson DR (eds) Developing Theories Of Intention: Social Understanding And Self-Control. Mahwah, NJ: Erlbaum; 1999. p77-94,

[22] Dawson G, McKissick FC. Self-recognition in autistic children. Journal of Autism and Developmental Disorders 1984;17: 383-94.

[23] Keenan JP, Christiana W, Malcolm S, Johnson A. Mirror-Self Recognition In Autism And Asperger's Syndrome: Implications For Neurological Correlates'. Poster Presented At The Eleventh Annual Cognitive Neuroscience Society Meeting, San Francisco. CA., April 2004.

[24] Pierce K., Muller R-A., Ambrose J., Allen G., Courchesne E. Face Processing Occurs Outside The Fusiform 'Face Area' In Autism: Evidence From Functional MRI'. Brain 2001;124: 2059-73.

[25] Keenan JP, McCutcheon NB, Pascual-Leone A. Functional Magnetic Resonance Imaging And Event Related Potential Suggest Right Prefrontal Activation For Self-Related Processing. Brain And Cognition 2001;47: 87-91. 
[26] Critchley HD, Daly EM, Bullmore ET. Et Al. The functional neuroanatomy of social behavior. Changes in cerebral blood flow when people with autistic disorder process facial expressions. Brain 2000;123: 2203-12.

[27] Pierce et al., (2001).

[28] Hobson (1990).

[29] Hobson \& Meyer (2005).

[30] Murray D, Lesser M. Lawson W. Attention, Monotropism And The Diagnostic Criteria For Autism. Autism. The International Journal Of Research And Practice 2005;9 (2): 139-56.

[31] Williams D. Autism And Sensing. The Unlost Instinct. London: Jessica Kingsley; 1998.

[32] Chiu PH, Kayali MA, Kishida KT, Tomlin D, Klinger LG, Klinger MR, Montague PR. Self Responses along cingulate cortex reveal quantitative neural phenotype for highfunctioning autism. Neuron 2008;57: 463-73.

[33] Tomlin D, Kayali M A, King-Casas B, Anen D, Camerer CF, Et Al. Agent-Specific Responses In The Cingulated Cortex During Economic Exchanges. Science 2006;5776: 1047-50.

[34] Chiu et al. (2008).

[35] Lombardo MV, Chakrabarti B, Bullmore ET, Sadek SA, Pasco G, Wheelwright SJ, Suckling J, MRC AIMS Consortium, Baron-Cohen S. (2009). Atypical Neural SelfRepresentation in Autism. Brain 2009. http://brain.oxfordjournals.org/cgi/content/ abstract/awp306v1(accessed 19 January 2010)

[36] Ruby P, Decety J. How Would You Feel Versus How Do You Think She Would Feel? A Neuroimaging Study On Perspective-Taking With Social Emotions. Journal Of Cognitive Neuroscience 2003;16: 988-99.

[37] Decety J, Sommerville JA. Shared representations between self and other: A social cognitive neuroscience view. Trends in Cognitive Science 2003;7: 527-33.

[38] Avikainen S, Forss N, Hair R. Modulated activation of the human SI and SII cortices during observation of hand actions. Neuron 2002;15: 640-6.

[39] Goldenberg G. Body Image And The Self. In: Feinberg TE, Keenan JP (eds.) The Lost Self. Pathologies Of The Brain And Identity. Oxford: Oxford University Press; 2005. p81-99.

[40] Jordan R, Powell S. Understanding And Teaching Children With Autism. Chichester: Wiley; 1995.

[41] Russell J. How Executive Disorders Can Bring About An Inadequate 'Theory Of Mind'. In: Russell J (Ed.) Autism As An Executive Disorder. Oxford: Oxford University Press. 1997.p281. 
[42] Russell (1997).

[43] Williams D, Happé F. Pre-Conceptual Aspects Of Self-Awareness In Autism Spectrum Disorder: The Case Of Action Monitoring. Journal Of Autism And Developmental Disorders 2009;39: 251-9.

[44] Mesulam MM. Principles Of Behavioral And Cognitive Neurology (2 ${ }^{\text {nd }}$ Ed.), Oxford: Oxford University Press; 2000.

[45] Devinsky O. Right Cerebral Hemisphere Dominance for a Sense of Corporeal and Emotional Self. Epilepsy \& Behaviour 2000;1: 60-73.

[46] Farrer C, Franck N, Georgieff N, Frith CD, Decety J, Et Al. Modulating The Experience Of Agency: A Positron Emission Tomography Study. Neuroimage 2003;18: 324-33.

[47] Tsakiris M, Constantine M, Haggard P. The Role Of Right Temporo-Parietal Junction In Maintaining A Coherent Sense Of One's Body. Neuropsychologia 2008;46: 3014-18.

[48] Saxe R, Baron-Cohen, S. Editorial: The Neuroscience Of Theory Of Mind. In: R. Saxe R, Baron-Cohen S. (eds.) Theory Of Mind. A Special Issue Of The Journal Social Neuroscience. Hove: Psychology Press; 2007.

[49] Frith U, Happè F. Theory Of Mind And Self Consciousness: What It Is Like To Be Autistic? Mind \& Language 1999;14: 1-22.

[50] Happè F. Theory Of Mind And The Self. In: Ledoux J, Debiec J, Moss H. (eds) The Self: From Soul To Brain, Annals Of The New York Academy Of Sciences 2003;1001: 134-144.

[51] Schore AN. Affect Dysregulation And Disorders of The Self. New York: Ww. Norton \& Company; 2003.

[52] Baron-Cohen S. Wheelwright S. The Empathy quotient: An investigation of adults with Asperger syndrome or high functioning autism, and normal sex differences. Journal of Autism and Developmental Disorders 2004;34: 163-75.

[53] Hobson (1993).

[54] Hobson RP. The Autistic Child's Appraisal Of Expressions Of Emotion. Journal Of Child Psychology And Psychiatry 1986; 27: 321-42.

[55] Yirmiya N., Sigman M., Kasari C., Mundy P. Empathy And Cognition In High Functioning Children With Autism. Child Development 1992;63: 150-60.

[56] Baron-Cohen S., Wheelwright S., Joliffe T. Is there a 'language of the eyes'? Evidence from normal adults and adults with autism or Asperger syndrome. Visual Cognition 1997;4: 311-31.

[57] Gaigg SB, Bowler DM. Free Recall And Forgetting Of Emotionally Arousing Words In Autism Spectrum Disorder. Neuropsychologia 2008;46: 2336-43. 
[58] Frith CD, Frith U. The Neural Basis Of Mentalizing. Neuron 2006;50: 531-34.

[59] Saxe \& Baron-Cohen (2007).

[60] Happé F., Brownell H., Winner E. Acquired Theory Of Mind Impairments Following Right Hemisphere Stroke. Cognition 1999;70: 211-40.

[61] Shamay-Tsoory SG, Tomer R, Berger BD., Goldsher D,Aharon-Peretz, J. Impaired "Affective Theory Of Mind" Is Associated With Right Ventromedial Prefrontal Damage. Cognitive And Behavioural Neurology 2005;18: 55-67.

[62] Chakrabarti B, Bullmore E, Baron-Cohen S. (2007) ‘Empathizing with basic emotions: Common and discrete neural substrates. In: Saxe R, Baron-Cohen S. (eds) Theory of Mind. A special issue of the Journal Social Neuroscience. Hove: Psychology Press; 2007. p. 364-384.

[63] Keysers C., Perrett I. Demystifying Social Cognition: A Hebbian Perspective. Trends In Cognitive Science 2004;8: 501-7.

[64] Rizzolatti G, Craighero L. The Mirror-Neuron Sys tem. Annual Reviews Of Neuroscience 2004; 27: 169-92.

[65] Kanner (1943).

[66] Asperger H. Die 'autistischen Psychopathen' im Kindesalter. Archiv für Psychiatrie and Nervenkrankheiten 1944;117: 78-136.

[67] Baron-Cohen, S. (2005) 'Autism - 'Autos': Literally, a Total Focus on the Self?' In: Feinberg TE., Keenan JP (eds.) The Lost Self. Pathologies of the Brain and Identity. Oxford: Oxford University Press. p166-80.

[68] Gillberg IC, Gillberg C. Asperger Syndrome - Some Epidemiological Considerations: A Research Note. Journal Of Child Psychology And Psychiatry 1989;30: 631-38.

[69] Frith U, De Vignemont, F. Egocentrism, Allocentrism, And Asperger Syndrome. Consciousness And Cognition 2005;14: 719-38.

[70] Frith U, De Vignemont F. (2005)

[71] Moore C, Lemmon K. The Self In Time: Developmental Perspectives. Hillsdale, NJ, Usa: Erlbaum; 2001.

[72] Nielsen M., Suddendorf T., Dissanayake C. Imitation And Self-Recognition In Autism. In: Rogers S., Williams JW. (Eds) Imitation And The Development Of The Social Mind: Autism And Typical Development. New York: Guilford Press; 2006. p138-56.

[73] Lind SE, Bowler DM. Delayed Self-Recognition In Children With Autism Spectrum Disorder. Journal Of Autism And Developmental Disorders 2009;39 (4): 643-650.

[74] Lind SE, Bowler DM. (2009). 
[75] Boucher J, Pons F, Lind S, Williams D. Temporal cognition in children with autistic spectrum disorders: Tests of diachronic thinking. Journal of Autism and Developmental Disorders 2007;37: 1413-29.

[76] Lind SE, Bowler DM. Episodic Memory And Autonoetic Consciousness In Autistic Spectrum Disorders: The Roles Of Self-Awareness, Representational Abilities And Temporal Cognition. In: Boucher J, Bowler DM. (eds), Memory In Autism: Theory And Evidence. Cambridge: Cambridge University Press; 2008. p166-188.

[77] Beitman BD, Nair J, Viamontes GI. 'Why Self-Awareness?' In: Beitman BD, J. Nair J. (eds.) Self-Awareness Deficits in Psychiatric Patients. Neurobiology, Assessment, and Treatment. New York: W.W. Norton \& Company; 2004. p3-23.

[78] Kircher T, David AS. (2003) p3.

[79] Kanner L. (1943).

[80] Tager-Flusberg, H. Current Theory And Research On Language And Communication In Autism. Journal Of Autism And Developmental Disorders 1996;26: 169-172.

[81] Hobson (1990).

[82] Jordan R, Powell S. (1995).

[83] Peeters G, Grobben G, Hendrickx,A., Van Den Eede S, Verlinden K. Self-Other And Third-Person Categorization In Normal And Autistic Children. Developmental Science 2003. 6: 166-172.

[84] Northoff G, Heinzel A. The Self In Philosophy, Neuroscience And Psychiatry: An Epistemic Approach', In: T. Kircher T., David A. (eds.) The Self In Neuroscience And Psychiatry. Cambridge: Cambridge University Press; 2003. p40-55.

[85] Lombardo MV, Barnes JL, Wheelwright SJ, Baron-Cohen S. Self-Referential Cognition And Empathy In Autism. PloS One 2007,2: e883.

[86] Lyons V, Fitzgerald M. Asperger Syndrome - A Gift Or A Curse? New York: Nova Science Publishers; 2007.

[87] Siegrist M. Inner Speech As A Cognitive Process Mediating Self-Consciousness And Inhibiting Self-Deception. Psychology Report 1995;76: 259-265.

[88] Whitehouse A.JO, Maybery MT, Durkin K. Inner Speech Impairments In Autism. Journal Of Child Psychology And Psychiatry 2006;47: 857-65.

[89] Hurlburt R, Happé F, Frith U. Sampling The Form Of Inner Experience In Three Adults With Asperger's Syndrome. Psychological Medicine 1994;24: 385-95.

[90] Grandin T. Thinking In Pictures: And Other Reports From My Life With Autism. New York: Vintage Books; 1995. 
[91] Kana RK, Keller TA, Cherkassky VL, Minshew NJ, Just MA. Sentence Comprehension In Autism: Thinking In Pictures With Decreased Functional Connectivity. Brain 2006;129: 2484-93.

[92] Kennedy DP, Redcay E, Courchesne E. Failing To Deactivate: Resting Functional Abnormalities In Autism. Proceedings Of The National Academy Of Science USA 2006;103: 8275-80.

[93] Perner J. Episodic Memory: Essential Distinctions And Developmental Implications. In: Moore C, Lemmon K.(Eds.) The Self In Time: Developmental Perspectives. Hillsdale NJ: Erlbaum; 2001. p181-202.

[94] Wheeler MA. Episodic Memory And Autonoetic Awareness. In: Tulving E, Craik FIM. (eds.) Oxford Handbook Of Memory. New York: Oxford University Press; 2000. p597-625.

[95] Nelson K, Fivush R. The Emergence Of Autobiographical Memory: A Social Cultural Developmental Theory. Psychological Review 2004;111: 486-511.

[96] Fujiwara E, Markowitsch HJ. Autobiographical Disorders. In: Feinberg TE, Keenan JP. (eds.) The Lost Self. Pathologies Of The Brain And Identity, Oxford: Oxford University Press; 2005. p65-80.

[97] Bowler DM, Gardiner JM, Grice SJ. Episodic memory and remembering in adults with Asperger syndrome. Journal of Autism and Developmental Disorders 2000;30: 295-304, p. 295.

[98] Millward C, Powell S, Messer D, Jordan R. Recall For Self And Other In Autism: Children's Memory For Events Experienced By Themselves And Their Peers. Journal For Autism And Developmental Disorders 2000;30: 15-28.

[99] Gardiner, J. M. Bowler, D. M, Grice SJ. Further Evidence Of Preserved Priming And Impaired Recall In Adults With Asperger's Syndrome. Journal Of Autism And Developmental Disorder 2003;33 (3) 250-69.

[100] Crane L, Goddard L. Episodic and semantic autobiographical memory in adults with Autism Spectrum Disorders. Journal of Autism and Developmental Disorders 2008; 38: 498-506.

[101] Boucher J, Bowler DM (eds) Memory in autism: theory and evidence. Cambridge: Cambridge University Press; 2008.

[102] Millward et al. (2000).

[103] Wheeler MA, Stuss DT, Tulving, E Toward A Theory Of Episodic Memory: The Frontal Lobes And Autonoetic Consciousness. Psychological Bulletin 1997;121: 331-54.

[104] Fink GR. In Search Of One's Own Past: The Neural Bases Of Autobiographical Memories. Brain 2003;126: 1509-10. 
[105] Shallice T, Fletcher P, Frith C, Grasby P, Frackowiak R, Dolan R. Brain Regions Associated With Acquisition And Retrieval Of Verbal Episodic Memory. Nature 1994;368: 633-35.

[106] Tulving E, Kapur S, Craik F, Moskovitch M, Houle S. Hemispheric Encoding/Retrieval Asymmetry In Episodic Memory: Positron Emission Tomography Findings. Proceedings Of The National Academy Of Science USA 1994;91: 2016-20.

[107] Fink GR, Markowitsch HJ, Reinkemeier M, Bruckbauer T, Kessler J, Heiss W-D. Cerebral Representation Of One's Own Past: Neural Networks Involved In Autobiographical Memory', Journal of Neuroscience 1996;16: 4275-82.

[108] Bruner J. Kalmar DA. Narrative and metanarrative in the construction of self. In: Ferrari M, Sternberg RJ. (eds.) Self-Awareness: Its Nature and Development. New York: Guilford Press; 1988.

[109] Dennett D. Consciousness Explained. Boston: Little, Brown; 1991.

[110] Schechtman M. The Constitution Of Selves. Ithaca: Cornell University Press; 1996.

[111] Ochs E, Capps L. Narrating The Self. Annual Review Of Anthropology 1996;25: 19-43.

[112] Gallagher S. Self-Narrative In Schizophrenia. In: Kircher T, David A. (eds.) The Self In Neuroscience And Psychiatry. Cambridge: Cambridge University Press; 2003. p336-360.

[113] Gallagher (2003).

[114] Loveland K. Mcevoy R, Tunali B, Kelley ML. Narrative Story Telling In Autism And Down’s Syndrome. British Journal of Developmental Psychology 1990;8: 9-23.

[115] Losh M, Capps L. Narrative Ability In Highfunctioning Children With Autism Or Asperger's Syndrome. Journal of Autism and Developmental Disorders 2003;33: 239-51.

[116] Colle L, Baron-Cohen S, Wheelwright S, Van Der Lely HK. Narrative discourse in adults with high-functioning Autism or Asperger Syndrome. Journal of Autism and Developmental Disorders 2008;38: 28-40.

[117] Tononi G, Sporns O, Edelman GM. Reentry And The Problem Of Integrating Multiple Cortical Areas: Simulation Of Dynamic Integration In The Visual System. Cerebral Cortex 1992; 2: 310-35.

[118] Belmonte M. Human, but more so: What the autistic brain tells us about the process of narrative. In: Osteen M. (ed.) Autism and representation. London: Routledge; 2008. p166-79.

[119] Viamontes GI, Beitman BD, Viamontes CT, Viamontes JA. Neural Circuits For SelfAwareness. Evolutionary Origins And Implementation In The Human Brain. In: Beit- 
man BD, Nair J. (eds) Self-Awareness Deficits In Psychiatric Patients. Neurobiology, Assessment, And Treatment. New York: W.W. Norton \& Company; 2004. p24-111.

[120] Decety \& Somerville (2003).

[121] Aylward EH, Minshew NJ, Field, K, Sparks BF, Singh N. Effects of age on brain volume and head circumference in autism. Neurology 2002;59: 175-83.

[122] Courchesne E, Townsend J, Akshoomoff NA,Et al. A new finding: impairment in shifting attention in autistic and cerebellar patients. In: Broman SH, Grafman J. (eds) Atypical cognitive deficits in developmental disorders: implications for brain function. Hillsdale, N.J.: Lawrence Erlbaum Associates; 1994. p101-137.

[123] Courchesne E, Press G, Yeung-Courchesne, R. Parietal lobe abnormalities detected with MR in patients with infantile autism. American Journal of Roentgeneology 1993;160: 387-393.

[124] Saitoh O, Karnds,CM, Courchesne E. Development Of Hippocampal Formation From 2 To 42 Years: MRI Evidence Of Smaller Area Dentate In Autism. Brain 2001;124: 1317-24.

[125] Aylward EH, Minshew NJ, Goldstein G, Honeycutt NA., Augustine AM, Yates KO, Barta PE, Pearlson GD. MRI volumes of amygdala and hippocampus in non-mentally retarded autistic adolescents and adults. Neurology 1999;53: 2145-50.

[126] Müller RA. The Study Of Autism As A Distributed Disorder. Mental Retardation And Developmental Disabilities Research Review 2007;13(1): 85-95.

[127] Courchesne E, Karns CM, Davis HR, Ziccardi R, Carper RA. et al. Unusual brain growth patterns in early life in patients with autistic disorder: an MRI study. Neurology 2001;57: 245-54.

[128] Carper RA, Courchesne E. Localized enlargement of the frontal lobe in early autism. Biological Psychiatry 2005;57 (2): 126-33.

[129] Fossati P, Hevenor S, Graham SJ. Et Al. In Search Of The Emotional Self: An fMRI Study Using Positive And Negative Emotional Words. American Journal Of Psychiatry 2003;160: 1938-45.

[130] Keenan JP, Gallup GG, Jr., Falk D. The Face In The Mirror: The Search For The Origins Of Consciousness. New York: Harper Collins; 2003.

[131] Kircher \& David (2001).

[132] Decety \& Somervie (2003).

[133] Mega MS, Cummings JL. Frontal Subcortical Circuits: Anatomy And Function. In: Salloway SP, Malloy PF, Duffy JD. (Eds) The Frontal Lobes and Neuropsychiatric Illness. Washington, DC: American Psychiatric Publishing; 2001. p15-32.

[134] Fossati et al. (2003). 
[135] McGilchrist I. The Master And His Emissary. The Divided Brain And The Making Of The Western World. New Haven and London: Yale University Press; 2010.

[136] Lyons V, Fitzgerald, M. ‘Did Hans Asperger (1906-1980) Have Asperger Syndrome? Journal Of Autism And Developmental Disorder 2007;37: 2020-21.

[137] Klin A, Volkmar F, Sparrow, S, Cicchetti DV, Rourke BP. Validity and Neuropsychological Characterization of Asperger Syndrome: Convergence with Nonverbal Learning Disabilities Syndrome. Journal of Child Psychology and Psychiatry 1995; 36, 1127-1140.

[138] Gunter HL, Ghaziuddin M, Ellis HE. Asperger Syndrome: Test Of Right Hemisphere Functioning And Interhemispheric Communication. Journal Of Autism And Developmental Disorders 2002;32: 263-81.

[139] Chiron C, Jambaque I, Nabbout R, Lounes R, Syrota A., Dulac O. The right brain hemisphere is dominant in human infants. Brain 1997;120: 1057-1065.

[140] Geschwind N, Galaburda AM. Cerebral Lateralization: Biological Mechanisms, Associations, And Pathology. Boston: MIT Press; 1987.

[141] Schore AN. The Experience-Dependent Maturation Of A Regulatory System In The Orbital Prefrontal Cortex And The Origin Of Developmental Psychopathology'. Development and Psychopathology 1996;8: 59-87.

[142] Fernald A. Intonation And Communicative Interest In Mother's Speech To Infants: Is The Melody The Message? Child Development 1989;60: 1497-1510.

[143] Meltzoff AN, Brooks R. 'Like Me' As A Building Block For Understanding Other Minds: Bodily Acts, Attention And Intention. In Malle BF, Et Al (Eds) Intentions And Intentionality: Foundations For Social Cognition. Boston: MIT Press; 2001. p171-192.

[144] Horwitz, B, Rumsey JM, Grady C.L, Rapoport SI. The Cerebral Metabolic Landscape In Autism. Intercorrelations Of Regional Glucose Utilization. Archives Of Neurology 1988; 45: 749-755.

[145] Just MA, Cherkassky VL, Keller TA, Minshew N. Cortical Activation And Synchronization During Sentence Comprehension In High-Functioning Autism: Evidence Of Underconnectivity. Brain 2004;127 (8): 1811-21.

[146] Casanova MF. White matter volume increase and minicolumns in autism. Annals of Neurology 2004;56: 453-54.

[147] Mizuno A, Villalobos ME, Davies MM, Dahl BC, Müller RA. Partially Enhanced Talamocortical Functional Connectivity In Autism. Brain Research 2006;1104 (1): 160-174.

[148] Courchesne E, Pierce K. Why the frontal cortex in autism might be talking only to itself: local overconnectivity but long-distance disconnection. Current Opinion in Neurobiology 2005;15: 225-30. 
[149] Belmonte M, Cook JEH, Anderson G, Rubinstein J, Greenough W, et al. Autism as a disorder of neural information processing: direction for research and targets for therapy. Molecular Psychiatry 2004a;9: 646-63.

[150] Kana et al. (2006).

[151] Just et al. (2007).

[152] Casanova MF, Buxhoeveden DP, Switala AE, Roy E. Minicolumnar pathology in autism. Neurology 2002a;58: 428-32.

[153] Casanova MF, Buxhoeveden DP, Switala AE, Roy E. Asperger's syndrome and cortical neuropathology. Journal of Child Neurology 2002b;17: 142-45.

[154] Keller TA., Kana RK, Just MA. A Developmental Study Of The Structural Integrity Of White Matter In Autism. Neuroreport 2007;18: 23-27.

[155] Turner KC, Frost L, Linsenbardt D, Mcilroy JR, Müller RA. Atypically Diffuse Functional Connectivity Between Caudate Nuclei And Cerebral Cortex. Behavioural And Brain Functions 2006;2: 34.

[156] Just MA., Cherkassky VL, Keller TA., Kana RK, Minshew N. Functional And Anatomical Cortical Underconnectivity In Autism: Evidence From A FMRI Study Of An Executive Function Task And Corpus Callosum Morphometry. Cerebral Cortex 2007;17: 951-61.

[157] Stuss DT, Picton TW, Alexander MP. Consciousness, Self-Awareness And The Frontal Lobes. In: Salloway SP, Malloy PF (eds.) The Frontal Lobes And Neuropsychiatric Illness. Washington, DC: American Psychiatric Publishing; 2001. P101-109.

[158] Courchesne \& Pierce (2005).

[159] Williams JHG, Whiten A., Suddendorf T, Perrett DI. Imitation, Mirror Neurons And Autism. Neuroscience \& Biobehavioral Reviews 2001;25: 287-95.

[160] Oberman LM, Ramachandran VS. The Simulating Social Mind: The Role Of The Mirror Neuron System And Simulation In The Social And Communicative Deficits In Autism Spectrum Disorders. Psychological Bulletin 2007;133(2): 310-27.

[161] Rizolatti \& Craighero (2004).

[162] Williams et al. (2001).

[163] Kaplan JT, Iacoboni M. Getting A Grip On Other Minds: Mirror Neurons, Intention Understanding, And Cognitive Empathy. In: Saxe R, Baron-Cohen S. (eds.) Theory Of Mind. A Special Issue Of The Journal 'Social Neuroscience. Hove: Psychology Press; 2007. P175-183.

[164] Pfeifer J, Iacoboni M, Mazziotta JC, Dapretto M. Mirror neuron system activity correlates with empathy and interpersonal competence in children. Social Neuroscience 2006; 6 (3-4): 175-83. 
[165] Kaplan \& Iacoboni (2007).

[166] Oberman \& Ramachandran (2007).

[167] Gallese V. The Roots Of Empathy: The Shared Hypothesis And The Neural Basis Of Intersubjectivity. Psychopathology 2003;36: 171-80.

[168] Ramachandran VS, Oberman LM. Broken Mirrors: A Theory Of Autism. Scientific American 2006; November, 39-45.

[169] Asendorpf JB, Baudonniere PM. Self-awareness and Other-awareness: Mirror selfrecognition and synchronic imitation among unfamiliar peers. Developmental Psychology 1993;29: 88-95.

[170] Uddin LQ, Kaplan JT, Molnar-Szakacs I, Zaidel E, Iacoboni M. Self-Face Recognition Activates A Frontoparietal 'Mirror' Network In The Right Hemisphere: An Event-Related fMRI Study. Neuroimage 2005; 25: 926-35.

[171] Uddin LQ, Molnar-Szakacs I, Zaidel E, Iacoboni M. Rtms To The Right Inferior Parietal Area Disrupts Self-Other Discrimination. Social Cognitive And Affective Neuroscience $2006 ; 1,65-71$.

[172] Dapretto M, Davies MS, Pfeifer JH, Scott AA, Sigman,M. Bookheimer SY, Iacoboni M. Understanding emotions in others: mirror neuron dysfunction in children with autism spectrum disorders. Nature Neuroscience 2006;9(1): 28-30.

[173] Theoret H, Halligan E, Kobyashi M, Fregni F, Tager-Flusberg H, Pascual-Leone A. Impaired Motor Facilitation During Action Observation In Individuals With Autism Spectrum Disorder. Current Biology 2005;15: 84-85.

[174] Nishitani NA, Vikainen S, Hari R. Abnormal Imitation-Related Cortical Activation Sequences In Asperger's Syndrome. Annals Of Neurology 2004;55: 558-62.

[175] Kennedy et al. (2006).

[176] Hobson RP. The Intersubjective Foundations Of Thought. In Braten S. (ed.) Intersubjective Communication And Emotion In Early Ontogeny. Cambridge: Cambridge University Press; 1998. p283-296.

[177] Iacoboni, M. 'Failure To Deactivate In Autism: The Constitution Of Self And Other'. Retrieved October 7, 2006, From http://www.awares.org/conferences/

[178] Happè F, Vital P. 'What Aspects Of Autism Predispose To Talent?', In: Happè F, Frith U.(eds.) Autism And Talent. London: Philosophical Transactions of The Royal Society; 2009. p1369-1376. 
Chapter 32

\title{
Critical Evaluation of the Concept of Autistic Creativity
}

\author{
Viktoria Lyons and Michael Fitzgerald \\ Additional information is available at the end of the chapter \\ http://dx.doi.org/10.5772/54465
}

\section{Introduction}

Autism Spectrum Disorders (ASD) are neurodevelopmental conditions that are associated with an astonishing combination of cognitive strengths and weaknesses with a substantial minority of individuals displaying some exceptional creative abilities, reaching genius proportions in some rare cases. Creativity is a multifactorial construct and neuroscience is only beginning to unravel some of the cognitive components involved in the creative process. In this chapter we contrast neuroscientific evidence from creativity research with models attempting to explain talent and creativity in ASD. Although there are no agreed definitions for creativity the formulation put forward by Griffiths [1] "Creativity is a mental journey between ideas or concepts that involves either a novel route or a novel destination" (p.6) seems to fit the picture very well. Various explanations and theories have been put forward to account for creativity ranging from unconscious mechanisms, cognitive processes, special abilities and personal traits to links with genetic processes and psychopathology.

The classical portrait of autism is that of rigid, stereotyped behaviours, a preference for sameness and a resulting lack of imagination. Therefore, the prevalent view is that creativity and imaginative thought are extremely difficult or impossible for individuals with ASD. There is substantial research evidence that almost all forms of imagination are impaired in autism including lack of pretend play, pragmatic language, comprehension and construction of narrative, theory of mind and experimental tests of creativity [2-6]. A significant challenge to this perceived lack of creativity is the enormous achievements that some people with ASD show in creative and scientific fields. Some theorists and clinicians have therefore challenged the view of impoverished creativity in ASD [7-12].

In this review the focus is on a subgroup of individuals on the autistic spectrum who display exceptional creative talents and abilities. The features of ASD that favour creativity in- 
clude narrow interests, great persistence, ability to see details within a whole, a fascination with facts (rather than people) and having savant type talents. While social imagination is impaired, autistic imagination of the Einsteinian type is amplified.

\section{Nature of autistic intelligence and creativity}

"Autistic intelligence" as described by Hans Asperger [13] is a sort of intelligence hardly touched by tradition and culture - "unconventional, unorthodox, strangely 'pure' and original, akin to the intelligence of pure creativity". As pointed out by Einstein "To raise new questions, new possibilities, to regard old problems from a new angle, requires imagination and makes real advance in science" [14 p. 40].

Individuals with ASD show great variation in IQ ranging from severe intellectual impairment to superior ability. In addition, intelligence as measured by traditional intelligence tests reveals a different intellectual profile in ASD than in the neurotypical population with peaks on Block Design and troughs in Comprehension that appears to be robust across IQ levels [15]. Individuals with ASD also generally display atypical cognitive processes when performing these tasks. More recent studies [16,17] revealed further evidence for a different nature of autistic intelligence including fast information processing despite poor measured IQ.

The relationship between intelligence and creativity is unclear and ranges from suggestions of totally distinct psychological entities to overlapping constructs to different labels for the same thing [18]. Guilford [19] in his 1950 landmark paper "Creativity" asserted that creative talent could not be understood in terms of "intelligence". Within the creativity literature, as noted by Lubart [20] "the dominant view is that certain intellectual abilities may be particularly useful in creative work, but no intellectual ability is devoted only to creativity" (p.288). Good general intelligence, domain-specific knowledge and special skills are necessary ingredients for creativity; however, these components alone are not sufficient for explaining creative processes [21].

Gardner's [22] model of multiple intelligences holds that intelligence is a collection of different intellectual capacities including linguistic, logical-mathematical, musical, bodily kinaesthetic, spatial intelligence and two forms of personal intelligence, - one oriented towards the understanding of other persons, the other towards an understanding of self. Autistic intelligence tends to be concentrated in the areas of music and logical-mathematical and spatial abilities. By nature, individuals with autism are extremely logical and analytical, and their thinking is concrete which makes them good mathematicians though lesser poets. The exception may be a minority of gifted individuals with ASD who have special literary talents as suggested by Ilona Roth [23] in her analysis of autism spectrum poets including Donna Williams, Tito Mukhopadhyay and Wendy Lawson. Roth makes the point that "poetry, with its dependence on intensely abstract, symbolic, and free-flowing forms of expression" ( $p$. 161) might be particularly suited to the autistic cognitive style. 
Many features of Asperger syndrome enhance creativity, but the ability to focus deeply on a topic and to take endless pains is characteristic. Hans Asperger [24] emphasized the intensity with which special interests are pursued already in his first lecture about children with "autistic psychopathology". It appears that these unique qualities of concentration and also perception as discussed in subsequent paragraphs in individuals with ASD may give rise to extraordinary creative abilities. Exceptionally gifted people like for example the animal scientist and author Temple Grandin [25] declares that her autism, as manifested in her acute visual/spatial mind and in her powers of concentration is what has made her success possible (p.188). People with Asperger syndrome live very much in their intellects, and certain forms of creativity benefit greatly from this [26]. Apart from good concrete intelligence additional characteristics of a gifted person with ASD include, ability to disregard social conventions, unconcern about the opinions of others and a sometimes-childlike naivety and inquisitiveness.

According Nancy Andreasen [27], who made a significant contribution to research on creativity, the personality traits that characterize creative individuals include "openness to experience, adventuresomeness, rebelliousness, individualism, ... persistence, curiosity, simplicity, ... the ability to see things in a different and novel way, indifference to social conventions, dislike of externally imposed rules, driven by own set of rules derived from within and a childlike manner" (p.30-32). Not surprisingly, the above two descriptions are strikingly similar.

\section{Cognitive processes involved in creativity}

Creativity is a multidimensional construct and cognitive neuroscience is only beginning to understand the many cognitive components involved in creative thinking including the neural substrates underlying these processes [for a review see 28]. Theories of creativity in general suggest that creativity is linked to attentional capacity [29] and associative or divergent thinking processes [30]. Mendelsohn [31] emphasised the specific role of "defocused" attention or a widened attentional ability in highly creative individuals, which is in contrast with the extremely narrow focus of attention ascribed to individuals with autism [e.g. 32]. Likewise, divergent thinking, which involves the production of a variety of responses [33] and assumes to depend on extensively connected neural networks also conflicts with the well reported neural underconnectivity and enhanced local networks found in autism. As pointed out by Nettle [34] "different domains of creativity require different cognitive profiles, with poetry and art associated with divergent thinking, schizophrenia and affective disorder, and mathematics associated with convergent thinking and autism" (p.1). It appears that other concepts of information processing need to be considered when attempting to elucidate the specific and unique mechanisms underlying autistic creativity. In the words of Allan Snyder [35] "The fact that genius might fall within the autistic spectrum challenges our deepest notions of creativity. Are there radically different routes to creativity: normal and autistic?" (p. 1403). 
The main current interpretation of special gifts and savant skills associated with autism include cognitive and psychological theories as well as various other models.

\section{Savant syndrome and creativity}

According to Treffert [36]: "Savant syndrome is a rare, but extraordinary condition in which persons with serious mental disabilities, including autistic disorder, have some 'island of genius' which stands in marked, incongruous contrast to overall handicap" (p.1351). Savant skills are found more commonly in ASD than in any other group [37] and are generally attributed to low-functioning autism but can also occur in individuals with normal and very high intelligence.

Theories put forward to explain savant skills strongly suggest a relationship with repetitive, obsessional and restricted behaviour [38]. Savants generally exhibit circumscribed interests usually within their skill area [39], which leads to considerable rehearsal, practice and training. Savant skills are also strongly associated with rote memory [40]. Pring [41] in her analysis of memory characteristics in savants argued against the rote memory explanation and instead proposed the existence of complex long-term memory structures in savants. In general, memory is considered an essential cognitive component of savant skills. In addition, researchers have suggested a role for 'implicit' or unintentional, learning in savant skill development [42,43]. Results of neuropsychological examinations of a calendar-calculating savant indicated that good memory, superior mental calculation and knowledge of calendar are the underlying elements for this specific talent [44]. Taken together, the classical portrait of the autistic savant is largely imitative and not very creative and some writers [e.g. 45] argued that true creativity is missing in savants "there are no savant geniuses about.... Their mental limitations disallow and preclude an awareness of innovative developments" ( $\mathrm{p}$. 177). In contrast, other theorists $[46,47]$ believe that savants, particularly those with Asperger syndrome with above average intelligence levels [48] can be extremely creative. Mottron et al [49] write that "Savant performance cannot be reduced to uniquely efficient rote memory skills .... and encompasses not only the ability for strict recall, requiring pattern completion, but also the ability to produce creative, new material within the constraints of a previously integrated structure" (p.1388).

Various pathological conditions such as frontotemporal dementia, dominant-hemisphere strokes, head injuries and infections may also result in the emergence of savant like abilities [e.g. 50]. Of particular interest is the fact that individuals with these diverse types of disorders and emerging savant skills also develop cognitive features and behavioural traits, which are characteristic of autism [51]. 


\section{Cognitive and psychological theories and explanations underlying special gifts and talents and savant skills in ASD}

The development of special gifts and talents in ASD has been associated in general with the ability to process local information. These abilities include detail-focused cognitive style (weak coherence) [52], enhanced perceptual functioning [53], an accentuated capacity for systemizing [54], privileged access to low-level perceptual processes [55] and various other psychological and physiological states.

\subsection{Weak central coherence}

A different cognitive style, the weak central coherence theory (WCC) proposed by Uta Frith [56] and Frith and Happé [57] has been suggested as an explanation for certain special abilities found in ASD. This exceptional part-based processing style is demonstrated in the superior ability individuals with ASD show on tasks such as block design and embedded figures resulting from deficits in integration processes that serve to draw information together as a meaningful whole [see 58]. According to Frith [59], the WCC particularly addresses the special gifts and talents and acute perceptual abilities in autism (e.g. hypersensitivity, visual and auditory abilities) and can explain the achievements of individuals with ASD syndrome in art, science, music, and many other areas. Local coherence, which is defined by close attention to mechanical or physical patterns, is exemplified in the work of Temple Grandin [60]. Atypical attentional mechanisms and abnormal neural connectivity have been suggested as possible cognitive and neural mechanisms underlying WCC.

\subsection{Enhanced perceptual functioning}

Also located at the level of perception is the model proposed by Mottron and Burack [61] and Mottron et al [62] which are based on enhanced perceptual functioning (EPF) suggesting that people with autism have an overdevelopment of low-level perceptual abilities at the expense of high-level processing mechanisms. This theory provides a convincing account of special abilities in ASD such as peaks of ability in visual and auditory modalities and also indicates that a variety of cognitive processes are required for the development of savant abilities. For example, Mottron et al [63] propose that enhanced detection of patterns, including similarity within and among patterns is contributing to creativity evident in savants. As far as neural correlates for their theory is concerned the authors suggest the notion of brain plasticity and an overfunctioning of brain regions involved in perception in autism [64].

EPF and WCC are similar in emphasizing detail focused processing bias and superior local processing. Superiority in local coherence may be specific to autistic creativity and as argued by Mills [65] "produces an imaginative faculty defined by close attention to mechanical or physical patterns not psychological or social rules" (p.126). 


\subsection{Systemizing theory}

This above interpretation appears to be in line with the "systemizing theory" put forward by Baron-Cohen et al. [66] which emphasises the superior ability in recognizing repeating patterns in stimuli (e.g. numerical, spatial, mechanical, auditory systemizing). The "systemizing theory", in contrast to the WCC model, predicts that individuals with ASD have a strong central coherence as indicated by their excellent skills in integrating information in areas such as astronomy and physics. This ability, however, does not apply to non-systemizable fields such as fiction. Similar to the WCC the systemizing model also posits excellent attention to detail in perception and memory. Baron-Cohen et al [67] further suggest that this excellent attention to detail is a consequence of sensory hypersensitivity found in individuals with ASD. Belmonte et al [68] posited that local overconnectivity in the posterior sensory parts of the cortex is responsible for the sensory 'magnification' in ASD.

\subsection{Low level information processing}

The work of Synder et al. [69] also provides an understanding of certain forms of creativity. According to Snyder and Mitchell [70] outstanding savant skills might be accomplished by accessing early stages of information processing. Their controversial model predicts the possibility of accessing non-conscious information by artificially disinhibiting the inhibiting networks associated with concept formation, using transcranial magnetic brain stimulation (TMS). By means of this method Snyder et al. [71] propose that this process will open "the door for restoration of perfect pitch, for recalling detail... and even enhancing creativity". A recent EEG study [72] attributing increased visual detail perception in autism to neural abnormalities related to low-level visual processing potentially supports this theory.

Snyders's theory [73] furthermore suggests the possibility that savant skills may be latent in everyone, but a "form of cortical disinhibition or atypical hemispheric imbalance" is required in order to access them (p. 1399). In support for his theory Snyder quotes the emergence of savant skills in individuals without any previous history for talents due to a variety of illnesses.

Snyder's model explains many of the talents and characteristics associated with Asperger geniuses including their childlike view of the world and lack of preconceptions, which is beneficial for developing new and original theories and perspectives. For example, Ludwig Wittgenstein, who had Asperger syndrome did spend little time reading other philosophers and felt most of them were wrong anyhow. He didn't want to "cloud his mind with false theories" [74].

\subsection{Primary process thought and disinhibition syndrome, reduced self-awareness - 'Flow'}

Theories of creativity $[75,76]$ also highlight the importance of primary process thought as found in dreaming, free association and psychosis in creative processes. Einstein suggested that creative scientists are the ones with "access to their dreams. Occasionally, a dream will actually provide the solution to a problem", as cited by Gregory [77] (p.226). Many mathe- 
maticians are intuitive thinkers and rely on the unconscious mind to a large extent, like for example the genius mathematician Poincare [78]. Freudian theory holds that primary processes or primitive thinking which creative persons have more access to are based on their weak defence mechanisms of repression. Individuals with Asperger syndrome have very weak defence mechanisms thus allowing them access to early childhood memories [79].

Low levels of repression or inhibition are associated with creativity and a number of theorists [80, 81] have suggested that creativity is "a disinhibition syndrome", i.e. highly creative individuals lack cognitive inhibition. Neural correlates of cognitive disinhibition are the frontal lobes and research indicates that creative individuals show less frontal-lobe activity during verbal association tasks [82]. Deficits in inhibition have been documented in autism [83] as well as in Attention Deficit Hyperactivity Disorder (ADHD) [84] a neurodevelopmental disorder that is associated with increased creativity.

Also relevant in this context maybe the concept of "flow" proposed by the psychologist Csíkszentmihályi [85]. The notion of "flow" indicates a familiar state of reduced self-awareness where temporal concerns (time, food, ego-self, etc) are ignored during periods where the individual is fully immersed in a task or process. According to Csíkszentmihályi "flow" is characterised by a feeling of great absorption, engagement and fulfilment and thought to be inherently reinforcing and rewarding [86]. As alerted to in the chapter "Atypical Sense of Self in Autism Spectrum Disorders: A Neuro-cognitive Perspective" (this book) [87] diminished self-awareness which is a characteristic of individuals with ASD and associated with right hemisphere dysfunction might be advantageous in the development of special talents in ASD as quoted by Happé and Vital [88] (p.1373).

To conclude, although no single theory can explain the cognitive mechanisms underlying savant skill development, prodigious memory, atypical perception and excellent attention to detail are fundamentally associated with savant like talents in individual with ASD.

\section{Neural basis of creativity in non-clinical populations}

The study of the neural basis of creativity is an area greatly neglected by scientific research and despite methodological difficulties associated with investigating creativity any account of creativity must include explanations about the neural correlates of creativity [89].

Neuroscientific approaches aiming to determine the physiological basis of creative thought, are assuming that creativity is a measurable trait. Creativity can be interpreted as physiological changes that are required for creative problem solving focussing on EEG measures of cortical activation [90]. Theories of creativity in general postulate that low levels of cortical activation contribute to creative inspiration. Imaging data [91] suggest that great creativity not only requires a high level of specialized knowledge (stored in temporal and parietal lobes) and divergent thinking (mediated by the frontal lobes) but also co-activation and communication between areas of the brain that normally do not show strong connections. Highly creative individuals also possess the ability to modulate neurotransmitters [92, 93] such as the norepinephrine system (located in the frontal lobes), indicated by a reduction of cerebral levels of norepinephrine during creative periods. Support for the role of frontal 
areas in a fluid analogy-making task comes from an fMRI study [94] indicating bilateral neural activations. A study measuring differences in cerebral blood flow between highly creative individuals and controls during a verbal task of creative thinking [95] implicated a neural network consisting of right and left fronto-temporal, parietal, and cerebellar regions in highly creative performances. These areas are involved in cognition, emotion, working memory and response to novelty.

\section{Neural basis of creativity in ASD}

We are not aware of any studies investigating directly the neural basis of creativity in autism apart from studies exploring savant skills in autism. Some of these support the Left Hemisphere (LH) dysfunction/ and Right Hemisphere (RH) compensation theory [e.g. 96] as indicated by hemispheric asymmetry. Research evidence of neuroanatomical abnormalities including atypical minicolumnar organization in ASD $[97,98]$ as well as neural hypotheses about abnormal connectivity [e.g. 99] support this theory.

\subsection{Hemispheric asymmetry}

Cerebral asymmetry refers to the lack of structural symmetry in left and right hemispheres in humans. Atypical cerebral asymmetry, a deviation from the normal pattern of cerebral asymmetry has been associated with special cognitive talents [100] and creativity [101] as cited by Smalley et al. [102]. For example, the capability for making distal or global verbal associations is one factor contributing to creativity and according to Brugger and Graves [103] the basis for this type of verbal creativity is "cerebral laterality in which an individual has a relative weakening of left hemisphere dominance and strengthening of availability of right hemisphere processing" [104] p. 138.

Atypical cerebral asymmetry has been associated with autism, dyslexia and ADHD [105], neurodevelopmental disorders considered to share regions of linkage overlap [106]. In addition, creativity in psychiatric populations is often associated with atypical cerebral asymmetry [107] and a RH “bias" [108].

Research evidence for atypical cerebral asymmetry in autism (e.g. increased size of some RH cortical structures) and reversed lateralization of language has been well documented [e.g. 109]. An imaging study by Herbert et al [110] found a "sizeable right-asymmetry increase" in subjects with autism. Individuals with autism had twice as much right-as left-asymmetrical cortex than the control sample. This finding was interpreted as a consequence of early abnormal brain growth abnormalities. According to the hypothesis put forward by Geschwind and Galaburda [111] the immaturity of the LH in utero makes it more susceptible to damage, which could result in a compensatory overdevelopment of the RH caused by neural migration and thus resulting in an anomalous RH-dominance. As the RH develops earlier than the LH, accelerated early brain development in autism may lead to anomalous lateralization of cognitive functions as suggested by Herbert et al [112]. 


\subsection{Right hemisphere processing and creativity}

Savant skills are linked to the RH [113], which is dominant for attention, visuospatial and emotional function. Various authors [114-115] have suggested that autistic savants have atypical LH dysfunction with $\mathrm{RH}$ compensation. Based on research evidence including imaging studies Treffert [116] speculated that "one mechanism in some savants, whether congenital or acquired is left brain dysfunction with right brain compensation." The notion of "paradoxical functional facilitation" as described by Kapur [117] denotes loss of function in one damaged brain area and enhanced function of another area, which as emphasized by Treffert is "central to explaining savant syndrome" (p.1356).

RH skills can be characterized as non-symbolic, artistic, concrete and directly perceived in contrast to LH skills that are more sequential, logical, and symbolic. For example musical, artistic, visual or spatial abilities (mathematics) are primarily RH skills.

The association between $\mathrm{RH}$ and creativity is based on research evidence demonstrating that the $\mathrm{RH}$ is more involved in production of mental images than the LH, perception and production of music, e.g. the right inferior frontal gyrus is known to be involved in musical pitch encoding and melodic pitch memory [118]. EEG studies show that highly creative individuals show more right than left-hemisphere activation during experimental studies [119], indicating that during the creative process creative individuals rely more on the RH. Lesion studies as well as unimpaired population studies have demonstrated that the RH is superior to the LH at noticing anomalies in objects [120]. Individuals with autism are well known for detecting even the smallest changes in the environment.

In sum, several lines of evidence suggest that atypical cerebral asymmetry which is a highly heritable trait [121] is associated with autism and linked to certain aspects of creativity. It is also likely that some of the structural brain abnormalities evidenced in autism are related to the special cognitive functioning that encourages great creativity. Neurological brain differences have been reported in the literature on creativity [122].

\subsection{Neuroanatomical abnormalities in autism}

Converging neuroscientific evidence has suggested that the neuropathology of ASD is widely distributed, involving impaired connectivity throughout the brain. Neuroanatomical abnormalities in autism include increase in cortical thickness [123], and increase in head and brain size [124]. Accelerated growth in brain size in early childhood in autism has been documented by a range of studies [125], which may be consistent with the asymmetric cerebral lateralization in autism as discussed above. The increased brain volumes in autism are believed to be the result of insufficient or abnormal prenatal pruning, which together with genetic factors are most likely to underlie these growth abnormalities [126]. In addition, there is evidence of higher birth weight [127] and faster body growth [128] as well as increased levels of growth hormones [129] in autism. These altered brain growth rates are considered to have a strong influence on patterns of brain connectivity and cerebral lateralization [130,131] and differential cognitive functioning. For example, the increased hippocampus size in autism [132] may be associated with enhanced visual-spatial, mathe- 
matical and mechanistic processing in autism as well as savant abilities such as calculation and memory. Imaging data of a reduced size of corpus callosum in autism [133] is consistent with the reduced interhemispheric brain connectivity reported in autistic individuals [134]. Neural underconnectivity [e.g. 135] provides support for the weak "central coherence theory" which postulates enhanced local and decreased global information processing in autism. Research on patterns of cortical connectivity also indicates that a specific minicolumnar phenotype found in autism may be beneficial for information processing and/or focused attention and may also offer an explanation for the savant abilities autism [136, 137].

To conclude, although neural mechanisms underlying savant skill and development are not well established, associating creativity with hemisphere lateralization and anatomical abnormalities in autism is supported by empirical evidence and also has some explanatory potential. Additional areas to explore are genetic factors and creativity found in other pathological conditions.

\section{Nature versus nurture}

Is great creativity a fortunate combination of specific traits, or do "creativity genes" exist? As speculated by Smalley et al [138] "genes that increase one's risk for certain psychiatric or learning disorders may also be 'enhancer' genes for creativity (and intuition)" (p.82). According to Gardner [139] it is extremely unlikely that there is such a thing as a "poetry gene or a music gene" since complex human behaviours typically have a "polygenic basis" (p. 175). Without doubt ASD have a polygenic basis and genetic factors not only contribute to specific skills but also to traits such as persistence, the capacity for concentration for extended periods, and curiosity about certain types of stimulation. Lykken et al [140] describe the concept of emergenesis, an extreme form of epitasis, in which a unique combination of genes may lead to qualitative shifts in capacity or ability that may apply to extremely gifted individuals with ASD.

The relationship between inherited talent and/or extensive practice is a very contentious aspect of superior ability in specific skills. The view propounded by Howe [141] emphasizing the overwhelming role of practice in the acquirement of special skills, is largely rejected by a majority of theorists who argue for the role of innate talent [142-144]. Special talents are essentially innate in predisposing to cognitive or physical qualities and are the key to understanding geniuses from Einstein to Mozart [145]. For example, research evidence from twin data [146] suggests a genetic basis for detail-focused cognitive style predisposing to talent in ASD. It is configuration of genes and variations in genetic inputs that are critical to the success in persons of great creativity. It is our belief that there are significant genetic underpinnings to creativity of genius proportions, which of course could not be expressed without environmental factors. 


\section{Novelty, ADHD and creativity}

The majority of theoretical conceptions of creativity agree that the main component of creativity is its novelty, uniqueness or unusualness that undoubtedly applies to the creativity displayed by gifted individuals with ASD. Novelty or sensation seeking behaviour is also strongly associated with ADHD. A significant degree of comorbidity between autism and ADHD has been documented [e.g. 147] in the literature. Although reported to have poor attention and concentration and being poor academic performers individuals with ADHD have a capacity to hyperfocus, which allows them to produce great works of art. For example the poetry of Lord Byron, who had ADHD [148] is an example for a work of genius in this area. As pointed out above, both autism and ADHD are associated with atypical cerebral asymmetry which is a highly heritable and complex phenotype linked to creativity and sharing regions of linkage overlap [149,150].

\section{Psychopathology}

There is a very close relationship between creativity (especially in literature and arts) and psychopathology, particularly mood disorder [151, 152]. An association of biochemical factors in psychosis and creativity has been suggested by Folley et al [153] indicating the noradrenergic system. This model also provides possible links between attention, divergent thinking, and arousal based on mechanisms that interact with structural and neurochemical systems of the brain and has the potential to explain the novelty seeking behaviour implicated in ADHD but may have less explanatory power as far as autism is concerned. According to Sternberg and Lubart [154] creativity and novelty must be coupled with appropriateness for something to be considered creative. Although schizotypal thought most likely leads to an increase in novel ideas, they may not always be appropriate.

In contrast, the nature of creativity displayed by individuals with ASD is associated with the distinctiveness of the autistic brain and its unique neural connectivity. In this context Temple Grandin [155] has stated, "it is likely that genius is an abnormality" (p178-179). However, she also believes that autistic intelligence is necessary in order to add diversity and creativity to the world: "It is possible that persons with bits of these traits are more creative, are possibly even geniuses...If science eliminated these genes, maybe the whole world would be taken over by accountants" (p.124).

\section{Conclusion}

The results of our evaluation suggest that many features of ASD are advantageous for great creativity. Creativity is an extremely complex and multifaceted construct and no cognitive theory or model of brain function has so far been able to fully account for it. We suggest that the distinctive gifts of perception, attention, memory and information 
processing combined with personality attributes can give rise to the extraordinary creativity seen in some individuals with ASD. It is our view that progress in elucidating the neural basis of autism may hold promises for a better understanding of autistic creativity and creativity in general. Autism Spectrum Disorders are mainly portrayed as negative phenomena, as a curse, but if they were an integral part of the mindset of highly creative individuals such as Einstein and Darwin who possessed autistic traits they could be regarded in some aspects as a gift [156].

\section{Author details}

Viktoria Lyons* and Michael Fitzgerald

*Address all correspondence to: viktorialyons@yahoo.co.uk

Trinity College Dublin, Ireland

\section{References}

[1] Griffiths TD. Scientific Commentary. Capturing Creativity. Brain 2008;131: 6-7.

[2] Baird G, Cox A, Charman, T, Baron-Cohen S. et al. A Screening Instrument for Autism at 18 Months of Age: A Six-Year Follow-up Study. Journal of the American Academy of Child and Adolescent Psychiatry 2000; 39: 694-702.

[3] Happé F. Communicative Competence and Theory of Mind in Autism: A Test of Relevance Theory. Cognition 1993;48: 101-119.

[4] Bruner J, Feldman C. Theories of Mind and the Problem of Autism. In: Baron-Cohen S, Tager-Flusberg H, Cohen DJ. (eds) Understanding Other Minds: Perspectives from Autism. Oxford: Oxford University Press; 1993. p 267-291.

[5] Baron-Cohen S. Theory of Mind and Autism: a Review. Special Issue of The International Review of Mental Retardation 2001; 23: 169.

[6] Craig J, Baron-Cohen S. Creativity and Imagination in Autism and Asperger Syndrome. Journal of Autism and Developmental Disorders 1999;29: 319-326

[7] Fitzgerald M. Autism and Creativity: Is there a link between autism in men and exceptional ability? New York: Brunner Routledge; 2004.

[8] Fitzgerald M. The Genesis of Artistic Creativity. London: Jessica Kingsley; 2005.

[9] Fitzgerald M, James I. The Mind of the Mathematician. Baltimore: John Hopkins Press; 2007. 
[10] Sacks O. An Anthropologist on Mars: Seven Paradoxical Tales. New York: Knopf; 1995.

[11] Treffert DA. The savant syndrome: an extraordinary condition. A synopsis: past, present, future. In: Happé F, Frith U. (eds.) Autism and talent. Philosophical transactions of The Royal Society 2009; vol. 364 (1522) p1351-1358.

[12] Mottron L, Dawson M, Soulières I. Enhanced perception in savant syndrome: patterns, structure and creativity. In: Happé F, Frith U. (eds.) Autism and talent. Philosophical transactions of The Royal Society 2009; vol. 364 (1522) p1385-1392.

[13] Asperger H. Die autistischen Psychopathen im Kindesalter. Archiv fuer Psychiatrie und Nervenkrankheiten 1944;11: 76-136.

[14] Jay E. Problem finding: understanding its nature and mechanism. Qualifying paper, Harvard Graduate School of Education, Cambridge, M.A.; 1989.

[15] Lincoln AJ, Allen MH, Kilman A. The assessment and interpretation of intellectual abilities in people with Autism. In: Schopler E, Mesibov GB (eds.). Learning and cognition in autism. New York: Plenum Press; 1995. p89-117.

[16] Dawson M, Soulieres I, Gernsbacher MA, Mottron L. The level and nature of autistic intelligence. Psychological Science 2007;18: 657-662.

[17] Scheuffgen K, Happé F, Anderson M, Frith U. High 'intelligence,' low 'IQ' Speed of processing and measured IQ in children with autism. Developmental Psychopathology 2000;12: 83-90.

[18] Sternberg RJ, O'Hara LA. Intelligence and Creativity. In: Sternberg RJ. (ed.) Handbook of Intelligence. Cambridge: Cambridge University Press; 2000.

[19] Guilford JP. Creativity. American Psychologist 1950;5: 444-454.

[20] Lubart T. In Search of Creative Intelligence. In: Sternberg J, Lautrey J, Lubart TI (eds.) Models of intelligence: international perspectives. Washington: APA; 2004.

[21] Heilman KM, Nadeau SE, Beversdorf DO. Creative Innovation: Possible Brain Mechanism. Neurocase 2003;9: 369-379.

[22] Gardner H. Frames of mind: The theory of multiple intelligences. New York: Basic; 1983.

[23] Roth I. Imagination and the Awareness of Self in Autistic Spectrum Poets. In: Osteen M. (ed.) Autism and Representation. New York: Routledge; 2008.

[24] Asperger H. Das psychisch abnorme Kind. Wiener Klinische Wochenzeitschrift 1938;49: 1314-1317.

[25] Grandin, T. Thinking in Pictures: and Other Reports from my Life with Autism. New York: Doubleday; 1995.

[26] Fitzgerald \& James, 2007. 
[27] Andreasen NC. The Creating Brain. The Neuroscience of Genius. New York: Dana Press; 2005.

[28] Abraham A, Windmann S. Creative cognition: The diverse operations and the prospect of applying a cognitive neuroscience perspective. Methods 2007; 22: 38-8.

[29] Mendelsohn GA. Associative and attentional processes in creative performance. Journal of Personality 1976;44: 341-369.

[30] Guilford JP. The nature of human intelligence. New York: McGraw-Hill; 1967.

[31] Mendelsohn 1976.

[32] Townsend J, Courchesne E. Parietal damage and narrow 'spotlight' spatial attention. Journal of Cognitive Neuroscience 1994;6: 220-232.

[33] Torrance EP. The Torrance Tests of Creative Thinking: Technical-norms manual. Bensenville, IL: Scholastic Testing Service; 1974.

[34] Nettle D. Schizotypy and mental health amongst poets, visual artists, and mathematicians. Journal of Research in Personality 2006;40: 876-890.

[35] Snyder A. Explaining and inducing savant skills: privileged access to lower level, less-processed information. In: Happé F, Frith U. (eds.) Autism and talent. Philosophical transactions of The Royal Society 2009; vol. 364 (1522) p1399-1406.

[36] Treffert 2009.

[37] Howlin P, Goode S, Hutton J, Rutter M. Savant skills in autism: psychometric approaches and parental reports. In: Happé F, Frith U. (eds.) Autism and talent. Philosophical transactions of The Royal Society 2009; vol. 364 (1522) p1369-1368.

[38] O'Connor N, Hermelin B. Talents and preoccupations in idiot-savants. Psychological Medicine 1991;21: 959-964.

[39] O'Connor \& Hermelin, 1991.

[40] Hill AL. Savants: Mentally retarded individuals with special skills. In Ellis N. (ed.) International review of research in mental retardation. New York: Academic Press; 1978. (9) p277-298.

[41] Pring L. Memory characteristics in individuals with savant skills. In: Boucher J, Bowler D. (eds.) Memory in Autism. Cambridge: Cambridge University Press; 2008.

[42] Treffert D. Extraordinary people: understanding 'idiot savants'. New York: Harper and Row; 1989.

[43] Hermelin B. Bright splinters of the mind: a personal story of research with autistic savants. London: Jessica Kingsley Press; 2001.

[44] Wallace GL, Happé F, Giedd JN. A case study of a multiply talented savant with an autism spectrum disorder: neuropsychological functioning and brain morphometry. 
In: Happé F, Frith U. (eds.) Autism and talent. Philosophical transactions of The Royal Society 2009; vol. 364 (1522) p1425-1432.

[45] Hermelin 2001.

[46] Treffert 2009.

[47] Mottron, Dawson \& Soulières 2009.

[48] Fitzgerald 2005.

[49] Mottron, Dawson \& Soulières 2009.

[50] Seeley WW, Matthews BR, Crawford RK, Gorno-Tempini ML, Foti D, Mackenzie IR, Miller BL. Unravelling Boléro: progressive aphasia, transmodal creativity and the right posterior neocortex. Brain 2008;131: 39-49.

[51] Heaton P. Wallace GL. Annotation: The savant syndrome. Journal of Child Psychology and Psychiatry 2004;45: 899-911.

[52] Frith U, Happè F. Autism: beyond "theory of mind". Cognition 1994;50: 115-132.

[53] Mottron, Dawson \& Soulières 2009.

[54] Baron-Cohen S, Ashwin E, Ashwin C, Tavassoli T, Chakrabarti B. Talent in autism: hyper-systemizing, hyper-attention to detail and sensory hypersensitivity. In: Happé F, Frith U. (eds.) Autism and talent. Philosophical transactions of The Royal Society 2009; vol. 364 (1522) p1377-1384.

[55] Snyder AW, Mitchell DJ. Is integer arithmetic fundamental to mental processing? the mind's secret arithmetic. London: Proceedings of the Royal Society 1999;266: 587-592.

[56] Frith U. Autism: Explaining the Enigma. Oxford: Blackwell; 1989.

[57] Frith \& Happè 1994.

[58] Happé F, Frith U. The Weak Coherence Account: detail-focused Cognitive style in Autism Spectrum Disorders. Journal of Autism and Developmental Disorders 2006;36: 5-25.

[59] Frith, U. (2004). Emanuel Miller lecture: Confusions and controversies about Asperger Syndrome. Journal of Child Psychology and Psychiatry 2004;45: 672-686, p. 680.

[60] Grandin 1995.

[61] Mottron L, Burack JA. Enhanced perceptual functioning in the development of autism. In: Burack JA, Charman T, Yirmiya N, Zelazo PR. (eds.) The development of autism: Perspectives from theory and research. New Jersey: Lawrence Erlbaum; 2001.

[62] Mottron L, Dawson M, Soulières I, Hubert B, Burack J. Enhanced Perceptual functioning in Autism: An Update, and eight Principles of Autistic Perception. Journal of Autism and Developmental Disorders 2006;3: 27-43.

[63] Mottron, Dawson \& Soulières 2009. 
[64] Mottron \& Burack 2001.

[65] Mills B. Autism and the Imagination. In: Osteen M. (ed.) Autism and Representation. New York: Routledge; 2008.

[66] Baron-Cohen S, Richler J, Bisarya D, Gurunathan N, Wheelwright S. The systemizing quotient: an investigation of adults with Asperger syndrome or high-functioning autism and normal sex differences. In: Frith U, Hill E. (eds.) Autism: Mind and Brain. Oxford: Oxford University Press; 2004. p161-186.

[67] Baron-Cohen et al. 2009.

[68] Belmonte MK, Allen G, Beckel-Mitchener A, Boulanger LM, Carper RA, Webb SJ. Autism and abnormal development of brain connectivity. Journal of Neuroscience 2004;24: 9228-31.

[69] Snyder AW, Bossomaier T, Mitchell DJ. Concept Formation: Object Attributes Dynamically Inhibited from Conscious Awareness. Journal of Integrative Neuroscience 2004;(3) 1: 31-46.

[70] Snyder \& Mitchell 1999.

[71] Snyder et al 2004.

[72] Vandenbroucke MWG, Scholte HS, van Engeland H, Lamme VAF, Kemner C. A neural substrate for atypical low-level visual processing in autism spectrum disorder. Brain 2008, 131(4): 1013-1024.

[73] Snyder 2009.

[74] Fitzgerald 2004.

[75] Freud S. Creative writers and daydreaming. In: Strachey J. (ed. \& trans.) Standard edition of the complete psychological works of Sigmund Freud. London: Hogarth Press; 1959. (9) p141-153.

[76] Kris E. Psychoanalytic explorations in art. New York: International Universities Press; 1952.

[77] Gregory FL. The Oxford Companion to the Mind. Oxford: Oxford University Press; 2004.

[78] Fitzgerald \& James, 2005.

[79] Lyons V, Fitzgerald, M. Early Memory and Autism. Letter to the Editor. Journal of Autism and Developmental Disorders 2005;35: 683.

[80] Eysenck H. Genius: The natural history of creativity. Cambridge: Cambridge University Press; 1995.

[81] Martindale C. Personality, situation, and creativity. In: Glover JA, Running RR, Reynolds CR (eds.) Handbook of creativity. New York: Plenum; 1989. p211-228. 
[82] Hudspith S. The neurological correlates of creative thought. Unpublished PhD. Dissertation, University of Southern California, Los Angeles, CA; 1985.

[83] Johnson KA, Robertson IH, Kelly SP. et al. Dissociation in performance of children with ADHD and high-functioning autism on a task of sustained attention. Neuropsychologia 2007;45: 2234-2245.

[84] Ozonoff, S. Components of executive function in autism and other disorders. In: Russell J. (ed.) Autism as an Executive Disorder. Oxford: Oxford University Press; 1997.

[85] Csíkszentmihályi M. Flow: the psychology of optimal experience. Ney York: Harper and Row; 1990.

[86] Csíkszentmihályi M, Lefevre J. Optimal experience in work and leisure. Journal of Personal and Social Psychology 1989;56: 815-822.

[87] Lyons V, Fitzgerald M. Atypical Sense of Self in Autism Spectrum Disorders: A Neuro-cognitive Perspective. In:

[88] Happé F, Vital P. What aspects of autism predispose to talent? In: Happé F, Frith U. (eds.) Autism and talent. Philosophical transactions of The Royal Society 2009; vol. 364 (1522): p1369-1376.

[89] Zeki S. Essays on science and society. Artistic creativity and the brain. Science 2001;293: 51-2.

[90] Martindale C. Creative imagination and neural activity. In: Kunzendorf R, Sheikh A. (eds.) Psychophysiology of mental imagery: Theory, research, and application. Amityville, NY: Baywood; 1990. p89-108.

[91] Heilman, Nadeau \& Beversdorf, 2003.

[92] Beversdorf DQ, Hughes JD, Steinberg BA, Lewis LD, Heilman KM. Noradrenergic modulation of cognitive flexibility in problem solving. Neuroreport 1999;10: 2763-7.

[93] Folley BS, Doop ML, Park S. Psychoses and creativity: is the missing link a biological mechanism related to phospholipids turnover? Prostaglandins, Leukotrienes and Essential Fatty Acids 2003;69: 467-476.

[94] Gaeke JG, Hansen PC. Neural correlates of intelligence as revealed by fMRI of fluid analogies. Neuroimage 2005;26: 555-64.

[95] Chavez-Eakle RA, Graff-Guerrero A, Garcia-Reyna JC, Vaugier V, Cruz-Fuentes D. Cerebral blood flow associated with creative performance: a comparative study. Neuroimage 2007;38: 519-28.

[96] Rimland B. Infantile Autism: the Syndrome and its implications for a neural theory of behavior. New York: Appleton-Century-Crofts; 1978.

[97] Casanova MF, Switala AE, Trippe J, Fitzgerald M. Comparative minicolumnar morphometry of three distinguished scientists. Autism, The International Journal of Research and Practice 2007;11: 55-569. 
[98] Casanova M, Trippe J. Radial cytoarchitecture and patterns of cortical connectivity in autism. In: Happé F, Frith U. (eds.) Autism and talent. Philosophical transactions of The Royal Society 2009; vol. 364 (1522) p1433-1436.

[99] Belmonte et al 2004.

[100] Geschwind N, Miller BL. Molecular approaches to cerebral laterality: development and neurodegeneration. American Journal of Medical Genetics 2001;101:379-381.

[101] Brugger P, Graves R.E. Right hemispatial inattention and magical ideation. European Archive for Psychiatry Clinical Neuroscience 1997;247: 55-57.

[102] Smalley SL, Loo SK, Yang MH, Cantor RM. Toward Localizing Genes Underlying Cerebral Asymmetry and Mental Health. American Journal of Medical Genetics Part B (Neuropsychiatric Genetics) 2004;135B: 79-84.

[103] Brugger \& Graves, 1997.

[104] Weinstein S, Graves RE. Are creativity and schizotypy products of a right hemisphere bias? Brain Cognition 2002;49: 138-151.

[105] Smalley et al 2004.

[106] Smalley SL, Kustanovich V, Minassian S, Stone JL, Ogdie MN. et al. Genetic linkage of attention-deficit/hyperactivity disorder on chromosome 16p13, in a region implicated in autism. American Journal Human Genetics 2002;71: 959-963.

[107] Overby LA III, Harris AE, Leek MR. Perceptual asymmetry in schizophrenia and affective disorder: Implications from a right hemisphere task. Neuropsychologia 1989;27: 861-870.

[108] Weinstein \& Graves 2002.

[109] Bigler ED, Mortensen S, Neeley ES, Ozonoff S, Krasny, L, Johnson M, Lu J, Provencal SL, McMahon W, Lainhart, JE. Superior temporal gyrus, language function, and autism. Developmental Neuropsychology 2007;31: 217-38.

[110] Herbert MR, Ziegler DA, Deutsch CK, O'Brien LM, Kennedy DN, Filipek PA, Barkardjiev AI, Hodgson J, Takeoka M, Makris N, Caviness Jr VS. Brain asymmetries in autism and developmental language disorder: a nested whole-brain analysis. Brain 2005;28: 213-226.

[111] Geschwind N, Galaburda AM. Cerebral Lateralization: Biological Mechanisms, Associations, and Pathology. Cambridge: MIT Press; 1987.

[112] Herbert et al 2005.

[113] Treffert D. The idiot savant: a review of the syndrome. American Journal of Psychiatry $2000 ; 45: 563-572$.

[114] Miller BL, Boone K, Cummings LR, Mishkin F. Emergence of artistic talent in frontotemporal dementia. Neurology 1998;51: 978-982. 
[115] Sacks O. Musicophilia: tales of music and the brain. New York: Knopf Publishing Group; 2007.

[116] Treffert 2009.

[117] Kapur N. Paradoxical functional facilitation in brain-behaviour research: a critical review. Brain 1996;119 1775-1790.

[118] Hyde K, Zatorre R, GriffithsTD, Lerch JP, Peretz I. Morphometry of the amusic brain: A two-site study. Brain 2006;129:2562-70.

[119] Martindale C, Hines D, Mitchell L, Covello E. EEG alpha asymmetry and creativity. Personality and Individual Differences 1984;5: 77-86.

[120] Smith SD, Dixon MJ, Tays WJ, Bulman-Fleming MB. Anomaly detection in the right hemisphere: The influence of visuospatial factors. Brain Cognition 2004;55: 458-62.

[121] Geschwind DH, Miller BL, DeCarli C, Carmelli D. Heritability of lobar brain volumes in twins supports genetic models of cerebral laterality and handedness. Proceedings of the National Academy of Sciences USA 2002;99: 3176-3181

[122] Herrmann N. The creative brain. Lake Lure, N.C: Applied Creative Services; 1988.

[123] Hardan AY, Muddasani S, Vemulapalli M, Keshavan MS, Minshew NJ. An MRI study of increased cortical thickness in autism. American Journal of Psychiatry 2006;163: 1290-92.

[124] Dissanayake C, Bui QM, Huggins R, Loesch DZ. Growth in stature and head circumference in high-functioning autism and Asperger disorder during the first 3 years of life. Development and Psychopathology 2006;18: 381-93.

[125] Courchesne E, Pierce K. Brain overgrowth in autism during a critical time in development: Implications for frontal pyramidal neuron and interneuron development and connectivity. International Journal of Developmental Neuroscience 2005; 23: 153-70.

[126] Courchesne E. Brain development in autism: Early overgrowth followed by premature arrest of growth. Mental retardation and Developmental Disabilities. Research Reviews 2004;10 106-11.

[127] Mraz KD, Green J, Dumont-Mathieu T, Makin S, Fein D. Correlates of head circumference growth in infants later diagnosed with autism spectrum disorders. Journal of Child Neurology 2007;22: 700-13.

[128] Dissanayake et al 2006.

[129] Mills JL, Hediger ML, Molloy CA, et al. Elevated levels of growth-related hormones in autism and autism spectrum disorder. Clinical Endocrinology 2007;67: 230-37.

[130] Crespi B, Badcock C. Psychosis and autism as diametrical disorders of the social brain. Behavioral and Brain Sciences 2008;31: 284-320. 
[131] Turner KC, Frost L, Linsenbardt D, McIlroy JR, Müller RA. Atypically diffuse functional connectivity between caudate nuclei and cerebral cortex. Behavioural and Brain Functions 2006;2 34 .

[132] Schumann KC, Hamstra J, Goodlin-Jones BL, Lotspeich LJ, et al. The amygdala is enlarged in children but nor adolescents with autism; the hippocampus is enlarged at all ages. Journal of Neuroscience 2004; 25: 6392-6401.

[133] Egaas B, Courchesne E, Saitoh O. Reduced size of corpus callosum in autism. Archives of Neurology 1995; 52: 794-801.

[134] Belmonte et al 2004.

[135] Just MA, Cherkassky VL, Keller TA. Kana, RK, Minshew N. (Functional and Anatomical Cortical Underconnectivity in autism: Evidence from a fMRI Study of an Executive function task and Corpus Callosum Morphometry. Cerebral Cortex 2007;17: 951-961.

[136] Casanova et al 2007.

[137] Casanova et al 2009.

[138] Smalley et al 2004.

[139] Gardner H. Extraordinary Minds. New York: Basic Books; 1997.

[140] Lykken DT. The mechanism of emergenesis. Genes, Brain \& Behavior 2006;5: 306-310.

[141] Howe MA. Genius Explained. Cambridge: Cambridge University Press; 1999.

[142] Happé F, Frith U. The beautiful otherness of the autistic mind. Introduction. appé F, Frith U. (eds.) Autism and talent. Philosophical transactions of The Royal Society 2009; vol. 364 (1522) p.1345-1350.

[143] Fitzgerald 2004.

[144] Fitzgerald 2005.

[145] Fitzgerald 2005.

[146] Happé \&Vital 2009.

[147] Goldstein S. Schwebach AJ. A comorbidity of pervasive developmental disorder and Attention Deficit Hyperactivity Disorder: results of a retrospective chart review. Journal of Autism \& Developmental Disorders 2004;34 (3): 329-339.

[148] Fitzgerald M. Did Lord Byron have Attention Deficit Hyperactivity Disorder? Journal of Medical Biography 2001;9: 31-33.

[149] Smalley et al 2002.

[150] Smalley et al 2004. 
[151] Andreasen NC. Creativity and mental illness: prevalence rates in writers and their first-degree relatives. American Journal of Psychiatry 1987;144: 1288-1292.

[152] Andreasen NC 1987.

[153] Folley et al 2003.

[154] Sternberg RJ, Lubart TI. Defying the crowd: Cultivating creativity in a culture of conformity. New York: Free Press; 1995.

[155] Grandin 1995.

[156] Lyons V, Fitzgerald M. Asperger Syndrome. A Gift or a Curse? New York: Nova Science Publishers; 2005. 




\section{Edited by Michael Fitzgevald}

The pace of research on Autism Spectrum Disorders (ASD) can only be described as extraordinary as this volume shows. It is extremely difficult for any single professional

to keep abreast of all the developments in this area. This volume gathers together leading researchers and expert clinicians from many different parts of the world to produce this "up-to-the-minute" volume. It gives an in depth view of many areas of research which may be unfamiliar to the clinician and indeed researcher focused on their own area of interest. The volume gives an in depth overview of the field of Autism Spectrum Disorders. 